

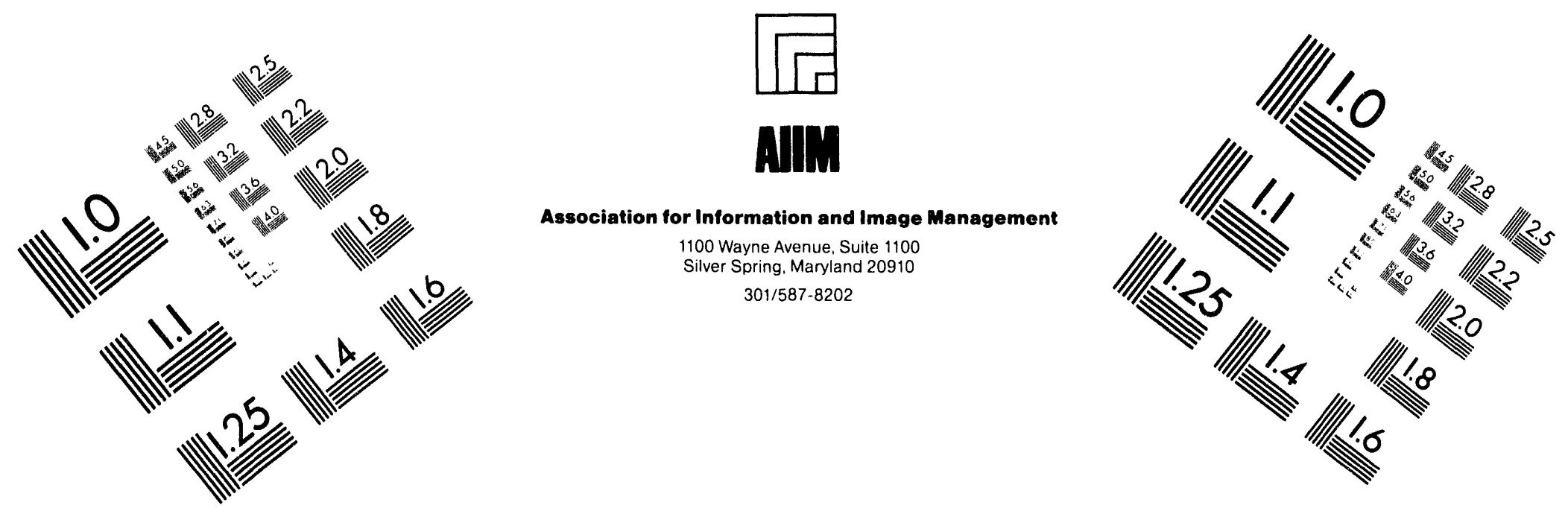

\section{Centimeter}

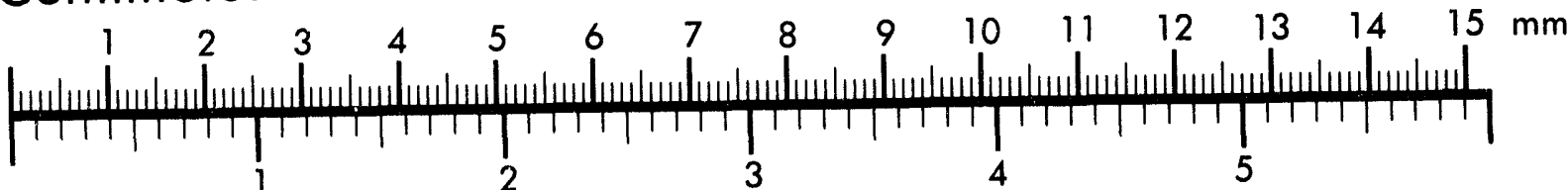
Inches
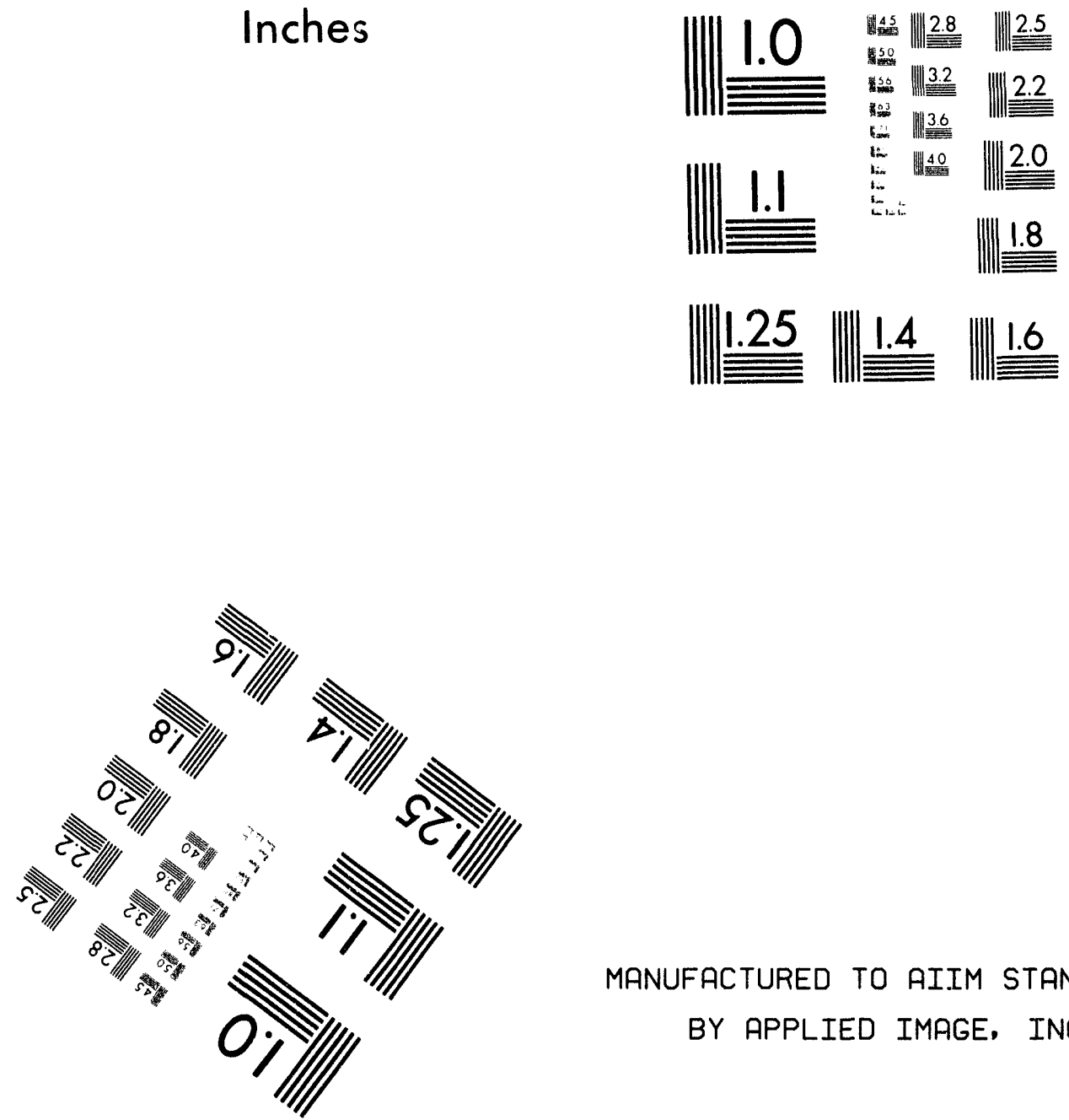

MANUFACTURED TO AIIM STANDARDS

BY APPLIED IMAGE, INC.

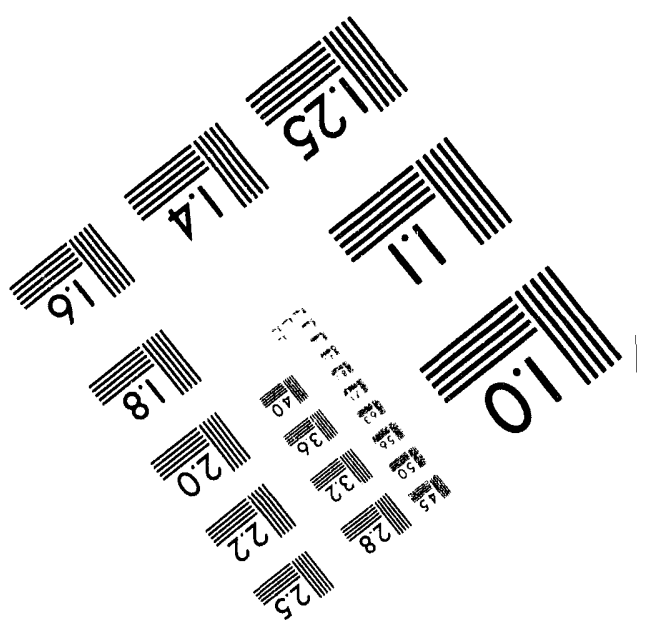



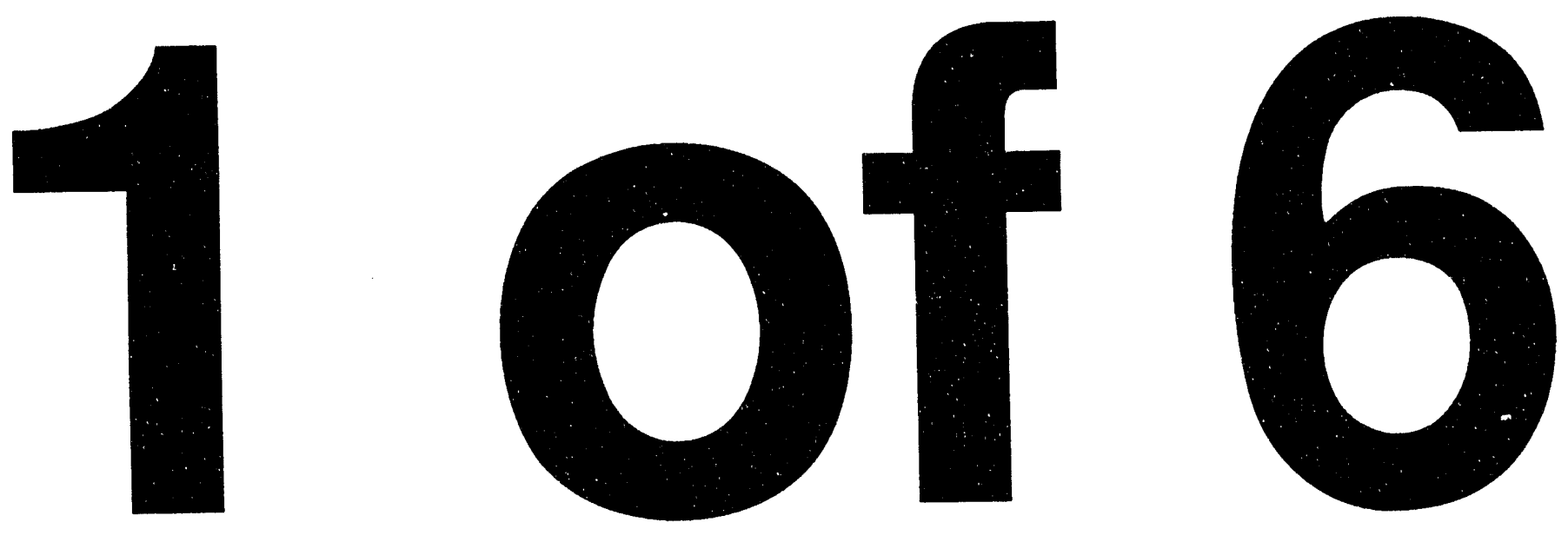


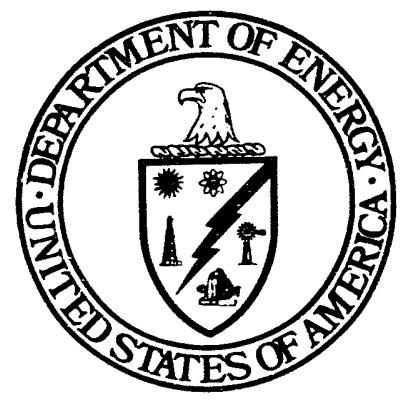

Department of Energy

\title{
Listing of Awardee Names Active Awards
}

\author{
As of July 1, $1994 \quad$ MASTFR
}

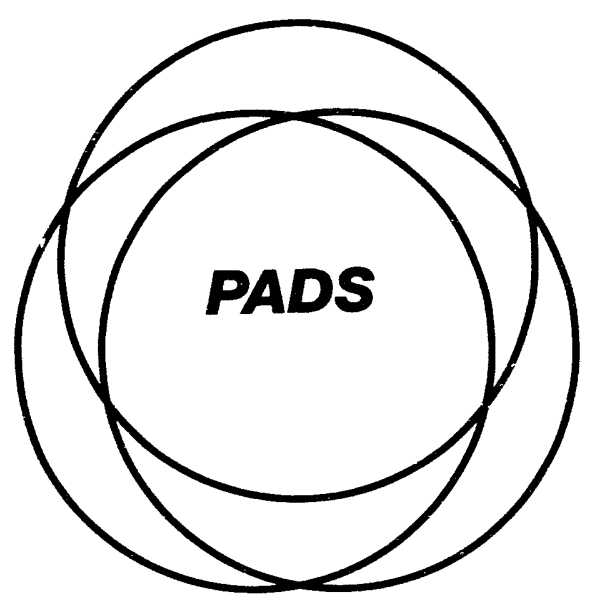

DTPIBUTION OF THIS DOCUMENT IS UNLIMITED

Office of Procurement and Assistance Management

Office of Management Review and Analysis

Washington, DC 20585 


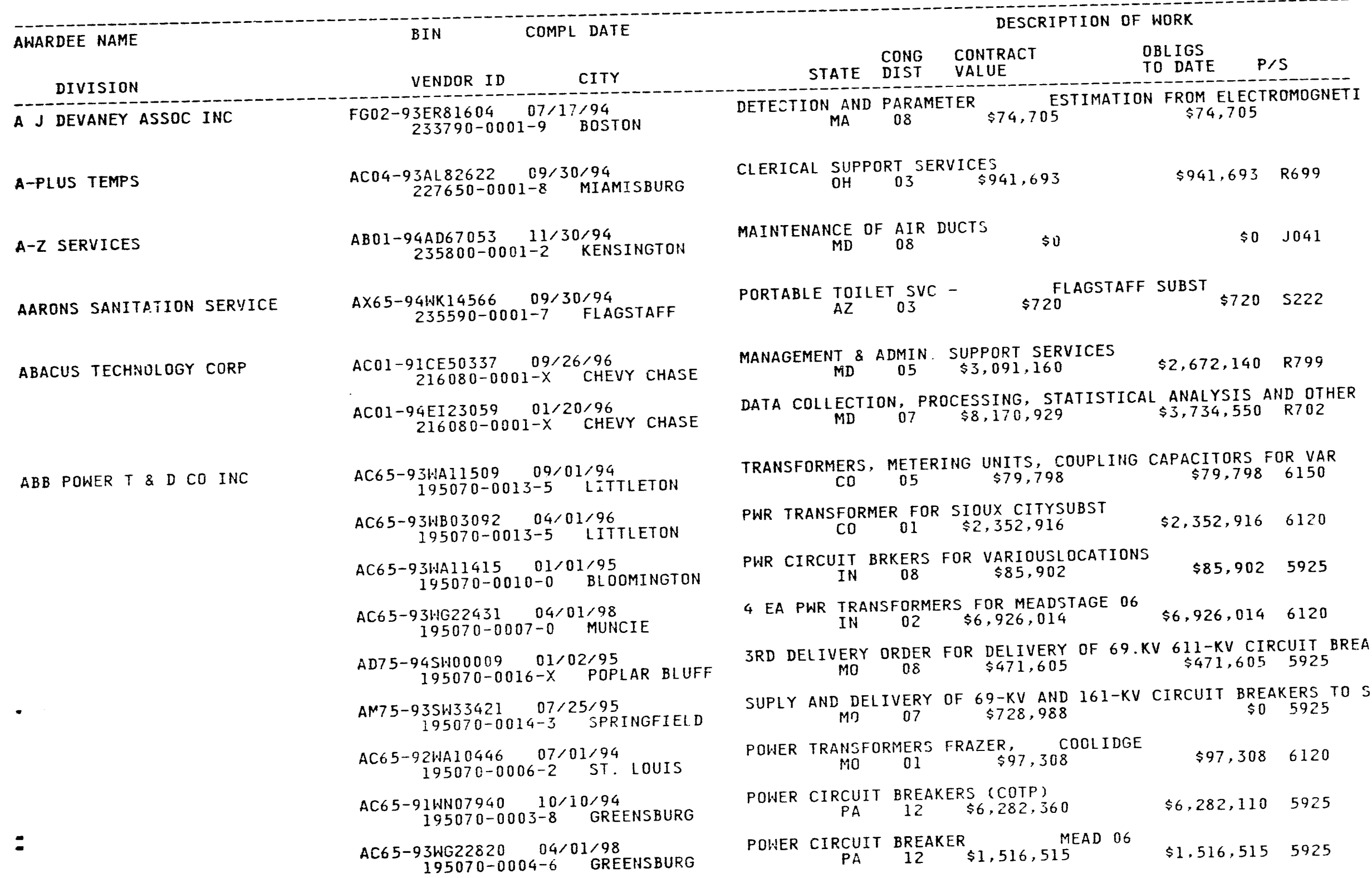




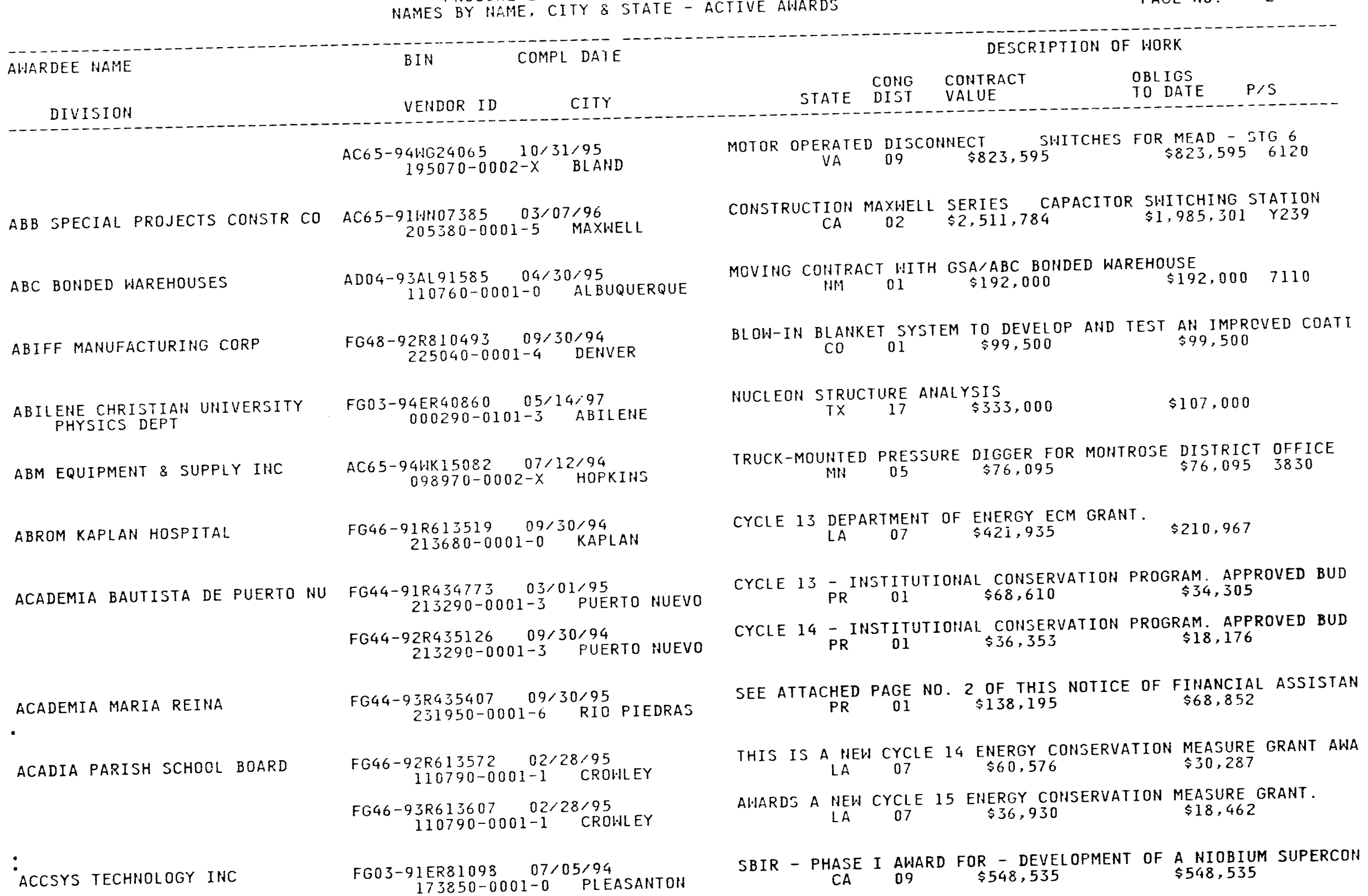




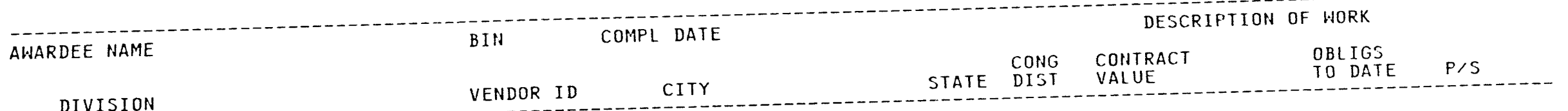

ACEEE

ACORN PUBLIC SCHOOLS

ACRION TECHNOLOGIES INC

ACTECH INC

ACUREX LJRP

EHVIRONMENTAL SYSTEMS ACUREX ENUIRONMENTAL CORP

AD AGENCY INC

ADA TECHNOLOGIES INC

:

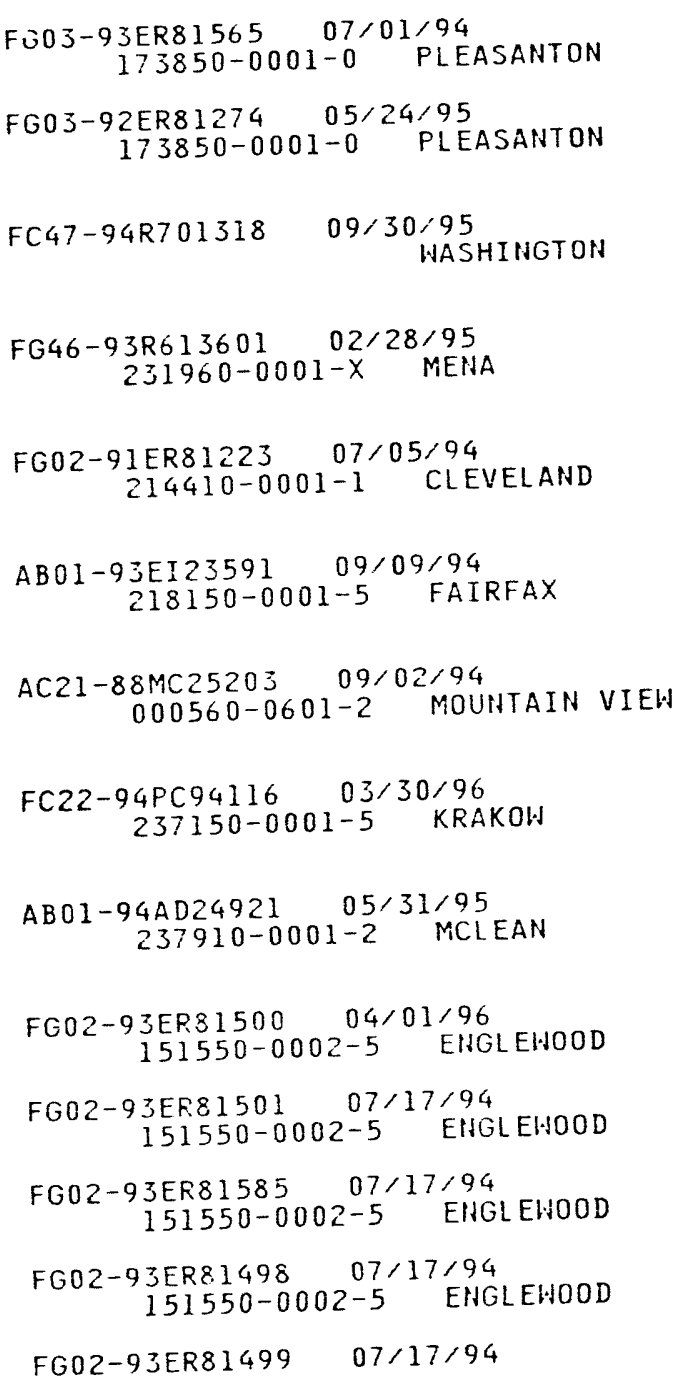
$\begin{array}{ccc}\text { AN EXTENDED INTERDIGITAL NIOBIUM SUPERCONDUCTING LINAC STRUC } \\ \text { CA } \\ 10 & \$ 74,925 & 97,925\end{array}$ SBIR PHASE I TO SHOW THE FEA SIBILITY OF A NEW RESONANT STR SBIR PHASE I; TO SHOW THE FEA SIBILITY OF $\$ 338,753$

See Attachment "A" for other remarks.

AHARDS A NEW CYCLE 15 ENERGY CONSERVATION MEASURE GRANT

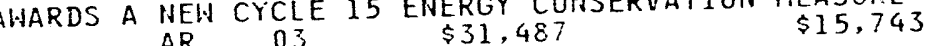

AHDFILL GAS RECOVERY FOR CNG VEHICLES \& FOOD GRADE CARBOI OH $\quad 19 \quad \$ 550,000$

BPA FOR ALL COMPONETS THAT COMPRISE PERSOHAL COMPUTERS \& OTH BPA FOR ALL COMPONETS THAT COMPRISE PERSOHAL COMPUTERS 9050 THERMAL-CHEMICAL DEGRALATION OF CERAMIS FILTER MATERIAL CA $12 \quad \$ 869,493$

CIOHS FROM COAL-FIRED HOME HEATING STORES THR EMISJIOHS REDUCTIOHS FROM $\$ 5,278,667 \quad \$ 1.304,616$

BPA FOR GRAPHIC PRODUCTION ANDVISUAL SERVICES

$$
\checkmark A \quad 08 \quad \$ 0
$$

$\$ 0 \quad T 001$

DEMONSTRATE THAT NOVEL FLUEGAS CONDITIONING PRODUCTS CAN TO DEMONSTRATE THAT NOVEL FLUEGAS CONDITIONHG $\$ 174,963$ RESEARCH OUTLINES A PLAN FOR DEVEL. TECHN. FOR CONVERTING

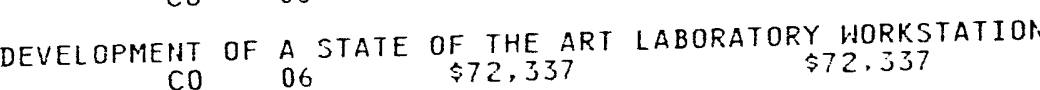
DEVELOP THE FOUNDATION FOR $\underset{\$ 73,967}{\text { EXTEND. THE TECHN. OF PLASMA }}$ A MUTI-PHASE PROGRAM FOR DEVELOPING A TRACE METAL 


COMPL DATE

\section{AWARDEE NAME}

BIN

VENDOR ID CITY

$151550-0002-5$ ENGLEWOOD

ADAMS COUNTY HOSPITAL

ADAMS COUNTY SCHOOL DIST \#12

ADAMS STATE COLLEGE

ADAMS 12 FIVE STAR SCHOOLS

$A D C$ LTD

ADDISON CONSTRUCTION CO

ADELAIDE CHILDRENS HOSPITAL HISTOPATHOLOGY DEPT

ADELPHI TECHNOLOGY INC

\section{ADELPHI UNIVERSITY}

:

ADG INDUSTRIAL

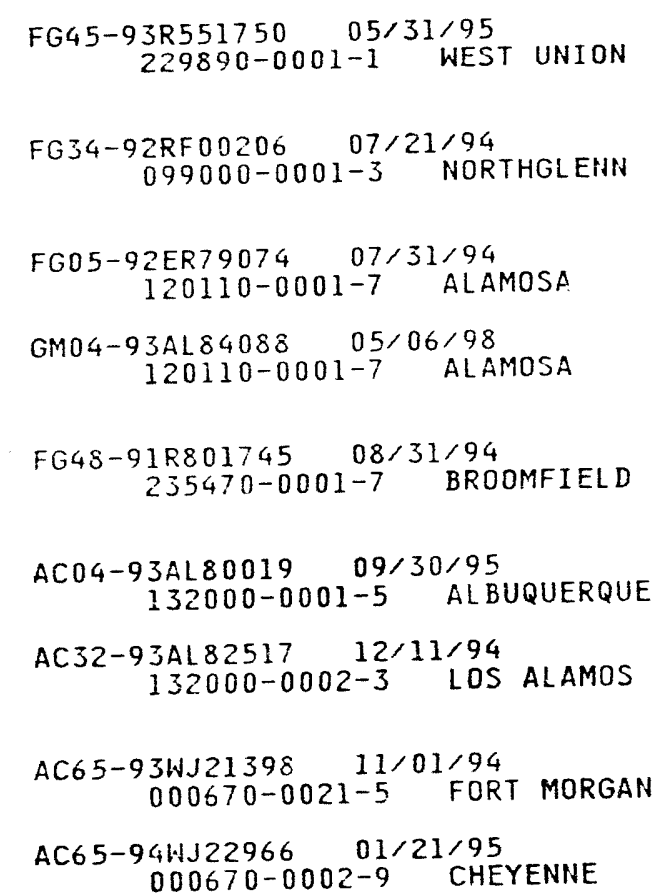

$A B 01-93 A D 63028 \quad 09 / 30 / 94$
PAGE NO. 4
DESCRIPTION OF WORK

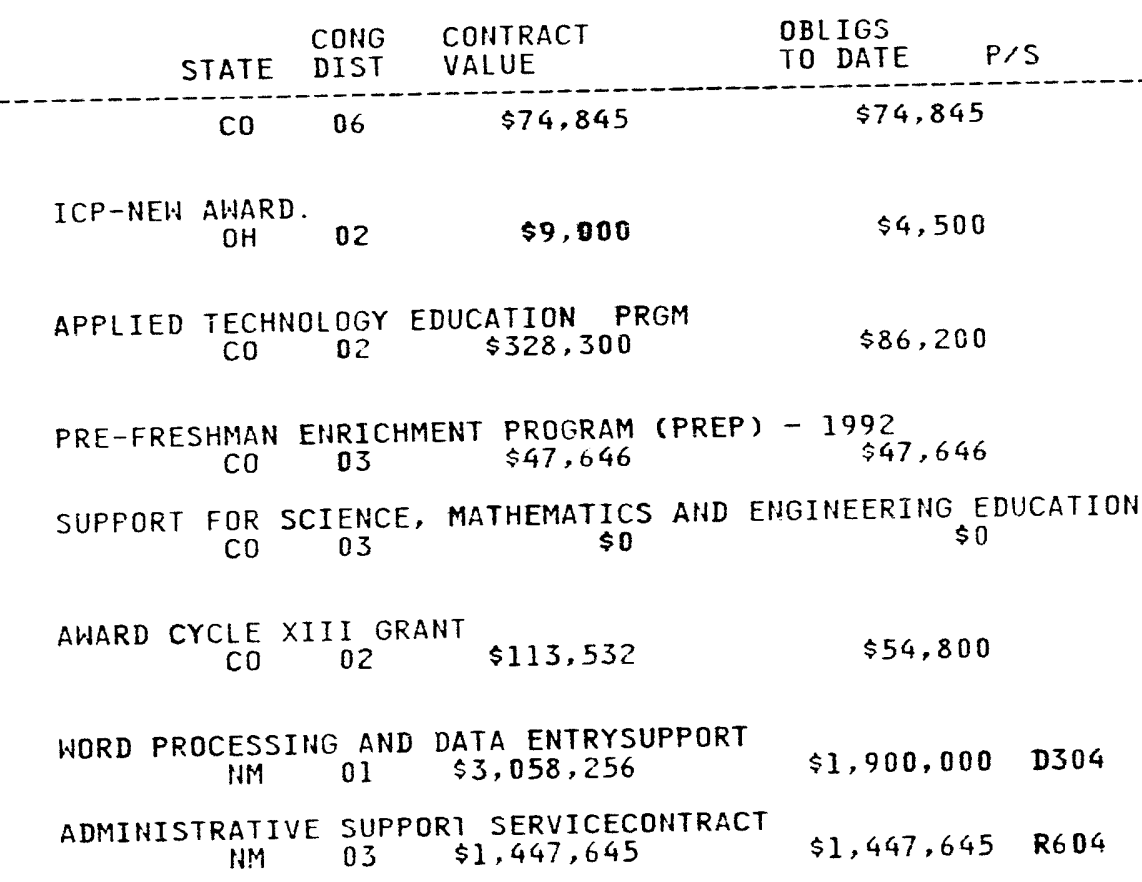

FT MORGAN SUBST DEOM \& FT MORGAN WEST SUBST, ST 01

AKRON SUBSTATION, STAGE $02, \$ 547,714 \quad \$ 547,714 \quad$ Y299

CORRELATION OF PHYSICAL AND GENETIC MAPS OF HUMAN

SBIR - PHASE I ANARD FOR - PARAMETRIC RADIATION AS AN INTENS

CA 11 \$544,489 $\$ 544,489$

DFVELOPMENT DF A PHENOMENOLOGICAL MODEL FOR COAL SLURRY

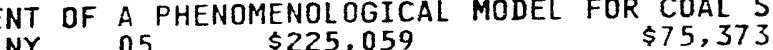

BPA FOR HARDWARE COMMODITIES 


AWARDEE NAME
DIVISION

BIN

\section{COMPL DATE

COMPL DATE

VENDOR ID CITY

$231070-0001-9$ DALE CITY

ADIRONDACK COMMUNITY COLLEGE

FG42-92R24381103/31/95 220880-0001-1 QUEENSBURY

ADMIRAL PEARY AREA VOC TECH

FG43-93R354200 12/31/94
$230880-0001-2$ EBENSBURG

AC85-93AP10030 06/30/95
$235390-0001-1$ JUIEAU

ADRIAN SUPPLY CO

ADVANCE SYSTEMS CONCEPTS ASSOC

ADVANCED COMBUSTION SYSTEMS

ADVANCED COMPUTER SYSTEM INC

ADVANCED CONTROL SYSTEMS

ADVANCED DATA CONCEPTS INC

ADVANCED FUEL RESEARCH INC

ADVANCED REACTOR CORP
FGO3-91ER81101 $12 / 31 / 94$
$214470-0001-3$ REDONDO BEACH

AC21-93MC30327 $09 / 30 / 94$
$234510-0001-6 \quad$ BELLINGHAM

$\begin{array}{cc}A C O 1-91 E I 21938 & 07 / 16 / 94 \\ 210420-0001-8 \quad \text { FAIRFAX }\end{array}$

AC75-925W29358 03/20/96 225110-0001-6 NORCROSS

AC04-93AL74199 07/20/96 $160660-0003-9$ ALBUQUERQUE

FG05-92ER8 $133905 / 25 / 95$
$000780-0002-5$ EAST HARTFORD

AC21-93MC 30040 09/13/96 000780-0002-5 EAST HARTFORD

FC02-93CH10565 03/31/97 000780-0002-5 EAST HARTFORD $000780-0001-7$ HARTFORD

FC02-92NE34267 09/30/96
FG05-91ER81151 07/05/95
TITLE III OF THE NATIONAL ENERGY CONSERVATION POLICY ACT NY $24 \quad \$ 444,392 \quad \$ 222,195$

TITLE III OF THE NATIONAL ENERGY CONSERVATION POLICY ACT TITLE III OF THE NATIONAL ENERGY CONSERVATION $\$ 55,821$

FURHISH 25 MUA TRANSFORMER ANDOPTIONS TO EKLUTHA POHERPLANT $\begin{array}{llll}\text { FURHISH } 25 \text { MUA TRANSFORMER ANDOPTIONS TO } & \$ 1,042,246 & 6120\end{array}$

SBIR - PHASE I AWARD FOR - ACCIDENT MANAGEMENT ADVISOR SYSTE CA 27 \$545.013 $\$ 545,013$

FUME GAS INCINERATION SYSTEM

WA $02 \quad \$ 119,746 \quad \$ 119,746 \quad 4540$

PROVIDE SUPPORT SVS FOR DATA \&MODELING ANALYSIS AND EVALUATI VA 10 \$1, 606,508 $\$ 987.786$ D302

FURNISH \& INSTALL A SUPERVISORY CONTROL AND DATA ACQUISITIOH $G A \quad 10 A \$ 5,401,840 \quad \$ 5,401,840 \quad 5975$

PROVIDE ADMIN AND TECH SAFEGUARDS AND SECURITY (S\&S) SUPPORT NM $\$ 17,977,690 \quad \$ 6,000,000 \quad R 408$

A RUGGED, STABLE FT-IR INTERFEROMETER FOR PROCESS MOHITORING $\begin{array}{lll}\text { STABLE FT-IR TNTERFEROMETER FOR } & \$ 338,058\end{array}$

RESEARCH TITLED "FOSSIL FUEL CONVERSION - MODELING AND

IN-SITE FT-IR MONITORING OF A BLACK LIQUOR BOILER

IN-SITU FT-IR DIAGHOSTICS FOR COAL LIQUEFACTION PROCESSES

CT $01 \quad \$ 549,629$

FIRST OF A KIND ENGINEERING TOSUPPORT COMMERICAL 


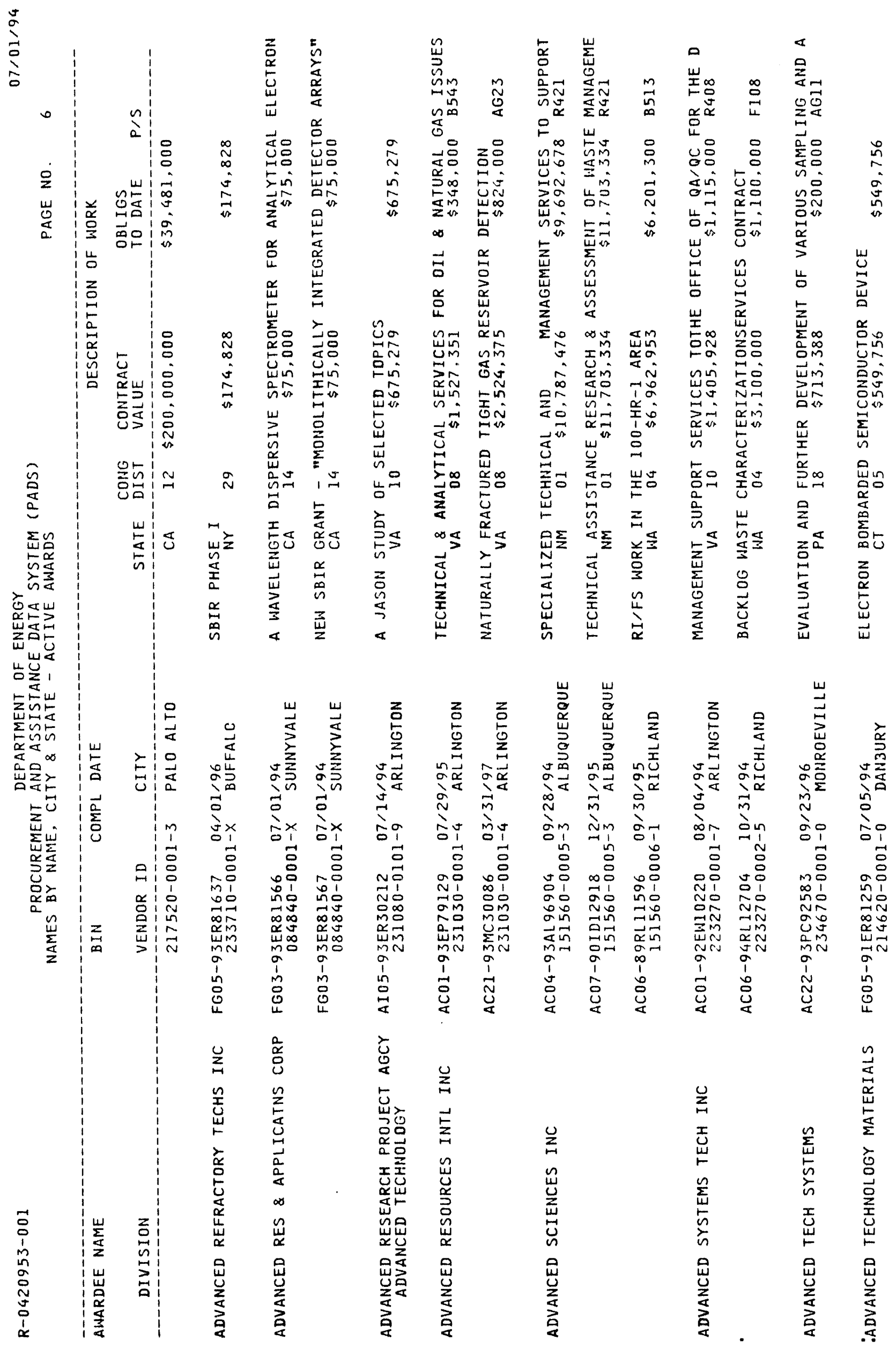




AWARDEE NAME
DIVISION

AEA O'DONNELL

AECL TECHNOLOGIES

AERODYNE RESEARCH INC

AEROJET-GENERAL CORP PROPULSION DIVISION

\section{AEROSPACE CORP}

EASTERN TECHNICAL DIV

\section{AEROTEST OPERATIONS INC}

AES CORP

AFEX CORP

AFFYMAX RESEARCH INSTITUTE

:

AGF DIRECT GAS SALES INC
COMPL DATE

DESCRIPTION OF WORK

BIN

VENDOR ID CITY

$-F G 05-92 E R 81327-02 / 17 / 95---1$
$214620-0001-0$ DANBURY

FG05-94ER81655 07/17/94
$214620-0001-0$ DANBURY

214620-0001-0 DANBUR

FG05-93ER81596 07/01/94
$214620-0001-0$ DANBURY

AC01-90FE61663 $07 / 31 / 94$
$228450-0001-4$ GREENBELT

$\begin{array}{cc}A C O 3-945 F 20218 & 08 / 31 / 94 \\ 237770-0001-9 & \text { ROCKVILLE }\end{array}$

FG02-93ER81522 $07 / 17 / 94$
$000900-0002-1 \quad$ BILLERICA

AR21-93MC30361 $11 / 30 / 94$
$127990-0203-X \quad$ SACRAMENTO

FG03-87ER $13812 \quad 02 / 29 / 96$
$000930-0302-1 \mathrm{EL}$ SEGUNDO

FG03-86ER60391001/09/95 000930-0004-9 LOS ANGELES

CRO1-83NE44484 03/15/30
$000950-0001-1$ SAN RAMON

ACO1-92PE79101 $09 / 29 / 94$
$225060-0001-1 \quad$ ARLINGTON

FG01-90CE15491 03/27/95 203790-0001-6 BRENHAM

FG03-92ER81275 07/21/94 220790-0001-2 PALO ALTO

$A D 21-93 M C 3031203 / 31 / 95$

PHASE 1, SBIR AWARD, MICRO PYROVIDICON
CONG CONTRACT

NEW SBIR PHASE I REAL-TIME X-RAY

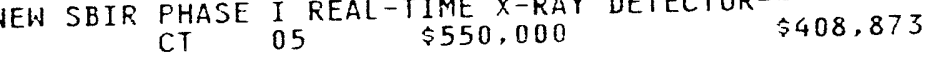

OBLIGS

DIAMOND BASED PHOTOMULTIPLIERS

$\$ 75,000$

$\begin{array}{lll} & \\ C T & 05 & \$ 75,000\end{array}$

$\$ 75,000$

SUPPORT FOSSIL ENERGY IN THE TECHNOLOGHY WORK AREA OF FLUE G SUPPORT FOSJIL ENERGY $\$ 1.237,331$ \$ $1.150,705$ R799

BASIC AHARD OF PLUTONIUM CONSUMPTIOH R\&D STUDY

$\begin{array}{llll}M D & 08 & \$ 991,573 & \$ 961,573 \quad \text { AG52 }\end{array}$

AN IN-SITU TESTER FOR SEAL $\begin{array}{rll}\$ 74,954 & \text { INTERGRITY OF MULTIPLE PANE } \\ \text { MA } & 05 & \$ 74,954\end{array}$

EM PLATELET-COOLED PLASMA ARC TORCH

$$
\begin{array}{ccc}
\text { ET-COOLED PLASMA ARC TORCH } & \$ 568,112 \text { AH92 } \\
\text { CA } 03 & \$ 568,112 & \$ 568,12
\end{array}
$$

A SHOCK TUBE STUDY OF THE REACTION OF HYDROXYL RADICALS WITH $\begin{array}{llll}C A & 27 & \$ 648,000 & \$ 552,638\end{array}$

MOLECULAR DETECTION BY MULTIPHOTON IONIZATION DISSOCIATION

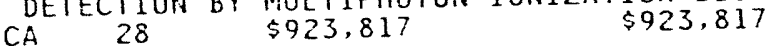

TO AHARD CONTRACT FOR DISPOSALOF SPENT NUCLEAR FUEL AND/OR

TECHNICAL SUPPORT SERVICES FORTHE OFFICE OF POLICY, PLANHIN VA $10 \quad \$ 2,072,490 \quad \$ 1,195,500 \quad$ R799

DETERMINE OPTIMUM CONDITIONS FOR THE AFEX PROCESS FOR THANOL TX 14 S $\$ 86,254$ $\$ 86,254$

SBIR PHASE I : TO DEVELOP SPAT IALLY DEFINED OLIGONUCLEOTIDE CA ${ }_{13} \$ 413,708 \quad \$ 413,708$

SUPPLY NATURAL GAS 


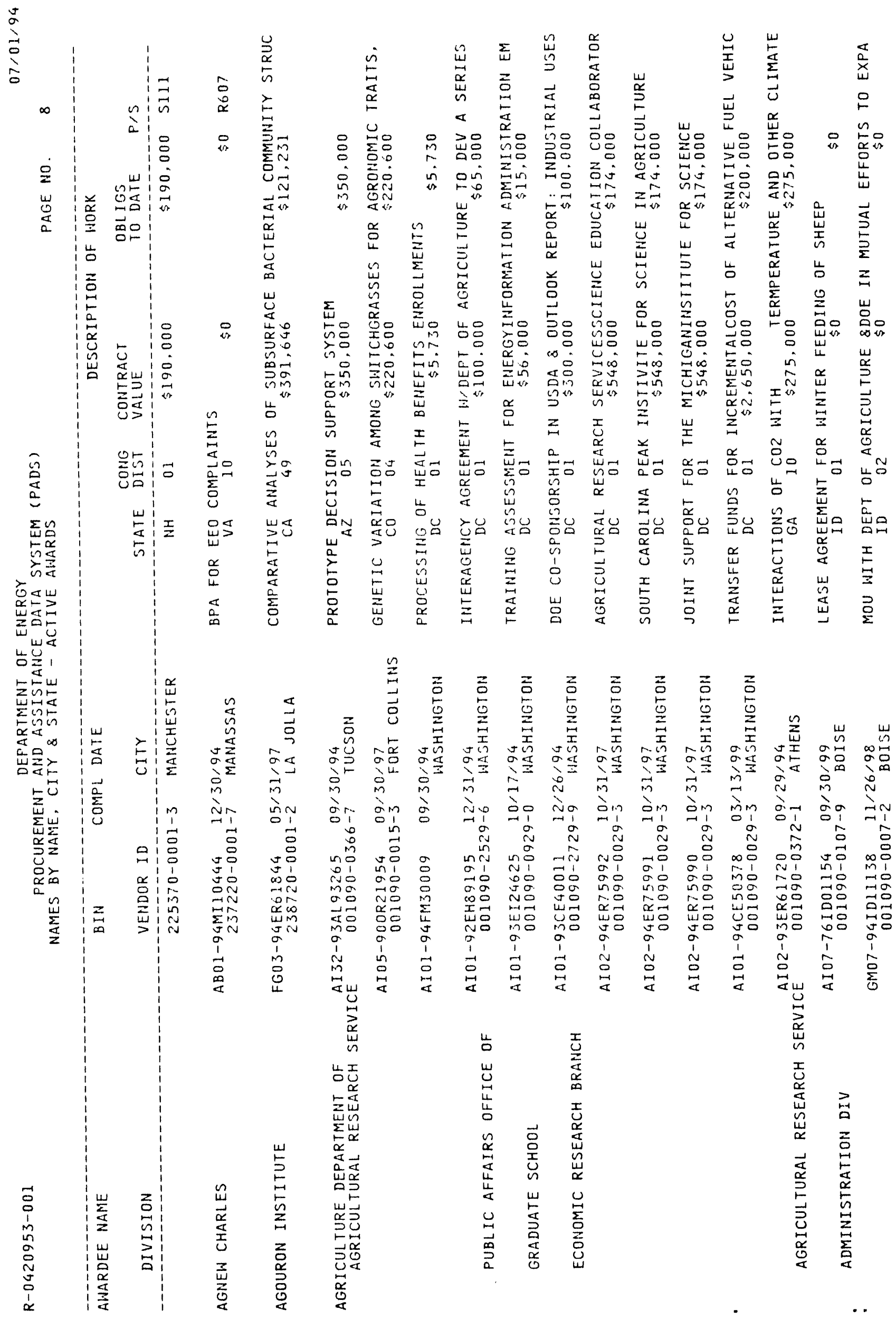




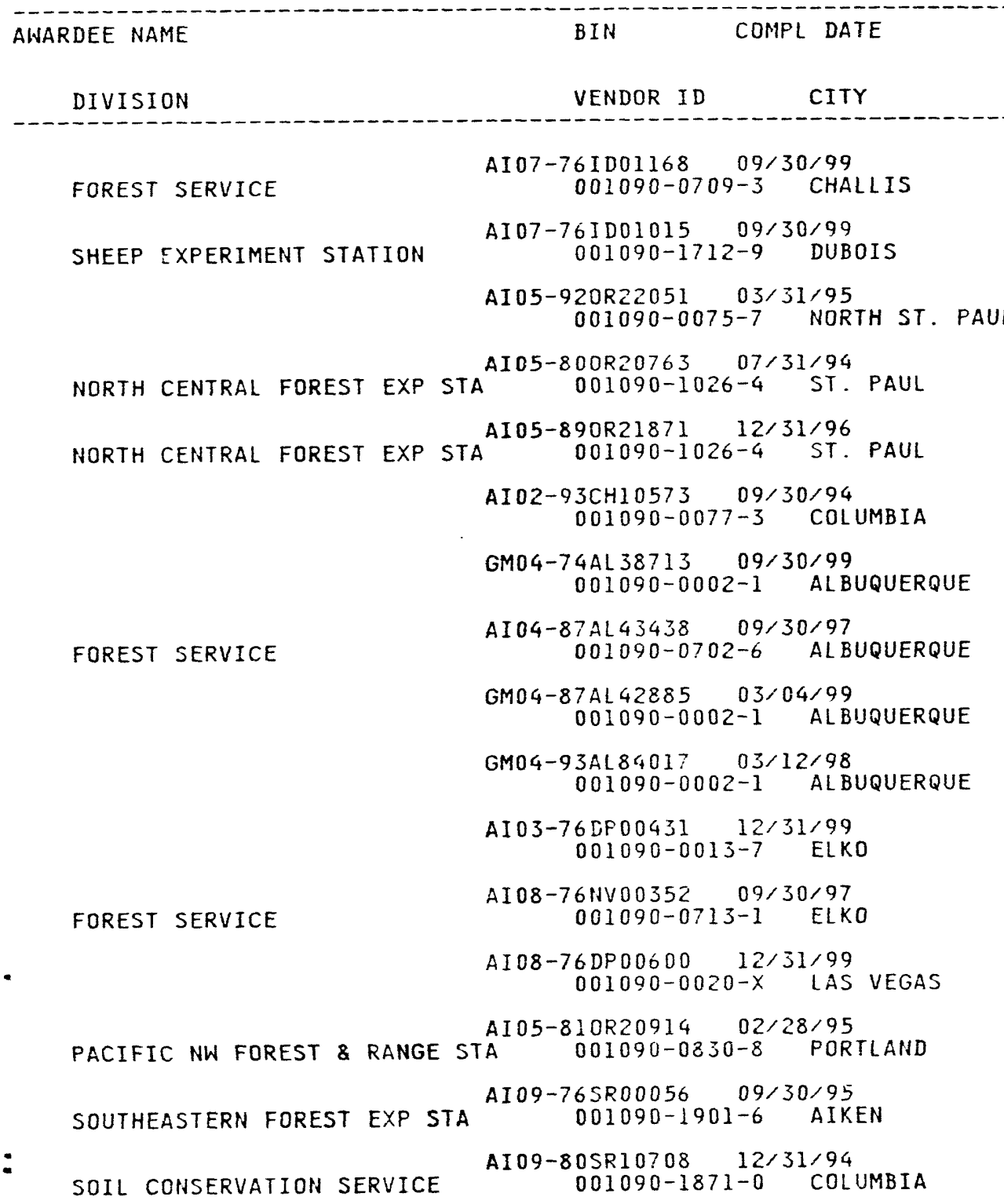

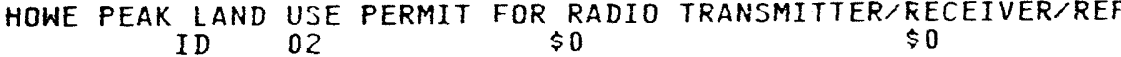
MONITORING STATION - SHEEP EXPERIMENT STATION DUBOIS IDAHO
ID
02 $\begin{aligned} & \text { BIRD AND MAMMAL USAGE OF HYBIRD POPLAR PLANTATION } \\ & \text { MN } \$ 250,000\end{aligned}$ ESTABLISHMENT OF POPULUS ENERGY FARMS SHORT ROTATION WOODY CROP TRAILS FOR ENERGY PRODUCTION IN NO MN $04 \quad \$ 602,390 \quad \$ 602,390$ ECOLDGICAL STUDY \& WILDLIFE MANAGMENT PLAN AGREEMENT AND UNDERSTANDING $\underset{N M}{\text { WHICH PROVIDES BASIS UNDER }} \underset{\$ 0}{\$ 0}$ STAFF SUPPORT AND OPERATING EXPENSES FOR AL'S REAL PROPERTY NM 01 \$275,000 $\$ 275,000$ DOA MAKES AVAILABLE TO THE DOETHE USE OF NATIONAL FOREST THIS AGREEMENT SETS FORTH THE GEN POLICY FOR THE USE OF CONSTRUCT ACCESS ROAD ONHATIONAL FOREST LAND
$\$ 18,206$ PERMIT TO INSTALL, OPERATE, AND MAINTAIN RADIOELECTRONIC E UTILIZE ERDA EQUIPMENT AIND CONTRACTOR PERSONNEL TO FIGHT W INCREASING THE BIOMASS PRODUCTION OF ALDER PLANTATIONS IN TH OR
ADMINISTER FOREST MANAGEMENT AT SRP $S C \quad 03 \quad \$ 49,906,228$ $\$ 49,906,228$ FOREST RESDURCE MANAGEMENT PROGRAM (SOIL SURVEY) 
PAGE NO.

AGRICULTURAL RESEARCH SERVICE OOTO90-0332-2 BUSHLAND

DEVELOPMENT AND TESTING FO WIND

$$
\text { TX } 13 \text { TST } \$ 90,300
$$
AGRICULTURAL RESEARCH SERVICE AI36-93CH10576 07/31/98 COLLE STATION

RESEARCH ON WIND/NYBRID ELECTRICAL GENERATION FOR

FDREST PRODUCTS LAB AI 02-89ER14068 D4/30/95 $001090-2338-2$ MADISON

FOREST SERVICE

AGUIRRE ENGINEERS INC AI05-940R22197 03/31/99 001090-0779-4 RHINELANDER 08 \$285,000

OBLIGS P/S AND WILDLIF

RAMAN MICROPROBE INVESTIGATIONCF MOLECULAR STRUCTURE AND EVALUATION DF GENETIC SELECTIDN CRITERIA FOR PDPULUS CLONES

SUPPORT SERVICES

CO $06 \quad$ \$O

$\$ 0 \quad R 408$

PB/TECH SUPPORT SERVICES

CO $02 \quad \$ 849,488 \quad \$ 89,488 \quad R 408$

WORD PROCESSING \& DATA ENTRY SUPPORT SERVICE

ACO3-93SF19692 06/14/95
$167430-0001-9$ SAN MATEO

FC07-941D13281 02/15/96
$236800-0001-4$ DEER PARK

TEL TECHNOL OGY PARTNERSHIP UNDER TRP , $1,165,880$

$\$ 270,574 \quad R 707$

DEFENSE DUL USE CRIT $\$ 2,410,123 \quad \$ 851,263$

MATERIALS COMPATIBILITY \& LUBRICANTS RESEARCH ON CFC-REFRIGE

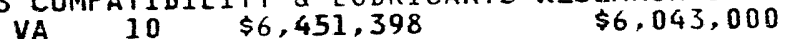

AIR CONDITIONING \& REFRIG TECH FG02-91CE23810 $09 / 30 / 95$ INGTON

AIR FLITE INC AC75-92SW31336 10/31/94
$163990-0002-5$ SHAWNEE

AERIAL TRANSMISSION IINE INSPECTION SERVICES
$\$ 72,000$
$\$ 72,000 \quad$ V211

USE OF AREA AT SHOS

AI08-76NV00489 03/31/96 DEE BASE CA 18 $\$ 0$ TATION AND C

AIR FORCE DEPARTMENT OF FLIGHT TEST CENTER

VANDENBERG AIR FORCE BASE AI32-94AL $96953 \quad 09 / 30 / 94$
DOL $230-5655-1 \quad$ LOMPOC

TECHNICAL SUPPORT FOR LIDAR MEASUREMENTS AI 32-93AL $93288 \quad 09 / 30 / 94$
$001230-5655-1 \quad$ LOMPOC

$\begin{array}{ccc}\text { MODIFY GROUP EQUIPMENT AT THE ANDENBERG TRACKING STA } \\ \text { CA } \\ \$ 20,000 & \$ 20,000\end{array}$ GMO4-84AL26919 09/07/99 MK 21 REENTRY VEHICLE, WPD, J.H. GRAYSON, 846-2222. $001230-2423-4$ SAN BERNARDIND CA 35
BALLISTIC MISSILE OFFICE 


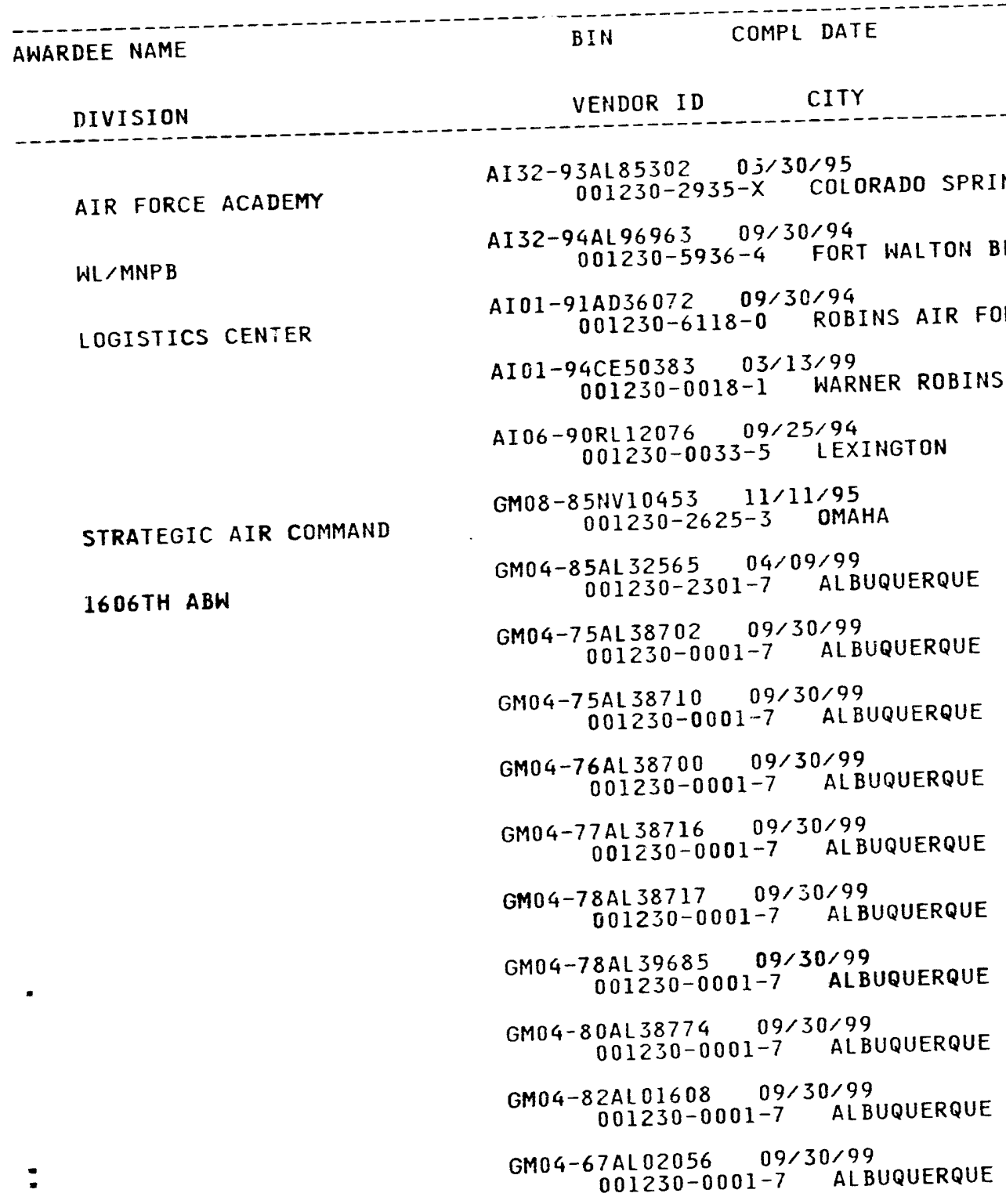

DESCRIPTION OF HORK

STATE DIST CONTRACT VALUE

OBLIGS

$P / S$ $\begin{array}{cccc}\text { SUPPORT OF DEVELOPMENT OF INSTRUMENTATION BASED ON } \\ \text { CO } 05 & \$ 6,000 & \$ 6,000\end{array}$ SOFTWARE RESEARCH SUPPORT $\quad \$ 75,000$ OH-SITE HARDWARE MAINTENANCE OF THE CSP SYS IA FOR ON-GOING, ON-SITE HARDWARE MAINTENANCE $\$ 355,000$ TRANSFER OF FUNDS FOR INCREMENTAL COST OF ALTERN GA 03 \$3,391,244 $\$ 351,373$ DEVELOFMENT OF HAHKS SOFTWARE IN SUPPORT OF MA $07 \quad \$ 395,000 \quad \$ 395,000$ AIR SAMPLING SUPPORT FOR SAFEGUARD $C$.

NE 02 \$0 \$O SERIOUS ACCIDENT/INCIDENTS ON KAFB - OPA D. JACKSON DEVELOP PRODUCE AHD PROCURE PARACHUTES FOR WEAPONS. AND DEVELDP, PRODUCE AHD PROCURE PARACHUTES FOR WEAPONS $\begin{gathered}\$ 0 \\ \$ 0\end{gathered}$ SUPPORT PROVIDED BY TACTICAL FIGHTER HEAPONS CENTER RANGE NM OI 01 \$O ESTABLISHMENT THAT ALO HAS EXCLUSIVE USE OF AND MAINTNENACE

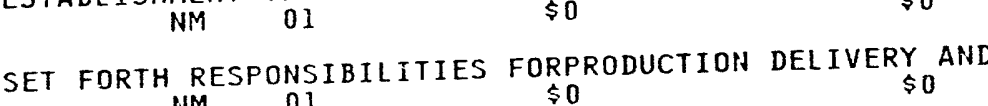
AGREEMENT WHERE KAFB MAY OBTAIN ASSISTANCE IN THE FORM OF NHA 01 \$O \$O AGREEMENT BETWEEN KAFB AND DOETO JOINTLY REPLACE THE $\begin{array}{clll}\text { NM } & 01 & \$ 0 & \\ \text { PROVIDE MUTUAL ASSISTANCE IN } & \begin{array}{l}\$ H \\ \text { NM }\end{array} & 01\end{array}$ FORM OF TECHNICAL AID IN ESTABLISH BASIS AND RESPONSIBILITIES FOR NEW MATERIAL STOCKNM OI \$O

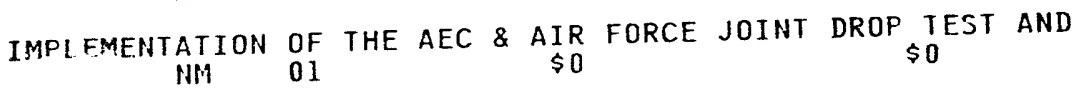




\section{AWARDEE NAME}

BIN
DESCRIPTION OF HORK

CONG CONTRACT STATE DIST VALUE OBLIGS TATE DIST VALUE
NM ARRANGEMENTS BY WHICH AEC THROUGH SANDIA
NM 01 VENDOR ID CITY CITY $\$ 0$ OOL $230-0001-7$ ALBUQUERQUE DEVELOPMENT \& INITIAL PRODUCTION OF AN ARMINE AND FUZINE GM04-82AL20530 09/30/99 001230-0001-7 ALBUQUERQUE WEAPONS LAB GM04-84AL30536 09/30/99 001230-2010-7 ALBUQUERQUE GM04-87AL436こI 04/23/99 001230-0001-7 ALBUQUERQUE GM04-76AL38666 01/01/99 001230-0001-7 AL BUQUERQUE GM04-76AL38690 01/01/99 001230-0001-7 ALBUQUERQUE GM04-76AL38698 01/01/99 001230-0001-7 ALBUQUERQUE GM04-76AL38686 01/01/99 001230-0001-7 ALBUQUERQUE

FTWC

WEAPONS LAB
GMO4-83AL22894 11/19/99 GM04-87AL43564 03/09/99 001230-0001-7 ALBUQUERQUE GMO4-82AL53612 03/30/99 001230-2010-7 ALBUQUERQUE GM04-87AL43781 12/10/99 001230-0001-7 ALBUQUERQUE GMO4-77AL 02172 10/01/99 [.01230-0001-7 ALBUQUERQUE GM04-88AL $53637 \quad 10 / 05 / 99$ 001230-0001-7 ALBUQUERQUE AI04-90AL64049 09/30/95 001230-0001-7 ALBUQUERQUE GMO4-89AL58775 08/30/94 001230-0001-7 ALBUQUERQUE AI04-90AL60600 04/01/96 001230-0001-7 AL BUQUERQUE

$$
\text { HM } 01 \text { \$O }
$$$$
\text { ร० }
$$

DELINEATES RESPONSIBILITIES OFTHE PARTIES FOR DESIGN, DEVEL NM 01

$$
\$ 0
$$

DESIGN DEVELOP \& TEST FOR BMO THE SECURE CHYPTOGRAPHIC UNIT NM 01 \$O

DEFINES RESPONSIBILITIES CF AEC SUPPORT TO AFLC STOCKPILE

$$
\mathrm{NM} 01
$$$$
\$ 0
$$

ESTABLISH RESPONSIBILITIES FORTHE PRODUCTION OF WH72 NM 01

$$
\$ 0
$$

$$
\text { \$0 }
$$

AFSC ASSISTANCE TO ALO FOR AECRESEARCH, DEVELOPMENT AND ESTABLISH $\underset{N M}{A} \underset{01}{A G R E E M E N T}$ FOR COMBINING \& ANALIZING DATA DEVELOPED$$
\text { ร0 }
$$

IMPLEMENTS THE USE PERMIT NO DACA09-4-79-348 COVERING THE

$$
\text { NM } 01
$$

SET FORTH THE DIVISION OF RESPONSIBILITIES AMONG $\underset{\$ 0}{\$ 0}$, AIR

$$
\text { NM } 01
$$

DEFINE JOINT RESPONSIBILITIES AND UNDERSTANDINGS OF THE

IN DEVELOPMENT PREPRODUCTION, PPJDUCTION DELIVERY REPAIR AND

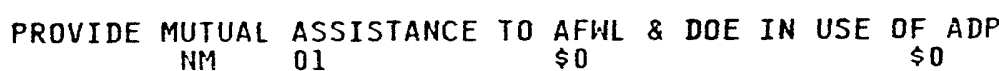

OPERATING INSTRUCTION SNLA SECURITY/1608TH SECURITY POLICE

SOUTH VALLEY SUFERFUND AGREEMENT

$\$ 5,343,426$

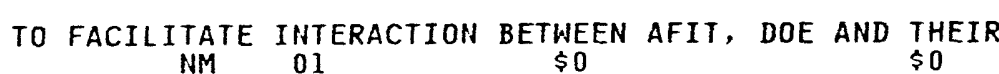

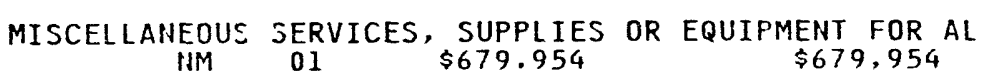




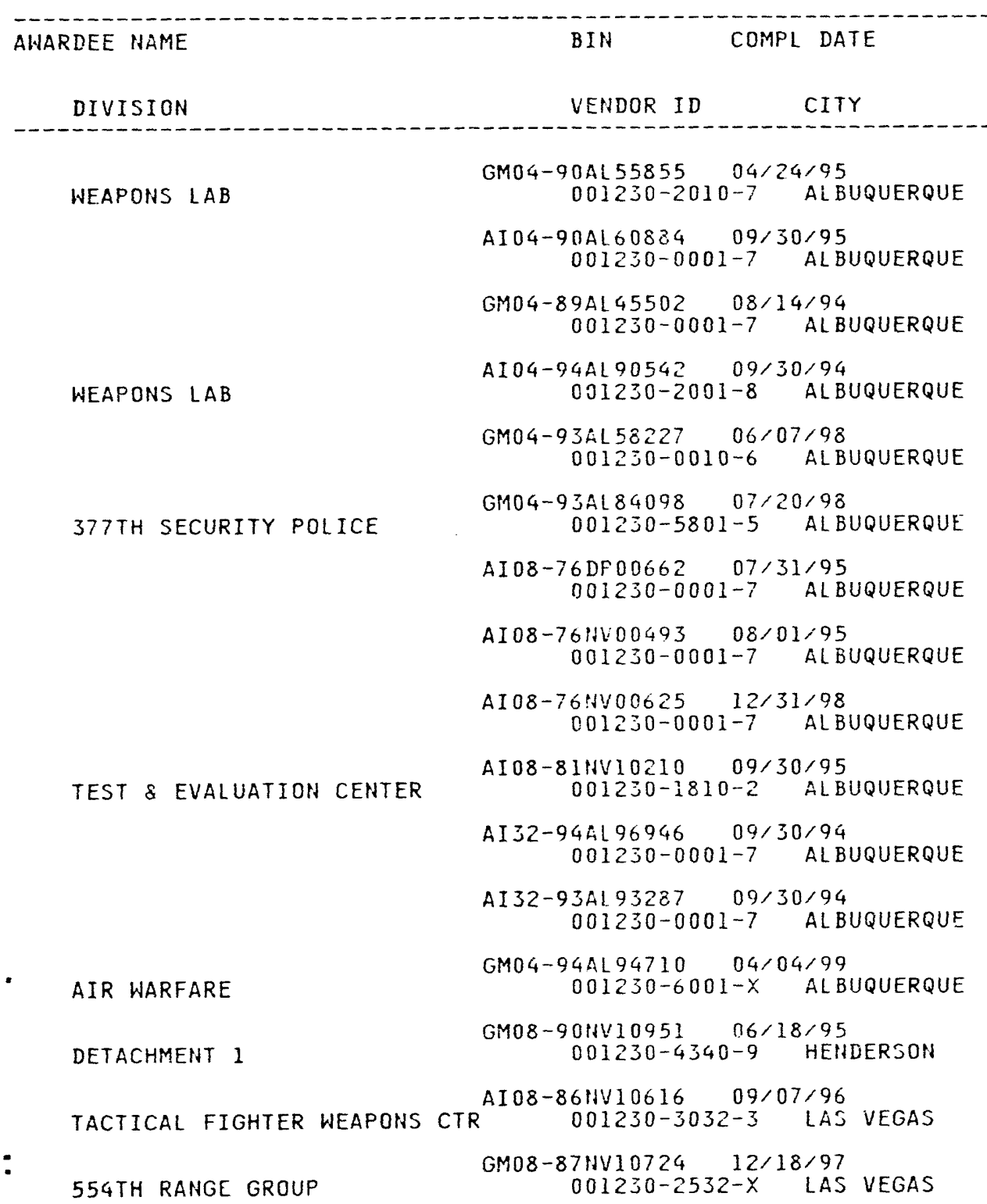

BASE SUPPORT AGREEMENT BETHEENDOE/AL AND THE AIR FORCE
HM
$\begin{gathered}\text { MI } \\ \$ 0\end{gathered}$

PROVIDE UTILITIES FOR AL COMPLEX NM 01 \$4,242,000

$\$ 4,242,000$

DEV PROD \& DEPLOY OF THE WHB $\$$ AND ASSOCIATED JOINT TELECOMMUNICATION SERVICES FORAL AND ITS CONTRACTORS
IIM OOI
$\$ 102,399$ MUTUAL ASSISTANCE \& EMERGENCY PUBLIC AFFAIRS

THIS AGREEMENT SETS FORTH THE GEHERAL POLICY FOR THE

HOST TENANT AGREEMENT TO PROVIDE LOGISTIC SUPPORT TO EG\&G

$$
\text { IM } 01 \text { \$1,041,000 \$1.041,000 }
$$

UNDERSTANDIHG FOR OVERHIGHT PARKING OF TRAHSIENT DOE SECUR
NM $O I$

MOHITORING STONE!HELL FLATS AREA AT TONEOPOAH TEST RANGE-P HM OI $O 1$ STATS AREA AT TONI

FACILITATE THE USE OF RESOURCES OF THE DOE REMOTE SENSING

COATING OF OPTICAL RECEIVERS

$$
\begin{array}{lll}
\text { OF OPTICAL RECEIVERS } & \\
\text { HM } 01 & \$ 10,000 & \$ 10,000
\end{array}
$$

LABOR AND OVERHEAD TO PROVIDE VISIBLE IMAGERY DATA ON THE IM 01 \$4,000 $\$ 4,000$ TO FACILITATE COOPERATIVE EFFORTS FOR THE MUTUAL EXCHANGE OF MOU TO DELIMEATE RESPONSIBILITY BETHEEN DOE/NV \& USAF DET 1

OPERATION AND MAIMTENANCE SUPPORT SERVICES TO BE PROVIDED TO NV 01 TENANCE SUPPORT SERVICES TO BE $\$ 0$

PERMIT FOR CONTRACTOR PERSONNEL TO USE ROAD FROM GATE 700 , N NV 01 \$O TO USE ROAD FROM $\$ 0$ 
ANARDE NAME

AWARDEE NAME

BIN
COMPL DATE

DESCRIPTI $N$ N OF WORK

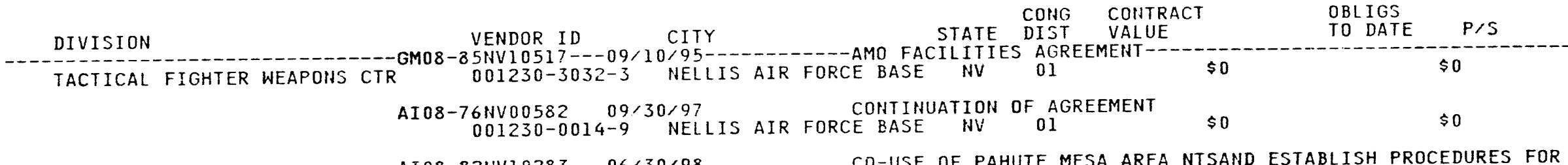

TACTICAL FIGHTER WEAPONS CTR AI08-821NV10283 $006 / 30 / 98$ CO-USE OF PAHUTE MESA AREA NTSAND ESTABLISH PROCEDUR

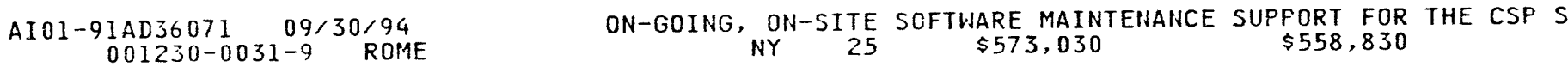

ON-GOING, ON-SITE SCFTHARE MAINTEHANCE SUPFORT FOR THE CSP S NY 25 ST $2573,030 \quad \$ 558,830$

$\begin{array}{llll}0 & 01230-0057-2 & \text { FAIRCHILD AIR FORCE BA* WA } 05 & \$ 0\end{array}$ AIR PRODUCTS \& CHEMICALS INC FR22-93PC90579 $12 / 31 / 20$

FCO $4-93 A L 94461 \quad 05 / 31 / 97$
$001260-0001-8$ ALLENTOWN AC22-91PC90018 09/30/94 001260-0001-8 ALLENTOHN

AIRBORME EXPRESS

ABO1-93AD62484 09/30/94 195720-0002-0 LANDOVER

ABO1-93AD63002 07/01/94 195720-0001-2 CHANTILLY

AIRPOL INC

FC22-91PC90542 08/31/94 $205140-0001-5$ WEST PADUCAH

AC22-9IPC90368 09/19/94 205140-0002-3 TETERBORO

AKRON UNIVERSITY OF
DELINEATES THE SECURITY RESPONSIBILITIES OF THE PARTIES WITH TX 13 \$O DOE AGREES TO MAKE ALERT PADS 4 AND 5 AVAILABLE TO SAC AHD

REPAYMENT AGREEMENT

$C A \quad 35 \quad \$ 0 \quad \$ 0$

NOVEL SELECTIVE SURFACE FLOW MEMBRANES FOR THE RECOVERY OF PA $15 \quad \$ 3,559,660$

ALTERHATE FUELS DEVELOPMENT

$$
\text { PA } 15 \quad \$ 24,707,500 \quad \$ 22,078,411 \text { AGI1 }
$$

BPA FOR AIRFREIGHT SHIPMENTS

$\begin{aligned} \text { MD } & 05 \\ \text { BPA FOR AIR FREIGHT } & \$\end{aligned}$

10 MW DEMONSTRATION OF GAS SUSPENSION ABSORPTION

POLISH RETROFIT INSTALLATION

$$
\begin{array}{lll}
\mathrm{NJ} & &
\end{array}
$$

DYNAMICS OF CHARGE-TRANSFER EXCITED STATES RELEVANT TO 


DIVISION
CHEMISTRY DEPT
CHEMISTRY DEPT
ALABAMA A \& M UNIVERSITY
BIOLOGY

BIOLOGY

ALABAMA POWER CO

ALABAMA SOUTHERN CMTY COLLEGE

ALABAMA STATE OF

ENERGY DIVISION

ENERGY DIVISION

ENERGY DIVISION

ALABAMA UNIVERSITY OF

:

BIN COMPL DATE

COMPL DATE

CITY

FG02-90ER14151 08,31/96

$001380-0101-4$ AKRON

FGO2-87CH10330 10/31/94
$001390-0602-8$ NORMAL

FG05-92ER54162 12/31/94 $001390-0002-X$ NORMAL

CRO1-83NE4436I 03/15/30 001420-0001-9 BIRMINGHAM

FG05-93ER79162 06/14/95 228580-0001-8 MOHROEVILLE

FG44-78CS60017 03/31/95 001430-0401-8 MONTGOMERY FG44-80R410074 06/30/95 $001430-0401-8$ MONTGOMERY FG44-80R430362 09/30/94 001430-0401-8 MONTGOMERY

$$
\begin{array}{r}
\text { FG02-91ER75658 } 09 / 29 / 94 \\
001450-0001-X \quad \text { BIRMINGHAM }
\end{array}
$$
FG02-91ER75678 09/29/94 O01450-0001-X BIRMINGHAM FG05-91ER45449 05/31/95 $001450-0001-X$ BIRMINGHAM FG05-93ER61573 03/31/95 $001450-0001-X$ BIRMINGHAM FC07-93ID13230 09/30/95 $001450-0001-x$ BIRMINGHAM FG05-88ER40467 12/31/94 001450-0702-2 HUNTSVILLE
DESCRIPTION OF MORK

CONG $\begin{aligned} & \text { CONT } \\ & \text { VALU }\end{aligned}$

TO DATE P/S $\$ 640,519$ MOLECULAR FIGENSTATE SPECTROSCOPY: APPLICATIDN TO THE OH $14 \quad \$ 557,261$

GENETIC EFFECTS OF PLUTONIUM IN DROSOPHILA $\$ 333,530$

HOT-FORGING OF REFR'CTORY CARBIDE: GRAPHITE COMPOSITES HOT-FORGING OF REFR:CTORY $\$ 153,027 \quad \$ 153,027$ DISPOSAL OF SPENT NUCLEAR FU $\underset{\$ 0}{\text { EL AND/OR HIGH LEVEL RADIOACT }}$

PRE-FRESHMAN ENRICHMENT PROGRAM (PREP) - 1993

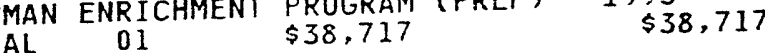

WEATHERIZATION OF LOW INCOME HOUSING PLUS TRAINING AND TEC WEATHERIZATION OF L $\$ 29,666,068 \quad \$ 26,897,553$ ENERGY EXTENSION SERVICE

GRANT PROGRAM FOR SCHOO!S, HOSPITALS, BUILDINGS OWNED BY UNI $\begin{array}{crcr}\text { AL } & 02 & \$ 14,448,459 & \$ 1,159,335\end{array}$

$\$ 750,000$

ALABAMA DDE/EPSCOR TRAINEESHIPGRANT

AL 06
ALABAMA DOE/EPSCOR PLANNING GRANT

ALABAMA DOE/EPSCOR PLANNING $\$ 222,384$

$\$ 120,000$

MIGRATIDN DF CONSTITUTIONALLY LIQUATED FILMS RADIOIMMUNOTOXIN THERAPY OF EXPERIMENTAL HUMAN GASTROINTESTI AL $06 \quad \$ 331,700$ EXPANDABLE PATTERN CASTING TECHNOLOGY LABORATORY
$\$ 265,744$ STUDY OF HIGH ENERGY NUCLEUS -NUCLEUS INTERACTIONS WITH A M 
DIVISION

VEHDOR ID CITY

STATE DIST VALUE

JER61730--09/14/96--
$001450-0002-8$ HUNTSVILLE

$\begin{array}{lll}\text { UMKEHR } & \text { OZONE COMPARISON } \\ \text { AL } & 05 & \$ 692,287\end{array}$

TD DATE FG02-92CHI0495 $12 / 31 / 94$
$001450-0003-6$ TUSCALOOSA

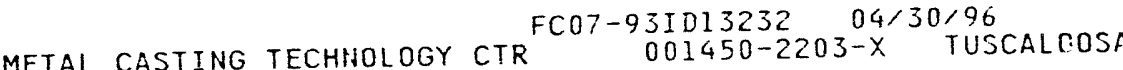
FG22-93PC93202 08/25/96

CHEMISTRY DEPT

MATHEMATICS DEPT

CHEMISTRY DEPT

PHYSICS \& ASTRONOMY DEPT

ALAMEDA HOSPITAL

ALAMEDA UNIFIED SCHOOL DIST

$$
\begin{gathered}
F G 49-92 R 914034 \quad 08 / 31 / 94 \\
222860-0001-8 \quad \text { ALAMEDA }
\end{gathered}
$$

ALASKA ELECTRIC LIGHT \& POHER

AC85-93AP10027 09/30/95 $001530-0001-5$ JUHEAU

FG22-93PC9342907/12/94 EHVIROHMENTAL SCIENCE \& MATH FG22-93PC934290101-5 AHCHORAGE

ALASKA STATE OF ENERGY \& POHER LEV DIV

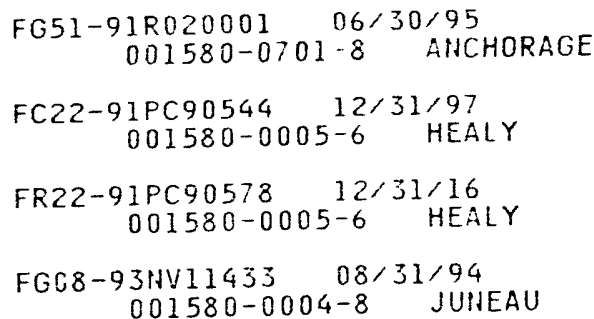

DESIGH \& COHSTRUCTION OF THE ADDITION TO THE BEVILLE ENERGY

DESIGN \& CONSTRUCTION 07 \$10,000,000 \$10,000,000

CLEAN FERROUS CASTING TECHNOLOGY RESEARCH PROJECT

$$
A L \quad 07 \$ 1,257,534
$$

MOLECULAR ACCESSIBILITY IN OXIDIZED AND DRIED COAL

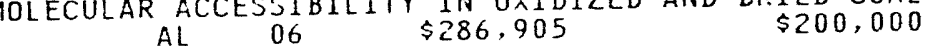

NEW GRANT - "LINEAR AND NONLINEAR STABILITY TOPICS IN PHYSIC

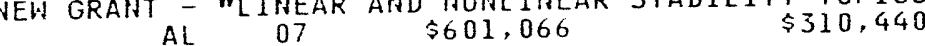

MAGNETIC RESOHANCE AND OPTICALSPECTROSCOPIC STUDIES OF RADI AL $07 \quad \$ 1,204,316 \quad \$ 850,597$

A COMPREHEHSIVE STUDY OF MESONPRODUCTIOH $\$ 2,180,450$

$$
A L \quad 07 \$ 2,552,450 \quad \$ 2,180,450
$$

TITLE III OF THE NATIONAL ENERGY CONSERVATION POLICY ACT I OF THE HATIONAL ENERGY CONJERVAT $\$ 107,148$

TITLE III OF THE HATIONAL ENERGY CONSERVATION FOLICY ACT CA THE NATIONAL ENERGY COHSERVATION $\$ 99,949$

SUBSTATION DPERATION AND LINE MAINTENANCE FOR THE 138 KV

ENERGY RELATED EQUIPMENT GRANT

THIS IS THE STATE OF ALASKA'S CONSOLIDATED SECP/EES PROGRAM HEALY CLEAN COAL PROJECT $\$ 461,138$

HEALY CLEAN COAL PROJECT

REPAYMENT AGREEMENT

$\$ 0$

$\$ 0$

PROJECT CHARIOT OVERSIGHT AND MOHITORING

AK $01 \quad \$ 441,672 \quad \$ 441,672$ 


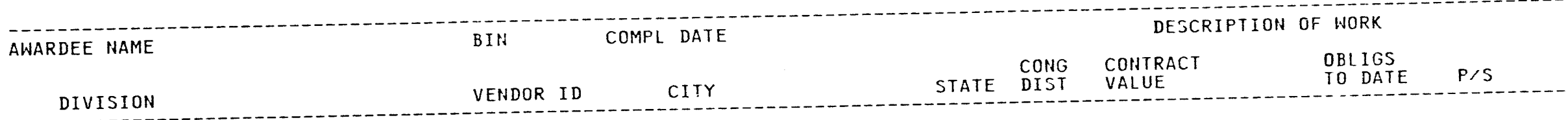

ALASKA UNIVERSITY OF GEOPHYSICAL INSTITUTE

$$
\begin{gathered}
\text { FG22-93BC14864006/30/96 } \\
001590-0001-7 \text { FAIRBANKS } \\
\text { FG51-92R020159009/30/94 } \\
001590-0001-7 \quad \text { FAIRBANKS }
\end{gathered}
$$$$
\text { 001590-0501-9 FAIRBANKS }
$$

ALBANY COLLEGE OF PHARMACY

FG42-92R243812 $07 / 31 / 95$
$220890-0001-5 \quad$ ALBAHY

FG44-92R435068 09/30/94
$222580-0101-3$ ALBAHY

BOARD OF RECENTS

ALBERS AIR CONDITIONING CORP

FG49-93CE15559 03/31/95
$234990-0001-6$ TEMPE

FG43-93R354201 12/31/94 $001670-0201-5$ PHILADELPHIA

ALBERT EINSTEIN MEI
NORTHERN DIV

ALBERT ENGINEERING

ALBERTA UNIVERSITY OF CHEMISTRY

\section{ALBUQUERQUE CITY OF POLICE DEPT}

POLICE DEPT

FGO1-89CE15312 07/06/94
$185350-0001-1$ YUKON

FG02-91ER61123 01/31/97
$205750-0101-1 \quad$ EDMONTON

GMO4-87AL53638 $09 / 25 / 99$
$126790-0201-6$

GMO4-94AL $94697 \quad 12 / 01 / 98$ $126790-0201-6$ ALBUQUERQUE
A STUDY OF THE MAGNETIC FIELD AHNIHILATION

THE EFFECT OF CLOUD: TCROPHYSICS ON CLIMATE SENSITIVITY

THE KATMAI SCIENTIFIC DRILLINGPROJECT

HYDROTHERMALLY TPEATED COALS FOR PULVERIZED COAL INJECTION

AY 01 \$69,336 $\$ 69,336$

STUDY OF HYDROCARBON MISCIBLE SOLVENT SLUG INJECTION PROCESS

$\begin{array}{lll} & \\ & & \end{array}$

CYCLE 14 ECM AWARD. $\$ 300,000 \quad \$ 150,000$

TITLE III OF THE NATIONAL ENERGY CONSERVATION POLICY ACT

CYCLE 14 - INSTITUTIOHAL CONSERVATIDN PROGPAM. APPROVED BUD

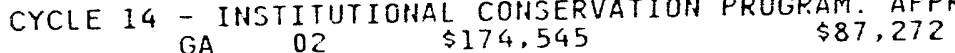

CONSTRUCTICH. FIELD TEST, AND FIRST PUBLIC DEMONSTRATION OF AZ 01 \$95,514 $\$ 95,514$

TITLE III OF THE NATIONAL ENERGY CONSERVATION POLICY ACT $\begin{array}{cr}\text { OF THE MATIONAL ENERGY CONSERVATION POLTCY } \\ \text { PA } 01 & \$ 128,920\end{array}$

DEVICE FOR HELL SITE MONITORING OF ROD PUMPED WELL
MULTI-COLUMN CAPILLARY GEL ELECTROPHORESIS AND LASER-INDUCED

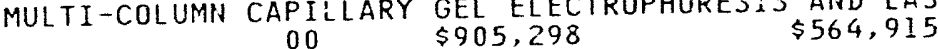

PROVIDES FOR THE LOAN OF AN ARMORED VEHICLE AT SNLA 3430 TO IM 01 \$O

THIS AGREEMENT SETS FORTH THE POLICY FOR THE USE OF THE CTA 


\section{AWARDEE NAME}

BIN

COMPL DATE

DESCRIPTION OF HORK

IVISION

VENDOR ID STATE DIST

CONTRACT VALUE

P.'S

POLICE DEPT

ALCOA FUJIKURA LTD

ALDEN CENTRAL SCHOOL DISTRICT

ALDERSON-BROADDUS COLLEGE

ALDINE INDEPENDENT SCHOOL DIST

ALEM ASSOC

ALEXANDER \& ALEXANDER

ALEXANDER LOCAL SCHOOL DIST

ALFRED UNIVERSITY CERAMICS COLLEGE OF

ALGERIA GOVERNMENT OF

ALISA SYSTEMS INC

ALL HALLOWS HIGH SCHOOL
GM04-93AL84096 07/06/98 126790-0201-6 ALBUQUERQUE

AM75-945H35005 12/05/95 172540-0003-3 BRENTHOOD FG42-93R253866 07/31/95
$230410-0001-6$ ALDEN

FG43-93R356203 01/31/95 $001770-0001-5$ PHILIPPI

FG46-93R613634 02/28/95 $223030-0001-7$ HOUSTON

FG02-94ER81656 07/17/94 $198580-0001-4$ BOSTON

AC01-91PR38151 09/26/94 $132040-0001-X \quad$ BAL TIMORE

FG45-93R551731 01/31/95
$229700-0001-6$ ALBANY

$F G 02-93 E R 12128 \quad 11 / 30.95$
$001850-0201-3 \quad$ ALFRED

FC02-93P010101 05/31/95 $234430-0001-0$ ALGIERS

AC34-94RF00741 02/28/95 $237250-0001-8$ GOLDEN

FG42-93R253867 07/31/95 230580-0001-4 NEW YORK PLAN, PARTICIPATE, AND SUPPORTTRAINING FOR MOVING $\underset{\$ 0}{\$ 0}$ ACTUAL NM 01 $\$ 0$

SUPFLY OF FIBER OPTIC GROUND WIRE TN $00 \quad \$ 419,900$

$\$ 419,900 \quad 6015$

CYCLE 15 AWARD. $\$ 23,250$ $\$ 11,625$

TITLE III OF THF NATIONAL ENERGY CONSERVATION POLICY ACT WV $01 \quad \$ 100,000 \quad \$ 50,000$

AWARDS A NEW CYCLE 15 ENERGY CONSERVATION MEASURE GRANT. TX $29 \quad \$ 130,626 \quad \$ 65,313$

ACTIVATED OPTICAL CERAMICS: A NEW CLASS OF MATERIALS FOR $M A \quad 01 \quad \$ 74,970 \quad \$ 74,970$

PROVIDE EMPLOYEE BENEFIT ACTURIAL RISH MGMT \& INS SUPPORT SE HD $03 \quad \$ 725,395 \quad \$ 725,395 \quad$ R799

ICP - NEW AWARD.

$\$ 5,628$

$\$ 2,814$

COMBUSTION SYNTHESIS \& ENGINEERING OF NANOPARTCLES NY $34 \quad \$ 607,110 \quad \$ 405,767$

CLIMATE CHANGE COUNTY STUDY

$00 \$ 21,000$

$\$ 20,000$

CV , ALISA ENHANCEMENTS TO CC:MAIL

CO $02 \quad \$ 93,421$

$\$ 93,421 \quad$ D307

CYLLE $15 \underset{N Y}{\text { AWARD. }} 16 \quad \$ 72,660$

$\$ 36,330$ 


DWARDEE NAME
DIVISION
BOARD OF EDUCATION

ALLEN \& HOSHALL INC

ALLIANCE TO SAVE ENERGY

ALLIANT HEALTHCARE SYSTEM

KOSAIR CHILDREN'S HOSPITAL

ALLIED FILM \& VIDEO

ALLIED-SIGNAL INC

BENDIX KANSAS CITY DIV

ALPINE BOARD OF EDUCATION

ALTAMIRA INSTRUMENTS INC

\section{ALUMINUM CO OF AMERICA \\ aLCOA LABS}

ALCOA LABS
COMPL DATE

DESCRIPTION OF WORK

BIN VALUE

OBLIGS

TO DATE P/S

VENDOR ID CITY

CYCLE 14 - INSTITUTIONAL CONSERVA

$\$ 9,848$

$\$ 5,016$

$2 R 43498609 / 36$
$220410-0101-1$

AC75-88SW22580 07/31/94

FG43-93R340423 09/30/96 002050-0001-0 WASHINGTON

FG44-91R434497 12/31/94 208530-0001-8 LOUISVILLE

FG44-93R435300 09/30/95 208530-0101-4 LOUISVILLE

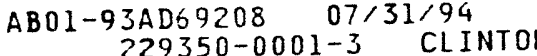

FG02-92CH10519 07/31/94 165680-0005-1 DES PLAINES

FCO4-93AL $94462 \quad 11 / 30 / 96$ 165680-0005-1 DES PLAINES

AC21-92MC29245 09/18/94 165680-0005-1 DES PLAINES

AC04-76DP00613 03/31/95 $165680-0101-5$ KANSAS CITY

FG42-94R263908 06/30/96 239010-0001-1 ALPINE

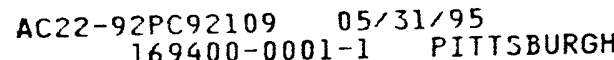

FC07-89ID12903 $12 / 31 / 94$
$002290-0101-7 \quad$ ALCOA CENTER FC07-89ID12848 07/29/96 $002290-0101-7$ ALCOA CENTER BROAD SPECTRUM OF ARCHITECTUAL\& ENGINEERING STUDIES, ANALSES
$\$ 500,000$

ALLIANCE DSM BEHAVIOR PROJECT

$$
\text { DSM BEHAVIOR PROJECT } \$ 75,000 \quad \$ 75,000
$$

CYCLE 13 - INSTITUTIONAL CONSERVATION PROGRAM. APPROVED BUD SEE ATTACHED PAGE NO. 2 OF THIS NOTICE OF FINANCIAL ASSISTAH VIDEO CASSETTE DUPLICATION SERVICES MD 08

THE ROLE OF WATER IN EHANCING THE ACTIVITY OF AN EMZYME THE ROLE OF WATER IN EHANCING \$821,887 INDUSTRIAL WASTE REDUCTION PROGRAM IL 06 \$1,247,066

$\$ 175,983$

HIGH TEMPERATURE, SIZE- SELECTIVE MEMBRANES IL $\$ 545,882 \quad \$ 436,707 \quad$ AG12 DPERATION OF GOVT FAC/PRODUCTION BLDGS $\begin{array}{llll}\text { MO GOVT FAC } & 05 \$ 9,767,026,914 & \$ 9,767,026,914 & \text { M181 }\end{array}$

CYCLE 16 AWARD. $\$ 129,489 \quad \$ 66,445$

DIRECT AROMATIZATION OF METHANE PA 14 \$340,930 $\$ 243,900$ AGII

PA 05 \$1, 155,441

\$924, 354

LOW TEMPERATURE ELECTROYLYSIS OF ALUMINUM PA $23 \quad \$ 4,7<0,785 \quad \$ 1,416,106$
LIGHTWEIGHT REFRACTORY AGGREGATES 
AWARDEE NAME

BIN

COMPL DATE

VENDOR ID

CITY

STATE DIST CONTRACT
VALUE

DESCRIPTION OF WORK

\section{DIVISION}

ALCOA LABS

ALCOA LABS

\section{ALZETA CORP}

ENGINEERING

ENGINEERING

AM PRO PROTECTIVE AGENCY INC

SECURITY

AMARILLO CITY OF

AMAX $R$ \& $D$ INC

AMER MUSEUM OF NATURAL HISTORY FG02-92ERI4265 06/30/95
FCO7-941D13219 07/31/96 002290-0101-7 ALCOA CENTER

FC07-94ID13237 06/11/00

FCO7-94ID13238 04/18/99 002290-0101-7 ALCOA CENTER

FC02-92CE41003 07/31/94 191360-0101-5 SANTA CLARA

AC04-89CE40918 10/31/94 191360-0101-5 SANTA CLARA

AC01-93SA10070 09/30/94 169040-0001-5 WASHINGTON

AC02-90CH10437 01/31/95 169040-0102-X LEMDNT

GMO4-85AL3131509/30/99 $132070-0001-0$ AMARILLO

GMO4-76AL02249 05/16/99 132070-0001-0 AMARILLO

GMO4-90AL42113 12/29/94 $132070-0001-0$ AMARILLO

AC22-92PC92208 09/29/96 $002400-0001-3$ GOLDEN

AC22-92PC92110 03/27/95 $002400-0001-3$ GOLDEN

AC22-93PC92206 11/05/94 002400-0002-1 PITTSBURGH

$2 E R 1426506 / 30 / 95$
$219850-0001-6$ NEW YORK
R\&D FOR ENERGY EFFICIENT ALUMINUM PRODUCTION - PILOT-SCALE PA $20 \quad \$ 3,004,738$ $\$ 1,000,000$

INITIATE \& INCREMENTALL: FUND PHASE I OF II "DEVELOPMENT OF PA 20 \$13,851,734

SPRAY-FORMING OF ALUMINUM - PHASE II

$$
\text { PA } 20 \stackrel{\$ 19,318,516}{20} \$ 1,621,000
$$

APPLICATION ASSEMSSMENT OF HIGH TEMPERATURE, LOW NO CA $13 \quad \$ 452,000 \quad \$ 156,000$

HIGH TEMPERATURE RADIANT COMBUSTION SYSTEM

$$
\text { CA I3 } \$ 1,801,568 \text { \$1, } 363,711 \text { AE32 }
$$

SECURITY PROTECTIVE FORCE SUPPORT SERVICES

$$
\begin{array}{llll}
\text { PROTECTIVE } & \text { FORCE SUPPORT SERVICES } \\
\text { DC OI } & \$ 9,493,024 & \$ 9,249,581 & 5206
\end{array}
$$

PROTECTIVE FORCE AND SECURITY SERVICES FOR ARGONNE NATIONAL

$$
\text { IL } 13 \text { \$1 } 4,416,747 \quad \$ 12,920,293 \quad \$ 206
$$

MOU BETWEEN CITY OF AMARILLO, TEXAS AND PANIEX PLANT.

TX 15 SO

DOE AND ITS DESIGNEES SHALL HAVE EXCLUSIVE USE OF THE AREA

MUTUAL AID IN FIRE FIGHTING

$\underset{T X}{13}$ IN FIRE FIGHTING SD

$\$ 0$

ENGINEERING DEVELOPMENT OF ADVANCED PHYSICAL FINE COAL CLEAN CO $05 \$ 14,979,194$

$\$ 6.248,912$ AG14

DEVELOPMENT OF VANADIUM-PHOSPATE CATALYSTS FOR METHANOL PROD CO $02 \begin{array}{lll}\$ 449,496 & \$ 335,496 \text { AGI }\end{array}$

BENCH-SCALE TESTING OF THE MICRONIZED MAGNETITE PROCESS

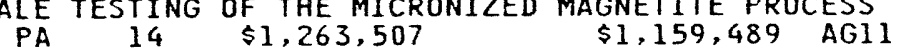

THE ROLE OF CARBON \& TEMPERA- TURE IN DETERIINING ELECTRICAL NY $17 \quad$ \$125,988 $\quad \$ 125,988$ 


\begin{tabular}{|c|c|}
\hline AWARDEE NAME & COMPL DATE \\
\hline DIVISION & VENDOR ID \\
\hline AMERASIA TECHNOLOGY INC & $\begin{array}{c}\text { AR21-94MC31177 01/21/95 } \\
239100-0001-0 \text { WESTLAKE VI }\end{array}$ \\
\hline AMERCOM INC & $\begin{array}{c}\text { FC02-92CE40998 } 09 / 30 / 96 \\
220070-0001-6 \text { CHATSWORTH }\end{array}$ \\
\hline \multirow[t]{4}{*}{ AMERICAN CHEMICAL SOCIETY } & $\begin{array}{c}\text { FG02-92ER75735 } 07 / 01 / 94 \\
002560-0001-8 \text { WASHINGTON }\end{array}$ \\
\hline & $\begin{array}{c}\text { FGO1-90CE90043 } 04 / 27 / 95 \\
002560-0001-8 \text { WASHINGTON }\end{array}$ \\
\hline & $\begin{array}{c}\text { FG02-94ER61837 } 04 / 30 / 95 \\
002560-0001-8 \text { WASHINGTON }\end{array}$ \\
\hline & $\begin{array}{c}\text { FG03-93ER61705 } 09 / 29 / 94 \\
002560-0007-7 \quad \text { KNOXVILLE }\end{array}$ \\
\hline
\end{tabular}

AMERICAN COLL OF NUCLEAR PHYS

FG02-93ER61671 08/14/94
$002570-0001-1$ WASHINGTON FG02-93ER61704 08/31/94 002570-0001-1 WASHINGTON

AMERICAN ELIN CORP

AMERICAN EXPRESS TRAVEL REL

AMERICAN FOUNDRYMEN'S SOC INC

AMERICAN GEOLOGICAL INSTITUTE

AMERICAN INDIAN SCI \& ENG SOC

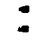

AC65-93WBO3091 $06 / 01 / 95$
$180720-0002-0 \quad$ WEIZ

ADOI-94FM30002 11/29/94
$236180-0001-4$ ARLINGTON

FC07-931D13240 03/31/95
$183760-0001-2$ DES PLAINES 002700-0002-X ALEXANDRIA

FG04-90AL62174 10/31/94
$139660-0001-6 \quad$ BOULDER

GM34-93RF00661 04/16/95 139660-0001-6 BOULDER
FG05-94ER75979 03/31/97
DESCRIPTION OF WORK

STATE DIST VALUE
EM-FIELD PORTABLE DETECTION OFVOCS USIIH A SAWGC SYSTEM

CONTINOUS FIBER CERAMIC COMPESITES (CFCC)

SUMMER SCHOOLS IH NUCLEAR CHEMISTRY

$\begin{array}{ccccc}\text { SUMMER SCHOOLS IN } & \text { NUCLEAR } & \text { CHEMISTRY } & \$ 544,026\end{array}$

PROJECT SEED- SUMMNER EDUCATIONAL EXPERIENCE FOR DISADVANTAG

$\begin{array}{ccc}\text { PROJECT SEED- SUMMNER EDUCATIONAL EXPERIENCE FOR DIS } \\ \$ 228,243 & \$ 201,972\end{array}$

THE 1994 BOC PRIESTLY $\quad \begin{gathered}\text { CONFERENCE } \\ \text { DC } 01\end{gathered}$ $\begin{array}{cccc}\text { INTERNATIONAL SYMPOSIUM OF CHEMISTS: } & \text { VIEWS OF IMAGING CENTER } \\ \text { TN } & 02 & \$ 5,000 & \$ 5,000\end{array}$

PROCEEDIGNS OF A HORKSHOP ON MOLECULAR NUCLEAR MEDICINE FRDNTIERS IN NUCLEAR MEDICINE SYMPOSIUM: NUCLEAR MEDICINE SHUNT REACTOR FOR RUGBY SUBST - SPLIT AWARD WITH 93 WA11263 PARTICIPATION IN THE TRAVEL AND TRANSPORTATION PAYMENT AND E

PROCESS MODELING OF A CUPOLA FURNACE
$\mathrm{Ii} \quad 06 \mathrm{~F}$ \$293,230
$\$ 85,430$

NEW AWARD ADMIMISTRATION AND IMPLEMENTATION OF RUSSIA-U.S. G NEW AWARD ADMINISTRATION AND IMPLEMENTATION $\$ 112,046$ SCHOLARSHIPS TO AMERICAN INDIAN STUDENTS IN SCIENCE

MOU TO LOAN COMPUTER EQUIPMENT 


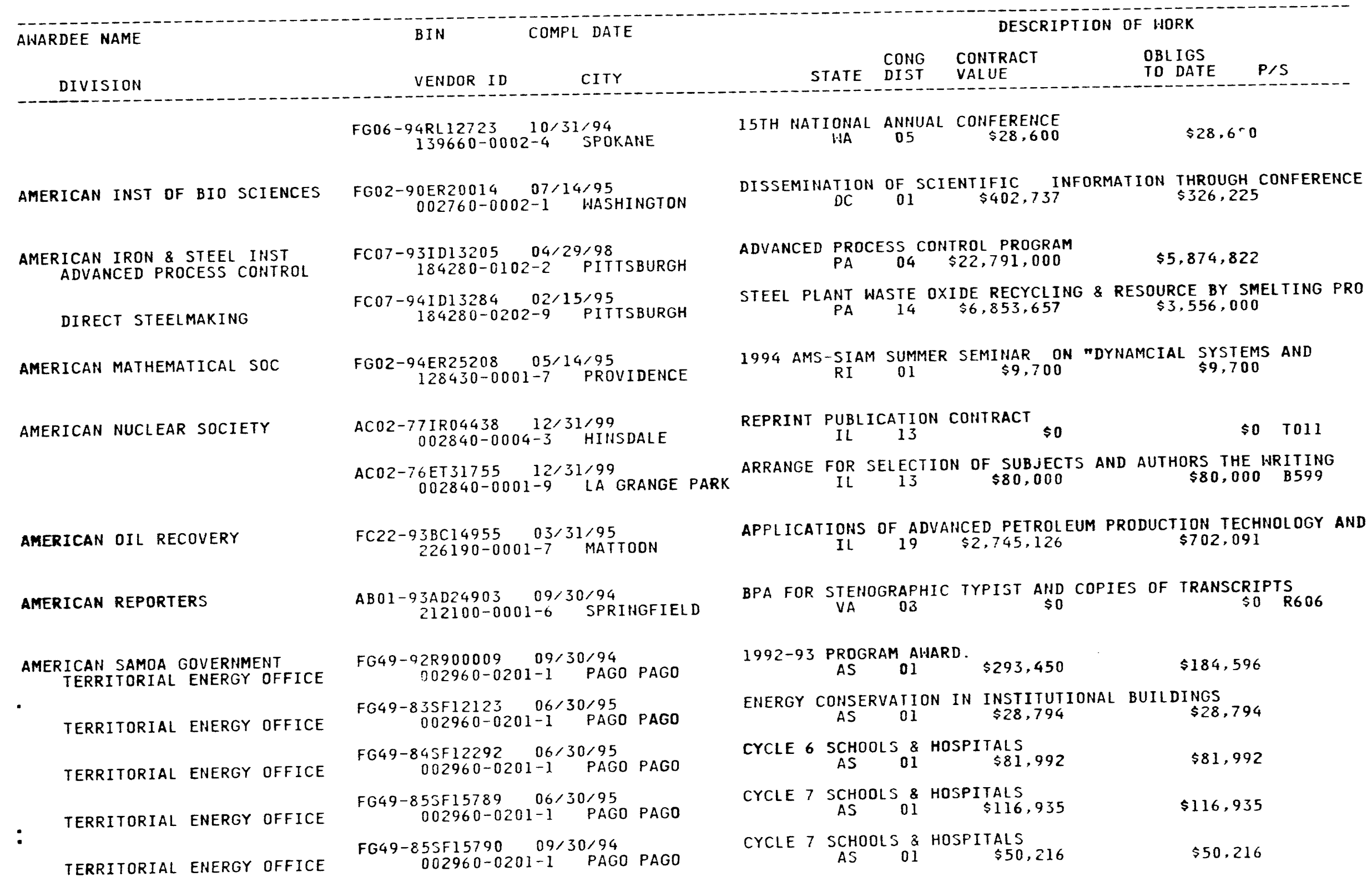


DIVISION

VENDOR ID

CITY CDNG

COHTRACT ALUE OBLIGS

\section{SSF18240 08/31/94} 002960-0001-9 PAGD PAGO

TERRITORIAL ENERGY OFFICE TERRITORIAL ENERGY OFFICE TERRITORIAL ENERGY OFFICE TERRITORIAL ENERGY OFFICE TERRITORIAL ENERGY OFFICE TERRITORIAL ENERGY OFFICE TERRITORIAL ENERGY DFFICE TERRITORIAL ENERGY OFFICE AMERICAN SOC FOR PHOTOBIOLOGY AMERICAN SOC OF ADVANCED FUEL ,AMERICAN SOLAR NETWORK LTD AMERICAN STATISTICAL ASSN AMERICAN SUPERCONDUCTOR CDRP

$$
\begin{gathered}
\text { FG49-90SF18703 08/31/94 } \\
002960-0201-1 \text { PAGO PAGO }
\end{gathered}
$$$$
002960-0201-1 \text { PAGO PAGO }
$$

FG49-91R913018 08/31/94 002960-0201-1 PAGO PAGO FG49-91R913017 09/30/94 FG49-92R914024 08/31/94 $002960-0201-1$ PAGO PAGO FG49-92R914023 08/31/94 $002960-0201-1$ PAGO PAGO

FG49-9JR915019 08/31/95 $002960-0201-1$ PAGC PAGO

FG05-94ER61787 01/31/95
$002470-0002-7 \quad$ AUGUSTA

FC 36-946010010 $03 / 31 / 95$
$237740-0001-8$ ALPINE

FG49-93CE15569 03/31/95 232570-0001-4 CARMICHAEL

FGO1-92EI $2293902 / 28 / 95$
$003050-0002-0$ ALEXANDRIA

FG02-93ER81605 07/17/94 233270-0001-8 HESTBOROUGH$$
\text { FG49-90SF18704 08/31/94 }
$$
$002960-0201-1$ PAGO PAGO FG49-93R915018 08/31/95 $002960-0201-1$ PAGD PAGO $\begin{array}{ccc}\text { GRANT PROGRAMS } & \text { FOR } & \text { SCHOOLS ANDH } \\ \text { AS } & 01 & \$ 80,409\end{array}$

GRANT PROGRAMS FOR SCHOOLS ANDHOSPITALS AND BUILDINGS OWHED GRANT PROGRAMS FOR TCHOOL $\$ 57,600$ $\$ 57,600$

GRANT PROGRAMS FOR SCHOOLS ANDHOSPITALS AND BUILDINGS ONNED ARANT PROGAMS FOR SCHOOL 01 \$38,49? $\$ 38,497$ ADMINISTRATIVE GRANT

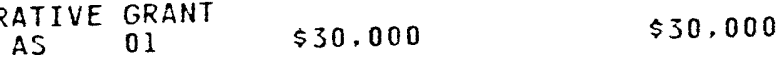

CYCLE XIII GRANT

CYCLE XIV ICP ADMINSTRATIVE GRANT AHARD. $\$ 30.000$

CYCLE XIV ICP GRANT AWARD.

CYCLE XV ICP ADMIHISTRATIVE GRAHT. $\$ 30.000 \quad \$ 30,000$

EXHIBIT I - BUDGET PLAN. EXHIBIT II - GENERAL GRANT PROVISIO I - BUDGET PLAN. $\begin{array}{r}\text { EXHIBIT } \\ \text { AS }\end{array}$

SYMPOSIUM ON PHOTOCHEMISTRY AND PHOTOBIOLOGY OF GENETIC MATE GA $10 \quad \$ 9,300 \quad \$ 9,300$

DEVELOP OF A NAT:L CERTIFIC. PROCESS FOR AFV TECHHIC. TRANG IX 23 \$125,000 $\$ 100,000$

SOLAR SKYLITE WATER HEATER

CA 51 \$ $\$ 99,294$

$\$ 99,294$

PROVIDE ASSISTANCE FOR THE PURPOSE OF CONDUCTING MEETINGS TO VA 08 \$188,325 $\$ 138,325$

OXIDE DISPERSION STRENGTHENED OF SILVER FOR USE IN HIGH$M A \quad 03 \quad \$ 75,000 \quad \$ 75,000$ 
$\frac{1}{2}$

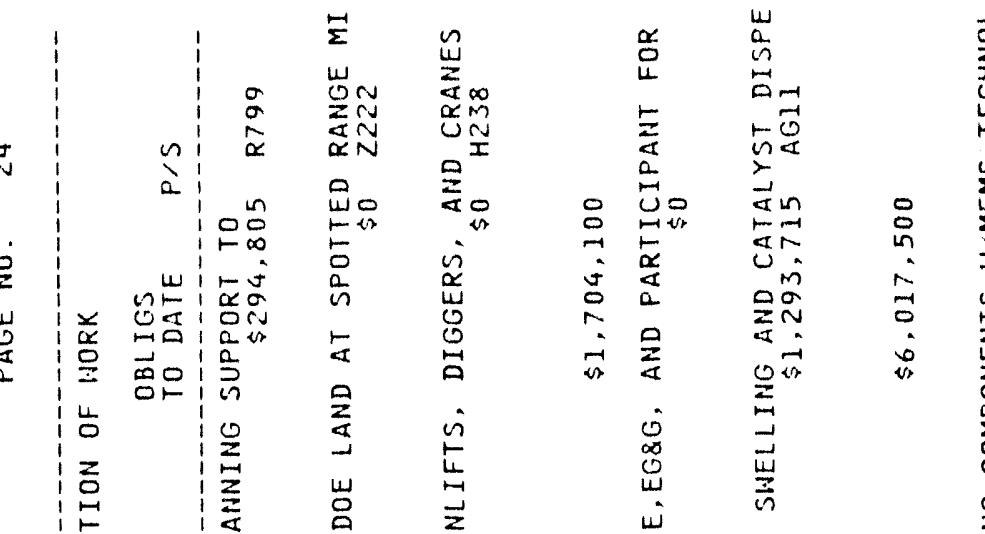

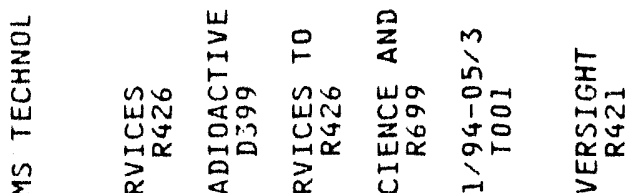

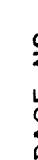

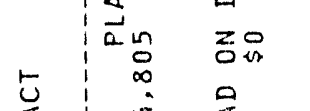

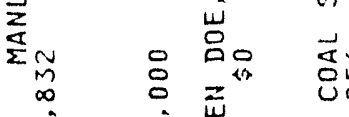

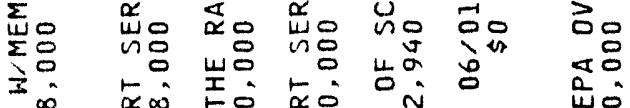

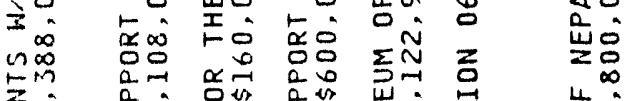

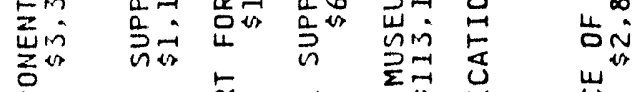

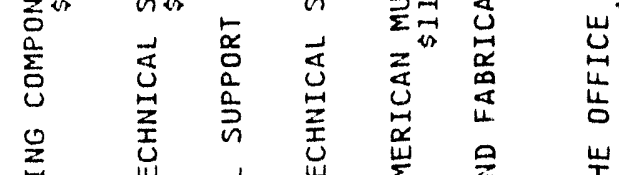

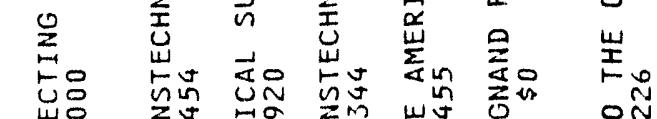

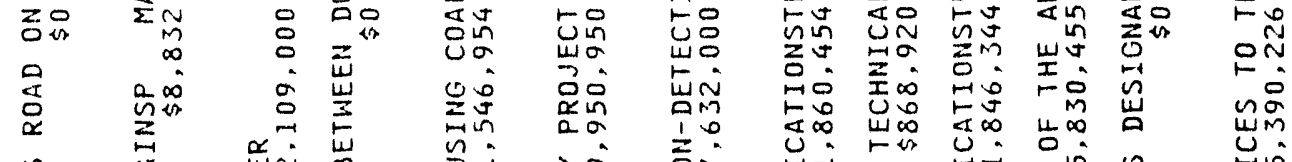

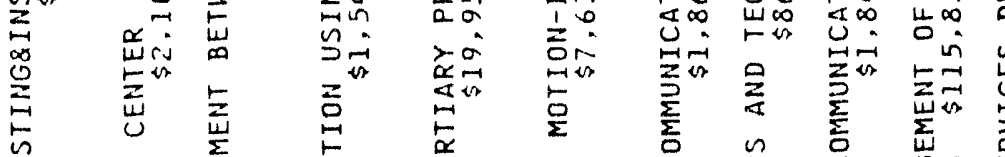
吃

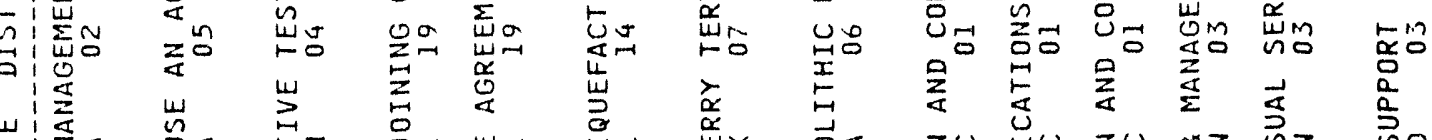

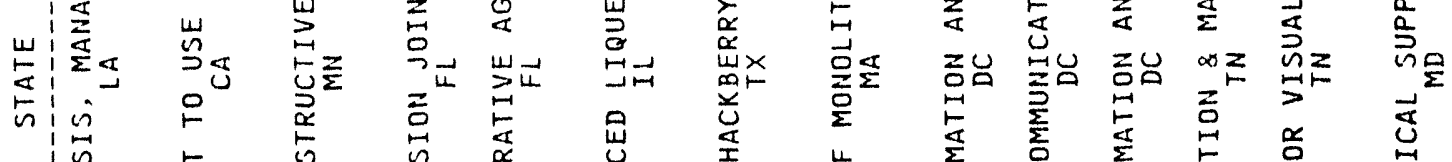

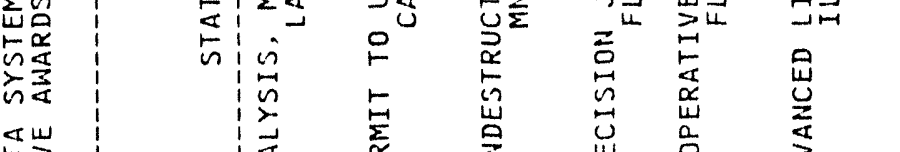

すष山

㟔।

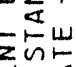

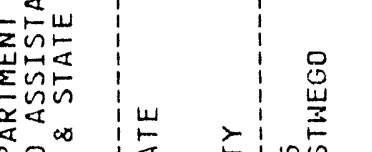
永

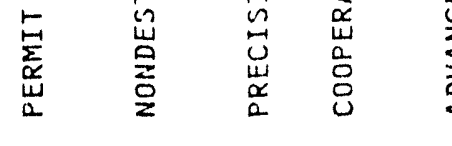

兵

i

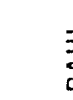
年

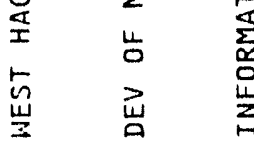

要的

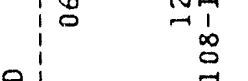

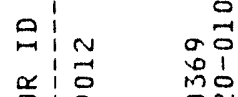

ôे वे

요

No mi nol तi

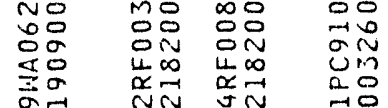

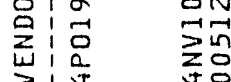

咅尔

Na

$\begin{array}{ll}0 & \infty \\ 1 & 1 \\ 1 & \infty \\ 0 & 0 \\ 0 & 0\end{array}$

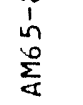

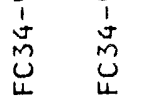

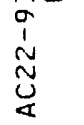

岸

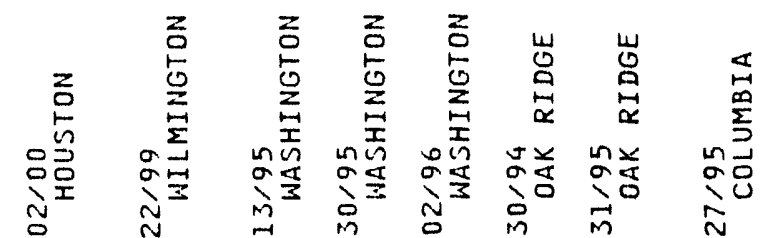

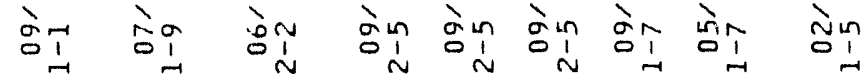

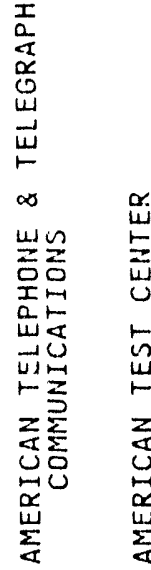

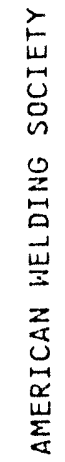

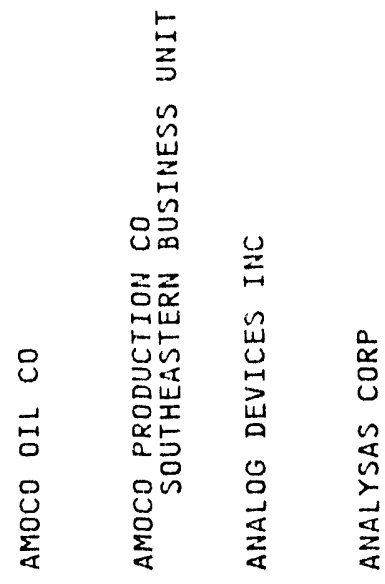




\begin{tabular}{|c|c|c|c|c|c|c|c|}
\hline AWARDEE NAME & BIN & 1 DATE & & & DESCR & F HORK & \\
\hline & ד תחסי & CTTY & STATE & $\begin{array}{l}\text { CONG } \\
\text { DIST }\end{array}$ & $\begin{array}{l}\text { COHTRACT } \\
\text { VALUE }\end{array}$ & $\begin{array}{l}\text { OBLIGS } \\
\text { TO DATE }\end{array}$ & $P / S$ \\
\hline
\end{tabular}

ANDCARE INC
ANDERTIY, / SMITH OPERATING CO
ANDERSON CITY OF
COMMUNITY SCHOOL CORP

ANDREW COLLEGE

ANDREWS PETROLEUM INC

ANN RILEY \& ASSOC LTD

AHNE ARUNDEL COMMUNITY COLLEGE

ANNE ARUNDEL MEDICAL CENTER

ANSON COUNTY HOSPITAL

ANTILLES MILITARY ACADEMY

:

APPALACHIAN \& OHIO POWER CO
FG05-94ER81657 07/17/94
$236060-0001-4$ DURHAM

FC22-93BC14952 07/05/96
$225670-0001-1$ TUSCALOOSA

FG45-94R562003 06/30/95 $238070-0101-8$ ANDERSON

FG44-92R435078 09/30/94
$197610-0001-X \quad$ CUTHBERT

SC92-93FE63084 09/30/94 $003620-0004-2$ COSTA MESA

AB 01-93AD24904 09/30/94 $77790-0001-X$ WASHINGTON

FG43-92R343200 02/28/95
$222530-0001-9$ ARNOL

FG43-93R353100 08/31/94
$222530-0001-9$ ARNOLD

FG43-92R343210 02/28/95 222550-0001-6 ANNAPOLIS

FG43-93R353200 03/31/95 222550-0001-6 ANNAPOLIS

FG44-93R435375 09/30/94 $231240-0001-3$ WADESBORO

FG44-91R434775 03/01/95 003830-0001-7 TRUJILLO ALTO

FC21-90MC26304 07/04/98

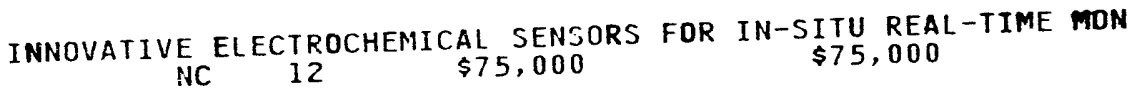

SANDSTONE OIL FI

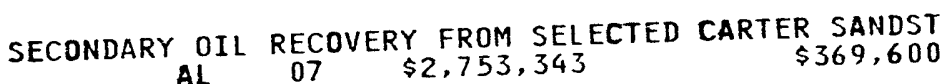

ICP - NEW AWARD ${ }_{02} \quad \$ 26,360 \quad \$ 14,620$

CYCLE 14 - INSTITUTIONAL CONSERVATION PROGRAM. APPROVED BUD $\$ 98,976$
CYCLE 14

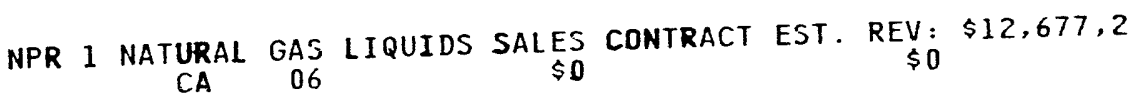
BPA FOR STEND-TYPISTS COPIES OF TRANSCRIPTS, \& $\underset{\$ 0}{\$ 0}$ RELATED CDURT

TITLE III OF THE NATIONAL ENERGY CONSERVATION POLICY ACT

TITLE III OF THE NATIONAL ENERGY CONSERVATION POLICY ACT

TITLE III OF THE NATIONAL ENERGY CONSERVATION POLICY ACT

MD 01 TATERGY CONSERVATION POLICY ACT

TITLE III OF THE NATIONAL ENERGY CONSERVATION POLICY $\$ 105,000$

SEE ATTACHED PAGE NO. 2 OF THIS NOTICE DF FINANCIAL ASSISTAN

$\$ 14,430 \quad \$ 7,215$

CYCLE 13 - INSTITUTIONAL CONSERVATION PROGRAM. APPROVED BUD

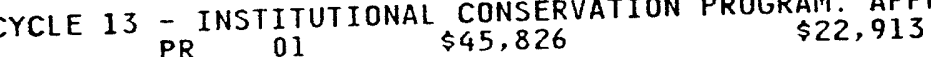

CLEAN COAL II - PFBC UTILITY DEMONSTRATION PROJECT 


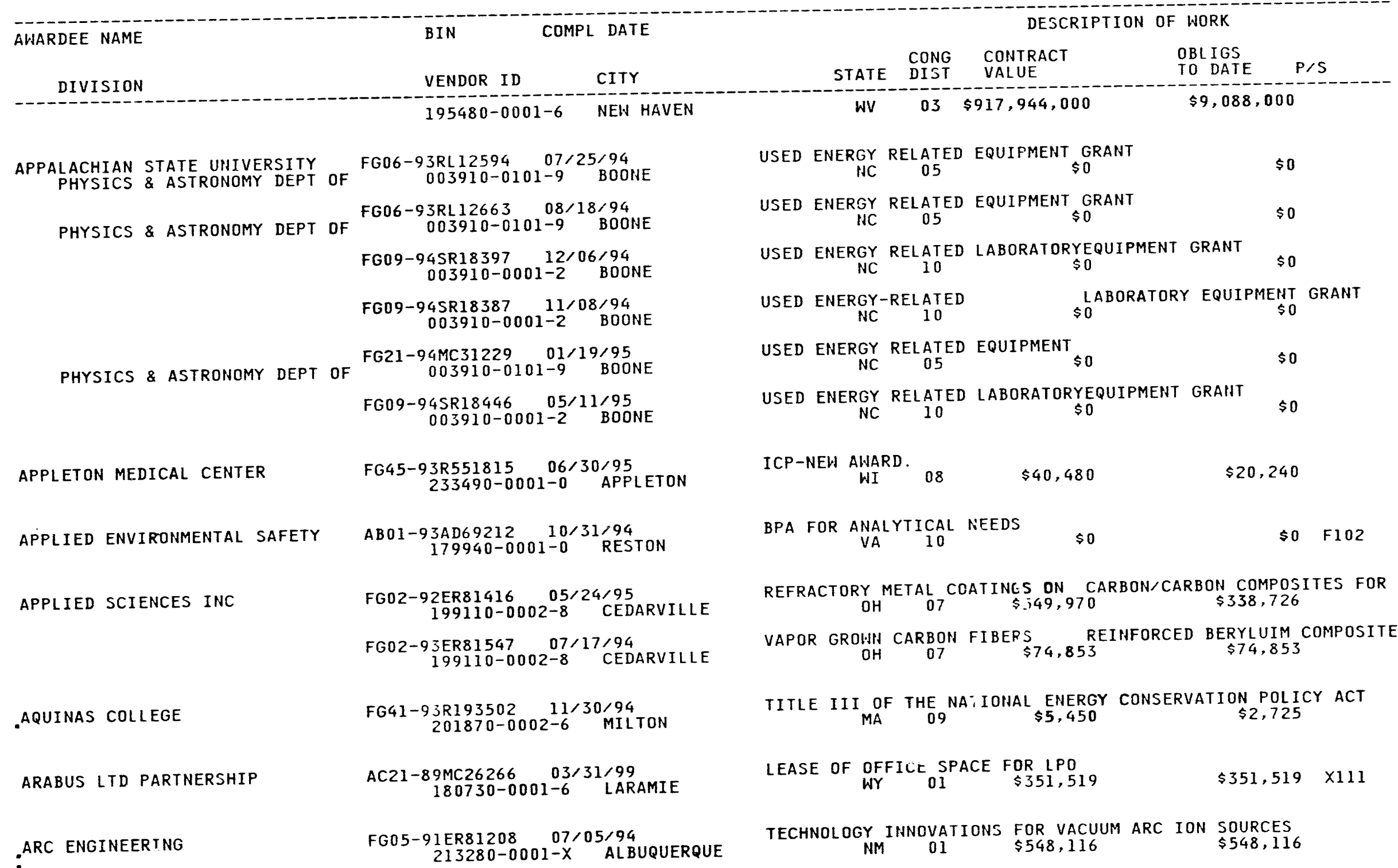




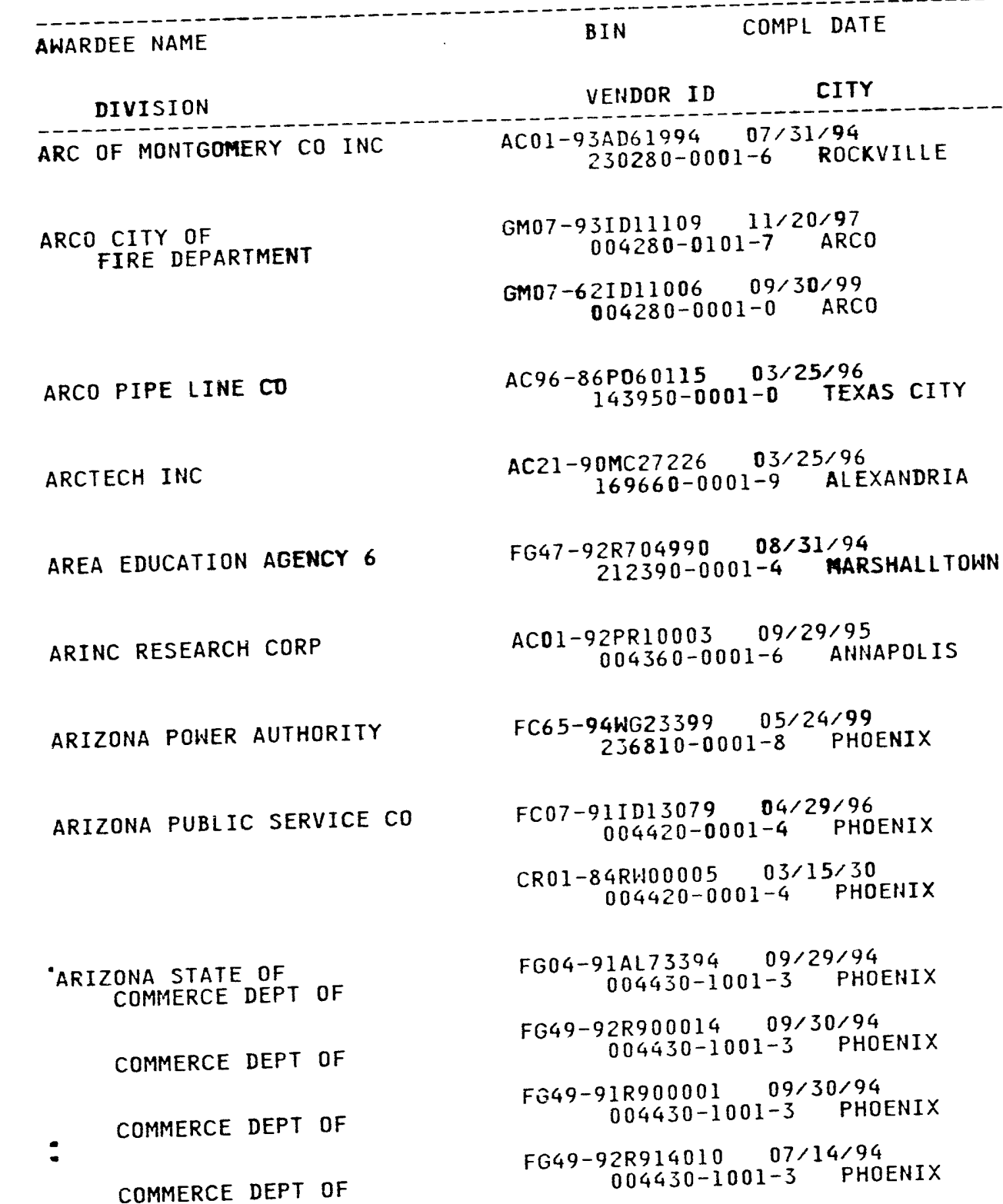

DESCRIPTION OF WORK

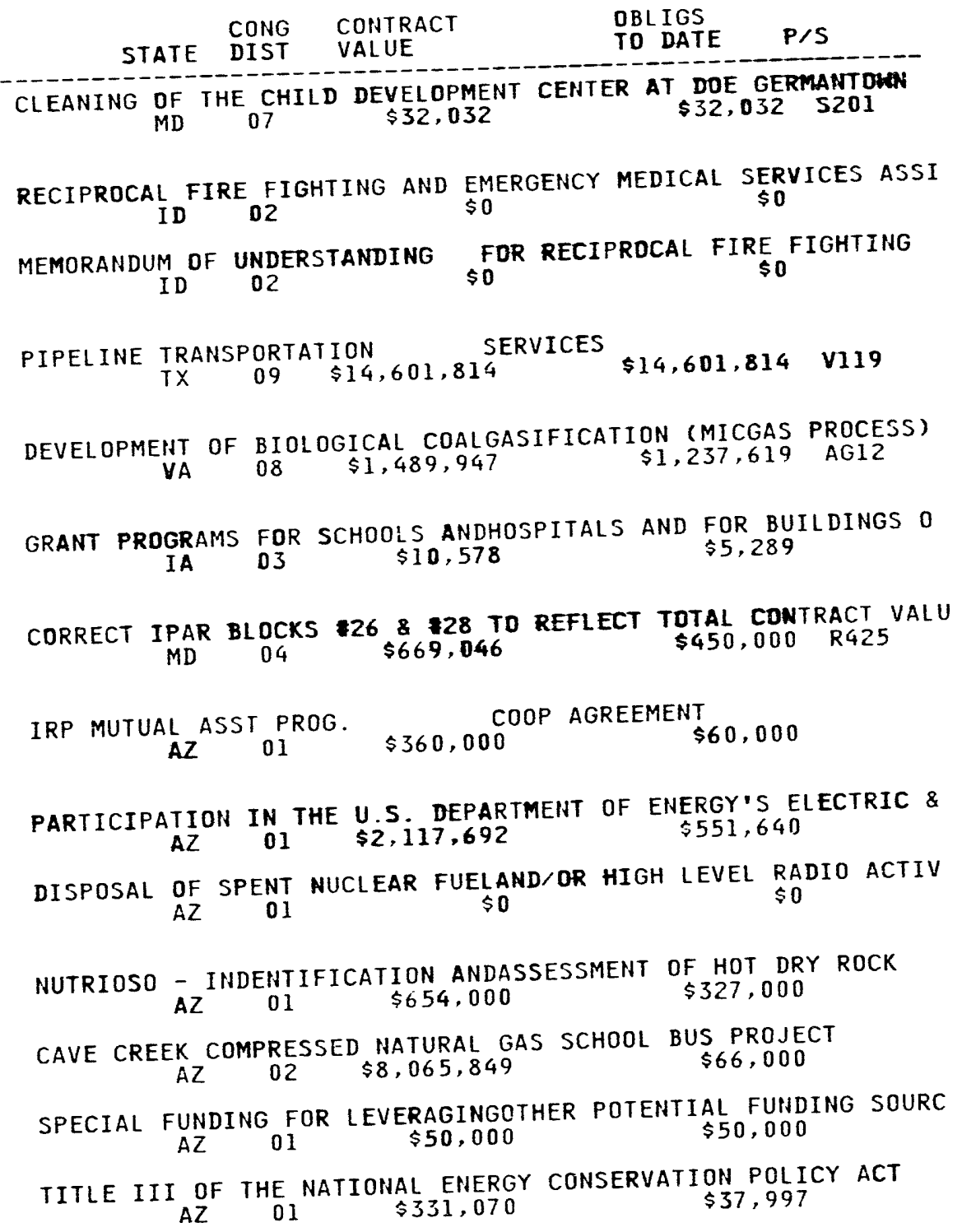


COMPL DATE

BIN

DIN

VENDOR ID

CITY

CONG CONTRACT

DESCRIPTION OF HORK

DIVISION

FG49-89SF18096 03/31/95
$004430-0001-8$ PHOENIX 004430-0001-8 PHO

COMMERCE DEPT OF

FG49-93R915017 06/30/95
$004430-1001-3$ PHOENIX

COMMERCE DEPT OF

FC65-93HG19551 09/30/98
$004430-1001-3$ PHOENIX

FG03-78CS60036 12/31/99 004430-0201-0 PHOENIX

ECONOMIC SECURITY DEPT

ARIZONA STATE UNIVERSITY

CHEMISTRY DEPT

FG02-86ER13558 06/30/95
$004440-0601-X$ TEMPE

FG02-87CH10315 12/31/94 004440-0001-1 TEMPE

BOTANY \& MICROBIOLOGY DEPT

FG02-89ER14031 05/31/95
$004440-0401-7$ TEMPE

FG02-89ER45398 06/14/95

CHEMISTRY DEPT

FG02-90ER14111 05/31/96 004440-0001-1 TEMPE

FG02-91ER61127 02/14/97
$004440-0001-1$ TEMPE

FG02-91ER14227 07/14/94

CHEMISTRY DEPT 004440-0601-X TEMPE

FG03-94ER45510 $11 / 30 / 96$
SOLID STATE SCIENCE CENTER FOR $004440-2301-1$

SOLID STATE SCIENCE CENTER FOR $004440-2301-1$ TEMPE

FG03-94ER25213 05/31/96 004440-0001-1 TEMPE

FG03-93ER14378 09/28/96
$004440-0601-X$ TEMPE

FG03-93ER14404 12/14/96 $004440-0601-X$ TEMPE

CHEMISTRY DEPT
WEATHERIZATION ASSISTANCE FOR LOW INCOME PERSONS

AZ $01 \quad \$ 7,540,216 \quad \$ 4,358,501$

ARIZONA CYCLE 15 ADMINISTRATIVE GRANT

$\$ 50.000$

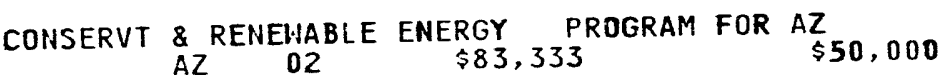

$\begin{array}{lll}\text { WEATHERIZATIUN PROGRAM INSTAL LATION OF INSULATION } & \$ 152,150\end{array}$ $\underset{A Z}{\operatorname{ELECTRONIC} \text { STRUCTURE AND REACTIVITIES DF CLUSTERS OF HEAVY }} \underset{\$ 01}{\$ 807,346}$

DESIGN AND CONSTRUCTION OF A CENTER FOR SCIENCE AND $A Z \quad 01 \$ 42,985,000 \quad \$ 25,000,000$

SPECIFIC MUTAGENESIS OF A CHLOROPHYLL-BINDING PROTEIN $A Z$ MZ $\$ 488,993 \quad \$ 486,113$

MOBILE IONS IN FAST ION $\$ 641,581$ CONDUCTING SYSTEMS

CONTINUUM DAMAGE MECHANICS II

$$
\text { DZ } 01 \text { MECHANICS } \$ 410,500 \quad \$ 268,441
$$

TIME OF FLIGHT MASS SPECTROMETRY OF DNA FOR RAPID SEQUENCE $A Z \quad 01 \quad \$ 1,216,433 \quad \$ 860,734$

GENERATION, DETECTION \& CHARACTERIZATION OF GAS-PHASE TRANSI

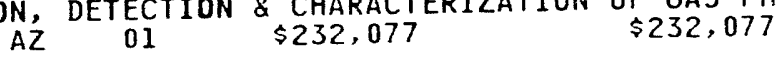

HIGH RESOLUTION INTERFACE NANOCHEMISTRY AND STRUCTURE

$$
\text { AZ } 01 \text { \$310,257 } \$ 99,391
$$
$\begin{array}{ccc}\text { A MICROAHALYTICAL (SIMS) STUDYOF THE TRACE } \\ \text { AZ } & \text { ELEMENT AND ISOT } \\ \$ 64,098 & \$ 180,000\end{array}$

CHARACTERIZING THE DYNAMICS OFSPATIO-TEMPORAL DATA
$\$ 105,965$

$$
\text { AZ } 010 \% 105,965 \quad \$ 105,965
$$

APPLICATION OF NEW CONCEPTS INSOLID ELECTROLYTES, AND PRESS

$$
A Z \text { OI } \$ 409,681 \text { \$133,018 }
$$

SUPRAMOLECULAR STRUCTURES FOR PHOTOCHEMICAL ENERGY CONVERSIO

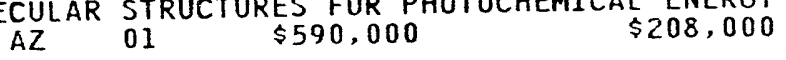




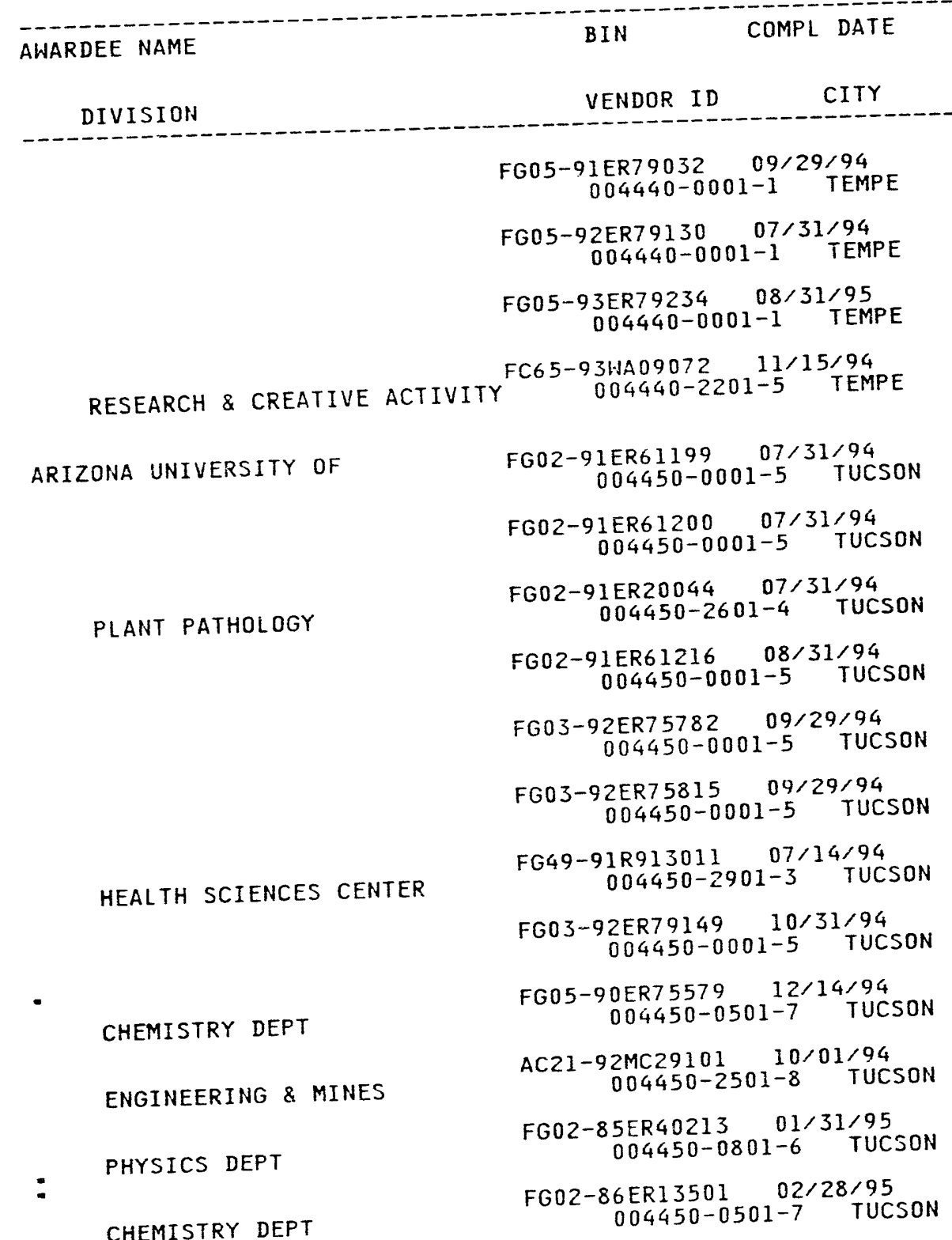

CONG CONTRACT

VALUE

TO DATE

$P / S$

AN ENERGY-FILTERED TEM FOR QUANTITATIVE ELECTRON MICROSCOPY

$\begin{array}{ll}\text { AZ } & \$ 663,077 \\ & \end{array}$

$\begin{array}{rrr}1992 & \text { INSTRUMENTATION } \\ \text { AZ } & \text { X-RAY CRYSTALLOGRAPHY OF PHOT } \\ & \$ 208,860 & \$ 208,860\end{array}$

1993 UNTVERSITY INSTRUMENTATION PROGRAM - CONTROLLED PLANAR

1993 UNIVERSITY INSTRUMENTATION PROGRAM -
AZ

CONVERSION OF RICE HULLS TO DIESEL FUEL

CONVERSION OF RICE HULLS TO $\$ 271,773$ DIESEL FUEL $\$ 124,081$

STOCHASTIC MODELING OF MACRODISPERSION IN UNSATURATED HETERO

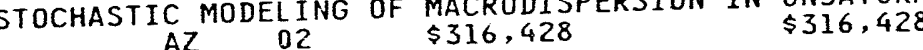

TRANSFDRT OF SUBSURFACE BACTERIA IN POROUS MEDIA

ROLE OF A PECTOLYTIC ENZYME INTHE PROGRAMMED RELEASE DF CELL

THE LAND COMPONENT OF THE GL OBAL CLIMATE $\begin{gathered}\text { SYSTEM WITH ADEQUAT } \\ \$ 369,240\end{gathered}$

AN ANALYTICAL THREE-DIMENSIONAL NEUTRON TRANSPORT BENCHMARK

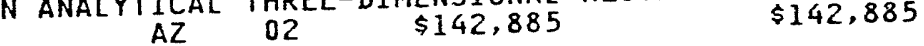

REACTOR SHARING $02 \quad \$ 13,900 \quad \$ 13,900$

CYCLE XIII GRANT

REACTOR INSTRUMENTATION UPGRADE

SOLAR VARIABILITY DETECTION FACILITY $\$ 240,167$

$\begin{array}{ccc}\text { EM-HIGH RESOLUTION SUBSURFACE IMAGING AND NEURAL NETWORK } & \text { NOLE } \\ \text { AZ } & \$ 353,244 & \$ 353,244 \text { AH92 }\end{array}$

THEORY \& PHENOMENOLOGY OF STRONG AND WEAK INTERACTION HIGH E

THEORY \& PHENOMENOLOGY $\$ 3,830,310 \quad \$ 2 \quad \$ 3,579,400$

CHEMICAL ACTIVATION OF MOLECULES BY METALS $\$$ AZ $\$ 866,683$ 


\begin{tabular}{|c|c|}
\hline & $\begin{array}{c}\text { FG02-92ER20066 05/31/95 } \\
004450-0001-5 \text { TUCSON }\end{array}$ \\
\hline & $\begin{array}{c}\text { FG02-92ER20079 06/30/95 } \\
004450-0001-5 \text { TUCSON }\end{array}$ \\
\hline & $\begin{array}{c}\text { FG02-92EK40733 } 06 / 30 / 95 \\
004450-0001-5 \text { TUCSON }\end{array}$ \\
\hline & $\begin{array}{c}\text { FG03-93ER25155 03/31/95 } \\
004450-0001-5 \text { TUCSON }\end{array}$ \\
\hline & $\begin{array}{c}\text { FG03-93ER75886 05/31/95 } \\
004450-0001-5 \text { TUCSON }\end{array}$ \\
\hline HEALTH SCIENCES CENTER & $\begin{array}{c}\text { FG49-93R915015 } 06 / 30 / 95 \\
004450-2901-3 \text { TUCSON }\end{array}$ \\
\hline & $\begin{array}{c}\text { FG03-93ER61526 } 11 / 30 / 95 \\
004450-0001-5 \text { TUCSON }\end{array}$ \\
\hline ARID LANDS OFFICE OF & $\begin{array}{c}\text { FC65-9411A10634 11/09/95 } \\
004450-3101-8 \text { TUCSON }\end{array}$ \\
\hline PLANT PATHOLOGY & $\begin{array}{c}\text { FG02-89ER]4038 } 06 / 30 / 96 \\
004450-2601-4 \text { TUCSON }\end{array}$ \\
\hline & $\begin{array}{c}\text { FG03-93ER12133 } 02 / 29 / 96 \\
004450-0001-5 \text { TUCSON }\end{array}$ \\
\hline PHYSICS DEPT & $\begin{array}{c}\text { FG03-93ER45488 } 03 / 31 / 96 \\
004450-0801-6 \text { TUCSON }\end{array}$ \\
\hline & $\begin{array}{c}\text { FG03-93ER40792 } 04 / 30 / 96 \\
004450-0001-5 \text { TUCSON }\end{array}$ \\
\hline & $\begin{array}{c}\text { FG03-93ER61595 04/30/96 } \\
004450-0001-5 \text { TUCSON }\end{array}$ \\
\hline & 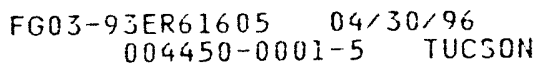 \\
\hline & $\begin{array}{c}\text { FG03-93ER14349 } 05 / 31 / 96 \\
004450-0001-5 \text { TUCSON }\end{array}$ \\
\hline & $\begin{array}{c}\text { FGOJ-93ER20120 07/14/96 } \\
004450-0001-5 \text { TUCSON }\end{array}$ \\
\hline
\end{tabular}
ENGINEERING THE PRODUCTION OF SUGAR ALCOHOLS IN TRANSGENIC ROLE OF ZEIN PROTEINS IN $\$ 279.121$ STRUCTURE \& $\begin{gathered}\text { ASSEMBLY OF } \\ \$ 279,121\end{gathered}$ PROBIHG VACUUM STRUCTURE IN NUCLEAR COLLISIONS

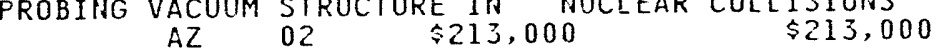
STUDIES OF SPATIAL AND TEMPORAL DISORDER IH MACROSCOPIC SYST

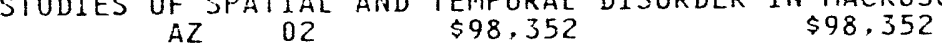
RESEARCH IN "DRIVE REINFORCEMENT NEURAL NETWORKS FOR REACTOR $\begin{array}{rrrr}\text { AZ } & 02 & \$ 107,341 & \$ 107,341\end{array}$

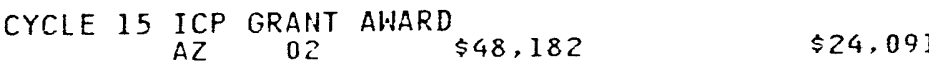
RESEARCH ON THE EFFECT OF BIOSURFOCTANTS ON BIODEGRIDATION

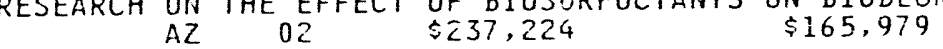
DEVELOPMENT OF A COMMERCIALLY ACCEPTABLE SYSTEM FOR UTILIZIN

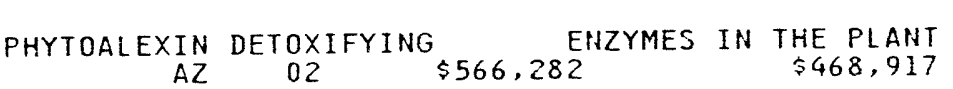
RESEARCH IN THE CREATION AND DESTRUCTION OF C60 AND OTHER FU AZ $02 \quad \$ 905,258 \quad \$ 594,201$

RESEARCH IN ARTIFICALLY STRUCTURED MAGNETIC MATERIALS AZ 02 A $\$ 270,000 \quad \$ 180,000$

$\begin{array}{ccc}\text { DYNAMIC BEHAVIOR OF EXCITED HADRONIC MATTER } \\ \text { AZ } 02 & \$ 303,000 & \$ 196,000\end{array}$ THE ACCESSIBILITY OF CHROMATINAS A BIOLOGICAL DOSIMETER FOR

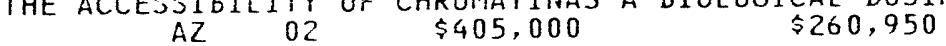
THEORETICAL STUDIES ON RADIATION-INDUCED TRANSFORMATIONS IN $A Z \begin{array}{lll}02 & \$ 290,000 & \$ 189,631\end{array}$

REJEARCH INTO "A MODEL APPROACH TO HYDRODENITROGENATION CATA AZ $02 \quad \$ 287,147$ T194,394

RESEARCH IN MOLECULAR CHARACTERIZATION OF THE ROLE OF A CALC $004450-0001-5$ TUCSON 


\section{AWARDEE NAME}

BIN

COMPL DATE

DESCRIPTION OF WORK

BIN

DIVISION

VENDOR ID CITY TUCSON

STATE DIST VALUE OBLIGS
TO DATE

P/S

APPLIED MATHEMATICS DEPT OF $004450-3001-1$

$\begin{array}{lcl}\text { AND } & \text { NUIILINEAR } & \text { PROCESSES } \\ A Z & 03 & \$ 507,150\end{array}$

$\$ 163.560$

FG03-93ER14396 09/30/96
$004450-0001-5$ TUCSON

FG03-93ER61727 09/14/96
$004450-0001-5$ TUCSON

CHEMICAL ENGINEERING DEPT

FG22-94PC94203 09/30/97
$004450-0401-0$ TUCSON

FGOJ-93ER $45500110 / 31 / 97$
$004450-0001-5$ TUCSON

FILM COOLING IN A FULSATING STREAM

$\$ 136,301$

$A Z$ TH 02 \$429,473

DE TROPOSPHERIC AN

THE FOCUS OF THE RESEARCH IS ON MID-LATITUDE TROPOSPH INTERACTIONS BETHEEN TRACE METALS, SODIUM AND SORBENTS IN CO
$\$ 199,999$

EARLY STAGES OF NUCLEATION

$\begin{array}{lll}\text { AG } & \text { NUCLEATION } \\ 02 & \$ 470,067 & \$ 124,339\end{array}$

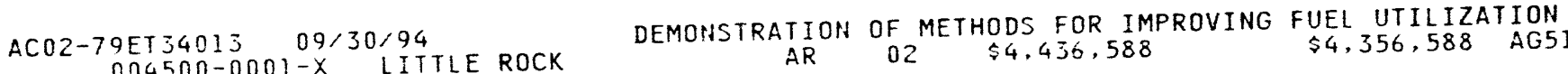

AKKANSAS POWER \& LIGHT CO $004500-0001-X$ LITTLE ROCK

AR $\quad 03$

$\$ 0$

$\$ 0$

CRO1-83NE44363 0 03/15/30
$004500-0001-X$ LITTLE ROCK

AHARDS $\$ 1,353,599$ NEW FEDERAL FUHDS UNDER NEW HEATHERIZATION $A R \quad 02 \$ 10,391,271 \quad \$ 8,404,153$

ARKANSAS STATE OF FG FG6-90R659000 03/31/95 $004510-0701-8$ LITTLE ROCK

DHS OFF OFF CG46-93R649300 06/30/95

AIDC ENERGY DFFICE DIV

ARKANSAS STATE UNIVERSITY CHEMISTRY \& BIOCHEMISTRY

CHEMISTRY \& BIOCHEMISTRY

CHEMISTRY OR64510-0501-5 LITTLE ROCK

AWARDS $\$ 29,413$ NEW DOE, AUTHORIZES $\$ 15,116$ NEW STATE
\$ FG06-94RL12766 02/06,95
$004520-0101-3$
STATE UNIVERSITY USED ENERGY RELATED LABORATORYEQUIPMENT GRANT $\begin{array}{ccccc}\text { FG09-935R18375 } 09 / 21 / 94 \\ 004520-0101-3 & \text { STATE UHIVERSITY USED ENERGY REIATED LABORATORYEQUIPMENT GRANT } & \text { AR } & 01 & \text { \$O }\end{array}$ $\begin{array}{ccccc}\text { FG09-94SR18402 } 12 / 15 / 94 & \text { USED ENERGY RELATED LABORATORYEQUIPMENT GRAHT } \\ 004520-0001-7 & \text { STATE UNIVERSITY } & \text { AR } & 01 & \text { SO }\end{array}$

FG09-945R18386 11/03/94 USED ENERGY RELATED LABORATORYEQUIPMENT GRANT 004520-0001-7 STATE UNIVERSITY ASED ENERGY RELATED LAB EQUIFMENT

$\begin{array}{rrr}\text { FG34-94RF00815 } 04 / 27 / 95 & \text { USED ENERGY RELATED LAB EQUIFMENT } \\ 004520-0201-X & \text { STATE UNIVERSITY AR OI } & \text { ST }\end{array}$

$\$ 0$

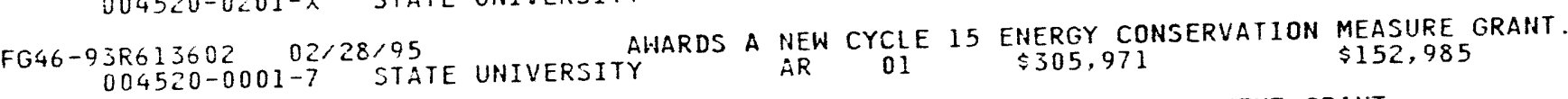

FG09-94SR18440 05/08/95 USED ENERGY RELATED LABORATORYEQUIPMENT GRAIT $004520-0101-3$ STATE UNIVERSITY AR OI 


\section{ARKANSAS TECH UNIVERSITY}

FG02-90CH10443 12/30/94 $151600-0001-1$ RUSSELLVILLE

ARKANSAS UNIVERSITY OF

$$
\begin{aligned}
& \text { FG02-91ER75659009/29/94 } \\
& 004530-0002-9 \text { LITTLE ROCK } \\
& \text { FG02-91ER75668 } 09 / 29 / 94 \\
& 004530-0002-9 \quad \text { LITTLE ROCK } \\
& \text { FG05-92ER40753 08/31/95 } \\
& \text { 004530-0002-9 LITTLE ROCK }
\end{aligned}
$$

ELECTRONICS \& INSTRUMENTATIOH $022-93 P C 93203 \quad 08 / 19 / 96$ TITE ROCK

$$
\text { FG05-93ER79163006/14/95 }
$$$$
\text { D04530-0003-7 PINE BLUFF }
$$

ARLINGTON TOWN SCHOOL DISTRICT FG41-94RI96006 05/31/96 $4 R 196006$
$099200-0001-0 \quad$ ARLINGTON

ARMCO INC

RESEARCH \& TECHNOLOGY

$$
\text { GM07-93IDI1107 02/07/98 }
$$$$
\begin{aligned}
& 3101110702 / 07 / 98 \\
& 16940-0101-1
\end{aligned}
$$

ARMS CONTROL \& DISARMAMENT AGC

$$
\begin{gathered}
\text { AI01-90DP50073 02/01/95 } \\
004610-0001-6 \text { WASHINGTON }
\end{gathered}
$$$$
\text { AI01-92DP50087 01/02/97 }
$$$$
\text { 004610-0001-6 WASHINGTON }
$$

ARMY DEPARTMENT OF

DEPARTMENT OF
CORPS DF ENGINEERS

CORPS OF ENGINEERS

\section{STRICOM}

$$
\begin{aligned}
& \begin{array}{c}
\text { AI08-93NV11442 09/16/98 } \\
004620-0983-1 \text { MOBILE }
\end{array} \\
& \text { AI96-94P019011 07/31/94 } \\
& \begin{array}{c}
\text { AI32-94AL } 96954 \quad 09 / 30 / 94 \\
004620-7988-0 \quad \text { OZARK }
\end{array} \\
& \begin{array}{r}
\text { AI } 04-90 A L 6584409 / 30 / 98 \\
004620-0056-7 \text { FORT CHAFFEE }
\end{array}
\end{aligned}
$$

DESIGN AND CONSTRUCTION OF A CENTER FOR ENERGY STUDIES

$$
\begin{array}{lll}
A R \quad 03 & \$ 1,050,000 & \$ 838,100
\end{array}
$$

ARKANSAS EPSCOR DOE TRAINEESHIPS

$$
A R \quad 02 \quad \$ 1,500,000
$$

$\$ 750,000$

ARKANSAS EPSCOR DOE PLANNING GRANT

$$
A R \quad 02 \quad \$ 183,268
$$

$\$ 120,000$

ULTRA - RELATIVISTIC HEAVY IONNUCLEAR PHYSICS

$\begin{array}{cccc}\text { AR } & 02 & \$ 88,000 & \$ 67,000 \\ \text { ELECTROSTATIC } & \text { BENEFICIATION DFCDAL } & \\ \text { AR } & 02 & \$ 323,418 & \$ 199,964 \\ \text { PRE-FRESHMAN ENRICHMENT } & \text { PRDGRAM (PREP) } & -1993\end{array}$

PRE-FRESHMAN ENRICHMENT PROGRAM (PREP) - 1993

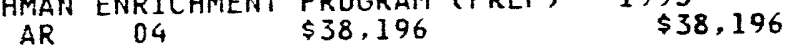

TITLE III OF THE NATIOHAL ENERGY CONSERVATION POLICY ACT

$$
\begin{aligned}
& \text { OF THE NATIONAL ENERGY CONSERVATION POLICY } \\
& \text { VT } 01 \text { \$108,000 }
\end{aligned}
$$

INTELLECTUAL PROPERTY ROYALITIES HOLDING COMPANY AGREEMENT

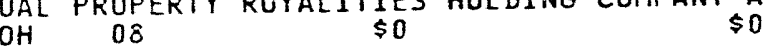

ACDA'S CONTINUED DEVELOPMENT AND MAINTENANCE OF AN ARM CONTR ACDA'S CONTINUED DEVELOPMENT AND MAINTENANCE OF AN A
DC 01

RESEARCH PROJECT ENTITLED CRISIS STABILITY AND THE OFFENSE $D$ PROJECT ENTITLED CRISIS STABILITY AND THE
DC $01 \quad \$ 150,000$

INTERAGENCY AGREEMENT FOR CORPS TO ACQUIRE PROJECT SALMON RE $\begin{array}{rrrr}\text { INTERAGENCY AGREEMENT FOR CORPS TO ACQUIRE } & \text { PROJECT } \\ \text { AL } & 01 & \$ 552,600 & \$ 552,600\end{array}$ REAL ESTATE SERVICE FOR COMMERCIALIZATION OF XMDEWS EXPERIMENTS AT THE AVIATION TEST BED AT FT. RUCKER, AL

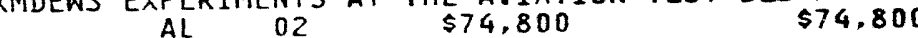
HOST RECRUIT TACTICAL TRAINING

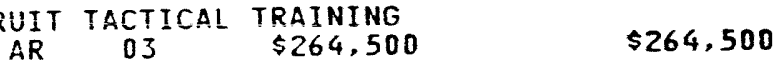




\section{DIVISION}

IERRA ARMY DEPOT

CORPS OF ENGINEERS

CDRPS OF ENGINEERS

CORPS OF ENGINEERS

CORPS OF ENGINEERS

WATERWAYS EXPERIMENT STATION

CORPS OF ENGINEERS

CORPS OF ENGINEERS

CORPS OF ENGINEERS

CORPS OF ENGINEERS

CORPS OF ENGINEERS

CORPS OF ENGINEERS

CORPS OF ENGINEERS

CORPS OF ENGINEERS

CORPS OF ENGINEERS

CORPS OF ENGINEERS
VENDOR ID CITY 3AD33231---09/30/97-

GM07-9 $31 D 11120 \quad 12 / 31 / 99$
$004620-7687-3 \quad$ HERLONG

AI 08-76DP00670 09/30/99 $004620-0919-X$ LOS ANGELES

A108-88NV10775 09/30/94 004620-0937-8 SAN FRANCISCO

GM08-85NV10451 01/22/96 004620-0937-8 SAIN FRANCISCO

AI34-93RF00662 10,31/94 $004620-0915-7$ GOLDEN

AI34-93RF00467 09/30/94 JRFO046? $09 / 30 / 94$
$004620-6615-0$ GOLDEN

AI01-91EW40008 12/30/95 004620-0931-9 WASHINGTON

AI01-92EW10372 09/30/97 004620-0931-9 WASHINGTON

AT01-93EH10842 02/15/98 004620-0931-9 WASHINGTON

ATO1-93EW1 0753 09/30/97 004620-0931-9 WASHINGTON

ATO1-93EW10755 09/30/97 $004620-0931-9$ WASHINGTON

AT01-93EW10754 09/30/97 $004620-0931-9$ WASHINGTON

AT01-93EH10756 09/30/97 004620-0931-9 HASHINGTON

AT21-92MC29208 $09 / 30 / 94$ $004620-0931-9$ WASHINGTON

AI21-91MC28086 10/01/96 004620-0931-9 WASHINGTON

AT21-92MC29235 02/12/95 $004620-0931-9$ WASHINGTON
CONG CONTRACT STATE DIST VALUE SUPPORT WASHFAX IV INTERIM (1)SYSTEM-
AZ OS TO DATE P/S FIRING RAIIGE USE AGREEMENT WITH SIERRA ARMY DEPOT REIMBURSE NV FOR ELECTRIC POHER USED AT ANGELS PEAK NEVADA B DESIGN REVIEW SUPPORT TO WASTEMANAGEMENT PROJECT OFFICE FORM PROCEDURAL FRAMEWORK FOR $\underset{\text { CA }}{\$ 05}$ PROVIDING DESIGN AND CONSTRUCT

MD/TECH ENGINEERING ASSISTANCE

$\$ 144,092$

MD/WASTE CEMENTATION

CO $02 \quad \$ 96,100,100$

TO DEVELOPE LINE ITEMS AND RELATED UNIT COST THAT ARE SPECIF TO DEVELOPE 01 \$ $\$ 1,740,000 \quad \$ 1,740,000$ PROVIDE DOE WITH A BOARD RAHGEOF TECHHICAL ASSISTANCE MAHAG PROVIDE DOE WITH A BOARD RANGEOF TECHNICAL ASSISTANC PROCUREMEHT OF TECHNICAL ANALYTICAL SERVICES IN GROUND FREEZ DC OFOCUREMENT OP $\$ 20,000 \quad \$ 20,000$ INDEPENDENT COST ESTIMATE REVIEH OF SELECTED ENVIRONMENTAL $R$ INDEPENDENT COST ESTIMATE $\$ 400,000 \quad \$ 400,000$ COST QUALITY ASSESSMENT (COMA)SUPPORT-TASK 200- REOBLIGATIO COST QUALITY ASSESSMENT $01,165,800 \quad \$ 1,165,800$

TASK 300-COST REVIEHS AT DOE OAK RIDGE AND SAVANNAH RIVER FI ASK 300-COST REVIENS 01 \$14,000 $\$ 414,000$ TASK- 100 USACE STAFF SALARIES AND EXPENSES TO SUPPORT HQ. FI DC $\quad 01 \quad \$ 500,000 \quad \$ 500,000$

ENVIRONMENTAL IMPACT STATEMENTFOR PINON PINE POHER PROJECT DC $01 \quad \$ 586,910 \quad \$ 586,910$ ENVIROHMENTAL TECHHICAL
DC ENVIRONMENTAL IMPACT STATEMENTFOR YORK FLUIDIZED BED 
DIVISION

CORPS OF ENGINEERS

CORPS OF ENGINEERS

CORPS OF ENGINEERS

CORPS OF ENGINEERS

EISENHOWER MEDICAL CENTER

CORPS OF ENGINEERS

IDAHO ARMY NATIONAL GUARD

1016TH FIELD SERVICE CO

CONSTRUCTION ENG RES LAB

AMCCOM

CORPS OF ENGINEERS

ENVIRONMENTAL HYGIENE AGENCY

TECOM

CORPS OF ENGINEERS

CORPS OF ENGINEERS

CORPS OF ENGINEERS AT21-92MC29250 11/11/94 04 WASHINGTON

AT21-93MC30333 09/30/95 004620-0931-9 NASHINGTON

AT21-93MC $30254 \quad 07 / 15 / 95$ 004620-0931\%9 HASHINGTON

AT01-94EH11230 07/22/94 004620-0931-9 HASHINGTON

AI09-925F.18272 01/17/98 004620-1109-7 FORT GORDON

AI 05-930R22109 09/30/94 004620-0971-8 FORT SHAFTER

GM07-9IIDI107201/23/97 004620-6373-9 POCATELLO

GM07-92IDI1095 04/08/99 004620-7273-8 POCATELLO

AI $05-920 R 2207509 / 30 / 94$ $004620-0805-3$ CHAMPAIGN

AI07-94IDI3j01 09/30/96 004620-7425-0 ROCK ISLAND

AI05-910R21996 05/27/97 $004620-0978-5$ LOUISVILLE

AI05-900R219]3 02/12/95 $004620-5551-5$ ABERDEEN

AI $04-94 A L 97246 \quad 10 / 31 / 94$ 004620-6251-1 ABERDEEN

AT21-9 $2 M C 2921205 / 05 / 95$
$004620-0979-3$ BALTIMORE

AI05-930R22137 12/31/94 $004620-0929-7$ VICKSBURG

AI01-92EW50602 09/30/96 004620-0929-7 VICKSBURG
ENVIRONMENTAL IMPACT STATEMENT(EIS) FOR TAMPA ELECTRIC IGCC

$$
\text { DC } 01 \text { \$443.360 } \$ 443.360
$$

TASK ORDER O10--ENVIRONMENTAL CHARACTERIZATIOH AND

$$
\text { DC } 01 \quad \$ 2.940 .038 \quad \$ 2.850 .000
$$

ENVIRONMEHTAL CHARACTERIZATIOHAND SITE REMEDIATIOH SUPPORT

$$
\text { DC } 01 \text { \$1,903,000 \$1,140,000 }
$$

TASK ASSIGHMENT 94-100 FOR MASTER INTERAGENCY AGREEMENT

$$
D C \quad 01 \quad \$ 10.000 \quad \$ 10.000
$$

TRAINING FOR DDEAMC RADIATION ACCIDENT/INCIDENT TEAM
GA 10 SURVEY OF WETLANDS IN HAWAII FOR THE HAWAII GEOTHERMAL PROJE
HI OI $\$ 78,000$

FIRING RANGE USE AGREEMENT FORIDAHO ARMY WATIOHAL GUARD HEA

INEL FIRING RANGE USE AGREEMENT WITH 1016 TH FIELD SERVICE CO

INTERAGENCY TASK FORCE ON ENERGY FACILITY SITING
IL
$\$ 975,000$

TREAT AND DISPOSE OF CONTAMINATED LOW LEVEL WASTE GENERATED IL 17 \$1.000.000 \$1.000.000

TO ESTABLISH AN IHTERAGENCY AGREEMENT WITH THE CORPS OF ENGI $\mathrm{KY} 03 \quad \$ 664.000 \quad \$ 664,000$

PERFORM AHALYTICAL TESTING OF NITROAROMATIC SAMPLES FROM THE $M D \quad 01 \quad \$ 496.750 \quad \$ 490.750$

INTERAGENCY AGREEMENT BETWEEN THE DEPT OF ENERGY \& THE U.S.

$$
M D \quad 01 \quad \$ 49.900 \quad \$ 49.900
$$

ENVIRONMENTAL TECHNICAL SUPPORT SERIVCES; TASK ORDER

$$
\text { MID } 03 \text { \$1 } 15.000 \text { \$115.000 }
$$

SUBBOTTOM HYDRO-ACOUSTIC SURVEY OF CLINCH RIVER AND POPLAR C

$$
M S 02 \text { \$137,150 \$137.150 }
$$

SITE CHARACTERIZATIOH - AHALYSIS COHE PENTROMETER SYSTEMS IS

$$
\text { MS } 02 \quad \$ 2,700,000 \quad \$ 2,000,000
$$




DWARDEE NAME
SECURITY OPERATION
CORPS OF ENGINEERS
CORPS OF ENGINEERS
CORPS OF ENGINEERS
CORPS OF ENGINEERS
CORPS OF ENGINEERS

CORPS OF ENGINEERS

ARDEC

CORPS DF ENGINEERS

CORPS OF ENGINEERS
COMPL DATE

\section{DESCRIPTION OF WORK}

BIN

COMPL DATE

VENDOR ID
AI08-9INVI1079--06/30/96004620-0029-X VICKSBURG

AI05-89ER $1399904 / 30 / 95$
$004620-4863-2$ DURHAM GM07-93ID11116 12/31/99 GM07-93ID11116 $12 / 31 / 99$
$004620-7586-9$ FORT BRAGG AIO2-94CH10592 09/30/94
$004620-0921-1$ OMAHA AI02-94CH10617 10/31/94 004620-0921-1 OMAHA

AI04-84AL27686 09/30/95 004620-0921-1 OMAHA

AI13-85ID12579 04/04/99 004620-0921-1 OMAHA

AI05-940R22141 09/30/97 004620-0916-5 HANOVER

AIO4-93AL $97292 \quad 09 / 27 / 94$
DOVER

AI01-92EH50616 12/30/94 004620-7182-0 PICATINNY ARSENA

GM04-84AL23521 09/30/99 $004620-0902-5$ ALBUQUERQUE

GMO4-72AL38701 09/30/99 004620-0002-8 ALBUQUERQUE GMO4-78AL38704 09/30/99 004620-0002-8 ALBUQUERQUE GM04-71AL $03028 \quad 09 / 30 / 99$ 004620-0002-8 ALBUQUERQUE GM04-87AL45505 09/02/99 004620-0002-8 ALBUQUERQUE GMO4-87AL52148 08/26/97 $004620-0902-5$ AL BUQUERQUE GM04-84AL53613 01/16/99 004620-0002-8 ALBUQUERQUE
CONG CONTRACT

OELIGS TO DATE P/S

STATE DIST VALUE FIELD AND LABORATORY INVESTIGATIO ENGINEERING ASSISTANCE AND FIELD AND LABORATORY $\$ \$ 339,358$ TNE MOLECULAR BIOLOGY OF ANAEROBICAROMATIC BIODEGRADATION
NC 02

INEL FIRNG RANGE USE AGREEMENTWITH FORT BRAGG \$0

DISPOSAL LOW-LEVEL WASTE AND LOW-LEVEL RADIOACTIVE ACTIVE DISPOSAL LOW-LEVEL WASTE AND LOW-LEVEL RADIOACTIVE DEVELOPMENT OF WORKPLAN FOR IMPLEMENTING $\begin{aligned} & \$ \text { REMOVAL ACTION } \\ & 02 \$ 4,000,000\end{aligned}$

REAL ESTATE SERVICES IN COLORADO
NE 02 \$1, 366,075
$\$ 1,366.075$

GRAND JUNCTION FACILITY LEASE TO THE CORPS OF 19.5 ACRES NE $\quad 02$

OF TRITIUM CONTAMI DEMONSTRATION OF CRYOGENIC IMMOBILIZATION OF TRITIUM $\$ 150,000$

THERMAL BATTERIES DISPOSAL FORDOE IN PICATINNY CUSTODY THERMAL BATTERIES DISPOSAL FORDOE IN PICATINNY CUSTO $\$ 39,000$
HJ EARTH PENETRATION RADAR IMAGING SYSTEM(EFRIS)

EARTH PENETRATION RADAR IMAGING SYSTEM(EPRISI $\$ 2,600,000$ CONSTRUCTION MANAGEMENT SERVICES

$\$ 0$

PROVIDES MUTUAL SUPPORT SERVICES BETWEEN DNA AND DOE ON A PERSHING II \& SETS FORTH RESPONSIBILITIES FOR DEVELOPMENT \&

SETS FORTH ARRANGEMENTS BY WHICH DOE, THROUGH SANDIA CORP NM 01 \$O

JOINT TEST HORKING GROUP, DEVELOP THE PLANS FOR AND THE CORPS HILL ACQUIRE LAND \& INTERESTS ON BEHALF OF DOE

ESTABLISH AN ARRANGEMENT BETWEEN THE DEPARTMENT DF THE ARMY NIM 01 
PACIFIC COMMAND

SPECIAL OPS COMMANE

CORPS OF ENGINEERS

WHITE SANDS MISSILE RANGE

CORPS OF ENGINEERS

FORT STEHART

CHARLESTON DISTRICT

CORPS OF ENGINEERS

CORPS OF ENGINEERS

CORPS OF ENGINEERS

CORPS OF ENGINEERS

CORPS OF ENGINEERS

CORPS OF ENGINEERS

CORPS OF ENGINEERS
GMO4-89AL57504 09/30/99 004620-4702-4 ALBUQUERQUE

GMO4-81ALO $02477 \quad 12 / 31 / 99$ 004620-0002-8 ALBUQUERQUE

GM04-93AL84048 04/12/98 004620-3402-X ALBUQUERQUE

GMO4-93AL $94684 \quad 08 / 24 / 98$ 004620-0002-8 AL BUQUERQUE

A132-91AL72727 09:30/94 004620-0902-5 ALBUQUERQUE

AI32-94AL96961 09/30/94 004620-5062-9 LAS CRUCES

AI22-93PC93025 09/28/93 004620-0922-X PITTSBURGH

AI09-89SR18094 02/28/95

AI09-85SRI4066 12/31/95 $004620-2534-9$ AIKEN

AT09-85SR15139 12/31/95 $004620-0934-3$ AIKEN

ATO9-85SR15140 12/31/95

AI05-920R22019 09/30/94 004620-0964-5 NASHVILLE

AI05-910R21995 09/30/94 004620-0964-5 HASHVILLE

A105-920R22065 09/30/95 004620-0964-5 WASHVILLE

AI05-910R22002 12/31/96 004620-0964-5 NASHVILLE

AI05-910R21917 12/31/99 004620-0964-5 NASHVILLE COOPERATIVE PROGRAM OF RESEARCH \& DEVELOFMENT INTENDED TO

ESTABLISH A PROGRAM AND FORUM FOR COMBINING AND ANALYZING NM 01

USE OF CTA LIVE FIRING RAHGE $\$ 0$

$\$ 0$ THIS AGREEMENT SETS FORTH THE GENERAL POLICY FOR THE
NM
01

REAL ESTATE SERVICES

$\$ 173,500$

$\$ 173.500$

CALIBRATION/UPGRADE SERVICES

$$
\text { NM } 02 \text { \$5,000 }
$$

ENVIRONMENTAL TECHNICAI SUPPORT SERVICES

$$
P A \$ 14 \quad \$ 166.955 \text { \$166.955 }
$$

HELICOPTER SERVICES

$$
\text { SC } 03 \text { SERVICES } \$ 20.000 \quad \$ 20.000
$$

PROJECT MANAGEMENT SERVICES $\$$

PROJECT MANAGEMENT SERVICES

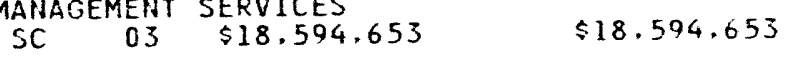

PROJECT MANAGEMENT SERVICES

$S C \quad 03 \$ 38,846,404 \quad \$ 38.276,404$

PREPARE REAL ESTATE PLANNING DOCUMENT FOR EACH FORK POPLAR C $\begin{array}{ccc}\text { REAL ESTATE PLANNING DOCUMENT FOR } & \text { EACH FORK } \\ \text { TN } 05 & \$ 108.165 & \$ 108.165\end{array}$

POLYCHLORINATED BIPHENYLS (FCB) SOURCE IDENTIFICATION PROJEC INATED BIFHENYLS (FCB) SOURCE IDENTIFICATIOH
IN 05

VALUE ENGINEERING SERVICES

$$
\text { TN } 05 \text { SERVICES } \$ 57,000
$$

$\$ 57.000$

EAST FOR POPLAR CREEK ENVIRONMENTAL RESTORATION ACTIVITIES TH $05 \quad \$ 255,000 \quad \$ 255,000$

ENVIRONMENTAL RESTORATION AND WASTE MANAGEMENT ACTIVITIES IN $05 \quad \$ 510,155 \quad \$ 510,155$ 


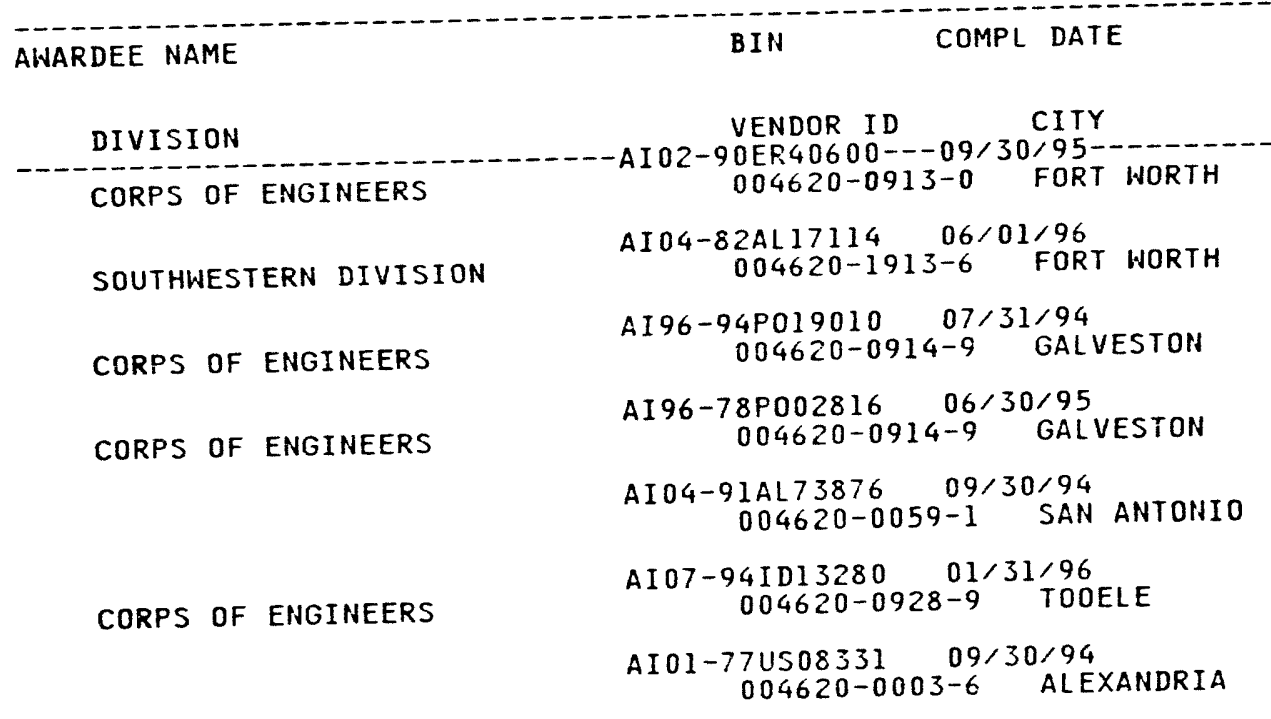

FOREIGN SCIENCE \& TECH CTR

AI01-90IN00528 $09 / 30 / 95$
$004620-5869-7 \quad$ CHARLOTTESVILLE

AI01-92CE50352 $06 / 30 / 97$
$004620-7308-4 \quad$ FORT BELVOIR

FUELS \& LUBRICANTS

AI05-820R20954 09/30/94 $004620-0010-9$ FORT LEHIS

GM04-94AL $9471103 / 30 / 99$ 004620-0010-9 FORT LEWIS

GM04-94AL $94700 \quad 01 / 21 / 99$ 004620-0010-9 FORT LEWIS

IST SPECIAL FORCES AIRBORNE

GM07-90ID11066 09/30/96 004620-6010-1 FORT LEWIS

AT06-94RL12183 07/26/95 004620-0961-0 WALLA WALLA

CORPS OF ENGINEERS

AT06-93RL12107 07/26/95 004620-0961-0 HALLA WALLA

CORPS OF ENGINEERS

AT06-92RL12106 07/26/95 004620-0961-0 WALLA WALLA

CORPS OF ENGINEERS

AT06-90RL12103 07/26/95 004620-0961-0 WALLA WALLA

DESCRIPTION OF WORK

CONG CONTRACT OBLIGS

TO DATE P/S STATE DIST VALUE TX 12 \$1,500,000 $\$ 450,000$ INTERAGENCY AGREEMENT WITH THECORP OF ENGINEERS INTERAGENCY AGREEMENT WITH THECORP OF $\$ 223,946,968$ REAL ESTATE SERVICE FOR $\begin{array}{r}\text { COMMERCIALIZATION OF } \\ \$ 20,000\end{array}$ FUNDING FOR REAL ESTATE ACQUISTION TX $09 \$ 173,526,168$

$\$ 173,502,668$ HOST RECRUIT TACTICAL TRAINING TX $20 \quad \$ 100,000 \quad \$ 100,000$ DISPOSAL OF RADIOACTIVE HASTE

UT $01 \quad \$ 400,000 \quad \$ 400,000$

ACQUISITION OF CRIDE OIL

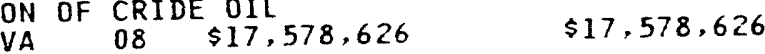

PROVIDE DOE OBLIGATION IN SUPPORT OF INTELLIGENCE COMMUNITY

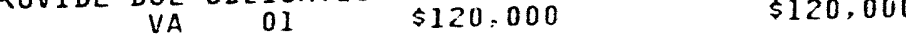
$\begin{array}{lll}\text { SUPPORT FROM U S ARMY FOR AMFAPROGRAM } & \$ 300,000\end{array}$ ENVIRONMENTAL AND ADMINISTRATIVE SUPPORT AT FORT LEHIS

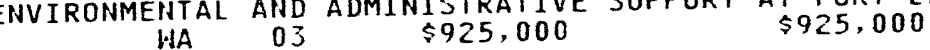
THIS AGREEMENT SETS FORTH THE GENERAL POLICY FOR THE SCHEDUL THIS AGREEMENT SETS FORTH THE GENERAL POLICY FOR THE SCHEDUL INEL FIRING RANGE USE AGREEMENT A/E SERVICES FOR DESIGN AND CONSTRUCTION OF THE
$\$ 1,437,000$ WA 05 \$1, 437,000 $\$ 1,437,000$

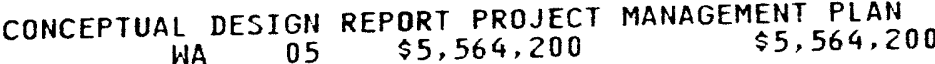
TECHNICAL AND MANAGEMENT EXPERTISE RELATIVE TO AND MANAGEMENT EXPERTISE RELATIVE
WA $05 \quad \$ 4,087,000$ ASSIGN TASK ORDER $\begin{aligned} 003-E M 1-01-00 & \$ 12,992,500\end{aligned}$ CORPS OF ENGINEERS 


\section{AWARDEE NAME}

BIN

COMPL DATE

DESCRIPTION OF HORK

DIVISION

VENDOR ID STATE DIST

CONTRACT

OBLIGS TO DATE

CORPS OF ENGINEERS

CORPS OF ENGINEERS

CORPS OF ENGINEERS

CORPS OF ENGINEERS

CORPS OF ENGINEERS

CORPS OF ENGINEERS

CORPS OF ENGINEERS

CORPS OF ENGINEERS

CORPS OF ENGINEERS

CORPS OF ENGINEERS

CORPS OF ENGINEERS

CORPS OF ENGINEERS

- corps of engineERs

CORPS OF ENGINEERS

CORPS OF ENGINEERS ARPEL
AT06-90RL12101 07/26/95
004620-0961-0 WALLA WALLA

AI06-90RL12074 07/26/95 004620-0961-0 WALLA WALLA

AT06-92RL12105 07/26/94 004620-0961-0 WALLA WALLA

AT06-94RL12112 07/26/95 004620-0961-0 WALLA WALLA

AT06-93RL12181 07/26/95 004620-0961-0 WALLA WALLA

AT06-93RL12111 07/26/95 004620-0961-0 WALLA WALLA

AT06-93RL12108 07/20/95 $004620-0961-0$ WALLA WALLA

AT06-93RL12178 07/26/95 004620-0961-0 HALLA WALLA

AT06-93RL12110 07/26/95 004620-0961-0 WALLA WALLA

AT06-93RL12179 09/15/94 $004620-0961-0$ HaLLA WALLA

AT06-93RL12180 06/30/95 $004620-0961-0$ WALLA WALLA

AT06-94RL12185 07/26/95 004620-0961-0 WALLA WALLA

AT 06-94PL12184 07/26/95 004620-0961-0 WALLA WALLA

AI04-33AL23266 09/30/94 004620-0940-8 HUNTINGTON

AI21-94MC $31340 \quad 07 / 04 / 94$ 004620-0940-8 HUNTINGTON

FG02-94FE63303 04/30/95 $238480-0001-6$ MOHTEVIDEO
ASSIGN TASK ORDER 001-PM-01-00

HA 05 \$2,102,000

$\$ 2.102 .000$

PERFORMANCE DF ENVIRONMENTAL RESTORATION

$$
\text { WA } 05 \text { SO }
$$

WELL HEAD SURVEYING TECHNICAL AND MANAGEMENT EXPERTISE FOR

RL STATE TRIBAL GOVERHMENTS PER REQUIREMENTS OF

PROVIDES PROGRAM SCHEDULING COST ESTIMATING SUPPORT AND

HA $05 \quad \$ 220,000 \quad \$ 220,000$

MAPPIHG/GIS. PROVIDE USACE TE CHNICAL SERVICES FOR MAFPING A HA. PROVIDE USACE TE CHNICAL SERVICES FOR M

A $/ E$ SERVICES FOR RESEARCH INTOPUREX PLANT FOR DEVELOPMENT HA 05 \$1,617,400

A $/ E$ SERVICES FOR DESIGN AND CONSTRUCTION OF THE WA 05 S $\$ 79,000 \quad \$ 79,000$

ORDNANCE AND EXPLOSIVE WASTE SURVEY AND REMEDIATION HA $05 \$ 2,811,000 \quad \$ 2,811,000$

PROVIDE TECHNICAL ASSISTANCE IN ACTIVITY BASED WA 05 \$316,800

TASK I PROJECT MOBILIZATION AND CONCEPTUAL DESIGN WA $05 \quad \$ 942,400 \quad \$ 942.400$

ACTIVITY BASED COST ESTIMATES AND BASELINE WA 05 \$350,000 $\$ 350,000$

A/E SERVILES FOR PROJECT W-252PHASE II LIQUID

HA $05 \quad \$ 300,000 \quad \$ 300,000$

REAL ESTATE SERV (PA) UMTRA
$04 \$ 1,930,010$
$\$ 1,930,010$

CLEAN COAL TECHMOLOGY COHSTRUCTION REVIEH-

WORKSHOP ON ENVIROHMENTAL REGULATIOHS AND AJSESSMENT IN 


BIN

COMPL DATE

DESCRIPTIDN OF WORK

DIVISION

BIN

CITY

STATE DIST

CONTRACT

VENDOR ID

VALUE

OBLIGS

$F / S$

\section{ART SERVICES INC}

ARTCOM PLUS INC

ARTCOM PLUS PHOTO

ARTHUR D LITTLE INC

ARTISAN GRAPHICS INC

ARTS UNIVERSITY OF THE

- asahina \& CO

ASAP SERVICES CORP

EASF SYYTEMS INC
$A B 01-94 A D 25706 \quad 06 / 30 / 95$
$111070-0001-7$ WASHINGTON

$A B 01-94 A D 2491105 / 31 / 95$

$111070-0001-7$ WASHINGTON

$A B O 1-94 A D 25704$
$227710-0001-6$

AB01-94AD24910 05/31/95 227710-0001-6 ARLINGTON

ABO1-94AD25719 $06 / 30 / 95$
ARLINGTON

AC01-90CE23821 $09 / 27 / 94$
$004770-0002-9$ CAMBRIDGE ACO1-92EH891j2 07/30/95
$004770-0002-9 \quad$ CAMBRIDGE AC02-92CE50343 $09 / 01 / 94$
$004770-0002-9 \quad$ CAMBRIDGE

AB01-94AD25705 $06 / 30 / 95$
$004810-0001-1 \quad$ ALEXANDRIA

FG43-94R364113 05/31/95
$237960-0001-0$ PHILADELPHIA

$A B 01-926 C 2030209 / 30 / 94$
$203250-0001-8 \quad 0 S A K A$

ABO1-94AD11113 $05 / 31 / 95$
$237480-0001-4$ WASHINGTON

AC01-94EI24251 09/18/94 BALTIMORE
BPA FOR GRAPHIC ARTS AND RELATED SERVICES. 07/01/94 THRU 06/ BPA FOR GRAPHIC FRODUCTION ANDOTHER VISUAL ART DELIVERABLES BPA FOR GRAPHIC FRODUCTION ANDOTHER VISUAL ART DELIVERABLE TOOI BPA FOR GRAPHIC ARTS AND RELATED SERVICES. 07/01/94 THRU 06/ BPA FOR GRAPHIC ARTS AND RELATED SERVICES. OT/O1/\$O TOOI BPA FOR GRAPHIC PRODUCTION ANDOTHER VISUAL ART DELIVERABLES VA 08 \$0

BPA FOR PHOTO AND RELATED SERVICES

$\$ 0 \quad$ T015

TECHNICAL SUPPORT SERVICES FORCE 42 MA 08 \$2,311,097

$\$ 2,311,097 \quad$ B 543

TECHNICAL SUPPORT SERVICES FOREH-24.

$\$ 4,730,000 \quad F 999$

MULTIFUEL REFORMERS FOR FUEL CELLS IN TRANSPORATION

MULTIFUEL REFORMERS FOR FUEL CELLS IN $\$ 2,003,153$ AG8

BPA FOR GRAPHIC ARTS AND RELATED SERVICES. 07/01/94 THRU 06/ BPA FOR GRAPHIC ARTS AND RELATED SERVICES. OT/01/\$O TOOI

TITLE III OF THE NATIONAL ENERGY CONSERVATION POLICY ACT I OF THE NATIONAL ENERGY CONSERVATION POL
PA
01

PROVIDE HITH PATENT SERVICES

\$0 R418

TEMPORARY SECRETARY

$\$ 0$

$\$ 0 \quad R 699$

HIGH-SPEED, HIGH-VOLUME, NON-IMPACT PRINTER $M D \quad 08 \quad \$ 358,842$ 


\begin{tabular}{|c|c|c|c|c|c|c|c|}
\hline AWARDEE NAME & BIN & DATE & & & DESCR & F WORK & \\
\hline DIVISION & VENDOR ID & CITY & STATE & $\begin{array}{l}\text { CONG } \\
\text { DIST }\end{array}$ & $\begin{array}{l}\text { CONTRACT } \\
\text { VALUE }\end{array}$ & $\begin{array}{l}\text { OBLIGS } \\
\text { TO DATE }\end{array}$ & $P / S$ \\
\hline
\end{tabular}

ASHE MEMORIAL HOSPITAL

CONTROLLER OFFICE OF THE

\section{ASHEBORO CITY OF}

BOARD OF EDUCATION

ASPEN CENTER FOR PHYSICS

ASPEN TECHNOLOGY INC

ASSOC RELOCATION MGMT CO INC
FG44-92R434987 08/30/94 208070-0001-9 JEFFERSON FG44-93R435376 09/30/95 208070-0201-1 JEFFERSON

FG44-92R434988 12/31/94 $220650-0101-1$ ASHEBORO $\begin{array}{rc}\text { FG03-93ER40766 } & 12 / 31 / 94 \\ 004990-0001-3 & \text { ASPEN }\end{array}$

AC22-94PC94053 03/30/97 005020-0001-5 CAMBRIDGE AC22-92PC92002 06/25/95 $005020-0001-5$ CAMBRIDGE

AC22-93PC93049 09/29/95 $005020-0001-5$ CAMBRIDGE

AD65-93WG23177 06/10/96 197260-0003-3 GLENDALE

AD65-93WJ22069 07/16/96 197260-0003-3 GLENDALE

AD65-93WA10784 02/11/98 197260-0089-0 CITRUS HEIGHTS

AD65-93WG22466 03/09/98 197260-0090-4 ROSEVIILE

AD65-93WA10896 04/01/96 197260-0091-2 SALINAS

AD65-93WJ21437 05/03/98 197260-0092-0 YUBA CITY

AD6 5-93HL $04110 \quad 02 / 05 / 98$ 197260-0088-2 AKRON

AD65-93WA11166 06/25/96 197260-0094-7 BRUSH
CYCLE 14 - INSTITUTIONAL CONSERVATION PROGRAM. APPRDVED BUD SEE ATTACHED PAGE NO. 2 OF THIS NOTICE OF FINANCIAL ASSISTAN NC 05 N $\$ 63,570$ $\$ 36,572$

CYCLE 14 - INSTITUTIONAL CONSERVATION PROGRAM. APPROVED BUD NC $04 \quad \$ 33,571$ $\$ 28,709$

NEW AWARD - \$6,000 FUNDING FOR1993 ASPEN WINTER ELEMENTARY CO $03 \quad \$ 12,000$ $\$ 12,000$

LICENSING AGREEMENT FOR ASPEN PLUS WITH MODEL MANAGER $\begin{array}{lllll}\text { MA } 08 & \$ 48,836 & \$ 48,836 & \text { D399 }\end{array}$ LICENSING AGREEMENT LICENSING AGREEMENT FOR ASPEN PLUS WITH MODEL MANAGER

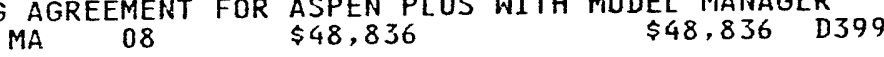

RELOCATION SVC $\underset{03}{\mathrm{H} K E I T H}$ JONES $\begin{array}{rrrr}\text { HOME RELOCATIONS SVCS FOR } & \text { RAY FORBES } & \$ 26,345 & \text { R499 }\end{array}$ HOME RELOCATION SVCS FOR DAVID RADOSEVICH

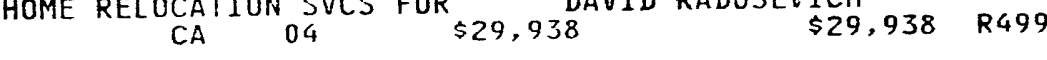
RELOCATION SVCS FOR $\$ 39,039$ EWARD MOED HOME RELOCATION FOR JIM FISHER $\begin{array}{llll}\text { OCATION FOR JIM FISHER } & \text { FIS } \\ \text { CA } & \$ 61,073 & \$ 61,073 & \text { R499 }\end{array}$

RELOCATION SVC FOR ROLLIE $\underset{\text { CA }}{02} \underset{30,656}{\text { ORTIZ }} \quad \$ 30,656 \quad$ R499 HOME RELOCATION SVCS FOR ERNEST BAIER $\begin{array}{cllll}C O & 04 & \$ 7,664 & \$ 7,664 & R 499\end{array}$ HOME RELOCATION SVCS LARRY
CO $\$ 26,943 \quad R 499$ 


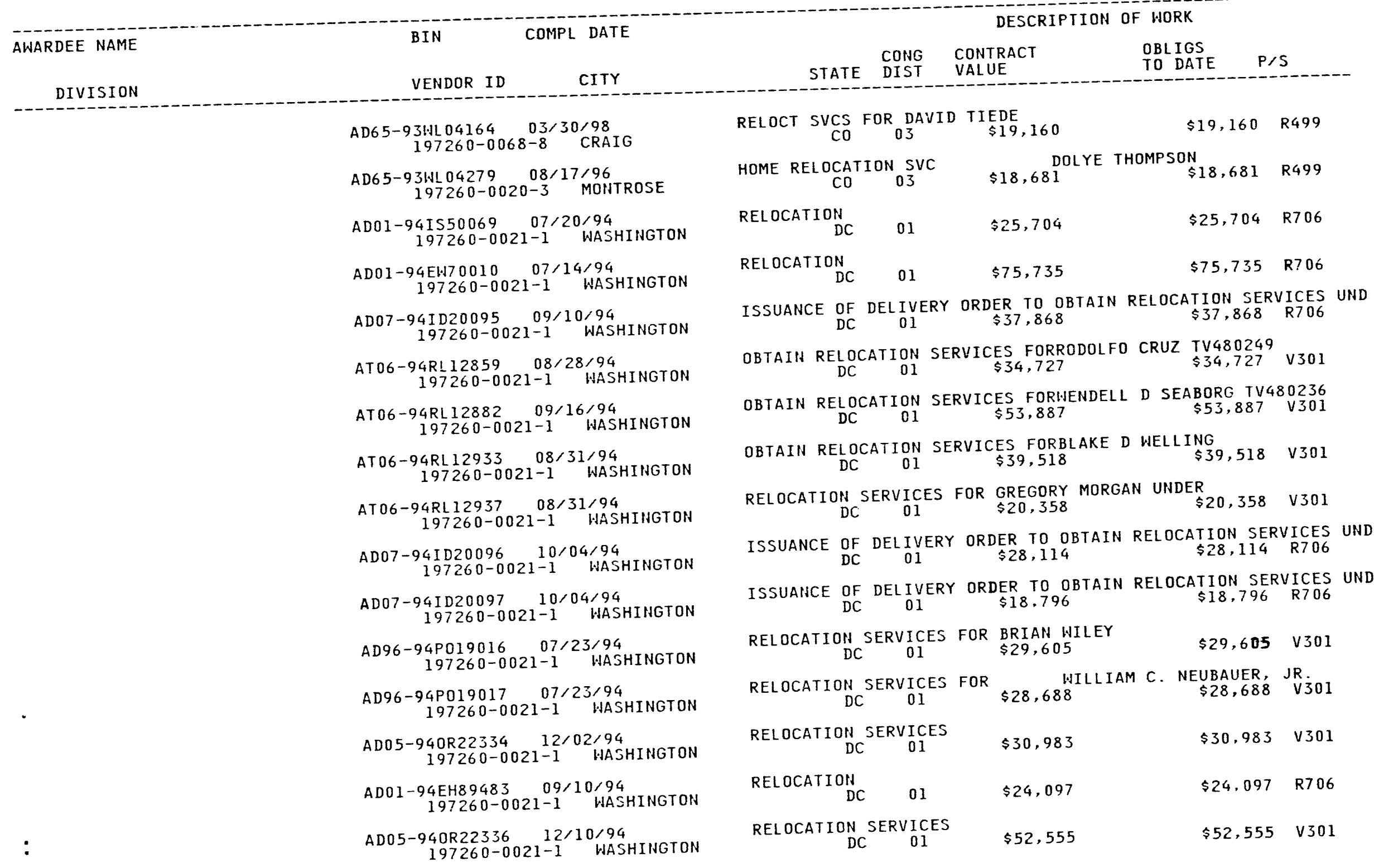




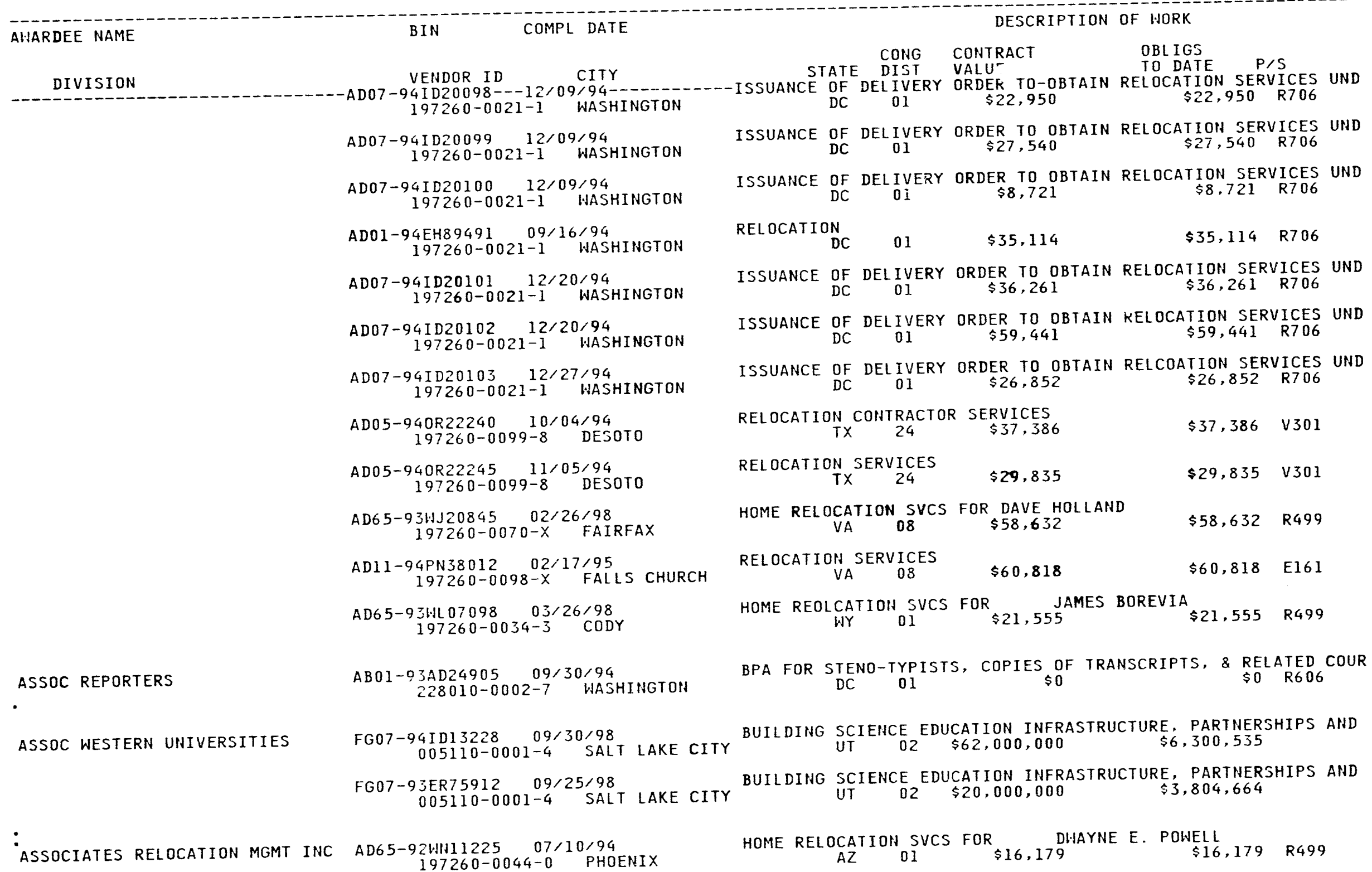




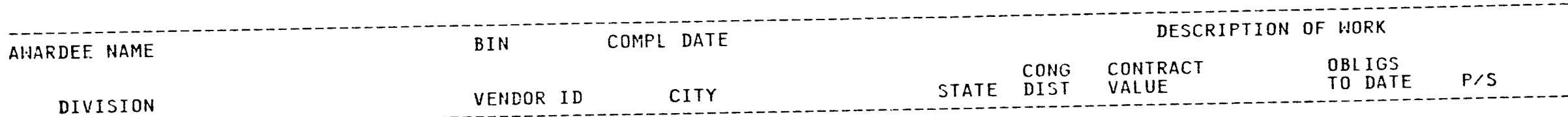
AD65-93WF01894 $12 / 28 / 95$
$197260-0027-0$ TEMPE AM01-91AD37074 07/22/94 $197260-0021-1$ WASHINGTON

ASTA ENGINEERING INC

ACO1-92RH00254 08/18/95
$195190-0003-8$ GERMANTOWN

AC01-9311510045 09/08/94 195190-0003-8 GERMANTOHN

ASTRO-ARC POLYSOUDE

FC34-94RF00862 05/02/95
$237840-0001-0$ SUH VALLEY

AT \& T TECHNOLOGIES INC AD01-94AD34161 $09 / 30 / 94$

SECURE COMMUIIICATION SYST UHIT II9380-0301-2 GREENSBORO FEDERAL SYSTEMS \& BUS UNIT

AT\&T TECHNOLOGIES INC

ADO1-94AD34227 07/08/94 $119380-0201-6$ GREENSBORO

$A B 02-85 C H 10227 \quad 12 / 31 / 99$ $19380-0001-3$ GREENSBORO

ATHENS CITY OF SCHOOL DISTRICT

ATHOL MEMORIAL HOSPITAL

FG45-94R562183 $12 / 31 / 95$
$238340-0101-5$ THE PLAINS

$F G 41-93 R 193500$ A1 $30 / 94$
$228300-0001-3$ ATHOL

FG44-92R410604 09/30/94 $005260-0201-8$ ATLANTA

HOUSIIHG AUTHORITY

ATLANTA PARTHERSHP OF BUS \& ED

:Atlanta uiniversity
HOM RELOCATION SVCS FOR $\begin{aligned} & \text { RONALD BECK } \\ & \text { AZ } \\ & 01\end{aligned}$

$\$ 26,198 \quad R 499$

RELOCATION REALTY SERVICES NATIONWIDE
$D C \quad 01$
\$0 $\quad$ 301

QUALITY ASSURAHCE TECHHICAL SUPPORT SERVICES FOR THE OFFICE MD 06 \$1,715,109 \$1,493,427 R408

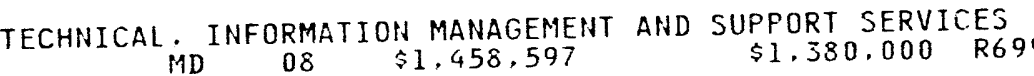

COOPERATIVE AGREEMENT BETHEEN DOE, EG\&G AND PARTICIPANT

DELIVERY ORDER FOR 2 STU-III SECURE DATA DEVICES

$\begin{array}{cccc}\text { DELIVERY ORDER FOR } & 2 \text { STU-1II SECURE DATA DEVICES } \\ 12 & \$ 3.990 & 5811\end{array}$

STU-III VOICE/DATA TERMINALS

NC $\begin{array}{llll}12 & \$ 75.810 & \$ 75.810 & 5805\end{array}$

UNIX SYSTEM V PROPRIETARY SOFTWARE AND SOFTWARE $\begin{gathered}\$ \text { ICENSE } \\ \$ 0\end{gathered}$

ICP-NEW AWARD

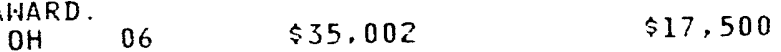

FY'93 ICP AHARD D $\quad \$ 6.800 \quad \$ 3,400$

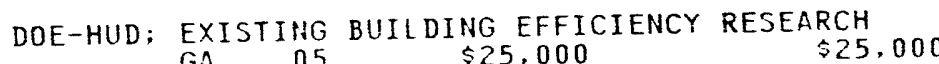

PROPOSAL TO IMPROVE SCIENCE, MATH AND TECHIOLOGY EDUCATION A GA $04 \quad \$ 19,400 \quad \$ 19,400$

MINORITY UNDERGRADUATE TRAINING IN ENERGY-RELATED CAREERS GA $05 \quad \$ 405,570 \quad \$ 359,040$ 


ALARDEE NAME

DESCRIPTION OF WORK

AHARDEE NAME

BIN

COMPL DATE

OBLIGS VEINDOR ID
DIVISION

VEHDOR ID CITY

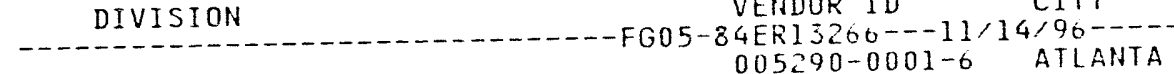

STATE COHG COHTRACT

\section{CHEMISTRY DEPT}

CHEMISTRY DEPT

ATLANTIC AEROSPACE ELECTRONICS FG05-87ER45306 11,30/94
$005290-0301-5$ ATLANTA FG22-90PC90292 08/31/94 $005200-0301-5$ ATLANTA

ATLAHTIC MANAGEMENT CENTER IHC AMO1-92AD10772 09/30/97 $003300-0002-0$ ALEXAHDRIA ADO1-93AD83251 07/31/94 003300-0002-0 ALEXANDRIA ADO1-94ADS3457 07/31/94 003300-0002-0 ALEXANDRIA AD01-94ADS3459 09/30,94 003300-0002-0 ALEXANDRIA ADO1-94AD85462 08/31/94 $003300-0002-0$ ALEXANDRIA

RL13-74GJ31003 07/23/94 $169720-0001-7 \quad M O A B$

ATM SECURITY \& INVESTIGATIONS

ATM SERVICE CO

ATMOSPHERIC ENVIRONMENTAL RES
AC04-89AL55418 09/15/94
$176260-0001-3$ ALBUQUERQUE

AC $04-93 A L 75636 \quad 09 / 06 / 95$ $170260-0001-3$ ALBUQUERQUE

FG02-93ER61549 01/14,96

FG02-86ER60485 11/30/95 005430-0001-X CAMBRIDGE

FG02-90ER61064 10/31/96 $005430-0001-X$ CAMBRIDGE 005430-0001-X CAMBRIDGE

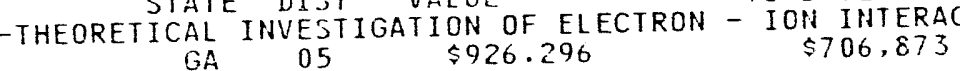

CERAMIC MATERIALS FROM PRECERAMIC ORGANOMETALLIC COPOLYMUS W GE 06 FAMIC MATERIALS FROM $\$ 559,865 \quad \$ 481,735$

ALTERNATIVE FORMULATIONS FOR REGENERABLE FLUE GAS CLEANUP CA GA $05 \quad \$ 174,270$

$\$ 159,955$

MULTI-SPECTUAL \& MULTI-SCALE DETECTIOH/DISCRIMINATION OF

$$
\begin{array}{lll}
M A & 0 & \$ 74,819
\end{array}
$$

PROVIDE TRAINING IN PROGRAM/PROJECT MANAGEMENT AND PROCUREME VA OE $\$ 25,425,426$ TRAINING $\$ 0 \quad \cup 006$ TRAINING VA 08 $\$ 78,784$ $\$ 78,784 \quad \cup 006$ TRAINING VA 08 $\$ 183.085$ $\$ 183.085 \quad 0006$ TRAINING COURSES $\$ 184,918$ $\$ 184,918 \quad 0006$ TRAINING COURSES

$\$ 59,054$ $\$ 59,054 \quad 0006$

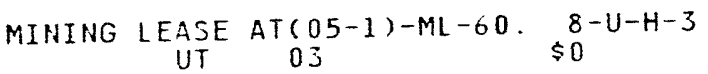

$\$ 0 \times 293$

SECURITY ESCORT SERVICES FOR DOE $/ A L$

$$
\begin{array}{llll}
\text { ESCORT SERVICES FOR DOE/AL } & \$ 2.067,801 & 5699
\end{array}
$$

ADMINISTRATIVE AND CLERICAL SERVICES IN SUFPORT OF PERSONIEL NM $01 \quad \$ 2,173,318 \quad \$ 1,086,658$ R408

ACCELRATED LINE-BY-LINE CALCULATINS FOR THE RADIATIVE MA 08 \$212,571 $\$ 136,782$

RADIATIVE CHEMICAL AND DYNAMICAL FEEDBACK FROCESSES MA 08 \$1,627,128 $\$ 1,472,786$ PROGRAM OI RADIATIVE TRANSFER MODEL DEVELOPMENT IN SUPPORT $M A \begin{array}{lll}\text { MA } 08 & \$ 1,254,850 & \$ 786,139\end{array}$ 


AHARDEE NAME
DIVISION

BIN

$$
\text { COMPL DATE }
$$

BIN

ATOM SCIENCES INC

ATOMIC ENERGY OF CANADA LTD

\section{ATOMIC SUPPORT AGENCY}

\section{AUBURN UNIVERSITY}

CHEMICAL ENGINEERING DEPT

MATERIALS ENGINEERING DEPT

CHEMICAL ENGINEERING DEPT

PHYSICS DEPT

PHYSICS DEPT

CHEMICAL ENGINEERING DEPT

CHEMICAL ENGINEERING DEPT

\section{FGO2-90ER61065 10/31/96 $005430-0001-X$ CAMBRIDGE}

FG05-94ER81659 07/17/94
$005440-0001-3$ OAK RIDGE

FC21-92MC2824512/31/94 005460-0001-0 CHALK RIVER FC02-91CHI0492 $09 / 30 / 96$
$005460-0002-9 \quad$ PINAWA

GMO4-76AL3868501/01/99 $171270-0001-8$ ALBUQUERQUE

FG05-85ER13357 $08 / 31 / 94$
$005530-0201-5 \quad$ AUBURN FG05-91ER54134 09/14/94 $005530-0501-4$ AUBURN

FG22-91FC91281 09/15/94 $005530-0201-5$ AUBURN FG05-85ER53206 10/31/94
$005530-0401-8$ AUBURN FG05-86ER53217 12/31/94 005530-0401-8 AUBURI

FG05-90ER54085 04/30/95 $005530-0001-2$ AUBURN

FG22-91PC91311 09,09/95 $005530-0201-5$ AUBURH

AC22-91PC91055 09/30/95 005530-0201-5 AUBUR!

FG03-935F19645 12,20/95
$005530-0001-2$ AUBURN

FG05-85ER53192 02/28/97 $005530-0001-2$ AUEUPH $\begin{array}{rrrr}\text { TESTBED MODEL AND DATA } & \text { ASSIMILATION FOR ARM } \\ \text { MA } & 08 & \$ 917.084 & \$ 578.573\end{array}$

SBIR GRANT FOR IMPROVED ANALYTICAL MEASUREMENTS TO MONITOR $N$ SBIR GRANT FOR IMPROVED ANALYTICAL MEASUREMENTS $\$ 74,982$

EM-SOIL TREATMENT TO REMOVE URANIUM AND RELATED MIXED EM-SOIL TREATMENT TO REMOVE $\$ 400,084 \quad \$ 300,847$

PRPARATION \& PACKAGING OF RADIOACTIVE HASTES \& DISPOSAL IN $00 \$ 39,494,445$

$\$ 3,794,000$ SET FORTH TERMS \& CONDITIONS FOR ALO EXCLUSIVE USE DF FC NM 01 \$O INTERFACIAL CHEMISTRY $\$ 943.855 \quad \$ 943.855$ "REPAIR HELDING OF FUSION REACTOR COMPONENTS" (NEN)

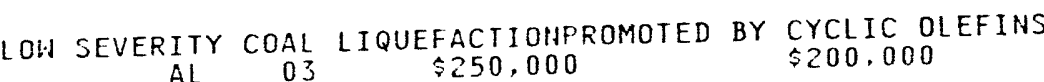
THEORETICAL STUUIES OF THE HEATING OF TOROIDAL PLASMAS HITH $\begin{array}{lll}03 & \$ 813.760 & \$ 792.091\end{array}$ THEORETICAL ATOHIC PHYSICS FORFUSION AL $03 \quad \$ 831,594$

EXPERIMENTAL STUDIES OF PLASMAFLUCTUATIONS USTHG ELECTRON $C$ AL 033 \$ $033.910 \quad \$ 833.910$

COUFIGURATION DIFFUSION OF ASFHALTENES IN FRESH DEACTIVATED $A L=\$ 245,912$ \$198,227

ROLE OF RESID SOLVENT IN CATALYTIC COPROCESSING USING FINELY $A L O 3 \$ 150,220 \quad \$ 150,000$ AGII

HIGH TEMPERATURE MATERIALS TECHHOLOGY RESEARCH FOR ADVANCED $A L$ OJ $\$ 120,000 \quad \$ 80,000$

EXPERTMEHTAL STUDIES OF RADIO FREQUENCY WAVE AND CONFINEMENT AL $03 \quad \$ 2,120,047 \quad \$ 1,480,603$ 


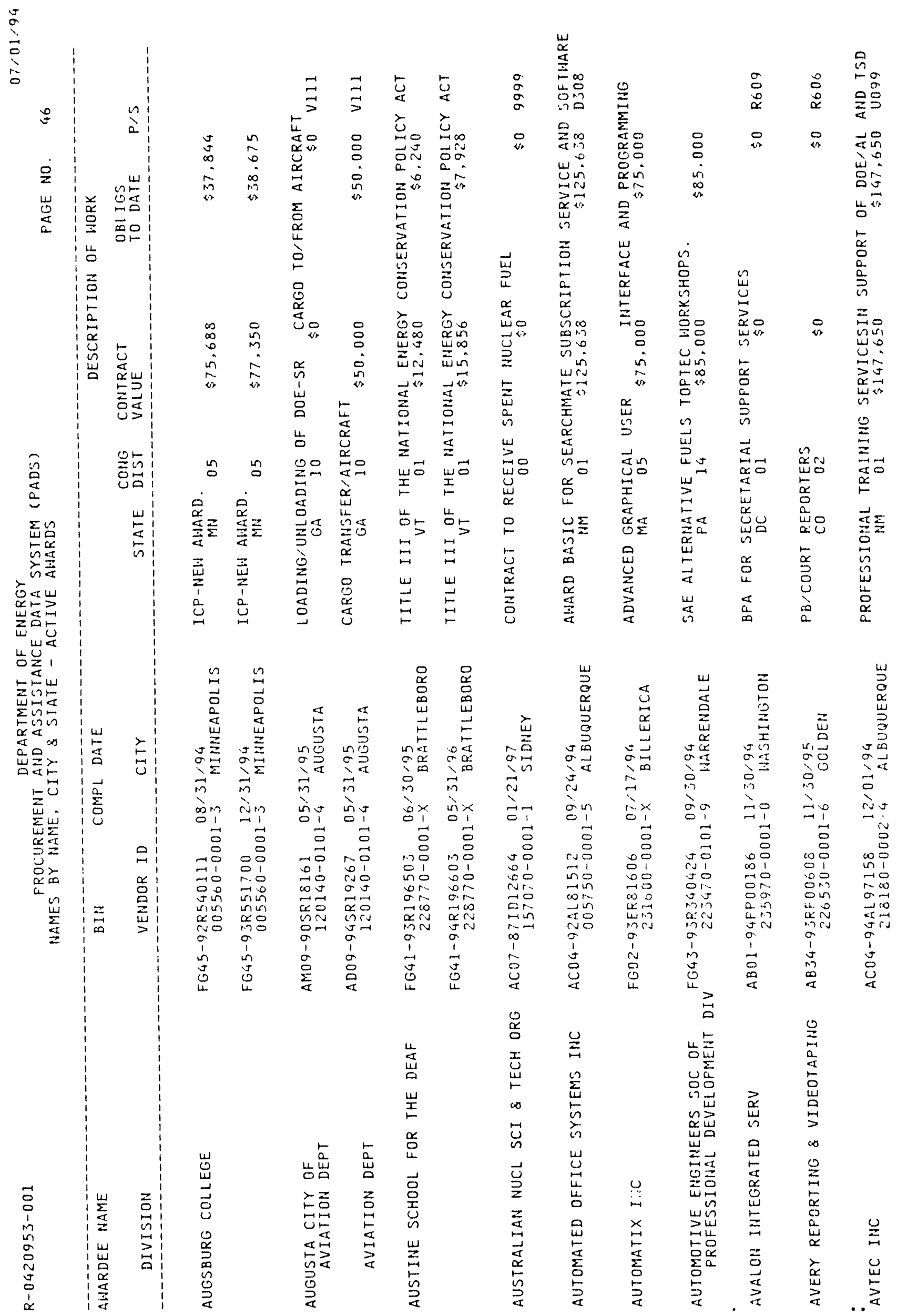




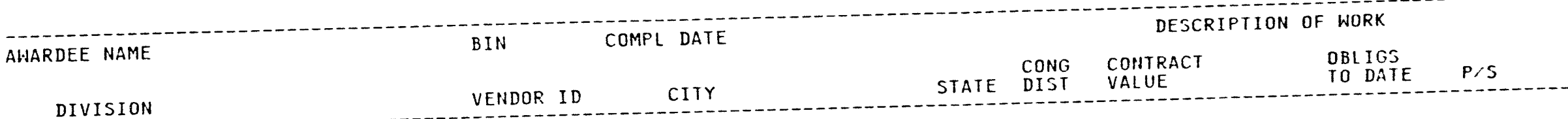

AWARE INC

AWD TECHNOLOGIES INC

B \& C CONSTRUCTION CO

B \& M TECHHOLOGICAL SVCS INC

B \& S TRANSPORT INC

B \& W HUCLEAR ENV SERVICE

BABCOCK \& WILCOX CO

RESEARCH \& DEVELOPMENT DIV

RESEARCH \& DEVELOPMENT DIV

$$
\begin{gathered}
\text { AC22-92PC92160 } 09 / 23 / 01 \\
005990-0001-5 \text { ALLIANCE } \\
\text { FR22-91PC90577 10/11/10 } \\
005990-0001-5 \text { ALLIANCE } \\
\text { FR22-94PC } 9430409 / 30 / 14 \\
005990-0001-5 \text { ALLIANCE }
\end{gathered}
$$$$
\text { ONC59103 } 05 / 24 / 95 \text { ALNCE }
$$

PARALLEL WAVELET METHODS FOR COMPUTATIOAL FLUID DYHAMICS MA 08 \$ $\$ 74.088$

74.088

RECYCLE OF CONCRETE
$\$ 289.571$ AH93

LOCATED IN THE FORRESTAL

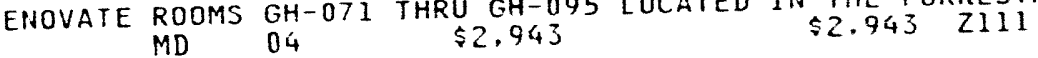
MATERIALS AHD EQUIPMEHT TO REHOVATE ROOMS ML LABOR, MATERIALS AND EQUIPMENT $\$ 4.550 \quad \$ 4,550 \quad \mathrm{Z111}$ TO PROVIDE SUPFORT SERVICE TO ASSIST ON - SIIE REVIEW $M A \quad 08 \quad \$ 450,000$ PROVIDE STOKER GRADE COAL FOR THE 200 AREA AND 300 AREA HA 04 \$7,500,000

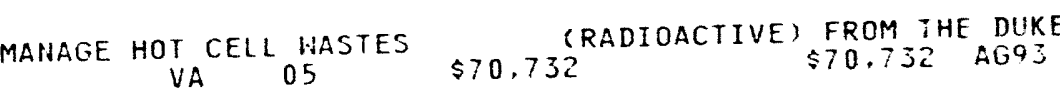

COOPERATIVE AGREEMEHT - DEVELOPMENT OF PROTOTYPE IIZNIN CONC

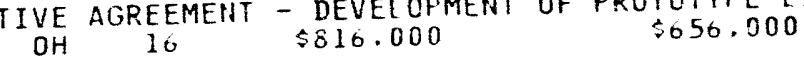
LNCB-CCT III OH $_{16} \$ 11.279,415 \quad \$ 5.442 .800$ EM "CHEMICAL DECONTAMINATIOH OF PROCESS EQUIPMENT USIHG $\mathrm{OH} 17 \quad \$ 861,90$ EM-DEVELOPMENT OF A LONG-TERM POST RADIATION MOHITOR $\begin{array}{cccc}O H & 17 & \$ 904.270 & \$ 741.647\end{array}$ ENGIMEERIMG DEVELOPMEHT OF ADVANCED COAL-FIRED LON-EMISSION $\mathrm{OH}$ 16 \$163.063.050 $\$ 4.143 .876$ AGI3 REPAYMENT AGREEMEN OH 16

$\$ 0$

REPAYMENT AGREEMENT $\$ 0$ so 
NUCLEAR EQUIPMENT DEPT

NAVAL NUCLEAR FUEL DIV

NAVAL NUCLEAR FUEL DIV

MAVAL NUCLEAR FUEL DIV

WAVAL NUCLEAR FUEL DIV

WAVAL NUCLEAR FUEL DIV

NAVAL NUCLEAR FUEL DIV

MAVAL NUCLEAR FUEL DIV

HAVAL HUCLEAR FUEL DIV

NAVAL NUCLEAR FUEL DIV

NAVAL NUCLEAR FUEL DIV

RESEARCH \& DEVELOPMENT DIV
ACO2-77ET37209 $12 / 31 / 94$
$005990-0402-9$ BARBERTOH

FR22-93PC93303 02/15/00 005990-0007-4 LORAIN

FCO2-92CE40945 09/29/94 D05990-0003-1 LYNCHBURG

ACQ5-850R21526 07/31/94 005990-0203-4 LYNCHBURG

AC07-88ID12701 07/01/95 005990-000j-1 LYHCHBURG

FCO7-88IDI2798 09/30/95 005990-000j-1 LYHCHBURG

AC11-89P1438184 12,31/94 $005990-0203-4$ LYINCHBURG

AC11-90FWj818: 01,$15 ; 90$ 0025990-0203-4 LYHCHBURO

AC11-9051139104 07/31/96 005990-0203-4 LYHCHBURG

AC11-92P1138191 07/31/95 $005990-0203-4$ LYNCHBURG

ACI1-93P113819401/01/96 005990-0203-4 LYIICHBURG

ACI1-93FN33193 10/30/96 005990-0203-4 LYIICHBURG

FC36-94G010011 09/30/98 005990-0203-4 LYHCHBURO

$A C 11-93 P H 38192 \quad 09 / 30 / 01$ 055990-0203-4 LYMCHBURG

AA11-90PN38188 07/24/10 055990-0203-4 LYIHCHBURG

CRO1-83HE44482 03/15/30 005990-0603-x LYICHBURG LARGE SCALE LMFBR STEAM GEHERATOR DEVELOPMENT (DOUBLE WALL OH 13 \$5 06 \$. 583 ESTABLISH RECOUFMENT/REPAYMENTPLAH OH $1 \mathrm{~J}$ $\$ 0$ $\$ 0$ CONTINUOUS FIBER CERAMIC COMPSISTE PRCGRAM (CFCC) PROCESS SCRAP LOTS $84-2$ THRU $84-5$ AHD RETURI THE URAHIUM REC VROCESS SCRAP L 05 \$A $\$ 9,405.752$ AAD RETURA THE URANIUM DEVELOPMENT OF RAIL/BARGE CASK $\checkmark A$ OG $06 \$ 23.669 .694$ $\$ 14.090 .960 \quad$ AG54 DEVELOPMENT OF HIGH PRESSURE HEAT EXCHANGE SYSTEMS
VA
05 FABRICATE NAVAL REACTOR CORES

$$
\text { VA OO \$119.702.917 \$119.702,917 } 1140
$$

FABRICATE HAVAL REACTOR CORES

$$
\text { VA NAVAL REACTOR CO \$132,394,448 \$132,241.948 } 4470
$$

FABRICATE HAVAL REACTOR CORES

VA OO \$486.105.686 \$486.105.486 4470

DEVELOP AHD FABRICATE STEAM GENERATOR
06 \$ $\$ 15.004 .400$

MAIUFACTURE THE LEAD SUBASSEMBLY OF THE HEH CONCEPT STEAM

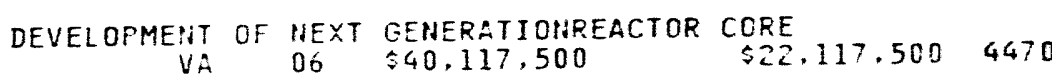

COMMERICAL OF MID-SIZE SUPERCONDUCTIVTY MAGNETIZ

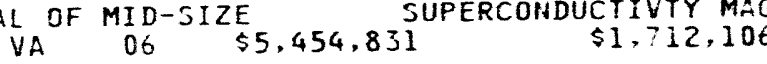

FABRICATE HAVAL REACTOR CORES

VA $06 \$ 214,389,900$

$\$ 214,315,295 \quad 4470$

FABRICATE NAVAL REACTOR CORES $\$ 0$

$\$ 0$

DISPOSAL OF SPENT NUCLEAR FUELAHD OR HIGH LEVEL RADIOACTIVE $\checkmark A$ SP 06 \$O $\$ 0$ 
AWARDEE NAME

BIN

COMPL DATE

DESCRIPTIOH OF HORK

DIVISION

VENDOR ID CITY OPCS9659-1 $005990-0005-8$

CITY

BABCOCK \& WILCOX IDAHO INC

AC07-921D13050 09/30/94 $216180-0001-2$ IDAHO FALLS

BABSON COLLEGE

BAKER UNIVERSITY

BALDWIN J KEVIN

BALTIMORE COUNTY OF BOARD OF EDUCATION

BOARD OF EDUCATION

BALTIMORE GAS \& ELECTRIC

BANGOR SCHOOL DEPARTMENT

BANNOCK CDUNTY OF SHERIFF'S DEPARTMENT

"BAHNOCK REGIONAL MEDICAL CTR

BAPTIST MEDICAL CENTER DE KALB

BBAPTIST MISSION SCHOOL

$$
\begin{gathered}
\text { FG } 41-92 R 19341507 / 31 / 94 \\
221430-0001-4 \quad \text { BABSON PARK }
\end{gathered}
$$

FG47-92R702697 08/31/94 223530-0001-0 BALDWIN CITY

$A B O 1-94 M I 10457 \quad 04 / 30 / 95$ $230270-0001-2$ HASHINGTON

FG43-93R353101 $08 / 31 / 94$
$006310-0301-0$ TOHSON

FG43-93R353201 03/31/95 $006310-0301-0$ TOHJON

CROI-83NE $44367 \quad 03 / 15 / 30$ 006320-0001-5 BALTIMORE

FG41-94R192606 06/30/96 BAIIGOR

GM07-921011089 09/30/99 006380-0101-3 POCATELLO $144040-0001-4$ POCATELLO

FG44-93R435274 08/31/95
$231810-0001-9$ FORT PAYNE

FG44-92R435167 10/31/94 225530-0001-4 CHARLOTTE AMALIE
GM07-91ID11084 09/30/99
MANAGE, OPERATE \& MAINTAIN GOVERIMENT-OHNED PRODUCTION \& SUP

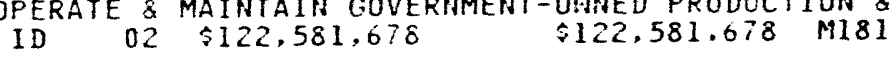

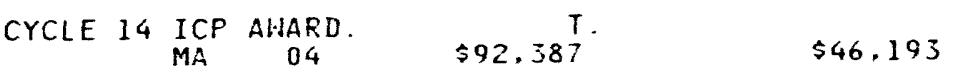

GRANT PROGRAMS FOR SCHOOLS ANDHOSPITALS AND FOR BUILDINGS 0 KS $02 \quad \$ 219.983 \quad \$ 100,000$

BPA FOR EEO IHUESTIGATIOHS 05/01/94-04/30/95 DC 01 \$0

今0 $\quad R 499$

TITLE III OF THE NATIONAL ENERGY CONSERVATION FOLICY ACT

TITLE III OF THE NATIONAL EHERGY COHSERVATIOH FOLICY ACT

DISPOSAL OF SPENT FUEL

$\$ 0$

$\$ 0$

FY'94 ICP AWARD.

$\$ 85,631$

$\$ 42,000$

INEL FIRING RAHGE USE AGREEMENT WITH BANNOCK CO. SHERIFF'S ID 02

MOU TO PROVIDE OPTIMAL CARE FOR EMPLOYEES OF THE INEL \& FOST ID 02

\$D

SEE ATTACHED PAGE NO. 2 OF THIS HOTICE DF FINANCIAL ASSISTAN $A L$ O $04 \quad \$ 27,065 \quad \$ 15,322$

CYCLE 14 - INSTITUTIOHAL COHSERVATION PROGRAM. APPROVED BUD - INSTITUTIOHAL COHSERVATION PROGRAM. APPRO
VI 01 \$11.922 
AWARDEE NAME

BIN

COMPL DATE

DIVISION

VENDOR ID

CITY

CDATE CONG

CONTRACT

VALUE

PAGE NO. 50

BARA KING PHOTOGRAPHIC INC

BARBER-SCOTIA COLLEGE

BAREISS ASSOC

BARNES KISSELLE RAISCH CHOATE

BARRY DR CAROLE JOYCE

BARSTOW COMMUNITY COLLEGE

BART ASSOC INC

BARTHOLOMEW CONSOL SCH CORP

BARTOL RESEARCH INSTITUTE

BARTON COUNTY COMMUNITY COLL

BASLER ELECTRIC CO

BATAVIA CITY OF

$$
\begin{array}{r}
\text { ABO1-94AD25720 06/30/95 } \\
\text { HYATTSVILLE }
\end{array}
$$

FG05-93ER79164 06,14/95 006460-0001-2 COHCORD

FC36-93CH10562 09/26/95 234580-0001-1 NEW PORT RICHEY

$A B 01-936 C 2031907 / 31 / 95$
$228930-0001-0$ DETROIT

$A B 04-92 A L 82551 \quad 10 / 30 / 94$ 229160-0001-1 AL BUQUERQUE

FG49-92R914035 08/31/95 223060-0001-8 BARSTOW

AC65-91WN0768 8 05/08/95 165890-0004-2 SACRAMENTO

AC65-901.1106702 05/25/95 $165890-0003-4$ FAIRVIEW

FG45-92R540014 02/28/95
$006700-0001-9$ COLUMBUS

FG02-91ER40626 01/31/95
$029830-0101-6$ NEWARK

FG47-92R702695 08/31/94 223350-0001-2 GREAT BEND

AM65-92WA09359 07/06/94 $181260-0001-5$ HIGHLAND

ACO2-76CHOO140 12/31/99
BPA FOR PHOTO AND RELATED SERVICES

$M D$ D

$\$ 0$

$\$ 0 \quad 1015$

PRE-FRESHMAN ENRICHMENT PROGRAM (PREP) - 1993

$$
\text { IIC } 08 \text { \$ } \$ 38,741 \text { ( }
$$

TECHHOLOGY TRANSFER FOR NEWLY DEVELOPED SENSORS

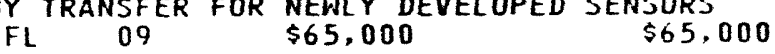

BPA FOR DOMESTIC PATENT APPLICATIONS

$\underset{M I}{\operatorname{DOMESTIC} \text { PATENT APPLICATIONS }}$

\$0 R418

$$
\begin{aligned}
& \text { PSYCHIATRIC EXAMINATIONS } \\
& \$ 35.000 \quad 0519
\end{aligned}
$$

TITLE III OF THE NATIONAL ENERGY CONSERVATION POLICY ACT

$$
\begin{array}{ccc}
\text { DF THE NATIONAL ENERGY CONSERVATION POLICY } \\
\text { CA } \\
35 & \$ 270,394 & \$ 135.197
\end{array}
$$

OL INDA-TRACY $500 \mathrm{KV} \mathrm{T.L.,} \mathrm{PART} 3$

$\begin{array}{lllll}C A & 04 & \$ 4,128,818 & \$ 4,128,818 & Y 219\end{array}$

$500 \mathrm{KV}$ TL. MAXWELL TO SACRAMENTO RIVER, CALIFORNIA

ICP - NEW AHARD $02 \quad \$ 121,018 \quad \$ 60,509$

NEW GRANT ENTITLED "PARTICLE THEORY AND COSMOLOGY"

GRANT PROGRAMS FOR SCHOOLS ANDHOSPITALS AND FOR BUILDIHGS 0

$$
\text { KS } 01 \quad \$ 172,130 \quad \$ 86,065
$$

LINE AND STATION RELAYS

$$
\text { IL } 21 \text { RELAYS } \$ 471,980 \quad \$ 155.290 \quad 6110
$$

ELECTRIC POHER AT GOVERHMENT-OWNED PUMPING STATIOHOH FOX RIV 
NAMES BY NAME, CITY \& ST

AISARDEE NAME

BIN

COMPL DATE

DIVISION

VENDOR ID

CITY 006760-0001-0 BATAVIA

BATESVILLE COMMUNITY SCH CORP

FG45-94R562001 06/30/95 $132300-0001-3$ BATESVILLE

BATH COUNTY OF SCHOOL DISTRICT

BATTELLE MEMORIAL IHSTITUTE COMPUTER INFORMA \& ED DEPT

COLUMBUS LABS

BAYAMON TECHNOLOGICAL UHIV

\section{BAYFRONT MEDICAL CENTER}

baylor college of medicine $006840-0001-6$ COLUMBUS
FG44-92R434960 07/31/94 $208580-0101-2$ ONINGSVILLE

W-7405-ENG-92 09/30/94 $006840-0301-5$ COLUMBUS

ATO2-89CH92990 09/30/94 $000840-0001-6$ COLUMBUS

FG02-90ER14135 0?/01/94
$000840-0201-9$ COLUMBUS AC22-91PC90366 07/31/94 $006840-0001-6$ COLUMBUS AC01-91EHO0151 10/30/94
$006840-0001-6$ COLUMBUS FG02-93ER40703 12/31/94
$000840-0001-6 \quad$ COLUMBUS AC22-93PC93251 02/27/95
$006840-0001-6$ COLUMBUS AC06-76RL01831 $09 / 30 / 97$
$006840-0002-4 \quad$ RICHLAND AC06-76RL01830 09/30/97 006840-0602-2 RICHLAND

FG44-85R432338 $12 / 31 / 94$
$132340-0001-8$ BAYAMON 132340-0001-8 BAYAMO FG44-92R434856 09/30/94
$224680-0001-3$ SAINT PETERSBURG

FGO3-94ER61830 02/28/97
$180640-0001-7$ HOUSTON
DESCRIPTION OF HORK

DESCRIPTION OF WORK

TO DATE P/S $\$ 15.000 \quad \$ 119$

STATE DIST VALUE

IL 15 :15.000

$\$ 3.000$

ICP - NEH AHARD.

$\$ 3.000$

CYCLE 14 - INSTITUTIONAL CONSERVATION PROGRAM. APFROVED BUD

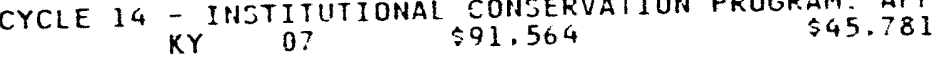

PERFORMS MULTI-PROGRAM WORK FOR DOE PRIMARILY IN IHE O4 PR $\mathrm{OH} \quad 15$ TO BATTELLE COLUMBUS LABORATORIESDECOMMISSIONING PROJECT AN INVESTIGATION OF HISTORY DEPENDENT DAMAGE IN TIME AN INVESTIGATION OF HISTORY DEPENDEHT DAMAGE INT

CHARACTERIZATION OF AIR TOXICSFROM A LABORATORY COAL FIRED OH IS OF AIR TOXICSFRO $\$ 999.902$ $\$ 999.902$ AGII

SUPPORT SEPVICES FOR DFFICE OFSECURITY EVAUATIONS

SUPPORT SERVICES FOR OFFICE OFSECURITY EVAUATIOHS

ADVANCED FESEARCH ON STRANDS AND CABLE FOR PARTICLE
$\$ 525,000$

OH 15 F $\$ 525.000$ FOAL FIRED POHER PLANTS UTIL

A STUDY OF TOXIC EMISIIOHS FROM COAL FIRED PONER PLANTS

USE PERMIT WA 04

$\$ 0$

S0 R699

BATIELLE MEMORIAL INSTITUTE/PACIFIC NORTHWEST WA O4\$5.013.473.817 \$3.448.870.511 M181

MONE PR $01 \quad \$ 295,360$

$\$ 88.843$

CYCLE 14 - IHSTITUTIONAL CONSERVATION PROGRAM. APPROVED BUD MAPPING AND CLONING CHROMOSOME

MAPPING AND 25 CL $\$ 1,401,648$

$\$ 494.700$ 


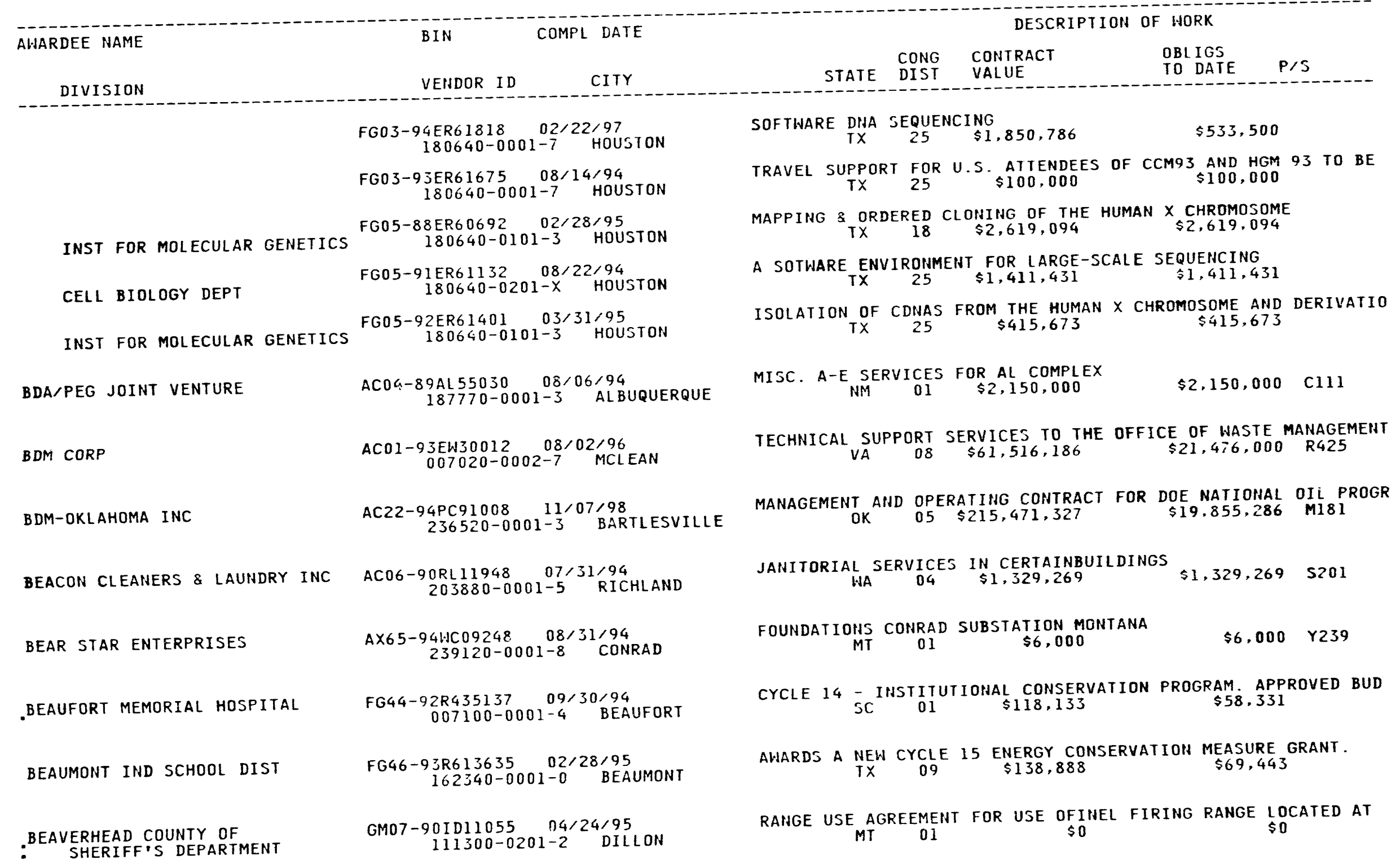




\section{AWARDEE NAME}

BECHTEL NATIONAL INC

BECHTEL HANFORD INC

BECHTEL PETROLEUM OPER INC

\section{BECKMAN RESEARCH INST}

\section{BEDFORD COUHTY OF} EDUCATION BOARD OF

BEECH GROVE CITY OF SCHOOLS

-BELDEN \& BLAKE CORP

BELEN CONSOLIDATED SCHOOLS

:BELL ATLANTIC BUSINESS SYSTEMS

BIN

COMPL DATE

VENDOR ID CITY STATE DONG VALUE

DESCRIPTION OF WORK

ACO3-89SF $17887 \quad 12 / 31 / 94$
$007170-0003-6$ SAN FRANCISCO

AC22-90PC89857 08/31/94 007170-0003-6 SAN FRANCISCO

AC22-91PC90027 10/31/94 $007170-0003-6$ SAN FRANCISCO

FR22-91PC90581 06/11/14 007170-0003-6 SAN FRANCISCO

AC05-910R21949 06/25/96 $007170-0002-8$ DAK RIDGE

AC22-93PC91029 01/31/97 007170-0005-2 SAN ANTONIO

AC06-93RL12367 06/30/98 007170-0204-7 RICHLAND $A C 01-85 F E 6052007 / 31 / 95$
$121740-0001-6$ TUPMAN FG03-91ER61137 08/31/94
$206480-0001-X$ DUARTE

FG44-90R434291 12/31/94 158250-0101-8 SHELBYVILLE

FG45-94R562005 06/30/95 238080-0101-1 BEECH GROVE

AC21-91MC28240 12/30/94 215510-0001-6 NORTH CANTON

GM04-92AL58229 04/01/97 $007310-0001-3$ BELEN

AC01-93AD32307 12/01/94 225860-0001-3 ROCKVILLE
AND FINAL DESIGHS IN SUPPORT OF THE MODULAR
CA

BASELINE DESIGN AND SYSTEM ANALYSIS $\$ 3,041,880 \quad$ AG12

BASELINE DESIGN/ECONOMICS FOR ADVANCED FISCHER-TROPSCH TECHN $\begin{array}{lll}0 & \$ 2,554,456 & \$ 2,461,351 \text { AGI }\end{array}$

REPAYMENT AGREEMENT \$O \$0

PROJECT MANAGEMENT CONTRACTOR FOR DOE'S FORMERLY UTILIZED SI PRO $\$ 337,913,736$ \$121,172,091 R408

REFINING \& END USE STUDY OF COAL LIQUIDS TX 21 \$4,558,385 \$1,501,916 AG14 ENVIRONMENTAL RESTORATION MANAGEMENT CONTRACT HA $04 \$ 861,645,463 \quad \$ 7,040,536 \quad$ F999

MANAGEMENT AND OPERATING CONTRACTORS AT THE NAVAL CAND OPERATING CONTRACTORS AT THE NAVAL

IMPROVEMENT \& AUTOMATION OF LIGATION-MEDICATED GENOMIC SEQUE

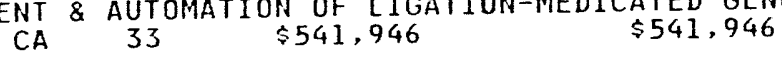

CYCLE 12 INSTITUTIONAL CONSERVATION PROGRAM. APPROVED FUNDED TN $04 \quad \$ 18,975 \quad \$ 11,385$

ICP - NEW AWARD i $\$ 23,000 \quad \$ 11,500$

HORIZONTAL DRILLING IN SHALLOHGEOLOGICALLY COMPLEX $\begin{array}{llll}0 \mathrm{H} & 16 & \$ 1,411,419 & \$ 903,308\end{array}$

SUPPORT FOR SCIENCE, MATHEMATICS AND ENGINEERING TO IMPROVE NM 03

MAINFRAME COMPUTER MAINTENANCEAGREEMENT

$M D \quad 08 \quad \$ 89,254$




\begin{tabular}{|c|c|}
\hline AWARDEE NAME & COMPL DATE \\
\hline DIVISION & $\begin{array}{cc}\text { VENDOR ID } & \text { CITY } \\
4-93 A L 9008-0-05 / 31 / 95-1 \\
225860-0001-3 & \text { ROCKVIL }\end{array}$ \\
\hline BELL DF PENNSYLVANIA & $\begin{array}{c}\text { ACDE-76NV00141 } 09 / 30 / 99 \\
193630-0002-8 \quad \text { ALTOONA }\end{array}$ \\
\hline BELL TELEPHONE CO & $\begin{array}{c}\text { AC08-76NV00153 } 12 / 31 / 99 \\
007380-0002-7 \text { RENO }\end{array}$ \\
\hline
\end{tabular}

FG47-93R702701 08/31/95 UNIFIED SCHOOL DISTRICT \$357 231980-0101-3 BELLE PLAINE

BELLEVUE CITY DF SCHOOL DISTRICT

SCHOOL DISTRICT

BELLEVUE INDEPENDENT SCH DIST

BELLMAHR SCHOOL DISTRICT

BELOIT MEMORIAL HOSPITAL

BELOIT SCHOOL DISTRICT

BEND RESEARCH INC

FG44-91R434499 07/01/94

$$
\begin{gathered}
\text { FG51-92R020153 } 05 / 31 / 95 \\
144120-0101-6 \text { BELLEVUE } \\
\text { FG51-93R020303 08/31/94 } \\
144120-0101-6 \text { BELLEVUE }
\end{gathered}
$$
$183 j 10-0001-3$ BELLEVUE

$$
\begin{gathered}
\text { FG42-94R26,3900 06/30/95 } \\
238930-0001-1 \quad \text { BELLMAWR }
\end{gathered}
$$$$
\begin{gathered}
\text { FG45-93R551816 06/30/95 } \\
099480-0001-3 \quad \text { BEIOIT }
\end{gathered}
$$$$
\begin{array}{r}
\text { FG45-93R551790 12/31/94 } \\
104590-0001-2 \text { BELOIT }
\end{array}
$$$$
\begin{array}{cc}
\text { FG03-91ER81230 09/30/94 } \\
007590-0001-8 \quad \text { BEND }
\end{array}
$$$$
\begin{array}{rc}
F G 03-91 E R 81228 & 07 / 05 / 95 \\
007590-0001-8 & \text { BEND }
\end{array}
$$$$
\text { FG03-91ER81229 03/30/95 }
$$$$
007590-0001-8 \text { BEIN }
$$$$
\begin{gathered}
\text { FG03-92ER81420 02/17/95 } \\
007590-0001-8 \text { BEND }
\end{gathered}
$$

DESCRIPTION OF WORK

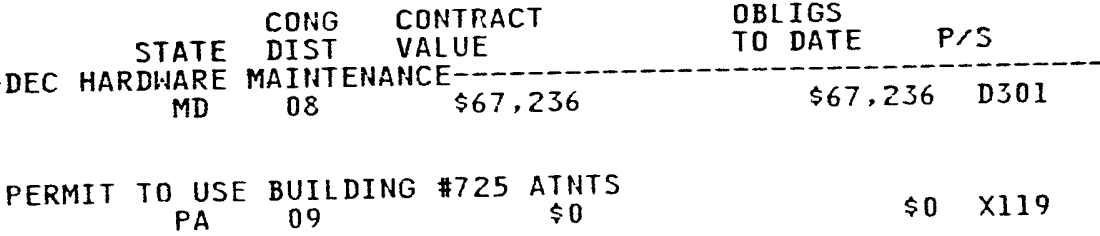

PRDVIDE TELEPHONE SERVICES AT NTS PAYMENT BEING MADE BY REEC

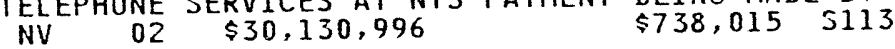

GRANT PROGRAMS FOR SCHOOLS ANDHOSPITALS AND FOR BUILDINGS O

$$
\begin{array}{llrl}
\text { KS } & 04 & \$ 57,260 & \$ 28,630
\end{array}
$$

CYCLE 14 ECM AWARD. INCLUDES $\$ 12,000$ TA CREDIT.

THIS AHARD INCLUDES THE ATTACHED APPENDICES, A, B, \& C; THE THIS AHARD INCLUDES THE ATTACHED APPENDICES, $\begin{gathered}A, \\ \$ 5,913\end{gathered}$

CYCLE 13 - INSTITUTIONAL CONSERVATION FROGRAM. APPROVED BUD CYCLE 16 AHARD.

$\begin{array}{llll} & & \$ 15,432 & \$ 10,802 \\ \text { ICP-NEW AHARD. } & & & \\ \text { HI } & 01 & \$ 70,600 & \$ 35,300 \\ \text { ICP-NEW AHARD. } & & & \\ \text { HII } & 01 & \$ 13,500 & \$ 6,750\end{array}$

SBIR - PHASE I AHARD FOR - DEVELOPMENT OF HOLLOW-FIBER MODUL

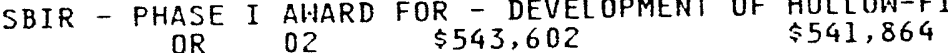

SBIR - PHASE I AHARD FOR - A MEMBRANE PROCESS FOR HOT-GAS CL

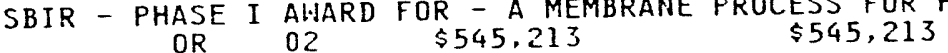

SBIR - PHASE I AWARD FOR - A CATALYTIC MEMBRANE REACTOR FOR $\begin{array}{lll}O R & 02 & \$ 542,117\end{array}$

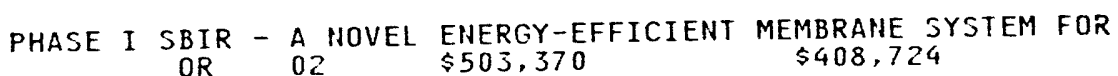




\begin{tabular}{|c|c|}
\hline AWARDEE NAME & COMPL DATE \\
\hline \multirow[t]{3}{*}{ DIVISION } & VENDOR ID \\
\hline & $\begin{array}{c}\text { FG03-94ER81660 } 07 / 17 / 94 \\
007590-0001-8 \text { BEND }\end{array}$ \\
\hline & $\begin{array}{c}\text { FG03-93ER81551 } 04 / 01 / 96 \\
007590-0001-8 \text { BEND }\end{array}$ \\
\hline BENTHOS INC & $\begin{array}{c}\text { FG02-93ER81524 } 07 / 17 / 94 \\
232950-0001-8 \text { NORTH FALMOUTH }\end{array}$ \\
\hline \multirow[t]{2}{*}{ BENTLEY COLIEGE } & $\begin{array}{c}\text { FG09-93SR18368 } 09 / 08 / 94 \\
007790-0001-3 \text { WALTHAM }\end{array}$ \\
\hline & $\begin{array}{c}\text { FG22-93PC93432 } 08 / 30 / 94 \\
007790-0001-3 \text { WALTHAM }\end{array}$ \\
\hline $\begin{array}{l}\text { BENTON COUNTY OF } \\
\text { SHERIFF'S OFFICE }\end{array}$ & $\begin{array}{c}\text { AC06-93RL12516 } 11 / 17 / 97 \\
007810-0202-8 \quad \text { RICHLAHD }\end{array}$ \\
\hline $\begin{array}{l}\text { BERGENFIELD CITY OF } \\
\text { BOARD OF EDUCATION }\end{array}$ & $\begin{array}{rl}\text { FG } 42-94 R 263909 & 06 / 30 / 96 \\
007930-0301-6 \quad \text { BERGENFIELD }\end{array}$ \\
\hline $\begin{array}{l}\text { BERH UNIVERSITY } \\
\text { PHYSICS INSTITUTE }\end{array}$ & $\begin{array}{r}\text { FG02-90ER.61054 } 09 / 14 / 94 \\
008030-0101-0 \text { SIDLERSTRASSE }\end{array}$ \\
\hline $\begin{array}{l}\text { BERNALILLO CDUNTY DF } \\
\text { SHERIFF'S DEPT }\end{array}$ & $\begin{array}{r}\text { GM04-93AL84031 } 01 / 04 / 98 \\
226850-0101-8 \text { ALBUQUERQUE }\end{array}$ \\
\hline $\begin{array}{l}\text { BERNALILLO TOWN OF } \\
\text { MUHICIPAL SCHOOLS }\end{array}$ & $\begin{array}{c}\text { GM04-92AL84064 } 08 / 05 / 97 \\
226820-0101-7 \quad \text { BERNALILLO }\end{array}$ \\
\hline MUNICIPAL SCHOOLS & $\begin{array}{r}\text { FG04-93AL } 9683707 / 08 / 96 \\
226820-0101-7 \quad \text { BERNALILLO }\end{array}$ \\
\hline BERNICE PAUAHI BISHOP MUSEUM & $\begin{array}{c}\text { FG02-9JCH10543 } 06 / 30 / 96 \\
228570-0001-4 \text { HONOLULU }\end{array}$ \\
\hline BERTRAND CHAFFEE HOSPITAL & $\begin{array}{c}\text { GM07-82ID1 } 1003 \quad 09 / 30 / 99 \\
003130-0001-7 \quad \text { SPRINGVILLE }\end{array}$ \\
\hline
\end{tabular}

CONG
STATE DIST

PHASE I SBIR-DEVELOPMENT OF A MEMBRANE-BASED HYBRID SYSTEM $F$

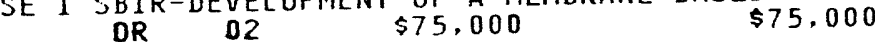

A NOVEL PROCESS FOR THE REMOVAL OF N2 FROM N2-CONTAINING NAT $\begin{array}{lll}O R & 02 \\ \$ 173,892 & \$ 173,892\end{array}$

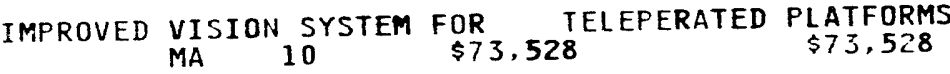

USED ENERGY RELATED LABORATORYEQUIPMENT GRANT \$O

ENERGY RELATED EQUIPMENT GRANT $\$ 0$

LAW ENFORCEMENT SERVICES TRAINING OF 5 INDIVIDUALS

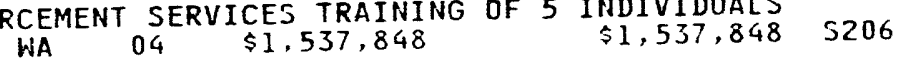

CYCLE 16 AHARD. $05 \quad \$ 63,314 \quad \$ 31,657$

SIMULATIONS OF THE CARBON CYCLE IN THE OCEANS (PART III) $00 \quad \$ 374,056 \quad \$ 237,016$

THIS AGREEMENT SETS FORTH THE GENERAL POLICY FOR THE CTA NM 01 SO

SO

SUPPORT FOR SCIENCE, MATHEMATICS, AND ENGINEERING TO IMPROVE EDUCATION OUTREACH NM 03 $\$ 25,000$ DESIGN AND CONSTRUCTION OF THEBISHOP SCIENCE CENTER HI $01 \$ 37,617,103 \quad \$ 10,000,000$

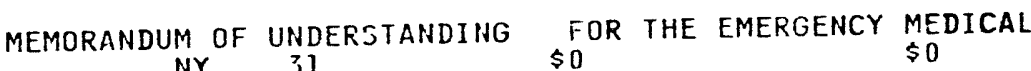




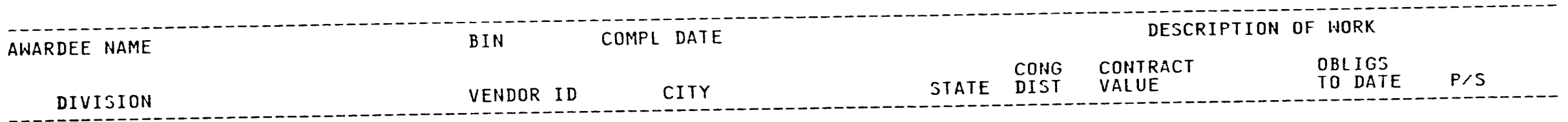

BESSEMER CARRAWAY MEDICAL CTR FG44-93R435291 $08 / 31 / 95$ BSEMER

BEST TEMPORARIES INC

BETHANY COLLEGE

BETHESDA HOSPITAL INC

BETHLEHEM STEEL CORP

BGS SYSTEMS INC

BIG BEAVER FALLS AREA SCHCUL

BIG BUTTE CATTLE ASSOCIATION

\section{BILL TYSON PAINTING}

\section{BINGHAM COUNTY OF} SHERIFF'S OFFICE

: SHERIFF'S OFFICE

FG43-93R356201 $01 / 31 / 95$
$008190-0001-9$ BETHANY

FG45-92R540170 04/30/95

FC21-91MC27362 01/26/9i

FR21-91MC28128 07/26/16

FC22-90PC89658 12/31/96

ACO1-89E121282 09/30/94
$005960-0002-2$ WALTHAM

FG43-92R344200 06/30/95 GMO7-92ID11065
$227800-0001-5$ ARCO

A $665-9311111576 \quad 09 / 08 / 94$ $234160-0001-3$ ELVERTA

GM07-94IDI1140 12/31/99

GM07-93ID11128 07/02/98
$A B 01-94 P 01020003 / 31 / 95$
$193740-0001-6$ $099530-0001-8$ CINCINNATI $137930-0002-8$ BUnivS HARBOR $137930-0002-8$ BURNS HARBOR SPARROWS POINT

SEE ATTACHED PAGE ND. 2 OF THIS NOTICE OF FINAHCIAL ASSISTAN $A L \quad 07 \quad \$ 199,999 \quad \$ 100,000$

TEMPORARY SECRETARIES

DC 01

$\$ 0 \quad R 699$

TITLE III OF THE NATIONAL ENERGY CONSERVATION POLICY ACT WV $01 \quad \$ 65,000 \quad \$ 48,750$

ICP - NEH AHARD

$\$ 21,923$

$\$ 10,961$

CLEAN COAL TECHNOLOGY III; BLAST FURNACE GRANULATED COAL IN $01 \$ 191,700,000 \quad \$ 30,064,530$ REPAYMENT AGREEMENT; BLAST
IN 01
$\$ 0$

INNOVATIVE COKE OVEN GAS CLEANING SYSTEM

MD $02 \quad \$ 45,239,781 \quad \$ 11,915,800$

SOFTWARE MAINTENANCE

$\$ 48,816$

$\$ 48,816 \quad 7030$ $218850-0001-4$ BEAVER FALLS

TITLE III DF THE NATIONAL ENERGY CONSERVATION POLICY ACT PA $04 \quad \$ 247,100$ $\$ 100,000$ $169360-0201-3$ BLACKFOOT $169360-0201-3$ BLACKFUOT AGREEMENT $\underset{\text { IDETHEEN }}{\text { BD }}$ THE BIG BUTTE CATTLE ASSOC \& $\underset{\$ 0}{\text { THE }}$ U.S. DOE

MISC PAINTING AT ELVERTA MAINT FAC

$C A \quad 02$ \$1,715

$\$ 1,715 \quad$ Y119

FIRING RANGE USE AGREEMENT WITH THE BINGHAM COUNTY SHERIFF'S ID 02 \$O

\$0

MOU TO MAINTAIN EGUIPMENT \& PERSONNEL FOR THE FURPOSE OF PRO ID 02 \$0 


\begin{tabular}{|c|c|c|c|c|c|c|c|}
\hline AHARDEE NAME & BIN & DATE & & & DESCF & F HORK & \\
\hline & VENDOR ID & CITY & STATE & $\begin{array}{l}\text { CONG } \\
\text { DIST }\end{array}$ & $\begin{array}{l}\text { CONTRACT } \\
\text { VALUE }\end{array}$ & $\begin{array}{l}\text { OBLIGS } \\
\text { TO DATE }\end{array}$ & $P / S$ \\
\hline
\end{tabular}

BINGHAM CTY DISASTER SERVICE

BID-IMAGING RESEARCH INC

BIO-PROCESS INNOVATION INC

BIOELECTROMAGNETICS SOCIETY

BIOENGINEERING RESOURCES INC

BIOLOGICAL SCI CURRIC STUDY

BIOMEDICAL RES FND OF NH LA

BIOSPHERICS INC

BIOTRACES INC

BIRMINGHAM BOARD OF EDUCATION

BISHOP CARROLL HIGH SCHOOL

$=$

BLACK \& VEATCH $\begin{array}{rc}\text { GM07-93IDI } 1127 & 07 / 02 / 98 \\ 231380-0001-0 & \text { BLACKFOOT }\end{array}$

AC21-93MC30173 12/22/94
$198500-0001-5 \quad$ LINCOLNSHIRE

FG01-94CE15594 04/13/96
$237410-0001-9$ HEST LAFAYETTE

FG02-94ER6178904/14/95 $184350-0001-X$ FREDERICK

FC02-90CE40939 09/30/94 237010-0001-8 FAYETTEVILLE

FCO4-94AL $98770 \quad 07 / 01 / 97$ 237010-0001-8 FAYETTEVILLE

FG03-93ER5158401/31/95 207020-0001-9 COLORADD SPRINGS

FG02-90CH10442 02/01/95
$199860-0001-7$ SHREVEPORT

AB01-93AD69211 $10 / 31 / 94$
$156510-0002-\times \quad B E L T S V I L L E$

FG05-93ER81602 04/01/96 $234440-0001-4$ GREENBELT

FG44-93R435289 08/31/94 008640-0001-4 BIRMINGHAM

FG47-93R70270308/31/95 232190-0001-0 HICHITA

AC65-91HA08492 09/10/94

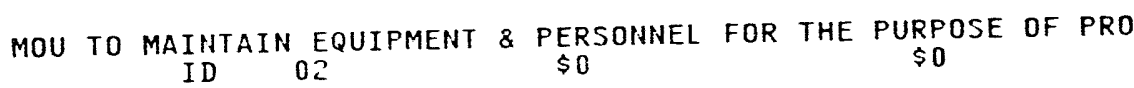

EM WASTE INSPECTION TOMOGRAPHY (HIT)

IL 10 \$3,156,993

$\$ 3,125,710 \quad$ AH93

DEVELOP A LOW-ENERGY CONTINUOUS REACTOR FOR THE PRODUCTION O IN $07 \quad \$ 92,022$ $\$ 92,022$

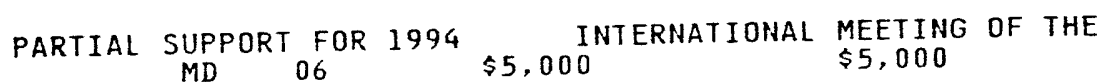

BIOLOGICAL CONVERSION OF $\underset{\$ 4,246,300}{\text { WASTE GASES }} \$ 2,088,000$

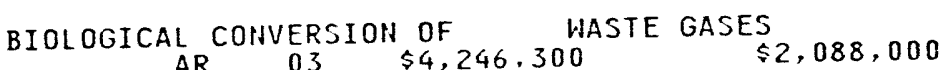

PRODUCTION OF ETHONOL FROM REFINERY WASTE GASES AR $03 \quad \$ 4,347,366$

$\$ 277.020$

NEW AWARD FOR PROJECT ENTITLED: "THE HUMAN GENOME PROJECT: $\begin{array}{lrl}\text { FOR PROJECT ENTITLED: } & \text { "THE HUMAN GENOME } \\ 05 & \$ 362,292 & \end{array}$

DESIGN AHD CONSTRUCTION OF A BIOMEDICAL REJEARCH INSTITUTE LA $04 \$ 37,037,500 \quad \$ 28,287,500$

BPA FOR AHALYTICAL NEEDS

$\$ 0$

$\$ 0 \quad F 102$

NEUTRON DETECTOR

MD $05 \quad \$ 547,688$

$\$ 153,781$

SEE ATTACHED PAGE NO. 2 OF THIS NOTICE OF FINANCIAL ASSISTAN

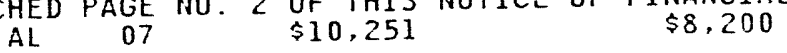

GRANT PROGRAMS FOR SCHOOLS ANDHOSPITALS AND FOR BUILDINGS 0 KS 04 \$7, 965 \$3.982

OPEN-END TITLE I, II, \& II A/E SERVICES 


AWARDEE NAME

DESCRIPTION OF HORK

AWARDEE NAME

COMPL DATE

\section{COHTRACT}

DIVISION

VENDOR ID

$008780-0003-8$ AURORA

STATE DIST VALUE

OBLIGS

008780-0003-8 AUROR

ACO4-84AL18855 06/01/95
$008780-0001-1$ KANSAS CITY

AC05-890R21802 04/30/95

008780-0001-1 KANSAS CITY

AC05-920R21987 07/31/94

$008780-0001-1$ KAHSAS CITY

AC06-91RL11977 05/31/95

008780-0001-1 KAHSAS CITY

BLACK RIVER UNION SCHOOL DIST

FG41-94R196604 05/31/96
$238880-0001-7$ LUDLOH

BLACKFOOT CITY OF
FIRE DEPARTMENT

\section{POLICE DEPARTMENT}

BLADEN COMMUNITY COLLEGE

BLAINE CITY OF

JOINT SCHOOL DISTRICT \#6I

BLAINE COUNTY OF

JOINT SCHOOL DISTRICT \#6I

\section{BLAIR INC}

BLAZE TECH CORP

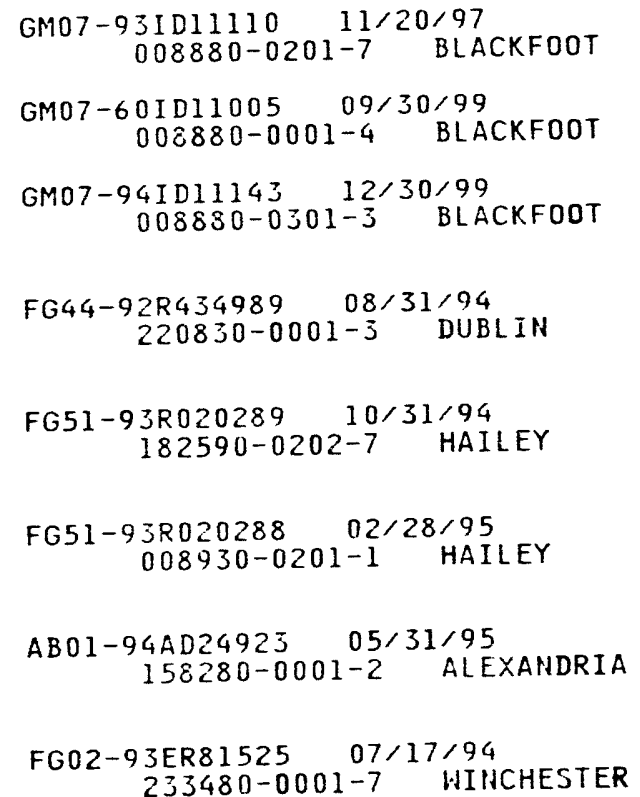

CO $06 \quad \$ 6,122,339$

WEAPOHS ASSEMBLY BAYS - AAO

$\$ 4.580,901 \quad 0123$

TITLES I \& II ENGINEERING SERVICES FOR PVIJF

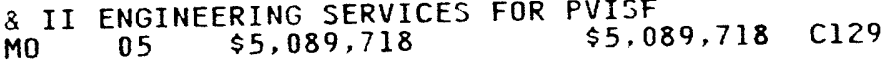
$\begin{array}{cccc}\text { TITLE I AND II A-E DESIGN SERVICES EMERGENCY NOTIFICATION SY } \\ \text { MO } & 05 & \$ 2.810,151 & \$ 2.810,151 \\ \text { C } 211\end{array}$

A/E FOR DESIGN ON PHASE I B-524 WITH OPTION FOR FURTHER MO 06 \$1, 984,039 \$1,984,039 C119

TITLE III OF THE NATIONAL ENERGY CONSERVATION FOLICY ACT VT 01 N $\$ 41,370$ $\$ 20,685$

RECIPRDCAL FIRE FIGHTING AHD EMERGENCY MEDICAL SERVICES ASSI ID 0 C MEMORANDUM OF UNDERSTANDIHG ID 02 $\$ 0$

RANGE USE AGREEMENT FOR RECIPROCAL FIRE FIGHTING RANGE USE AD 02 $\$ 0$

$\$ 0$

CYCLE 14 - INSTITUTIONAL CONSERVATION PROGRAM. APPROVED BUD NC ${ }_{03} \quad \$ 31,345 \quad \$ 28,210$

CYCLE 15 TAS AHARD.

$\$ 19,600$

$\$ 9,800$

CYCLE 15 ECM AHARD.

$\$ 139,637$

$\$ 61,881$

BPA FOR VISUAL SERVICES DESIGNAND FABRICATION 06/01/94-05/3 VA 08 $\$ 0$ \$0 T001

LOW NO BURNER FOR COAL COMBUSTION MA $07 \quad \$ 75,000$

$\$ 75,000$ 


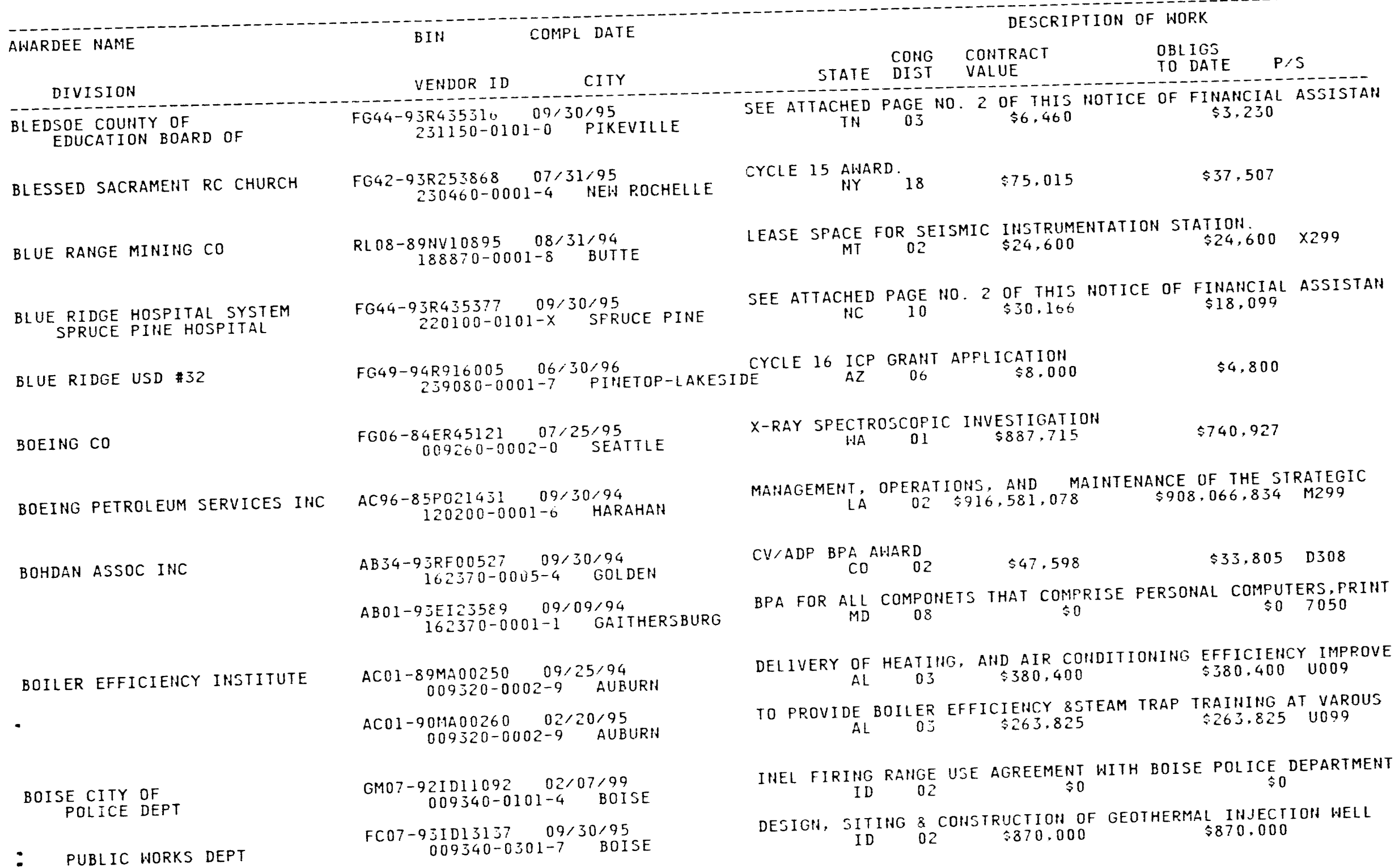




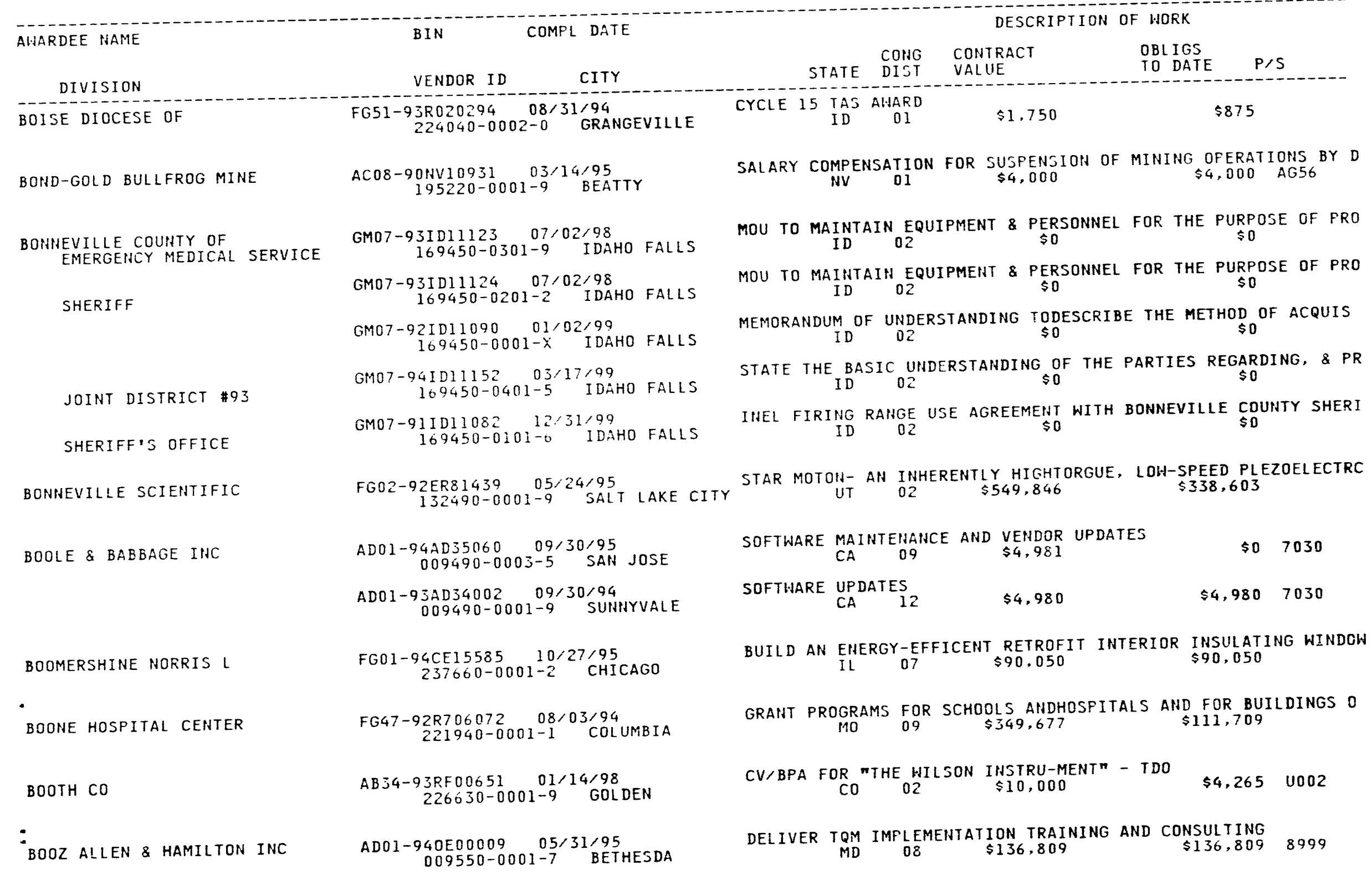




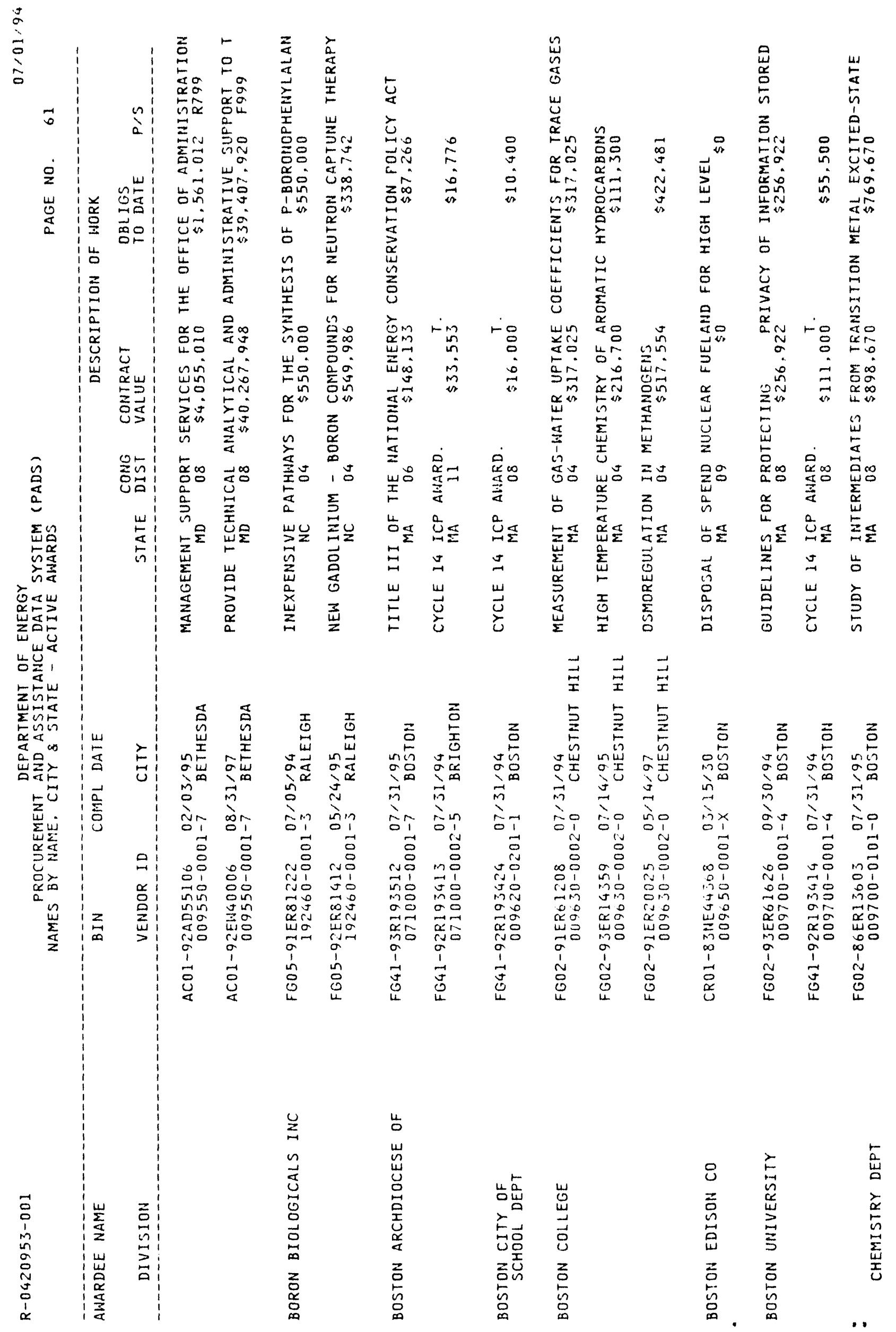




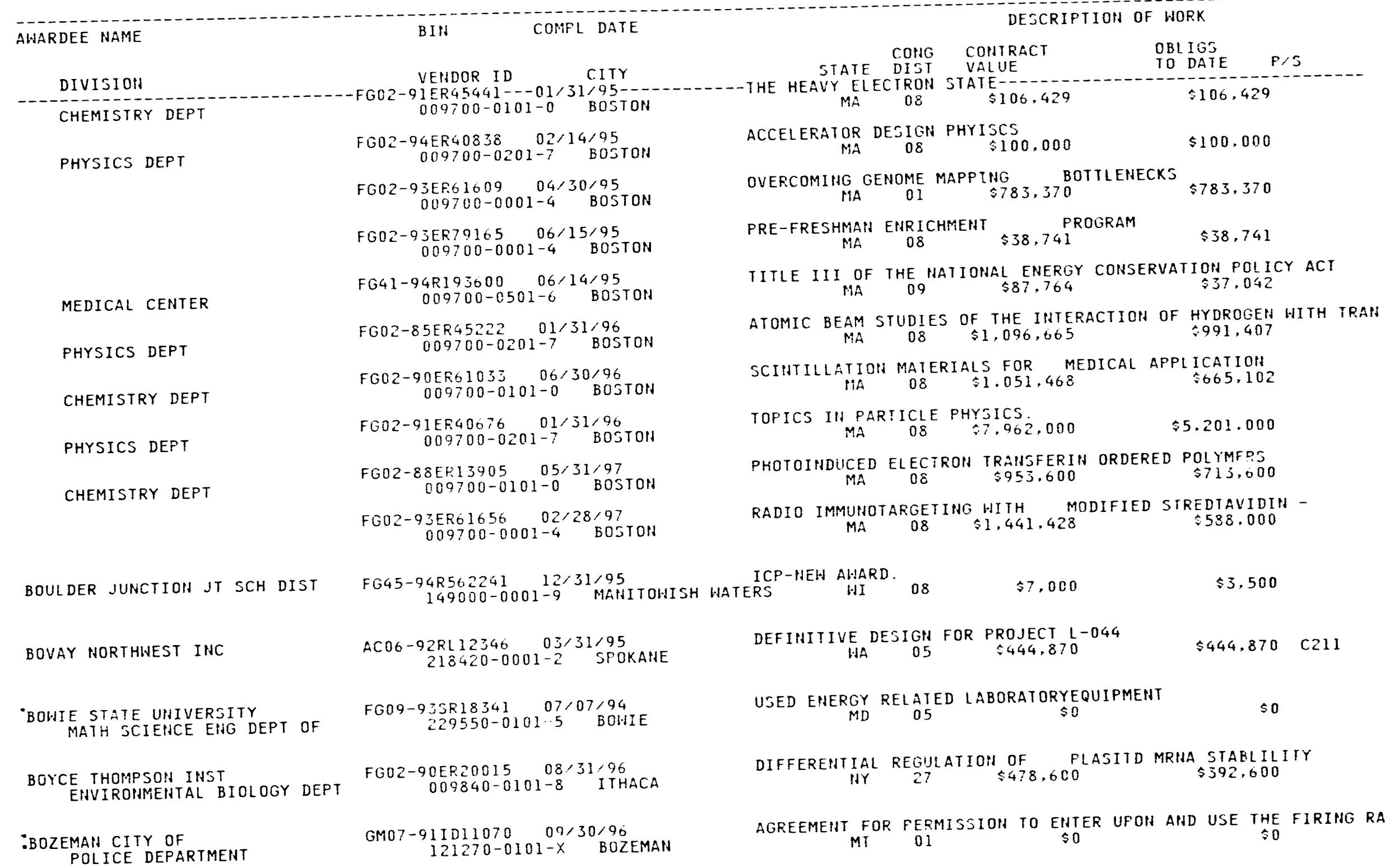




AWARDEE NAME
DIVISION
BP AMERICA
RESEARCH \& DEVELOPMENT

BRADLEY MEMORIAL HOSPITAL

BRANCO ENTERPRISES INC

\section{BRANDEIS UNIVERSITY}

CHEMISTRY DEPT

\section{BRAVO ENERGY TRADING NA}

BREAKWATER SCHOOL

BRECKINRIDGE MEMORIAL HOSPITAL

BREEDEN INC

BREVARD COLLEGE
COMPL DATE

\section{DESCRIPTION OF HORK}

BIN

STATE DIST

CONTRACT

TO DATE

$P / S$

VENDOR ID

CITY

FG02-92ID13138 07/01/95

216670-0101-9 CLEVELAND

FG41-94R191600 05/30/96 037920-0001-6 SOUTHINGTON

AC75-935W33521 $10 / 31 / 94$
$235370-0001-4$ SPRINGFIELD

FG02-86ER13486 02/28/95
$010030-0001-0$ WAL THAM

FGO2-89ER14027 07/14/96

$010030-0201-3$ HALTHAM

FG02-92ER40706 12/31/94

O10030-0001-0 WAL THAM

FG02-91EF61235 08/31/94

$010030-0001-0$ HAL THAM

FG02-93ER45495 06/30/97

010030-0001-0 HAL THAM

SC92-94FE63271 10/01/94 $176370-0002-8$ LOHG BEACH

FG41-93RI92515 06/30/95 207710-0001-4 PORTLAND

FG44-93F.435301 09/30/95 $010180-0001-1$ HARDINSBURG

FGO1-92CE15524 11/30/94
$220090-0001-3$ BAY ST. LOUIS

FG44-92R434992 08/31/94
$220660-0001-9$ BREVARD
MEMBRANE/DISTILLATION HYBRID PROCESS FOR PROPAHE/PROPYLENE S $\mathrm{OH} 21 \quad \$ 3.816 .125$

$\$ 1,313.217$

TITLE III OF THE NATIONAL ENERGY COHSERVATION PCLICY ACT $\begin{array}{lll}\text { OF THE NATIONAL ENERGY CONSERVATION PCLICY } & \$ 96,218\end{array}$

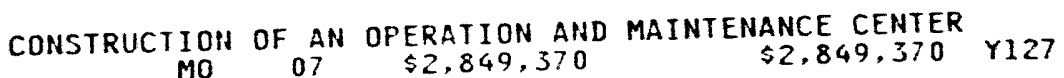

EFFECT OF LIGHT ON FERMENTATION AND DEVEL OFMENT OF MECHANISTIC STUDIES OF EXCITEDSTATE CHEMICAL REACTIDHS

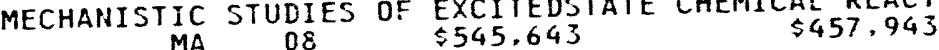
RESEARCH IN ELEMENTARY
PARIICLE PHYSICS.
$\$ 1.507,999$ $\begin{array}{ccc}\text { MECHANISMS OF REPAIR OF DOUBLE-STRAND CHROMOSOMAL BREAKS } & \\ 08542,27 & \$ 531,900 & \end{array}$ ORDERING IN CRYSTALLINE AND QUASICRYSTALLINE ALLOYS: AN ORDERING IN CRYSTALLINE AND $\$ 240,000$ OASTCRYSTALLINE ALLOY NPR-1 CRUDE OIL SALES CONTRACTEST REV: $\$ 11530976.40$

TITLE III OF IHE NAIIONAL ENERGY CONSERVATION POLICY ACT

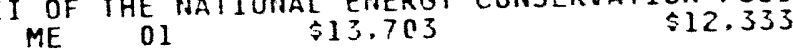

SEE ATTACHED PAGE HO. 2 OF THIS NOTICE OF FINANCIAL ASSISTAN

DEVELOP SLANT LEG OIL PLAT FORM CYCLE 14 - INSTITUTIONAL CONSERVATION PROGRAM. AFPROVED BUD
HC KY 02 HO. 2 \$88,056 $\$ 44,028$ 


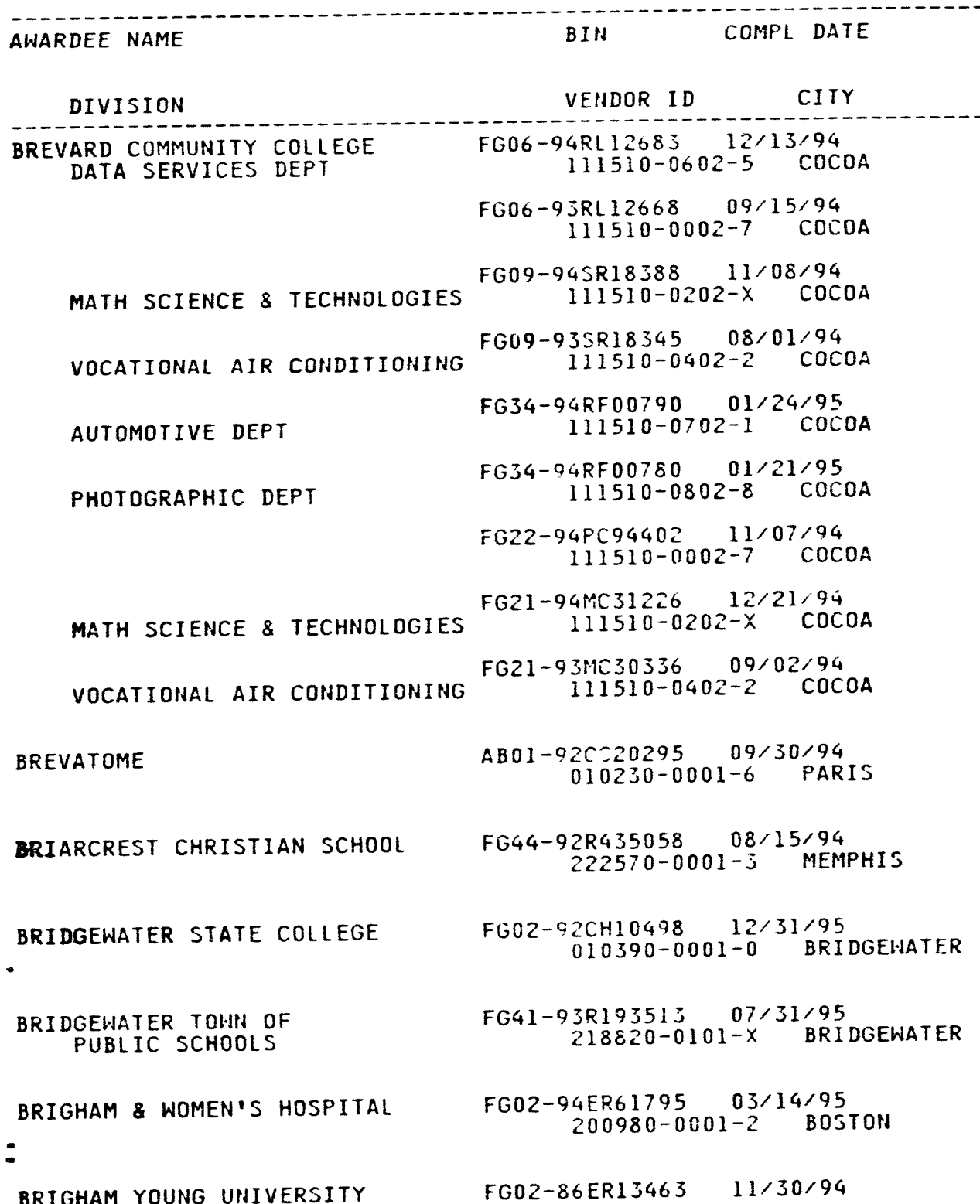

DESCRIPIION OF HORK

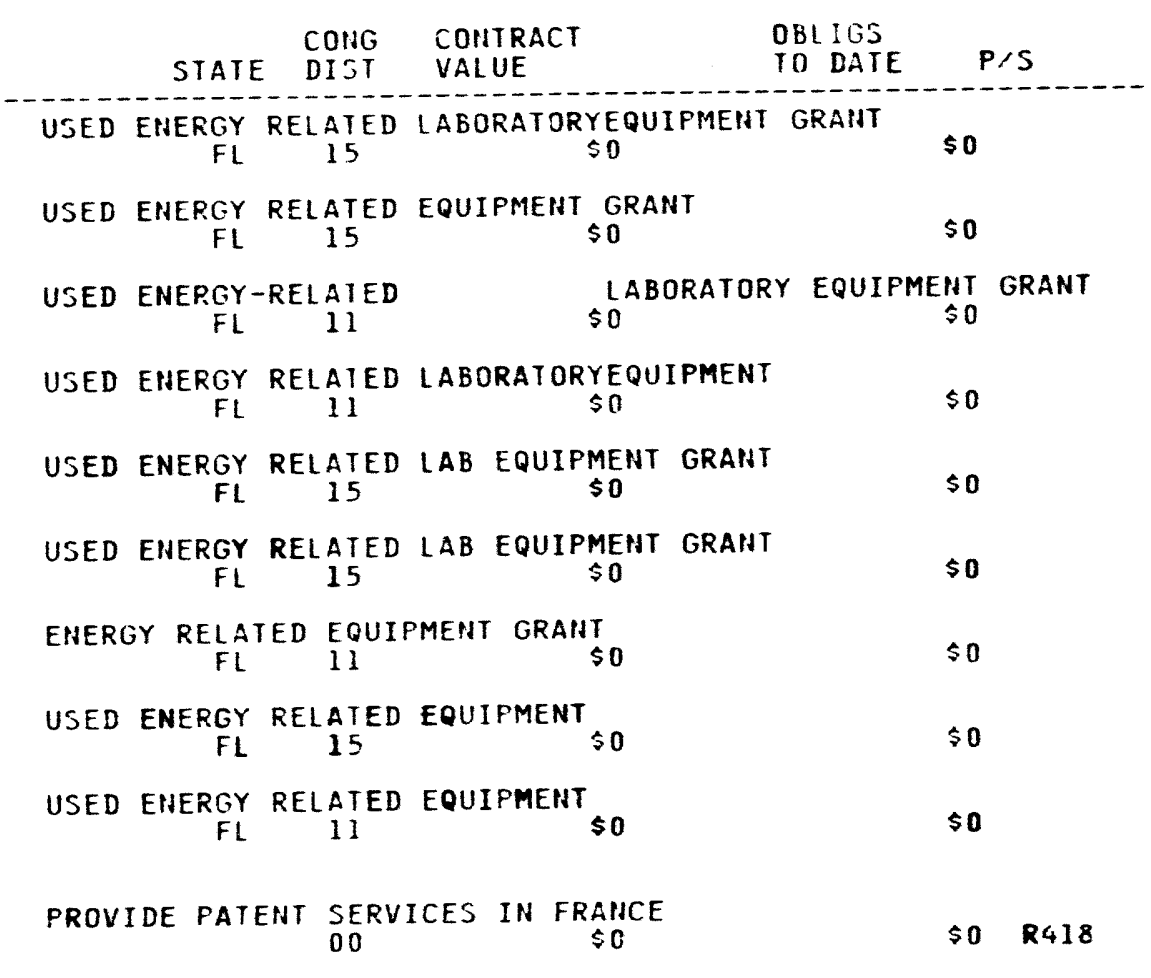

CYCLE 14 - INSTITUTIOHAL CONSERVATION FROGRAM. AFPROVED BUD OLD COLONY CENTER FOR
MA OOS $\$ 10,000,000$

TITLE III OF THE NATIONAL ENERGY CONSERVAIION POLICY ACT INTERNATIONAL HORKSHOP ON
MA HUMAN CHROMOSOME 9 TO BE HEL D STUDIES IN LIQUID MEMBRAHE TRANSPORT 


\section{AWARDEE NAME}

BIN

COMPL DATE

DESCRIPTION OF HORK

DIVISION

VENDOR ID CITY

$010420-0001-8$ PROVO

FG03-93ER45479 10/31/94
$010420-0001-8$ PROVO

$F G 22-91 P C 91308 \quad 09 / 14 / 94$
$010420-1201-6 \quad$ PROVO

MECHANICAL ENGINEERING DEPT $F G 22-93 P C 93226 \quad 09 / 29 / 96$
$010420-1201-6 \quad$ PROVO

MECHANICAL ENGINEERING DEPT

BRINK ELECTRIC CONSTRUCTION CO AC65-921 1 H109394 04/15/96
$132540-0015-3$ SACRAMENTO AC65-9114107387 $12 / 15 / 95$
$132540-0015-3 \quad$ SACRAMENTO AC65-91WH07388 $12 / 20 / 95$
$132540-0015-3 \quad$ SACRAMENTO AC65-9411C08968 06/15/95
$132540-0024-2$ RUGBY AC65-93HC08493 12/02/94 $132540-0023-4$ PIERRE

AC65-93WC08226 08/20/94 $132540-0022-6$ HATERTOWN

AC65-94AC08969 10/07/95 132540-0022-6 WATERTOWH

AC65-931J321110 10/15/94 132540-0016-1 THERMOPOL IS AC65-921.1J16973 10/15/94 132540-0017-X TORRINGTON AC65-93WJ21533 $10 / 15 / 95$ $132540-0017-x$ TORRINGTON

BRISTOL BOROUGH SCHOOL DIST

FG4J-93R354202 12/31/94 010470-0001-6 BRISTOL

:

FG $43-93 R 35550003 / 31 / 95$
$213600-0101-8$ BRISTOL
STATE DIST VONTRACT

UT $03 \quad \$ 798,184$

OBLIGS

$\$ 798,184$

NEH GRANT - MMICROSTRUCTURAL DEPENDENCE DF CAVITATION DAMAGE

UT 03 T $\$ 85.831$ \$85.831

PARTICULATE BEHAVIOR IN A CONTROLLED-PRDFILE PULVERIZED COAL UT 033 IN $\$ 274,282$ CONTRLLLD-PROFILE PULVERI

INVESTIGATIOH OF MINERAL TRANSFORMATION AND ASH DEPOSITION D UT 03 \$ $\$ 200,000 \quad \$ 200.000$

CONSTRUCTION OF OLINDA TRACY $500 \mathrm{KV}$ TRANSMISSION LINES, FOR CA $03 \$ 15,882.466 \quad \$ 15.882 .466$ Y299

CONSTRUCTION OLINDA SUBSTATIONSTAGE 01 (COTP)

CA 03 \$15,195,053 $\$ 15,195,053 \quad$ Y219

TRACY SUB... STAGE 01 - COTP ALAMEDA COUNTY

CA $03 \$ 14,526,372 \quad \$ 14,526,372 \quad$ Y219

REGBY SUBSTATION. STAGE OG

$$
\begin{array}{lccc}
\text { ND } & 01 & \$ 1 &
\end{array}
$$

PIERRE SUBST, STG $7 \quad \$ 496.544 \quad \$ 496.544 \quad 2239$

WATERTOWN SUBST, ST $18 \$ 404,837 \quad \$ 404,837 \quad 2239$

SUMMIT-WATERTOHN 115-KV TRANSMISSION LINE REBUILD

THERMOPOLIS SUBST, STG 06

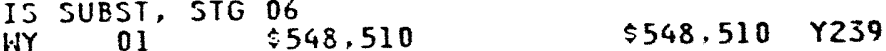

CONSTR OF GERING-LIMESTONE 69-KV T.L. $\$ 10.018,772 \quad$ Y239 HY \& HE IISKV TLIHE CONDUCTOR REPAIR 8 REMOVE 13.8 KV TLINE
WY

TITLE III OF THE NATIOHAL ENERGY CONSERVATION FOLICY ACT $P A \quad 08 \quad \$ 92,000 \quad \$ 58.550$

TITLE III OF THE NATIONAL ENERGY CONSERVATION POLICY ACT SCHOOL SYSTEM
TITLE III OF THE NATIONAL ENERGY CONSERVATION POLICY 


\begin{tabular}{|c|c|}
\hline AWARDEE NAME & COMPL DATE \\
\hline DIVISIOH & VENDOR ID \\
\hline BRISTOL-PLYMOUTH REG VOC HS & $\begin{array}{c}\text { FG41-93R193507 11/30/94 } \\
228340-0001-8 \text { TAUNTON }\end{array}$ \\
\hline BRITISH COAL CORP & $\begin{array}{c}\text { GI01-91FE62384 } 09 / 20 / 94 \\
215440-0001-4 \text { CHEL TENHAM }\end{array}$ \\
\hline BROADDUS HOSPITAL FOUNDATION & $\begin{array}{c}\text { FG43-91R336204 08/31/94 } \\
197890-0001-4 \text { PHILIPPI }\end{array}$ \\
\hline BROCKTON PUBLIC SCHOOLS & $\begin{array}{c}\text { FG41-92R193422 } 07 / 31 / 94 \\
010580-0001-2 \text { BROCKTOR }\end{array}$ \\
\hline & $\begin{array}{c}\text { FG41-93R193514 07/31/95 } \\
010580-0001-2 \text { BROCKTOH }\end{array}$ \\
\hline BROOMFIELD CITY OF & $\begin{array}{r}\text { FG34-91RF00116 } 04 / 30 / 96 \\
010780-0001-8 \quad \text { BROOMFIELD }\end{array}$ \\
\hline BROWN \& CO & $\begin{array}{c}A C 01-93 F M 39017 \\
158310-0002-8 \quad \text { ARLIHGTON }\end{array}$ \\
\hline $\begin{array}{l}\text { BROWN UNIVERSITY } \\
\text { CHEMISTRY DEPT }\end{array}$ & $\begin{array}{c}\text { FG02-84ER13235 } 04 / 14 / 97 \\
010890-0102-9 \text { PROVIDENCE }\end{array}$ \\
\hline GEOLOGICAL SCIENCES DEPT & $\begin{array}{c}\text { FG02-85ER60304 } 01 / 31 / 97 \\
010890-0302-1 \quad \text { PROVIDENCE }\end{array}$ \\
\hline PHYSICS DEPT & $\begin{array}{c}\text { FG02-86ER } 45267 \quad 11 / 30 / 95 \\
010890-0502-4 \text { PROVIDENCE }\end{array}$ \\
\hline PHYSICS DEPT & $\begin{array}{c}\text { FG02-87ER40334 12/31/94 } \\
010890-0502-4 \text { PFOVIDEHCE }\end{array}$ \\
\hline PHYSICS DEPT & $\begin{array}{c}\text { FG02-88ER40452 04/30/95 } \\
010890-0502-4 \text { FROVIDENCE }\end{array}$ \\
\hline BIOLOGY \& MEDICINE & $\begin{array}{c}\text { FG02-88ER13918 } 05 / 14 / 95 \\
010890-0902-X \quad \text { PR.OVIDENCE }\end{array}$ \\
\hline EOLOGICAL SCIEI & $\begin{array}{r}\text { FGO2-90ER14144 } 08 / 14 / 94 \\
010390-0302-1 \quad \text { PROVIDEHCE }\end{array}$ \\
\hline
\end{tabular}

FY. 93 ICP AHARD

INTERHATIONAL AGREEMENT GREENHOUSE GASES FROM FOJJIL FUELS

OOO $\$ 594,030 \quad \$ 507,030$

TITLE III OF THE WATIOHAL EHERGY COHSERVATION POLICY ACT

WV $02 \quad \$ 70.250 \quad \$ 35.125$

CYCLE 14 ICP AHIARD.

$\begin{array}{cccc}\text { MA } & 11 & \$ 56,600 & \$ 36,392 \\ \text { TITLE III OF THE HATIONAL EHERGY } & \text { COHSERVATION POLICY ACT } \\ \text { MA } & 09 & \$ 35.650 & \$ 25.863\end{array}$

HATER MGT. PROGRAMS/MUHICIFALISUBJ. RUHOFF HATER FR. ROCKY CO $02 \$ 72.064 .640 \quad \$ 70.000 .000$

AUDITING \& ANALYSES SUPPORT SERVICES

$$
\begin{array}{lllll}
8 & \text { AHALYSES SUPPORT } & \text { SERVICES } & \$ 677.000 & R 799
\end{array}
$$

PHOTOCHEMICAL GENERATION OF THE OPTOACOUSTIC EFFECT MODEL VALIDAIIOH RESEARCH: CO MPARISON OF SIMULATED AHD OBSE
RI 01

SURFACES AHD THIH FILMS STUDIED BY FICOSECOHD ULTRASOUNICS

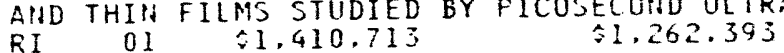

HUCLEAR EXCITATIONS AHD REACTION MECHAHISMS

NUCLEAR EXCTTATIONS AHD REACTION MECHANISMS $\$ 643.530$ RESEARCH \& DEVELOPMENT OF A HELUIM-4-BASED SOLAR HEUTRINO DELTA-AMINOLEVULIHATE $\$ 638.067$ BIOSYNTHESIS IN OXYEENIC GRAIN-BJUHDARY TRAHSPORT AND RELATED PROCESSES IN NATURAL $\begin{array}{ll}\text { RI } & 01 \\ \text { RI } & \$ 427,337\end{array}$ 
DESCRIPTION OF WORK

AWARDEE NAME
COMPL DATE

STATE DONG

FG02-91ER40688 10/31/96 010890-0002-2 PROVIDENCE FG02-93ER79166 06/14/95 010890-0002-2 PROVIDENCE

ENGINEERING DEPT FG22-90PC90307 12/31/94 $010890-0202-5$ PROVIDENCE FG22-91PC91305 08/31/94 $010890-0202-5$ PROVIDENCE

ENGINEERING DEPT

ENGINEERING DEPT

ENGINEERING DEPT

PHYSICS DEPT

PHYSICAL PLANNING DEPT

BROWNING-FERRIS INDUST OF WV

BROWNING-FERRIS INDUSTRIES INC SERVICE GROUP

BRUNEAU-GRANDVIEW JT DIST \#365

- BRUNSHICK COUNTY OF SCHOOLS

BRYAN CITY OF

EBUENA VISTA COUNTY hOSPITAL
AC21-94MC31273 06/29/97 MORGANTOWN

FG22-94PC94218 $08 / 31 / 97$
$010890-0202-5 \quad$ PROVIDENCE FG22-92PC92544 08/12/95 $010890-0202-5$ PROVIDENCE FG21-93MC $30117 \quad 08 / 30 / 94$ $010890-0502-4$ PROVIDENCE FG41-91R195302 11/30/94 010890-0402-8 PROVIDENCE

AX65-94HK14565 $09 / 30 / 94$
$162390-0102-3$ RIFLE

GM07-94ID11154 03/17/99 236980-0001-6 GRANDVIEW

FG44-93R435378 09/30/94 231280-0101-4 SOUTHPORT

FG46-93R613621 08/31/94 232630-0001-2 BRYAN 232200-0001-0 STORM LAKE
FG47-93R707025 08/31/94
PRE-FRESHMAN ENRICHMENT
RI 01

REACTIVITY OF YOUHG CHARS VIA ENERGETIC DISTRIBUTION RI $01 \quad \$ 200,000$ $\$ 200,000$ CHARACTERIZATION OF POROSITY VIA SECOHDARY REACTIOHS
RI 01

$\begin{array}{rrrr}\text { KINETICS AND MECHANISMS OF NOX- CHAR REDUCTION } \\ \text { RI } 01 & \$ 229,000 & \$ 200,000\end{array}$ VAPORS PRESSURES AND HEATS OF VAPORIZATION OF PRIMARY COAL T RI 01 \$194,819 \$194,819

USED ENERGY RELATED EQUIPMENT $\$ 0$

\$0 TITLE III OF THE NATIOHAL ENERGY CONSERVATION POLICY ACT

NONHAZARDOUS SOLID HASTE PICKUP AND DISPOSAL $\begin{array}{llll}\text { HV } & 01 & \text { PICKUP AND DISPOSAL } & \\ \$ 59,769 & \$ 19,923 & 5205\end{array}$

PORTABLE TOILET - RIFLE SUBST

$\$ 993 \$ 222$

STATE THE BASIC UHDERSTANDING OF THE PARTIES REGARDING, \& PR ID 01

$\$ 0$ $\$ 0$

SEE ATTACHED PAGE NO. 2 OF THIS NOTICE OF FINANCIAL ASSISTAN NC 07 \$14.088 $\$ 7,060$

$\begin{array}{ccccc}\text { AWARDS A NEH } & \text { CYCLE } & 15 & \text { TECHNICAL ASSISTANCE GRANT. } \\ \text { TX } & 08 & \$ 39,188 & \$ 19,594\end{array}$

GRANT PROGRAMS FOR SCHOOLS ANDHOSPITALS AND FOR BUILDINGS 0 IA $05 \quad \$ 6,960 \quad \$ 3,480$ 


\begin{tabular}{|c|c|c|c|c|c|c|c|}
\hline AWARDEE NAME & BIN & DATE & & & DESCF & $=$ WORK & \\
\hline DIVISION & VENDOR ID & CITY & STATE & $\begin{array}{l}\text { CONG } \\
\text { DIST }\end{array}$ & $\begin{array}{l}\text { CONTRACT } \\
\text { VALUE }\end{array}$ & $\begin{array}{l}\text { OBLIGS } \\
\text { TO DATE }\end{array}$ & $P / S$ \\
\hline
\end{tabular}

BUHL JOINT SCHOOL DIST $\$ 412$

$F G 51-93 R 020297 \quad 10 / 31 / 94$
$226280-0001-6 \quad$ BUHL

BUNCOMBE COUNTY OF

BOARD OF EDUCATION

EDUCATION BOARD OF

BURCHETTE \& CO INC

BURLE INDUSTRIES INC

BURLINGTON INDUSTRIES INC LEES COMMERCIAL CARPETS

BURNS \& MCDONHELL ENG CO

BURNS \& ROE INC

INDUSTRIAL SERVICE CORP

BURNS CONCRETE INC

BURTON TECHHOLOGY INC

BUTTE COUNTY OF

EMERGENCY SERVICE

:

SHERIFF
FG44-92R434993 07/31/95 $220110-0101-3$ ASHEVILLE

FG44-93R435379 09/30/95 220110-0101-3 ASHEVILLE

$A B 01-94 A D 24917 \quad 05 / 31 / 95$ $159340-0001-2$ ALEXANDRIA

$A D 01-941550108 \quad 07 / 25 / 95$
$235070-0001-6 \quad$ LANCASTER

AD01-94AD64827 08/12/94 $096250-0202-2$ GREENSBORO

AC05-900R21860 09/30/94 $011290-0001-X$ KANSAS CITY

ACO1-93PRI0018 10/05/96 $011300-0003-6$ ORADELL

AC22-89PC88400 07/31/94 011300-0106-7 PITTSBURGH

GM07-921D13155 10,31/94 $22387000001-3$ IDAHO FALLS

FG01-94CE15613 04/27/96 $177880-0001-9$ RALEIGH

GM07-93ID11125 07/02/98

GM07-93ID11126 07/02/98
CYCLE 15 ECM AWARD

ID $\quad 02 \quad \$ 61,600 \quad \$ 30,800$

CYCLE 14 - INSTITUTIONAL CONSERVATION PROGRAM. APFROVED BUD NC 11 \$ $\$ 59,022 \quad \$ 29,511$

SEE ATTACHED PAGE NO. 2 OF THIS NOTICE OF FINAHCIAL ASSISTAN NC $1 \mathrm{I}$. $\$ 70,088$ \$40,000

BPA FOR GRAPHIC PRODUCTION \& VISUAL SERVICES VA 08 $\$ 0$ $\$ 0 \quad \mathrm{~T} 001$

13" MONOCHROME MONITOR

$\$ 254$ $\$ 254 \quad 7042$

BROADL OOM CARPEY

$\$ 5,252$

$\$ 5,252 \quad 7220$

Y-12 STEAM PLANT ASH HANDLING AND DISPOSAL

$$
\begin{array}{lllll}
M & \text { PLANT ASH } & \text { HANDLING AND } & \text { DISPOSAL } \\
05 & \$ 2,465,809 & \$ 2,465,809 & \text { C219 }
\end{array}
$$

SUPPORT SERVICES TO DEVELOP INDEPENDENT COST ESTIMATION FOR NJ $02 \quad \$ 5,826,755 \quad \$ 1.250,000 \quad$ R425

OPERATION MAINTENANCE AND PROGRAM IMPLEMENTATION SUPPORT SER $P A$ i $\$ 96,140,731 \quad \$ 89,509,474$ R425

LICENSE FOR PLACEMENT AND OPERATION OF A CONCRETE BATCH PLAN ID 02 $\$ 0$

DEVELOP WEAR RESISTANT COMPOSITE STRUCTURE OF VITREOUS FIBER NC $04 \quad \$ 94,494$

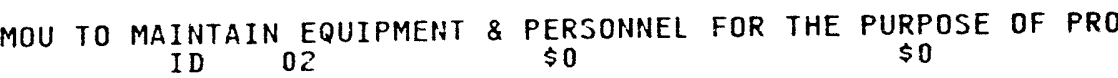

MOU TO MAINTAIN EQUIPMENT \& PERSONNEL FOR THE PURPOSE DF PRO ID 02 \$O 


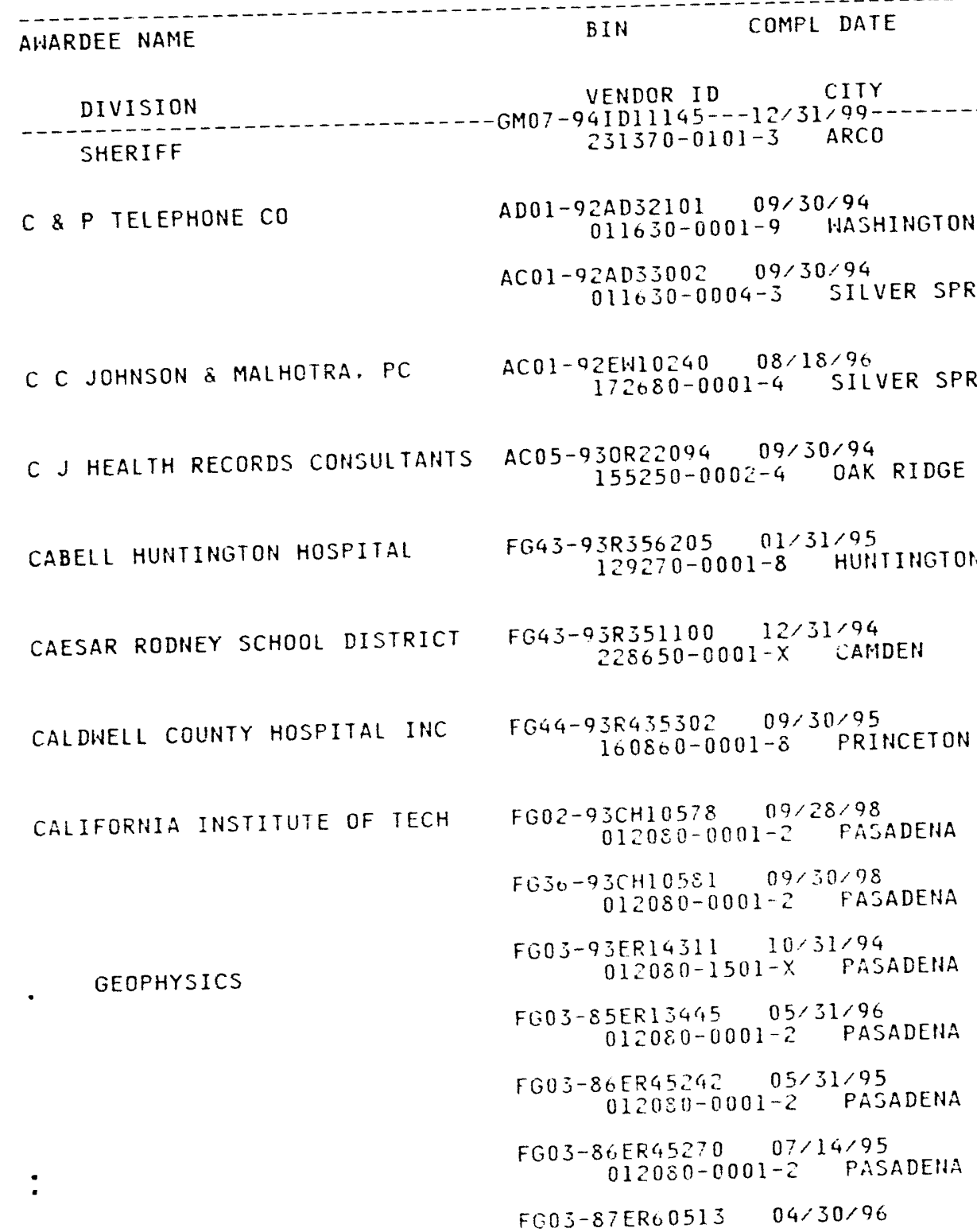

DESCRIPTION OF HORK

CONG CONTRACT
STATE DIST VALUE STATE DIST
RANGE USE AGREEMEIT
ID 01

TELEPHONE MAINTENAHCE

$\$ 13.409$

INTRA LATA COMMUNICATION SERVICES $M D \quad 08 \quad \$ 2.672 .576$

OBLIGS

TO DATE P/S 0 50 $13.409 \quad 0399$ TECHNICAL \& ADM SUPFORT SERVICE FOR EM, OECE MAIL AND FILE SERVICES, SECURITY PROCESSING. ETC LEGAL LIBRA IILE III OF THE HATIONAL ENERGY CONSERVATION POLICY ACT TITLE III OF THE NATIOHAL ENERGY COHSERVATION POLICY TITLE III OF THE NATIOHAL EMERGY CONSERVATION FOLICY ACT SEE ATTACHED PAGE NO. 2 OT THIS NOTICE OF FINANCIAL ASSIJTAN SEE ATTACHED PAGE NO. 2 OI $\$ 12.335$ \$ 167

EMZYME CATALYSTS FOR A BIOTECHHOLOGY-BASED CHEMICAL BIOCATALYSIS BY DESIGH $\$ 356,713 \quad \$ 181,713$

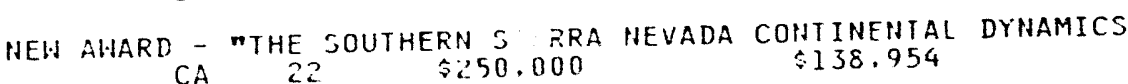

"INFRARED SPECTREJCOPY AHD HYDROGEN ISTOPE GEOCHEMISTRY OF H CA 22 STRUCTURES AND PROPERTIES

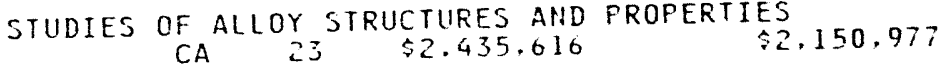

"THE KINITECS OF SHORT RANGE ORDERING IN UHDER COOLED ALLOYS CA 22 $\$ 805.450 \quad \$ 701,391$ RESONANT MULTIPHOTON IONIZATION SPECTOR OF MOLECULES \& MOLEC 


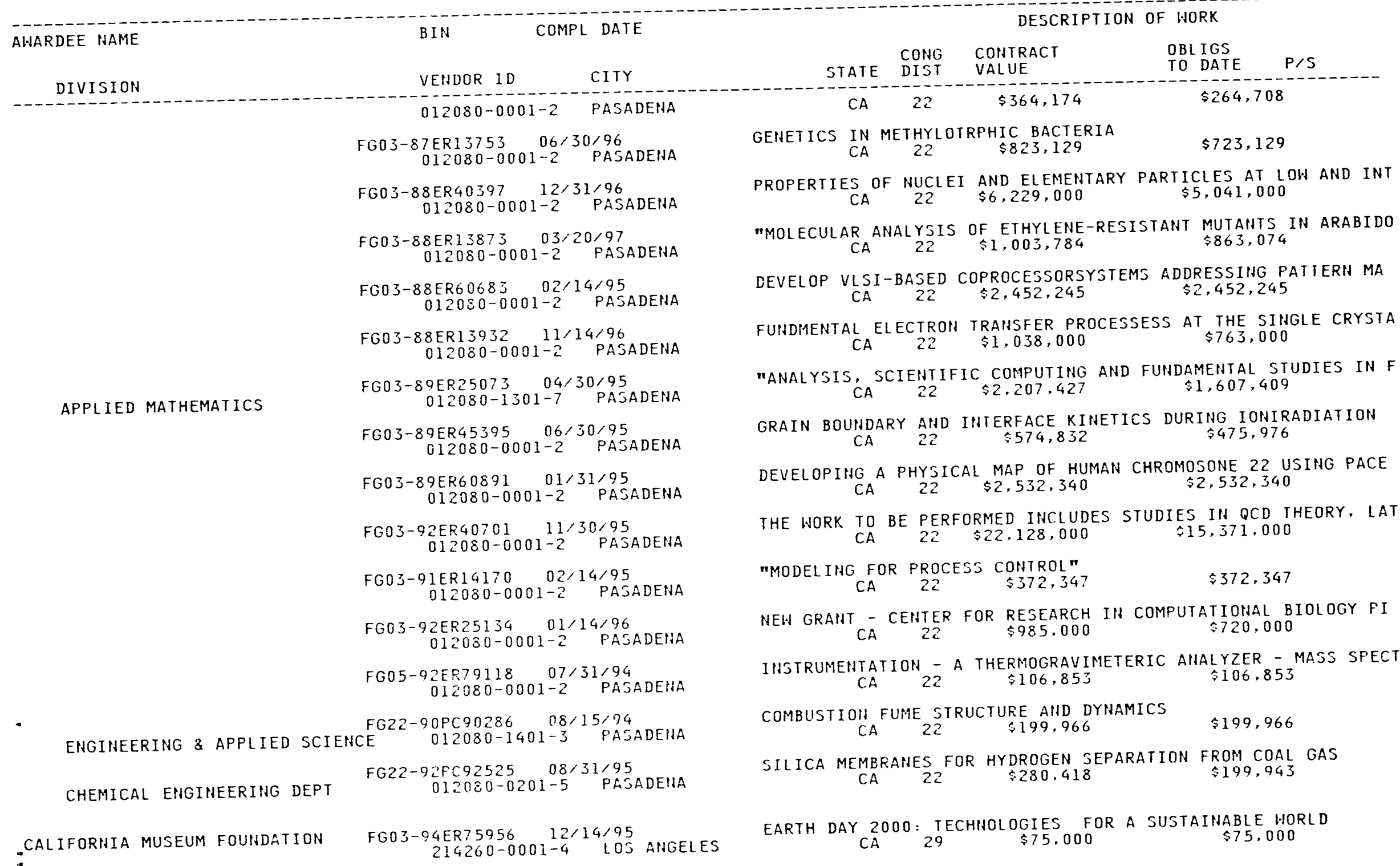


DESCRIPTION OF HORK

\section{AWARDEE NAME}

BIN

CONG CONTRACT

STATE DIST VALUE

EPIDERMIOLOGICAL STUDIES AT ETEC

CA 08 \$ $\$ 42,000$ OBL IGS
TO DATE

$\mathrm{P} / \mathrm{S}$

$\$ 841.361$

CALIFORIIA PUBLIC HEALTH FDN FG03-915F18983 10/31/94 $215380-0101-2$ BERKELEY

HEAL TH SERVICES DEPT OF

GM04-92AL58224 09/02/97 $012120-0007-2$ LIVERMORE

CALIFORNIA STATE OF

$F G 03-78 C 56003512 / 31 / 99$

ECOMOMIC OPPORTUNITY OFFICE OF 012120-0902-9 SACRAMENTO

FG03-90SF18300 07/31/94

$012120-0002-1$ SACRAMENTO

$\begin{array}{ccc}\text { FGO3-94SF20191 } 09 / 30 / 94 \\ \text { WATER RESOURCES COHTROL BOARD } 012120-2002-2 & \text { SACRAMENTO }\end{array}$

FG49-92R914054 08/31/95

ENERGY COMMISSION

$012120-0702-6$ SACRAMENTO

INTEGRATED WASTE MGMT BOARD

FG49-92R900018 09/30/94

012120-1902-4 SACRAMENTO

FG49-92R900017 09/30/94 012120-0502-3 SACRAMENTO

WATER RESDURCES DEPT

FG49-90SF1868409/30/94 012120-0702-6 SACRAMENTO

ENERGY COMMISSION

FG49-80C569085 09/30/94
$012120-1002-7 \quad$ SACRAMENTO

PLANHING \& RESEARCH OFFICE OF

FG49-91R913039 09/30/94

ENERGY COMMISSION

$012120-0702-6$ SACRAMENTO

FG49-92R914055 09/30/95 $012120-0702-6$ SACRAMENTO

ENERGY COMMISSION

ENERGY COMMISSION

ENERGY COMMISSION

CALIFORNIA STATE UHIVERSITY

FG49-93R915030 08/31/95 $012130-0010-6$ CHICO
MUTUAL ASSISTANCE AND EMERGENCY MANAGEMENT $\$ 0$

CA 09 SO

$\begin{array}{clll}\text { WEATHERIZATION PROGRAM INSTAL LATION OF INSULATION } \\ \text { CA } & 03 & \$ 647,000 & \$ 647,000\end{array}$

ENVIRONMENTAL MONITORING

$$
\text { CA } \quad 03 \quad \$ 12,396,304 \quad \$ 5,696,304
$$

SITE INVESTIGATION \& ESTABLISHMENT OF A PROCEDURAL FRAMEHORK SITE INVESTIGATION \& ESTABLISHMENT OF A PROCEDURAL $\$ 120,422$

TITLE III OF THE NATIONAL ENERGY CONSERVATION POLICY ACT

TA 03 TALE $\$ 229,104 \quad \$ 114,582$

INCREASING PAPER INDUSTRY COMPETIVENESS THROUGH MIXED PAFER

$$
\text { CA } 03 \quad \$ 827,327 \quad \$ 100,000
$$

MICE3 GRANT TO DEMONSTRATE ULTRASONIC DISHING TECHNOLOGY

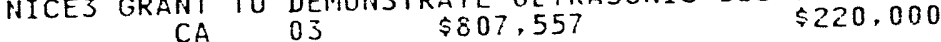

GRANT PROGRAMS FOR SCHOOLS ANDHOSPITALS AND BUILDINGS SWNHED

CALIFORNIA ENERGY EXTENSION SERVICE

$$
\text { CA OJ } \checkmark 29,296,970
$$

$\$ 7.380,130$

CALIFORNIA ADMINISTRATIVE GRANT

$\$ 236,009$

THERGY CONSERVATICH POLICY ACT

$\begin{array}{lll}\text { TITLE III OF THE NATIONAL } & \$ 241,456 & \$ 120,728\end{array}$

CA $03 \quad \$ 5,122,444 \quad \$ 2,283,096$

INSTITUTIONAL CONSERVATION PROGRAM, CYCLE 15, STATE OF CALIF CA 01 \$228,310

$\begin{array}{cccc}\text { PRE-FRESHMAN ENRICHMENT PROGRAM (PREP) } & \text { PRA } \\ \text { CA } & 02 & \$ 37.883 & \$ 37.883\end{array}$

A NEW METHOD FOR DETERMINING THERMODYNAMIC PROPERTIES OF CAR $\begin{array}{llll}\text { CA } & 04 & \$ 240,317 & \end{array}$

$$
\begin{array}{llll}
C A & 03 & \$ 418,111 & \$ 104,741
\end{array}
$$

CALIFORNIA COORDINATING AGENCYCYCLE 15 GRANT
FG49-93R91502908/31/96 $012120-0702-6$ SACRAMENTO $012120-0702-6$ SACRAMENTO

FG03-93ER79167 06/14/95 FG03-92ER14307 09/14/95 


\begin{tabular}{|c|c|c|c|c|c|c|c|}
\hline AHARDEE NAME & BIN & L DATE & & & DESCF & F WORK & \\
\hline DIV & VENDOR ID & CITY & STATE & $\begin{array}{l}\text { CONG } \\
\text { DIST }\end{array}$ & $\begin{array}{l}\text { CONTRACT } \\
\text { VALUE }\end{array}$ & $\begin{array}{l}\text { OBLIGS } \\
\text { TO DATE }\end{array}$ & P/S \\
\hline
\end{tabular}

FG03-86ER13521 05/31/95 $012130-0002-5$ FULLERTON FG03-92ER75317 01/31/95 $012130-0002-5$ FULLERTON FG03-93ER61728 09/14/96 D1 $130-0002-5$ FULLERTON FG03-94ER79243 06/14/96 012130-0003-j

TRUSTEES BOARD OF

ENGINEERING \& COMPUTER SCI

TRUSTEES BOARD DF

TRUSTEES BOARD OF

"CALIFORNIA UHIVERSITY OF NUCLEAR EHGINEERING

PHYSICS DEPT

FG03-93ER79170 06,14/95

FG49-89SF18331008/31/94

FG49-91R913035 08/31/95

FG49-92R914036 08/31/94

FG02-90ERI3033 09/30.94 $012140-7402-2$ BERKELEY

AT03-76ER70025 12/31/99 $012140-2002-X$ BERKELEY

AT03-76ER70088 12/31/99 $012140-0002-9$ BERKELEY
CORRELATED PROCESSES IN ATOMICPHYSICS

$$
\text { CA } 39 \quad \$ 429,402 \quad \$ 429,402
$$

NEW GRANT: CIMATEC (CIENCIA, MATEMATICA, TECNOLOGIA)

$$
\begin{array}{lll}
\text { CA } & 39 & \$ 90,790
\end{array}
$$

LABORATORY STUDIES OF THE SENSITIVITY OF TROPOSPHERIC OZONE

$$
\text { CA } 39 \quad \$ 565.632 \quad \$ 240,007
$$

PRE-FRESHMAN ENRICHMENT PROGRAM (PREP) FOCUS: PHYSICS-2

$$
\begin{array}{llll}
C A & 13 & \$ 37,581 & \$ 37.581
\end{array}
$$

GRANT PROGRAMS FOR SCHOOLS ANDHOSPITALS AND BUILDINGS OWNED

$$
\text { CA } 32 \quad \$ 611,344 \quad \$ 305,669
$$

NEW ALIARD FOR A PERIOD OF THREE (3) YEARS PROJECT TITLEN A H $C A \quad 30 \quad \$ 358,571 \quad \$ 103,790$

FGO3-94ER61797 $03 / 14 / 97$
$012130-0011-4$ LOS ANGELES

$012130-0011-4$ LOS ANGELES

$\begin{array}{rl}\text { FGO3-87ER } 40347 & 03 / 31 \\ 012130-0306-7 & \text { NORTHRIDGE }\end{array}$

FG05-92ER79061 $09 / 30 / 94$
$012130-0006-8 \quad$ HORTHRIDGE $012130-1713-0$ SEAL BEACH $012130-0013-0$ SEAL BEACH $012130-1713-0$ SEAL BEACH

AT03-76ER70201 12/31/99 CI2140-0002-9 12/31/99
FRE-FRESHMAN ENRICHMENT PROGRAM (PREP) 93

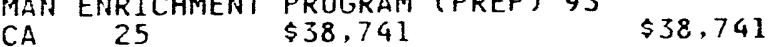

"THEORETICAL RESEARCH IN INTERMEDIATE - ENERGY NUCLEAR PHYSI CA $23 \quad \$ 515,000 \quad \$ 515,000$

PRE-FRESHMAN EHRICHMENT PROGRAM (PREP) - $1992 . \$ 40.000$

$$
C A \begin{array}{lll}
C 3 & \$ 40,000 & \$ 40.000
\end{array}
$$

GRANT PROGRAMS FOR SCHOOLS ANDHOSPITALS AND BUILDINGS OWNED CA 42 \$670,309 $\$ 335,152$

CYCLE XIII GRANT $32 \quad \$ 548,769 \quad \$ 274,384$

TITLE III OF THE NATIONAL ENERGY CONSERVATION POLICY ACT $C A$ SA 32 \$36, 088 \$178,044 $\begin{array}{ccc}\text { NUCLEAR EHGINEERING RESEARCH MIGRATION OF FISSION PRODUCTS I } \\ \text { CA } 08 & \$ 404,136 & \$ 403,451\end{array}$

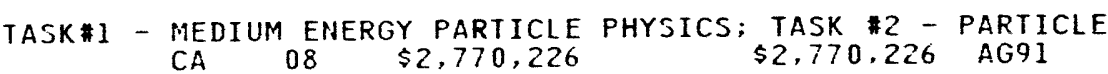

$\begin{array}{cccc}\text { INTRA AND INTERMOLECULARENERGYTRANSFER STUDIES } & \\ & \$ 906,284 & \$ 906,284 & \text { AG91 }\end{array}$

FOURIER SPACE COMPUTER SIMULATION OF CRYSTALLINEIMPERFECTION

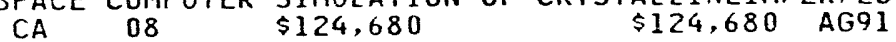




\section{DESCRIPTION OF WORK}

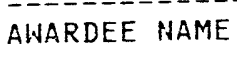

BIN

COMPL DATE

CONG CONTRACT

VENDOR ID

CITY

STATE DIST VALUE

DIVISION

ATO3-76ER70230 $12 / 31 / 99$
$012140-0002-9$ BERKELEY

AT03-76ET53055 12/31/99

012140-0002-9 BERKELEY

AT03-76EV70124 12/31/99

$012140-0002-9$ BERKELEY

AT03-77EV70264 12/31/99

MEDICINE DEPT

012140-1902-1 BERKELEY

AHREHCE LIVERMORE NATL LAB

W-7405-ENG-48 $09 / 30 / 97$
$012140-7502-9$ BERKELEY

AMO3-76SF00010 12/31/99 012140-0002-9 BERKELEY

AC03-76SF00098 09/30/97 012140-0002-9 BERKELEY

AT03-76SF90247 12/31/99 012140-0002-9 BERKELEY

ATO3-76SF90249 12/31/99
$012140-3702-X \quad$ BERKELEY

FG03-85ER1 $3375 \quad 02 / 29 / 96$ 012140-0002-9 BERKELEY

FG03-85ERI3419 08/14/94 012140-0002-9 BERKELEY

FG03-86ER60406 01/31/95 012140-0002-9 BERKELEY

FG03-86ER $13518 \quad 04 / 30 / 96$ 012140-0002-9 BERKELEY

FG03-87ER40323 12/31/94 012140-0002-9 BERKELEY

FG03-87ER13727 08/14/96 $012140-2002-x$ BERKELEY

PHYSICS DEPT

FG03-87ERI3742 06/30/96 $012140-2002-X$ BERKELEY
CHRONIC IRRADIATION AHD BRAIN DEVELOPMENT

CA $08 \quad \$ 218,620 \quad \$ 218.620$ AG91

A STUDY OF PLASMA HEATING AND FIELD REVERSAL BY INTENSE ELEC CA 08 \$1,081,989

STUDIES ON DNA IRRADIATION ANDDNA SYNTHESIS IN VIVO

INFLUEHCE OF LATE RADIATION EFFECTS ON THE IMMUNOLOGIC PARAM INFLUEHCE OF LATE RADIATIOH EFFECTS ON THE IMUN $\$ 1,227,498$ AG91

OPERATION OF LAHRENCE LIVERMORE LABORATORY

CA $0818,157,781,159 \quad 18,120,203,387 \quad M 18$ THIS IS A BASIC CONTRACTWITH $\underset{\$ 0}{\text { PROJECTS AGREEMENT }} \underset{\$ 0}{S}$ AG9

OPERATION OF BERKELEY NATIOHALLABORATORY

CA $08 \$ 2,340,383,734 \quad \$ 2,303,340,728$ AZ11

DETERMINATION OF THE MICROB
CA
08 $\begin{gathered}\$ 88,750 \\ \text { IAL CONTENT OF GCEAN SEDIMENTS } \\ \$ 88,750\end{gathered}$ $\begin{array}{cccc}\text { WATER COLUMN BIOLOGY OF THE NORTH PACIFIC CENTRAL GYRE } \\ \text { CA } & 08 & \$ 18,000 & \$ 18,000\end{array}$

TRANSCRIPTION CONTROL ELEMENTSAHD MANIPULATION OF CHLOPOPLA $C A=08 \$ 1,043,124 \quad \$ 932,124$

DIFFUSION/DISPERSIDN TRANSPORTOF CHEMICALIY - REACTING SPEC CA 08 \$ $\$ 1,157,665$ $\$ 1,157.665$

"STRUCTURE AND STABILITY DF NUCLEIC ACIDSn

"DETERMINATION OF GENES AND PHENOTYPES OF BACTERIA NECESSARY
$\$ 643,901$

NEH SRG: "MEDIUM ENERGY MESON RESEARCH" (FORMERLY SRC ER CA 08 \$2,105,000 \$1,853,000

" SELF - GENERATED STOCHASTIC HEATING IN AN RF DISCHARGE " CA $08 \quad \$ 851,522$

MPHYTOCHROME FROM GREEN PLANTS: ASJAY, PURIFICATION AND CHA CA $\begin{array}{r}08 \\ \$ 826,000\end{array}$ 
AWARDEE NAME

BIN

COMPL DATE

VENDOR ID CITY

DIVISION

PHYSICS DEPT

PHYSICS DEPT

PHYSICS DEPT

PHYSICS DEPT

FG03-88ERI3882 04/30/96

FG03-89ER52154 10/31/94

FG03-89ER51110 11/09/94
ZERI3793--12/14/96----
$012140-2002-X$ BERKELEY

$012140-2002-X$ BERKELEY

$012140-2002-X$ BERKELEY

$012140-2002-x \quad$ BERKELEY

FGO3-90ER40571 11/14/94 012140-0002-9 BERKELEY

FG03-90ER60999 $01 / 31 / 96$ 012140-0002-9 BERKELEY

FGOJ-91ER61125 01/31/97 $012140-0002-9$ BERKELEY

FG03-91EF.14200 07/31/94 $012140-0002-9$ BERKELEY

FG03-92ER61393 10/31/95 $012140-0002-9$ BERKELEY

FGOJ-92ER61458 02/14/95 $012140-0002-9$ BERKELEY

FG03-92ERI4276 08/31/94 012140-0002-9 BERKELEY

FG03-92ER14287 07/31/95 $012140-0002-9$ BEFKELEY

FG03-92ER25135 07/31/94 $012140-0002-9$ BERKELEY

FG03-92ER25140 02/14/05 012140-0002-9 BERKELEY

FGO3-93ER25177 07/31/96 012140-0002-9 BERKELEY

FG05-92ER79131 07/31/94 012140-0002-9 BERKELEY

FG22-90FC90287 08/31,04 $012140-0002-9$ BERKELEY
DESCRIPTION OF HORK

CONG CONTRAC

OBLIGJ

D DATE $P / S$ STATE DIST VALUE CA 08 \$ $\$ 905.485$

$\$ 686,735$ "ANALYSIS OF PROTEINS ESSENIRAL DOE AGRPBACTERIA MEDIATED DN CA $08 \quad \$ 698,184$ $\$ 600,394$

COMPUTER MODELIHG OF (ESE) FEATURES WITH THE ULTIMATE AIM OF $C A$ OE \$1,775,109

\$1.755.109

"COOPERATIVE PROGRAM TO ANALYZE heAT TRANSFER AT HIGH beta I CA $08 \quad \$ 1,419,000 \quad \$ 1,419,000$

PROGRAM OF STUDY OF RAVE PARTICLE PRODUCTION IN HIGH ENERG $C A$ SA $\$ 449.000 \quad \$ 449,000$

THE PROJECT INVESTIGATES A NUMBER OF ALGORITHMIE FROBLEMS IH CA 08 \$ $\$ 1,595,231$ \$1, 224,181

NEW AHARD OF A PROJECT ENTITLES NULTRASENSITIVE FLUORESCENCE CA 08 \$1,903,847 \$1,221,867

HEH GRANT - EXPERIMEHTAL MEASUREMENT OF THERMAL CONDUCTIVITY CA 08 S 0841.213 \$341.213

PATHWAYS TO GENETIC SCREENING:PATIENT KNOHLEDGES-PATIEHT $P$ CA 08 PATHWYYS T456.939 RESEARCH TO UHDERSTAND THE ROLE OF DNA REPAIR IN CONTROLLING CA $08 \quad \$ 326.738 \quad \$ 326,738$

FUNDING TO SUPPORT IMPROVED STRATEGIES FOR COLLECTING MT DAT $C A \quad 08 \quad \$ 68,402 \quad \$ 68,402$

THERMAL RADIATION AND CONDUCTION IN MICROSCALE STRUCTURES

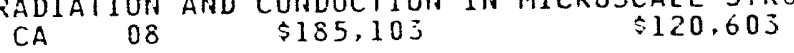

GIGABIT TESTBED RESEARCH

CA $08 \quad \$ 458,930 \quad \$ 458.929$

RESEARCH TO DEVELOP HPCC - ADAPTIVE METHODOI OGIES FOR COMPUT $C A \begin{aligned} & 08 \\ & \text { S }\end{aligned}$

NEH GRANT "GEOMETRIC ANALYSIS OF MULTICOMPONENT HAVE EQUATIO $C A \quad 08 \quad \$ 150,000 \quad \$ 50,000$

INSTRUMENTATION - PURCHASE OF A PHOFHORIMAGER SYSTEM FOR PLA CA 08 S163,067

OXIDATION OF COAL AND COAL FYRITE: MECHAMISMS AHD INFLUENCE CA 08 \$200.000 $\$ 200.000$ 


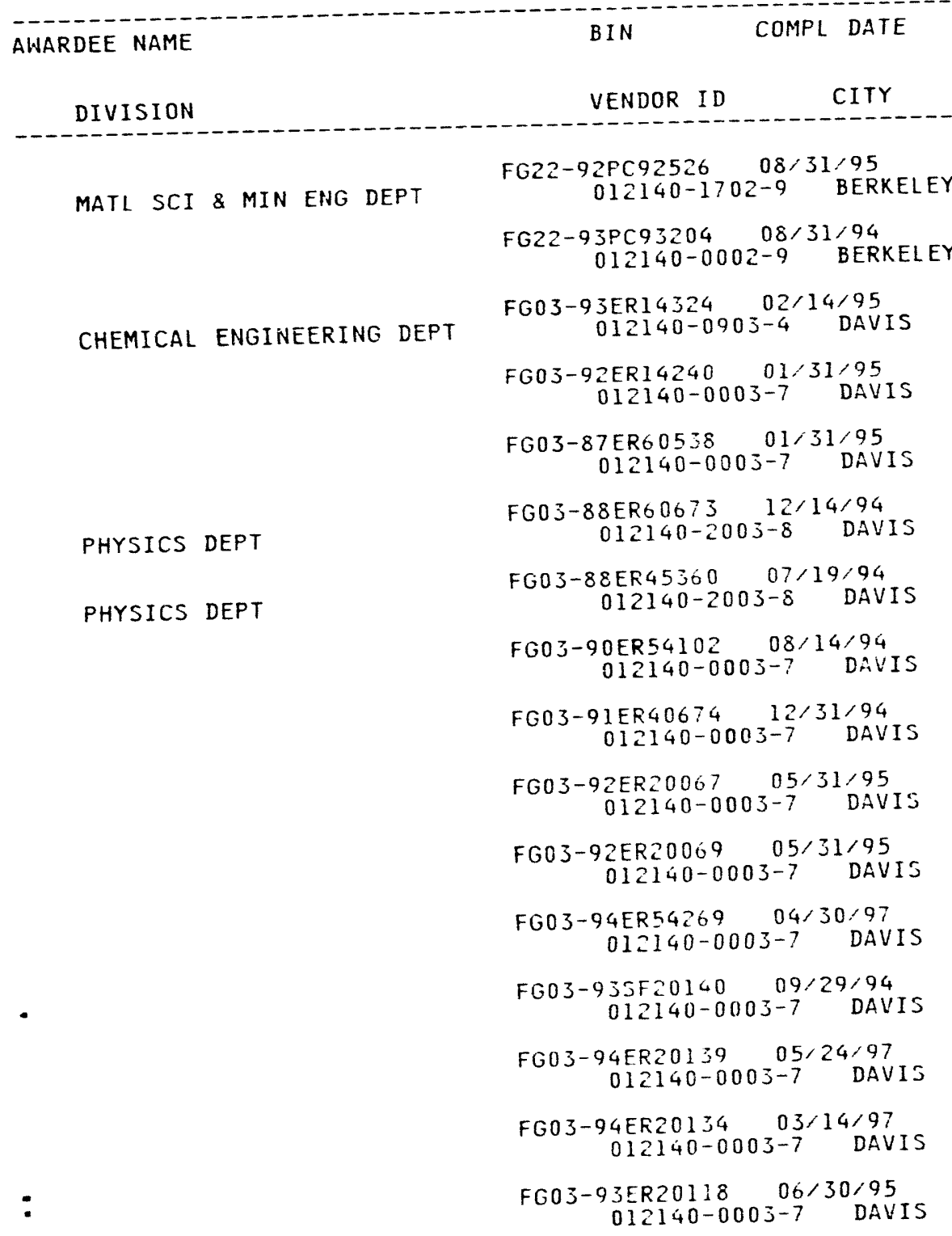

DESCRIPTION OF HORK

CONG CONTRACT
STATE DIST VALUE

OBLIGS

$P / 2$

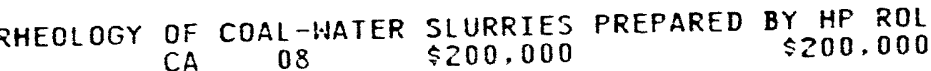

ANALYSIS OF MICROALGAE POHDS FOR CONVERSION OF CO2 TO BIOMAS

ANALYSIS OF MICROALGAE PONDS FOR CONVERSION $\$ 100,000$

CHARACTERIZATION OF THE METAL-SUPPORT INTERFACE IN SUPPORTED CHARACTERIZATION OF THE METAL 04 S182,000 $\$ 182,000$

NEW GRANT " EHERGETICS OF SILICATE MELTS FROM THERMAL DIFFUS NEN GRANT " ENERGETICS OF STITCATE MELTS FROM THERMA $\$ 196.752$

STUDIES OF THE REPAIR OF RADIATION-INDUCED GENETIC DAMAGE IN STUDIES OF THE REFAIR OF RAD,700 04 \$1,806,700

INEUTRON SCATTER STUDIES OF CHROMATIN STRUCTURES RELATED TO MINEUTRON SCATTER STUDIES OF $\$ 2.269,347$ ?

nINUESTIGATIOH OF THE RATE-CONTROLLING MEZHANISM(S) FOR HIGH NUESTGATION OF THE RATE-CONTROLLING MESHANTSMS $\$ 752,905$

"IMJECTION OF COMPACT TOROIDS FOR TOKAMAK FUELING AND CURREN CA $04 \quad$ \$871.907 $\quad \$ 871.907$

RESEARCH OH QUAHTUM GRAVITY

$$
\begin{array}{lll}
\text { OH QUANTUM GRAVITY } \\
\text { CA } 04 & \$ 5.515 .000 & \$ 3.861,000
\end{array}
$$

ROLE OF MALATE METABOLISM IN PLANT CELL DIFFERENIIATION

ROLE OF MALATE METABOL \$351,000 \$236.000

CELLULOSE BINDING PROTEINS OF CLOSTRIDIUM CELLULOVORANS CA $04 \quad \$ 321,000$

$\widehat{S} 12,000$

NEW AWARD - DIAGNOSTIC OF THE SPATIAL \& VELOCITY DISTRIBUTIO NEW AWARD - DIAGNOSTIC OF $0491.816 \quad \$ 96,404$

HUF USER'S GRAHT AHARD - IOH DECAY IHSTABILITY LARGE SCALE. CA 03 \$112.563 $\$ 112.563$

IEH ALARD - REGULATION OF EMBRYONIC DEVELOFMENT IN HIGHER PL CA RUARACTERIZATIOH OF VASCULAR PLASMODE CELLULAR AND MOLECULAR $\begin{gathered}\$ 351.000 \\ \text { CA } 04\end{gathered}$ PROIEIN TRANSLOCATION AND ASSEMBLY IN CHLOROPLASTS

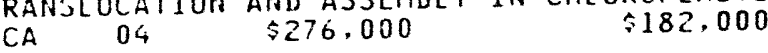




\section{AWARDEE NAME}

DIVISION

COMPL DATE

PHYSICS DEPT

CHEMISTRY DEPT

PHYSICS DEPT
BIN

COMPL DATE

VENDOR ID CITY $012140-0003-7$ DAVIS

FG05-92ER79120 07/31/94 012140-0003-7 DAVIS

FG22-94PC94204 06/30/97 012140-0003-7 DAVIS

FG03-84ER45083 01/31/96 $012140-2004-6$ IRVINE FG03-85ER45196 03/31/95 $012140-1004-0$ IRVINE

FG03-86ER13469 07/31/94 $012140-0004-5$ IRVINE

FG03-86ERI3514 03/14/96 012140-0004-5 IRVINE

FG03-86ER13525 05/31/96 012140-0004-5 IRVINE

FG03-89ER60849 07/31/95 012140-2004-6 IRVINE

FG03-90ER45420 06/30/96 012140-0004-5 IRVINE

FG03-90SF18785 09/29/94 $012140-0004-5$ IRVINE FG03-92ER54145 12/31/94 012140-0004-5 IRVINE FG03-91ER40679 01/31/95 012140-0004-5 IRVINE FG03-91ER61227 08/31/94
$012140-0004-5$ IRVINE FG03-92ER75805 09/29/94
$012140-0004-5$ IRVIIIE FG03-92ER79147 09/29/94 $012140-0004-5$ IRVINE FG03-93ER54220 07/31/96
$012140-0004-5$ IRVINE
DESCRIPTION OF WORK

CONG CONTRACT STATE DIST VALUE OBLIGS

TO DATE P/S INTERACTIVE EFFECTS DF ELEVATED CO2, DROUGHT, AND HIGH TEMPE INSTRUMENTATIOH-HYBRID VLSI PARTICLE DETECTOR PRECISION BOND CA O4 \$147,508 \$147,508 FUNDAMENTAL KINETICS OF SUPERCRITICAL COAL LIQUEFACTION: EF $C A{ }_{04} \$ 183,458$ SURFACE EXCITATIONS AND THEIR INTERACTION WITH LOW ENERGY CA 40 \$1.596,525 \$1,188,76I RAMAN SPECTROSCOPY OF MOLECULAR ADSORBATES CA 40 \$1, 465,783 $\$ 1,465,783$ RESEARCH IN CHEMICAL KINETICS SYNTHESIS AND CHEMISTRY OF UTRIUM AND LANTHANIDE METAL CA 40 \$1,016,200 $\$ 887,013$ THE BIOGENERGETIES OF SALT TOLERANCE" $\$ \$ 999,582$ SURFACE CHEMISTRY INVESTIGATION OF COLLOID TRANSPORT IN PACK CA $40 \quad \$ 665,816 \quad \$ 515,782$ NEH
CA 40
$\$ 495,849$
$\$ 405.712$

MINORITY UHDERGRADUATE TRAINING IN ENERGY-RELATED CAREERS

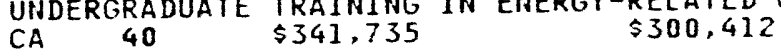

"THE DIFFUSION OF BEAM IONS DURING AUXILIARY HEATING IN TFTR THE DIFFUSION OF BEAM $\$ 185,409$ \$ $\$ 185,409$ FROHT END ELECTRONICS DEVELOPMENT FOR SSC DETECTORS CA $40 \quad \$ 8,225,300 \quad \$ 8,055,300$ NEW GRANT - A CENTER OF EXCELLENCE FOR THE MEDICAL APPLICATI 40 SA $\$ 1,476,000$
A NEW GRANT - UNIVERSITY REACTORSHARING PROGRAM CA $40 \quad \$ 19,700$ GRANT TO FUND REPLACEMENT $\$ 82,750 \quad \$ 82,750$ NEH GRANT - "THERMOHUCLEAR FUSION IN A STAGED Z-PINCH" NEH GRANT - "THERMOHUCLEAR FUSTON IN A STAGED $\$ 420.169$ 
AWARDEE NAME

BIN

VENDOR ID

CITY
STATE DIST VALUE

\section{FG05-92ER79125 07/31/94
$012140-0004-5$ IRVINE} FG03-94ER54271 05/31/97
$012140-0004-5$ IRVINE FG03-93ER61543 12/31/95 $012140-0005-3$ LA JOLLA

PHYSICS DEPT

BIOLOGY DEPT

PHYSICS DEPT

PHYSICS DEPT PHYSICS DEPT
FGO3-93ER40759 11/14/95
$012140-2005-4$ LA FC03-92ER54144 04/14/98 $012140-0005-3$ LA JOLLA

FG03-90ER61061 10/31/94 012140-0005-3 LA JOLLA

FG03-85ER53199 10/31/94 $012140-0005-3$ LA JOLLA

FG03-86ER13497 03/31/96 012140-0605-1 LA JOLLA

FG03-86ER45230 11/30/94 012140-0005-3 LA JOLLA

FG03-86ER60429 03/14/97 $012140-0005-3$ LA J0LLA

FG03-86ERI3527 11/30/96 $012140-0005-3$ LA JOLLA

FG03-87ER13685 04/30/96 $012140-0005-3$ LA JOLLA

FGOJ-87ER45332 03/14/96 $012140-2005-4$ LA JOLLA

FG03-88ER53275 11/14/94 $012140-2005-4$ LA JOLLA 012140-2005-4 LA JOLLA

FG03-90ER60981 05/31/96 $012140-0005-3 \quad L A$ JOLLA
FG03-90ER40546 12/31/96
1992 INSTRUMENTATION - AN ULTRAHIGH VACUUM VARIABLE TEMPERAT $C A \quad 40 \quad \$ 334,375 \quad \$ 334,375$ THEORETICAL STUDIES OF ALPHA AND ENERGETIC - PARTICLE PHYSIC

APPLICATION SEEKS FUNDS TO CONTINUE MEASUREMENTS OF DIC CA 41 F $\$ 43,832$ \$411.995

THEORETICAL PROBLEMS IN ACCELERATOR PHYSICS

THEORTICAL PROBLEMS IN ACCELERATOR PHYSICS $\$ 263.000$

SAH DIEGO CO-CENIER FACIIITY OPERATION AND SUPPORT FOR CONDU CA 41 \$27,713,476 $\$ 12,124,705$

"DIAGNOSTIC MODELING OF THE ARM EXPERIMENTAL CONFIGURATION" CA
MODEL OF THE ARM EXPERIMENTAL CONFIGUT

STUDIES OF PLASMA TRANSPORT RELEVANT TO TANDEM MIRROR $C A \quad 41 \quad \$ 2,103,428 \quad \$ 2,101,940$

CHARACTERIZATION AND BIOSYHTHESIS OF COMPLEX PROTEIN - BOUND $C A \quad 41 \quad \$ 841,142 \quad \$ 841,142$

"INVESTIGATION OF SUPER CONDUCTIVITY AND MAGNETISM D AND $S E$ $C A \quad 41 \$ 3.017 .053 \quad \$ 3.017 .055$

STRUCTURE AND FUNCTION OF THE HUMAN METALLDTHIOHISM GENE FAM $\begin{array}{lll}C A & 41 & \$ 1,135,048\end{array}$

"PREMIXED TURBULENT COMBUSTION
CA 41
$\$ 529.266$
$\$ 417.758$

"EXPERIMENTAL AND THEORETICAL STUDY OF FUEL DROPLETS SUBJECT CA $41 \quad \$ 1.134 .880 \quad \$ 994,950$

"PREPARATION AHD CHARACTERIZATION OF SUPERLATTICES"

"INUESTIGATIONS IN THE THEORY OF anOMALOUS TRAHSPORT AND MAD $\begin{array}{llll}C A & 41 & \$ 2,107,239 & \$ 2,080,062\end{array}$

RESEARCH I' THEORETICAL \& EXPERIMENTAL PHYSICS DEVOTED TO IN CA $41 \quad \$ 5,849,704 \quad \$ 4.084,704$

THE PRIMARY FOCUS OF THIS PROPOSAL IS THE SHIPBDARD MEASUREM CA $450 \begin{aligned} & \$ 474,783 \\ & 4343,836\end{aligned}$ 
VENDOR ID CITY NEER $40580--12 / 31 / 94-\cdots-1$ 012140-0005-3 LA JOLLA

FG03-90ER14138 07/31/98 $0 I 2140-0005-3$ LA JOLLA

FG03-90ER14148 08/14/96 $012140-0005-3$ LA JOLLA

FG03-91ER61116 07/31/94 $012140-0005-3$ LA JOLLA

FG03-91ER14176 07/14/94 $012140-0005-3$ LA JOLLA

FG03-91ER61171 10/31/94 $012140-0005-3$ LA JOLLA

FG03-9IER14188 06/14/96 $012140-0005-3$ LA JOLLA

FG03-91ER61198 10/31/94 $012140-0005-3$ LA JOLLA

FG03-91ER61215 08/31/95 012140-0005-3 LA JOLLA

FG03-91ER61222 08/31/94 $012140-0005-3$ LA JOLLA

FG03-92ER40718 03/31/95 012140-0005-j LA JOLLA

FG03-92ERo $1410 \quad 04 / 30 / 95$ DI2140-0005-3 LA JOLLA

FG03-92ER14272 06/14/95 012140-0005-j LA JOLLA

FG03-94ER25192 12/31/95

APPLIED MECH \& ENG SCI DEPT

FG03-93ER40793 02/14/96 012140-0005-3 LA JDLLA

FGO3-93ERE $1592 \quad 04 / 30 / 96$ 012140-0005-3 LA JOLLA
FG03-9JER61690 09/29/96 $012140-0005-3 \quad$ LA JOLLA
CONG COHTRACT STATE DIST VALUE THE EINSTEIN-PODOLSKY-ROSEH PHENOME
EXPERIMENTAL RESEARCH ON THE EINTH

OBLIGS

TO DATE P/S $\$ 140,000$ SIO0,000 NONPERIODIC, BROADBAND SIGNALS: SIGNAL PROCESSING IN CHAOS TRAVELING-HAVE CONVECTION IN FLUID MIXTURES

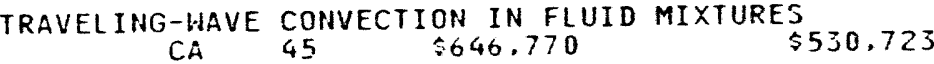
OCEANIC CO2 MEASUREMENTS FOR THE HOCE HYDROGRAPHIC SURVEY IN CA 45 \$286.177 $\$ 286.17 \%$

"KNOT INVARIANTS AND THE THERMODYHAMICS OF LATIICE GAS AUTOM CA 41 \$290,000 $\$ 290,000$

COMPARATIVE STUDIES IN THE FIELD OF MOUSE AND HUMAN LEUKEMIA CA 45 \$6 72,798 IN $\$ 672,798$

FORMATIOH OF THREE-DIMEHSIONALSINGULARITIES, REGULARIZATION CA 41 \$772.823 $\$ 375.130$

ATMOSPHERIC AHD CLIMATE RESEARCH ON ROLE OF CLOUD. HATER VAP CA 41 \$3, 393,415 $\$ 1,997,388$

STUDIES OF OCEAN PREDICTABILITY AT DECADE-TO-CENTURY TIME SC CA 41 \$376.766 \$376.760

SYMMETRIC TRUNCATION OF THE SHALLOH WATER EQUATIONS $C A \quad 41$ \$339,708 $\$ 339.700$

CONTINUAIION OF SUPPORT FOR THE INTECAMPUS INSTITUTE FOR RES $C A \quad 41$ \$ $\$ 30,000$ $\$ 830,000$

QUALITY ASSURAHCE DF MEASUREMENTS OF OCEAHIC CARBOH DIOXIDE CA 41 \$722,875 $\$ 722.875$

HEW GRAHT - NHEW MOLECULAR PRECURSORS FOR LOH-TEMPERATURE RO CA 41 \$3 32.966 \$234.191

NUMERICAL STUDIES OF THO-PHASEAND PARTICULATE FLOH

$$
\text { CA } 49 \text { \$128.050 \$63.050 }
$$

THE SCOPE OF WORK INCLUDES NUMERICAL SIMULATIONS AND APPROPR CA 41 \$375,000 HERE THE GRANTEE PROPOSES TO STUDY THE STRUCTURES OF THE LMD HIERARCHY OF THERMOHALINE CIRCULATION MODELS

OF THERMOHALINE CIRCULATION MODELS
CA 49


PROCUREMENT AND ASSISTANCE DATA

NAMES BY NAME, CITY \& STATE - ACTIVE AWARDS

PAGE NO. 79

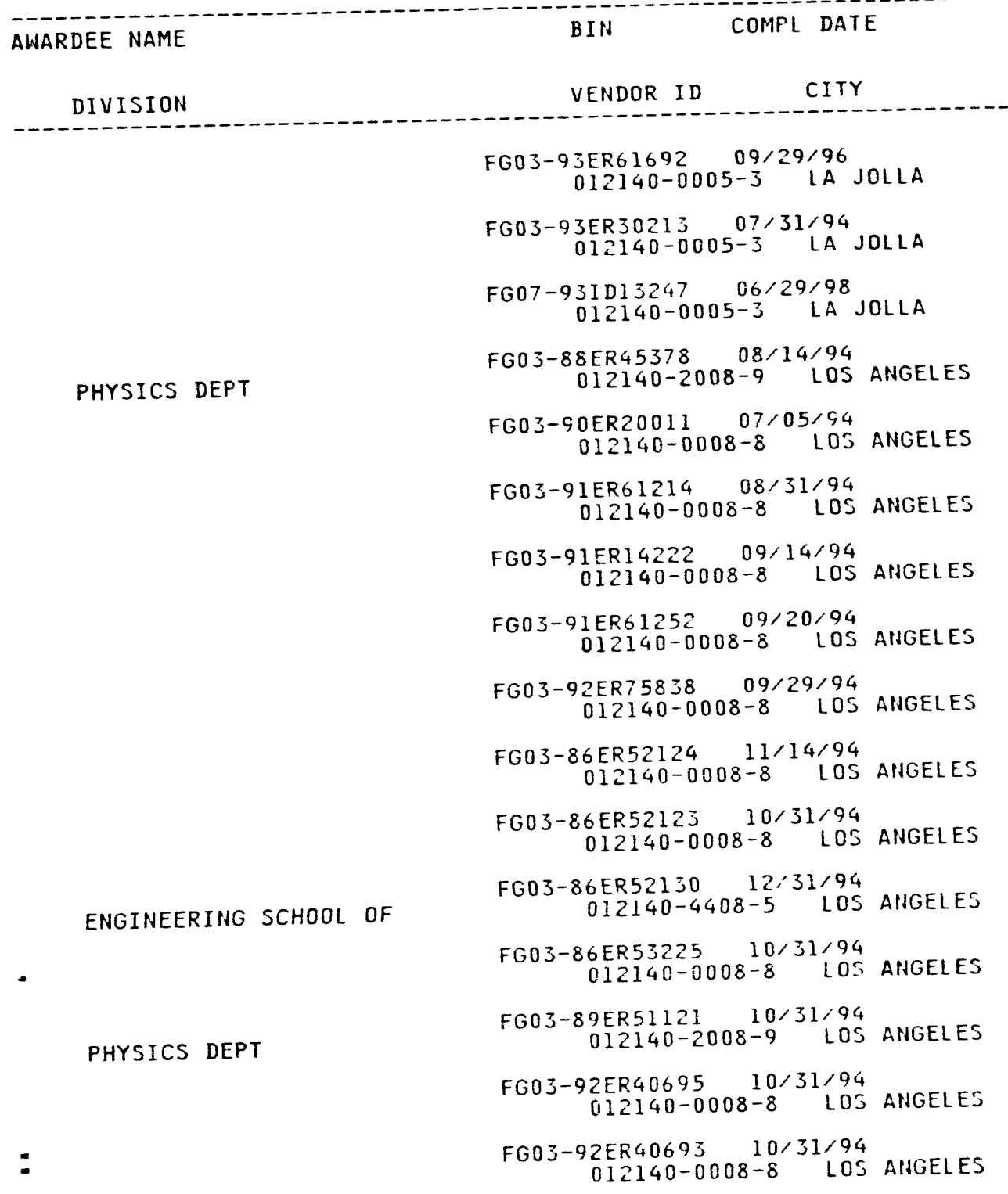

DESCRIPTION OF HORK

$\begin{array}{ll}\text { CONG CONTRACT } & \text { OBLIGS } \\ \text { STATE DIST VALUE } & \text { TO DATE }\end{array}$

TO DATE

$P / S$

CELL-SURFACE PROTEINS AS MARKERS FOR THE PHYSIOLOGICAL STATE CA $49 \quad \$ 465.982$ \$ 460.797

AN ASSESSMENT OF THE RESEARCH MEEDS FOR ADVANCED FUEL CELLS

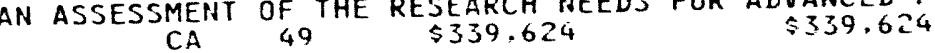

$\begin{array}{cll}\text { MODELS OF GEOTHERMAL BRIME CHEMISTRY } & \$ 331,170 \\ \text { CA } 49 & \$ 2,056.171\end{array}$

QUANTUM FLUXUATIONS IN MESOSCOPIC SYSTEMS $\$ 338,390$ CARBON DIOXIDE AHD THE STOMATAL CONTROL OF WATER BALANCE AHD

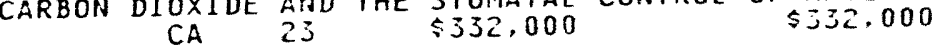
NEW GRANT - DEVELOPMENT OF AN ADVANCED FINITE-DIFFERENCE ATM CA 23 SA $\$ 432,529 \quad \$ 432,529$ NEW GRANT - "OXYGEN AND CATIONDIFFUSION IN OXIDE MINERALS" NA $2{ }_{2 j} \quad \$ 103.765 \quad \$ 103.765$ NEH GRANT - CO2 EXCHANGE, ENVIROHMENTAL PRODUCTIVITY INDICES NEH GRANT CA CO2 23 STHAS $\$ 421.031$ \$383.355 NEW AWARD - THE GOAL OF THIS PROJECT IS TO REBUILD FISSION-R NEW AWARD CA 23 \$100.000 \$100.000 DEVELOPMENT AHD AHALYSIS OF FUSIOH BREEDER BLAHKER CA 23 FINESSE KEY ISSUES OF FUSION HUCLEAR TECHIOLOGY DEVELOPMENT FINESSE: KEY ISSUES OF FUSION HUCLEAR TECHINOLOGY DEV "PLASMA SURFACE INTERACTION IHMAGHETIC FUSION SYSTEMS" CA 28 \$2.356.001 $\$ 2.356 .001$ MAGNETIC FUSION THEORY \& EXPERIMENTAL RESEARCH 3 DEVELOPMENT CA $23 \quad \$ 26,151,467 \quad \$ 26,106,467$

RECIPROCAIING PROBE MEAJUREMENTS AHD COHTROL OF EDGE PLASHAS CA 23 \$2,653,175 IEW GRAHT - "ACCELERATOR RESEARCH \& DEVELOFMENT" FI - DAVID CA 233 \$960,000 IEW GRANT - THEORETICAL AND EXPERIMENTAL STUDIES IN ACCELERA O12140-0008-8 LOS AHGELES 
DIVISION

PHYSICS DEPT

PHYSICS DEPT

CHEMISTRY DEPT

PHYSICS DEPT

CHEMISTRY DEPT

BIN

BIN
COMPL DATE VENDOR ID CITY $012140-0008-8$ LOS ANGELES

FG03-86ERI3490 06/30/95 012140-0008-8 LOS ANGELES

FG03-86ER45264 06/30/95 $012140-0008-8$ LOS ANGELES FG03-87ER60504 02/28/95 $012140-0008-8$ LOS AIIGELES FG03-89ER140j3 05/31/95 $012140-2008-9$ LOS ANGELES FG03-89ER14066 05/14/95 012140-2008-9 LOS ANGELES FG03-91ER12114 01/14/95 $012140-0008-8$ LOS ANGELES FG03-92ER40727 05/31/95 $012140-0008-8$ LOS ANGELES FG03-93ER54224 08/14/95 $012140-0008-8$ LOS ANGELES FG03-86ER52134 12/31/95 012140-0008-8 LCS ANGELES FG03-85ER13363 07/31/96 012140-1008-3 LOS ANGELES FG03-87ER60548 03/14/96 012140-0008-8 LOS ANGELES FG03-87ER13686 03/14/96 012140-0008-8 LOS ANGELES FG03-88ER52150 01/31/96 012140-2008-9 LOS ANGELES FGO3-90ER14117 02/29/96 012140-1008-3 LOS ANGELES FG03-90ER20016 08/31/96 $012140-0008-8$ LOS ANGELES FG03-91ER40662 01/14/96 DI2140-0008-8 LOS ANGELES $\begin{array}{lll}\text { CONG } & \text { CONTRACT } & \text { OBLIGS } \\ \text { STATE DIST VALUE } & \text { TO DATE }\end{array}$ STATE DIST VALUE TO DATE P'S NEW GRANT - "SYHCHRONOU \$\$ MOLECULAR BIOLOGY AHD GENETICSOF THE ACELATE UTILIZING MOLECULAR BIOLOGY AND GENETICSOF THE ACELATE $\$ 783,320$ MAMORPHIZATION OF METALLIC ALLOYS UNDER PROTON AND NEUTRON I CA 23 \$ $\$ 861,584 \quad \$ 861,584$

THEORETICAL PROBLEMS IN LASER SPECTROSCOPY OF ATOMS AND MOLE BASIC STUDIES OF TRANSPORT PROCESSES IN POROUS MEDIA CA 23 \$505,414 $\$ 505,414$

"HIGH-RESOLUTION RAMAN SPECTROSCOPY OF COMPLEXES AND CIUSTER CA $23 \quad \$ 565,712 \quad \$ 565,712$

NEW GRANT EXPERIMENTAL. THEORETICAL AND \& COMPUTATIONAL STUD CA 23 \$ $\$ 00,082 \quad \$ 600,082$

NEW GRANT - "EXPERIMENTAL. THEORETICAL AND COMPUTATIONAL STU NEN GRANT NEW GRANT - "HIGH BETA TOKAMAKS" - PI - STEVEN COHLEY - $8 / 25$
CA
23

"THE PISCES EXPERIMENTAL PROGRAM"
CA $23 \quad \$ 13,081,010$
$\$ 10,218.586$

THE CHEMISTRY OF THE ETHYNYL RADICAL IN ITS XZST AND AZTT ST CA $28 \quad \$ 903,150 \quad \$ 903,150$

HUMAN GENE FOR RADIATION HYPERSENSITIVITY $\$ 1,536,365$

$$
\text { CA } 23 \quad \$ 1,674,679 \quad \$ 1,536,365
$$

"HAVE tURBULEHCE and SELF-LOCALIZATION IN CONTINOUS MEdia" $C A \quad 23 \quad \$ 741,920 \quad \$ 653.920$

"HUCLEAR SYSTEMS AND IESTING PROGRAM FOR ITER" (NEW GRANT CA 23 \$1.218.000 $\$ 708.000$

SEVERAL CAREFULLY CHOSEN BIMETALLIC SURFACES, COMPOSED OF A CA 42 CHOSEN BIMETALLIC SURFACES, $\$ 518,365 \quad$ COMPOSE

NEW GRANT - "THE GIBBERELLIN A20 3B-hydroxylase: ISOLATION

NE 23 SA $\$ 42,000$ \$348,000

NEW GRANT - PREVIOUSLY CONTRACT DE-AT03-88ER40384. "HIGH NEN GRANT CA $23 \quad \$ 8,133,000 \quad \$ 5,438,000$ 


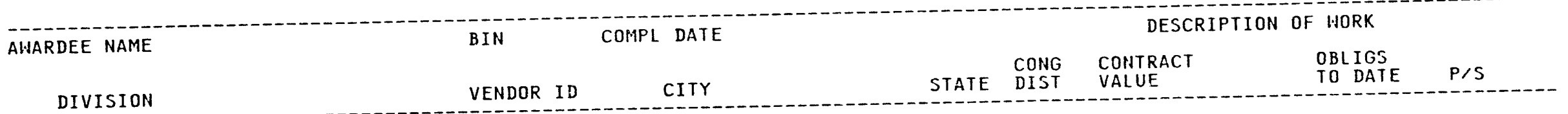

PHYSICS DEPT

PHYSICS DEPT

PHYSICS DEPT

PHYSICS DEPT

PHYSICS DEPT
FG03-93ER14355 06/14/96 $012140-0008-8$ LOS ANGELES

FG03-93ER40796 02/29/96 $012140-2008-9$ LOS ANGELES

FG03-93ER61686 08/31/96 $012140-0008-8$ LOS ANGELES

FG03-93ER61688 08/31/96 012140-0008-8 LOS ANGELES

FG03-85ER51069 12/14/96 $012140-0008-8$ LOS ANGELES

FC03-86ER52126 11/30/96 $012140-0008-8$ LOS ANGELES

FG03-89ER $14049 \quad 10 / 31 / 96$ $012140-2008-9$ LOS ANGELES

FG03-91ER54115 11/14/96 012140-0008-8 LOS ANGELES

FG03-88ER $13828 \quad 03 / 31 / 97$ $012140-2008-9$ LOS ANGELES

FG03-88ER40420 01/31/97 $012140-2008-9$ LOS ANGELES

FG03-88ER40424 01/31/97 $012140-2008-9$ LOS ANGELES

FC03-87ER60615 10/31/98 $012140-0008-8$ LOS ANGELES

FG0 $3-86$ ER40271 12/31/96 $012140-0012-6$ RIVERSIDE

FG03-92ER40738 07/14/94 $012140-0012-6$ RIVERSIDE

FG03-92ER40746 01/31/95 $012140-0012-6$ RIVERSIDE

FG03-94ER40837 01/31/97 $012140-0012-6$ RIVERSIDE
LINEAR KIIETIC THEORY AND PARTICLE TRANSPORT IN STOCHASTIC M CA 23 THY AND PARTICL $\$ 222,314$

NEW GRANT-EXPERIMENTAL INVESTIGATION DF NONLINEAR PL

NEW GRANT - NIMPACT OF GLOBAL CLIMATE CHANGE ON ECOSYSTEM $\begin{array}{llll} & & \\ \text { CA } & 23 & \$ 523,636 & \$ 352,390\end{array}$

NEW GRANT - "GENETIC CONTROL MECHANISM FOR ADAPTIVE STRATEGI

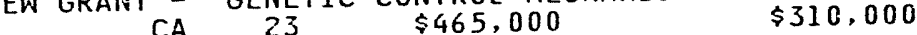
ADVANCED LIMITER TEST II (ALT-II) PUMP LIMITER EXPERIMENT IN

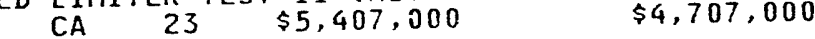

FUSION REACTORS = PHYSICS AND TECHNOLOGY. THE GRANTEE PROPO

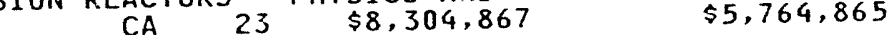

ENERGY RELATED STUDIES UTILIZING MICROLINE THERMOCHRONOLOGY

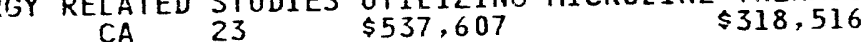

NEW GRANT - RADIATION EFFECTS AND MICROMECHANICS OF SIC/SIC $\begin{array}{lll}\text { CA RADIATION EFFECTS } & 23 & \$ 747.000\end{array}$ $\$ 407,000$

-TIME-RESOLVED LASER STUDIES ON THE PROTON PUMP MECHANISM OF CA $23 \quad \$ 1,182,180 \quad \$ 908,130$ "PARTICLE PHYSICS" $23 \quad \$ 3,351,000 \quad \$ 2,527,000$ "INTERMEDIATE ENERGY AND NUCLEAR PHYCISC"

$23 \$ \$ 5,864,000 \quad \$ 4,307,000$ NEW COOPERATIVE AGREEMENT FOR A "BIOMEDICAL AND ENVIRONMENTA 作 "CENTRAL COLLISIONS OF HEAVY ION PHYSICS 作 $\$ 2,462,000$ THE PRIMARY GOAL OF THE PROJECT IS THE SEARCH FOR NEUTRINO $O$ CA $36 \quad \$ 204,000 \quad \$ 204,000$ NEW GRANT - A STUDY OF QUARK AND GLUON JETS AND OF THE LONG

NEW AWARD - HIGH ENERGY PHYSICS $\$ 200,000$

$\$ 958,000$ 


\section{AWARDEE NAME}

DIVISION IN

\section{DESCRIPTION OF WORK}

\section{CONG CONTRACT
STATE DIST VALUE}

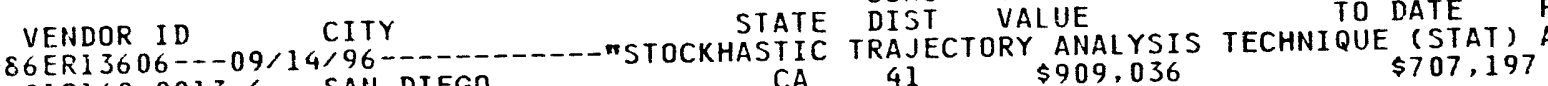

OBLIGS

P/S $012140-0013-4$ SAN DIEGO AC03-76SF0101209/30/94 $012140 \% 0015-0$ SAN FRANCISCO FG03-89ER60873 07/14/95 012140-2015-1 SAN FRANCISCO

PHYSICS DEPT FG03-94ER61796 03/14/95 012140-0015-0 SAN FRANCISCO FG03-84ER45108 03/31/96

PHYSICS DEPT 012140-2016-X SANTA BARBARA

PHYSICS DEPT

FG03-85ER45197 02/28/97 $012140-2016-x$ SAHTA BARBARA FG03-85ER13317 11/30/94 012140-0016-9 SAITA BARBARA FG03-85ER13314 10/31/94 $012140-0016-9$ SANTA BARBARA

FG03-87ER45288 12/14/95 012140-0016-9 SANTA BARBARA

FG03-87ER13738 09/14/98 $012140-2016-x$ SANTA BARBARA

PHYSICS DEPT FG03-87ER45331 02/29/96 $012140-2016-x$ SAITTA BARBARA.

PHYSICS DEPT

FG03-88ER1384203/31/95 $012140-2016-X$ SANTA BARBARA PHYSICS DEPT FG03-89ER14048 12/31/95 CHEMICAL \& NUCLEAR ENGINEERING 012140-5816-7 SANTA BARBARA ELECTRICAL \& COMPUTER EHG

FG03-90ER40545 08/31/95 012140-7316-6 SANTA BARBARA FG03-90ER61062 10/31/96 012140-0016-9 SAllTA BARBARA FG03-91ER40618 03/31/96 012140-0016-9 SAIITA BARBARA
STUDIES OF BASIC RADIOBIOL OGICAL MECHANISMS TRANSFERED F

CARBORANYL AMINO ACIDS FOR SITE SPECIFIC NEUTRON CAPTURE THE $C A$ A 05 \$1, 210,390 $\$ 696,240$

WEW GRANT - "INTERNATIONAL WORKSHOP ON HUMAN Y CHROMOSOME" $P$ NEW GRANT - "INTERNATIONAL 12 \$1,000 $\$ 11,000$

RESEARCH ON THE THEORY OF PATTERN FORMATION IN SYSTEMS FAR $F$

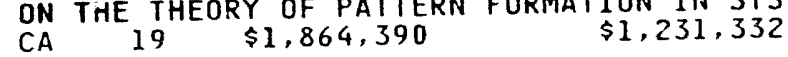
$\begin{array}{rll}\text { NUMERICAL SIMULATION OF QUANTUM MANY-BODY SYSTEMS } \\ \text { CA } 19 & \$ 1,275,408 & \$ 1,100,808\end{array}$ STUDIES RELATED TO CATALYTIC ACTIVATION $C A \quad 19 \quad \$ 1,476,500 \quad \$ 1,115,292$ INVESTIGATINS OF FLUID DYNAMICPHENOMENA NEAR-LIQUID INTERFA

"POLYMERS AT SURFACES"

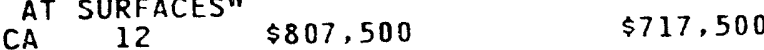

"BIFURCATIONS AND PATTERNS IN NONLINEAR DISSIFATIVE SYSTEMS" CA $19 \quad \$ 1,447,640 \quad \$ 1,017,430$

"MOLECULAR PROPERTIES OF THIN ORGANIC INTERFACIAL FILMS" $\begin{array}{ccc}\text { CR PROPERTIES OF THIN ORGANIC INTERFACIAL FIL } \\ \text { CA } 19 & \$ 1,432,060 & \$ 1,246,660\end{array}$

"ENERGY TRANSFORMATION IN DRGAN OMETALLIC COMPLEXES" "ENERG TA $19 \quad \$ 765,400 \quad \$ 765,400$ THE ACTIVATION AHD DECOMPOSITION OF ALKANES ON GROUP VIII TR

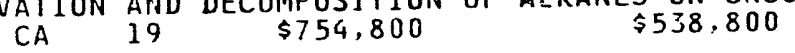
THIS HORK IS AN ANALYTICAL ANDNUMBERICAL STUDY OF A NEW TEC $\begin{array}{ccc}\text { THIS HORK IS AN ANALYTICAL ANDNUMBERICAL STUDY OF A } \\ \text { CA } 19 & \$ 130,000 & \$ 130,000\end{array}$ NEW CA $\quad 19 \quad \$ 1,153,497 \quad \$ 645,073$ HIGH ENERGY USER GROUP

PHYSICAL MODELLING OF SEDIMENTARY BASINS, MAGMA MECHANICS AN

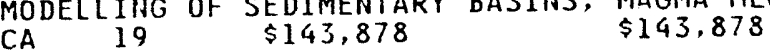
19 \$853,831 $\$ 853,831$ 


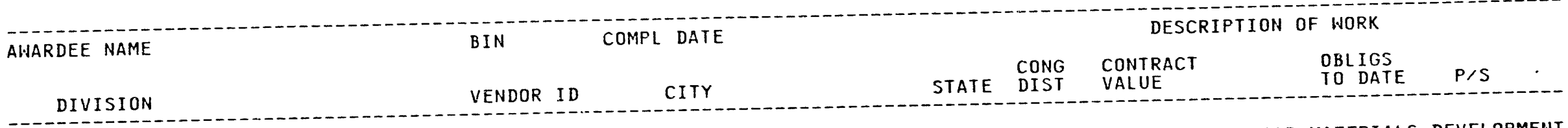
012140-0016-9 SANTA BARBARA

FG03-93ERI2138 08/14/96 $012140-0016-9$ SANTA BARBARA

FG03-93ER25186 09/29/95 $012140-0016-9$ SANTA BARBARA

FG05-91ER75039 09/29/94 012140-0016-9 SANTA BARBARA

NATURAL SCIENCES DIV FG03-84ER13245 03/31/95 $012140-4917-6$ SANTA CRUZ

PHYSICS DEPT

$$
\text { FG03-87ER45324 01/31/95 }
$$$$
012140-2017-8 \text { SAITA CRUZ }
$$

FG03-92ER40689 10/31/94 $012140-0017-7$ SANTA CRUZ $A B 34-93 R F 00644$ 02/01/98
$012140-7520-7$ GOLDEN

LWRENCE LIVERMORE NATL LAB

GMO4-86AL53651 09/30/99 SANDIA NATIONAL LABORATORIES 012140-4318-6 ALBUQUERQUE W-7405-ENG-36 09;30/97 $012140-3607-4$ LOS ALAMOS

LOS ALAMOS NATIONAL LAB

CALLOWAY COUNTY OF EDUCATION BOARD OF CAMBER CORP

CAMDEN DIOCESE OF FG44-93R435303 $09 / 30 / 95$ $A C 01-94 E H 4043404 / 28 / 97$
$227780-0002-x$ SEAL BEACH AC01-93CE16072 04/07/97 $227780-0001-1$ CAMBRIDGE

FG42-94R263911 06/30/96 $216510-0006-9$ CHERRY HILL FG42-94R263918 06/30,96 $216510-0006-9$ CHERRY HILL
FG03-94ER54275 05/31/97

FUNDAMENTAL STUDIES FOR FUSIONREACTOR MATERIALS DEVELOPMENT CA $\$ 930,000$ $\$ 180,000$

PHOTO-INDUCED ELECTRON TRANSFER FROM A CONDUCTING POLYMER TO CA $22 \quad \$ 900,000 \quad \$ 296,130$

LATTICE GAUGE THEORY ON A MASSIVELY PARALLEL COMPUTING FACIL GAUGE THEORY ON A MASSIVELY PARALLEL COMPUTI
$\$ 90,405$

SHARED TIME-RESOLVED SPECTROMETER FOR PHOTOCATALYSIS AND MAT TONOPLAST TRANSPORT AND SALT TOLERANCE IN PLANTS

"EXPERIMENTAL STUDIES OF CRITICAL BEHAVIOR IN SYSTEMS WITH Q "EXPERIMENTAL STUDIES OF CRITICAL BEHAVIOR NEW GRAII - "RESEARCH IN HIGH ENERGY PHYSICS" P.I. A. SEIDEN $\begin{array}{lll} & \end{array}$

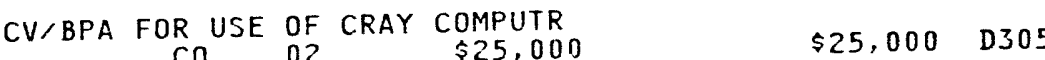
SET FORTH THE UNDERSTANDING AND RESPONSIBILITIES FOR JOINT MEO OF LANL BR OF $U$ OF $C A$

SEE ATTACHED PAGE NO. 2 OF THIS NOTICE OF FINANCIAL ASSISTAN
SEE ATTACHED PAGE NO. 2 OF THIS NOTICE OF FINANCIAL $\$ 31,251$

TECHNICAL SUPPORT SERVICES FORTHE OFFICE OF PROGRAM SUPPORT OT \$1.718.181 \$600,000 R408 SUPPORT SERVICES FOR COHSERVATION AND RENEHABLE ENERGY

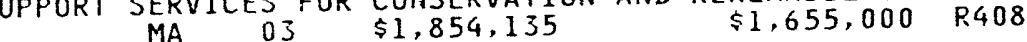

CYCLE 16 AHARD.

$\$ 29,202$

$\$ 20,669$ 


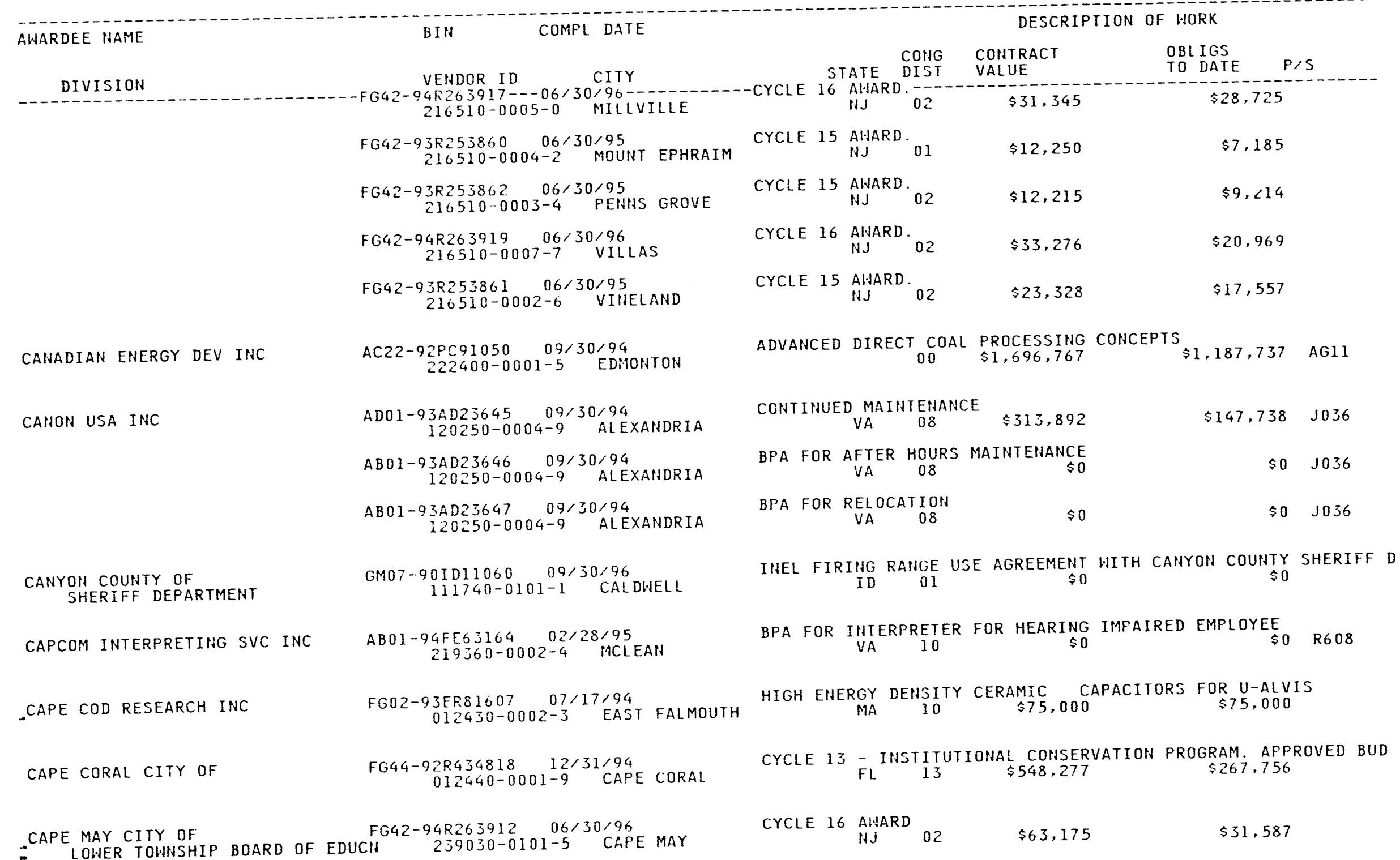




AWARDEE NAM
DIVISION
CAPITAL LIGHT

CAPITAL LIGHTING \& SUPPLY

CAPITOL POLICE U S

CAPITOL RADIO WHOLESALERS INC

CAPSTONE CORP

CARDINAL CSD

CARDINAL HILL REHAB HOSPITAL

CARDINAL LOCAL SCHOOL DISTRICT

CARDINAL SPELLMAN SCHOOL

\section{CAREER BLAZERS}

CARIBOU COUNTY OF

SHERIFF'S DEPT

CARLSBAD CITY OF

CARNEGIE INST OF WASHINGTON
COMPL DATE

CONG CONTRACT

VENDOR ID CITY AC01-94AD67046 04/12/95

$237360-0001-4$ ALEXANDRIA

GMOS-89NV10857 03/14/99
$181820-0001-7$ WASHINGTON

$181820-0001-7$ WHASH

AB01-94AD69239 $12 / 31 / 94$
$012540-0003-8$ GAITHERSBURG

$012540-0003$

ACO1-94EW10812001/27/96
$236700-0001-1$ FALLS CHURCH

FG47-93R707026 $08 / 31 / 94$
$232030-0001-6 \quad$ ELDON FG44-93R435304 $09 / 30 / 95$
$233820-0001-6 \quad$ LEXINGTON-FAYET

FG45-93R551760 06/30/95
$012610-0002-1 \quad$ MIDDLEFIELD

$F G 47-93 R 70350908 / 31 / 95$
$223680-0002-X \quad$ OMAHA

AB01-93AD00014 10/31/94 $234430-0001-9$ WASHINGTON

GM07-93IDI1112 12/31/99 227300-0101-8 SODA SPRINGS

ACO4-86AL24138 06/05/96 $155270-0001-3$ CARLSBAD

GM04-88AL53633 06/15/99 $155270-0001-3$ CARLSBAD

FG02-94ER20133 01/14/97 $012710-0002-4$ HASHINGTOH
TO DATE P/S PURCHASE FLUORESCENT LIGHT FIXIURES AND LAMPS FOR DOE GERMAN
VA 08

MOU TO OFFICIALLY IDENTIFY THEDUTIES AND SUPPORT $\underset{\$ 0}{\text { ACTIVITIES }}$

$$
\text { DC } 01 \text { \$O }
$$

BPA FOR ELECTRONIC SERVICES AND SUPPLIES

$\$ 0 \quad 5999$

TECHNICAL AND ADMINISTRATIVE SUPPORT SERVICES

$$
\begin{aligned}
& \text { AND ADMINISTRATIVE SUPPORT SERVICES } \\
& \text { VA } 02 \quad \$ 1,000,000
\end{aligned}
$$

GRANT PROGRAMS FOR SCHOOLS ANDHOSPITALS AND FOR BUIDLINGS IA $03 \quad \$ 5,902$ $\$ 2,951$

SEF THIS NOTICE OF FINANCIAL ASSISTALI

ICP-NEW AWARD

13 $\$ 88,166 \quad \$ 41,420$

GRANT PROGRAMS FOR SCHOOLS ANDHOSPITALS AND FOR BUILDINGS 0 $\mathrm{HE} \quad 02 \quad \$ 13,636 \quad \$ 12,270$

BPA FOR TEMPORARY SECRETARIAL WORK
DC OI

ค $0 \quad R 699$

FIRING RAHGE USE AGREEMENT WITH CARIBOU COUNTY $\$ 0$

WATER PIPELINE, MAINTENANCE AND SERVICE. NO COST TO THE GOVE

PROVIDES FOR COORDINATIOH OF EMERGENCY PLANS, EXERCISES, NM 02

SO

PRODUCTION OF LIFOPHILLIC MATERIALS FROM PLANTS

$$
\text { DC } 01 \$ 1,433,065 \quad \$ 535,673
$$


AWARDEE NAME

DIVISION BIN COMPL DATE

CONG CONTRACT DESCRIPTION OF WORK

FG02-94ER20143 05/31/97 $012710-0002-4$ WASHINGTON FG02-93ER14400 09/14/96 $012710-0002-4$ WASHINGTON

CARNEGIE-MELLON UNIVERSITY CHEMICAL ENGINEERING DEPT

PHYSICS DEPT

CHEMISTRY DEPT

METALLURGICAL ENG \& MATLS

ELECT \& COMPUTER ENGINEERING

METALLURGICAL ENG \& MATLS

PHYSICS DEPT

CHEMICAL ENGINEERING DEPT

:
FG02-85ER13396 02/14/98 $012730-0201-6$ PITTSBURGH

FG02-87ER40315 11/30/95 $012730-1001-9$ PITTSBURGH FG02-87ER40324 12/31/95 $012730-0301-2$ PITTSBURGH FG02-88ER45358 07/31/94 $012730-1801-X$ PITTSBURGH FG02-89ER14042 08/14/95 9ER14042 $0872730-1601-7$ PITTSBURGH FG02-90ER45423 07/31/96 $012730-1801-X$ PITTSBURGH FG02-91ER40682 10/31/94 012730-1001-9 PITTSBURGH FG02-92ER45474 07/31/96 $012730-0001-3$ PITTJBURGH FG02-94ER61809 03/31/95 $012730-0001-3$ PITTSBURGH FG02-94ER $30226 \quad 05 / 31 / 95$ 012730-0001-3 PITTSBURGH FG02-93ER14345 04/14/96 $0127>0-0201-6 \quad$ PITTSBURGH FG02-93ER61711 09/14/94 $012730-0001-3$ PITTSBURGH FG02-93ER61712 09/14/94 $012730-0001-3$ PITTSBURGH FG22-92PC92152 03/13/95 MECHANICAL ENGINEERING DEPT
012730-0701-8 PITTSBURGH
MOLECULAR BASIS OF DISEASE
DC 01

$\begin{array}{ccc}\text { WATER-ROCK INTERACTION IN THE EARTH'S UPPOER MANTLE } \\ \text { DC } 01 & \$ 276,285 & \$ 92,095\end{array}$

STRATEGIES FOR OPTIMAL PLANT REDESIGN IN A CHANGING ENVIRONM

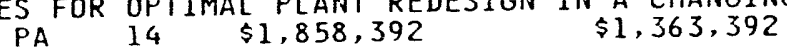

EXPERIMENTAL MEDIUM ENERGY PHYSICS

$$
\text { PA MEDIUM ENERGY PHYSICS } \$ 5,324,000 \quad \$ 5,887,000
$$

NUCLEAR RESEARCH WITH HEAVY IONS

$\$ 1,420.000$

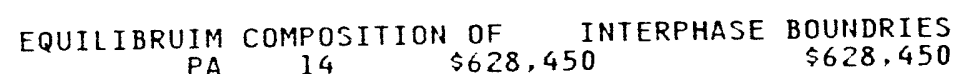

RESEARCH ON A RECONFIGURABLE MODULAR MANIPULATOR SYSTEM PA $14 \quad \$ 613,798 \quad \$ 495,266$

THE ROLE OF MICROSTRUTURE PHENOMENA IN MAGNETIC THIN PA $14 \quad \$ 534,569 \quad \$ 357,200$

HIGH ENERGY PHYSICS \& SSC CALORIMETER SUB SYSTEM R\&D

PRESSURE INDUCED AMORPAIZATIOHOF SILICA AHACOGNES A PROBE

PA I4 $\$ 382,181$ \$

3RD INTERNLT'L WORKSHOP ON CHROMOSOME 16 TO BE HELD PA 14 14 $\$ 37,730 \quad \$ 37,730$

THE ECONOMIC COST OF ACID
PA

THE GRANTEE WILL DEVELOP A RESEARCH PROGRAM EMPHASIZING

PA 14 \$284,339 \$173,630

BARRIERS TO ENER TECH ADOPTIONAN ANAL OF EVIDENCE FROM SELEC PA $14 \quad \$ 132,810 \quad \$ 132,810$

INVERSE METH FOR PARAMETERIZATION OF ENER USE MODS: AN APPLN PA $14 \quad \$ 130,821 \quad \$ 130,821$

THE PHYSICS OF COAL LIQUID SLURRY ATOMIZATION

THE PHYSICS OF COAL LIQUTD $\$ 449,505 \quad$ PA 


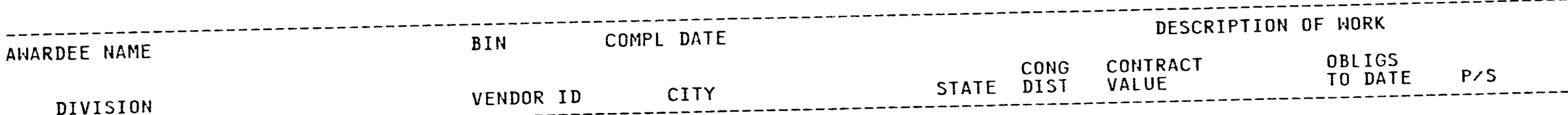

AC22-92FC91346 09/29/95 ENGINEERING \& PUB POLICY DEPTS 012730-0501-5 PITTSBURGH AC21-92MC29094 $08 / 19 / 95$ $012730-0001-3$ PITTSBURGH AC21-92MC29104 01/29/95 $012730-0001-3$ PITTSBURGH FG22-92PC92541 09/03/95 012730-0001-3 PITTSBURGH AR21-93MC30362 10/29/94 012730-1901-6 PITTSBURGH

ROBOTICS INSTITUTE

CARNEY HOSPITAL

\section{CAROLINA DAY SCHOOL}

CAROLINA POWER \& LIGHT CO

CARRIZOZO CITY OF MUNICIPAL SCHOOLS

MUNICIPAL SCHOOLS

CARROLL COLLEGE

CARROLL COUNTY HOSPITAL

carson county of

$$
\begin{gathered}
\text { FG41-92R193423 } 07 / 31 / 94 \\
012740-0001-7 \quad \text { BOSTON } \\
\text { FG41-93R193510 } 07 / 31 / 95
\end{gathered}
$$

FG44-93R435380 09/30/94 231290-0001-1 ASHEVILLE

CRO1-83NE44481 03/15/30 012790-0002-3 RALEIGH

FG04-92AL82977 05/31/95 $012890-0101-4$ CARRIZOZO

GMO4-92AL58234 05/08/97 012890-0101-4 CARRIZOZ0

FG45-93R551791 12/31/94 097170-0002-6 WAUKESHA

FG44-93R435317 09/30/95 231160-0001-8 HUHTINGDON

GM04-85AL $32386 \quad 04 / 23 / 95$ 228360-0001-5 AMARILLO$$
\text { 012740-0001-7 BOSTON }
$$

DEVELOPMENT OF THE INTEGRATED ENVIRONMENTAL CONTROL MODEL

DEVELOPMENT AND APFLICATION OFOPTIMAL DESIGN CAPABILITY FOR

$$
\text { PA } 14 \quad \$ 694,109 \quad \$ 515,000 \quad \text { AG12 }
$$

EM-MOBILE WORKSYSTEM FOR DECONTAMINATION AND

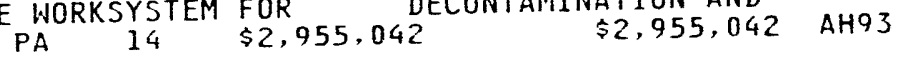

EXPLORING THE FUNDAMENTALS OF RADICAL ASSISTED NOX REDUCTION PA $14 \quad \$ 199,640$ $\$ 199,640$

EM - ASBESTOS PIPE-IHSULATION REMOVAL SYSTEM

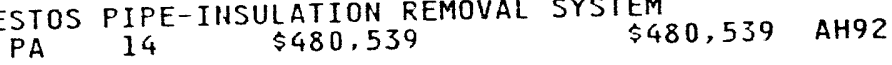

CYCLE $14 \underset{\mathrm{MA} \text { ICP AHARD. }}{11} \quad \$ 151,030^{\mathrm{T}} . \quad \$ 80,358$

TITLE III OF THE NATIONAL ENERGY CONSERVATION POLICY ACT $M A N 08$ \$11,040

SEE ATTACHED PAGE NO. 2 OF THIS NOTICE OF FINANCIAL ASSISTAN NC 11 \$868 $\$ 434$

DISPOSAL OF SPENT NUCLEAR FUELAND/OR HIGH LEVEL RADIOACTIVE NC 04 $\$ 0$ $\$ 0$

EXTENDED EDUCATION PROGRAM

$\begin{array}{lll}\text { EDUCATION PROGRAM } \\ \text { NM } & 02 & \$ 51,500\end{array}$

$\$ 51,500$ SUPPORT FOR SCIENCE, MATHEMATICS, AND ENGINEERING TO IMPROVE

ICP-NEW AHARD.

WII 04

$\$ 12,700$

$\$ 6.350$

SEE ATTACHED PAGE NO. 2 OF THIS NOTICE OF FINANCIAL ASSISTAN TN 08 \$136, $\$ 80$ $\$ 59,384$

COOPERATIOH OF EMERGENCY MATTERS OF MUTUAL CONCERN $\$ 0$ 


AWARDEE NAME

DESCRIPTION OF WORK

\section{DIVISION}

VENDOR ID

CITY

CONG CONTRACT

VALUE

CYCLE 15 ICP GRANT AWARD

CYCLE 16 ICP GRANT APPLICATION

$\$ 32.500$

FG49-93R915005 06/30/95
$229450-0001-6$ CASA GRANDE FG49-94R916007 06/30/96

CASA GRANDE

CASCADE NATURAL GAS CORP

$A D 06-93 R L 1270809 / 30 / 94$
$225800-0002-X \quad$ KENNEWICK

AD06-93RLI2566 09/30/94 225800-0001-1 SEATTLE

CASE WESTERN RESERVE UNIV RADIOLOGY DEPT

RADIOLOGY DEPT

FG02-88ER60617 10/31/96 013070-0701-5 CLEVELAND

FG02-88ER60658 12/31/94 013070-0701-5 CLEVELAND

FG02-93ER45496 06/30/97 O13070-0001-0 CLEVELAND

FG02-93ER14383 09/14/94 013070-0001-0 CLEVELAND CASPER COLLEGE
BIOLOGY

CASS COUNTY SHERIFF DEPT

FG09-94SR18392 11/15/94 4SR18392 11/15/94
$235340-0101-X \quad$ CASPER

GM33-88AL53636 02/15/99 8 AL $5363602 / 15 / 99$
$179700-0001-0$ KAHSAS CITY

CASSIA COUNTY OF JOINT SCHOOL DISTRICT \#151 JOINT SCHOOL DISTRICT \#151

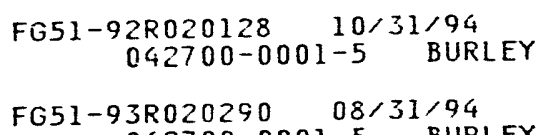
FG51-92R020128 $10 / 31 / 94$
$042700-0001-5$ BURLEY

FG51-93R020290 08/31/94 $042700-0001-5$ BURLEY

FG44-92R434994 12/31/94 013210-0001-4 SALISBURY

$:$

CATAWBA MEMORIAL HOSPITAL
FG44-93R435381 09/30/94

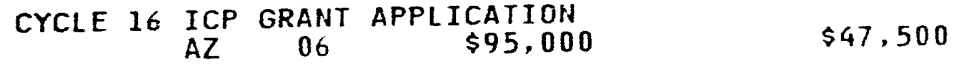

INSTALL NATURAL GAS LINE SERVICE TO 1167 WAREHOUSE WA $04 \quad \$ 4,500 \quad \$ 4,500$

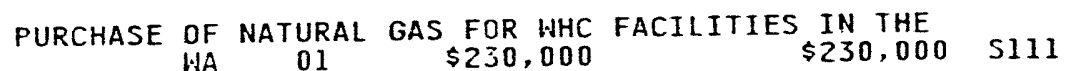
WA 01

IONIZATION OF LIQUIDS $\underset{20}{\mathrm{OH}} \stackrel{\$ 669,738}{\$ 522,738}$

MUTAGENICITY OF RADON AND RADON DAUGHTERS

$\begin{array}{ccc}\text { MUTAGENICITY OF RADON AND } & \text { OH } \\ 21 & \$ 1,347,814 & \$ 1,287,814\end{array}$

DISLOCATIONS AND POLYTYPIC $\underset{\$ 32,870}{\text { TRANSFORMATIDNS IN SIC }}$ APPLICATIONS OF IN SITU $\underset{\$ 2}{\&}$ SPE-CTROSCOPIC TECHNIQUES FOR THE

USED ENERGY RELATED LABORATORYEQUIPMENT GRANT \$0

DESCRIBES THE RELATIONSHIP BETHEEN THE SHERIFF'S $\underset{\$ 0}{\$ 0}$ DFICE AND CYCLE 14 ECM GRANT.

$$
\$ 87,665
$$

CYCLE 15 TAS AHARD.

$\$ 3,400$

$\$ 1,700$

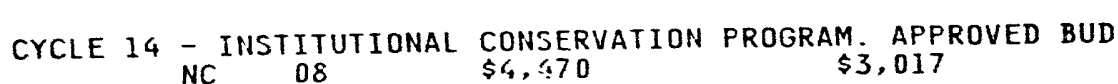
CYCLE 15- INSTITUTIONAL CONSERVATION PROGRAM. APFROVED BUDG 


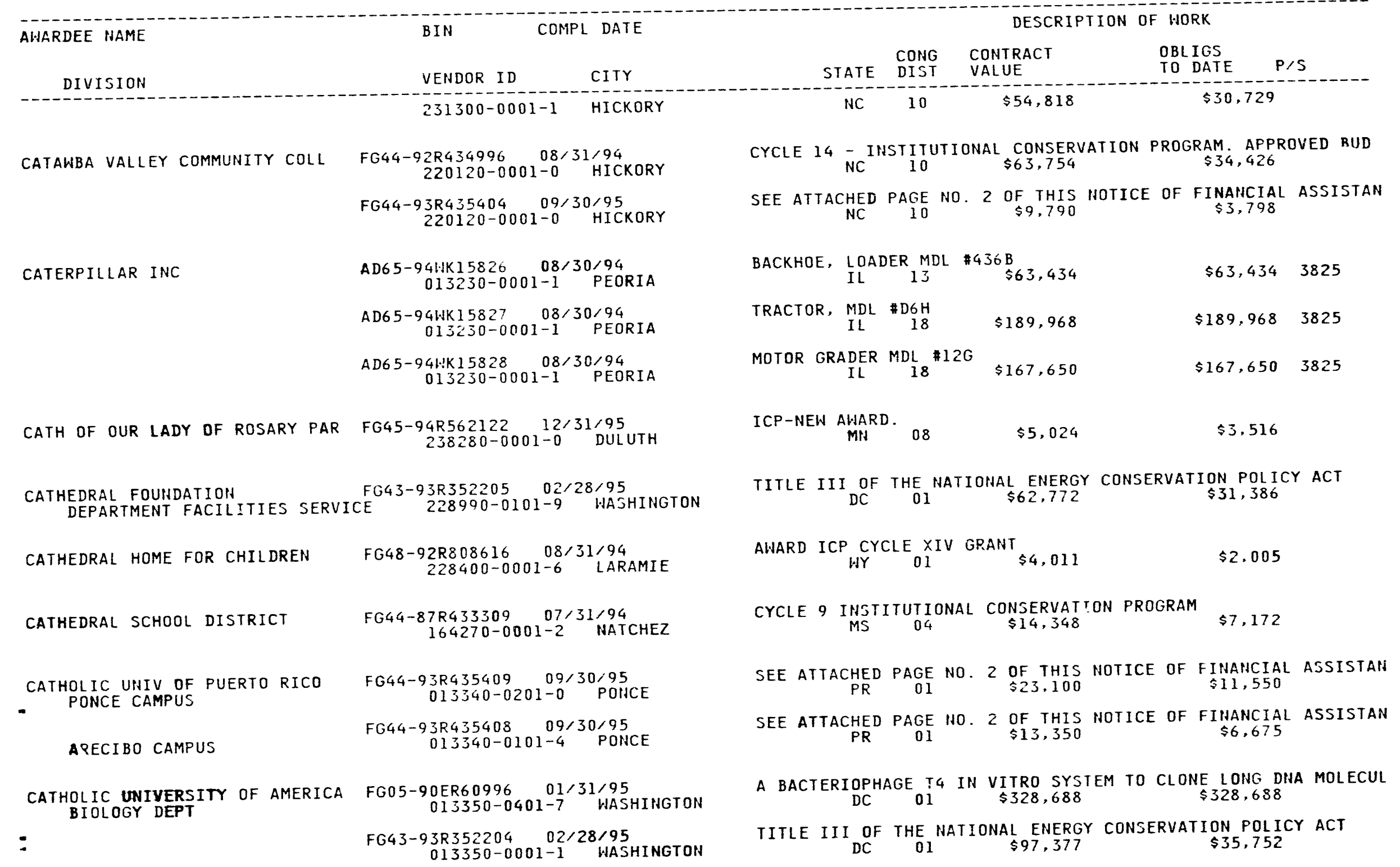




\begin{tabular}{|c|c|c|c|c|c|c|c|}
\hline AWARDEE NAME & BIN & DATE & & & DESCF & F WORK & \\
\hline DIVISION & VENDOR ID & CITY & STATE & $\begin{array}{l}\text { CONG } \\
\text { DIST }\end{array}$ & $\begin{array}{l}\text { CONTRACT } \\
\text { VALUE }\end{array}$ & $\begin{array}{l}\text { OBLIGS } \\
\text { TO DATE }\end{array}$ & $P / S$ \\
\hline
\end{tabular}

FG43-90R323201 $07 / 31 / 94$
$171320-0001-2$ ELKTON FG43-89R313203 07/31/94 $171320-0001-2$ ELKTON

CEDARS-SINAI MEDICAL CENTER

CELERON GATHERING CORP

CELLULAR ONE

CEMCOM RESEARCH ASSOC

CENTRAL ARKANSAS UNIVERSITY OF CENTRAL FLORIDA UNIVERSITY OF CENTRAL INTELLIGENCE AGENCY LIAISON FINANCE OFFICE

$:$

LIAISON FINANCE OFFICE
FG03-92ER61402 03/31/95
$013560-0001-0$ LOS ANGELES FG49-86SF16152 10/31/95 $013560-0001-0$ LOS ANGELES

SC92-94FE6 $3272 \quad 10 / 01 / 94$ $166500-0002-7$ BAKERSFIELD SC92-93FE63073 09/30/94 $166500-0002-7$ BAKERSFIELD

AD01-93AD34098 09/30/94 194440-0002-8 GREENBELT

AMO1-93AD34049 09/30/94 $194440-0002-8$ GREENBELT

FG05-92ER81351 05/24/95 222120-0001-4 BALTIMORE

FG46-93R613604 02/28/95 111840-0001-8 COHHAY

FG44-92R434895 09/30/94 ORLANDO

AIO1-90MA35072 09/30/94 013970-0201-8 WASHINGTON AI01-90DP00523 09/23/95 $013970-0001-5$ HASHINGTON A101-93P010092 09/30/95 013970-0201-8 WAJHINGTON
TITLE III OF THE NATIONAL ENERGY CONSERVATION POLICY ACT NONE

MD $01 \quad \$ 92,000$

MD $\quad 01$

$\$ 90,000$

$\$ 50,000$

THE ULTIMATE GOAL OF THIS RESEARCH IS TO CREATE A DNA MAP OF

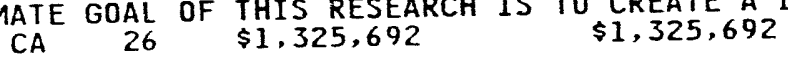

CYCLE 8 SCHOOLS \& HOSPITALS

$$
\begin{array}{lrr}
\text { SCHOOLS \& HOSPITALS } & \$ 409,400 & \$ 266,000
\end{array}
$$

NPR-I CRUDE OIL SALES CONTRACTEST REV \$13770971.43 NPR-1 CRUDE OIL SALES CONTRACTEST. REV: $\$ 14,289,491.22$ CA 20 $\$ 0$

CELLULAR TELEPHONE SERVICES

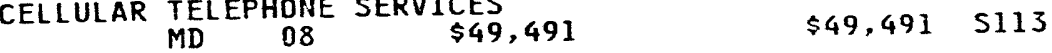

CELLULAR TELEPHONE SERVICE FORDOE HEADQUARTERS $M D \quad 08 \quad \$ 50,044$

CONYERSION OF SOX CONTROL RESIDUE AND WASTE OILY EMULSION TO

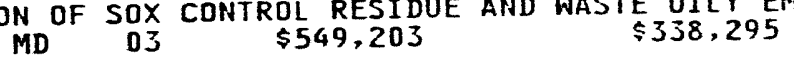

AWARDS A NEW CYCLE 15 ENERGY CONSERVATION MEASURE GRANT.

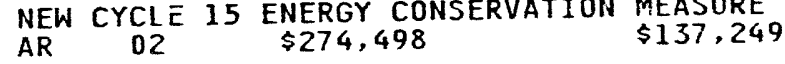

CYCLE 14 - IHSTITUTIONAL CONSERVATION PROGRAM. APPROVED BUD

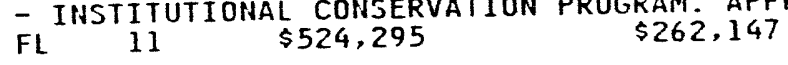

MAINTENANCE FOR ONE ITS-H LASERFAX TERMINAL

DC OI $\$ 180,000$ \$170,000

IS BETWEEN DOE AND CIA PROVIDING FUNDS FOR DESIGN AND ENGINE DC $\begin{array}{llll}\text { OIN CIA PROVIDING FUNDS FOR } \\ \$ 50,000\end{array}$

TECH SUPPORT ASSOC HITH PERFORMANCE OF EMPIRICAL ESTIMATION

$$
\begin{array}{llll}
\text { DC } & 01 & \$ 75,000 & \$ 75,000
\end{array}
$$


DESCRIPTION OF WORK

AWARDEE NAME

COMPL DATE

CONG CONTRACT

DIST VALUE

OBLIGS
TO DATE

$P / S$

VENDOR ID

CITY

INITIATE NEW IA WITH $\underset{\$ 10}{\text { CIA FOR INTELLIIGENCE-RELATED }}$

ESTABLISHMENT OF INTERAGENCY AGREE WITH THE CIA

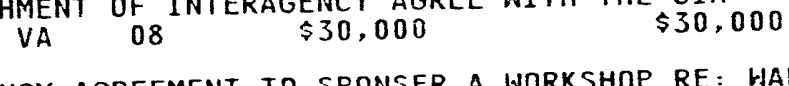

INTERAGENCY AGREEMENT TO SPONSER A WORKSHOP RE: HARNIHG COMM

$$
\text { VA } 08 \text { S } \$ 300,000
$$

$\$ 30.000$

ESTABLISH IA WITH CIA FOR TESTING AND EVALUATION OF R\&D PROT VA 09 $\$ 50,000$

$\$ 50,000$

PROVIDE SUPPORT FOR A COUNTERTERRORISM COMMUNITY-WIDE COMMUN VA 08 \$100,000 \$100,000

COUNTERTERRORISM BOARD

AI01-93IN00624 09/30/94 013970-0403-7 ARLINGTON

AI01-93IN00616 07/02/98 $013970-0003-1$ ARLINGTON

ASST DIR FOR LIAISON OFF FIN AI01-91AD36216 $013 / 01 / 96$ ROSLYN

CENTRAL RESEARCH INST OF JAPAN ACO2-89CH10424 $03 / 31 / 96$

CENTRAL STATE HOSPITAL

FG44-91R434686 07/01/94 $014080-0001-6$ MILLEDGEVILLE

FG04-87AL43437 09/29/96 01403437
$014090-0102-4$

CHEMISTRY DEPT

FG42-92R243814 07/31/94

CENTRAL SUFFOLK HOSPITAL 220900-0001-5 RIVERHEAD

CENTRAL WASHINGTON UHIVERSITY FG51-92R020156 12/31/94 ELLENSBURG FG06-93ER79172 06/14/95 $014150-0001-8$ ELLENSBURG

ECENTRAL WYOMING COLLEGE

FG48-92R808615 08/31/94 $224300-0001-6 / 31 / 94$
OBTAIN UPGRADED ADP EQUIPMENT FOR ACCESS AND USE DF THE CAMS

$$
\begin{array}{lll}
V A & 07 & \$ 15,937
\end{array}
$$$$
\$ 15,937
$$

ADP SUPPLIES \& SERVICES
VA
$\$ 4,900,000$
$\$ 4,212,000$

JOINT PROGRAM BETWEEN DOE \& CENTRAL RESEARCH INSTITUTE OF $00 \$ 20,000,000$ ऽ AG91

CYCLE 13 - INSTITUTIONAL CONSERVATION PROGRAM. APPROVED BUD GA INSTITUTIONAL CONOF, $\$ 279.99$ $\$ 139,997$

GRANT FOR HISTORICALLY BLACK COLLEGES AND UNIVERSITIES

OH $O 7$ TALLY BLALK LUL

CYCLE 14 AWARD.

$\$ 201,160$

CYCLE 14 ECM AHARD. INCLUDES $\$ 10,000$ PRE-AWARD COSTS

PRE FRESHMAN ENRICHMENT PROGRAM

$\$ 185,515$

$$
\text { HA } 04 \quad \$ 56.083
$$

$\$ 39,754$

AWARD ICP CYCLE XIV GRANT

$\$ 7,450$

$$
\$ 14,900
$$


$R-0420953-001$

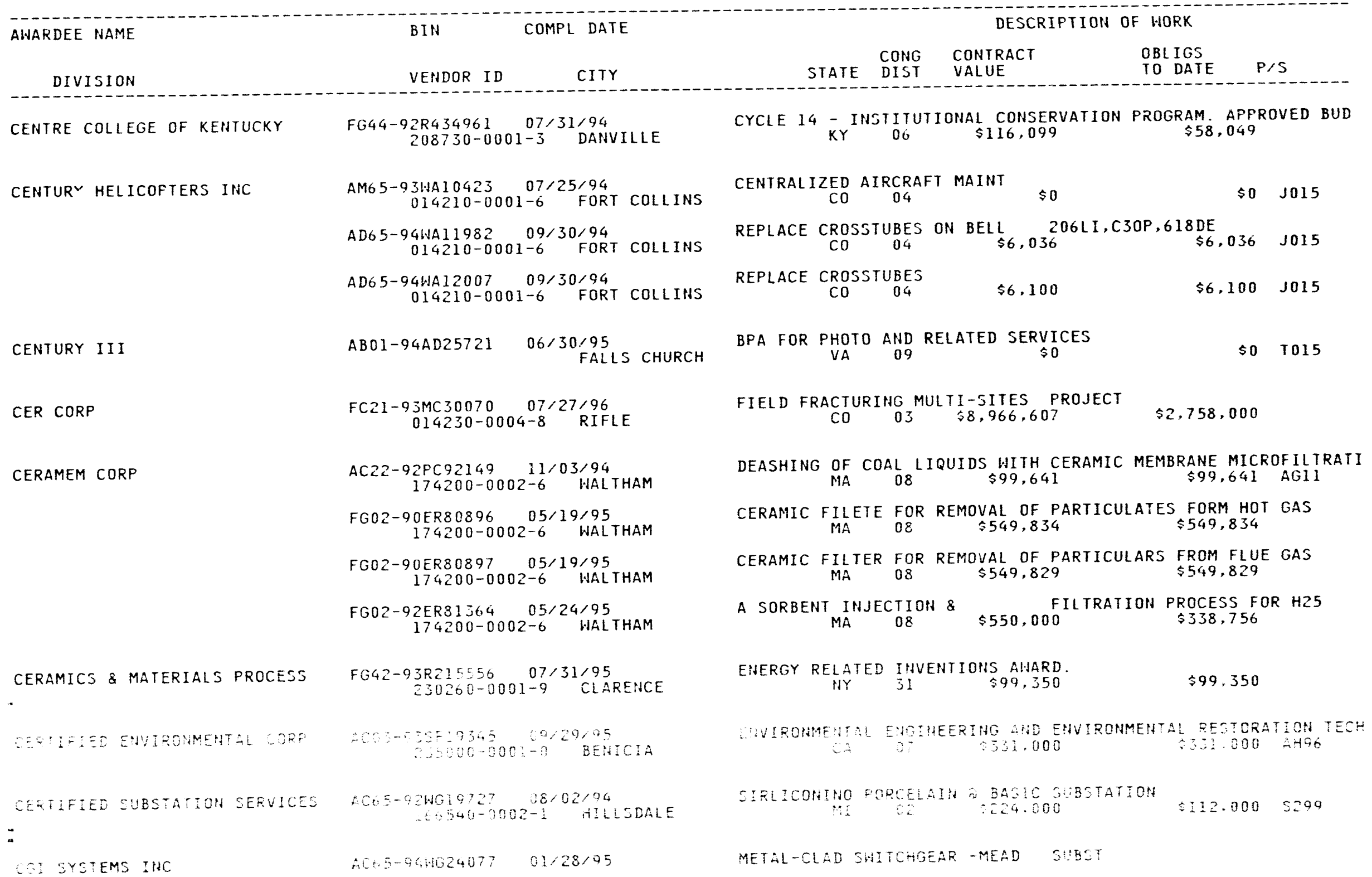


AWARDEE NAME COMPL DATE

DESCRIPTION OF WORK

AWARDEE NAME

BIN

BIN

CITY

STATE DIST

DIVISION

VENDDR ID PARAMOUNT

CA 02

$\$ 176,994$

OBLIGS $159350-0001-6$

FG06-94RL12852 $06 / 26 / 95$
$014340-0101-6 \quad$ CHADRON

CHADRON STATE COLLEGE IRTC DEPT

CHAMA TOWN DF MUNICIPAL SCHOOLS

CHAMPION INC

CHANDLER REGIONAL HOSPITAL

CHANG CORP

CHAR-FUEL ASSOC LTD

CHARLES G WILLIAMS CONSTR INC

CHARLES RIVER ANALYTICS INC

CHARLOTTE CENTRAL SCHOOL

CHAS H THOMPKINS INC

CHATTAHOOGA CITY OF EDUCATIOH BOARD OF

CHEATHAM COUNTY OF SCHOOL SYSTEM
GMO4-92AL58228 04/02/97 226730-0101-8 CHAMA

AB01-94AD25716 06/30/95 $154220-0001-3$ WASHINGTON

FG49-93R915006 06/30/95 232270-0001-6 CHANDLER

ABO4-93AL90118 05/21/95 228540-0001-3 AL BUQUERQUE

FC21-92MC29268 09/30/94 $225400-0001-X$ ENGLENOOD

AC65-92WA09981 $08 / 31 / 94$
$222040-0002-7$ GOLDEN

FG02-91ER81178 07/05/94 $214320-0001-2$ CAMBRIDGE

FG41-94R196611 05/31/96 238910-0001-4 CHARLOTTE

AC01-92AD66150 08/05/95 $210770-0001-4$ WASHINGTON

FG44-93R435318 09/30/95 $100040-0201-6$ CHATTANODGA

FG44-93R435319 09/30/95

USED ENERGY RELATED LABORATORYEQUIPMENT GRANT

$$
\text { NE } 03
$$

$\$ 0$

SUPPORT FOR SCIENCE, MATHEMATICS AND ENGINEERING TO IMPROVE NM OS

$\$ 0$

BPA FOR METAL ENGRAVING

$\$ 0$

$\$ 0 \quad 9999$

CYCLE 15 ICP GRANT AWARD

AZ 01 ANARD $\$ 80,937$

$\$ 40,468$

BPA FOR COMPUTER SOFTWARE AND RELATED SUPPLIES

IIM 01 \$282,000

$\$ 282,000 \quad 7030$ PILOT SCALE DEMOHSTRATION OF CHARFUEL COAL REFINING PROCESS CO $06 \quad \$ 2,160,747$

ADMIN CHG TO PROPERLY SET FORTH CONT COMPL DATE CO 05 \$ $155.824 \quad \$ 155,824 \quad 2111$

A NEURAL NETHORK APPROACH TO NUCLEAR PLANT MONITORING.

$$
\begin{aligned}
& \text { NETWORK APFROACH TO } \\
& \text { MA } 08 \text { \$550,000 }
\end{aligned}
$$

$\$ 550,000$

TITE III OF THE MATIONAL ENERGY CONSERVATION POLICY ACT

$$
\text { IIT THE NATIONAL ENERG }
$$$$
\$ 7,361
$$

ELEVATOR MAINTENANCE FOR GTN FACILITY

$$
\text { MC } 01 \quad \$ 1,554,813
$$

SEE ATTACHED PAGE NO. 2 OF THIS NOTICE OF FINANCIAL ASSISTAN TN $03 . \$ 34,621$ $\$ 17,310$

SEE ATTACHED PAGE NO. 2 OF THIS NOTICE OF FINAHCIAL ASSISTAN TH 05 N 2 \$26,519 NOTICE OF FINANCIAL 


\section{AWARDEE NAME}

BIN

COMPL DATE

DESCRIPTION OF HOKK

DIVISIDN

VENDOR ID

STATE DIST

CONTRACT VALUE

OBLIGS

TO DATE P/S

CHELSEA HOUSING AUTHORITY

FG41-92R110448 09/30/95 $225520-0001-0$ CHELSEA

CHEMAT TECHNOLOGY INC

CHEMICAL WASTE MANAGEMENT INC

CHERSKOV FLAYNIK \& WASSERBAUER

CHESHIRE TOWN OF

PUBLIC SCHOOLS

CHESTER COUNTY BD OF EDUCN

CHESTNUT HILL ACADEMY

CHEVERUS HIGH SCHOOL

CHEVRON USA INC

CHICAGO CITY OF

PLANNING DEPT

EDUCATION BOARD OF
FG03-93ER81570 07/09/94 $233860-0001-0$ INORTHRIDGE

AC21-92MC29105 07/07/94 $181280-0002-0$ ANDERSON

AC02-92CHI0536 09/24/94 $224390-0001-9$ CHANNAHON

FG41-93R191501 08/31/95

FG44-93R435341 09/30/95 014980-0001-0 HENDERSON

FG43-94R364100 05/31/95 238560-0001-1 PHILADELPHIA 230430-0001-3 PORTLAND

FG41-94R192613 06/30/96 PORTLAND 015030-0004-4 HOUSTON

FCO2-94CE16071 07/31/95

FC02-90CE27504 07/31/94 $015070-0301-3$ CHICAGO

FG45-93R551528 08/31/95 015070-0101-0 CHICAGO
FG41-93R192511 06/30/95

SC91-94FE63054 11/01/94
DOE/HUD INTATIVEF O $\$ 25,000 \quad \$ 25,000$

CAPACITIVE ENEROY STORAGE USING HIGH SURFACE AREA TRANSITION $\begin{array}{ll}\text { CA } 25 & \$ 75,000\end{array}$

EM-VAC*TRAX-MOBIL VACUUM, THERMAL TREATMENT SYSTEM $S C$ SC $\$ 501,401$ TACUUM $\$ 501,401$ AH92

SUPPORT SERVICES PATENT PREPARTION AND PROSECUTIOH IL 11 \$63,000 $\$ 50,000$ R499

INITIAL AWARD.

SEE ATTACHED PAGE NO. 2 OF THIS NOTICE OF FINANCIAL ASSISTAH TH 07 N $\$ 30.735 \quad \$ 15,367$

TITL III OF THE NATIONAL ENERGY CONSERVATION POLICY ACT PA THE NATIONAL $\$ 2$ ENERGY CONSERVATION $\$ 3,985$

TITLE III OF THE NATIONAL ENERGY CONSERVATION POLICY ACT

FY:94 ICP AWARD .

NOSR NATURAL GAS SALES CONTRACT ESTIMATED REVENUE: $\$ 4,011,43$ TX 08 $\$ 0$ STUDIES IN URBAN ENERGY CONSERVATION-URBAN CONSORTIUM IL 01 \$ $\$ 941,000 \quad \$ 941,000$

RESEARCH IN ENERGY CONSERVA- TION FOR MUNICIPALITIES IL 01 \$5,920,389 $\$ 5,920,389$

ICP-NEW AHARD.
IL 

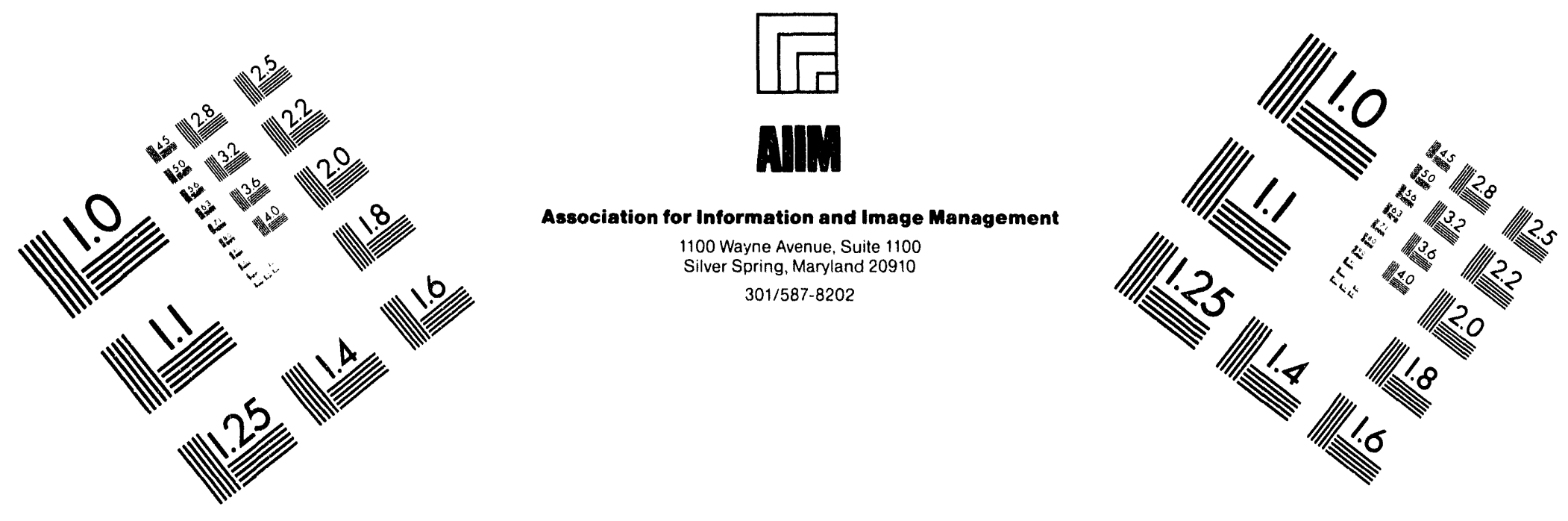

\section{Centimeter}

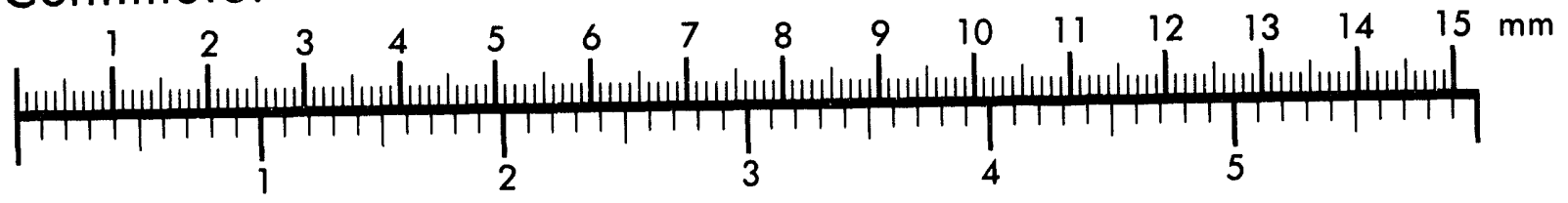
Inches
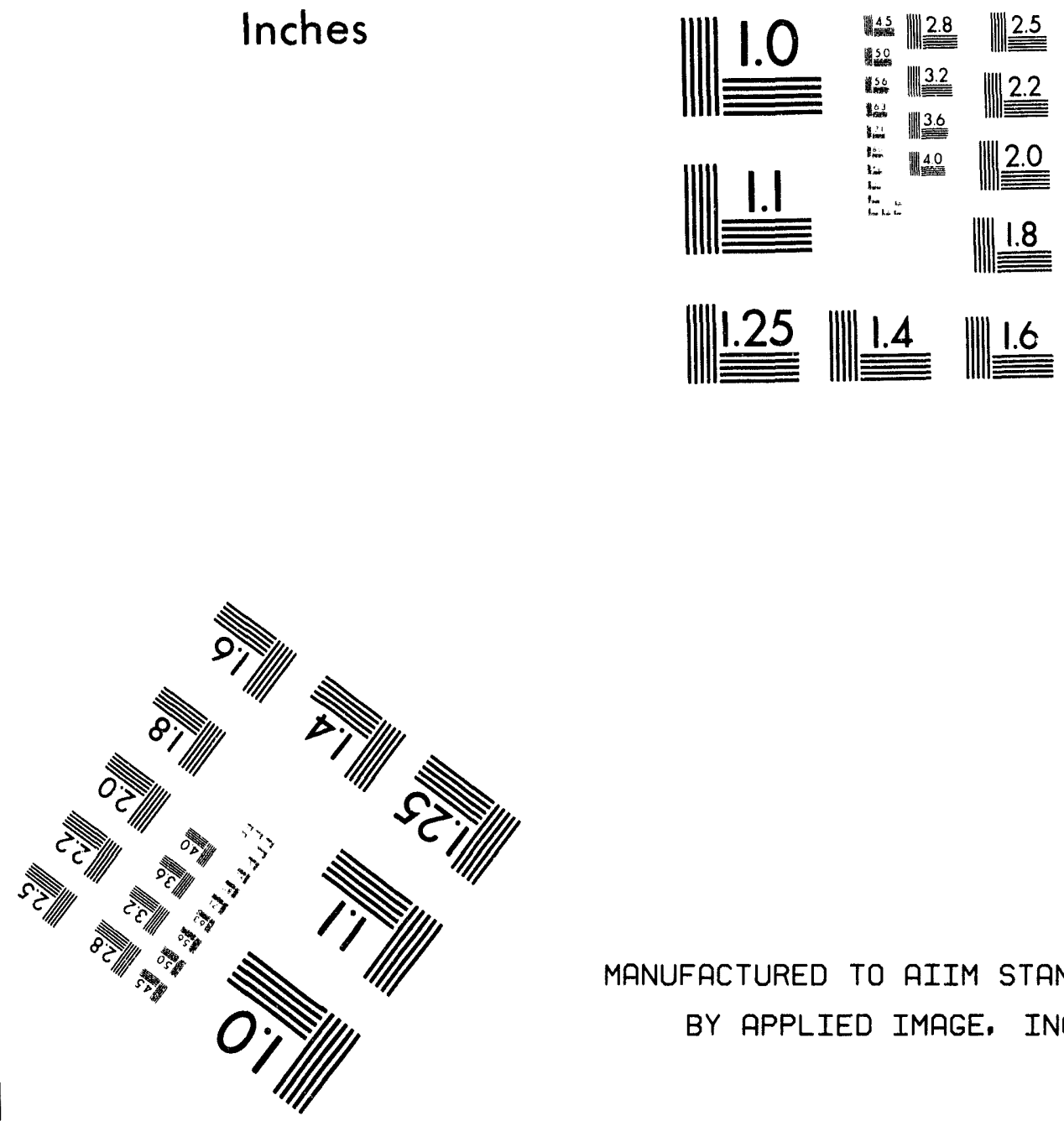

MANUFACTURED TO AIIM STANDARDS

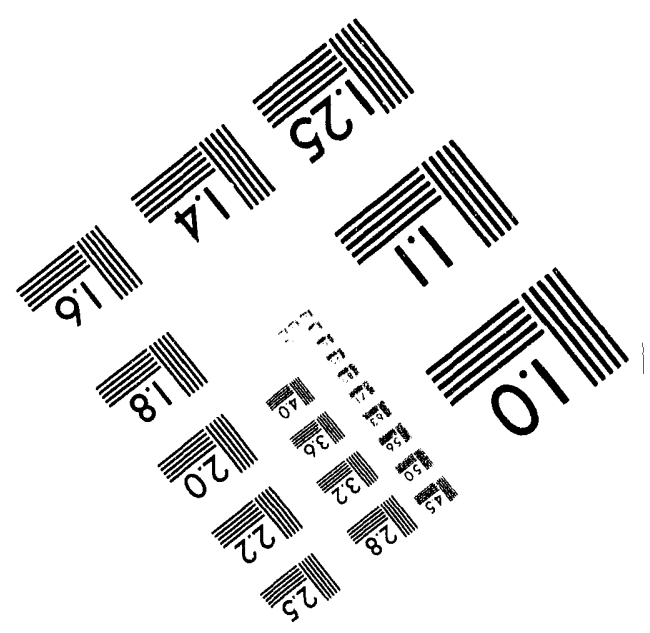



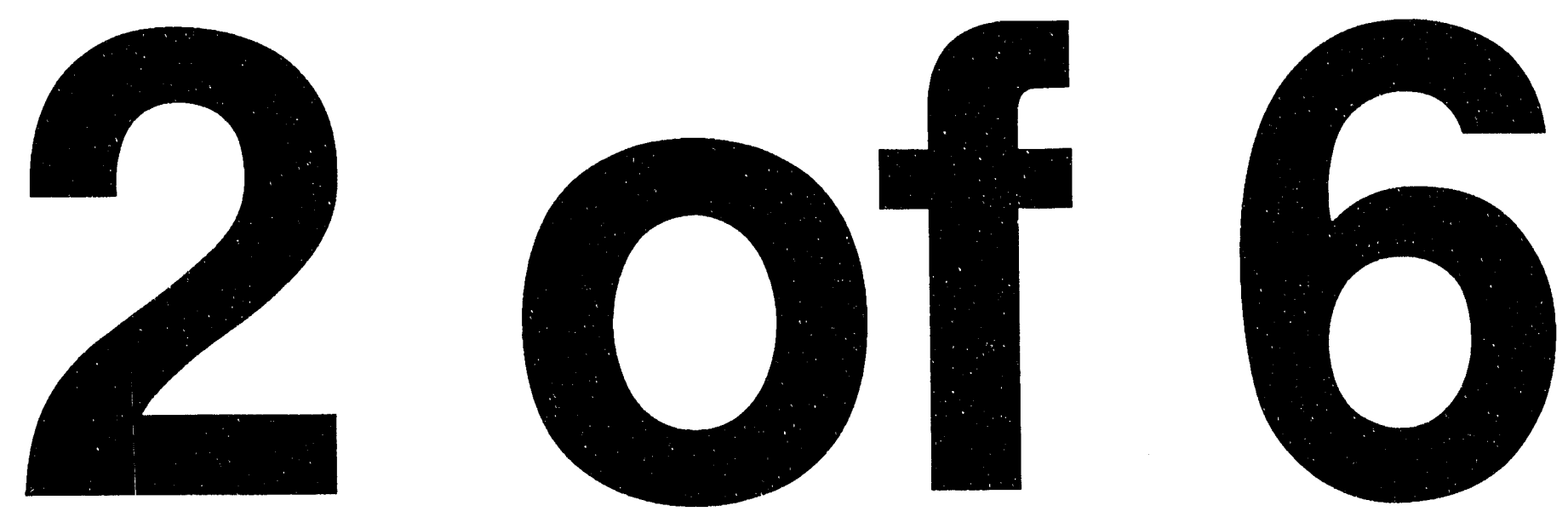


AWARDEE NAME BIN COMPL DATE

DESCRIPTION OF WORK

AWARDE NAME

VENDOR ID

CITY

STATE CONG CONTRACT

OBLIGS

STATE

CHICAGO UNIVERSITY OF ARGONHE NATIONAL LAB-WEST

MEDICINE DEPT

PHYSICS DEPT

MOLECULAR GENETICS \& CELL BI

JAMES FRANCK INST

PHYSICS DEPT

BIDLOGICAL SCIENCES

ENRICO FERMI INSTITUTE

RADIOLOGY DEPT
GM07-93ID11114 12/31/99 015130-1904-X IDAHO FALLS

FG02-92ER14244 07/15/96 $015130-0002-0$ CHICAGO $\begin{array}{cc}\text { FG02-86ER6 } 0408 & 12 / 31 / 94 \\ 015130-1402-1 & \text { CHICAGO }\end{array}$ FG02-86ER60437 01/31/96
$015130-1102-2$ CHICAGO FG02-86ERI $3546 \quad 06 / 04 / 96$ $015130-1602-4$ CHICAGO FG02-87ER13679 11/30/95 $015130-1702-0$ CHICAGO FG02-87ER13726 05/14/96 $015130-1102-2$ CHICAGO

FG02-88ER60678 04/14/97 $015130-1302-5$ CHICAGO

FG02-90ER40560 10/31/95 $015130-0502-2$ CHICAGO FG02-91ER40606 12/14/96 $015130-0002-0$ CHICAGO

FG02-91ER20024 05/14/97 $015130-0002-0$ CHICAGO

FGD2-92ER25119 04/30/95 015130-0002-0 CHICAGO

FG02-92ER14305 06/14/95 015130-0002-0 CHICAGO

FG02-94ER61840 05/31/97 $015130-0002-0$ CHICAGO

FG02-94ER61816 03/31/97 $015130-1502-8$ CHICAGO

FG02-93ER61588 11/30/94 $015130-0002-0$ CHICAGO
INEL FIRING RANGE USE AGREEMENT WITH ARGONNE NATIOHAL LABORA ID 02 \$O

$\begin{array}{cccc}\text { DEVELOPMENT OF SYNCHROTRON } & X \text { X-RAY MICRO- } & \text { SPECTROSCOPIC } \\ \text { IL } & 01 & \$ 500,738 & \$ 358,427\end{array}$

CORRELATION OF CHROMOSONE PATTERNS IN HUMAN LEUKEMIC CELLS

$$
\text { IL } 01 \quad \$ 2,824,039 \quad \$ 2,824,039
$$

ELECTRON MICROSCOPE DEVELOPMENT

$\$ 2,703,635$

ORGANIZATION AND REGULATION OFTHE GENES FOR NITROGEN

$$
\text { IL } 01 \quad \$ 861,130 \quad \$ 766,130
$$

QUANTUM DYHAMICS OF FAST CHEMICAL REACTIONS

$$
\text { IL } 01 \text { S } \$ 805,001 \quad \$ 703,001
$$

FUNDAMENTALS AND TECHNIQUES OFNON-IMAGING OPTICS

$$
\text { IL } 01 \text { \$1,414,490 } \$ 1,216,490
$$

SPECIFICITY OF MUTATION INDUCED BY IONIZING RADIATION

$$
\text { IL } 01 \text { \$ } \$ 838,380 \quad \$ 627,635
$$

$\begin{array}{ccc}\text { PARTICIPATION IN HIGH ENERGY PHYSICS } & \$ 5.174,000\end{array}$

NUCLEAR PHYSICS AND ASTROPHYSICS

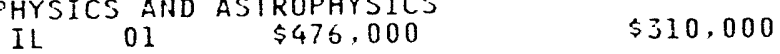

SIGHAL TRANSDUCTION IN PLANT DEVELOPMENT: CHEMICAL \& BIOCHEM

$$
\text { IL } 01 \quad \$ 551,337 \quad \$ 45,624
$$

STUDIES OF COMPLEXITY IN FLUIDSYSTEMS

$$
\text { IL COMPLEXITY IN FLUIDSYSTEMS } \$ \$ 45,500,500
$$

$\begin{array}{cccc}\text { BOHD SELECTIVE CHEMISTRY } & \text { BEYOND THE ADIABOTIC } \\ \text { IL } & 01 & \$ 323,000 & \$ 323,000\end{array}$

DEVELOPMENT OF DOPAMINE RECEPTOR RADIOPHARMACATICALS IL 01 \$ $\$ 690,000 \quad \$ 225,000$

METHODOLOGY FOR EVAULATION OF DIAGNOSTIC PERFORMANCE IL 01 \$556,730 $\$ 176,411$

DEVELOPEMNT OF GAMMA-EMITTING RECEPTOR-BINDING RADIO IL $01 \quad \$ 262,680 \quad \$ 262,680$ 


\begin{tabular}{|c|c|c|c|c|c|c|c|}
\hline AWARDEE NAME & BIN & L DATE & & & $\mathrm{DESCI}$ & F WORK & \\
\hline DIVISION & VENDOR ID & CITY & STATE & $\begin{array}{l}\text { CONG } \\
\text { DIST }\end{array}$ & $\begin{array}{l}\text { CONTRACT } \\
\text { VALUE }\end{array}$ & $\begin{array}{l}\text { OBLIGS } \\
\text { TO DATE }\end{array}$ & $P / S$ \\
\hline
\end{tabular}
ENDOR ID

FG02-93ER61655 01/31/97
$015130-0002-0$ CHICAGO

CHEMISTRY DEPT

ARGONNE NATIONAL LAB

CHICOPEE CITY OF

CHILDREN'S HOSP OF MICHIGAN CARING FOR KIDS PROJECT

CHILDREN'S HOSPITAL CORP

CHILE GOVERNMENT OF

CHIPPEWA VALLEY HOSPITAL

CHOCTAW COUNTY MEDICAL CENTER

¿HOWAN COLLEGE

:
AC22-91PC91056 03/26/96
$015130-0402-6 \quad$ CHICAGO

FG45-90R520258 11/30/94

$015130-0002-0$ CHICAGO

FG45-92R540079 12/31/94

$015130-0002-0$ CHICAGO

FG45-93R551533 08/31/95

$015130-0002-0$ CHICAGO

$W-31109-E N G-38 \quad 09 / 30 / 94$
$015130-0101-9$ LEMONT

FG41-93R193515 07/31/95

FG02-91CH10473 08/06/94
$211850-0101-8$ DETROIT

FG02-94ER61794 03/15/95 $121400-0001-3$ BOSTOH

FCO2-94P010140 11/16/95 $235770-0001-5$ SANTI IAGO

$\begin{array}{cc}\text { FG45-0?R551817 06/30/95 } & 06 / 30 \\ 219760-0001-7 & \text { DURAND }\end{array}$

FG44-92R435108 09/30/94 $223170-0001-4$ ACKERMAN

FG44-92R434997 08/31/94 220670-0001-2 MURFREESBORO

FG44-93R435382 09/:0/95 220670-0001-2 MURFREESBORO $\begin{array}{clll}\text { RECEPTOR - DNA BINDING TO } & \text { TARGET AUGER ELECTRON } \\ \text { IL } & 01 & \$ 1,030,000 & \$ 405,000\end{array}$

MOLECULAR CATALYTIC COAL LIQUID COHVERSION

$\begin{array}{rrrr}\text { IL } 01 & \$ 363,573 & \$ 363,573 \text { AGI }\end{array}$

ICP - NEW AWARD IL $\$ 238,890 \quad \$ 119,444$

ICP - NEW AWARD IL $_{01} \$ 523,760 \quad \$ 261,879$

ICP-NEW AWARD.
IL

OPERATION OF ARGONNE NATIONAL LABORATORY

$$
\text { IL AR } 13 \$ 8,327,788,126 \quad \$ 7,992,944,491 \quad M 181
$$

TITLE III OF THE NATIONAL ENERGY CONSERVATION POLICY ACT

$$
\text { MA } 02 \text { THE NATIONAL ENERGY CONSERVATION POL } \$ 50.467
$$

FACILITIES \& EQUIPMENT FOR A POSITRON EMMISSION TOMOGRAPHY $\begin{array}{lll}M I & 13 & \$ 10,156.000\end{array}$

FIRST INTERNATION WORKSHOP ON HUMAN CHROMOSOME 1

$$
\text { MA } 09 \text { \$10,915 } \$ 10,915
$$

CHILE CLIMATE CHANGE COUNTY STUDY

$\$ 26,500$

ICP-NEW AHARD

HI $03 \quad \$ 96,840 \quad \$ 48,420$

CYCLE 14 - INSTITUTIONAL COHSERVATION PROGRAM. APPROVED BUD MS 01 \$ $\$ 5,258 \quad \$ 3,155$ CYCLE 14 - INSTITUTIONAL CONSERVATION PROGRAM. APPROVED BUD
NC 01

SEE ATTACHED PAGE NO. 2 DF THIS NOTICE OF FINANCIAL ASSISTAN NC 01 \$8, 920 \$6, 467 


AWARDEE NAME

BIN COMPL DATE

DESCRIPTION OF WORK

DIVISION

VENDOR ID

STATE CONG

COPTRAC

OBLIGS PO DATE P/S
TO

CHRIST THE KING CHURCH

CHRISTIAN COUNTY OF

BOARD OF EDUCATION

CHRISTINA SCHOOL DISTRICT

CHUBBUCK CITY OF

POL ICE DEPARTMENT

CHURCH OF THE SACRED HEART

CH2M HILL INC

CIMARRON PUBLIC SCHOOLS

CINCINNATI CITY DF
SCHOOL DISTRICT

SCHOOL DISTRICT

. CINCINNATI TECHHICAL COLLEgE

CINCINNATI UNIVERSITY OF PHYSICS DEPT

- CHEMISTRY DEPT

FG02-91ER4545908/31/95

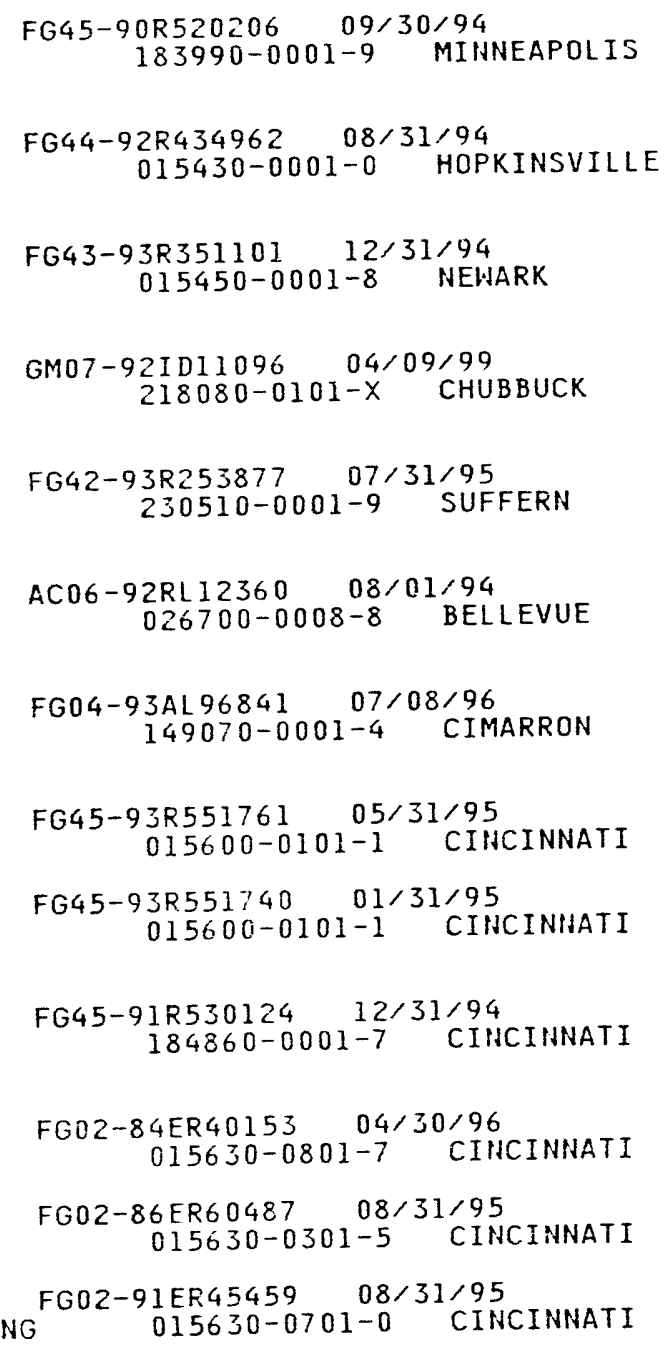

ICP - NEW AWARD MN $_{05} \$ 19,756 \quad \$ 13,829$

CYCLE 14 - INSTITUTIONAL CONSERVATION PROGRAM. APPROVED BUD

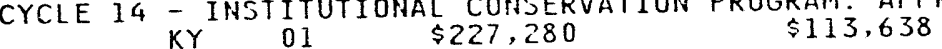

TITLE III OF THE NATIONAL ENERGY CONSERVATION POLICY ACT I

$\begin{array}{rrr}\text { INEL FIRING RANGE USE AGREEMENT WITH CHUBBUCK } \\ \text { ID } & 02 & \$ 0\end{array}$ I D 02

CYCLE 15 AHARD.

$\$ 22,855$

$\$ 11,427$

FOR DESIGN OF $L-045 \mathrm{H} 300$ AREA TREATMENT

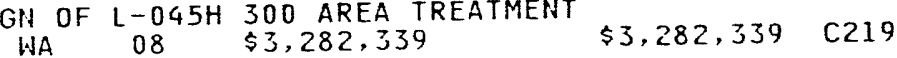

EDUCATION OUTREACH

$$
\underset{N M}{N O U T R E A C H}
$$

$\$ 25,000$

ICP-NEW AWARD

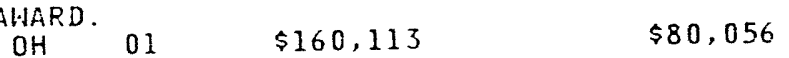

ICP-NEW AWARD

OH $01 \quad \$ 8 J, 666$

$\$ 41,831$

ICP-NEW AWARD
OH

01
$\$ 230,134$
$\$ 108,331$

INELASTIC STRONG INTERACTIONS AT HIGH ENERGIES

DEVELOPMENT OF MORE EFFICACIOUS TC-99M ORGAN IMAGING AGENTS ROLE INTERFACIAL PRDPERTIES ONTHE STEADY STATE \& NON-STEADY $\begin{array}{ccc}\text { ROLE INTERFACIAL PROPERTIES ONTHE STEADY STATE } & \$ 270,800 \\ O H \quad 01 & \$ 389,717 & \$ 270 N-\end{array}$ 


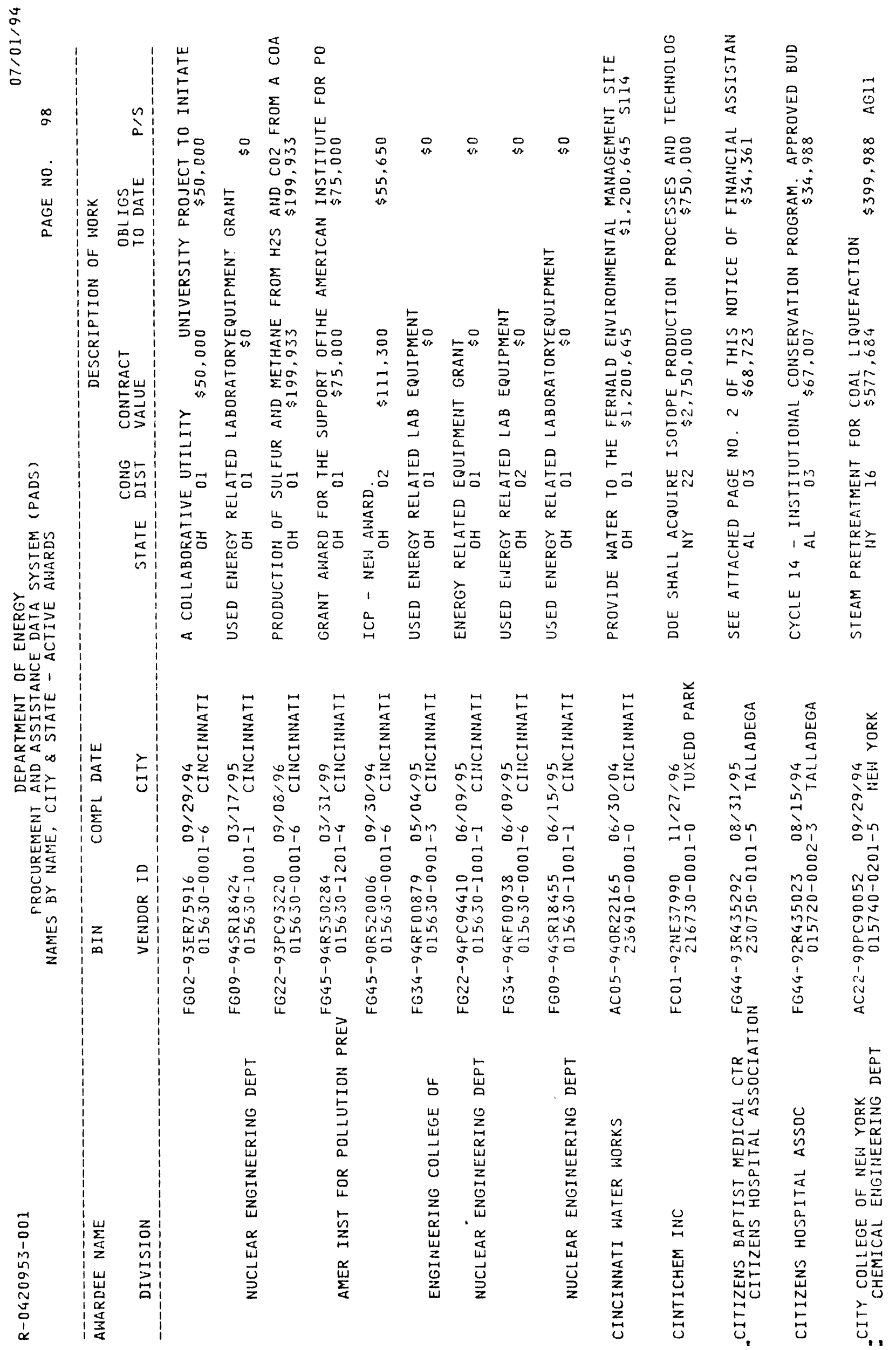




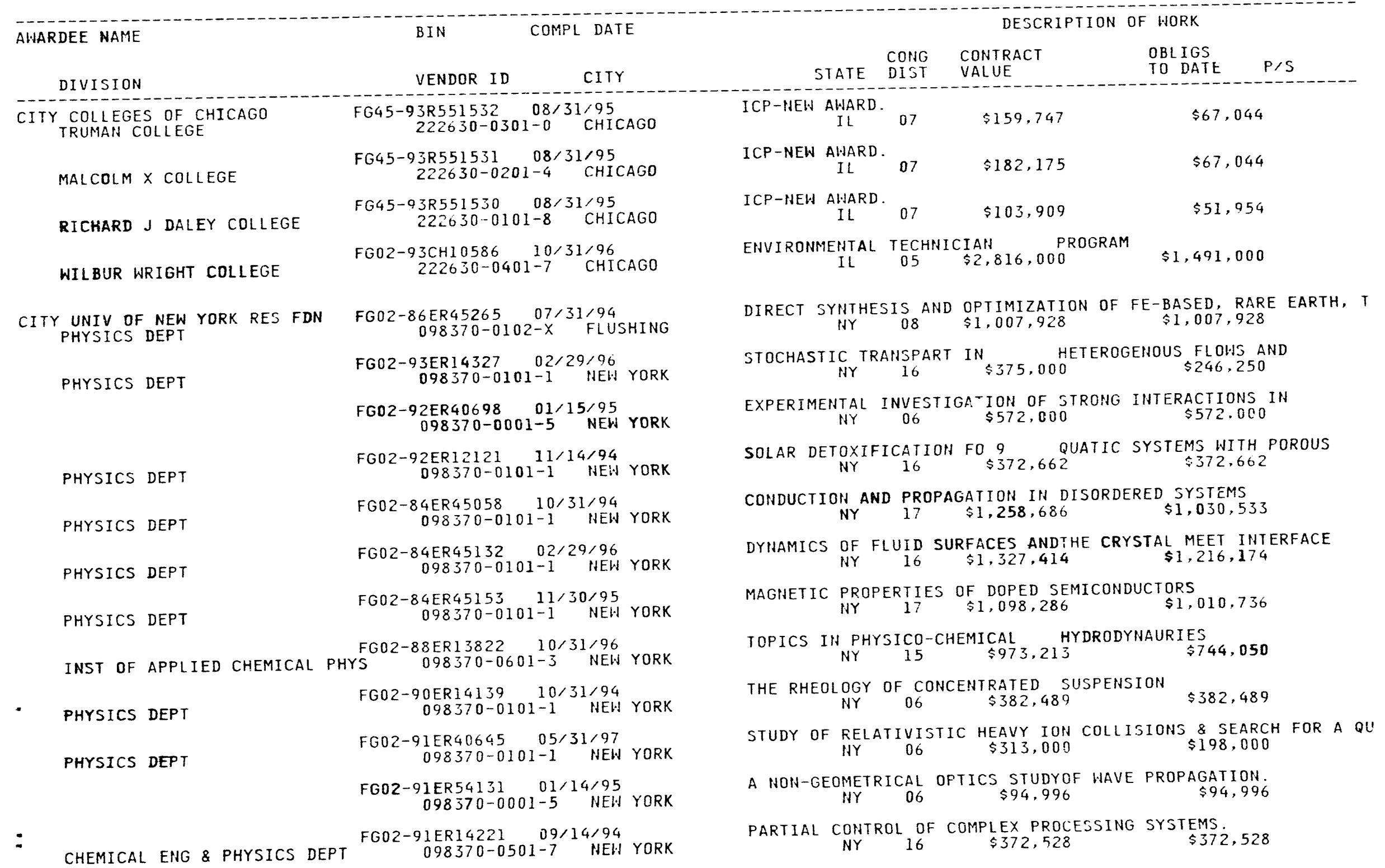




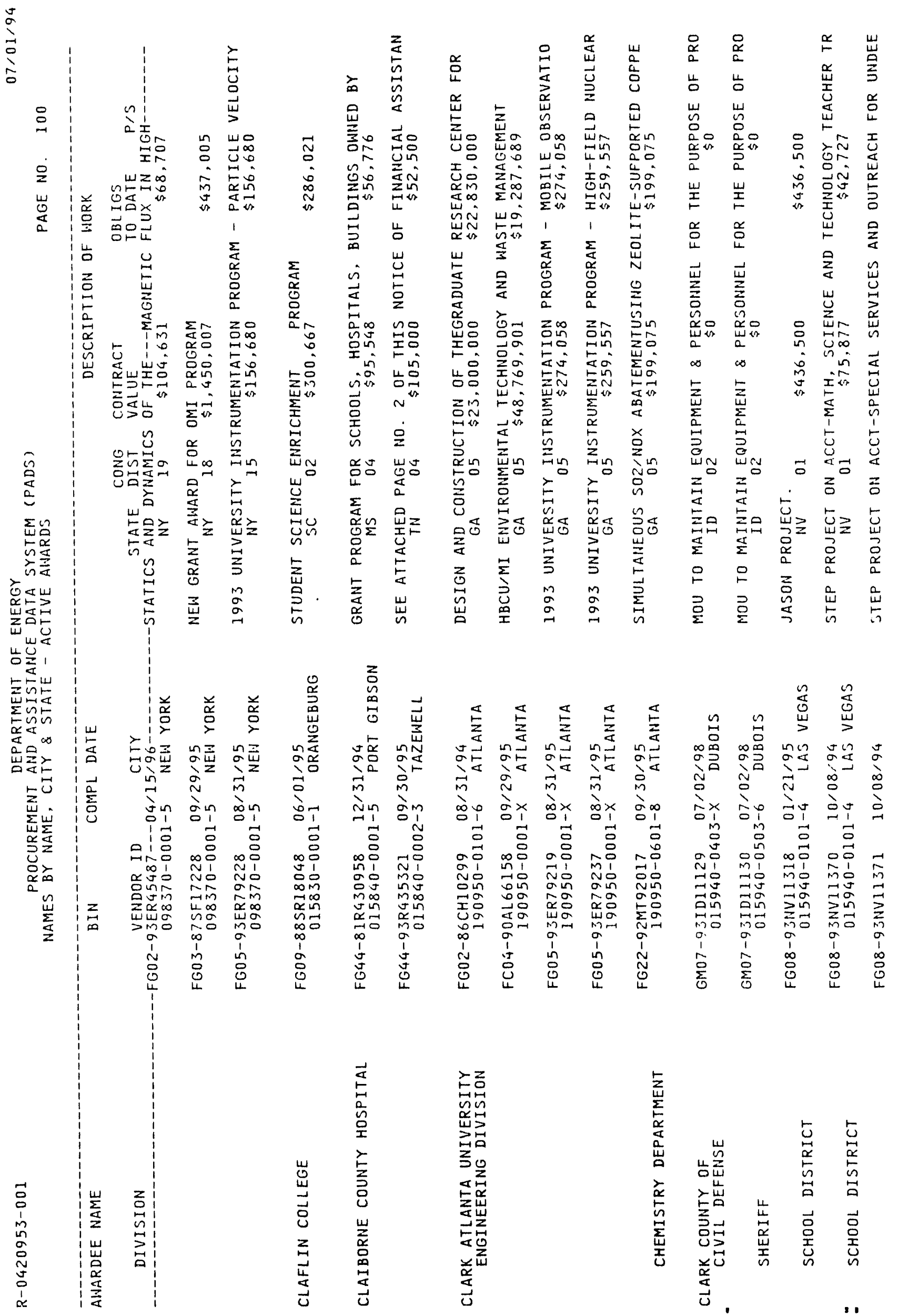




\begin{tabular}{|c|c|}
\hline AWARDEE NAME & COMPL DATE \\
\hline DIVISION & VENDOR ID \\
\hline SCHOOL DISTRICT & $015940-0101-4$ \\
\hline SCHOOL DISTRICT & $\begin{array}{r}\text { GMO8-91NV11412 } 11 / 27 / 95 \\
015940-0101-4 \text { LAS VEGAS }\end{array}$ \\
\hline SCHODL DISTRICT & $\begin{array}{r}\text { FG08-93NV11455 09/30/94 } \\
015940-0101-4 \text { LAS VEGAS }\end{array}$ \\
\hline SCHOOL DISTRICT & $\begin{array}{r}\text { FG49-93R915002 } 05 / 31 / 95 \\
015940-0101-4 \text { LAS VEGAS }\end{array}$ \\
\hline SCHOOL DISTRICT & $\begin{array}{r}\text { FG } 49-94 R 916000 \quad 05 / 31 / 96 \\
015940-0101-4 \text { LAS VEGAS }\end{array}$ \\
\hline SCHOOL DISTRICT & $\begin{array}{c}\text { FG49-94R916001 } 05 / 31 / 96 \\
015940-0101-4 \text { LAS VEGAS }\end{array}$ \\
\hline CLARK DONALD ASSOC & $\begin{array}{c}\text { AC65-90WL } 0257201 / 09 / 95 \\
015950-0001-1 \text { LAS VEGAS }\end{array}$ \\
\hline CLARK UNIVERSITY & $\begin{array}{c}\text { FGO2-9JER14388 } 09 / 14 / 94 \\
016010-0001-4 \text { WORCESTER }\end{array}$ \\
\hline & $\begin{array}{c}\text { FG09-94SR18414 02/02/95 } \\
016010-0001-4 \text { WORCESTER }\end{array}$ \\
\hline CHEMISTRY DEPT & $\begin{array}{r}\text { FG02-88ER40417 } 01 / 31 / 97 \\
016010-0102-9 \text { UPTON }\end{array}$ \\
\hline CLARKE COLLEGE & $\begin{array}{r}\text { FG47-93R707027 } 08 / 31 / 94 \\
112110-0001-X \quad \text { DUBUQUE }\end{array}$ \\
\hline $\begin{array}{l}\text { CLARKSON UNIVERSITY } \\
\text { - CHEMICAL ENGINEERING DEPT }\end{array}$ & $\begin{array}{r}\text { FG02-86ER13552 } 11 / 30 / 95 \\
016060-0101-9 \text { POTSDAM }\end{array}$ \\
\hline CHEMICAL ENGINEERING DEPT & $\begin{array}{c}\text { FG02-88ER13919 05/31/97 } \\
016060-0101-9 \text { POTSDAM }\end{array}$ \\
\hline CHEMICAL ENGINEERING DEPT & $\begin{array}{c}\text { FG02-90ER61029 } 04 / 30 / 96 \\
016060-0101-9 \text { POTSDAM }\end{array}$ \\
\hline \multirow[t]{2}{*}{ RY DEPT } & $\begin{array}{c}\text { FG02-94ER61784 01/31/96 } \\
016060-0501-4 \quad \text { POTSDAM }\end{array}$ \\
\hline & FG22-91PC91297 \\
\hline
\end{tabular}

DESCRIPTION OF HORK

STATE DONG CONTRACT

NV 01 \$36, 379

OBLIGS
TO DATE P/S $\$ 29,919$ MOU TO IMPLEMENT A COOPERATIVEEFFORT AT REACHING NATIONAL E EDUCATIONAL OUTREACH TO SUPPORT THE WHITNEY MESA EDUCATIONAL NV 01 \$115,000 $\$ 30,000$

CYCLE 15 ICP GRANT AWARD

IV $02 \quad \$ 327,201$

$\$ 163,596$

CYCLE 16 ICP GRANT APPLICATION

$\$ 21,100$

CYCLE 16 ICP GRANT APPLICATION

$\$ 102,212$

ADMIN SUPPORT SERV.

$$
\begin{array}{lllll}
\text { NV } & 01 & \$ 1,197,268 & \$ 1,197,268 & \text { R699 }
\end{array}
$$

$\begin{array}{llll}\text { NOVER ALUMINUM AND SULFUR } & \text { BATTERIES } & \\ \text { MA } & 03 & \$ 198,796 & \$ 66,733\end{array}$

USED ENERGY RELATED LABORATORYEQUIPMENT $\$ 00$

NUCLEAR STRUCTURE RESEARCH AT THE HIGH FLUX BEAM REACTOR NYY OI $\$ 1,119,000 \quad \$ 784,000$

GRANT PROGRAMS FOR SCHOOLS ANDHOSPITALS AND FOR BUILDINGS 0 IA $02 \quad \$ 14,000 \quad \$ 7,000$

ANALYSIS AND COMPUTER TOOLS FOR SEPARATION PROCESSES NY $13 \quad \$ 509,404 \quad \$ 445.284$

DROPLET MOTIOH! IN NUMERICALLY SIMULATED TURBULENCE
NY STUDY OF THE ATMOSPHENIC CHEMISTRY OF RADO PROGENY IN NY 30 \$1,040,000 $\$ 865,050$ ORGANIZATION \& OPERATION OF THE 6TH INTERNAT'L. SYMPOSIUM A COMPUTATIONAL MODEL FOR COALTRANSPORT AND COMBUSTION 


AWARDEE NAME
DIVISION
VENDOR ID
$016060-9001-2$

SC ENERGY RESEARCH \& DEV CTR FC21-92MC29061 03/30/97 $016240-0301-x$ CLEMSON

CLEVELAND AREA HOSPITA:

FG46-92R613540 10/31/94 211160-0001-6 CLEVELAND

CLEVELAND CATHOLIC DIOCESE

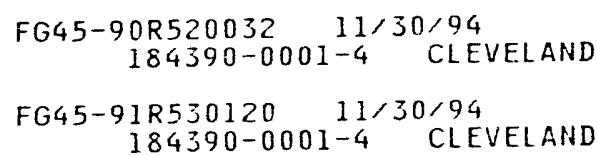

CLEVELAND ELEC ILLUMINATING CO CROI-85RW00046 03/15/30 124500-0001-1 CLEVELAND

FG45-92R540187 07/31/94 016360-0001-0 CLEVELAND
DESCRIPTION OF HORK

CONG CONTRACT
STATE DIJT VALUE

$\$ 301,130$

OBLIGS
TO DATE P/S $\$ 200.000$

FOR COAL PROCESSING: METAL CARBIDES AND NITRI IYY 30 TOL $\$ 283,690 \quad \$ 200,000$ ASH \& PULVERIZED COAL DEPOSITION IN COMBUSTORS \& GAJIFIERS NY $26 \quad \$ 331,600 \quad \$ 200,000$

TITLE III OF THE NATIONAL ENERGY CONSERVATION POLICY ACT $\mathrm{NM} 03 \quad \$ 27,704 \quad \$ 13,852$

EXTEND SCALE MODEL TO ASSESS EFFECTS OF SURFACANTS AND ADAPT

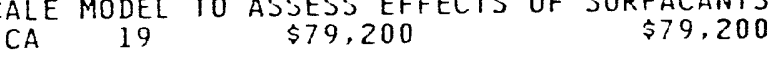

CHARACTERIZATION AND THERMOPHYSICAL PROPERTIES AND BI-BASED SC 03 \$O76,205 $\$ 308,723$

THE MAGNESIUM CHELATION STEP IN CHLOROPHYLL BIOSYNTHESIS

$\begin{array}{ccc}\text { USED ENERGY RELATED LABORATORYEQUIPMENT } & \$ 30 \\ \text { SC } & 03 & \$ 0\end{array}$

$\begin{array}{llll}\text { ADVANCED GAS TURBINE SYSTEMS RESEARCH } & \\ \text { SC } & 0 j & \$ 10,970,799 & \$ 6,470,799\end{array}$

THIS IS A NEH CYCLE 14 ENERGY CONSERVATION MEASURE GRANT AWA

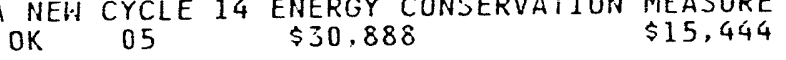

$I C P-N E W{ }_{D H}^{A W A R D} 20 \quad \$ 107,438 \quad \$ 52,916$

$\begin{gathered}\text { ICP-NEW AWARD. } \\ \text { OH }\end{gathered} 19 \quad \$ 131,033 \quad \$ 65,516$

DISPOSAL OF SPENT NUCLEAR AND/OR HIGH LEVEL RADIOACTIVE WAST $\mathrm{OH} \quad 20 \mathrm{SO} \quad \$ 0$

ICP - NEW AHARD.

OH 21

$\$ 90,805$ 


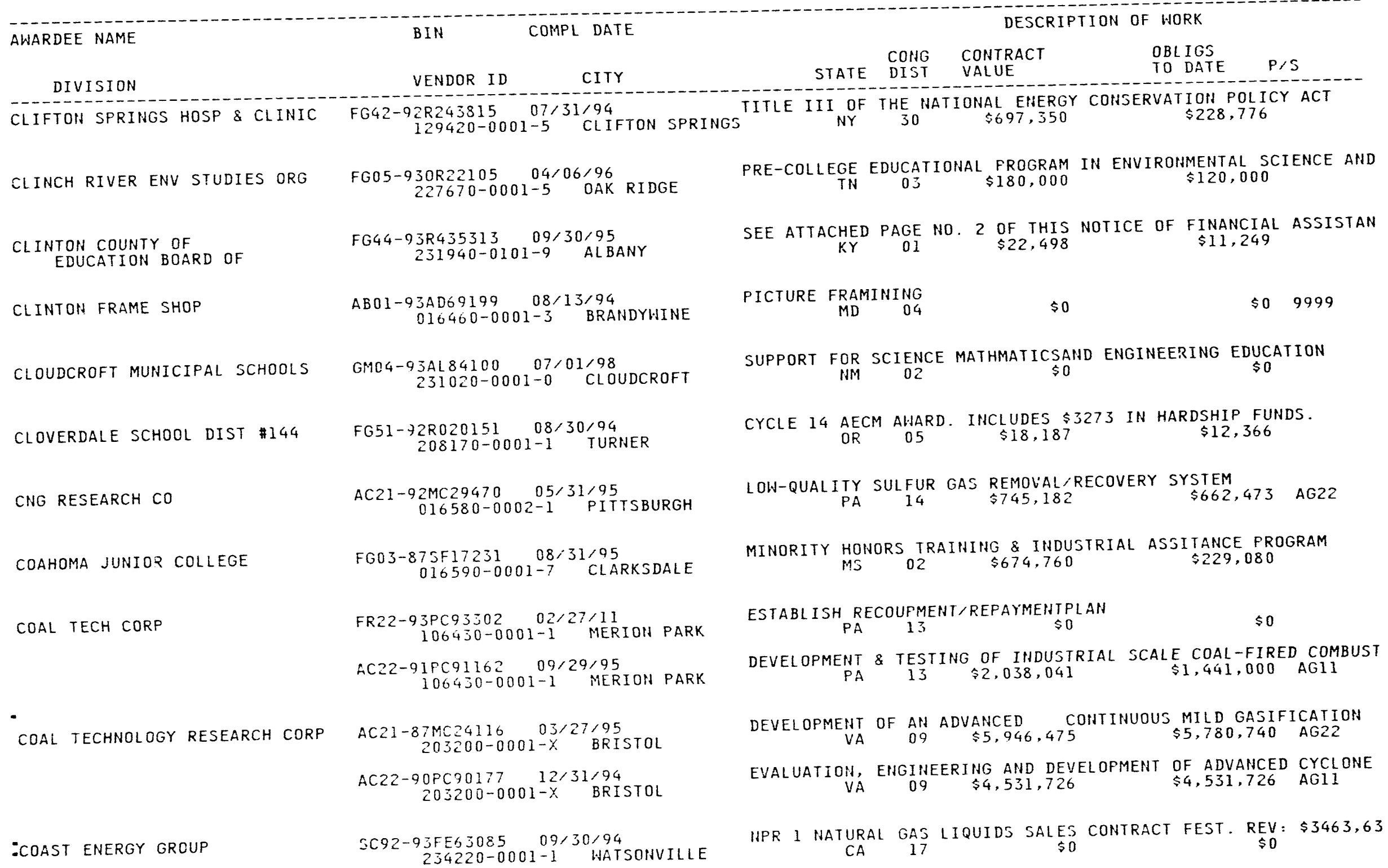




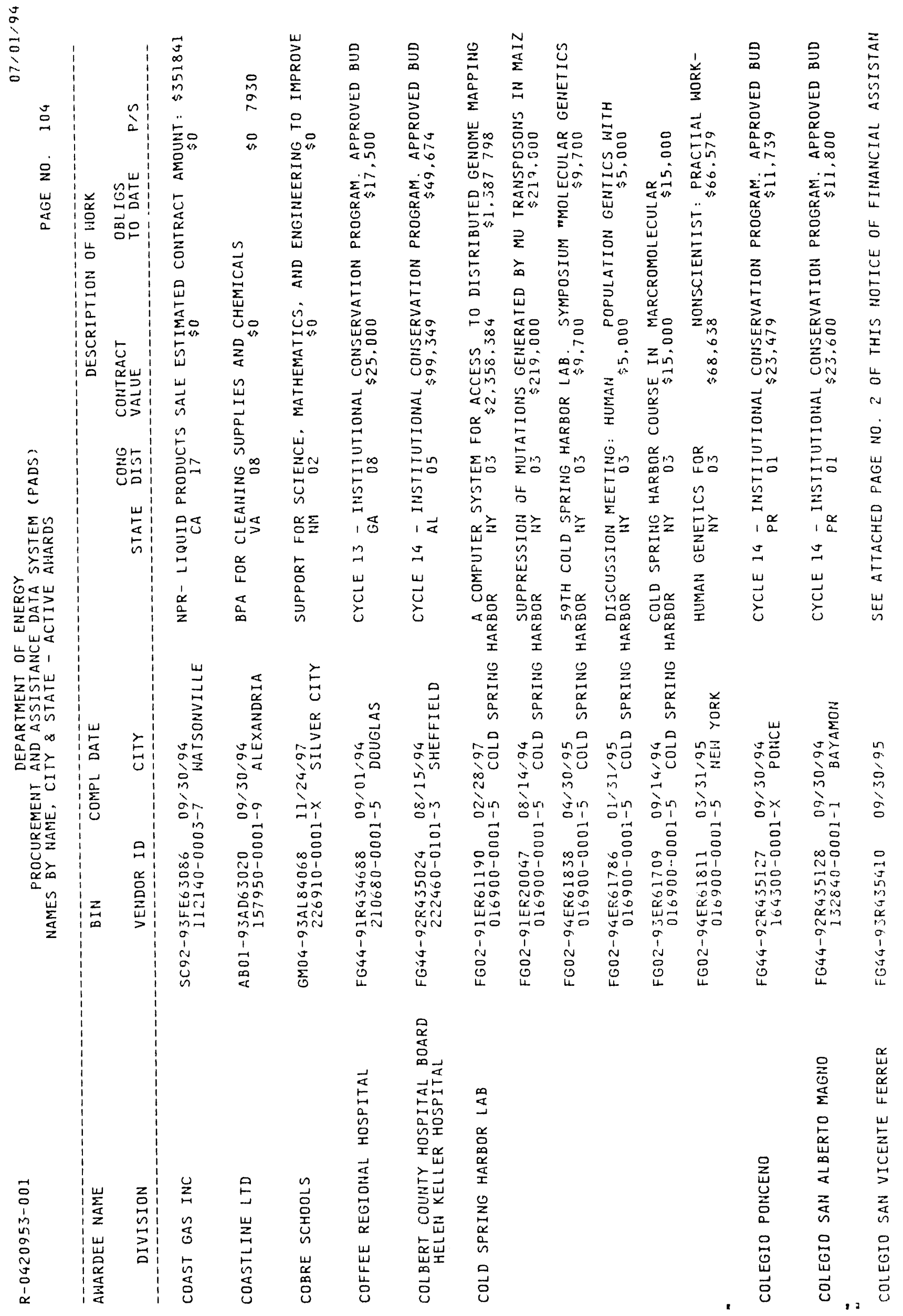




AHARDEE NAME

BIN

COMPL DATE

VENDOR ID CITY

$179240-0002-X \quad$ BAYAMON

COLEGIO SANTO DOMINGO

FG44-92R435129 09/30/94 $2 R 435129009 / 30 / 94$
$100200-0001-4 \quad$ BAYAMON

COLEMAN RESEARCH CORP

EARTH SCIENCES

$$
\begin{array}{r}
\text { AC21-92MC28135 08/23/94 } \\
224170-0101-2 \text { DENVER }
\end{array}
$$

AC21-92MC29106 08/28/94 224170-0002-4 LAUREL

ACO1-93EW10279 01/18/98 $224170-0003-2$ FAIRFAX

DIGITAL SIGNAL

COLLABORATIVE RESEARCH INC RESEARCH \& DEVELOPMENT

AC21-93MC30176 07/12/94 224170-0205-1 SPRINGFIELD

FG02-9,2ER61399 03/14/95 217800-0101-0 BEDFORD

FG02-93ER81608 07/17/94 $217800-0002-2$ WALTHAM

COLORADO CENTER FOR ENVIR MGMT FG34-91RF00117 07/07/96 TECH \& REGULATORY INTEGRATIOH

COLORADO LOCAL INITIATIVE GRP FG34-92RF00475 08/06/96 $2 R F 00475$
$223440-0001-1 \quad$ BROOMFIELD

COLORADO PUBLIC SERVICE CO

SC07-791D01370 12/31/97 017170-0002-9 PLATTEVILLE

COLORADO SCHOOL OF MINES

PHYSICS DEPT

: PHYSICS DEPT

\author{
FG01-92FE62672 09/29/97 \\ FG02-86ER45253 02/28/95 \\ $017190-1101-X$ GOLDEN \\ FG02-88ER53276 08/14/94 \\ FG02-89ER14079 01/31/95
}

DESCRIPTION OF WORK

$\begin{array}{ll}\text { CONG } & \text { COHTRACT } \\ \text { STATE DIST VALUE }\end{array}$

$\$ 22,248$

TO DATE

$\$ 11,124$
CYCLE 14 - INSTITUTIONAL CONSERVATION PROGRAM. APPROVED BUD PR
01

FRACTURE DETECTION, MAFPING, AHD AHALYSIS OF NATURALLY $\begin{array}{lll}\text { CO } 01 & \$ 883.909 & \$ 883.909 \\ & & \end{array}$

$\begin{array}{lllll}\text { EM-GEOPHYSICAL DATA FUSION FORSUBSURFACE IMAGIHG } & \\ \text { MID } & \text { N5 } & \$ 1,096,478 & \$ 1,078,360 & \text { AH93 }\end{array}$

TECHNICAL AND PROGRAM SUPPORT SERVICES FOR THE OFFICE OF VA 10 10 $\$ 47,996,145 \quad \$ 34,737,011$ R425

EM RESEARCH ENTITLED "3 $34 \begin{array}{ll}\text { DIMENSIONAL INTEGRATED } \\ \text { VA }\end{array}$

CHIMERA-FREE, HIGH COPY NUMBERYAC LIBRAIRES ADN EFFICIENT MA $\begin{array}{lll}08 & \$ 912.790 & \$ 912.790\end{array}$

$\begin{array}{ccc}\text { NON-RADIOACTIVE SELECTION } & \text { SYSTEM BASED OH ENZYME } \\ 07 & \$ 75,000 & \$ 75.000\end{array}$

DEV \& IMPLEMENT PROGRAMS TO BRING WASTE PREVENTION,REDUCII CO $05 \quad \$ 6,780,000 \quad \$ 6,780,000$

IMPACT ASSISTANCE FOR RF WORK-ERS. VENDORS \& LOCAL BUSINESS CO 05 \$ $\$ 1,747,489 \quad \$ 1,747.489$

RECEIVE \& STORE SPENT HTGR $\underset{04}{\text { FUEL ELEMENTS }}$

FIELD INSTITUTE PROGRAM ON ENERGY AHD MINERALS OPPORTUNITIE: CO 05 \$1,214,592 \$150,000 FUNDAMENTAL OPTICAL STUDIES OFCOMPETITIVE ADSORPTION AND CO 05 \$954,631 395,631 FUSION GAMMA RAY DIAGHOSIICS OF ALFHA PARTICLE PRODUCTION $C O \quad 05 \quad \$ 203.952$ \$203.952 COMPUTATIOHAL METHODS FOR IMPROVING THE RESOLUTION OF 
AWARDEE NAME

BIN COMPL DATE

DESCRIPTION OF HORK

DIVISION

VENDOR ID CITY STATE CONG CONTRACT

$017190-1601-1$ GOLDEN

C0 $05 \quad \$ 683,846$

OBLIGS

GEOPHYSICS DEPT

CHEMICAL ENGINEERING

FG02-92ER14294 07/31/95
$017190-1301-2$ GOLDEN FG03-93ER40789 04/30/96 017190-0001-8 GOLDEN

FG03-93ER14363 07/31/95 017190-0001-8 GOLDEN

CHEMICAL ENG \& PETROLEUM

FG06-94RL12760 02/06/95 017190-1701-8 GOLDEN

FG09-94SR18381 10/17/94 017190-0001-8 GOLDEN

CHEMICAL ENGINEERING FG34-94RF0078I 02/06/95
$017190-1301-2$ GOLDEN

FG21-92MC29248 05/18/96 017190-0001-8 GOLDEN

PETROLEUM ENGINEERING

AC22-93BC14891 09/28/96 017190-1901-0 GOLDEN

COLORADO STATE OF

HEALTH DEPT

FC04-82AL16257 09/30/94 17200-0101-4 DEHVER

HEALTH DEPT

FC34-79AL1120900/30/94 017200-0101-4 DEIJVER

HEALTH DEPT

FG34-92RF0034900/30/94 017200-0101-4 DENVER

FG34-93RF00672 09/30/94 017200-0101-4 DENVER

HEALTH DEPT

FG48-92R801773 08/31/94

ENERGY CONSERVATION UFFICE OF 017200-0801-9 DENVER FG48-90R801741 09/30/94 $017200-0001-8 / 30 / 94$ DEN

$=\quad$ LOCAL AFFAIRS DEPT FG48-94R801800 03/31/95
$017200-0401-3$ DEIIVER rG48-93R810499 09/30/95 $\begin{array}{rrrr}\text { COMPOSITION } & \text { DEPENDENCE } & \text { OF } & \text { FLUID THERMOPHYSICAL } \\ \text { CO } & 05 & \$ 347,554 & \$ 225,832\end{array}$ MEASUREMENTS OF NUCLEAR REACTIONS AT VERY LOW ENERGIES TO CO

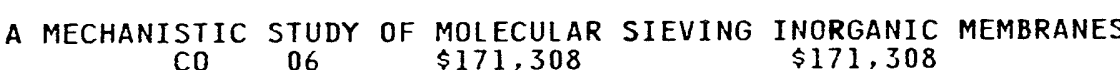
USED ENERGY RELATED LABORATORYEQUIPMENT GRANT USED ENERGY RELATED LABORATORYEQUIPMENT
CO
05 $\underset{\$ 0}{ }$

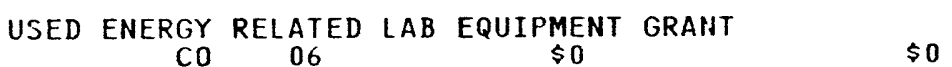
$\begin{array}{ccc}\text { CONSORTIUM FOR HYDRATE } & \text { RESEARCH ON THE USE OF } & \text { RO } \\ \text { CO } & \$ 150,000 & \$ 120,000\end{array}$ INTERDISCIPLINARY STUDY OF RESERVOIR COMPARTMENTS $\begin{array}{lllll}\text { CO } 05 & \$ 944,364 & \$ 753,266 & \text { AG11 }\end{array}$

COLORADO UMTRA SITES

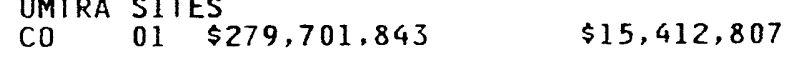

ENVIRONMENTAL MONITORING PROGRAM

$\$ 9,860,836$

RFO HEALTH INITIATIVES $\underset{\text { CO }}{01} \underset{\$ 6,815,653}{\text { DUTLINED IN AGREEMENT IN }}$

DG/FY93 FUHDING FOR STATE AND LOCAL TRAINING IN EMERGENCY

$\begin{array}{rccc}\text { CO } & 01 & \$ 408,269 & \$ 408,269 \\ \text { AWARD CYCLE XIV ICP } & \text { CCORDINATING AGENCY GRANT } \\ \text { CO } & 01 & \$ 40,551 & \$ 21,911\end{array}$ ICP PHASE II STATE ADMIN OBLIGATION

$\$ 90,750$

ALISRD FY94 WEATHERIZATION GRANT
CO OI \$5, 464, 158
$\$ 5,464,158$

NICE 3 - TECHHOLOGY TRANSFER OF RADIATION-CURED COATING FOR 


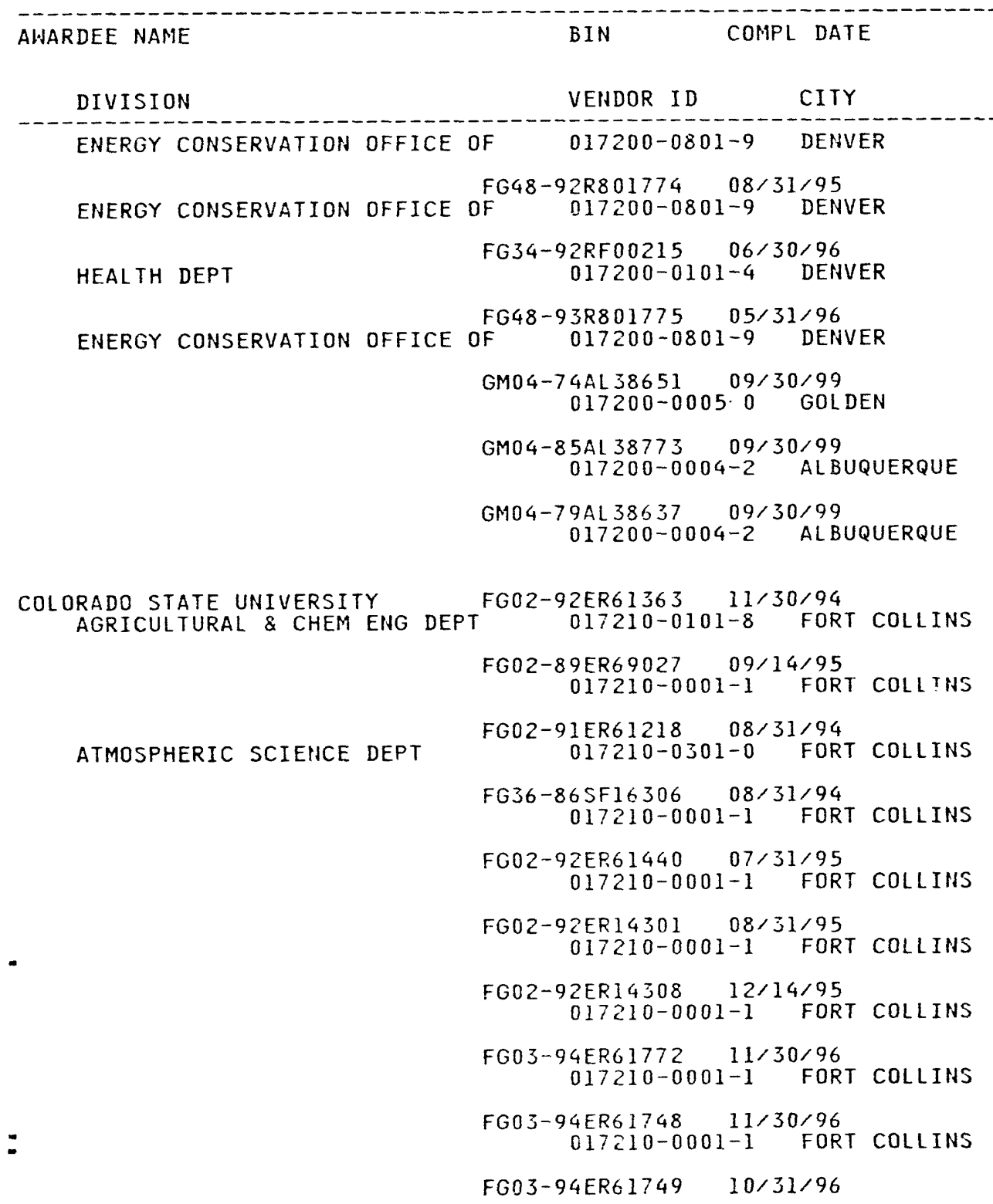

AWARD CYCLE XIV ICP COORDINATING AGENCY GRANT
CO

OVERSIGHT ASSESSMENT \& REMED. BY DOE a RFO-PER IA BTWN CO 01 \& $\$ 826,289$ \$ $\$ 26,289$

AWARD CYCLE XV ICP COORDINATING AGENCY GRANT

$$
\begin{array}{rrr}
C O & \text { ICP } & \text { COORDINATING AGENCY GRANT } \\
\$ 1,096,132 & \$ 567,535
\end{array}
$$

TO ACQUIRE A BUFFER ZONE SURROUNDING THE PLANT FOR SAFETY

$$
\begin{array}{llll}
C O & 05 & \$ 0 & \$ 0
\end{array}
$$

$\underset{\text { IMPLEMENTATION OF THE URANIUM MILL TAILINGS REMEDIAL }}{\text { OM }}$ OI

DOE PROVIDES ASSISTANCE TO THESTATE OF COLORADO $\underset{\$ 0}{\text { IN }} \underset{\$ 0}{\text { IN }}$ ITS

THE USE OF ARM DATA TO TEST ANIMPROVED PARAMETERIZATION OF

$$
\text { NM } 01 \text { \$0 } \$ 0
$$

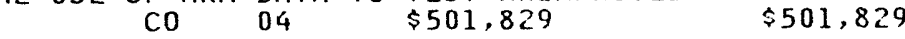

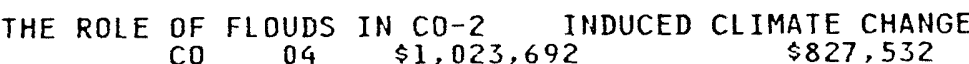

DEVELOPMENT OF AN ADVANCED FINITE-DIFFERENCE ATMOSPHERIC GEN

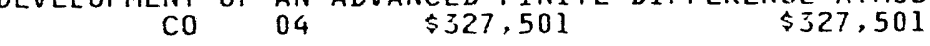

TESTING, EVALUATION AND OPTIMIZATION OF SOLAR HEATING AND CO CO $04 \$ 3,090,583 \quad \$ 2,806,669$

PARAMETERIZATIONS IN HIGH RESOLUTIN ISOPYCNAL HIND-

$$
\text { CO } 04 \quad \$ 173,636 \quad \$ 112,984
$$

PHOTOINDUCED CHARGE SEPARATIONIN LINKED DONOR-CHROMOPHORE-

$$
\text { CO } 04 \quad \$ 344,050 \quad \$ 234,050
$$

IN SITU SCANNING MICROSCOPY IMAGING OF DYE SENSITIZATION CO D4 $\$ 325,000 \quad \$ 221,430$

ON THE FUMDAMENTAL ROLE OF DAYVERSUS NIGHT RADIATION DIFFER

$$
\text { CO } 04 \quad \$ 430,000 \quad \$ 116,400
$$

A MODELIHG AND REMOTE SENSING STUDY OF THE RADIATIVE HEATING CO $04 \quad \$ 724,201 \quad \$ 200,379$

EXPLICIT SIMULATION AND PARAMETERIZATION OF MESOSCALE CONVEC 


AWARDEE NAME

DESCRIPTION OF WORK

DIVISION

VENDOR ID

017210-0001-1 FORT COLLINS

CHEMISTRY DEPT

CHEMISTRY \& CHEMICAL ENG
FG03-94ERI4405 10/31/96 017210-0401-7 FORT COLLINS

FG03-93ER14341 03/31/96 017210-2301-1 FORT COLLINS

FG03-93ER40788 04/14/96 017210-0001-1 FORT COLLINS

FG03-93ER61601 03/14/96 017210-0001-1 FORT COLLINS

FG03-93ER14369 11/30/95 017210-0001-1 FORT COLLINS

FG22-93PC93206 08/31/96 017210-0001-1 FORT COLLINS

FG48-91R801748 07/31/94 $017210-0001-1$ FORT COLLINS

COLORADO UNIVERSITY OF

$$
\begin{gathered}
\text { FG02-85ER53207 10/31/94 } \\
017220-0001-5 \quad \text { BOULDER }
\end{gathered}
$$
$F G 02-85 E R 1345107 / 31 / 95$
$017220-0401-0 \quad$ BOUL DER FG02-86ER40269 02/14/95 $017220-1201-3$ BOUL DER

PHYSICS DEPT

FG02-86ER40302 04/30/95

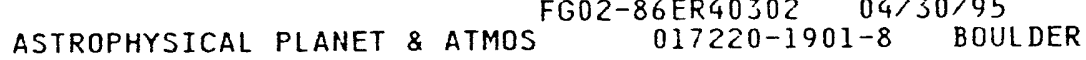
FG02-87ER53244 11/23/94

ASTROPHYSICAL PLANET \& ATMOS FG02-87ER53244 $017220-1901-8$ BOULDER FG02-90ER61019 07/14/94 $017220-0001-5$ BOULDER

GEOLOGICAL SCIEHCES DEPT

FCO2-92ER14233 $12 / 31 / 94$

$017220-2001-6$ BOULDER

FGO2-9IERG06:2 10/31/94

$017220-0001-5$ BOULDER

FGOJ-S4ER618?5 03/31/95 TO DATE

STATE DI
$\$ 638,063$ $\$ 194,000$

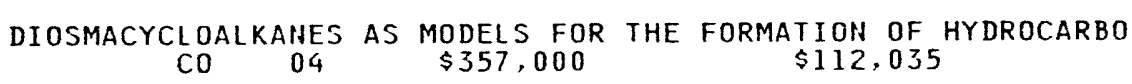
$\begin{array}{ccc}\text { NEW AWARD FOR PROJECT ENTITLED"STUDY OF IMPROVED METHODS FO } \\ \text { CO } 04 & \$ 313,000 & \$ 190.500\end{array}$

NEW GRANT TO FUND RESEARCH IN COLLIDER PHYSICS.

CO 04 \$1, $130,000 \quad \$ 682,400$

EFFECTS OF VEGETATION ON RADONTRANSPORT PROCESSES IN SOIL

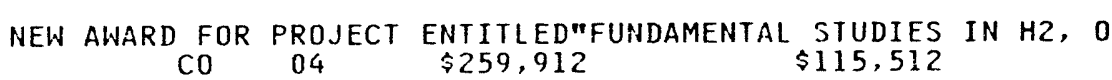

TEMPERATURE EFFECTS ON CHEMICAL STRUCTURE AND MOIION IN COAL CO 045 ON $\$ 199,935$ STRUCTURE AND MOTION

AHARD CYCLE XIII GRANT

$\$ 82,830$

$\$ 41,415$

DETRAPPING/RETRAPPING ORBITS IN STELLARATORS

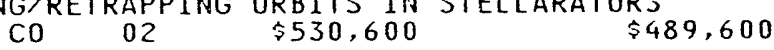

NEW AWARD (GRANT) 11 MONTHS

$\$ 613,713$

PION-NUCLEUS INTERACTIONS AND NUCLEON TRANSFER REACTIONS

PARTICLE DYNAMICS IN A WAVE WITH VARIABLE AMPLITUDE

CO 02 \$1,104,000 $\$ 1,094,000$

REVERSED FIELD PINCH EXPERIMENTS

$\$ 715,919$

THERMOBALINE CIRCULATIONS AND GLOBAL CLIMATE CHANGE

CHRYSTAL CHEMISTRY OF $\begin{gathered}\text { HYDROXYL \& WATER IN SILICATE } \\ \text { CO } 02\end{gathered}$

DETECTOR RESEARCH \& DEVELOFMENT ASSOCIFTED WITH SDC DETECTOR SYMPOSIUM ON HUMAN GENOME PROJECT 


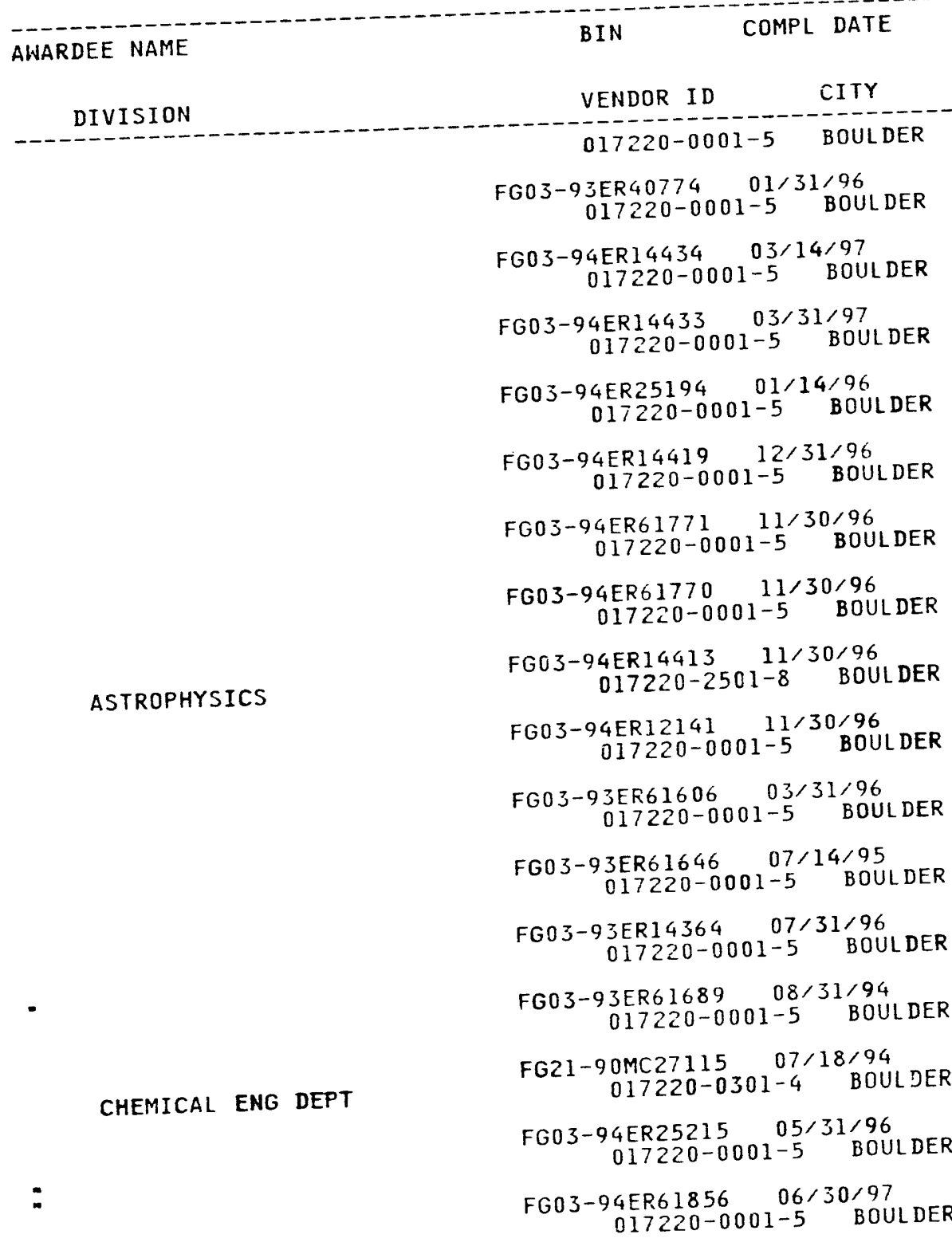

\section{DESCRIPTION OF WORK \\ DESCRIPTION OF WORK}

STATE DIST VONTRE

TO DATE P/S $\$ 3,500$
NEW GRANT - THEORETICAL NUCLEAR PHYSICS

$\$ 375,000$

MECHANISTIC ASPECTS OF PHOTOCONVERSION AT $\$$ SEMICOND

$$
\begin{array}{ccc}
\text { CO } & 02 & \$ 310,000 \\
\text { TIME-RESOLVED FTIR EMISSION STUDIES OF LASER PHOTOFR } & \$ 315,000 & \$ 103,000
\end{array}
$$

ANALYSIS \& DEVELOPMENT OF INTERMEDIATE MODELS OF ATN

$$
\text { CO } 02 \text { DEVELOPMENT OF INTERMEDIATE MODEL } \$ 56,74.5
$$

SEISMIC ABSORPTION AND MODULUSMEASUREMENTS IN POROUS ROCKS

SEISMIC ABSORPTION AND $\$ 408,107 \quad \$ 145,369$

TONDS THE UNDERSTANDING AND PARAMETERIZATION OF HIGH LATIT THE UNDERSTANDING AND PARAMETERIZATION $\$ 194,678$
02537,333

IMPACT OF AIR-SEA INTERACTION ON TROPICAL CLOUD LIFE-CYCLES IMPACT OF AIR-SEA INTERACTION

PHYSICS OF CORRELATED SYSTEMS

TO-DIMENSIONAL SYNTHESIS: UL TRATHIN POROUS MEMBRANES

TWO-DIMENSIONAL SYNTHESIS: $\$ 900,000 \quad \$ 291,000$

NEW APPROACHES TO RECOGNIZING FUNCTIONAL DOMAINS IN BIOLOGIC NEW APPROACHES TO RECOGNIZING FUNCTIONAL DOMAINS $\$ 202,399$ THERMOHALINE CIRCULATIONS AND GLOBAL CLIMATE CHANGE CO $02 \quad \$ 165,000 \quad \$ 165,000$

"LASER PHOTOELECTRON SPECTROSCOPY OF IONS" P.I.

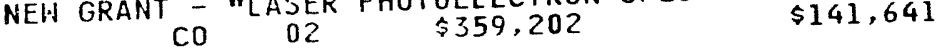

GLACIER CALVING, DYNAMICS, ANDSEA LEVEL \$214,107

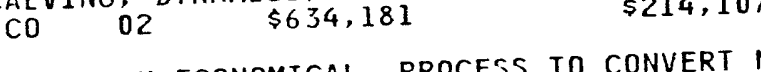

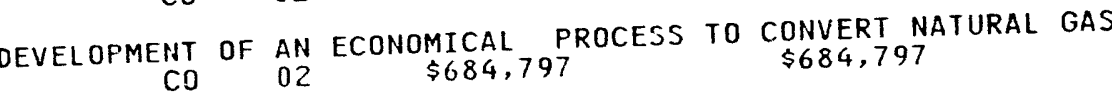
$\begin{array}{ccc}\text { NUMERICAL NIETHODS FOR SOME SIRUCTURED MATRIX ALGEBRA PROBLEM } \\ \text { CO } 02 & \$ 97,000 & \$ 97,000\end{array}$ $\$ 97,000$

CONFIRMATORY ANALYSIS OF SAGE-1 AND -II DATA IN RESPONSE TO CD $02 \quad \$ 649,832$
$\$ 216,623$ 


\begin{tabular}{|c|c|c|}
\hline AWARDEE NAME & COMPL DATE & DESCRIPTION OF HORK \\
\hline DIVISION & VENDOR ID & $\begin{array}{l}\text { CONTRACT } \\
\text { VALUE }\end{array}$ \\
\hline \multirow{4}{*}{ COMPUTATIONAL MATHEMATICS } & $\begin{array}{rl}F G 03-94 E R 61857 & 06 / 30 / 96 \\
017220-0001-5 \quad B O U L D E R\end{array}$ & 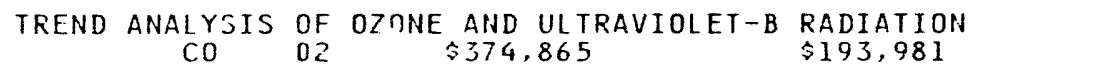 \\
\hline & $\begin{array}{c}\text { FG03-94ER25217 } 06 / 30 / 95 \\
017220-0001-5 \text { BOUL DER }\end{array}$ & $\begin{array}{c}\text { FAST ALGORITHMS FOR TRANSPORT MODELS } \\
\text { CO } 02\end{array}$ \\
\hline & $\begin{array}{c}\text { FG03-93ER25165 } 05 / 31 / 96 \\
017220-2402-\times \text { DENVER }\end{array}$ & 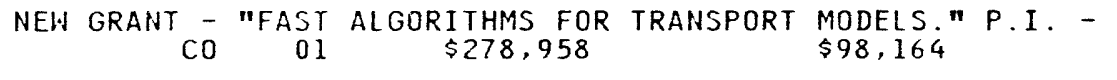 \\
\hline & $\begin{array}{l}\text { GM04-81AL38643 } 09 / 30 / 99 \\
017220-0004-X \quad \text { ALBUQUERQUE }\end{array}$ & 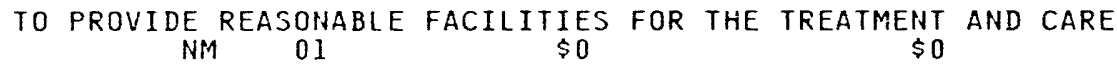 \\
\hline COLORADO-UTE ELEC ASSOC INC & $\begin{array}{c}\text { FC21-89MC25137 } 10 / 18 / 11 \\
017250-0002-4 \quad \text { NUCLA }\end{array}$ & 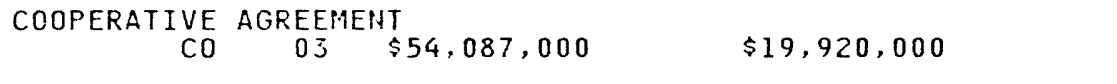 \\
\hline \multirow[t]{2}{*}{$\begin{array}{l}\text { COLUMBIA BASIN CMTY COLLEGE } \\
\text { COLUMBIA BASIN COLLEGE FDH }\end{array}$} & $\begin{array}{c}F G 06-94 R L 12731 \quad 12 / 02 / 94 \\
162630-0101-1 \quad \text { PASCO }\end{array}$ & $\begin{array}{cccc}\text { ENVIRONMENTAL SCIENCE SCHOLARSHIP } & \text { SNDOWMENT } \\
\text { WA } & 04 & \$ 40,000 & \$ 40,000\end{array}$ \\
\hline & $\begin{array}{c}F G 06-93 R L 12691 \quad 09 / 30 / 94 \\
162630-0001-5 \text { PASCO }\end{array}$ & $\begin{array}{cccc}\text { SUPPORT FOR HAZARDOUS MATERIALS MANAGEMENT CURRICULUM } \\
\text { WA } & 04 & \$ 129,000 & \$ 129,000\end{array}$ \\
\hline $\begin{array}{l}\text { COLUMBIA COUHTY HOSP DIST \#I } \\
\text { MEDICAL LOGISTICS }\end{array}$ & $\begin{array}{r}\text { FG51-92R020117 } 10 / 31 / 94 \\
196200-0101-X \quad \text { DAYTON }\end{array}$ & $\begin{array}{c}\text { CYCLE } 14 \underset{\text { WA }}{\text { ECM }} \text { AHARD. } 05 \\
\text { WA }\end{array}$ \\
\hline COLLMBIA GAS OF PENNSYIVANIA & $\begin{array}{rl}\text { AC22-89PC } 89476 & 09 / 18 / 94 \\
017320-0002-6 & \text { PITTSBURGH }\end{array}$ & $\begin{array}{rcccc}\text { NATURAL GAS } & \text { SERVICE TO } & \text { BUILDINGS } & 900,901,902,903,920,921, \& \\
\text { PA } & 18 & \$ 297,000 & \$ 297,000 & 5111\end{array}$ \\
\hline COLUMBIA GRAPHICS CORP & $\begin{array}{l}\text { ABO1-94AD25696 } 06 / 30 / 95 \\
017360-0001-2 \quad B E L T S V I L L E\end{array}$ & $\begin{array}{c}\text { BPA FOR GRAPHICS ARTS } \\
\text { MD } 05\end{array}$ \\
\hline \multirow[t]{2}{*}{ COLUMBIA SERVICES GROUP INC } & $\begin{array}{c}\text { AC01-92DP } 30458 \quad 08 / 23 / 95 \\
202710-0003-1 \quad \text { GERMANTOHN }\end{array}$ & 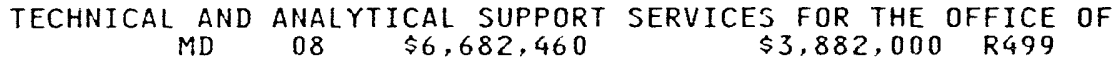 \\
\hline & $\begin{array}{r}\text { ACO1-92AD00071 } 06 / 23 / 97 \\
202710-0002-3 \quad \text { ARLINGTON }\end{array}$ & $\begin{array}{ccccc}\text { SUPPORT SERVICES TO CONDUCT A SECRET DOCUMENT INVENTORY } \\
\text { VA } 10 & \$ 837,982 & \$ 278,751 & \text { R612 }\end{array}$ \\
\hline \multirow[t]{2}{*}{$\begin{array}{l}\text { COLUMBIA UNIVERSITY } \\
\text { LAMONT DOHERTY GEOL OBSV }\end{array}$} & $\begin{array}{c}\text { FC22-93BC14961 } 10 / 30 / 95 \\
017420-0305-2 \text { NEW ORLEANS }\end{array}$ & $\begin{array}{l}\text { DYNAMIC ENHANCED RECOVERY TECHNIQUES } \\
\text { LA } 03 \text { \$ } \$ 19,505,410\end{array}$ \\
\hline & $\begin{array}{c}\text { FG02-85ER60372 } 08 / 14 / 94 \\
017420-0301-X \text { NEH YORK }\end{array}$ & 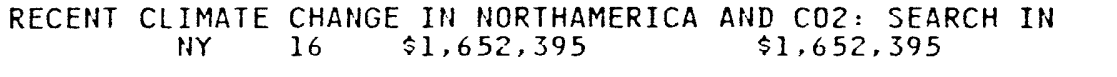 \\
\hline
\end{tabular}




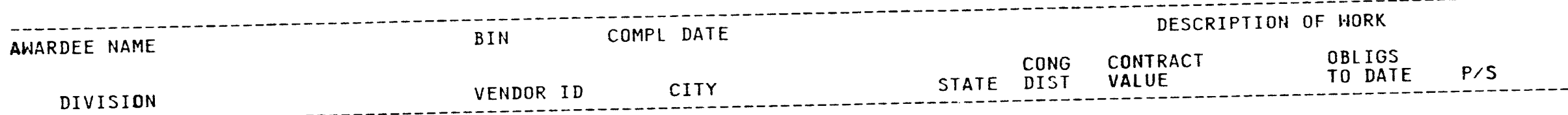

APPLIED PHYS \& NUCL ENG DEPT FG02-89ER53297 09/24/94 $017420-0101-7$ NEW YORK FG02-91ER14225 $07 / 14 / 94$ 017420-0001-0 NEH YORK FG02-91EF61247 09/14/94 $017420-0001-0$ NEH YORK FG02-92ER61397 08/31/94 017420-0001-0 NEW YORK FG02-92ER61477 09/14/94 017420-0001-0 NEH YORK FG05-91ER79035 09/29/94 $017420-0001-0$ HEH YORK FG42-92R243816 07/31/94 $017420-1901-3$ NHEW YORK

TRUSTEES

CHEMISTRY DEPT

$$
\text { FG02-92ER40699 11/30/94 }
$$
$017420-0001-0$ NEW YORK FG02-87ER13653 12/31/94 $017420-0201-3$ NEH YORK

FG02-91ER14226 12/31/94 017420-0001-0 NEW YORK

FG02-92ER54168 10/31/94 017420-0001-0 NEH YORK APPLIED PHYS \& NUCL ENG DEPT FG02-86ER53222 $02 / 23 / 95$ YORK

CHEMISTRY DEPT

FG02-86ER13492 02/28/95 $017420-0201-3$ NEH YORK FG02-91ER61202 07/31/95 017420-0001-0 HEH YORK FG02-92ER61451 05/31/95 017420-0001-0 NEW YORK AC22-92BC14884 09/29/95 : SCHOOL OF MINES
HIGH BETA AND CONFINEMEN

$$
\text { AND CONFINEMENT } 16 \text { S1,565,525 } \$ 1,565,525
$$

SINGLE COLISION STUDIES OF HOT ATOM ENERGY TRANSFER \& CHEMI

$$
\text { NY } 16 \text { STUDIES } \$ 302,000 \text { \$302,000 }
$$

THE EFFECTS OF CHANGING LAHD USE ON ORGANIC CARBON \& NITROGE

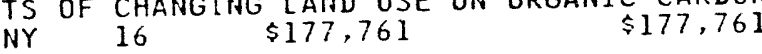

MEASURMENTS OF CARBON DIOXIDE IN THE SOUTHERN OCEAN ALONG

$$
\text { NY OF } 17 \text { CARBON DIOXIDE TN THE SOUTHERN } \$ 279,836
$$

THE IMPACT OF RECENT MODEL IMPROVEMENTS ON GISS GCM

PURCHASE OF A VERY HIGH RESOLUTION PULSED LASER SPECTROSCOPY

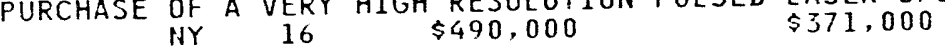

TITLE III DF THE NATIONAL ENERGY CONSERVATION POLICY ACT

$$
\text { ITLE III NF THE NATIONAL } \$ 95,241 \text { I6 }
$$

HIGH ENERGY PHYSICS RESEARCH

NY 16 \$3,963,000 \$3,963,000

UTILIZATION OF MAGNETIC EFFECTS AS A MEANS OF ISOTOPE

$$
\text { NY } 16 \quad \$ 590.201 \quad \$ 590,201
$$

CHARGE GEIERATION \& SEPARATIONAT LIQUID INTERFACES

GENERATION \& SEPARATIONAT LIQUID INTERFACES
NY
16

HIGH BETA PLASMA THEROY $\$ 199,974 \quad \$ 174,000$

HIGH BETA TOKANTAK RESEARCH
HY 16
$\$ 7,780,848$

ENERGY PARTITIONING IN ELEMENTARY GAS PHASE PROCESSES HY $16 \quad \$ 786,780$

STEADY-STATE \& TRANSIENT MODELLING OF TRACER \& NUTRIENT DIST NY 16 \$ 16 \$511,789 $\$ 375,243$ CHROMATOGRAPHIC SYSTEM FOR
NY

SURFACTANT LOSS CONTROL IN CHEMICAL FLOODING - SPECTROSCOPIC NY $16 \quad \$ 602,232 \quad \$ 602,232$ AG11 
DIVISION

VENDOR ID CITY 3ER61539---12/31/95-- Y N

FG02-93ER40764 12/14/95 017420-0001-0 NEW YORK

PHYSICS DEPT

FG02-86ER40281 11/14/95 017420-0701-5 NEW YORK

MICROELECTRONICS SCIENCE LAB FG02-90ERI4104 12/14/95 $017420-2001-1$ NEW YORK FG02-91ER40669 10/14/95 $017420-0001-0$ NEW YORK

FG02-84ER13287 01/31/96 $017420-0301-X \quad$ NEW YORK

LAMONT DOHERTY GEOL OBSER

FG02-87ER53257 05/31/96 $017420-1501-8$ NEW YORK

ELECTRICAL ENGIHEERING DEPT

FGO2-90ER61022 04/30/96 HEALTH SCIEHCES GENETICS \& DEV 017420-1401-1 NEW YORK FG02-93ER14339 03/31/96 017420-0001-0 NEW YORK

$F G 02-93 E R 61594 \quad 04 / 30 / 96$ 017420-0001-0 NEW YORK FG02-93ER61719 09/14/96 017420-0001-0 NEW YORK

FG02-93ER61716 $08 / 31 / 96$ 017420-0001-0 IHEW YORK

FG02-94ER40841 12/31/96 017420-0001-0 HEH YORK

FG02-88ER45341 12/31/96 $017420-1601-4$ NEN YORK

SCHOOL OF MINES MEALTH SCIENCES GENETICS \& DEV OE2-88ER60631111/30/96 $017420-1401-1$ HEH YORK FG02-94ER40819 12/31/96 017420-0001-0 HEW YDRK
FG02-90ER14162 01/31/97 $017420-0201-3$ HEW YORK
CONG CNTRACT

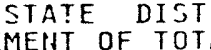

VALUE

$\$ 722,130$

TO DATE P/S $\$ 633,868$
PARTIAL PRES3

NY 17 THEORY OF ULTRA-DENSE MATTER AHD THE DYHAMICS OF HIGH
NY 16 RELATIVISTIC HEAVY IOH PHYSICSPROGRAM
NY
16

$\$ 4,988,000$

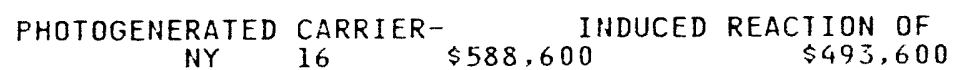
INUERSE FREE-ELECTRON LASER BFAT-WAVE ACCELERATOR RESEARCH.
NY 16 ENERGETICS OF SILICATE MELTS FROM THERMAL DIFFUSION STUDIES HY 30 \$1, 092,142 $\$ 976,110$

IDN TEMPERATURE GRADIENT NY INSTABILITY AND ANOMALOUS
NY 16 \$1,758,000 $\$ 1,549,000$

NANODOSIMETRY AND NANODOSIMET-RIC BASED MODELS OF RADIATION

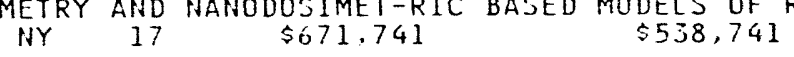

MODEL STUDIES IN HYDROCARBON OXIDATION
HY
$\$ 289,287$ GEOCHEMICAL CONSTRAINTS ON OCEAN GENERAL CIRCULATION
NY

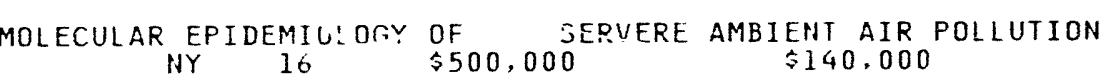
$\begin{array}{lllll}\text { SOUTHERN OCEAN CONTROL ON } & \text { CURRENT ICE SHELF EVOLUTION } \\ & 08 & \$ 364.873 & \$ 117.553\end{array}$ $\begin{array}{ccccc}\text { EXPERIMENTAL STUDY OF E+E- ANNIHILATION AT THE HIGHEST } & \text { AHY } \\ \text { INY } & 15 & \$ 256,536 & \$ 81,375\end{array}$ PROTONS AND LATTICE DEFECTS INPEROVSKITE-RELATED OXIDES
NY RADIATION PHYSICS AND BIOLOGY/RADIOLOGICAL RESEARCH NY 16 \$6,167,014 \$4,964,764

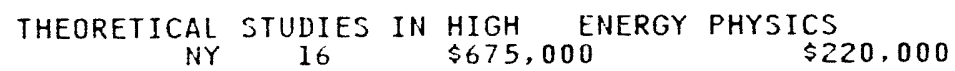

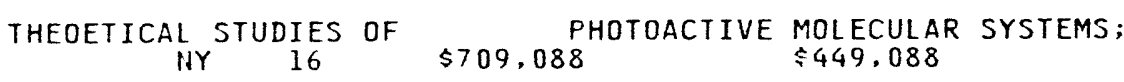

CHEMISTRY DEPT 


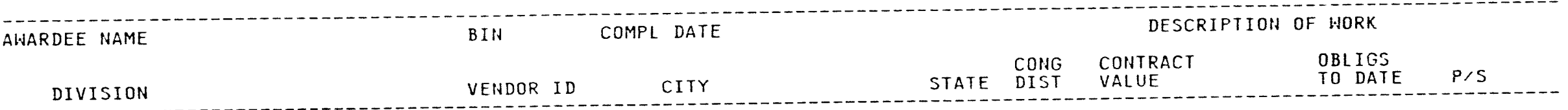

HEALTH SCIENCES GENETICS \& DEV O O17420-1401-1 NEW YORK FG02-94ER61758 11/30/95 017420-0002-9 PALISADES

COLUMBUS COLLEGE

FG44-91R434690 08/01/94 017470-0001-9 COLUMBUS FG44-93R435347 09/30/95 017470-0001-9 COLUMBUS FG $44-93 R 435346 \quad 09 / 30 / 94$ $017470-0001-9$ COLUMBUS

COMBUSTION ENGINEERING INC

FC22-90PC89655 03/27/95 $017520-0007-2$ BIRMINGHAM AC03-93SF19682 09/30/94 $017520-0005-6$ HIHDSOR AC03-895F17886 12/31/94 $017520-0005-6$ HIIIDSOR AC03-915F19090 09/30/94 $017520-0005-6$ HINDSOR AC22-89PC8 $8654 \quad 12 / 31 / 95$ $017520-0005-6$ HINDSOR AC22-91PC91160 12/31/94 $017520-0005-6$ WINDSOR AC22-92PC92159 08/31/94 $017520-0005-6 \quad$ WINDSOR

FC21-91MC26308 05/20/01 $017520-0006-4$ SPRINGFIELD

COMBUSTION POWER CO INC AC21-90MC27423 10/06/95 017540-0001-0 MENLO PARK
CHROMOSOME-SPECIFIC DNA'S/STS

liY $17 \quad \$ 2,270,095 \quad \$ 1,484,088$

SEASONAL STUDY OF CARBON DIOXIDE IN THE SOUTHERN IHY 16 \$3 $\$ 30,000 \quad \$ 160.050$

CYCLE 13 - INSTITUTIONAL CONSERVATION PROGFAM. APPROVED BUD GA $03 \quad \$ 409.544 \quad \$ 195,998$

SEE ATTACHED PAGE NO. 2 OF THIS NOTICE OF FINANCIAL ASSISTAN SEE ATTACHED PAGE NO. 23273,493 N 32 \$136,665

SEE ATTACHED PAGE NO. 2 OF THIS NOTICE OF FINANCIAL ASSISTAR GA $03 \quad \$ 6.671 \quad \$ 3,335$

COMMERCIAL DEMONSTRATION OF WSA-SNOX TECHNOLOGIES INITIATIVE HORK FOR PU COHSUMPTION
31
$\$ 4,309,379$
$\$ 4,309,379 \quad A G 53$

PRELIMINARY AND FINAL DESIGNS IN SUPPORT OF THE MODULAR HIGH CT $01 \$ 56,668,400 \quad \$ 8,575,908$ AG92

DBTAIN NRC CERTIFICATION FCP THE BALANCE DF PLANT FOR CE'S $S$ $C T$ O 1 \$3j,945,323 $\$ 15,978,636$ AG51 COMBUSTIOH CHARACTEKIZATION OFBENEFICIATED COAL BAJED FUELS
CT OTI

DEVELOPMENT AND TESTING OF INDUSTRIAL-SCALE COAL-FIRED COMBU ENGINEERING DEVELOPMENT OF ADVANCED COAL-FIRED LOH-EMISSION CT DEVELPMENT $0112,200,544 \quad \$ 3,307,320$ AGI3 IGCC REPOHERING PROJECT
IL $20 \$ 270,700,000$

MOVING GRANULAR BED FILTER DEVELOPMENT FROGRAM $C A \quad 11$ \$3, $019,628 \quad \$ 1,509,387$ AG14

DISASTER RECOVERY SERVICES FORIBM 3090 SC 03 S $\$ 258,000 \quad \$ 258,000 \quad$ DJ06 

COMMERCE DEPARTMENT OF AIO2-92ER61366 $11 / 30 / 94$
NATIDNAL OCEANIC \& ATMOS ADMIN $017570-1203-6$ BOULDER NATIONAL OCEANIC \& ATMOS ADMIN AIN2-94ER61759 $017570-1203-6$ BOULDER NATIONAL OCEANIC \& ATMOS ADMIN $017570-1203-6$ BOULDER NATIONAL OCEANIC \& ATMOS ADMIN AIO3-94ER61760 $017570-1203-6$ BOULDER MATIONAL OCEANIC \& ATMOS ADMIN $017570-1203-6 \quad$ BOULDER

AI03-93ER75891 05/14/95 $017570-0003-8$ BOULDER AI03-93ER61726 09/14/94 $017570-0003-8$ BOULDER

GM04-83AL $38648 \quad 09 / 30 / 99$ NATIONAL OCEANIC \& ATMOS ADMIN 017570-1222-2 GOLDEN

AI $01-92$ CE16059 09/30/94 $0175>0-0015-1$ WASHINGTON AI $01-92$ CE16058 12/27/94

CENSUS BUREAU OF THE CENSUS BUREAU OF THE

AI01-91PR38166 09/30/96
$017570-0415-7 \quad$ HASHINGTON AI $01-91$ CE40982 11/30/94 $017570-0415-7$ WASHINGTON

AI01-94AD11109 05/31/95 017570-0015-1 WASHINGTON

AI01-94EI24841 09/30/94 017570-0015-1 HASHINGTON

AI01-94EI24163 12/31/94 $017570-0015-1$ HASHINGION $017570-0015-1$ HASHINGTON

SHIPBOARD MEASUREMENTS DF THE CLOUD-CAPPED MARINE BOUNDARY $\begin{array}{ll}\text { MEAJUREMENTS OF THE CLOUD-CAPPED MARTNE } \\ \text { CO } 02 & \$ 758,400\end{array}$ WORKING GROUP VIII FROTOCOL
CO 02
$\$ 53,175$
$\$ 53,175$

NEW INTERAGENCY AGREEMENT FOR A PROJECT ENTITLED: "EVALUATI CO $02 \quad \$ 102,500 \quad \$ 102,500$ NEW INTERAGENCY AGREEMENT FOR A PROJECT ENTITLED: "ADDITIOH CO $02 \quad \$ 400,700 \quad \$ 20,000$ NEW INTERAGENCY AGREEMENT FOR A PROJECT ENTITLED: "CONTINUE CO $02 \quad \$ 972,400 \quad \$ 200,000$

NEW INTERAGENCY AGREEMENT FOR PROJECT ENTITLED "NCCSET WUMME CD $02 \quad \$ 203.550 \quad \$ 203,550$

NEW INTERAGENCY AGREEMENT FOR A PROJECT ENTITLED: "REEVALUA $\begin{array}{lll}C O & 02 & \end{array}$ $\begin{array}{ccc}\text { PROVIDE METOROLOGICAL DATA FROM METROPOLITAN DENVER MESONET } \\ \text { CO } & 05 & \$ 0\end{array}$ SUPPORT FROM DEPARTMENT OF COMMERCE TO CARRY OUT THE PROVISI DC $01 \quad \$ 1,175,000 \quad \$ 718,000$

FINANCIAL ASSISTANCE TO DOC INSUPPORT OF SINGLE AUDIT CLEAR DC 01 \$ $\$ 833,000 \quad \$ 83,000$

FUND DOE PARTICIPATION IN CFFRREPORT DC DI \$104,000

$\$ 104,000$

AI01-93AD34030 09/30/94 017570-0015-1 HASHINGTON
PRODUCT CODE ALLOCATIONS OF EMISSONS TOHN AGES AND OTHER DAT DC 01 \$386,000 AGES AND
$\$ 253,000$

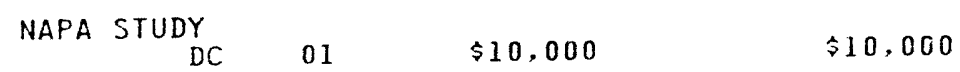

$\begin{array}{ccc}\text { ESTABLISH AN ECOHOMIC BULLETINBOARD (EBB) } \\ \text { DC } 01 & \$ 1,216 & \$ 1,216\end{array}$

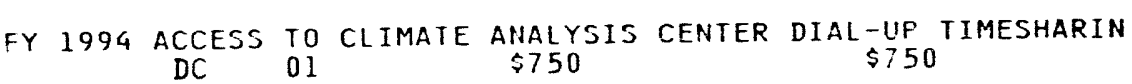

$\begin{array}{rrr}1994 \text { CENSUS IMPORT/EXPORT TAPES } & \$ 7,250\end{array}$ 

ALUE

DEBT MGMT DATA BASE SYSTEM

AI $01-93 F M 80031 \quad 09 / 30 / 94$ $017570-3315-7$ WASHINGTON

AI01-94AD35037 09/30/95 $017570-0033-X \quad$ CAMP SPRINGS

AI 01-94EI24201 09/30/95 017570-1405-5 GERMANTOWN

NATIONAL WEATHER SERVICE

AI01-93EI23457 09/30/94 017570-0005-4 GERMANTOHN

AI 01-9411510250 09:30/97

MATIONAL OCEAHIC \& ATMOS ADMIN $017570-1213-3$ SILVER SPRING

AI02-94ER6175j 11/30/98 NATIONAL OCEANIC \& ATMOS ADMIN $017570-1213-3$ SILVER SPRING

AI 05-900R21956 09/30/94 NATIONAL OCEANIC \& ATMOS ADMIN 017570-1201- $x$ AJHEVILLE NATIONAL OCEANIC \& ATMOS ADMIN AI05-90ER60952 03/14/96 NATIOHAL OCEANIC \& ATMOS ADMIN $017570-1201-x$ ASHEVILLE NATIONAL WEATHER SERVICE AI08-921IV11059 12/31/96 VEG ATMOS TURBULENCE \& DIFF LAB AI05-76EV03688 $0175730 / 99$ RIDGE

AI01-88GC2013901/04/95 017570-002j-2 ARLINGTOH

AI01-94GC12161 03/31/95 $017570-0014-3 \quad$ SFRIHGFIELD

AIO1-93AD33660 09/30/94 $017570-0014-3 \quad$ SPRINGFIELD NATIONAL TECH INFORMATION SVC AI05-920R22010 $12 / 31 / 94$ SPRINGFIELD NATIOHAL TECH INFORMATION SVC
AI05-82TC11274 09/30/98 $21 C 11274$
$017570-1314-8 \quad$ SPRINGFIELD
ONE YEAR'S SUPPORT FOR REPORTING REQUIREMENTS TO CREDIT BUPE DC 01 T2,000

$\begin{array}{ccc}\text { ACCESS TO CLIMATE ANALYSIS CENTER } & \$ 850 & \$ 0 \\ \text { MD } 07 & \end{array}$

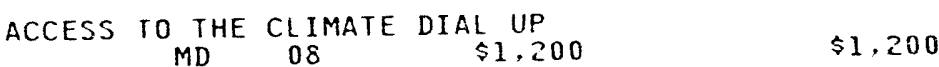

$\begin{array}{ccc}\text { ACCESS TO CLIMATE DIAL-UP SERVICES } & \$ 1,200 \\ \text { MD } 06 & \$ 1,200 & \$ 1,200\end{array}$

NTERAGENCY AGREEMENT TO REVIEW THE SAFETY OF SPACE MISSIONS $\begin{array}{rrrr}M D & \text { AGREEMENT TO REVIEN THE SAFETY } & \$ 150,000\end{array}$

SUPPORT FOR OPERATIONS OF THE HMO/GAW QUALITY CONTROL/

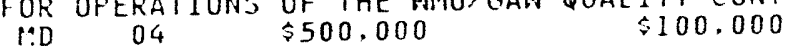

$\begin{array}{rll}\text { GLOBAL HISTORICA! CLIMATE NETHORK } & \$ 496,000\end{array}$

DATA PREPARATION AND ANALYSIS FOR ANHEX III, USA/FRC COOFERA NC $11 \$ 1,495,000$ \$1.221,000

CLIMATOLOGICAL AEROLOGICAL REFERENCE DATA SET

$$
\text { IIC } 11 \text { \$1,765,000 \$1.747.000 }
$$

SUPERSEDES CONTRACT NO. DE-AI08-87NV10566

$$
\text { INV } 01 \text { \$15.823,000 }
$$
CONTINUATION OF ATMOSPHERIC TURBULEHCE \& DIFFUSION RE

PROVIDE FOR INTERAGENCY AGREE-MENT TO COVER U.S. PATENT VA $10 \$ 4,065,930 \quad \$ 4.066 .930$ SUBSCRIPTIOH $08 \quad \$ 250 \quad \$ 250$

MEDLAR ONLINE SERVICE $\$ 3,000 \quad \$ 3,000$

ORNL ACCESS TO ELECTRONIC MEDIA FRODUCTION

$\begin{array}{lll} & \end{array}$

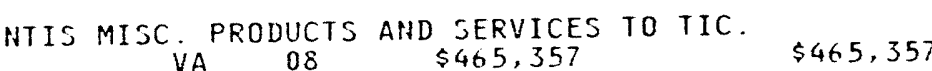


AWARDEE NAME

BIN.

COMPL DATE

DESCRIPTION OF WORK

DIVISION

VENDOR ID

CITY

STATE DIST

CONTRAC
VALUE

COMMONHEALTH EDISON CO

CRO1-83NE $4337203 / 15 / 30$
$017630-0001-X$ CHICAGO

(0)

ACO2-76ET10802 06/30/99
$017630-0001-X \quad$ CHICAGO

DISPOSAL OF IP

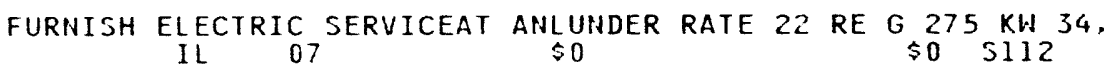

$017630-0001-X$ CHICAGO

COMMONWEALTH SCI \& IND RES ORG

FG02-92ER61373 11/30/94

0I7670-0002-2 MORDIALLOC VICTORIA

COMMUNICATION NETWORK SYSTEMS

COMMUHITY HOSP DF WILLIAMS CTY MONTPELIER DIVISION

COMMUNITY MEDICAL CENTER

COMMUNITY SCHOOL DISTRICT \#10

COMPA INDUSTRIES INC

COMPOSITE PARTICLES INC

COMPOSITE TECHNOLOGY DEVELOP

COMPREHENSIVE TECH INTL INC

COMPUCHEM LABORATORIES
AC02-93CH10593 09/30/94

217580-0002-3 BILLIHGS

FG45-94R562197 12/31/95

$184420-0101-8$ BRYAN

FG42-93R253855 06/30/95 017840-0002-7 TOMS RIVER

FG41-93R192508 06/30/95 220620-0001-4 READFIELD

ACO1-91EH89098 08,18,94 $213950-0001-8$ ANAHEIM

ACO1-92EH89216 09/09/96 213950-0002-6 ROCKVILLE FCO2-93CE41035 c 09/30/97
$234970-0001-9$ EMMAUS

FG03-93ER81586 04/01/96 $159450-0001-9$ BOUL DER

ACO1-90MA34225 02/28/95 194840-0001-0 CHAHTILLY

AD01-94AD15032 09/30/94 235740-0001-4 DURHAM
A PRECISE PASSIVE NARROH- BEAMFILTER INFRARED RADIOMETER AND OO $\$ 213,720$

$\$ 213,720$

TECHNICAL SUPPORT SERVICES FORTHE SOUTHERN GREAT PLAINS

$$
\text { OK } 06 \quad \$ 1,382,922 \quad \$ 1,105,869 \text { \$216 }
$$

ICP-NEW AWARD

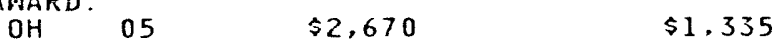

TITLE III OF THE NATIONAL ENERGY CONSERVATION POLICY ACT HJ $04 \quad \$ 423,959 \quad \$ 200,000$

TITLE III OF THE NATIONAL ENERGY CONSERVATION POLICY ACT ME $01 \quad \$ 35,968 \quad \$ 17,984$

SUPPORT SERVICES FOR OFFICE OFENVIRONMENT SAFETY \& HEALTH $\begin{array}{llll}C A & 02 & \$ 2,169,420 & \$ 2,118,946 \quad \text { R799 }\end{array}$

TECHNICAL SUPPORT SERVICE FOR THE OFFICE OF EH-30 $M D \quad 08 \quad \$ 2,370,020 \quad \$ 1,965,000 \quad R 409$

HIGH-VALUE SCRAP TIRE RECYCLE

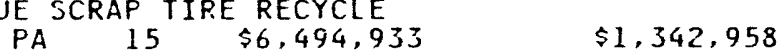

PHASE I - RADIATION RESISTAHT RF FEEDTHROUGH INSULATORS FOR

ADP INFORMATION SYSTEMS SUPPORT

$$
\text { VA } 08 \quad \$ 22,409,571 \quad \$ 17,893,785 \quad \text { D303 }
$$

SPECIMEN TEST AHD LABORATORY WORK FOR THE DRUG-FREE WORKPLAC IIC $04 \quad \$ 20,788 \quad \$ 20,788$ Q301 


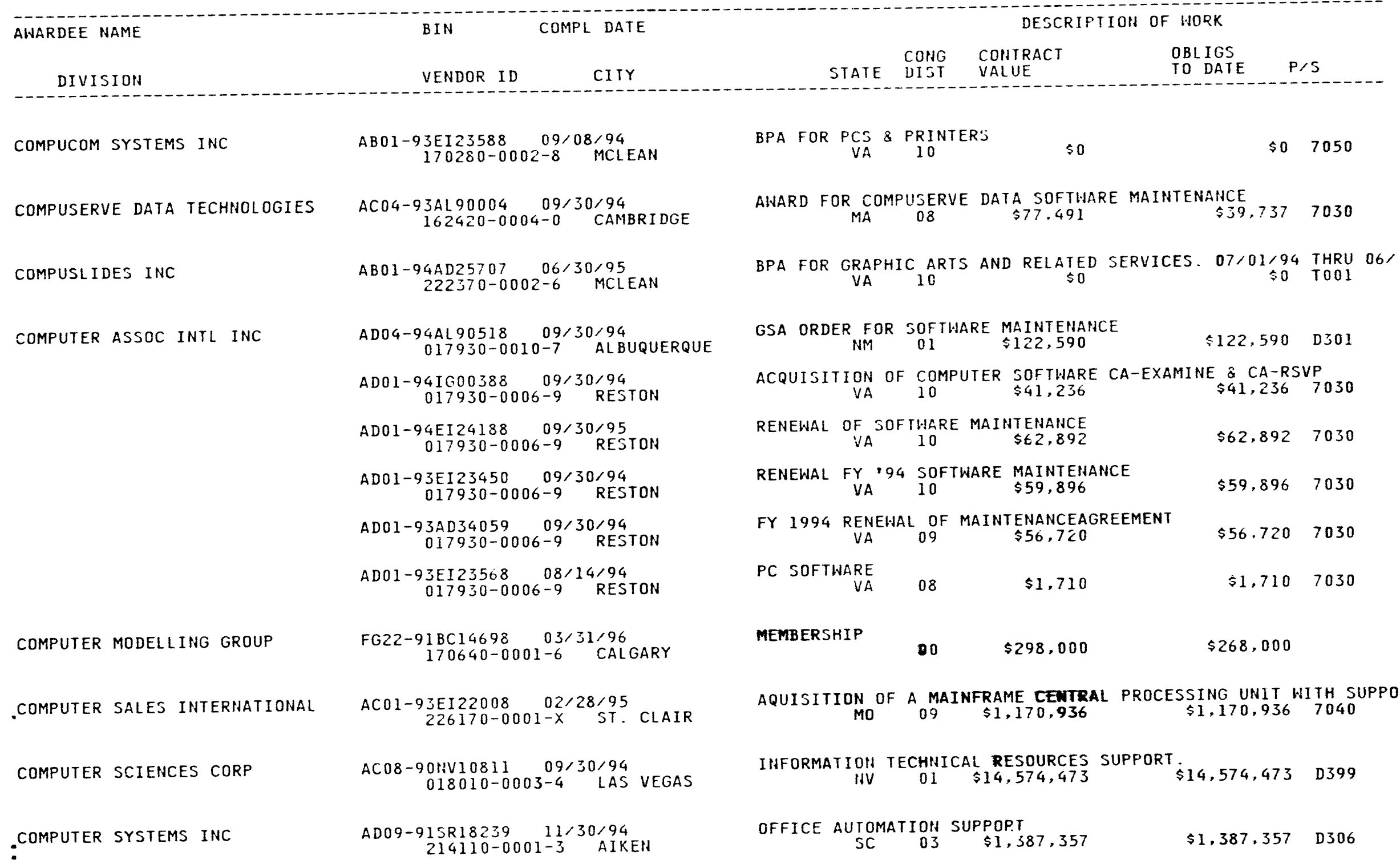




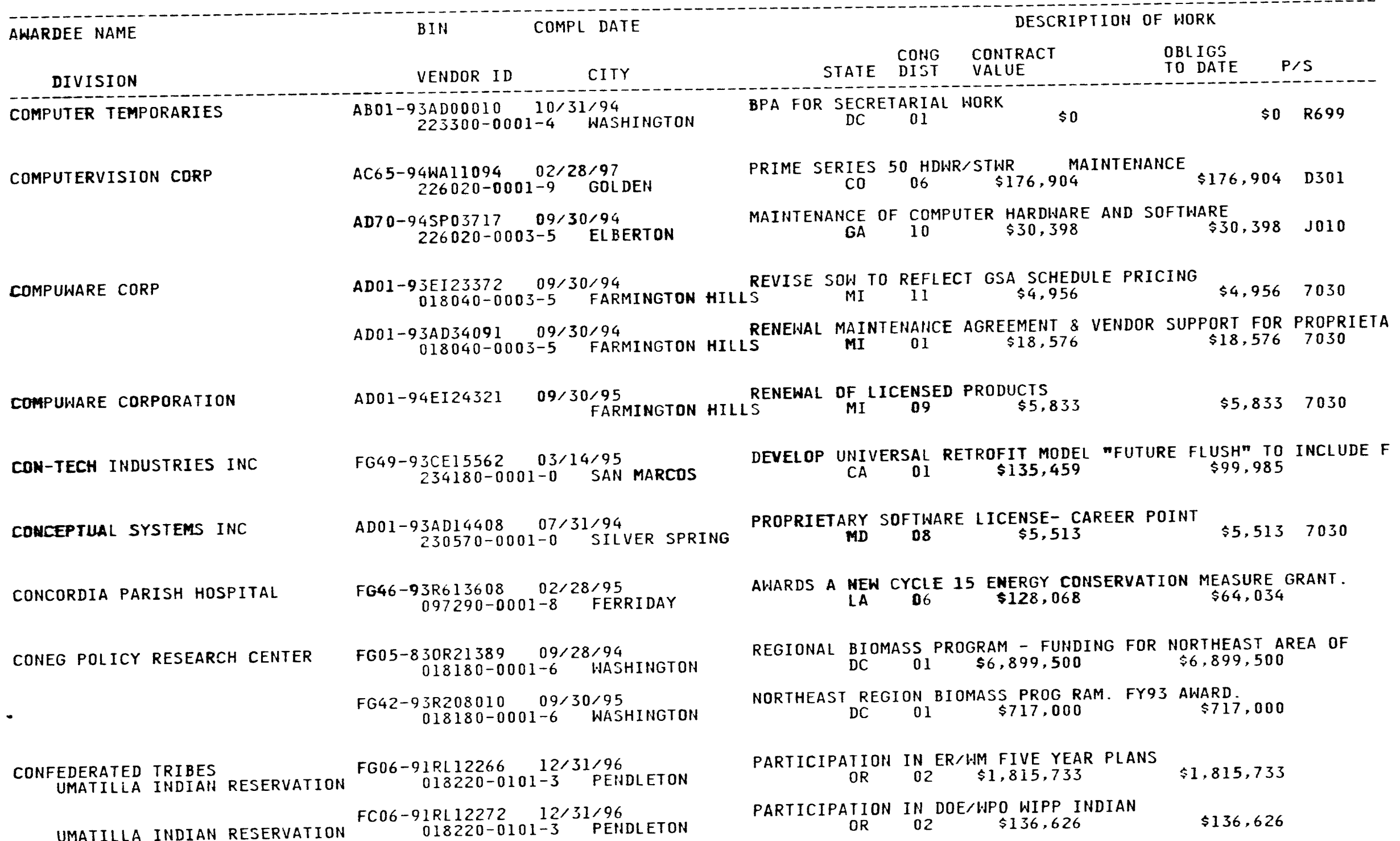

CONFERENCE BOARD
FC01-93RW00284 08/02/98 


AWARDEE NAME

DESCRIPTION OF WORK

DIVISION

VENDOR ID

CITY

$018230-0002-9$ FRANKFORT

CONFERENCE OF RADIATION CONTRL

CONGRESSIONAL QUARTERLY INC

CONNECTICUT COLLEGE

CONNECTICUT STATE OF

SOCIAL SERVICES DEPT OF

CONNECTICUT UNIVERSITY OF

CHEMISTRY DEPT

METALLURGY DEPT

- MEchanical ENG DEPT
FG08-94NV11514 04/14/99 $018250-0001-8$ FRANKFORT

AD01-93AD34008 09/30/94 $106510-0001-7$ WASHINGTON

FG41-92R191400 08/31/94
$164340-0001-4$ NEW LONDON FG41-94R191601 05/30/96 $164340-0001-4$ NEW LONDON

FC01-91EI22780 08/07/96 $018310-0008-3$ HARTFORD

FG41-94R130134 03/31/99 $018310-4008-5$ HARTFORD

FG41-93R130144 09/30/94 $018310-0008-3$ HARTFORD

FG41-93R130112 06/30/98 $018310-0008-3$ HARTFORD

FG05-92ER79109007/31/94

FG02-86ER13622 11/30/94 $018320-0402-3$ STORRS

FG02-92ER40716 11/30/94 018320-0002-8 STORRS FG02-92CH10501 $09 / 30 / 95$
$018320-0002-8$ STORRS FG02-87ER45318 06/30/95
$018320-0802-9 \quad$ STORRS

FG02-88ER $13894 \quad 02 / 28 / 95$

\section{DESCRIPTION OF WORK}

CONG CONTRACT

KY $06 \quad \$ 499,830$

TO DATE $\$ 94,674$

GRANT FOR RADIOLOGICAL EMERGENCY MANAGEMENT PROGRAM FACILITA $K Y \quad 06 \quad \$ 50,000 \quad \$ 10,000$

ACCESS TO HASHINGTON ALERT SYSTEM

$\$ 39,700$

TITLE III OF THE NATIONAL ENERGY CONSERVATION POLICY ACT

$$
\text { CT } 02 \quad \$ 83,000 \quad \$ 41,500
$$

TITLE III OF THE HATIONAL ENERGY CONSERVATION POLICY ACT

$$
\text { CT } 02 \quad \$ 90,000 \quad \$ 45,000
$$

COLLECTION \& AHALYSIS OF DATA RELATED TO THE ENERGY INFO ADM
CT
01 FY 1994 WEATHERIZATION.
$01 \$ 2,311,519$
$\$ 2,311,519$

GRANT PROGRAMS OF SCHOOLS AND HOSPITAL AND BUILDINGS ONNED CT $01 \quad \$ 2,419,770 \quad \$ 49,999$

FY 1993 AWARD.
CT
$\$ 728,556$
$\$ 172,495$

1992 INSTRUMENTATIDN - AUTOMATED DNA SEQUENCER
CT
02 STRUCTURAL \& ELECTRONIC PROPERTIES OF IRON \& COBALT CT $02 \quad \$ 609,964 \quad \$ 609,964$ INVESTIGATIONS IN PARTICLE FIELD THEORY
CT
02 INITAL PLANING \& DESIGN ACTIVITIES FOR THE ADVANCED CT $02 \$ 20,400,000 \quad \$ 10,400,000$

A COHERENT MODEL OF MARTENSITIC NUCLEATION AND CT 02 \$1,066,039 $\$ 886,146$

MICROMECHANICAL VISOPLASTIC SIRESS-STRAIN MODEL WITH CT $02 \quad \$ 315,344 \quad \$ 315,344$ 


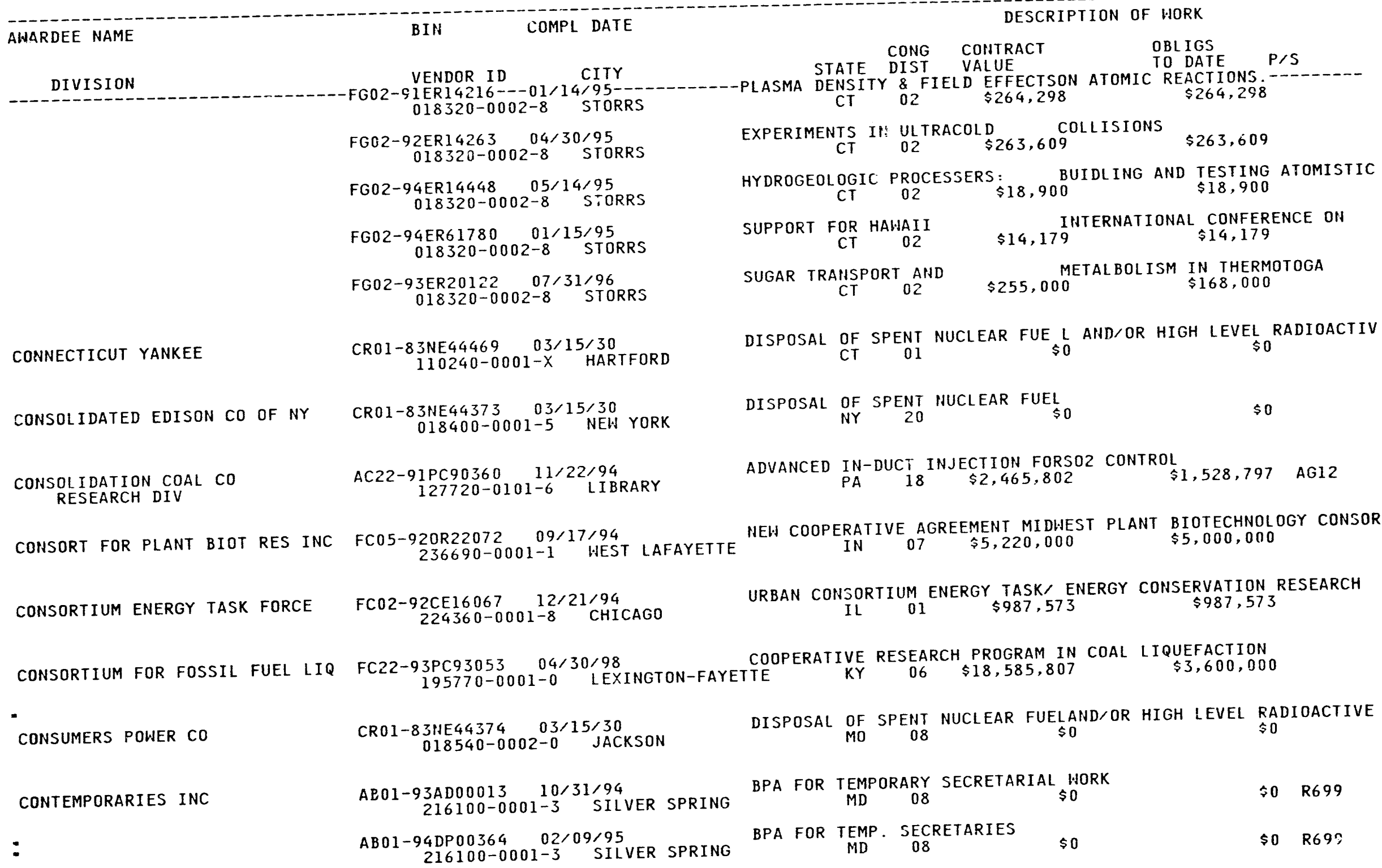




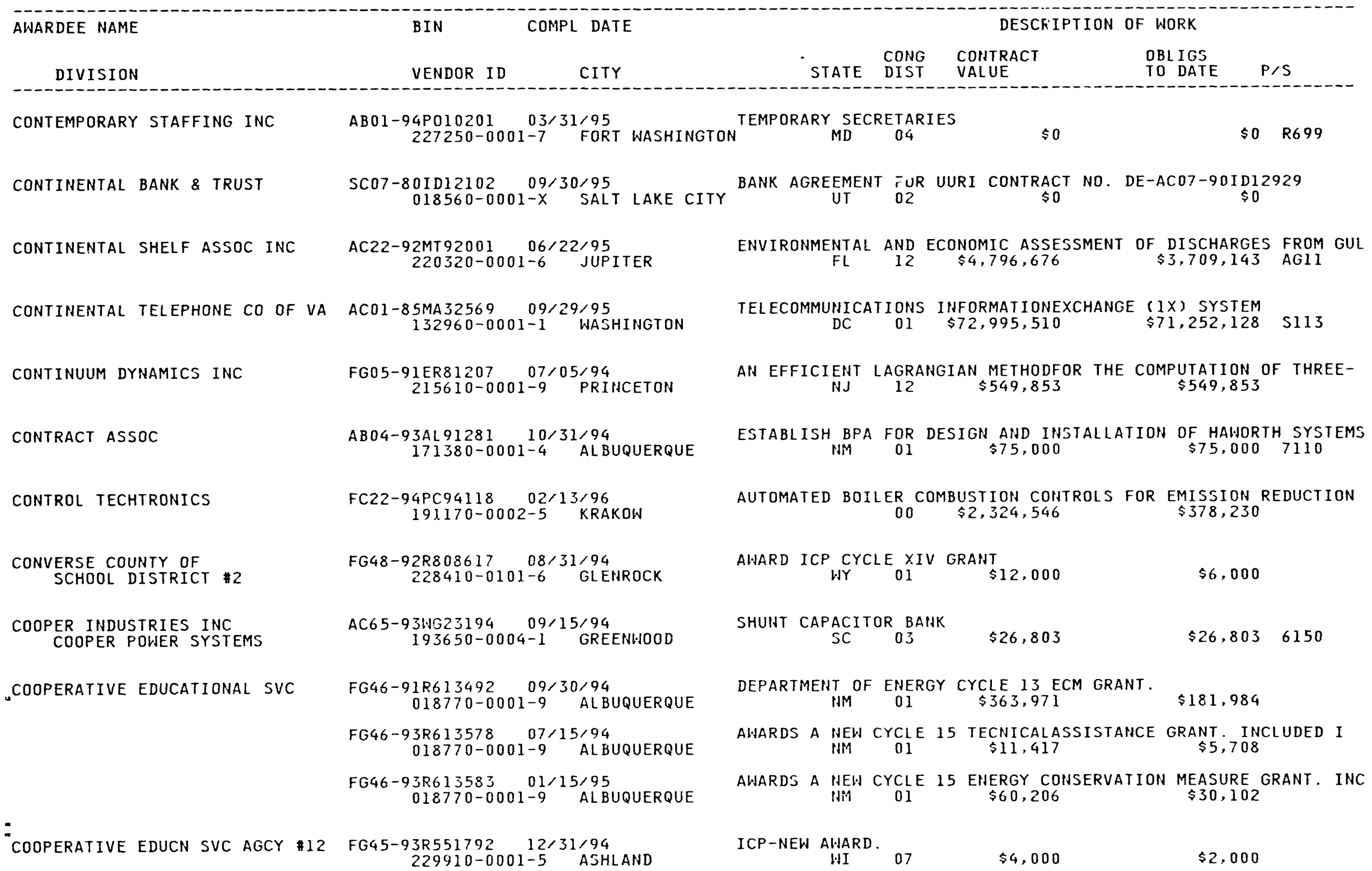




AWARDEE NAME

\section{AWARDEE NAME}

BIN

COMPL DATE

DESCRIPTION OF WORK

DIVISION

VENDOR ID

CITY

STATE DING CONTRACT

OBLIGS

\section{COOPERS \& LYBRAND}

TQM SERVICES

COOSA VALLEY TECHNICAL INST

COPPER QUEEN COMM HOSPITAL

CORE TECHNOLOGIES INC

CORNELL UNIVERSITY

BIOCHEMISTRY MOLECULAR CELL

AGRONOMY DEPT

$$
\begin{aligned}
& \begin{array}{c}
\text { AC65-901NA06914 08/25/94 } \\
018780-0002-0 \text { DENVER }
\end{array} \\
& \begin{array}{rc}
A D 09-935 R 18351 & 08 / 26 / 95 \\
018780-0012-8 & \text { AIKEN }
\end{array} \\
& \text { AD01-94AD81013 08/31/94 } \\
& \text { 018780-0011-X ARLINGTON } \\
& \text { AD01-94AD81014 04/06/95 } \\
& 018780-0011-X \text { ARLINGTON } \\
& \text { AD65-93:UG22580 08/31/94 } \\
& \text { 018780-0111-6 ARLINGTON } \\
& \text { AD01-94EI25064 09/30/94 } \\
& \text { OI8780-0011-X ARLINGTON }
\end{aligned}
$$

FG44-90R434334 07/30/95
$199360-0001-3$ ROHE

FG $49-94 R 916009 \quad 06 / 30 / 96$
BISBEE

FG03-93ER81561 $07 / 01 / 94$
$233510-0001-4$ TACOMA

FG01-90CE21010 12/31/94
$018890-0001-9$ ITHACA

FG02-84ER13233 05/31/96

FG02-84ERI
$018890-1701-9$ ITHACA

FG02-84ER13214 05/15/95
$018890-0101-5$ ITHACA

FG02-85ER13370 05/14/96

AGRICULTURE \& LIFE SCI COLL OF $018890-2001-X$ ITHACA

FG02-85ER.45211 05/31/95

- MATERIALS SCIENCE \& ENGRG DEPT 018890-0901-6 ITHACA

FG02-86ER $13495 \quad 12 / 31 / 95$
MGT, FINANCIAL, \& PROGRAM ANALYSIS SUPPORT SERVS

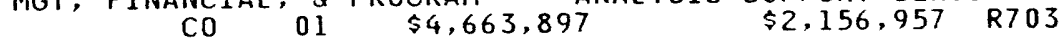

TOTAL QUALITY ASSESSMENT AND PLANNING TRAINING

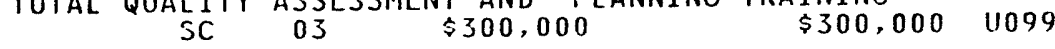

DELIVER TQM IMPLEMENTATION SERVICES

$\$ 526,374 \quad 8999$

DELIVER TQM IMPLEMENTATION SERVICES

$\$ 587,296 \quad 8999$

TQM IMPLEMENTATION PHASE

$$
\begin{aligned}
& \text { EMENTATION PHASE } \\
& \text { VA. } 10
\end{aligned}
$$

$\$ 333,568 \quad R 499$

DELIVER TQM INPLEMENTATION SERVICES

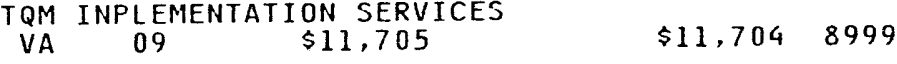

CYCLE 12 INSTITUTIOHAL CONSERVATION PROGRAM. APPROVED FUNDED

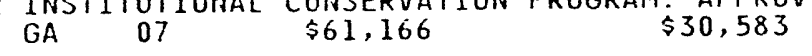

$\begin{array}{llll}\text { CYCLE } 16 & \text { ICP } & \text { GRANT APPLICATION } \\ & \text { AZ } & 05 & \$ 27,800\end{array}$

$\$ 16,680$

DIRECT SULFUR REDUCTION IN ADVANCED COAL CONVERSION PROCESSE WA $\$ 66 \quad \$ 75,000 \quad \$ 75,000$

INNOVATION EDUCATIONAL PROGRAMFOR RESIDENTAL ENERGY EFFIFIE NY $28 \quad \$ 183,924 \quad \$ 98,910$

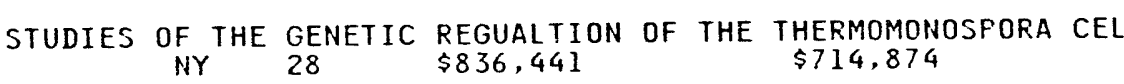
EFFECTS OF FREEZING AND COLD ACCLIMATION ON THE PLASMA MEMBR

MICROBIAL ECOLOGY OF THERMOPHILIC ANAEROBIC DIGESTION

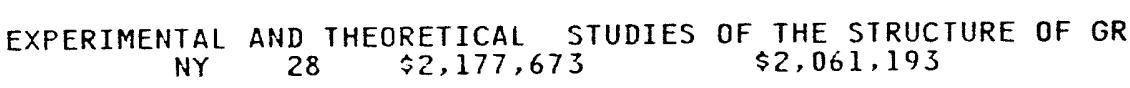

ANAEROBIC METABOLISM OF AROMATIC COMPOUNDS BY PHOTOTROPHIC 


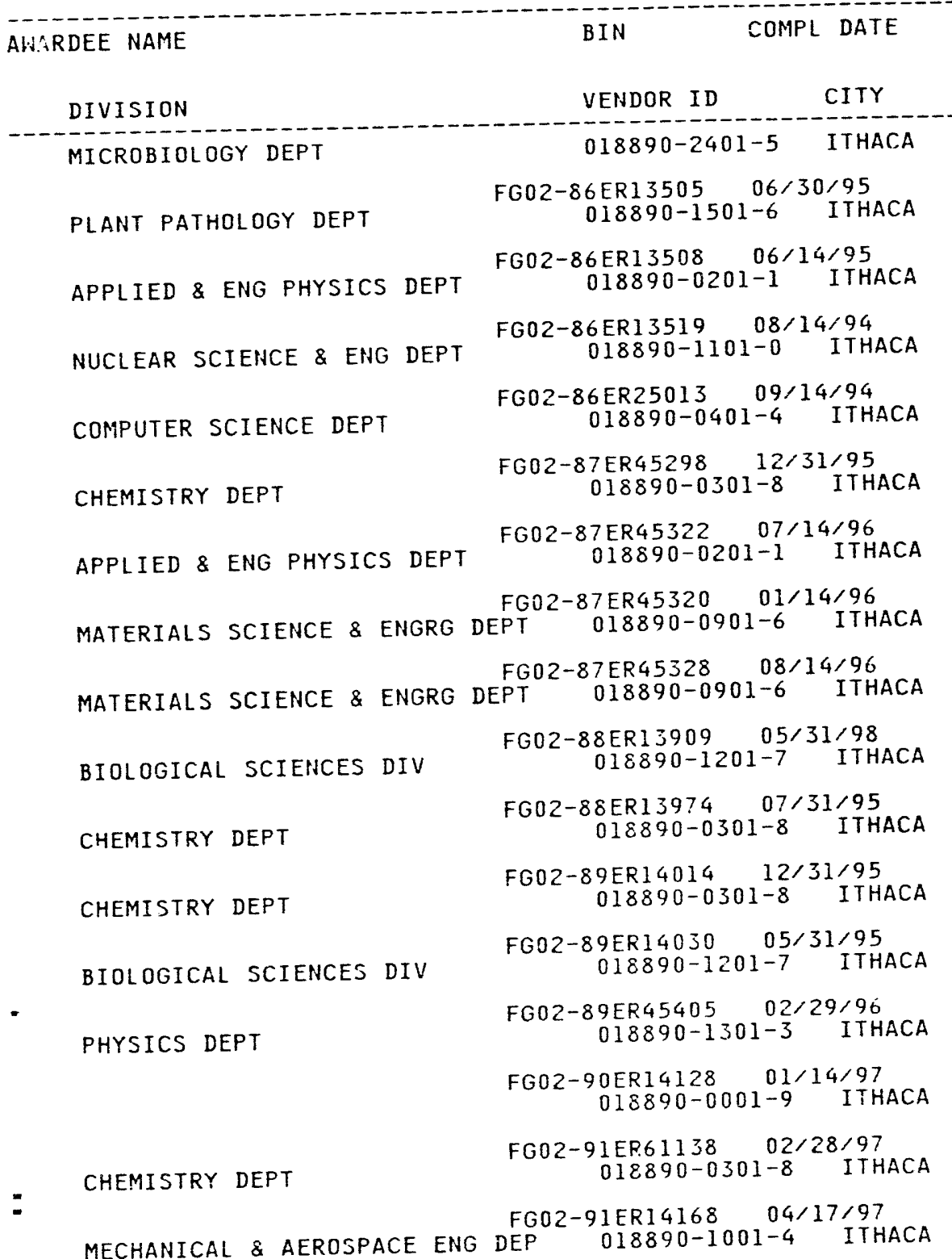

DESCRIPTION OF WORK

CONG CONTRACT
STATE DIST VALUE

NY $28 \quad \$ 607,500$

OBLIGS P/S $\$ 517,950$

MECHANSIMS OF INHIBITION OF VIRAL REPLICATION IN PLANTS NY $28 \quad \$ 702,000 \quad \$ 615,000$ THE DETECTION OF THE ETHYNYL RADICAL FOR STUDIES OF THE
$\$ 744,688$ EXPERIMENTAL STUDY OF INTERACTIONS OF HIGHLY CHARGED IONS EXPERIMENTAL STUDY OF $\$ 2,159,392 \quad \$ 2,159,392$ LARGE SCALE COMPUTATIONAL PROBLEMS IN NUMERICAL OPTIMIZATION LARGE SCALE COMPUTATIONAL $28 \quad \$ 917,290 \quad \$ 915,288$ $\begin{array}{rrr}\text { SYNTHESIS AND PROPERTIES OF NOVEL CLUSTER PHASES } \\ \text { NY } 28 & \$ 1,382,811 & \$ 1,120,705\end{array}$ UHV-STEM STUDIES OF NUCLEATIONAND GRONTH OF THIN METAL AND NY 28 \$1,218,996 $\$ 991,664$ STUDIES OF THE III-V $\begin{gathered}\text { COMPOUNDSIN THE MEGABAR REGIME } \\ \$ 1,138,676\end{gathered}$

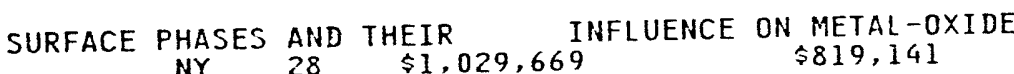
STRUCTURE AND FUNCTION OF THE SELF-INCOMPATABILITY PROTEINS NT $28 \quad \$ 887,876 \quad \$ 683.876$

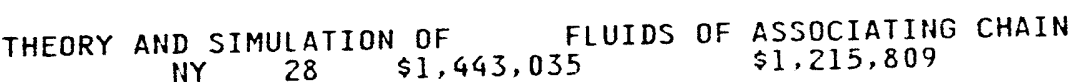
EXACT CALCULATIONS OF PHASE AND MEMBRANE EQUILIBRIA FOR NY 28 SXACT CALC $\$ 714,953 \quad \$ 583,953$ MOLECULAR ANALYSIS OF $\$ 497,604$ CYTOPLASMIC MALE STERILITY THE GEOMETRY OF DISORDER: THEORETICAL INVESTIGATIONS OF NY 28 THE GEOMETRY OF DISORD $\$ 436,552$ $\begin{array}{llll}\text { REACTION AND DIFFUSION IN } & \text { TURBULENT COMBUSTION } \\ \text { NY } & 28 & \$ 660,094 & \$ 460,823\end{array}$ SUBCELLULAR BORON DISTRIBUTIONIN BNCT USING ION MICROSCOPY

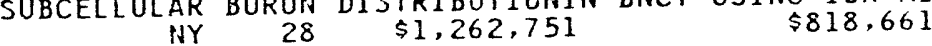

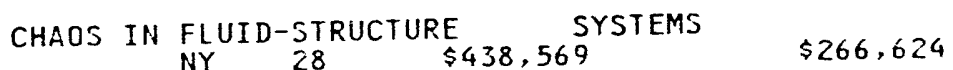




\begin{tabular}{|c|c|c|c|c|c|c|c|}
\hline AWARDEE NAME & BIN & DATE & & & DESCI & F HORK & \\
\hline DIVISION & VENDOR ID & CITY & STATE & $\begin{array}{l}\text { CONG } \\
\text { DIST }\end{array}$ & $\begin{array}{l}\text { CONTRACT } \\
\text { VALUE }\end{array}$ & $\begin{array}{l}\text { OBLIGS } \\
\text { TO DATE }\end{array}$ & $\mathrm{P} / \mathrm{S}$ \\
\hline
\end{tabular}

PLANT BREEDING DEPT OF

$$
\begin{aligned}
& \text { FG02-91ER20037 07/31/94 } \\
& 018890-2601-8 \text { ITHACA } \\
& \text { FG02-91ER14205007/31/94 } \\
& 018890-1301-3 \text { ITHACA } \\
& \text { FG02-90ER12988 09/09/94 } \\
& 018890-1101-0 \text { ITHACA } \\
& \text { FG02-92ER40731005/31/95 }
\end{aligned}
$$

PHYSICS DEPT

NUCLEAR SCIENCE \& ENG DEPT

ELECTRICAL ENGINEERING DEPT

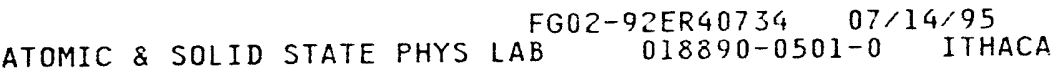

FG02-92ER75780 09/29/94 $018890-0001-9$ ITHACA

APPLIED MATH

FG02-94ER25199 02/14/97 018890-1901-1 ITHACA

FG02-93ER61567 03/31/96 018890-0001-9 ITHACA

FG02-93ER25164 05/31/96 $018890-1901-1$ ITHACA

APPLIED MATH

PLASMA STUDIES LAB

NUCLEAR SCIENCE \& ENG DEPT

NUCLEAR SCIENCE \& ENG DEPT

FG02-93ER54221 07/31/96 $018890-0801-X$ ITHACA

FG02-93ER79216 09/29/94 $018890-1101-0$ ITHACA

FG02-93ER75921 09/29/94 018890-1101-0 ITHACA

FG05-93ER79224 08/31/95 $018890-0001-9$ ITHACA

FG05-93ER79240 08/31/95 0I8890-0001-9 ITHACA

OFFICE OF SPONSORED PROGRAMS AC22-91PC90183 $12 / 13 / 94$
FG22-93PC93216 08/24/96 $018890-1001-4$ ITHACA
MECHANISMS \& GENETIC CONTROL OF INTERSPECIFIC CROSSING BARR

PHOTOCHEMICAL DYNAMICS OF SURFACE ORIENTED MOLECULES.

NY $28 \quad \$ 400,000 \quad \$ 400,000$

UNIVERSITY REACTOR INSTRUMENTATION PROGRAM

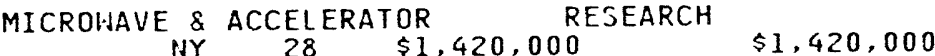

INVESTIGATIN OF THE COHERENT SYNCROTRON DYNAMICS SPECTRUM NY 28 \$650,000 $\$ 450,000$

APPLICATINS OF A COLD NEUTRON BEAM AND DESIGN STUDIES FOR A NY $28 \quad \$ 262,037 \quad \$ 262,037$

IINEAR, NON-MODAL PHENOMENA INNUMERICAL ANALYSIS AND APPLIED NY $28 \quad \$ 225,000 \quad \$ 72,750$ ACCURATE RESTORATION OF DHA
NY SEQUEHCES
28

IN DYNAMICAL

NUMERICAL ANALYSIS OF
NY 28 $\quad \begin{gathered}\text { BIFURCATIONS IN DYNAMIR } \\ \$ 197,000\end{gathered}$

IOH RINGS FOR MAGNETIC FUSION

NY $28 \$ 1,432,772 \$ \$ 354,782$

UPGRADED IHSTRUMENTATION FOR THE CORNELL TRIGA REACTOR $\begin{array}{lll}\text { IIY } 28 & \$ 32,200 & \$ 24,200\end{array}$

CORINELL TRIGA REACTOR SHARING PROGRAM

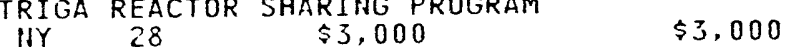

1993 UNIVERSITY INSTRUMENTATION PROGRAM - A TUNABLE VACUUM U WY $26 \quad \$ 236,325 \quad \$ 236,325$

1993 UNIVERJITY INSTRUMENTATION PROGRAM - A MASS SPFCTROMETE NY 28 \$132,000 $\$ 132,000$

DENSE IHCLIHED FLOHS-THEORY AND EXPERIMENT HYY $28 \quad \$ 695,695 \quad \$ 597,320$ AG11

FLUID DYNAMICS OF PRESSURIZED. ENTRAINED COAL GASIFIERS

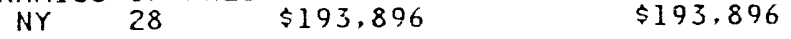




AWARDEE NAME

\section{DIVISION VENDOR ID}

VENDOR ID CITY KNIGHT LAB SUBMICRON FACILITY

CORPORATE VISIONS INC

$A B D 1-94 A D 25708 \quad 06 / 30 / 95$ 129490-0001-0 WASHINGTON

$A B 01-94 A D 24916 \quad 05 / 31 / 95$ 129490-0001-0 WASHINGTON

CORPUS CHRISTI ROMAN CATH CHU

COTTER CORP

FG42-93R253869 $07 / 31 / 95$
$230470-0001-8$ PORT CHESTER

RL13-74GJ31009 07/23/94 $169760-0001-1$ LAKEWDOD

RL13-74GJ31010 07/23/94 169760-0001-1 LAKEWOOD

RL13-74GJ31018 07/23/94 169760-0001-1 LAKEHOOD

RLI3-74GJ31033 07/23/94 169760-0001-1 LAKEHOOD

RL13-74GJ31035 07/23/94 169760-0001-1 LAKEWOOD

$R L 13-74 G J 31038 \quad 07 / 23 / 94$ 169760-0001-1 LAKEWOOD

COTIONWOOD JOINT SCH DIST \#242 GMO7-94IDI1147 01/06/99 $236500-0001-6$ COTTONWOOD

COUNCIL ENERGY RESOURCE TRIBES FG03-93ER75932 0 09/14/94

FG03-93ER75931 09/14/94 C19100-0003-5 DEIIVER

COUNCIL GREAT LAKES GOVERNORS

COURT PAMELA MEADE
FG05-830R21390 09/28/94 $019110-0001-2$ MADISON

$A B 34-93 R F 00605 \quad 11 / 30 / 95$
DESCRIPTION OF WORK

CONG COHTRACT OBLIGS
STATE STATE DIST VALUE TOERY CONSERVATION POLICY ACT-$\begin{array}{rll}\text { TITLE III OF THE NATIONAL ENERGY CONSERVATION POL ICY } & \$ 47,985\end{array}$

BPA FDR GRAPHIC ARTS AND RELATED SERVICES. 07/01/94 THRU 07/ $D C \quad 01$ \$O $\$ 0$ TOOI

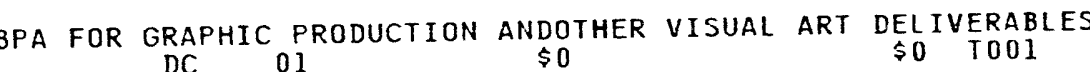
DC 01 \$O

CYCLE 15 AWARD.

NY $18 \quad \$ 48,765$

$\$ 24,382$

MINING LEASE AT $(05-1)-M L-60 . \underset{06}{8-C-J D-7 A} \quad \$ 0 \times 293$

MINING LEASE AT $(05-1)-M L-60 . \$ 8-C-J D-8 \quad \$ 0 \times 293$

MINING LEASE AT $(05-1)-M L-60 . \underset{06}{8-C-5 R-13 A} \quad \$ 0 \times 293$

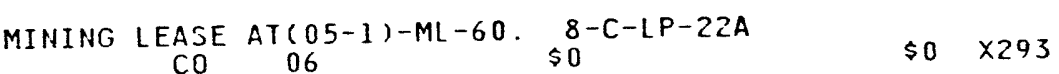

MINING LEASE AT $(05-1)-M L-60 . \underset{00}{8-C-B L-23 A} \quad \$ 0 \times 293$

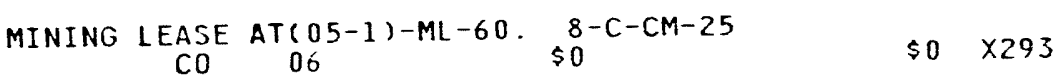

MOU TO STATE BASIC UNDERSTANDING OF THE PARTIES REGARDING \& ID 01

CERT TRIBAL ENERGY POLICY

$$
\text { CO } 01 \text { P } \$ 66,560
$$

$\$ 66,560$

TRIBAL INTERIISHIP PROGRAM
CO
01
$\$ 288,618$
$\$ 170,809$

REGIONAL BIOMASS PROGRAM - FUNDING FOR GREAT LAKES STATES. HI $02 \quad \$ 5,488,500 \quad \$ 5,488,500$

PB/COURT REPORTERS 


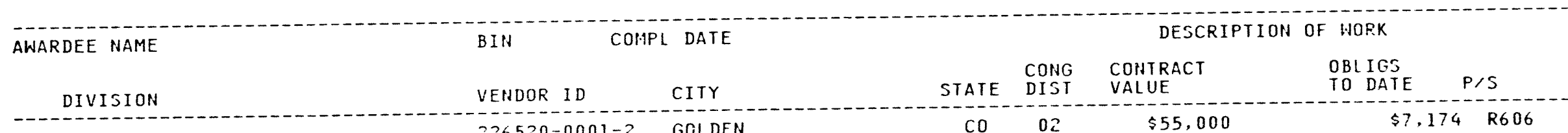

COVALENT ASSOC INC

COVINGTON COUNTY DF SCHOOL DISTRICT

COX \& ASSOC

COYNE TEXTILE SERVICES

CQ INC/COMBUSTION ENGIHEERING

CRATERS OF THE NATL MONUMENT

CRAWFORD COUNTY COMM SCH CORP

CRAY COMMUNICATIOHS INC

- CRAY RESEARCH INC

CREARE INC

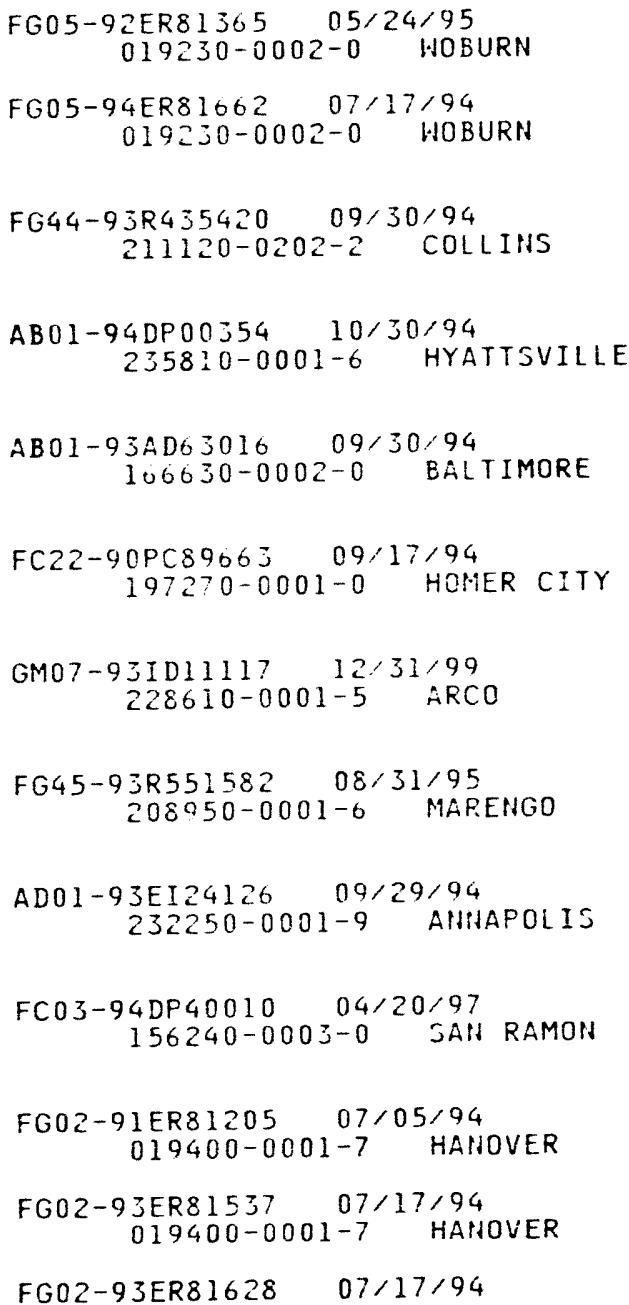

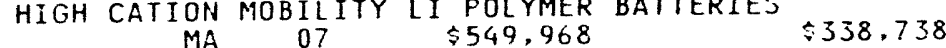

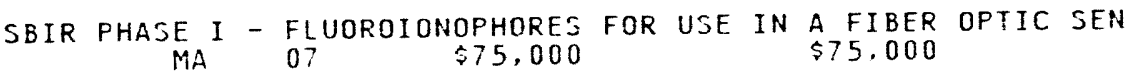

SEE ATTACHED PAGE NO. 2 OF THIS NOTICE OF FINANCIAL ASSISTAN MS $04 \quad \$ 17,945$ \$12.561

SECRETARIAL SERVICES
MD 07
ร 0
$\$ 0 \quad R 699$

BPA FOR RENTAL OF SMOCKS, COVERALLS

$\$ 0 \quad 3209$ ADVANCED COAL COHVERSION PROCESS DEMOHSTRATIOH. DEVELOPMENT
PA O O4 $\$ 23,946.004$ FIRING RANGE USE AGREEMENT HITH CRATERS OF THE MOOH HATIONAL
ID

ICP-HEW AWARD.
III D9
$\$ 12,080$
$\$ 10,872$

PURCHASE COMMUNICATIONS HARDHARE AIID ESTABLISH FY94 MAINTENA

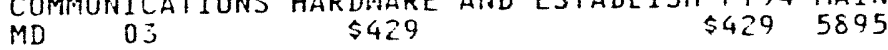

HIGH PERFORMANCE PARALLEL PROCESSOR COMPUTING

$$
\begin{array}{lll}
\text { CA } 10 & \$ 25.689 .326 & \$ 8,468,000
\end{array}
$$

FABRICATION OF TUNGSTEN AND TUNGSTEN-MOLYBDENIM ALLOY

$$
\mathrm{NH} 02 \quad \$ 548,271 \text { \$ } 02 \text { \$ }
$$

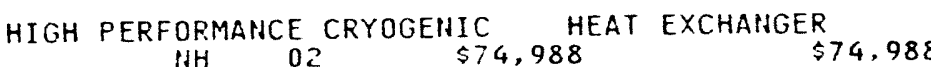

HELIUM-COOLED DIVERTORS WITH LOW-ACTIVATION MATERIALS AND 


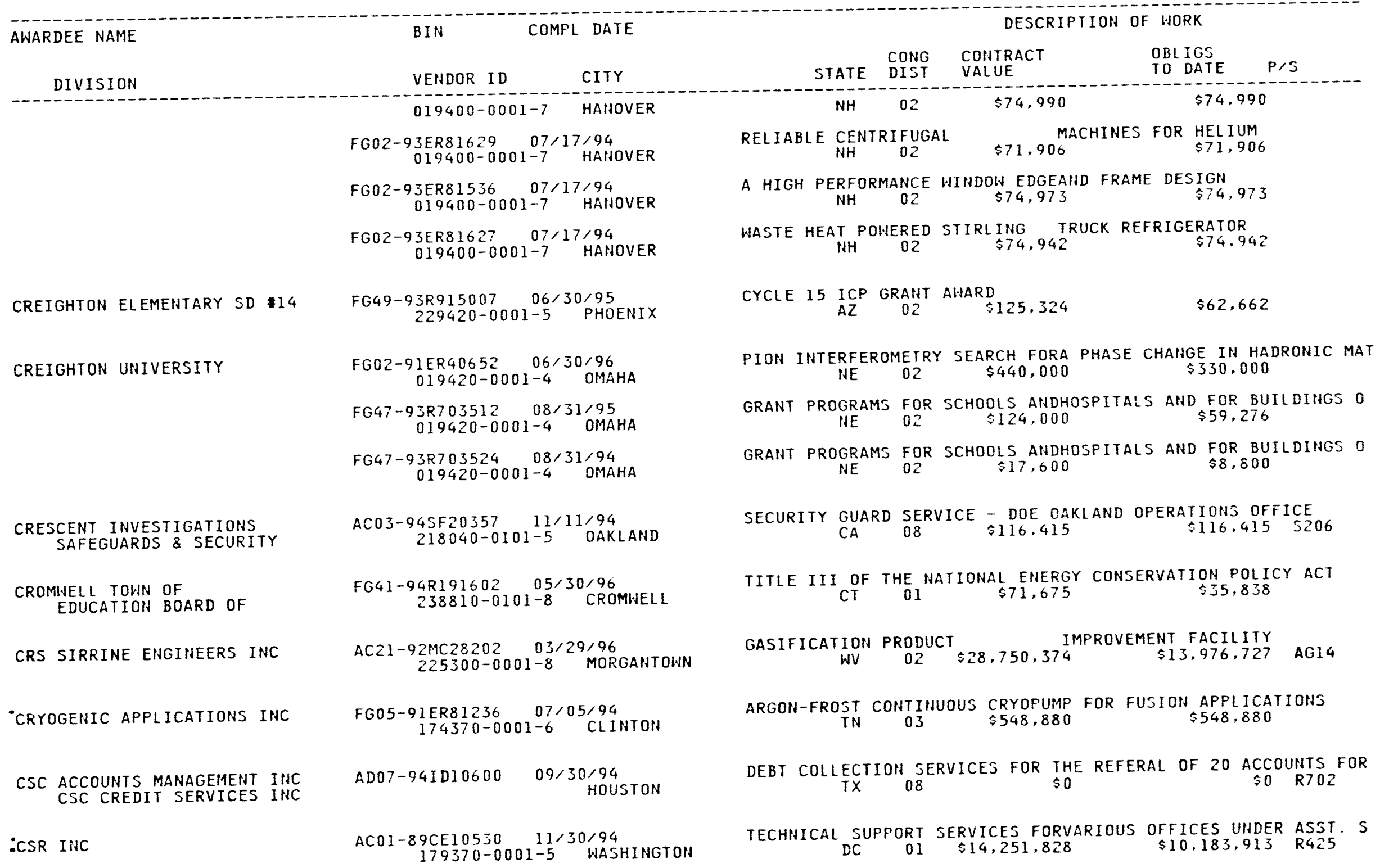




\section{AWARDEE NAME}

BIN

COMPL DATE

DESCRIPTION OF HORK

DIVISION

VENDOR ID

STATE CONG CONTRACT

OBLIGS TD DATE

$P / S$

CSS LABORATORIES INC

AMO1-92AD32235 04/29/95
$219050-0001-4$ IRVINE

AMO1-92AD32493 08/10/95 219050-0001-4 IRVINE

CSX TRANSPORTATION INC GMO4-86AL37761 $12 / 15 / 99$
$180030-0001-7$ LARGO CUBA INDEPENDENT SCHOOLS

CUBA TOWN OF MUNICIPAL SCHOOLS

CUDAHY SCHOOL DISTRICT

CUERO COMMUNITY HOSPITAL

CUMMINS CHESAPEAKE INC

CURTIS LEWIS \& ASSOC

CUSTOM COALS INTERNATIONAL

ECYBERNET SYSTEMS CORP
FG46-94R613650 12/31/95 GM04-92AL84057 04/01/97
$226800-0101-X$ CUEA

FG45-93R551793 12/31/95
$197690-0001-9$ CUDAHY FG46-92R613554 02/28/95
$223340-0001-9$ CUERO

AB01-93AD66358 09/30/94 226550-0001-3 BAL TIMORE

$A B 01-94 A D 67056 \quad 01 / 31 / 95$ 226550-0001-3 BALTIMORE

ABO1-94MI10419 12/06/94 $235900-0001-5$ HASHINGTON

FC22-93PC92643 03/21/96 225770-0001-4 STOYSTOWN FR22-93PC92649 12/21/15 225770-0001-4 STOYSTOWN

FG02-92ER81391 09/24/95 221220-0001-5 AllH ARBOR
80486 MICROCOMPUTER HORKSTATIONS
CA $40 \quad \$ 3,123,940$

$\$ 0 \quad 7010$

80486 MICROCOMPUTER WORKSHOPS

CA $08 \quad \$ 6,434,170$

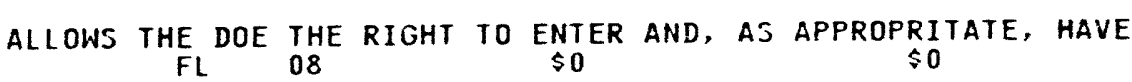

TITLE III OF THE NATIONAL ENERGY CONSERVATION POLICY ACT NM $03 \quad \$ 13,850 \quad \$ 6,925$ SUPPORT FOR $\underset{N M}{\substack{\text { SCIENCE, MATHEMATICS, AND ENGINEERING } \\ \$ 0}} \underset{\$ 0}{\text { TO IMPROVE }}$

ICP-NEW AWARD.
WI

WI $04 \quad \$ 6.800$

$\$ 3,400$

THIS IS A NEW CYCLE 14 ENERGY CONSERVATION MEASURE GRANT AWA
TX
$\$ 14$ BPA FOR MAINTENAHCE DF EMERGENCY DIESEL GENERATOR MAINTENANCE OF EMERGENCY GENERATOR MD 08 \$O $\mathbf{0} \mathbf{0 3 6}$ BPA FOR EEO INVESTIGATIONS $\$ 0 \quad \mathbf{J 0 3 6}$

$\$ 0 \quad R 607$

SELF SCRUBBING COAL: AN INTER GRATED APPROACH TO CLEAN AIR

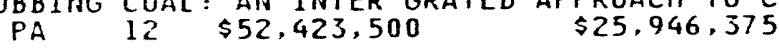

REPAYMENT AGREEMENT

so

$\$ 0$

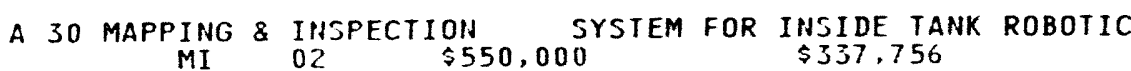




\begin{tabular}{|c|c|}
\hline AWARDEE NAME & COMPL DATE \\
\hline DIVISION & VENDDR ID \\
\hline CYBORG INC & $\begin{array}{c}\text { ACO1-93FE62806 } 06 / 01 / 96 \\
172790-0002-9 \text { BETHESDA }\end{array}$ \\
\hline \multirow[t]{2}{*}{ D-Q UNIVERSITY } & $\begin{array}{c}\text { FG49-92R914037 08/31/94 } \\
206050-0001-8 \text { DAVIS }\end{array}$ \\
\hline & $\begin{array}{c}\text { FG04-91AL72587 10/31/94 } \\
206050-0001-8 \text { DAVIS }\end{array}$ \\
\hline \multirow[t]{2}{*}{ DAGGETT \& GREENHOOT } & $\begin{array}{c}\text { R007-88ID12731 } 07 / 31 / 99 \\
149210-0001-8 \text { ENTERPRISE }\end{array}$ \\
\hline & $\begin{array}{c}\text { RLOT-901D10389 } 07 / 31 / 97 \\
149210-0001-8 \quad \text { ENTERPRISE }\end{array}$ \\
\hline DAILCO INC & $\begin{array}{c}\text { AC65-9JWC08010 09/15/94 } \\
234240-0001-9 \text { MILES CITY }\end{array}$ \\
\hline DAIRYIAND POWER COOP & $\begin{array}{c}\text { CRO1-83NE44377 } 03 / 15 / 30 \\
020210-0001-X \text { LA CROSSE }\end{array}$ \\
\hline $\begin{array}{l}\text { DALLAS COUNTY OF } \\
\text { HOSPITAL }\end{array}$ & $\begin{array}{c}\text { FG47-92R704983 } 08 / 31 / 94 \\
212490-0101-3 \text { PERRY }\end{array}$ \\
\hline DAMES \& MOORE & $\begin{array}{r}\text { ACO4-90AL57502 } 06 / 21 / 95 \\
020340-0010-2 \text { AL BUQUERQUE }\end{array}$ \\
\hline DAN KAIN TROPHIES INC & $\begin{array}{c}A B O 1-94 A D 25717 \\
133070-0001-2 \quad 06 / 30 / 95 \\
\text { ARLINGION }\end{array}$ \\
\hline \multirow[t]{2}{*}{ DAHA COLLEGE } & $\begin{array}{r}F G 47-93 R 703517 \quad 08 / 31 / 95 \\
020370-0001-4 \quad \text { BLAIR }\end{array}$ \\
\hline & $\begin{array}{r}F G 47-93 R 703522 \quad 08 / 31 / 94 \\
020370-0001-4 \quad \text { BLAIR }\end{array}$ \\
\hline -DANBURY HOSPITA & $\begin{array}{c}\text { FG41-92F.191401 } 08 / 31 / 94 \\
020410-0001-5 \text { DAHBURY }\end{array}$ \\
\hline
\end{tabular}

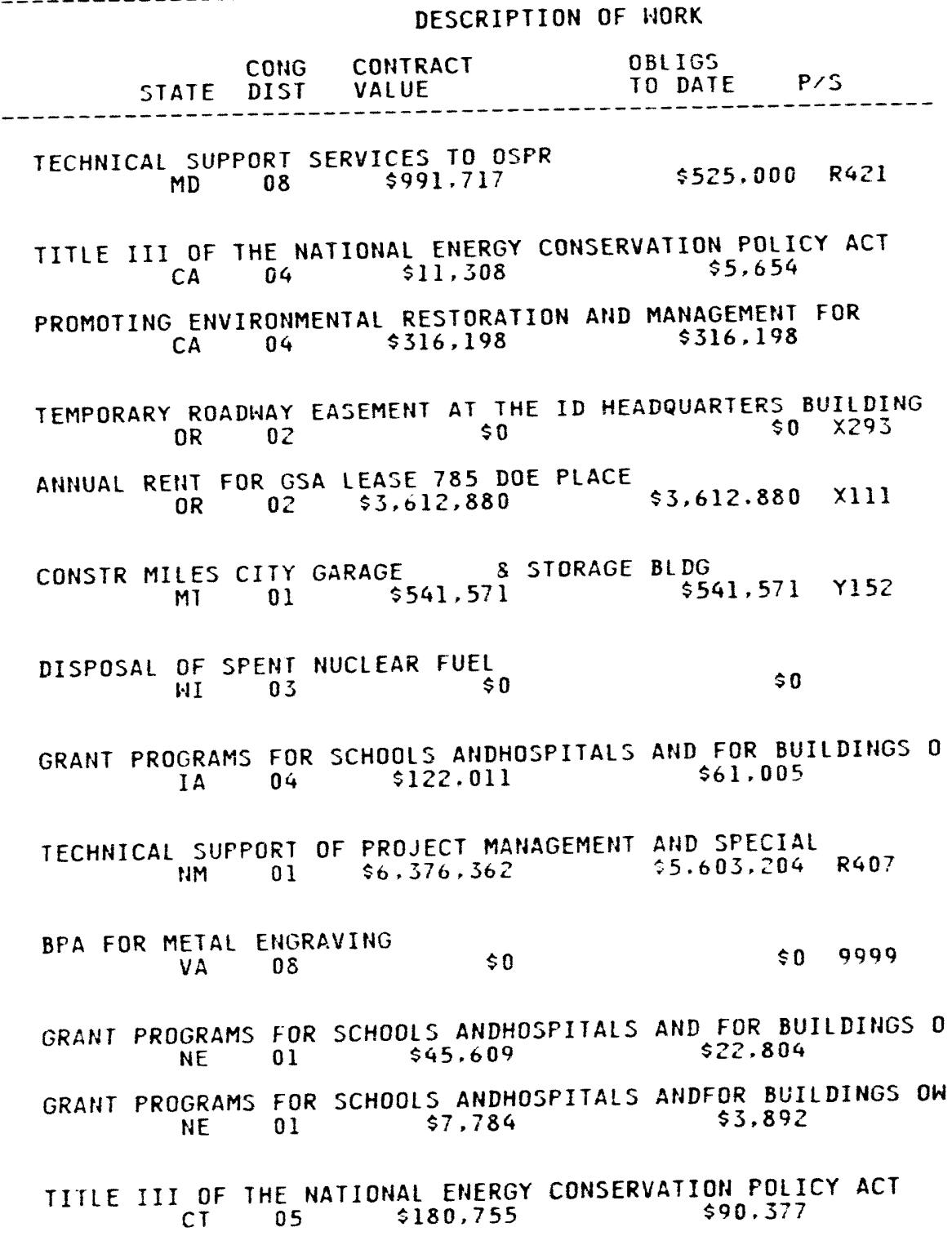


AWARDEE NAME

BIN
COMPL DATE

VENDOR ID

CITY

020410-0001-5 DANBURY

FG41-94R191603 05/30/96 020410-0001-5 DANBURY

DANOFF \& DONNELLY

$A B 01-94 M I 10462 \quad 04 / 30 / 95$ 218240-0001-4 ELLICOTT CITY

$=609-94 S R 18407 \quad 01 / 10 / 95$ $106570-0001-9$ DANVILLE

DANVILLE AREA COMMUNITY COLL

DARTMOUTH COLLEGE

PHYSICS \& ASTRONOMY DEPT

FG02-85ER53194 12/31/96
$020510-0201-0$ HANOVER

FG02-86ER13528 11/30/94 020510-0401-3 HAHOVER

THAYER SCHOOL OF ENGINEERING

FG02-86ER45260 07/14/95

THAYER SCHOOL OF ENGINEERING 020510-0401-3 HAHOVER

CHEMISTRY DEPT

FG02-86ER.13592 03/14/96
$020510-0501-X$ HAHOVER

FG02-87ER45311 08/30/96

THAYER SCHOOL OF ENGINEERING

PHYSICS DEPT

FG02-87ER45330 09/14/94
$020510-0101-4$ HALOVER

FG02-91ER20032 05/31/95 020510-0001-8 HAHOVER

FG02-91ERI4220 09/14/94 020510-0001-8 HANOVER

FG05-92ER79126 07/31/94 020510-0001-3 HAHOVER

DATA GENERAL CORP

ADO4-94AL $91212 \quad 09 / 30 / 94$ $020530-0001-5$ AL BUQUERQUE

AC04-9JAL $91020 \quad 04 / 30 / 95$ 020530-0002-3 MCLEAN

\section{CONG CONTRACT}

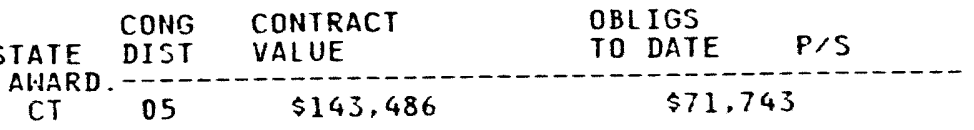
TITLE III OF THE NATIONAL ENERGY CONSERVATION POLICY ACT

BPA FOR EEO INUESTIGATION 05/01/94-04/30/95 \$0 $\quad 8499$

USED ENERGY RELATED LABORATORYEQUIPMENT
IL
15

NONL INEAR MAGNETOHYDRODYNAMICS

$$
\mathrm{NH} 02 \quad \$ 790,700
$$

$\$ 639,700$

SUBCOOLED SPRAYS IN VAFOR ENVIRONMENT

$$
\mathrm{NH} 02 \text { \$1.020.909 } \$ 1,020,909
$$

STUDY OF ROLE OF GRAIN JOUNDARIES ON STRENGIH DUCTILITY AND $\mathrm{NH} 02 \quad \$ 1.226 .942$

$\$ 1,066,491$

PHOTOEXCITED CHARGE PAIR ESCAPE AHD RECOMBINATIOH
NH 02
$\$ 717,688$

THE STUDY OF THE STRUCTURE ANDPROPERTIES OF GRAIN BOUHDARIES NH 02 \$8 $\$ 86,414$ \$711,529

EXCITON IH SEMICONDUCTING SUPERLATTICES, QUANTUM HELLS. $\mathrm{NH} 02 \quad \$ 768.242$ \$768.242

IRON REGULAIIONS OF GENE EXPRESSION IN THE BRADYRHIZOBIUM JA NH 02 \$267.000 \$267.000

MIXING \& SETTLING IN COHTINUOUS METAL PRODUCTIOH. $\mathrm{NH}$ O2 $\$ 275,000$ S275,000

1992 INSTRUMENTATION ACQUISITIOH OF A HIGH TEMPERATURE SINGL HH $02 \quad \$ 211.601 \quad \$ 211.601$

GSA ORDER FOR SOFTWARE MAINTENANCE

$$
\text { NM } 013 \$ 133,948 \quad \$ 133,948 \quad 0301
$$

DATA GEHERAL HARDHARE

VA $10 \quad \$ 714.985$

SUPPORT

$\$ 714,985 \quad$ D301 


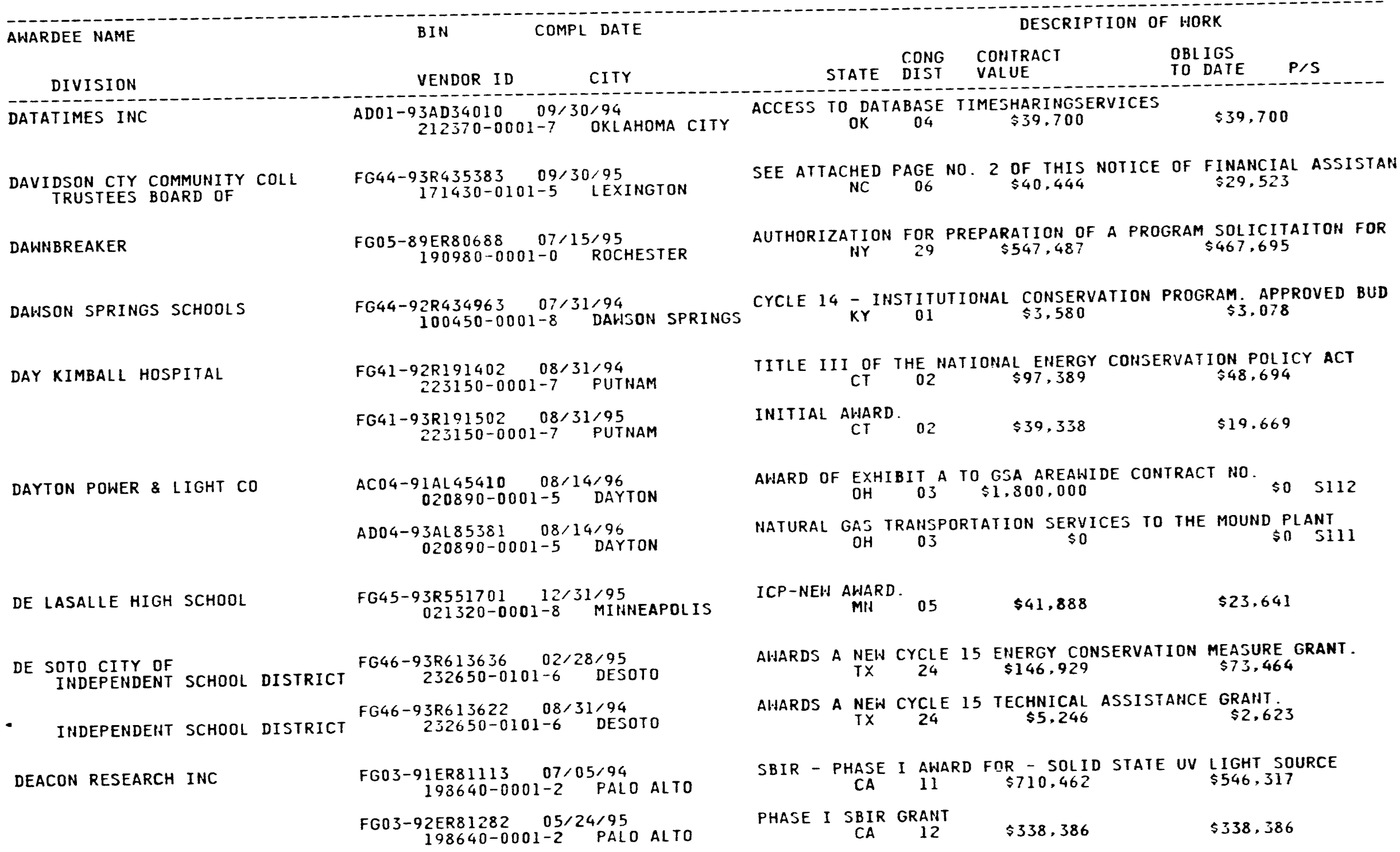




\section{AWARDEE NAME}

BIN

COMPL DATE

DESCRIPTION OF WORK

DIVISION

VENDOR ID CITY

J $5.200-0002-X$ VIENNA

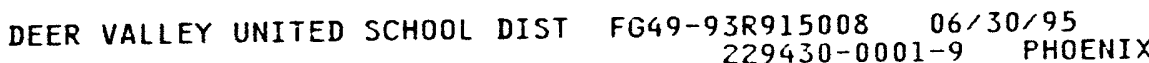

DEFENSE ANALYSES INSTITUTE FOR AI05-910R21993 03/31/95
$040000-0002-0$ WASHINGTON

DEFENSE ATOMIC SUPPORT AGENCY GMO4-76AL38664 01/01/99
$167020-0001-4$ ALBUQERQUE GM04-76AL38691 01/01/99 $167020-0001-4$ AL BUQUERQUE GM04-77AL00701 10/01/99 $167020-0001-4$ ALBUQUERQUE

DEFENSE COMMUNICATIONS AGENCY

AI01-89MA34191 09/30/94 021180-0004-9 WASHINGTON $\begin{array}{cc}\text { AI01-90DP00202 } & 06 / 27 / 95 \\ 021180-0004-9 & \text { WASHINGTON }\end{array}$ AI01-93AD34096 09/30/97
$021180-0003-0 \quad$ SCOTT

A101-91AD32061 09/30/96 021180-0005-7 ARLINGTON

DEFENSE CONTRACT ADMIN SERVICE MGMT AREA

-DEFENSE COURIER SERVICE

GM04-86AL58206 09/30/94 195030-0101-3 SEATTLE

AI01-92AD33084 09/30/97 194360-0001-4 FORT MEADE

DEFENSE DEPARTMENT OF GMO4-80AL38657 $09 / 30 / 99$
$021190-0010-7 \quad$ GOLDEN GMO4-83AL38659 09/30/99 021190-0008-5 AL BUQUERQUE GM04-52AL38660 09/30/99

$\begin{array}{ccccc}\text { CYCLE 15 } & \text { ICP GRANT AWARD } \\ & \text { AZ } & 03 & \$ 50,824 & \$ 25,412\end{array}$

COORDINATION IN CONTINUOUS FIBER CERAMIC COMPOSITES DC $01 \quad \$ 60,000 \quad \$ 60,000$ CATALOGING ALL MATERIAL IN TH E ATOMIC WPHS PROGRAM NM 01 I ESTABLISH GUIDELINES FOR TRANSFERRING DASA MATERIALS AND NM 01 $\$ 0$ $\$ 0$

PARTICIPATE IN PRODUCT CHANGE PROPOSALS TO AEC DESIGNED NM 01 $\$ 0$

ESTABLISH INTERAGENCY AGREEMENT WITH THE DEFENSE COMMUNICATI DC 01 \$707,185 $\$ 707,185$

PROVIDE ASSISTANCE HITH THE DOE EMERGENCY MANAGEMENT FACILIT DC 01 \$1,000,000 $\$ 80,000$ MAINTENANCE REPAIR OF DOE HQ'SWASHFAX IV SYSTEM FOR FY'S 19 LEASE \& MAINTENANCE OF WASHFAXIV INTERIM (I) SYSTEM $\begin{array}{lll}\text { VA } 10 & \$ 20,646 & \$ 20,646\end{array}$

CONCERNING CORPORATE ACO PROGRAM FOR MORRISON KNUDSEN HA 01 $\$ 0$

PROVIDE INTERAGENCY AGREEMENT FOR DELIVERY OF CRYPTO

$$
M D \quad 05 \quad \$ 5,000 \quad \$ 5,000
$$

AGREEMENT FOR STORAGE FACILITIES FOR WAREHDUSES NOS 343,344 CO 05

$\$ 0$

$\$ 0$

TO DELINEATE RESPONSIBILITIES TO BE ASSUMED IN CONNECTION IM 0

$\$ 0$

MUTUAL OPERATING AGREEMENT TO FURNISH GROUND INSTRUMENTATION 


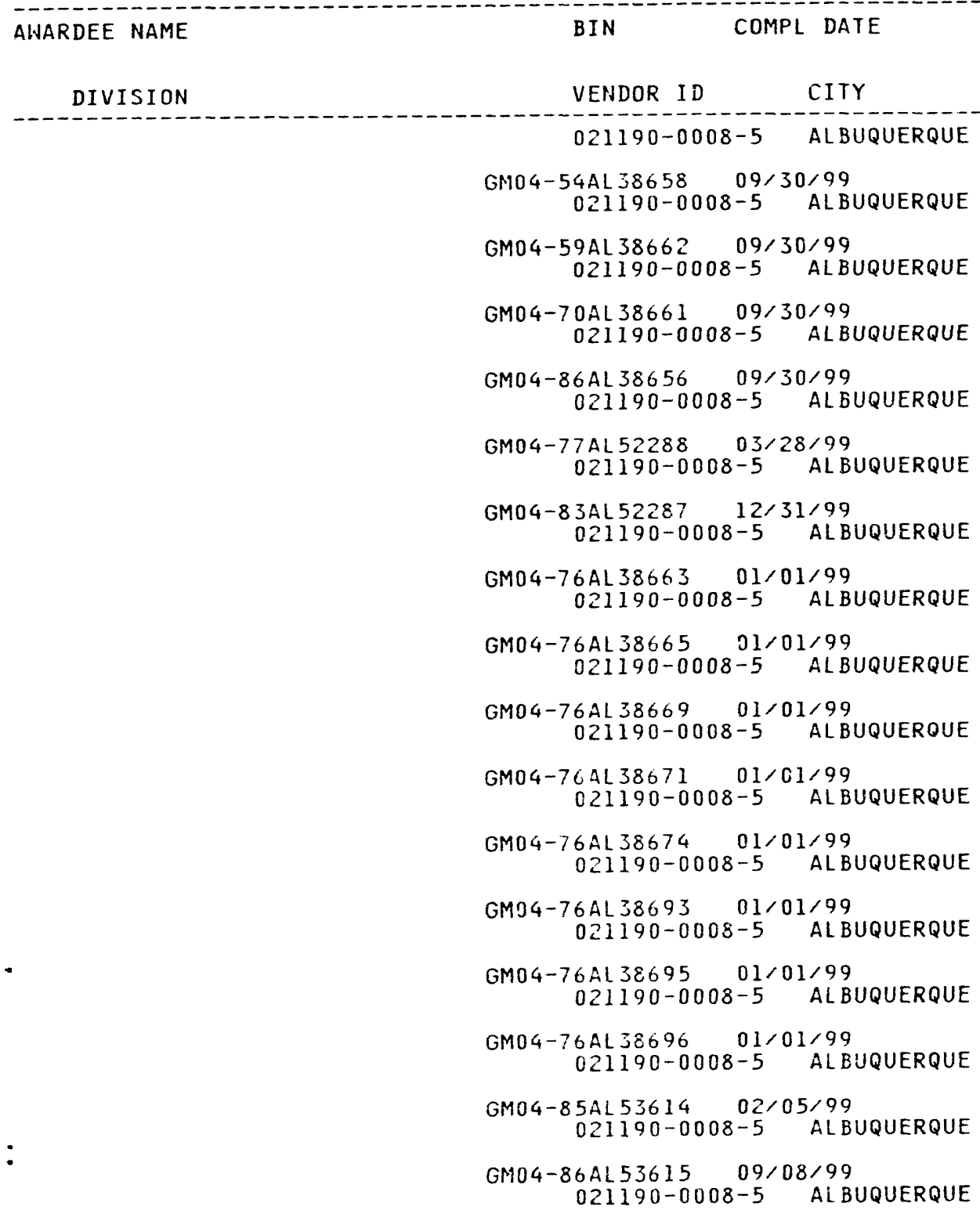

政

CONG CONTRACT
STATE DIST VALUE

DESIGN DEVELOPMENT \& PRODUCTION OF STOCKFILE CONTAINERS \&

PROVISIONING MAINTENANCE AND SUPPLY OF BASE SPARES AND MOD/

OUTLINES PRUVISIONS FOR AEC INAN DEPRATIONAL REVIEW OF NEW

DEFINES \& ELINEATES SUPPORT OFTHE PARTIES IN THE CONDUCT

USN WILL $\underset{N M}{P R O V I D E}$ SUPPORT SERVICES FOR DOE'S NORTHEASTERN

DELIHEATES RESPONSIBILITIES OFTHE PARTIES FOR THE DESIGN

IM 01 \$O

CATALOGING ALL MATERIAL IN THEATOMIC WPNS PROGRAM

MISSILE \& ROCKET RESPONSIBILITIES $\$ 01$

SALE, TRANSFER \& DELIVER OF ATOMIC WPNS TO DOD

ACCIDENT $\underset{N M S P O N S E}{\operatorname{Rin}}$ INVOLVING $\underset{\$ 0}{\operatorname{NUCLEAR}}$ WPNS IN CUSTODY OF DOD

JOINT TASK FORCE 2 - GUIDANCE IN PROVIDING ASSISTANCE TO

ALO PROVIDES REIMBURSABLE ASSISTANCE TO DOD/WSEG.
INM 01

PROVIDES REIMBURSABLE ASSISTANCE TO DEPUTY DIRECTOR TEST AND

PROVIDES AUTHORITY FOR THE JOINT ATOMIC WEAPOHS PUBLICATION

$\begin{array}{cccc}\text { ESTABLISH AN ARRANGEMENT BETHEEH THE DEPARTMENT } & \text { OF } \\ \text { NM } & 01 & \$ 0 & \$ 0\end{array}$

FROM A MANAGEMENT AND PROCEDURAL FRAMENORK FOR PRDVIDING O21190-0008-5 ALBUQUERQUE 


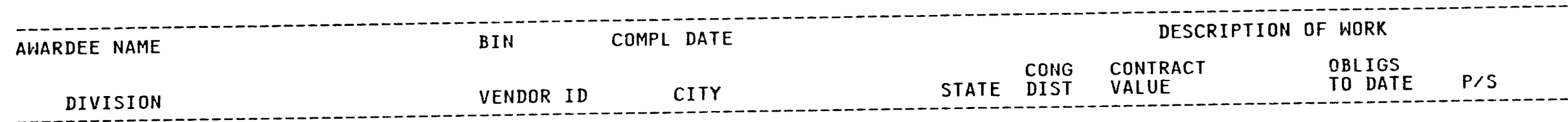

GM04-76AL $03486 \quad 09 / 04 / 99$
$021190-0008-5$ ALBUQUERQUE
GM04-78AL53653 09/12/99
$021190-0008-5$ ALBUQUERQUE
GM04-93AL84086 $06 / 23 / 98$
$021190-3408-7$ AL BUQUERQUE
AI08-76DP00630 $09 / 30 / 99$
$021190-0013-1$ LAS VEGAS
AI01-92PRI0001 $09 / 30 / 94$

DEFENSE CONTRACT AUDIT AGENCY AI01-92PRI0001 $09 / 30 / 94$ OLANDRIA

DEFENSE FUEL SUPPLY CENTER

DEFENSE INFO SYSTEM AGENCY

DEFENSE LOGISTICS AGENCY

DEFENSE FUEL SUPPLY CENTER

DEFENSE NUCLEAR AGENCY

$:$

FIELD COMMAND

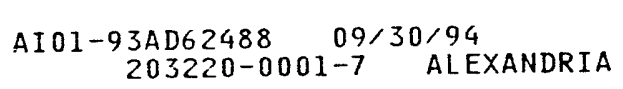

OUTLINES THE PRECEDURES FOR EMPLOYING NUCLEAR WEAPONS NM 01 \$ 00 THIS AGREEMENT ESTABLISHES POLICIES PROCEDURES, AND THIS AGREEMENT SETS FORTH THE GENERAL POLICY FOR THE $\begin{array}{ccc}\text { NM } & 01 & \$ 0 \\ \text { ESTABLISH PROCEDURES FOR COORDINATING USE OF RADIO FREQUEN }\end{array}$ NV 01 \$O CONTRACT
AUDIT COVERAGE $\&$ RELATED AUDIT SERVICES
SA $\$ 21,038,123$ TO OBTAIN GASOLINE, ETC. FROM DFSC AWARDED CONTRACTORS VIA U VA 08 \$O TELECOMMUNICATION HARDWARE \& CIRCUTS

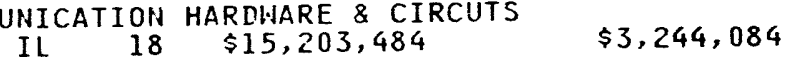

CONTINUATION OF AGREEMENT

$$
\text { CA OF AGREEMENT \$O }
$$

CONTRACT ADMINSTRATION SERVICES IN SUPPORT OF DOE CONTRACTS VA 08 \$4,929,098 $\$ 4,929,098$

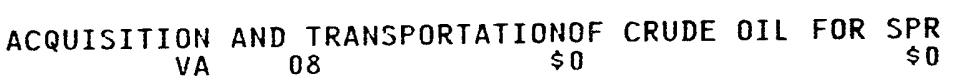
BASIC O.A.O. HO 9, FOR THE ACQUISITION AND DELIVERY TO THE $S$ VA $08 \$ 431,452,422 \quad \$ 400,249,939$ $\underset{\text { CONTINUATION OF AGREEMENT }}{\text { VA }}$ WEAPON INVENTORY AND RECONCILIATION BETWEEN DOE AL/DNA IRMD $\begin{array}{ccc}\text { NAI } & 01 & \$ 0 \\ \text { SUPPORT OF AEC TEST READINESS FACILITIES AT BARKING SANDS }\end{array}$ NM 01 \$ AIR SERVICE WITH THE LIMITATIONS OF AVAILABLE EQUIPMENT AND 


BIN COMPL DATE

AWARDEE NAME

DIVISION

VENDOR ID

CITY

STATE DIST

DESCRIPTION OF WORK

DIVISION

FIELD COMMAND

AI 04-92AL80021 09/30/94 O21210-0001-1 ALBUQUERQUE

AI08-86NV10596 07/17/96 021210-0001-1 AL BUQUERQUE

GM08-92NV10998 07/15/97
$021210-0101-8$ ALBUQUERQUE

DEFENSE RESEARCH TECHNOLOGIES

FG05-92ER81354 05/24/95
$198770-0001-6 \quad$ ROCKVILLE

DEKKER/PERICH \& ASSOC

AC04-94AL80553 05/08/99
$237700-0001-3$ ALBUQUERQUE

AC04-94AL85503 05/08/99 237700-0001-3 AL BUQUERQUE

DELAMARE STATE COLLEGE

$$
\begin{gathered}
\text { FG43-9 } 3 R 351200 \quad 12 / 31 / 94 \\
021360-0001-2 \text { DOVER } \\
\text { FG43-92R341202 06/30/95 } \\
021360-0001-2 \text { DOVER }
\end{gathered}
$$

DELAWARE STATE OF $\begin{array}{cc}\text { FG43-92R340405 } 06 / 30 / 95 \\ \text { O21380-0401-5 DOVER }\end{array}$

FG43-93R340413 03/31/95

HEALTH \& SOCIAL SVCS DEPT OF $021380-0703-0$ NEH CASTLE

DELAWARE TECH \& CMTY COLL

DELAWARE UNIVERSITY OF

MARINE STUDIES COLLEGE OF

MARINE STUDIES COLLEGE OF

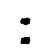

FG43-92R341203 12/31/94 021390-0002-1 GEORGETOWN

FG02-93ER61695 $08 / 31 / 96$
$021400-0703-4 \quad$ LEHES

FG02-93ER61696 08/31/96 021400-0703-4 LEWES

FG02-87ER13776 12/14/96
$021400-0703-4 \quad$ LEHES
PRINTING SERVICES FOR AL

$$
\text { NM } 01 \text { \$50,000 }
$$

$\$ 50,000$

REIMBURSE DOE FOR RADIOLOGICALMONITORING ON CACTUS CRATER, NM 01

$\$ 0$

AGREEMENT COVERING THE CONTRACTUAL ARRANGEMENTS ON JA.

NM 01

THE DEVELOPMENT OF AN OXYGEN SENSOR FOR CONTINUOUS MEASUREME

$$
\text { MD } 08 \quad \$ 549,524
$$

$\$ 338,455$

AWARD FOR A-E MISCELLANEOUS SERVICES

AWARD FOR A-E MISCELLANEOUS SERVICES

\$0 $\quad 219$

$\$ 0 \quad 4219$

TITLE III OF THE NATIONAL ENERGY CONSERVATION POLICY ACT

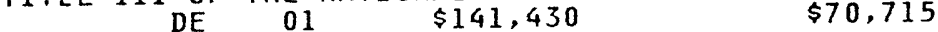

TITLE III OF THE NATIONAL ENERGY CONSERVATION POLICY ACT

$$
\begin{aligned}
& \text { OF THE NATIONAL ENERGY CONSERVATION POLICY } \\
& \text { DE }
\end{aligned}
$$

FY 192 ICP GRANT AWARD.

TITLE IV, PART A, WEATHERIZATION ASSISTANCE FOR LOW INCOME P

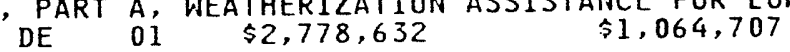

TITLE III OF THE NATIONAL ENERGY CONSERVATION POLICY ACT

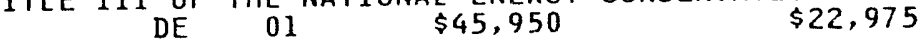

CONTROL OF LIGHT SATURATED PHOTOSYHTHESIS BY THE

DEGRADATION OF PARTICULATE ORGANIC MATTER IN COASTAL

$$
\text { DE } 01 \quad \$ 312,264 \quad \$ 100,775
$$

METABOLIC MECHANISMS OF PLANT GROWTH AT LOW WATER POTENTIALS MECHANISMS OF PLANT GRONTH AT L
$\$ 809,892$ 


\begin{tabular}{|c|c|}
\hline AWARDEE NAME & COMPL DATE \\
\hline DIVISION & $\begin{array}{l}\text { VENDOR ID CITY } \\
-84 \text { ER } 40163-04 / 30 / 97-1\end{array}$ \\
\hline PHYSICS DEPT & $021400-0301-2$ NEWARK \\
\hline & $\begin{array}{c}\text { FG02-84ERI3290 } 12 / 14 / 96 \\
021400-0001-3 \text { NEHARK }\end{array}$ \\
\hline CHEMICAL ENGINEERING DEPT & $\begin{array}{c}\text { FG02-85ER13436 } 01 / 31 / 95 \\
021400-0101-X \text { NEWARK }\end{array}$ \\
\hline CHEMISTRY \& BIOCHEMISTRY & $\begin{array}{c}\text { FG02-89ER14080 09/14/95 } \\
021400-1001-9 \text { NEWARK }\end{array}$ \\
\hline PHYSICS DEPT & $\begin{array}{c}\text { FG02-90ER45413 } 06 / 18 / 95 \\
021400-0301-2 \text { NEWARK }\end{array}$ \\
\hline & $\begin{array}{c}\text { FG02-91ER14187 } 06 / 14 / 97 \\
021400-0001-3 \text { NEHARK }\end{array}$ \\
\hline OCEANOGRAPHY PROGRAM & $\begin{array}{c}\text { FG02-91ER61184 } 07 / 31 / 94 \\
021400-1101-5 \text { NEWARK }\end{array}$ \\
\hline CHEMISTRY \& BIOCHEMISTRY & $\begin{array}{c}\text { FG02-92ER14273 } 06 / 30 / 95 \\
021400-1001-9 \text { NEWARK }\end{array}$ \\
\hline & $\begin{array}{c}\text { FG05-92ER79114 } 07 / 31 / 94 \\
021400-0001-3 \text { NEWARK }\end{array}$ \\
\hline CHEMICAL ENGINEERING DEPT & $\begin{array}{c}\text { AC22-93PC92116 } 08 / 23 / 96 \\
021400-0101-X \text { NEHARK }\end{array}$ \\
\hline & $\begin{array}{c}\text { AC22-90PC } 90050 \quad 09 / 25 / 94 \\
021400-0001-3 \text { NEHARK }\end{array}$ \\
\hline CHEMICAL ENGINEERING DEPT & $\begin{array}{c}F G 22-93 P C 93205 \quad 09 / 26 / 96 \\
021400-0101-x \text { NEWARK }\end{array}$ \\
\hline DELAWARE VALLEY COLLEGE & $\begin{array}{c}\text { FG02-93ER79173 } 06 / 14 / 95 \\
097350-0001-6 \quad \text { DOYLESTOWN }\end{array}$ \\
\hline DELMARVA POWER \& LIGHT CO & $\begin{array}{c}\text { FCO2-93CHI 0569 05/31/98 } \\
228980-0001-9 \text { NEHARK }\end{array}$ \\
\hline DELMONT CONSULTANT INC & $\begin{array}{c}\text { AC65-89WK05018 11/08/94 } \\
183120-0001-1 \text { MONTROSE }\end{array}$ \\
\hline ELPHI RESEARCH INC & $A C 21-92 M C 29107$ \\
\hline
\end{tabular}

DESCRIPTION OF WORK

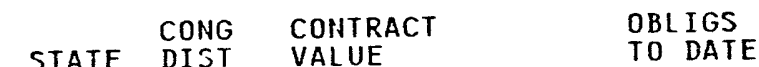
$\begin{array}{cl}\text { CONG CONTRACT } & \text { TO DATE PTE DIST VALUE } \\ \text { STATE } & \text { P TS } \\ 1984 / 1985 \text { SUMMER PROGRAMS OF THE LEHES CENTER FOR PHYSICS-- }\end{array}$ DE $\quad D 1$ $\$ 622,000$ $\$ 452,000$

A SURFACE SCIENCE INVESTIGATION OF POTENTIAL INTERMEDIATES, A SURFACE SCIENCE INVESTIGATION OF POTENTIAL $\$ 99,763$ THE GENERALIZED VAN DER WAALS THEORY OF PURE FLUIDS AND DEVELOPMENT OF AN EXP DATABASE\& THEDRIES FDR PREDICATION OF FUNDAMENTAL MAGNETIC STUDIES OF IRON-RORE-EARTH-METALLOID FUNDAMENTAL MAGNETIC STUDIES $\$ 207,026 \quad$ IRON-RORE $\$ 207,026$ LINEAR \& NONLINEAR SPECTROSCOPIC PROBING OF SOLUTE INTERACTI LINEAR \& ${ }_{D E} 01$ S $\$ 479,223 \quad \$ 313,223$ THE EFFECT OF BACTERIA \& MICROBIAL METABOLITES ON MINERAL DI THE EFFEC 01 D $\$ 351,643 \quad \$ 351,643$ OXIDATIN CATALYSIS WITH TRISCPYRAZOLYL BORATE METAL 1992 INSTRUMENTATION SYNTHESIS, CHARACTERIZATION AND REACTIV 1992 INSTRUMENTATION SYNTHESIS, CHARACTERIZATION AND
DE 01 SUPERCRITICAL CATALYSTS OF LIGHT HYDROCARBON CONVERSION DESIGN SYNTHESIS AND CHARACTERIZATION OF NOVEL FINE-PARTICLE DESIGN SYNTHESIS AND CHARACTERIZATION OF NOVEL FINE-PARTIC
DE 01 \$ $\$ 370,000,000$ AGII $\begin{array}{ccc}\text { A STUDY OF SHORT CONTACT TIME DIRECT COAL LIQUEFACTION USING } & \begin{array}{l}\text { LIQU } \\ \text { DE } 01\end{array} \\ \$ 227,992 & \$ 200,000\end{array}$

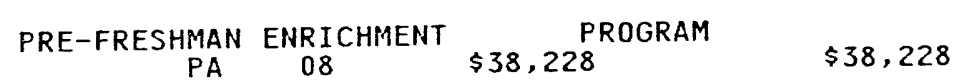
DEVELOPMENT OF A DISPATCHABLE PHOTOVALTAIC PEAK SHARING
DE
01

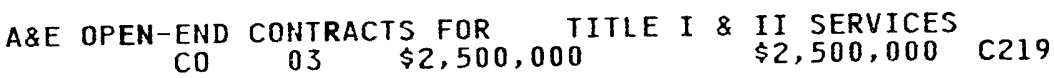
EM-DEVELOPMENT STUDIES FOR A NOVEL WET OXIDATION PROCESS 


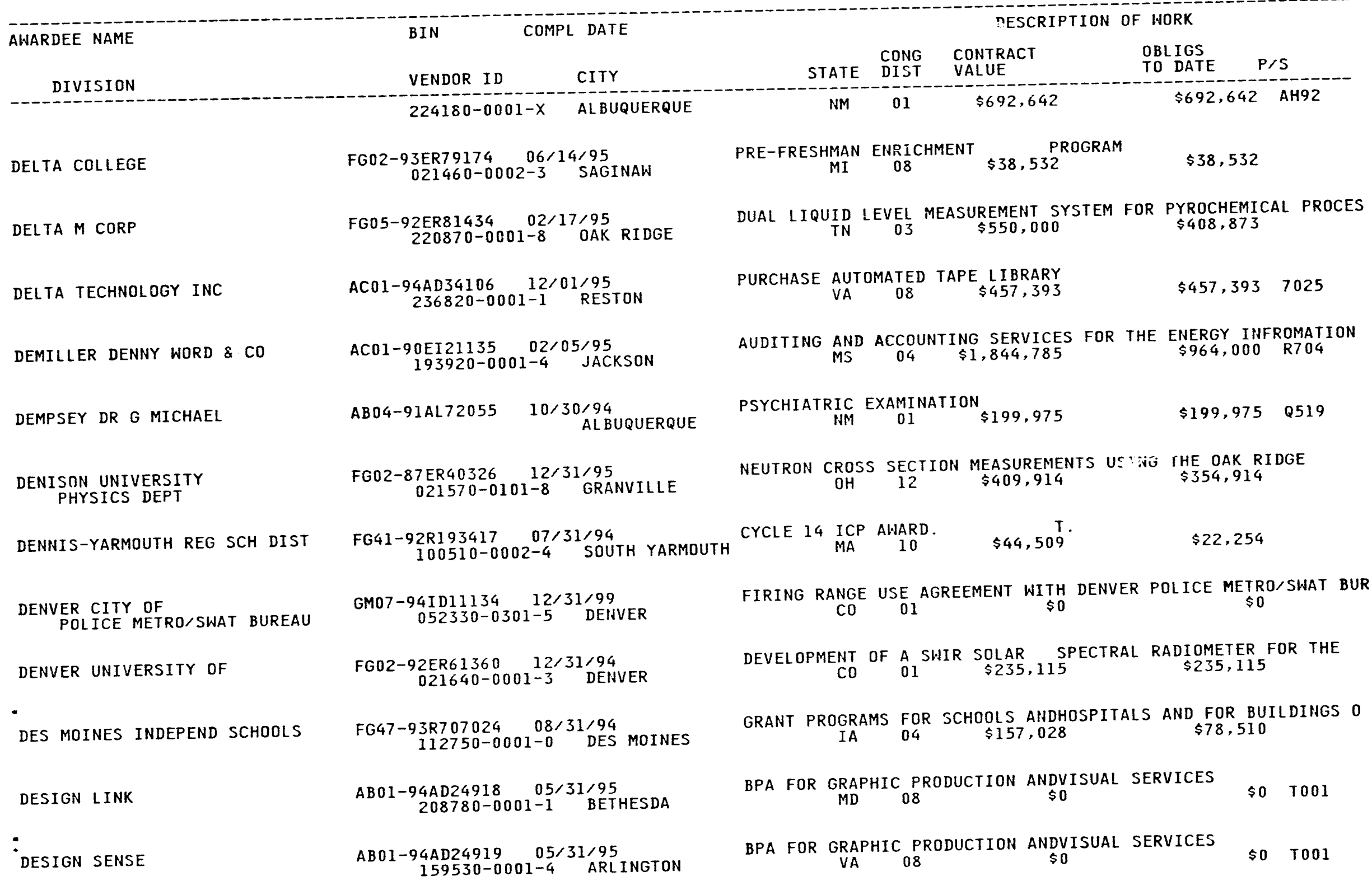


AWARDEE NAME

BIN

COMPL DATE

COMPL DATE

CITY STATE DIST VALUE

DESCRIPTION OF WORK

DIVISION

$20 R 21879 \quad 05 / 13 / 95$

AC05-920R21879 $05 / 13 / 95$
$218740-0001-8$ DECATUR

DESMEAR SYSTEMS INC

DETECTRONICS

FG03-94ER81663 $07 / 17 / 94$
LIVERMORE
FG03-93ER81486 07/29/94
$231850-0001-3 \quad$ LIVERMORE

DETROIT EDISON CO

DEVELOPMENT TECHNOLOGIES INC

DEVELOPMENT WORKSHOP

DEVER DESIGNS INC

DEXTER TOWN OF MUNICIPAL SCHOOLS

DFVLR

DHL WORLDWIDE EXPRESS

DIAL PAGE INC

DIAMOND OPTIMUM SYSTEMS
CR01-83NE44378 $03 / 15 / 30$
$021910-0002-9$ NEWPORT

ACO1-94EH89343 11/21/96
$235850-0001-0$ WASHINGTON

AC07-79ID12071 09/30/94 021960-0001-9 IDAHO FALLS

ABOI-94AD24928 05/31/95

GM04-93AL84062 11/24/97 226890-0101-2 DEXTER

GIO1-90CE32005 12/31/95
$193610-0001-25000$ SOLN90

$A B 01-93 A D 63001 \quad 07 / 01 / 94$ $164410-0003-2$ LANDOVER

ACO5-930R22098 09/30/94 234130-0001-2 KNOXVILLE

AC01-93AD33611 09/30/95 234300-0001-7 WOODLAND HILLS
MISCELLANEDUS ARCHITECT-ENGINEERING SERVICES

$$
\text { GA } 04 \quad \$ 505,204
$$

$\$ 505,204 \quad \mathrm{C} 111$

$\begin{array}{ccc}\text { PHASE I SBIR GAMMA RAY SPECTOMETERS } \\ \text { CA } & 09 & \$ 73,241\end{array}$

$\$ 73,241$

PHASE I -

$\$ 69,755$

$\begin{array}{rrrr}\text { DISPOSAL OF } & \text { SPENT NUCLEAR FUEL } \\ \text { MI } & 09\end{array}$

ADMINISTRATIVE SUPPORT SERVICES FOR THE OFFICE OF ENVIRONMEN DC O1 \$1,084,335 \$450,000 R699

JANITORIAL MAINTENANCE SERVICE
ID $02 \quad \$ 4,961,889$

$\$ 2,814,716 \quad \$ 201$

BPA FOR GRAPHIC PRODUCTION ANDVISUAL SERVICES
MD

\$0 T001

SUPPORT FOR SCIENCE, MATHEMATICS, AND ENGINEERING TO IMPROVE

INTL. AGREEMENT SMALL POWER SYSTEMS
00

$\$ 120,400$

BPA FOR AIR FREIGHT

$\$ 0$

$\$ 0 \quad$ V111

SUPPLY AND MAINTENANCE PAGERS TO DOE, OAK RIDGE, TN
TN 01

$$
\begin{aligned}
& \text { TiN } 01 \quad \begin{array}{l}
\$ 0,000 \\
\end{array} \\
& \text { CA } 01 \quad \begin{array}{lll} 
& &
\end{array}
\end{aligned}
$$




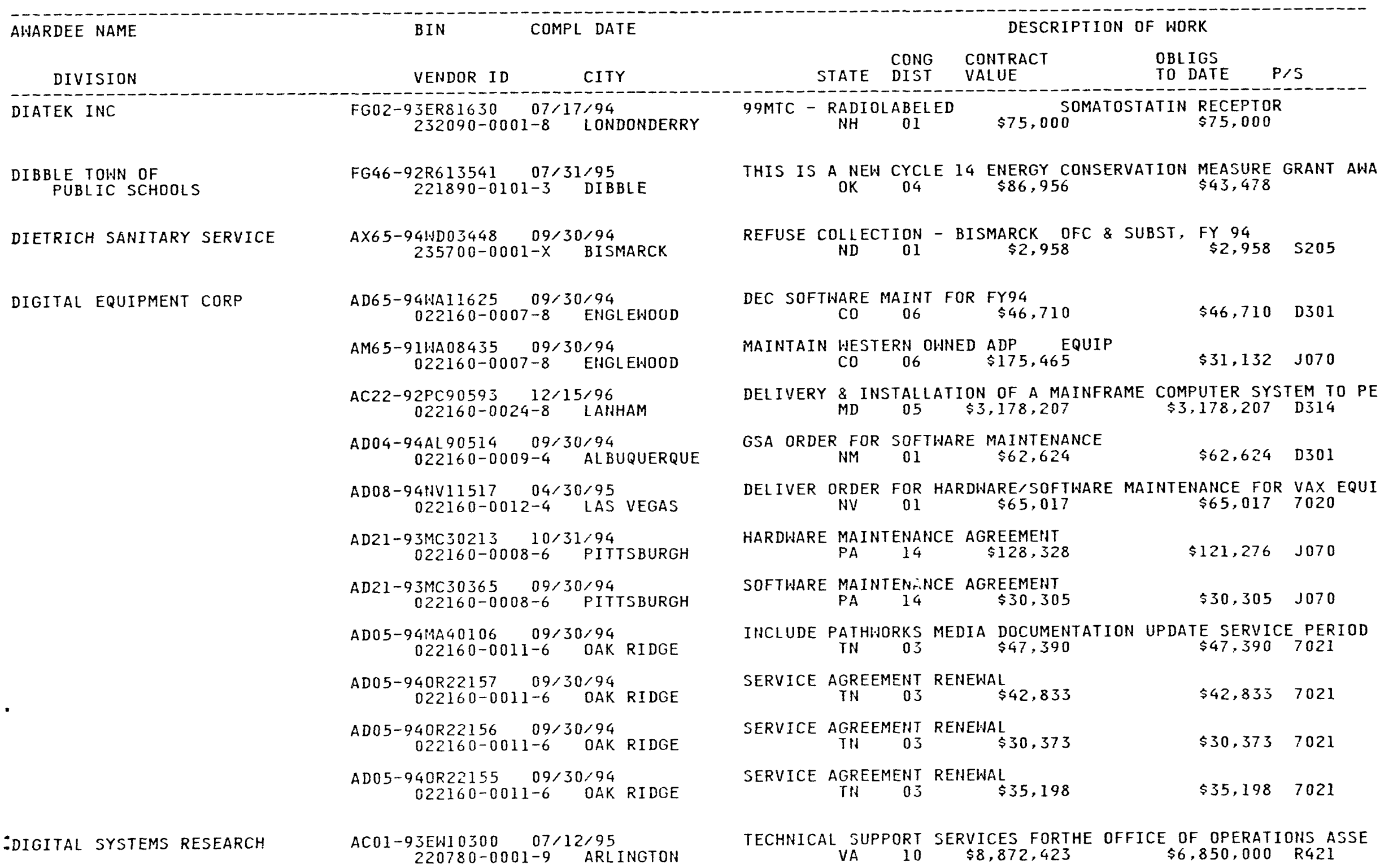


$R-0420953-001$

DEPARTMENT OF ENERGY

PROCUREMENT AID ASSISTANCE DATA SYSTEM (PADS)

$07 / 01 / 94$ NAMES BY NAME CITY \& STATE - ACTIVE AHARDS

PAGE NO. 140

\begin{tabular}{|c|c|c|}
\hline AWARDEE NAME & COMPL DATE & DESCRIPTION OF HORK \\
\hline DIVISION & VENDOR ID & $\begin{array}{l}\text { CONTRACT } \\
\text { VALUE }\end{array}$ \\
\hline DILLARD UNIVERSITY & $\begin{array}{c}\text { FG05-93ER79175 } 06 / 14 / 95 \\
208720-0001-X \text { NEW ORLEANS }\end{array}$ & $\begin{array}{cccc}\text { PRE-FRESHMAN ENRICHMENT } & \text { PROGRAM } & \text { (PREP) } & 1993 \\
\text { LA } & 02 & \$ 39,420 & \$ 39,420\end{array}$ \\
\hline DILLON POLICE DEPT & $\begin{array}{l}\text { GMO7-90ID11054 04/24/95 } \\
195810-0001-1 \quad \text { DILLON }\end{array}$ & 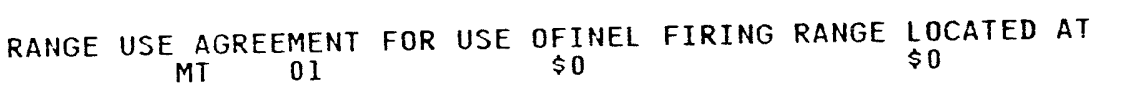 \\
\hline DIMENSIONS INTERNATIONAL INC & $\begin{array}{c}\text { AC02-90ER35086 09/14/94 } \\
203260-0001-1 \text { GERMANTOWN }\end{array}$ & 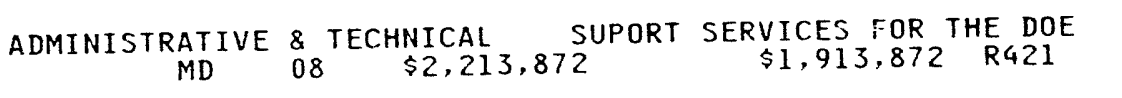 \\
\hline DIPLOMATIC LANGUAGE SERVICES & $\begin{array}{c}A B 01-94 A D 2116003 / 30 / 95 \\
226950-0001-4 \quad \text { ARLINGTON }\end{array}$ & $\begin{array}{rc}\text { TRANSLATION } & \text { SERVICES } \\
\text { VA } & 08\end{array}$ \\
\hline DISTRICT OF COLUMBIA GOVT OF & $\begin{array}{c}\text { FCOI-91EI22722 } 08 / 19 / 96 \\
022330-0003-X \text { WASHINGTON }\end{array}$ & $\begin{array}{ccc}1991-1992 \text { STATE HEATING OIL AND PROPANE PROGRAM } \\
\text { DC } 01 & \$ 33,554 & \$ 16,218\end{array}$ \\
\hline ENERGY OFFICE & $\begin{array}{c}\text { FG43-92R340409 } 09 / 30 / 97 \\
022330-0603-8 \text { HASHINGTON }\end{array}$ & DOE ORGAHIZATION ACT OF 1977 DC 01 ACT \\
\hline ENERGY OFFICE & $\begin{array}{l}\text { FG43-91R322003 09/30/94 } \\
022330-0603-8 \text { WASHINGTON }\end{array}$ & 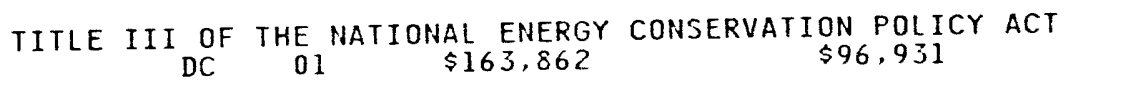 \\
\hline ENERGY OFFICE & $\begin{array}{c}\text { FG43-91R322001 } 09 / 30 / 94 \\
022330-0603-8 \text { WASHINGTON }\end{array}$ & 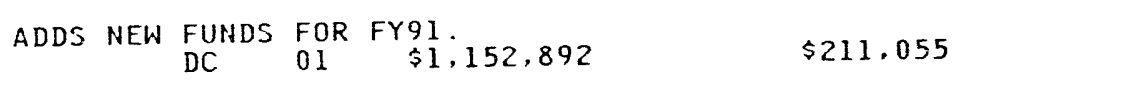 \\
\hline ENERGY OFFICE & $\begin{array}{c}\text { FG43-91R322002 } 09 / 30 / 94 \\
022330-0603-8 \text { WASHINGTON }\end{array}$ & $\begin{array}{l}\text { ENERGY EXTENSION SERVICE. } \\
\text { DC } 01\end{array}$ \\
\hline ENERGY OFFICE & $\begin{array}{c}\text { FG43-93R340427 } 09 / 30 / 98 \\
022330-0603-8 \text { WASHINGTON }\end{array}$ & 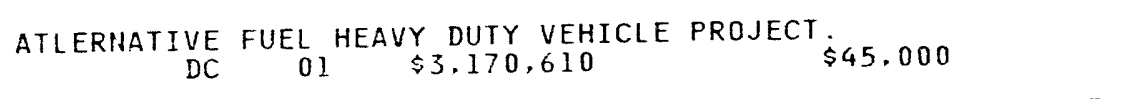 \\
\hline ENERGY OFFICE & $\begin{array}{c}\text { FG } 43-93 R 34041403 / 31 / 95 \\
022330-0603-8 \text { WASHINGTON }\end{array}$ & $\begin{array}{cccc}\text { TITLE IV, PART A. WEATHERIZATION ASSISTANCE FOR LOW INCO } \\
\text { DC } & 01 & \$ 2.045 .015 & \$ 1,230,903\end{array}$ \\
\hline CORRECTIONS DEPT & $\begin{array}{c}\text { AI01-94AD40140 03/31 } / 95 \\
022330-0201-6 \quad \text { LORTON }\end{array}$ & $\begin{array}{r}\text { FURNITURE REPAIRS } \\
\text { VA } 08\end{array}$ \\
\hline DIVERSIFIED ENGIHEERING CORP & $\begin{array}{c}\text { ACO5-890R21848 09/21/94 } \\
190990-0001-4 \text { HASHINGTON }\end{array}$ & MISC. A-E SERVICES \\
\hline DIVERSIFIED OPERATING & $\begin{array}{c}\text { FC22-93BC14954 07/31/95 } \\
236330-0001-1 \text { NEW RAYMER }\end{array}$ & $\begin{array}{l}\text { ADVANCED SECONDARY RECOVERY DEMONSTRATION FOR THE SOONER } \\
\begin{array}{cccc}\text { CO } 04 & \$ 1,577,186 & \$ 788,593\end{array}\end{array}$ \\
\hline
\end{tabular}


$R-0420953-001$

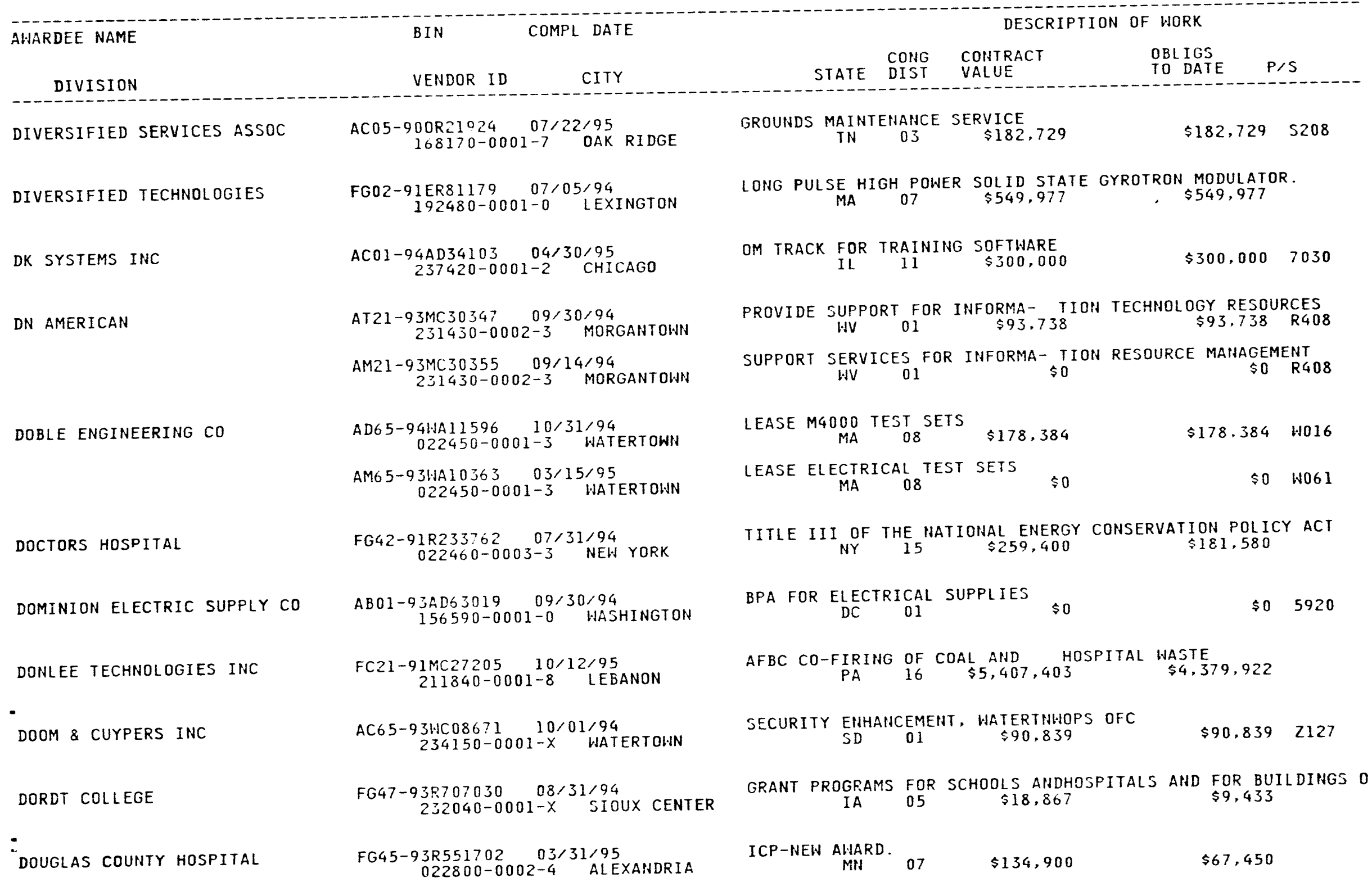




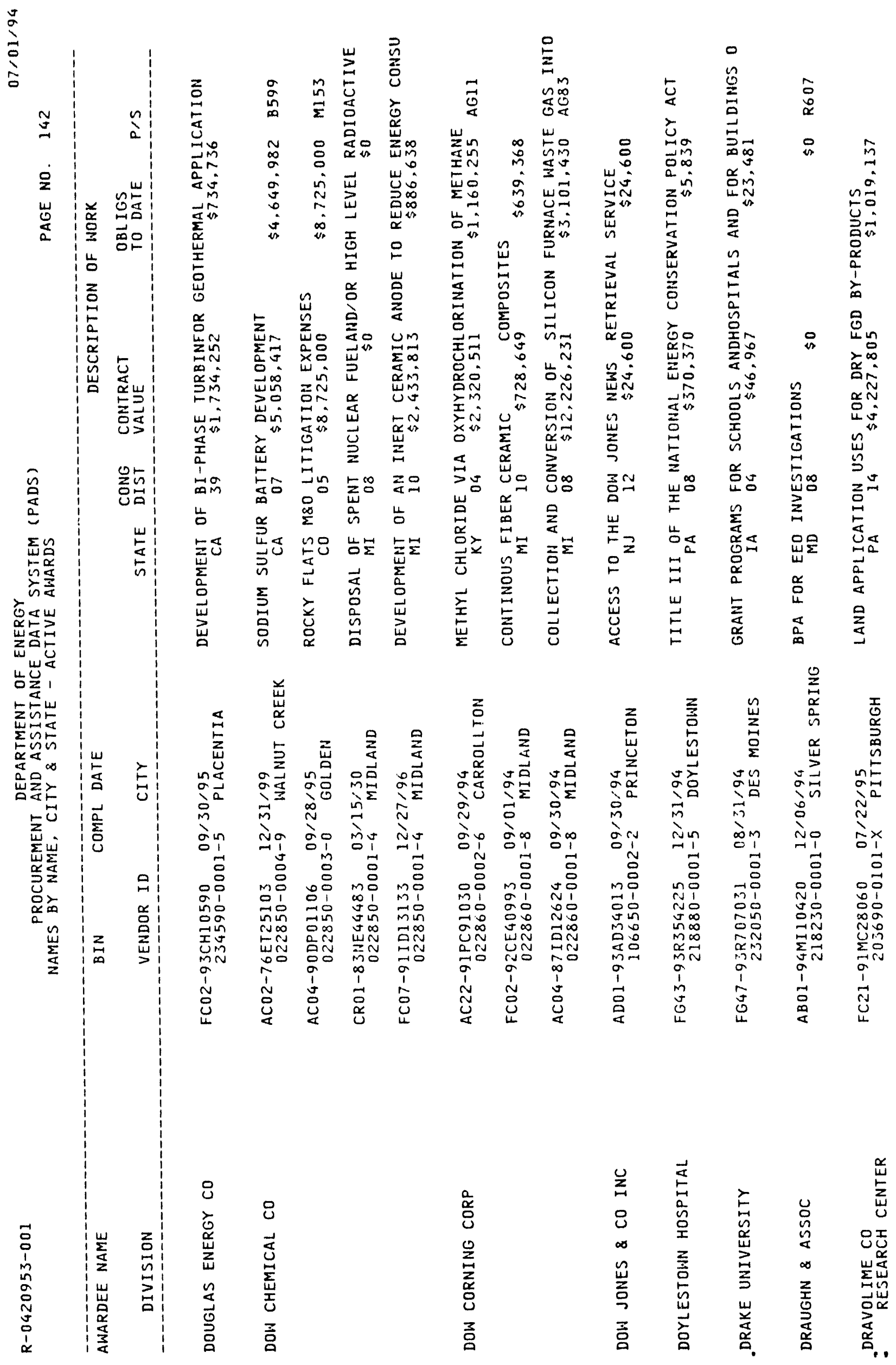




\section{AWARDEE NAME}

BIN

COMPL DATE

STATE DIST CONTRACT

DIVISION

VENDOR ID

CITY

CTATE DONST CONTRACT

OBL IGS

$\mathrm{P} / \mathrm{S}$

DREXEL UNIVERSITY

PHYSICS DEPT

DRYING TECHNOLOGY INC

DUBOIS CITY OF

DUCHESNE COUNTY OF

SCHOOL DISTRICT

DUCK RIVER ELECTRIC

DUKE POWER CO

\section{DUKE UNIVERSITY}

BOTANY DEPT

BOTANY DEPT
FG02-91ER40615 01/31/96 023000-0501-8 PHILADELPHIA

FG04-87AL44658 09/30/94 023000-0001-6 PHILADELPHIA

FG22-94PC94215 07/31/97 $023000-0001-6$ PHILADELPHIA

ACO1-92EI22374 09/30/94 $191900-0001-3$ WASHINGTON

ACO1-93AD34000 09/30/94 191900-0001-3 WASHINGTON

FG46-92R699203 08/31/94 $224780-0001-6$ SILSBEE

RL 07-76ID01287 09/30/99 023070-0001-1 DUBOIs

FG48-91R807663 10/31/94 228380-0101-9 DUCHESNE

ACO2-81FE10013 03/31/95 $023120-0001-6$ SHELBYVILLE

CRO1-83NE44379 03/15/30 023140-0001-3 CHARLOTTE

FC02-89ID12862 07/31/94 023140-0001-3 CHARLDTTE FG05-94ER25214 05/31/97
$023150-0002-5$ DURHAM

FG05-87ER13737 02/29/96 023150-1002-0 DURHAM

FG05-87ER6057509/14/96 $023150-1002-0$ DURHAM
NON-ACCELERATOR PARTICLE PHYSICS

PA 01 \$ $1,169,700$

$\$ 875,100$

THE STUDY OF FUEL PROPERTY EFFECTS ON ENGINE RELATED COMBUSPA $02 \quad \$ 1,337,346 \quad \$ 1,077,284$

CONVERSION OF COAL WASTE TO WASTE-CLEANING MATERIALS PA 02 W $\$ 233.893 \quad \$ 200,000$

SITE LICENSE FOR QUARTELY MACROECONOMIC MADEL AND INITIAL FO

ECOHOMETRIC FORCASTIIIG AND MODELING SUBSCRIPTION

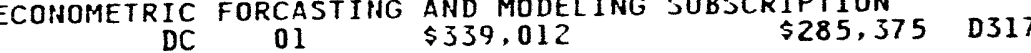

ENERGY RELATED INUENTIONS PROJECT - DESIGN, BUILD, AND INSTA

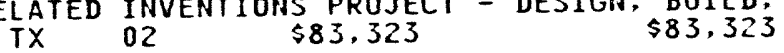

INEL TELEMENTRY SYSTEM

$\$ 0$

$\$ 0 \quad \times 293$

CYCLE XIII AWARD

$\$ 13,200$

$\$ 2,700$

BASE ELECTRICAL POWER FOR CFFF
TN $\$ 1,404,400$

$\$ 1,404,400 \quad \$ 112$

DISPOSAL OF NUCLEAR SPENT FUEL

\$0

DEVELOPMENT \& TEST OF HEAT PUMPS IN NEW APPLICATIONS FOR IND NC 09 \$2,200,000 $\$ 217,765$

EXTEHSIVE CHAOS ${ }_{\text {NC }} 02 \quad \$ 148,560 \quad \$ 46.693$

MOLECULAR STUDIES OF FUNDTIONAL ASPECTS OF HIGHER PLANT MITO NC $02 \quad \$ 608,933 \quad \$ 524,533$

INTEGRATAION OF EXPERIMENTAL AND MODELING APPROACHES IN THE NC $02 \quad \$ 3,667,548$ \$2, 645,564 


\section{AWARDEE NAME}

BIN

\section{COMPL DATE}

\section{DESCRIPIION OF WORK}

CONG CONTRACT OBLIGS STATE DIST VALUE TO DATE P/S

VENDOR ID CITY OER6 0654--01/31/97--
$023150-0502-7$ DURHAM EXTRAPOLATION MODELING CTR

CHEMISTRY DEPT FG05-88ER13931 12,31/94 023150-0202-8 DURHAM

BOTANY DEPT FG05-89ER14005 03/14/95 023150-1002-0 DURHAM FG05-90ER14141 08/14/96
$023150-0002-5$ DURHAM FG05-90ER40592 11/14/95
$023150-0702-X$ DURHAM

PHYSICS DEPT FG05-90ER6108200/14/94 $023150-0002-5$ DURHAM

PHYSICS DEPT

PHYSICS DEPT

CHEMISTRY DEPT FG05-91ER40619 02/28/97
$023150-0702-X \quad$ DUP.HAM FG05-91ER4066511/30/94 023150-0702-X DURHAM FG05-92ER6́1388 $02 / 14 / 95$
$023150-0202-8$ DURHAM FG05-92ER25145 $09,14 / 95$
$023150-0002-5$ DURHAM FG05-92ER61493 $09 / 19 / 95$
$023150-0002-5$ DURHAM FG22-93PC93219 09/22/96 023150-0002-5 DURHAM

FG44-85R432268 12/31/94 $023150-0002-5$ DURHAM

FG44-86R432416 12/31/94
$023150-0002-5$ DURHAM FG 44-93R435384 09/30/95
$023150-0002-5$ DURHAM FG44-93R435385 09/30/95 023150-1102-7 DURHAM

FG05-94ER61882 01/31/97 023150-0002-5 DURHAM DETERMINATION OF THE CELI \& MUCOUS DISTRIBUIION IN
$\$ 152,224$

STUDIES OF MULTIFREQUENCY PHASE-RESOLVED FLUORESCENCE SPECTR NC $02 \quad \$ 485,445 \quad \$ 423.890$

MOLECULAR GENETIC AND PHYSIOLOGICAL ANALYSIS OF PHOTOINHIBIT NC 02 AND PHYSIO,130 $\$ 616,130$

ANALYTICAL AHD EXPERIMENTAL STUDY OF INSTABILITIES IN BUOYAN NC $\$ 421,000$ \$235,000 NUCLEAR PHYSICS AT EXTREME ENERGY DENSITY

$\begin{array}{ll}\text { IIC } 02 & \$ 1.607 .000 \\ 1.062,000\end{array}$

TERRESTRIAL SOURCES AND SINKS IN THE GLOBAL CARBON BALANCE:

$$
\text { IIC SOURCS AND } \$ 391.746
$$

STUDIES OF NUCLEAR STRUCTURE USING NEUTRONS AND CHARGED PART HC $02 \$ 11,802,000$ \$8,042,000

HYBRID CENTRAL TRACKING
NC
TRACKING $\$ 2,728,129$
$\$ 2.728 .129$

NEH AWARD - PREPARRATIOH OF OLIGONUCLEOTIDE ARRAYS FOR HYBRI HC 02 \$734,550 $\$ 734,550$

HIGH RESOLUTION NUMERICAL METHODS FOR COMPRESSIBLE MULTI-PHA NC $02 \quad \$ 165,000 \quad \$ 105,000$

MODELING THE RESPONSE OF PLANTS AND ECOSYSTEMS TO CO2 AND CL IIC RO2 \$1,275,000 $\$ 825,000$

NOVEL CARBOH ION FUEL CELLS

NC 02 \$200,000 $\$ 200,000$

NONE

HC 02 \$200,000

$\$ 128.723$

$\begin{array}{lllll}\text { CYCLE } 8 \text { NC } & 02 & \$ 192,478 & \$ 96.239\end{array}$

SEE ATTACHED PAGE NO 2 OF THIS NOTICE OF FINANCIAL ASSISTAN HC 02 \$85,814 \$40,000

SEE ATTACHED PAGE NO. 2 OF THIS NOTICE OF FINANCIAL ASSISTAN IIC PAGE NO. 2 OF THIS NOTICE OF FINANCIAL

ONE STEP PCR SEQUENCING
$\$ 175,957$
$\$ 55,000$ 


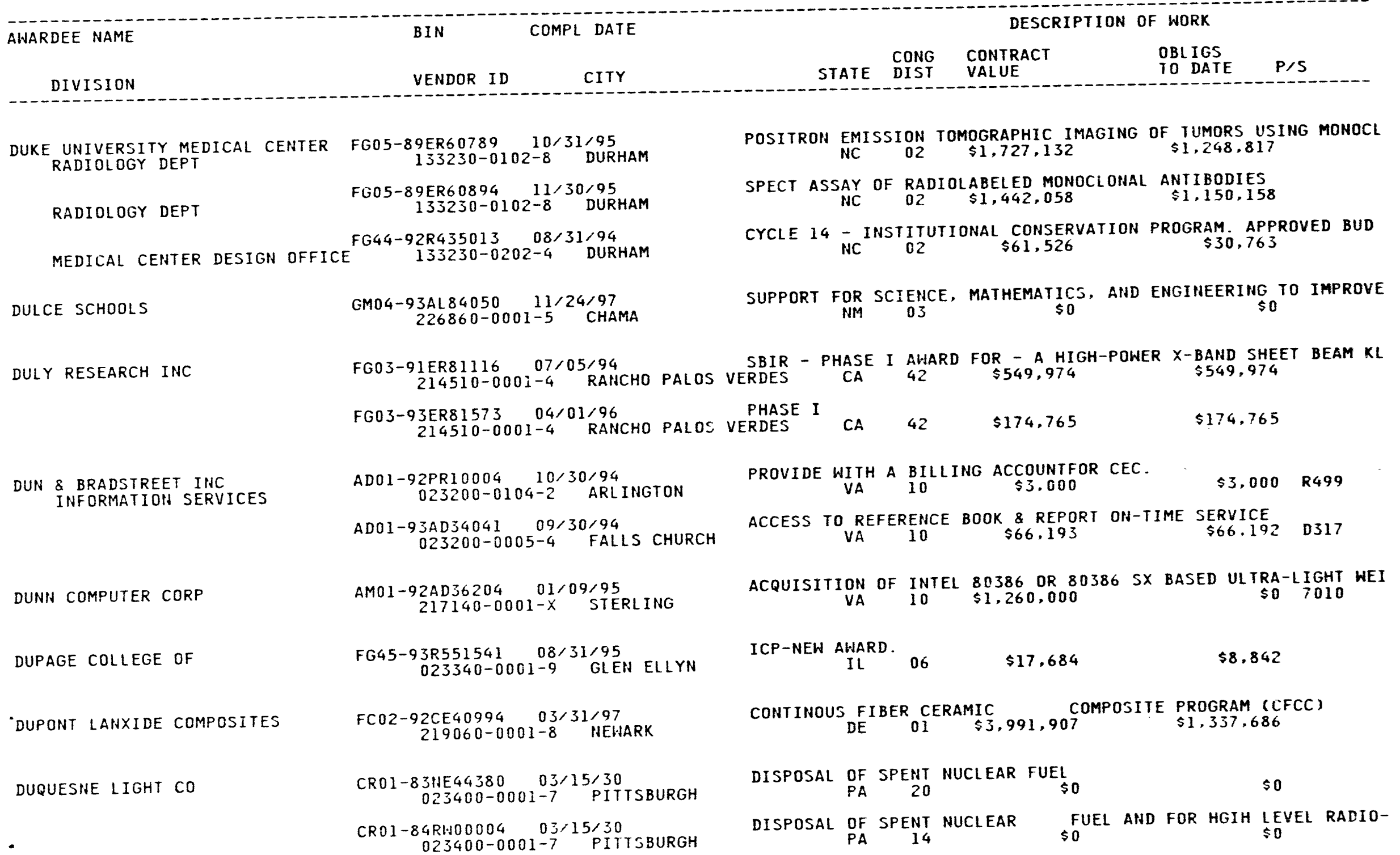




\begin{tabular}{|c|c|}
\hline AWARDEE NAME & COMPL DATE \\
\hline DIVISION & VENDOR ID \\
\hline DURABILITY & $\begin{array}{r}\text { FG01-93CE15589 } 03 / 19 / 95 \\
234060-0001-0 \quad \text { BLACKSBURG }\end{array}$ \\
\hline DWIGHT'S ENERGY DATA INC & $\begin{array}{c}\text { ACO1-92EI } 22054 \quad 12 / 11 / 94 \\
216760-0001-1 \text { RICHARDSON }\end{array}$ \\
\hline DWL ARCHITECTS \& PLANNERS INC & $\begin{array}{c}\text { ACO4-89AL55020 02/28/95 } \\
179980-0001-5 \text { AL BUQUERQUE }\end{array}$ \\
\hline DYLAKOR INC & $\begin{array}{c}\text { ADO1-93AD34090 09/30/94 } \\
227730-0001-3 \text { HOODLAND HILL }\end{array}$ \\
\hline DYLANTIC INC & $\begin{array}{cc}\text { AC07-93ID13214 } & 12 / 10 / 94 \\
225820-0001-9 & \text { IDAHO FALLS }\end{array}$ \\
\hline DYNAIR ELECTRONIC INC & $\begin{array}{c}A C D 4-92 A L 81562 \quad 12 / 31 / 94 \\
225240-0001-X \text { SAN DIEGO }\end{array}$ \\
\hline \multirow[t]{4}{*}{ DYNAMIC DECISIONS INC } & $\begin{array}{c}\text { AMOI-93EI23386 } 09 / 07 / 96 \\
221170-0001-0 \text { NEH YORK }\end{array}$ \\
\hline & $\begin{array}{c}\text { AMO1-92AD32234 } 07 / 12 / 95 \\
221170-0001-0 \text { NEH YORK }\end{array}$ \\
\hline & $\begin{array}{r}\text { AM21-92MC29465 } 09 / 30 / 94 \\
221170-0001-0 \text { NEW YORK }\end{array}$ \\
\hline & $\begin{array}{c}A B O 1-93 E I 2359009 / 09 / 94 \\
221170-0003-7 \text { JOHNSON CITY }\end{array}$ \\
\hline DYNAMICS TECHNOLOGY INC & $\begin{aligned} \text { FG03-91ER81117 } 07 / 05 / 94 \\
023620-0001-X \quad \text { TORRANCE }\end{aligned}$ \\
\hline DYNCORP & $\begin{array}{c}\text { ACO1-94AD32229 04/21/97 } \\
237440-0001-X \text { RESTON }\end{array}$ \\
\hline$=$ DYNMCDERMOTT PETROLEUM OPS CO & $\begin{array}{c}\text { AC } 96-93 P 01800003 / 31 / 98 \\
226010-0002-3 \text { HARAHAN }\end{array}$ \\
\hline
\end{tabular}




AWARDEE NAME

BIN

DESCRIPTION OF HORK

DIVISION

E \& S ENVIRONMENTAL CHEM INC COMPL DATE

CONG CONTRACT

OBL IGS $\checkmark E N D O R$ ID CITY

STATE DIST VALUE

TO DATE P/S

G02-92ER30196 03/15/96

2ER30196 03/15/96
$218170-0001-2$ CORVALLIS

E A PEDERSEN CO

$E$ G \& G MOUND APPLIED TECH

E I DUPONT DE NEMOURS \& CO INC FIBERS ADV MATERIALS SYSTS

E-MAX INSTRUMENTS INC

EA ENGINEERING SCI \& TECH INC

EAGLE RESEARCH GROUP INC

EAST ANGLIA UNIVERSITY CLIMATIC RESEARCH UNIT

EAST CAROLINA UNIVERSITY

EEAST HARTFORD PUBLIC SCHOOLS
AC65-89WC03739 08/01/94 023690-0004-X GRAND FORKS

ACO4-88DP43495 09/30/96 $176610-0001-6$ MIAMISBURG

FCO2-92CE40999 09/30/96 $023370-0104-0$ NEWARK

AC09-76SR00001 09/30/94 $023370-0001-X$ AIKEN

AC65-93WA11282 03/09/95 ENGLEWOOD

ACO1-93CE50340 01/22/96 227260-0001-0 SILVER SPRING

ACU1-93IN00623 09/29/94 023890-0001-0 ARLINGTON

FG02-86ER60397 11/30/94 NORWICH NB

FG44-94R435405 12/31/95 $4 R 435405$
$024010-0001-1 / 31 / 95$
GREENVILLE FG44-91R434665 01/30/95 1R434665 024010-0001-1 GREENVILLE FG44-92R434998 08/30/94 $024010-0001-1$ GREENVILLE

FG41-94R191604 05/30/96 216930-0001-6 EAST HARTFORD
INCROP., OF AN ORGANIC ACID REPRE. INTO MAGIC (MODEL OF

OR $05 \quad \$ 680,000$

$\$ 500,000$

CONSTRUCTION OF GRAND FORKS SUBSTATION. STAGE 06 ND $01 \quad \$ 2,245,174 \quad \$ 2,245,174 \quad Y 239$

MANAGER AND OPERATE THE GOVERNMENT-OWNED MOUND PLANT $O H \quad 03 \$ 1,458,725,178 \quad \$ 1,081,459,178 \quad M 181$

CONTINOUS FIBER CERAMIC
DE 01

OPERATION AND CONSTRUTION OF SRP

$$
\text { SC } 0313,397,197,179 \quad 11,897,147,179 \quad M 181
$$

DIGITAL FAULT RECORDERS VARIOUSITES

$$
\begin{array}{llll}
\text { CO } & 06 & \$ 201,883 & \$ 201,883
\end{array} 6625
$$

TECHNICAL AND ANALYTICAL SUPPORT SERVICES TO THE OFFICE OF $T$ $M D \quad 02 \quad \$ 3,939,553 \quad \$ 2,518,500 \quad R 425$

SUPPORT SERVICES IN THE AREA OF THREAT ASSESSMENT STUDIES FO VA 09 \$N TO4, 154 THEAT ASSES,285 R423

DETECTION OF COZ-INDUCED CLIMATE CHANGE

$00 \$ 1,220,586 \$ 1,220,586$

SEE ATTACHED PAGE NO. 2 OF THIS NOTICE OF FIMANCIAL ASSISTAN NC PAG NO. 2 OF THIS NOTICE OF FINANCIAL

CYCLE 13 - INSTITUTIONAL CONSERVATION PROGRAM. APPROVED BUD NC $01 \quad \$ 681,071 \quad \$ 340,535$

CYCLE 14 - INSTITUTIONAL CONSERVATION PROGRAM. APPROVED BUD HC 01 \$75,642 $\$ 37,821$

TITLE III OF THE NATIONAL ENERGY CONSERVATION POLICY ACT CT THE NATIONAL ENERGY CONSERVATION POL $\$ 11,965$ 


\begin{tabular}{|c|c|}
\hline AWARDEE NAME & COMPL DATE \\
\hline DIVISION & VENDOR ID \\
\hline EAST JASPER SCHOOL DISTRICT & $\begin{array}{c}\text { FG44-93R435424 } 09 / 30 / 95 \\
223190-0001-1 \text { HEIDELBERG }\end{array}$ \\
\hline EAST MISSISSIPPI JUNIOR COLL & $\begin{array}{rl}F G 44-87 R 433312 & 08 / 30 / 94 \\
164440-0001-7 & \text { SCOOBA }\end{array}$ \\
\hline EAST PENNSBORO AREA SCH DIST & $\begin{array}{c}\text { FG43-93R354204 } 12 / 31 / 94 \\
230890-0001-6 \text { ENOLA }\end{array}$ \\
\hline EAST STROUDSBURG UNIVERSITY & $\begin{array}{c}\text { FG43-93R354205 } 12 / 31 / 94 \\
204480-0001-6 \text { EAST STROU }\end{array}$ \\
\hline EAST TENNESSEE ECONOMICS CNCL & $\begin{array}{c}\text { FG05-940R22204 02/03/95 } \\
237970-0001-4 \text { OAK RIDGE }\end{array}$ \\
\hline EAST TENNESSEE MECH CONTRACTOR & $\begin{array}{c}\text { ACS5-930R21885 } 09 / 21 / 94 \\
123180-0002-6 \quad \text { KNOXVILLE }\end{array}$ \\
\hline & $\begin{array}{c}\text { AC05-930R22096 05/31/95 } \\
123180-0002-6 \quad \text { KNOXVILLE }\end{array}$ \\
\hline EAST TENNESSEE NATURAL GAS CO & $\begin{array}{c}\text { AC } 05-760 R 0402209 / 30 / 94 \\
024250-0001-1 \text { OAK RIDGE }\end{array}$ \\
\hline EAST TEXAS MEDICAL CENTER & $\begin{array}{c}\text { FG46-93R613623 } 08 / 31 / 94 \\
232660-0001-3 \text { TYLER }\end{array}$ \\
\hline EAST WEST CENTER & $\begin{array}{c}\text { FG01-91IE11052 } 03 / 30 / 95 \\
024270-0001-9 \text { HOHOLULU }\end{array}$ \\
\hline \multirow[t]{3}{*}{-} & $\begin{array}{c}\text { FG01-93EP10050 02/23/96 } \\
024270-0001-9 \text { HONOLULU }\end{array}$ \\
\hline & $\begin{array}{c}\text { FG02-93P010089 } 09 / 27 / 96 \\
024270-0001-9 \text { HOHOLULU }\end{array}$ \\
\hline & $\begin{array}{c}\text { FG02-93P010091 } 09 / 19 / 94 \\
024270-0001-9 \text { HONOLULU }\end{array}$ \\
\hline & $\begin{array}{c}\text { FGO3-92SF19167 } 07 / 30 / 94 \\
024270-0001-9 \text { HONOLULU }\end{array}$ \\
\hline
\end{tabular}

CYCLE $9 \underset{\text { INSTITUTIONAL CONSERVATION PROGRAM }}{\$ 47,047}$

$\$ 32,925$

TITLE III OF THE NATIONAL ENERGY CONSERVATION POLICY ACT
$\$ 75,000$

TITLE III OF THE NATIONAL ENERGY CONSERVATION POLICY ACT DESCRIPTION OF HORK

$\begin{array}{llll}\text { PA } & 10 & \$ 112,000 & \$ 56,000\end{array}$

EQUIPMENT GRANT FOR TECHNOLOGY2020 INITIATIVE RESULTING FRO

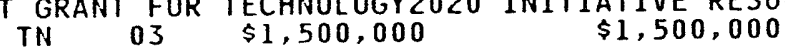

PHASE II CONSTRUCTION OF CLASSIFIED WASTE STORAGE FACILITY A TN 01 \$1,365,233 $\$ 1,365,233$ YIII TASK ORDER CONTRACT FOR OFFICECONSTRUCTION CONTRACT AT JOE NATURAL GAS FOR OAK RIDGE FACILITIES TN $0 \begin{array}{llll}03 & \$ 78,240,134 & \$ 78,146,134 & \$ 112\end{array}$ AWARDS A NEW CYCLE 15 TECHNICAL ASSISTANCE GRANT.
$\$ 50,000$ PACIFIC ISLANDS ENERGY SECURITY PROJECT $\begin{aligned} & \$ 446,080 \\ & \$ 315,806\end{aligned}$ EMERGING ENERGY SECURITY ISSUES AND OPPORTUNITIES FOR THE $U$. EMERGING ASIAN-PACIFIC ECONOMIC $\begin{gathered}\text { ENERGY SUPPORT PROJECT } \\ \$ 50,000\end{gathered}$ SUPPORT OF THE 1993 "INTERAMERICAN PETROLEUM AND HI 01 \$75,500 $\$ 47,000$ THERMAL COAL REQUIREMENTS AND PROSPECTS FOR CLEAN COAL TECHN $\begin{array}{rrrr}\text { THERMAL COAL REQUIREMENTS } \\ \text { HI } & 01 & \$ 277,754 & \$ 256,548\end{array}$ 


\begin{tabular}{|c|c|c|c|c|c|c|c|}
\hline AWARDEE NAME & BIN & L DATE & & & DESCF & F WORK & \\
\hline & VENDOR ID & CITY & STATE & $\begin{array}{l}\text { CONG } \\
\text { DIST }\end{array}$ & $\begin{array}{l}\text { CONTRACT } \\
\text { VALUE }\end{array}$ & $\begin{array}{l}\text { OBLIGS } \\
\text { TO DATE }\end{array}$ & $\mathrm{P} / \mathrm{S}$ \\
\hline
\end{tabular}

\section{EASTERN COLLEGE}

EASTERN IDAHO REGIONAL MED CTR

EASTERN ILLINOIS UNIVERSITY MATH DEPARTMENT

\section{EASTERN MENNONITE COLLEGE}

EASTERN SHORE HOSPITAL CENTER

EASTERN WASTE INDUST SVCS

EASTMAN KODAK CO

EASTON SCHDOL DEPT
FG43-94R364101 05/31/95
$024310-0001-X \quad$ ST. DAVIDS

024310-0001-X ST

1 ID11074 09/30/97
$206060-0001-1$ IDAHO FALLS

FG05-92ER79069 11/14/94
$024350-0101-0$ CHARLESTON

FG43-93R355501 03/31/95 024390-0001-9 HARRISONBURG

FG43-92R343211 02/28/95 $024430-0001-X$ CAMBRIDGE

AC01-92AD66151 08/31/95 220770-0001-5 FREDERICK

AD65-94WA11611 09/30/94 024480-0006-9 ENGLEWOOD

AD65-94WA11683 12/31/94 $024480-0004-2$ ROCHESTER

ADO4-94AL $9212209 / 30 / 95$ 024480-0004-2 ROCHESTER

AD01-93AD23648 09/30/94 024480-0002-6 ARLINGTON AB01-93AD23649 $09 / 30 / 94$
$024480-0002-6$ ARLINGTON AB01-93AD23650 09/30/94 $024480-0002-6$ ARLINGTON

FG41-93R192517 06/30/95
$024490-0001-1 \quad$ EASTON
TITLE III OF THE NATIONAL ENERGY CONSERVATION POL ICY ACT

$$
P A \quad 07 \quad \$ 36,990 \quad \$ 18,495
$$

MOU TO PROVIDE OPTIMAL CARE FOR THE EMPLOYEES OF THE (INEL) ID 02

PRE-FRESHMAN ENRICHMENT PROGRAM (PREP) - 1992

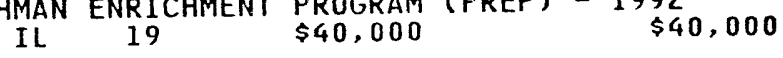

TITLE III OF THE NATIONAL ENERGY CONSERVATION POLICY ACT

$$
\text { VA } 06 \text { \$185,812 } \$ 100,000
$$

TITLE III OF THE NATIONAL ENERGY CONSERVATION POLICY ACT

$$
\text { MD } 01 \text { THE NATIONAL ENERGY CONSERVATION } \$ 48,807
$$

TRASH REMOVAL SERVICES FOR GTN

MD $06 \quad \$ 358,000$

$\$ 357,999 \quad \$ 205$

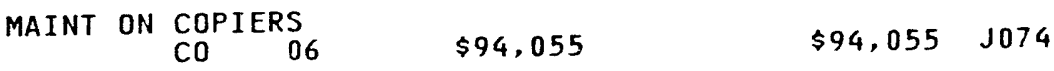

MAINTENANCE ON PRINTERS 28 \$37,376 $28,37,376 \quad 7035$

MAINTENANCE ON KODAK COPIERS

CONTINUED MAINTENANCE $\$ 881,979 \quad \$ 577,950 \quad J 036$

BPA FOR AFTER HOURS \& MUSIC REPAIRS ON KODAK EQPT $\underset{\$ 0}{\$ 0}$ J036 BPA TO CONTINUE COPIER RELOCATION SERVICES ON KODAK EQPT VA 09 \$0

TITLE III OF THE NATIONAL ENERGY CONSERVATION POLICY ACT

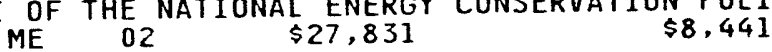




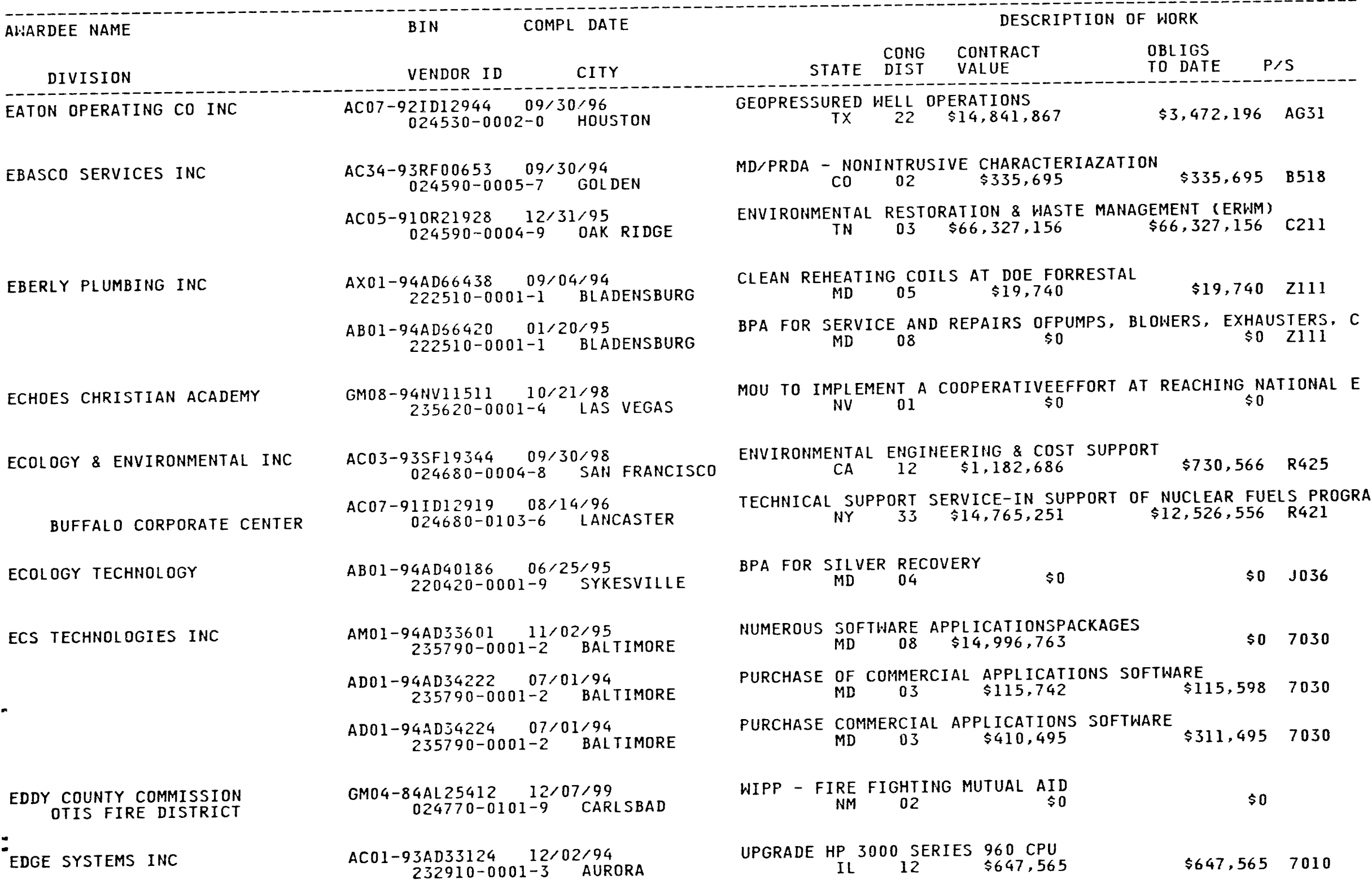




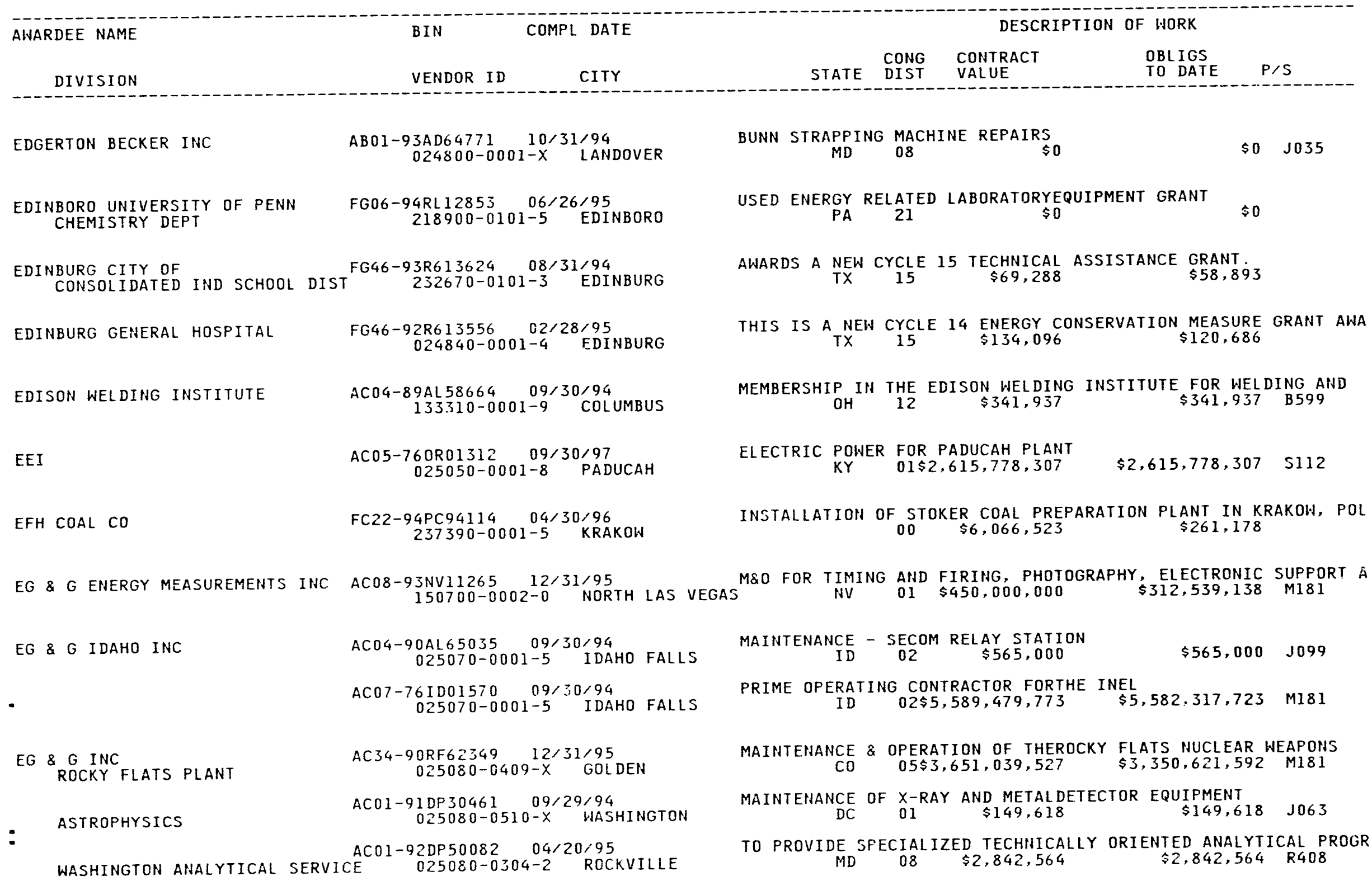




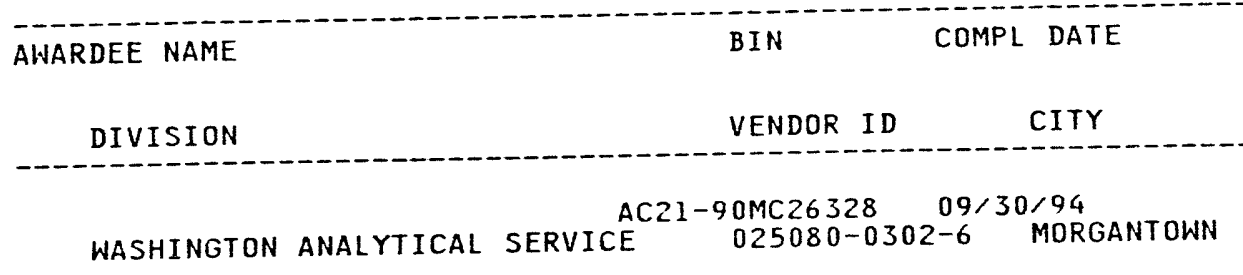

EG \& G SPECIAL PROJECTS INC

EGGEN WEED CONTROL INC

\section{EGGHEAD SOFTWARE}

EGYPT ARAB REPUBLIC OF

EHOVE BOARD DF EDUCATION

EIC LABORATORIES INC

ACO1-93SA10161 07/31/94 $35 A 1016107 / 31 / 94$
$230990-0001-9$ WASHINGTON AC65-92WG17240 12/16/94
$216800-0001-2 \quad$ SCOTTSDALE AB01-93EI23616 $07 / 11 / 94$
$183430-0004-8$ FCO2-93P010100 09/29/95
$234420-0001-7 \quad$ GIZAH FG45-92R540178 12/31/94
$209700-0001-4$ FG02-93ER81609 07/17/94
$025140-0002-5 \quad$ NORWOOD AC21-92MC29108 $09 / 29 / 94$
$025140-0002-5 \quad$ NORHOOD

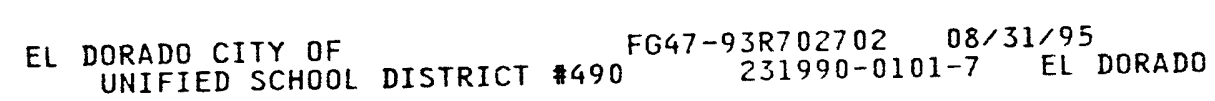

EL PASO SCIENCE MUSEUM $-$

ELBA CITY OF

ELEANOR ROOSEVELT INSTITUTE $-$
FG03-94ER75954 12/14/95
$236420-0001-0$ EL PASO

FL07-80ID44002 $12 / 15 / 99$
$025260-0001-7$ ELBA

FG03-94ER61819 $01 / 31 / 97$
$207010-0001-5$ DENVER

FG03-93ER61670 00/14/95
$207010-0001-5$ DEHVER
ON-SITE SUPPORT SERVICES FOR THE MORGANTOWN ENERGY

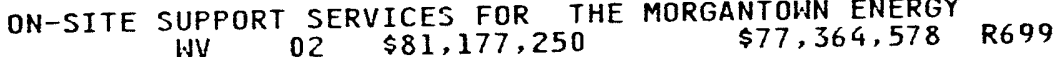

CORRECTIVE \& PREVENTIVE MAINTENANCE, MODIFICATION TO INSTALL

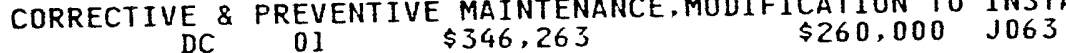

BASE YEAR OF WEED REMOVAL AND STERILIZATION OF VARIOUS

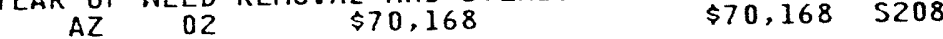

BPA FOR PC SOFTWARE/UPGRADES 07 \$0

$\$ 0 \quad 7035$

CLIMATE CHANGE COUNTRY STUDY.

$I C P-N_{O H}{ }_{O H A R D}$ OS $\$ 70,824 \quad \$ 35,412$

ELECTROCHRONIC GLAZINGS DEPOSITED BY HIGH RATE

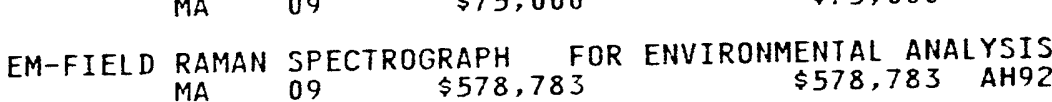

GRANT PROGRAMS FOR SCHOOLS ANDHOSPITALS AND FOR BUILDINGS 0 $\begin{array}{llll}\text { KS } & 04 & \$ 187,130 & \$ 93,565\end{array}$

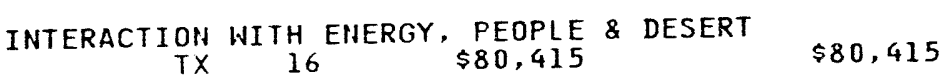

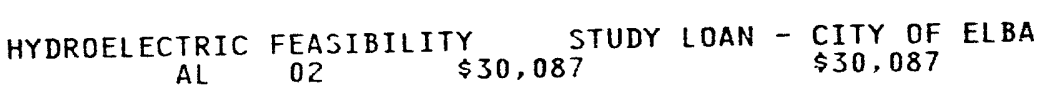

HUMAN GENOME LIBRARIES
CO 01

WORKSHOP ON IDENTIFICATION OF TRANSCRIBED SEQUENCES 


\begin{tabular}{|c|c|c|c|c|c|c|c|}
\hline AWARDEE NAME & BIN & DATE & & & DESCR & WORK & \\
\hline & VENDOR ID & CITY & STATE & $\begin{array}{l}\text { CONG } \\
\text { DIST }\end{array}$ & $\begin{array}{l}\text { CONTRACT } \\
\text { VALUE }\end{array}$ & $\begin{array}{l}\text { OBLIGS } \\
\text { TO DATE }\end{array}$ & $P / S$ \\
\hline
\end{tabular}

ELECTRIC POWER RESEARCH INST

\section{ELECTRO ENERGY INC}

ELECTROCHEMICAL SOCIETY

ELECTRONIC BALLAST SYSTEMS

\section{ELEMENTARY WORKSHOP}

ELIZA COFFEE MEMORIAL HOSPITAL FG44-93R435296 08/31/94 $230780-0001-X$ FLORENCE

ELKHART COMMUNITY SCHOOLS

ELMORE COMMUNITY HOSPITAL

FG45-92R540017 08/31/95
$208910-0001-1$ ELKHART

FG45-93R551583 08/31/95 208910-0001-1 ELKHART

FG44-92R435022 08/15/94 222140-0001-1 WETUMPKA

ELMORE MEDICAL CENTER HOSPITAL : ELMWOOD OFFICE PARK

RL $96-88 P 01415003 / 31 / 98$
$169820-0001-X$ HARAHAN
GM04-81AL53610 09/29/99
$025290-0001-8$ PALO ALTO FC06-93RL12450 09/30/98 FG22-94PC93256 02/23/99 025290-0001-8 PALO ALTO

FG02-93ER1437909/14/94 $233970-0001-7$ DANBURY

FG02-87CE34012 05/31/96 025350-0002-4 PENNINGTON

FG44-93R410609 09/30/94 235320-0001-6 MIAMI

FG43-92R341204 06/30/95 219380-0001-3 WILMINGTON
ADVANCE IN AREAS OF MUTUAL INTEREST, THE SAFETY, $\underset{\$ 0}{\$ 0}$ RELIABILITY DESIGN OF A SPENT FUEL DRY TRANSFER SYSTEM FOR USE WITH

CO-FUNDING OF THE EPRI HIGH SULFUR TEST FACILITY CA 12 \$10,498,700

$\$ 800,000$

$\begin{array}{lccc}\text { RESEARCH IN } & \text { BIPOLAR } & \text { NICKEL-EMTAL HYDRIDE } \\ \text { CT } & 05 & \$ 194,378 & \$ 194,378\end{array}$

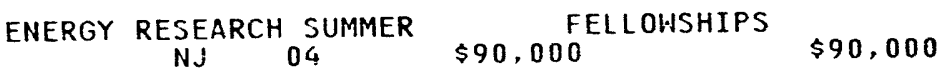

ELECTRONIC HID
FL
21

TITLE III OF THE NATIONAL ENERGY CONSERVATION POLICY ACT DE $01 \quad \$ 72,500 \quad \$ 37,200$

SEE ATtACHED PAGE NO. 2 OF THIS NOTICE OF FINANCIAL ASSISTAN AL 05 \$15,600 $\$ 10,920$

ICP - NEW AWARD

$\$ 195,300$

$\$ 76,776$

ICP-NEW AWARD.

$\$ 104,592$

$\$ 50,000$

CYCLE 14 - INSTITUTIONAL CONSERVATION PROGRAM. APPROVED BUD $A L \quad 03 \quad \$ 119,732 \quad \$ 100,000$

AWARD CY XIII ALLOCATION

$$
\begin{aligned}
& \text { XIII ALLOCATION } \\
& \text { ID } \\
& \text { OI }
\end{aligned}
$$

$\$ 37,023$

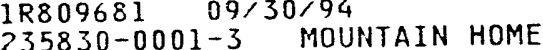

OFFICE SPACE LEASE $\$ 2, \$ 12,166,094$

$\$ 8,086,253 \times 111$ 


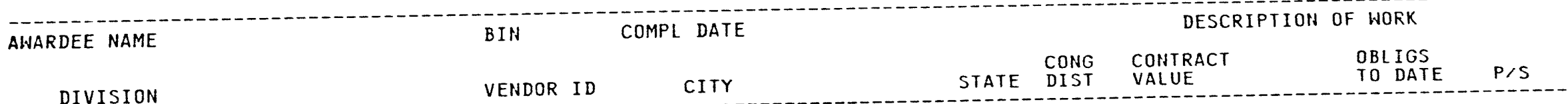

ELTRON RESEARCH INC

EMEC CONSULTANTS

EMERY WORL DWIDE

EMORY UNIVERSITY FG05-91ER25105 $08 / 31 / 94$ MATHEMATICS \& COMPUTER SCIENCE 025880-0501-2 ATLAN

CHEMISTRY DEPT

EMP ASSISTANCE PROGRAM INC

EMPORIA STATE UNIVERSITY

$-$

EMRICH \& DITHMAR
FG05-86ERI3568 06/30/97
$025880-0201-3$ ATLANTA

FG05-93ER61737 03/24/97 $025880-0001-0$ ATLANTA

FG02-93ER8 $1506 \quad 07 / 17 / 94$
$025710-0003-9 \quad$ BOULDER

FG02-90ER80910 11/19/94 025710-0002-0 AURORA

FG02-91ER81164 07/05/94 025710-0002-0 AURORA

FG02-91ER81165 09/05/95 025710-0002-0 AURORA

$\begin{array}{cc}\text { FG02-92ER8 } 1344 & 05 / 24 / 95 \\ 025710-0002-0 & \text { AURORA }\end{array}$

$\begin{array}{cc}\text { ABO1-93AD6 } 2487 & 09 / 30 / 94 \\ 025840-0006-7 & \text { SCRANTON }\end{array}$

$A B 01-93 A D 63000 \quad 07 / 01 / 94$ $025840-0005-9$ STERLING

AC65-93HA08719 09/30/94 $234020-0001-6$ DEIVER

FG47-92R?02696 08/31/94 $025900-0001-4$ EMPORIA

AC02-92CH10507 09/21/94 2CH10507 09/21/94
FC07-91ID13104 c 12/31/95
$211830-0001-4$
EXPORT
NATURAL GAS CONVERSION TO ETHYLENE IN SUTEMEDIATE PEROVSKIT SOLID ELECTROYTES FOR INTERMEDIATE TEMPEATURE PLROVSKIT SOLID ELECTROYTES 14
$\$ 549,775$ DISTRIBUTED FIBER OPTICS SENSOR FOR REVERSIBLE DETECTION OF IL 14 \$ $\$ 549,949$ $\$ 549,949$

SPONTANEOUS NATURAL GAS OXIDATIVE DIMERIZATION ACROSS MIXED IL 14 \$549,995 $\$ 549.993$

ELECTROCHEMICAL NATURAL GAS REDUCTION TO ALCHOLS $\begin{array}{lll}\text { IL } 14 & \$ 549,979 & \$ 338.737\end{array}$

ELECTROLYSIS OF NEODYMIUM OXIDES PA 20 \$2,869,813

$\$ 948,140$

BPA FOR AIRFREIGHT SHIPMENTS

\$0 V111

BPA FOR AIR FREIGHT

$\$ 0$

$\$ 0 \quad$ V111

NETWORK BASED HIGH-PERFORMANCECONCURRENT COMPUTING GA $05 \quad \$ 137,140 \quad \$ 137,140$

THEORETICAL STUDIES OF COMBUSTION DYNAMICS

THE DEVELOPMENT OF NEW NEURODENGERATING COCAINE ANALYOGUES $F$ $G A \quad 04 \quad \$ 631,205 \quad \$ 263,147$

EMP ASSIST PRGMS FOR WAPA

$\$ 84,218 \quad 0999$

GRANT PROGRAMS FOR SCHOOLS ANDHOSPITALS AND FOR BUILDINGS 0 KS $05 \quad \$ 147,197 \quad \$ 70,911$

SUPPOET SERVICES - PATENT PREPARTATION \& PROSECUTION

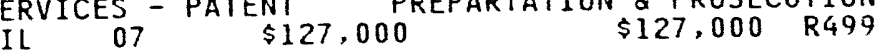




\begin{abstract}
AWARDEE NAME
\end{abstract}
BIN

COMPL DATE

VEHDOR ID

CITY

STATE CONG
DESCRIPTION OF WORK

VALUE
OBLIGS
TO DATE
DIVISIDN

ENCOAL CORP

ENCDRE COMPUTER CORP

ENCORE TEMPORARY INC

ENERGAS CO

ENERGETICS INC

ENERGY \& ENV RESEARCH CORP

\section{FC21-90MC27339 09/17/94 202910-0001-0 GILLETTE \\ FR21-90MC27356 09/17/14 202910-0001-0 GILLETTE} AD65-94WG24121 $10 / 30 / 94$
$226060-0001-3$ FORT LAUDERDALE

ABO1-94DP00367 02/09/95 4DP00367 02/09/95
$216110-0001-7$ WASHINGTON AB01-94AD38002 10/31/94 $216110-0001-7$ WASHINGTON

AC04-93AL 93313 09/30/97 $93 A L 93313$
$065350-0002-8$ AMARILLO

AC01-94FE63260 03/27/96 AFE63260 $03 / 27 / 96$
$025930-0001-5 \quad$ COLUMBIA ACO1-90EH89030 05/03/95 $025930-0001-5$ COL UMBIA ACO1-91EH89028 01/24/96 $025930-0001-5$ COLUMBIA

ACO1-93CE40969 01/18/95 $025930-0001-5$ COLUMBIA

AC21-92MC29231 $08 / 27 / 94$ 2MC29231 $02530-0001-5$ COLUMBIA

AC22-90PC90165 08/25/94
$025980-0001-3$ IRVINE FC22-91PC $90547 \quad 12 / 31 / 94$
$025980-0001-3 \quad$ IRVINE FC22-87PC79796 12/31/94

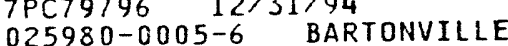

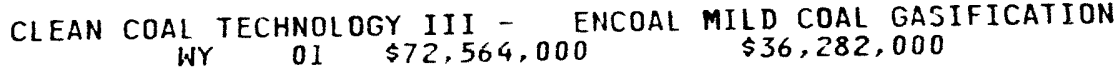

REPAYMENT AGREEMENT; CLEAN $\underset{\text { COAL TECHNOLOGY III; ENCOAL }}{\$ 01}$ SO

MAINTENANCE ON CONCEPT $32 / 87$ SYSTEM

FL 01 \$ $\$ 73,692$

$\$ 73.692 \quad 7010$

BPA FOR TEMP. SECRETARIES

DC. SECRETARIES 01 \$0

\$0 $\quad R 699$

BPA FOR TEMPORARY SECRETARIES

$\$ 0$

$\$ 0 \quad R 699$

TRANSPORTATION OF NATURAL GAS TO THE DOE PANTEX PLANT.
$\$ 0$

TECHNICAL SUPPORT TO THE OFFICE OF FE, OFFICE OF OAR TECHNICAL SUPPORT SERVICES FORTHE OFFILE OF NEPA PROJECT AS MD 03 \$3.662.847 \$3.662,847 B510 TECHNICAL, ANALYTICAL \& ADMINISTRATIVE SUPPORT SERVICES TD T $\dot{M D}{ }_{03} \$ 6,667,354 \quad \$ 6,667,354$ R408

ENGINEERING/SCIENTIFIC ANALYSIS; ENVIRONMENTAL HEALTH MD 05 SCIENTIFIC 429,308 \$130,000 R425

EM-SUPPORT SERVICES FOR ENVIRONMENTAL RESTORATION AND $M D \quad 03 \quad \$ 2,693,356 \quad \$ 2,693,356 \quad R 425$

UTILIZATION OF COAL-WATER FUELS IN FIRE-TUBE BOILERS

EVALUATION OF GAS REBURNING AND LOW NOX BURNERS ON A WALL FI CA 40 今16,174,582 $₹ 8,087,290$

ENHANCING THE USE OF COALS BY GAS REBURNING SORBENT INJECTIO $\begin{array}{lrrr}\text { THE USE OF COALS BY GAS REBURNING SORBENT } \\ \text { IL } & 17 & \$ 42,204,998 & \$ 18,747,816\end{array}$ 


AWARDEE NAME
DIVISION
PROCESS DEVELOPMENT

ENERGY \& ENVIR ANALYSIS INC

ENERGY \& ENVIR RES CORP

ENERGY CONCEPTS CO

ENERGY CONVERSION DEVICES INC

\section{ENERGY DEPARTMENT OF} ROCKY FLATS

\section{WESTERN AREA POWER ADMIN}

WESTERN AREA POWER ADMIN

SAVANHAH RIVER OPERATIONS OFF

- haterways EXPERIMENT Station PITTSBURGH NAVAL REACTORS OF IDAHO OPERATIONS OFFICE 三 IDAHO OPERATIONS OFFICE

$$
\text { COMPL DATE }
$$

CONG COHTRACT STATE DIST VALUE OBLIGS VENDOR ID CITY CITY 025980-0306-3 BRILLIANT

AC21-93MC28139 05/17/96 025960-0001-6 ARLINGTON

FR22-91PC90582 06/11/14
$025980-0001-3$ IRVINE

AC21-87MC23299 07/31/95 025980-0003-X ORRVILLE

FG01-91CE15404 03/10/95 026030-0001-2 AHHAPOLIS

FGOI-93CE15557 08/22/95 026030-0001-2 ANIJAPOLIS

FCO2-93CH10571 09/01/97
$233470-0001-3$ TROY

GM13-90GJ30007 12/29/95 026050-1720-6 DENVER

PM34-87AL45748 09/30/94 $026050-0715-4$ LOVELAND

AI34-92AL8259100/30/96 026050-0715-4 LOVELAND

GM04-83AL53646 02/16/99 $026050-1218-2$ LARGO

GM07-94ID11139 11/12/98 GM07-841D11017 09/30/99
$026050-1904-7$ IDAHO FALLS GM07-86ID11020 09/30/99 026050-0504-6 IDAHO FALLS GM07-81ID11019 09/30/99 026050-0504-6 IDAHO FALLS OF TOXIC EMISSIDNS-FROM A COAL-FIRED POHER PLANT $18.42,820$

RESEARCH ENTITLED "DEVELOPMENTOF A GAS INFORMATION SYSTEM

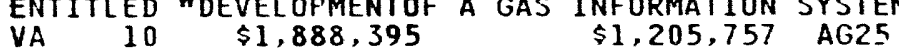

REPAYMENT AGREEMENT FOR EVALUATION OF GAS REBURNING AIND LOH CA 40 \$0

AFBC SYSTEMS CONCEPTS APPLICABLE TO THE COMMERCIAL

STEAM-METHANE REFORMING IN MOLTER CARBONATE SALT

$\begin{array}{llll}M D & 04 & \$ 71,964 & \$ 71,964\end{array}$

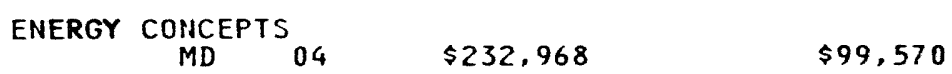

DEVELOPMENT OF A ROOF TOP PHOTOVOLTAIC SYSTEM

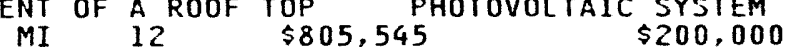

PROGRAMMATIC AGREEMENT FOR ENVIRONMENTAL RESTORATION

FIRM ELECTRIC SERVICE FOR ROCKY FLATS PLANT

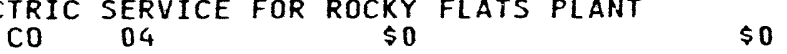

ELECTRIC TRANSMISSION SERVICE TO ROCKY FLATS PLANT $\mathrm{CO} \quad 04 \quad \$ 323,024 \quad \$ 0$

ESTABLISHES AN AGREEMENT BETWEEN THE PARTIES CONCERNING THE $\mathrm{FL} 08$ \$0 $\$ 0$

MEMORANDUM OF AGREEMENT BETWEEN U.S. ARMY ENGINEER WATERWAYS ID 02

MEMORANDUM OF UNDERSTANDING TODOCUMENT THE RELATIONSHIP \&

MEMORANDUM OF UNDERSTANDING BETWEEN ID \& AL THAT ESTABLISHES ID 02 \$0

MEMORANDUM OF UNDERSTANDING TOESTABLISH PROCEDURES FOR REVI 


AWARDEE NAME \\ COMPL DATE}

VENDOR ID CITY

DIVISION IDAHO DPERATIONS OFFICE

IDAHO OPERATIONS OFFICE

IDAHO OPERATIONS OFFICE

IDAHO OPERATIONS OFFICE PITTSBURGH NAVAL REACTORS OFF CHICAGO OPERATIONS OFFICE CHICAGO OPERATIONS OFFICE CHICAGO OPERATIONS OFFICE WESTERN AREA POWER ADMIN

ENERGY FUELS NUCLEAR INC _ENERGY INC

ENERGY INTERNATIONAL INC

$$
\text { : }
$$

ENERGY MATERIALS RESEARCH
GM07-85IDII021---09/30/99$026050-0504-6$ IDAHO FALLS GM07-86ID11018 09/30/99 026050-0504-6 IDAHO FALLS GM07-88ID11028 09/30/99 026050-0504-6 IDAHO FALLS GM07-88ID11030 09/30/99 026050-0504-6 IDAHO FALLS GM07-94ID11135 12/31/99 026050-1904-7 IDAHO FALLS GM07-76ID11007 09/30/99 $026050-0402-3$ CHICAGO GM07-78ID11008 09/30/99 $026050-0402-3$ CHICAGO

GM07-89ID11037 09/30/99 $026050-0419-8$ CHICAGO

AI04-89AL4574? 09/30/94 026050-0701-4 ALBUQUERQUE GM04-90AL65012 03/19/95 $026050-0001-X$ AL BUQUERQUE GMO4-85AL42530 09/30/99 026050-0001-X ALBUQUERQUE

RL13-74GJ31004 07/23/94 R007-86ID12659 09/30/99
$154260-0001-8 \quad$ IDAHO FALLS AC22-92PC92108 09/24/95 155480-0001-2 PITTSBURCH AC22-93PC93157 01/23/96 155480-0001-2 PITTSBURGH

FG45-93R551901 12/31/95
CONG CONTRACT OBLIGS STATE DIST VALUE TO DATE P/S

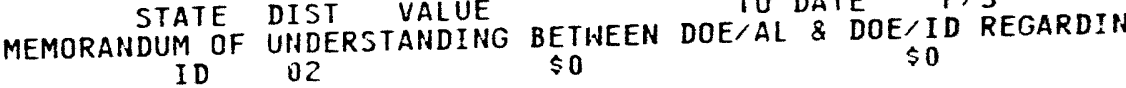
$\$ 0$ MOU BETWEEN NY STATE ENERGY RESEARCH \& DEVELOPMENT MEMORANDUM OF UNDERSTANDING BETHEEN SAN FRANCISCO OPERATIONS ID 02 $\$ 0$ MEMORANDUM OF UNDERSTANDIHG ONTHE STEEL INITIATIVE BETWEEN I D 02 $\$ 0$ $\$ O$ FIRING RANGE USE AGREEMENT WITH THE IDAHO BRAHCH OF THE PITT $\begin{array}{cll}\text { ID } & 02 & \$ 0 \\ \text { MEMORANDUM OF UNDERSTANDING } & \text { BETHEEN CH-DOE \& ID-DOE ON THE }\end{array}$ IL 02 MOU BETWEEN THE CHICAGO OPERATION OFFICE \& THE INEL RELATING IL 02 S $\$ 0$ THE MOU ESTABLISHES THE ENVIROHMENTAL SAFETY \& HEALTH FUNCTI IL 02 S \$0 \$O POST 89 FIRM ELECTRIC CAPACITY\& ENERGY LOS ALAMOS
$\$ 0$ ENHENCE LEVEL OF OVER SIGHT OFDAILY ACTIVITIES AT THE SNL, NM 01 \$0 ESTABLISH QUIDELINES FOR AUTHORIZED REFRESENTATIVES IN THE NM 01 $\$ 0$ $\$ 0$

MINING LEASE AT $(05-1)-M L-60.8-U-E-4$ CO 01 $\$ 0$

$\$ 0 \times 293$

LETTER AGREEMEHT HITH ENERGY, INC. ALLOHING ID, DOE TO PLACE ID 02 so

TECHHOLOGY DEVELOPMENT FOR COBALT FISCHER-TROPSCH CATALYSTS IMPROVEMENT OF STORAGE, HANDLING, \& TRANSPORTABILITY OF FINE

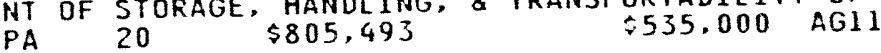
PRODUCTION OF SILICON SHEET DIRECTLY FROM BULK POLYCRYSTALLI 


\begin{tabular}{|c|c|}
\hline AWARDEE NAME & COMPL DATE \\
\hline DIVISION & VENDOR ID \\
\hline & $026210-0002-9$ \\
\hline ENERGY PRODUCTS DIJTRIBUTION & $\begin{array}{c}A D O 1-94 A D 6647100 / 18 / 94 \\
238760-0001-7 \text { TOWSON }\end{array}$ \\
\hline \multirow[t]{4}{*}{ ENERGY RESEARCH CORP } & $\begin{array}{c}A C 21-92 M C 2926108 / 30 / 94 \\
026280-0001-6 \quad \text { DAHBURY }\end{array}$ \\
\hline & $\begin{array}{c}\text { AC21-90MC27168 } 10 / 31 / 94 \\
026280-0001-6 \quad \text { DANBURY }\end{array}$ \\
\hline & $\begin{array}{c}\text { FG05-92ER81330 } 05 / 24 / 95 \\
026280-0001-6 \quad \text { DAHBURY }\end{array}$ \\
\hline & $\begin{array}{cc}\text { AC21-93MC } 30054 & 02 / 20 / 95 \\
026280-0001-6 & \text { DAHBURY }\end{array}$ \\
\hline
\end{tabular}

ENERGY SCIENCE LABORATORIES

ENERGY TECHNOLOGY SUPPORT UNIT

ENG COMP OPTECONOMICS INC

ENG SCI SOFTWARE INC

ENGELHARDT INST OF MOL BIOL

ENGINEERING RES CONSULTING INC

ENGINEERING RESOURCES CO INC

$=$
FG03-92ER81283 $05 / 24 / 95$
$199880-0001-4$ SAN DIEGO

GI01-93CE16084 $03 / 31 / 98$ FOR
$154270-0001-1$ HARWELL OXFORDSHIRE

STATE DI

DESCRIPTION OF WORK

TO DATE P/S

PROVIDE SILVER TINT SOLAR FILM

$\$ 91,372$

TRACE ELEMENT EMISSIONS

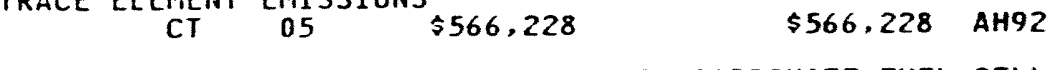

SIMULATED COAL GAS FUELED MOLTEN CARBONATE FUEL CELL $\begin{array}{llll}\text { COAL GAS FUELED } & \text { MOLTEN CARBONATE FUEL CELL } \\ \text { CT } & 05 & \$ 46,935,911 & \$ 35,497,415\end{array}$

COAL-GAS MULTIPLE CONTAMINANT POLISHING

$$
\text { MULTIPLE CONTAMINANT POLISHING } \$ 55 \text { \$549,986 } \$ 338,742
$$

RESEARCH ENTITLED "MOLTEN CARBONATE FUEL CELL ANALYSIS" $\begin{array}{llll}\text { ENTITLED } & \text { "MOLTEN } & \text { CARBONATE FUEL CELL ANALYSIS } \\ \text { CT } & 05 & \$ 650,008 & \$ 650,008 \text { AGI2 }\end{array}$

THERMAL COMPOSITE PLASMA FACING MATERIAL
CA
$\$ 590.109$
$\$ 338,405$

FOR A CO-OPERATIVE PROGRAMME TO ESTABLISH AND OFERATE :EA I AC21-92MC29109 $07 / 31 / 95$
$223920-0001-8$ ANINAPOLIS $\underset{\text { EM-ROAD TRAHSPORTABLE }}{\text { MD }} \begin{aligned} & \text { ANALYTICAL LABORATORY SYSTEM } \\ & \$ 6,104,721\end{aligned}$

FG02-92ER14247 02/28/95
$177980-0001-1 \quad$ SMITHFIELD

SMALL STRAIN HETEROGENEOUS DEFORMATION IN POLYCRYSTALS

FG02-93ER61538 12/14/94
$225890-0001-4$ MOSCOW

SEQUENCING OF DNA BY HYBRIDIZATION WITH $00 \$ 103,350$ \$103, 350

ACO2-92CHI0511 09/29/95
$215010-0001-2$ TULLAHOMA

SUPPORT SERVICES AS REQUIRED TO ASSIST IN PROJ. SCOPING, TN $04 \quad \$ 2,954,654 \quad \$ 1,444,865$ R799

FG05-94ER81664 07/17/94
$234070-0001-4$ FAYETTEVILLE

IN-SITU MICROBIAL METAL IMMOBILIZATION

$A R$ OS $\$ 75,000$

$\$ 75,000$
BIOLOGICAL UPGRADING OF COAL LIQUIDS
ENGINEERING RESOURCES LTD

AC22-91PC91059 09/23/94 


\section{AWARDEE NAME}

BIN

COMPL DATE

VENDOR ID

CITY

026440-0002-5 FAYETTEVILLE

AC22-92PC92118 09/23/94 026440-0002-5 FAYETTEVILLE

ENGINEERING SERVICES GROUP PC

ACO5-930R21880 12/13/95 30R21880 12/13/95
$225850-0001-X \quad$ KNOXVILLE

ENGINEERING SYSTEMS MGMT INC

ENGINEERING TECHNOLOGIES

ENI IMC

ENTERPRISE ADVISORY SERVICES

\section{ENTERPRISES MANAGEMENT LTD}

:

ENVAIR
ACO1-93EH89331 09/15/94 215480-0002-7 GAITHERSBURG

ACO2-93CE5021209/29/94 215480-0002-7 GAITHERSBURG

FG05-91ER81097 07/05/94 214590-0001-3 FAYETTEVILLE

AC 34-93RF00647 09/20/96 234140-0001-6 GOLDEN

ACO1-93CE75j16 03/22/95 199980-0002-5 SILVER SPRING

ACO1-92CE16054 09/24/94 $199980-0001-7$ HOUSTON

ACO1-94FE62999 01/01/95 $199980-0001-7$ HOUSTON

AC05-940R22107 03/16/95 199980-0001-7 HOUSTON

AC04-94AL85382 04/12/96 $199980-0001-7$ HOUSTON

ACO4-93AL73177 09/27/98 199980-0001-7 HOUSTON

AC01-93AD83093 12/29/94 $226490-0001-5$ BETHESDA

FG06-93ER61552 02/28/95
DESCRIPTION OF HORK

OBLIGS

CONG CONTRACT

VALUE

O DATE P/S $\$ 350,700$ AGI1

BENCH SCALE DEMONSTRATION OF BIOLOGICAL PRODUCTION OF ETHANO

AR $03 \quad \$ 318,300 \quad \$ 318,300$ AGI

MISCELLANEOUS A-E SERVICES

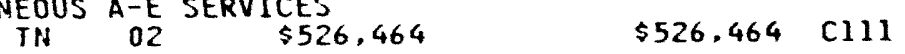

TECHNICAL MANAGEMENT AND ADMINISTRATIVE SUPPORT SERVICES TO MD 08 \$1,098,017 \$785,000 R699

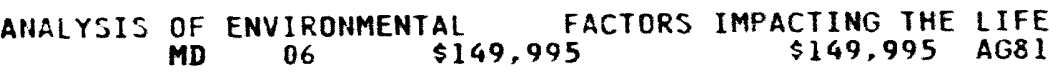

BIOCONVERSION OF COAL SYNGAS TO FUEL OXYGENATES

$$
\text { AR } \underset{03}{\$ 550,000}
$$

ADMINISTRATIVE SUPPORT SERVICES

$$
\text { CO } 02 \text { \$14.931.659 \$2,623.434 R699 }
$$

PROVIDE SUPPORT SERVICES FOR THE OFFICE OF FEDERAL MANAGEMEN

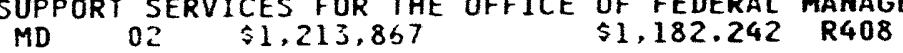
MANAGEMENT SUPPORT SERVICES FOR THE OFFICE OF TECHIIICAL AND TECHIICAL AND ADMINISTRATIVE SUPPORT SERVICES FOR NPRC TE $07 \quad \$ 1,735,681 \quad \$ 1,735,681 \quad R 799$ PROJECT CONTRLS AND TECHNICAL AND ADMINISTRATIVE SUPPORT SER TX 22 T75.311 $\$ 52.000$ R408 TECHHICAL SERVICES FOR THE OFFICE OF ENVIRCHMENT, SAFETY AND MANAGEMENT AHD TECHNICAL
TX 22 SERVICE TO SUPPORT FPMD IN
$\$ 5,314,017$ PROVIDE MANAGEMENT PRACTICUM $M D$ MS PRACTICUM $\$ 58,365$ $\$ 58,365 \quad 0008$ ACP RESEARCH AND SCIENIIFIC COORDINATION 


AWARDEE NAME
DIVISION

ENVIROCARE OF UTAH

\section{ENVIROGEN INC}

ENVIROMANAGEMENT \& RES INC

ENVIRONMENTAL PROTECTION AGCY NATIONAL AIR \& RAD ENV LAB

\section{REGION VIII}

REGION VIII

$$
\text { COMPL DATE }
$$
226620-0001-5 KENHEHICK

ACO5-940R22074 03/17/96
$237020-0001-1$ CLIVE 237020-0001-1 CLIVE

FG05-94ER81665 07/17/94 235990-0001-8 LAWRENCEVILLE

AC01-93EP79166 08/04/95 $215080-0001-8$ SPRIHGFIELD AC01-93FN80005 08/16/96 215080-0001-8 SPRINGFIELD

AI 05-920R22039 12/31/94 026670-1901-6 MONTGOMERY

AI34-93RF00646 09/30/94 $026670-0514-7$ GOLDEN

AI34-93RF00643 06/29/98 026670-0514-7 GOLDEN

AT01-93EW53021 09/30/94 026670-0005-6 HASHINGTON

AI 01-91EH89125 09/30/94 02667C-0005-6 WASHINGTON

AIO1-92EW50664 09/30/94 026670-0005-6 WASHINGTON

AI01-92E1450669009/30/94 $026670-0005-6$ WASHINGTON

AI01-94AD38003 09/30/94 026670-0005-6 WASHINGTON

AI01-92EH40102 12/30/94 026670-0005-6 WASHINGTON AI01-93EW30125 01/31/95
$026670-0005-6$ WASHINGTON

AI01-90EH89073 05/01/95
DESCRIPTION OF HORK

OBLIGS

TO DATE P/S $\$ 370,939$

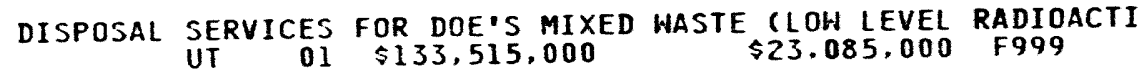

OPTIMIZATION OF PROL ONGED MICROBIAL ACTIVITY DURING IN SITU

$$
\begin{aligned}
& \text { NJ OF PROL ONGED MICROBIAL ACTIVITY DURING } \\
& 12
\end{aligned}
$$

INFORMATIOH MAHAGEMENT SUPPORTSERVICES TO THE OFFICE OF DOM VA 08 \$1,069,809 $\$ 683,000$ R699

PROVIDE INFO MANAGEMENT AND DOCUMENT PRODUCTION SYSTEM OF WO

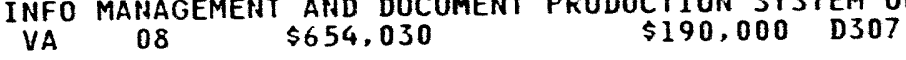

CHARACTERIZE RADIOLOGICALLY CONTAMINTATED SOIL SAMPLES $\begin{array}{llll}A L & 02 & \$ 295,000 & \$ 295,000\end{array}$ MD/WIND TUNHEL CERTIFICATION

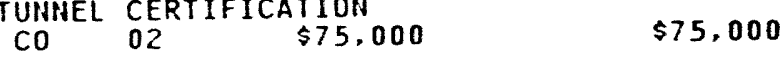

MD/FLUIDIZED BED INCINERATION VERIFICATION MODEL

$$
\text { CO } 02 \text { BED } \$ 534,746 \quad \$ 534,746
$$

ELECTROSMATIC PURGING OF HEAVYMETALS \& FULLY SATURATED SOIL

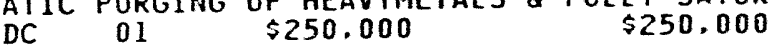

CO-SPONSER DEVELOPMENT OF ARAR'S ASSISTANCE DATABASE HHICH H

$$
\begin{array}{lll}
D C & 01 & \$ 450,000
\end{array}
$$

INTERAGENCY AGREEMENT FOR PNEUMATIC FRACTURING \& TECHIOLOGY

$$
\text { DC AGREEMENT FOR PNEUMATIC FRACTURING \& } \$ 250,000
$$

TO SPONSOR A CONFERENCE IN COLLABORATION WITH THE ENVIROHMEN DC 01 \$125,000 $\$ 125,000$ CORRECT COMPLETION DATES $\$ 50,000 \quad \$ 50,000$

IA W/EPA TO EVALUATE \& RECOMMEND IMPROVEMENT TO DOE'S MGMT 0 DC EVALATE \& $\$ 2,000,000 \quad \$ 830,000$

EPA WILL PROVIDE TECHNICAL ANALYSES OF ISSUES ASSOCIATED WIT EPA TRAINING SEMINARS, HORKSHOPS 


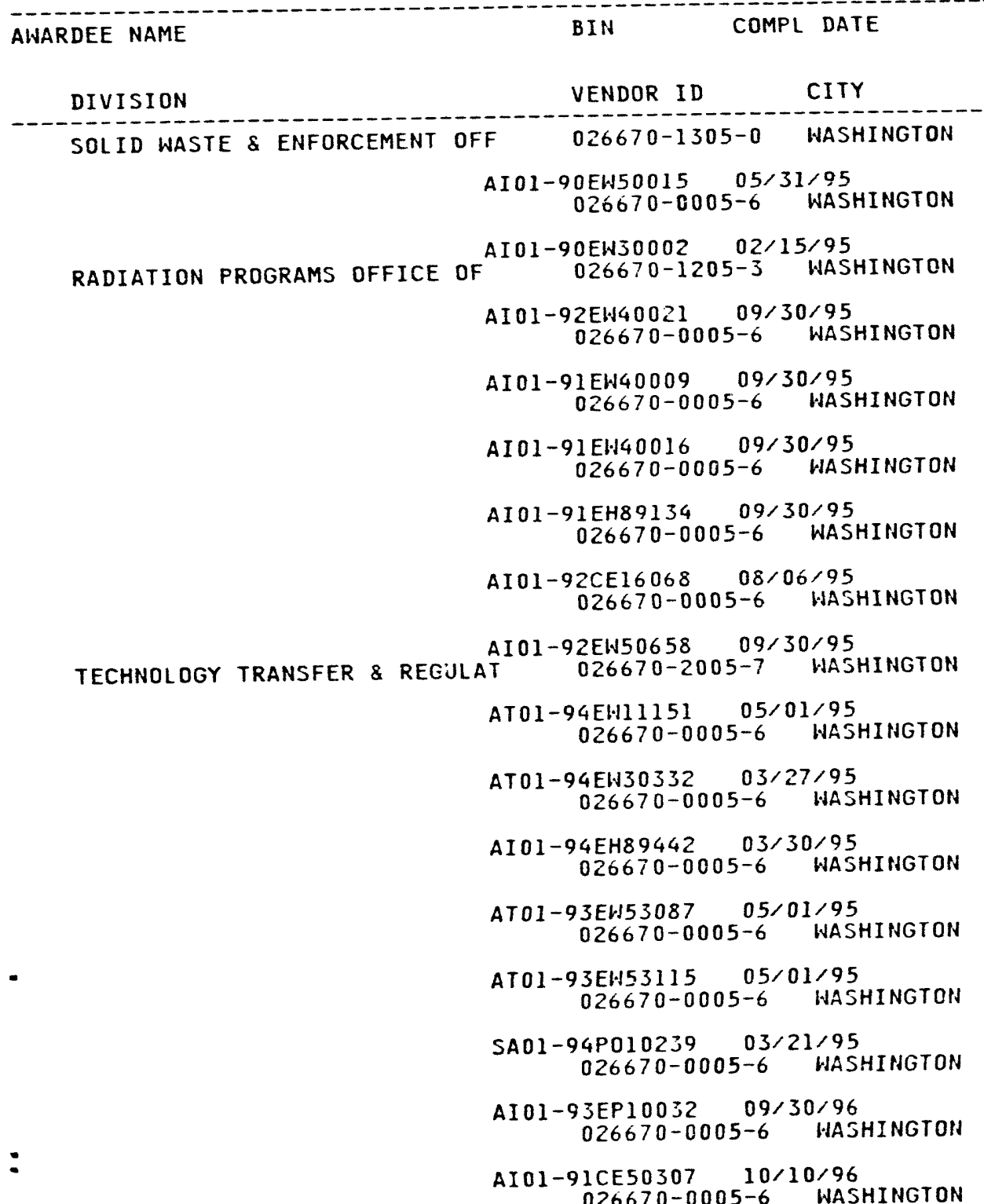

DESCRIPTION OF WORK

STATE CONG CONTRACT OBLIGS

DC 01 \$300,000 $\$ 300,000$

COOPERATIVELY FUND LIAISON TO ASSIST EPA AND DOE POINTS OF $C$ $D C$ O $\$ 640,000 \quad \$ 200,000$

SUPPORT FOR TASKS RELATING TO THE RADIOACTIVE MIXED WASTE MA DC 01 \$ $\$ 1,825,000 \quad \$ 1,365,000$

ESTABLISHMENT OF A NATIONAL PRIORITY SYSTEM FOR FEDERAL FACI DC 01 NATIS5,000 $\$ 155,000$

FUNDING FOR PATHHAY MODEL HORK

DC $01 \quad \$ 1,150,000 \quad \$ 850,000$

TO EXAMINE ISSUES RELATED TO INCINAERATION OF RADIACTIVE AND $D C$ OI $\$ 415,000 \quad \$ 415,000$

INTERAGENCY AGREEMENT WITH EPATO DEVELOP A SERIES OF TRNG $W$ DC 01 A $\$ 300,000 \quad \$ 300,000$

INTERAGENCY AGREEMENT/EPA DEVELOPMENT OF INTL ENV TECHNOLOGY DC 01 AGREEMENT/EPA DEVELOPMENT O $\$ 100.000$ \$100,000

INTERAGENCY AGREEMENT FOR TECHNICAL SUPPORT TO INTEGRAIED DE DC $\begin{array}{ccc}01 & \$ 15,000 & \$ 1,500\end{array}$

TASK ASSIGNMENT UNDER EPA MASTER INTERAGENCY AGREEMENT FOR T DC 01 I $\$ 25,000$ \$25,000

SUPPORT FOR THE OFFICE OF PROGRAM INTEGRATION

DC 01 T725,232

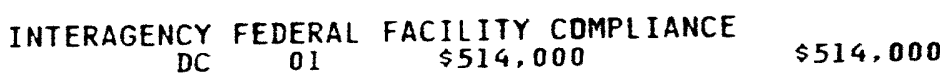
DC
01
. 000

TECHNICAL SUPPORT TO THE OFFICE TECHHOLOGY DEVELOPMENT-TASK

SITE EMERGING TECH TASK 93-502

$\begin{array}{lrrr}D C & 01 & \$ 600,000 & \$ 600,000\end{array}$

"FUNDS IN" INTERAGENCY AGREEMENT FOR $\$ 75,000$ \$0

IA FUNDS DUT: SUPPORT FOR "ESTIMATION OF COSTS OF CLIMATE CH DC 01 \$900,000 $\$ 150,000$

SUPPORT FROM EPA FOR AMFA PROGRAM ELEMENTS

DC 01 \$600,000 $\$ 200,000$ 


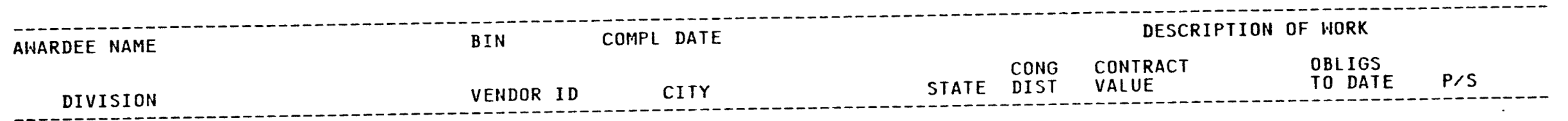

\begin{tabular}{|c|c|}
\hline & $\begin{array}{c}\text { AI } 02-93 \text { ER7 } 5890 \quad 10 / 31 / 96 \\
026670-0005-6\end{array}$ \\
\hline & $\begin{array}{c}\text { AI02-93ER7 } 5889 \quad 10 / 31 / 97 \\
026670-0005-6 \text { WASHINGTON }\end{array}$ \\
\hline & $\begin{array}{c}\text { AI01-93EW10529 } 02 / 15 / 98 \\
026670-0005-6 \quad \text { WASHINGTON }\end{array}$ \\
\hline & $\begin{array}{c}\text { AT01-93EH10825 02/15/98 } \\
026670-0005-6 \text { WASHINGTON }\end{array}$ \\
\hline & $\begin{array}{c}\text { ATO1-93EH30228 } 02 / 15 / 98 \\
026670-0005-6 \text { WASHINGTON }\end{array}$ \\
\hline & $\begin{array}{c}\text { AI05-900R21945 09/30/95 } \\
026670-0011-0 \text { GULF BREEZE }\end{array}$ \\
\hline & $\begin{array}{c}\text { AI22-90PC90164 12/31/94 } \\
026670-0010-2 \text { RALEIGH }\end{array}$ \\
\hline & $\begin{array}{l}\text { AI01-92EW50604 01/31/97 } \\
026670-0003-X \text { LAS VEGAS }\end{array}$ \\
\hline & $\begin{array}{c}\text { AI01-92EH89250 08/20/94 } \\
026670-0003-X \text { LAS VEGAS }\end{array}$ \\
\hline & $\begin{array}{l}\text { AI08-91NV10963 } 06 / 30 / 96 \\
026670-0003-X \text { LAS VEGAS }\end{array}$ \\
\hline & $\begin{array}{c}\text { AIO1-92EW50646 } 12 / 31 / 95 \\
026670-0002-1 \quad \text { CINCINNATI }\end{array}$ \\
\hline ENVIRONMENTAL RES GROUPS INC & $\begin{array}{l}\text { AC65-92WA07693 11/05/94 } \\
216690-0001-X \text { LITTLETON }\end{array}$ \\
\hline ENVIRONMENTAL RISK LTD & $\begin{array}{ccc}\text { FG05-94ER8 } 1666 & 07 / 17 / 94 \\
236070-0001-8 & \text { BLOOMFIELD }\end{array}$ \\
\hline ENVIRONMENTAL SCI \& RES FDN & $\begin{array}{c}\text { AC07-941D13268 10/31/98 } \\
237710-0001-7 \text { IDAHO FALLS }\end{array}$ \\
\hline ENVIRONMENTORS PROJECT & $\begin{array}{c}\text { FG02-94ER75982 } 03 / 14 / 97 \\
237050-0001-2 \text { WASHINGTON }\end{array}$ \\
\hline
\end{tabular}

DOE FUNDING TO SUPPORT EPA UNDERSTANDING NATIONS EPA SUMMER INSTITUTE IN HANDS-ON ENVIRONMENTAL SCIENCE DC $01 \quad \$ 611,887$

INTERAGENCY WITH EPA TO PROVIDE DOE WITH A BROAD RANGE OF RE DC $01 \$ 40,000,000$ \$O

ADDENEUM TO IA WITH EPA FOR CONTINUED EPA FUNDING FOR SUPPOR DC $01 \quad \$ 490,000 \quad \$ 490,000$

TASK ORDER FOR WASTE ISOLATIONPILOT PLANT LAND WITHDRAWAL A DC $01 \quad \$ 6,515,000 \quad \$ 6,515,000$

COLLABORATIVE RESERACH ON BIOREMEDIATION OF CONTAMINATED SIT FL $01 \quad \$ 20,000 \quad \$ 20,000$ INTERAGENCY AGREEMENT AWARD

NC 02 \$2,467,000 $\$ 2,467,000$

DEVELOPMENT AND IMPLEMENTATIONOF A QUALITY ASSURANCE MONITO NV OI $\$ 1,000,000 \quad \$ 575,000$

ENHANCEMENT OF CAP88-PC DOSE/RISK ASSESSMENT SOFTWARE PACKAG NV 01 \$160,000 $\$ 160,000$

PROVIDE COMPREHENSIVE RADIOLOGICAL SURVEILLANCE AND SAFETY $P$ NV OI \$16,655,359 \$16,655,359

EPA SUPERFUND - INHOVATIVE TECHNOLOGY EVALUATION - INHOVATIVE TECHNOLOGY EVALUATION
$\begin{aligned} & \text { OH } \\ & 04\end{aligned} 1,700,000$

CONSERVATION AND RENEWABLE ENERGY PREFERENCE CUSTOMER

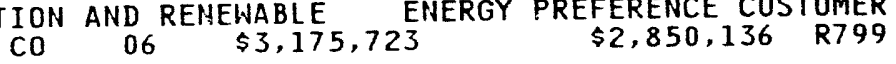

$\begin{array}{llll}\text { CT } & 01 & \$ 74,783 & \$ 74,783\end{array}$

ENVIRONMENTAL SURVEILLANCE, MONITORING \& RADIOECOLOGY \& WILD ID $02 \quad \$ 6,213,255 \quad \$ 1,150,000 \quad B 510$

ENVIRONM. PROJECT ENABLES INN-ER-CITY SCHOOL STUDENT TO WORK $\begin{aligned} \text { PROJECT ENABLES INN-ER-CITY SCHOOL STUDENT } & \$ 62,711\end{aligned}$ 
AWARDEE NAME

BIN

COMPL DATE

VENDOR ID

CITY STATE CONG CONTRAC DESCRIPTION OF WORK

DIVISION

SC91-94FE63055 11/01/94
$227530-0002-6$ DENVER 227530-0002-6

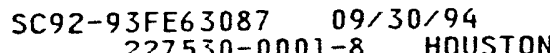
SC92-94FE63273 10/01/94 227530-0001-8 HOUSTON

EQUITABLE GAS CO

ERC INC

ERM PROGRAM MANAGEMENT CO

ERVING PUBLIC SCHOOLS

ESEERCO

ESPANOLA HOSPITAL

\section{ESSEX CORP}

SYSTEMS EFFECTIVENESS DIV

ESTANCIA TOWN OF

MUNICIPAL SCHOOLS

ETHIOPIA TRANSITIONAL GOVT OF
ADI1-91PN38189 04/17/97
$216580-0001-3$ PITTSBURGH

FG05-92ER81435 02/17/95 215090-0001-1 TULLAHOMA

FG05-93ER81557 08/01/94 215090-0001-1 TULLAHOMA

AC07-93IDI3210 10/06/97 225070-0001-5 ENGLEHOOD FG41-93R193516 $07 / 31 / 95$
$232360-0001-5$ ERVING

FG05-91CE35045 03/31/95 026890-0001-6 NEW YORK

GM32-94AL $96949 \quad 04 / 08 / 99$ 026960-0001-8 ESPANOLA

AC04-93AL $91124 \quad 03 / 14 / 96$ 136790-0102-9 MCLEAN

GM04-93AL 84054 11/24/97 226870-0101-5 ESTANCIA

FC02-93P010097 09/29/95 234390-0001-X ADDIS ABABA NOSR NATURAL GAS CONDENSATE SALES CONTRACT EST. $\underset{01}{\text { REVENUE: }} \underset{\$ 0}{\$ 0}$ \$4,

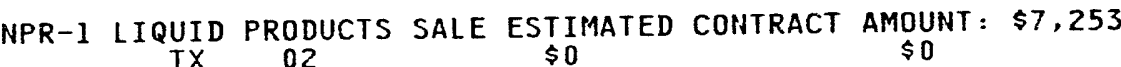

NPR-1 CRUD EOIL SALES CONTRACTEST REV $\$ 1487588.23$

NATURAL GAS UTILITY SUPPLIES AND SERVICES

$$
\text { PA } 14 \underset{\$ 1,280,906}{\$ 1,280,906} \$ 111
$$

SBIR PHASE I A LIGHT METAL FUELED NONEQUILIBRIUM PLASMA GENE TN $04 \quad \$ 549,942 \quad \$ 408,835$

A T-LAYER MHD GENERATOR

$$
\underset{T N}{\text { MHD }} \underset{04}{\text { GENERATOR }} \$ 674,877 \quad \$ 174,961
$$

TECHNICAL AND MANAGERIAL SUPPORT SERVICES

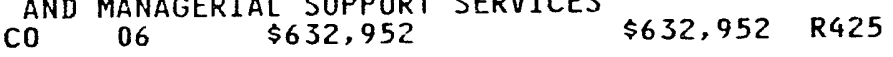

TITLE III OF THE NATIONAL ENERGY CONSERVATION POLICY ACT

$$
M A T 01 \quad \$ 128,493 \quad \$ 64,246
$$

DEMONSTRATION OF HIGH PHASE ORDER (HPO) POWER TRANSMISSION 0 NY $15 \$ 11,312,965 \$ \$ 2,600,000$

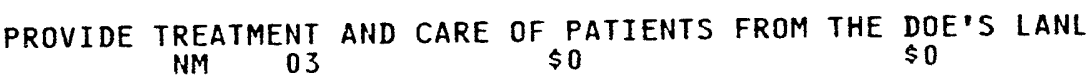
$\$ 0$

LETTER CONTRACT FORLOGISTICAL AND TECHNICAL SUPPORT SERVICES VA $10 \$ 16,319,003 \quad \$ 3,100,000$ R706

SUPPORT FOR SCIENCE, MATHEMATICS, AND ENGINEERING TO IMPROVE HM 01 $\$ 0$ $\$ 0$

CLIMATE CHANGE STUDY $\$ 00$

$\$ 109,300$ 


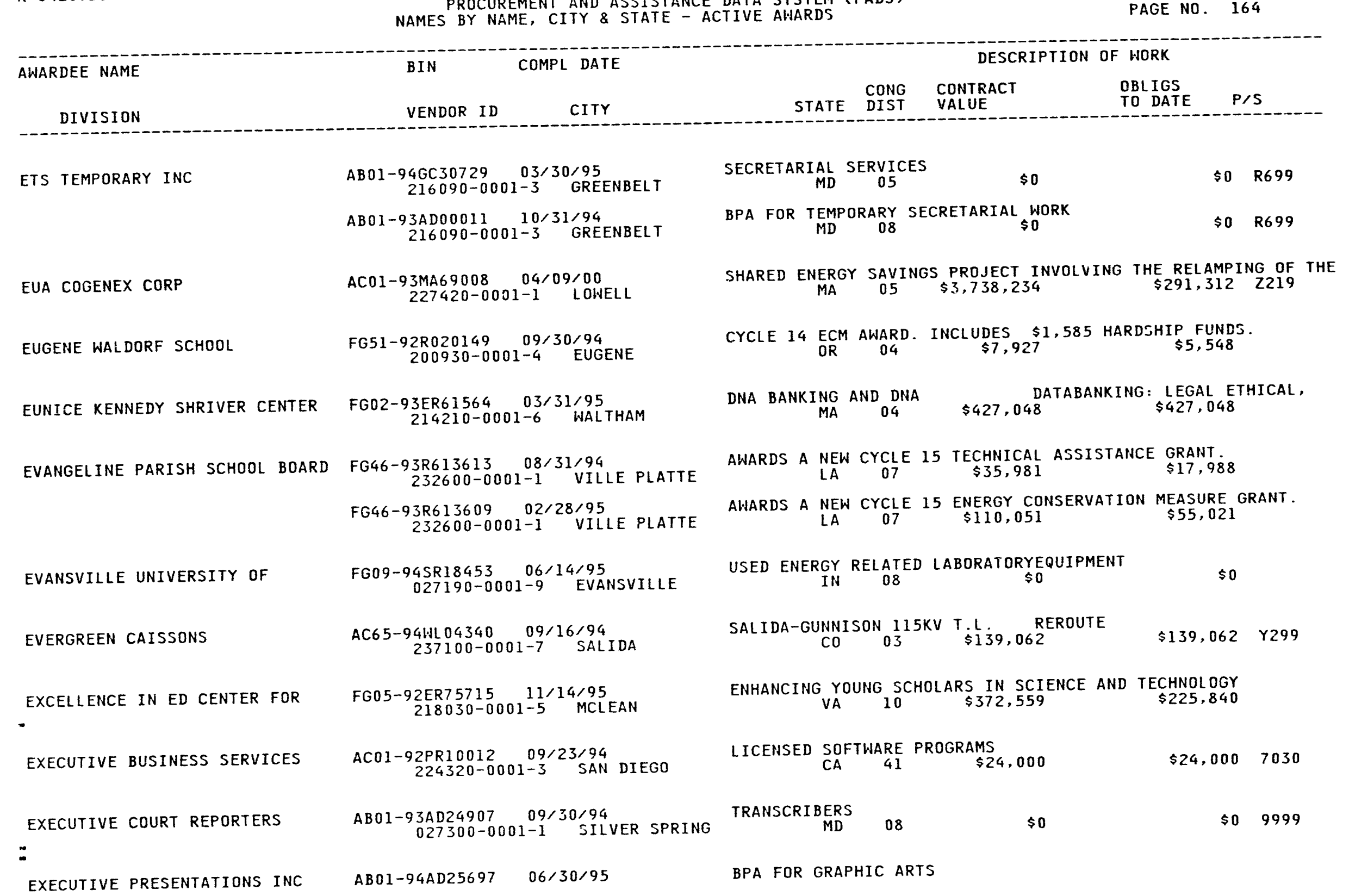




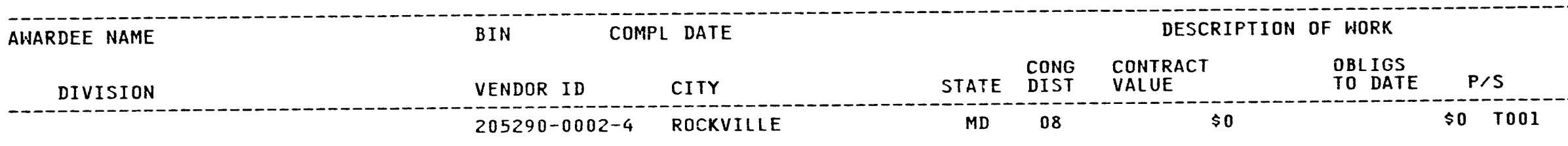

ABO1-94AD24927 05/31/95 205290-CD02-4 ROCKVILLE

EXETER ASSOC INC

EXPLORATORIUM

EXPLUS INC

EXXON RES \& DEV LABORATORIES

EYE FOUNDATION HOSPITAL

EYE RESEARCH INSTITUTE

F D SERVICES INC

- F r lauran corp

FACILITIES MANAGEMENT CO

:

ACO1-90MA00256 01/31/95

FGD3-94ER75957 12/14/94

FG03-93ER61583 03/31/95

AB01-94AD24935 05/31/95
$227980-0001-7$ ASHBURN

AC22-94PC94051 06/12/95

FG44-93R435293 08/31/95 $230760-0001-2$ BIRMINGHAM FG02-91ER61229 $08 / 31 / 94$
$213520-0001-6$ BOSTON

AC01-92FE62316 09/30/97 $224630-0001-5$ GREENVILLE

AC05-940R22241 08/30/94 221950-0002-3 KNOXVILLE

AMO1-93EW10424 02/25/95
$227510-0001-0$ LANDOVER ADO1-94EW11021 02/25/95 $227510-0001-0$ LAHDOVER
106770-0002-2 SILVER SPRING $213530-0001-X$ SAN FRANCISCO 213530-0001-X SAN FRANCISCO $239070-0001-3$ BATON ROUGE BPA FOR GRAPHIC PRODUCTION ANDOTHER VISUAL $\$ 0$

ART DELIVERABLES

TECHNICAL ASSISTANCE IN THE CONDUCT OF INTERVENTIONS BEFORE $M D \$ 08 \$ 2,846,152 \quad \$ 2,060,000$ R499

LAGOON RESTORATION PROJECT

$$
\begin{array}{lll}
\text { CA } 08 & \$ 45,670
\end{array}
$$

THE HUMAN GENOME: SCIENCE AND THE SOCIAL CONSEQUENCES INTER $C A \quad 05 \quad \$ 150,199$ $\$ 148,740$

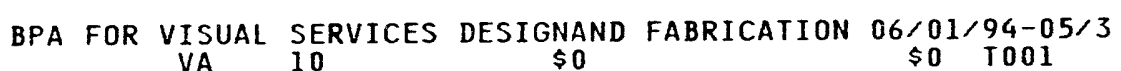

BENCH SCALE SLURRY CATALYST TESTING

$\$ 2,000,0 J 0 \quad A G 11$ SEE ATTACHED PAGE NO. $\underset{\text { AL }}{2 \underset{06}{\$ 107,201}}$ THIS NOTICE OF FINANCIAL ASSISTAN

ADO1-94EW11162 02/21/95 $227510-0001-0$ LAMDOVER
CENTER OF EXCELLENCE-MICROLASER MICROSCOPE
MA 09 \$999,177

$\$ 999,177$

MANAGEMENT AND OPERATING CONTRACT IN SUPPORT OF THE NAVAL PE TN $02 \quad \$ 204,513 \quad \$ 204,513 \quad$ Y 224

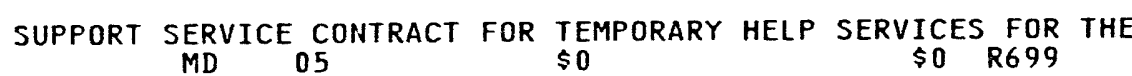

TEMPORARY HELP DERVICES DELIVERY ORDER

$\$ 16,318 \quad R 699$

SUPPORT SERVICES: SECRETARY I $\left(5400\right.$ HRS a $\$ 20.86$ PER ${ }^{H R},-\$ 1$ SC $04 \quad \$ 44,202,455 \quad \begin{aligned} & \$ 40,070,678 \\ & \text { M299 }\end{aligned}$

EXPANSION OF PARKING LOT AND REPAIR OF COLLAPSED DRAINGAGE D 


\begin{tabular}{|c|c|}
\hline AWARDEE NAME & COMPL DATE \\
\hline \multirow[t]{5}{*}{ DIVISION } & VENDOR ID \\
\hline & $\begin{array}{c}\text { ADO1-93EW10597 02/25/95 } \\
227510-0001-0 \quad \text { LANDOVER }\end{array}$ \\
\hline & $\begin{array}{c}\text { AD01-93EW1 } 063902 / 25 / 95 \\
227510-0001-0 \quad \text { LANDOVER }\end{array}$ \\
\hline & $\begin{array}{c}\text { AC01-93EW10667 07/28/95 } \\
227510-0001-0 \quad \text { LANDOVER }\end{array}$ \\
\hline & $\begin{array}{r}\text { ADO1-94EW11285 } 02 / 25 / 95 \\
227510-0001-0 \quad \text { LAHDOVER }\end{array}$ \\
\hline
\end{tabular}

FAIRCHILD SPACE \& DEFENSE CORP

FAIRFIELD BOARD OF EDUCATION

FAIRFIELD COUNTY SCHOOLS

FAIRFIELD MEMORIAL HOSPITAL

FAIRFIELD UNIVERSITY

PHYSICS DEPT

FAIRMONT STATE CDLLEGE

- fairhar electronics inc

FALCON MICROSYSTEMS INC
ACO1-94NE32172 $03 / 28 / 96$
$237230-0001-0$ GERMANTOWN

FG41-93R191504 08/31/95
$027640-0001-4$ FAIRFIELD

FG44-92R435139 09/30/94 $214690-0001-6$ HINHSBORO

FG44-93R435439 09/30/95 027680-0001-9 WINNSBORO

FG02-91ER40673 11/14/94
$183140-0001-9$ FAIRFIELD

FG09-94SR18420 02/16/95
$027740-0001-7$ FAIRMONT

AB01-94AD69240 12/31/94 225740-0001-3 KENSINGTON $172920-0008-8$ GOLDEN

ADO4-94AL90586 04/02/95 $172920-0001-0$ LANDOVER

AB 04-93AL90115 05/21/95
$A B 34-93 R F 00462 \quad 07 / 31 / 94$
DESCRIPTION DF HORK

OBL IGS CONG CONTRACT
STATE DIST VALUE
TEMPORARY HELP SERVICE DELIVERY ORDER

MD $\quad 05 \quad \$ 201,977 \quad \$ 201,977 \quad$ R699

TEMPORARY HELP SERVICES DELIVERY ORDER

$M D \quad 05 \quad \$ 380,210 \quad \$ 380,210 \quad R 699$

SUPPORT SERVICES ADMINISTRATIVE CLERICAL SUPPORT SERVICE FOR $M D$ O5 \$4,205,386 $\$ 3,363,513$ R699

TEMPORARY HELP SERVICES TO OFFICE OF ENVIROMENTAL MANAGEMENT

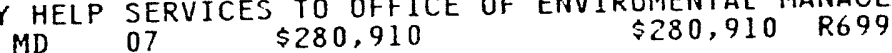

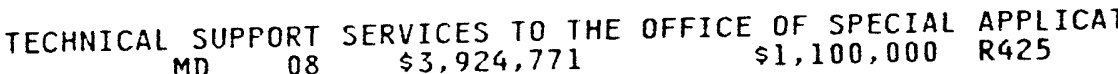

INITIAL AWARD.
CT

CYCLE 14 - INSTITUTIONAL CONSERVATION PROGRAM. APPROVED BUD $\begin{array}{llll}\text { SC } & 05 & \$ 67,809 & \$ 38,990\end{array}$

SEE ATTACHED PAGE NO. 2 OF THIS NOTICE OF FINANCIAL ASSISTAN SC 05 \$109,859 $\$ 54,929$

SSC \& G-2 CALORIMETER \& OPTICAL CALIBRATION R\&D

SSC \& G-2 CALORIMETER \& $\$ 127,700 \quad \$ 127,700$

USED ENERGY RELATED LABORATORYEQUIPMENT

$$
\text { WV RELATED LABORATORYEQUTPMENT } 01 \text { \$0 }
$$

BPA FOR ELECTRONIC SERVICES AND SUPPLIES

\$0 5999

CV/ADP BPA AWARD

$\$ 483,967 \quad D 308$

ORDER FOR 28 MAC QUADRA 6505

$\$ 122,150 \quad 7050$

BPA FOR COMPUTER SOFTWARE AND RELATED SUPPLIES 


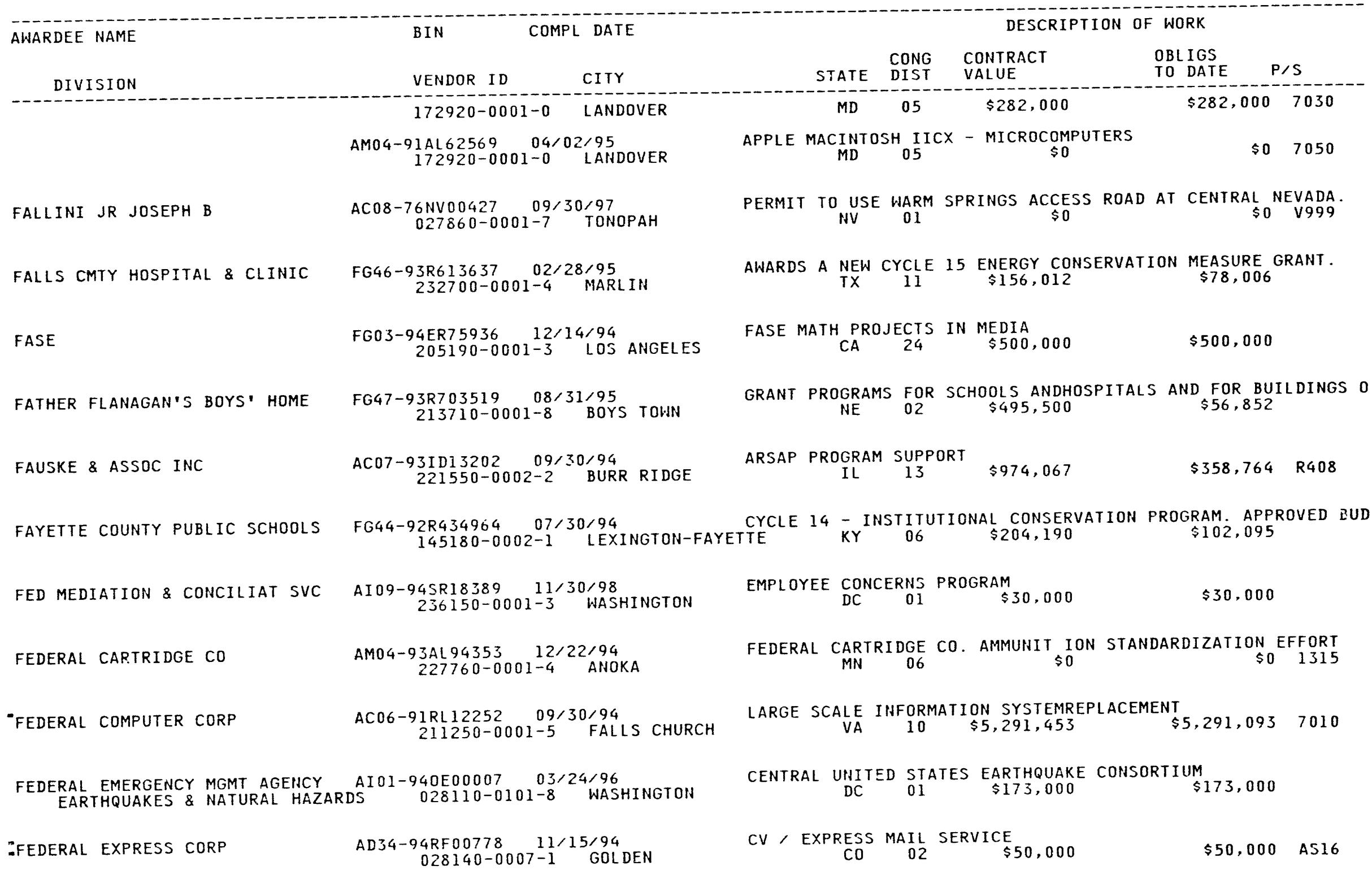




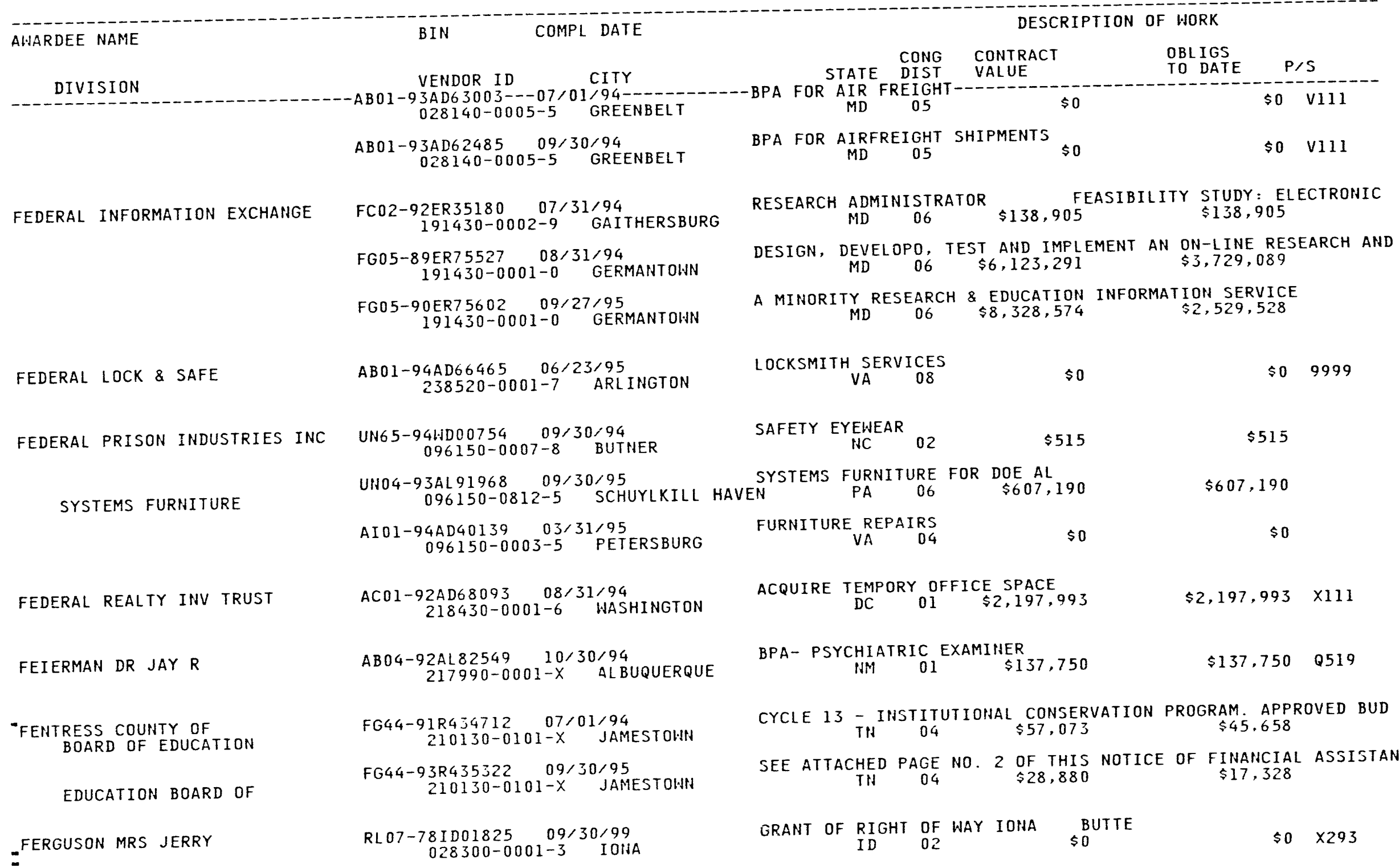




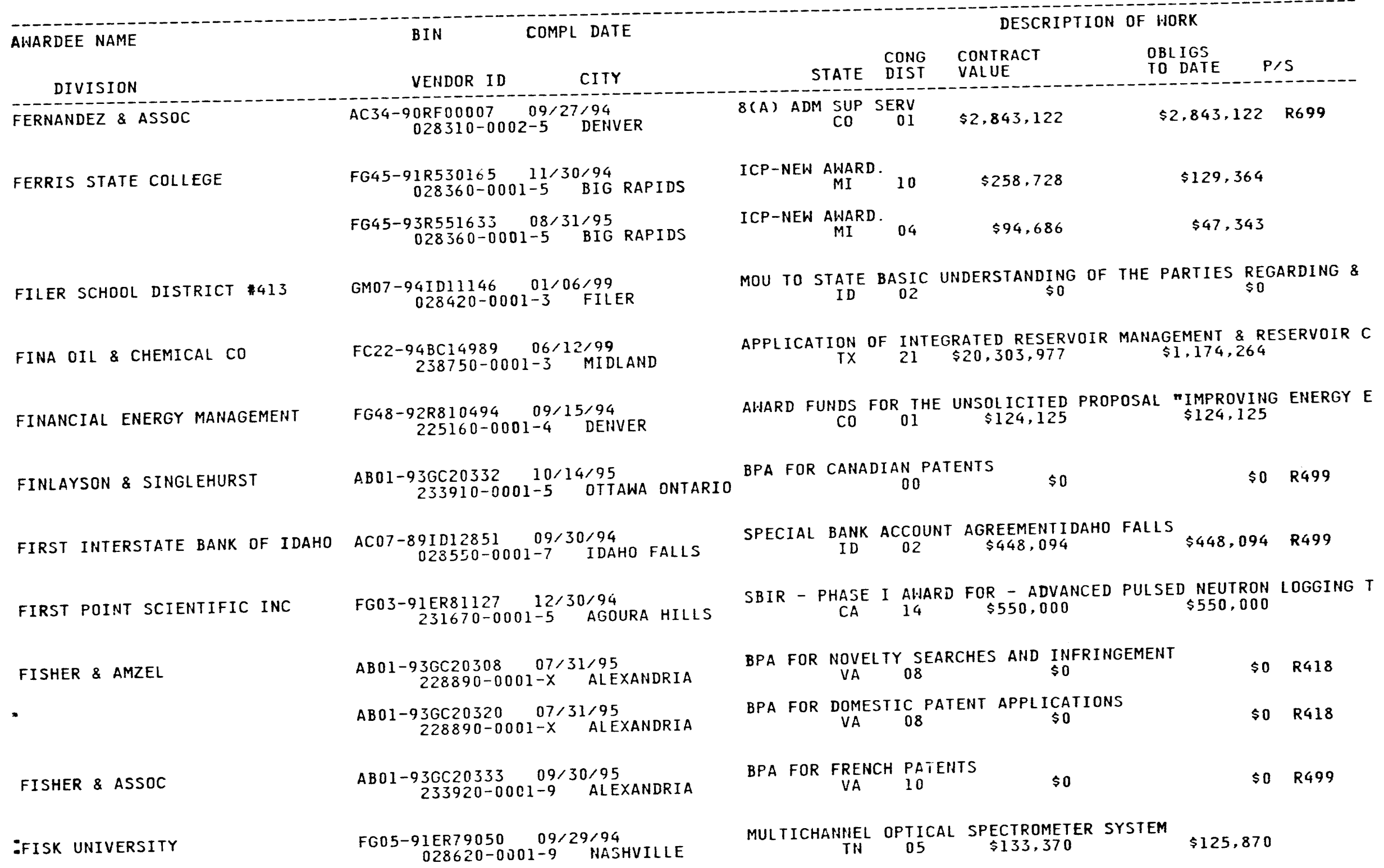




AWARDEE NAME
DIVISION

BIN COMPL DATE VENDOR ID CITY 028620-0001-9 NASHVILLE

FLEHR HOHBACH TEST ALBRITTON

ACO3-90SF18705 09/29/94 204200-0001-1 SAN FRANCISCO

PATENT-LEGAL SERVICES STATE CONG CONTRACT DESCRIPTION OF HORK

FLEMING COUNTY OF BOARD OF EDUCATION

\section{FIINT CITY OF}

COMMUNITY SCHOOLS

FLORENCE COUNTY SCHOOL DIST

FLORIDA A \& M UNIVERSITY PSYCHOLOGY DEPARTMENT

\section{PHARMACOLOGY}

COOPERATIVE EDUCATION

PHYSICS DEPT

PHYSICS DEPT

PHYSICS DEPT

- Florida atLantic UNIVERSity PHYSICS DEPT

FLORIDA COUNTY SCHOOL BOARD

-FLORIDA INTERNATIONAL UNIV
FG44-92R434965 07/31/94 028720-0101-8 FLEMINGSBURG FG45-93R551634 08/31/95
$217300-0101-7 \quad$ FLINT

FG45-93R551794 12/31/94

FG03-925F19487 09/24/94 $124790-0501-1$ TALLAHASSEE

FG05-89ER75523 09/28/94 $124790-0301-9$ TALLAHASSEE

FG04-90AL65852 09/29/94 124790-0401-5 TALLAHASSEE FG09-94SR18429 03/27/95 $124790-0101-6$ TALLAHASSEE

FG34-94RF00857 05/03/95 $124790-0101-6$ TALLAHASSEE

FG21-94MC $31237 \quad 07 / 05 / 95$ 124790-0101-6 TALLAHASSEE

FG05-89ER45392 11/30/94 $028860-0101-5$ BOCA RATON GM04-93AL84091 09/28/98
$236490-0001-6 \quad$ LARGO

FGO3-90SF18715 $09 / 29 / 94$
$028880-0002-4$ 229920-0001-9 FLORENCE

THEORETICAL STUDIES OF METALLIC ALLOYS $\mathrm{FL} \quad 14 \quad \$ 304,668$
CYCLE 14 - INSTITUTIOHAL CONSERVATION PROGRAM. APPROVED BUD $\mathrm{KY} \quad 07 \quad \$ 88,640 \quad \$ 44,319$

ICP-NEW AWARD

MI 05

$\$ 118,000$

$\$ 59,000$

ICP-NEW AHARD.

WI

$\$ 4,100$

$\$ 2,050$

NEN - THE IDENTIFICATION AND EDUCATION OF BLACK CULTURALLY D PREDOCTORAL TRAINING PROGRAM IN ENVIRONMENTAL TOXICOLOGY

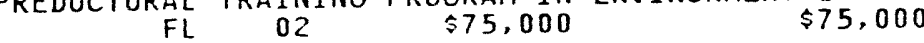
HBCU GRANT FOR CO-OP PROGRAM

USED ENERGY RELATED LABORATORYEQUIPMENT \$0 \$0

USED ENERGY RELTED LAB EQUIPMENT

USED ENERGY RELATED EQUIPMENT FL $\quad 02$

$\$ 0$

$\$ 304,668$

TECH ASSIST IN SUPPORT DF SCIENCE, MATH \& ENG. EDUCATION $\mathrm{FL} \quad 08$ $\$ 0$

MINORITY UNDERGRADUATE TRAINING FOR ENERGY-RELATED CAREERS

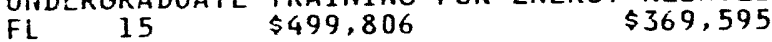




BIN COMPL DATE

AWARDEE NAME

VENDOR ID

CITY

STATE DINT

DIVISION

PAGE HO. 171

CRO1-83NE44383 $03 / 15 / 30$
$028900-0001-X \quad$ MIAMI

CRO1-83NE4G471 $03 / 15 / 30$
$028900-0001-X$ MIAMI

CROI-83NE44472 03/15/30 028900-0001-X MIAMI

FLORIDA POWER CORP

FLORIDA SOLAR ENERGY CENTER

CR01-83NE44382 03/15/30 028910-0001-3 CRYSTAL RIVER

FG01-90CE21048 06/21/95
$028920-0001-7$ CAPE CANAVERAL

FC36-86SF16305 01/01/95 028920-0001-7 CAPE CANAVERAL

FLORIDA STATE OF

GOVERNOR'S ENERGY OFFICE

FG44-80R430363 09/30/94 028940-0301-3 TALLAHASSEE

FG04-90AL65778 07/31/95

HEALTH \& REHABILITATIVE SVCS $028940-0501-6$ TALLAHASSEE

FG04-90AL66151 08/31/95

HEALTH \& REHABILITATIVE SVCS $028940-0501-6$ TALLAHASSEE

FG44-92R410600 09/30/95

HOUSING \& COMMUNITY DEV DIV OF 028940-0601-2 TALLAHASSEE

GOVERNOR'S ENERGY OFFICE

- FLoRIdA STATE UNIVERSITY BIOLOGICAL SCIENCES DEPT

\section{:}

FG44-80C569090 $06 / 30 / 95$
$028940-0301-3$ TALLAHASSEE

FG05-91ER6115908/31/94

FG05-92ER40742 07/31/94 028950-0001-8 TALLAHASSEE

FG05-92ER40750 09/29/94 028950-0001-8 TALLAHASSEE

FG05-92ER61500 09/29/94 028950-0001-8 TALLAHASSEE $028950-0101-4$ TALLAHASSEE
DISPDSAL OF SPENT NUCLEAR FUEL

DISPOSAL OF SPENT NUCLEAR FUELAND/OR HIGH LEVEL RADIOACTIVE

$\begin{array}{ccccc}\text { DISPOSAL OF SPENT NUCLEAR FUELAND/OR HIGH LEVEL RADIOACTIVE } & \text { FL } \\ \text { FL } & 18 & \$ 0 & \$ 0\end{array}$

\$0

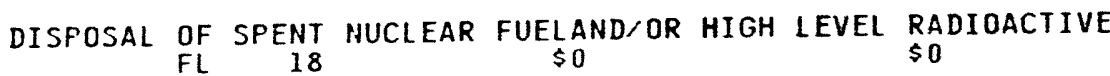

$\mathrm{FL} 18$ \$0

DAYLIGHT AVAILABILITY RESEARCH

$\$ 782,505$

CONDUCT RESEARCH AT A SOLAR COOLING RESEARCH FACILITY $\mathrm{FL} \quad 11 \$ 10,406,500 \quad \$ 2,977,009$

GRANT PROGRAM FOR SCHOOLS, HOSPITALS, BUILDINGS DWNED BY UNI $\mathrm{FL} \quad 02 \$ 92,147,247$

$\$ 4,577,595$

$\begin{array}{llll}\text { AGREEMENT IN PRINCIPLE FOR ENVIRONMENTAL } & \text { OVERSIGHT, } \\ \text { FL } & 02 & \$ 565,656 & \$ 224,486\end{array}$

EPIDEMIOLOGY STUDY OF PINELLASPLANT

$\$ 86,993$

SOLAR WEATHERIZATION ASSISTANCE PRODUCTS
FL 02
$\$ 866,000$

ENERGY EXTENSION SERVICE

$F L \quad 02 \quad \$ 5,166,530 \quad \$ 3,560,459$

PHYSIOLOGICAL \& PHYLOGENETIC STUDY O MICROBES FROM GEOCHEMIC $\mathrm{FL} \quad 02 \underset{1,027,930}{\$ 1,027,930}$

EXPERIMENTAL HIGH ENERGY PHYSICS

$\$ 1,200,000$ $\begin{array}{ccc}\text { THEORETICAL NUCLEAR PHYSICS GROUP OF THE SUPERCOMPUTER COMPU } \\ \mathrm{FL} \\ 02 & \$ 120,000 & \$ 120,000\end{array}$

MULTIPLE ALIGNMENT AND HOMOLOGSEQUENCE DATABASE COMPILATION

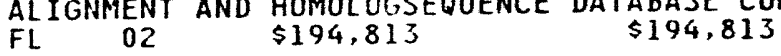




\section{(2)}

PHYSICS DEPT

BIOLOGICAL SCIENCES DEPT

OCEANOGRAPHY DEPARTMENT OF

FG05-93ER25147 11/14/94 028950-0001-8 TALLAHASSEE

FG05-86ER40273 02/28/95 028950-0701-2 TALLAHASSEE

FG05-86ER1357506/30/95 028950-0101-4 TALLAHASSEE

FG05-92ER61416 04/30/95 028950-0901-5 TALLAHASSEE

FG05-92ER61430 04/30/95 028950-0001-8 TALLAHASSEE

FG05-92ER4073509/29/95 028950-0001-8 TALLAHASSEE

FG05-92ER40751 09/29/95 028950-0001-8 TALLAHASSEE

FG05-94ER61865 05/31/95 028950-0001-8 TALLAHASSEE

FG05-87ER60517 11/30/95 028950-0201-0 TALLAHASSEE

\section{CHEMISTRY DEPT}

CHEMISTRY DEPT

BIOLOGICAL SCIENCES DEPT INST OF MOLECULAR BIOPHYSICS

- PHYSICS DEPT

CHEMISTRY DEPT PHYSICS DEPT

FG05-87ER40329 02/29/96 028950-0201-0 TALLAHASSEE

FG05-90ER61039 08/14/96 028950-0101-4 TALLAHASSEE

FG05-86ER60473 10/31/96 028950-0301-7 TALLAHASSEE

FG05-87ER40319 12/31/96 028950-0701-2 TALLAHASSEE

FG05-85ER45208 04/30/97 028950-0201-0 TALLAHASSEE

FG05-91ER45443 03/14/97 028950-0701-2 TALLAHASSEE

FG05-93ER54192 10/31/97 028950-0001-8 TALLAHASSEE
RESEARCH TOWARD A HETEROGENEOUS NETWORKED COMPUTER CLUSTER

$$
F L \text { FL } \$ 998,780 \quad \$ 674,127
$$

RESEARCH IN THEORETICAL NUCLEAR PHYSICS

$$
\text { FL } 02 \text { THEORETICAL NUCLEAR PHYSICS } \$ 1,038,000
$$

GUARD CELL BIOCHEMISTRY: RESP ONSE TO ENVIRONMENTAL STIMULI

$$
\begin{array}{lll}
F L & & \$ 825,452
\end{array} \$ 825,452
$$

TUDIES OF BOTTOM BOUNDARY LAYER PROCESSES ALONG

$$
\mathrm{FL} 02 \quad \begin{aligned}
& \$ 218,777 \\
& \$ 214,216
\end{aligned}
$$

TRANSFORMATION RATES AND RATE OF DISSOLVED, COLLOIDAL AND PA

$$
\mathrm{FL} \quad 02 \quad \$ 451,508 \quad \$ 179,975
$$

SUPPORT OF EXPERIMENTAL NUCLEAR PHYSICS AT THE SUPERCOMPUTER $\mathrm{FL} 02 \quad \$ 312,000 \quad \$ 204,000$

LIQUID HELIUM FIUID DYNAMICS STUDIES

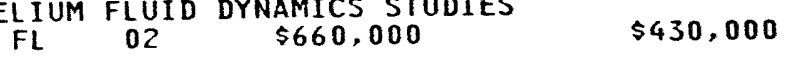

NEW AWARD - THIRD INTERHATIONAL CONFERENCE ON BIOINFORMATICS

$$
\mathrm{FL} \quad 02 \text { \$14,550 } \$ 14,550
$$

ENERGY TRANSFORMATION IN MOLECULAR ENVIRONMENTAL RESEARCH

$$
\mathrm{FL} 02 \quad \$ 973,460 \quad \$ 823,160
$$

RESEARCH IN ACTINIDE CHEMISTRY

FL 02 \$1,019,000

$\$ 889,000$

DOE SUBSURFACE MICROBIAL CULTURE COLLECTION

$$
\text { FL } \quad 02 \quad \$ 991,654 \text { MICROBIAL }
$$

SELECTIVE EXCITATION, RELAXATION, AND ENERGY CHANHELING IN M FL $02 \quad \stackrel{\$ 469,885}{ }$

$\begin{array}{llll}\text { RESEARCH IN ELEMENTARY PARTICLE PHYSICS } & \\ & \text { FL } & 02,212,000\end{array}$

HE-ATOM SURFACE SCATTERING APPARATUS FOR STUDIES OF CRYSTALL $\mathrm{FL} 02 \quad \$ 1,566,830 \quad \$ 1,276,070$ HEAVY FERMIONS AND OTHER HIGHLY CORRELATED ELECTRON SYSTEMS
FL
02 $\mathrm{FL} \quad 02 \quad \$ 431,899$

FLUID DYNAMICS OF SUPERCRITICAL HELIUM WITHIN CABLED-IN-COND $\mathrm{FL} \quad 02 \quad \$ 800,000 \quad \$ 240,000$ 
BIN COMPL DATE

VENDOR ID CITY STATE CONG CONTRACT DESCRIPTION OF WORK

FG02-85CE40740 12/31/94 028970-1302-8 GAINESVILLE

COLIEGE OF ENGINEERING

FG02-86NE37967 05/31/95

NUCLEAR ENGINEERING SCI DEPT

FC02-93CH10564 01/31/96 028970-0002-3 GAINESVILLE

FG03-93SF20139 09/29/95 028970-0002-3 GAINESVILLE

FG05-94IS40025 09/30/94 028970-0002-3 GAINESVILLE

PHYSICS DEPT

FG05-86ER40272 02/28/97 028970-0802-4 GAINESVILLE

FG05-86ER13574 06/30/96 028970-1702-3 GAINESVILLE

MICROBIOL OGY DEPT

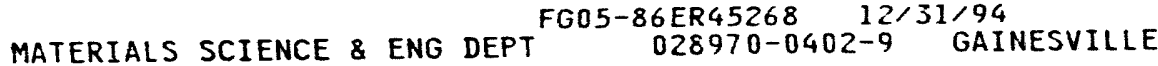

MICROBIOLOGY DEPT

FG05-86ER13581 07/31/95 028970-1702-3 GAINESVILLE

FG05-86ER45280 10/31/95 028970-0802-4 GAINESVILLE

PHYSICS DEPT

CHEMISTRY DEPT

CHEMISTRY DEPT
FG05-88ER13881 $12 / 31 / 94$
$028970-0102-X$ GAINESVILLE

FG05-89ER14018 04/30/97 028970-0102-X GAINESVILLE

FG05-91ER45462 03/24/95 028970-0002-3 GAINESVILLE

FG05-94ER61785 01/14/95 $028970-0002-3$ GAINESVILLE

FG05-93ER7587104/14/95 $028970-0002-3$ GAINESVILLE

FG05-93ER75880 04/14/95 3ER75880 $04 / 14 / 95$
$028970-0002-3$ GAINESVILLE

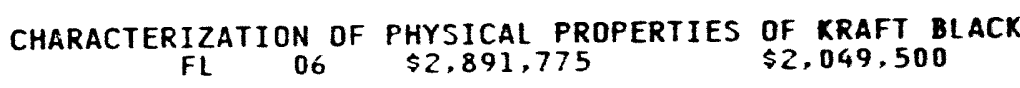

REMOTE TELEPRESENCE ROBOTICS SYSTEM FOR INSPECTION AND MAINT $\mathrm{FL} 06 \quad \$ 8,784,060 \quad \$ 4,570,500$

DEVELOPMENT OF ON-LINE YISCOMETERS FOR KRAFT BLACK FL 05 \$314,460 $\$ 300,000$

PLASMA SPECTROSCOPY AND EXPERIMENTAL DIAGNOSTIC DEVELOPMENT, $\begin{array}{lll}\text { FL } 05 & \$ 132,478 & \$ 132,478\end{array}$

IAEA TRAINING COURSE ON NUCLEAR TECHNIQUES IN INSECT CONTROL FL $06 \quad \$ 87,000 \quad \$ 87,000$

RESEARCH IN THEORETICAL AND EXPERIMENTAL ELEMENTARY PARTICLE $F L \quad 06 \$ 9,761,678 \quad \$ 7,961,678$

PYRUVATE DECARBOXYLASE: A KEY ENZYME FOR ALCOHOL PRODUCTION $\mathrm{FL} 06 \quad \$ 832,280$ \$718,93?

STUDIES OF HEAVY FERMION SYSTEMS

$\$ 799,972$

GENE-ENZYME RELATIONSHIPS IN SOMATIC CELLS AND THEIR ORGANIS FL ${ }_{06}$ \$911.866 $\$ 911,866$

H-RAY SCATIERING STUDIES OF NONEQUILIBRIUM ORDERING PROCESSE $F L$ O6 $\$ 586.036 \quad \$ 524.236$

DEVELOPMENT OF LASER EXCITED ATOMIC FLUORESCENCE AND IONIZAT FL $06 \quad \$ 664.340 \quad \$ 664,340$

THE GLOW DISCHARGE AS AN ATOMIZATION AND IONIZATION SOURCE

FL $06 \quad \$ 732,240 \quad \$ 544,240$

QUANTUM-CONFINEMENT EFFECTS AND OPTICAL BEHAVIOR OF INTERMED

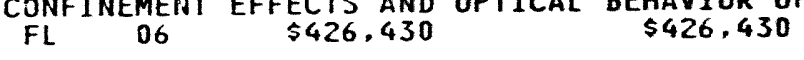

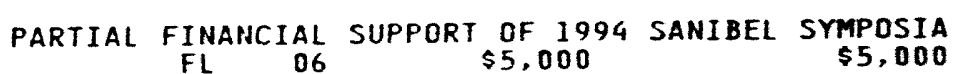

DROPLET-VAPOR CORE REACTORS WITH MHO POWER CONVERSION UNITS FL 05 R $\$ 48,344$

$\$ 48,344$

AN INNOVATIVE FUEL DESIGN COHCEPT FOR IMPROVED LIGHTWATER RE $\mathrm{FL} \quad 06 \quad \$ 100,000 \quad \$ 100,000$ 


ANARDEE NAME

AWARDEE NAME

AWARDEE NAME BIN COMPL DATE

DESCRIPTION OF HORK

DIVISION COLLEGE OF ENGINEERING

VENDOR ID CITY 91 PC $90182--12 / 12 / 94---1302-8$ GAINESVILLE
$028970-1302-8$. FG44-92R434896 09/30/94 028970-0002-3 GAINESVILLE

FLOUR BLUFF ISD FG46-92R613558 09/30/94
$223240-0001-6$ CORPUS CHRISTI

FLOYD COUNTY SCHOOL BOARD

FG44-92R434966 07/31/94 029050-0002-3 PRESTONSBURG

FG44-93R435314 09/30/95 029050-0002-3 PRESTONSBURG

FLUOR DANIEL ENV REST MGMT CO

ACO5-920R21972 11/30/97
$226590-0001-8$ FERNALD

FLUOR DANIEL INC

$$
\begin{gathered}
\text { AC05-910R21964 } 09 / 05 / 94 \\
170520-0002-4 \text { IRVINE } \\
\text { AC07-94ID13206 12/31/96 } \\
170520-0002-4 \text { IRVINE }
\end{gathered}
$$

FM TECHNOLOGIES INC

FG05-91ER81245 07/05/94
$174560-0001-8$ FAIRFAX

$A C 65-92 W B 0272907 / 12 / 94$
$203300-0001-2$ GOLDEN

ACO1-92AD64622 $04 / 30 / 95$
$029260-0002-2$ HASHINGTON

AC07-90ID13019 09/18/94 029260-0101-0 DEARBORN

SCIENTIFIC RESEARCH LABS

FORREST COUNTY AGRIC HIGH SCH

FG44-85R.432247 07/01/94 $129800-0001-9$ BRDOKLYN $=$
FORREST COUNTY SCHOOL DISTRICT FG44-85R432248
$129810-0001-2$ HATTIESBURG
CONG CONTRACT

OBLIGS

TO DATE P/S STATE DIST VALUE OF-GRANULAR FLOW DOWN ON INCLINED
RESEARCH ENTITIED "STUDIES OF FL 06 \$728.345 $\$ 648,560$ AG11 CYCLE 14 - INSTITUTIONAL CONSERVATION PROGRAM. APPROVED BUD THIS IS A NEW CYCLE 14 ENERGY CONSERVATION MEASURE GRANT AWA

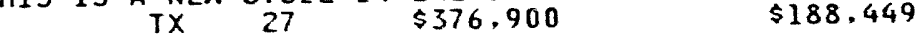

CYCLE 14 - INSTITUTIONAL CONSERVATION PROGRAM. APPROVED BUD $\begin{array}{lll}\mathrm{KY} \text { TY } & \$ 75,375 & \$ 66,346\end{array}$ SEE ATTACHED PAGE NO. 2 OF THIS NOTICE OF FINANCIAL ASSISTAN KY $05 \quad \$ 3,950 \quad \$ 1,822$

ENVIRONMENTAL RESTORATION MANAGEMENT CONTRACTOR FOR THE FERN $O H \quad 08 \$ 2.461 .692 .245 \quad \$ 691,759,960 \quad M 300$

A-E SERVICES FOR RECONFIGURATION OF THE DDE NUCLEAR WEAPONS CA $40 \$ 143,718,101 \quad \$ 73.718 .101 \quad$ C219 MISCELLANEOUS A-E SERVICES AT THE INEL - CY 1994-98 MODULATOR SYSTEMS FOR HEAVY ION FUSIOH ACCELERATORS $\begin{array}{lll}\text { VA } & \text { MS } \$ 549,558 & \$ 549,558\end{array}$

SUMMIT-WATERTOWN T.L. PROJECT ENVIRONMENTAL STUDY

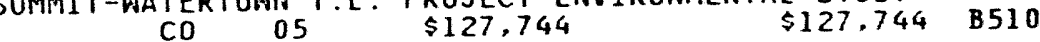

LEASING OF I3 VEHICLES $\$ 96,670 \quad \$ 96,670 \quad 9999$ R\&D FOR MODULAR ELECTRIC VEHICLE PROGRAM (MEVP) MI $16 \quad \$ 9,610,986 \quad \$ 7,068,000 \quad$ AG83 NONE MS $05 \quad \$ 28,256 \quad \$ 14,126$ GRANT PROGRAM FOR SCHODLS AND HOSPITALS AND BUILDINGS OWNED MS 05 \$ $\$ 50,736 \quad \$ 25,366$ 


\begin{tabular}{|c|c|c|}
\hline AWARDEE NAME & COMPL DATE & DESCRIPTION OF WORK \\
\hline DIVISION & VENDOR ID & STATE DIST \\
\hline FORT ATKINSON SCHOOL DISTRICT & $\begin{array}{c}\text { FG45-93R551795 12/31/94 } \\
029360-0001-7 \text { FORT ATKINSON }\end{array}$ & $\begin{array}{c}\text { ICP-NEW AWARD. } \\
\text { WI }\end{array} 09$ \\
\hline $\begin{array}{l}\text { FORT HALL TOWN OF } \\
\text { POLICE DEPT }\end{array}$ & $\begin{array}{r}\text { GM07-93ID11106 } 12 / 30 / 99 \\
225050-0101-4 \text { FORT HALL }\end{array}$ & $\begin{array}{rrr}\text { INEL FIRING RANGE USE AGREEMENT WITH FORT HALL POLICE DEPT } \\
\text { ID } & 02 & \$ 0\end{array}$ \\
\hline FORT HAYS STATE UNIVERSITY & $\begin{array}{c}\text { FG02-91CH10471 } 07 / 28 / 94 \\
101130-0001-4 \text { HAYS }\end{array}$ & 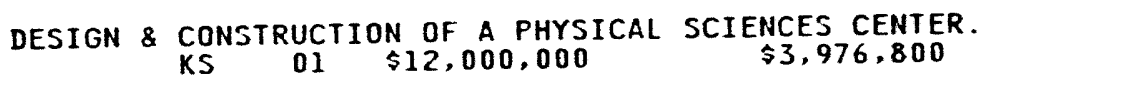 \\
\hline FORT LEWIS COLLEGE & $\begin{array}{l}\text { GMO4-94AL94708 02/22/99 } \\
145210-0001-0 \text { DURANGO }\end{array}$ & SUPPORT FOR SCIENCE, MATHEMATICS AND ENGINEERING EDUCATION \\
\hline FORT OSAGE R-I SCHOOL DISTRICT & $\begin{array}{l}\text { FG47-92R706065 } 08 / 03 / 94 \\
221780-0001-0 \text { INDEPENDENCE }\end{array}$ & $\begin{array}{clcc}\text { GRANT PROGRAMS FOR SCHOOLS ANDHOSPITALS AND FOR BUILDINGS } 0 \\
\text { MO } & 05 & \$ 220,968 & \$ 110,484\end{array}$ \\
\hline FORT VALLEY STATE COLLEGE & $\begin{array}{l}\text { FG05-88ER75424 09/24/94 } \\
029510-0001-4 \text { FORT VALLEY }\end{array}$ & 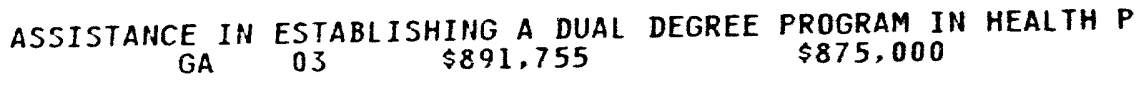 \\
\hline FORT WORTH MUS OF SCI \& HIST & $\begin{array}{c}\text { FG03-94ER75955 08/11/95 } \\
236600-0001-9 \text { FORT WORTH }\end{array}$ & 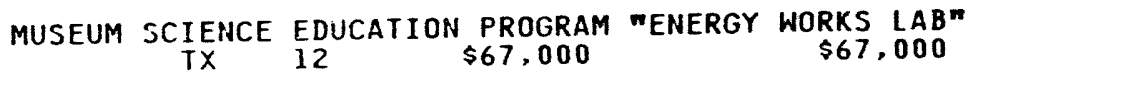 \\
\hline FOSTER MILLER ASSOC INC & $\begin{array}{l}\text { FG02-92ER81370 } 05 / 24 / 95 \\
029570-0002-4 \text { WALTHAM }\end{array}$ & $\begin{array}{ccc}\text { STEAM GENERATOR UPPER BUNDLE INTER TUBE INSPECTIN AND } \\
\text { MA } 08 & \$ 549,929 & \$ 338,685\end{array}$ \\
\hline & $\begin{array}{c}\text { FG02-94ER81667 } 07 / 17 / 94 \\
029570-0002-4 \text { WALTHAM }\end{array}$ & 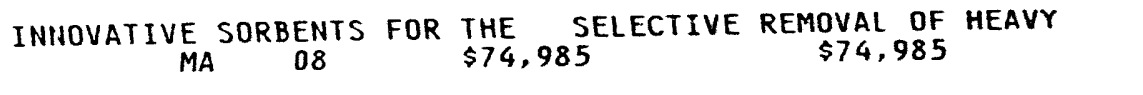 \\
\hline FOSTER WHEELER DEV CORP & $\begin{array}{l}\text { AC21-86MC21023 } 12 / 31 / 94 \\
029590-0001-3 \text { LIVINGSTON }\end{array}$ & $\begin{array}{ccccc}\text { PRESSURIZED FLUIDIZED-BED } & \text { COMBUSTION (PFBC) - } & \text { SECOND } \\
\text { NJ } & 11 & \$ 42,161,130 & \$ 37,945,017 & \text { AGI3 }\end{array}$ \\
\hline & $\begin{array}{c}\text { AC22-91PC91154 04/30/95 } \\
029590-0001-3 \text { LIVINGSTON }\end{array}$ & 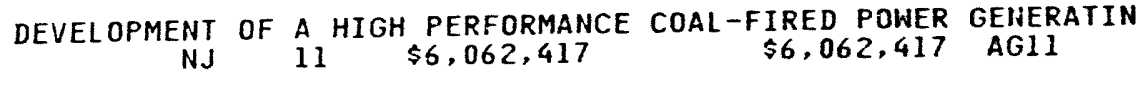 \\
\hline FOSTER WHEELER USA CORP & $\begin{array}{r}\text { ACO1-94PR10017 10/04/96 } \\
121930-0001-8 \text { LIVINGSTON }\end{array}$ & $\begin{array}{clcc}\text { SUPPORT SERVICES TO DEVEL OP INDEP COST ESTIMATE OF DOE WASTE } \\
\mathrm{NJ}\end{array}$ \\
\hline & $\begin{array}{c}\text { ACO1-94PR10016 10/18/96 } \\
121930-0001-8 \text { LIVINGSTON }\end{array}$ & $\begin{array}{c}\text { TECHNICAL SUPPORT SERVICES FORINDEPENDANT COST ASSESSMENTS } \\
\text { NJ } 11 \\
\$ 4,985,284\end{array}$ \\
\hline
\end{tabular}




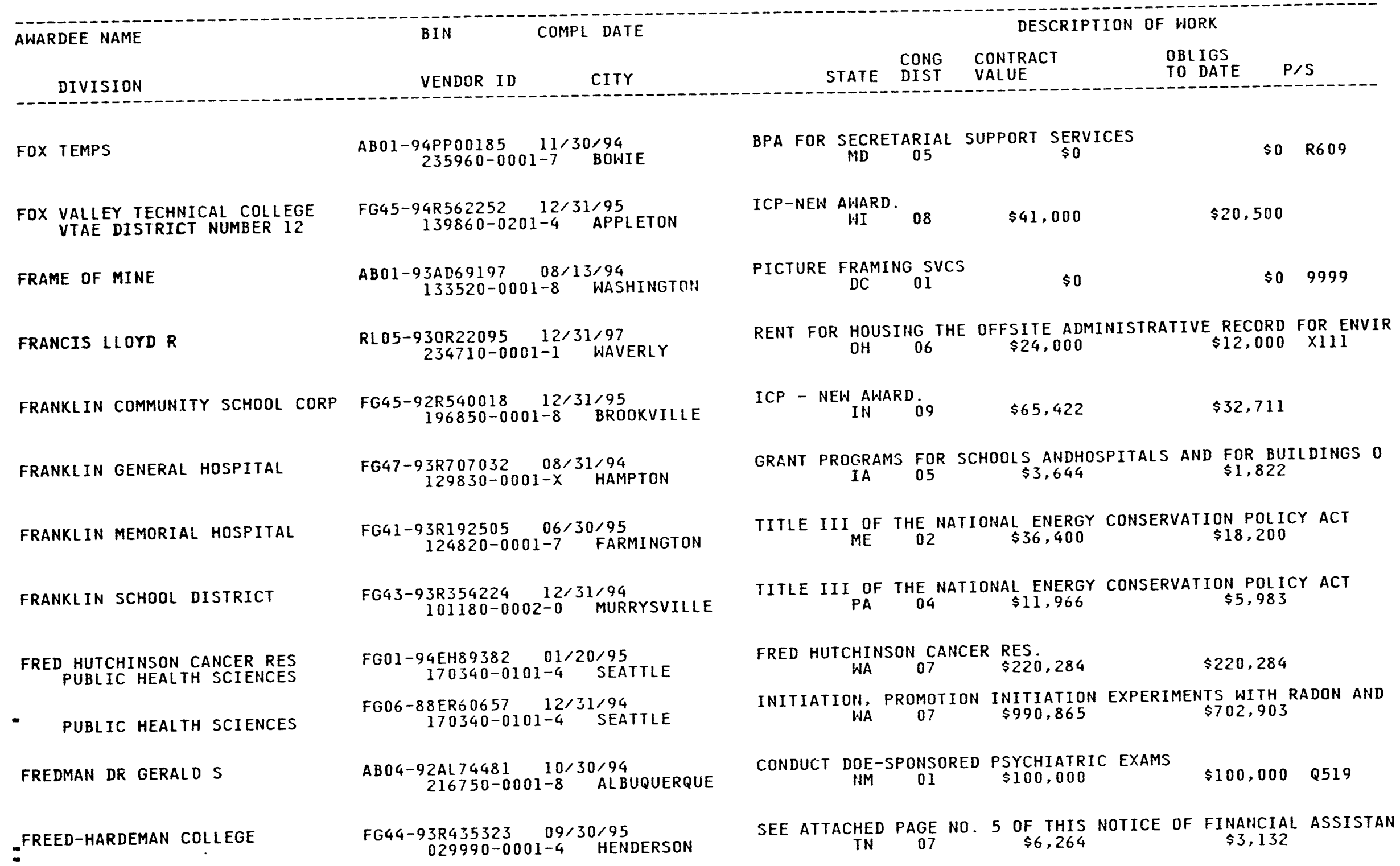




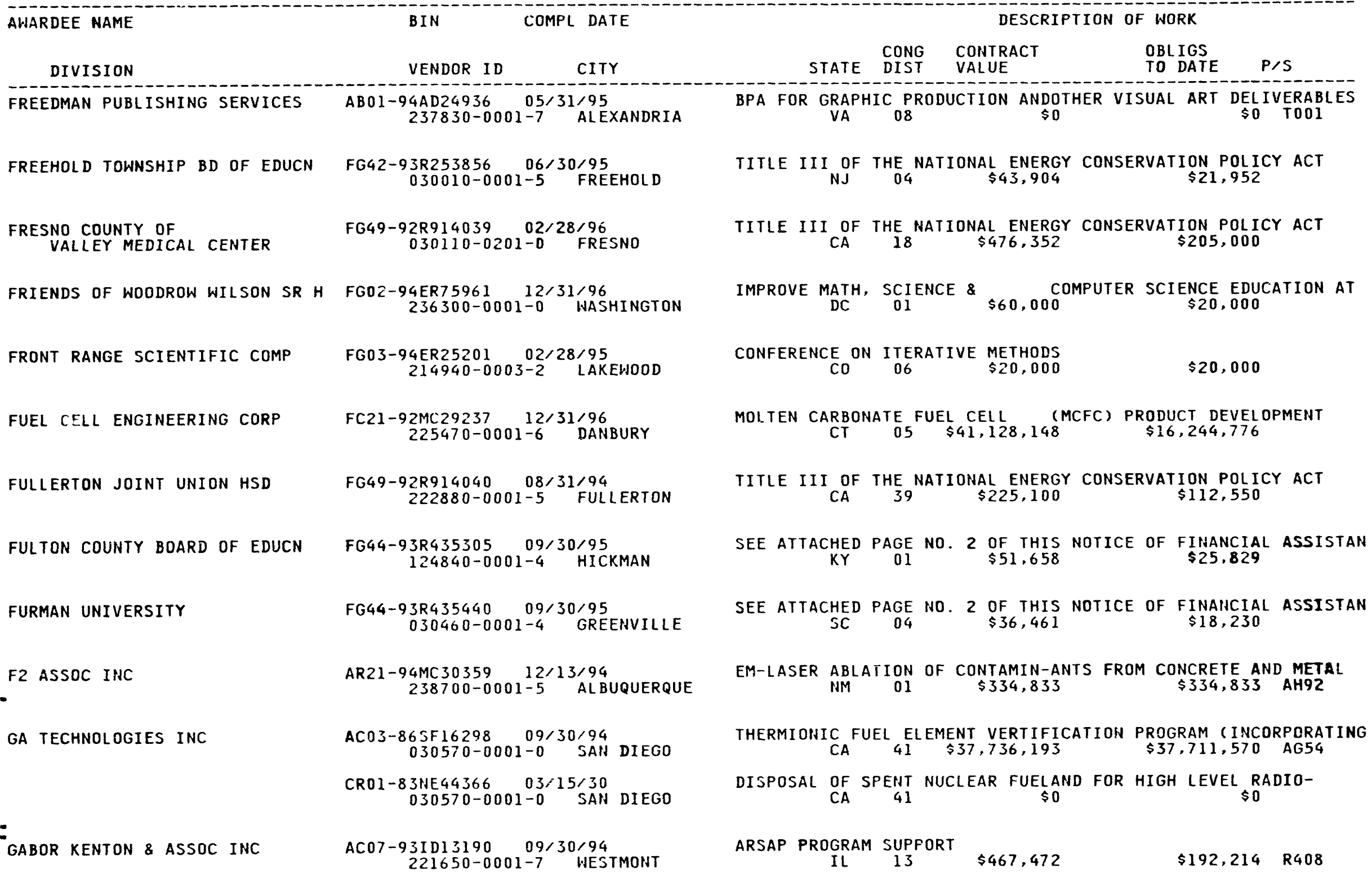




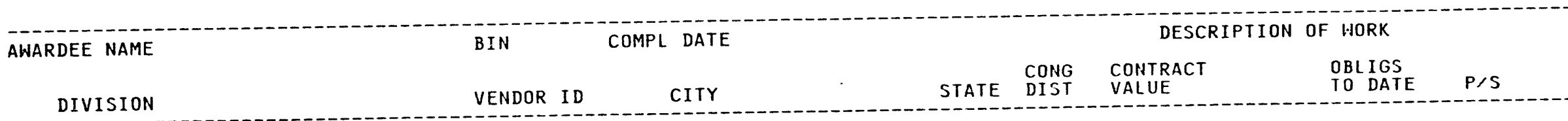

GALLEGOS SANITATION INC

GALLIHER \& HEUGLEY INC

GALLUP TOWN OF

MUNICIPAL SCHOOLS

GALVESTON COLLEGE

SAMBIA GOVERNMENT OF THE

GAMBRELL'S CLEANERS

GAMMA CORP

GARCIA CONSULTING INC

GARDNER-ZEMKE CO

GARFIELD SCHOOL DISTRICT

:

GARRETT CERAMIC COMPONENTS
AX65-94HJ22318 09/30/94 235450-0001-X FORT COLLINS

$A B 01-93 A D 63 \cap 21 \quad 09 / 30 / 94$ $234500-0001-2$ WASHINGTON

GM04-92AL58238 04/02/97 226740-0101-1 GALLUP

FG46-93R613625 08/31/94 030760-0001-2 GALVESTON FC02-93P010096 $09 / 29 / 95$
$234380-0001-6 \quad$ BANJUL

AB01-94AD69252 07/05/95 $204960-0001-2$ WASHINGTON

$A B 01-93 A D 63008 \quad 07 / 31 / 94$ 204960-0001-2 WASHINGTON

$A B 01-93 A D 69203 \quad 07 / 05 / 94$ 204960-0001-2 MASHINGTOH

ACO1-93IE11130 04/21/95 $3 I E 1113004 / 21 / 95$
$182320-0001-5$ FALLS CHURCH

AC01-92DP00191 07/30/97 $224260-0001-5$ ARLINGTON

AC65-93WL 04210 11/30/94 030920-0003-X VERHAL

FG48-91R807664 10/31/94 $1 R 807664 \quad 10 / 31 / 94$
$119620-0001-X \quad$ PANGUITCH

FG02-92CE41002 07/31/94
TRASH REMOVAL SVCS

CO $\quad 04 \quad \$ 4,735$

$\$ 4,735 \$ 205$

BPA FOR LUMBER SUPPLIES AND MATERIALS

$\$ 0 \quad 5510$

SUPPORT FOR SCIENCE, MATHEMATICS AND ENGINEERING $\underset{\$ 0}{\$ 0}$ TO IMPROVE

$\begin{array}{rrr}\text { AWARDS A NEH CYCLE } & 15 \text { TECHNICAL ASSISTANCE GRANT. } \\ & \$ 20,781 & \$ 10,390\end{array}$

CLIMATE CHANGE COUNTRY STUDY.

DONTRY \$157,000

$\$ 134,000$

BPA FOR CLEANING SERVICES

BPA FOR DRY CLEANING AND LAUNDE: ING

$\$ 0 \quad \$ 209$

BPA FOR CLEANING SERVICES

$\$ 0 \quad \$ 209$

SUPPORT SERVICE PROVIDING ENERGY EMERGENCY SIMULATIONS $\begin{array}{lll}\text { SERVICE PROVIDING ENERGY EMERGENCY } & \$ 115,000 \\ \text { BA } & \$ 389,479 & \end{array}$

SUPPORT FOR CLASSIFIED MAILROOM CONTROL AND MANAGEMENT; PERS VA $10 \quad \$ 5,896,475 \quad \$ 2,675,567 \quad$ R421

FLAMING GORGE SUBST
UT 03
$\$ 1,397,992$
$\$ 1,397,992 \quad Y 239$

CYCLE XIII AWARD

$\$ 26,409$

$\$ 13,790$

CONTINOUS FIBER CERAMIC COMPONETS 


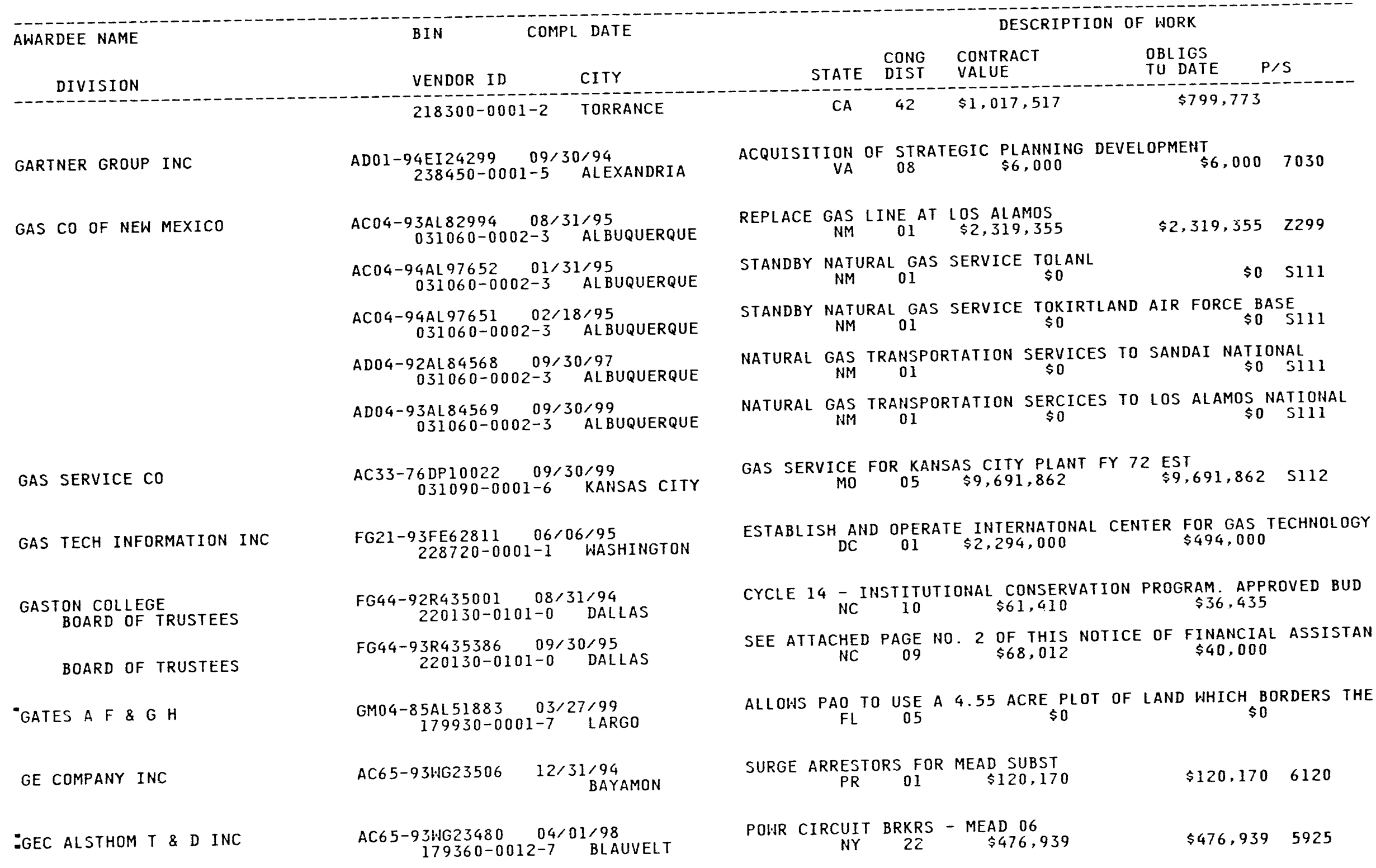




\begin{abstract}
AWARDEE NAME
\end{abstract}
BIN

COMPL DATE

VENDOR ID CITY 179360-0007-0 WATERTOWN

GEISINGER WYOMING VALLEY MED

FG43-92R344209 09/30/94 $219270-0001-7$ WILKES-BARRE

GEM TECHNOLOGY IHC

GENERAL ATOMIC CO

GENERAL ATOMICS

PROCESS DEVELOPMENT DEPT

. GENERAL EYNAMICS CORP

GENERAL ELECTRIC CO

ADVANCED NUCLEAR TECHNOLOGY ADVANCED NUCLEAR TECHIOLOGY

$$
\begin{array}{r}
\text { AC06-94RL12863 01/08/95 } \\
238440-0001-1 \quad \text { RICHLAND }
\end{array}
$$

AC03-91SF18601 09/30/95 205270-0001-9 SAN DIEGO

AC01-88ID12698 12/31/94 $031270-0001-4$ SAII DIEGO

AC03-84SF11962 09/30/94 $031270-0001-4$ SAN DIEGO

ACO3-89ER51114 10/31/98 $031270-0001-4$ SAN DIEGO

AC03-89ER52153 10/31/97 $031270-0001-4$ SAN DIEGO

AC03-89SF17885 12/31/94 $031270-0201-7$ SAN DIEGO

FG03-92ER54150 01/31/95 $031270-0001-4$ SAN DIEGO

AC04-93AL94418 07/21/96 $031270-0001-4$ SAN DIEGO

FC02-93CH10554 08/09/95 $233460-0001-X$ SAN DIEGO

AC03-93SF19681 09/30/94 031310-0019-8 SAll JOSE ACO3-895F17445 12/31/94 $031310-4719-4$ SAN JOSE

AC03-90SF18494 09/30/95 $031310-4719-4$ SAN JOSE
DESCRIPTION OF WORK

CONG CONTRACT

$\begin{aligned} & \text { STATE DIST } \text { VALUE } \\ & \text { WATERTOWN STATIC VAR SYSTEM, - FURNISH } \\ & \text { SD OI } \$ 8,485,970\end{aligned}$

TO DATE

INSTALL
$\$ 8,485,970 \quad 6150$

CONSERVATION POL ICY ACT

$P A \quad I 1$ \$ $\$ 78,194 \quad \$ 66,832$

INTEGRATED INFORMATION SYSTEM TO SUPPORT THE ANALYSIS WA $04 \quad \$ 300,000 \quad \$ 300,000 \quad B 506$

INERTIAL CONFINEMENT FUSION TARGET SUPPORT

$$
\begin{array}{llll}
\text { CONFINEMENT FUSION TARGET } & \text { SUPPORT } & \$ 25,782,000 & \text { AZ12 }
\end{array}
$$

DEVELOPMENT OF A LEGAL WEIGHT TRUCK CASK AND OVERHEIGHT TRUC CA $44 \quad \$ 21,163,605 \quad \$ 16,494,992$ AG54 HIGH TEMPERATURE GAS REACTOR TECHNOLOGY PROGRAM CA 41 \$33,238,324 $\$ 30,314,889$ AG51 $\begin{array}{ccc}\text { OPERATION OF D-III FACILITIES AND OTHER RELATED RESEARCH } \\ \$ 492,292,154 & \$ 212,479,246 \text { AG } 92\end{array}$ OPERATION OF D-III FACILITIES AND OTHER RELATED RESEARCH -

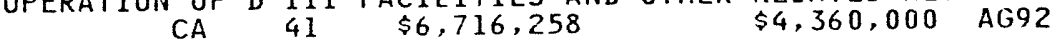
PRELIMINARY AND FINAL DESIGNS IN SUPPORT OF THE MODULAR HIGH CA 41 \$241,657,336 $\$ 51,888,209 \quad A G 92$

FUSION PROGRAMS IN APPLIED PLASMA PHYSICS DEFENSE HIGH LEVEL WASTE CASK PROGRAM FINAL DESIGN AND NRC CA $41 \quad \$ 727,717 \quad \$ 200,000$ AZ13 DESIGN AND BUILD POOL TYPE SUPERCONDUCTING FAULT CURRENT CA $49 \$ 3,425,800 \quad \$ 2,281,027$

INITIATE PU CONSUMPTION EFFORT $C A \quad 13 \$ 4,281,75$ $\$ 4,281,751 \quad A G 53$ BASIC CONTRACT FOR RESEARCH AND DEVELOPMENT OF A REFERENCE $C A \quad 07 \$ 50,912,649 \quad \$ 45,621,559$ AG53 DESIGN AND NRC CERTIFICATION OF ALWR PLANT CA $13 \$ 140,554,221 \quad$ $\$ 45,371,000$ AG53 


\begin{tabular}{|c|c|c|c|c|c|c|c|}
\hline AWARDEE NAME & BIN & L DATE & & & $\mathrm{DESCF}$ & F WORK & \\
\hline DIVISION & VENDOR ID & CITY & STATE & $\begin{array}{l}\text { CONG } \\
\text { DIST }\end{array}$ & $\begin{array}{l}\text { CONTRACT } \\
\text { VALUE }\end{array}$ & $\begin{array}{l}\text { OBLIGS } \\
\text { TO DATE }\end{array}$ & $\mathrm{P} / \mathrm{S}$ \\
\hline
\end{tabular}

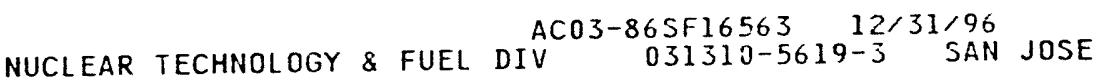

CRO1-83NE44426 03/15/30 031310-0019-8 SAN JOSE

CRO1-83NE $4438403 / 15 / 30$ 031310-0025-2 SUNNYVALE

AC65-94WG21877 04/30/98 031310-0048-1 FORT EDWARD

FC02-92CEG1000 07/01/94

CORP RESEARCH \& DEV DEPT $031310-0721-4$ SCHENECTADY

FCO2-93CH10589 09/30/95 $031310-0021-X$ SCHENECTADY

FG02-94ER1214201/14/97 $031310-0721-4$ SCHENECTADY

CORP RESEARCH \& DEV DEPT AC21-88MC25003 $01 / 31 / 95$
RESEARCH \& DEVELOPMENT CENTER $031310-2921-8$
SCHENECTADY AC21-93MC30174 09/30/94 031310-0721-4 SCHENECTADY CORP RESEARCH \& DEV DEPT AC21-93MC30244 07/24/95 $031310-5321-6$ SCHENECTADY POWER GENERATION ACO3-89SF $17787 \quad 12 / 31 / 94$ RESEARCH \& DEVELOPMENT CENTER

ENVIRONMENTAL SERVICES $A C 21-87 M C 2317009 / 30 / 94$
$031310-4442-X \quad$ LEBANON AC65-91\%N07917 02/12/98 $031310-0061-9$ BAYAMON

FG51-93R020312 08/31/94 $224190-0001-3$ EVERETT

AC21-86MC23165 10/24/94 $031360-0103-6$ INDIANAPOL IS

GENERAL MOTORS CORP
ALLISON GAS TURBINE DIV
"SMALL PLANT DESIGN STUDIES AND ABWR DESIGN VERIFICATION PRO CA I3 $\$ 87,900,972$

DISPOSAL OF SPENT NUCLEAR FUEL $\$ 39,926,560$ AG53

DISPOSAL OF SPENT NUCLEAR FUEL CA 10 $\$ 0$

(1) $500 \mathrm{KV}$ SERIES CAPACITOR BANK

$\$ 2,452,692 \quad 5910$

CONTINOUS FIBER CERAMIC COMPOSITES PROGRAM

100 MVA GENERATOR UTILIZING HIGH TEMPERATURE WINDINGS NY 21 \$3,164,990 $\$ 1,500,000$

EVAPGRATION THROUGH TANGSTEN TO ACHIEVE HIGH-RATE VAPOR NY $28 \quad \$ 899,956$

ENHANCED DURABILITY FOR HIGH TEMPERATURE DESULFURIZATION NY 23 \$1, 118,222 $\$ 1,118,222$ AG12 EM - RAPID SURFACE SAMPLING AND ARCHIVAL RECORD (RSSAR) RESEARCH TITLED "ADVANCED TURBINE SYSTEMS (ATS) PROGRAM NY $21 \$ 4,629,360 \quad \$ 3,414,859$ AG23 $\begin{array}{rrrr}\text { ENGINEERING STUDIES FOR THE SP-100 SPACE REACTOR POHER } \\ \text { PA } & 13 & \$ 8,994,652 & \$ 3,273,968 \text { AG54 }\end{array}$ INTEGRATED OPERATION OF A PRESSURIZED FIXED-BED GASIFIER PA 16 \$26,825,800 $\$ 22,835,907$ AG13

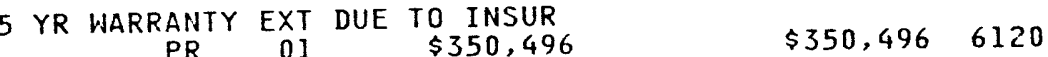

CYCLE 15 ECM AHARD.
HA 02
$\$ 271,752$
$\$ 135,876$ $\begin{array}{llll}\text { RESEARCH ENTITLED ADVANCED COAL-FUELED GAS TURBINE } \\ \text { IN } 06 & \$ 39,319,915 & \$ 28,437,090 \text { AG }\end{array}$ ADVANCED TURBINE SYSTEMS - PFOGRAM CONCEPTUAL DESIGH AND 


\begin{tabular}{|c|c|}
\hline AWARDEE NAME & COMPL DATE \\
\hline DIVISION & VENDOR ID \\
\hline ALLISON GAS TURBINE DIV & $031360-0103-6$ \\
\hline GENERAL SERVICE ADMINISTRATION & $\begin{array}{l}09 / 30 / 94 \\
\text { HUNTSVILLE }\end{array}$ \\
\hline \multirow[t]{11}{*}{$\begin{array}{l}\text { GENERAL SERVICES ADMIN } \\
\text { IRM }\end{array}$} & $\begin{array}{c}\text { AD03-935F20040 09/29/94 } \\
031400-3645-0 \text { OAKLAND }\end{array}$ \\
\hline & $\begin{array}{c}\text { AI04-93AL } 9127409 / 30 / 95 \\
031400-0008-1 \text { DENVER }\end{array}$ \\
\hline & $\begin{array}{r}\text { GM04-87AL } 5820209 / 24 / 97 \\
031400-0040-5 \text { GOLDEN }\end{array}$ \\
\hline & $\begin{array}{l}\text { RL13-64GJ31043 12/31/96 } \\
031400-0035-9 \text { GRAND JUNCTION }\end{array}$ \\
\hline & $\begin{array}{c}\text { AI01-90CE50056 } 12 / 07 / 95 \\
031400-0022-7 \text { WASHINGTON }\end{array}$ \\
\hline & $\begin{array}{l}\text { AI01-92CE50342 } 12,15 / 96 \\
031400-0022-7 \text { WASHINGTON }\end{array}$ \\
\hline & $\begin{array}{c}\text { AI01-90CE73515 09/30/94 } \\
031400-0022-7 \text { HASHINGTON }\end{array}$ \\
\hline & $\begin{array}{c}\text { AI01-91AD36258 } 06 / 18 / 96 \\
031400-0022-7 \text { WASHINGTON }\end{array}$ \\
\hline & $\begin{array}{c}\text { AI01-941G00389 } 09 / 30 / 94 \\
031400-0022-7 \text { WASHINGTON }\end{array}$ \\
\hline & $\begin{array}{c}\text { AI01-93AD33519 } 09 / 30 / 97 \\
031400-0022-7 \quad \text { WASHINGTON }\end{array}$ \\
\hline & $\begin{array}{c}\text { AI01-93AD64797 09/29/94 } \\
031400-0022-7 \text { WASHINGTON }\end{array}$ \\
\hline AUTOMOTIVE COMMODITY CENTER & $\begin{array}{c}\text { AD07-93ID10529 } 12 / 29 / 94 \\
031400-3222-6 \text { WASHINGTON }\end{array}$ \\
\hline AUTOMOTIVE COMMODITY CENTER & $\begin{array}{c}\text { AD07-93ID10528 } 12 / 29 / 94 \\
031400-3222-6 \text { WASHINGTON }\end{array}$ \\
\hline AUTOMOTIVE COMMODITY CENTER & $\begin{array}{c}\text { AD07-93ID10525 } 12 / 29 / 94 \\
031400-3222-6 \text { WASHINGTON }\end{array}$ \\
\hline
\end{tabular}

DATA ENTRY AND ANCILLARY $\begin{gathered}\text { SERVICES FOR THE PERIOD } \\ \$ 91,000\end{gathered}$

GSA/GSA CONTRACTOR, CSC TO PERFORM INDEPENDENT ANALYSIS OF D PURCHASE SUPPLIES FROM DENVER GSA SELF SERVICE STORE BASIC \& SUPPLEMENTAL GSA INTERAGENCY FLEET MANAGEMENT

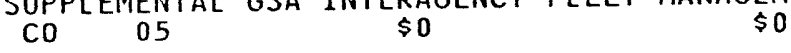
SPACE LEASE (GS-08B-04296) CO 030 (GS-08B-04296) \$0 \$0 $\times 111$

INTERAGENCY AGREEMENT WITH GSATO PROVIDE ACQUISITION OF FED DC 01 \$22,350,000 $\$ 8,788,000$ SECONDARY DISTRIBUTION OF GAS MILEAGE GUIDE DC $01 \quad \$ 836,000 \quad \$ 476,650$ SUPPORT FOR THE FEDERAL RELIGHTING INITIATIVE

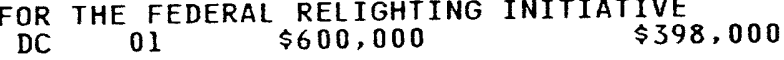
AN IA FOR INDEPENDENT MGMT REVIENS. INTERNAL CONTROL EVALUAT AN IA FOR INDEPENDENT MGMT REV 01 \$382,000 $\begin{array}{ccc}\text { INTERAGENCY } & \text { AGREEMENT FOR EQUIPMENT \& SUPPLIES } \\ 01 & \$ 1,500 & \$ 1,500\end{array}$ SUPPORT COMSEC EQUIPMENT
DC
$01 \$ 6,480,000$
$\$ 2,113,137$

PROVIDE INTERAGENCY AGRREMENT FOR REDISTRIBUTION OF EXCESS $F$ DC OI $\$ 16,463$ $\$ 16,463$

PURCHASE SEVEN UTILITY, PASSENGER CARGO (4X4) $\begin{array}{llll}\text { DC } 01 & \$ 105,000 & \$ 105,000 & 2310\end{array}$

PURCHASE 29 UTILITY WAGONS $(4 \times 4)$ $\$ 363,000 \quad 2310$ $\begin{array}{ccccc}\text { PURCHASE TWO } & 3 / 4-T O N \text { PICKUPS, EXTENDED CAB } & (4 \times 4) \\ \text { DC } & 01 & \$ 28,000 & \$ 28,000 & 2310\end{array}$ 


\section{AD07-93ID10522 12/29/94}

AUTOMOTIVE COMMODITY CENTER

AUTOMOTIVE COMMODITY CENTER

AD07-93ID10518 12/29/94

$031400-3222-6$ WASHINGTON

AD07-93ID10517 12/29/94

AUTOMOTIVE COMMODITY CENTER

AUTOMOTIVE COMMODITY CENTER

AUTOMOTIVE COMMODITY CENTER

AUTOMOTIVE COMMODITY CENTER

AUTOMOTIVE COMMODITY CENTER

AUTOMOTIVE COMMODITY CENTER

AUTOMOTIVE COMMODITY CENTER

AUTOMOTIVE COMMODITY CENTER

AUTOMOTIVE COMMODITY CENTER

AUTOMOTIVE COMMODITY CENTER

AUTOMOTIVE COMMODITY CENTER

AUTOMOTIVE COMMODITY CENTER$$
\text { TER }
$$

AUTOMOTIVE COMMODITY CENTER

:

AD07-93ID10515 12/29/94 $031400-3222-6$ WASHINGTON

AD07-93ID10514 12/29/94 031400-3222-6 WASHINGTON

AD07-93ID10513 12/29/94 $031400-3222-6$ WASHINGTON

AD07-93ID10512 I2/29/94 $031400-3222-6$ WASHINGTON

AD07-94ID10580 05/10/96 $031400-3222-6$ HASHINGTON AD07-94ID10581 05/10/96 $031400-3222-6$ WASHINGTON AD07-94ID10582 05/10/96 $031400-3222-6$ WASHINGTON AD07-94ID10583 05/10/96 AD07-941010584 05/10/96 $031400-3222-6$ WASHINGTON AD07-94ID10585 05/10/96 $031400-3222-6$ WASHINGTON AD07-94IDI0586 05/10/96 AD07-94ID10587 05/10/96

AD07-94ID10588 05/10/96 AUTOMOTIVE COMMODITY CENTER $031400-3222-6$ WASHINGTON
PURCHASE THELVE $3 / 4$-TON PICKUPS, 2-DOOR CAB $(4 \times 4)$

$\$ 206,289 \quad 2310$

PURCHASE SIX VAN CARGO, COMPACT ( $4 \times 2$ ) DC 01 ( $\$ 43,541$

$\$ 43,541 \quad 2310$

PURCHASE THIRTEEN VAN WAGONS. PASSENGER DC OI $\$ 162,287$

$\$ 162,287 \quad 2310$

PURCHASE THREE PICKUPS, 2-DOORCAB ( $4 \times 2)$ DC 01 $\$ 0$

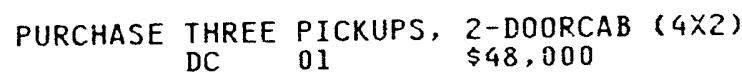

$\$ 48,000 \quad 2310$ PURCHASE THREE PICKUPS, $\underset{\text { DC }}{2-D O O R C A B} \underset{\$ 0}{2}(4 \times 2)$ $\$ 0$

PURCHASE 33 COMPACT PICKUPS $(4 \times 2)$

$\$ 330,000 \quad 2310$

PURCHASE $121 / 2-T O N$ PICKUPS, $4 \times 2$, BI-FUEL (LNG)

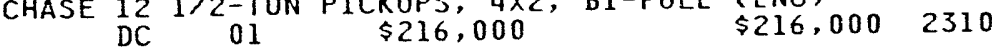
PURCHASE TWO 3/4-TON PICKUPS, $4 \times 2$ EXTENDED CAB $\begin{array}{lllll}D C & 01 & \$ 30,000 & \$ 30,000 & 2310\end{array}$ PURCHASE THO 1-TON PICKUPS, $4 \times 2$ EXTENDED CAB $\begin{array}{lllll}D C & 01 & \$ 32.000 & \$ 32,000 & 2310\end{array}$

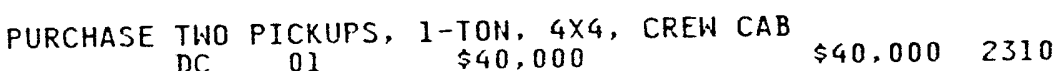
PURCHASE SIX PASSENGER VANS, WITH ALTERNATE FUEL PREP PACKAG DC ODI $\$ 94,000 \quad \$ 94,0002310$ PURCHASE EIGHT UTILITY WAGONS, $4 \times 4$, PTI

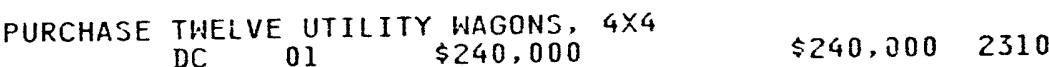
PURCHASE ONE CARRYALL, $4 \times 4,52,000 \quad \$ 22,000 \quad 2310$ IURCHASE ONE SINGLE AXLE TRUCKTRACTOR, $4 \times 2$ DC 01 S $\$ 51,000 \quad \$ 51,000 \quad 2310$ 


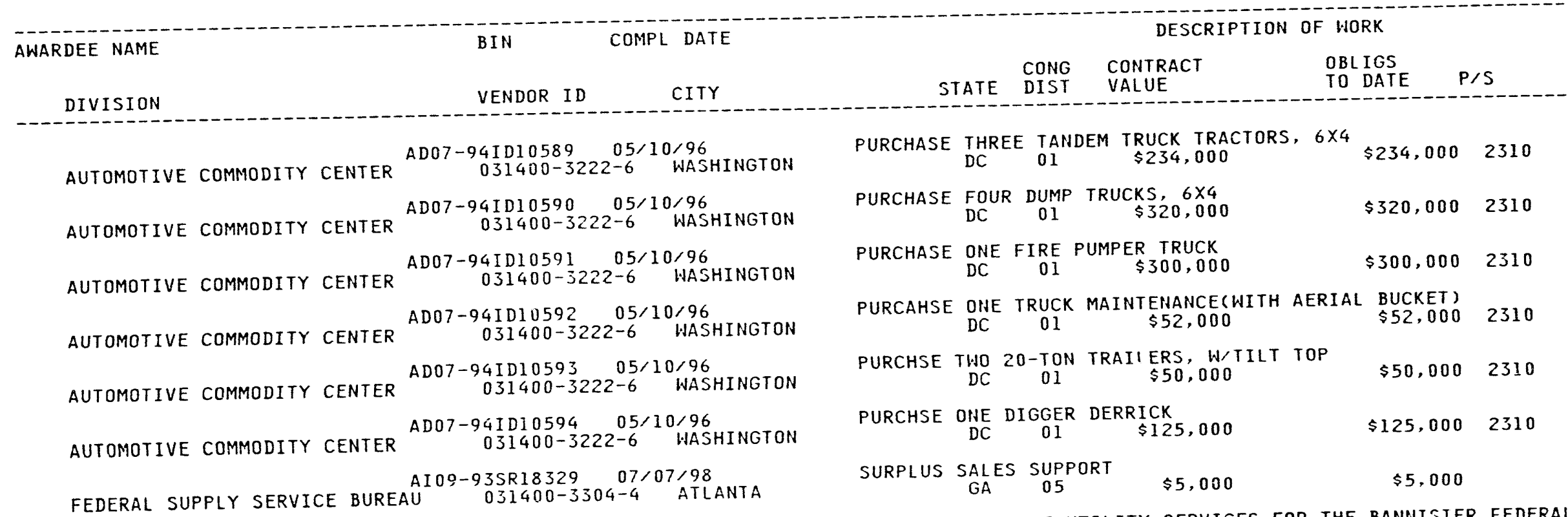

GM33-89AL53604 02/13/99 031400-0013-8 KANSAS CITY GM04-85AL38642 09/30/99 D31400-0002-2 ALBUQUERQUE

ALBUQUERQUE FLEET MGT CTR GMO4-87AL44641 06/01/97 $031400-3102-5$ AL BUQUERQUE REGION 7

AI 04-93AL91695 10/01/94 $031400-3402-4$ AL BUQUERQUE GMO4-88AL42102 06/02/99 031400-0044-8 AMARILLO

AI04-93AL91151 12/31,95 $031400-0010-3$ FORT WORTH

ADMIN OFFICE DF AI65-94WJ24276 09/30/94
$031400-3710-4$ FORT WORTH ADMIN OFFICE OF AI65-94WJ24446 09/30/94 WORTH AD06-93RL12623 09/30/94 $031400-0043-X$ BREMERTON

DOE WILL PROVIDE UTILITY SERVICES FOR THE BANNISTER FEDERAL MO O5

COVERS ADP AND OFFICE AUTOMATION TECHNICAL OR PRODUCTION IIM $^{\text {AND }} 01$ $\$ 0$

BASIC AND SUPPLEMENT GSA INTERAGENCY FLEET MANAGEMENT SYSTEM IMM 01 TO $\$ O$

DESIGN \& CONSTRUCTION SERVICESFOR ESTABLISHING A DOE-AL ESTABLISHES THE BASIC AND SUPPLEMENTAL GSA INTERAGENCY FLEET TX 13 THABLISHES THE BASIC AND SUPPLEMENTAL GSA INTERAGEN $\$ 0$ 70 EACH MONOCULAR NIGHT VISIONDEVICES $T_{1 X} 12 \quad \$ 646,433 \quad \$ 646,433$ FORKLIFT PURCHASE $\begin{array}{lll}\text { FORKLIFT } & \text { PURCHASE } & \$ 36,744\end{array}$ ADP SUPPORT ACTIVITY WA $06 \quad \$ 570,279$ $\$ 570,279 \quad \mathrm{D} 306$ 


AWARDEE NAME

\section{DIVISION \\ GENOA TOWN OF PUBLIC SCHOOLS}

GENOMIC RESEARCH INSTITUTE FOR

GEO-MICROBIAL TECH INC

GEOPHEX LTD

GEORGE H LANIER MEMORIAL HOSP

GEORGE MASON UNIVERSITY

GEORGE WASHINGTON UNIVERSITY

PHYSICS DEPT

: PHYSICS DEPT
BIN COMPL DATE

VENDOR ID O31400-0017-0 RICHLAND AI01-86FE61051 09/30/95 $031400-0033-2$ CASPER FG47-92R703498 $08 / 31 / 94$
$223630-0101-X \quad$ GENOA

FG02-93ER61566 04/14/96
$224490-0001-1$ GAITHERSBURG FG02-93ER61674 08/15/94 224490-0001-1 GAITHERSBURG

AC22-92PC92119 09/28/94 $138100-0001-3$ OCHELATA

AR21-93MC $30358 \quad 03 / 29 / 95$ 235040-0001-5 RALEIGH

FG44-89R43415308/31/94 $031630-0001-0$ VALLEY

FC02-90ER12951 $08 / 31 / 94$
$101250-0001-4$ FAIRFAX FG05-94ER61848 02/28/95 $101250-0001-4$ FAIRFAX

FG02-93ER14320 12/31/95 031640-0001-4 WASHINGTON FG03-93ER6157908/31/94 031640-0001-4 WASHINGTON FG05-86ER40270 01/31/95 031640-0501-6 WASHINGTON FG05-86ER40285 12/31/94 031640-0501-6 WASHINGTON FG05-89ER75516 09/14/94
DESCRIPTION OF WORK

CONG CONTRACT
STATE DIST VALUE LEASE OF GSA OWNED VEHICLES-
WA 04
$\$ 100,000$
PROVIDE MOTOR POOL SERVICES TONPR-

OBLIGS $-1$ PROVIDE MOTOR POOL SERVICES TONPR
WY 01
$\$ 2,674,000$

$\$ 1,892,000$ GRANT PROGRAMS FOR SCHOOLS ANDHOSPITALS AND FOR BUILDINGS 0 $\mathrm{NE} \quad 03 \quad \$ 18,543 \quad \$ 9,271$ IDENTIFICATION OF GENES IN ANONYMOUS DNA SEQUENCES MD $08 \quad \$ 195,585 \quad \$ 195,585$ GENOME SEQUENCING AND
MD 08 MICROBIOLOGICAL RECOVERY OF METALS FROM SPENT COAL LIQUEFACT $0 \mathrm{~K} \quad 05 \quad \$ 192,304 \quad \$ 192,304$ AG61

EM - GEOPHYSICAL AIRBORNE $\underset{\text { NC }}{04} \underset{\$ 468,723}{\text { UNMANNED SURVEY SYSTEM }} \underset{\$ 468,723}{\text { SH92 }}$ AH92

CYCLE 11 INSTITUTIONAL CONSERVATION PROGRAM. APPROVED BUDGE $A L \quad 03 \quad \$ 57,941 \quad \$ 37,661$

NUCLEAR ENGINEERING RESEARCH GRANT AN EXAMINATION OF THE FAC VA $10 \quad \$ 219,545 \quad \$ 219,545$

CONSTRUCTION OF AN INTEGRATED DATABASE TO SUPPORT GENOMIC SE VA $11 \quad \$ 48,500$ $\$ 48,500$

TO STUDY NEW HIGH TEMP. $\underset{01}{\text { DC }} \underset{0246,500}{\text { PLASMAS \& SAMPLE INTRODUCTION }}$ NEW AWARD FOR PROJECT ENTITLED: "ADJUDICATING GENETIC TESTI INVESTIGATIONS OF THE STRUCTURE AND ELECTROMAGNETIC INTERACT DC 01 \$1,281,000 $\$ 1,279,000$ A PROGRAM IN MEDIUM ENERGY NUCLEAR PHYSICS

DC OI $\$ 1,520,000 \quad \$ 1,520,000$ 1989 INSTRUMENTATION GRANT - INSTRUMENTATION FOR A MULTI-WIR 


\begin{tabular}{|c|c|}
\hline AWARDEE NAME & COMPL DATE \\
\hline DIVISION & VENDOR ID \\
\hline PHYSICS DEPT & $031640-0501-6$ \\
\hline $\begin{array}{c}\text { GEORGETOWN COUNTY OF } \\
\text { SCHOOL DISTRICT }\end{array}$ & $\begin{array}{r}\text { FG44-93R435444 } 09 / 30 / 95 \\
231630-0101-7 \text { GEORGETO }\end{array}$ \\
\hline GEORGETOWN UNIVERSITY & $\begin{array}{c}\text { ACO2-87NVI0649 08/31/96 } \\
031680-0001-9 \text { WASHINGTO }\end{array}$ \\
\hline GEORGIA ACADEMY FOR THE BLIND & $\begin{array}{c}\text { FG } 44-93 R 43536509 / 30 / 95 \\
232460-0001-8 \text { MACON }\end{array}$ \\
\hline GEORGIA EMERGENCY MGMT AGENCY & $\begin{array}{c}\text { FG09-93SR18289 } 01 / 27 / 98 \\
226570-0001-0\end{array}$ \\
\hline \multirow[t]{3}{*}{ GEORGIA POWER CO } & $\begin{array}{c}\text { CR01-83NE } 44386 \\
031740-0001-7\end{array}$ \\
\hline & $\begin{array}{c}\text { CRO1-83NE44478 } 03 / 15 / 30 \\
031740-0001-7 \text { ATLANTA }\end{array}$ \\
\hline & $\begin{array}{rc}\text { CRO1-83NE } 44479 & 03 / 15 / 30 \\
031740-0001-7 & \text { ATLANTA }\end{array}$ \\
\hline
\end{tabular}

GEORGIA RESEARCH FDN UNIV OF

SAVANNAH RIVER ECOLOGY LAB INSTITUTE OF ECOLOGY

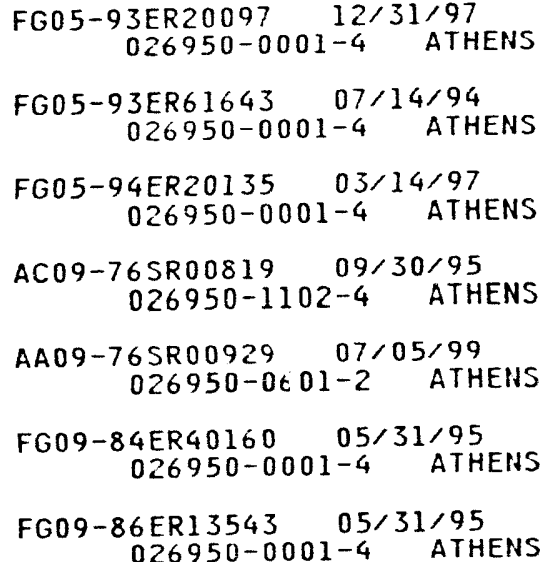

DESCRIPTION OF WORK

CONG CONTRACT
STATE DIST VALUE

$\$ 182,551$

OBLIGS

TO DATE P/S

DC

01

SEE ATTACHED PAGE NO. 2 OF THIS NOTICE OF FINANCIAL ASSISTAN SC 01 NO. 2 OF9,750

RESEARCH, DEVELOPMENT AND DEMONSTRATION OF A FUEL CELL BATTE $D C \quad 01 \quad \$ 5,349,269 \quad \$ 3,551,574$ AZ11

SEE ATTACHED PAGE NO. 2 OF THIS NOTICE OF FINAHCIAL ASSISTAN GA 08 \$5, $224 \quad \$ 2,712$

ENVIRONMENTAL MONITORING AND EMERGENCY PREPAREDNESS

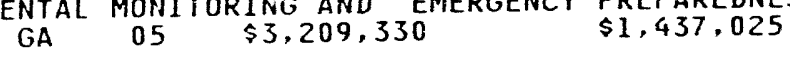

DISPOSAL OF $\underset{\text { GPENT NUCLEAR FUELAND/OR HIGH LEVEL }}{\substack{\text { RADIOACTIVE } \\ \$ 0}}$

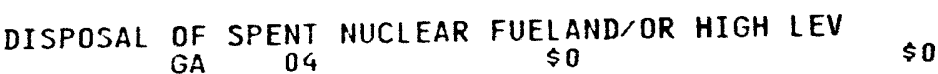
DISPOSAL OF SPENT NUCLEAR FUELAND/OR HIGH LEVEL RADIOACTIVE GA 04 SPEN $\$ 0$

NEW AWARD - THE CENTER FOR PLANT AND MICROBIAL COMPLEX CARBO GA 10 \$4,450,000 $\$ 1,720,000$

SYMPOSIUM ENTITLED "PARADIGMS AND PARADOXES OF THE MICROBIAL $G A \quad 10 \quad \$ 6,000 \quad \$ 6,000$ POST-TRANSCRIPTIONAL. REGULATION OF THE R/B GENE FAMILY IN MA GA 10 \$306,000

OPERATION OF SR ECOLOGY LABORATORY AND PERFORM ECOLOGICAL GA $10 \$ 108,651,81$

MASTER APPENDICES B C AND D FOR SPECIAL RESEARCH SUP $G A$ IO $\$ 0$

HIGH ENERGY HADRON-HADRON COLLISIONS

$$
\text { GA } 10 \quad \$ 279,000 \quad \$ 228,000
$$

SOYBEAN RIBULOSE BISPHOSPHATE CARBOXYLASE SMALL SUBUNITI GA $10 \quad \$ 922,958$ \$ 0 \$58,958 


\begin{tabular}{|c|c|c|}
\hline AWARDEE NAME & COMPL DATE & DESCRIPTION OF HORK \\
\hline DIVISION & VENDOR ID & $\begin{array}{l}\text { OBL IGS } \\
\text { TO DATE }\end{array}$ \\
\hline BOTANY DEPT & $\begin{array}{r}\text { FG09-86ER13602 08/18/95 } \\
026950-0301-3 \text { ATHENS }\end{array}$ & 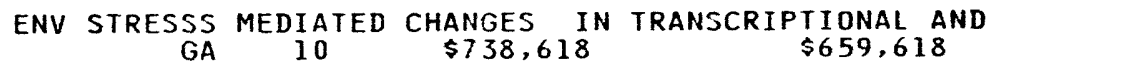 \\
\hline PHYSICS \& ASTRONOMY DEPT & $\begin{array}{r}\text { FG09-87ER45291 } 11 / 30 / 94 \\
026950-1001-x \text { ATHENS }\end{array}$ & 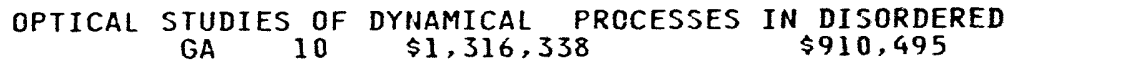 \\
\hline CHEMISTRY DEPT & $\begin{array}{r}\text { FG09-87ER13811 } 07 / 15 / 94 \\
026950-0401-X \text { ATHENS }\end{array}$ & $\begin{array}{c}\text { POTENTIAL ENERGY SURFACES FOR CHEMICAL REACTIONS } \\
\text { GA } 10 \quad \$ 719,178\end{array}$ \\
\hline BIOCHEMISTRY DEPT & $\begin{array}{rl}F G 09-88 E R 13901 & 04 / 14 / 95 \\
026950-0201-7 \text { ATHENS }\end{array}$ & 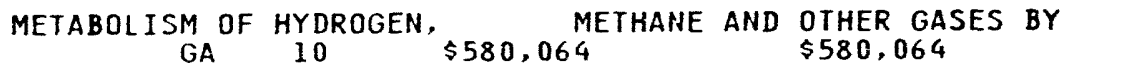 \\
\hline & $\begin{aligned} \text { FG09-88ER13970 } 09 / 14 / 96 \\
026950-0001-4 \text { ATHENS }\end{aligned}$ & 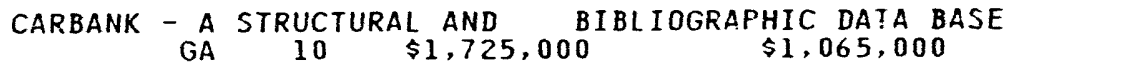 \\
\hline BIOCHEMISTRY DEPT & $\begin{array}{r}\text { FG09-91ER } 20045 \quad 08 / 14 / 94 \\
026950-0201-7 \text { ATHENS }\end{array}$ & 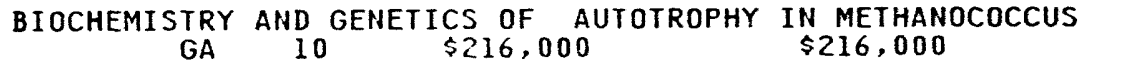 \\
\hline & $\begin{array}{r}\text { FG05-94ER20149 } 06 / 30 / 97 \\
026950-0001-4 \text { ATHENS }\end{array}$ & 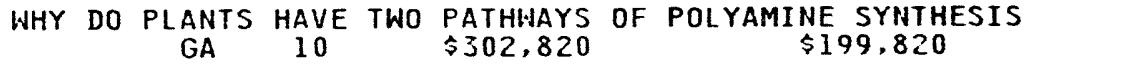 \\
\hline $\begin{array}{l}\text { GEORGIA STATE OF } \\
\text { OFFICE OF ENERGY RESOURCES }\end{array}$ & $\begin{aligned} \text { FG44-79R410044 } 09 / 30 / 94 \\
031790-0501-7 \text { ATLANTA }\end{aligned}$ & $\begin{array}{cccc}\text { GRANT PROGRAM FOR } & \text { SCHOOLS, HOSPITALS, BUILDINGS OWNED BY UNI } \\
\text { GA } & 05 & \$ 21,518,940 & \$ 1,082,930\end{array}$ \\
\hline NATURAL RESOURCES DEPT & $\begin{aligned} \text { FG44-93R410615 } 09 / 30 / 95 \\
031790-1001-0 \text { ATLANTA }\end{aligned}$ & $\begin{array}{c}\text { DEMONSTRATION OF AUTOMATED DYEBATH REUSE IN CARPET MANUFACTU } \\
\text { GA }\end{array} 09 \begin{array}{ll}\$ 868,037 & \$ 38,000\end{array}$ \\
\hline OFFICE OF ENERGY RESOURCES & $\begin{aligned} \text { FG44-78CS60045 } 03 / 31 / 95 \\
031790-0501-7 \text { ATLANTA }\end{aligned}$ & $\begin{array}{cccc}\text { WEATHERIZATION OF LOW INCOME HOUSING PLUS TRAIUING AND TEC } \\
\text { GA } & 05 & \$ 47,573,936 & \$ 30,963,753\end{array}$ \\
\hline OFFICE OF ENERGY RESOURCES & $\begin{aligned} & \text { FG44-80C569091 } 06 / 30 / 95 \\
& 031790-0501-7 \text { ATLANTA }\end{aligned}$ & $\begin{array}{l}\text { ENERGY EXTENSION SERVICE } \\
\qquad \text { GA } 05 \$ 10,469,025\end{array}$ \\
\hline NATURAL RESOURCES DEPT & $\begin{array}{c}\text { FC09-92SR18268 } 09 / 30 / 96 \\
031790-1003-7 \text { WAYNESBORO }\end{array}$ & $\begin{array}{ccc}\text { INVESTIGATION OF TRITIUM IN } \\
\text { GA GORDON AND OIHER AQUIFERS IN }\end{array}$ \\
\hline \multirow[t]{3}{*}{ GEORGIA STATE UNIVERSITY } & $\begin{array}{c}\text { FG09-91ER20043 } 07 / 31 / 94 \\
031800-0001-5 \text { ATLANTA }\end{array}$ & 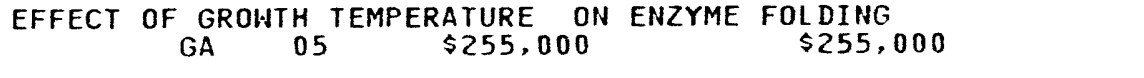 \\
\hline & $\begin{array}{rl}\text { FG } 44-93 R 435351 & 09 / 30 / 95 \\
031800-0001-5 \text { ATLANTA }\end{array}$ & 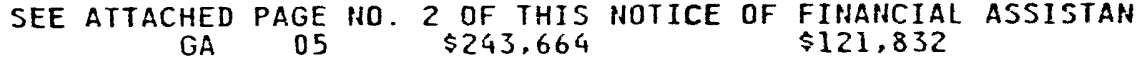 \\
\hline & $\begin{aligned} \text { FG05-84ER40162 } 05 / 31 / 96 \\
031800-0001-5 \text { ATLANTA }\end{aligned}$ & 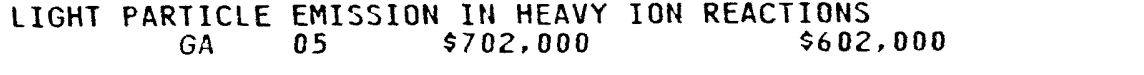 \\
\hline
\end{tabular}




\section{A'VARDEE NAME}

BIN COMPL DATE

DESCRIPTION OF WORK

\section{DIVISION}

VENDOR ID CITY

$031800-0001-5$ ATLANTA

STATE CONG CONTRACT

031800-0001-5 ATLANTA

GA $04 \quad \$ 222,000$

OBL IGS

O DATE P/S

GEORGIA TECH RESEARCH CORP

$$
\begin{gathered}
\text { FG02-89ER12883 } 07 / 31 / 94 \\
097540-0001-8 \text { ATLANTA } \\
\text { FG05-85ER45194 12/31/94 } \\
097540-0001-8 \text { ATLANTA } \\
\text { FG05-86ER45234 } 10 / 31 / 94 \\
097540-0101-4 \text { ATLANTA } \\
\text { FG05-87ER40330 } 02 / 14 / 96 \\
097540-0101-4 \text { ATLANTA } \\
\text { FG05-87ER52141 } 04 / 30 / 96 \\
097540-1301-2 \text { ATLANTA } \\
\text { FG05-87ER13708 } 06 / 30 / 96 \\
097540-0001-8 \text { ATLANTA } \\
\text { FG05-88ER45369 } 07 / 31 / 94 \\
097540-0101-4 \text { ATLANTA } \\
\text { FG05-91ER54122 06/30/95 } \\
097540-1901-0 \text { ATLANTA } \\
\text { FG05-92ER79146 } 09 / 29 / 94 \\
097540-2001-9
\end{gathered}
$$

PHYSICS DEPT

PHYSICS DEPT

FUSION RESEARCH CENTER

PHYSICS DEPT

MECHANICAL ENGINEERING

NEELY NUCLEAR RESEARCH CENTER $097540-2001-9$ ATLANTA

FG05-92ER75807 09/29/94 097540-0001-8 ATLANTA

FG05-94ER75984 03/14/95 $097540-0001-8$ ATLANTA

FG05-93ER75879 04/30/95 097540-1901-0 ATLANTA

FG04-93AL $9464909 / 29 / 96$ 097540-1401-9 ATLANTA

AEROSPACE ENGINEERING

FG09-86SR15156 09/30/95 097540-0001-8 ATLANTA

FG22-90PC90293 09/30/94 097540-1701-8 ATLANTA

FG22-91PC91287 08/31/94
LOW ENRICHED URANIUM (LEU) FUEL CONVERSION

CHED URANIUM (LEU) FUEL CONVERSION
05

A CARBANION APPROACH TO POLYACETYLENE
GA
06

$\$ 772,642$

THE STRUCTURE OF REACTIVITY OFHETEROGENEOUS SURFACES AND TH GA 06 \$1,862,397 \$1,706,358

NUCLEAR STRUCTURE FROM RADIOACTIVE DECAY

$\$ 667,880$

FUSION STUDIES PROGRAM
GA 06
$\$ 650,000$
$\$ 400,000$

STATISTICAL FLUCTUATIONS IN LASERS

$G A \quad 05 \quad \$ 908,949$

$\$ 591,187$

GROWTH, STRUCTURE AND STABILITY OF EPITAXIAL OVERLAYERS GA 05 AND $\$ 495,434 \quad \$ 495,434$

SUPPORT OF U.S. ITER ACTIVITY (NEW GRANT)

$\$ 322,196$

UNIVERSITY REACTOR INSTRUMENTATION PROGRAM

$$
\text { GA REACTOR INSTRUMENTATION PROGRAM } \$ 51,514
$$

DOE REACTOR SHARING PROGRAM

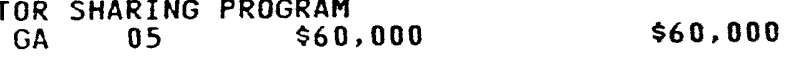

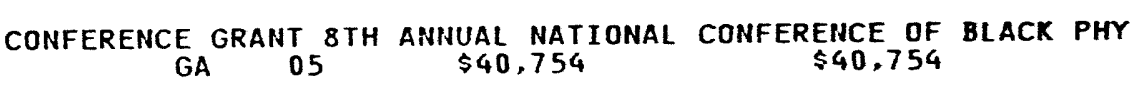

RESEARCH ON LASER HEATING OF THERMOLUMINSCENT MATERIALS GA 05 \$150,000 $\$ 150,000$

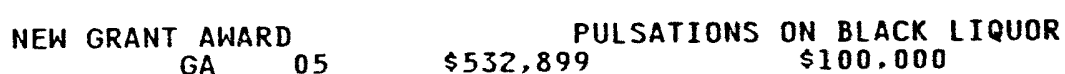

DOCUMENTATION OF NUCLEAR WASTEMANAGEMENT TECHNOLOGY GA $05 \quad \$ 50,000 \quad \$ 50,000$

HIGH TEMPERATURE MEMBRANE FOR HZS AND SO2 SEPARATIONS

SUPERCRITICAL THERMODYNAMICS OF SULFUR AND NITROGEN SPECIES 


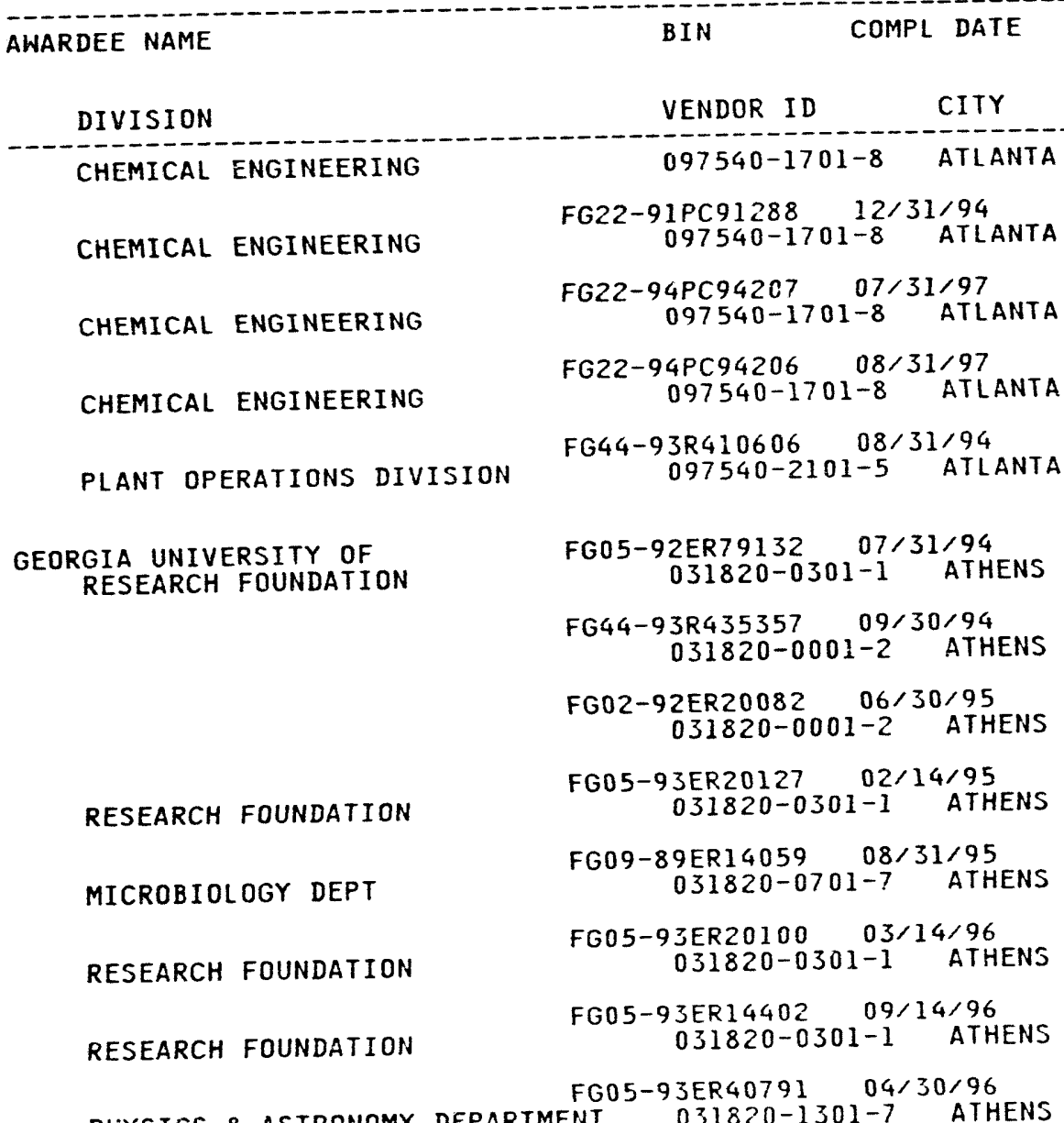

RESEARCH FOUNDATION

RESEARCH FOUNDATION

: CHEMISTRY DEPT
FG05-93ER20114 06/14/96
$031820-0301-1$ ATHENS FG05-93ER20115 06/14/96
$031820-0301-1$ ATHEN

FG05-94ER14428 01/14/97
$031820-0401-8$ ATHENS

FG05-93ER20099 01/31/98

\section{DESCRIPTION OF HORK}

CONG CONTRACT
STATE DIST VALUE

OBLIGS

TO DATE P/S

GA $05 \quad \$ 313,105$

$\$ 200,000$

HIGH TEMPERATURE ELECTROCHEMICAL SEPARATION OF H25 FROM COAL GA 05 S $\$ 199,977$ $\$ 199,977$

HIGH TEMPERATURE ELECTROCHEMICAL POLISHING OF HYDROGEN SULFI GA 05 \$199,951 \$199,951 SUPERCRITICAL FLUID REACTION FOR COAL PROCESSING

SEE ATTACHED PAGE NO. 2 OF THIS NOTICE OF FINANCIAL ASSISTAN $\begin{array}{cccc}\text { TICAL FLUID REACTIDN FOR COAL PROCESSING } \\ \text { GA } & 05 & \$ 220,000 & \$ 200,000\end{array}$ GA 05 N 2 \$59,962 $\$ 59,962$

1992 INSTRUMENTATION PHOSPHORIMAGER AHD PD DENSITOMETER IMAG GA $10 \quad \$ 136,100 \quad \$ 136,100$

SEE ATTACHED PAGE NO. 2 OF THIS NOTICE OF FINANCIAL ASSISTAN $G A \quad 10 \quad \$ 59,100 \quad \$ 29,550$ MECHANISMS OF LIGNIN BIOSYHTHESIS DURING MICROBIOLOGY AND BIOCHEMISIRY OF ANAEROLI FERMENTATION THE C GA $10 \quad \$ 527,300 \quad \$ 527,300$ HERMICELLULASES FROM THE ETHANOLOGENIC THERMOPHILE: GA 10 \$402,119 $\$ 402,119$ NEH AWARD - NITROGEN CONTROL OF CHLOROPLAST DIFFERENTIATION

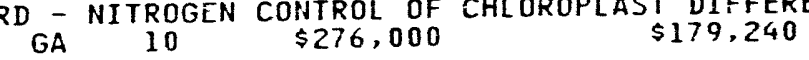

GA $10 \quad \$ 263,474 \quad \$ 94,936$

STUDIES OF THE SPIN-ISOSPIN RESPONSE OF THE NUCLEAR CONTINIU $\begin{array}{lll}\text { GA THE SPIN-ISOSPIN RESPONSE OF THE NUCLEAR } \\ 10 & \$ 174,000 & \$ 110,000\end{array}$ STRUCTURES AND FUNCTIONS OF OLIGOSACCHARINS GA 10 STRUCTURAL STUDIES OF COMPLEX CARBOHYDRATES OF PLANT CELL WA GA 10 \$1,229,366 \$814,366

THEORETICAL STUDIES OF ELEMENTARY HYDROCARBON SPECIES AND T $G A \quad 10 \quad \$ 284,997$ $\$ 94,999$ NEW AWARD ENTI'LED DEVELOPMENTOF INNOVATIVE TECHNIQUES AND 

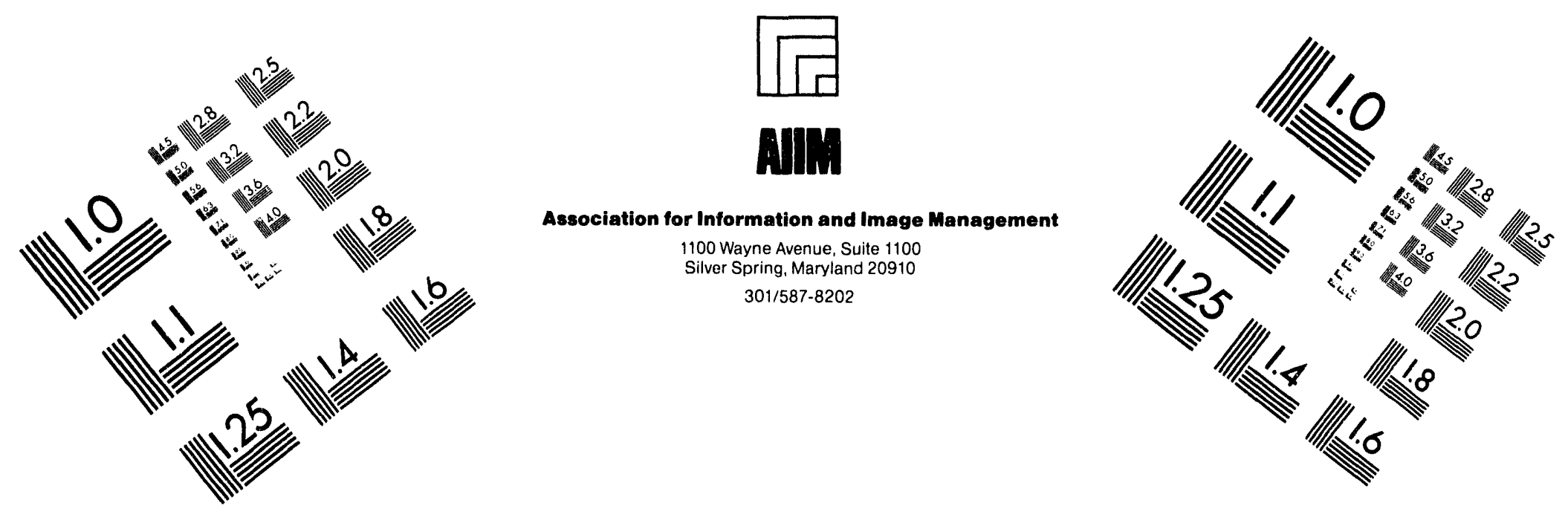

Centimeter

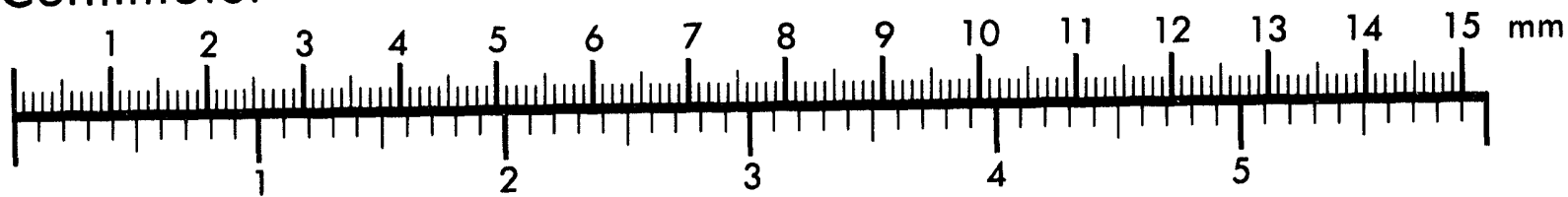

Inches
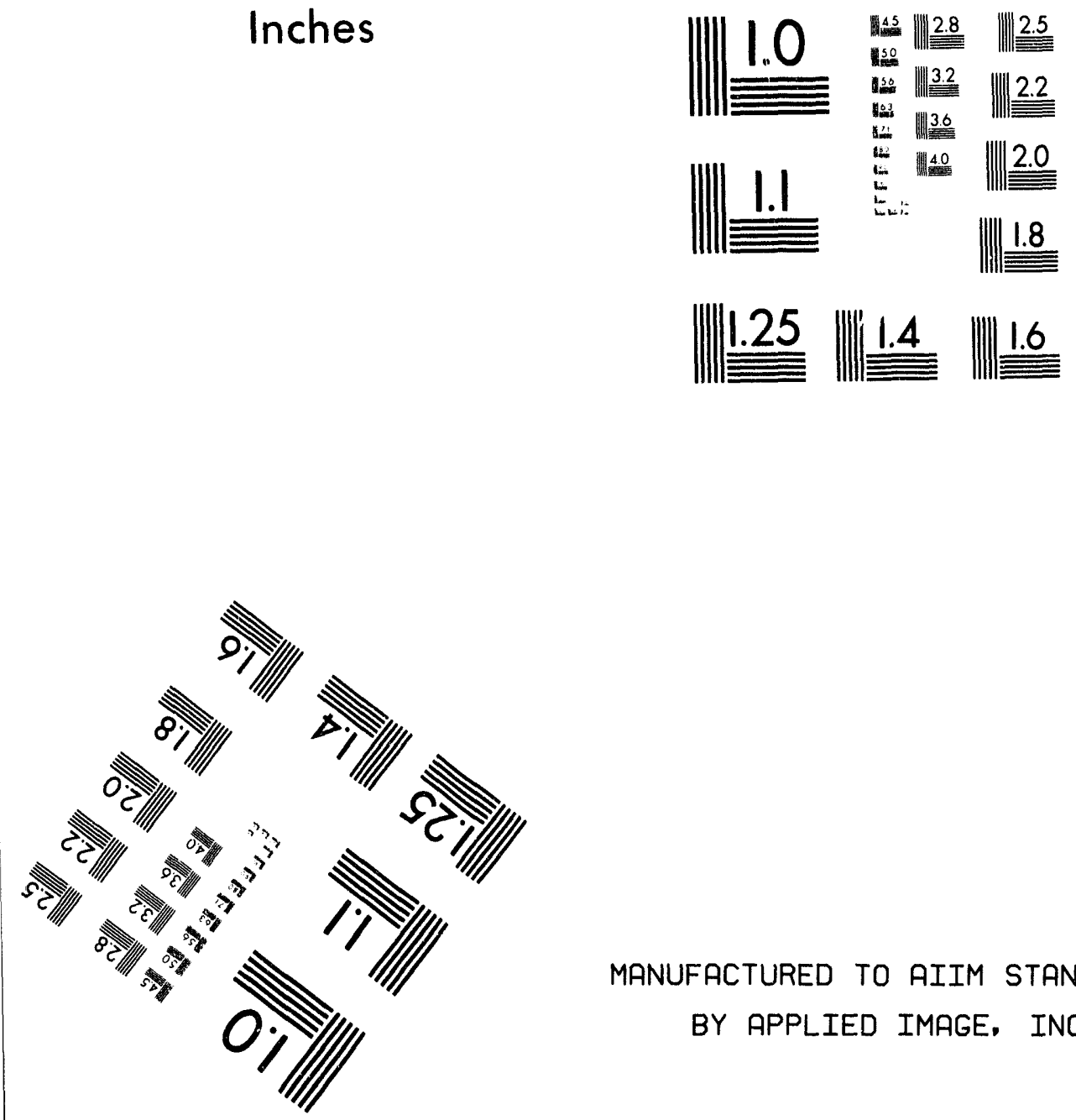

MANUFACTURED TO AIIM STANDARDS

BY APPLIED IMAGE, INC.

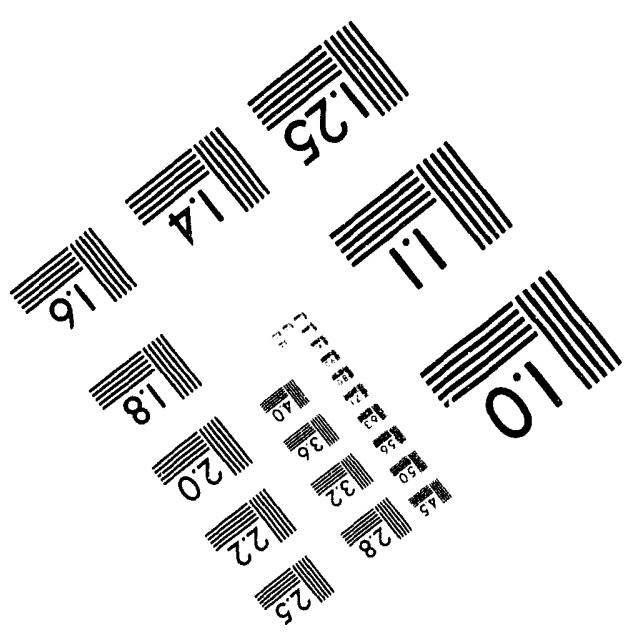



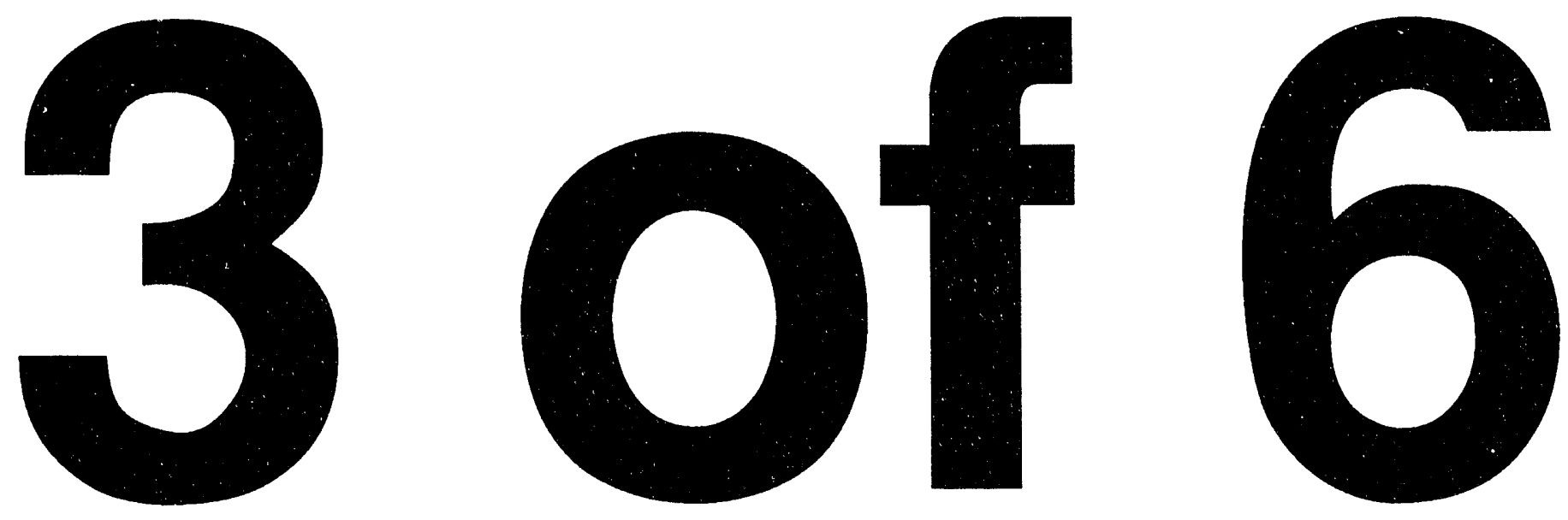


BIN COMPL DATE

AWARDEE NAME
DIVISION
COASTAL PLAIN EXP STATION

GEOSCIENCES ELECTRONICS CORP

GEOTHERMAL FOOD PROCESSORS

GEOTHERMAL TECHNOLOGY ORG

GERMANTOWN SCHOOL DISTRICT

GIFFORD MEDICAL CENTER

GILBERT/COMMONWEALTH INC

GINER INC

VENDOR ID CITY

BIN COMPL DATE

$031820-1201-0$ ATHENS

AC21-88MC25105 $12 / 31 / 94$
$178040-0001-4$

FM03-79SF10752 06/01/99
$031860-0001-7$ FERNLEY

GM07-88ID12690 08/31/99 $170780-0001-3$ SANTA ROSA

FG45-93R551818 $06 / 30 / 95$
$031930-0001-9 \quad$ GERMANTOWN

FG41-93R196509 06/30/95
$228810-0001-0$ RANDOLPH FG41-94R196610 05/31/96
$228810-0001-0$ RANDOLPH

AC05-870RZ1722 $08 / 30 / 94$
$032090-0002-6 \quad$ READING

AC01-93FE62704 $09 / 30 / 95$
$032090-0002-6 \quad$ READING

AC22-88PC88851 $02 / 26 / 95$
$032090-0002-6 \quad$ READING

AC21-89MC25177 01/21/96 $032090-0002-6$ READING

$A C 01-94 F E 6274701 / 02 / 97$
$032090-0002-6 \quad$ READING

FG02-93ER81610 07/17/94
$032170-0001-3$ WALTHAM

FG02-93ER14386 09/14/94 $032170-0001-3$ WALTHAM
PAGE NO. 190

DESCRIPTION OF WORK $P / S$ STATE DIST VALUE

FABRICATE AVAILABLE EXISTING WIRELESS MHD TOOLS

$\begin{array}{llll}\text { AVAILABLE EXISTING WIRELESS MWD } & \text { TOOLS } \\ \text { CA } 21 & \$ 980,492 & \$ 766,863 & \text { AG23 }\end{array}$

LOAN GUARANTEE FOR VEGETABLE DEHYDRATION PLANT

DOE AGREEMENT WITH GEOTHERMAL TECHNOLOGY ORGANIZATION FOR

CA 01

\$0

ICP-NEW AWARD.
HI 09
$\$ 8,000$
$\$ 4,000$

TITLE III OF THE NATIONAL ENERGY CONSERVATION POLICY ACT

TITLE III OF THE NATIONAL ENERGY CONSERVATION POLICY ACT

$\begin{array}{llll}V T & 01 & \$ 34,910 & \$ 17,455\end{array}$

ARCHITECT ENGINEER SERVICES FOR THE BETHEL VALLEY LIQUID LOW

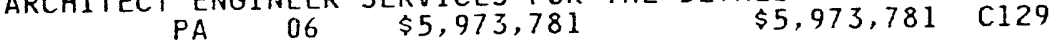

TECHHICAL SUPPORT SVCS FOR OF COF FOSSIL ENERGY TO PROVIDE A $\begin{array}{cccc}\text { PA } & 19 & \$ 2,382,822 & \$ 672,992 \\ \text { SCALE UP TEST AND SUPPORTING RESEARCH FOR THE DEVELOPMENT OF }\end{array}$ $\begin{array}{cccc}\text { SCALE UP TEST AND SUPPORTING RESEARCH FOR THE DEVELOPMEN } \\ \text { PA } 06 \text { \$10,749,856 } & \$ 10,749,856 \text { AGI } 1\end{array}$

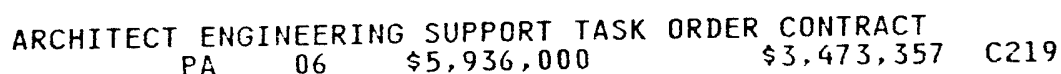
$\begin{array}{cccc}\text { SUPPORT SERVICES TECHNICAL ANDENGINEERING ANALYSIS. } & \\ \text { PA } 19 & \$ 4,681,879 & \$ 1,248,683 & R 425\end{array}$ MOLDED TIC BI POLAR PLATES FORHIGH VOLTAGE BATTERY AND FUEL $\begin{array}{lll}M A & 08 & \$ 74,978\end{array}$

$\begin{array}{rccc}\text { BIFUNCTIONAL AIR ELECTRODES } & \text { FOR METAL-AIR BATTERIES } \\ \text { MA } & 07 & \$ 90,749 & \$ 90,749\end{array}$ 


\section{AWARDEE NAME}

BIN

COMPL DATE

DESCRIPTION OF HORK

DIVISION

VENDOR ID

STATE CONG CONTRACT

OBLIGS TO DATE $\mathrm{P} / \mathrm{S}$

GLEN RIDGE BOARD OF EDUCATION

FG42-94R263901 06/30/95 238940-0001-5 GLEN RIDGE

CYCLE 16 AWARD.

3ER61722 09/29/94

3ER61722 09/29/94
$233450-0001-6$ CALVERTON

GLOBAL ENV SOC INC INST OF

GLDBAL FOUNDATION INC

GLOBAL MANAGEMENT SYSTEMS INC

GLOBAL OUTPOST INC

GLOBAL RIVER ENVIRONMENTAL

GODDARD COLLEGE

GOHEIR

\section{GOLD BAR MINE}

GOLDEN TECHNOLOGIES INC

GOLDER ASSOC INC
FG05-94ER40830 01/19/95
$236590-0001-9$ CORAL GABLES

ACO1-94ER35216 02/23/95

$237340-0001-7$ BETHESDA

FG03-93SF19964 09/30/94

235220-0001-3 COLLEGE PARK

FG02-94ER75987 03/31/97 $237210-0001-3$ ANN ARBOR

FG41-94R196600 05/31/95 $123290-0001-4$ PLAINFIELD

FG41-93R130542 09/30/94 235430-0001-2 PROVIDENCE

AC08-90NV10930 03/14/95 195210-0001-5 BEATTY

FC34-94RF0086905/02/95 235090-0002-1 GOLDEN

FC02-93CH1056I 03/28/97 235090-0001-3 PITTSBURGH

AT65-94WJ24293 04/18/95 191980-0010-1 LAKEWOOD

AC06-89RL11615 12/31/94 $191980-0001-2$ REDMOND
VARIABILITY AND PREDICTABILITYOF THE COUPLED OCEANMD $05 \quad \$ 100,000 \quad \$ 100,000$

CONFERENCE GRANT AWARD (NONPROFIT)

$$
\mathrm{FL} 18 \text { \$4,000 }
$$

LAN/WANG SUPPORT TO THE OFFICEOF ENERGY RESEARCH

$$
\begin{array}{cccc}
\text { SUPPORT TO THE OFFICEOF ENERGY RESEARCH } & \text { REA } \\
08 & \$ 984,048 & \$ 300,000 & 7010
\end{array}
$$

TELEROBOTIC SIMULATION OF THE COMMERCIAL SPACE POWER SYSTEM MD $04 \quad \$ 150,000 \quad \$ 75,000$

TOWARDS A VISION OF COMMUNITY LOCALLY AND GLOBALLY GREEN MI 13 \$450,000 $\$ 145,500$

TITLE III OF THE NATIONAL ENERGY CONSERVATION POLICY ACT VT 01 \$10,000 $\$ 5,000$

GRANT PROGRAM FOR SCHOOLS AND HOSPITAL AND BUILDINGS OWENED RI 01 \$2,388,956 $\$ 30,000$

SALARY COMPENSATION FOR SUSPENSION OF MINING OPERATIONS BY D NV $01 \quad \$ 3,000 \quad \$ 3,000$ AG56 $\underset{\text { COOPERATIVE }}{\text { COGREEMENT BETWEEN }} \underset{\$ 06}{\text { DOE, EG\&G AND PARTICIPANT }} \underset{\$ 0}{\$ 0}$

CATALYTIC MEMBRANE PROGRAM

PA $18 \quad \$ 2,307,642$

$\$ 1,577,000$

TAKS ORDER 001. GEOTECH SVCS ON BIG GEORGE CARTER MOUNTAIN A/E SERVICES (TASKS I II AND III) FOR THE

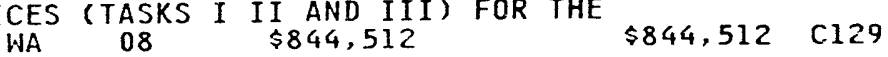




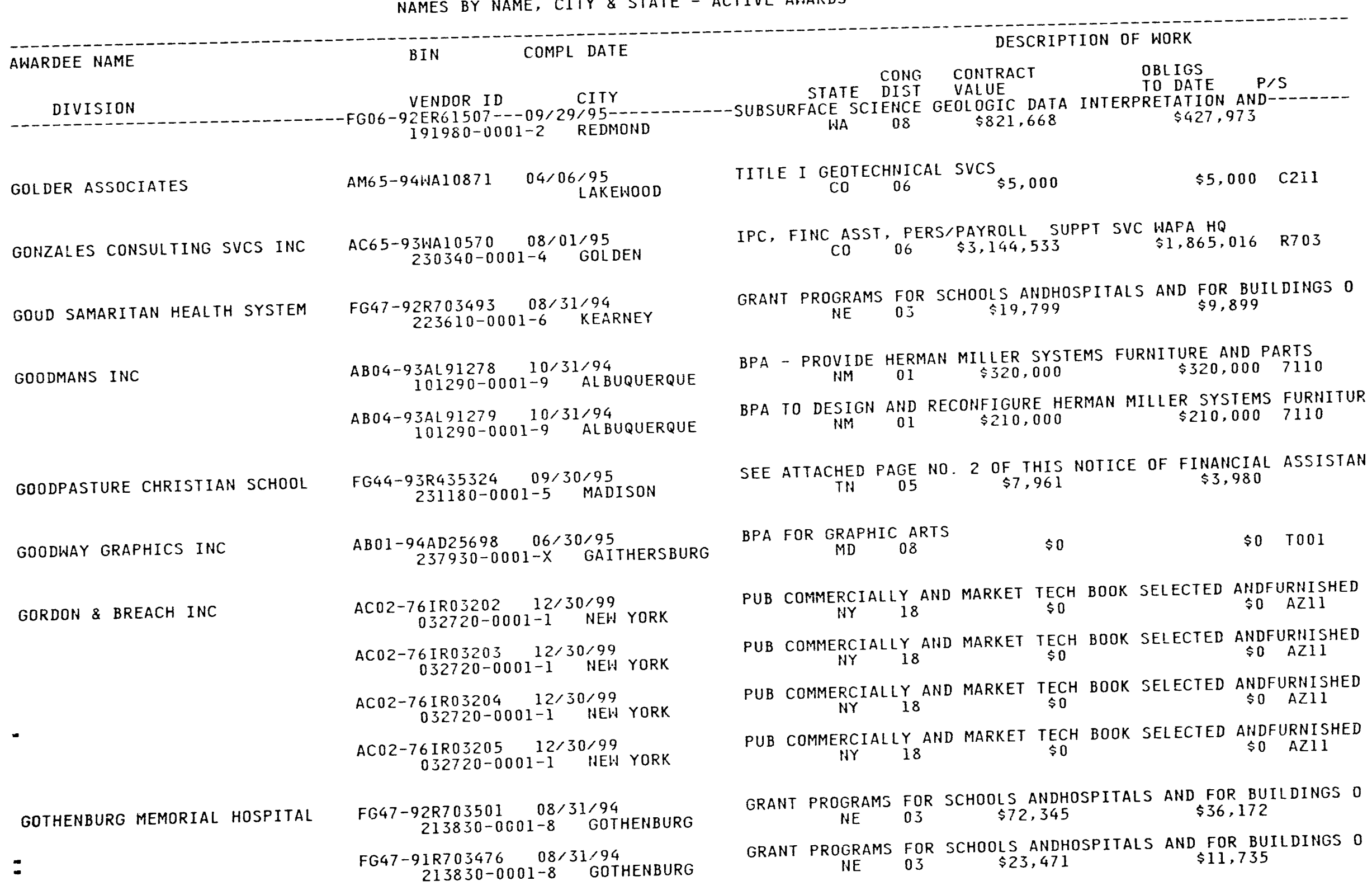




\section{AWARDEE NAME \\ DIVISION}

BIN

COMPL DATE

DESCRIPTION OF WORK

VENDOR ID

CITY

STATE CONG CONTRACT

OBL IGS
TO DATE

$\mathrm{P} / \mathrm{S}$ GOVERNMENT COMPUTER SALES INC AMOI-92AD36215 04/12/95
$218410-0001-9$

GOVERNMENT MICRO RESOURCES

AM08-90NV10943 09/28/94 193830-0001-5 CHANTILLY AM07-92ID13061 01/10/95 193830-0001-5 CHANTILLY

AM01-93AD33089 04/05/96 $1938 \div 0-0001-5 \quad$ CHANTILLY

GOVERNMENT MICRO RESOURCES INC

AD08-94NV11595 07/15/94 193830-0001-5 CHANTILLY

AD01-94AD34233 07/15/94 193830-0001-5 CHANTILLY

AD08-94NV11616 07/19/94 193830-0001-5 CHANTILLY

AD07-94ID10602 08/28/94 193830-0001-5 CHANTILLY

GOVERNMENT PRINTING OFFICE DOCUMENTS SUPERINTENDENT OF

GOVERNMENT TECHNOLOGY SERVICE GOVERNMENT TECHNOLOGY SVCS INC
AI09-91SR17273 09/30/94 032870-0101-9 HASHINGTON

AMOI-91MA35161 09/15/94 164570-0001-0 CHANTILLY

$A B 34-93 R F 00461 \quad 09 / 30 / 94$ $184840-0007-9$ GOLDEN

AD96-93P018040 08/08/94 $184840-0001-X \quad$ CHANTILLY

AD96-94P019014 08/08/94 184840-0001-X CHANTILLY

CR01-83NE44385 03/15/30 $032910-0002-1 \quad$ PARSIPPANY
INDEFINITE QUANTITIES CONTRACTFOR COLOR PRINTERS

WA 01 \$0

$\$ 0 \quad 7025$

ADP HARDWARE, SOFTHARE AND PERIPHERALS

$$
\text { VA } 08 \quad \$ 5,247,754
$$

$\$ 248,754 \quad 7010$

ACQUISITION OF DESKTOP MICORCOMPUTERS, LAPTONS\& PERIPHERALS VA 08 $\$ 0$ $\$ 07010$ AWARD OF THREE-YEAR INDEFINITEDEL IVERY/INDEFINITE QUANTITY
VA 08
$\$ 6,861,079$

PURCHASE EQUIPMENT TO SUPPORT LANS AND MICROPROCESSOR SYSTEM
VA 08 DELIVERY ORDER FOR APPLE MACIIITOSH COMPUTERS, PERIPHERALS AN $\begin{array}{lllll}\text { VA } & 10 & \$ 144,109 & \$ 144,109 & 7021\end{array}$ PURCHASE COMPUTER EQUIPMENT FOR TDD, WMD \& ERD.

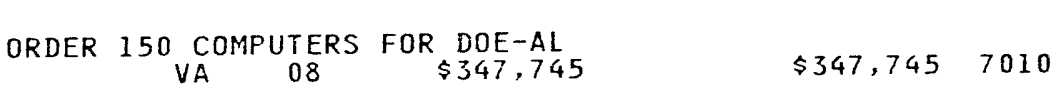
$\begin{array}{ccc}\text { PURCHASE MISCELLANEOUS } & \text { PUBLICATIONS AND SUBSCRIPTIONS } \\ \text { DC } 01 & \$ 10,000 & \$ 10,000\end{array}$

PORTABLE COMPUTERS WITH INTEL 80386 MICROPROCESOR TECHNOLOGY VA $10 \$ 1,743,000$ \$O 7010

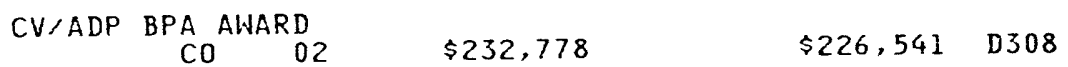
0003 AA ADVANCED SYSTEM WITH OFFICE AUTOMATION INSTALLED VA 08 \$1,054,186 \$1,054,186 7021

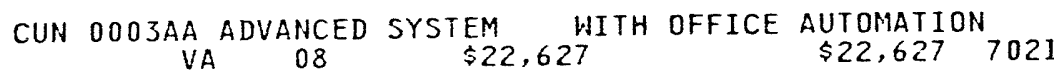

DISPOSAL OF SPENT NUCLEAR FUELAND/OR HIGH LEVEL RADIOACTIVE NJ 11 \$O 


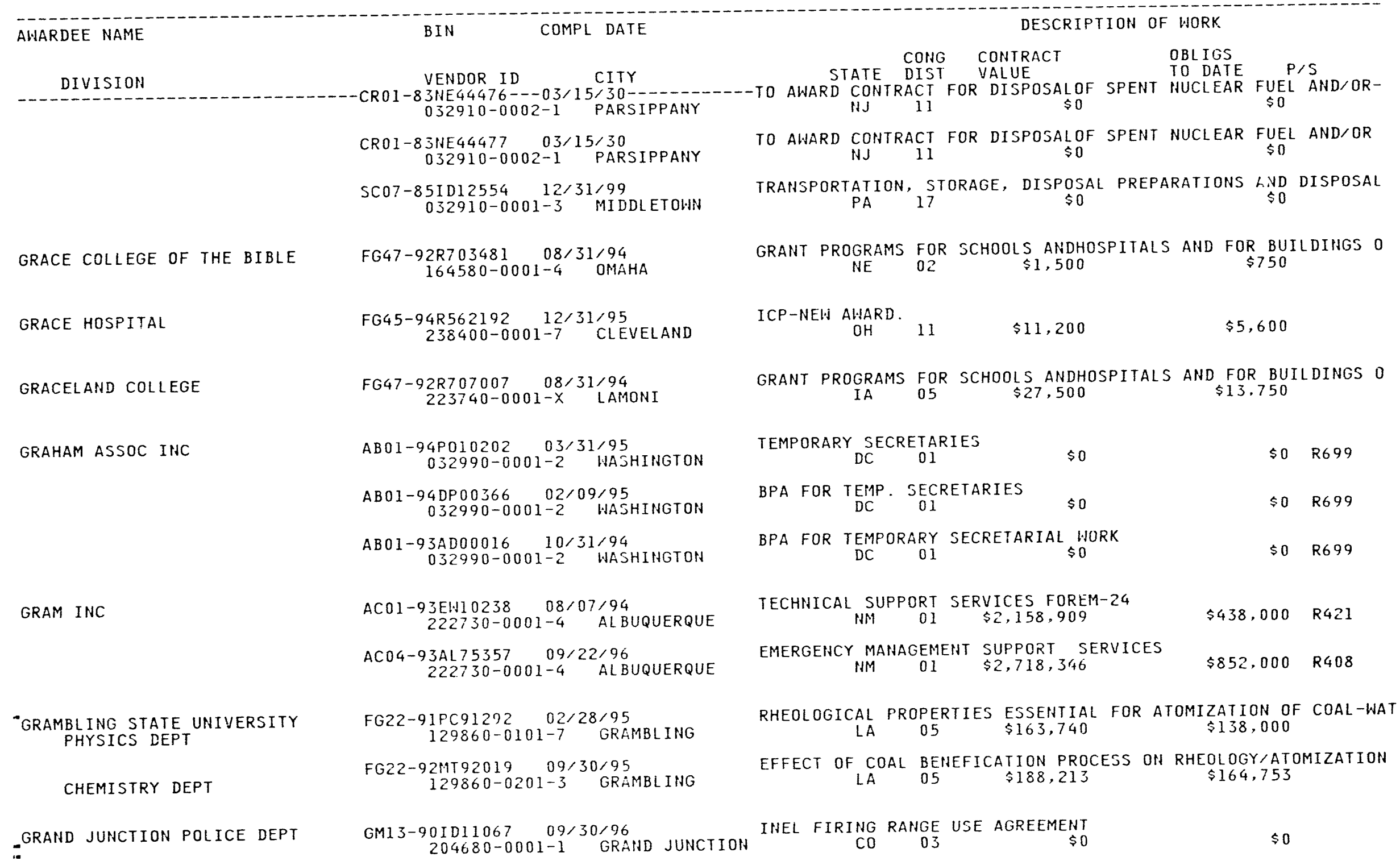




AWARDEE NAME
DIVISION

GRAND VALLEY NATIONAL BANK

GMI3-936J84094 09/30/95

SPECIAL BANK ACCOUNT AGREEMENTFOR GEOTECH LETTER-OF-CREDIT
$\$ 0$

$231050-0001-1$ GRAND JUNCTION

GRANT COUNTY BOARD OF EDUCN

FG43-92R346201 $07 / 31 / 95$
$033160-0002-X \quad$ PETERSBURG

FG43-93R356200 $01 / 31 / 95$

$033160-0002-X$ PETERSBURG

GRANT MEDICAL CENTER

GRAPHIC ARTS INC

GRAPHIC VISIONS ASSOC INC

GRAPHICA INC

GRATIOT COMMUNITY HOSPITAL

GRAVELL ROBERT B

-GREAT DIVIDE DEFENSE PRODUCTS

GREAT LAKES BIBLE COLLEGE

-gREAT LAKES GOV INC COUNCIL OF
FG45-90R520027 11/30/94
$172980-0001-2$ COLUMBUS

$A B 01-94 A D 25709 \quad 05 / 31 / 95$ 033230-0001-3 ALEXANDRIA

AB01-94AD25699 06/30/95 033260-0002-2 GAITHERSBURG $113790-0001-7$ LANHAM FG45-90R520056 02/28/95
$033320-0001-2$ ALMA

$A B 01-92 G C 2027307 / 22 / 94$
$163220-0001-2$ SILVER SPRING 163220-0001-2 SILVER SFRING

AMO4-93AL $94354 \quad 12 / 22 / 94$ 203860-0002-6 ONTARIO 219680-0001-1 LANSING

FG45-93R530280 09/28/94 235270-0001-1 CHICAGO
AB01-94AD24937 05/31/95

AB01-93GC20309 07/31/95

FG45-93R551636 08/31/95
TITLE III OF THE NATIONAL ENERGY CONSERVATION POLICY ACT

$$
\text { WV } 02 \quad \$ 86,900 \quad \$ 30,157
$$

TITLE III OF THE NATIONAL ENERGY CONSERVATION POLICY ACT WV 01 \$62,076 $\$ 31,038$

ICP - NEW AWARD ${ }_{\text {OH }}$ i2 $\$ 321,644 \quad \$ 125,000$

BPA FOR GRAPHIC ARTS AND RELATED SERVICES. 07/01/94 THRU 06/ BPA FOR GRAPHIC ARTS $M D \quad 08$

$\$ 0 \quad T 001$

BPA FOR GRAPHIC PRODUCTION \& OTHER VISUAL ART DELIVERABLES $M D \quad 08$ $\$ 0$ \$0 TOOI

ICP - NEW AWARD. MI io $\$ 55,000$

$\$ 27,500$

BPA FOR NOVELTY SEARCHES ETC

$\$ 0 \quad \mathrm{R} 418$

BPA FOR NOVELTY SEARCHES AND $\underset{\$ 0}{\text { INFRINGEMENT }}$

$\$ 0 \quad$ R418

GREAT DIVIDE DEFENSE PRODUCTS INC. AMMUNITION $\begin{array}{lll}C A & 36\end{array}$

ICP-NEW AWARD

$\$ 25,358$

$\$ 12,679$

FISCAL YEAR 1993 FUNDING OF $\$ 694,000$ FOR CONTINUATION OF THE IL 07 \$694,000 $\$ 694,000$ 


\section{AWARDEE NAME}

BIN

COMPL DATE

CITY

STATE

DIST

DESCRIPTION OF WORK

GREATER LAUREL BELTSVILLE HOSP FG43-93R353102 08/31/94

GREEN LOCAL SCHOOL DISTRICT

GREENBAR CORP

$$
\begin{gathered}
\text { AB34-93RF00536 09/30/94 } \\
204260-0002-1 \text { GOLDEN } \\
\text { AB34-93RF00535009/30/94 } \\
204260-0002-1 \text { GOLDEN } \\
\text { AC34-94RF00749001/27/97 } \\
204260-0002-1 \quad \text { GOLDEN }
\end{gathered}
$$
204260-0001-3 THORNTON

GREENE COUNTY HOSPITAL

GREENE MEMORIAL HOSPITAL

GREENFIELD TOWN OF

GREenVille technical COLlege $232440-0001-0$ LAUREL

FG45-93R551748 01/31/95 229880-0001-8 GREENSBURG$$
\text { AD65-94WA11901 03/29/95 }
$$$$
\text { 204260-0001-3 THORNTON }
$$

AD65-94HA1189703/29/95

AD65-94WA11888 03/29/95 204260-0001-3 THORNTON

AC75-90SW26941 11/30/94 204260-0001-3 THORNTON

FG44-91R434745 08/01/94
$145400-0001-2$ EUTAW

$$
\begin{gathered}
\text { FG47-92R704987 08/31/94 } \\
223690-0101-1 \quad \text { JEFFERSON }
\end{gathered}
$$

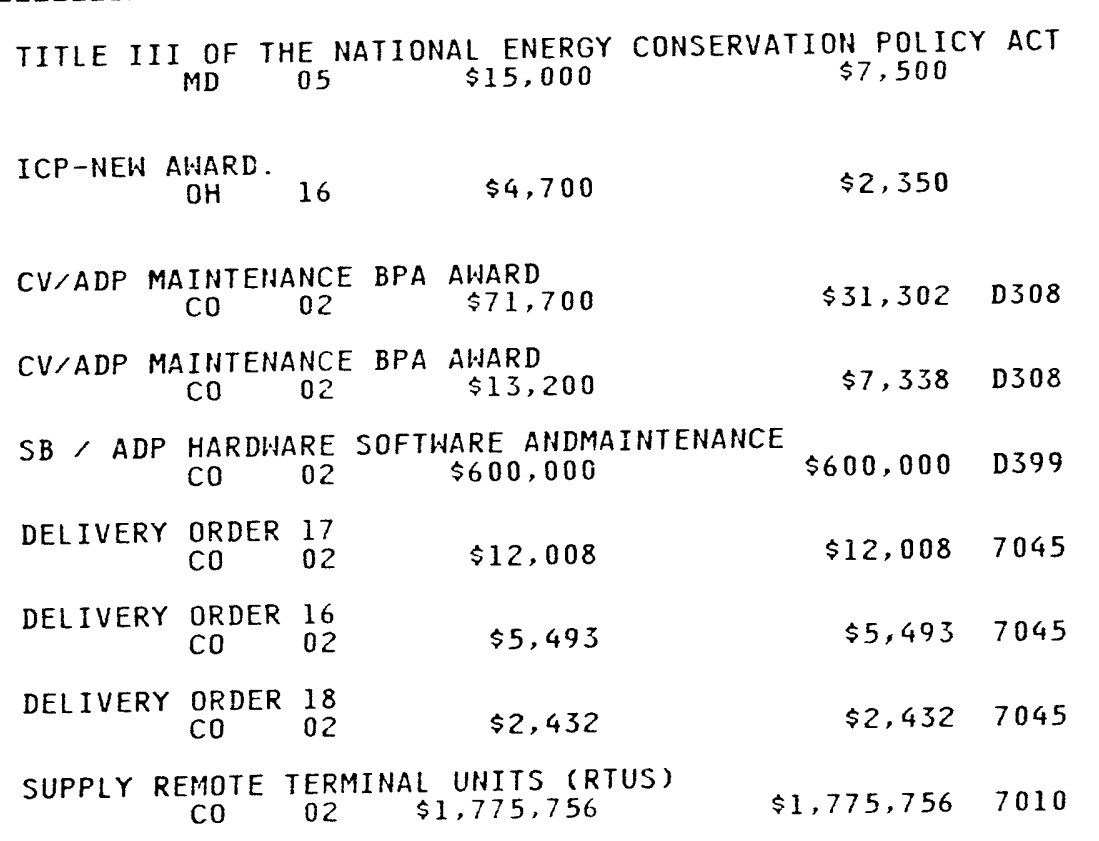

CYCLE 13 - INSTITUTIONAL CONSERVATION PROGRAM. APPROVED BUD $\begin{array}{lll}\text { AL } 07 & \$ 14,147 & \$ 7.073\end{array}$

GRANT PROGRAMS FOR SCHOOLS ANDHOSPITALS AND FOR BUILDINGS 0 IA $05 \quad \$ 62,315 \quad \$ 31,157$

FG45-93R551751 0 05/31/95
$230300-0001-X \times$ XNIA

ICP-NEW AWARD
OH 07
$\$ 27,000$
$\$ 13,500$

FG41-92R193428 07/31/94 221460-0001-5 GREENFIELD

CYCLE 14 ICP AWARD.

$\$ 162,004$

$\$ 80,000$

FG03-87SF17226 $09 / 29 / 95$
$033730-0001-7 \quad$ GREENVILLE
NEW GRANT AWARD FOR SCHOLARSHIPS UNDER THE OMI PROGRAM $S C \quad O 4 \quad \$ 835,931 \quad \$ 365,308$ 


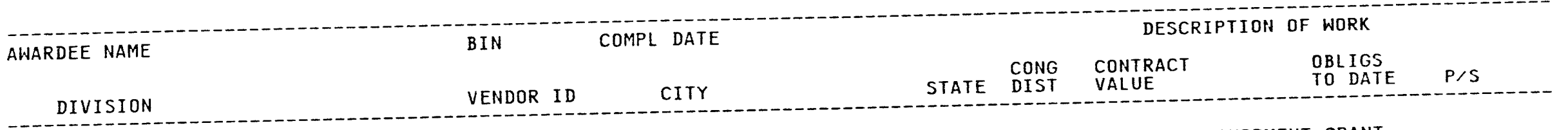

FG06-94RL12775 03/13/95

INSTITUTIONAL DEVELOPMENT

INSTITUTIONAL DEVELOPMENT

INSTITUTIONAL DEVELOPMENT

INSTITUTIONAL DEVELOPMENT

INSTITUTIONAL DEVELOPMENT

INSTITUTIONAL DEVELOPMENT

GRISWOLD TOWN OF

GROTON SCHOOL

GRUMMAN AEROSPACE CORP ADVANCED DEVELOPMENT PROGRAMS

ADVANCED ENERGY SYSTEMS DEPT

GS SANITATION SVC

GSI TRANSCOMM
FG09-94SR18400 12/12/94 4 SR 18400
$033730-0001-7$

FG09-93SRI8367 08/26/94 $033730-0101-3$ GREENVILLE

FG34-94RF0078901/24/95 033730-0101-3 GREENVILLE

FG44-90R434355 07/20/94 $033730-0001-7$ GREENVILLE

FG06-94RL12851 05/15/95 $033730-0101-3$ GREENVILLE

FG34-94RF00909 06/06/95 $033730-0101-3$ GREENVILLE

FG06-94RLI2843 06/26/95 033730-0101-3 GREENVILLE

FG41-93R191505 08/31/95 $232330-0001-4$ JEWETT CITY

FG41-91R193321 c $08 / 31 / 94$
$214670-0001-9$ GROTON

FG02-89ER51124 02/28/95 034040-0202-4 PRINCETON AC07-88ID12753 09/30/94 03012753
$03040-0101-X \quad 30 / 94$
BETHPAGE

AX65-94WC08852 09/30/94 $236380-0001-X$ HATERTOWN

FG01-93CE15580 09/21/95 234860-0001-2 PITTSBURGH
USED ENERGY RELATED LABORATORYEQUIPMENT GRANT

$\$ 0$ \$O

USED ENERGY RELATED LABORATORYEQUIPMENT GRANT

USED ENERG RELATED LABORATORYEQUIPMENT GRANT \$0

USED ENERGY RELATED LABORATORYEQUIPMENT

USED ENERGY RELATED LAB EQUIPMENT GRANT

CYCLE 12 INSTITUTIONAL CONSERVATION PROGRAM. APPROVED FUNDED $\begin{array}{lll}\text { SC } 04 & \$ 254,330 & \$ 121,699\end{array}$

USED ENERGY RELATED LABORATORYEQUIPMENT GRANT

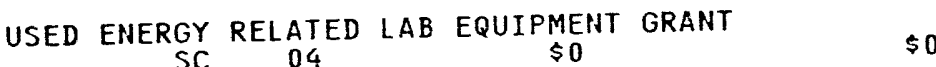

USED ENERGY RELATED LABORATORYEQUIPMENT GRANT

$$
\text { SC } 04 \text { \$0 }
$$

$\$ 0$

INITIAL AWARD.

$$
\text { CT } 02 \quad \$ 80,256
$$

$\$ 40,128$

TITLE III OF THE NATIONAL ENERGY CONSERVATION POLICY ACT.

STABILITY OF TRANSPORT SIMULATIONS OF SECOND REGION NJ $07 \quad \$ 991,884 \quad \$ 991,884$

SPACE NUCLEAR POHER SUPPLY

$$
\begin{array}{lllll}
\text { NY } & 04 & \$ 1,227,326 & \$ 1,227,326 & \text { AC21 }
\end{array}
$$

GARBAGE PICKUP

SD 01

$\$ 1,440$

$\$ 1,440 \quad \$ 205$

MEIKSIN COMMUNICATION SYSTEM FOR UNDERGROUND MINE

$\begin{array}{lll} & \end{array}$ 


\section{AWARDEE NAME \\ GUAM GOVERNMENT OF \\ ENERGY OFFICE \\ ENERGY OFFICE \\ ENERGY OFFICE}

GUADALUPE MEDICAL CENTER

GUILFORD COLLEGE

GULF COAST COMMUNITY COLLEGE NATURAL SCIENCES

GULF STATES UTILITIES CO

GUSTAVUS ADOLPHUS COLLEGE

GUTTENBERG MUNICIPAL HOSPITAL
BIN

COMPL DATE

DESCRIPTION OF WORK

VENDOR ID STATE DIST VALUE

OBLIGS

CITY FOR ESTABIISHMEIT

NM $\quad 02$

2 BI. ISHMENT OF AN

\$0

$\mathrm{P} / \mathrm{S}$

9AL53634 10/05/99
$179460-0001-4 \quad$ CARLSBAD

FG49-81R910232 08/31/94
$034140-0201-9$ AGANA

FG49-84SF15138 09/30/94 034140-0201-9 AGANA

FG49-88SF17627 07/31/94 034140-0201-9 AGANA

FG44-90R434240 06/30/95 159680-0001-5 GREENSBORO

FG34-94RF00929 06/06/95 034270-0101-6 PANAMA CITY

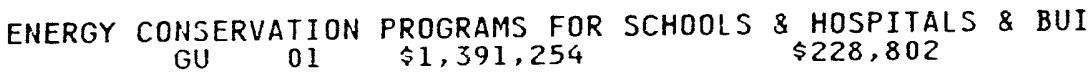
CONSOLIDATED ENERGY GRANT

$$
\text { GU } 011 \% 5,932,383 \quad \$ 1,182,468
$$

GRANT PROGRAMS FOR SCHOOLS ANDHOSPITALS AND BUILDINGS OWNED GU 01 \$81,954 $\$ 81,954$

CYCLE 12 INSTITUTIONAL CONSERVATION PROGRAM. SPECIAL TERMS HC $06 \quad \$ 394,349 \quad \$ 198,927$

USED ENERGY RELATED LAB EQUIPMENT GRANT $\mathrm{FL} \quad 01$ \$O

AC $96-93 P 01831701 / 28 / 95$ HACKBERRY

AC96-9?:018018 $01 / 28 / 95$ 101450-0004-4 HACKBERRY

AC96-93P018020 01/28/95 $101450-0002-8$ NEH IBERIA

AC96-93P01801901/25/95 $101450-0003-6$ PLAQUEMINE

FC02-89ID12860 09/30/94 $101450-0001-X \quad$ BEAUMONT

CROI-84RWO0010 03/15/30 $101450-0001-X$ BEAUMONT

AC96-91P016064 06/30/95 101450-0006-0 WINNIE

FG45-93R551703 12/31/94 $034420-0001-7$ ST. PETER

FG47-92R704985 08/31/94

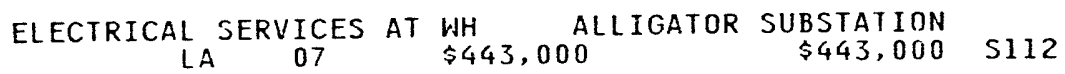

ELECTRICAL SERVICES AT HH RHIS $\$ 33,000 \quad \$ 112$

ELECTRICAL SERVICE AT WI LA 03 AT $\$ 329,000$ $\$ 329,000 \quad \$ 112$

ELECTRICAL SERVICE AT BC LA 08 AT $\$ 387,000$ $\$ 387,000 \quad 5112$

DEVELOPMENT \& TEST OF HEAT PUMPS IN NEW APPLICATIONS FOR IND TX 09 \$ $\$ 1,275,000$ $\$ 452,245$

TO AWARD CONTRACT FOR DISPOSALOF SPENT NUCLEAR FUEL AND $/ O R$ $T X \quad 01$

$\$ 1,119,000 \quad \$ 112$

ELECTRICAL SERVICES AT BH $T \times \quad 09 \quad \$ 1,119,000$

$\$ 47,954$

ICP-NEW AWARD. MN 02 


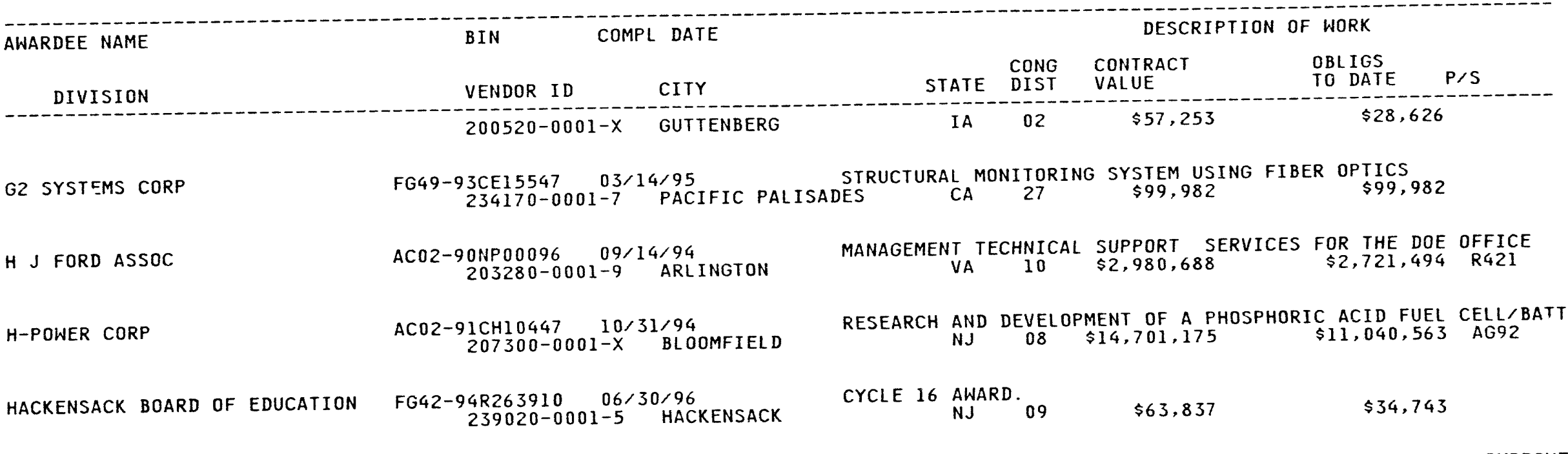

HAGERMAN MUNICIPAL SCHOOLS GM04-93AL84063 11/24/97 $178060-0001-1$ HAGERMAN

HAGERSTOWN GOODWILL INDUSTRIES AC01-93AD67033 07/31/94 232300-0001-3 HAGERSTOWN

HAGUE INTERNATIONAL

HAHNEMANN UNIVERSITY

HAIMSON RESEARCH CORP

:
AC21-93MC $30318 \quad 10 / 29 / 96$ 034650-0002-1 KENNEBUNK AC21-94MC31327 $05 / 09 / 95$
$034650-0001-3$ SOUTH PORTLAND

FG02-93CH10540 12/04/96 034660-0001-7 PHILADELPHIA

FG43-93R354207 12/31/94 034660-0001-7 PHILADELPHIA

FG03-92ER81284 02/17/95
$220800-0001-2$ PALO ALTO

FG03-92ER81285 05/24/95 220800-0001-2 PALO ALTO

FG03-93ER81487 08/01/94 220800-0001-2 PALO ALTO
SUPPORT FOR SCIENCE, MATHEMATICS, AND ENGINEERING $\underset{\$ 0}{\$ 0}$ TO IMPROVE

PROVIDE FLOOR CLEANING AND TRASH PICK- UP SERVICES FOR GERMA $M D \quad 08 \quad \$ 190,915 \quad \$ 190,915 \quad \$ 201$

EVALUATION OF CERAMIC HEAT EX-CHANGER COMPONENTS ME 01 \$ $\$ 1,588,110 \quad \$ 200,000$ AG14 HIGH PRESSURE COAL-FIRED CERAMIC AIR HEATER FOR GAS

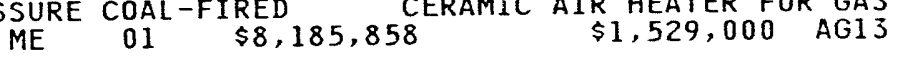

DESIGN \& CONSTRUCTION OF AN AMUBLATORY CARE \& TEACHING $P A \quad 02 \$ 68,434,000 \quad \$ 10,000,000$

TITLE III OF THE NATIONAL ENERGY CONSERVATION POLICY ACT PA $02 \quad \$ 326,155 \quad \$ 91,211$

PHASE I - SBIR, A17 CHZ TAVELING WAVE RELATIVISTIC KLYSTRON PHASE I SBIR GRANT - AN 11.4 GHZ 800 MW PHASE STABLE RF FENE

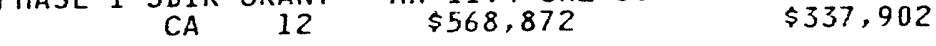

A 17 GIGAHERTZ HIGH GRADIENT ACCELERATOR STRUCTURE FOR A COM $C A \$ 12 \quad \$ 674,919 \quad \$ 171,973$ 
$\frac{5}{2}$

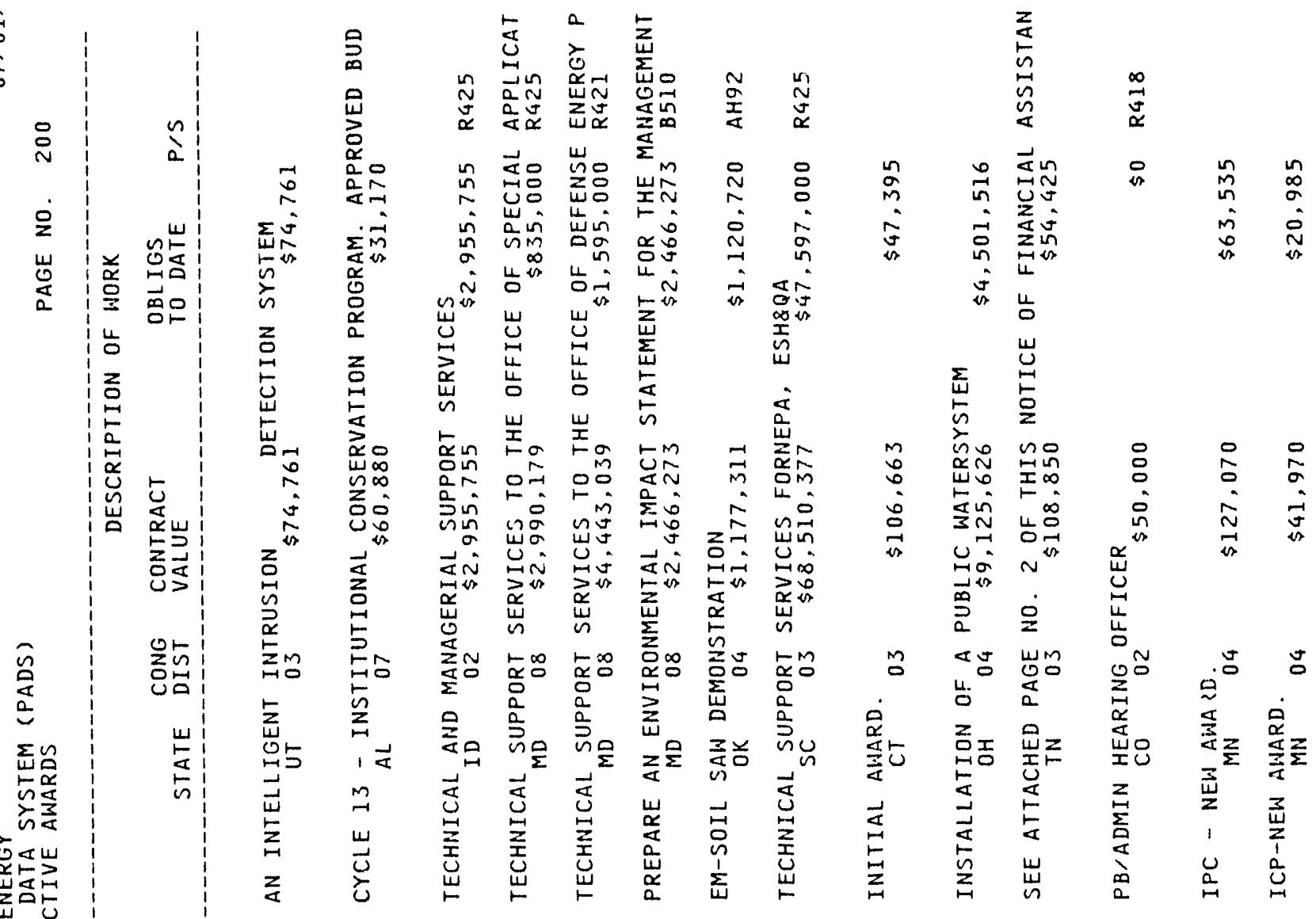

岂岕

o

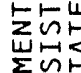

$\sum_{i n n}$

$\alpha<$

w

㟔全主

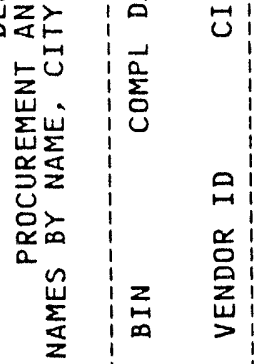

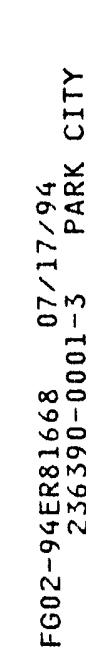

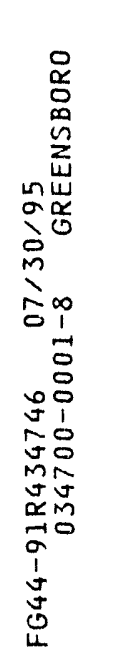

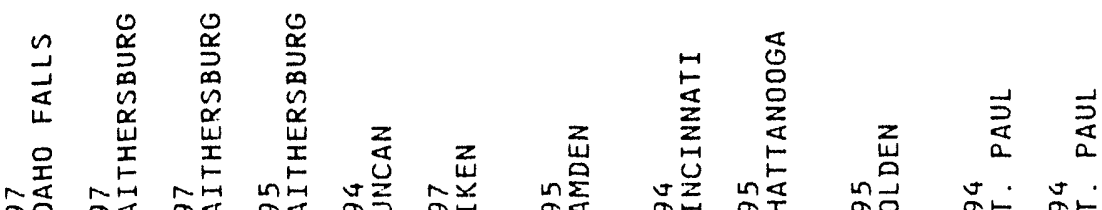

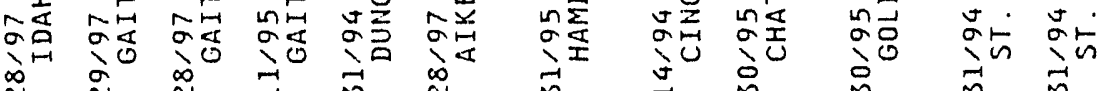

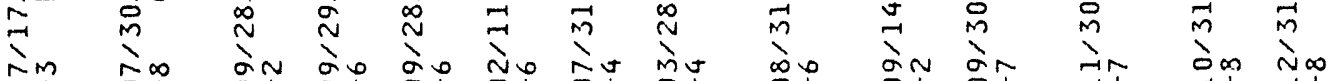

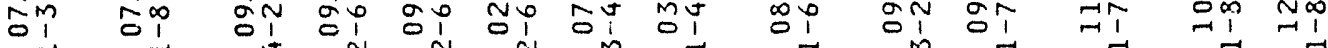

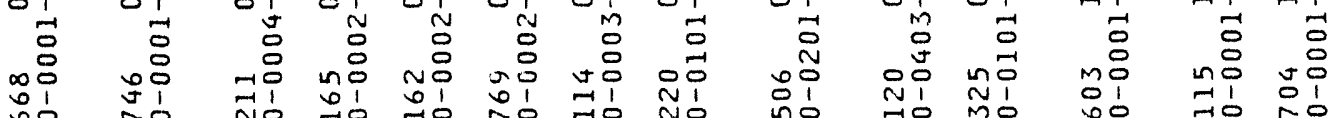

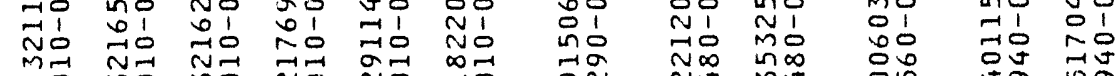

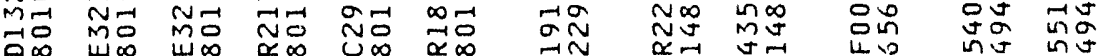

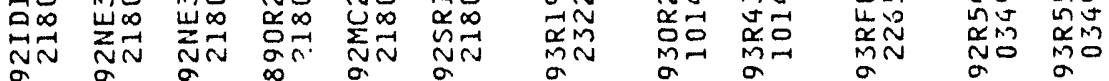

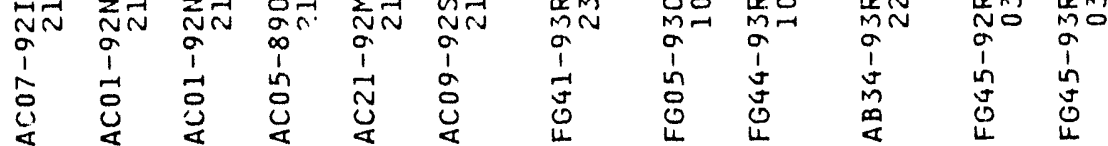

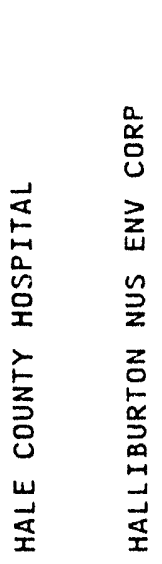
产
岃

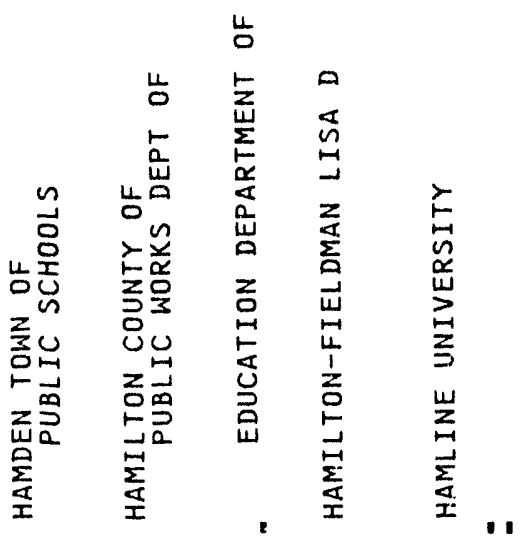

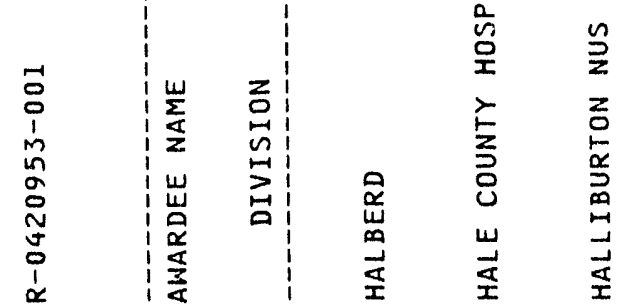




\begin{tabular}{|c|c|c|}
\hline AWARDEE NAME & COMPL DATE & DESCRIPTION OF WORK \\
\hline DIVISION & VENDOR ID & $\begin{array}{l}\text { OBLIGS } \\
\text { TO DATE }\end{array}$ \\
\hline \multirow[t]{2}{*}{$\begin{array}{l}\text { HAMMOND CITY OF } \\
\text { SCHOOLS }\end{array}$} & $\begin{array}{c}\text { FG } 45-93 R 551594 \quad 08 / 31 / 95 \\
034970-0101-5 \text { HAMMGND }\end{array}$ & $\underset{\text { IN }}{\text { ICP-NEW AWARD. }} 01$ \\
\hline & $\begin{array}{c}\text { FG45-94R562006 } 06 / 30 / 95 \\
034970-0101-5 \text { HAMMOND }\end{array}$ & ICP - NEW IN $^{\text {AWARD }} \dot{O}_{I}$ \\
\hline HAMPTON UNIVERSITY & $\begin{array}{c}\text { FG03-90SF } 18720 \quad 09 / 29 / 94 \\
129930-0001-2 \text { HAMPTON }\end{array}$ & $\begin{array}{c}\text { MINORITY UNDERGRADUATE TRAINING IN ENERGY-RELATED CAREERS } \\
\qquad \begin{array}{ccc}\text { VA } 01 & \$ 487,471 & \$ 375,036\end{array}\end{array}$ \\
\hline CHEMISTRY DEPT & $\begin{array}{c}\text { FG05-86ER13589 } 07 / 31 / 94 \\
129930-0301-1 \text { HAMPTON }\end{array}$ & THE USE OF ION $\underset{\text { VA }}{\text { CHROMATOGRAPHY }} \underset{\text { O1 }}{\$ 403,654}-$ D.C. PLASMA ATOMIC EMISSION \\
\hline \multirow[t]{5}{*}{ PHYSICS DEPT } & $\begin{array}{rl}\text { FG05-87ER } 40380 & 12 / 31 / 94 \\
129930-0401-8 \text { HAMPTON }\end{array}$ & $\begin{array}{c}\text { THE HAMPTON UNIVERSITY GRADUATE STUDIES (HUGS) AT THE CONTIN } \\
\text { VA } \\
01\end{array}$ \\
\hline & $\begin{array}{c}\text { FG05-91 ER40620 03/31/97 } \\
129930-0001-2 \text { HAMPTON }\end{array}$ & $\begin{array}{rrr}\text { CEBAF DRIFT } & \text { CHAMBER } & \text { DEVEL OPMENT } \\
\text { VA } & 01 & \$ 540,000\end{array}$ \\
\hline & $\begin{array}{c}\text { FG05-94ER } 4085805 / 31 / 97 \\
129930-0001-2 \text { HAMPTON }\end{array}$ & $\begin{array}{ccc}\text { SELECTED PROBLEMS IN NUCLEAR/HIGH ENERGY PHYSICS } \\
\text { VA } 01 & \$ 156,000 & \$ 50,000\end{array}$ \\
\hline & $\begin{array}{c}\text { FG22-92MT92018 05/31/95 } \\
129930-0001-2 \text { HAMPTON }\end{array}$ & 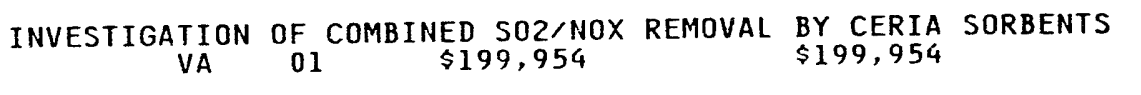 \\
\hline & $\begin{array}{c}\text { FG22-93MT } 9300509 / 01 / 96 \\
129930-0001-2 \text { HAMPTON }\end{array}$ & $\begin{array}{cccc}\text { SIMULTANEOUS REMOVAL OF H2S AND NH3 IN COAL GASIFICATION PRO } & \begin{array}{c}\text { GLS } \\
\text { VA }\end{array} 01 & \$ 199,700 & \$ 199,700\end{array}$ \\
\hline HANCOCK COUNTY BD OF EDUCN & $\begin{array}{l}\text { FG44-93R435326 } 09 / 30 / 95 \\
035060-0001-2 \text { SNEEDVILLE }\end{array}$ & 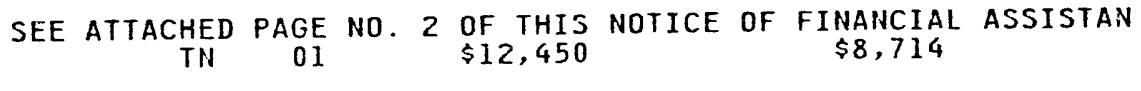 \\
\hline HANCOCK-AVOCA CSD & $\begin{array}{c}\text { FG47-93R707033 } 08 / 31 / 94 \\
232060-0001-7 \quad \text { AVOCA }\end{array}$ & $\begin{array}{clcl}\text { GRANT PROGRAMS FOR SCHOOLS ANDHOSPITALS AND FOR BUILDINGS } 0 & \text { FOR } \\
\text { IA } & 04 & \$ 8,622 & \$ 4,311\end{array}$ \\
\hline HANDS ON SCIENCE OUTREACH INC & $\begin{array}{l}\text { FG02-92ER75695 } 10 / 31 / 94 \\
217760-0001-3 \text { ROCKVILLE }\end{array}$ & $\begin{array}{ccccc}\text { SCIENCE ENRICHMENT } & \text { THROUGH } & \text { INFORMAL SCIENCE IN THREE } & \text { SCH } \\
\text { MD } & 08 & \$ 172,822 & \$ 172,822\end{array}$ \\
\hline HANFORD ENVIRONMENTAL HEALTH & $\begin{array}{l}12 / 31 / 94 \\
\text { RICHLAND }\end{array}$ & 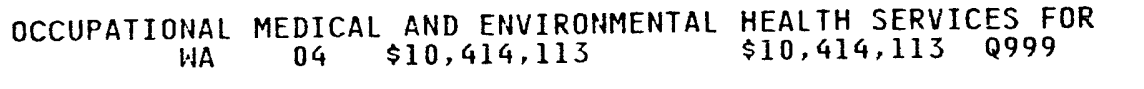 \\
\hline HARDESTY INC & $\begin{array}{c}A B 01-94 A D 6705812 / 31 / 94 \\
193400-0002-1 \text { JESSUP }\end{array}$ & 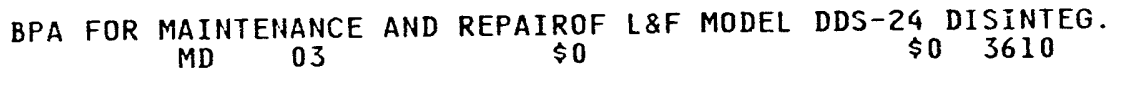 \\
\hline
\end{tabular}




\begin{tabular}{|c|c|c|c|c|c|c|c|}
\hline AWARDEE NAME & BIN & L DATE & & & DESCR & F WORK & \\
\hline DIVISION & VENDOR ID & CITY & STATE & $\begin{array}{l}\text { CONG } \\
\text { DIST }\end{array}$ & $\begin{array}{l}\text { CONTRACT } \\
\text { VALUE }\end{array}$ & $\begin{array}{l}\text { OBLIGS } \\
\text { TO DATE }\end{array}$ & $P / S$ \\
\hline
\end{tabular}

HARDIN COUNTY OF

EDUCATION BOARD OF

HARDIN MEMORIAL HOSPITAL

HARFORD COUNTY PUBLIC SCHOOLS

HARL INGEN CITY OF INDEPENDENT SCHOOL DISTRIC

HARPER HOSPITAL

HARRIS CORP

HARRIS CANADA INC

HARRIS CANADA INC

- CONTROLS \& COMPOSITION

HARRISON MEMORIAL HOSPITAL

-HARRY DEJULIO INTL FIRE DISTR
FG44-93R435327 09/30/95 231190-0101-5 SAVANNAH

FG44-93R435306 09/30/95 035270-0001-1 ELIZABETHTOWN

$$
\begin{gathered}
F G 43-91 R 333202011 / 30 / 94 \\
035340-0001-3 \text { BEL AIR } \\
\text { FG43-92R3433203002/28/95 } \\
035340-0001-3 \text { BEL AIR } \\
\text { FG43-9 } 3 R 353202003 / 31 / 95 \\
035340-0001-3 \text { BEL AIR } \\
\text { FG43-9 } 3 R 35310308 / 31 / 94 \\
035340-0001-3 \text { BEL AIR }
\end{gathered}
$$

FG46-93R613626 08/31/94 32613626
$232680-0101-7$ HARLINGEN

FG45-93R551637 08/31/95 217310-0001-4 DETROIT

AD65-93WJ22239 08/14/94 035450-0508-9 CALGARY

AD65-94WK15991 09/30/94 035450-0508-9 CALGARY

AC65-90WH12360 12/31/94 035450-0301-9 MELBOURNE

FG44-90R43421207/31/94 197470-0001-6 CYNTHIANA

AC65-93WL 04144 11/22/94 229580-0001-X FARMINGTON
SEE ATTACHED PAGE NO. 2 OF THIS NOTICE OF FINANCIAL ASSISTAN

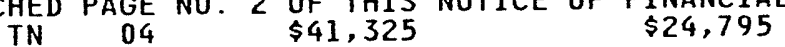

SEE ATTACHED PAGE NO. 2 OF THIS NOTICE OF FINANCIAL ASSISTAN KY $02 \quad \$ 85,295 \quad \$ 42,647$

TITLE III OF THE NATIONAL ENERGY CONSERVATION POLICY ACT MD $02 \quad \$ 38,000 \quad \$ 21,250$

TITLE III OF THE NATIONAL ENERGY CONSERVATION POLICY ACT $\begin{array}{cccc}\text { MD } & 02 & \$ 95,000 & \$ 47,500 \\ \text { TITLE III OF THE NATIONAL ENERGY } & \text { CONSERVATION POLICY ACT } \\ \text { MD } & 02 & \$ 67,500 & \$ 33,750\end{array}$ TITLE III OF THE NATIONAL ENERGY CONSERVATION POLICY ACT

AWARDS A NEW CYCLE 15 TECHNICAL ASSISTANCE GRANT.

ICP-NEW AWARD.
MI

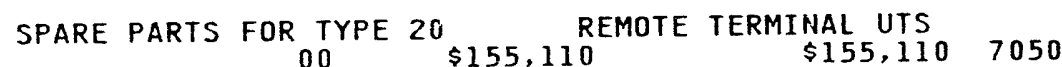
SPARE PARTS FOR HARRIS SYSTEM

$\$ 37,988 \quad 5820$

SCADA $00 \$ 37,988$

$\$ 11,664,520 \quad 9999$

CYCLE 12 INSTITUTIONAL CONSERVATION PROGRAM. APPROVED FUNDED

$\begin{array}{ccccc}K Y & 06 & & \$ 65,086 \\ \text { PAINTING OF } & \text { ELECT } & \text { EQUIP FOR } & \text { SLCAO } \\ & 03 & \$ 153,086\end{array}$ $\$ 32,543$

$\$ 153,086 \quad 7239$ 


\begin{tabular}{|c|c|}
\hline AWARDEE NAME & COMPL DATE \\
\hline DIVISION & VENDOR ID \\
\hline \multirow[t]{4}{*}{$\begin{array}{l}\text { HARVARD UNIVERSITY } \\
\text { MATHEMATICS DEPT }\end{array}$} & $\begin{array}{c}\text { FG02-88ER25065 } 07 / 31 / 94 \\
035710-0902-9 \text { CAMBRI DGE }\end{array}$ \\
\hline & $\begin{array}{c}\text { FG02-93ER61672 } 08 / 14 / 94 \\
035710-0102-8 \quad \text { CAMBRIDGE }\end{array}$ \\
\hline & $\begin{array}{c}\text { FG02-93ER61708 } 09 / 14 / 94 \\
035710-0102-8 \quad \text { CAMBRIDGE }\end{array}$ \\
\hline & $\begin{array}{c}\text { FG05-92ER79127 } 07 / 31 / 94 \\
035710-0002-1 \quad \text { CAMBRIDGE }\end{array}$ \\
\hline ORGANISMIC \& EVOLUT BIOL D & $\begin{array}{l}\text { FG02-84ER60257 } 11 / 30 / 94 \\
\text { DEPT } 035710-1002-7 \text { CAMBRIDGE }\end{array}$ \\
\hline PHYSICS DEPT & $\begin{array}{r}\text { FG02-85ER45190 } 10 / 31 / 94 \\
035710-1202-x \text { CAMBRIDGE }\end{array}$ \\
\hline PRES \& FELLOWS OF HARVARD & $\begin{array}{l}\text { FG01-94EH89439 } 05 / 31 / 95 \\
\text { COLL } 035710-2202-5 \text { CAMBRIDGE }\end{array}$ \\
\hline \multirow[t]{2}{*}{ MEDICINE SCHOOL OF } & $\begin{array}{c}\text { FG02-88ER6 } 0688 \quad 01 / 31 / 95 \\
035710-1602-5 \text { CAMBRIDGE }\end{array}$ \\
\hline & $\begin{array}{c}\text { FG02-88ER45379 } 03 / 14 / 95 \\
035710-0002-1 \quad \text { CAMBRIDGE }\end{array}$ \\
\hline APPLIED SCIENCES DIV & $\begin{array}{c}\text { FG02-89ER45401 } 08 / 31 / 95 \\
035710-0102-8 \text { CAMBRIDGE }\end{array}$ \\
\hline CHEMISTRY DEPT & $\begin{array}{c}\text { FG02-84ER13289 } 11 / 30 / 95 \\
035710-0402-7 \text { CAMBRIDGE }\end{array}$ \\
\hline PHYSICS DEPT & $\begin{array}{c}\text { FG02-84ER40158 } 05 / 31 / 96 \\
035710-1202-x \text { CAMBRIDGE }\end{array}$ \\
\hline CHEMISTRY DEPT & $\begin{array}{r}\text { FG02-90ER14132 } 06 / 14 / 96 \\
035710-0402-7 \text { CAMBRIDGE }\end{array}$ \\
\hline PHYSICS DEPT & $\begin{array}{l}\text { FG02-91ER40654 10/31/96 } \\
035710-1202-x \text { CAMBRIDGE }\end{array}$ \\
\hline GENETICS DEPT & $\begin{array}{c}\text { FG02-87ER60565 } 01 / 31 / 97 \\
035710-1902-4 \quad \text { CAMBRI DGE }\end{array}$ \\
\hline PHYSICS DEPT & $\begin{array}{r}\text { FG02-88ER } 13861 \quad 02 / 28 / 97 \\
035710-1202-X \quad \text { CAMBRI DGE }\end{array}$ \\
\hline
\end{tabular}

DESCRIPTION OF HORK

STATE CONG CONTRACT

MATHEMATICS AND STRING THEORY

MA 08 \$1,969,762

TO DATE

$\mathrm{P} / \mathrm{S}$

$\$ 1,969,762$

ROLE OF BAROCLINIC WAVE ARPLITUDE AND TRANSPORT MA 08 \$52,500 $\$ 52,500$

INVEST. OF THE EFFECT OF NATULPHENOMENA AND INDTL ACT ON MA $08 \quad \$ 507,501 \quad \$ 167,755$

1992 INSTRUMENTATION - UHV THIN FILM DEPOSITION SYSTEM $M A \quad 08 \quad \$ 250,800 \quad \$ 250,800$

THE EFFECT OF ELEVATED CO2 ON PLANT COMMUNITIES
MA 08 \$1,815,828

FUNDAMENTAL PROPERTIES OF SPIN-POLARIZED QUANTUM SYSTEMS MA 08 \$ $1,893,744$ \$ $1,893,744$

23RD DOE/NRC NUCLEAR AIR CLEANING AND TREATMENT CONFERENCE MA 09 NOSAR AIR CLEANING AND TREATMENT $\$ 69,937$ CONFE

$\begin{array}{ccc}\text { CHARACTERIZATION \& MODIFICA- TION OF PHASE T7 DNA } \\ 08 & \$ 2,308,488 & \$ 2308,488\end{array}$

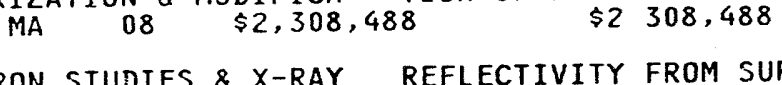

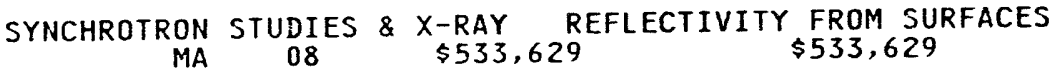

MEASUREMENTS OF CRYSTAL GROHTHKINETICS AT EXTREME DEVIATIONS MA 08 \$550,144 $\$ 438,400$

MODEL STUDIFS OF HYDRODESULFURIZATION \& HYDRODENITROGENATION MA $08 \quad \$ 959,514 \quad \$ 856,514$

THE THEORY OF HIGH ENERGY COLLISION PROCESSES
08 \$2, 590,000 $\begin{array}{cccc}\text { LASER SPECTROSCOPY OF } & \text { HYDROCARBON RADICALS } \\ \text { MA } & 02 & \$ 643,500 & \$ 540,500\end{array}$ WARM LIQUID CALORIMETRY, FRONTEND ELECTRONICS, \& SILICON DRI MA $08 \$ 14,395,000 \quad \$ 8,344,000$

GENOMIC SEQUENCE COMPARISON
MA 08 \$1,944,327 $\$ 1,944,327$

THEORETICAL STUDIES OF HIGHLY IONIZED SPECIES

MA 08 \$1,262,000 $\$ 976,000$ 


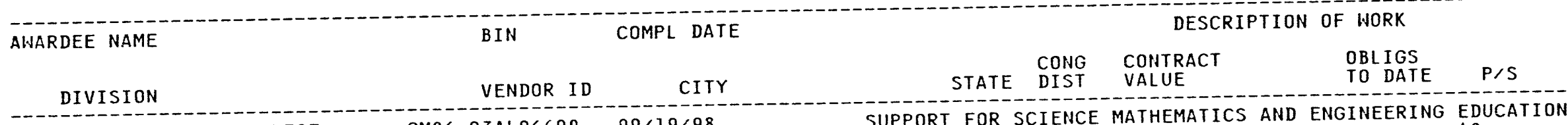

HASKELL INDIAN JR COLLEGE GMO4-93AL $94690009 / 19 / 98$
$234090-0001-1$
LALIRENCE

HASTINGS CATHOLIC SCHOOLS

FG47-92R703482 $08 / 31 / 94$
$223560-0001-1$ HASTINGS

FG47-93R703532 08/31/94 $223560-0001-1$ HASTINGS

HATCH TOWN OF MUNICIPAL SCHOOLS GM04-93AL84067 11/24/97

HAWAII STATE OF FG49-92R900012 09/30/94 BUSINESS ECON DEV \& TOURISM 035900-1001-0 HONOLULU FG49-90SF18664 09/30/94 BUSINESS \& ECONOMIC DEV DEPT FG49-935900-0701-X HONOLULU COMMUNITY SERVICES

ENERGY DFFICE

ENERGY OFFICE

PLANNING \& ECON DEV DEPT BUSINESS DEPT OF

HAWAII UNIVERSITY OF NATIONAL ENERGY INSTITUTE

PHYSICS \& ASTRONOMY DEPT
FG49-91R900002 08/31/94

FG49-92R914017 08/31/94 035900-0301-4 HONOLULU

FC03-925F19168 06/30/95

FG49-89SF18099 03/31/95 035900-0201-8 HONOLULU

FG49-94R900023 06/30/95 035900-1101-7 HONOLULU

FG01-90CE50310 06/21/95

FG03-93ER61541 12/31/95 035920-0002-0 HOHOLULU

AMO3-76SF00235 12/31/99 $035920-0202-3$ HONOLULU

FG03-88ER60663 01/31/97 $035920-0002-0$ HOHOLULU $035900-0901-2$ HONOLULU 035900-0301-4 HONOLULU 035920-1702-0 HONOLULU
SUPPORT FOR SCIENCE MATHEMATICS AND ENGINEERING EDUCATION $\begin{array}{lr}\text { FOR } & \text { SCIENCE } \\ \text { KS } & 01\end{array}$ $\$ 0$

GRANT PROGRAMS FOR SCHOOLS ANDHOSPITALS AND FOR BUILDINGS O $\mathrm{NE} \quad 03$ \$3,780 $\$ 1,890$

GRANT PROGRAMS FOR SCHOOLS ANDHOSPITALS AND FOR BUILDINGS 0 NE $03 \quad \$ 6,400 \quad \$ 3,200$

SUPPOKT FOR SCIENCE, MATHEMATICS, AND ENGINEERING TO IMPROVE NM 02

$\$ 0$

TO SUPPORT ALTERNATIVE TRANSPORTATION FUELS ACTIVITIES $\mathrm{HI} 02 \quad \$ 13,250 \quad \$ 13,250$

GRANT PROGRAMS FOR SCHOOLS ANDHOSPITALS AND BUILDINGS OWNED HI $01 \quad \$ 60,000 \quad \$ 30,000$

CREATE AND RECOMMEND LEVERAGING STRATEGY PLAN FOR HAWAII $\mathrm{HI} \quad 0 \mathrm{I} \quad \$ 50,000 \quad \$ 50,000$

CYCLE XIV ICP ADMINISTRATIVE GRANT.
$\$ 30,000$

COHDUCT A COMPREHENSIVE ENERGYSTUDY FOR THE STATE INCLUDING $\mathrm{HI} 01 \quad \$ 2,332,740$

$\$ 1,435,000$

WEATHERIZATION ASSISTANCE FOR LOW INCOME PERSONS

$$
\text { HI } 01 \text { ASSISTANCE FOR LOW INCOME } \$ 1,711,790 \quad 150,992
$$

NEW GRANT AWARD. PROGRAM YEAR 1994-95.

$$
\text { HI } 02 \quad \$ 3,146,503 \quad \$ 150,800
$$

ALCOHOL TRANSPORTATION FUELS DEMON. PROJ. $\$ 7773,478$

NEW AWARD - "MEASUREMENT OF DIC, ALKALINITY AND PH ON THE WO HI MEAREMENT $\$ 549,997$ AL $\$ 360,135$

THIS IS A BASIC CONTRACTHORK DEFINED BY SEPARATE PRO $\$ 00$ AG91

AN INVESTIGATION OF RADON RELEASE AND MOBILITY IN SUBSURFACE HI $01 \quad \$ 627,062 \quad \$ 397,816$ 


\begin{tabular}{|c|c|}
\hline AWARDEE NAME & COMPL DATE \\
\hline DIVISION & $\begin{array}{l}\text { VENDOR ID } \\
\text {-92ER20078--06/30/95-- }\end{array}$ \\
\hline \multirow[t]{3}{*}{ PLANT MOLECULAR PHYSIO DEPT } & $035920-2102-8$ HONOLULU \\
\hline & $\begin{array}{c}\text { FG03-94ER40833 } 11 / 30 / 98 \\
035920-0002-0 \text { HONOLULU }\end{array}$ \\
\hline & $\begin{array}{c}\text { FG49-85SF15758 } 09 / 30 / 94 \\
035920-0002-0 \text { HONOLULU }\end{array}$ \\
\hline \multirow[t]{2}{*}{ HAWORTH INC } & $\begin{array}{c}\text { AD03-88SF17553 } 09 / 30 / 94 \\
174640-0001-3 \text { HOLLAND }\end{array}$ \\
\hline & $\begin{array}{c}\text { AB 04-93AL91280 10/31/94 } \\
174640-0004-8 \text { AL BUQUERQUE }\end{array}$ \\
\hline HAYES INTERNATIONAL INC & $\begin{array}{c}\text { FC34-94RF00863 05/02/96 } \\
237980-0001-8 \text { KOKOMO }\end{array}$ \\
\hline HAYES SEAY MATTERN \& MATTERN & $\begin{array}{c}\text { ACO5-910R21999 } 01 / 31 / 95 \\
036030-0001-3 \text { ROANOKE }\end{array}$ \\
\hline HAYWARD UNIFIED SCHOOL DIST & $\begin{array}{c}\text { FG49-92R914041 08/31/94 } \\
222890-0001-9 \text { HAYWARD }\end{array}$ \\
\hline HAZARDOUS \& MEDICAL WASTE SVC & $\begin{array}{c}\text { ACO1-94EH89386 } 04 / 17 / 97 \\
237460-0001-7 \text { SILVER SPRING }\end{array}$ \\
\hline HCA MEDICAL CENTER HOSPITAL & $\begin{array}{c}\text { GM04-93AL84019 12/11/97 } \\
236470-0001-9 \text { LARGO }\end{array}$ \\
\hline \multirow[t]{5}{*}{ HEA! TH \& HUMAN SVCS DEPT OF } & $\begin{array}{c}\text { AI01-92PR10002 } 09 / 30 / 94 \\
036100-0005-8 \text { WASHINGTON }\end{array}$ \\
\hline & $\begin{array}{c}\text { AIO1-93EH89304 } 09 / 30 / 94 \\
036100-0008-2 \text { ATLANTA }\end{array}$ \\
\hline & $\begin{array}{c}\text { AI01-93EH89303 } 09 / 30 / 94 \\
036100-0008-2 \text { ATLANTA }\end{array}$ \\
\hline & $\begin{array}{c}\text { AI01-93EH40410 09/30/97 } \\
036100-0008-2 \text { ATLANTA }\end{array}$ \\
\hline & AI01-93CE41062 \\
\hline
\end{tabular}

DESCRIPTION OF WORK

\section{STATE CONG CONTRACT} STATE DIST VALUE HI 01 ON THE SIRUCTURE. \begin{tabular}{l}
$\$ 280,151$ \\
\hline STRCTURE
\end{tabular}

RESEARCH IN HIGH ENERGY PHYSICS
HI

CYCLE 7 SCHOOLS \& HOSPITALS

HI HOOLS \& HOSPITALS
SI

OBLIGS TO DATE POS
FUNCTION AND SYSTHESIS OF $V$ SYSTEMS FURNITURE, 369 HORKSTATION OF VARIOUS CONFIGURATION MI $04 \quad \$ 1,220,132 \quad \$ 1,220,1327110$ BPA TO PROVIDE HAHORTH SYSTEMSFURNITURE AND PARTS

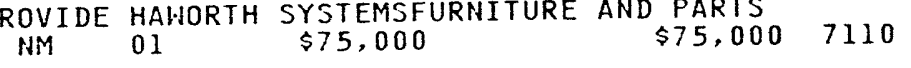

CODPERATIVE AGREEMENT BETWEEN DOE, EG\&G, AND PARTICIPANT FOR IN 05 \$0

SELECT A-E FOR FACILITIES CAPABILITY ASSURANCE PROGRAM (FY-9 $\checkmark A \quad 06 \quad \$ 4,095,529 \quad \$ 4,095,529$ C211

TITLE III OF THE NATIONAL ENERGY CONSERVATION POLICY ACT $C A \quad 09 \quad \$ 108,712 \quad \$ 54,356$

INFORMATION MANAGEMENT ADMINISTRATIVE \& TECHNICAL SUPPORT S MD 08 \$1.997,922 $\$ 500,000 \quad \mathrm{R} 612$

EMERGEHCY RADIOLOGICAL TREATMENT CENTER $\mathrm{FL} \quad 08$ $\$ 0$

AUDIT SERVICES OF EDUCATIONAL INSTITUTIONS $\begin{array}{llll}\text { DC } & 01 & \$ 622,614 & \$ 22,614\end{array}$ PROGRAM DIRECTION BY THE CENTERS FOR DISEASE CNOTROL IMPLEME $G A$ O4 $\$ 12,400,000$ $\$ 12,400,000$

INCTERAGENCY AGREEMENT CFY 93 PROJ SUPPORT FOR ENERGY RELATE GA $05 \$ 26,600,000 \quad \$ 22,680,000$

THE AGENCY FOR TOXIC SUBSTANCES DISEASE REGISTRY WILL CONDUC $G A$ OS $\$ 15,000,000$ $\$ 10,072,000$

ASSESSMENT OF PUBLIC HEALTH RISKS ASSOCIATED WITH MUNICIPAL, 


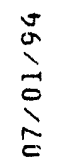

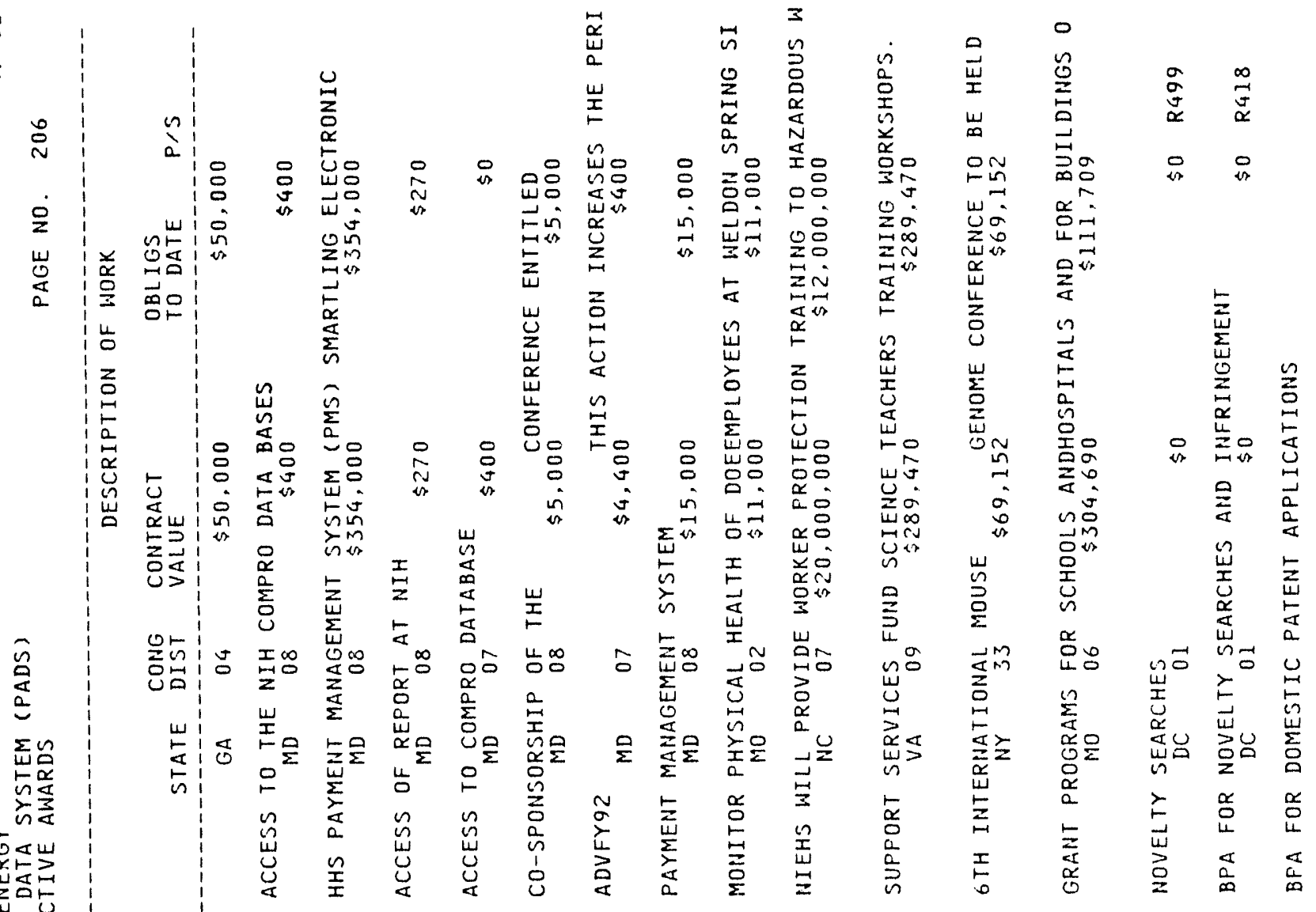

嵌

运

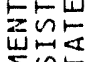

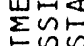

舟我

㟧是

㟧

$\frac{a}{2}$

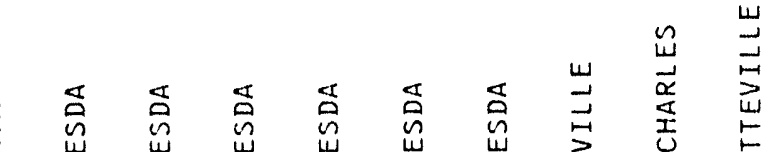

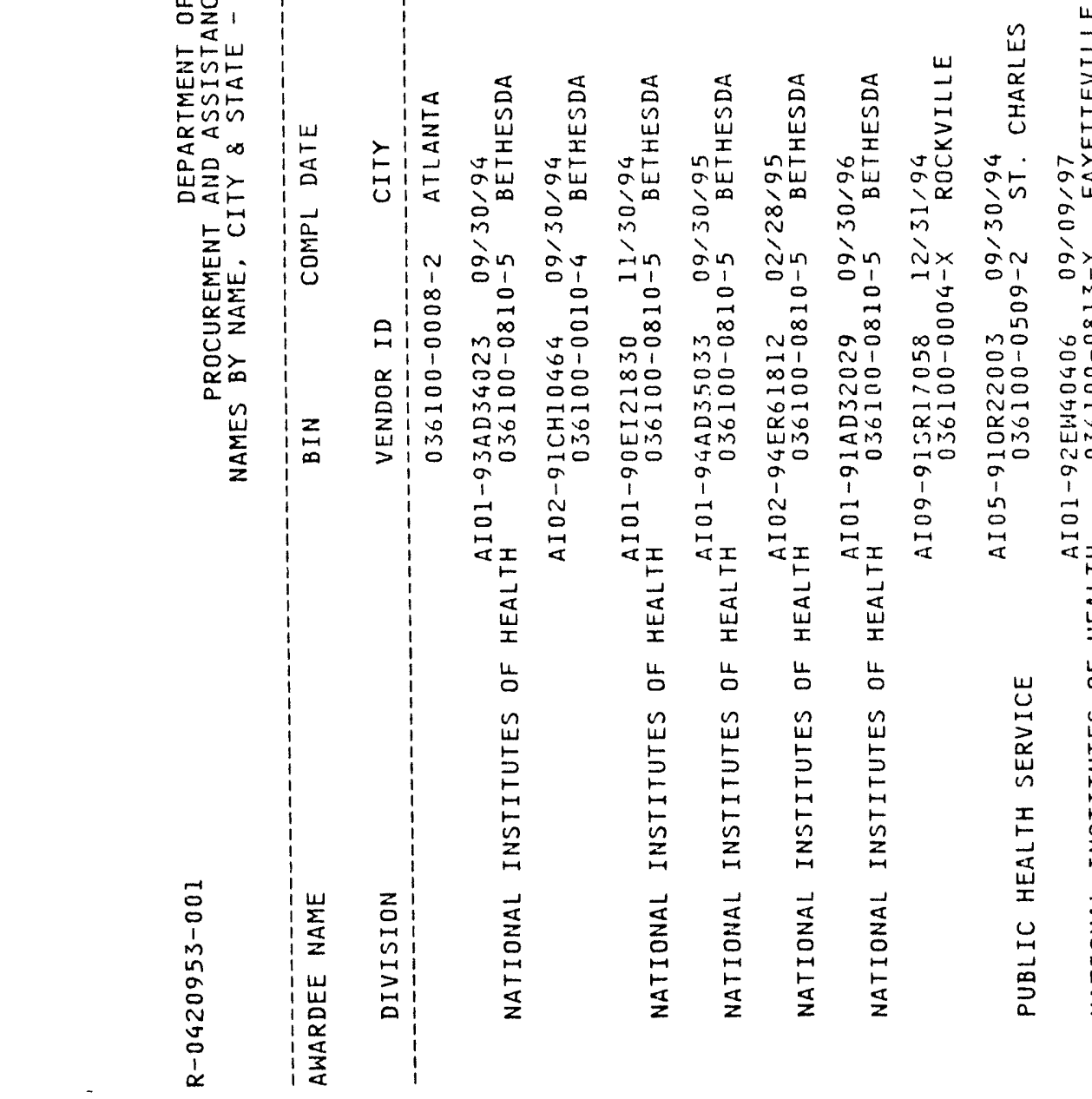

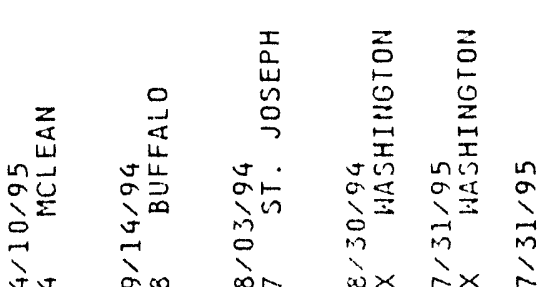

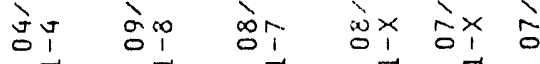

$\begin{array}{lllll}-1 & -1 & -1 & 1 & 0 \\ 0 & 0 & 0 \\ 0 & 0 & 0 & 0\end{array}$

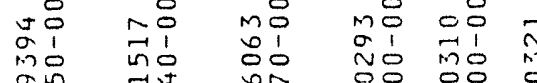

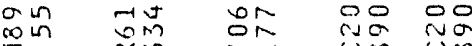
虽 品

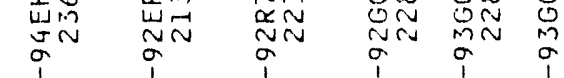
落 2
$\mathbb{N}$
0
0

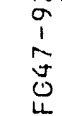




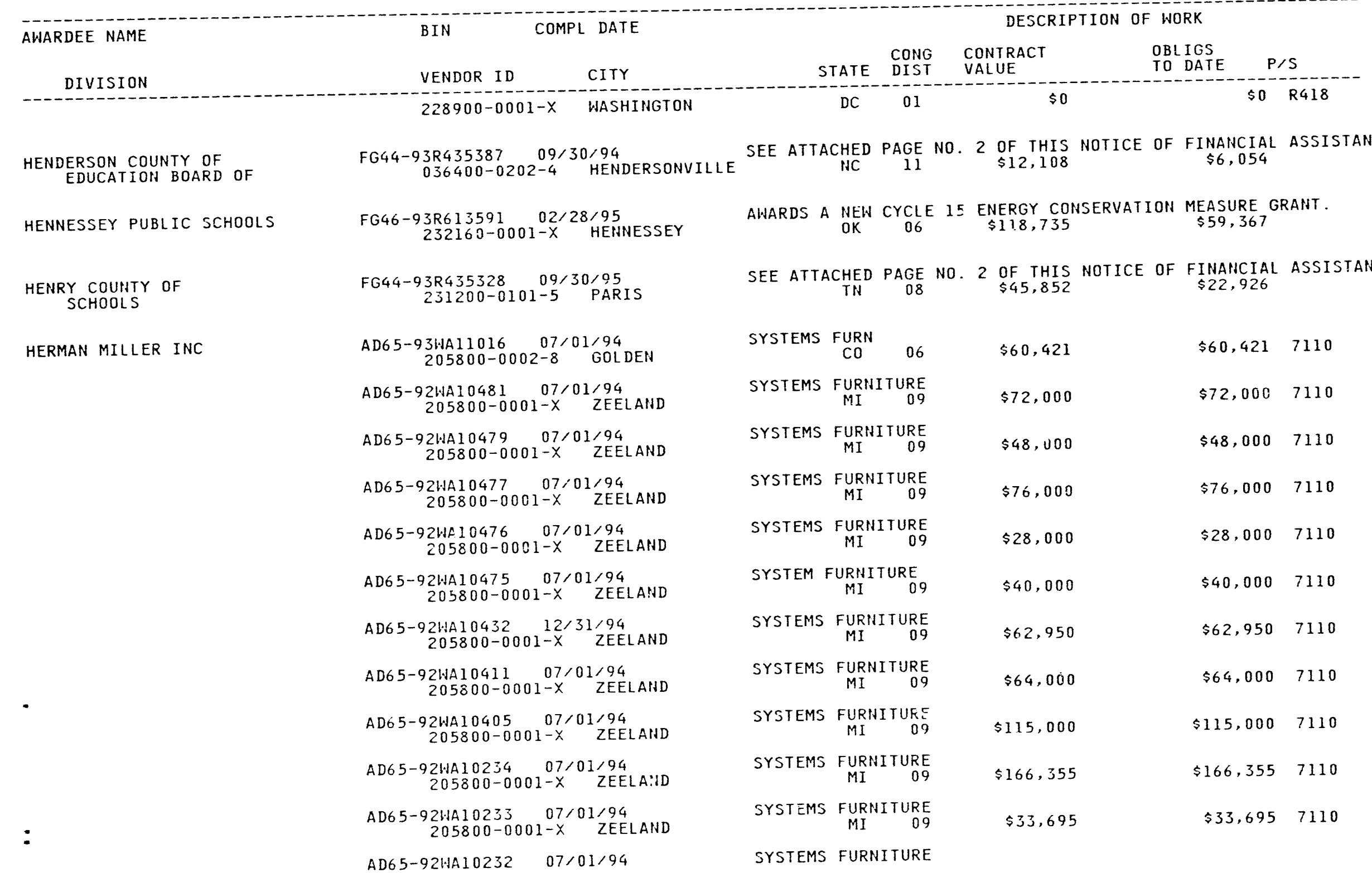

SEE ATTACHED PAGE NO. 2 OF THIS NOTICE OF FINANCIAL ASSISTAN DESCRIPTION OF WORK 


\begin{tabular}{|c|c|c|c|c|c|}
\hline \multirow{2}{*}{$\begin{array}{l}\text { AWARDEE NAME } \\
\text { DIVISION }\end{array}$} & \multirow{2}{*}{$\begin{array}{lc}\text { BIN } & \text { COMPL DATE } \\
\text { VENDOR ID } & \text { CITY }\end{array}$} & \multicolumn{4}{|c|}{ DESCRIPTION OF WORK } \\
\hline & & $\begin{array}{ll}\text { CONG } \\
\text { STATE } & \text { DIST }\end{array}$ & $\begin{array}{l}\text { CONTRACT } \\
\text { VALUE }\end{array}$ & $\begin{array}{l}\text { OBLIGS } \\
\text { TO DATE }\end{array}$ & $P / S$ \\
\hline & $205800-0001-x$ & MI & $\$ 141,390$ & $\$ 141,390$ & $\because 110$ \\
\hline & $\begin{array}{r}\text { AD65-92WG21136 09/20/94 } \\
205800-0001-X \text { ZEELAND }\end{array}$ & $\begin{array}{c}\text { SYSTEMS FURN } \\
\text { MI }\end{array}$ & $\$ 176,000$ & $\$ 176,000$ & 7110 \\
\hline & $\begin{array}{c}\text { AD65-924IG21137 } 09 / 30 / 94 \\
205800-0001-x \quad \text { ZEELAND }\end{array}$ & $\begin{array}{cr}\text { SYSTEMS FURNITURE } \\
\text { MI } \quad 09\end{array}$ & $\$ 660,000$ & $\$ 660,000$ & 7110 \\
\hline \multirow[t]{8}{*}{ HEWLETT-PACKARD CO } & $\begin{array}{cc}A D 01-93 A D 34047 & 09 / 30 / 94 \\
036640-0010-2 & \text { ROCKVILLE }\end{array}$ & SOFTWARE LICENSE & $\$ 46,086$ & $\$ 46,086$ & 7030 \\
\hline & $\begin{array}{c}\text { AD03-935F20161 } 09 / 30 / 94 \\
036640-0010-2 \quad \text { ROCKVILLE }\end{array}$ & $\begin{aligned} \text { PROCUREMENT OF } & \times 400 \mathrm{H} \\
\text { MD } & 08\end{aligned}$ & $\begin{array}{l}\text { HARDIJARE AND/OR } 3 \\
\quad \$ 26,763\end{array}$ & $\begin{array}{l}\text { SOFTWARE } \\
\$ 26,760\end{array}$ & 7022 \\
\hline & $\begin{array}{c}\text { ADO3-93SF19992 } 09 / 30 / 94 \\
036640-0010-2 \text { ROCKVILLE }\end{array}$ & $\begin{array}{c}\text { HARDWARE MAINTENANCE } \\
\text { MD } 08\end{array}$ & $\begin{array}{l}\text { SUPPORT FOR HP } 3 \\
\$ 33,000\end{array}$ & $\begin{array}{r}3000 / 950 \\
\$ 33,000\end{array}$ & D301 \\
\hline & $\begin{array}{c}\text { AD03-93SF19991 } 09 / 30 / 94 \\
036640-0010-2 \quad \text { ROCKVILLE }\end{array}$ & $\begin{array}{c}\text { SOFTWARE MAINTENANCE } \\
\text { MD } 08\end{array}$ & $\begin{array}{l}\text { SUPPORT FOR HP3O } \\
: 30,000\end{array}$ & $000 / 950 \$ 30,000$ & D301 \\
\hline & $\begin{array}{c}\text { AD04-94AL90502 } 09 / 30 / 94 \\
036640-0010-2 \quad \text { ROCKVILLE }\end{array}$ & $\begin{array}{c}\text { HEHLETT-PACKARD EQUIP } \\
\text { MD } 08\end{array}$ & $\begin{array}{l}\text { PMENT MAINTENANCE } \\
\$ 41,312\end{array}$ & $\$ 41,312$ & 7030 \\
\hline & $\begin{array}{c}\text { AD04-94AL90515 } 09 / 30 / 94 \\
036640-0010-2 \quad \text { ROCKVILLE }\end{array}$ & $\begin{array}{l}\text { HEWLETT-PACKARD SOFTH } \\
\text { MD } 08\end{array}$ & $\begin{array}{l}\text { WARE MAINTENANCE } \\
\$ 31,019\end{array}$ & $\$ 31,019$ & 7030 \\
\hline & $\begin{array}{c}\text { ADO4-94AL90664 } 05 / 02 / 95 \\
036640-0010-2 \quad \text { ROCKVILLE }\end{array}$ & DISC DRIVES, $\underset{\text { MD }}{\underset{08}{\text { COMPUTER }}}$ & $\begin{array}{l}\text { R RACK, } \\
\qquad 25,480\end{array}$ & $\begin{array}{l}\text { SUPPORT } \\
\$ 25,480\end{array}$ & 7035 \\
\hline & $\begin{array}{c}\text { AD05-940R22158 } 09 / 30 / 94 \\
036640-0030-7 \quad \text { OAK RIDGE }\end{array}$ & HARDWARE MAINTENANCE & $\$ 28,134$ & $\$ 28,134$ & 7021 \\
\hline HIGH PLAINS CONSTRUCTION & $\begin{array}{c}\text { AC65-9411L } 04393 \quad 10 / 11 / 94 \\
238530-0001-0 \text { CRAIG }\end{array}$ & $\begin{array}{r}\text { CRAIG MTNCE FACILITY } \\
\text { CO } \\
03\end{array}$ & $\begin{array}{l}\text { REMODEL \&SITE IM } \\
\$ 345,815\end{array}$ & $\begin{array}{l}\text { MPROVEMENTS } \\
\qquad 345,815\end{array}$ & Y299 \\
\hline \multirow[t]{3}{*}{ HIGH POWER INC } & $\begin{array}{c}\text { AC65-91HC05611 08/01/94 } \\
169520-0011-9 \text { WOLF POINT }\end{array}$ & $\begin{array}{c}\text { CONSTRUCTION OF NEW W } \\
\text { MT } 02\end{array}$ & $\begin{array}{l}\text { WORLFPOINTSUBSTAT } \\
\$ 2,412,363\end{array}$ & $\begin{array}{r}\text { TION, STAGE } 02 \\
\$ 2,412,363\end{array}$ & Y239 \\
\hline & $\begin{array}{cc}\text { AC65-941.624197 } 09 / 30 / 95 \\
169520-0001-1 \quad \text { BOULDER CITY }\end{array}$ & $\begin{array}{c}\text { CONSTRUCTION OF MEAD } \\
\text { NV OI }\end{array}$ & $\begin{array}{l}\text { SUB, STG OE } \\
\$ 9,791,791\end{array}$ & $\$ 9,791.791$ & Y239 \\
\hline & $\begin{array}{c}\text { AC65-93HG21343 } 03 / 10 / 95 \\
169520-0001-1 \quad \text { BOULDER CITY }\end{array}$ & CONSTRUCTIJN OF MEAD & $\begin{array}{l}\text { SUBST STG } 05 \\
\$ 6,747,544\end{array}$ & $\$ 6.747,544$ & Y234 \\
\hline
\end{tabular}




\begin{tabular}{|c|c|c|c|c|c|c|c|}
\hline AWARDEE NAME & BIN & $\angle$ DATE & & & DESCRIP & F WORK & \\
\hline DIVISION & VENDOR ID & CITY & STATE & $\begin{array}{l}\text { CONG } \\
\text { DIST }\end{array}$ & $\begin{array}{l}\text { CONTRACT } \\
\text { VALUE }\end{array}$ & $\begin{array}{l}\text { OBLIGS } \\
\text { TO DATE }\end{array}$ & $P / S$ \\
\hline & $145580-000$ & PH.'STONSBURG & KY & 07 & $\$ 53.000$ & $\$ 26$ & \\
\hline
\end{tabular}

HIGHLINE COMMUNITY COLLEGE

HILL COUNTY ELECTRIC COOP INC

HISPANIC ASSN OF COLL \& UNIV

HISTORY ASSOC INC

HIWASSEE COLLEGE

HMJ CORP

HOBART \& WILLIAM SMITH COLLEGE

BRISTOL GYM

HOBBS INC

HOCKING TECHNICAL COLLEGE

$=$ HOLBROOK USD
FG51-92R020144 10/31/94

102020144
$231490-0001-7$ DES IOINES

AC 35-915C00003 11/13/94
$215530-0001-3$
ITASCA

FG03-93ER75605 06/30/96 $233210-0001-6$ SAN ANTONIO

ACO1-93EH89246 08/24/95 037050-0004-7 ROCKVILLE

AC02-91NP00092 09/14/94 037050-0004-7 ROCKVILLE

FG44-93R435329 09/30/95 231250-0001-7 MADISONVILLE

FG02-92ER81355 06/03/95 $169880-0002-X$ KENSINGTON

FG42-92R243819 07/31/94 037110-0001-0 GENEVA FG42-93R253870 07/31/95
$037110-0101-7$ GENEVA

AB 1-94AD25715 05/31/95 $208760-0001-4$ WASHINGTON

FG45-94R562182 12/31/95 $238140-0001-3$ NELSONVILLE

FG47-92R706070 08/03/94 221540-0001-0 COLUMBIA
CYCLE 14 ECM AWARD.

$\$ 94,363$

$\$ 47,181$

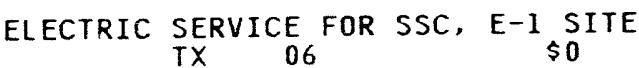

$\$ 0 \quad \$ 112$

DOE/HACU CONNECTIONS: THE HIS PANIC SERVING INSTITUTIONS MOL TX 20 \$1, 056,743 $\$ 289,301$

TECHNICAL SUPPORT SERVICES FORTHE MANAGEMENT OF THE EPIDEMI $M D \quad 08 \quad \$ 2,106,981 \quad \$ 1,275,000 \quad R 421$

HISTORY OF THE NPR PROGRAM

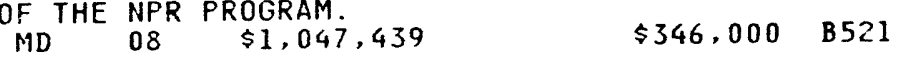

SEE ATTACHED PAGE NO. 2 OF THIS NOTICE OF FINANCIAL ASSISTAN TN 02 \$129,750 $\$ 59,384$

$\begin{array}{cccc}\text { DECHLOVINATION SYSTEM FOR } & \text { MHD SPENT SEẼ } \\ \text { MD } & 08 & \$ 550,000 & \$ 338,756\end{array}$

TITLE III OF THE NATIONAL ENERGY CONSERVATION POLICY ACT NY $31 \quad \$ 106,775 \quad \$ 53,387$

CYCLE $15 \underset{\text { NYARD. }}{\text { AWY }} 27 \quad \$ 86,178 \quad \$ 43,089$

BPA: GRAPHIC ARTS AND RELATED SERVICES RESPONDING TO WASHING DC 01 $\$ 0$ \$O TOOI

ICP-NEW AWARD

$\mathrm{OH} .06$

$\$ 21,163$

$\$ 10,581$

GRANT PROGRAMS FOR SCHOOLS ANDHOSPITALS AND FOR BUILDINGS 0 MO $\quad 09 \quad \$ 10.208$ $\$ 5,104$ 


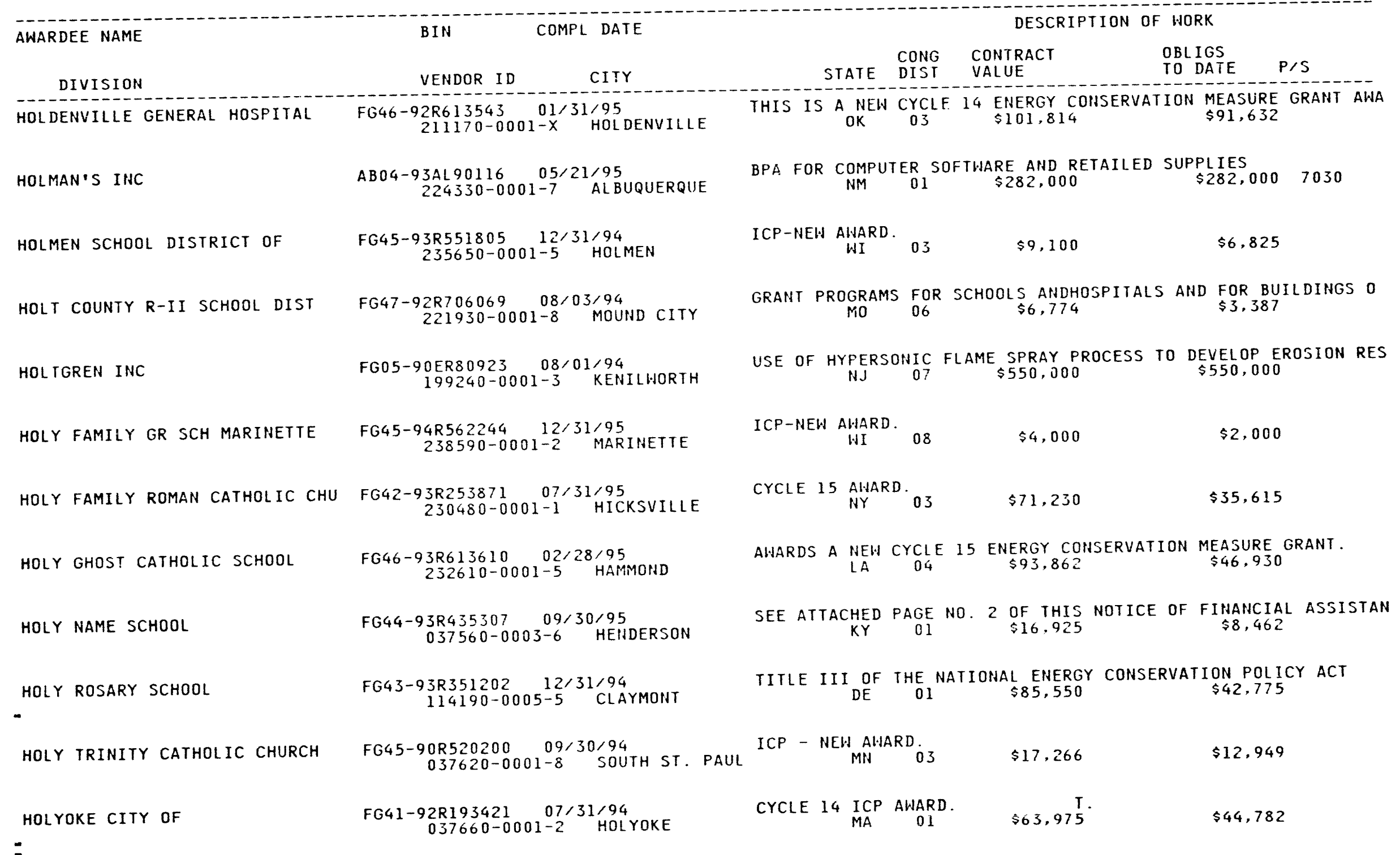

:

HONDO TOWN OF
GMO4-92AL84060 07/22/97
SUPPORT FOR SCIENCE, MATHEMAIICS, AHD ENGINEERING TO IMPROVE 


AWARDEE NAME
DIVISION
MUNICIPAL SCHOOLS

HOPE GAS INC

HOPKINS FRENCH CROCKETT

HORIZON DATA CORP

HORSESHOE BEND DISTRICT \#

HOSPITAL LINEN SVC FACILITY

HOUSTON ADVANCED RESEARCH CTR

HOUSTON COMMUNITY COLLEGE

-HOUSTON LIGHTING \& POWER CO

HOUSTON UNIVERSITY OF

:
COMPL DATE

DESCRIPTION OF WORK

BIN

CITY

VENDOR ID RUIDOSO

$226980-0101-1$

$A C 21-92 M C 29074008 / 31 / 02$
$222740-0001-8$ CLARKSBURG

AC07-91ID10416 10/31/95

$173020-0101-4$ IDAHO FALLS

$\begin{array}{cc}A L 01-90 C E 90053 & 09 / 11 / 94 \\ 203240-0001-4 & \text { RESTON }\end{array}$

GMO7-94IDI1150 $03 / 17 / 99$
$236960-0001-9$ HORSESHOE BEND

FG43-93R354208 12/31/94

209810-0001-0 PITTSBURGH

FG03-93ER61725 09/14/94

$232750-0001-2$ THE WOODLANDS FG03-87SF17229 08/23/96
$164690-0001-0$ HOUSTON

FG03-90SF18627 12/05/94 $164690-0001-0$ HOUSTON

FG03-90SF18716 09,29/94

164690-0001-0 HOUSTON

AC96-91P016062 09/24/94
$157560-0002-X$ FREEPORT

CRO1-87RH00129 03/15/30

157500-0001-1 HOUSTON

FGO3-93ER40805 $07 / 31 / 94$
$038080-0001-5$ HOUSTON

FG03-94ER14426 02/14/9?
$038080-0001-5$ HOUSTOH
CONG CONTRACT

NM 02

OBLIGS

TO DATE P/S $\$ 0$

$\$ 0$

PROVIDE NATURAL GAS TRANSPORTATION

WV 01 \$750,000 $\$ 335,000 \quad \$ 111$

PATENT ATTORNEY SERVICES

ID $02 \quad \$ 80,000 \quad$ R418

POWER INFORMATION CENTER

VA $10 \quad \$ 1,789,754 \quad \$ 1,018,472$ R408

STATE THE BASIC UNDERSTANDING OF THE PARTIES REGARDING, \& PR ID 01 $\$ 0$

TITLE III OF THE NATIONAL ENERGY CONSERVATION POLICY ACT PA $14 \quad \$ 235,000$ $\$ 75,000$

1993 WORKSHOP ON SEQUENCING BYHYBRIDIZATION

NEW GRANT AHARD FOR OMI PROGRAM
TX 18 \$1, 490,773

$\$ 503,810$

SUMMER AND TRANSFER ACHIEVEMENT READINESS (STAR) PROGRAM IN $T X \quad 18$ \$1,993,630 \$1,419,778

MINORITY UNDERGRADUATE TRAINING IN ENERGY-RELATED CAREERS TX $18 \quad \$ 1,386,585 \quad \$ 353,369$

OBLIGATION OF FUNDS FOR ELECTRICAL SERVICES AT BM $T X \quad 14 \$ 1,546,000 \quad \$ 1,546,000 \$ 112$

DISPOSAL OF SPENT NUCLEAR FUELAND OR HIGH LEVEL RADIOACTIVE TX 18 \$O

NEW GRANT ENTITLED "GEM MUON SYSTEM R\&D."

$\begin{array}{cc}\text { "CHARGE SEPARATION IN PHOTOREDOX REACTIONS." } \\ \text { TX } 18 & \$ 365,000\end{array}$ 


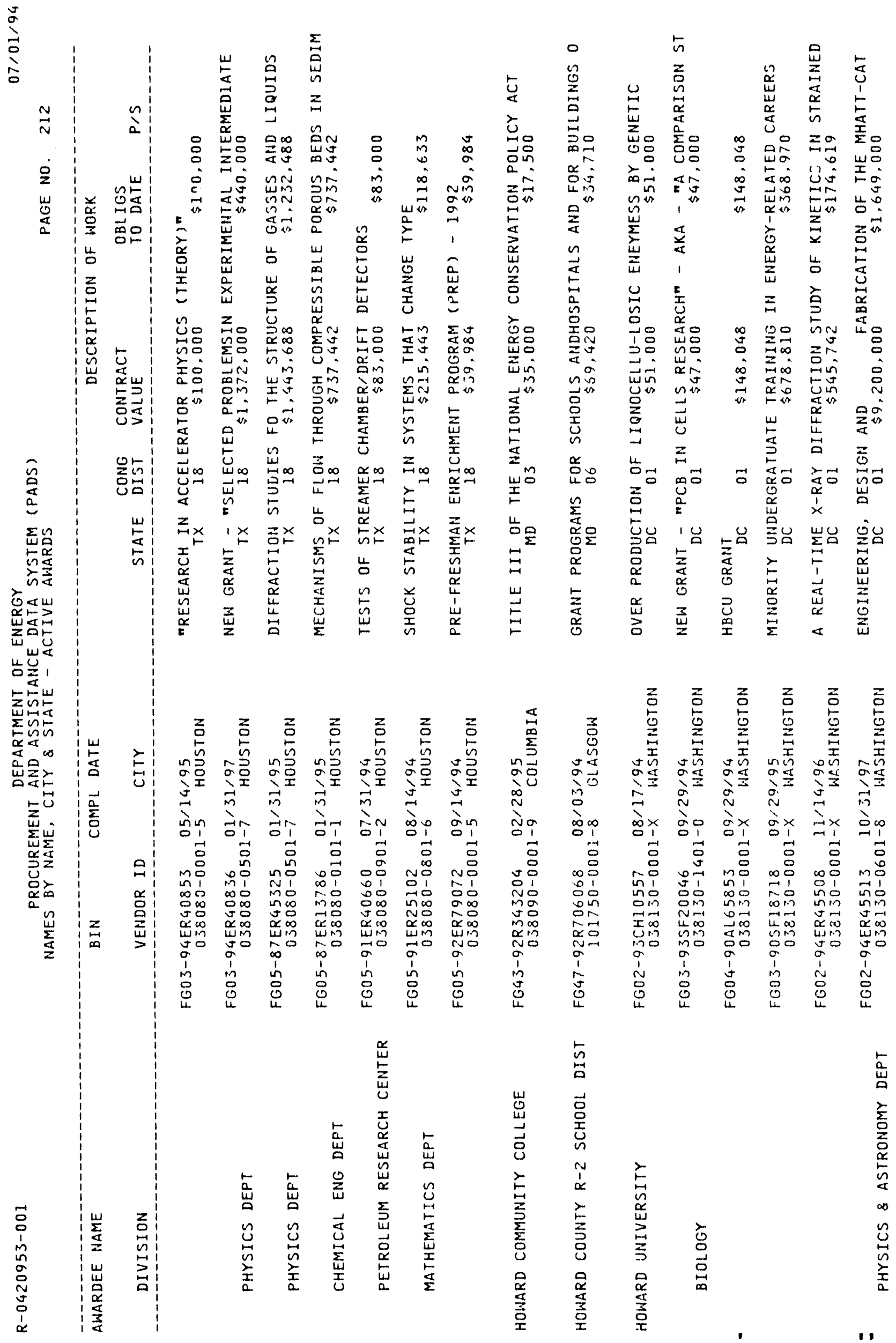




\section{AWARDEE NAME}

BIN

COMPL DATE
VENDOR ID 4ER40854---05/31/98$038130-0001-X$ WASHINGTON

HOWE FURNITURE CORP

HUBBARD THURMAN TUCKER HARRIS

HUFFMAN LABORATORIES INC

HUGHES AIRCRAFT CO

ELECTRON DYNAMICS DIVISION

HUGHES EASTERN CORP

HUGO PUBLIC SCHOOLS

HUNTINGTON COUNTY OF COMMUNITY SCHOOL CORP

COMMUNITY SCHOOL CORP

- HUNTWAY REFINING CO

HUTCHINSON HOSPITAL

HYATT COURT REPORTING

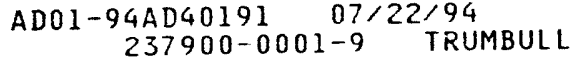

\section{STATE DONG CONTRAC}

DESCRIPTION OF HORK

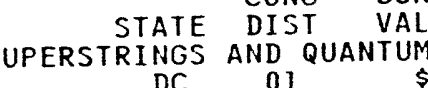
DC AND QUANTUM- 01 \$300, 000 \$300, 000 SUPERFIEL DS- - $\$ 75,000$ CONFERENCE TABLES

$\$ 15,533$

$\$ 15,533 \quad 7110$

BPA FOR NOVELTY SEARCHES AIND INFRINGEMENT TX 03 $\$ 0$

$\$ 0 \quad R 418$

BPA FOR DOMESTIC PATENT APPLICATIONS

$$
T X \quad 03
$$

\$0 R418

\section{MICROANALYTICAL DETERMINATIONS}

$$
\text { CO } 06 \quad \$ 177,291
$$

$\$ 106,718 \quad 6640$

ADVANCED NICKEL-METAL HYDRIDE CELL DEVELOPMENT

$$
\begin{array}{lrl}
\text { NICKEL-METAL HYDRIDE } & \text { HA } \\
\text { CA } & \$ 198,184 & \$ 81,483
\end{array}
$$

UTILIZATION OF THE MICROFLORA INDIGENOUS TO AND PRESENT IN 0

$$
\text { MS } 03 \text { \$3,855,399 \$1,033,818 }
$$

AWARDS A NEW CYCLE 15 ENERGY COHSERVATION MEASURE GRANT.

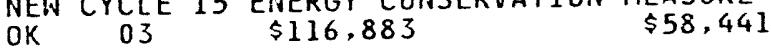

$\underset{\text { ICP-NEW AWARD. }}{\text { IC }} 04 \quad \$ 57,014 \quad \$ 28,507$

ICP - NEW IN AWARD ${ }_{04} \quad \$ 9,600 \quad \$ 4,800$

NPR-1 CRUDE OIL SALES CONTRACTEST REV $\$ 2265924.30$ $C A \quad 09$

GRANT PROGRAMS FOR SCHOOLS ANDHOSPITALS AND FOR BUILDINGS 0 KS

FOR SCHOD $\$ 177,348$

$\$ 88,674$

PB/COURT REPORTERS

CO 02

$\$ 0 \quad R 606$ 


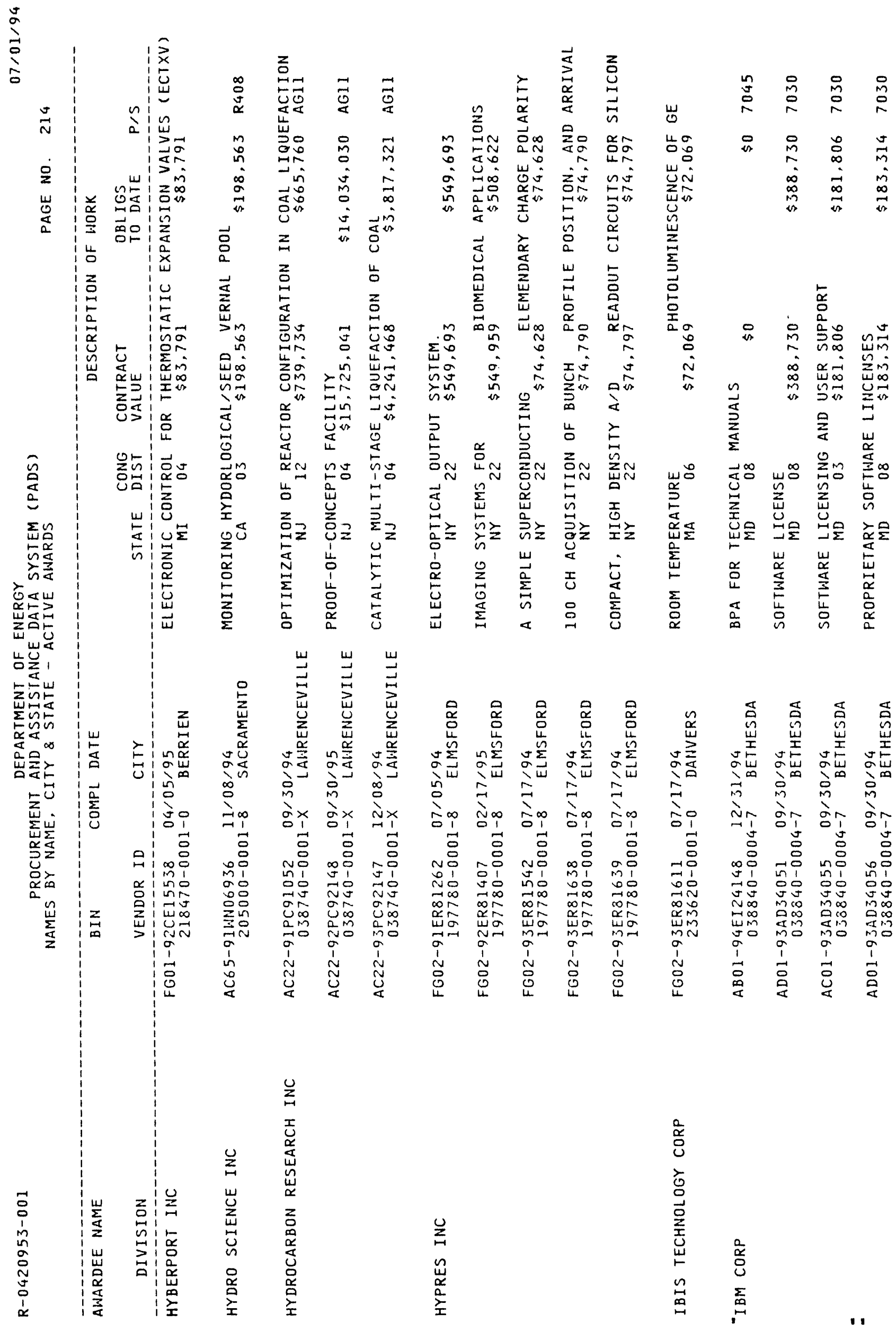




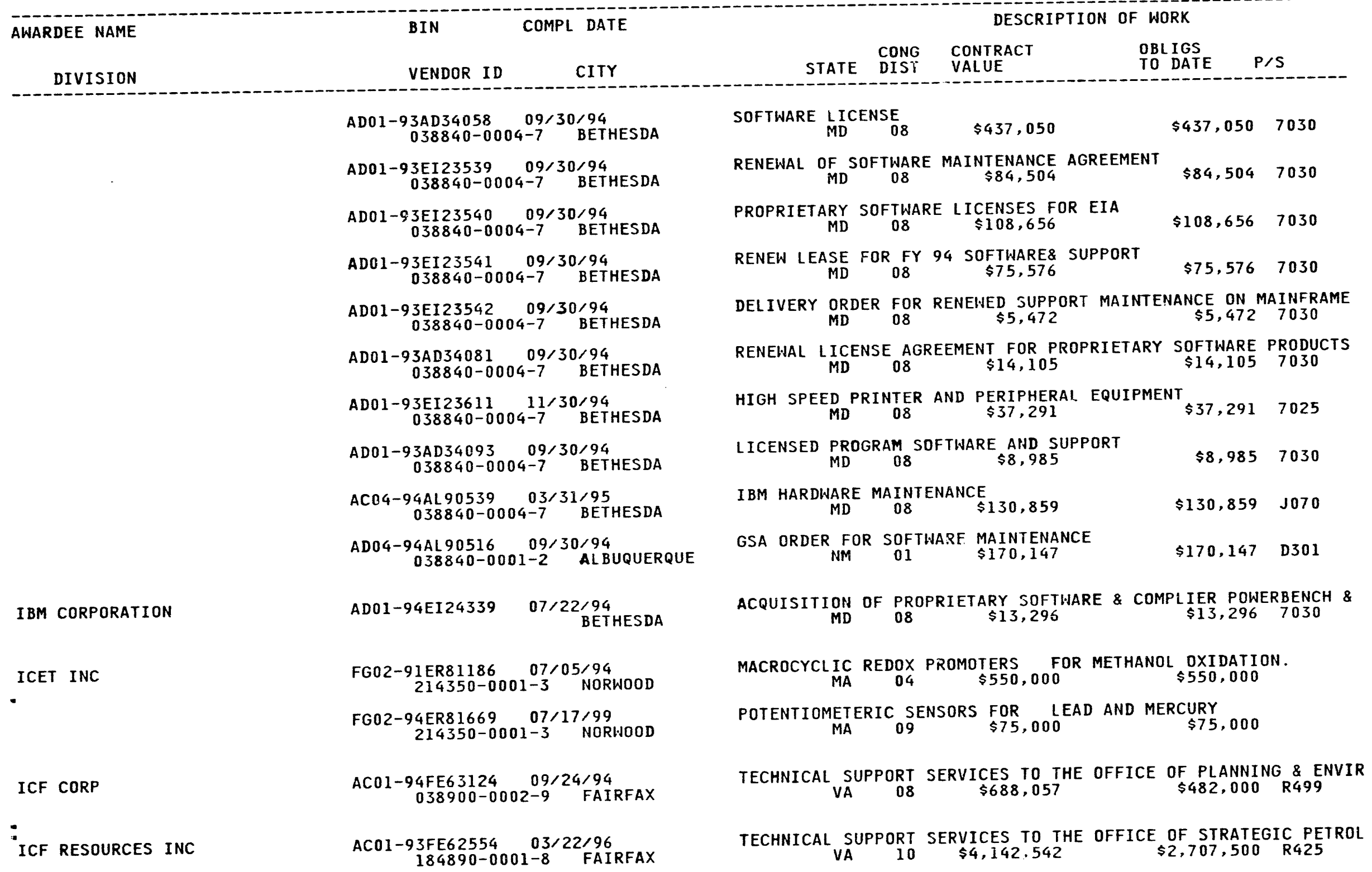




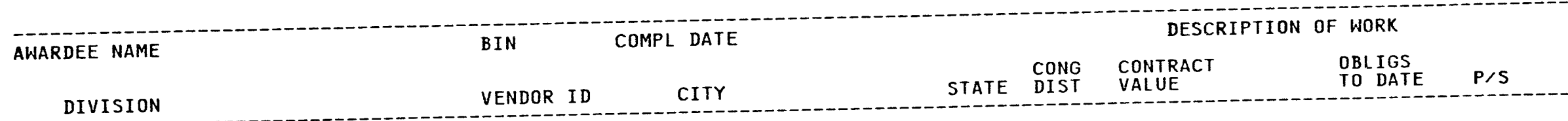

IDAHO ARMY NATIONAL GUARD 126 TH ENG CO

IDAHO FALLS CITY OF FIRE DEPARTMENT

POLICE DEPT

POLICE DEPT

IDAHO FALLS CONSOLIDATED HOSP

\section{IDAHO POWER CO} $=$ IDAHO STATE OF

\begin{tabular}{|c|c|}
\hline $\begin{aligned} A C 01-93 F E 62701 \\
184890-000\end{aligned}$ & $\begin{array}{l}09 / 25 / 94 \\
1-8 \text { FAIRFAX }\end{array}$ \\
\hline $\begin{array}{r}\text { AC22-92MT92004 } \\
184890-000\end{array}$ & $\begin{array}{l}08 / 31 / 94 \\
1-8 \text { FAIRFAX }\end{array}$ \\
\hline $\begin{array}{r}C 21-92 M C 28138 \\
184890-000\end{array}$ & $\begin{array}{l}07 / 22 / 95 \\
1-8 \text { FAIRFAX }\end{array}$ \\
\hline
\end{tabular}

GM07-90ID11052 09/30/96 O38940-0102-X IDAHO FALLS

GM07-93ID11108 11/20/97 $11 / 20 / 97$
$038970-0301-5 \quad$ IDAHO FALLS AC07-81ID12214 01/28/99 038970-0001-6 IDAHO FALLS

RL 07-76ID01421 09/30/99 038970-0001-6 IDAHO FALLS

GM07-81ID11009 09/30/99 038970-0001-6 IDAHO FALLS GM07-91ID1 1083 09/30/97 38970-0201-9 IDAHO FALLS GM07-91ID11087 09/30/97 038970-0201-9 IDAHO FALLS GM07-91ID11091 09/24/99 038970-0001-6 IDAHO FALLS

GM07-80ID11004 09/30/99 038980-0001-X IDAHO FALLS

$\begin{array}{cc}\text { AC07-86ID12588 } & 10 / 31 / 96 \\ 039010-0001-1 \quad B O I S E\end{array}$

FC04-85AL20535 09/30/94 039020-0301-4 BOISE

FG07-90ID13046 09/30/94 $\begin{array}{cccc}\text { TECHNICAL SUPPORT SERVICES TO THE OFFICE OF PLANNING \& ENVIR } \\ \text { VA } 08 & \$ 999,903 & \$ 974,239 & \text { R425 }\end{array}$ $\begin{array}{ccc}\text { STATE AND NATIONAL ENERGY ENVIROMENTAL RISK ANALYSIS SYSTEMS } \\ \text { VA } 10 & \$ 194,792 & \$ 194,792\end{array}$ VA $10 \quad \$ 194,792$

DEVELOPMENT OF A NATURAL GAS SYSTEMS ANALYSIS MODEL
$1001,827,910$

MEMORANDUM OF UNDERSTANDING WITH THE 126TH ENGR CO \& DOE'S I ID 02

$\$ 0$

RECIPROCAL FIRE FIGHTING AND EMERGENCY MEDICAL SERVICES ASSI ID 02 \$0 \$O

RECIPROCAL FIRE FIGHTING ASSISTANCE AGREEMENT $\$ 0 \quad \$ 0 \quad \$ 202$ WEATHER STATION ON TOWER AT AIRPORT $\$ 0 \times 293$ MEMORANDUM OF UNDERSTANDING FOR RECIPROCAL FIRE FIGHTING ID 02 $\$ 0$

$\$ 0$

INEL FIRING RANGE USE AGREEMENT

$\$ 0$

MEMORANDUM BETHEEN THE U.S. DEPARTMENT OF ENERGY FIELD OFFIC MOU TO DESCRIBE THE METHOD OF $\underset{\text { ID }}{\text { ACQUISITION }}$ BY DOE-ID OF A TRA

OF ENERGY

MEMORANDUM OF UNDERSTANDING $\underset{\$ 0}{\text { BETWEEN DEPARTMENT } 0}$ CONTRACT FOR POWER DELIVERY TOTHE INEL ID $02 \quad \$ 30,000,000 \quad \$ 5,000,000 \quad 5112$

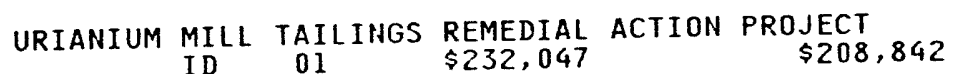
STATE ENVIRONMENTAL MONITORINGPROGRAM HEALTH AGREEMENT 


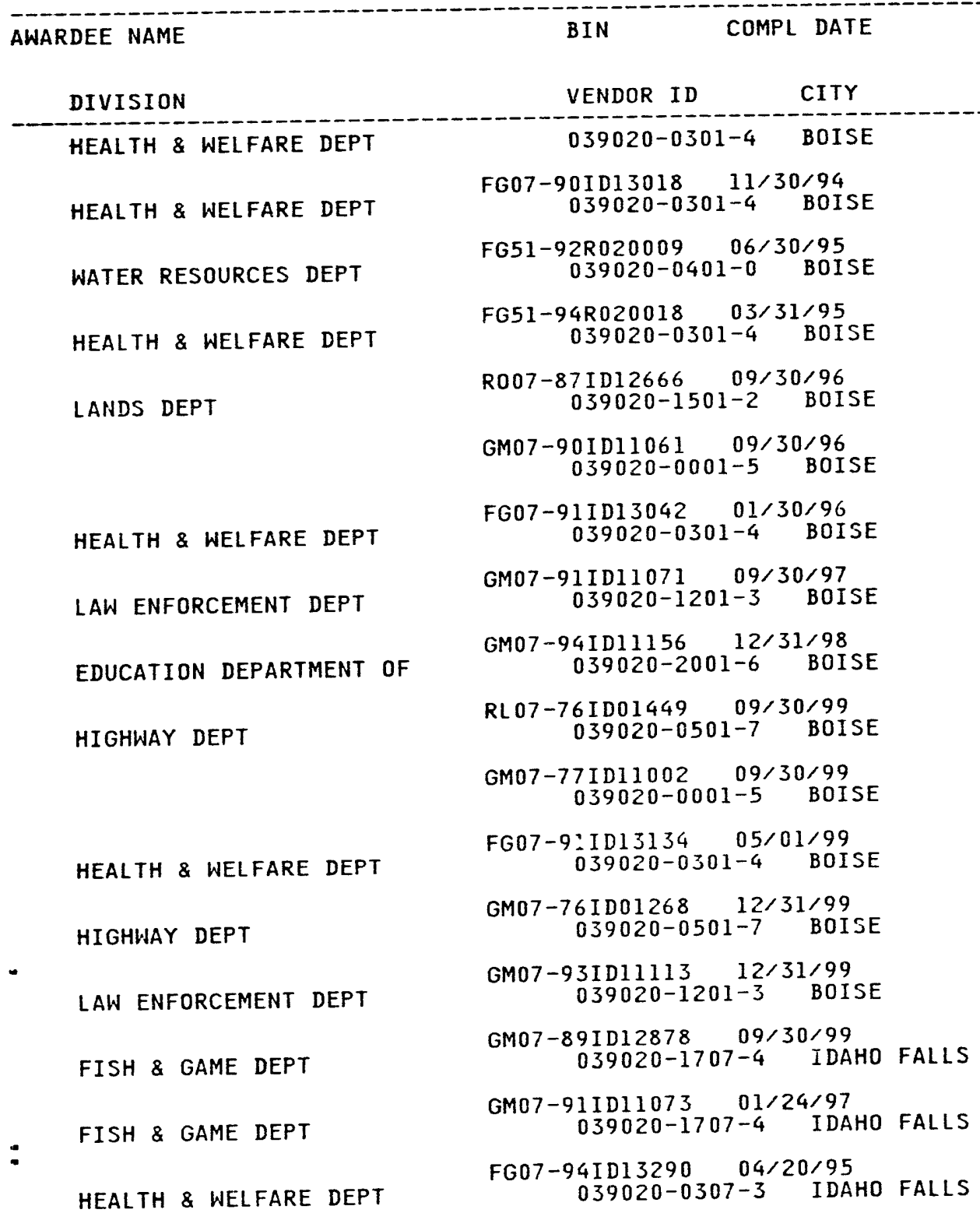

ENVIRONMENTAL OVERSIGHT AND MONITORING

ENVIRONMENTAL OVERSIGHT,

DESCRIPTION OF WORK

THIS IS THE STATE OF IDAHO CONSOLIDATED SECP/EES PROGRAM AWA

TITLE IV, PART A, WEATHERIZATION ASSISTANCE FOR LOW INCOME $P$ $\begin{array}{llll}\text { PART A, WEATHERIZATION ASSISTANCE } & \text { FOR } \\ \text { ID } & 01 & \$ 1,995,193 & \$ 1,995,193\end{array}$

TEMPORARY PERMIT FROM STATE OFIDAHO GRANTING IDAHO OPERATIO

MOU BETWEEN STATE OF IDAHO \& THE (DOE-ID) (INEL) IN PLANNING CONDUCT RESEARCH AND DEVELOPMENT ACTIVITIES AT THE INEL WHIC $\mathrm{ID} 02 \quad \$ 3,081,354 \quad \$ 2,474,370$

FIRING RANGE USE AGREEMENT

$\$ \mathbf{0}$

MOU TO STATE THE BASIC UNDERSTANDING OF THE PARTIES REGARDIN

MAINTENANCE OF ROADS ON NRTS
$\$ 0$
\$0 $\times 293$

MEMORANDUM OF UNDERSTANDING $\underset{\$ 0}{\text { FOR THE ENVIRONMENTAL }}$ $\begin{array}{cccc}\text { IMPLEMENTATION OF THE INTERAGENCY } & \text { AGREEMENT BETWEEN ENVIRONM } \\ \text { ID } & 02 & \$ 11,195,835 & \$ 4,150,771\end{array}$

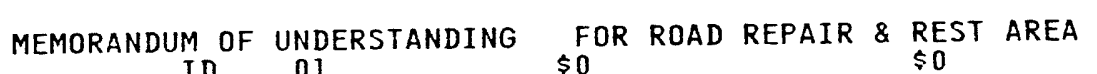

SPECIAL DEPUTIZATION OF FEDERAL OFFICERS \& CONTRACTOR PROTEC AGREEMENT $\underset{\text { IDETWEEN }}{02}$ IDAHO DEPARTMENT OF FISH \& GAME \& THE INEL

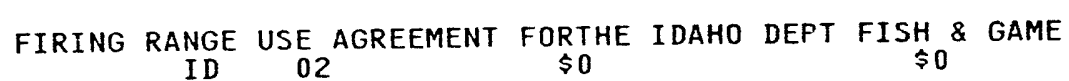
TECHNOLOGY DEVELOPMENT FOR ENVIRONMENTAL RESTORATION AND WAS HEALTH \& WELFARE DEP 


\begin{tabular}{|c|c|}
\hline AWARDEE NAME & COMPL DATE \\
\hline DIVISION & VENDOR ID \\
\hline HIGHWAY DEPT & $\begin{array}{c}\text { RL } 07-84 I D 1253409 / 30 / 99 \\
039020-0509-2 \quad \text { RIGBY }\end{array}$ \\
\hline \multirow[t]{3}{*}{ IDAHO STATE UNIVERSITY } & $\begin{array}{c}\text { ACO7-94ID13289 } 09 / 30 / 94 \\
039030-0002-7 \quad \text { IDAHO FALLS }\end{array}$ \\
\hline & $\begin{array}{c}\text { FG51-93R020298 } 10 / 31 / 94 \\
039030-0001-9 \text { POCATELLO }\end{array}$ \\
\hline & $\begin{array}{c}\text { FG07-90ID13044 09/30/95 } \\
039030-0001-9 \quad \text { POCATELLO }\end{array}$ \\
\hline
\end{tabular}

$\begin{array}{cc}\text { IDAHO TRANSPORTATION DEPT } & \text { GM07-94ID11141 } \\ \text { FEDERAL HIGHWAY ADMINISTRATI } & \text { BOISE }\end{array}$

IDAHO UNIVERSITY OF AGRICULTURAL ENGINEERING

AGRICULTURAL ENGINEERING

AGRICULTURAL ENGINEERING

IDEAL REPROGRAPHICS CO

IGNACIO UJT SCHOOL DISTRICT

IGR ENTERPRISES INC

:

IIT RESEARCH INSTITUTE

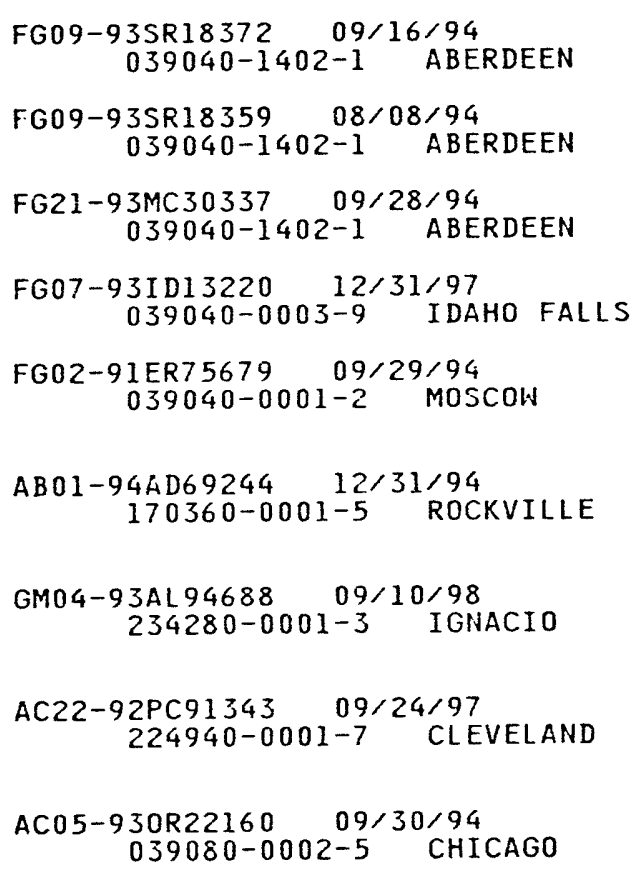

\section{WEATHER STATION SITE}

ID 02

DESCRIPTION OF WORK

TQM SERVICES, DECISION CENTER SUPPORT AND OTHER SERVICES ID $02 \quad \$ 27,422$

CYCLE 15 ECM AWARD.

INDIAN AMERICAN SCHOLARSHIP PROGRAM

$$
\text { ID } 02 \quad \$ 246,000
$$

$\$ 2,321$

OBLIGS

TO DATE P/S

IDAHO TRANSPORTATION CONSORTIUM ORGANIZED TO TAKE ADVANTAGE

$$
\text { ID } 02
$$

USED ENERGY RELATED LABORATORYEQUIPMENT

ID 02
USED ENERGY RELATED LABORATORYEQUIPMENT

ID 02

USED ENERGY RELATED EQUIPMENT
ID
02

$$
\text { ID } 02
$$

MAGNETICALLY CONTROLLED DEPOSITION OF METALS UTILIZING GAS P ID $02 \quad \$ 500,000$ $\$ 500,000$

STATE OF IDAHO PLANNING GRANT ENERGY PRODUCTION \& ENVIROHMEN $\begin{array}{lll}\text { ID } 01 & \$ 736,138 & \$ 620,000\end{array}$

BPA FOR DUPLICATION OF VIDEO CASSETTES

$$
M D \quad 08
$$

SUPPORT FOR SCIENCE, MATHEMATICS, AND ENGINEERING EDUCATION CO 03

IGR COMBINED NOX/SOX CONTROL TECHNOLOGY

$$
\mathrm{OH} \text { i1 } \$ 1,919,917
$$

$\$ 922,000 \quad A G 21$

IN-SITU SOIL HEATING USING ELECTROMAGNETIC SYSTEM

$\begin{array}{ccccc}\text { SOIL HEATING USING ELECTROMAGNETIC } & \text { SYSTEM } & \\ \text { IL } & 01 & \$ 863,756 & \$ 850,000 & A G 93\end{array}$




\section{AWARDEE NAME}

BIN

COMPL DATE

\section{DESCRIPTION OF WORK}

DIVISION

VENDOR ID

CITY

CONG CONTRACT
STATE DIST VALUE

OBLIGS DO P/S

ILLINOIS INSTITUTE OF TECH FG02-94ER40840 $03 / 31 / 97$ CHCAGO $039130-0001-1$ CHICAG

METALLURGICAL \& MATERIAL ENG $\begin{array}{cc}\text { FG06-94RL12682 } & 02 / 06 / 95 \\ 039130-1301-6 & \text { CHICAGO }\end{array}$ CHEMICAL ENGINEERING DEPT FG22-94PC94208 05/31/97 4 PC $9420805 / 31 / 97$
$039130-0901-9$ CHICAGO $A C 22-92 B C 1488309 / 20 / 95$ $039130-0901-9$ CHICAGO CHEMICAL ENGINEERING DEPT FG22-92PC9254909/30/94 $039130-1101-3$ CHICAGO

BIOLOGY DEPT

ENVIRONMENTAL ENGINEERING

$$
\begin{gathered}
\text { FG22-93PC93207 08/31/95 } \\
039130-1201-X \text { CHICAGO }
\end{gathered}
$$

FG45-93R551534 08/31/95 $039130-0001-1$ CHICAGO

METALLURGICAL \& MATERIAL ENG FG06-94RL12850 05/15/95 $039130-1301-6$ CHICAGO METALLURGICAL \& MATERIAL ENG $\begin{array}{cc}\text { FG09-94SR18458 } 06 / 21 / 95 \\ 039130-1301-6\end{array}$ CHICAGO

ILLINOIS POWER CO

$$
\begin{aligned}
& \text { CRO1-84RW00008 03/15/30 } \\
& 096420-0001-6 \text { DECATUR }
\end{aligned}
$$$$
\text { FG45-90R520285 12/31/94 }
$$$$
\text { FG45-88R591422 04/30/95 }
$$$$
\text { 039140-1307-9 PONTIAC }
$$

- correctional center

$$
\text { FC22-92PC92521 08/31/95 }
$$

FC22-92PC92521 $08 / 31 / 95$
ENERGY \& NATURAL RESOURCES DPT 039140-0101-1 SPRINGFIELD

$$
\text { FG45-93R53028309/30/94 }
$$

ENERGY \& NATURAL RESOURCES DPT 039140-0101-1 SPRINGFIELD

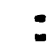

$\underset{\text { PARTICIPATION IN HIGH ENERGY PHYSICS }}{\$ 195,000} \stackrel{\$ 65,000}{01}$

USED ENERGY RELATED LABORATORYEQUIPMENT GRANT

$$
\text { IL } 01
$$

SO

HYDRODYNAMIC MODELS FOR SLURRYBUBBLE COLUMN REACTORS

SURFACTANT - ENHANCED ALKALINEFLOODING FOR LIGHT OIL RECOVE IL $01 \quad \begin{array}{lll}\$ 565,030 & \$ 450,000 & \text { AG62 }\end{array}$

BIOCATALYTIC REMOVAL OF ORGANIC SULFUR FROM COAL

IL OI $\$ 145,581 \quad \$ 139,857$ DEVELOPMENT AND EVALUATION OF A MN OXIDE ABSORBENT
IL 01 \$1 ICP-NEW AWARD.

$$
\text { IL } 01
$$

$\$ 139,910$

$\$ 67,044$

$\begin{array}{ccc}\text { USED ENERGY RELATED LABORATORYEQUIPMENT GRANT } \\ \text { IL } & 01 & \$ 0\end{array}$ USED ENERGY RELATED LABORATORYEQUIPMENT
IL
02 DISPOSAL OF SPENT NUCLEAR FUELAND FOR HIGH LEVEL $\underset{\$ 0}{\text { RADIOACTIVE }}$

ICP - NEW AWARD

$\$ 14,694$

$\$ 7,347$

ICP - NEW AWARD is

$\$ 46,136$

$\$ 23,067$

SUPPORT OF HIGH SULFUR COAL DESULFURIZATION RESEARCH IL $20 \quad \$ 5,578,665 \quad \$ 2,446,229$ HEAVY DUTY STATE/MUNICIPAL VEHICLE ALTERNATIVE FUEL DEMONSTR IL $20 \quad \$ 359,500 \quad \$ 80,500$ PROVIDES GRANTS TO STATETO DEVELOPE AND IMPLEMENT COMPREHENS IL $20 \quad \$ 34,265,324 \quad \$ 16,419,612$ 
AWARDEE NAME

COMPL DATE

DESCRIPIION OF HORK

DIVISION

BIN

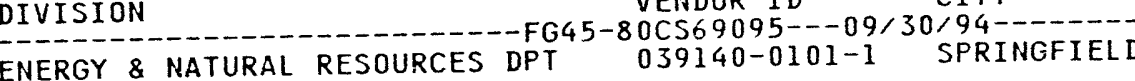

FG45-89CH1040903/31/95

DEPT OF COMMERCE \& CMTY AFFS $039140-1801-1$ SPRINGFIELD

$039140-1801-1 / 31 / 95 / N$

COMMERCE \& CMTY AFFS DEPT OF r $039140-1801-1 \quad$ SPRINGFIELD

ILLINOIS UNIVERSITY OF

ENGINEERING DEPARTMENT

NUCLEAR PHYSICS DEPT OF

NUCLEAR ENGINEERING DEPT

CHEMISTRY DEPARTMENT

CHEMISTRY DEPARTMENT

PHYSIOLOGY \& BIOPHYSICS DEPT

= NuCleAR ENGINEERING DEPT
FG02-88ER53269 07/14/94 $039170-3401-8$ CHAMPAIGN

FG02-90ER54092 09/30/94 $039170-3701-7$ CHAMPAIGN

FG02-90ER61081 09/14/94 039170-0001-6 CHAMPAIGN

FG02-91ER20056 08/31/94 039170-0001-6 CHAMPAIGN

FG02-91ER20046 08/31/94 $039170-0001-6$ CHAMPAIGN

FG02-90ER12972 09/29/94 $039170-2001-7 \quad$ CHAMPAIGN

FG02-92ER75781 09/30/94 039170-0001-6 CHAMPAIGN

FG02-93ER75873 09/30/94 039170-0001-6 CHAMPAIGN

FG02-88ER13949 11/30/94 039170-0501-8 CHAMPAIGN

FG02-92ER75729 10/31/94 039170-0001-6 CHAMPAIGN

FG02-87ER13716 08/31/95 039170-0501-8 CHAMPAIGN

FG02-88ER13948 02/28/95 $039170-2401-2$ CHAMPAIGN

FG02-89ER5215901/14/95 $039170-2001-7$ CHAMPAIGN

FG02-90ER61017 07/14/95
OBL IGS

STATE CONG CONTRACT

STATE DIST VALUE
F ILLINOIS ENERGY EXIENSI

IL 20 \$8,639,275

TO DATE P/S

$\$ 4,210,917$

FY8 9 WEATHERIZATION GRANT FOR THE STATE OF ILLINOIS

IL $20 \$ 94,337,177$

$\$ 68,121,667$

DOE FUNDS OF $\begin{gathered}\$ 50,000 \\ 20\end{gathered} \begin{array}{r}\$ 50,000 \\ \$ 50,000\end{array}$

METHODS FOR TESTING TRANSPORT MODELS

$\begin{array}{lll}\text { IL } 19 & \$ 363,800\end{array}$

$\$ 363,800$

HEL IUM TRANSFORT STUDIES

$\$ 134,219$

$\$ 134,219$

TERRESTRIAL SOURCES AND SINKS IN GLOBAL CARBON BALANCE IL $19 \quad \$ 399,796$ $\$ 399,796$

REGULATION OF TOXIC HEAVY METAL RESISTANCE SYSTEMS IN THE SO IL $19 \quad \$ 303,000 \quad \$ 303,000$

GENETICS OF SOLVENT PRODUCING CLOSTRIDIA.

$$
\text { IL } 19 \quad \$ 309,088
$$

$\$ 309,088$

UNIVERSITY REACTOR INSTRUMENTATION PROGRAM

$$
\text { IL } 19 \quad \$ 475,500
$$

$\$ 195,500$

DEVELOPMENT OF ADVANCED DIRECTPERCEPTION DISPLAYS FOR

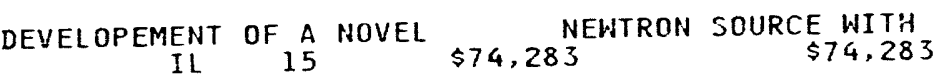

THIN FILMS UNDER CHEMICAL $\$$ STRESS

NUCLEAR PONER ENGINEERING EDUCATION PROGRAM

IL $19 \quad \$ 100,000 \quad \$ 100,000$

BIOCHEMICAL AND BIOPHYSICAL STUDIES OF THE E. COLI AEROBIC IL $19 \quad \$ 869,916$

$\$ 750,916$

MECHANISMS OF PROTON PUMPING IN BACTERIORHODOSIN IL $19 \quad \$ 506,980$ $\$ 506,980$

PARTICLE REFLECTION AND TRITIUM DECAY IN THE WALLS OF IL 19 \$197,509

DETERMINING THE CHEMICAL COMPOSITION OF CLOUD 
DESCRIPTION OF WORK

DIVISION

CHEMICAL ENGINEERING DEPT

PLANT BIOLOGY DEPT

DECISION \& CONTROL LAB

ENERGY RESOURCES CENTER

CHEMICAL ENGINEERING DEPT
BIN

CITY

VENDOR ID

CITY

039170-0001-6 CHAMPAIGN

FG02-91ER25099 01/31/95 039170-0001-6 CHAMPAIGN

FG02-91ER20057 08/31/95 039170-0001-6 CHAMPAIGN

FG02-93ER61534 12/31/95 039170-0001-6 CHAMPAIGN

FG02-91ER45439 12/31/95 039170-0001-6 CHAMPAIGN

FG02-91ER40677 10/31/95 039170-0001-6 CHAMPAIGN

FG02-87ER1376903/14/96 039170-0401-1 CHAMPAIGN

FG02-90ER20008 05/31/96 $039170-2501-9$ CHAMPAIGN

FG02-90ER14146 08/14/96 039170-0001-6 CHAMPAIGN

FG02-93ER14393 09/14/96 039170-0001-6 CHAMPAIGN

FG02-88ER13939 06/14/97 $039170-3501-4$ CHAMPAIGN

FG02-92ER20095 09/14/97 039170-0001-6 CHAMPAIGN

FG02-85ER13311 07/31/94 039170-1402-5 CHICAGO

FG02-85ER13384 07/14/94 039170-0402-X CHICAGO

FG02-92ER25133 08/31/94 039170-0002-4 CHICAGO

FG02-93ER61603 05/31/96 039170-0002-4 CHICAGO

FG02-93ER25168 09/14/96 $039170-0002-4$ CHICAGO

STA

STATE DIS

\section{CONTRACT}

$\$ 2,144,472$

OBLIGS

TO DATE P/S

NUMERICAL METHODS FOR MOLECULAR DYNAMICS.

$\$ 2,144,472$ IL 19 MLCHANISM \$236,999 $\$ 236,999$ MUDELING $19 \quad \$ 286,596 \quad \$ 181,396$ MATERIALS SCIENCE RESEARCH

$$
\begin{array}{ll}
\text { IL } 19 & \$ 31,670,068
\end{array} \$ 26,307,190
$$
IL $19 \quad \$ 8,777,900 \quad \$ 6,199,900$

EVALUATION OF MIXING RULES FORVISCOSITY AND THERMAL IL $19=\$ 568,034 \quad \$ 494,934$

REGULATION OF CELL DIVISION INHIGHER PLANTS

IL 19 \$516,000 $\$ 422,000$

STRESS INDUCED PHASE TRANSFORMATIONS

$$
\text { IL } \quad{ }_{19} \$ 206,880 \quad \$ 66,169
$$

MODEL BUILDING AND CONTROL OF LARGE SCALE SYSTEMS
IL

ANTHROPOGENIC IMPACTS ON PHOTOSYNTHETIC ACTIVITY: IL $19 \quad \$ 1,417,840 \quad \$ 495,760$

BOILING OF AQUEOUS POLYMER SOLUTIONS $\begin{aligned} \text { IL } 07 & \$ 800,737\end{aligned}$

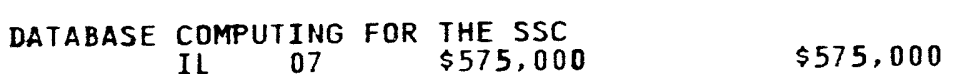

MECHANISM \& STRUCTURE OF THE PLANT PLASMA MEMBRANE CA++-ATP

MODELING OF COUPIED PROCESSES IN SUBSURFACE TRANSPORT OF

DEVELOPMENT OF A MUON SUBSYSTEM FOR SOLENOIDAL SSC DETECTOR $\begin{array}{ccc}\text { ELECTRON TRANSFER } & \text { ACTIVATION OF COORDINATED } \\ \text { IL II } 19 & \$ 603,570 & \$ 371,100\end{array}$ SHOCK TUBE STUDIES OF HIGH TEMPERATURE HYDROCARBON PYROLYSIS

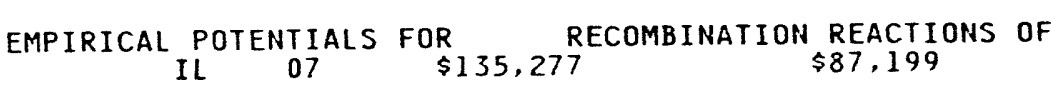
APPROXIMATION IN THE $\underset{07}{\substack{\text { PERFORMANCE EVALUATION DF QUEU } \\ \$ 27,551}}$ 


\begin{tabular}{|c|c|c|c|c|c|c|c|}
\hline AWARDEE NAME & BIN & L DATE & & & DESCF & F WORK & \\
\hline & VENDOR ID & CITY & STATE & $\begin{array}{l}\text { CONG } \\
\text { DIST }\end{array}$ & $\begin{array}{l}\text { CONTRACT } \\
\text { VALUE }\end{array}$ & $\begin{array}{l}\text { OBLIGS } \\
\text { TO DATE }\end{array}$ & $P / S$ \\
\hline
\end{tabular}

FG02-93ER61679 08/31/96 $039170-0002-4$ CHICAGO

CHICAGO CIRCLE

HEALTH SERVICES CENTER

HEALTH SERVICES CENTER

FG45-89R510452 08/31/94 039170-2102-1 CHICAGO

FG45-89R510464 08/31/94 $039170-2720-X \quad$ CHICAGO

FG45-91R530026 12/31/94
$039170-0002-4$ CHICAGO

FG45-91R530031 $12 / 31 / 94$
$039170-2720-X$ CHICAGO

FG45-92R540081 12/31/94 $039170-0002-4$ CHICAGO

FG02-84ER52111 04/30/95

ELECTRICAL ENGINEERING DEPT

MICROBIOLOGY DEPT

CHEMISTRY DEPARTMENT

CHEMICAL ENGINEERING DEPT

PHYSIOLOGY \& BIOPHYSICS DEPT

MICROBIOLOGY DEPT

PLANT BIOLOGY DEPT

NUCLEAR REACTOR LABORATORY

NUCLEAR ENGINEERING DEPT

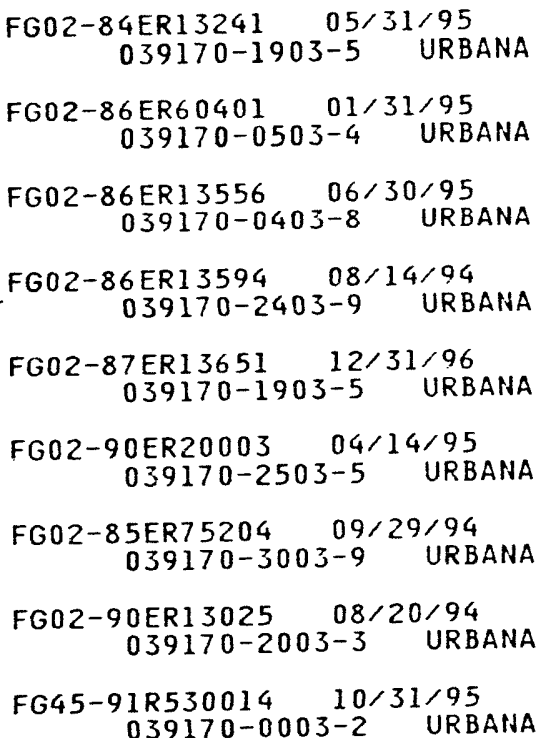

EVOLUTION OF A METABOLIC
IL 07
$\begin{gathered}\$ 473,820 \\ \text { GENE }\end{gathered}$

APPLICATIOH OF RAILGUN PRINCIPLE TO HIGH VELOCITY HYDROGEN P ILN OF RAILGUN PRINCIPLE TO HIGH VELOCITY
IL $19 \quad \$ 1,988,045$

GENETICS OF THE METHANOGENIC BACTERIUM, METHANOCOCCUS VOLTAE IL 19 \$758,303 $\$ 758,303$

FLUORINE-18 LABE:ED ANDROGENS AND PROGESTINS; IMAGING IL 19 \$1, 228,460 \$1,218,633

GAS-LIQUID FLOW IN PIPELINES
$\$ 982,507$

$\$ 982,507$

STUDY PHOTOSYNTHESIS IN INTACTPLANTS

$\$ 839,200$

HYDROGEN-INDEPENDENT MEIHANOGENIC SYSTEMS

IL $19 \quad \$ 667.770 \quad \$ 593,595$

GENETIC STUDIES ON CYTOPLASMICMALE STERILITY IN MAIZE

UNIVERSITY REACTOR SHARING

NUCLEAR ENGINEERING RESEARCH "DEVELOPMENT OF ADVANCED NODAL IL $19 \quad \$ 178,420 \quad \$ 178,420$

ICP-NEW AHARD. HE CONTINUED ADMINISTRATION OF IL $19 \quad \$ 55,254 \quad \$ 27,627$ 


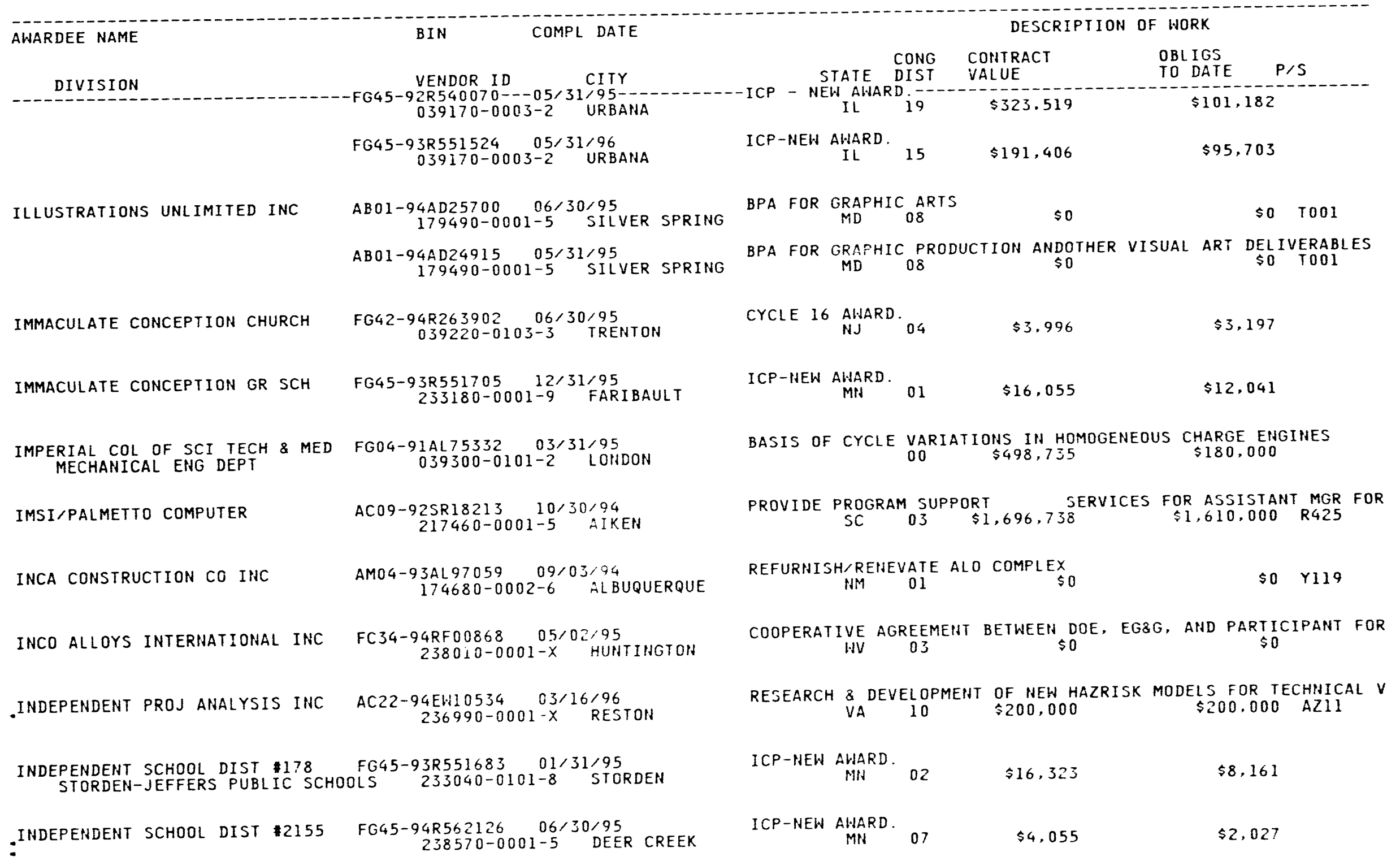


$R-0420953-001$

DEPARTMENT OF ENERGY

PROCUREMENT AND ASSISTANCE DATA SYSTEM (PADS) NAMES BY NAME, CITY \& STATE - ACTIVE AHARDS

PAGE NC. 224

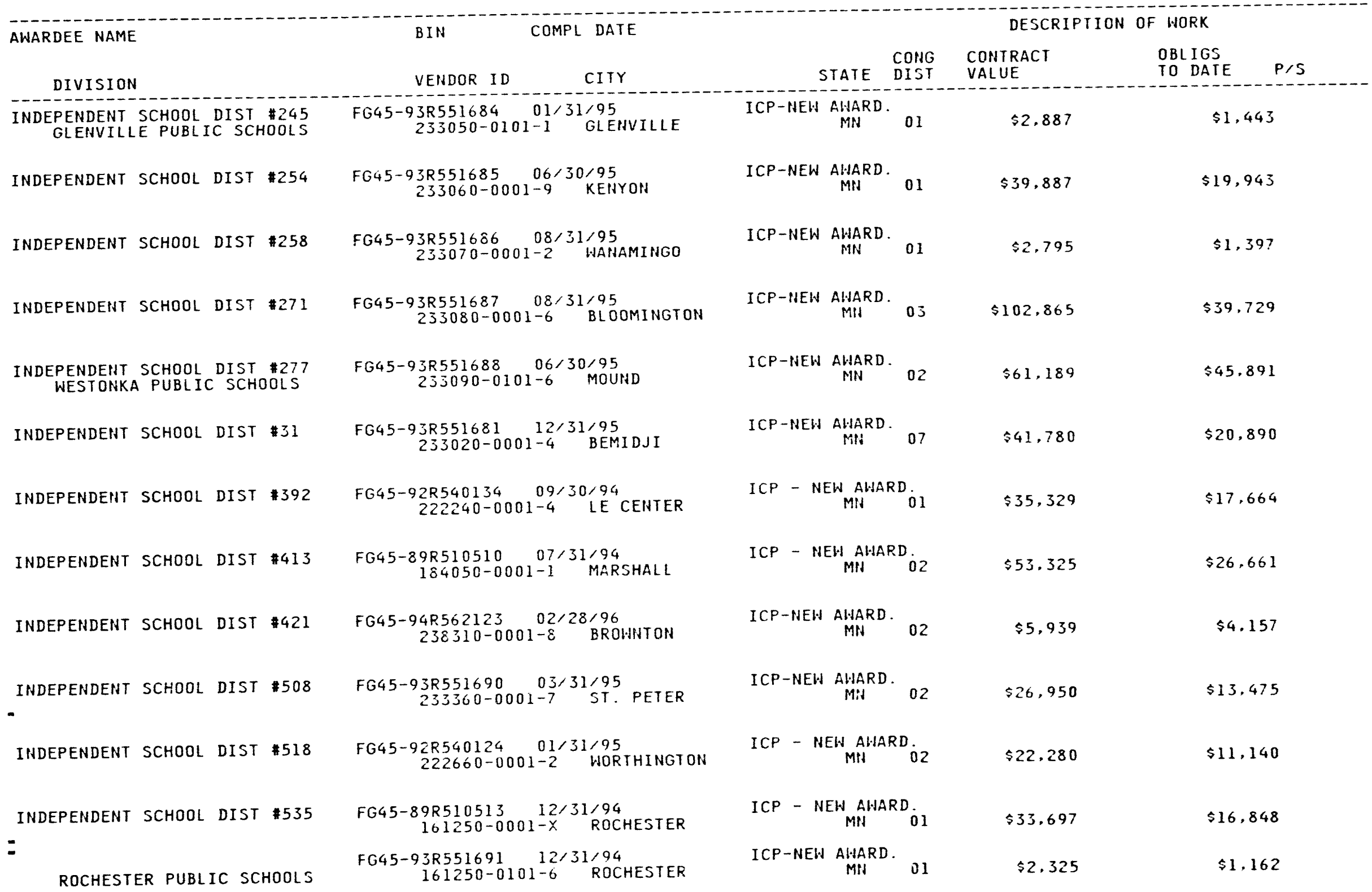




\section{AWARDEE NAME}

BIN

COMPL DATE

DESCRIPTION OF WORK

DIVISION

VENDOR ID

CITY STATE DIST

CONTRACT

OBL IGS TO DATE $P / S$

INDEPENDENT SCHOOL DIST $\$ 547$

INDEPENDENT SCHOOL DIST $\$ 550$

INDEPENDENT SCHOOL DIST $\$ 625$

SAINT PAUL PUBLIC SCHOOLS INDEPENDENT SCHOOL DIST $\$ 697$ INDEPENDENT SCHOOL DIST $\$ 699$ INDEPENDENT SCHOOL DIST $\$ 701$ HIBBING PUBLIC SCHOOLS

INDEPENDENT SCHOOL DIST $\$ 716$

INDEPENDENT SCHOOL DIST $\$ 769$

INDEPENDENT SCHOOL DIST $\$ 836$ INDEPENDENT SCHOOL DIST $\$ 861$ $-$

WINONA PUBLIC SCHOOLS

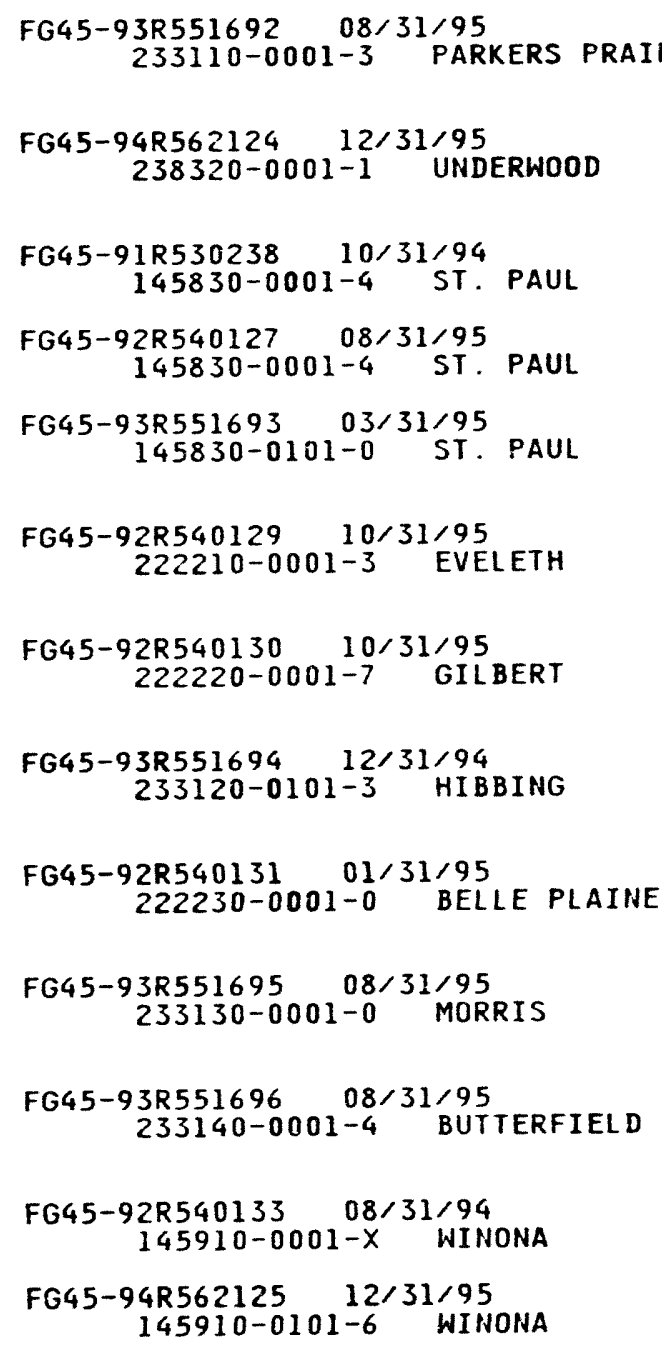

ICP-NEW AWARD MN 07 


\section{AWARDEE NAME}

BIN

COMPL DATE

DESCRIPTION OF WORK

VENDOR ID STATE

CONG

CONTRACT VALUE

INDEPENDENT SCHOOL DIST $\$ 880$

INDEPENDENT SCHOOL DIST 91

INDEPENDENT SCHOOL DIST \#911

INDEPENDENT SCHOOL DIST $\$ 912$

INDEPENDENT SCHOOL DISTRICT

INDIAN MOUNTAIN SCHOOL

INDIAN TOWNSHIP PASSAMAQUODDY

INDIANA MICHIGAN ELECTRIC

INDIANA STATE OF

\section{COMMERCE DEPARTMENT OF}

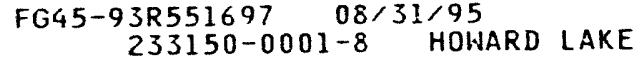

FG41-94R110901 06/30/98

PRINCETON

CR01-83NE44440 03/15/30 039580-0001-0 COLUMBUS

FC01-91EI22789 08/07/96 $039590-0001-4$ INDIANAPOLIS

FG45-93R530281 09/30/95 039590-0101-0 IHDIANAPOL IS

FG45-93R530278 03/31/95

\begin{tabular}{|c|c|c|c|c|}
\hline ICP-NEW & $\underset{\text { MN }}{\text { AWARD. }}$ & 02 & $\$ 41,374$ & $\$ 20,686$ \\
\hline ICP-NEW & $\begin{array}{l}\text { AWARD. } \\
\text { MN }\end{array}$ & 08 & $\$ 790$ & $\$ 513$ \\
\hline ICP-NEW & $\begin{array}{l}\text { AWARD } \\
\text { MN }\end{array}$ & 08 & $\$ 82,162$ & $\$ 41,081$ \\
\hline ICP-NEW & $\begin{array}{l}\text { AWARD. } \\
\text { MNN }\end{array}$ & 08 & $\$ 16,095$ & $\$ 10,461$ \\
\hline$I C P-N E$ & $\begin{array}{c}\text { EW AWARD } \\
\text { MN }\end{array}$ & 07 & $\$ 11,837$ & $\$ 5,918$ \\
\hline ICP-NEW & $\begin{array}{l}\text { AHARD } \\
\text { MN }\end{array}$ & 08 & $\$ 187,724$ & $\$ 93,861$ \\
\hline ICP-NEW & $\begin{array}{l}\text { AWARD. } \\
\text { MN }\end{array}$ & 01 & $\$ 44,834$ & $\$ 22,416$ \\
\hline
\end{tabular}

TITLE III OF THE NATIONAL ENERGY CONSERVATION POLICY ACT CT $06 \quad \$ 11,500 \quad \$ 5,750$

FY 94 WEATHERIZATION PROJECT.

$M E \quad 02 \quad \$ 22,665$

$\$ 22,665$

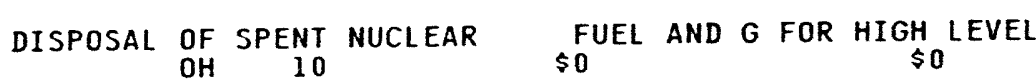

1991-1996 STATE HEATING OIL AND PROPANE PROGRAM FOR THE COLI IN 10 \$22,920 $\$ 11,460$

ELECTROGRAPHIC SCREENING PROCESS FOR PICTURE TUBE MANUFACTUR IN 10 \$1,000,000 $\$ 348,000$

FY 93 FUHDING TO SUPPORT THE WEATHERIZATION ASSISTANCE PROGR IN 02 \$50,000 $\$ 50,000$ 


AWARDEE NAME
COMISION

INDIANA STATE UNIVERSITY GRADUATE STUDIES SCHOOL OF

INDIANA UNIV \& PURDUE UNIV

INDIANA UNIV OF PENNSYLVANIA CHEMISTRY DEPT

INDIANA UNIVERSITY CHEMISTRY DEPT

PHYSICS DEPT

CHEMISTRY DEPT

CHEMISTRY DEPT

CHEMISTRY DEPT

PHYSICS DEPT

- CHEMISTRY DEPT

CHEMISTRY DEPT

CHEMISTRY DEPT

: PHYSICS DEPT
BIN

COMPL DATE VENDOR ID CITY 9CHIO410--03/31/95--
$039590-0001-4$
INDIANAPOLIS FG45-89R510558 06/30/95 039590-0101-0 INDIANAPOL IS

FG02-91CH10469 06/30/95 039600-0102-9 TERRE HAUTE

FG45-94R562014 06/30/95 238290-0001-4 INDIANAPOLIS

FG06-93RL12661 08/18/94 039610-0401-3 INDIANA

FG02-86ER13570 05/31/95 039620-0101-8 BLOOMINGTON FG02-84ER45147 10/31/95 039620-0601-X BLOOMINGTON FG02-86ER13616 08/31/94 039620-0101-8 BLOOMINGTON FG02-87ER60530 11/30/94 039620-0101-8 BLOOMINGTON FG02-87ER13702 01/31/97 039620-0101-8 BLOOMINGTON FG02-87ER40365 03/31/96 039620-0601-X BLOOMINGTON FGO2-88ER40404 12/31/96 039620-0101-8 BLOOMINGTON FG02-88ERI3906 12/31/94 039620-0101-8 BLOOMINGTON FG02-91ER14175 11/30/94 039620-0101-8 BLOOMINGTON FG02-91ER40661 10/31/94 039620-0601-X BLCOMINGTON FG02-92ER40714 03/14/95
DESCRIPTION OF WORK

$\begin{array}{ll}\text { CONG CONTRACT } & \text { OBLIGS } \\ \text { STATE DIST VALUE } & \text { TO DATE }\end{array}$

TO DATE PIS FUIDING FOR THE STATE-OF INDIANA'S LOW-INCOME WEATHE IN $10 \$ 32,356,863 \quad \$ 32,344,863$

FY 1989 FUNDING FOR ADMINISTRATION OF THE INSTITUTIONAL CONS IN 10 \$1, 132, 450 $\$ 290,961$

DESIGN \& CONSTRUCTION OF AN ADVANCED TECHNOLOGY CENTER IN $07 \$ 17,000,000 \quad \$ 4,814,414$

ICP - NEW AWARD.

$\$ 22,000$

$\$ 11,000$

USED ENERGY RELATED EQUIPMENT GRANT

METAL ALKOXIDES-MODELS FOR METALOXIDES. ALKOXIDE LIGANDS IN IL $09 \quad \$ 996,228$ \$996,228

HIGH-RESOLUTION ELECTRON ENERGY LOSS STUDIES OF SURFACE VIBR IN $09 \quad \$ 965,066 \quad \$ 922,666$

FREE RADICAL AND REARRANGEMENTREACTIONS IN COAL
IN
09 $\stackrel{\$ 724,535}{\$ 724,535}$ TOXIC ORGANIC COMPOUNDS FROM ENERGY PRODUCTION

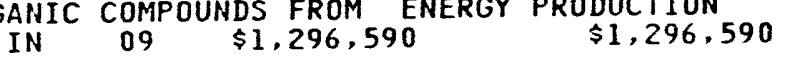
A MODEL APPROACH TO VANADIUM IN CRUDE OILS AND THEIR THEORETICAL STUDIES OF INTERMEDIATE - ENERGY NUCLEAR PHYSICS IN 09 \$2,192,000 $\$ 1,871,000$ STUDIES OF ENERGY DISSIPATION PHENOMENA IN LOW TO THE MOLECULAR PRECURSOR $\underset{\text { IN }}{09} \underset{579,700}{\text { STRATEGY FOR THE PRODUCTION }}$

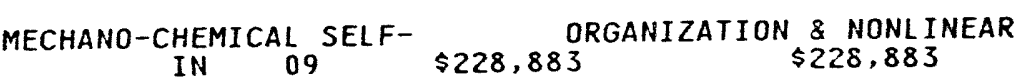
CENTRAL \& FORWARD TRACKING HITH WIRE CHAMBERS.

IN $09 \quad \$ 6,420,200 \quad \$ 6,420,200$ CHARATERIZATON OF THE MULTIFRAGMENT DECAY CHANNEL IN HIGHLY 


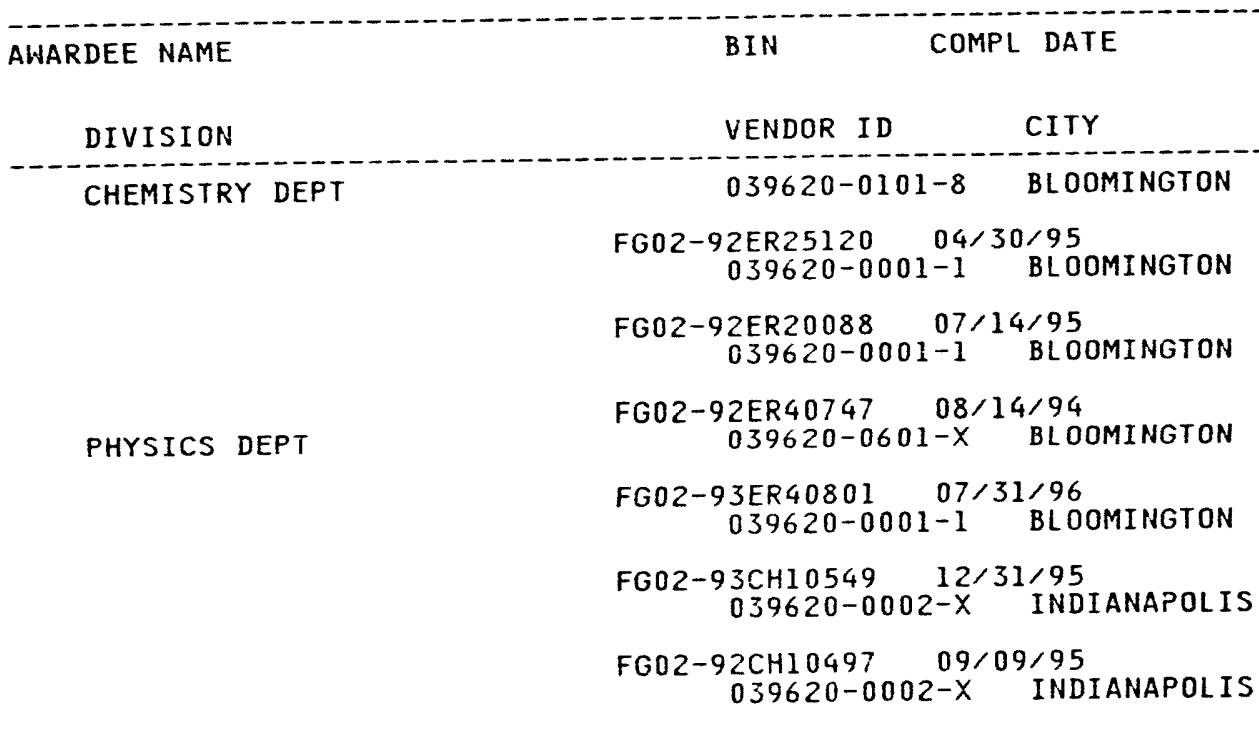

INDIANA VOCATIONAL TECH COLL

\section{INDUSTRA INC}

INDUSTRIAL FILTER \& PUMP MFG

INDUSTRIAL FOAM PRODUCTS INC

INDUSTRIAL HOSPITAL

INDUSTRIAL MACHINERY EQUIPMENT

$=$

INDUSTRIAL PHOTOGRAPHIC PROD
FG45-93R551585 $08 / 31 / 95$
$208770-0002-6$ FORT WAYNE

FC36-93CH10563 09/28/94
$235100-0001-3 \quad$ SEATTLE

FG02-92ER81349 $02 / 17 / 95$
$221200-0001-8$ CICERO

FG02-93ER81519 07/17/94
$221200-0001-8$ CICERO

FGO1-94CE15597 11/25/95
$238300-0001-4$ PERRYILLE

FG44-93R435411 09/30/95 233840-0001-3 SAN JUAN

$A B 01-93 A D 66356 \quad 09 / 30 / 94$ $170830-0001-8$ UPPER MARLBORO

\section{DESCRIPTION OF WORK}

STATE DIST VONTRACT

IN 08 \$258,000

OBLIGS

TO DATE P/S $\$ 258,000$

APPROXIMATIN OF ATTRACTORS ANDAPPLICATIONS

IN $08 \quad \$ 297,000$

$\$ 297,000$

PHYLOGENETIC ANALYSIS OF HYPER THERMOPHILIC NATURAL

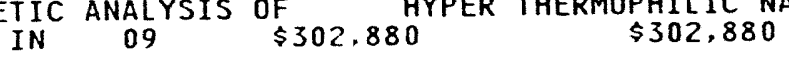

PARTIAL SNAKE EXPERIMENTS AT THE AGS
IN 08 \$190,000 $\$ 190,000$

NONL INEAR BEAM DYNAMICS IN HIGH ENERGY ACCELERATORS IN 09 \$315,000

$\$ 100,000$

DESIGN AND CONSTURCTION OF THECANCER RESEARCH TREATMENT IN $10 \$ 12,800,000 \quad \$ 10,000,000$

DESIGN AND CONSTRUCTION OF A CANCER RESEARCH

$$
\text { IN } 10 \$ 20,000,000 \quad \$ 10,000,000
$$

ICP-NEW AWARD

IN $04 \quad \$ 118,856$

$\$ 59,428$

BLACK LIQUOR RECORCVERY
WA
07
$\$ 63,214$

ALTERNATIVES (BASIC AWARD)

DURABLE, LOW COST CERAMIC MATERIALS FOR USE IN HOT GAS

A METHODE FOR THE REDUCTION OFDUST CAKE ADHESION IN CERAMIC IL 05 REDUCTION OFDUST CAKE ADHESION $\$ 75,000 \quad \$ 75,000$

COMPLETION OF ENGINEERING DESIGN FOR GIBBAL WALL TM SYSTEM R MO $08 \quad \$ 99,950 \quad \$ 99,950$

SEE ATTACHED PAGE NO. 2 OF THIS NOTICE OF FINANCIAL ASSISTAN

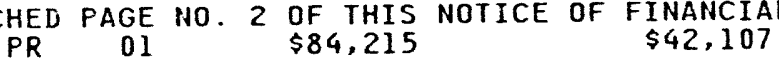

BPA FOR MAINTENANCE OF EMERGENCY DIESEL GENERATOR

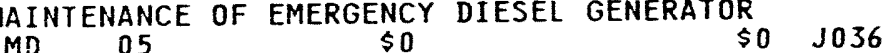

$A B 01-94 A D 69247 \quad 04 / 04 / 95$

BPA FOR VIDEO CASSETTE TAPE 


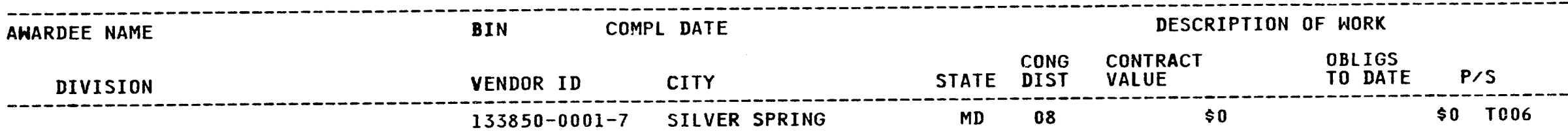

INFORMATION CONSUMER PROF ENQ AT65-94WN14750 03/31/95 238710-0001-9 SACRAMENTO

INFORMATION DIMENSIONS INC

INFORMATION HANDLING SERVICES

INFORMATION MGMT CONSULTANTS

INGLEWOOD GROUP INC

\section{INRAD INC}

INSITEC INC

INSTITUTE FOR ADVANCED STUDY PHYSICS

INSTITUTE OF GAS TECHNOLOGY

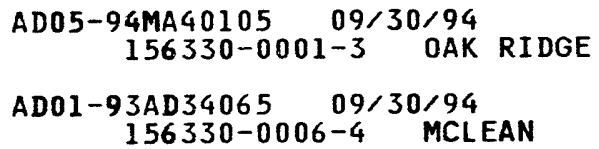

AD01-94AD63050 12/31/94 039860-0003-8 ENGLEWOOD

ADO1-94EH89402 07/31/94 039860-0003-8 ENGLEWOOD

AD01-93AD21146 09/30/94 039860-0003-8 ENGLEWOOD

ACO1-94ER25182002/15/95 236940-0001-1 MCLEAN

AC34-94RF00824 07/29/94 237330-0001-3 GOLDEN

FG05-90ER8 092708/01/94 199250-0001-7 NORTHVALE

FG03-91ER81124 09/30/94 133870-0002-2 SAN RAMON

FG02-90ER40542 11/30/96 039990-0301-4 PRINCETON

AC22-93PC92114 06/23/96 $040040-0001-7$ CHICAGO

FC21-92MC28178 09/30/95 040040-0001-7 CHICAGO

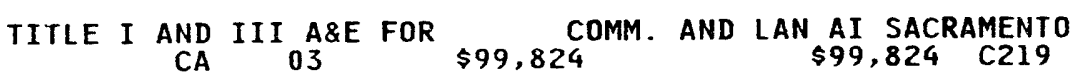

MAINTENANCE SUBSCRIPTION RENEWAL

$\$ 32,926 \quad 7021$

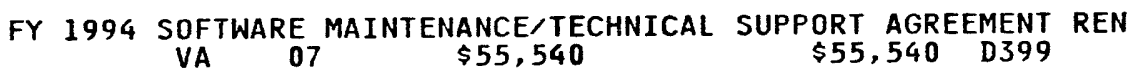

CD ROLM SUBSCRIPTIONS

$\$ 9,095$

$\$ 9,0959999$

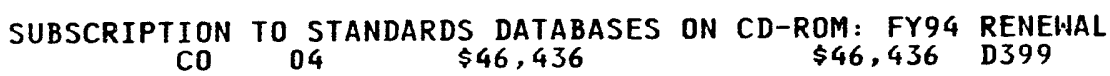
$\underset{\text { OBTAIN MONTHLY UPDATES OF IND USTRY \& FEDERAL SPECIFICATION }}{\text { ON }}$

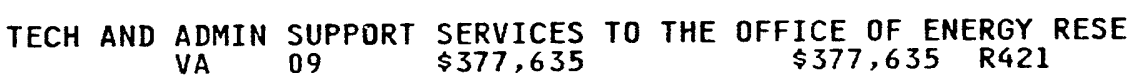

JB / SUPPORT TO DOE / RFO MANAGER

$\$ 169,371 \quad R 409$

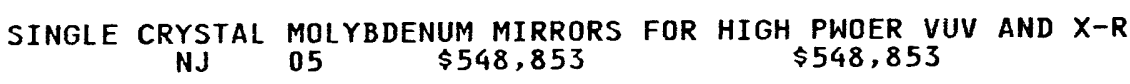
SBIR - PHASE I AWARD FOR - IN SITU MEASUREMENTS OF PARTICULA

PROBLEMS IN PARTICLE THEORY

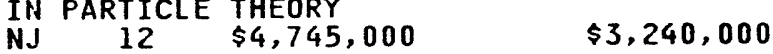

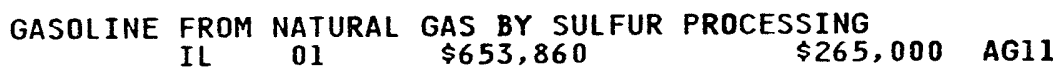
EVALUATION OF HIGH-EFFICIENCY GAS-LIQUID CONTRACTORS FOR IL OF HIGH-EFFICIENCY GAS-LIQUID CONTRACTORS
$01 \quad \$ 2,485,632$ 


\section{AWARDEE NAME}

DIVISION

BIN

VENDOR ID

CITY

INSTITUTE OF NUCLEAR POWER

$A C 01-94 N E 2076912 / 08 / 94$
$169890-0001-5$ MOSCOW

INTEGRATED APPLIED PHYSICS INC FG03-92ER81287 $02 / 17 / 95$ PAENA

FG03-93ER81575 07/01/94 $174710-0004-X$ PASADENA

INTELLIGENETICS INC

INTELLISENSE CORP

INTER-AMERICAN UNIV OF PR MATH DEPT

INTERA INC

INTERACTIVE SOFTWARE

INTERFET CORP/RMD INC

INTERIOR DEPARTMENT OF FISH \& WII DLIFE SERVICE

RECLAMATION BUREAU OF

GEOLOGICAL SURVEY

:
FG03-94ER61871 06/30/96
$238800-0001-8$ MOUNTAIN VIEW

FG02-92ER81371 $05 / 24 / 95$
$220860-0001-4 \quad$ BOSTON

FG05-92ER79096 05/14/95 040190-0101-4 SAN JUAN

$A C 21-92 M C 29111$
$224570-0001-7$

$A C 01-93 A D 3361309 / 20 / 94$
$234000-0001-9$ DENVER

FG02-93ER81612 07/17/94 233880-0001-8 WATERTOWN

GM08-86NV10535 01/07/96 040250-0704-5 ANCHORAGE

$\begin{array}{cc}\text { AI65-93WG21675 } 02 / 28 / 95 \\ 040250-0422-4 & \text { PHOENIX }\end{array}$

AI03-93ER1433803/14/95 040250-0817-3 MENLO PARK

AI65-94WN13897 11/30/98 $040250-0428-3$ SACRAMENTO
DESCRIPTION OF WORK

TOLIGS STATE DINT VONTRACT $\mathrm{P} / \mathrm{S}$

TO PROVIDE CONTINUED MEMBERSHIP IN INPO FOR DDE AND TO ASSIS $05 \$ 2,000,000 \quad \$ 2,000,000$ R425

PHASE I - SBIR GRANT - POWER CONDITIONING FOR EFFICIENT, EFF HIGH CURRENT HIGH BRIGHTNESS FERROELECTRIC ELECTRON SOURCE $F$

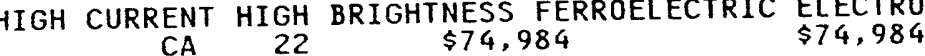

THE BIOSCI ELECTRONIC NEWSGROUP NETWORK FOR THE BIOLOGICAL S CA $14 \quad \$ 171,113 \quad \$ 82,838$

INEXPENSIVE MINIATURE POSITIONING INSTURMENT FOR MA $\$ 549,990 \quad \$ 38,746$

PRE-FRESHMAN ENRICHMENT PROGRAM (PREP) - 1992

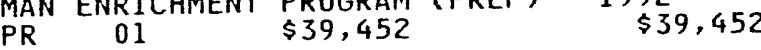

EM-IN-SITU DECONTAMINATION OF SAND AND GRAVEL ACQUIFERS BY

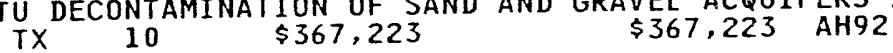

PERPETUAL SOFTWARE LICENSE

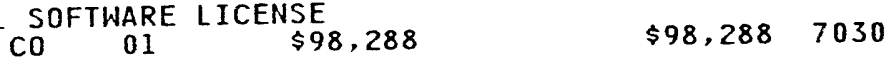

NOVEL DETECTOR FOR X-RAY ABSORPTION SPECTROSCOPY $\begin{array}{ll}\$ 75,000 & \text { ABSORPTION } \$ 75,000\end{array}$

MEMORANDUM OF UNDERSTANDING TOIDENTIFY EACH DEPARTMENT'S IN $\begin{array}{ccc}\text { AK } & 01 & \$ 0 \\ \text { HANGER SPACE, MAINTENANCE, FUEL, FOR PAO HELICOPTER }\end{array}$

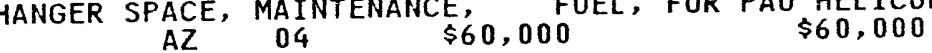
NEW INTERAGENCY AGREEMENT FOR PROJECT ENTITLED "MASS AND ENE ACCT SVCS IN SUPPORT OF COTP

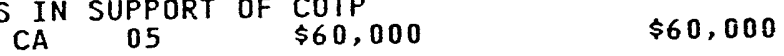




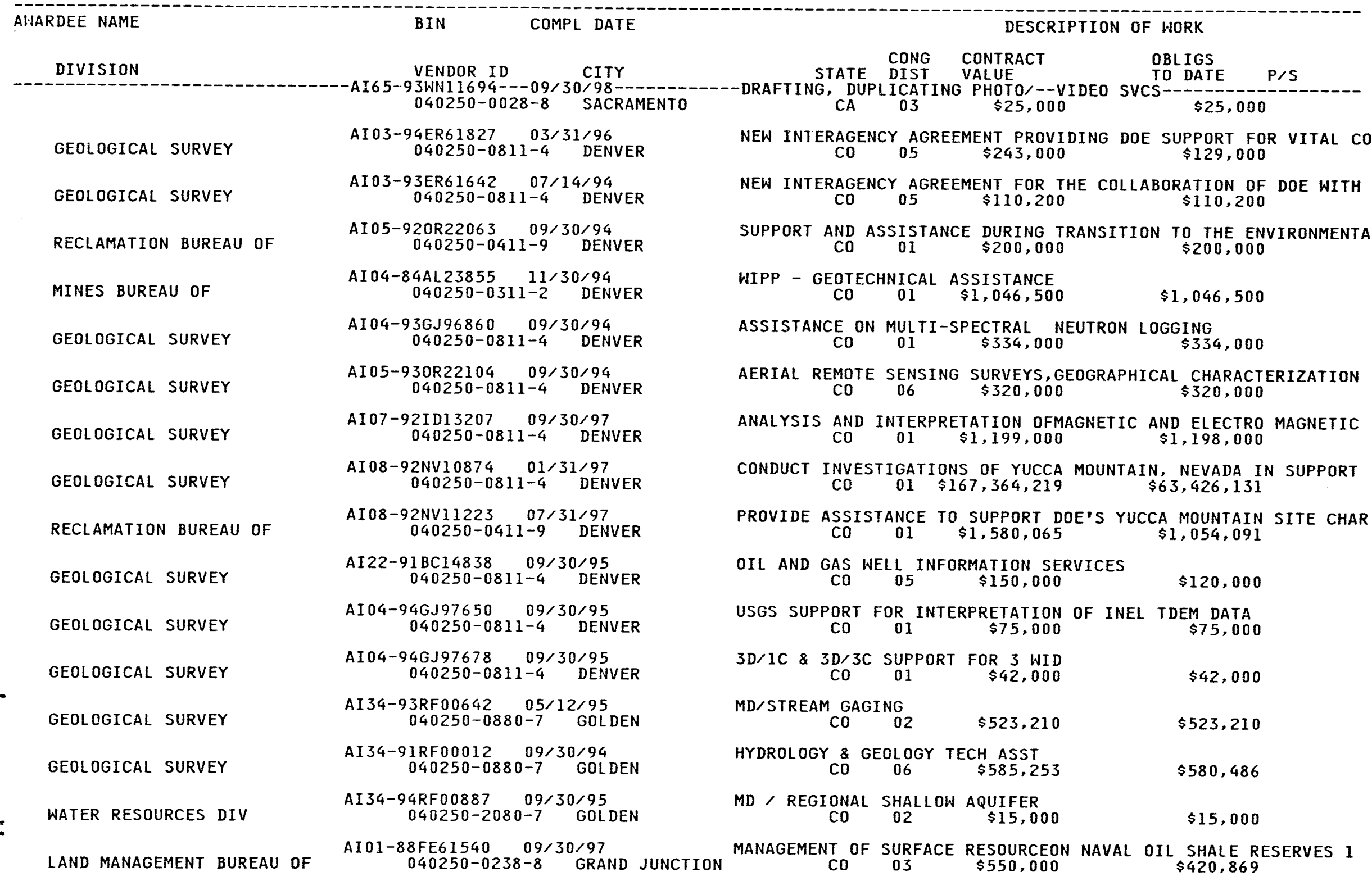




BIN

\section{COMPL DATE}

STATE DIST VALUE

OBLIGS TO DATE P/S

VENDOR ID

CITY ALUE

DIVISION

A104-90AL63756 09/30/94

PROVIDE AMENDED HITHDRAWAL PPL MANENT LAND

AND MANAGEMENT BUREAU OF OAL63756 09/30/94
$040250-0238-8$ GRAND JUNCTION AI 04-90AL65851 09/30/94
$040250-0238-8$ GRAND JUNCTION DOCUMENTATION, PERMANENT LAND TRANSFER

LAND MANAGEMENT BUREAU OF

LAND MANAGEMENT BUREAU OF AII3-88GJ30008 $05 / 31 / 98$
$040250-0238-8$ GRAND JUNCTION

AI $04-91 A L 72780 \quad 09 / 30 / 95$
$040250-0287-6 \quad$ LAKEWOOD

AI $01-94$ CE $5037904 / 13 / 99$ $040250-0032-6$ WASHINGTON

AI01-93AD15029 09/30/94

DRUG PROG COORDINATION STAFF 040250-2932-4 WASHINGTON AI07-76ID01473 $09 / 30 / 99$
$040250-1132-8$ WASHINGTON AI08-88NV10776 09/30/94 $040250-0332-5$ WASHINGTON

MINES BUREAU OF

MINES BUREAU OF

AI22-94PC94651 $08 / 14 / 94$
$040250-0332-5$ WASHIHGTON GM07-76ID01239 09/30/99
$040250-0007-5$ BOISE AI07-76ID01132 09/30/99
$040250-0007-5$ BOISE GM07-87ID11022 12/31/99 AIRCRAFT SERVICES AI07-81IDI2319 $02 / 20 / 96$
$040250-0013-X$ IDAHO FALLS AI 07-81ID12306 09/30/95 AI07-81 040250-0013-X IDAHO FALLS GM07-83ID11011 09/30/99 040250-0213-2 IDAHO FALLS

LAND MANAGEMENT BUREAU OF GM07-72ID11045 $12 / 31 / 95$
$040250-0213-2$ IDAHO FALLS INSTALLATION OF GEOPHYSICAL TEST RANGE

PREPARE ENVIRONMENTAL DOCUMENTATION, LAND TRANSFERS, AND PREPARE ENVIRONMENTAL DOCUMENTATION, LAND \$RANSFERS, $\$ 165,043$ $\begin{aligned} & \text { INTERAGENCY TRANSFER OF FUNDS FOR INCREMENTAL COST } \\ & \$ 8,988,368 \$ 200,000\end{aligned}$ ASSESSMENT CHARGE WHICH ALLOWSDOE TO RIDE DOI'S COLLECTION ASSESSMENT CHARGE WHICH ALLOWSDOE TO RIDE DOT $\$ 3,500$ RE INSTALLATION OF AIR SAMPLING UNIT AT CRATERS OF DC OI THE WASTE MANAGEMENT PROJECT OFFICE DESIGN REVIEW SUPPORT TO THE HASTE MANAGEMENT PROJ
$\$ 56,018$ STUDIES ON LOW RANK COAL (LRC)STABILITIES $\$ 80,000$

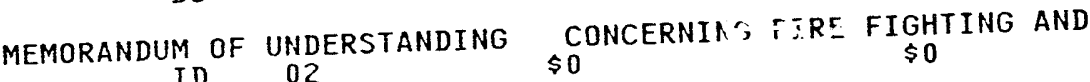
ADMINISTRATION OF GRAZING LANDS WITHIN THE NRTS $\underset{\$ 0}{\&}$ FIRE FIGHT MOU) BETHEEN DEPT OF INTERIOR, AIRCRAFT SERVICES \& DOE-ID (MOU) BETHEEN DEPT OF TNTERIOR, THIS INTERAGENCY AGREEMENT IS TO COMBINE TWO SEISMIC AND TWO LIQUID \& SOLID WASTE AT INEL GEOHYDROLOGIC STUDIES OF DEVELOP A COMMUNICATION SITE MANAGEMENT PLAN FOR EAST BUTTE CONCERNS MANAGEMENT OF GRAZINGACTIVITIES ON THE INEL (NRTS) ID 02 MANAGEMENT OF GRAZINGACTI 


\section{DIVISION}

LAND MANAGEMENT BUREAU OF

LAND MANAGEMENT BUREAU OF

FISH \& WILDLIFE SERVICE

GEOLOGICAL SURVEY

GEOLOGICAL SURVEY

GEOLOGICAL SURVEY

GEOLOGICAL SURVEY

LAND MANAGEMENT BUREAU OF

NATIONAL PARK SERVICE

LAND MANAGEMENT BUREAU OF

INDIAN AFFAIRS BUREAU OF

LAND MANAGEMENT BUREAU OF

NATIONAL PARK SERVICE

LAND MANAGEMENT BUREAU OF

GEOLOGICAL SURVEY

FISH \& WILDLIFE SERVICE
BIN COMPL DATE

CONG CONTRACT $\begin{array}{lll}\text { CONG CONTRACT } & \text { OBLIGS } \\ \text { STATE DIST VALUE } & \text { TO DATE P/S }\end{array}$ VENDOR ID
CITY 040250-0213-2 IDAHO FALLS ID 02 INEL FIRING RANGE USE AGREEMENT WITH BUREAU OF LAN GM07-92ID11088 09/30/99 AI05-93ER7589302/28/95 040250-0789-4 LAFAYETTE

AI05-870R21741 09/30/94 040250-0858-0 ST. CHARLES

AI05-940R22207 09/30/94 040250-0858-0 ST. CHARLES AI02-93CHI0579 07/31/94 040250-0891-2 LINCOLN

AI 02-94CH10618 09/30/94 040250-0891-2 LINCOLN

GM04-68AL38640 09/30/99 040250-0048-2 ALBUQUERQUE GMO4-90AL63418 11/07/94 040250-0248-5 ALBUQUERQUE GM04-94AL $94712 \quad 04 / 18 / 99$ 040250-1148-4 ALBUQUERQUE GM04-83AL53630 06/17/99 040250-0244-2 CARLSBAD GM32-87AL37160 06/23/99 040250-2715-1 LOS ALAMOS AIO4-90AL66317 09/30/94 040250-0241-8 SANTA FE AI32-89AL57311 09/30/94 040250-1141-7 SANTA FE AI 08-92NV11144 12/31/97 040250-0209-4 CARSON CITY AI08-91NV11040 07/31/96
$040250-0814-9$ GM08-83NV1033906/06/98 040250-0714-2 LAS VEGAS
THEORY AND USE OF MODERN MICROSCOPICAL METHODS WITH APPLICAT LA 07 T422,073 $\quad \$ 422,073$

GEOHYDROLOGY - WELDON SPRING SITE REMEDIAL ACTION PROJECT ( $W$ MO 09 \$873,900 $\$ 873,900$

TECHNICAL SUPPORT IN THE AREASOF GEOLOGY, GEOCHEMISTRY, AND MO 02 \$126,700 $\$ 126,700$

TECHNICAL SUPPORT FOR GROUNDWATER LEVEL MONITORING NE 02 \$19,500 $\$ 19,500$ WELL DEVELOPMENT, AQUIFER TESTING, WATE SAMPLING AT NE $\$ 13,000 \quad \$ 13,000$ PROVIDES RESPONSE FOR ROCKY FLATS EMERGENCY SITUATION INVOLPROVIDES RESPONSE FOR ROCKY FLATS EMERGENCY SITUATIC \$O THE PURPOSE OF THIS MOU IS TO PROVIDE FOR THE CONDUCT BY DOE THIS AGREEMENT SETS FORTH THE GENERAL POLICY FOR THE SCHEDUL PROVIDES FOR THE WITHDRAWAL OFPUBLIC LANDS ADMINISTERED BY

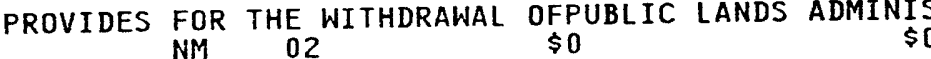
PROVIDE A PROCEDURE BY WHICH DOE \& BIA WILL COORDINATE AND NM 03 PR IA TO ASSIST IN THE ADMINISTRATIVE LAND WITHDRAWAL PATROLLING AND ENFORCEMENT RESTRICTIONS ON DOE PROPERTY NM $03 \quad \$ 374,321 \quad \$ 374,321$ ROAD MAINTENANCE AGREEMENT FORTV PEAK COMMUNICATIONS SITE I NV $02 \quad \$ 500 \quad \$ 500$ PROVIDE PRE AND POST SHOT GEOLOGICAL, GEOPHYSICAL AND HYDROL NV $01 \$ 16,000,000 \quad \$ 9,923,190$ RADIO REPEATER STATION LOCATEDON HAYFORD PEAK NEAR PAHRANAG NV 01 \$O 


\begin{tabular}{|c|c|c|c|c|c|c|c|}
\hline AWARDEE NAME & BIN & 1 DATE & & & $\mathrm{DESCI}$ & F HORK & \\
\hline DIVISION & VENDOR ID & CITY & STATE & $\begin{array}{l}\text { CONG } \\
\text { DIST }\end{array}$ & $\begin{array}{l}\text { COHTRACT } \\
\text { VALUE }\end{array}$ & $\begin{array}{l}\text { OBLIGS } \\
\text { TO DATE }\end{array}$ & $P / S$ \\
\hline
\end{tabular}

LAND MANAGEMENT BUREAU OF

LAND MANAGEMENT BUREAU OF

INDIAN AFFAIRS BUREAU OF

MINES BUREAU OF

MINES BUREAU OF

FISH \& WILDLIFE SERVICE

MINES BUREAU OF

GEOLOGICAL SURVEY

GEOLOGICAL SURVEY

GEOLOGICAL SURVEY

WATER RESOURCES DIV

GEOLOGICAL SURVEY

- geological survey

GEOLOGICAL SURVEY

GEOLOGICAL SURVEY
AI08-76NV00441 $09 / 30 / 99$
$040250-0214-0$ LAS VEGAS

AI $08-93 N V 1122410 / 31 / 97$
$040250-0225-6 \quad$ RENO

GM04-94AL $94693 \quad 10 / 20 / 98$ 040250-2794-1 OKLAHOMA CITY

AI05-930R22161 09/14/94
$040250-0302-3 \quad$ ALBANY

AI07-93ID13253 03/31/95
$040250-0302-3 \quad$ ALBAHY

AI05-930R22088 01/31/95 040250-0736-3 PORTLAND

AI22-90PC91508 09/27/95 040250-0323-6 PITTSBURGH

AI09-91SR18214 12/31/94
$040250-0801-7$ AIKEN

AI09-91SR18222 09/30/96 040250-0801-7 AIKEN

AI09-92SRI8286 09/30/96

AI09-85SRI4095 12/31/98

AI05-870R21707 09/30/95 040250-0847-5 KNOXVILLE

AI05-940R22196 09/30/95 040250-0847-5 KNOXVILLE

AI05-940R22164 09/30/95 040250-0847-5 KNOXVILLE

AI05-940R22140 06/30/95 040250-0847-5 KNOXVILLE

AI05-930R22127 12/31/94 $040250-0821-1$ NASHVILLE
FIRE SUPPRESSION AGREEMENT

$N V \quad 01$ \$O

$\$ 0$

PROCESSING AND ADMINISTERING RIGHT-OF-WAY RESERVATIONS ASSOC NV $02 \quad \$ 50,000 \quad \$ 10,000$

SUPPORT SCIENCE, MATH \&ENG EDUCATION

$\$ 0$

TECHNICAL ASSISTARCE IN EVALUATION OF TREATABILITY OF CONTAM OR 05 S $\$ 90,000 \quad \$ 90,000$

AGREEMENT WITH BUREAU OF MINESTO COOPERATE \& COLLABORATE ON $O R \quad D^{2} \$ 1,119,400 \quad \$ 552,000$

$\begin{array}{rll}\text { ECOLOGICAL SURVEYS } & \$ 583,960 & \$ 583,960\end{array}$

UTILITY SERVICES, FACILITY OPERATING AND MAINTENANCE SERVICE PA 14 \$9,289,500 $\$ 9,289,500$

SEISMIC ATTENUATION STUDY

SC $03 \quad \$ 526,064 \quad \$ 526,064$

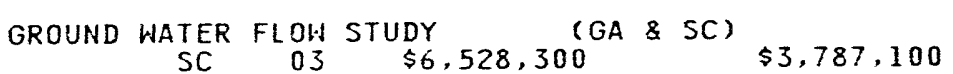

DELINEATION OF 100 -YEAR FLOOD PLAIN FOR SELECTED STREAMS AT SC 03 \$343,000 $\$ 343,000$

RADIOCOMMUNICATION OF SRP PERIMETER GAMMA MOHITORING $S C \quad 03 \quad \$ 450,900 \quad \$ 450,900$

COLLECT SURFACE WATER DISCHARGE DATA
TN 02

INSTALLATIOH AHD OPERATION OF SURFACE WATER STAGE GAGES AT T TN 02 \$65,862 $\$ 65,862$

SEEPAGE AND SPRING INVEHTORY AT THE DAK RIDGE K-25 SITE

SEEPAGE AND SPRING INVENTORY OF BEAR CREEK VALLEY TN $02 \quad \begin{array}{ll}\$ 75,000 & \$ 175,000\end{array}$

TRITIUM TRACER TEST ANALYSIS

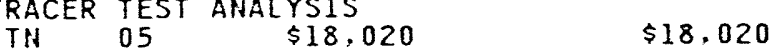




\section{DESCRIPTION OF WORK}

\section{AWARDEE NAME}

BIN

VENDOR ID CITY

DIVISION

I $05-9$

040250-0821-1 NASHVILLE

GEOLOGICAL SURVEY

WATER RESOURCES DIV

RECLAMATION BUREAU OF

AI05-920R22025 09/30/96

040250-2021-1 NASHVILLE

AI32-77DP03856 01/10/97

040250-0403-8 AMARILLO

AI01-88FE61541 09/30/97

$040250-0020-2$ MOAB

LAND MANAGEMENT BUREAU OF

MINERALS MANAGEMENT SERVICE

GEOLOGICAL SURVEY

GEOLOGICAL SURVEY

GEOLOGICAL SURVEY

GEOLOGICAL SURVEY

GEOLOGICAL SURVEY

GEOLOGICAL SURVEY

GEOLOGICAL SURVEY

GEOLOGICAL SURVEY

GEOLOGICAL SURVEY

MINES BUREAU OF
AI01-88FE61542 09/30/94 040250-0252-3 VERNAL

AI21-92MC29078 05/11/95 040250-1986-8 HERNDON

AI01-93RW00278 11/15/97 $040250-0826-2$ RESTON

AI 01-91CE31020 06/03/96 040250-0826-2 RESTON

AI01-92EW50631 09/03/95 040250-0826-2 RESTON

AI0I-94EI $24838 \quad 04 / 12 / 95$ 040250-0026-1 RESTON

AI01-93EW53093 07/19/98 040250-0826-2 RESTON 040250-0826-2 RESTON

AI07-94ID13282 12/31/96 $040250-0826-2$ RESTON

AI09-76SR00060 11/30/98 040250-0826-2 RESTON

AT21-93MC $30139 \quad 02 / 28 / 97$ 040250-0826-2 RESTON 040250-0826-2 RESTON

AI08-91NVI1080 06/30/96 $040250-0379-1$ SPOKANE
AI05-87ER60578 06/30/96

AI21-92MC29214 02/28/97
STATE DIST VALUE

TN 05 MONITORING- $\$ 44,120$ TO DATE P/S SURFACE WATER DISCHARGE DATA

$\$ 106,750$ FURNISHING WATER FOR ERDA L OS ALAMOS PROJECT AND RELATED TX $13 \quad \$ 685,081 \quad \$ 685,081$

MANAGEMENT OF SURFACE $\underset{\text { UT }}{\text { RESOURCES ON }} \underset{\$ 55,46,462}{\text { NAV OL SHALE }}$ MANAGEMENT OF SURFACE RESOURCES ON NAVAL OIL SHALE RESERVE 2 UT 03 \$93,113 $\$ 93,113$ OIL AND GAS RESOURCE ATLAS SERIES, OFFSHORE NORTHERN GULF VA $10 \quad \$ 2,400,000 \quad \$ 1,555,000$

PROVIDE THE OFC OF CIVILIAN RADIOACTIVE WASTE MGMT WITH TECH VA $10 \quad \$ 629,031 \quad \$ 228,574$ $\begin{gathered}\text { INTERAGENCY AGREEMENT } \\ \text { VA } 10\end{gathered} \$ 1,640,000 \quad \$ 1,640,000$ TECHNOLOGY ASSESSMENT - TECHNOLOGY TRANSFER $\begin{array}{lll}\text { VA } 10 & \$ 1,150,000 & \$ 1,150,000\end{array}$ MAINTAINING THE URANIUM RESOURCE ASSESSMENT DATA SYSTEM AND VA $09 \quad \$ 60,000 \quad \$ 60,000$

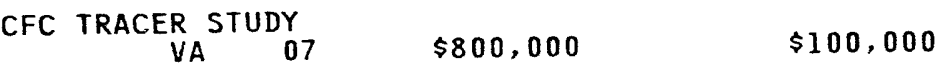
GAS TRANSPORT IN SOILS AND ITSRELATION TO RADON AVAILABILIT VA 10 \$1,217,600 $\$ 1,030,950$

LOW-LEVEL WASTE TO PROVIDE SUPORT TO DOE IN ASSISTING STATES VA $10 \quad \$ 50,000 \quad \$ 50,000$

PERFORMHYDROLOGICAL STUDIES ATSRP
VA $10 \quad \$ 5,568,691$ USGS WESTERN BASINS GEOLOGY AND RESOURCE STUDIES VA $10 \quad \$ 1,736,000 \quad \$ 693,000$

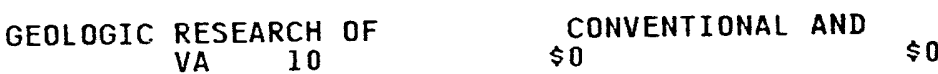
MINE SAFETY SERVICES AND SURVEILLANCE IN SUPPORT OF WEAPONS WA $05 \quad \$ 35,000 \quad \$ 28,597$ 


AWARDEE NAME
DIVISION
GEOLOGICAL SURVEY

INTERIORS UNLIMITED INC

INTERLINE RESOURCES

INTERMAGNETICS GENERAL CORP FG02-90ER80925 02/14/95 ADVANCED SUPERCONDUCTORS INC 040280-0102-1 WATERBURY ADVANCED SUPERCONDUCTORS INC FO2-90ER80926 $05 / 19 / 95$ HATERBURY FG02-91ER81153 03/30/95 $040280-0002-5$ WATERBURY FG02-91ER81155 03/30/95 $040280-0002-5$ WATERBURY

ADVANCED SUPERCONDUCTORS INC FG02-92ER81461 $05 / 24 / 95$ WATERBURY ADVANCED SUPERCONDUCTORS INC FG02-92ER81331 $0402 / 24 / 95$ WATERBURY ADVANCED SUPERCONDUCTORS INC FG2-93ER81513 $07 / 17 / 94$

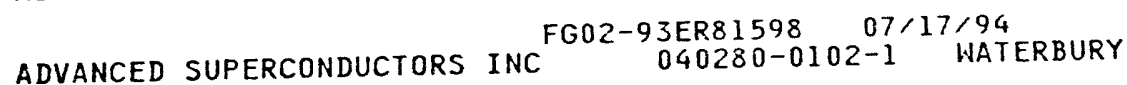
-intermountain gas co
$A D 07-91$ ID $1306002 / 29 / 96$
$217600-0001-9$ BOISE

INTERMTN CONSUMER PRO ENG INC AT65-9411N14752 $09 / 01 / 94$
$232930-0002-9$
SACRAMENTO

AM65-93HN09876 08/31/94 232930-0001-0 MIDVALE

AM65-93WL $03664 \quad 08 / 31 / 94$

\section{DESCRIPTION OF WORK}

TO DATE P/S

HYDORLOGIC INVESTIGATION

WA ${ }_{06} \$ 1,082,777 \quad \$ 1,082,777$

PROVIDE ALL LABOR, MATERIALS AND EQUIPMENT TO PAINT FIRST AN DC $010 \begin{array}{llll} & & & \end{array}$

NPR. 3 LIQUID PRODUCTS SALES CONTRACT TOTAL EST REV: $\$ 512,46$ UT 03 $\$ 0$

$\$ 0$

CHANGES IN NB3SN CONDUCTORS MADE BY THE "INTERNAL TIN" CT 05 T549,975 \$549,975 A MORE ECONOMICAL LOW LOSS NBSSN FOR PULSED FIELD
$\$ 422,525$ AN IMPROVED METHOD OF INTRODUCING ADDITIONAL ALLOYING ELEMEN

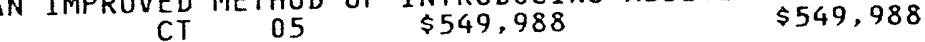
IMPROVED PROPERTIES IN NB $46.5 \mathrm{WT} \because$ TI MATERIALS FOR THE SSC

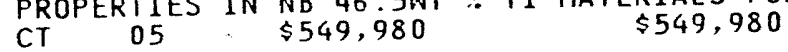
HIGH STRENGTH MENO \& MULTI- FILAMENT 2212 BSCCO FABRICATION OF N63AI SUPERCONDUCTING STRANDS USING $\begin{array}{llll}C T & 05 & \$ 545,069 & \$ 338,722\end{array}$ METHOD OF IMPROVING INTERNAL- TIN NBSSN FOR FUSION THERMO-MECHANICAL PROCESSING OF NBTI BASED SUPERCONDUCTIORS $C T \quad 05 \quad \$ 75,000 \quad \$ 75,000$

NATURAL GAS SERVICE TO INEL

A TE TITLE I AND III SUCS FOR COMMUNICATION SYST RELCOATION

A-E SVC FOR SAO $03 \quad \$ 50,000$

A-E SVCS FO SLCAO 


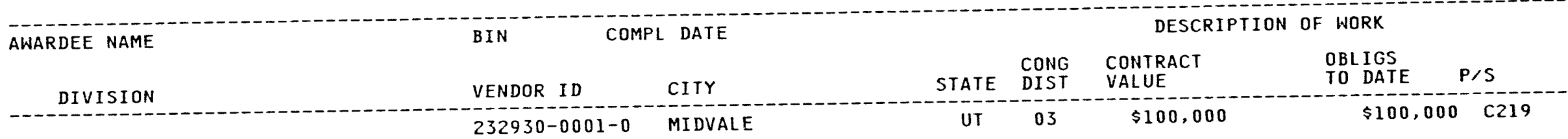

INTERNATIONAL ATOMIC ENERGY AG GI0I-93AN00013 $12 / 14 / 97$
$040330-0003-8$ GI $01-93 N E 20717$
$040330-0003-8$

INTERNATIONAL DATA PROD CORP

$A B 01-93 E I 23587 \quad 09 / 08 / 94$ $101830-0002-1$ GAITHERSBURG

INTERNATIONAL DATA PRODUCTS

AM01-92AD32620 08/12/95 $101830-0002-1$ GAITHERSBURG

AMO1-91EI21398 02/27/96 $101830-0002-1$ GAITHERSBURG

INTERNATIONAL DEV COOP AGCY AI01-92CE33020 04/22/97

US TRADE \& DEVELOPMENT PROGRAM 040400-0101-X WASHINGTON

INTERNATIONAL FALLS MEM HOSP

INTERNATIONAL INST ENERGY CONS

INTERNATIONAL RADIATION DETECT

INTERNATIONAL TECHNOLOGY CORP

INTERMET SYSTEMS INC

INTERPHASES RESEARCH

3PO10085 12/30/94
$215630-0001-6 \quad$ WASHINGTON 145960-0003-4 CINCINNATI

ACO1-92MA21100 10/31/94 FG45-94R562128 $12 / 31 / 95$
$238330-0001-5$ $\begin{aligned} & \text { ICP-NEN AHARD. } \\ & \text { INTERNATIONAL FALLS }\end{aligned}$

EQUIPMENT AND TECHNICAL ASSISTANCE FOR NONPROLIFERATION AND IAEA NUCLEAR SAFETY ASSISTANCEPROGRAM OO $\$ 200,000$ $\$ 1.500,000$

IAEA NUCLEAR SAFE

BPA FOR PC'S \& PRINTERS
MD
08

$\$ 0$

$\$ 200,000$

EXECUTE CONTRACT AMARD FOR SOLICITATION NUMBER DE-FB01-91AD3 $M D \quad 08 \$ 11,202,600$ $\$ 0 \$ 010$

NUMEROUS ITEMS OF ADP HARDWARE\& SOFTHARE MD 08 \$1,661,390

\$0 7010

FINESSE OFFICE IN WORLD BANK DC 01 W $\$ 450,000$

$\$ 450,000$

ASIA REGIONAL CONFERENCE TO ADDRESS ALTERNATIVE DC $01 \quad \$ 20,000 \quad \$ 20,000$

SBIR - PHASE I AWARD FOR - SILICON JUNCTION DIODE ABSOLUTE R CA $42 \quad \$ 308,381 \quad \$ 308,381$

PROVIDES ENVIRONMENTAL SUPPORTSERVICES TO INCLUDE PROGRAMMA NV $01 \$ 54,539,790 \quad \$ 39,465,051$ B510

TECHNICAL SUPPORT SERVICES TO THE OFFICE OF PLANNING AND OH 01 \$1,752,062

$\$ 154,000$ R425

ACD1-93FE62490 03/30/96 204890-0002-9 GAITHERSBURG

PAR 720 DTD $04 / 20 / 94$ COMPLETION DATE OF 01/31/94 INCORRECT; MD 08 \$11, 490,093 $\$ 10,924,155$ R605

NANOSCALE PROCESSING FOR TERNARY SEMICONDUCTORS 
R-0420953-001

PROCUREMENT AND ASSISTANCE DATA SYSTEM (PADS) NAMES BY NAME, CITY \& STATE - ACTIVE AWARDS

PAGE NO. 238

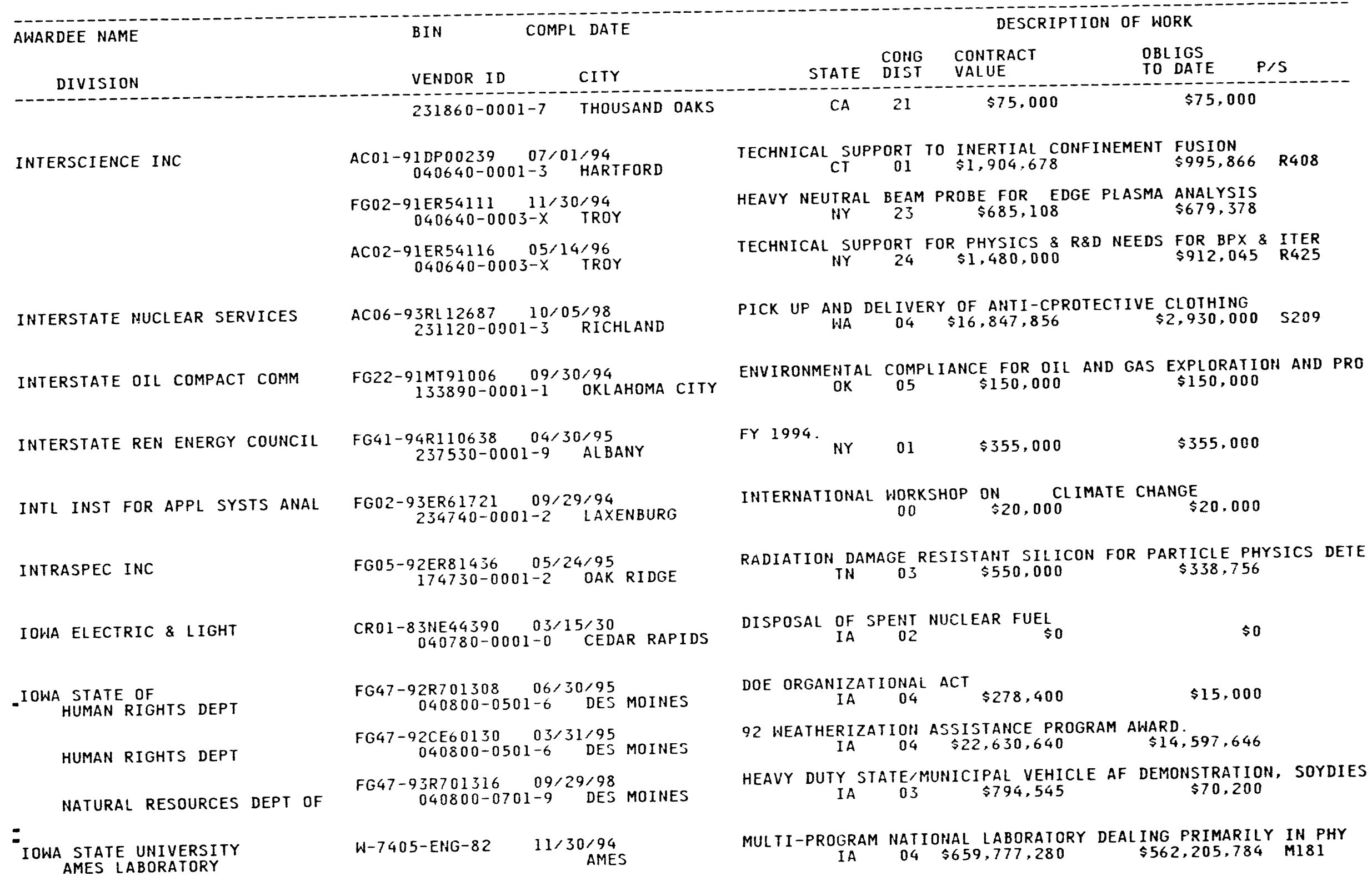




AWARDEE NAME BIN COMPL DATE

DIVISION

VENDOR ID

CITY

STATE CONG CONTRACT VALUE

OBLIGS TO DATE

$P / S$
RL 02-76CH0O144 12/31/99
$040810-0001-8$ AMES RL 02-76CHO0145 12/31/94 $\begin{array}{ll}\text { RL 02-76CHO0146 } & 12 / 31 / 99 \\ 040810-0001-8 & \text { AMES }\end{array}$

RL 02-76CHOO148 12/31/94 RLO2-78CHOO149 12/31/99 040810-0001-8 AMES AC02-76IR03192 12/30/99 040810-0001-8 AMES

\section{MIGH ENERGY PHYSICS}

\section{PHYSICS DEPT}

PHYSICS DEPT

NUCLEAR ENGINEERING DEPT

PHYSICS DEPT

FG02-87ER40371 06/14/96
$040810-1101-X$ AMES

FG02-91ER4063404/30/95 $040810-0601-6$ AMES

FG02-92ER40692 11/14/94

FG02-87ER75360 07/31/94
040810-0801-9 AMES FG02-92ER75700 11/14/94
$040810-0001-8$ AMES

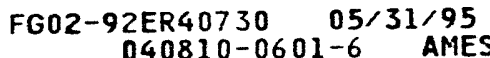

LAND LEASE

\section{$\$ \mathbf{0}$}

$\$ 0$

$\$ \mathbf{0}$

LAND LEASE

LAND LEASE CONTRACT - RESEAR $\underset{\text { IA }}{\text { CH }}$ CHL BL (SPEDDING $\underset{\$ 0}{\text { HALL }}$ X299

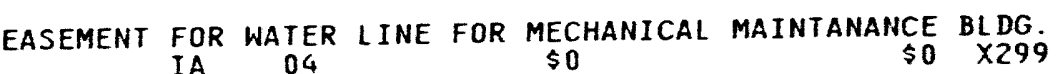

PUB COMMERCIALLY AND MARKET $\underset{\text { TECH }}{\text { TO BO }}$ BOOK FURNISHED $\underset{\$ 0}{\text { BY COMMISSIO }}$ AZII

RESEARCH IN INTERMEDIATE $/$ HIGH ENERGY PHYSICS

$$
\text { IA INTER } \$ 1,237,500 \quad \$ 1,075,500
$$
FG02-92ER61473 07/31/95
$040810-0001-8$ AMES

FG02-92ER75772 11/30/94 FG02-92ER79153 09/29/94 040810-0001-8 AMES

FG02-94ER40817 10/31/96
VERY HIGH ENERGY GAMMA RAY ASTROPHYSICS

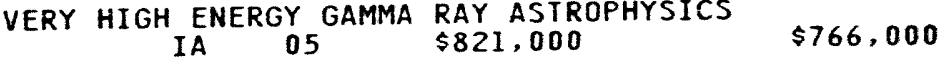

RELATIVISTIC HEAVY ION PHYSICS

$\$ 646,000$

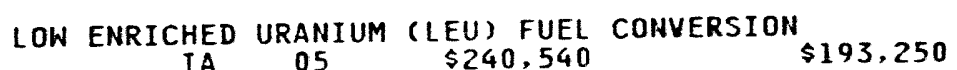

NEURAL NETWORK RECOGNITION OF NEUCLEAR POWER PLANT

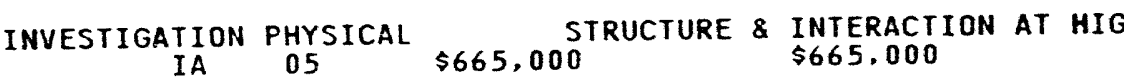

MODELING OF LAND-SURFACE $\underset{\text { IA }}{\$}$ ATMOSPHIRE DYNAMICS FOR

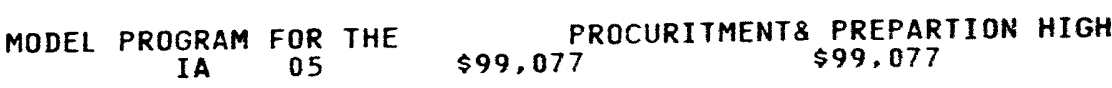

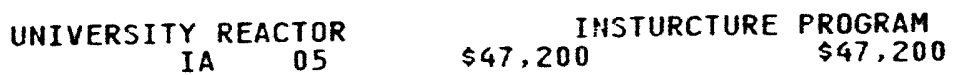

INVESTIGATION IN EXPERIMENTAL AND THEORETICAL HIGH ENERGY IA $05 \quad \$ 2,785,000 \quad \$ 785,000$ IA 05 \$171,885 \$171,885 


\section{DIVISION}

BIN

VENDOR ID

ZOOLOGY DEPT

$$
\begin{gathered}
\text { FG02-93ER20124-- } 08 / 14 / 95- \\
\text { 040810-0501-X AMES } \\
\text { AC04-93AL96849 } 09 / 30 / 94 \\
\text { 040810-0001-8 AMES } \\
\text { FCO6-87RL10933 } 09 / 30 / 96
\end{gathered}
$$

AGRICULTURAL EXPERIM STATION FCO6-87RL10933 $040810-1001-3$ AMES FC21-91MC28081 11/30/94
$040810-0401-3$ AMES

MECHANICAL ENGINEERING DEPT FG22-94PC94210 08/31/97 MECHANICAL ENGINEERING DEPT

MINING \& MINERALS RES INST

MINING \& MINERALS RES INST

MINING \& MINERALS RES INST

\section{BOTANY DEPT}

MATH DEPARTMENT

IOWA UNIVERSITY OF PHYSICS \& ASTRONOMY DEPT

- CHEMISTRY DEPT

PHYSICS \& ASTRONOMY DEPT

\section{= CHEMISTRY DEPT}

FG22-92PC $2530 \quad 08 / 31 / 95$
$040810-1401-9$ AMES FG22-92PC92531 08/31/95 FG22-93PC93209 08/31/96
B40810-1401-9 AMES FC65-93WA10699 $03 / 30 / 96$ FG02-94ER25220 06/30/96 040810-1301-2 AMES

FG21-93MC30116 07/16/94 040820-0201-4 IOWA CITY FG02-88ER13935 12/31/94 040820-0301-0 IOWA CITY FG02-86ER40286 05/31/95 040820-0201-4 IDWA CITY FG02-91ER40664 02/28/95 040820-0001-1 IOWA CITY FG06-94RL12842 03/19/95
$040820-0301-0$ IOWA CITY FG06-94RL12765 02/06/95 FG02-94ER20147 06/30/97
CONG CONTRACT OBLIGS STATE DIST VALUE PAT

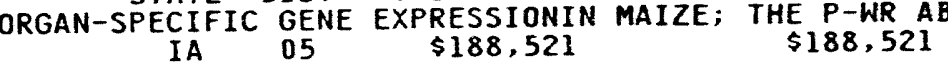
MEMBERSHIP OF CENTER FOR NON- DESTRUCTIVE EVALUATIONIA OF CENTER FOR $\$ 35,000$ S $\$ 35,000$ R415 IOWA COMMODITY IRRADIATION PROJECT

$$
\text { IA } 04 \text { \$11,782,011 } \$ 5,000,003
$$

SPECTRAL METHODS APPLIED TO FLUIDIZED BED COMBUSTORS PRESSURE FLUCTUATIONS AS A DIAGNOSTIC TOOL FOR FLUIDIZED BOI IA 04 \$251,445 $\$ 199,995$ BED MATERIAL AGGLOMERATION DURING FLUIDIZED BED COMBUSTION IA 04 \$200,000 $\$ 200,000$ HETERONUCLEAR NMR PROBES OF COAL STRUCTURE AND REACTIVITY IA $04 \quad \$ 301,498$ DEVELOPMENT OF A GAS-PROMOTED OIL AGGLOMERATION PROCESS IA $04 \quad \$ 256,880 \quad \$ 200,000$ ASBR PROCESS - SWINE WASTE TO BIOGAS
IA
04 REGULATION OF CAROTENOID BIOSYNTHESIS: IA $05 \quad \$ 244,510 \quad \$ 80,510$ PIEMAN PROBLEMS FOR NONLINEAR CONSERVATION LAWS IN ONE AND IA 05 \$38,172 $\$ 38,172$

USED ENERGY RELATED EQUIPMENT SYNTHESIS AND CHEMISTRY OF CATIONIC D METAL ALKYL CONFIGURATION SPACE FADDEEV CALCULATIONS

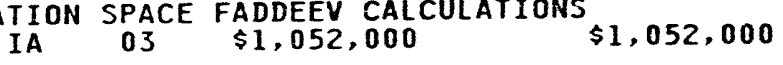

R\&D ON THE SCINTILLATING FIBERCALORIMETRY FOR THE SSC DETECT IA 03 \$1,435,000 $\$ 1,435,000$ USED ENERGY RELATED LABORATORYEQUIPMENT GRANT IA 01

$\$ 0$ USED ENERGY RELATED LABORATORYEQUIPMENT GRANT 


\section{AWARDEE NAME \\ CHEMISTRY DEPT \\ CHEMISTRY DEPT}

BIN

VENDOR ID

COMPL DATE

040820-0301-0 IOWA CITY

PHYSICS \& ASTRONOMY DEPT

ISOTRON CORP

IVINSON MEMORIAL HOSPITAL

$J$ BUSEK CO INC

$J$ COOPER \& ASSOC

JACKSON \& TULL CHARTERED ENG

-JACKSON COUNTY BOARD OF EDUCN

JACKSON COUNTY OF HOSPI TAL

:JACKSON MADISON CTY SCH SYST

FG06-94RL12?57 02/06/95
$040820-0201-4$ IOWA CITY
FG09-94SR18416 02/02/95
$040820-0301-0$ IOWA CITY
FG34-94RF00813 04/27/95
$040820-0301-0$ IOWA CITY

AC21-93MC30162 08/31/94 231040-0001-8 NEW ORLEANS

FG48-92R808613 08/31/94
$228390-0001-6$ LARAMIE

FG02-91ER8 $117403 / 30 / 95$
$169900-0002-3$ NEEDHAM

FG02-91ER81175 07/05/94 169900-0002-3 NEEDHAM

FG02-92ER81362 05/24/95 $169900-0002-3$ NEEDHAM

$A B 01-94 M I 10421 \quad 12 / 06 / 94$
$235910-0001-9$ WASHINGTON

FC34-94RF00867 05/02/97
$238790-0001-8 \quad$ SEABROOK.

FG44-90R434214 $01 / 31 / 95$
$097720-0002-4$ MCKEE

FG47-92R704996 09/30/94 O41370-0203-7 MAQUOKETA

FG44-93R435330 09/30/95 $3 R 43533009 / 30 / 95$
$231210-0001-2 \quad$ JACKSON
DESCRIPTION OF HORK

OBL IGS

TO DATE P/S STATE DIST VALUE $\$ 0$

USED ENERGY RELATED LABORATORYEQUIPMENT GRANT

IA 01

IA 01 \$O \$O

USED ENERGY RELATED LAB EQUIPMENT

IA OI

$\triangle 0$

$\mathbf{\$ 0}$

EM ELECTROKINETIC DECONTAMINA-TION OF CONCRETE

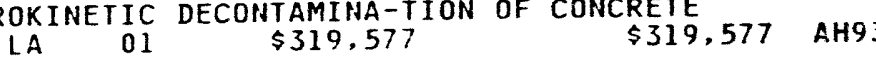

AWARD ICP CYCLE XIV GRAHT

$$
\underset{H Y}{C Y C L E} \text { XIV GRANT } \$ 35,000 \quad \$ 19,950
$$

ELECTRIC ISOLATOR FOR WATER COOLED HIGH VOLTAGE SYSTEMS.

MA $09 \quad \$ 548,951 \quad \$ 548,951$

CERAMIC/METAL ELEMENTS FOR MHDSIDEWALLS.

$$
\text { MA } 09 \quad \$ 549,991
$$

LOH COST, FLEXIBLE, MHD GEHERATOR TEST FACILITY

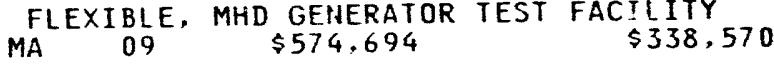

DC 01 \$O

50

$\$ 0 \quad$ R607

COOPERATIVE AGREEMENT BETWEEN DOE, EG\&G, AND PARTICIPANT $M \underset{04}{\text { A }}$ \$0

CYCLE 12 INSTITUTIONAL CONSERVATION PROGRAM. APPROVED FUNDED

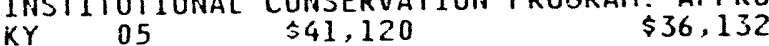

GRANT PROGRAMS FOR SCHOOLS ANDHOSPITALS AND FOR BUILDINGS 0

$$
\text { IA } 02 \quad \$ 29,103 \quad \$ 12,853
$$

SEE ATTACHED PAGE NO 2 OF THIS NOTICE OF FINANCIAL ASSISTAN

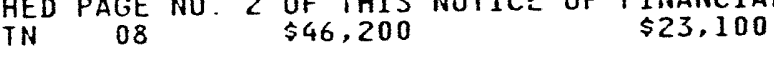




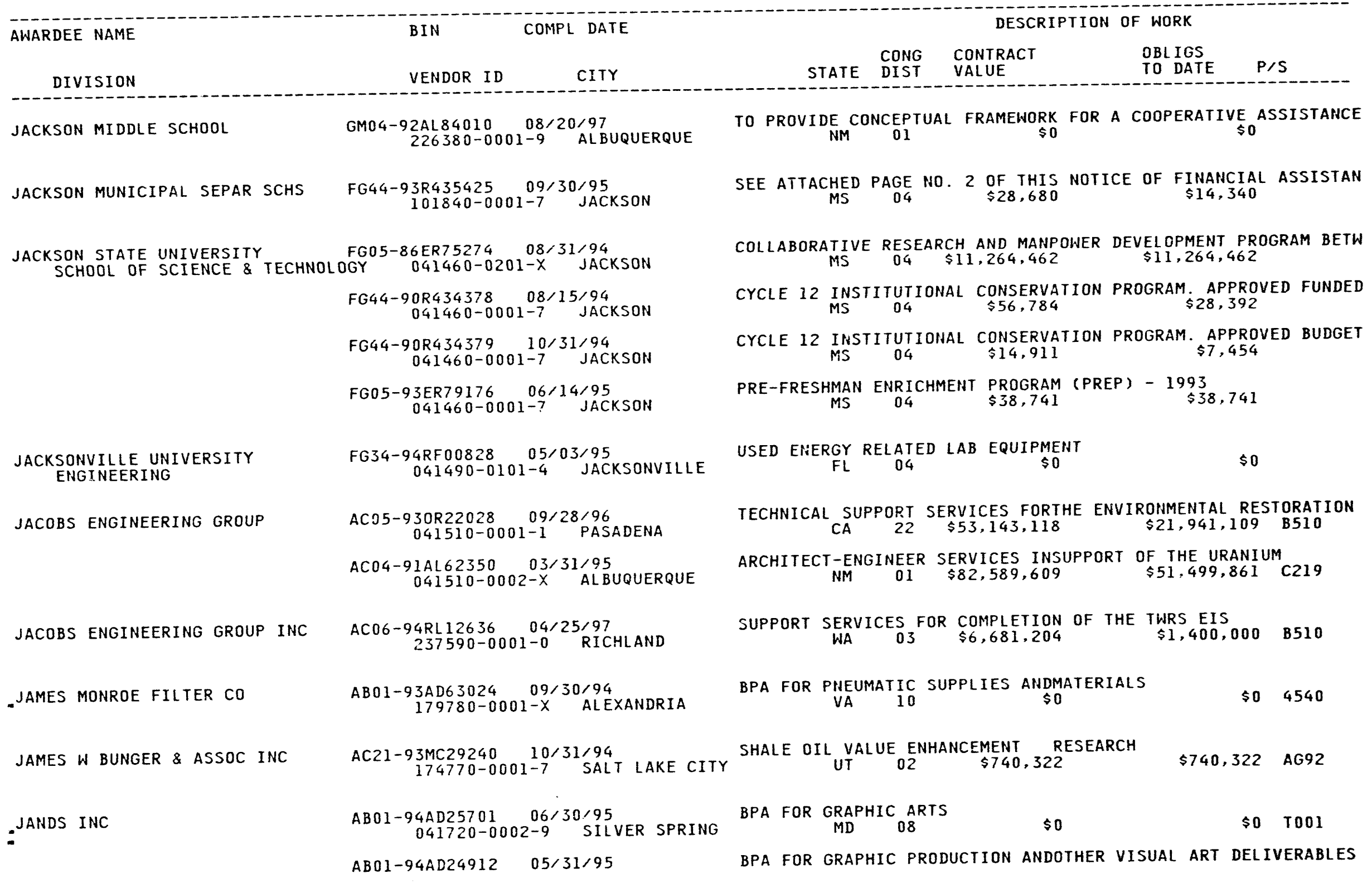




\section{AWARDEE NAME \\ DIVISION}

JASEN TODD A ESQ

JASON ASSOC CORP

JEFFERSON COUNTY AIRPORT

JEFFERSON COUNTY MEMORIAL HOSP

JEFFERSON COUNTY OF

SHERIFF

CIVIL DEFENSE

JEFFERSON CTY PUB SCH DIST R-I

JEFFERSON CTY PUBLIC SCHOOLS

JEFFERSON DAVIS PARISH SCH BD

JEMEZ MOUNTAIN SCHOOLS

JEMEZ VALLEY SCHOOLS
NAMES BY NAME, CITY \& STATE - ACTIVE AWARDS

NO 243

BIN COMPL DATE DESCRIPTION OF WORK

STATE DIST CONTRAC VALUE

TO DATE P/S

VENDOR ID CITY SILVER SPRING

$041720-0002-$

MD 08

\$0

PB/ADMINISTRATIVE HEARING OFFICER

$$
\begin{array}{lrlll}
\text { STRATIVE } & \text { HEARING } & & & \\
\mathrm{C} 0 & 02 & \$ 50,000 & \$ 8,760 & \mathrm{R} 418
\end{array}
$$

226510-0001-9 GOLDEN

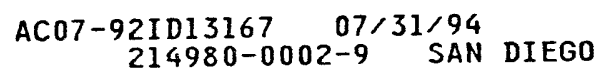

FG03-92ER81315 05/24/95 14980-0001-0 LITTLETON GMO4-80AL53652 07/11/99
$180780-0001-4$ GOLDEN

FG47-93R703514 $08 / 31 / 95$
$231730-0001-3$ FAIRBURY

GM07-93ID11121 07/02/98
$041950-0203-6$ RIGBY

GM07-93ID11122 $07 / 02 / 98$
$041950-0303-2$ RIGBY

FG34-93RF00649 $02 / 15 / 98$
$151520-0002-4$ GOLDEN

FG03-92SF19531 09/29;94 $101880-0001-1$ LOUISVILLE

FG46-93R613614 02/28/95 146020-0002-9 JENNINGS

GM04-92AL84049 07/21/97 226750-0001-9 JEMEZ SPRINGS

GM04-93AL84065 11/24/97 226760-0001-2 BERNALILLO
INTEGRATION COORDINATION CONTRACTOR SUPPORT FOR ENVIRONMENTA

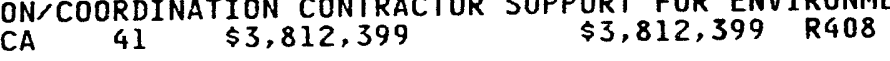

REINTERPRETATION OF WELLBORE LOG DATA USING NEURAL NETWORK B

$$
\text { CO } 06 \quad \$ 322,083 \quad \$ 322,083
$$

SET FORTH AN AGREEMENT BETWEENTHE JEFFERSON COUNTY AIRPORT

$$
\text { CO } 05
$$$$
\$ 0
$$

GRANT PROGRAMS FOR SCHOOLS ANDHOSPITALS AND FOR BUILDINGS 0 NE $\quad 03 \quad \$ 35,090 \quad \$ 17,545$

MOU TO MAINTAIN EQUIPMENT \& PERSONNEL FOR THE PURPOSE OF PRO I D 02 $\$ 0$

MOU TO MAINTAIN EQUIPMENT \& PERSONNEL FOR THE PURPOSE OF PRO ID 02

DG/TECH EDUCATION PROGRAM FOR $\mathrm{K}-12$ STUDENTS IN ARVADA WEST $\begin{array}{lll}\text { CO } 06 & \$ 311,254 & \$ 235,866\end{array}$

MATH/SCIENCE LEADERSHIP DEVELOPMENT AND RECOGNITION PROGRAM KY $03 \quad \$ 58,950 \quad \$ 49,543$

AWARDS A NEW CYCLE 15 ENERGY CONSERVATION MEASURE GRANT.

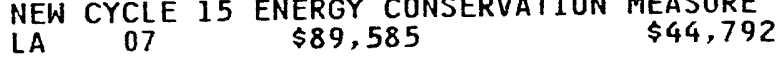

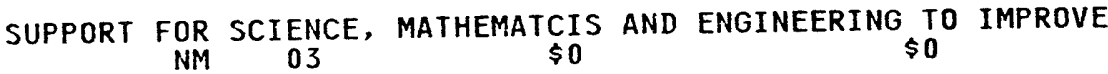
NM 03

SUPPORT FOR SCIENCE, MATHEMATICS AND ENGINEERING TO IMPROVE NM 03 


\begin{tabular}{|c|c|}
\hline AWARDEE NAME & COMPL DATE \\
\hline DIVISION & VENDOR ID \\
\hline JERICHO SCHOOL DISTRICT & $\begin{array}{c}\text { FG41-94R196601 05/31/95 } \\
238870-0001-3 \text { JERICHO }\end{array}$ \\
\hline JERRY $R$ BERGESON \& ASSOC & $\begin{array}{c}\text { ACO1-89FE61762 } 09 / 26 / 94 \\
137870-0002-X \text { TUPMAN }\end{array}$ \\
\hline \multirow[t]{2}{*}{ JESSAMINE COUNTY BD OF EDUCN } & $\begin{array}{c}\text { FG44-90R434215 07/01/94 } \\
101900-0001-5 \text { NICHOLASVILLE }\end{array}$ \\
\hline & $\begin{array}{c}\text { FG44-91R434509 07/01/94 } \\
101900-0001-5 \text { NICHOLASVILLE }\end{array}$ \\
\hline JET PROCESS CORP & $\begin{array}{c}\text { FG05-93ER81599 } 10 / 01 / 94 \\
214630-0001-4 \text { NEW HAVEN }\end{array}$ \\
\hline JIL SYSTEMS INC & $\begin{array}{c}\text { AC02-92NP00878 } 05 / 07 / 95 \\
220500-0001-4 \quad \text { ARLINGTON }\end{array}$ \\
\hline JOAQUIN MANUFACTURING CORP & $\begin{array}{c}\text { AC65-94WK15263 08/26/94 } \\
176900-0001-0 \text { COMMERCE CITY }\end{array}$ \\
\hline $\begin{array}{l}\text { JOHN BROWN E \& C INC } \\
\text { LAWRENCE ALLISON \& ASSOC WES }\end{array}$ & $\begin{array}{l}\text { ACO1-86FE60896 } 09 / 30 / 94 \\
\text { ST } 226320-0101-3 \text { CASPER }\end{array}$ \\
\hline $\begin{array}{l}\text { JOHN D ARCHBOLD HOSPITAL } \\
\text { EHGINEERING SERVICES }\end{array}$ & $\begin{array}{c}\text { FG44-93R435359 } 09 / 30 / 95 \\
232450-0101-0 \text { THOMASVILLE }\end{array}$ \\
\hline JOHA R BAYLESS CO & $\begin{array}{c}\text { FG03-92ER81290 02/17/95 } \\
214520-0001-8 \text { MALIBU }\end{array}$ \\
\hline JOHN HILE \& SONS INC & $\begin{array}{c}A C 02-77 \text { IR0 } 4219 \\
042420-0001-4\end{array}$ \\
\hline $\begin{array}{l}\text { JOHNS HOPKINS UNIVERSITY } \\
\text { ARTS \& SCIENCES SCHOOL OF }\end{array}$ & $\begin{array}{r}\text { FG02-85ER40211 } 08 / 31 / 94 \\
042440-1401-2 \text { BALTIMORE }\end{array}$ \\
\hline PHYSICS DEPT & $\begin{array}{r}\text { FG02-86ER53214 } 12 / 31 / 96 \\
042440-0801-2 \text { BALTIMORE }\end{array}$ \\
\hline
\end{tabular}

INDEPENDENT RESERVOIR ENGINEERING STUDIES AT THE NAVAL PETRO DESCRIPTION OF WORK

CYCLE 12 INSTITUTIONAL CONSERVATION PROGRAM. APPROVED FUNDED CYCLE 13 - INSTITUTIONAL CONSERVATION PROGRAM. APPROVED BUD

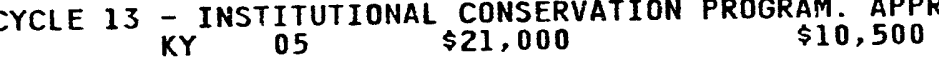

JET VAPOR DEPOSITION OF ULTRA-THIN PLATINUM CATALYST LOADING CT 03 T $\$ 75,000$ P $\$ 75,000$

DEVELOP \& IMPLEMENT AN INTERGRATED DATA PROCESSING DEVELOP \& IMPLEMENT AN $\$ 2,068,916 \quad$ INTERGRATED $\$ 1,704,389$ R408 PURCHASE VAN TRAILER $\$ 44,702 \quad \$ 44,702 \quad 2330$

MANAGEMENT \& OPERATION OF NPOSR-CUW

WY $01 \underset{\$ 91,329,711}{\$ 89,276,978}$ M299

SEE ATTACHED PAGE NO. 2 OF THIS NOTICE OF FINANCIAL ASSISTAN GA PAGE NO. 2 OF T175,000

PHASE I SBIR AWARD TO JOHN R BAYLESS CO TITLE: HIGH ENERGY

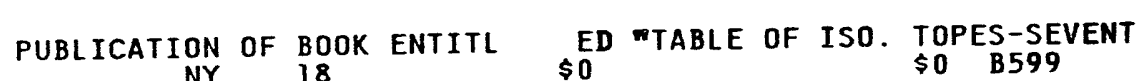
RESEARCH IN THEORETICAL PHYSICS

$\$ 985,000$

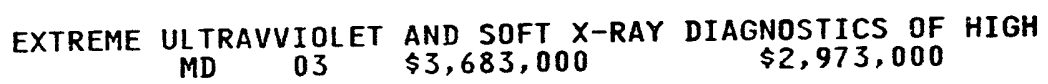




AKARDE NAME

COMPL DATE

DESCRIPTION OF WORK

AWARDEE NAME

BIN

CONG CONTRACT OBLIGS
TO

CITY

VENDOR ID FO2-87ER13777-- $02 / 28 / 97---D$
$042440-0401-7$ BALTIMORE

CHEMICAL ENG DEPT

HYGIENE \& PUBLIC HEALTH

PHYSICS DEPT

ENVIRONMENTAL HEALTH DEPT

BIOLOGY DEPT

MECHANICAL ENG DEPT

EARTH \& PLANETARY SCI DEPT

GEOGRAPHY \& ENVIRONMENTAL

CHEMISTRY DEPT

MEDICINE SCHOOL OF

BIOLOGY DEPT

MATERIALS SCI \& ENGINEERING

HYGIENE \& PUBLIC HEALTH

HYGIENE \& PUBLIC HEALTH

JOHNSON \& TOWERS INC FG02-88ER60636 01/31/96 04 TMORE
$042440-1101-3$ BALTIMORE FG02-88ER40413 12/31/94 042440-0801-2 BALTIMORE FG02-88ER60655 02/29/96 042440-1301-6 BALTIMORE FG02-89ER14011 12/31/94 $042440-0201-4$ BALTIMORE FG02-89ER14043 06/30/95 $042440-1801-8$ BALTIMORE FG02-89ERI4074 08/31/94 042440-0601-X BALTIMORE $\begin{array}{cc}\text { FG02-90ER6 0946 } 06 / 30 / 96 \\ 042440-1901-4 & \text { BALT IMORE }\end{array}$ FG02-91ER14189 06/14/97 $042440-0501-3$ BALTIMORE FC02-91ER61230 08/31/94 042440-1201-X BALTIMORE FG02-92ER20080 06/30/95 042440-0201-4 BALTIMORE FG02-94ER12140 11/30/96 042440-2001-2 BALTIMORE FG05-93ER79217 08/31/95 042440-1101-3 BALTIMORE FG43-93R353203 03/31/95 $042440-1101-3$ BALTIMORE FG02-93ERI4342 03/31/96 $042440-0002-X$ LAUREL

$A B 01-94 A D 66430 \quad 09 / 30 / 94$ $170850-0003-1$ BALTIMORE
PREDICTION OF THERMODYNAMIC--
MD
03

TO DATE $\$ 814,768$ S $\$ 635,158$ AND CARCINOGENESIS: AN INTEGRATED MD $07 \quad \$ 3,483,462 \quad \$ 2,967,430$ STUDIES OF RELATIVISTIC HEAVY ION COLLISIONS MD 07 \$1, 170,000 $\$ 1,170,000$ REGIONAL AEROSOL DEPOSTION IN HUMAN UPPER AIRWAYS HYDROGEN/SULFUR METABOLISM IN THE HYPERTHERMOPHILIC MD $07 \quad \$ 363,333 \quad \$ 363,333$

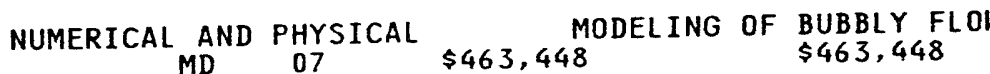
TUDY OF TRACE METALBEHAVIOR SHEET SILICATE REACHRTEM/AEM MDTODY $03 \quad \$ 536,844 \quad \$ 536,844$ REDUCTIVE OBILIZATION OXIDE - BOUND METALS $\$ 342,173$ THEORETICAL STUDIES OF NONADIABETIC \& SPIN-FORBIDDEN PROCESS MD $07 \quad \$ 604,500 \quad \$ 424,500$ DEVELOPMENT OF A HUMAN GENOME DATA BASE. $\$ 8,707,000$ HOW NITRITE, AMMONIA \& SUFITE CROSS THE INNER ENVELOPE MD 07 \& $\$ 309,000 \quad \$ 210,000$ ULTRA SONIC AND DIELECTRIC NONINVASIVE DIAGNOSTICS FOR MD 07 \$990,095 $\$ 358,006$ 1993 UNIVERSITY INSTRUMENTATION PROGRAM - BIOIMAGING INSTRUM MD 07 \$162,000 \$162,000

TITL III OF THE NATIONAL ENERGY CONSERVATION POLICY ACT MD 03 \$160,000 $\$ 107,200$ Q-BRANCH RAMAN SCATTERING AND MOOBRN KINETICS THEORY $M D=55 \quad \$ 284,000 \quad \$ 184,150$

EMERGENCY DIESEL GENERATOR SYSTEM 


\begin{tabular}{|c|c|}
\hline AWARDEE NAME & BIN COMPL DATE \\
\hline DIVISION & VENDOR ID \\
\hline $\begin{array}{l}\text { JOHNSON CTRLS WORLD SVCS INC } \\
\text { LABORATORY SUPPORT DIVISION }\end{array}$ & $\begin{array}{c}\text { AC05-900R21907 09/27/95 } \\
207380-0101-5 \text { OAK RIDGE }\end{array}$ \\
\hline JOHNSON MEMORIAL HOSPITAL & $\begin{array}{c}\text { FG45-93R551586 } 03 / 31 / 95 \\
232550-0001-7 \text { FRANKLIN }\end{array}$ \\
\hline JOHNSON VAUGHN & $\begin{array}{c}\text { RL 07-78ID01826 09/30/99 } \\
042590-0001-2 \text { NEWTON }\end{array}$ \\
\hline JOHNSTON \& ROACH & $\begin{array}{c}\text { ABO4-93AL84940 } 10 / 20 / 94 \\
217190-0002-6 \quad \text { PASCO }\end{array}$ \\
\hline JOINT SCHOOL DISTRICT $\# 60$ & $\begin{array}{c}\text { GM07-94ID11155 } 03 / 17 / 99 \\
114640-0001-8 \text { SHELLEY }\end{array}$ \\
\hline JOINT VENTURE ORGANIZATION II & $\begin{array}{c}A C 01-94 F E 62717001 / 31 / 9 ? \\
178100-0003-9 \text { G :HERSBURG }\end{array}$ \\
\hline JONES HI TECH INC & $\begin{array}{c}\text { AC65-93WA11097 } 04 / 01 / 95 \\
234530-0001-3\end{array}$ \\
\hline JORWAY CORP & $\begin{array}{c}\text { FG02-90ER81026 05/19/95 } \\
101910-0001-9 \text { WESTBURY }\end{array}$ \\
\hline JSA HEALTHCARE CORP & $\begin{array}{c}\text { ADO1-93AD15031 } 09 / 30 / 94 \\
234290-0001-7 \text { COLUMBIA }\end{array}$ \\
\hline \multirow[t]{5}{*}{ JUPITER CORP } & $\begin{array}{c}\text { ACO1-93ANO0028 } 09 / 12 / 94 \\
206890-0001-4 \quad \text { WHEATON }\end{array}$ \\
\hline & $\begin{array}{c}\text { AC01-93EP79133 10/27/94 } \\
206890-0001-4 \text { HHEATON }\end{array}$ \\
\hline & $\begin{array}{c}\text { ACO1-93EH60001 } 12 / 14 / 94 \\
206890-0001-4 \text { WHEATON }\end{array}$ \\
\hline & $\begin{array}{c}\text { ACO2-91NP00115 } 03 / 31 / 95 \\
206890-0001-4 \text { WHEATON }\end{array}$ \\
\hline & ACO1-94NE20842 \\
\hline
\end{tabular}
COMPL DATE

\section{DESCRIPTION OF HORK}

STATE DIST VALUE
OPERATION AND MAINTENANCE OF THE ORO WATER PLANT AND OTHER
TN $03 \quad \$ 40,188,873$ ICP-NEW AWARD.

$\underset{I N}{\operatorname{IN}} 06 \quad \$ 91,010 \quad \$ 45,505$

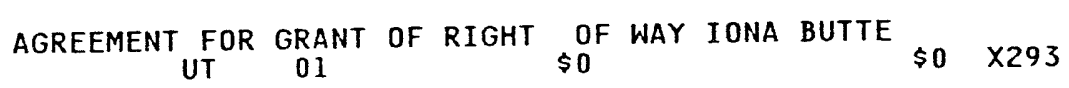
BLANKET PURCHASE AGREEMENT - HEARING OFFICER

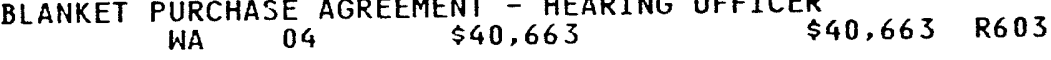
STATE THE BASIC UNDERSTANDING OF THE PARTIES REGARDING, \& PR TECHNICAL SUPPORT SERVICES FORTHE OFFICE OF GAS AND PETROLE TECHNICAL SUPPORT SERVICES FORTHE OFFICE OF GAS AND PETROLE
MD 08
$\$ 3,062,792$

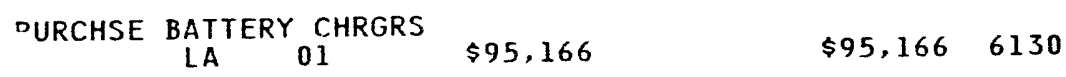

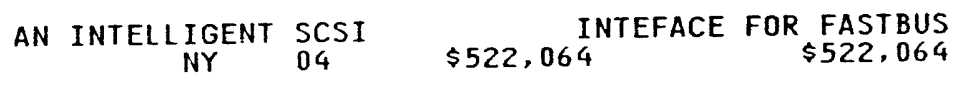
COLLECTION AND TRANSPORTATION OF URINE SPECIMENS FOR LABORAT MD $05 \quad \$ 118,381 \quad \$ 60,000$ SUPPORT SERVICES TECHNICAL \& ADMINISTRATIVE SUPPORT SERVICE $\begin{array}{llll}M D & 08 & \$ 934,363 & \$ 934,363 \\ \text { R421 }\end{array}$ $\begin{array}{cccc}\text { TECHNICAL SUPPORT } & \text { SERVICES TO THE OFFICE OF EP } \\ \text { MD } 08 & \$ 1,587,033 & \$ 267,000 & R 406\end{array}$ TO PROVIDE TECHNICAL AND SUPPORT SERVICES TO THE OFFICE OF ENGINEERING DESIGN OVERSIGHT, TECHNICAL ANALYSIS SYSTEMS, SY SPECIALIZED TECHNICAL \& ADMINISTATIVE SUPPORT SERVICES TO TH 


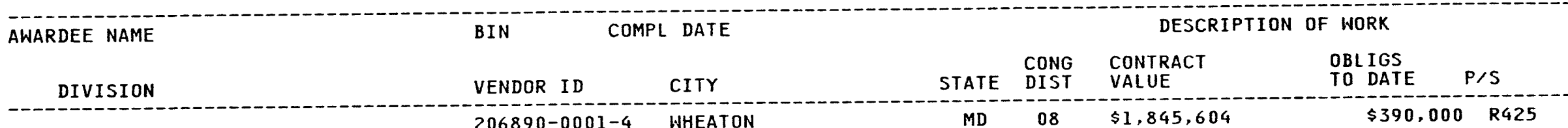

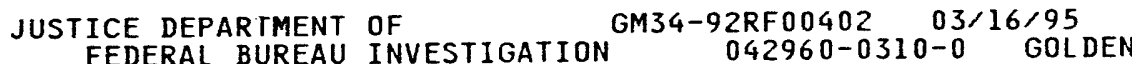
AI 01-91AD32015 09/30/96 042960-0001-2 WASHINGTON AI01-93AD34011 09/30/98 042960-0001-2 WASHINGTON GM07-76ID11013 09/30/99 FEDERAL BUREAU INVESTIGATION $042960-0301-1$ WASHINGTON GM04-83AL53647 06/15/99 FEDERAL BUREAU INVESTIGATION U S ATTORNEY'S OFFICE GMO4-83AL 53648 02/14/99 GM07-91ID11078 06/12/97 UNITED STATES MARSHAL SERVICE $942960-0709-2$ BOISE GM33-85AL53641 10/30/99 FEDERAL BUREAU INVESTIGATION $042960-0307-0$ KANSAS CITY GM04-91AL58218 06/19/96

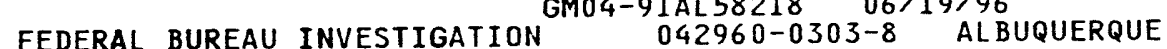
GM04-83AL $3865209 / 30 / 99$ FEDERAL BUREAU INVESTIGATION 042960-0303-8 ALBUQUERQUE GM04-84AL $3865509 / 30 / 99$ FEDERAL BUREAU INVESTIGATION $042960-0303-8$ ALBUQUERQUE GM04-93AL84022 01/19/98 042960-0803-X ALBUQUERQUE GM04-93AL84046 01/04/98 042960-0303-8 ALBUQUERQUE FEDERAL BUREAU INVESTIGATION GM32-76AL53627 06/11/99 FEDERAL BUREAU INVESTIGATION $042960-0306-2$ LOS ALAMOS GM32-76AL53626 01/30/99 - federal bureau investigation
MOU WITH FBI $\underset{\text { CO }}{\text { FB }}$

$\$ 0$

$\$ 0$

ACCESS TO JURIS ON-LINE DATA BASE

$\$ 49,300$ ACCESS TO THE NATIONAL CRIME INFORMATION CENTER AND THE NATI
DC 01

MEMORANDUM OF UNDERSTANDING BETHEEN THE FBI, DOE $\&$ DOD FOR $\mathrm{DC} \quad \mathrm{O}$

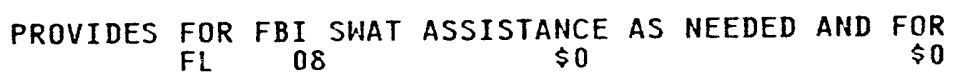
TO RECOGNIZE THAT THE AO WILL COORDINATE PROSECUTION OF INEL FIRING RANGE USE AGREEMENT $\$ 0$ PROVIDES A CLEAR BASIS FRO CONTINGENCY RESPONSE PLANNING, TO DEAL EFFECTIVELY WITH THREATS, AND CRIMINAL $\underset{\$ 0}{\text { ACTS }} \underset{\$ 0}{\text { ASSOCIAT }}$

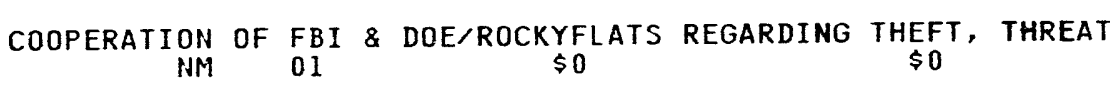

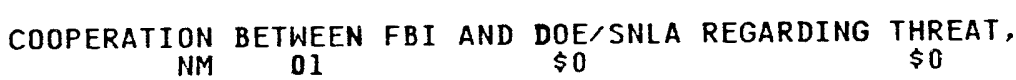
THIS AGREEMENT SETS FORTH THE GENERAL POLICY FOR THE CTA THIS AGREEMENT SETS FORTH THE GENERAL POLICY FOR THE CTA SETS FORTH RESPONSIBILITIES OFEACH AGENCY WITH REGARD TO SUPPORT TO BE RENDERED TO LANL $\underset{03}{\$ 0}$ DOE COMPLEX DURING LIMITED MUTUAL ASSISTANCE AND INCIDENTRESPONSE AND RESOLUTION 


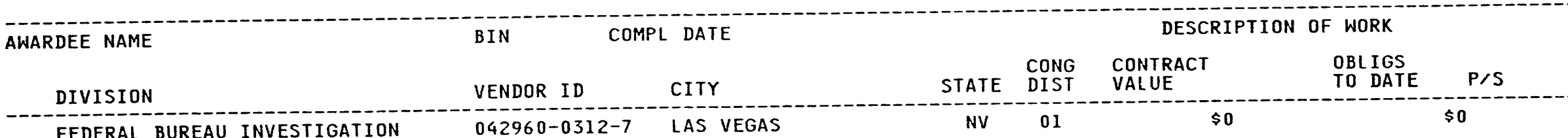

FEDERAL BUREAU INVESTIGATION

FEDERAL BUREAU INVESTIGATION

GM04-88AL53616 09/09/99

GM04-85AL53622 01/04/99

FEDERAL BUREAU INVESTIGATION 04-85A2960-0305-4 AMARILLO

$K$ \& M ENG \& CONSULTING CORP

K-RAY INC

KAHN-OPPENHEIMER INC

KAI TECHNOLOGIES INC

KAISER ENGINEERS INC

KAISER FOUNDATION HEALTH

\section{KAISER PERMANENTE}

MOANALUA MEDICAL CENTER

- KALAMAZOO PUBLIC SCHOOL DIST

KALAMAZOO VALLEY INT SCH DIST

KANSAS CITY CITY OF

POLICE DEPT
AC21-90MC27346 09/30/94
$203890-0001-9$ WASHINGTON

AC21-94MC30330 04/28/95 237500-0001-8 MORGANTOHN

AB01-94AD25718 06/30/95 $183440-0001-7$ WASHINGTON

AC21-94MC29249 $02 / 08 / 95$

AC22-88PC88881 $09 / 30 / 94$ $178120-0001-X \quad$ PITTSBURGH

$\begin{array}{cc}\text { FG } 49-91 R 913029 & 02 / 28 / 95 \\ 176910-0001-4 & \text { OAKLAND }\end{array}$

FG49-93R915027 08/31/95 232280-0101-6 HONOLULU

FG45-92R540212 01/31/95 $043150-0001-9$ KALAMAZOO

FG45-93R551638 08/31/95 232810-0001-0 KALAMAZOO

GM33-87AL53635 02/11/99 $043220-0201-3$ KANSAS CITY
CONFIRMS THE GENERAL PRACTICE, PROCEDURE AND AUTHORITY THAT $O H$ O 03 \$O PROVIDES $\underset{T X}{\text { BASIS }} \underset{13}{\text { FOR CONTINGENCY- RESPONSE PLANNING, COORDINA }}$

TECHNICAL AND ENGINEERING $\underset{\text { DC }}{01} \underset{\$ 6,848,407}{\text { ASSISTANCE FOR THE OFFICE OF }}$

BUILDING CLEANING AND REPAIR

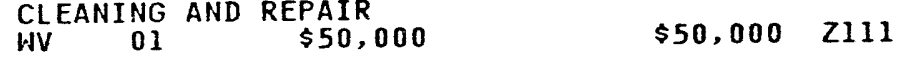

BPA FOR METAL ENGRAVING

$\$ 0 \quad 9999$

EM - RESEARCH TITLED "ELECTRO-MAGNETIC MIXED WASTE $\begin{array}{cccc}\text { MA } & 07 & \$ 618,270 & \$ 499.932\end{array}$ AH92

ENGINEER DEVELOPMENT OF ADVANCED PHYSICAL FINE COAL CLEANING PA $14 \quad \$ 8,871,427 \quad \$ 8,871,427$ AG11

CYCLE XIII GRANT

$\$ 776,901$

CYCLE XV ECM GRANT AWARD

$$
\begin{array}{lcrr}
\text { ECM } & \text { GRANT } & \text { AWARD } \\
\text { HI } & 01 & \$ 245,808 & \$ 122,904
\end{array}
$$

ICP - NEW AWARD.

$\$ 170,445$

$\$ 85,222$

ICP-NEW AWARD.

MI $06 \quad \$ 141,410$

$\$ 70,705$

PROVIDES FOR THE LOAN OF 2 LIGHT ARMORED VEHICLES TO THE 


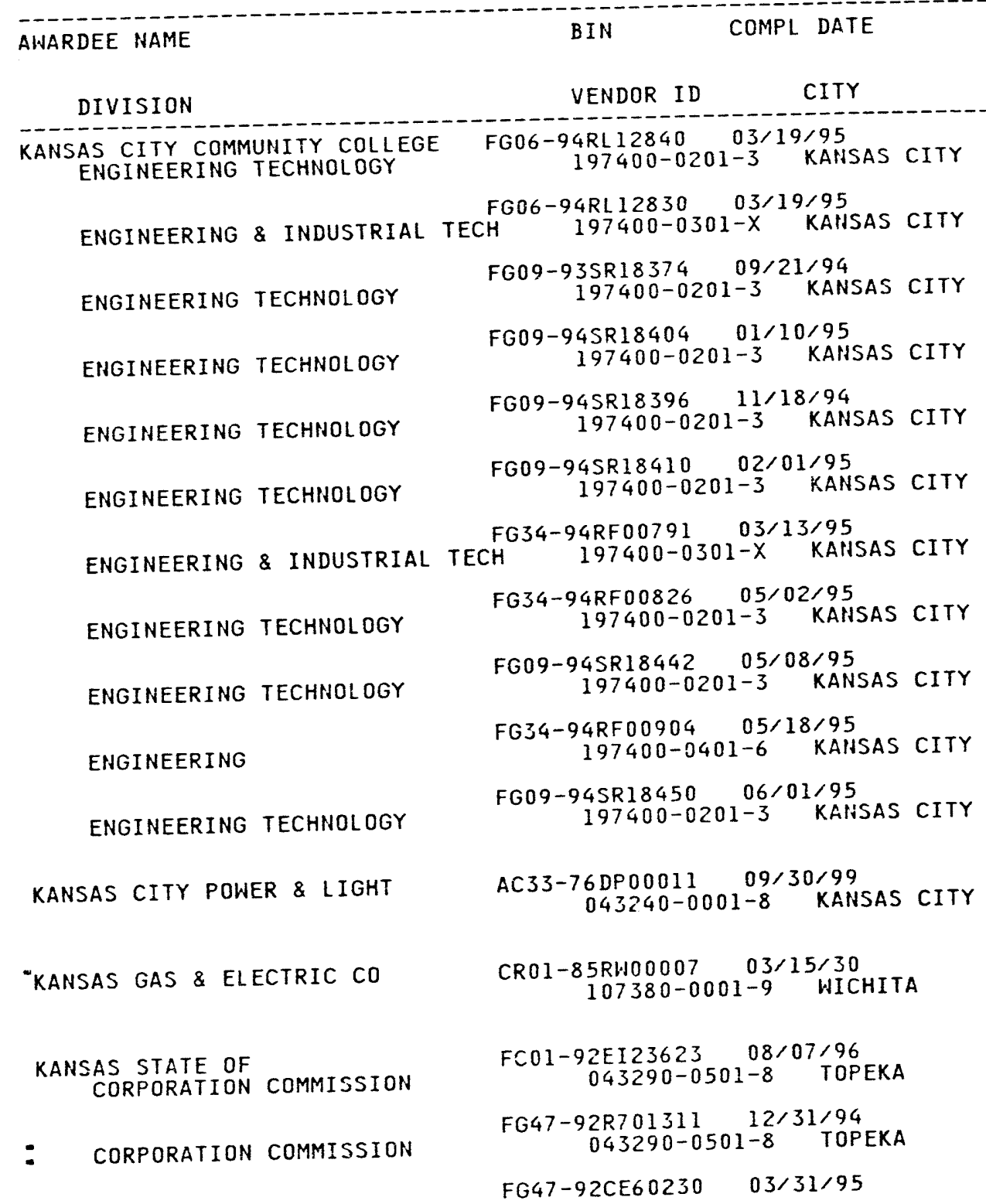

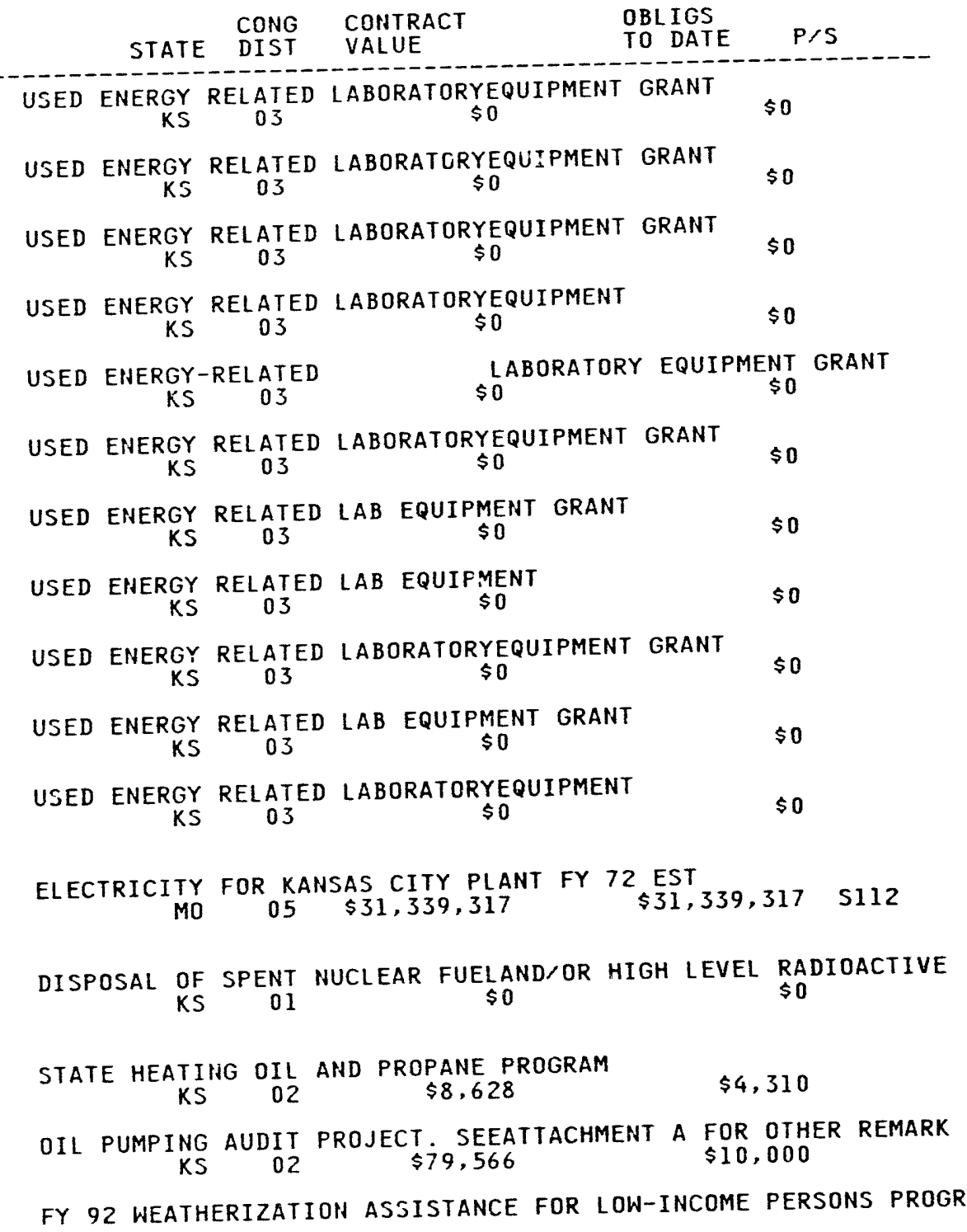




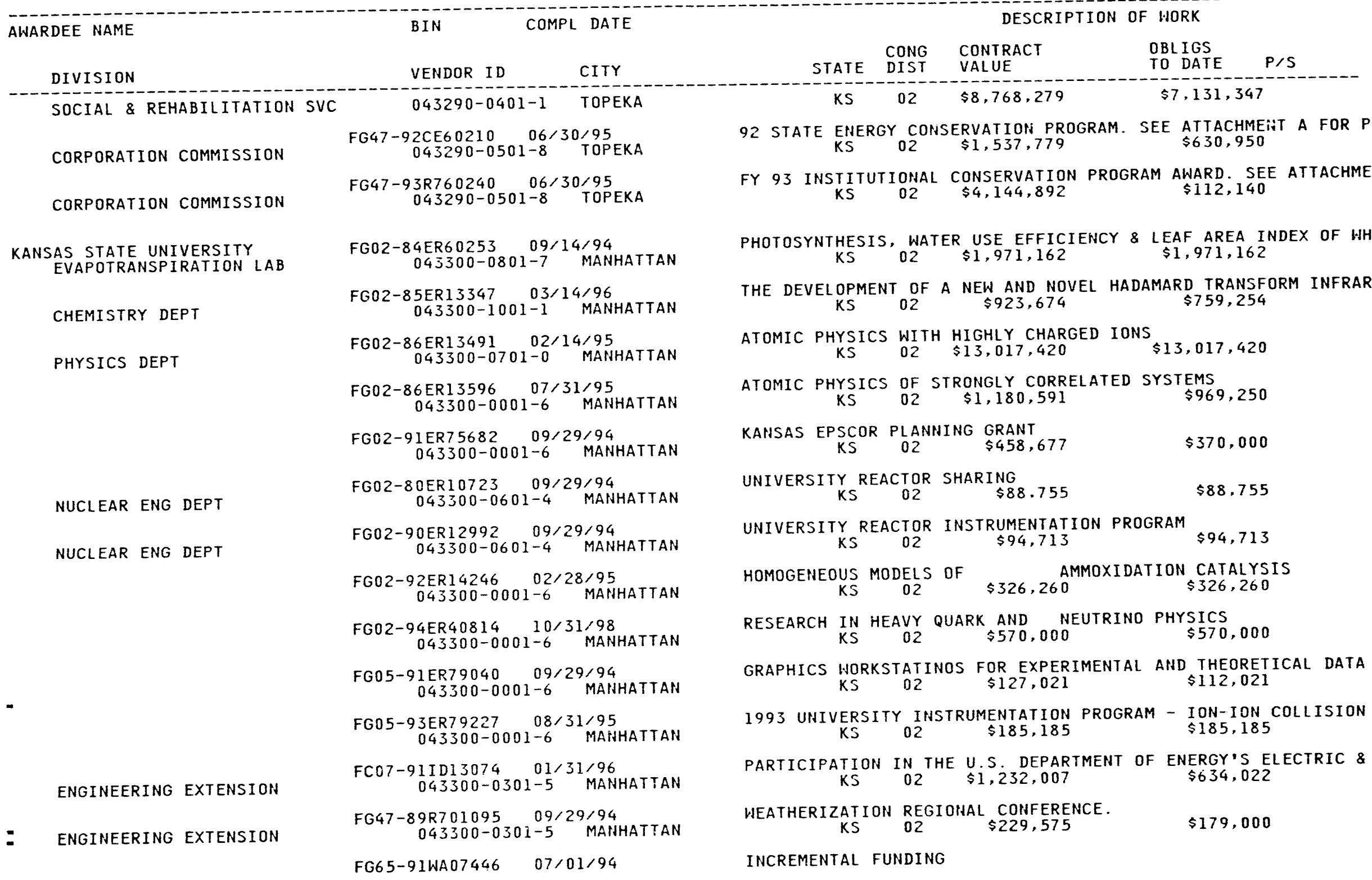




\section{AWARDEE NAME \\ DIVISION}

BIN \\ DESCRIPTION OF WORK}

VENDOR ID

VENDOR ID

CITY

$043300-0001-6$ MANHATTAN

KANSAS UNIVERSITY OF

MEDICAL CENTER

PHYSICS DEPT

PHYSICS \& ASTRONOMY DEPT

CHEMICAL \& PETRO ENG DEPT

RESEARCH INC CENTER FOR

RESEARCH INC CENTER FOR

\section{KAPIOLANI MEDICAL CENTER}

KAPL INC

:

KAW CITY PUBLIC SCHOOLS

$$
\begin{gathered}
\text { FG02-92ER61392 03,31/95 } \\
043310-0002-8 \quad \text { KANSAS CITY }
\end{gathered}
$$

FG47-93R702704 08/31/95 043310-1002-3 KANSAS CITY

FG01-91CE15511 09/25/94 $043310-0001-x$ LALRENCE

FG02-85ER40214 04/30/95 $043310-0801-0$ LAWRENCE

FG02-89ER40506 04/30/96 $043310-0401-5$ LAWRENCE

FGO2-90ER $1415901 / 14 / 96$ 043310-0001-X LAWRENCE

FG02-94ER25212 05/31/95 $043310-0001-x$ LAWRENCE

FG02-93ER79177 06/14/95 $043310-0001-X$ LAWRENCE

AC22-92BC14881 09/24/95 $043310-0101-6$ LAHRENCE

FG22-92PC92532 07/06/94 043310-1101-1 LAHRENCE

FC22-93BC1495? 12/31/98 $3 B C 1495712 / 31 / 98$
$043310-1101-1 \quad$ LAWRENCE

GMO4-94AL94728 06/19/99

LAWRENCE

FG49-93R915028 $08 / 31 / 95$
$232390-0001-6$ HONOLULU

AC12-76SN00052 09/30/95 6 SN00052
$231540-0001-1$ SCHENECTADY

FG46-93R613587 08/31/94
STATE CONG CONTRACT

KS $02 \quad \$ 48,068$

OBL IGS TO DATE P/S $\$ 48,068$

HUMAN GENETICS EDUCATION FOR MIDDLE ANS SECONDARY SCIENCE KS $03 \quad \$ 594,000 \quad \$ 594,000$

GRANT PROGRAMS FOR SCHOOLS ANDHOSPITALS AND FOR BUILDINGS 0 KS $03 \quad \$ 83,200 \quad \$ 4,658$

OPTIMIZATION AND SCALE UP OF FERMENTATION PROCESS FOR PRODUC KS 02 \$110.000 $\$ 95.000$

POLE MODEL PREDICTIONS FOR WEAK AND ELECTRO-WEAK DECAYS OF $H$ KS 02 \$961,000 $\$ 961,000$

DETECTOR DEVELOPMENT AND RESEARCH IN HEAVY-ION NUCLEAR

KS $02 \quad \$ 728,000 \quad \$ 525,000$

ATOMIC PHYSICS IH STRONG $\underset{\text { KS }}{02}$ FIELDS $\$ 409,000 \quad \$ 313,000$

WORKSHIP ON ALGORITHMS FOR MACROMOLECULAR MODELING

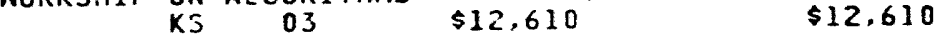

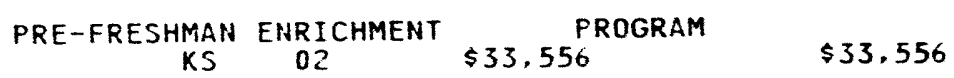

IMPROVING RESERVOIR CONFORMANCE USING GELLED POLYMER SYSTEMS KS 02 \$1,311.568 $\$ 707,123$ AGI1

FISCHER-TROPSCH SYNTHESIS IN SUPERCRITICAL REACTION MEDIA KS 02 \$149,033 $\$ 140,000$

IMPROVED DIL RECOVERY IN FLUVIAL DOMINATED DELTAIC RESERVOIR

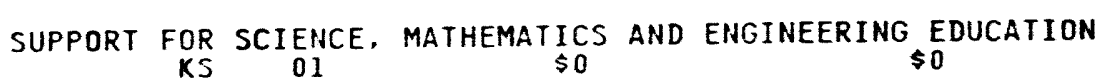

CYCLE XV $\underset{\text { HI }}{\text { ECM }} \underset{01}{\text { GRANT. }} \quad \$ 117,725 \quad \$ 58,426$

OPERATE AND MAINTAIN GOVERNMENT-OWNED LABORATORY AND CONDUCT NY $23 \$ 5,181,240,230 \quad \$ 5,176,617,838$ M181

AWARDS A NEW CYCLE 15 TECHNICAL ASSISTANCE GRANT. IT INCLUD 


AWARDEE NAME
DIVISION

KAY ELECTRIC COOPERATIVE

KELASTIC MINE BEAM CO

KELLY TEMPORARY SERVICES

KEMPER MILITARY SCH \& JR COLL ACADEMIC AFFAIRS

KENNEBEC VALLEY MEDICAL CENTER

KENROB \& ASSOC INC

\section{KENSINGTON ASSOC}

KENT STATE UNIVERSITY PHYSICS DEPT

PHYSICS DEPT

- liquid cRystal institute

\section{KENTON CITY OF} SCHOOLS

$\Xi^{\text {KENTUCKY COMMONWEALTH OF }}$

BIN COMPL DATE

DESCRIPTION OF WORK

VENDOR ID CITY

KAW CITY

$232140-0001-2$

STATE

DIST

ONG
CONTRA
VALUE

URACT
$\$ 7,720$

OBLIGS

TO DATE P/S

AC02-93CH10546 06/30/98
$229330-0001-6$ BLACKWELL

FG01-93CE15394 09/21/95 234610-0001-9 GREENSBURG

AB01-94PP00184 11/30/94 236110-0001-9 WASHINGTON

FG06-94RL12761 03/13/95 236930-0101-4 BOONVILLE

FG41-91R192307 12/31/94 $043600-0001-4$ AUGUSTA

AC01-94RW00267 05/22/96 237890-0001-9 WASHINGTON

ABO1-94MI10460 04/30/95 218250-0001-8 SILVER SPRING

FG02-86ER40251 12/31/96 $043750-0201-8$ KENT

FG02-89ER40531 08/14/94 $043750-0201-8$ KENT FG45-92R530276 09/30/94
$043750-0301-4$ KENT

FG45-93R551762 06/30/95 $233390-0101-4$ KENTON

FG44-93R410611 09/30/94 $209730-0001-5$ FRANKFORT TO PROVIDE ELECTRIC UTLILTY SERVICES TO THE ARM PROGRAM OK 05 SO

DEVELOPMENT OF THE VARIABLE WALL MINING MACHINE FOR THE MARK PA 12 \$146,627 $\$ 83,000$

BPA FOR SECRETARIAL SUPPORT $\underset{\text { SERVICES }}{\$ 0}$

\$0 R609

USED ENERGY RELATED LABORATORYEGUIPMENT GRANT MO 06 $\$ 0$

\$0

TITLE III OF THE NATIONAL ENERGY CONSERVATION POLICY ACT.

$$
\text { ME } 01 \text { \$81,156 } \$ 36,961
$$

COMMUNICATIONS NETWORK AND CO MPUTER OPERATIONS SUPPORT SERV DC $01 \$ 14,516,622 \quad \$ 1,200,000$ R425

BPA FOR EEO INVESTIGATIONS 05/01/94-04/30/95 $\begin{array}{cr}\text { EEO INVESTIGATIONS } & 05 \% 01 \\ \text { MD } & 08\end{array}$

\$0 R499

THEORETICAL INTERPRETATION OF DATA OBTAINED IN HIGH ENERGY $O H=11 \quad \$ 632,958 \quad \$ 467,958$

NUCLEUS-NUCLEUS COLLISIONS ANDTHE NUCLEAR EQUATIOIN OF STATE $\mathrm{OH} 11 \quad \$ 444,000 \quad \$ 444,000$

POLYMER DISPERSED FERROELECTRIC SMECTIC-C* DISPLAY TECHNOLOG OH 11 \$191,551 $\$ 99,993$

ICP-NEW AWARD.

$\mathrm{OH}^{04} \quad \$ 31,006$

$\$ 15,503$

ALTERNATE FUEL WRECKER DEMONSTRATION $\underset{K Y}{\text { FUEL }} \underset{03}{\text { WRECKER }} \begin{gathered}\text { DEMONSTRA } \\ \$ 51,000\end{gathered}$ 


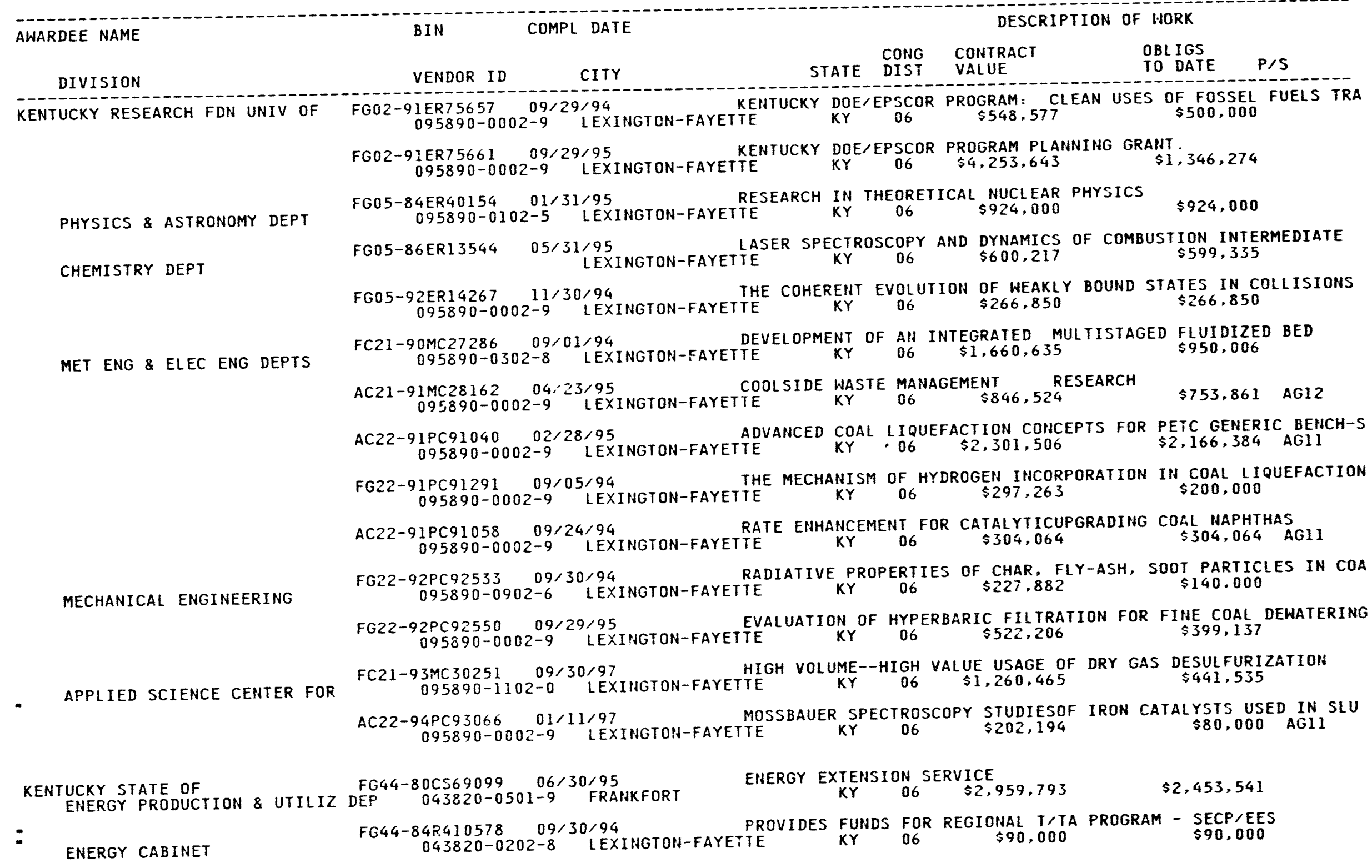




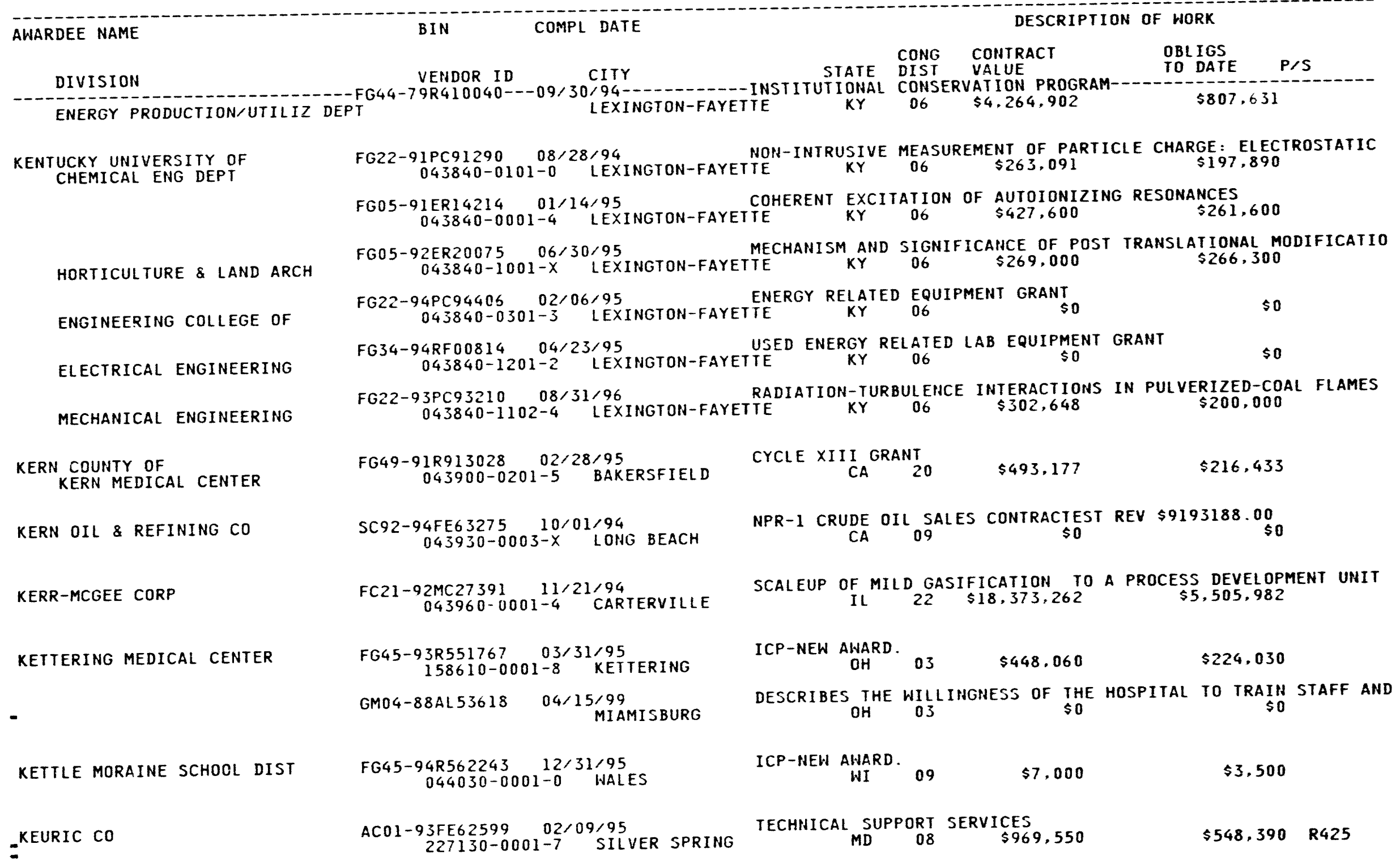




\section{AWARDEE NAME}

KEVRIC CO INC

KEYSTONE CONSTRUCTION INC

\section{KIFI TELEVISION}

KIMBALL COUNTY HOSPITAL

KIMBERLY SCHOOL DISTRICT $\$ 414$

KIMCO INCORPORATED

KING PATRICK T

KING'S DAUGHTERS HOSPITAL

-KINGMAN COMMUNITY HOSPITAL

KINGSWELL INC

EKIRA INC
BIN

COMPL DATE

\section{DESCRIPTION OF WORK}

VENDOR ID CITY SONG CONIRACT VALUE

OBLIGS TO DATE $P / S$

PROVIDE SPECIALIZED TECHNICAL AND ADMINISTRATIYE SUFPORT

ACO1-93DP40003 12/31/94 $217960-0001-9$ SILVER SPRING

PROVIDE SPECIALIZED TECHNICAL AMD

RATIVE SUFPOR

$4 W 10439208 / 01 / 94$

$4 W L 0439208 / 01 / 94$
$225150-0001-0$
MONTROSE

MONTROSE POWER OPERATIONS CENTER DISPATCH OFFICE EXPANSION \&

$61001110 \quad 09 / 30 / 99$

GRANT OF RIGHT OF HAY FOR INELTELEMETRY STATION CO $03 \quad \$ 52,744 \quad \$ 52,744 \quad$ Y299

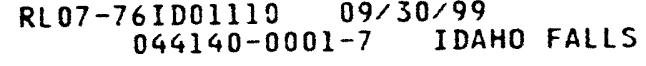

GRANT PROGRAMS FOR SCHOOLS ANDHOSPITALS AND FOR BUILDINGS 0 NE $\quad 03 \quad$ \$60,304 $\$ 30,152$

CYCLE 14 ECM AWARD.

$$
\$ 7,150
$$

$\$ 3,575$ $\begin{array}{ccccc}\text { CONCRETE } & \text { APRON WATERTOHN } \\ \text { SD } & 01 & \$ 11,000 & \text { SERVICE CENTER, SD } & \text { SP } \\ \$ 11,000 & \text { Y239 }\end{array}$

PROVIDE SUPPORT SERVICES TO OFFICE OF PATENTS IN THE OFFICE

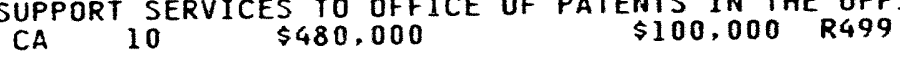

ICP-NEW AWARD.

$\$ 17,500$

$\$ 8,750$

CYCLE 14 - INSTITUTIONAL CONSERVATION PROGRAM. APPROVED BUD $\begin{array}{lll}\text { MS OS } \$ 78,860 & \$ 35,000\end{array}$

CYCLE 13 - INSTITUTIONAL CONSERVATION PROGRAM. APPROVED BUD MS $05 \quad \$ 62,250 \quad \$ 30,000$

GRANT PROGRAMS FOR SCHOOLS ANDHOSPITALS AND FOR BUILDINGS 0 KS $04 \quad$ \$58,987 $\$ 29,493$

BPA FOR AIR CONDITIONING SUPPLIES AND MATERIALS $\underset{\$ 0}{\$ 0} \underset{\text { MD }}{4120}$

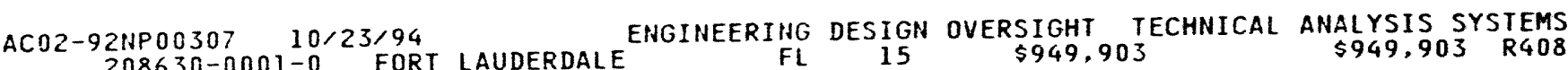


$R-0420953-001$

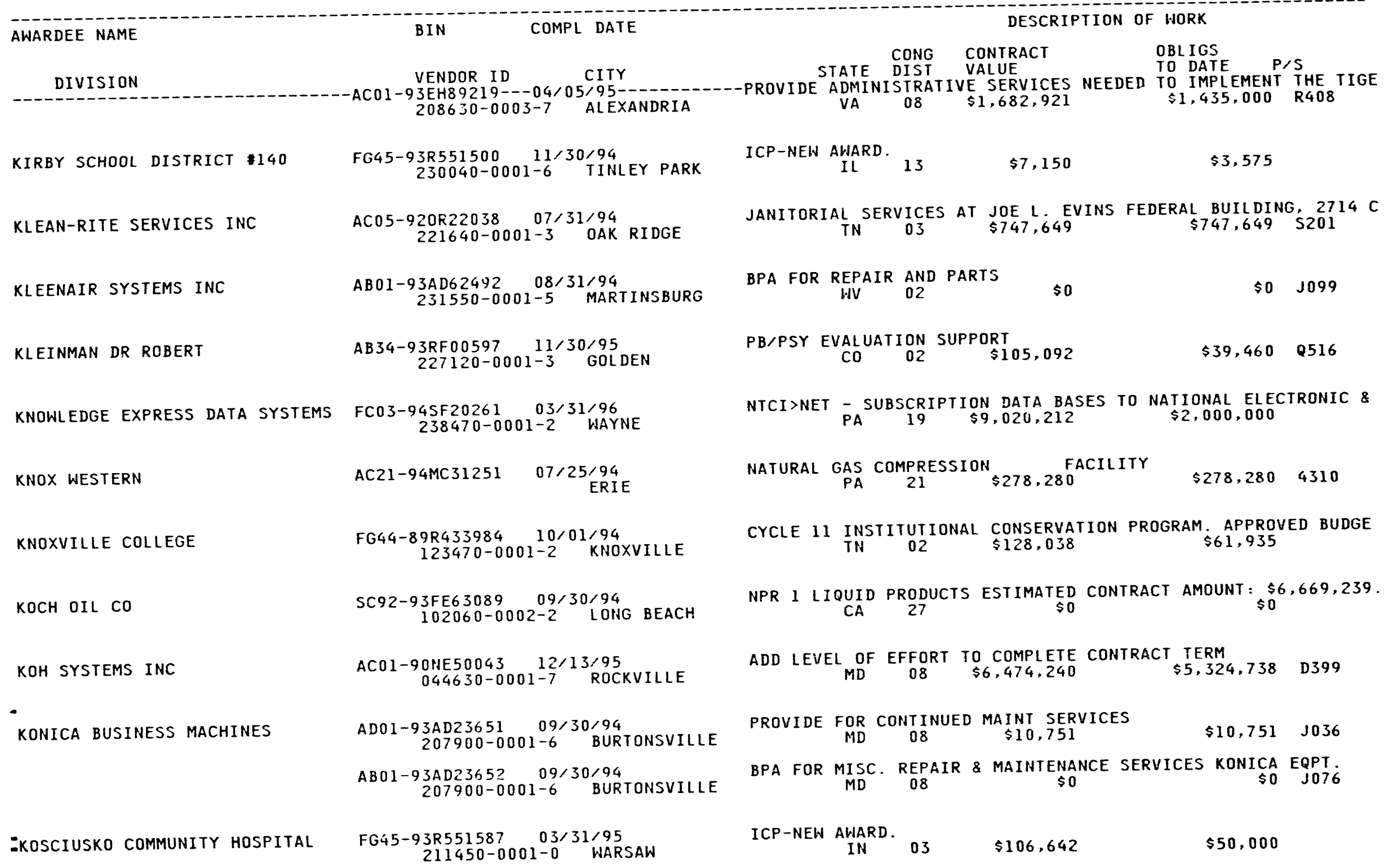




\section{AWARDEE NAME}

BIN

COMPL DATE

VENDOR ID

CITY

CONG DIST VALU

DESCRIPTION OF WORK

KOSSUTH COUNTY HOSPITAL

FG47-92R704986 09/01/94

$212610-0001-3$ ALGONA

KPI CORP

KPMG PEAT MARWICK

\section{KRA CORP}

\section{KRALL ASSOC}

$L$ \& S DIESEL SERVICE INC

LA CROSSE SCHOOL DISTRICT

LA CUEVA VOLUNTEER FIRE DEPT

LA JOYA INDEPENDENT SCH DIST

$$
\begin{aligned}
& \begin{array}{c}
\text { AC65-94WN1 } 424809 / 29 / 94 \\
176960-0002-0 \text { ANAHEIM }
\end{array} \\
& \begin{array}{cc}
\text { AC65-93WG23862 07/01/94 } \\
176960-0002-0 \text { ANAHEIM }
\end{array} \\
& \text { AC65-94NG24083 12/31/96 } \\
& \begin{array}{l}
4 \text { WG24083 } 12 / 31 / 96 \\
176960-0002-0
\end{array} \\
& \begin{array}{c}
\text { ACOI-93IG00312 06/20/95 } \\
229030-0001-8 \text { WASHINGTON }
\end{array} \\
& \text { ACO1-94RW0029103/31/96 } \\
& \text { 229030-0001-8 WASHINGTON }
\end{aligned}
$$

AC01-93EI23799 01/02/98 $231060-0001-5$ SILVER SPRING

FG03-88ER53280 10/31/96 $17 / 31 / 96$
$178150-0001-0$ DEL MAR

AB01-93AD66357 09/30/94 170880-0001-6 GLEN BURNIE AB01-93AD63026 09/30/94 $170880-0001-6$ GLEN BURNIE

$$
\begin{gathered}
\text { FG45-94R562242 } 12 / 31 / 95 \\
045010-0001-5 \text { LA CROSSE }
\end{gathered}
$$

GM32-94AL $96930 \quad 11 / 30 / 98$ $236230-0001-9$ LOS ALAMOS

FG46-93R613627 08/31/94 $045060-0001-3$ LA JOYA
SWITCHBOARDS FOR TRACY SUB, STAGE O4

"A PROGRAM OF FRC THEORY RESEARCH"
GRANT PROGRAMS FOR SCHOOLS ANDHOSPITALS AND FOR BUILDINGS 0 IA $\quad 06 \quad$ \$48,626 $\$ 24,313$ CA $38 \quad \$ 730,068$

$\$ 730,068 \quad 6150$ SWITCHBRDS FOR
CA
38 $\underset{\$ 364,497}{\text { SUBST }}$ $\$ 364,497 \quad 6150$

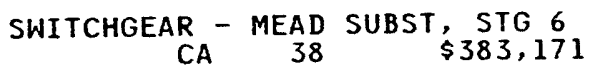
$\$ 383,171 \quad 6150$

NATIONWIDE AUDIT SUPPORT SERVICES FOR THE OFFICE OF INSPECTO $\begin{array}{lll}\text { DC } 01 \quad \$ 22,638,091 & \$ 2,959,400 & R 704\end{array}$ PROVIDE PROFESSIONAL AUDIT SERVICES FOR THE OFFICE OF CIVILI DC $01 \quad \$ 729,654 \quad \$ 35,000$ R408

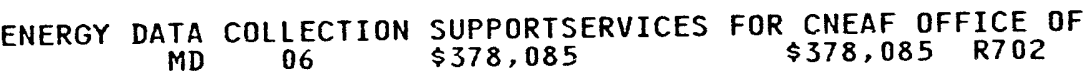

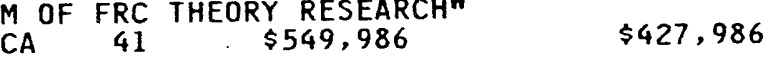

BPA FOR MAINTENANCE OF EMERGENCY DIESEL GENERATOR $\begin{array}{cllll}\text { MD } & 02 & \$ 0 & \$ 0 & J 036 \\ \text { TOOLS AND TEST } & \begin{array}{l}\text { EQUIPMENT } \\ \text { MD }\end{array} & \$ 0 & \$ 0 & 9999\end{array}$ ICP-NEW AWARD.
WI $\begin{array}{lll}\text { MUTUAL AID ASSISTANCE } & \\ \mathrm{NM} & \$ 0 & \$ 0\end{array}$

AWARDS A NEW CYCLE 15 TECHNICAL ASSISTANCE GRANT. TX $15 \quad \underset{\$ 45,494}{\$ 36,393}$ 


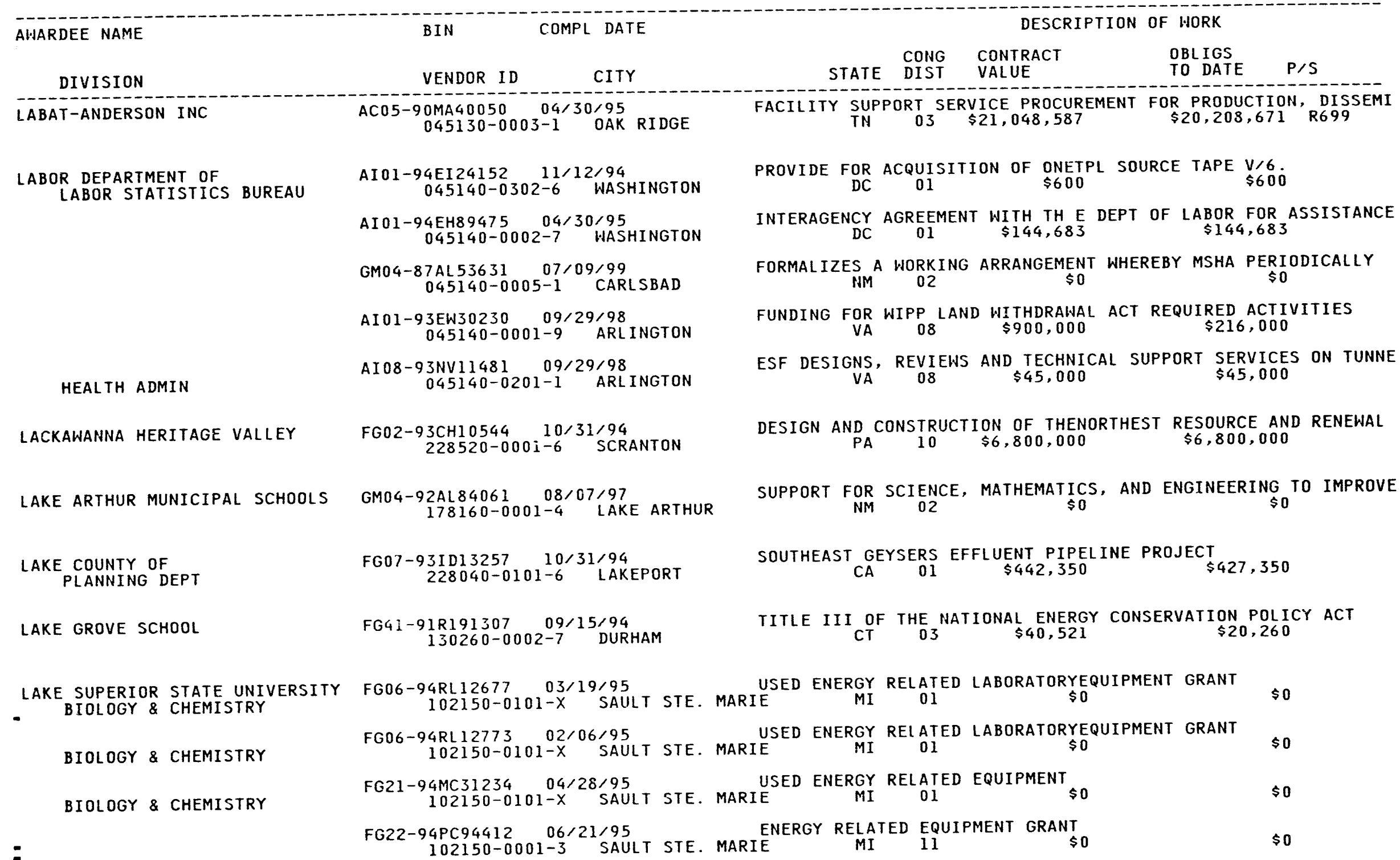




\section{AWARDEE NAME}

DIVISION

LAKESHORE HOSPITA:

LAM ASSOC INC

LAMB ENGINEERING \& CONSTR CO

LANE COLLEGE

LANGSTON UNIVERSITY

-LANIER WORLDWIDE INC

LANSING SCHOOL DISTRICT

¿LARSON \& TAYLOR
BIN

COMPL DATE

STATE DIST VONTRAC

DESCRIPTION OF WORK

VENDOR ID CITY

FG $44-91 R 434748 \quad 08 / 01 / 94$
$176970-0001-6 \quad$ BIRMINGHAM

FG44-92R435025 08/15/94

$176970-0001-6$ BIRMINGHAM

ACO1-93PR $10026001 / 31 / 95$
$206880-0003-7$

$A C 01-93 A D 6472502 / 29 / 96$
$206880-0001-0 \quad$ ROSSLYN

AC01-93AD64713 03/31/95
$206880-0001-0$ ROSSLYN

$A C 01-92 A D 6462104 / 30 / 95$
$206880-0001-0$ ROSSLYN

AC01-92PR10006 06/30/95
$206880-0001-0 \quad$ ROSSLYN

AC01-94AD24932 12/31/97

206880-0001-0 ROSSLYN

AC65-94WG23939 12/17/94
$114910-0001-5$ SALT LAKE CITY

FG05-93ER79178 06/14/95
$045680-0001-7$ JACKSON

FG04-87AL43435 09/29/96 $095900-0001-0$ LANGSTON

$A B 01-93 A D 2366409 / 30 / 94$
$045740-0001-5$ ARLINGTON $F G 45-88 R 59131609 / 30 / 94$
$045790-0002-1 \quad$ LANSING

ABO1-93GC20303 09/30/94 $045900-0001-6$ ARLINGTON

SUPPORT SERVICES FOR PHOTOGRAPH SERVICES

ICP NEW AWARD.
CYCLE 13 - INSTITUTIONAL CONSERVATION PROGRAM. APPROVED BUD $\$ 99,355$

$\$ 49,677$

CYCLE 14 - INSTTTUTIONAL CONSERVATION PROGRAM. APPROVED BUD AL $06 \quad \$ 142,054 \quad \$ 7 \mathrm{i}, 027$

AUTOMATED DATA PROCESSING SUPPORT SERVICES

$\begin{array}{lll} & \end{array}$

LABOR AND MOVING SERVICES FOR THE DOE

$\begin{array}{llll}\text { SELF SERVICE SUPPLY STORE CONTRACT } & \$ 800,000 & \$ 216\end{array}$

$\$ 1,227,300 \quad \$ 299$

COURIER AND MESSENGER SERVICESIN THE WASHINGTON D.C. METROP

VA 10 \$2,999,111 $\$ 2,092,759$ R602

FACILITIES MGMT SUPPORT SERVICES

$\$ 708,208 \quad R 799$

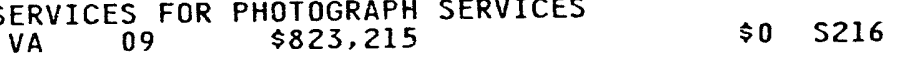

KOFA SUBST, STG $1 \frac{1}{01} \quad \$ 1,875,000 \quad \$ 1,875,000 \quad Y 239$

PRE-FRESHMAN ENRICHMENT PROGRAM (PREP) - $1993.58,501$

GRANT FOR HISTORICALLY BLACK COLLESGES AND UNIVERSITIES

$$
\begin{array}{lll}
\text { HISTORICALLY } & \text { BLACK COLLESGES AND } \\
0 \mathrm{~K} & \$ 63,744 & \$ 63,744
\end{array}
$$

BPA FOR MISCELLANEDUS REPAIRS AND SERVICE ON COPIERS

BPA FOR MISCELLANEOUS REPAIRS AND SERVICE ON COPIERS $\$ \$ 010$

$M I \quad 06 \quad \$ 282,957$

$\$ 141,478$

PROVIDE FOREIGN PATENT SERVICES FOR 34 COUNTRIES VA 10 
AWARDEE NAME

DIVISION

LAS VEGAS CITY OF

WEST PUBLIC SCHOOLS

CITY SCHOOLS

WEST PUBLIC SCHOOLS

WEST PUBLIC SCHOOLS

LASER DIAGNOSTICS

- LAWRENCE \& MEMORIAL HOSPITAL

FACILITIES ENGINEERING

$\because$ LAWRENCE COUNTY BD OF EDUCN
BIN

COMPL DATE

COMPL DATE

VENDOR ID CITY $045900-0001-6$ ARLINGTON

$A B 01-92 G C 20274 \quad 07 / 22 / 94$ 045900-0001-6 ARLINGTON

$A B 01-926 C 20298 \quad 09 / 30 / 94$ 045900-0001-6 ARLINGTON $A B 01-93 G C 2031207 / 31 / 95$ $045900-0001-6$ ARLINGTON $A B 01-93 G C 20323 \quad 07 / 31 / 95$ 045900-0001-6 ARLINGTON $A B 01-93 G C 20331 \quad 07 / 31 / 95$ 045900-0001-6 ARLINGTON $A B 01-93 G C 20337 \quad 09 / 30 / 95$ 045900-0001-6 ARLINGTON

GMO4-93AL84072 11/24/97 $107460-0201-7$ LAS VEGAS FG46-93R613579 07/15/94 107460-0101-0 LAS VEGAS FG46-93R613584 01/15/95 $107460-0201-7$ LAS VEGAS FG46-94R613651 12/31/95 $107460-0201-7$ LAS VEGAS

FG02-94ER81671 07/17/94 235950-0001-3 LOS ALAMOS

FG41-92RI91404 08/31/94 $222830-0001-7$ NEW LONDON FG41-93RI91500 08/31/95 222830-0101-3 NEW LONDON

FG44-92R435026 08/15/94 $146230-0002-8$ MOULTON
DESCRIPTION OF WORK $\begin{array}{lll}\text { CONG } & \text { CONTRACT } & \text { OBLIGS } \\ \text { STATE DIST VALUE } & \text { TO DATE }\end{array}$ STATE DIST VALUE
PROVIDE FORIEGN PATENT SERVICES FOR THIRTEEN COUNTRIES-
\$O RA TO BPA FOR THE PERFORMANCE OF NOVELTY SEARCHES, INFRINGEMENT SE VA 08 \$0 $\$ 0$ R418

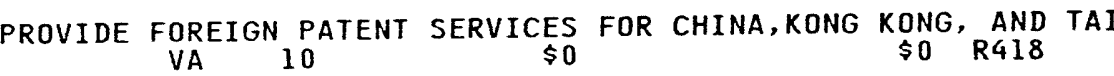
BPA FOR NOVELTY SEARCHES AND INFRINGEMENT VA 08 $\$ 0$
\$0 R418

BPA FOR DOMESTIC PATENT APPLICATIONS

$\$ 0 \quad R 418$

BPA FOR PATENTS AND CLASSIFIEDINVENTIONS

$\$ 0 \quad$ R418

BPA FOR PATENTS IN 39 COUNTRIES

\$0 R499 SUPPORT FOR $\underset{N M}{\substack{\text { SCIENCE, MATHEMATICS, } \\ \$ 0}} \underset{\$ 0}{\$ 0}$ AND ENGINEERING TO IMPROVE

AWARDS A NEW CYCLE 15 TECNICALASSISTANCE GRANT. INCLUDED I

AWARDS A NEW CYCLE 15 ENERGY CONSERVATION MEASURE GRANT. INC NM 03 \$137,710 $\$ 68,854$

TITLE III OF THE NATIONAL ENERGY CONSERVATION POLICY ACT NM $03 \quad \$ 145,060 \quad \$ 72,528$ REAL TIME MONITORING OF HEAVY METALS USING $\underset{\text { NM }}{\substack{\text { LASER-INDUCED } \\ \$ 75,000}}$

TITLE III OF THE NATIONAL ENERGY CONSERVATION POLICY ACT CT $02 \quad \$ 69,980 \quad \$ 34,990$

INITIAL AWARD.

$C T \quad 02 \quad \$ 26,903$

$\$ 13,451$

CYCLE 14 - INSTITUTIONAL CONSERVATION PROGRAM. APPROVED BUD AL $05 \quad \$ 39,240 \quad \$ 19,620$ 


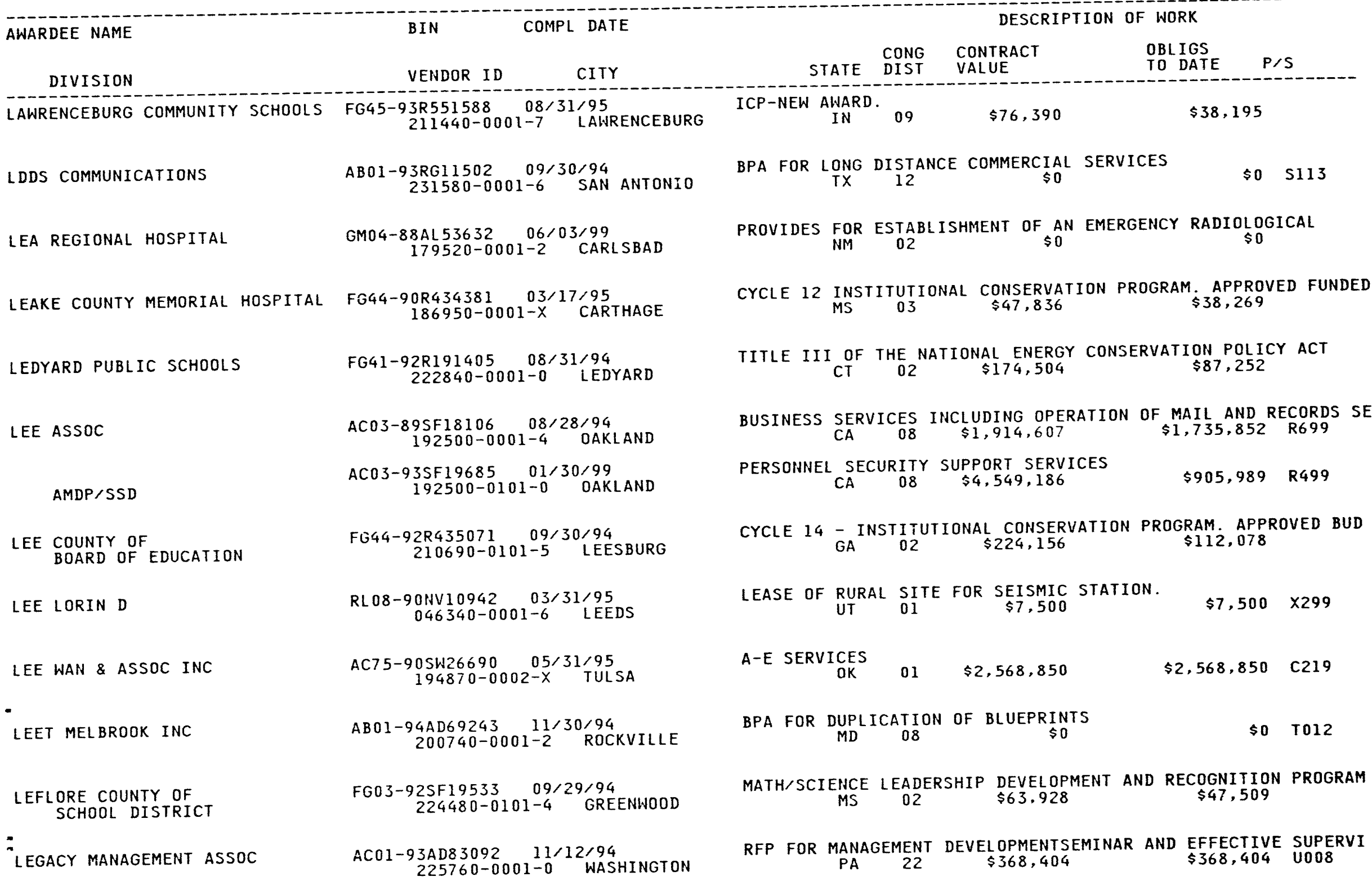




\begin{tabular}{|c|c|c|c|c|c|c|c|}
\hline AWARDEE NAME & BIN & DATE & & & DESCR & F WORK & \\
\hline חIVTSION & VENDOR ID & CITY & STATE & $\begin{array}{l}\text { CONG } \\
\text { DIST }\end{array}$ & $\begin{array}{l}\text { CONTRACT } \\
\text { VALUE }\end{array}$ & $\begin{array}{l}\text { OBLIGS } \\
\text { TO DATE }\end{array}$ & $P / S$ \\
\hline
\end{tabular}

LEGENT CORP

\section{LEGI-SLATE INC}

LEHIGH UNIVERSITY

CHEMISTRY DEPT

METALLURGY \& MATERIALS ENG DEP $046450-0601-0 \quad$ BETHLEHEM

FG02-86ER45269 07/14/95

CHEMISTRY DEPT

CHEMISTRY DEPT

FG02-86ER13580 $06 / 14 / 96$
$046450-0201-5$ BETHLEHEM

FG02-90ER20009 05/31/95 $046450-0001-2$ BETHLEHEM

MATERIALS SCIENCE \& ENG DEPT FG02-90ER45419 $06 / 30 / 95$ BLEHEM

FGU2-92ER54141 10/31/95 046450-0001-2 BETHLEHEM

FG02-93ER14350 05/31/96 046450-0001-2 BETHLEHEM

FG02-93ER79179 06/14/95 046450-0001-2 BETHLEHEM

FG02-93ER $1437308 / 31 / 96$ 046450-0001-2 BETHLEHEM

FG03-925F19520 09/30/94 046450-0001-2 BETHLEHEM
RENEWAL OF MAINTENANCE AGREEMENT FOR FY 1994

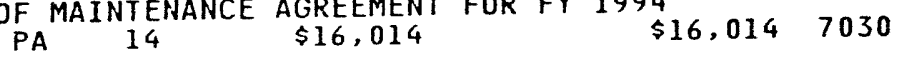

RENEWAL OF SOFTWARE MAINTENANCE

$\$ 16,548 \quad 7030$

RENEWAL OF MAINTENANCE AGREEM ENT FOR FY 1994

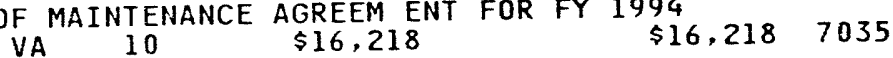

ACCESS TO LEGI-SLATE DATA BASE

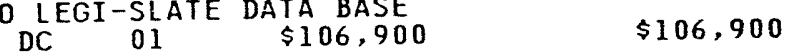

POLYMER-BASED CATALYSTS
PA 15

$\$ 680,702$

EFFECT OF PAINT DEFECTS ON STRUCTURAL PHASE TRANSITIONS

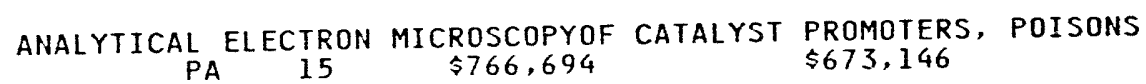

MECHANISM \& CONTROLLING CHARACTERISTICS OF CATALYTIC
$\$ 1,016,878$

POST TRANSCRIPTIONAL REGULA- TION OF CHLOROPLAST GENE EXPRE

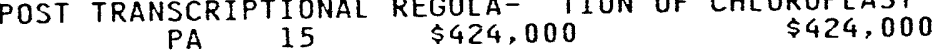

ROLE OF STRUCTURE ON ION MONEMENT IN GLASSES

ELECTRON CYCLOTRON HEATING \& CURRENT DRIVE IN TOROIDAC

$\begin{aligned} \text { ELCTRON } \\ \text { PACLOTRON HEATING } 15\end{aligned} \$ 190,000 \quad \$ 140,000$

MOLECULARE STRUCTURES AND REACTIVITY OF MIXED METAL

$\begin{array}{cccc}\text { PA } & 15 & \$ 280,370 & \$ 171,369\end{array}$

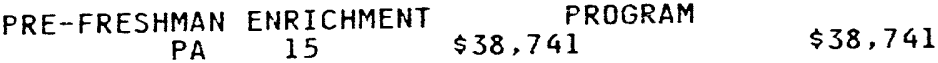

SURFACE \& SOLUTION INTERFACE GEOCHEMISTRY OF LEAD SULFIDE \& $\begin{array}{lll}\text { PA } 15 & \$ 345,916 & \$ 222,952\end{array}$

MATH/SCIENCE LEADERSHIP DEVELOPMENT AND RECOGNITION PROGRAM

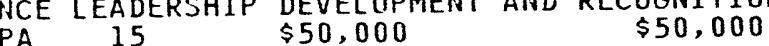




\section{AWARDEE NAME}

BIN

DIVISION

VENDOR ID CITY OID $4110350-0001-2$ BETHLEHEM FC07-91ID13103 $02 / 28 / 95$ 046450-0901-X BETHLEHEM

PHYSICS DEPT

CHEMISTRY DEPT

FG22-89PC89785 09/30/94 046450-0201-5 BETHLEHEM

FG22-91PC91301 09/11/94 046450-0001-2 BETHLEHEM

FG21-92MC29228 09/07/95 046450-0201-5 BETHLEHEM

CHEMISTRY DEPT

MATERIALS SCIENCE \& ENG DEPT $\begin{array}{cc}\text { FG22-92PC92542 } 01 / 14 / 95 \\ 046450-0501-4\end{array}$ BETHLEHEM

CHEMISTRY DEPT

FG22-93PC93222 $08 / 30 / 96$ $046450-0201-5$ BETHL EHEM

LEKTROX CO

LEMOYNE-OWEN COLLEGE

FINANCE \& ADMIN

LENOIR COMMUNITY COLLEGE

LEOMINSTER PUBLIC SCHOOLS

LEONARD BLOOM LAW OFFICES

:
FG05-92ER81342 02/17/95
$222100-0001-7$ NEW PORT RICHEY

FG44-93R435388 $09 / 30 / 94$
$220140-0001-8$ KINSTON

FG44-92R435003 07/31/95
$220140-0001-8$ KINSTON

FG41-92R193427 07/31/94
$200210-0001-8 \quad$ LEOMINSTER

FG41-93R193518 07/31/95 200210-0001-8 LEOMINSTER

$A B 01-926 C 2027207 / 22 / 94$
$169920-0001-2$ TOWSON

$A B 01-936 C 20307 \quad 07 / 31 / 95$
$169920-0001-2$ TOWSON
FG44-92R435059 $08 / 15 / 94$
$046550-0101-1$ MEMPHIS
DESCRIPIION OF WORK

CONG CONTRACT OBLIGS
STATE DIST VALUE TO DATE P/S METALS INITATIVE HOLDING COMPANY AGREEMENT BETWEEN THE U.S. PA 15 \$O MOLTEN METAL ANALYSIS BY LASERPRODUCED PLASMAS $15 \$ 3,318,257$ SINGLE ELECTRON CHEMISTRY OF COALS

$\$ 199,118$ ETHANOL SYNTHESIS AND WATER GAS SHIFT OVER BY FUNCTIONAL SUL
PA I
$\$ 200,000$

SELECTIVE METHANE OXIDATION OVER PROMOTED OXIDE CATALYSTS PA 15 \$562,082 $\$ 285,271$ ROBOTIC WELD OVERLAY COFTTNGS FOR EROSION CONTROL $\begin{array}{cccc}\text { PA } & 15 & \$ 195,318 & \$ 185 \\ & & \end{array}$

NO DECOMPOSITION IN NON-REDUCING ATMOSPHERES $\$ 200,000$ IMPROVED CONTROL OF PULP AND CHIP REFINING THROUGH DIRECT ME CYCLE 14 - INSTITUTIONAL CONSERVATION PROGRAM. APPROVED BUD $\begin{array}{lll}\text { TN } 07 & \$ 253,196 & \$ 162,804\end{array}$

SEE ATTACHED PAGE NO. 2 OF THIS NOTICE OF FINANCIAL ASSISTAN NC OI $\$ 588 \quad \$ 294$

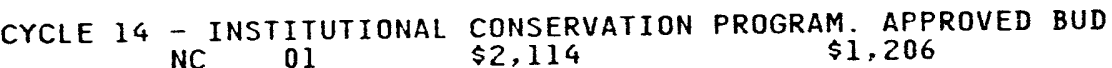

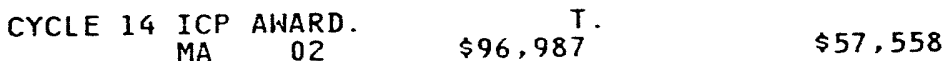

TITLE III OF THE NATIONAL ENERGY CONSERVATION POLICY ACT OF THE NATIONAL ENERGY CONSERVATION POLICY
MA
01

BPA FOR NOVELTY SEARCHES AND INFRINGEMENTS SEARCHES $\begin{array}{cl}\text { MD } 02 & \$ 0 \\ & 02\end{array}$

BDA 02 FOR NOVES AND
R499

\$0 R418 
$R-0420953-001$

\begin{tabular}{|c|c|c|c|c|c|c|c|}
\hline AWARDEE NAME & BIN & $L$ DATE & & & DESCR & F WORK & \\
\hline DIVISION & VENDOR ID & CITY & STATE & $\begin{array}{l}\text { CONG } \\
\text { DIST }\end{array}$ & $\begin{array}{l}\text { CONIRACT } \\
\text { VALUE }\end{array}$ & $\begin{array}{l}\text { OBLIGS } \\
\text { TO DATE }\end{array}$ & $P / S$ \\
\hline
\end{tabular}

LEWIS COUNTY OF
SCHOOLS

\section{LEWIS UNIVERSITY}

LEWIS-CLARK STATE COLLEGE

LEXINGTON CHILDREN'S MUSEUM

LEXINGTON SCHOOL DISTRICT $\# 2$

FG44-93R435331 09/30/95 $93 R 435331$
$146300-0101-8$ HOHENWALD
FG45-93R551523 11/30/94 3R5
$15910-0001-8$ ROMEOVILLE
FG06-93ER79180 06/24/95 IER79180 06/24/95
I $46330-0001-2$ LENISTON 224280-0001-2 LEXINGTON-FAYETTE

SEE ATTACHED PAGE NO.

TN 01

ICP-NEW AWARD

$$
\underset{I L}{A W A R D} \cdot 13 \quad \$ 14,054
$$

PRE FRESHMAN ENRICHMENT PROGRAM

$$
\text { ID } 01 \quad \$ 57,708
$$

$\$ 38,472$

ENERGY QUEST IS A TWO-YEAR ENERGY EDUCTION PROJECT COMPOSED FG44-93R435442009/30/95
$231620-0001-7$ WEST COLUMBIA FG44-93R435441 09/30/95
$233890-0001-1$ BATESBURG

LEXINGTON/RICHLAND CTY SD

LIBRARY OF CONGRESS AI01-92CE50341 $12 / 01 / 94$
ICE $046880-0301-3$ WASHINGTON CONGRESSIONAL RESEARCH SERVICE
CORY OF CONGRESS

FEDLINK NETWORK OFFICE

$$
\text { AI01-94IS30017 07/31/94 }
$$$$
\begin{gathered}
\text { AI01-93AD34063 09/30/94 } \\
046880-0201-7 \text { WASHINGTON }
\end{gathered}
$$$$
\begin{gathered}
\text { AI05-92ER61486 08/30/94 } \\
046880-0001-4 \quad \text { WASHINGTON }
\end{gathered}
$$$$
\begin{gathered}
\text { AI21-94MC31323 09/30/94 } \\
046880-0001-4 \text { WASHINGTON }
\end{gathered}
$$

LICENSING EXECUTIVES SOCIETY

$$
\begin{gathered}
\text { FG06-92RL12385 09/30/94 } \\
216880-0001-1 \text { NORWALK }
\end{gathered}
$$$$
\begin{gathered}
\text { FC22-91PC90548 08/07/94 } \\
205510-0001-5 \text { OAKLAND }
\end{gathered}
$$

-LIFAC NORTH AMERICA

$$
\text { FR22-91PC90583 11/07/13 }
$$

SEE ATTACHED PAGE NO. 2 OF THIS NOTICE OF FINANCIAL ASSISTAN SC $02 \quad \$ 77,211 \quad \$ 38,605$

SEE ATTACHED PAGE NO. 2 OF THIS NOTICE OF FINANCIAL ASSISTAN

$$
\begin{array}{rr}
\text { SHED PAGE NO. } 2 \text { OF THIS NOTICE OF FINANCIAL } \\
\$ 111,320
\end{array}
$$

PROVIDE TECHNICAL AND RESEARCHASSISTANCE TO THE UNITED STAT TECHNICAL AND RESEARCHASSISTANCE TO THE UNITE
DC OI $\$ 250,000$

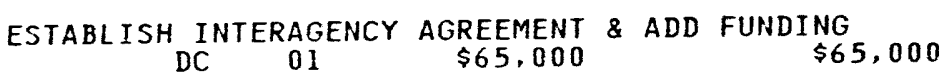

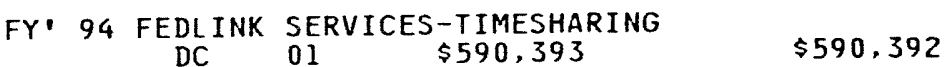

PILOT RESIDENT FELLOW PROGRAM IN SCIENCE AND TECHNOLOGY; BIO

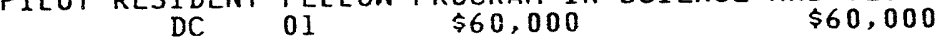

PROVIDE PERIODICALS SUBSCRIP- TION SERVICES

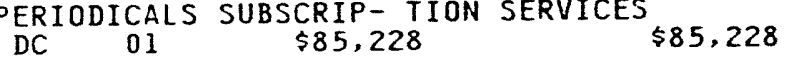

INVENTOR ASSISTANCE INITIATIVES

$\$ 49,485$

$\$ 49,485$

LIFAC SORBENT INJECTION DESULFURIZATION DEMONSTRATION PROJEC $C A T 08 \$ 20,755,222 \quad \$ 10,636,864$

REPAYMENT AGREEMENT FOR COOPERATIVE AGREEMENT DE-FC22-90PC 90 


\section{AWARDEE NAME}

BIN

COMPL DATE

VENDOR ID

COMPL DATE

205510-0001-5 OAKLAND

LIFE SCIENCES RESEARCH FDN

FG05-89ER14025 05/31/95

$\begin{array}{ll}9 E R 14025 & 05 / 31 / 95 \\ 183080-0001-0 \quad B A L T I M O R E\end{array}$

FG43-92R340408 09/27/94
$046930-0001-9$ ERIE

AB01-94AD25724 06/30/95 046940-0001-2 SILVER SPRING

\section{LIGHT INC}

\section{LIGHT SCULPTING INC}

LIGHTWAVE ELECTRONICS CORP

LIMBACH \& LIMBACH

LIMESTONE SCHOOL DEPARTMENT

LINAC SYSTEMS

- LINCOLN COUNTY HOSPITAL DIST

LINCOLN COUNTY OF EDUCATION BOARD OF

$$
\begin{aligned}
& 92 C E 1554308 / 14 / 95 \\
& 218110-0001-0 \text { MILWAUKEE }
\end{aligned}
$$
174900-0001-7 MOUNTAIN VIEW FG03-92ER81291 02/17/95

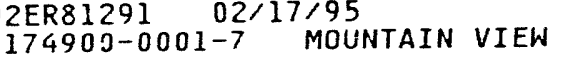

AC03-92SF19602 09/30/94 225200-0001-5 SAN FRANCISCO

FG41-94R192615 06/30/96 LIMESTONE

FG03-91ER81254 09/05/94 215370-0001-2 SAN DIEGO 238020-0001-3 CALIENTE $3 R 43533209 / 30 / 95$
$180800-0102-2$ FAYETTEVILLE

¿LINCOLN COUNTY SCHOOL DISTRICT
GM08-93NV11469 09/16/98

$115090-0002-X$ PANACA
FG03-93ER81489 08/01/94

FG49-94R916002 05/31/96

FG44-93R435332 09/30/95

\section{DESCRIPTION OF WORK}

OBLIGS

TO DATE P/S

CA 08

DEVELOPING RESEARCH CAPABILITIES IN ENERGY BIOSCIENCES $M D \quad 03 \quad \$ 2,375,352$

DESIGN, BUILD \& TEST A NEW OZONE MONITOR MEASURING PA 21 $\$ 94,262$ $\$ 94,262$

BPA FOR PHOTO AND RELATED SERVICES $M D \quad O G$ $\$ 0$

$\$ 0 \quad$ T015

DEVELOP RAPID FABRICATION SYSTEM PROTO TYPES BY IRRADIATION WI $05 \quad \$ 134,588 \quad \$ 99,538$

MODE-LOCKED PULSE PRE-AMPLIFIER FOR PHOTOCATHODE ILLUMINATIO CA $12 \quad \$ 659,488$ PRE-AMPL $\$ 172,401$

PHASE I AWARD FOR LIGHTWAVE ELECTRONICS CORP. TITLE: ALL SO $C A \$ 12 \quad \$ 549,805 \quad \$ 408,724$ PROVIDE SUPPORT SERVICES TO OFFICE OF PATENTS IN THE OFFICE
CA 06 \$ $\$ 1,510,000$

FY'94 ICP AWARD ${ }_{\text {ME }} \quad \$ 35,362 \quad \$ 8,644$

SBIR - PHASE I AWARD FOR - WIRE SHADOW EMITTANCE SCANNER FOR $\begin{array}{cccc}\text { CYCLE } & 16 & \text { ICP } & \text { GRANT APPLICATION } \\ \text { NV } & 02 & \$ 73,766\end{array}$ $\$ 36,883$

SEE ATTACHED PAGE NO. 2 OF THIS NOTICE OF FINANCIAL ASSISTAN TN 04 \$50,393 $\$ 25,196$

MOU TO IMPLEMENT A COOPERATIVEEFFORT AT REACHING EDUCATION NV 02 \$0 


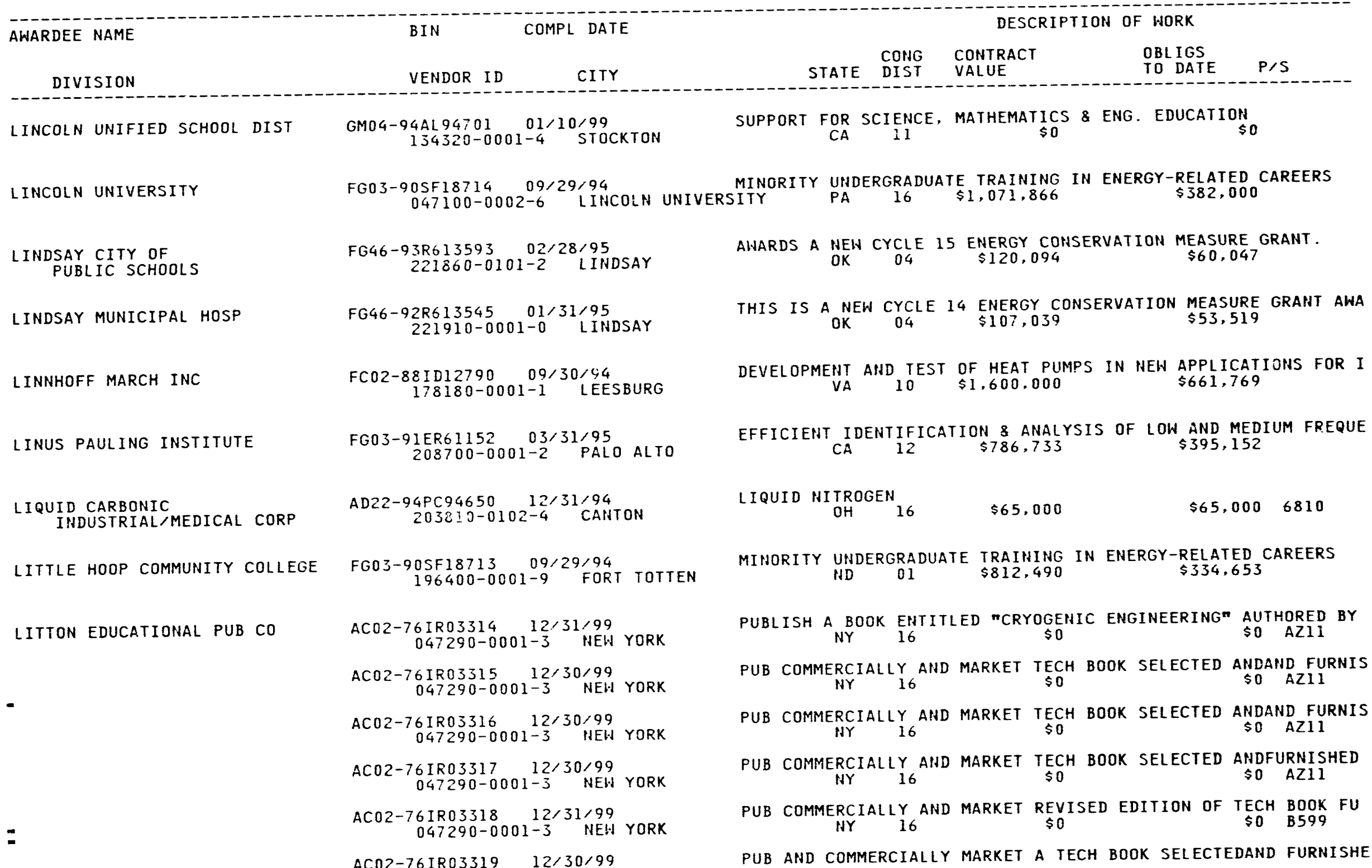




\begin{tabular}{|c|c|}
\hline AWARDEE NAME & COMPL DATE \\
\hline \multirow[t]{4}{*}{ DIVISION } & VENDOR ID \\
\hline & $047290-0001-3$ \\
\hline & $\begin{array}{c}\text { ACO2-76IR03320 12/30/99 } \\
047290-0001-3 \text { NEN YORK }\end{array}$ \\
\hline & $\begin{array}{c}\text { AC02-76IR03321 } 12 / 30 / 99 \\
047290-0001-3 \text { NEW YORK }\end{array}$ \\
\hline LITWIN ENGINEERS \& CONSTR INC & $\begin{array}{c}\text { ACO2-91CE40954 } 09 / 30 / 94 \\
182470-0001-6 \text { HOUSTON }\end{array}$ \\
\hline \multirow[t]{2}{*}{$\underset{\text { EFM }}{\text { LIVERMORE CITY OF }}$} & $\begin{array}{r}\text { FG03-93SF19633 12/31/94 } \\
047320-0101-7 \quad \text { LIVERMORE }\end{array}$ \\
\hline & $\begin{array}{c}\text { GMO4-93AL58222 } 05 / 22 / 98 \\
047320-0001-0 \text { LIVERMORE }\end{array}$ \\
\hline LOCAP & $\begin{array}{c}\text { AC } 96-81 P 010088 \quad 03 / 09 / 98 \\
047430-0001-7 \quad \text { HOUSTON }\end{array}$ \\
\hline \multirow[t]{2}{*}{ LOCKHEED ENV SYST \& TECH CO } & $\begin{array}{c}\text { ACO7-941D13307 11/11/94 } \\
237760-0001-5 \text { IDAHO FALLS }\end{array}$ \\
\hline & $\begin{array}{r}\text { AC07-94IDI3312 } 06 / 28 / 96 \\
237760-0102-X \text { LAS VEGAS }\end{array}$ \\
\hline $\begin{array}{l}\text { LOCKHEED MISSILES \& SPACE CO } \\
\text { APPLIED PHYSICS }\end{array}$ & $\begin{array}{c}\text { FG03-91SF18864 07/30/94 } \\
047470-0502-1 \quad \text { PALO ALTO }\end{array}$ \\
\hline LOCKWOOD GREENE TECHNOL OGIES & $\begin{array}{c}\text { AC05-930R22097 08/22/98 } \\
227540-0001-1 \quad \text { OAK RIDGE }\end{array}$ \\
\hline \multirow[t]{3}{*}{ LODESTAR RESEARCH CORP } & $\begin{array}{c}\text { FG02-88ER53263 } 10 / 31 / 96 \\
166260-0001-2 \text { BOULDER }\end{array}$ \\
\hline & $\begin{array}{c}\text { FGO2-92ER54169 07/31/95 } \\
166260-0001-2 \quad \text { BOULDER }\end{array}$ \\
\hline & $\begin{array}{c}\text { FG03-93ER54212 } 01 / 31 / 95 \\
166260-0001-2 \text { BOULDER }\end{array}$ \\
\hline
\end{tabular}

PUB COMMERCIALLY AND MARKET THIRD EDITION OF TECH BOOK FURNI

NY 16 AND MARKET ${ }_{\$ 0}$ EDITION OF TEC \$O AZ11

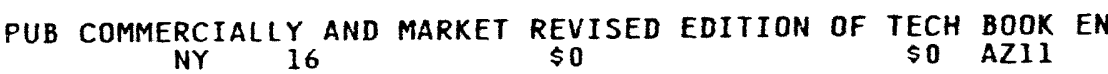

DISIGN, FABRICATION \& TESTING OF A CHEMICAL HEAT PUMP.

$$
\underset{T X}{\text { FABRICATION \& TESTING OF A CHEMICAL HEAT PUMP }} \begin{aligned}
& \text { HET } \\
& \$ 1,344,843
\end{aligned}
$$

GREENVILLE ROAD IMPROVEMENTS

MUTUAL ASSISTANCE AND EMERGENCY MANAGEMENT

$C A \quad 09$ \$O

ENGINEERING STUDY OF LOCAP FAC

$$
\text { TX } \begin{array}{lllll}
\text { NT } & 18 & \$ 4,140,000 & \$ 4,140,000 & \text { VI19 }
\end{array}
$$

FEASIBILITY STUDY FOR TREATMENT OF ALPHA CONTAMINATED MIXED $\begin{array}{ccc}\text { ID } & 02 & \$ 248,000 \\ \text { ENVIRONMENTAL GAS SAMPLE CHARACTERIZATION }\end{array}$

$$
\text { NV } 01 \text { SAMPIE CHARACTERIZATION } \$ 162,449 \quad H 399
$$

NLUF USER-APPLICATION OF A HIGH-DENSITY GAS LASER TARGET TO

$$
C A \quad 11 \quad \$ 109,933 \quad \$ 109,933
$$

MISCELLANEOUS A-E SERVICES FOROAK RIDGE OPERATIONS

$$
\text { TN } 03 \begin{array}{llll}
\$ 17,066,368 & \$ 5,147,510 & 6219
\end{array}
$$

FUNDAMENTAL STUDIES OF FUSION PLASMAS $\$ 3,716,000$

RESEARCH HILL JOIN THE PPL/MITTFTR SCATTERLY TEAM \& ANALYZE CO $02 \quad \$ 483,924 \quad \$ 310,953$ NEW AWARD FOR PROJECT ENTITLED"FUNDAMENTAL STUDIES OF FUSIO
CO
02 


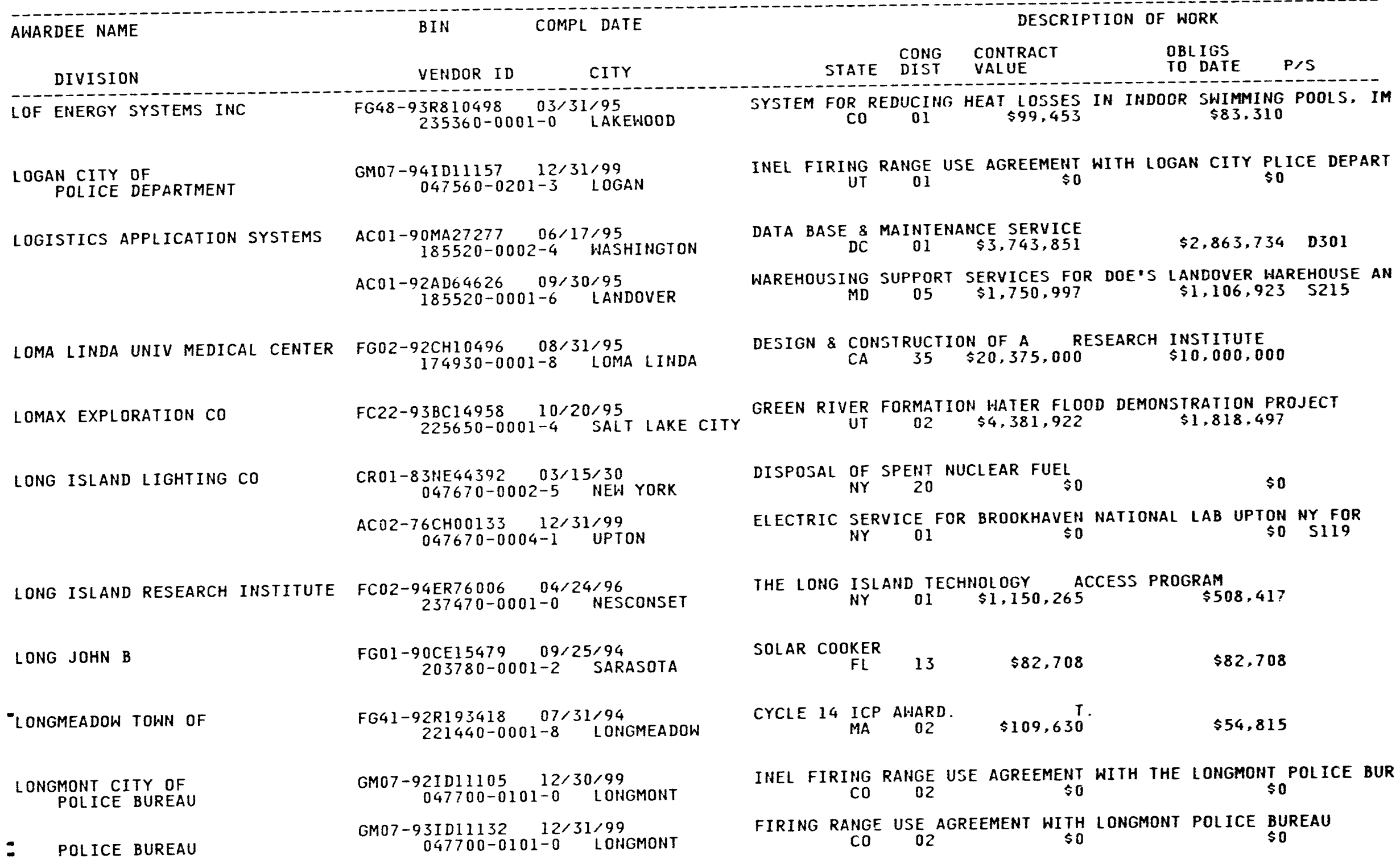




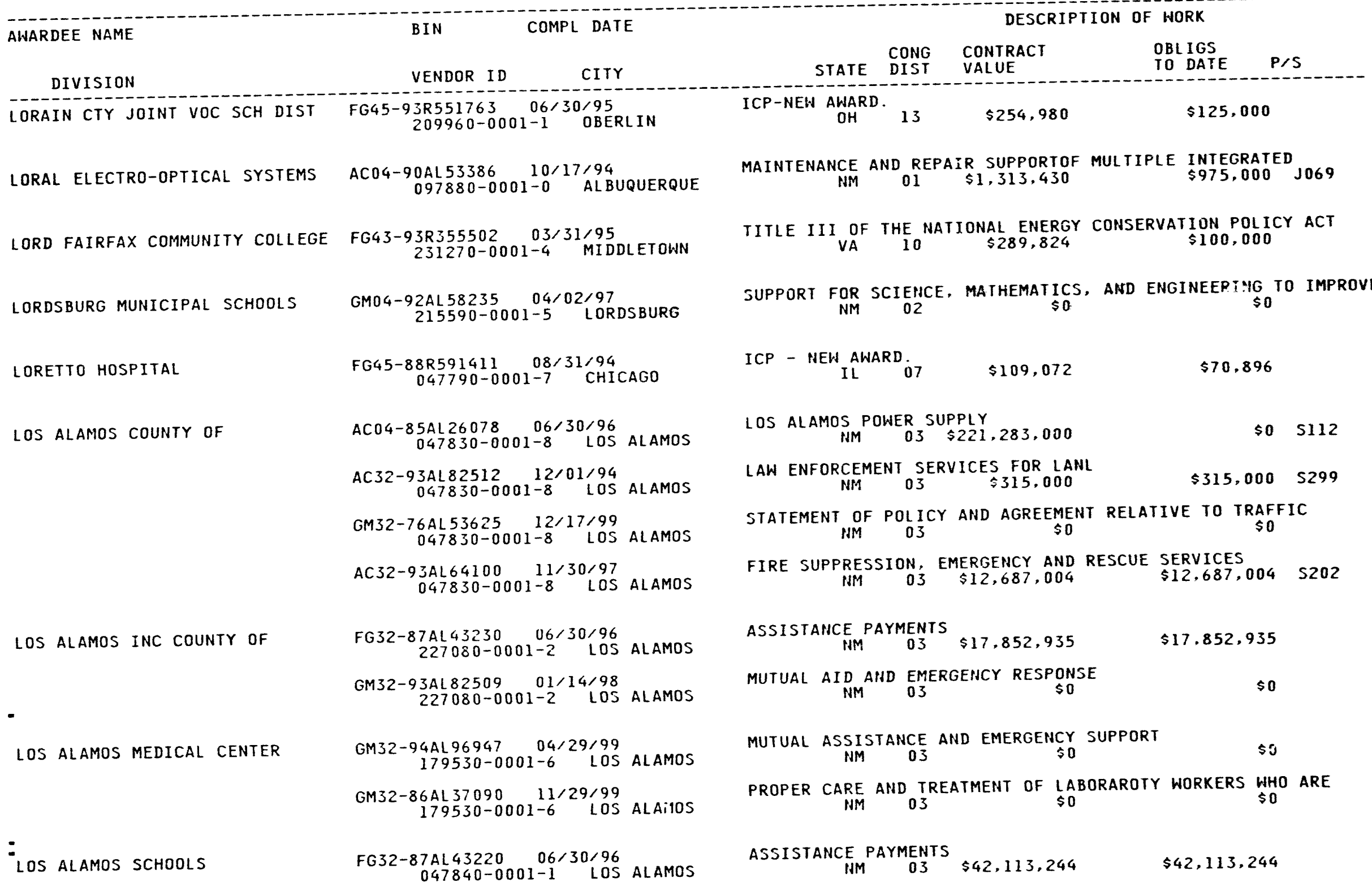




\begin{tabular}{|c|c|c|c|c|c|c|c|}
\hline VARDEE NAME & BIN & L DATE & & & DESCR & = WORK & \\
\hline DIVISION & VENDOR ID & CITY & STATE & $\begin{array}{l}\text { CONG } \\
\text { DIST }\end{array}$ & $\begin{array}{l}\text { CONTRACT } \\
\text { VALUE }\end{array}$ & $\begin{array}{l}\text { OBLIGS } \\
\text { TO DATE }\end{array}$ & Prs \\
\hline
\end{tabular}

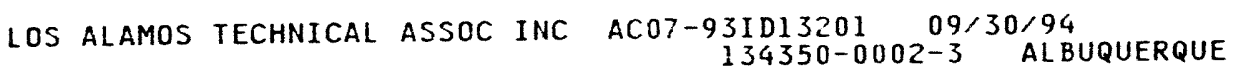

LOS ANGELES CITY OF

WATER \& POWER DEPT

IOS ANGELES UNIFIED SCH DIST

ENERGY PROGRAMS OFFICE

BUSINESS MANAGERS OFFICE

LOS BANOS COMMUNITY HOSPITAL

LOUDON COUNTY OF

BOARD OF EDUCATION

LOUISIANA BOARD OF REGENTS

LOUISIANA POWER \& LIGHT

LOUISIANA STATE OF

OFFICE OF CONSERVATION

SOCIAL SERVICE DEPARTMENT OF

FC07-94ID13309 05/31/96
$047850-0101-1$ LOS ANGELES

FG49-88SF17751 08/31/94 $047870-0001-2$ LOS ANGELES

FG49-90SF18676 08/31/94 047870-0501-4 LOS ANGELES

FG49-86SF16171 12/31/94 047870-0001-2 LOS ANGELES

FG49-895F18324 02/28/95 $047870-0401-8$ LOS ANGELES

FG49-92R914043 08/31/94
$222900-0001-9$ LOS BANOS FG44-91R434717 07/01/94
$210140-0101-3$ LOUDON

FG02-91ER75669 $09 / 29 / 95$
$223880-0001-7 \quad$ BATON ROUGE

CRO1-84RH00009 03/15/30 047940-0002-2 NEH ORLEANS

FG22-91MT91004 09/30/94
$04 / 960-0501-3$ BATON ROUGE FG46-90R659001 03/31/95 047960-0601-X BATON ROUGE FG46-93R649301 06/30/95 047960-0301-0 BATON ROUGE

FG96-87P013145 12/31/94

PROVIDE TECHNICAL SUPPORT ARSAP BY DEVELOPING AN IODINE ANAL ECHNICAL SUPPORT ARSAP BY DEVELOPING AN
$\$ 366,057$

TESTING AND EVALUATION OF ELECTRIC VEHICLES

$$
\text { CA } 2525 \$ 32,459 \quad \$ 112,288
$$

GRANT PROGRAMS FOR SCHOOLS ANDHOSPITALS AND BUILDINGS OWNED

$$
\text { CA } 25 \quad \$ 345,781 \quad \$ 192,889
$$

GRANT PROGRAMS FOR SCHOOLS ANDHOSPITALS AND BUILDINGS OHNED

$$
\text { 25A } \$ 444,782 \quad \$ 222,391
$$

CYCLE 8 SCHOOLS \& HOSPITALS

$$
\text { CA } 25 \quad \$ 308,626 \quad \$ 159,314
$$

GRANT PROGRAMS FOR SCHOOLS ANDHOSPITALS AND BUILDINGS ONNED

$$
\text { CA } 25 \quad \$ 207,293 \quad \$ 103,646
$$

TITLE III OF THE NATIONAL ENERGY CONSERVATION POLICY ACT

$$
C A \text { I8 } \$ 0
$$

CYCLE 13 - INSTITUTIONAL CONSERVATION PROGRAM. APPROVED BUD

$$
\begin{aligned}
& \text { TN INSTITUTIONAL CONSERVATION PROGRAM. } \\
& 02 \\
& \$ 37,418
\end{aligned}
$$

DOE/EPSCOR PLANNING GRANT FOR LOUISIANA.

$$
\text { LA } 06 \text { \$2,261,890 } \$ 1,139,530
$$

DISPOSAL OF SPENT NUCLEAR FUELAND OR HIGH LEVEL RADIOACTIVE LA 02 


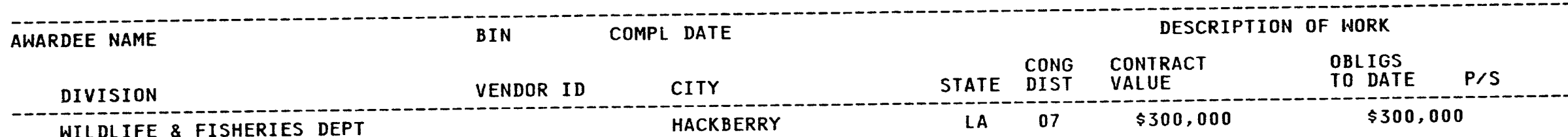

WILDLIFE \& FISHERIES DEPT

LOUISIANA STATE UNIVERSITY NUCLEAR SCIENCE CENTER 047970-1001-0 BATON ROUGE FG02-93CH10542 05/30/98 047970-0001-5 BATON ROUGE FG05-84ER4015905/14/95 047970-0001-5 BATON ROUGE

ZOOLOGY \& PHYSIOLOGY DEPT

FG05-86ER60393 01/31/95
$047970-0601-3$ BATON ROUGE FG05-87ER60503 11/14/94 047970-0201-8 BATON ROUGE

CHEMISTRY DEPT

BASIN RESEARCH INSTITUTE

PHYSICS \& ASTRONOMY DEPT

PHYSICS \& ASTRONOMY DEPT

CHEMISTRY DEPT

PHYSICS DEPT

CONCURRENT COMPUTING LAB

FG05-87ER13748 12/14/94 047970-0901-2 BATON ROUGE

FG05-88ER4044503/31/95

FG05-88ER40481 10/31/94 047970-0401-0 BATON ROUGE

FG05-91ER14167 12/31/94 047970-0201-8 BATON ROUGE

FG05-91ER40617 02/28/95 047970-0501-7 BATON ROUGE

FG05-92ER14291 08/14/95 047970-0001-5 BATON ROUGE 047970-1301-X BATON ROUGE FG05-93ER14367 12/14/94

CLINCAL INFORMATIONS SYSTEMS FG06-94RL12670 $12 / 21 / 94$ O47970-1501-2 BATON ROUGE FG09-93SR $18370 \quad 09 / 16 / 94$ 047970-1401-6 BATON ROUGE

: PENNINGTON BIOMEDICAL RES
FG02-89ER12896 08/31/94 047970-0401-0 BATON ROUGE

FG05-92ER45477 03/14/95 047970-0001-5 BATON ROUGE

NUCLEAR ENGINEERING RESEARCH ACTINIDE BEHAVIOR IN IFR NUCLEAR ENGINEERING
LA 06 DESIGN \& CONSTRUCTION OF A CENTER HAS COASTED ENERGY \& DESIGN \& CONSTRUCTION \$13,150,000 06 \$A THE STRUCTURE OF NUCLEI FAR FROM BETA STABILITY LA 06 \$1,237,000 \$1,092,000 MUTAGENIC EFFECT OF TRITIUM ONDNA OF DROSOPHILA MELANOGASTE MUTAGENIC EFFECT OF TRITIUM A PHYSICO-CHEMICAL STUDY OF SOME AREAS OF FUNDAMENTAL SIGNIF LA 06 \$1,245,000 $\$ 947,650$

AMMONIUM SILICATE DIAGENESIS AND ITS INFLUENCE ON THE INTERP $\begin{array}{lll}\text { LA } 06 & \$ 310,429 & \$ 310,429\end{array}$

A SEARCH FOR DIRECT LEPTONS INNUCLEON-NUCLEUS AND NUCLEUS-N LA 06 \$1, 442,000 \$1,098,000

MEASUREMENTS OF SOLAR PROTON-PROTON FUSION NEUTRINOS WITH A LA $06 \quad \$ 469,752 \quad \$ 469,752$

STUDIES OF METAL AMMONIA INTERACTIONS WITH AROMATIC SUBSTRAT $\begin{array}{lll}\text { LA } 06 & \$ 461,000 & \$ 361,000\end{array}$

RESEARCH IN PARTICLE PHYSICS

$$
\begin{array}{lll}
\text { IN PARTICLE } & \text { PHYSICS } & \$ 2,372,744
\end{array}
$$

RESEARCH ON GALLIUM ZEOLITES FOR LIGHT PARAFFIN AROMATIZATIO LA $06 \quad \$ 230,392 \quad \$ 189,952$

RESEARCH ON EMBEDDED MIICROCLUSTERS IN ZEOLITES AND CLUSTER B LA $06 \quad \$ 145,800 \quad \$ 144,126$

SENSITIZED LUMINSECENCE IN ORGANIZED MEDIA LA 06 ( $\$ 93,000$ $\$ 93,000$ USED ENERGY RELATED LABORATORYEQUIPMENT GRANT LA $\underset{04}{\operatorname{Re}} \underset{\$ 0}{ }$ USED ENERGY RELATED LABORATORYEQUIPMENT LA RO 06 \$0 \$0 AC21-89MC26366 10/17/94 


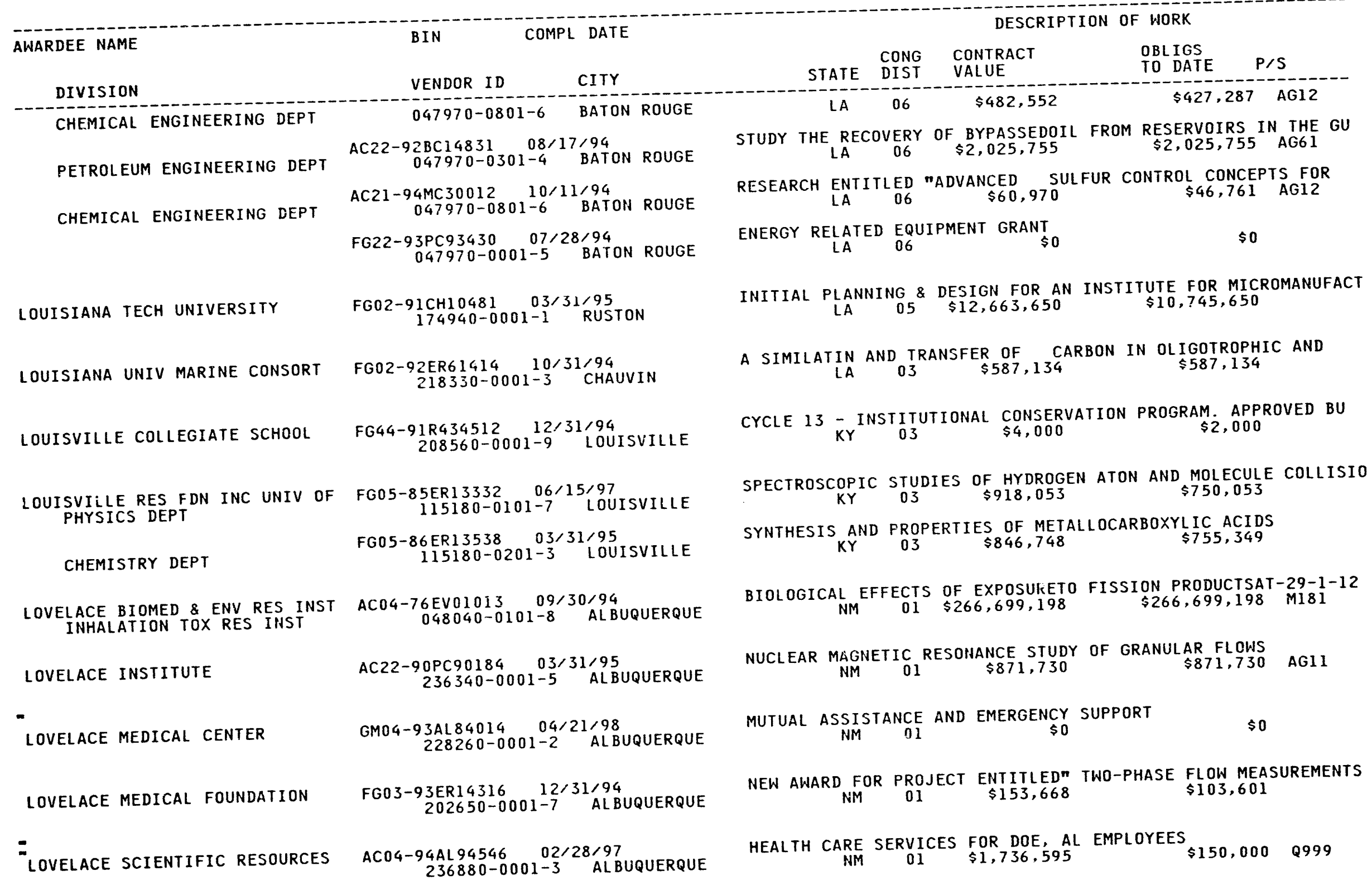




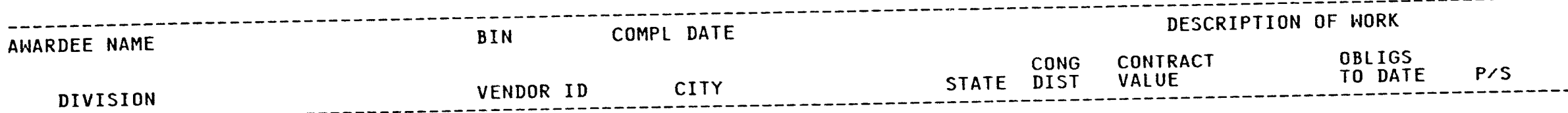

LOVING TOWN OF

MUNICIPAL SCHOOLS

MUNICIPAL SCHOOLS

LOVINGTON PUBLIC SCHOOLS 1 BLDG

LOWELL UNIVERSITY OF

PHYSICS DEPT

ELECTRICAL ENGINEERING

RADIATION LABORATORY

RADIATION LABORATORY

RADIATION LABORATORY

PHYSICS DEPT

LOWER SAVANNAH COUNCIL OF GOVT

LOWNDES COUNTY BOARD OF EDUCN GMO4-92AL84080 07/21/97
$226830-0101-0$ LOVING GMO4-93AL84104 07/01/98
$226330-0101-0$ LOVING

FG46-94R613652 12/31/95 $F G 02-94 E R 4084804 / 30 / 95$
$048090-0001-6 \quad$ LOWELL FG02-86ER40246 11/30/94
$048080-0101-2 \quad$ LOHEL FG02-91ER14179 10/31/94 048080-0501-8 LOWELL

FG02-86ER75277 09/30/94 048080-0401-1 LOWELL

FG02-91ID13083 09/29/94 048080-0401-1 LOWELL

FG02-91IDI3141 09/15/94 FG02-92ER40723 05/31/95
$048080-0101-2 \quad$ LOWELL

FG09-93SR18344 08/31/94
$217770-0001-7 \quad$ AIKEN FG09-92SR18267 12/31/94
$217770-0001-7$ AIKEN

FG44-92R435027 08/31/94 048140-0001-4 HAYNEVILLE

FG44-92R435028 08/15/94 048140-0001-4 HAYHEVILLE SUPPORT FOR SCIENCE, MATHEMATICS, AND ENGINEERING TO IMPRDVE NM 0 $\$ 0$ SUPPORT FOR SCIENCE MATHEMATICS AND ENGINEERING EDUCATION NM 02 $\$ 0$

TITLE III OF THE NATIONAL ENERGY CONSERVATION POLICY ACT NM $02 \quad \$ 8,976 \quad \$ 4,488$

SINGLE PARTICLE AND COLLECTIVEFHENOMENA IN NUCLEI

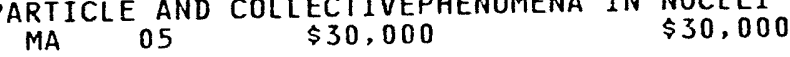

NEUTRON STUDIES IN THE ACTINIDE REGION

$$
\text { MA } 05 \text { \$1,276,000 } \$ 1,161,000
$$

STABILITY \& HEAT TRANSFER IN TIME-MODULATED FLOHS

$$
\text { MA } 05 \text { \& } \$ 230,053 \quad \$ 230,053
$$

EVALUATION FOR LOH-ENRICHED UR:NIUM FUEL CONVERSION MA $05 \quad \$ 125,493$

$\$ 125,493$

UNIVERSTITY REACTOR INSTRUMENTATION PROGRAM

$$
\text { MA } 050 \text { REACTOR INSTRUMENTATION PROGRAM } \$ 115,723
$$

UNIVERSITY REACTOR SHARING

$$
\begin{array}{lll}
\text { MA } & \text { REACTOR } & \text { SHARING } \\
& \$ 32,000 & \$ 32,000
\end{array}
$$

BETA AND GAMMA DECAY HEAT MEASUREMENTS.

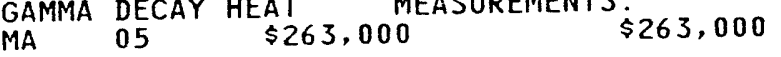

ESTABLISH SR REGIONAL DIVERSIFICATION INITIATIVE

CONDUCT OF A REGIONAL SOLID WASTE MANAGEMENT FEASIBILITY

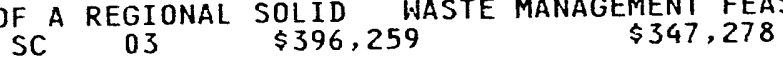

CYCLE 14 - INSTITUTIONAL CONSERVATION PROGRAM. APPROVED BUD

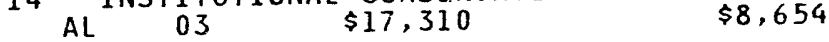

CYCLE 14 - INSTITUTIONAL CONSERVATION PROGRAM. APPROVED BUD

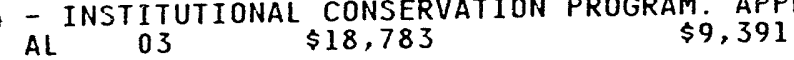




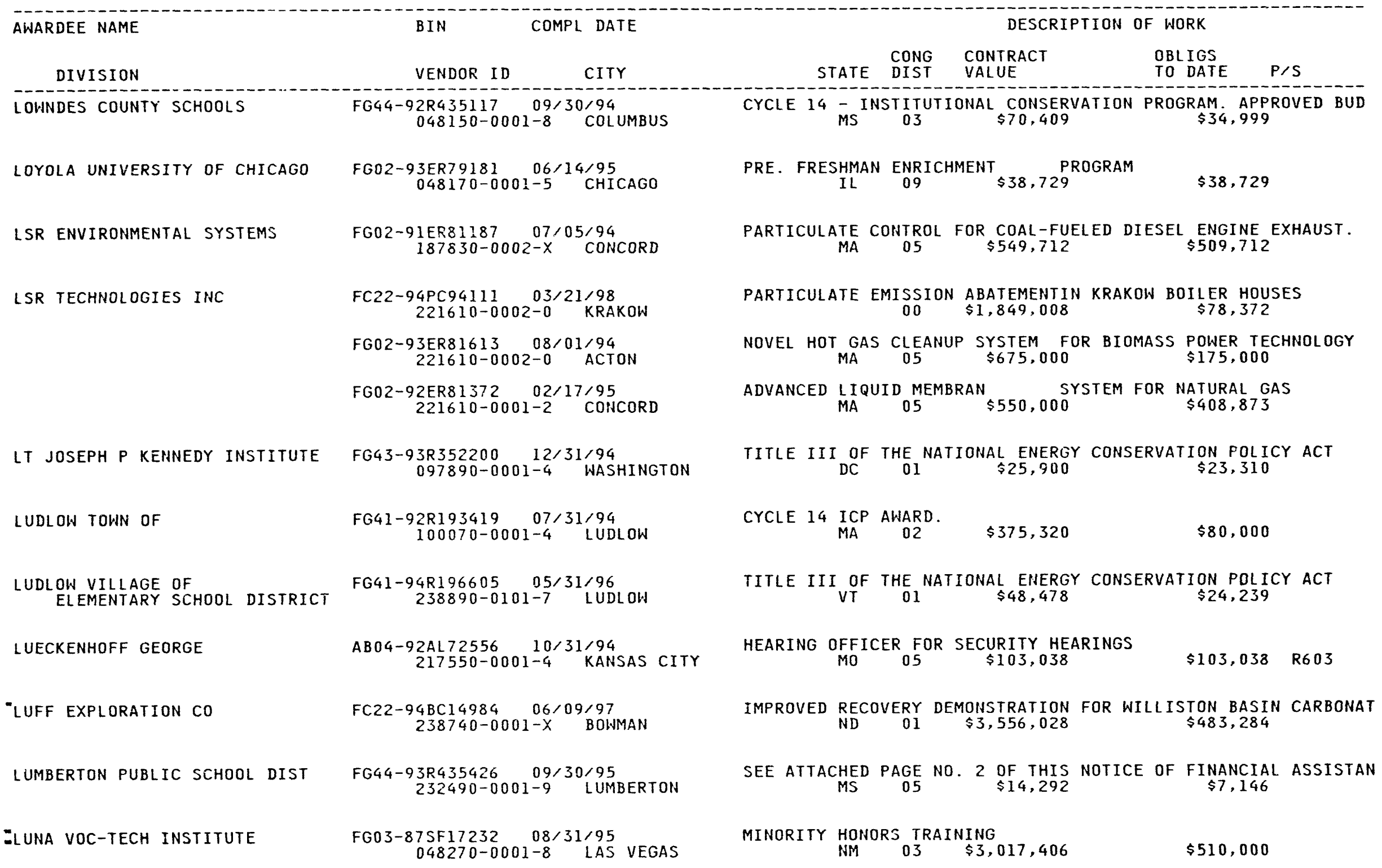




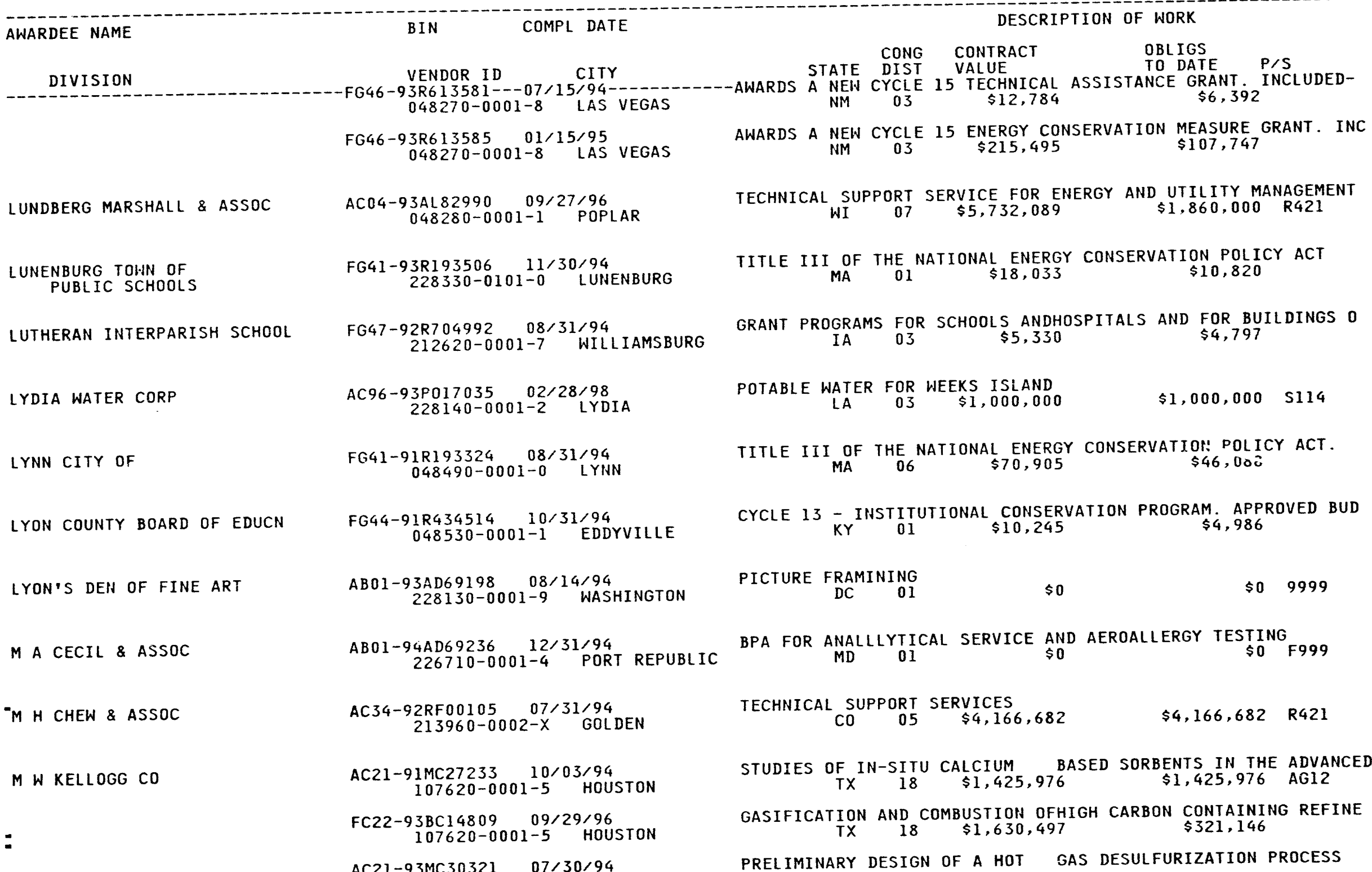




AWARDEE NAME
DIVISION
M-C POWER CORP

MAAS OPTICIANS INC

MAC TECHNICAL SERVICES CO

\section{MACVICAR DESIGN \& COMM}

MADERA CITY OF

EDUCATION OFFICE OF

MADERA COMMUNITY HOSPITAL

MADISON COUNTY HOSPITAL

-MADISON METROPOLITAN SCH DIST

MAGDALENA TOWN OF

- MUNICIPAL SCHOOLS

MUNICIPAL SCHOOLS
NAMES BY NAME, CITY \& STATE - ACTIVE AWARDS

PAGE NO. 276

BIN COMPL LATE

VENDOR ID CITY

HOUSTON

$107620-0001-5$

AC21-90MC27394 09/14/94 202790-0001-4 BURR RIDGE

FC21-92MC28065 03/31/96 202790-0001-4 BURR RIDGE

$A B 01-94 A D 69233 \quad 02 / 28 / 95$ 216770-0002-3 ROCKVILLE

ACO2-90CH10415 12/25/94
$158670-0002-8 \quad$ LEMONT

AC06-92RL12434 09/30/95 158670-0001-X RICHLAHD

$A B 01-94 A D 2492905 / 31 / 95$ 237880-0001-5 ARLINGTON FG49-92R914044 08/31/94
$222910-0101-9$ MADERA

FG49-90SF18677 06/30/95 202240-0001-2 MADERA

FG45-93R55175205/31/95 229900-0001-1 LONDON

FG45-93R551823 12/31/94 229930-0001-2 MADISON

FG45-93R551796 06/30/95 229930-0001-2 MADISON

FG04-92AL82976 05/31/95 219100-0101-5 MAGDALENA

GMO4-92AL58236 07/21/97 219100-0101-5 MAGDALENA
DESCRIPTION OF WORK

\begin{tabular}{lc} 
NTRACT & OBLIGS \\
\hline$\$ 790,737$ & TO DATE
\end{tabular}

$\begin{array}{clll}\text { SIMULATED COAL GAS MOLTEN } & \text { CARBONATE FUEL CELL POWER } \\ \text { IL } & 13 & \$ 55,393,218 & \$ 38,055,322\end{array}$ MOLTEN CARBONATE FUEL CELL (MCFC) PRODUCT DEVELOPMENT IL 13 \$16,213,294 $\$ 6,032,967$

BPA FOR SAFTEY GLASSES

$\$ 0$

$\$ 0 \quad 6540$

PROVIDES SUPPORT SERVICES FOR TECHNICAL EXPERTISE FOR THE BASIC CONTRACT FOR GENERAL SUPPORT SERVICES

$$
\begin{array}{lllll}
\text { WTRACT } & \text { FOR } & \text { GENERAL } & \text { SUPPORT } & \text { SERVICES } \\
\text { WA } & 04 & \$ 72,832,549 & \$ 54,562,963 & R 425
\end{array}
$$

BPA FOR GRAPHIC PRODUCTION ANDVISUAL SERVICES VA 08 $\$ 0$ $\$ 0 \quad T 001$

TITLE III OF THE NATIONAL ENERGY CONSERVATION POLICY ACT $C A \quad 19 \quad \$ 113,478 \quad \$ 56,739$

GRANT PROGRAMS FOR SCHOOLS ANDHOSPITALS AND BUILDINGS OWNED CA $30 \quad \$ 380,159$ $\$ 190,079$

$$
\text { OH } 15
$$

$\$ 17,850$

$\$ 8,925$

ICP-NEW AWARD.
WI

$\$ 195,220$

$\$ 97,608$

ICP - NEW AHARD

$\$ 5,000$

$\$ 2,500$

EXTENDED EDUCATION PROGRAM

NM $03 \quad \$ 51,500$

$\$ 51,500$

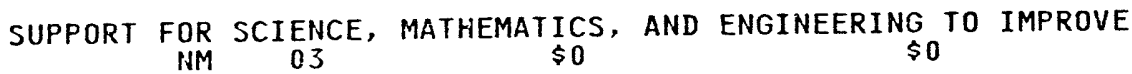

ICP-NEW AWARD 


\begin{tabular}{|c|c|c|c|c|c|c|c|}
\hline AWARDEE NAME & BIN & DATE & & & DESC & F WORK & \\
\hline TVISION & VENDOR ID & CITY & STATE & $\begin{array}{l}\text { CONG } \\
\text { DIST }\end{array}$ & $\begin{array}{l}\text { CONTRACT } \\
\text { VALUE }\end{array}$ & $\begin{array}{l}\text { OBLIGS } \\
\text { TO DATE }\end{array}$ & $P / S$ \\
\hline
\end{tabular}

MAHARISHI INTERNATIONAL UNIV

MAHNOMEN COUNTY \& VILLAGE HOSP NURSING CENTER

MAINE METAL PRODUCTS ASSN

MAINE SCHOOL ADMIN DIST \#17

MAINE SCHOOL ADMIN DIST \#20

MAINE SCHOOL ADMIN DIST \#40

MAINE SCHOOL ADMIN DIST $\$ 56$

MAINE SCIENCE \& TECH COMM

MAINE STATE OF

HOUSING AUTHORITY

maine state planning office
FG47-93R707034 08/31/94 115240-0001-9 FAIRFIELD

FG45-93R551706 12/31/94 048960-0101-X MAHNOMEN

FG04-94AL99286 03/31/96 237870-0001-1 PORTLAND

FG41-93R192514 06/30/95 218120-0001-4 SOUTH PARIS

FG41-94RI92603 $06 / 30 / 96$
FORT FAIRFIELD

FG41-93R192510 06/30/95
$210610-0001-X$ WAL DOBORO FG41-94R192604 06/30/96 WALDOBORO

FG41-94R192605 $06 / 30 / 96$
SEARSPORT

FCO2-93ER75945 $09 / 29 / 95$
$234750-0001-6 \quad$ AUGUSTA

FG41-93R130212 06/30/98
$049060-0001-0$ AUGUSTA

FG41-93R130242 06/30/38 049060-0001-0 AUGUSTA

FG41-94R130234 03/31/99 049060-0801-1 AUGUSTA

FC01-91EI22786 08/07/96
$049060-0701-5$ AUGUSTA
GRANT PROGRAMS FOR SCHOOLS ANDHOSPITALS AND FOR BUIDLINGS 0 IA $\quad 03 \quad$ SCHOOL $\$ 49,850 \quad \$ 24,925$

$\underset{\text { ICP-NEW AWARD }}{\text { MN }}$

$\$ 70,868$

$\$ 63,781$

TRP 1373 ENVIRONMENTALLY CONSCIOUS MFG ME $01 \$ 1,180,813 \quad \$ 502,000$

TITLE III OF THE NATIONAL ENERGY CONSERVATION POLICY ACT $\mathrm{ME} \quad 02 \quad \$ 13,617 \quad \$ 6,808$

TITLE III OF THE NATIONAL ENERGY CONSERVATION POLICY ACT FY'94 ICP AWARD ${ }_{\text {ME }}$ O1 $\$ 107,771 \quad \$ 42,000$ FY.94 ICP AWARD ${ }_{\text {ME }}$ O2 $\$ 54,892 \quad \$ 27,446$ MAINE DOE/EPSCOR IMPLEMENTATION PROGRAM

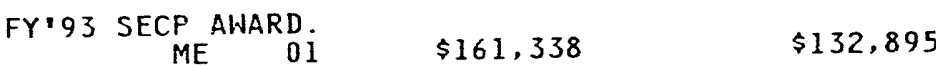
AWARD NEW FY.93 FUNDS. $\$ 401,654 \quad \$ 51,500$ FY 1994 GRANT AWARD WEATHERIZATION. $\$ 2,959,394$ $\begin{array}{ccc}1991-1992 \text { STATE HEATING OIL } \& \text { PROPANE PROGRAM } \\ \text { ME } 01 & \$ 42,633 & \$ 21,294\end{array}$ 


\begin{tabular}{|c|c|c|c|c|c|c|c|}
\hline AWARDEE NAME & BIN & 1 DATE & & & DESCF & F WORK & \\
\hline DIVIS & VENDOR ID & CITY & STATE & $\begin{array}{l}\text { CONG } \\
\text { DIST }\end{array}$ & $\begin{array}{l}\text { CONTRACT } \\
\text { VALUE }\end{array}$ & $\begin{array}{l}\text { OBLIGS } \\
\text { TO DATE }\end{array}$ & $P / S$ \\
\hline
\end{tabular}

MAINE UNIVERSITY OF

MAINE YANKEE ATOMIC POWER

\section{MAITLAND BROS}

MALAMUT OPTICIANS

\section{MAMARONECK UFSD}

MANAGEMENT GRAPHICS INC

- manhattan college NUCLEAR ENGINEERING SCHOOL OF ENGINEERING

MANSFIELD UNIVERSITY PHYSICS

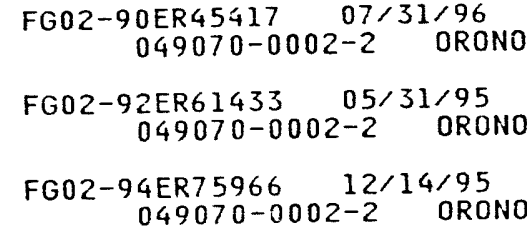

$A B 01-94 A D 69234 \quad 02 / 28 / 95$ 216780-0001-9 ROCKVILLE FG42-93R253876 07/31/95
$230500-0001-5$ MAMARONECK

ADOI-94AD64808 03/31/95 222500-0002-6 MINNEAPOLIS

AD01-94AD25693 08/30/94 222500-0002-6 MINHEAPOL IS

FG02-90ER13043 09/29/94 $049370-0301-1$ NEW YORK

FG02-91ID13082 09/29/94 $049370-0202-3$ RIVERDALE

FG34-94RF0081905/01/95 049490-0101-9 MANSFIELD

FG43-94R364104 05/31/95 STRUCTURE ADHESION AND $\begin{gathered}\text { STABILITY OF METAL/OXIDE AND } \\ \text { ME } 02\end{gathered}$

ADSORPTOON CONTROL OF ORGANIC CARBON BUROAL IN OCEAN ME $02 \quad \$ 336,024 \quad \$ 336,024$

PRE-SERVICE TEACHER ENHANCEMENT PROGRAM $M E \begin{array}{lll}M 2 & \$ 95,082 & \$ 41,711\end{array}$

DISPOSAL OF SPENT NUCLEAR

$\begin{array}{llllll}\text { CONSTRUCTION } & 24-\text { INCH } & \text { BRINE } & \text { DISPOSAL PIPELINE } & \text { PIS } \\ & 14 & \$ 9,366,000 & \$ 9,366,000 & Y 299\end{array}$

BPA FOR SAFETY GLASSES

BPA FOR SAFETY GLASSES
MD

$\$ 0 \quad 6540$

CYCLE 15 AWARD.

CYCLE $15 \underset{\text { NYARD. }}{\text { AWAR }} 18 \quad \$ 242,734 \quad \$ 121,365$

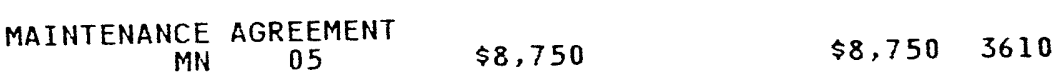

$\begin{array}{lll}\text { SLIDE RECORDER } & & \\ M N & \$ 16,915 & \$ 16,915 \quad 9999\end{array}$

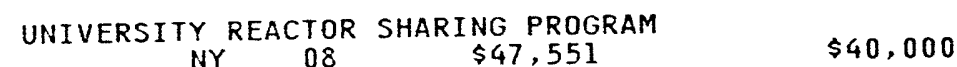

$\begin{array}{cccc}\text { UNIVERSTITY REACTOR INSTRUMENTATION PROGRAM } & \text { NY } \\ \text { NY } & 17 & \$ 90,745 & \$ 90,745\end{array}$

USED ENERGY RELATED LAB EQUIPMENT

$\$ 0$

TITLE III OF THE NATIONAL ENERGY CONSERVATION POLICY ACT 


\begin{tabular}{|c|c|c|c|c|c|c|c|}
\hline AWARDEE NAME & BIN & 1 DATE & & & DESCF & F WORK & \\
\hline DIVISION & VENDOR ID & CITY & STATE & $\begin{array}{l}\text { CONG } \\
\text { DIST }\end{array}$ & $\begin{array}{l}\text { CONTRACT } \\
\text { VALUE }\end{array}$ & $\begin{array}{l}\text { OBLIGS } \\
\text { TO DATE }\end{array}$ & P/S \\
\hline
\end{tabular}

MANUFACTURING \& TECH CONVERSN

MANUFACTURING SCIENCES CORP DENVER OFFICE

MARATHON CSD

MARC INDUSTRIES

MARGARET R PARDEE MEM HOSPITAL

MARIAN HEIGHTS ACADEMY

MARINE BIOLOGICAL LABORATORY

EMARINE SAFETY SYSTEMS INC $\begin{array}{cc}\text { AC21-89MC26288 } & 06 / 01 / 95 \\ 174970-0002-0 & \text { SANTA FE SPRINGS }\end{array}$

BROAD AGENCY ANNOUCEMENT) SONIC ENHANCED ASH

FC05-9OCE40893 03/31/95

FG05-90ER80943 05/19/95

174970-0001-2 COLUMBIA

AC22-90PC90155 05/31/95

$174970-0001-2$ COLUMBIA

FC34-94RF00733 06/30/96 233940-0102-0 GOLDEN

AC21-93MC $30170 \quad 07 / 17 / 94$ 233940-0001-6 OAK RIDGE

FG42-93R253872 07/31/95 230590-0001-8 MARATHON

AC65-92WF01778 09/30/94 136970-0002-0 LAS VEGAS

FG44-92R435004 08/30/94 220680-0001-6 HENDERSONVILLE

FG45-93R55158908/31/95 232560-0001-0 FERDINAND

FG02-85ER13361 04/30/95
049670-0001-0 WOODS HOLE FG02-92ER61438 05/31/95 049670-0001-0 WOODS HOLE

FG46-93R699306 08/31/95 $231970-0001-3$ HOUSTON
$\begin{array}{llll}C A & 34 & 54,166,874 & \end{array}$

PULSED COMBUSTION PROCESS FOR BLACK LIQUOR GASIFICATION COOP

$$
M D \text { O } \$ 10,732,802 \quad \$ 9,957,679
$$

AN ADVANCED CONCEPT FOR COST-EFFECTIVE PRESSURIZED FLUIDIZED

$$
M D 0_{3} \$ 549,946 \quad \$ 549,946
$$

DEVELOPMENT AND TESTING OF COMMERCIAL SCALE COAL-FIRED COMBU

MD $03 \quad \$ 2,360,745 \quad \$ 2,360,745$ AG1

STAGE I AND II OF THE NATIONALCONERSION PILOT PROJECT AT RF $C O \quad 02 \$ 1,077,236 \quad \$ 988,000$

EM ADVANCED TECHNOLOGIES FOR DECONTAMINATION AND CONVERSION TN $03 \quad \$ 937,790 \quad \$ 931,992$ AH93

CYCLE 15 AWARD.

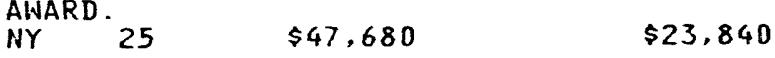

JANITORIAL SUPPORT SERVICES

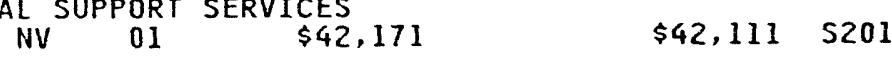

CYCLE 14 - INSTITUTIONAL CONSERVATION PROGRAM. APPROVED BUD $\begin{array}{lll}\text { - INSTITUTIONAL CONSERVATION PROGRAM, APP } & \text { PC } \\ \text { NC } 11 & \$ 19,939 & \$ 40,000\end{array}$

ICP-NEW AWARD.

$\$ 57,967$

$\$ 28,983$

SUMMER INVESTIGATIONS INTO THEISOLATION CULTIVATION AND MET MA $10 \quad \$ 149,430 \quad \$ 149,430$

ORIGINS \& FATES DF DISSOLVED ORGANIC MATTER ALONG THE NEW

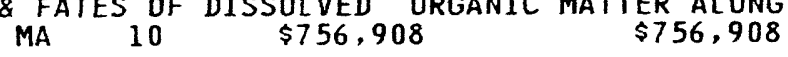

AWARDS $\$ 99,000$ NEW DOE FUNDS TO DEVELOP AN ON-BOARD PUMPS-AN $T X \quad 25 \quad \$ 99,000 \quad \$ 99,000$ 


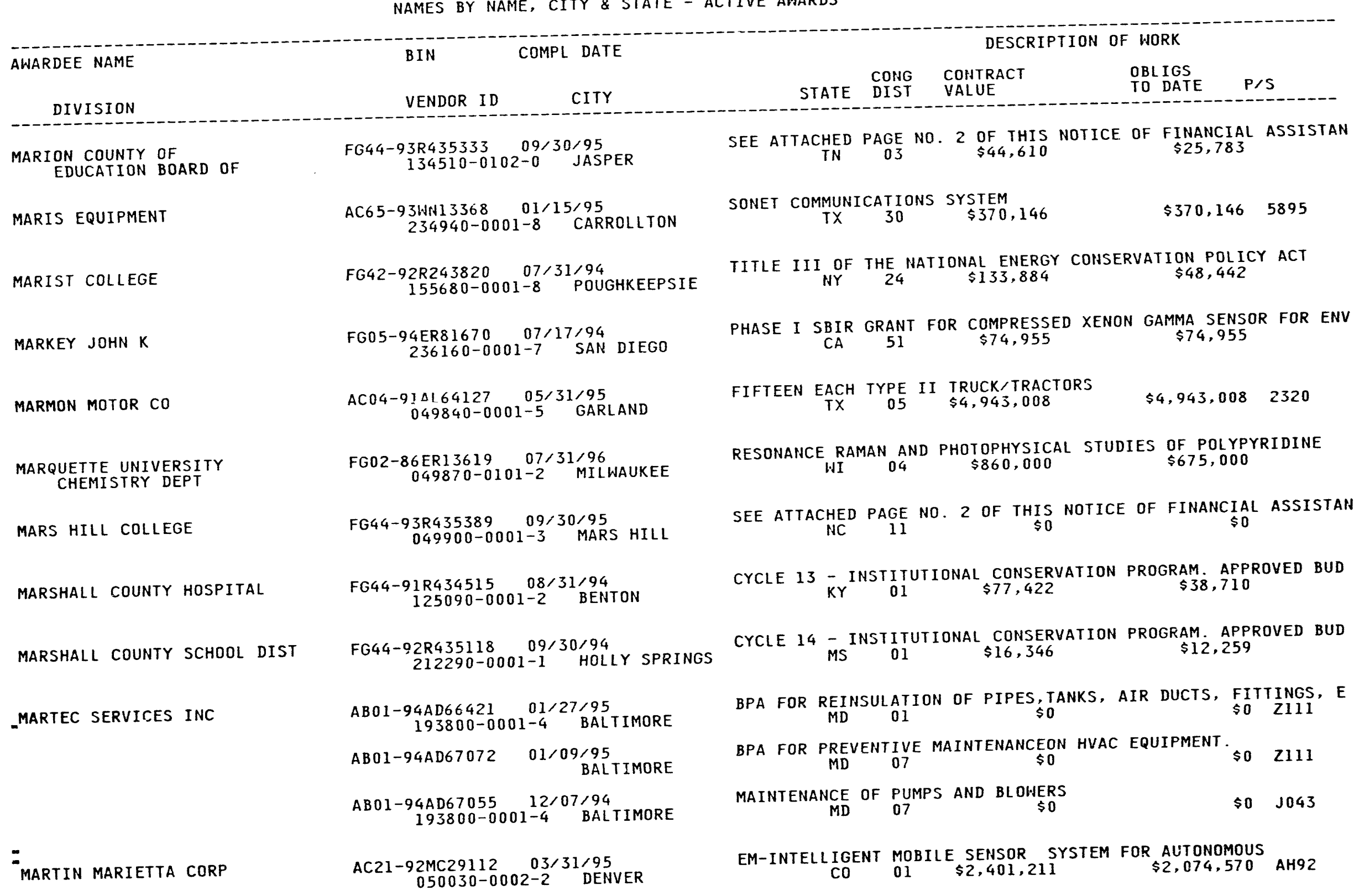


PROCUREMENT AND ASSISTANCE DATA SYSTEM (PADS) NAMES BY NAME, CITY \& STATE - ACTIVE AWARDS
PAGE NO. 281

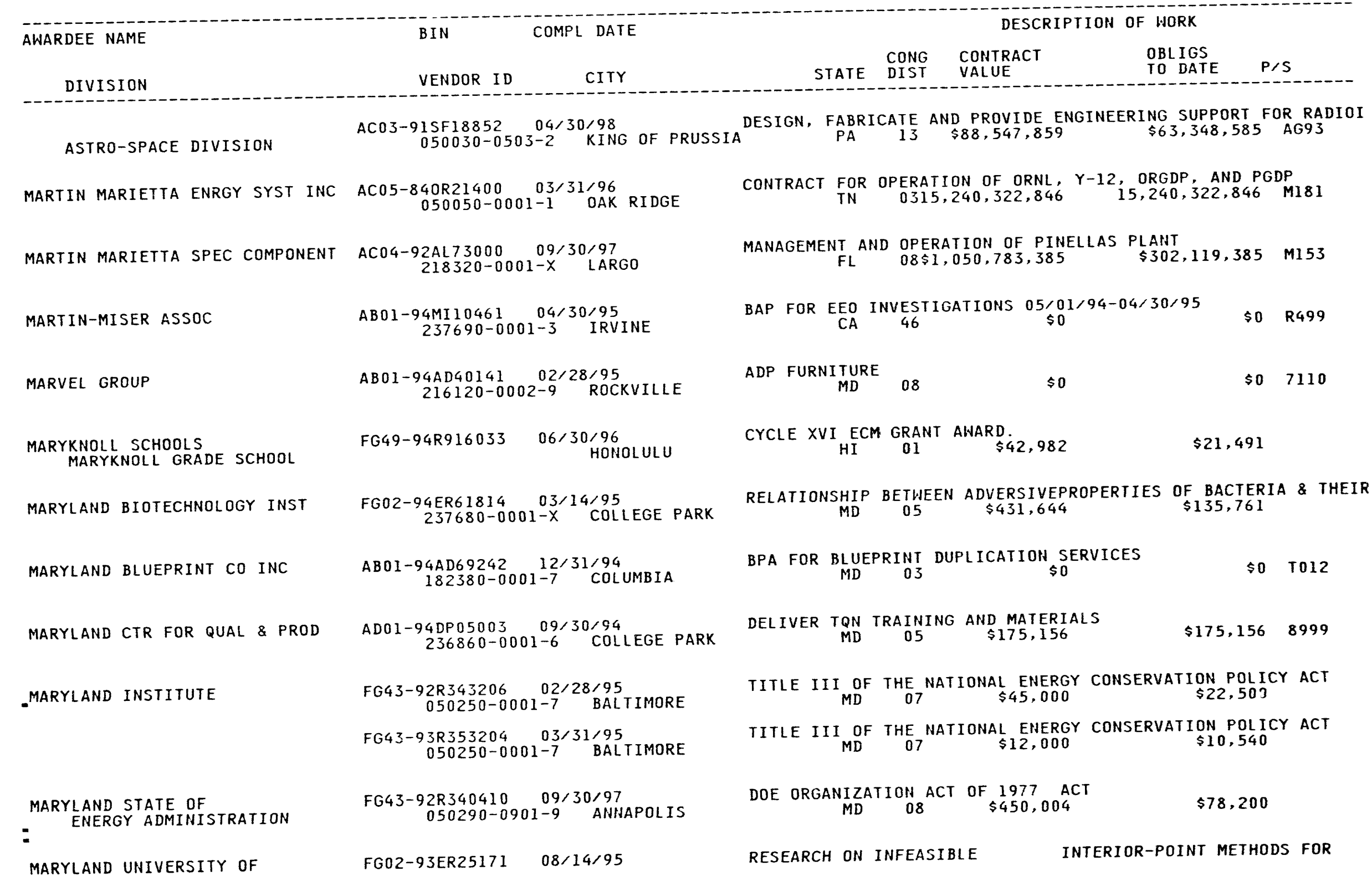




AWARDEE NAME BIN COMPL DATE

DIVISION
MATHEMATICS \& STATISTICS DEPT

ELECTRICAL ENGINEERING DEPT FG05-89ER14090 $09 / 14 / 95$ FG43-93R353208 03/31/95
$050300-0001-1$ BALTIMORE FG02-94ER25198 02/28/97 $050300-0001-1$ BALTIMORE FG02-94ER14441 04/14/97 050300-0004-6 COLLEGE PARK

FG02-93ER40762 11/30/95 050300-0004-6 COLLEGE PARK

FG02-93ER54197 11/14/95 050300-0004-6 COLLEGE PARK

CHEMISTRY \& NUCLEAR ENG FG02-81ER10228 $09 / 15 / 94$
$050300-0304-5 \quad \mathrm{COL}$ 050300-0304-5 COLLEGE PARK

RESEARCH \& ADMIN OFFICE OF FG02-90ER12986 09/19/94 050300-2804-8 COLLEGE PARK FG02-92ER20083 06/30/95 050300-0004-6 COLLEGE PARK

FG02-92ER54178 08/14/94 050300-0004-6 COLLEGE PARK

FG02-94ER40834 01/31/97 050300-0004-6 COLLEGE PARK

FG02-94ER40855 05/31/95 050300-0004-6 COLLEGE PARK

FG02-94ER61746 10/31/96 050300-0004-6 COLLEGE PARK

MECHANICAL ENGINEERING DEPT FG02-93ER14336 03/31/96 050300-0904-3 COLLEGE PARK FG02-93CE23803 $07 / 31 / 94$
$050300-0904-3$ COLLEGE PARK FG02-93ER20106 05/19/96 050300-0004-6 COLLEGE PARK

\section{DESCRIPTION OF WORK}

STATE CONG CONTRACT

STATE DIST

OBLIGS
TO DATE P/S $\$ 95,844$

$\$ 95,844$

PULSE PROPAGATION IN INHOMOGENEOUS OPTICAL WAVEGUIDES $M D \quad 03 \quad \$ 501,639$ $\$ 404,088$

TITLE III OF THE NATIONAL ENERGY CONSERVATION POLICY ACT

DISCRETE AND CONTINUOUS MODELSOF CONSERVATION LAWS MD $03 \quad \$ 222,280 \quad \$ 75,803$ CONTAMINANT DISPERSAL IN BOUNDED TURBULENT SHEAR FLOWS $\begin{array}{rcc}\text { MD } & 05 & \$ 407,999 \\ \text { HADRONIC \& NUCLEAR PHYSICS }\end{array}$

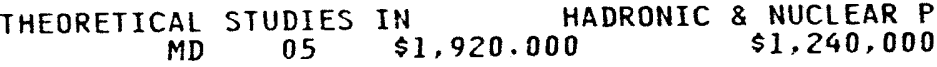
MARYLAND CONTROLLED FUSION RESEARCH PROGRAM $\begin{array}{rll}\text { MD } 05 & \$ 1,427,000 & \$ 927,000\end{array}$ UNIVERSITY REACTOR SHARING.

$$
\text { MD }{ }_{05}^{\text {REACTOR }} \text { SHARING } \$ 187,170 \quad \$ 185,900
$$

UNIVERSITY REACTOR INSTRUMENTATION PROGRAM

MD $05 \quad \$ 90,495 \quad \$ 85,995$

STRUCTURE \& REGULATION OF L-GLUTAMATE DEHYDROGENQUE

MD $05 \quad \$ 271,053$

STUDIES OF THE LONGITUDINAL INSTABILITY HITH AS ELECTRON

RESEARCH IN NUCLEAR STRUCTURE

ACCELERATOR RESEARCH STUDIES

A STUDY OF LONGWAVE RADIATION CODES FOR CLIMATE STUDIES;

$M D$ MD $\$ 1,068,918 \quad \$ 291,000$ $\begin{array}{cccc}\text { CHARATERIZATION OF METAL } & \text { CUTTING DYNAMICS } \\ \text { MD } & 05 & \$ 204,658 & \$ 135,771\end{array}$ EHD ENHANCEMENT OF IN-TUBE CONDENSATION AND EVAPORATION $M D$ O5 $\$ 115,000$ \$115,000

MECHANISMS OF TRANSCRIPTINAL GENE REGULAITON IN THE MD $05 \quad \$ 255,067 \quad \$ 165,517$ 


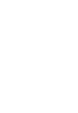

METEOROL OGY DEPT

FG02-93ER25166 05/31/96 050300-0004-6 COLLEGE PARK

FG02-93ER61633 06/30/95 050300-1104-8 COLLEGE PARK

CHEMISTRY \& BIOCHEMISTRY

FG02-93ER40802 04/30/96 050300-2304-6 COLLEGE PARK

FG02-93ER61691 08/31/96 050300-0004-6 COLLEGE PARK

FG03-93SF20142 09/29/94 050300-2804-8 COLLEGE PARK

RESEARCH \& ADMIN OFFICE OF

FG05-92ER5414901/31/95 050300-2604-5 COLLEGE PARK

PLASMA RESEARCH LAB

CHEMISTRY \& BIOCHEMISTRY

BOTANY DEPT

PLASMA RESEARCH LAB

MECHANICAL ENGINEERING DEPT

ELECTRICAL ENGINEERING DEPT

PHYSICAL SCIENCE \& TECH INST

- MEChanical ENGINEERING DEPT

PLASMA RESEARCH LAB

METEOROLOGY DEPT

:

ELECTRICAL ENGINEERING DEPT

FG05-92ERI4230 02/14/95 050300-2304-6 COLLEGE PARK

FG05-86ER13461 01/31/95 050300-2104-3 COLLEGE PARK

FG05-87ER52147 07/14/95 $050300-2604-5$ COLLEGE PARK FG05-88ER13838 07/31/94 050300-0904-3 COLLEGE PARK FG05-88ERI3846 12/31/95 050300-0704-0 COLLEGE PARK FG05-88ERI3902 12/14/94 050300-3004-2 COLLEGE PARK

FG05-88ER13977 12/14/94 $8 E R 13977$ 12/14/94
$050300-0904-3$ COLLEGE PARK FG05-89ER53301 09/14/95 050300-2604-5 COLLEGE PARK FG05-90ER61075 10/31/94 050300-1104-8 COLLEGE PARK FG05-91ER40642 05/31/95
$050300-0704-0$ COLLEGE PARK
A PROPOSAL FOR THE DEVELOPMENTOF TECHNIQUES FOR IMPLEMEN-

$$
M D \quad 05 \quad \$ 900,000 \quad \$ 576,000
$$

ICRCCM PHASE II VERIFICATION AND CALIBRATION OF RADIATION

$$
\text { MD } 11 \text { T5 } \$ 571,855 \text { A } \$ 571,855
$$

REACTION MECHANISM STUDIES OF HEAVY ION INDUCED REACTIONS MD 05 \$631,000 $\$ 418,000$

VALIDATION OF SOIL MOISTURE INGCMS - AMIP DIAGHOSTIC

$$
\text { MD } 05 \text { \$150,000 } \$ 50,000
$$

NLUF USER'S GRANT AWARD - DEVELOPMENT OF DENSITY AND TEMPERA NLUF USER'S GRANT ANARD $\$ 157,259 \quad \$ 157,259$

MEASUREMENT OF IMPURITY ION DENSITIES AND ENERGIES IN THE DI MD

ODD-ELECTRON ORGANOMETALLIC CHEMISTRY DF RELEVANCE TO HYDROC $\begin{array}{ccc}\text { MD } & 05 & \$ 224,413\end{array}$

IDENTIFYING CALCIUM CHANNELS AND PORTERS IN PLANT MEMBRANES $M D \$ 05 \quad \$ 525,251$ \$525,251

MICROHAVE GENERATION FOR MAGNETIC FUSION ENERGY APPLICATIONS $M D \quad 05 \$ 2,635,283 \quad \$ 2,285,283$

LAGRANGIAN ANALYSIS OF CONTAMINANT DISPERSAL IN BOUNDED TURB

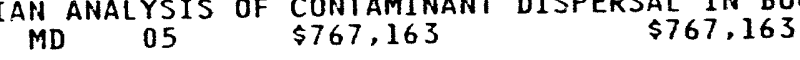

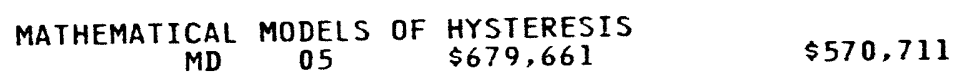

THERMOPHYSICAL PROPERTIES OF SUPERCRITICAL FLUIDS AND FLUID MD $05 \quad \$ 701,920 \quad \$ 701,920$

MECHANICS OF REDUNDANTLY DRIVEN ROBOTIC SYSTEMS

NONL INEAR DYNAMICS AND PLASMA TRANSPORT

A STUDY OF LONGWAVE RADIATION CODES FOR CLIMATE STUDIES: VA MD $05 \quad \$ 890,615 \quad \$ 890,615$

ACCELERATOR RESEARCH STUDIES

MD $05 \quad \$ 4,569,500 \quad \$ 4,569,500$ 


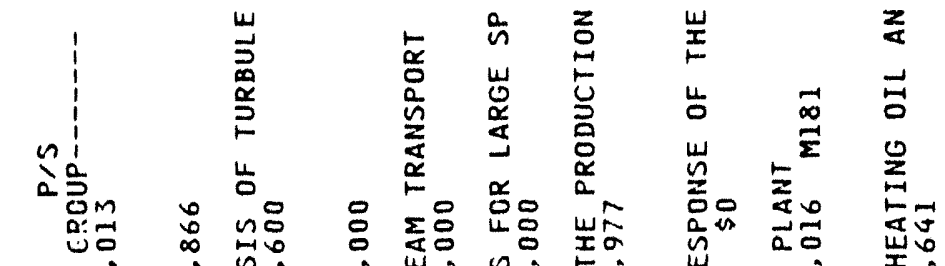

$$
\begin{aligned}
& \begin{array}{ll}
\infty & \\
0 & \infty \\
0 & 0 \\
0 & 0 \\
0 & 0 \\
0 & 0 \\
0 & 0
\end{array}
\end{aligned}
$$

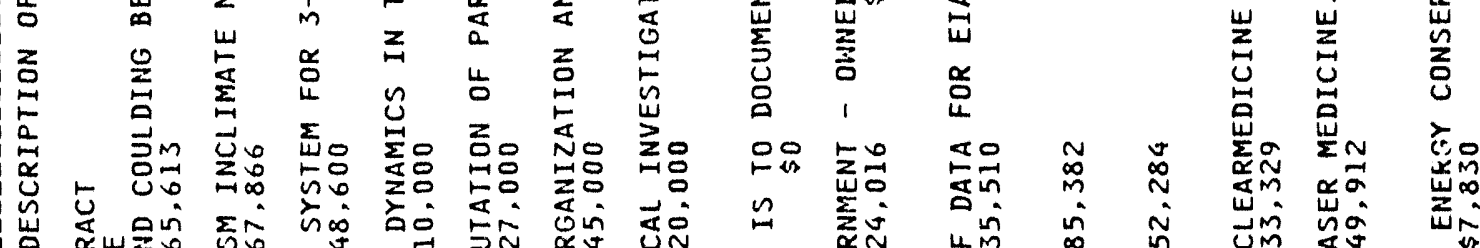

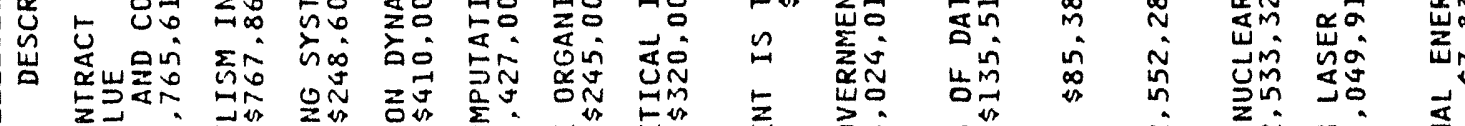

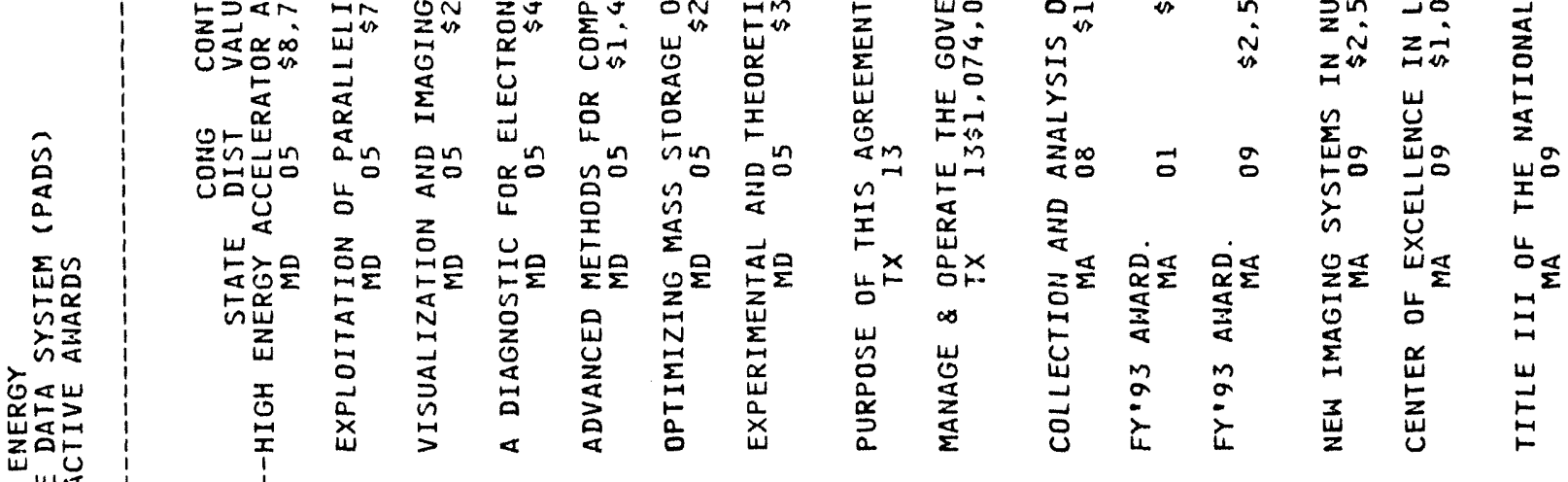

覀।

点言岕

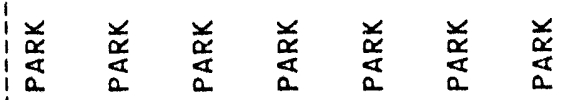

语行

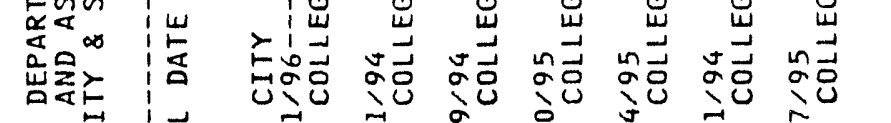

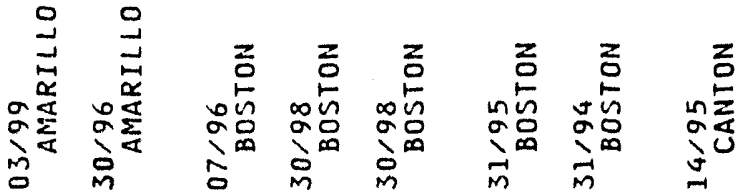

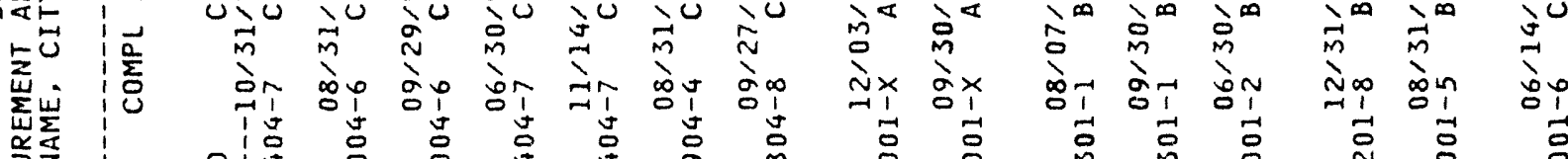

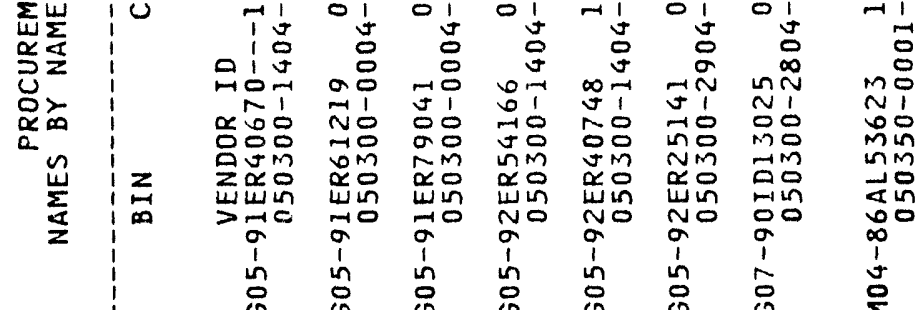

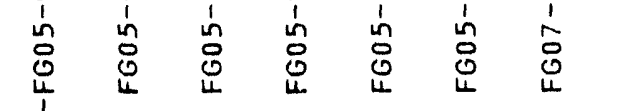

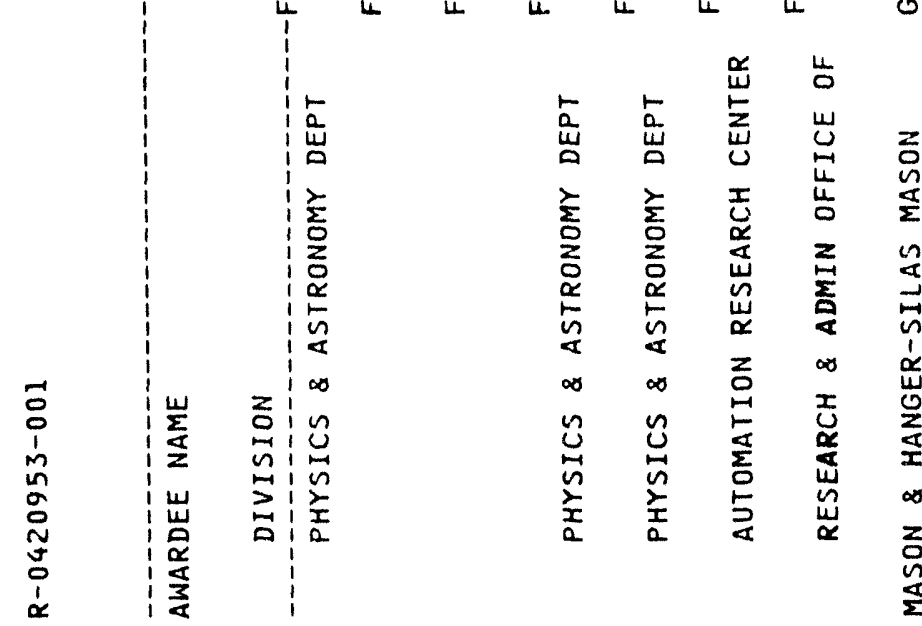

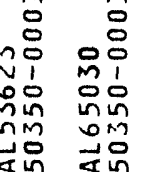

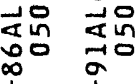

范吉

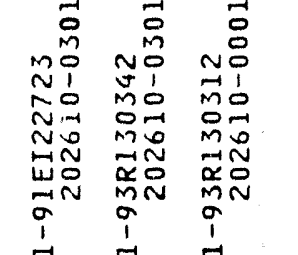

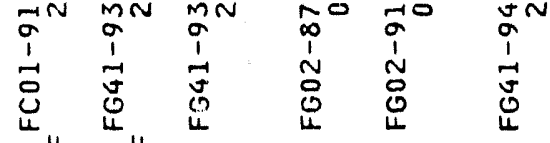
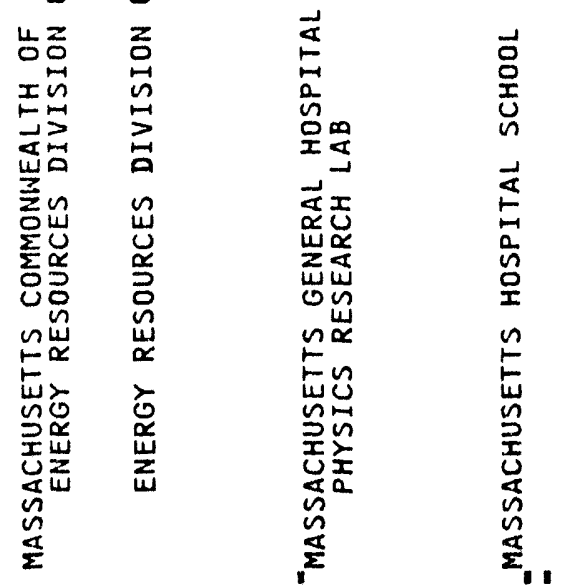

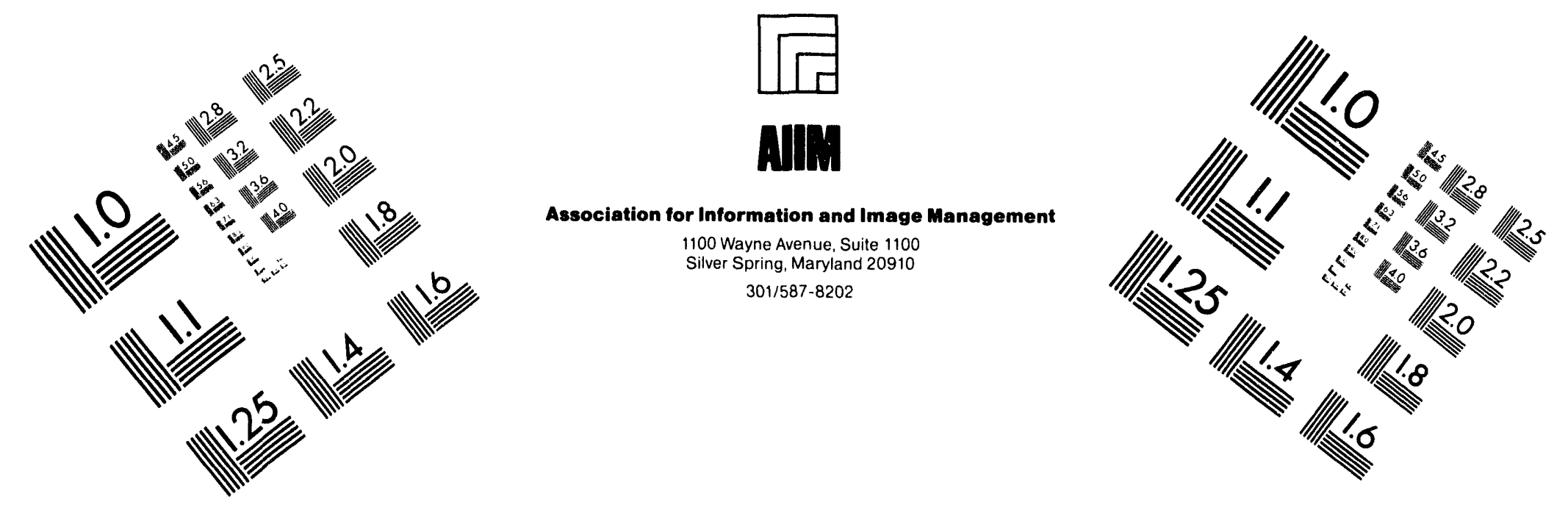

\section{Centimeter}

1

Inches
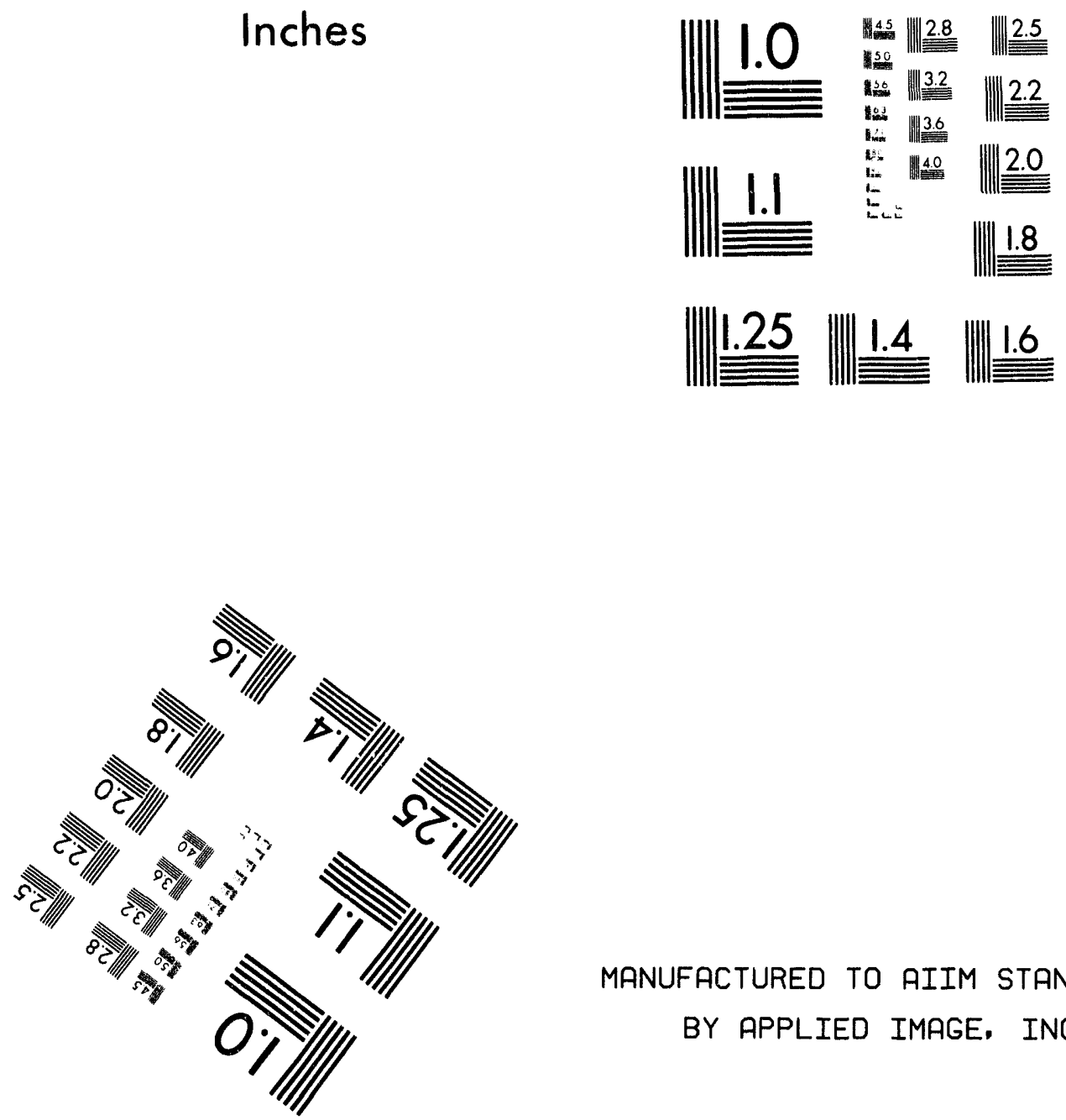

MANUFACTURED TO AIIM STANDARDS

BY APPLIED IMAGE, INC.

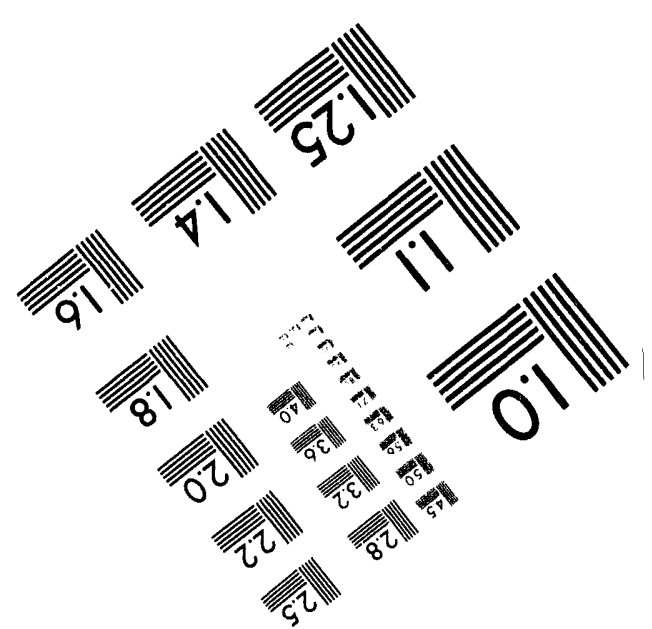



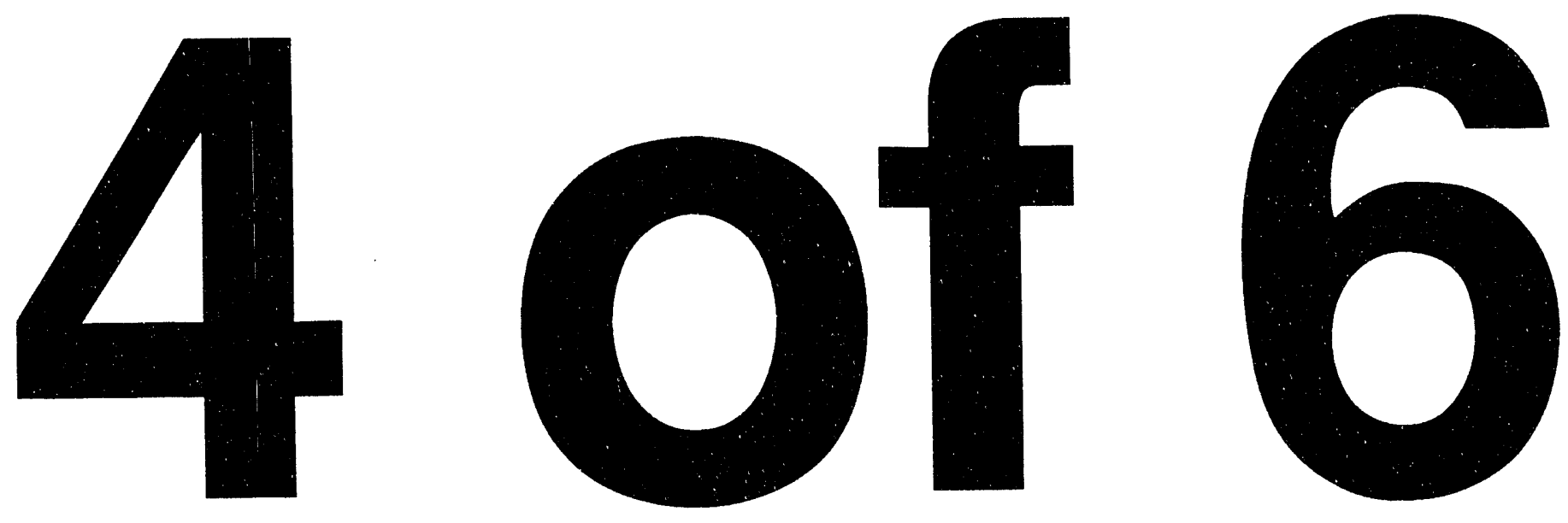


\section{AWARDEE NAME}

IN

COMPL DATE \\ DESCRIPTION OF HORK}

STATE DIST CONTRACT

VENDOR ID CITY

FG02-85ER13454 08/14/94

$050440-0301-8$ CAMBRIDGE

MASSACHUSETTS INST OF TECH

CHEMISTRY DEP

CHEMICAL ENGINEERING DEPT

FG02-88ER13896 09/14/94 050440-0201-1 CAMBRIDGE

FG02-88ER25066 08/19/94 $050440-2401-5$ CAMBRIDGE

MATHEMATICS DEPT

FG02-89ER60874 07/31/94
$050440-4401-6$ CAMBRIDGE

FG02-90ER45422 07/31/94 050440-4001-0 CAMBRIDGE

CIVIL ENGINEERING DEPT

FG02-91ER61220 08/31/94

METEOROLOGY \& PHYS OCEAN DEPT 050440-2101-6 CAMBRIDGE

NUCLEAR REACTOR LAB

FG02-80ER10770 09/15/94 050440-2601-8 CAMBRIDGE

FG02-90ER12971 09/29/94 050440-2501-1 CAMBRIDGE

NUCLEAR ENGINEERING DEPT

FG02-92ER75727 07/31/94
$050440-2501-1$ CAMBRIDGE

FG02-92ER75787 09/29/94 050440-2501-1 CAMBRIDGE

NUCLEAR ENGINEERING DEPT

FG02-93ER20129 09/09/94
$050440-3601-3$ CAMBRIDGE FG03-93SF19706 07/01/94
$050440-0001-9$ CAMBRIDGE FG05-91ER79031 c 09/29/94
$050440-0001-9$ CAMBRIDGE FG05-92ER79111 07/31/94
$050440-0001-9$ CAMBRIDGE FG05-92ER79115 07/31/94 050440-0001-9 CAMBRIDGE FG05-92ER79128 07/31/94
$050440-0001-9$ CAMBRIDGE FG22-91PC91293 09/17/94

UNIVERSITY REACTOR INSTRUMENTATION PROGRAM THERMODYNAMICS AND RATE OF METHA

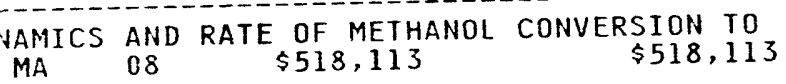

TO DATE P/S

MACK \& STATISTICAL HYDRODYNAMICS

$\begin{array}{llll}\text { MA } 08 & \$ 341,575 & \$ 341,575\end{array}$

GEOMETRY AND ELEMENTARY PARTICLE PHYSICS

$\begin{array}{cccc}M A & 08 & \$ 1,665,199 & \$ 1,665,199\end{array}$

$\begin{array}{ccc}\text { EPITHERMAL NEUTRON CAPTURE } & \text { THERAPY } & \$ 974,492\end{array}$

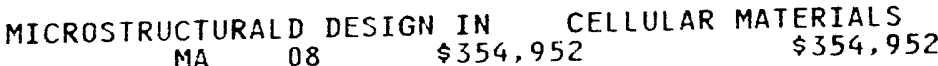

DEVELOPMENT OF AN ADVANCED REPRESENTATION OF CUMULUS CONVECT MA 08 \$271,690 $\$ 271,690$

UNIVERSITY REACTOR SHARING
MA 08
$\$ 880,600$
$\$ 880,600$

$M A \quad 08 \quad \$ 260,700 \quad \$ 260,700$

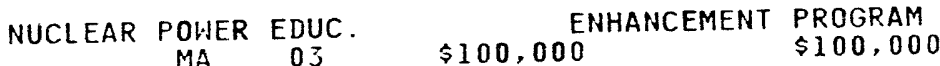

IMPROVING HUMAN RELIABILITY THROUGH BETTER NUCLEAR POWER $M A{ }_{03} \$ 281,780 \quad \$ 281,780$

INT'L SYMPOSIUM ON CELLULAR \& MOLECULAR BIOLOGY OF MA $08 \quad \$ 3,500$ NEW GRANT FOR ASSESSMENT OF FACTORS OF SOCIOECONOMIC ACCEPTA SECOND GENERATION DIGITAL IMAGING SPECTROPHOTOMETER
MA 08

INSTRUMENTATION - AN AREA DETECTOR FOR HIGH RESOLUTION MACRO $M A \quad 08 \quad \$ 230,710 \quad \$ 230,710$

INSTRUMENTATION - HPLC-DAD/APCI-MS AND DIRECT FLAME SAMPLING $M A \begin{aligned} & M A \\ & 0\end{aligned} \quad \$ 355,940 \quad \$ 355,940$

MESOLUTION EELS MICROANALYSIS SYSTEM $\begin{array}{ccc}\text { INSTRUMENTATION - HIGH } & \text { RESOLUTION EELS MICROANALYS } \\ \text { MA } 08 & \$ 119,775 & \$ 119,775\end{array}$

DIRECT CATALYTIC DECOMPOSITIONOF NITRIC OXIDE 


\section{AWARDEE NAME}

BIN

COMPL DATE

DESCRIPTION OF WORK

DIVISION

BIN COMPL DATE

050440-0001-9 CAMBRIDGE

FG22-91PC91294 09/30/94 $050440-0001-9$ CAMBRIDGE

CHEMISTRY DEPT

PLASMA FUSION CENTER

MATERIALS SCI \& ENG DEPT

PHYSICS DEPT

NUCLEAR REACTOR LAB

MECHANICAL ENGINEERING DEPT

MATERIALS SCI \& ENG DEPT

CIVIL ENGINEERING DEPT

MATERIALS SCI \& ENG DEPT

MECHANICAL ENGINEERING DEPT

NUCLEAR ENGINEERING DEPT

CHEMICAL ENGINEERING DEPT

PHYSICS DEPT

$=$
FG02-88ER13914 11/30/94 O50440-0301-8 CAMBRIDGE 050440-4801-1 CAMBRIDGE FG02-91ER45458 12/31/94 050440-1901-1 CAMBRIDGE

FG02-94ER75993 11/10/94 $050440-3301-4$ CAMBRIDGE

FG02-93ER75859 05/31/95 $050440-2601-8$ CAMBRIDGE

FG02-85ER13335 08/31/95 $050440-2001^{-X}$ CAPABRIDGE

FG02-86ER45261 06/30/95 050440-1901-1 CAHIORIDGE

FG02-89ER60846 07/31/95 $050440-4001-0$ CAMBRIDGE

FG02-89ER45396 06/21/95 050440-1901-1 CAMBRIDGE

FG02-89ER14085 09/14/95 $050440-2001-x$ CAMBRIDGE

FG02-92ER75710 04/14/95 $050440-2501-1$ CAMBRIDGE

FG02-92ER14262 04/30/95 $050440-0201-1$ CAMBRIDGE

FG02-92ER45473 05/14/95 050440-3301-4 CAMBRIDGE FG02-92ER75785 05/15/95 $050440-2501-1$ CAMBRIDGE

FG02-92CH10518 09/30/95 $050440-0001-9$ CAMBRIDGE
FG02-91ER40648 11/30/94
CONG CONTRACT

MA $08 \quad \$ 198,725 \quad \$ 198,725$

OBLIGS

$P / S$

THE ROLE OF CORE STRUCTURE ON CHAR REACTIVITY

O8 $\$ 199.982$

INTERFACIAL SYSTEMS FOR PHOTOCHEMISTRY ENERGY

$\begin{array}{rlcl}\text { MA } & 08 & \$ 1,581,220 & \$ 1,581,220 \\ & \end{array}$

EXPERIMENTAL \& THEORETICAL
ST STUDIES OF HIGH GRADIE

OXIDES \& SURFACE MAGINETISM.

$08 \quad \$ 398,400 \quad \$ 398,400$

DOE NEW ENGLAND REGIONAL SCIENCE BOHL

A STUDY OF THE REDESIGN OPTIONS FOR THE MIT RESEARCH

MA 03 \$ $148,484 \quad \$ 148,484$

THE DEVELOPMENT OF A FRICTIOH MODEL PREDICTING THE SLIDIHG B

$\$ 655,310$

STRUCTURAL DISORDER AHD TRANSPORT IN TERNARY OXIIDES WITH

$\begin{array}{llll}M A & 08 & \$ 983,531 & \$ 983,531\end{array}$

COLLOIDS IN GROUNDHATER: THEIR MOBILIZATION SUBSURFACE

COLLOIDS IN GROUNDHATER: $\$ 623,970$ THETR MOBILIZATION $\$ 509,410$

RADIATION DISORDER AND APERIODICITY IN PRADIATED

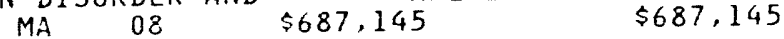

RHEOLOGICAL AHD FLOW CHARACTERISTICS OF DENSE

EXPERIMENTAL EVALUATION OF INSTRUMEHTED SYNTHESIS METHOD

$\begin{array}{ll}050,000 & \$ 260,000\end{array}$

SOLUBILIZATION OF TRACE ORGANICS IN BLOCK COPOLYMER

$03 \quad \$ 267,003 \quad \$ 267,003$

SYSTEMATIC GLOBAL RENORMALIZATION GROUP STUDIES MA $03 \quad \$ 130,743 \quad \$ 130,743$

CONCEPTUAL DESIGN OF A WATER- COOLED REACTOR WITH PASSIVE MA 03
0246,162 RATINAL ENHANCEMENT OF EMZYME PERFORMANCE IN ORGANIC SOLVENT 


\section{AWARDEE NAME}

PLASMA FUSION CENTER

ENERGY LAB

CHEMICAL ENGINEERING DE

CHEMICAL ENGINEERING DEPT

NUCLEAR ENGINEERING DEP

MATERIALS SCI \& ENG DEPT

APPLIED BIOLOGICAL SCI DEPT

CHEMISTRY DEPT

CHEMISTRY DEPT

- earth resources lab

EARTH \& PLANETARY SCI DEPT

CHEMISTRY DEPT
BIN

COMPL DATE

VENDOR ID

CITY

STATE DIST VALUE

DESCRIPTION OF WORK

ALUE

OBLIGS

$\mathrm{P} / \mathrm{S}$

SUPERCONDUCTIVING BITTER MAGNETS

$\$ 492,500$

ECONOMICS OF GLOBAL CHANGE RESEARCH PROGRAM

$\begin{array}{llll} & & \end{array}$

FUNDAMENIAL RESEARCH TO PROVIDE BETTER INSIGHT INTO THE FUEL 050440-0001-9 CAMBRIDG

FG04-87AL44875 08/31/95 050440-0001-9 CAMBRIDGE

FC21-92MC29264 09/30/95 050440-0901-6 CAMBRIDGE

FG22-92PC92534 03/07/95 050440-0201-1 CAMBRIDGE

AC22-92PC92111 09/30/95 050440-0201-1 CAMBRIDGE

ACO2-76ET37241 12/31/95 $050440-2501-1$ CAMBRIDGE

AC02-78ET51013 12/31/95 050440-0001-9 CAMBRIDGE

FG02-85ER45179 11/30/95 050440-1901-1 CAMBRIDGE

FG02-86ER60448 10/31/95 050440-4101-7 CAMBRIDGE

FG02-86ER13564 11/30/95 050440-0301-8 CAMBRIDGE

FG02-89ER14035 12/31/95 $050440-0301-8$ CAMBRIDGE

FG02-93ER14322 01/14/96 050440-4701-5 CAMBRIDGE

FG02-86ER13636 09/14/96 050440-0801-X CAMBRIDGE

FG02-87ER13671 01/31/96 050440-0301-8 CAMBRIDGE

FG02-87ER45307 05/31/96 050440-1901-1 CAMBRIDGE $\begin{array}{cccc}\text { MA } & 08 & \$ 728,468 & \$ 728 \\ & \text { FLUIDIZED BEDS }\end{array}$

$\begin{array}{lll}\text { SCALING OF PRESSURIZED } & \text { FLUIDIZED BEDS } \\ \$ 286,177 & \$ 397,440 & \$ 286,177\end{array}$

SELECTIVE CATALYTIC REDUCTION OF SULFUR DIOXIDE TO ELEMENTA $M A \quad 08 \quad \$ 199.999 \quad \$ 199,999$

CONVERSION OF LIGHT HYDROCARBON GASES TO METAL CARBIDES FOR $M A P O S \quad \$ 539,808$ $\$ 353,035$ AGI 1

FAST REACTOR BLANKET STUDIES

EXPERIMENTAL PROGRAM OF THE ALCATOR TOKAMAKS

MA $08 \$ 374,670.694 \quad \$ 310.211,285$ AG51

MECHANISMS OF THE OXIDATION OFMETALS AND ALLOYS

MA 08 \$2,034,959 \$1,851,902

COMPARATIVE MUTAGENESIS OF HUMAN CELLS IN VITRO AND IN VIVO MA MUTAGENESTS OF HUMAN

$\$ 2,980,820$

CHEMISTRY OF BIMETALLIC CYCLOPENTADIENYL COMPLEXES MA 08 \$1, 193,818 \$1, 048,750

HIGH PRESSURE HETEROGENEOUS CATALYSIS IN A LOW PRESSURE $\begin{array}{lll}\text { MA } & 08 & \$ 622,859\end{array}$

IN-SITU PERMEABILITY DETERMINATION USING BOREHOLE AND SPECTROSCOPIC AND DYNAMICAL STUDIES OF HIGHLY ENERGIZED \$1,303,500 \$1, 153,500 GRAIN BOUNDARY HONSTOICHIOMETRY IN

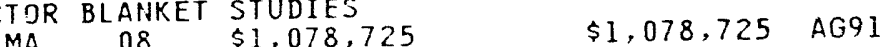

$\$ 1,078,725 \quad A G 91$

ELCTROSELSMIC CHARACTERIZATIONOF LITHOLOGY \& FLUID TYPE IN

$\begin{array}{lll}\text { MA } 08 & \$ 993,260 & \$ 887,147\end{array}$


$\stackrel{i}{0}_{0}^{0}$

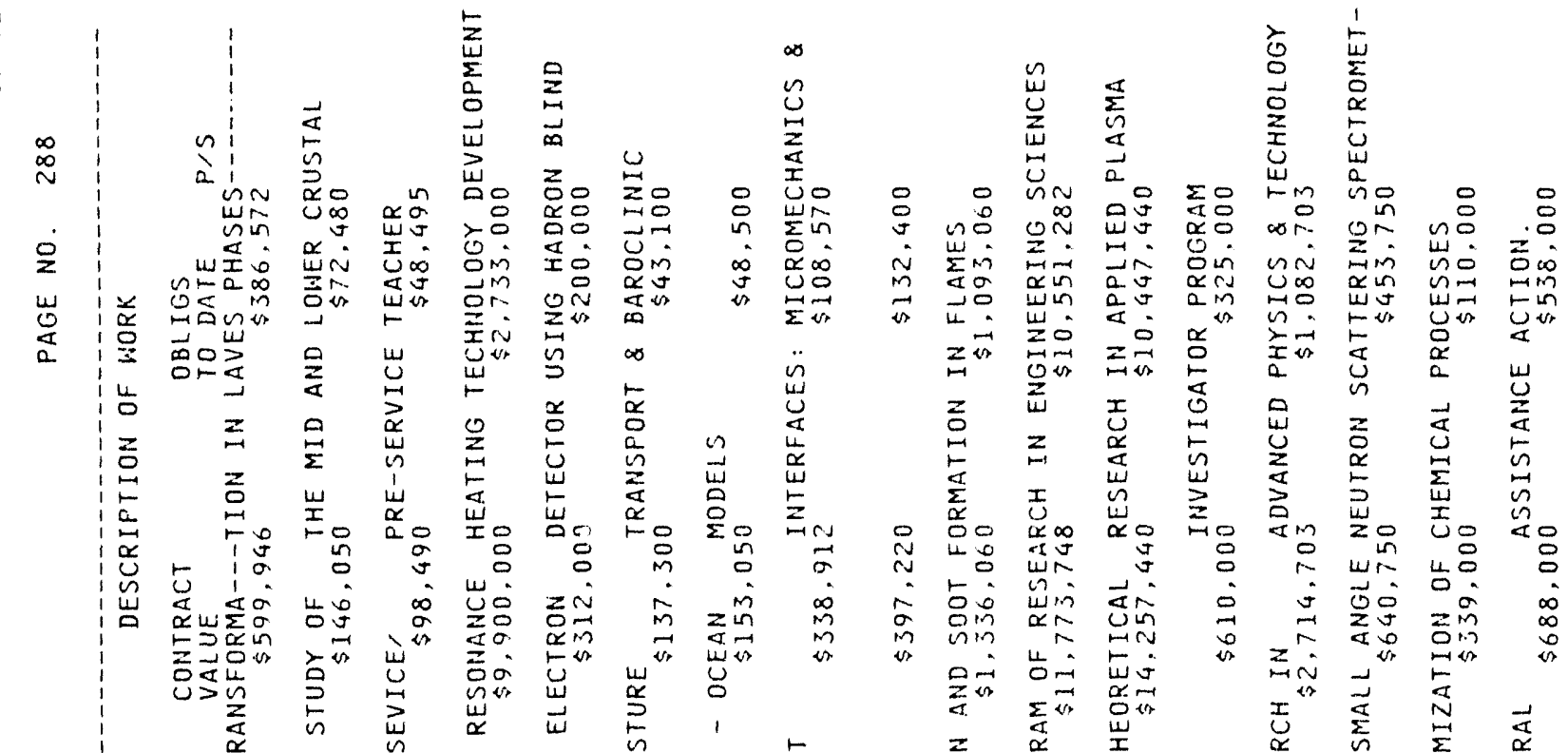

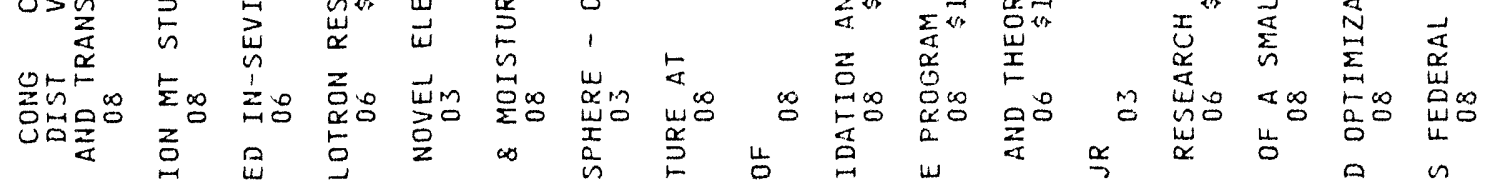

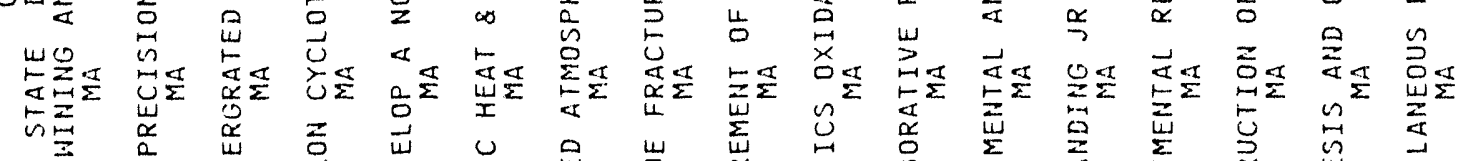

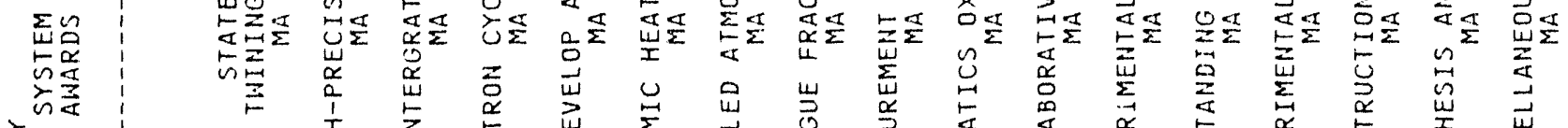
广。 政 至它

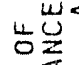

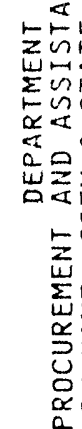
a

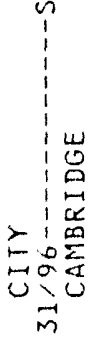

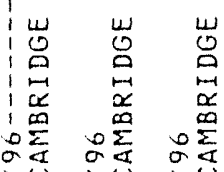

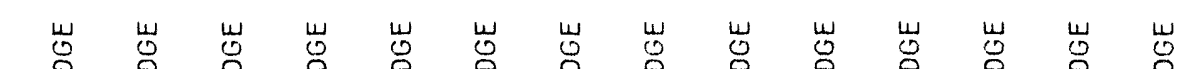

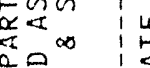

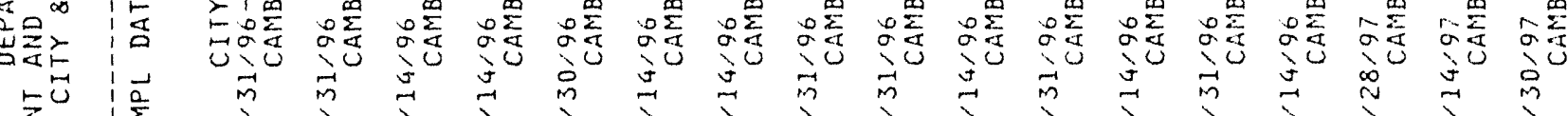

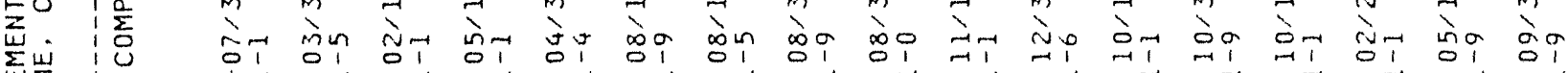
崖崖

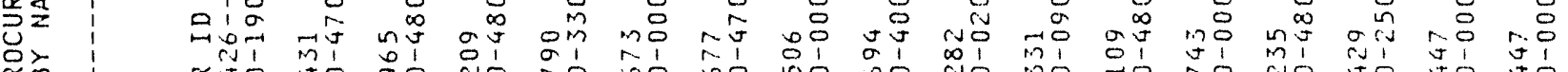

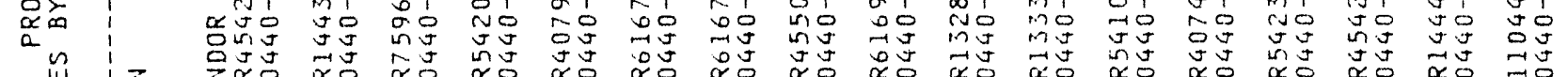
嵌

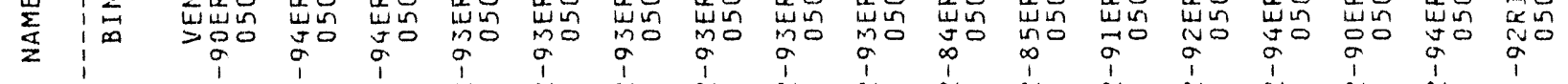

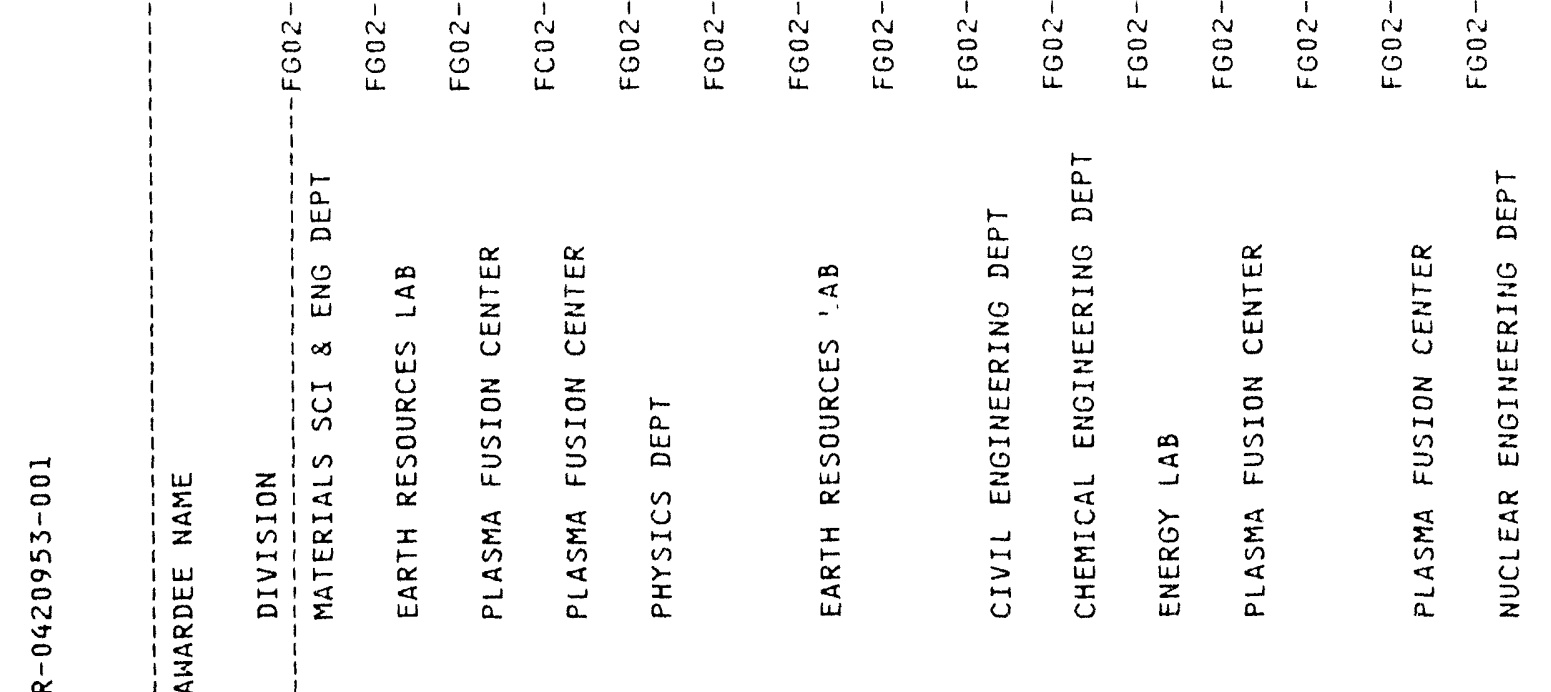




\begin{tabular}{|c|c|c|c|c|c|c|c|}
\hline AWARDEE NAME & BIN & $\angle$ DATE & & & DESCI & F WORK & \\
\hline DIVISION & VENDOR ID & CITY & STATE & $\begin{array}{l}\text { CONG } \\
\text { DIST }\end{array}$ & $\begin{array}{l}\text { CONTRACT } \\
\text { VALUE }\end{array}$ & $\begin{array}{l}\text { OBLIGS } \\
\text { TO DATE }\end{array}$ & $\mathrm{P} / \mathrm{S}$ \\
\hline
\end{tabular}

PLASMA FUSION CENTER

MASSACHUSETTS STATE OF COMMUNITIES \& DEVELOPMENT

MASSACHUSETTS UNIVERSITY OF

MATH \& STATISTICS DEPT

CHEMISTRY DEPT

PHYSICS \& ASTRONOMY DEPT

MICROBIOLOGY DEPT

GEOLOGY \& GEOGRAPHY

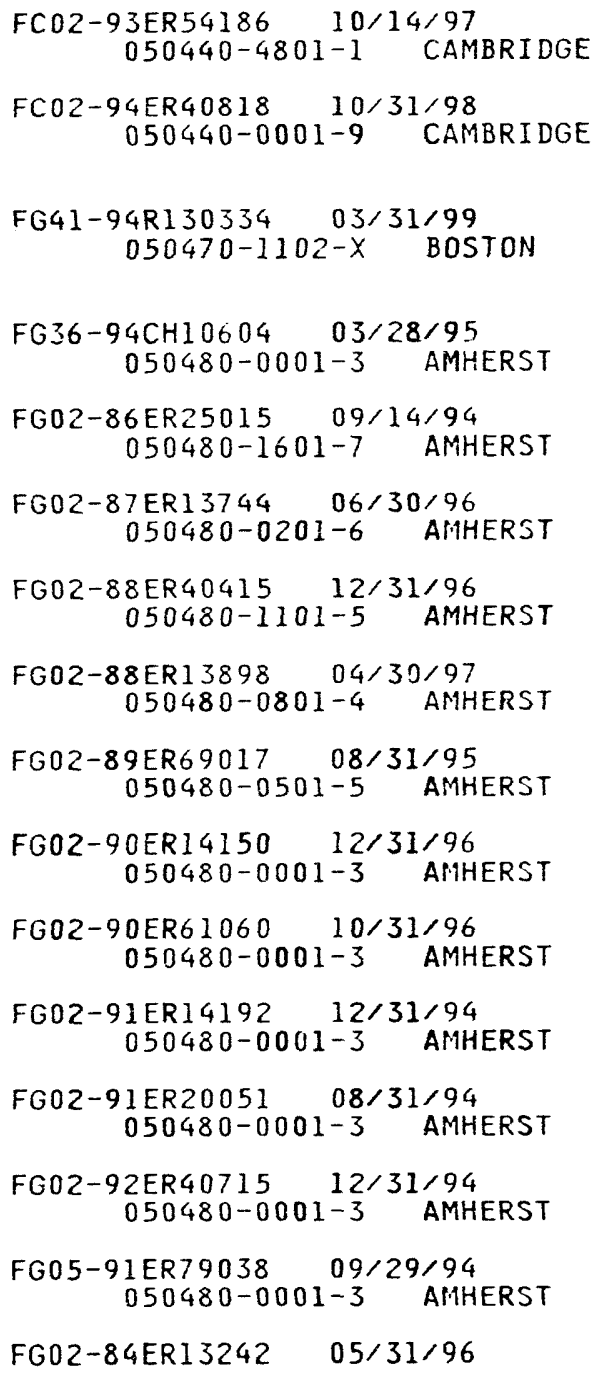

FCD2-93ER54186 10/14/97 050440-4801-1 CAMBRIDGE FC02-94ER40818 10/31/98 050440-0001-9 CAMBRIDGE

FG41-94R130334 03/31/99 $050470-1102-X$ BOSTON

FG36-94CH10604 03/28/95 $050480-0001-3$ AMHERST

FG02-86ER25015 09/14/94 050480-1601-7 AMHERST

FG02-87ER13744 06/30/96 050480-0201-6 AMHERST

FG02-88ER40415 12/31/96 050480-1101-5 AMHERST

FG02-88ER13898 04/30/97 050480-0801-4 AMHERST

FG02-89ER69017 08/31/95 050480-0501-5 AMHERST

FG02-90ER14150 12/31/96 050480-0001-3 AMHERST

FG02-90ER61060 10/31/96 050480-0001-3 AMHERST

FG02-91ER14192 12/31/94 050480-0001-3 AMHERST

FG02-91ER2005108/31/94 050480-0001-3 AMHERST

FG02-92ER40715 12/31/94 050480-0001-3 AMHERST

FG05-91ER79038 09/29/94 050480-0001-3 AMHERST

FG02-84ERI3242 05/31/96

FUSION DEVELOPMENT \& TECHNOLOGY

$$
\text { MA } 06 \$ 57,508,392 \quad \$ 19,294,392
$$

LABORTORY FOR NUCLEAR SCIENCE (BATES CERN-L3)

$$
\text { MA } 08 \$ 125,662,600 \quad \$ 19,227,600
$$

FY'94 AWARD

$$
\text { MA } 01 \quad \$ 6,095,209 \quad \$ 6,095,209
$$

FENESTRATION RESEARCH

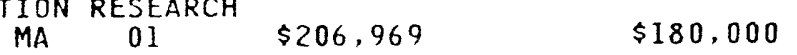
COMPUTATION AND GRAPHICS IN MATHEMATICAL RESEARCH
MA $01052,764,321$

THEORY OF DISSOCIATION $\begin{gathered}\text { DYNAMICS OF SMALL MOLECULES ON } \\ \$ 529,907\end{gathered}$ MEDIUM ENERGY NUCLEAR PHYSICS

$$
\text { MA } 01 \text { N } \$ 3,064,000 \quad \$ 2,278,000
$$

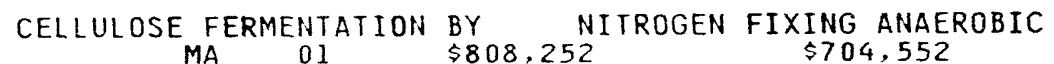

CLIMATE SYSTME RESEARCH: STUDIES OF GLOBAL END REGIONAL THEORETICAL STUDIES OF SOLID FLUID PHASE EQUILIBRIA $\begin{array}{cccc}M & \text { STUDIES OF } & \text { SOLID FLUID PHASE EQUILIBRIA } \\ \text { MA } & 01 & \$ 568,000 & \$ 378,000\end{array}$

GROUND-BASES MILLIMETER WAVE CLOUD OROFILING RADAR SYSTEM
MA 01 \$ $\$ 1,629,750$

$$
\text { MA } 01 \text { \$1,629,750 \$1,141,187 }
$$

PROBING FLAME CHEMISTRY WITH MBMS, THEORY, $\underset{\$ 242,000}{\$ 242,000}$.

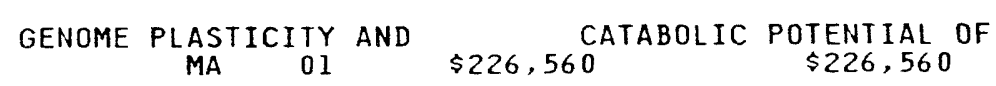

$\begin{array}{rrr}\text { HIGH ENERGY PHYSICS } & \$ 466,000 & \$ 466,000\end{array}$

POLARIMETRIC HIGH-PONER TRANSMITTER AND DATA ACQUISITION INS MA $01 \quad \$ 319,500 \quad \$ 290,000$

INVESTIGATION OF THE STRUCTUREOF PHOTOSYNTHEITC REACTION CE 


\begin{tabular}{|c|c|}
\hline AWARDEE NAME & COMPL DATE \\
\hline DIVISION & VENDOR ID \\
\hline CHEMISTRY DEPT & $050450-0202-4$ \\
\hline ENVIRONMENTAL SCIENCES & $\begin{array}{c}\text { FGO9-94SR18441 } 05 / 08 / 95 \\
\text { PROGRAM } 050480-1702-1 \text { BOSTON }\end{array}$ \\
\hline NUCLEAR MEDICINE DEPT & $\begin{array}{r}\text { FG02-94ER61850 05/31/95 } \\
050480-0903-7 \quad \text { HORCEST }\end{array}$ \\
\hline MASTER DATA CENTER & $\begin{array}{c}\text { ACO1-926C20266 } 09 / 30 / 95 \\
050520-0001-4 \text { SOUTHFI }\end{array}$ \\
\hline \multirow[t]{5}{*}{ MATERIALS \& ELECTROCHEM RES } & $\begin{aligned} \text { FG02-91ER81094 07/05/94 } \\
128900-0001-X \text { TUCSON }\end{aligned}$ \\
\hline & $\begin{array}{c}\text { FG02-92ER81271 05/24/95 } \\
128900-0001-X \text { TUCSON }\end{array}$ \\
\hline & $\begin{array}{r}\text { FG02-92ER81272 } 02 / 17 / 95 \\
128900-0001-\times \text { TUCSOH }\end{array}$ \\
\hline & $\begin{array}{r}\text { FG02-93ER81481 } 07 / 17 / 94 \\
128900-0001-\times \text { TUCSOH }\end{array}$ \\
\hline & $\begin{array}{c}\text { FG02-93ER81482 07/17/94 } \\
128900-0001-X \text { TUCSOH }\end{array}$ \\
\hline
\end{tabular}

MATHEMATICAL ASSN OF AMERICA

MATHEMATICAL SCIENCES RES INST

MATHTECH INC

MATSI INC

MAURER ENGINEERING INC

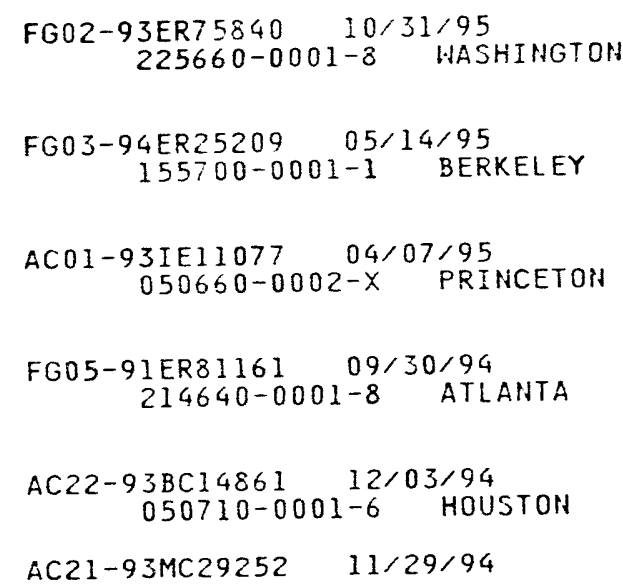

DESCRIPTION OF WORK

\begin{tabular}{|c|c|c|c|c|}
\hline STATE & $\begin{array}{l}\text { CONG } \\
\text { DIST }\end{array}$ & $\begin{array}{l}\text { CONTRACT } \\
\text { VALUE }\end{array}$ & $\begin{array}{l}\text { OBLIGS } \\
\text { TO DATE }\end{array}$ & $P / S$ \\
\hline MA & 01 & $\$ 1,095,330$ & $\$ 982$, & \\
\hline
\end{tabular}

$\begin{array}{rccc}\text { USED ENERGY RELATED LABORATORYEQUIPMENT GRANT } & \\ \text { MA } & 09 & \$ 0 & \$ 0\end{array}$

CONTINUING EDUCATION COURSE SOCIETY OF NUCLEAR MEDICINE

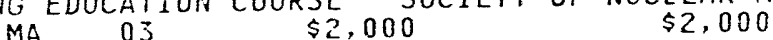

$\begin{array}{cccc}\text { MAINTANANCE OF DOE'S FOREIGN PATENT PORTFOLIO } & \\ \$ 320,000 & \$ 320,000 & \text { R499 }\end{array}$

NOVEL JOINING METHOD FOR GRAPHITE \& CARBOH-CARBON COMPOSITES

NOVEL JOINING METHOD FOR GRAPHITE \& CARBON-CARBON

THE DEVELOFMENT OF SIC/SIC CERAMIC COMPOSITES FOR

THE DEVELOPMENT OF A PROCESS TO SYNTHESIZE TUBLAR FULLERNES

THE DEVELOPMENT OF A PROCESS TO SYNTHESIZE TUB $\$ 408.873$

$\begin{array}{ccc}\text { IHHOVATIVE SIC FIBER COMPOSITEFILTERS } & \$ 75,000 & \$ 75,000\end{array}$

DOPING OF CVD SIC TO ENHANCE THERMAL CONDUCTIVITY

CAREER INFORMATION IN THE MATHEMATICAL SCIENCES (CIMS)

$$
\text { DC } 01 \quad \$ 225.000 \quad \$ 147,750
$$

MESOSCOPIC MODELING OF NONEQUILIBRIUM SYSTEMS

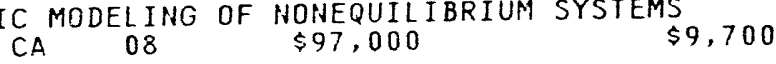

TECHNICAL, ANALYTICAL AND COMPUTER SUPPORT SERVICES TO THE

GRAPHITE AND METAL OXIDE CATALYST SUPPORTS FOR RECHARGEABLE

GRAPHITE

HORIZOHTAL OIL WELL APPLICATIONS AND OIL RECOVERY ASSESSMENT $\begin{array}{cccc}\text { TX } & \begin{array}{c}\text { HELL APPLICATIONS AND OIL RECOVERY ASSESSME } \\ 18\end{array} & \$ 258,916 & \$ 258,916\end{array}$

DEVELOPMENT OF A NEAR-BIT MWD SYSTEM 


BIN COMPL DATE

AWARDEE NAME
DIVISION

MAXWELL LABORATORIES INC

MAXWELL TOWN OF

MUNICIPAL SCHOOLS

MAYAK PRODUCTION ASSN

MAYFIELD CITY SCHOOLS

MCALLEN INDEPENDENT SCH DIST

MCBRIDE \& ASSOC INC

\section{MCCARTHY CONSTRUCTIOH}

MCCOOK COMMUNITY COLLEGE

MCCRACKEN COUNTY BD OF EDUCN

MCMIHIN COUHTY OF

: EDUCATION BOARD OF

VENDOR ID CITY
$050710-0001-6$ HOUSTOH
$94 I D 1331100 / 28 / 96$
$050770-0001-8$ SAH DIEGO

GM04-93AL $84079 \quad 11 / 25 / 97$ 227150-0101-0 MAXWELL

ACO1-93NE32169 12/07/97 226080-0001-0 CHELYABIHSK

FG45-93R551735 12/31/94
$229850-0001-7$ MAYFIELD

FG46-93R613628 08/31/94 050850-0001-3 MCALLEN

$A B 04-93 A L 9011 ? \quad 05 / 21 / 95$ 93AL9011? 05/21/95
$223890-0001-0$ ALBUQUERQUE AM04-92AL81715 09/28/94 223890-0001-0 ALBUQUERQUE

AD04-94AL90587 09/30/94 223890\%0001-0 ALBUQUERQUE

AC65-9411114377 04/21/95 $237520-0001-5$ SACRAMENTO

FG47-93R703528 08/31/94 $3 R 703528$
$102590-0001-9$ MCCOOK

FG44-93R435312 09/30/95 207270-0001-2 PADUCAH

FG44-93P435334 09/30/95 231220-0101-2 ATHEHS
PAGE NO. 291 


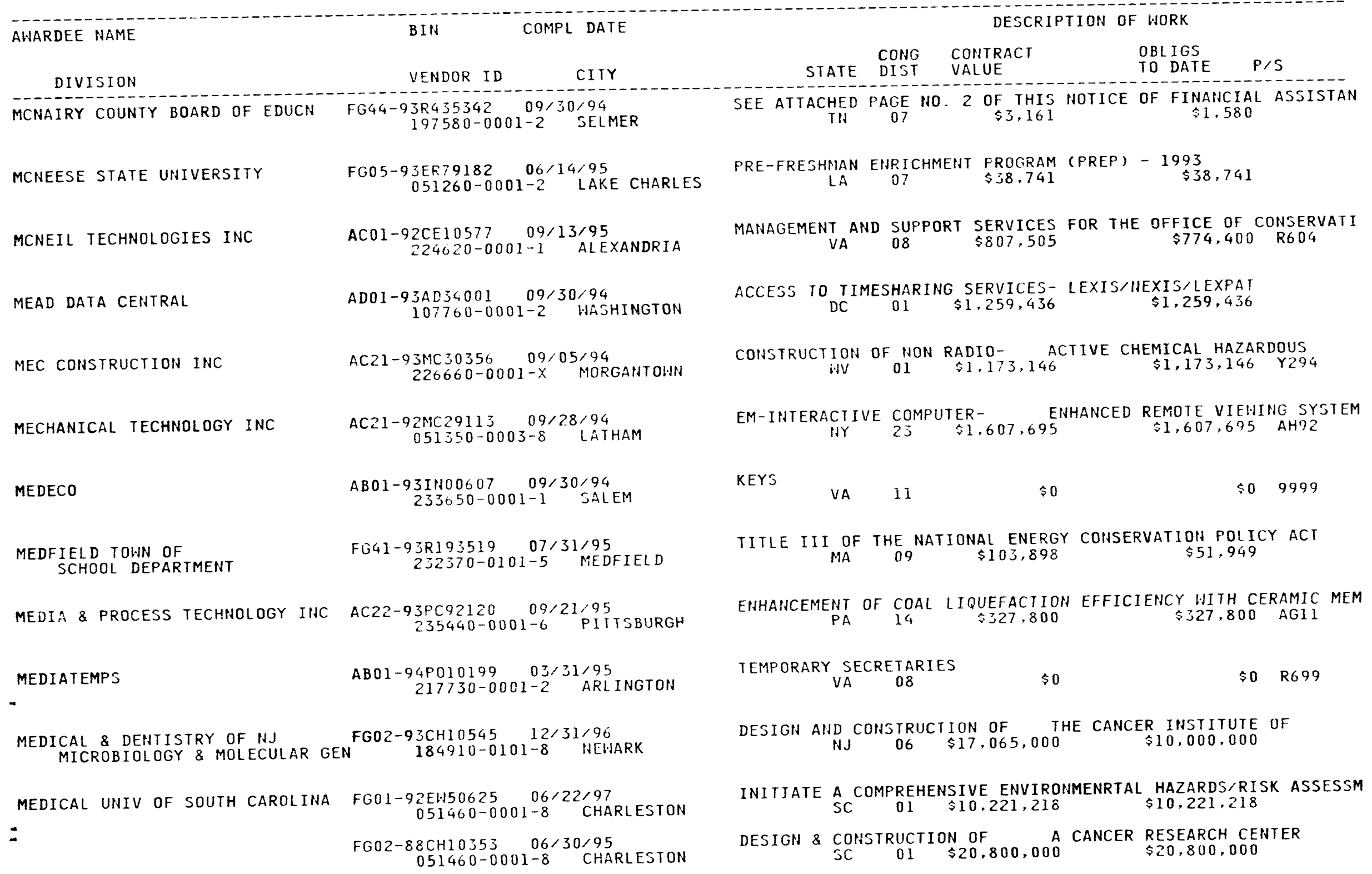


BIN COMPL DATE

AWARDEE NAME

COMPL DATE

CITY

STATE DIST

DESCRIPTION OF WORK

FG02-91CH10468 08/01/95 051460-0001-8 CHARLESTON FG09-91SR18217 04/29/97 051460-0001-8 CHARLESTON

MEHARRY MEDICAL COLLEGE BIOCHEMISTRY DEPT

GRADUATE STUDIES SCHOOL

BIOCHEMISTRY DEPT

MEHTA TECH INC

MELROE CD

MEMBRANE TECHNOLOGY \& RES INC

MEMBRANE TECHNOLOGY \& RESEARCH

MEMORIAL HOSPITAL

MEMORIAL MIDDLE SCHOOL

:

MEMPHIS STATE UNIVERSITY

$$
\begin{gathered}
\text { FG05-85ER13339 } 02 / 29 / 96 \\
051490-0101-5 \text { NASHVILLE }
\end{gathered}
$$$$
\text { FG05-89ER75530 09/28/95 }
$$$$
\text { FG05-92ER20087 07/14/95 }
$$$$
\text { 051490-0101-5 NASHVILLE }
$$

AC65-93WA10866 09/01/94 230350-0001-8 ELDRIDGE AD65-941.1324539 $09 / 29 / 94$
$237860-0001-8 \quad$ FARGO

FCO4-94AL98769 04/15/97 051540-0001-3 MENLO PARK

FCO2-90CE40903 12/31/94 051540-0001-3 MENLO PARK AC21-92MC28133 04/28/95 051540-0001-3 MENLO PARK AC21-93MC30179 02/24/95 $051540-0001-3$ MENLO PARK

FG44-93R43534309/30/94 051610-0009-0 CHATTANOOGA

FGO4-93AL96840 07/08/96
$230870-0001-9$ LAS VEGAS

FG05-92ER79097 09/30/94
DESIGN \& CONSIRUCTION OF GAZESCARDIAC RESEARCH INST. IBIOMED $S C \quad 01 \$ 30,000,000 \quad \$ 5,965,200$

CANCER \& BIRTH DEFECTS SURVEILLANCE SYSTEM FOR SC $01 \quad \$ 4,659,967$ \$2,760,924

RESPIRATORY ENZYMES OF THIOBACILLUS FERROOXIDANS

$05 \$ 1,140,623 \quad \$ 782,623$

TRAINING OF MIHORITIES IN THE LIFE SCIENCES

$\begin{array}{lll} & \end{array}$

BIOCHEMISTRY OF DISSIMILATORY SULFUR OXIDATION

$\begin{array}{lll}\text { TN } 05 & \$ 258,402 & \$ 169,673\end{array}$

DIGITAL FAULT RECORDERS VARIOUS SITES

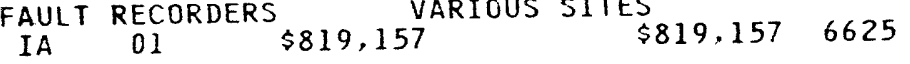

$\$ 25,604$

$\$ 25,604 \quad 3930$

DEVELOPMENT OF PERVAPORATION TO RECOVER AND REUSE VOLATILE $C A$ I1 $\$ 1,911,096 \quad \$ 416,473$

MEMBRANE VAPOR RECOVERY SYSIEM
CA $\$ 767,896$
$\$ 642,896$

OW-QUALITY NATURAL GAS SULFURREMOVAL/RECOVERY SYSTEM

$\begin{array}{llll}C A & 11 & \$ 933,748 & \$ 933,748\end{array}$

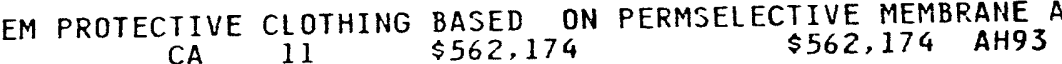

SEE ATTACHED PAGE NO. 2 OF THIS NOTICE OF FINANCIAL ASSISTAN TN $03 \quad \$ 18,318 \quad \$ 9,159$

EDUCATION OUTREACH

NM $03 \quad \$ 81,350$

$\$ 25,000$

PRE-FRESHMAN ENRICHMENT PROGRAM (PREP) - 1992 

PROCUREMENT AND ASSISTANCE DATA SYSTEM
NAMES BY NAME, CITY \& STATE - ACTIVE AWARDS

PAGE NO. 294

\begin{tabular}{|c|c|}
\hline AWARDEE NAME & COMPL DATE \\
\hline DIYISION & VENDOR ID \\
\hline CHEMISTRY DEPARTMENT & $051750-0101-9$ \\
\hline & $\begin{array}{c}\text { FG05-94ER } 14460006 / 30 / 95 \\
051750-0001-2 \text { MEMPHIS }\end{array}$ \\
\hline MERCER UNIVERSITY & $\begin{array}{c}\text { FG44-92R435072 } 09 / 30 / 94 \\
051950-0001-8 \text { MACON }\end{array}$ \\
\hline MERCY HEALTH CENTER & $\begin{array}{c}\text { FG47-93R707035 08/31/94 } \\
052000-0004-1 \quad \text { CORNING }\end{array}$ \\
\hline MERCY HEALTH SYSTEM & $\begin{array}{c}\text { FG45-94R562250 } 12 / 31 / 95 \\
238430-0002-6 \text { JANESVILLE }\end{array}$ \\
\hline $\begin{array}{l}\text { MERCY HEALTH SYSTEMS } \\
\text { MERCY HOSPITAL OF TIFFIN }\end{array}$ & $\begin{array}{c}\text { FG45-94R562196 } 12 / 31 / 95 \\
238430-0101-4 \text { TIFFIN }\end{array}$ \\
\hline MERCY HOSPITAL & $\begin{array}{c}\text { FGC4-93R435308 } 09 / 30 / 95 \\
052020-0014-6 \text { OHENSBORO }\end{array}$ \\
\hline MERCY MED CTR OF SPRINGFIELD & $\begin{array}{c}\text { FG45-91R530130 } 10 / 31 / 94 \\
052080-0001-6 \text { SPRINGFIELD }\end{array}$ \\
\hline MERCY MEMORIAL HOSPITAL & $\begin{array}{c}\text { FG45-93R551639 } 08 / 31 / 95 \\
123550-0001-8 \text { MOHROE }\end{array}$ \\
\hline MERIDIA EUCLID HOSPITAL & $\begin{array}{c}\text { FG45-93R551765 } 09 / 30 / 95 \\
184580-0001-6 \quad \text { EUCLID }\end{array}$ \\
\hline $\begin{array}{l}\text {-MERIDIAN CITY OF } \\
\text { JOINT SCHOOL DISTRICT \#2 }\end{array}$ & $\begin{array}{c}\text { FG51-93R020286 } 09 / 30 / 94 \\
231640-0101-0 \text { MERIDIAN }\end{array}$ \\
\hline JOINT SCHOOL DISTRICT \#2 & $\begin{array}{c}\text { FG51-93R020291 } 10 / 31 / 94 \\
231640-0101-0 \text { MERIDIAN }\end{array}$ \\
\hline \multirow[t]{2}{*}{ MERIDIAN CORP } & $\begin{array}{c}\text { AC01-94SA10056 01/31/96 } \\
052120-0003-3 \text { GERMANTOWN }\end{array}$ \\
\hline & ACOI-93AN00027 \\
\hline
\end{tabular}

DESCRIPTION OF WORK

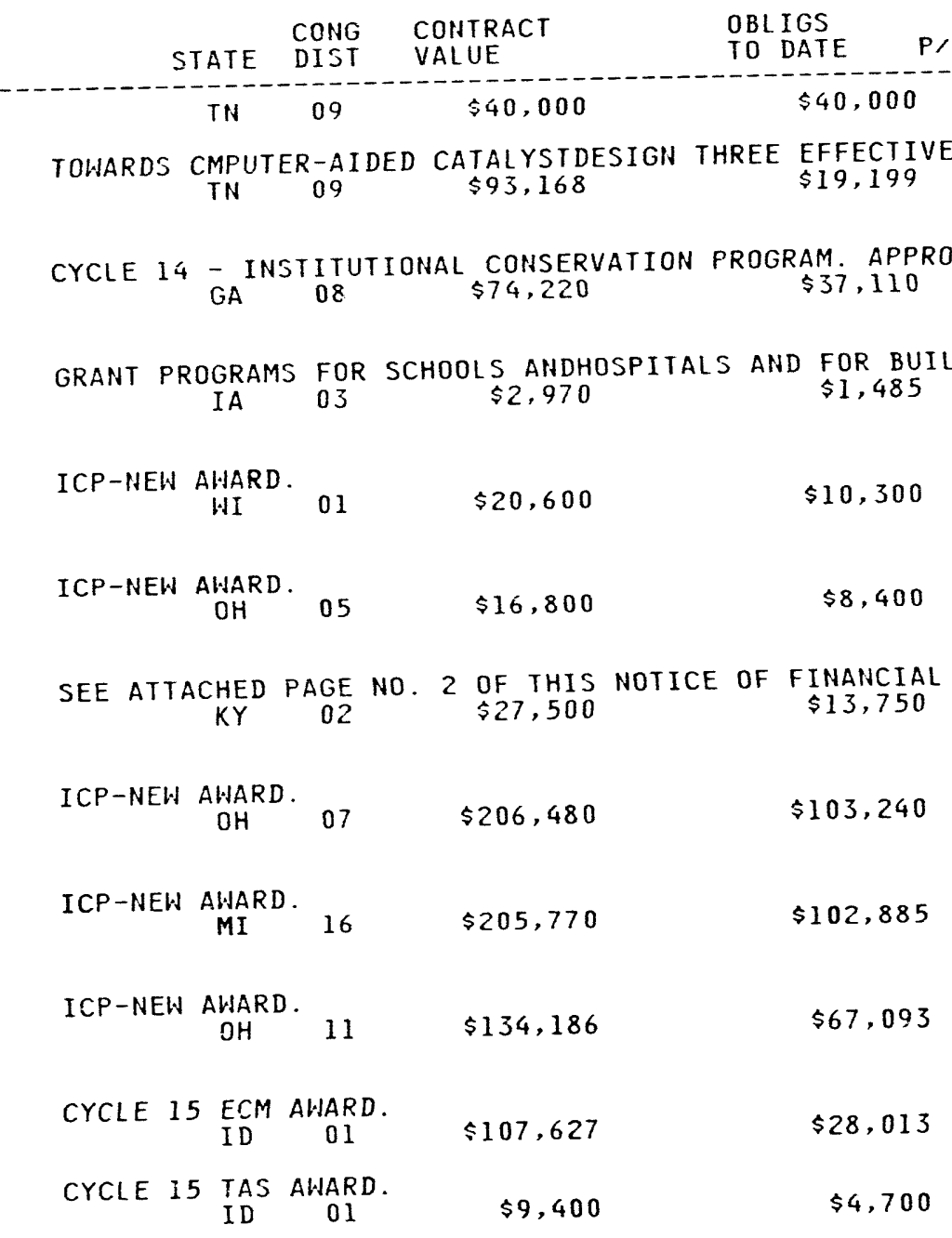

TECHNICAL SUPPORT SERVICES FORTHE OFFICE OF CLASSIFICATION

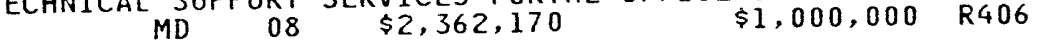
SPECIALIZED TECHNICAL, PROGRAMMANAGEMENT, AND CLERICAL SUPP 


\section{AWARDEE NAME}

DIVISION

B IN

COMPL DATE

BIN

$052120-0002-5$ ALEXANDRIA

AC01-90DP30414 12/14/94

052120-0002-5 ALEXANDRIA

AC01-92DP50081 04/01/97

MERZ \& MCLELLAN

MESA COUNTY OF

SHERIFF'S DEPT

MESA VISTA SCHOOLS

MESABI REGIONAL MEDICAL CENTER

MESCALERO APACHE ELEMENTRY SCH

META INC

-

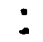

METABOLIX INC

GMO4-93AL84070 11/24/97 227000-0001-3 ESPANOLA

FG45-92R540136 02/28/95 222260-0001-1 HIBBING

FG04-93AL 96835 07/08/96 230830-0001-4 RUIDOSO

ACO1-92NE20406 03/03/95

ACO1-90मA60870 03/11/95 194830-0001-7 ARLINGTON

AC02-9011P00028 12/21/94 194830-0001-7 ARLINGTON

AC02-92NP00945 06/11/95 194830-0001-7 ARLINGTON

FG02-93ER81526 07/17/94
052120-0002-5 ALEXANDRIA

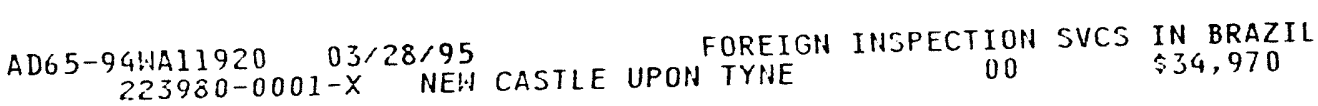

\section{CONG CONTRACT}

STATE DIST VALUE

$\$ 1,446,467$

SUPPORT SERVICES TO DP FOR TECH

VA 10 \$11,713,329

FOREIGN INSPECTION SERVICES INAUSTRIA

AD65-94WA11900 03/21/95
$223980-0001-X$ NEW CASTLE UPON TYHE

00

$\$ 10,647$

FOREIGN INSPECTION SERVICES AM65-92WA08769 09/14/94
$223980-0001-X$ HEW CASTLE UPON TYNE

00

$\$ 0$

GMO4-94AL94706 $01 / 10 / 99$
$236510-0201-5$ GRAHD JUNCTION EMERGEHCY LAH ENFORCEMENT

SUPPORT FOR SCIENCE, MATHEMATICS, AND ENGINEERING TO IMPROVE $194830-0002-5$ GAITHERSBURG
IMM 03 SCIENCE, MATHEMATICS

ICP - NEW AWARD.

$\$ 49,436$

$\$ 24,718$

EDUCATIOH OUTREACH

$\mathrm{NII} \mathrm{OZ}$

$\$ 75,000$

$\$ 25,000$ SERVICES FOR DEVELOPING NUCLEAR $\$ 1,630,000$ R408 CONFERENCING AND AUDIO VISUAL SUPPORT $\$ 3,413,215$ T016 PROVIDE ADMINISTRATIVE AND MANAGERIAL SUPPORT FOR THE OFFICE VA $10 \quad \$ 6,973,632 \quad \$ 6,973,632$ R408 TECHNICAL \& ADMINISTRATIVE SUPPORT SERVICES FOR THE NPR VA 10 \$ $\$ 1,927,712$

POLYMERIZATION OF CHIRAL ACIDS
SUPPORT SERVICES FOR DEVELOPIHG NUCLEAR SAFETY STANDARDS

ENZYME CATALYZED 


\begin{tabular}{|c|c|c|c|c|c|c|}
\hline AWARDEE NAME & BIN & $L$ DATE & & & DESCRIP & F HORK \\
\hline \multirow[t]{2}{*}{ DIVISION } & VENDOR ID & CITY & STATE & $\begin{array}{l}\text { COHG } \\
\text { DIST }\end{array}$ & $\begin{array}{l}\text { CONTRACT } \\
\text { VALUE }\end{array}$ & $\begin{array}{l}\text { OBLIGS } \\
\text { TO DATE }\end{array}$ \\
\hline & $233240-0001-7$ & CAMBRIDGE & MA & 08 & $\$ 75,000$ & $\$ 75,000$ \\
\hline
\end{tabular}

METALOR USA REFINING CORP

METALS RECOYERY INDUSTRIES

METHODIST HOSP OF MIDDLE MS

METHODIST HOSPITAL

METHODIST HOSPITALS INC

METHODIST HOSPITALS OF MEMPHIS

METRO DISPOSAL

.METRO RESTORATION CO INC

METROLASER

$=$

MEYER LONBERG ASSOC
FC07-92ID13081 $11 / 30 / 94$
$224560-0001-3$
NORTH ATTLEBOROUG

D OF ELECTROLYTIC PROCESSES FOR THE PRODUCTION OF MATERIAL GM02-92CH10512 02/01/99
$219130-0001-X$ EAST CHICAGO

HOLDING COMPANY AGREEMENT UNDER DOE'S METALS INITIATIVE

FG44-935435427 09/30/95
$212300-0001-1 \quad$ LEXINGTON

FG44-91R434628 09/01/94 $052270-0011-5$ JACKSONVILLE

FG46-93R613638 02/28/95 $052270-0009-3$ LUBBOCK

$F G 45-93 R 551590 \quad 03 / 31 / 95$
$227450-0001-2$ GARY

FG44-92R435060 08/15/94
$102700-0001-1$ MEMPHIS

FG44-93R435335 09/30/95 $102700-0001-1$ MEMPHIS

AX65-9411D03450 09/30/94 235720-0001-7 WEST FARGO

AX01-94AD67088 06/30/95
$224350-0001-4$ OAKTON

AX01-94AD66479 07/08/94 224350-0001-4 OAKTON

FG03-94ER81672 $07 / 17 / 94$
$236400-0001-3$ IRVINE

FG02-93ER81614 07/17/94
SEE ATTACHED PAGE NO. 2 OF THIS NOTICE OF FIHAHCIAL ASSISTAN MS 02 NO. $2 \underset{\$ 66,338}{\text { OF }}$

CYCLE 13 - INSTITUTIONAL CONSERVATION PROGRAM. APPROVED BUDD

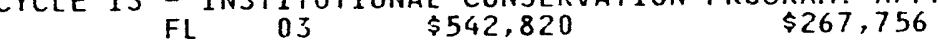

AWARDS A NEW CYCLE 15 ENERGY CONSERVATION MEASURE GRANT. $T X \quad 19 \quad \$ 242,340 \quad \$ 121,170$

ICP-NEW AWARD

IN $01 \quad \$ 136,994$

$\$ 69,650$

CYCLE 14 - INSTITUTIONAL CONSERVATION PROGRAM. APPROVED BUD TN 09 \$169,816 \$84,908

SEE ATTACHED PAGE NO. 2 OF THIS NOTICE OF FINANCIAL ASSISTAN TN 09 \$26,370 $\$ 13,185$

REFUSE COLLECTION - FARGO SUBST FY 94

$\$ 540 \$ 205$ PROVIDE ALL LABOR AND EQUIPMENT
VA
10 $\quad \begin{aligned} & \$ 25,000 \\ & \text { TO PERFORM ELECTRICAL WORK } \\ & \$ 25,000 \text { Z111 }\end{aligned}$

RENOVATE ROOM GH-099 LOCATED IN THE FORRESTAL BLDG.

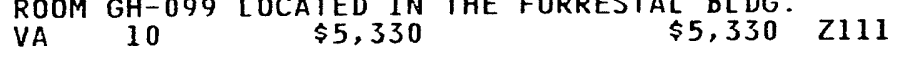

DEVELOP A HOLOGRAPHIC VERIFICATION TAG FOR SECURE INVENTORY CA $40 \quad$ \$73,748 $\quad \$ 73,748$

LIQUTO CRYSTAL SMART WINDOWS 


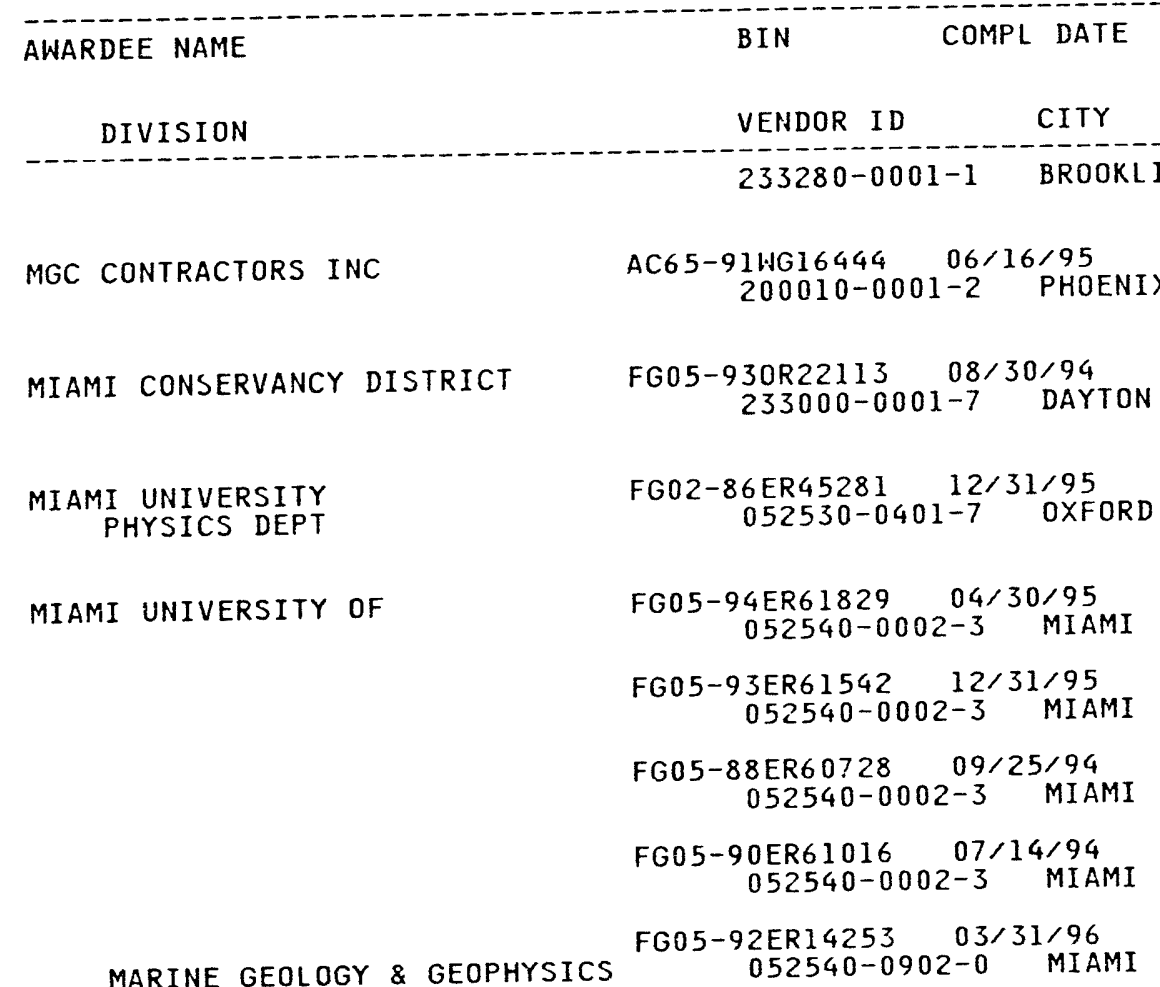

MIAMI VALLEY EMERGENCY MGMT

MIAMISBURG CITY OF

FIRE DEPARTMENT

MICAH TECHNICAL INDUSTRIES
GM04-88AL53617 08/03/99 230950-0001-4 MIAMISBURG GM04-89AL53640 $11 / 03 / 99$
$052560-0002-0 \quad$ LARGO

GMO4-89AL53619 10/03/99 052560-0101-9 MIAMISBURG GM04-76AL03589 10/01/99 052560-0001-2 MIAMISBURG FG04-93AL 97147 08/11/96 052560-0201-5 MIAMISBURG

AC65-93WJ22563 08/01/94

\section{DESCRIPTION OF WORK}

CONTRACT

STATE DIST VALUE

$\$ 50,000$

OBLIGS

TO DATE $P / S$

MA 0

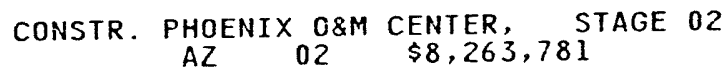

$\$ 8,263,781 \quad Y 111$

GREAT MIAMI CLEANUP AND THE WELLHEAD PROTECTION PROGRAM

$$
\begin{array}{rrr}
\text { MH } \\
04 & \$ 116,090 & \$ 116,090
\end{array}
$$

INVESTIGATION OF MAGNETIC ANISOTROPY AND SPIN WAVE MODES IN $\mathrm{OH} 08 \quad \$ 429,810 \quad \$ 389,640$

THE ROLES OF ADVECTIVES AND INSITU GROWTH IN DETERMINING TH $\mathrm{FL} 18 \quad \$ 81,480 \quad \$ 81,480$ THE CARBON DIOXIDE SYSTEM IN THE OCEANS
FL
18

LAELING CELLULAR ELEMENTS OF BLOOD WITH TECHNETIUM-99M $\begin{array}{llll}\mathrm{FL} & 18 & \$ 236,725 & \$ 236,725\end{array}$

USE OF A LASER BASED MONITORING TECHNIQUE TO MEASURE TRACE $G$ $18 \$ 340,196 \quad \$ 340,098$

TESTING THE CORRELATION BETHEEN SEQUENCE STRATIGRAPHY, SEISM $\mathrm{FL} \quad 18 \quad \$ 752,704 \quad \$ 450,387$

DESCRIBES THE RELATIONSHIPS, NOTIFICATION PROCEDURES AND

$$
\text { OH } 03 \text { THE RELATIONSHIPS, } \$ 0
$$

$\$ 0$

PROVIDE FOR MUTUAL AID OF THE PARTIES IN PROVIHIG EMERGENCY

FL 08

$\$ 0$

$\$ 0$

PROVIDES FOR FULL SUPPORT TO MOUND FROM THE CITY OF

AGREEMENT TO ALTER THE WATER SUPPLY TO THE CANAL AND PROVIDE OH OH

MOUND INITIATIVE BRIDGE FUNDING

$O H$ OS $\$ 900,000$

$\$ 700,000$

SWITCHBOARDS FOR FT MORGAN WEST SUBST 


\section{AWARDEE NAME} COMPL DATE

BIN

COMPL DATE

VENDOR ID CITY

235190-0001-6 CORONA

MICHAEL BAKER JR INC

$$
\begin{aligned}
& \begin{array}{c}
\text { AT22-94PC94608 06/02/95 } \\
191090-0001-1 \text { CORAOPOLIS }
\end{array} \\
& \text { AT22-93PC93579 12/31/94 } \\
& 191090-0001-1 \text { CORAOPOLIS } \\
& \text { AM22-89PC89500 09/18/94 } \\
& \text { 191090-0001-1 CORAOPOLIS } \\
& \begin{array}{c}
\text { AT22-94PC94606 01/31/95 } \\
191090-0001-1 \text { CORAOPOLIS }
\end{array} \\
& \text { AT22-92PC92928 12/31/94 } \\
& 191090-0001-1 \text { CORAOPOLIS } \\
& \text { AT22-92PC92929 12/31/94 } \\
& 191090-0001-1 \text { CORAOPOLIS } \\
& \text { AT22-93PC93609 11/30/94 } \\
& \text { 191090-0001-1 CORAOPOLIS } \\
& \begin{array}{c}
\text { AC22-92PC92117 09/28/94 } \\
160050-0001-2 \text { LANSING }
\end{array} \\
& \begin{array}{c}
\text { FC01-91EI22720 } 08 / 06 / 96 \\
052610-0802-6 \quad \text { LANSING }
\end{array} \\
& \begin{array}{c}
\text { FG45-93R530282 } 09 / 30 / 95 \\
052610-0802-6 \quad \text { LANSING }
\end{array} \\
& \begin{array}{c}
\text { FG45-76C560204 } 09 / 30 / 94 \\
052610-0302-4 \quad \text { LAHSING }
\end{array} \\
& \begin{array}{c}
\text { FG45-80CS69104 09/30/94 } \\
052610-0302-4 \quad \text { LANSING }
\end{array} \\
& \begin{array}{c}
\text { FG45-89CH10411 } 03 / 31 / 95 \\
052610-0202-8 \quad \text { LANSING }
\end{array} \\
& \begin{array}{c}
\text { FG45-89R510368 } 09 / 30 / 94 \\
052610-0802-6 \quad \text { LANSING }
\end{array} \\
& \begin{array}{c}
\text { FG45-92R530273 } 10 / 31 / 94 \\
052610-0802-6 \quad \text { LANSING }
\end{array}
\end{aligned}
$$

MICHIGAN BIOTECHNOLOGY INST

MICHIGAN STATE OF

PUBLIC SERVICE COMI.ISSION

PUBLIC SERVICE COMMISSION

COMMERCE DEPT

COMMERCE DEPT

CSA BUREAU \& DEPT OF LABOR

PUBLIC SERVICE COMMISSION

PUBLIC SERVICE COMMISSION

\section{DESCRIPTION OF WORK}

Is

(5)

DELIVERY ORDER \#058 - TITLE IIDESIGN ELECTRICAL SUBSTATIONS A/E SERVICES, FACILITY CONDITION, INSPECTIONS, AND SURVEYS

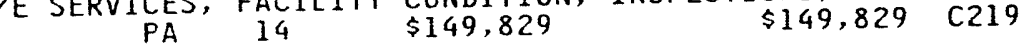
A/E ENGIHEERING SERVICES
PA 14
$\$ 0$
\$0 $\quad 219$

DELIVERY ORDER 057 - SUPERVISION OF CONSTRUCTION BLDG 84 - C $\begin{array}{clcc}\text { PA } & 14 & \$ 101,602 & \$ 101,602 \\ \text { DELIVERY ORDER } & \# 046 & \text { - A/E SERVICES - SITE SAMPLING AND ANALY }\end{array}$ $\begin{array}{ccccc}\text { DELIVERY } & \text { ORDER } & \# 046 & \text { - A/E SERVICES - SITE } \\ \text { PA } & 14 & \$ 809,694 & \$ 809,694 & \text { C219 }\end{array}$ DELIVERY ORDER \#47 PA 14
$\$ 115,839$
$\$ 115,839 \quad 219$

DELIVERY ORDER \#054 - DEVELOPMENT OF FACILITY STANDARDS

BIOCONVERSION OF COAL-FIRED SYNTHESIS GAS TO LIQUID FUELS

1991-1992 EIA STATE HEATING OIL PROGRAM

1991-1992 MI 03 S $\$ 38,471$ \$19,236

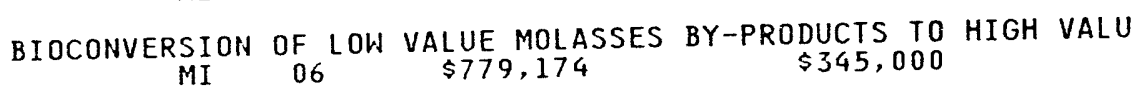

PROVIDES GRANTS TO STATE TO DEVELOP AND IMPLEMENT COMPREHE $\begin{array}{lll}\text { GRANTS TO STATE TO DEVELOP AND } \\ M I & \$ 13,593,688\end{array}$

STATE OF MICHIGAN ENERGY EXTENSION SERVICE

1989 WEATHERIZATION ASSTSTANCEFOR LOW INCOME PERSONS - STATE

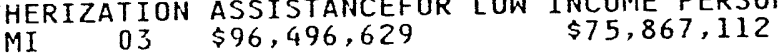

FY 1989 FUNDING FOR ADMINISTRATION OF THE INSTITUTIONAL CONS

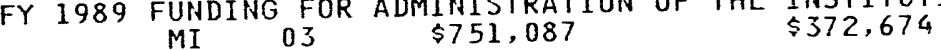

FUNDING FOR PUBLIC HOUSING PERFORMANCE CONTRACTING PROJECT

$\begin{array}{cccc}\text { FUNDING } & \text { FOR } & \text { PUBLIC } & \text { HOUSING PERFORMANCE CONTRACTING } \\ & 06 & \$ 40,000 & \$ 40,000\end{array}$ 
AWARDEE NAME

BIN

COMPL DATE

DESCRIPTION OF WORK

DIVISION

VENDOR ID

CITY

STATE CONG CONTRACT OBLIGS
TO DATE

$P / S$

MICHIGAN STATE UNIVERSITY

FG02-94ER $4084904 / 30 / 95$
$052630-0003-0$ BATAVIA

METALLURGY MECH \& MAT SCI DEPT $052630-0701-9051 / 97$ EAST LANSING

FG02-85ER13369 06/30/96

052630-0001-4 EAST LANSING

MICROBIOLOGY DEPT

FG02-87ER60524 12/31/95 052630-1601-8 EAST LANSING

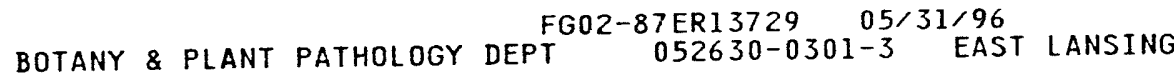

BIOCHEMISTRY DEPT

CHEMISTRY

PHYSICS \& ASTRONOMY DEPT

DOE PLANT RES LAB

PHYSICS DEPT

BOTANY \& PLANT PATHOLOGY DEPT $002-92$ ER20094 $09214 / 97$ - $0301-3$ EAST LANSING

BIOCHEMISTRY DEPT

ENGINEERING RESEARCH DEPT

FG02-93ER20108 05/31/96

052630-1401-5 EAST LANSING

FG02-93ER25167 06/14/96

$052630-0601-2$ EAST LANSING

FG02-93ER20111 06/14/95

052630-0001-4 EAST LANSING

FG02-93ER79183 06/14/95

052630-0001-4 EAST LANSING

FG02-93ER20121 07/31/96

$052630-1401-5$ EAST LANSING
BRIDGING $\underset{\text { IL }}{\text { SUPPORT FOR SSC }}{ }_{14} \underset{14}{\text { EMPLOYEE }} \$ 31,000$

DYNAMIC RECRYSTALLIZATION DURING HIGH TEMPERATURE LOW CYCLE $M I \quad 06 \quad \$ 872,598 \quad \$ 696,827$

MOLECULAR CLONING OF THE GENESENCODING CERTAIN KEY ENZYMES $M I \quad 06$ OF $\$ 790,123 \quad \$ 704,124$

RESEARCH ON MALIGNANT TRANSFORMATION OF DIPLOID HUMAN $M I 0_{0} \$ 1,725,001 \quad \$ 1,448,251$

ROLE OF ACYL CARRIER PROTEIN ISOFORMS IN PLANT LIPID MI ${ }_{06} \underset{\$ 59,813}{\$ 565,143}$ STRUCTURE OF SURFACE GLYCOCONJUGATES OR RHIZOBIUM MI ${ }_{06} \$ \$ 70,049 \quad \$ 470,049$

DIRECT PROBING OF CHROMATOGRAPHY COLUMNS BY MI $06 \quad \$ 514,375 \quad \$ 424,375$

THEORETICAL STUDIES OF BREAK DOWN IN RADOM MEDIA INTERDISCIPLINARY RESEARCGH PROGRAM IN THE FUNDAMENTAL MI $06 \quad \$ 18,977,550 \quad \$ 12,457,550$ SSC CALORIMETER R\&D
MI 06
$\$ 372,000$
$\$ 372,000$

A NATINAL COOPERATIVE FOR GENETIC ENGINEERING OF PLANT

1 CARBON METABOLISM IN $\begin{aligned} & \text { MNEROBIC BACTERIA: REGULATION } \\ & \$ 320,996\end{aligned}$

A SCALABLE COMMUNICATIONS $\underset{\$ 297,894}{\text { LIBRARY FOR }} \underset{\$ 19 \text { DISTRIBUTED }}{\$ 190,489}$

MINORITY SUMMER RESEARCH PROGRAM IN THE PLANT SCIENCES MI 08 \$ $\$ 147,150 \quad \$ 147,150$

PRE-FRESHMAN ENRICHMENT $\underset{\text { MI }}{08} \underset{18,744}{\text { PROGRAM }} \$ 38,744$

STRUCTURE-FUNCTION RELATION- SHIP OF ADP-GLUCOSE PYROPHOR-

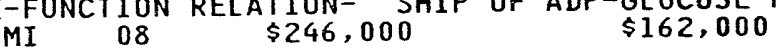


ANA

AWARDEE NAME

DIVISION

PROCUREMENT AND ASSISTANCE DATA SYSTEM (PADS)

NAMES BY NAME, CITY \& STATE - ACTIVE AWARDS

BIN COMPL DATE

VENDOR ID

CITr STATE DIST
PAGE NO. 300
CHEMICAL ENGINEERING

ENGINEERING RESEARCH DEPT

MICHIGAN TECHNOLOGICAL UNIV

INST OF WOOD RESEARCH

INST OF WOOD RESEARCH

INST OF WOOD RESEARCH

IN'ST OF WOOD RESEARCH

INST OF WOOD RESEARCH

METALLURGICAL ENG DEPT VALUE

DESCRIPTION OF WORK

$31 / 95$

$052630-0001-4$ EAST LANSING

FG02-93ER61681 08/31/96

052630-0001-4 EAST LANSING

FG22-93MT93002 08/22/94 052630-2001-5 EAST LANSING

FG22-93PC93213 09/02/96 052630-0601-2 EAST LANSING

FG05-91ER79045 09/29/94 552640-0001-8 HOUGHTON FC07-93ID13231 09/29/94 052640-0001-8 HOUGHTON

FG09-93SR18373 09/16/94 052640-0501-X HOUGHTON FG09-93SR18349 08/01/94 052640-0501-X HOUGHTON FG02-92ER75773 11/30/94 552640-0001-8 HOUGHTON

FG22-94PC94400 10/17/94 052640-0501-X HOUGHTON

FG21-93MC $30338 \quad 10 / 17 / 94$ $052640-0501-X \quad$ HOUGHTON

FG21-94MC $3122801 / 19 / 95$ 052640-0501-X HOUGHTON

FG02-92ERI4282 10/31/95 52640-0001-8 HOUGHTON

FG02-87ER45315 06/30/96 052640-0201-0 HOUGHTON

FG22-93PC93214 08/31/96
$052640-0201-0$ HOUGHTON

AC22-93BC14892 09/28/96 052640-0001-8 HOUGHTON

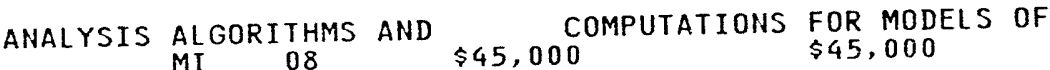

EFFECT OF MUTRIENT LIMITATION ON GENOMIC REARRANGEMENTS IN MI 08 \$306,599 \$109,132

PARTICIPATION IN A HYDKOCYCLONE DEVELOPMENT CONSORTIUM MI 08 \$75,000 $\$ 75,000$

RATE INHIBITION OF STEAM GASIFICATION BY ABSORBED HYDROGEN

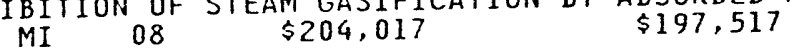

MOLECULAR BEAM EPITAXY EQUIPMENT FOR SYSTEMATIC INTERFACE RE

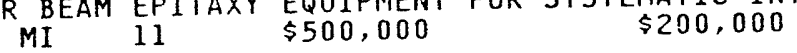

$\begin{array}{ccc}\text { ALTERNATIVE } & \text { GRANULAR MOLDING MEDIA } & \text { MI } \\ \text { MI } & 0147,766 & \$ 71,994\end{array}$

USED ENERGY RELATED LABORATORYEQUIPMENT
$\$ 0$

USED ENERGY RELATED LABORATORYEQUIPMENT MI 11 \$0

MICHIGAN TECHNDLOGICAL UNIV. PRE-SERVICE TEACHER

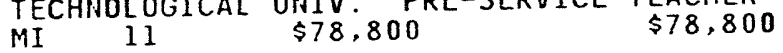

$\begin{array}{cl}\text { ENERGY RELATED EQUIPMENT GRANT } \\ \text { MI } & 11\end{array}$

$\begin{array}{rrr}\text { USED ENERGY RELATED EQUIPMENT } \\ \text { MI } & 01 & \$ 0\end{array}$

USED ENERGY RELATED EQUIPMENT
MI

THEORETICAL HYPERFINE STRUCTURE CONSTANTS FOR

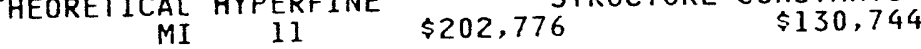

EFFECTS OF GRADIENTS ON BOUNDARY STABILITY

MI $11 \quad \$ 940,451 \quad \$ 854,428$

SEPARATION OF FLUE-GAS SCRUBBER SLUDGE INTO MARKETABLE PRODU MI 01 \$200,000 $\$ 200,000$

VISUAL DISPLAY OF RESERVOIR PARAMETERS AFFECTING EOR $\begin{array}{ccc}\text { DISPLAY OF RESERVOIR PARAMETERS AFFECTING EOR } \\ \begin{array}{c}\text { MI } \\ 01\end{array} & \$ 1,023,103 & \$ 818,482\end{array}$ 


\begin{tabular}{|c|c|c|c|c|c|c|c|}
\hline AWARDEE NAME & BIN & L DATE & & & DESC & F WORK & \\
\hline VISION & VENDOR ID & CITY & STATE & $\begin{array}{l}\text { CONG } \\
\text { DIST }\end{array}$ & $\begin{array}{l}\text { CONTRACT } \\
\text { VALUE }\end{array}$ & $\begin{array}{l}\text { OBLIGS } \\
\text { TO DATE }\end{array}$ & $\mathrm{P} / \mathrm{S}$ \\
\hline
\end{tabular}

GEOLOGY \& GEOL ENG DEPT

FC22-94BC14983 04/27/97 $052640-0301-7$ HOUGHTON

MICHIGAN UNIVERSITY OF NUCLEAR ENGINEERING DEPT

MATERIALS SCIENCE \& ENG

MATERIALS SCIENCE \& ENG

CHEMISTRY DEPT

ATMOSPHERIC \& OCEANIC SCNCES

$\begin{array}{ccc}\text { FG02-80ER10724 } & 09 / 15 / 94 \\ \text { MICHIGAN MEMORIAL-PHOENIX PROJ } 052650-1301-6 & \text { ANN ARBOR }\end{array}$ NUCLEAR ENGINEERING DEPT NUCLEAR ENGINEERING DEPT

CHEMISTRY DEPT

CHEMISTRY DEPT

CHEMISTRY DEPT
FG02-87ER4033909/30/94 052650-0501-3 AIIN ARBOR

FG02-88ER45366 07/31/94 052650-2401-8 ANI ARBOR

FG02-88ER45367 08/31/94 052650-2401-8 ANN ARBOR

FG02-90ER60984 07/31/94 052650-2101-9 AHH ARBOR

FG02-91ER2510909/14/94 FG02-92ER75789 09/29/94 052650-0501-3 ANIN ARBOR

FG02-93ER79193 09/29/94 $052650-0001-1 \quad$ AIN ARBOR

FG05-91ER79042 09/29/94 $052650-0001-1 \quad$ ANN ARBOR

FG05-92ER79112 07/31/94 $052650-0001-1$ AlN ARBOR 052650-2101-9 ANH ARBOR

FG09-93SR18376 09/27/94 052650-2101-9 AHIN ARBOR

FG09-935R $18350 \quad 08 / 01 / 94$ 052650-2101-9 ANN ARBOR

FG22-91PC91295 08/28/94 052650-0001-1 AIIN ARBOR $052650-0201-4$ ANII ARBOR

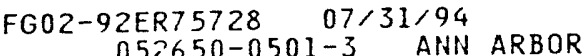

FG06-93RL12666 09/05/94
RECOVERY OF BYPASSED OIL IN THE DUNDEE FORMATION USING HORIZ MI $01 \quad \$ 2,616,819$ $\$ 800,000$

FAST NEUTRON CROSS SECTION MEASUREMENTS

$\begin{array}{lll}M I & 02 & \$ 1,311,000\end{array}$ $\begin{array}{clcc}\text { THE STRUCTURAL BASIS } & \text { FOR } \\ \text { MI } & 02 & \$ 689,075 & \text { FATIGUE INITIATION IN } \\ \$ 664,275 & \end{array}$

A STATISTICAL-MACHANICS STUDY OF SOLID-SOLID INTERFACES ICAL-MACHANICS STUDY OF SOLID-SOLID TNTERFA
MI $02 \quad \$ 801,958$ GENE SEQUENCING BY SCANNING MOLECULAR EXCITION MICRSCOPY $\begin{array}{llll}M I & 02 & \$ 768,173 & \$ 768,173\end{array}$ SPECTRAL METHODS \& SUM ACCELERATION ALGORITHMS.

$\begin{array}{lll}\text { MI } 02 & \$ 158,745 & \$ 158,745\end{array}$ UNIVERSITY REACTOR SHARING

$$
\text { MI } \quad 02 \quad \$ 421,200
$$

CONTINUING NUCLEAR ENGINEERINGEDUCATION ASER ENHANCED MIRONAVE PLASMA ISOTYPE SEPARATION $\begin{array}{ll}\$ 316,522 & \$ 316,522\end{array}$ UNIVERSITY REACTOR INSTRUMENTATION PROGRAM $\begin{array}{llll}M I & 02 & \$ 35,000 & \$ 35,000\end{array}$ CONSTRUCTION OF A FAST $\begin{aligned} X-R A Y & \$ 345,600 \\ & \$ 32\end{aligned}$ 1992 INSTRUMENTATION MULTIUSERNETWORK FOR ADVANCED REAL-TIM 992
INSTRUMENTATION MULTIUSERNETWORK FOR ADVANCED USED ENERGY RELATED EQUIPMENT GRANT $\$ 0$ \$0 USED ENERGY RELATED LABORATORYEQUIPMENT GRANT USED EHERGY RELATED LABORATORYEQUIPMENT MI RELATED LABORATORYEQU 13 $\$ 0$ \$O

A NOVEL IHTEGRATED TREATMENT SYSTEM FOR COAL WASTEWATERS

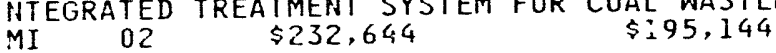




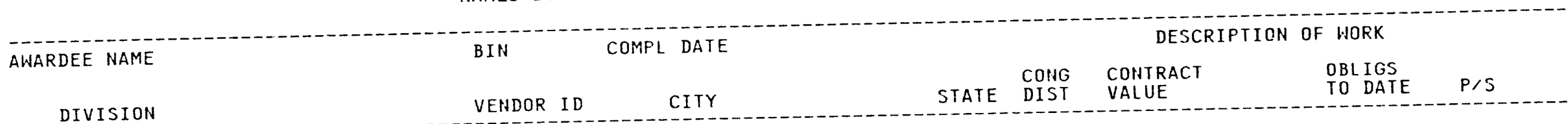

AC02-76ER01112 12/31/94 052650-0001-1 ANN ARBOR

FG02-89ER13996 11/14/94 052650-2101-9 ANN ARBOR

CHEMISTRY DEPT

CHEMISTRY DEPT

RESEARCH DEVELOPMENT \& ADMIN

NUCLEAR ENGINEERING DEPT

NUCLEAR ENGINEERING DEPT

NUCLEAR ENGINEERING DEPT

NUCLEAR ENGIN EERING DEPT

PHYSICS DEPT

NUCLEAR ENGINEERING DEPT

EHGINEERING DEPT OF

BIOLOGY DEPT

- nuClear engineERING DePT

CHEMISTRY DEPT

CHEMISTRY DEPT
FG02-91ERI4190 12/31/94
$052650-2101-9$ ANH ARBOR

FG02-90ER12932 11/30/94

FG02-92ER75712 11/14/94 052650-0501-3 ANN ARBOR $052650-0501-3$ ANN ARBOR

FG02-93ER75870 11/14/94 052650-0501-3 ANN ARBOR

FG02-93ER75869 11/14/94 052650-0501-3 ANIN ARBOR

FG02-85ER45189 01/31/95 $052650-0601-X$ ANN ARBOR

FG02-86NE37969 05/31/95 052650-0501-3 AINI ARBOR

FG02-89ER60820 07/31/95 $052650-2801-3$ AlH ARBOR

FG02-92ER20068 06/30/95 052650-2201-5 All ARBOR

FG02-94ER61813 03/15/95 $052650-0501-3$ AlNI ARBOR

FG06-94RL12844 04/17/95 052650-2101-9 AllH ARBOR

FG34-94RF00784 02/27/95 $052650-2101-9$ AINI ARBOR

FG21-94MC $31230 \quad 01 / 23 / 95$ 052650-2101-9 AllN ARBOR 052650-2901-X AIIN ARBOR

FG02-92ER75709 11/14/94
HIGH \& LOH FNERGY PHYSICS STUDIES USING AN 83 IN CYCLOTRON $M I \quad 02 \quad \$ 7,771,234$ $\$ 73,445,034$ AG91

HADAMARD TRANSFORM IMAGING

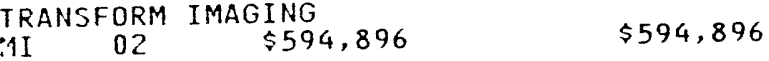

HYDROGEN INDUCED C-C, C-N, \& C-S BOND ACTIVATION ON PT \& NI O2 $\$ 424,912 \quad \$ 424,912$

NUCLEAR ENGINEERING RESEARCH

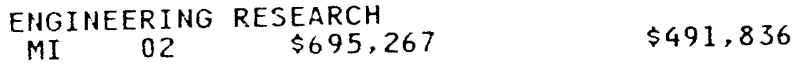

SIMULATION-BASED DIAGNOSTICS AND CONIROL FOR NUCLEAR

MI $\$ 263,531 \quad \$ 263,531$ ADVANCED COMPUTATIONAL METHODSFOR NODAL DIFFUSION,
$\$ 200,00 \mathrm{C}$

VARITIONAL TECHHIQUES IN REACTOR PHYSICS CALCULATION MI 02 \$107,866 $\$ 107,866$

FUEL CONTINEMENT AND STABILITY IN GAS NUCLEAR

MI 02 \$50,000 $\$ 50,000$

GROWTH AND DYHAMICS OF SCALE INVARIANT MATTER

$\begin{array}{llll}\text { MI } & 02 & \$ 1,574,527 & \$ 1,429,511 \\ & \end{array}$

DEVELOPMENT OF A SEMI-AUTONUMOUS MOBILE ROBOT FOR RE $\$ 4,411,500$

THE IHFLUENCE OF INTERFACIAL PROPERTIES ON TWO-PHASE LIQUID

$M I \quad 02 \quad \$ 695.232 \quad \$ 574,484$

MOLECULAR GENETUS NF $\$ 242.540$ MYOSIN MOTORS IN $\$ 79,703$

99 SYMPOSIUM ON PADIATIONS MEASUREMENTS AND APPLICATION MI $\$ 5,000 \quad \$ 5,000$

USED ENERGY RELATED LABORATORYEQUIPMENT GRANT

$\begin{array}{ccc}\text { MI } & 13 & \$ 0 \\ & & \\ \text { USED ENERGY RELATED } & \text { LAB EQUIPMENT GRANT }\end{array}$

USED ENERGY RELATED EQUIPMENT

$\$ 0$

$\$ 0$

CHEMISTRY DEPT 
DIVISION

VENDOR ID VENDOR ID CITY $052650-0001-1$ ANN ARBOR

FG22-93PC93212 08/31/95 052650-0001-1 ANN ARBOR

HOSPITALS

CHEMISTRY DEPT

CHEMISTRY DEPT

HUMAN GENETICS DEPT

PHYSICS DEPT

MATERIALS SCIENCE \& ENG

PHYSICS DEPT

INTERNAL MEDICINE DEPT
FG45-93R551649 08/31/95
$052650-3001-8$ ANH ARBOR

FG45-93R551648 08/31/95 052650-0001-1 ANH ARBOR

FG34-94RF0084505/03/95

FG34-94RF00925 06/06/95 052650-2101-9 ANN ARBOR

FG02-87ER60533 11/30/95 052650-1801-8 AIN ARBOR

FG02-91ER40675 12/31/95 052650-0601-X AHN ARBOR

FG02-93ER12130 10/31/95 052650-0001-1 AHN ARBOR

FG02-94ER61792 11/30/95 052650-0001-1 ANH ARBOR

FG22-92PC92537 11/03/95 052650-0001-1 ANH ARBOR

FG02-87ER453U2 04/30/96 052650-2401-8 AINI ARBOR

FG02-90ER45416 06/30/96 052650-0601-X AIN ARBOR

FG02-93ER61569 03/31/96 052650-0001-1 ANN ARBOR

FG02-93ER61660 07/31/96 052650-2701-7 ANN ARBOR

FG02-93ER61666 08/14/96 052650-0001-1 AIIH ARBOR

FG02-85ER45184 11/30/96 052650-0501-3 ANII ARBOR $052650-2101-9$ ANN ARBOR
CONG COHTRACT

STATE DIST VALUE

OBLIGS

T DATE P/S

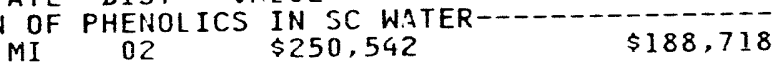

BIOLOGICAL DETERMINANTS OF PHOTOBIOREACTOR. DESIGN

$$
\begin{aligned}
& \text { MI } \\
& 02
\end{aligned}
$$

10.602

ICP-NEW AWARD.

$\$ 263,325$

$\$ 131,662$

ICP- NEW AWARD,

$\$ 55,650$

$\$ 27,825$

USED ENERGY RELATED LAB EQUIPMENT

$\$ 0$

USED ENERGY RELATED LAB EQUIPMENT GRANT

MI 13
STUDIES OF HUMAN MUTATION

$$
M I \quad 02 \$ 2,580,590
$$

$\$ 2,375,090$

EXPLOIT SECONDARY VERTEX INFORMATION AT THE CDF DETECTOR.

FEASIBILITY OF A NOVEL APPROACH FOR FAST ECONOMICAL MI 02 N450,882

THE ROLE OF PATENTS IN TECHNOLOGY TRANSFER IN THE

NOVEL CATALYSTS FOR UPGRADING COAL-DERIVED LIQUIDS

MICROMECHANICAL \& MICR ITIRUCTURAL STUDIES OF CERAMIC SUPERPL

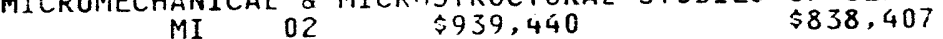

SYNCHROTRON STUDTES NF NARROW BAND MATERIALS

MI 02 \$585,000 $\$ 427,300$

GENOME TECHNOLOGY AND ITS IMPLICATIONS

MI 13 A $\$ 225,000 \quad \$ 147,750$

MOLECULAR MECHANISMS OF ENHANCED ( 18 FO FLUORODEOXY GLOUCOSE

$$
\begin{aligned}
& \$ 414,982 \\
& \$ 127,100
\end{aligned}
$$

CHANGES IN THE FLUX OF CAREON BETWEEN PLANTS AND SOIL

$$
\text { MI THE FLOX OF } \$ 414,666 \quad \$ 155,775
$$

THE INFLUENCE OF GRAIN BOUNDARY CHEMISTRY AND STRUCTURE ON I MI $02 \quad \$ 1,566,064 \quad \$ 1,299,125$ 


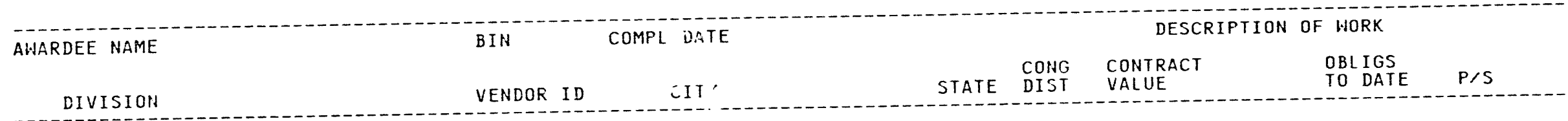

CYCLOTRON

CHEMISTRY DEPT

PHYSICS DEPT

MICROSENSOR SYSTEMS INC

MICROWAVE INC

MIDAC CORP

MIDDLE TENIESSEE STATE UNIV CHEMISTRY \& PHYSICS DEPT

MIDDLESEX BOARD OF EDUCATION

\section{MIDDLETON TOWN OF} PUBLIC SCHOOLS

MIDDLETOWN REGIONAL HOSPITAL

\section{FG02-87ERG $0561 \quad 12 / 31 / 96$
$052650-2501-4$ AHN ARBOR} FG02-90ER61085 12/23/96 $052650-2101-9$ AHH ARBOR

FG02-94ER40822 12/14/96 052650-0001-1 ANI ARBOR FG02-94ER14412 11/30/96 052650-0001-1 AHN ARBOR

FG02-85ER1344203/31/9? 052650-0001-1 Aill ARBOR

FG02-94ER40813 10,31/98 $052650-0601-X$ ANH ARBOR

FG05-94ER81673 07/17/94 $188140-0002-6$ BOHL ING GREEN

RL08-89NV10886 07/31/94 098070-0001-7 LAS VEGAS

FC34-94RF00866 c $05 / 02 / 95$
$238000-0001-6$ IRVINE

FG05-86ER4029306/30/95 052870-0101-0 MURFREESBORO

FG42-93R253857 06/30/95 $130480-0001-1$ MIDDLESEX

FG42-94R263913 06/30/96 $130480-0001-1$ MIDDLESEX

FG41-92R193420 07/31/94 221450-0101-8 MIDDLETON

FG45-93R551736 05/31/95 $134700-0001-8$ MIDDLETOWN

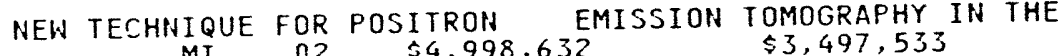
NAMOMETER SCALE EXUTON SPECTROSCOPY PHOTOCHEMISTRY ACCELERATOR PHYSICS R\&D MI $02 \quad \$ 250,000$ APPLICATIONS ICP MAGNETIC SECTOR MASS SPECTRO. TO MI $02 \quad \$ 457,056 \quad \$ 97,000$ $\begin{array}{lll}\text { ENERGY TRANSFER PROPERTIES ANDMECHANISMS } & \text { \$1, 077,466 }\end{array}$ POLARIZED SCATTERING EXPERIMENTS - HIGH ENERGY HIGH FREQUEHCY SAW DEVICES AS NOVEL DETECTOR FOR PORTABLE GC

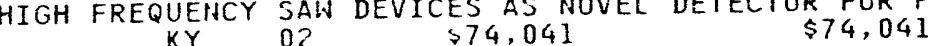

LEASE OF COMMUNICATIONS SITE ON MT. POTOSI

$\$ 3,240 \$ 3,240 \times 119$ COOPERATIVE AGREEMENT BETWEEN DOE, EG\&G, AND PARTICIPANT FOR $C A \quad 47$ \$O NUCLEAR STRUCTURE STUDIES VIA NEUTRON INTERACTIONS $\begin{array}{llll}\text { CYCLE } 15 & \text { A.JARD. } \\ \mathrm{NJ} & 07 & \$ 27,327 & \$ 13,663\end{array}$ CYCLE 16 AHARD. $07 \quad \$ 87,273 \quad \$ 43,636$

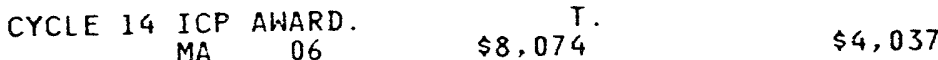

ICP-NEW AWARD. $\$ 32,280 \quad \$ 16,140$




\begin{tabular}{|c|c|}
\hline AWARDEE NAME & COMPL DATE \\
\hline DIVISION & VENDOR ID \\
\hline \multirow[t]{2}{*}{ MIDWEST POWER SYSTEMS INC } & $\begin{array}{r}\text { FC21-91MC27364 } 09 / 30 / 99 \\
211300-0001-X \text { DES MOINES }\end{array}$ \\
\hline & $\begin{array}{r}\text { FR21-91MC28120 07/01/17 } \\
211300-0001-x \text { DES MOINES }\end{array}$ \\
\hline $\begin{array}{l}\text { MIDHEST RESEARCH INST } \\
\text { NATIONAL RENEWABLE ENERGY }\end{array}$ & $\begin{array}{ccc}A C D 2-83 C H 10093 & 09 / 30 / 98 \\
L A B & 053000-0301-8 \quad G O L D E N\end{array}$ \\
\hline MIDHEST SANITATION & $\begin{array}{c}\text { AX65-94WD03449 } 09 / 30 / 94 \\
235710-0001-3 \quad \text { JAMEST OWN }\end{array}$ \\
\hline MILES COLLEGE & $\begin{array}{rl}\text { FG } 44-88 R 433605 & 12 / 31 / 94 \\
053070-0002-2 & \text { BIRMINGHAM }\end{array}$ \\
\hline MILFORD TOWN OF & $\begin{array}{c}\text { FG41-92R191308 09/30/94 } \\
216940-0001-x \text { MILFORD }\end{array}$ \\
\hline MILLER REPORTING CO INC & $\begin{array}{c}\text { ABO1-93AD24906 09/30/94 } \\
179540-0001-X \text { WASHINGTON }\end{array}$ \\
\hline MILWAUKEE PUBLIC SCHOOL DIST & $\begin{array}{c}\text { FG45-93R551819 } 06 / 30 / 95 \\
053370-0001-2 \text { MILWAUKEE }\end{array}$ \\
\hline MINATOME CORP & $\begin{array}{c}R L 13-746 J 3100209 / 16 / 94 \\
169990-0001-8 \text { HOUSTON }\end{array}$ \\
\hline MINERALOGICAL SOC OF AMERICA & $\begin{array}{c}\text { FG02-93ERI4399009/14/94 } \\
231690-0001-2 \text { WASHINGTON }\end{array}$ \\
\hline MINNESOTA CHILDREN'S MUSEUM & $\begin{array}{c}\text { FG02-94ER75949 } 12 / 14 / 95 \\
237580-0001-7 \text { ST. PAUL }\end{array}$ \\
\hline MINNESOTA STATE OF & $\begin{array}{c}\text { FCO1-91EI22719 } 08 / 07 / 96 \\
053480-0001-9 \text { ST. PAUL }\end{array}$ \\
\hline & FG45-89CH10412 \\
\hline
\end{tabular}

PCFB REPOWERING PROJECT

IA $04 \$ 202,959,000 \quad \$ 5,958,000$

REPAYMENT AGREEMENT - PCFB $\underset{\$ 0}{\text { REPOWERING PROJECT }} \$ 0$

OPERATION OF SOLAR ENERGY RESEARCH INSTITUTE

$\begin{array}{cccc}\text { OPERATION OF SOLAR ENERGY RESEARCH } & \text { INSTITUTE } \\ \text { CO } & 02 \$ 1,291,938,975 & \$ 1,063,991,306 & \text { AG71 }\end{array}$

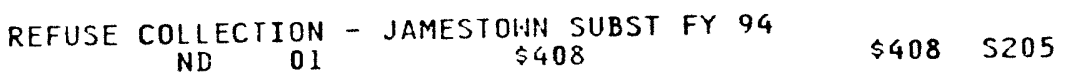

CYCLE X - INSTITUTIONAL CONSERVATION PROGRAM. APPROVED BUDG

NEW AWARD CT $_{\text {CT }} 03 \quad \$ 43,357 \quad \$ 21.678$

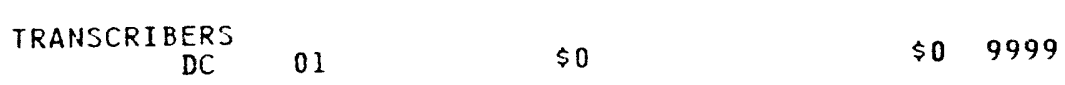

ICP-NEH AHARD.
WI TS $\$ 532,010 \quad \$ 266,005$

MINING LEASE AT $\underset{T X}{22}(05-1)-M L-60 . \underset{\$ 0}{8-U-C W-2 A} \quad \$ 0 \quad \times 293$

$\begin{array}{rlrr}\text { WORKSHOP ON HEALTH EFFECTS OF MINERAL BUSTS } & \\ \text { DC } & 01 & \$ 10,000 & \$ 10,000\end{array}$

LEARNING FROM LENNA: $\quad \$ 95,060$ DUCKWEED CLEANS UP

COLLECTION AND ANALYSIS OF DATA FOR EIA STATE HEATING OIL AN

FY89 FUNDING FOR THE STATE OF MINNESOTA'S LOW-INCOME 


AWARDEE HAME

DESCRIPTION OF WORK

AWARDEE HAME

VEHDOR ID

CITY $053480-1201-7$ ST. PAUL

JOBS \& TRAINING DEPT OF

FG45-89R510540 06/30/95 $053480-0801-X$ ST. PAUL

PUBLIC SERVICES DEPT

MIHHESOTA UHIVERSITY OF

FG02-92ER45465 12/31/94
$053490-0001-2$ MIHNEAPOLIS

FG02-89ER14036 06/30/96

MECHANICAL ENGINEERING DEPT FGO2-89ER $053490-1101-4$ MINHEAPOLIS

FG02-85ER13433 $08 / 31 / 96$

053490-1101-4 MIPHNEAPOL IS

FG02-86ER13512 04/14/97 $053490-2201-6$ MINHEAPOLIS

MICROBIOLOGY DEPT

CHEMISTRY DEPT

CHEMISTRY DEPT

PHYSICS \& ASTRCNOMY

MINNESOTA SUPERCOMPUTER INST

PHYSICS DEPT

AEROSPACE ENG \& MECH

FG02-86ER13515003/31/95 $053400-0401-8$ MININEAPOL IS FG02-86ERI3579 06/30/95 $05 \div 490-0401-8$ MINHEAPOLIS FGO2-87ER40328 01/31/96 053490-1701-2 MINHEAPOL IS FG02-87ER25035 07/31/96 053490-2501-5 MINHEAPOLIS FG02-87ER40362 05/31/97 $053490-1601-6$ MINNEAPOLIS FG02-87ERI3793 08/31/98 053400-2601-1 MINNEAPOL Is FGO2-88ER45337 $10 / 31 / 95$ 053490-0301-1 MIHNEAPOL IS FG02-88ER40405 09/30/94 55\$490-1701-2 MIHHEAPOL IS PHYSICS \& ASTRONOMY GRAY FRESHWATER BIOLOGICAL FG02-88ERI3362 02/28/95 053490-2701-8 MINNEAPOLIS FG02-89ER4539106/30/95 - CHEMICAL ENG \& MATERIALS SCI FG02-89ER453910301-1 MIIHEAPOLIS FG02-89ER40528 08/31/95
CONG CONIRACT OBLIGS TO DATE P/S \$ $54,341,637$

Mir

$04 \$ 72,636,722$

AHARD OF FY 1989 FUNDING FOR ADMINISTRATION OF THE IHSTITUT MN $19894 \quad \$ 628,028 \quad \$ 317,431$

CFYSTALLINE-AMORPHOUS $\underset{05}{\$ 364,730}$ INIERFACES \& AMORPHOUS FILMS

COUNCIL ON ENERGY ENGINEERING RESEARCH

$$
\begin{array}{ll}
\text { ON ENERGY ENGINEERING RESEARCH } \\
\text { MNS } 05
\end{array}
$$

ANALYTICAL AND EXPERIMENTAL STUDIES FOR THERMAL PLASMA INN $05 \quad \$ 1,460,357 \quad \$ 1.176,493$

THE MECHANISM OF SHITCHING FROM AN ACIDOGEHIC TO A BUTANOLMII $05 \quad \$ 975.070 \quad \$ 869,250$

STATE TO STATE OF MOLECULAR ENERGY TRANSFER MII 05 \$ $\$ 64.460 \quad \$ 961.190$ VARIATIONAL TRANSITION STATE THEORY
Fin 05 $\$ 823,740$
$\$ 718,740$

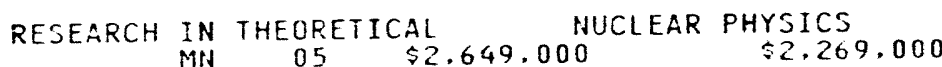

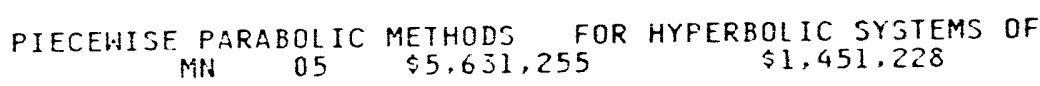

PION- AHD PROTOH-HUCLEUS INTERACTIONS AT IHTERMEDIATE ENERGY MN $05 \$ 2,042,000 \quad \$ 1,587,000$

LUBRICATED PIPELINING
MIN 05
$\$ 994,268$
$\$ 649,268$

A STUDY OF SCALE CRACKIHG AND ITS EFFECTS ON OXIDATION AND MiN 05 \$1.297.699 $\$ 1.216 .578$

HUCLEAR PHYSICS STUDIES AT INTERMEDIATE EHERGIES GENETICS OF BACTERIA THAT UTILIZE ONE CARBON COMPOUNDS MIN $05 \quad \$ 527,514 \quad \$ 527,514$ THEORY OF THE STRUCTURAL AND ELECTORNIC PROPERTIES OF SOLID
MH
05

PROGRAM TO STUDY THE C.AARACTERISTICS OF HIGH ENERGY 


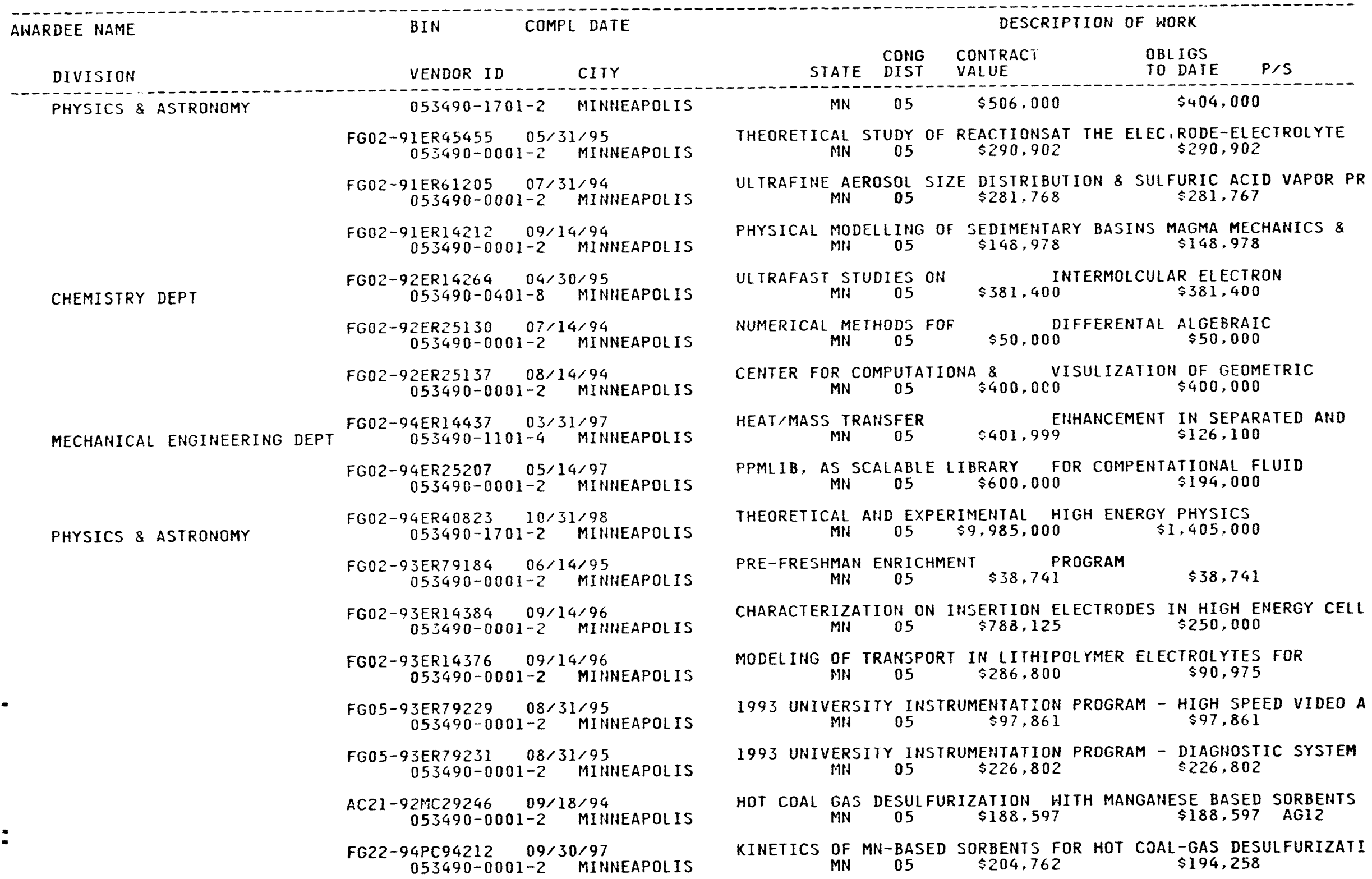




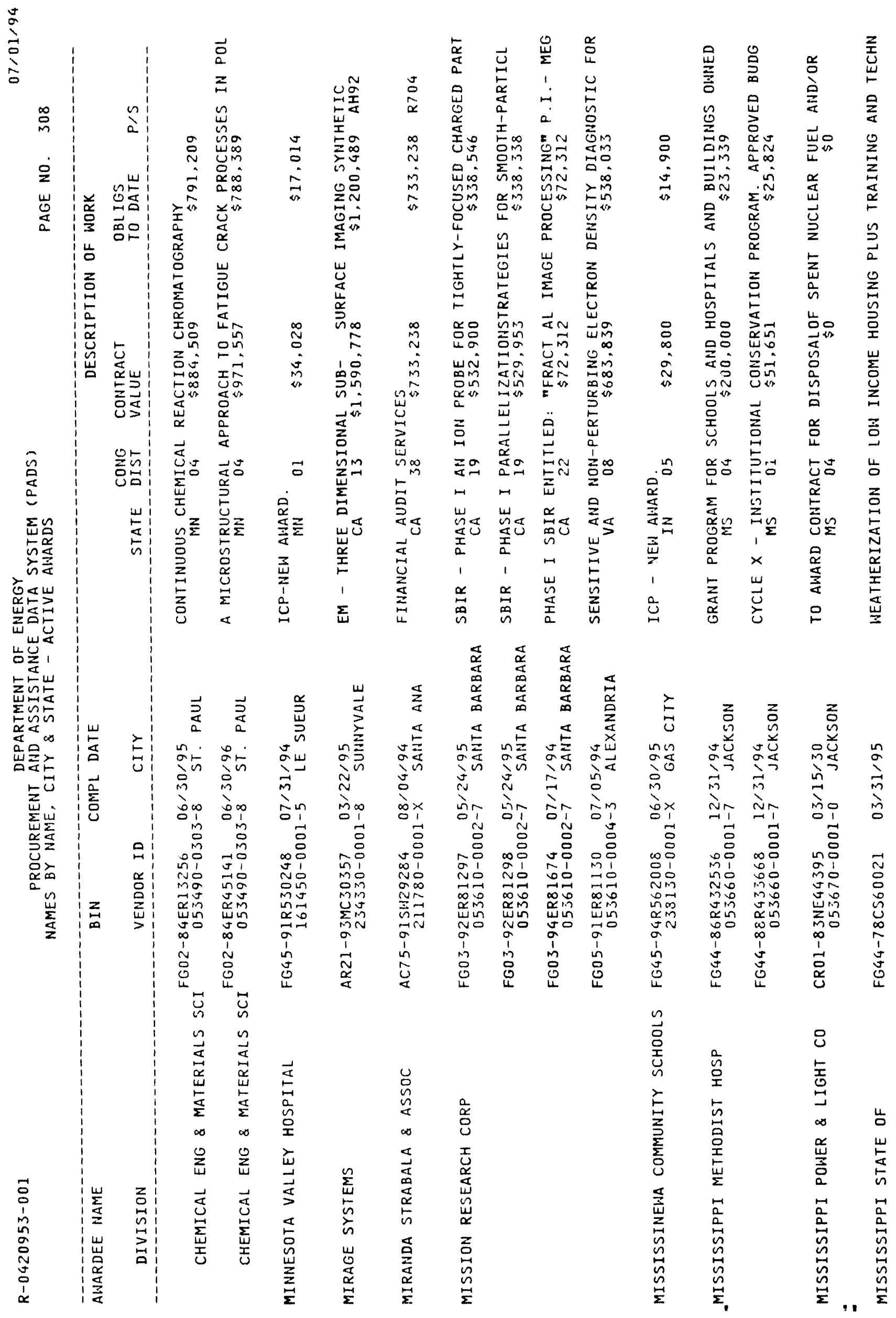




AWARDEE NAME

COMPL DATE

DESCRIPTION OF NORK

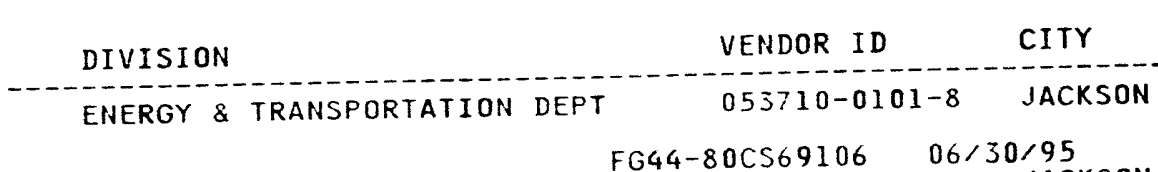

ENERGY \& TRANSPORTATION DEPT FG44-80C569106 $06 / 30 / 95$ JACKSON

ENVIRONMENTAL QUALITY FG08-91NV10971 01/08/96
$053710-0401-7$ JACKSON

MISSISSIPPI STATE UNIV PHYSICS \& ASTRONOMY

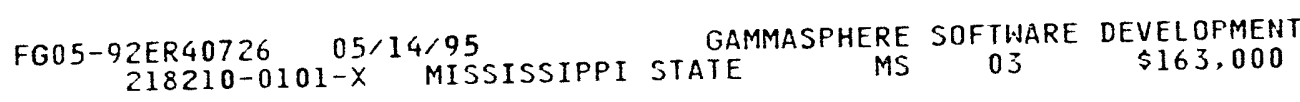

OBLIGS
TO DATE
$\$ 19,209,056$

STATE OF MISSISSIPPI ENERGY EXTENSION SERVICE - NO REMARKS

OVERSIGHT OF REMEDIAL INVESTIGATION AND FEASIBILITY STUDY OF

MISSISSIPPI STATE UNIVERSITY

FG02-93CH10575 $03 / 31 / 98$
$053720-0005-8$ MISSISSIPPI STATE

USE OF MISSISSIPPI STATE UNIVERSITY DIAGNOSTIC 053720-0005-8 MISSISSIPPI STATE OF MISSISSIPPI STATE $\$ 24,027,000$

$\begin{array}{ccccccc}\text { FG02-93ER75928 } 09 / 29 / 94 & \text { MISSISSIPPI DOE EPSCOR PROGRAM } & \$ 20,000\end{array}$ MECHANICAL \& NUCLEAR ENG DEPT 053720-0305-7 MISSISSIPPI STATE GERERAIZED GRID AND CFD TECHNOLOGY IN OCEAN/ATMOSPHERIC MOD ENGINEERING RES CTR FOR CFS FG05-93ER25187 08/31/96
$053720-0905-5$ MS 03 TE $\$ 1,110,806 \quad \$ 351,077$ FG02-91CH10479 12/31/94
$053720-0006-6 \quad$ STARKVILLE FG44-92R435119 09/30/94
$053720-0006-6$ STARKVILLE DESIGN \& CONSTRUCTION OF A DIAGNOSTIC INSTRUMENTATION \& ANAL MS 03 Y $\$ 7,953,600$ CONSERVATION PROGRAM. APPROVED BUD

MISSISSIPPI UNIVERSITY OF

PHYSICS \& ASTRONOMY DEPT CHEMICAL ENGINEERING DEPT - MISSISSIPPI VALLEY STATE UNIV MISSOURI STATE OF ENERGY DIV

$\div$
FG44-92R435124 09/30/94 $053730-0002-7$ UNIVERSITY FG05-91ER40622 03/31/95 $053730-0602-5$ UNIVERSITY FG22-93PC93227 09/29/96 $3 P C 93227$
$053730-0702-1$

FG44-90R434384 08/15/94 $053740-0001-2$ ITTA BENA FG47-92CE60330 06/30/95
$053800-0301-X \quad$ JEFFERSON CITY FG47-93R760344 06/30/95 053800-0101-7 JEFFERSON CITY
MS 03 \$92,000

CYCLE 14 - INSTITUTIONAL CONSERVATION PROGRAM. APPROVED BUD $\begin{array}{lll}M S & \end{array}$ EXPERIMENTAL STUDY OF HEAVY FLAVOR PHYSICS AND SSC R\&D MJ 01 \$793,000 $\$ 779,200$

ROLE OF CHAR DURING REBURNING OF NITROGEN OXIDE

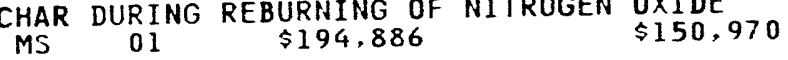

CYCLE 12 IHSTITUTIONAL CONSERVATION PROGRAM. APPROVED FUNDED MS 02 TISTITUTIONAL CONSERVATIO $\$ 38,205$

2 ALLOCATIONS - HEATHEPIZATION ASSISTANCE FOR LON INCOME PE FY93 ICP ECM FOR COORDINATING AGENCY GRANT

MO 04 \$ $1,105,995 \quad \$ 540,000$ 
AWARDEE NAME

BIN

COMPL DATE

DESCRIPTION OF WORK

DIVISION

BIN

DEPT OF NATURAL RESOURCES

PUBLIC SERVICE COMMISSION

NATURAL RESOURCES DEPT

NATURAL RESOURCES DEPT

MISSOURI UNIVERSITY OF

PHYSICS DEPT

CHEMISTRY DEPT

BIOCHEMISTRY DEPT

CHEMISTRY DEPT

RADIOLOGY DEPT OF

$$
\text { VENDOR ID }
$$$$
\text { STATE CONG CONTRACT }
$$$$
\text { STATE. DIST }
$$

OBLIGS PO DATE P/S

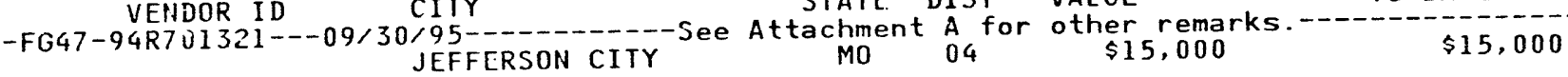

FC01-91EI22790 08/08/96 1991-92 STATE HEATING OIL AND PROPANE PROGRAM

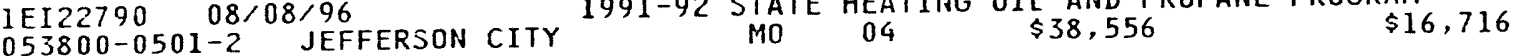

FG47-92R701310 11/30/98

SEE ATTACHMENT A FOR OTHER REMARKS. FG05-930R22073 $01 / 31 / 98$
$053800-0105-X$ ST. CHARLES

OVERSIGHT OF ENVIRONPENTAL RESTORATION AND WASTE MANA

WASTE MANAGEMENT

GMO4-85AL38771 09/30/99 053800-0003-7 ALBUQUERQUE

DESCRIBES RELATIONSHIP BETWEENDOE \& STATE EMERGENCY MGMT

$$
\text { NM } 01
$$

FG02-93ER75851 $08 / 31 / 94$ $053810-0001-4$ COLUMBIA

FG02-84ER53175 05/31/97 053810-0901-1 COLUMBIA

FG02-86ER60400 08/31/94 053810-0301-3 COLUMBIA

FG02-87ERI3713 04/30/96 053810-1501-1 COLUMBIA

FG02-88ER13880 05/14/97 053810-0301-3 COLUMBIA

FG02-89ER60875 10/31/95 053810-2001-5 COLUMBIA

FG02-91ER12107 09/14/94 053810-0001-4 COLUMBIA

FG02-91ER20036 07/14/94 053810-0001-4 COLUMBIA

FG02-80ER10725 09/29/94 053810-1001-X COLUMBIA

RESEARCH REACTOR FACILITY

RESEARCH REACTOR FACILITY

FG02-83ER75102 09/15/94 053810-1001-X COL UMBIA

FG02-90ERI2970 09/29/94 053810-1001-X COLUMBIA

: RESEARCH REACTOR FACILITY

FGO2-90ER13035 09/30/94

DOE/UTILITY NUCLEAR EDUCATION ENHANCEMENT PROGRAM MO $09 \quad \$ 50,000$ IN MFE PLASMA RESEARCH COLLISION PROCESSES OF INTEREST IN MFE PLASMA RESEAR
MO 09 BIFUNCTIONAL CHELATES OF RH-105 AND AU-199 AS POTENTIAL MO $09 \quad \$ 964,454 \quad \$ 964,454$

GENETIC \& MOLECULAR BIOLOGY OFHYDROGEN METABOLISM IN SULFATE $\begin{array}{lll}\text { MOLECULAR BIOLOGY } & \$ 696,000 & \$ 604,390\end{array}$

LATE TRANSITIN METAL H-OXO AND H-IMIDO COMPLEXES

PRODUCTIONS OF RADIOLABELED MONOCLONAL ANTIBODY CONYUGATES $\begin{array}{cccc}\text { PRODUCTIONS OF } & \text { RADIOLABELED MONOCLONAL ANTIBODY } \\ \text { MO } & 09 & \$ 1,186,782 & \$ 984,698\end{array}$

STUDY OF POTENTIAL HIGH BAND- GAP PHOTOVOLT'AIC MATERIALS FOR MO 09 \$966,882 $\$ 966,882$

MOLECULAR ANALYSIS OF NUCLEAR-CYTOPLASMIC INTERACTIONS AFFEC $\begin{array}{llll}M O & 09 & \$ 270,957 & \$ 270,957\end{array}$

UNIVERSITY REACTOR SHARING

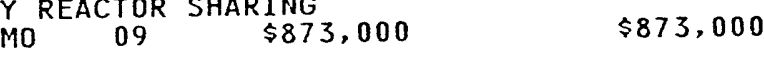

UNIVERSITY REACTOR SHARING

$$
\text { MO } 09 \quad \begin{aligned}
& \text { REACTOR } \\
& \text { SHARING }
\end{aligned}
$$

UNIVERSITY REACTOR INSTRUMENTATION PROGRAM

$$
\text { MO REACTOR INSTRUMENTATION PROGRAM } \$ 375,000
$$

NUCLEAR ENGINEERING RESEARCH "INVESTIGATIONS OF REACTIONS \& 


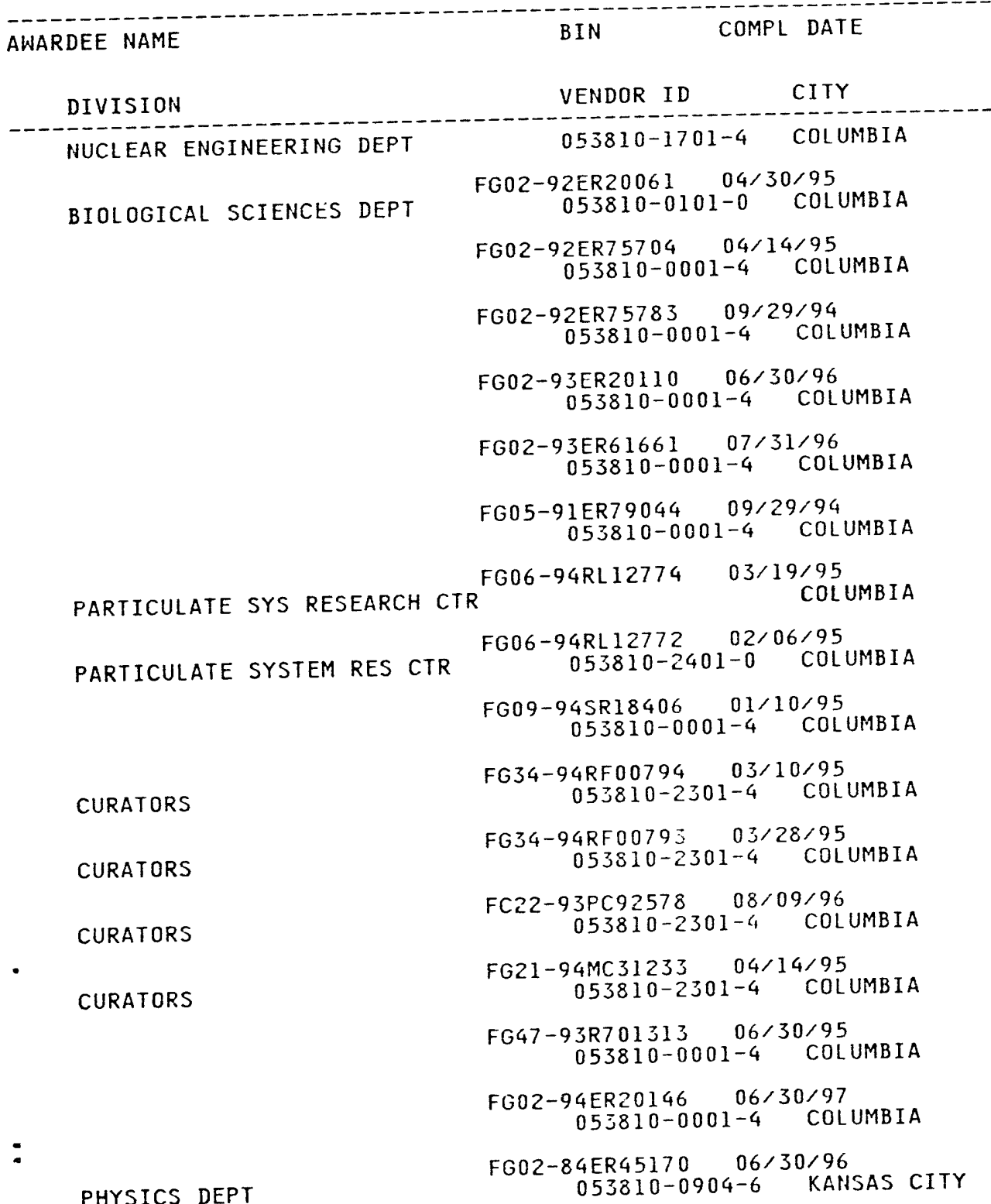

DESCRIPTION OF WORK

STATE DOHG CONTRACT

MO $09 \quad \$ 175,222$

OBLIGS
TO DATE P/S

$\begin{array}{ccc}\text { DOSAGE ANALYSIS OF GENE } & \text { EXPSION IN MAIZE } \\ \text { MO } 09 & \$ 279,120 & \$ 279,120\end{array}$

A DSP-BASED, SMART ANALOG-TO- DIGITAL CONVERTOR FOR NUCLEAR

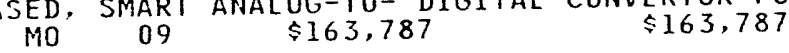

MEASUREMENTS OF PHYSICS - CHEMICAL PROPERTIES \& INTER-

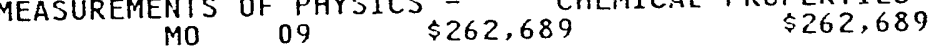

POSITION EFFECT AS A DETERMINENT VARIEGATED

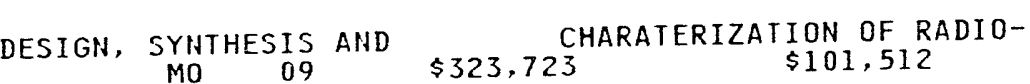

MULTI-BEAM MOSSBAUER SCATTERING FACILITY $\begin{gathered}\$ 126,065 \\ 09\end{gathered}$

USED ENERGY RELATED LABORATORYEQUIPMENT GRANT \$0

$$
\text { MO } 09 \text { \$O }
$$

USED ENERGY RELATED LABORATORYEQUIPMENT GRANT $\$ 00$

USED ENERGY RELATED LABORATORYEQUIPMENT

USED ENERGY RELATED LAB EQUIPMENT GRANT

$$
\begin{array}{ccc}
\text { MO } & \$ 9 \\
\text { MGY RELATED LAB EQUIPMENT GRANT }
\end{array}
$$

USED ENERGY RELATED LAB EQUIPMENT GRANT

THE CONSORTIUM FOR COAL LOG PIPELINES

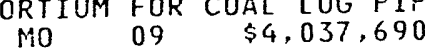

$\$ 108,000$

USED ENERGY RELATED EQUIPMENT

1993 ENERGY RELEATED INVENTIONS FROGRAM.

$\$ 89,467$

CELLULOSE SYHTHSIS AND $\$ 242,050$

160,050

THEORETICAL STUDIES ON THE STRUCTURES OF INSULATING AND META MO 09 \$1,253,491 $\$ 1,227,686$ 
(1) AHARDEE NAME

$---$

BIN

COMPL DATE

FG02-88ER $13819 \quad 08 / 31 / 94$ $8 E R 1381908 / 31 / 94$
$053810-0302-1 \quad$ ROLLA

CHEMISTRY DEPT

NUCLEAR REACTOR FACILITY

NUCLEAR REACTOR FACILITY

PHYSICS DEPT

MITRE CORP

MITSUBISHI CORP ELECTRIC CO

MITSUI CO

-MK-FERGUSON CO POWER DIV

POWER DIV

= POWER DIV

POWER DIV

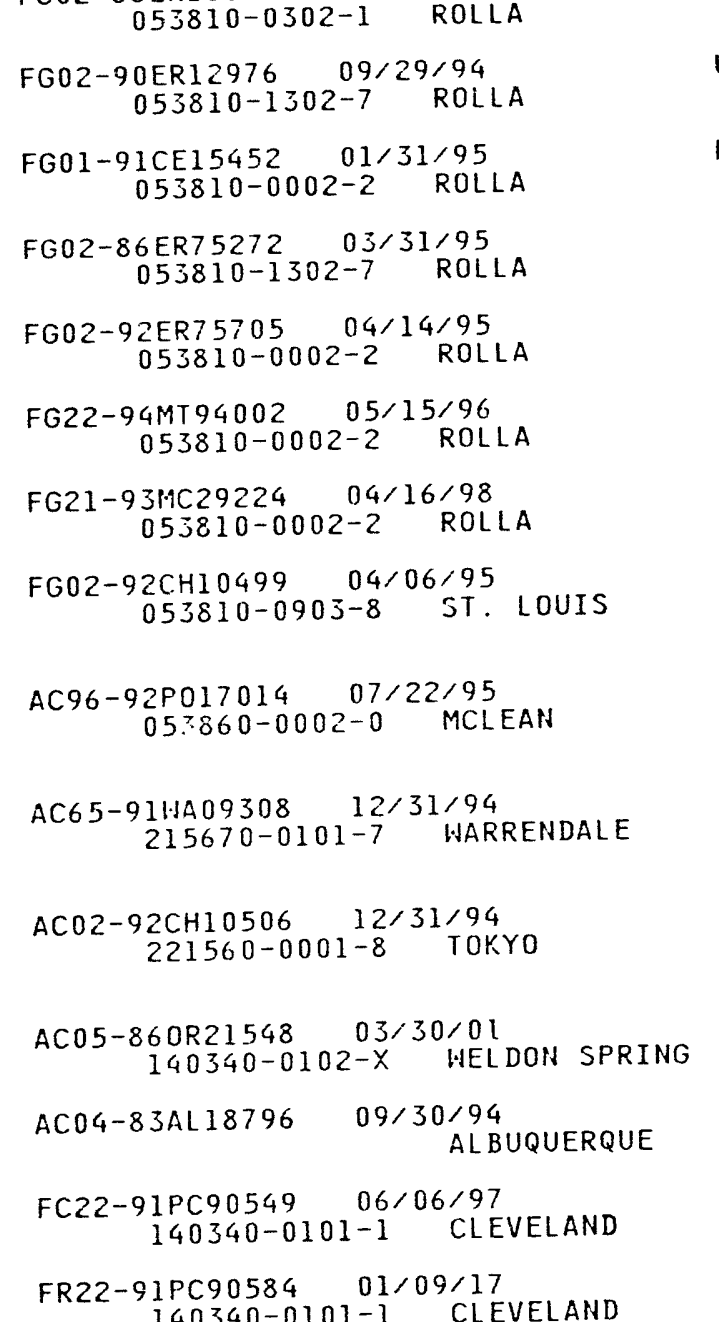

PAGE NO. 312

$07 / 01 / 94$ 


AWARDEE NAME
DIVISION
MK-FERGUSON OF IDAHO CO
POWER DIV

MK-FERGUSON OF OAK RIDGE CO

MOBILE INFIRMARY MEDICAL CTR

MOBILECOMM

MODELL DEVELOPMENT CORP

MODERN TECHNOLOGIES CORP

MOGEN PHILIP C

MOLECULAR DYNAMICS

- MOLECULAR MEdicine CENTER for

MOLONEY ELECTRIC CORP

MOLTECH CORP

\section{COMPL DATE}

BIN

CITY

CONG

DESCRIPTION OF HORK

VALUE

OBL IGS

$P / S$

MANAGEMENT \& OPERATIHG CONTRACT FOR CONSTRUCTION MANAGEMEN

ID $02 \$ 402,319,427$ \$401,186,774 MI81

157960-0101-9 IDAHO FALLS

AC05-910R21900 09/30/97

OAK RIDGE

FG44-91R434750 08/01/94 $188950-0001-3$ MOBILE

AMO1-93AD34048 09/30/94 234120-0001-9 ARLINGTON

AD01-93AD34099 12/31/94 $234120-0001-9$ ARLINGTON

FC07-90ID12915 01/16/95 192830-0001-3 FRAMINGHAM

AC05-920R21966 10/01/94 $20 R 21966$ 10101/94
$217500-0001-6$ DAYTON

$A B 04-91 A L 7255510 / 31 / 94$
$207540-0001-X \quad$ ALBUQUERQUE

FG03-92ER81299 05/25/95 221010-0001-6 SUNNYVALE

FG02-87CH10320 12/31/94
$158370-0001-1$ NEHARK AC65-93WJ21959 03/30/95 $136980-0001-6$ TORONTO

FG02-91ER81218 07/05/94 187370-0002-0 STOHY BROOK
MANAGEMENT AND OPERATING CONTRACT FOR CONSTRUCTION MANAGEMEN TN $03 \$ 439,046,925 \quad \$ 439,046,925$ MI99

CYCLE 13 - INSTITUTIONAL CONSERVATION PROGRAM. APPROVED BUD

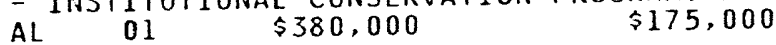

NUMERIC PAGER SERVICE ON NATIOHWIDE BASIS FOR DOE HEADQUARTE VA 08 \$62,400 $\$ 0 \quad 5805$

LEASE 150 NUMERIC PAGERS WITH NATIONWIDE MESSAGING SERVICE $F$

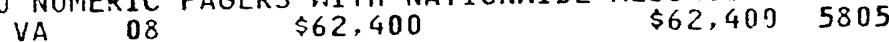

ASSESSMENT AND DEVELOPMENT OF AN INDUSTRIAL WET OXIDATION SY $M A \quad 05 \quad \$ 2,872,220 \quad \$ 1,917,683$

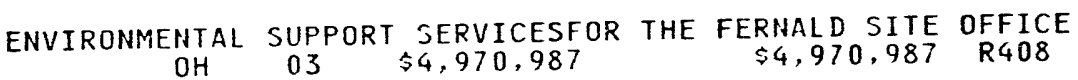

HEARING OFFICER

$$
\text { NMFICER } 01 \quad \$ 175,475
$$

$\$ 175,475 \quad R 603$

RAPID HIGH-THROUGHOUT DNA SEQUENCING USING CONFOCAL FLUORESC

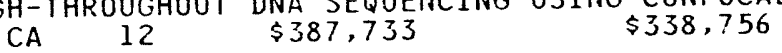

DESIGN AND CONSTRUCTION OF A NEW FACILITY FOR THE CENTER NJ 10 \$1 000,000 \$10,500,000

MOBILE POWER TRANSFORMER

$\$ 487,720 \quad 6120$ NY $01 \quad \$ 549,832 \quad \$ 549,391$
$00 \quad \$ 487,720$

ADVANCED BIOSENSORS FOR ANALYTICAL CHEMICAL MEASUREMENTS. 


\begin{tabular}{|c|c|}
\hline AWARDEE NAME & COMPL DATE \\
\hline DIVISION & VENDOR ID \\
\hline MOLTEN METAL TECHNOLOGY INC & $\begin{array}{c}\text { AC21-93MC } 30171009 / 30 / 94 \\
235020-0001-8 \text { WAL THAM }\end{array}$ \\
\hline MONONGAHELA POWER CO & $\begin{array}{cc}\text { AD21-88MC25112 } & 08 / 11 / 96 \\
054210-0001-X & \text { MORGANTOWN }\end{array}$ \\
\hline MONROC & $\begin{array}{cc}\text { GM07-8IID12217 } & 10 / 31 / 94 \\
054230-0001-7 & \text { IDAHO FALLS }\end{array}$ \\
\hline MONROE COUNTY MEDICAL CENTER & $\begin{array}{c}\text { FG47-93R707036 } 08 / 31 / 94 \\
232070-0001-0 \quad \text { ALBIA }\end{array}$ \\
\hline $\begin{array}{l}\text { MOHROE COUNTY OF } \\
\text { EDUCATION BOARD OF }\end{array}$ & $\begin{array}{c}\text { FG44-93R435336 } 09 / 30 / 95 \\
054290-0103-1 \text { MADI SONVILLE }\end{array}$ \\
\hline MOHROE SYSTEMS FOR BUS IHC & $\begin{array}{c}A D 01-93 A D 23653 \quad 09 / 30 / 94 \\
223100-0002-7 \quad \text { ARLINGTON }\end{array}$ \\
\hline MONTANA COLL OF MINERAL SCI & $\begin{array}{r}\text { FG07-93IDI3271 } 09 / 29 / 94 \\
054400-0001-1 \quad \text { BUTTE }\end{array}$ \\
\hline MONTANA DOUBLE CAR WASH & $\begin{array}{c}\text { ABO1-94AD62494 09/30/94 } \\
235840-0001-7 \text { WASHINGTON }\end{array}$ \\
\hline MONTANA POWER CO & $\begin{array}{c}\text { AC22-91ID12999 } 09 / 30 / 94 \\
121420-0001-0 \text { BUTTE }\end{array}$ \\
\hline $\begin{array}{l}\text { MONTANA STATE OF } \\
\text { - } \quad \text { NATURAL RESOUR CONSERV DEPT }\end{array}$ & $\begin{array}{l}\text { FG48-94R803725 } 12 / 31 / 94 \\
054420-0202-X \text { HELENA }\end{array}$ \\
\hline NATURAL RESOUR CONSERV DEPT & $\begin{array}{l}\text { FG48-94R803724 } 12 / 31 / 94 \\
054420-0202-X \text { HELEHA }\end{array}$ \\
\hline NATURAL RESOUR CONSERV DEPT & $\begin{aligned} \text { FG48-94R803723 } 12 / 31 / 94 \\
054420-0202-X \text { HELENA }\end{aligned}$ \\
\hline \multirow[t]{2}{*}{$\because \quad$ NATURAL RESOUR CONSERV DEPT } & $\begin{array}{r}\text { FG48-92R803698 } 06 / 30 / 97 \\
054420-0202-X \text { HELENA }\end{array}$ \\
\hline & FG48-90R803679 \\
\hline
\end{tabular}

DESCRIPTION OF WORK

CONG CONTRACT

CONG CONTRACT OBLIGS

EM - RECYCLE OF CONTAMINATED SCRAP META

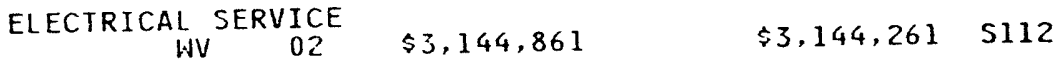

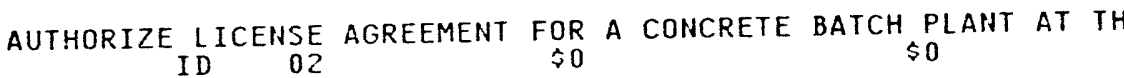

GRANT PROGRAMS FOR SCHOOLS ANDHOSPITALS AND FOR BUILDINGS IA $03 \quad \$ 4,242 \quad \$ 2,121$

SEE ATTACHED PAGE HO. 2 OF THIS NOTICE OF FINANCIAL ASSISTAN TH 02 \$ 18.893 \$ 13,225

MAINTEHAHCE SERVICES FOR MONROE COPIERS

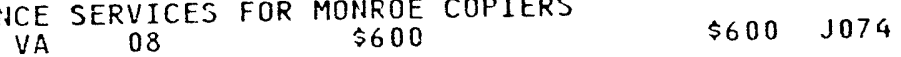

MULTI-PURPOSE SCIENCE LABORATORY

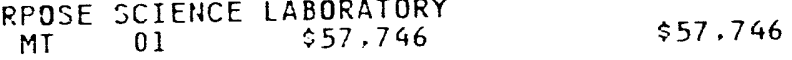

CAR WASHING

DC 01

$\$ 0$

$\$ 0 \quad 9999$

TO PROVIDE ELECTRICAL UTILITIES FOR MSE

MI 01 \$2, 900,000

$\$ 0 \quad \$ 112$

AWARD 1994 MARKETING PROGRAM FUNDS

$$
\begin{aligned}
& 94 \text { MARKETING PROGRAM FUHDS } \\
& M T
\end{aligned}
$$

AHARD 1994 PROGRAM ASSISTANCE FUNDS

$$
\begin{aligned}
& 94 \text { PROGRAM ASSISTANCE FUND } \\
& M T \text { \$ } 01
\end{aligned}
$$

AHARD 1994 TECHWICAL ASSISTANCE FUNDS

$\$ 156,098$

AHARD CYCLE XIV ICP COORDINATING AGENCY GRANT

$\begin{array}{ccc}\text { MT } & 01 & \begin{array}{c}\text { ICP } \\ \text { CONDINATING AGENCY GRANT }\end{array} \\ \$ 226,728 & \end{array}$

AWARD FY9O ALLOCATION FOR ICP PHASE II ADMINISTRATION 


AWARDEE NAME

\section{COMPL DATE}

BIN

VENDOR ID CITY

NATURAL RESOUR CONSERV DEP

MONTANA STATE UNIVERSITY

$054420-0202-X$ HELENA

GM07-91ID11077 05/22/97 $054430-0001-2$ POCATELLO

FG05-91ER79046 09/29/96 $054430-0001-2$ BOZEMAN

MONTANA TECH $164960-0001-8$ BUTTE

MONTANA UNIVERSITY OF FG02-91ER75681 09/29/95
$054440-0001-6 \quad$ MISSOULA

MONTEREY BAY AQUARIUM RESEARCH FG03-92ER61449008/31/95 $220800-0001-6$ PACIFIC GROVE

MONTESSORI CMTY SCHOOL INC

FG41-94R193607 06/14/95 $238840-0001-2$ SCITUATE

MONTGOMERY ELEVATOR CO

AC05-900R21927 06/30/95 8\$940-0001-0 KHOXVILLE

MONTGOMERY HOSPITAL

FG43-93R354209 12/31/94 054640-0001-1 $12 / 31 / 94$ HORPISTOWH

MOODY INDEPENDENT SCHOOL DIST

-MOORE PUBLIC SCHOOLS

MOOSE LAKE COMMUNITY HOSPITAL

MMORA TOHH OF

INDEPENDENT SCHOOLS

FG46-93R61 $3588008 / 31 / 94$
$054820-0001-x \quad$ MOORE

FG45-93R551707 12/31/94 233190-0001-2 MOOJE LAKE FG04-92AL82979 05/31/95
FG09-94SR18395 11/17/94

DESCRIPTION OF HORK

CONG COHTRACT

$\$ 1,701,388$

TO DATE P/S

MT

01

$\$ 88,201$

USE AGREEMENT WITH THE US FOREST SERVICE ID 02 \$O 50

NMR AND DIELECTRIC INSTRUMENTATION TO DETERMINE LIQUID CRYST MT 01 \$113.135 $\$ 103,240$

USED ENERGY RELATED
MT
01

LABORATORY EQUIPMENT GRANT

१०

PLANHING GRANT FOR THE STATE OF MONTANA.

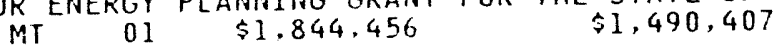

NEH - A NEW INSTRUMENT FOR MEASURING SINKING RAIES OF MARINE

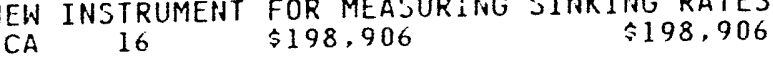

TITLE III OF THE NATIONAL ENERGY CONSERVATION POLICY ACT MA $10 \quad \$ 3.000 \quad \$ 1,500$

ELEVATOR MAINTENANCE SERVICES

TH $02 \quad \$ 99,909 \quad J 039$

TITLE III OF THE NATIONAL ENERGY CONSERVATION POLICY ACT PA $13 \quad \$ 212,500 \quad \$ 75.000$

AWARDS A NEH CYCLE 15 TECHHICAL ASSISTANCE GRANT.
$\$ 11,273$

AWARDS A NEW CYCLE 15 TECHHICAL ASSISTANCE GRANT.

ICP-HEW AHARD. $08 \quad \$ 30,288$

$\$ 15,144$
EXTENDED EDUCATION PROGRAM

IMM $03 \quad \$ 51,500$ 


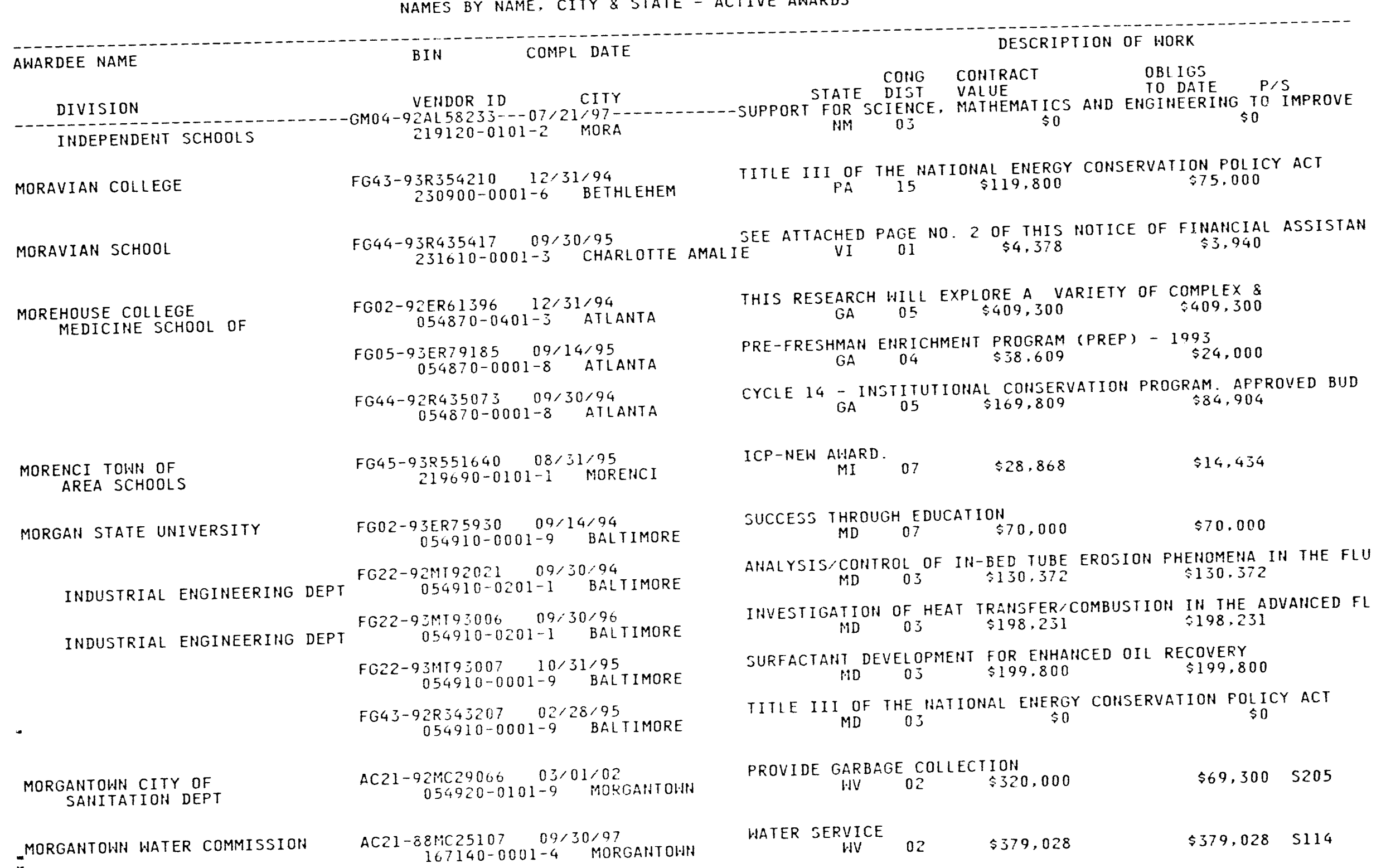




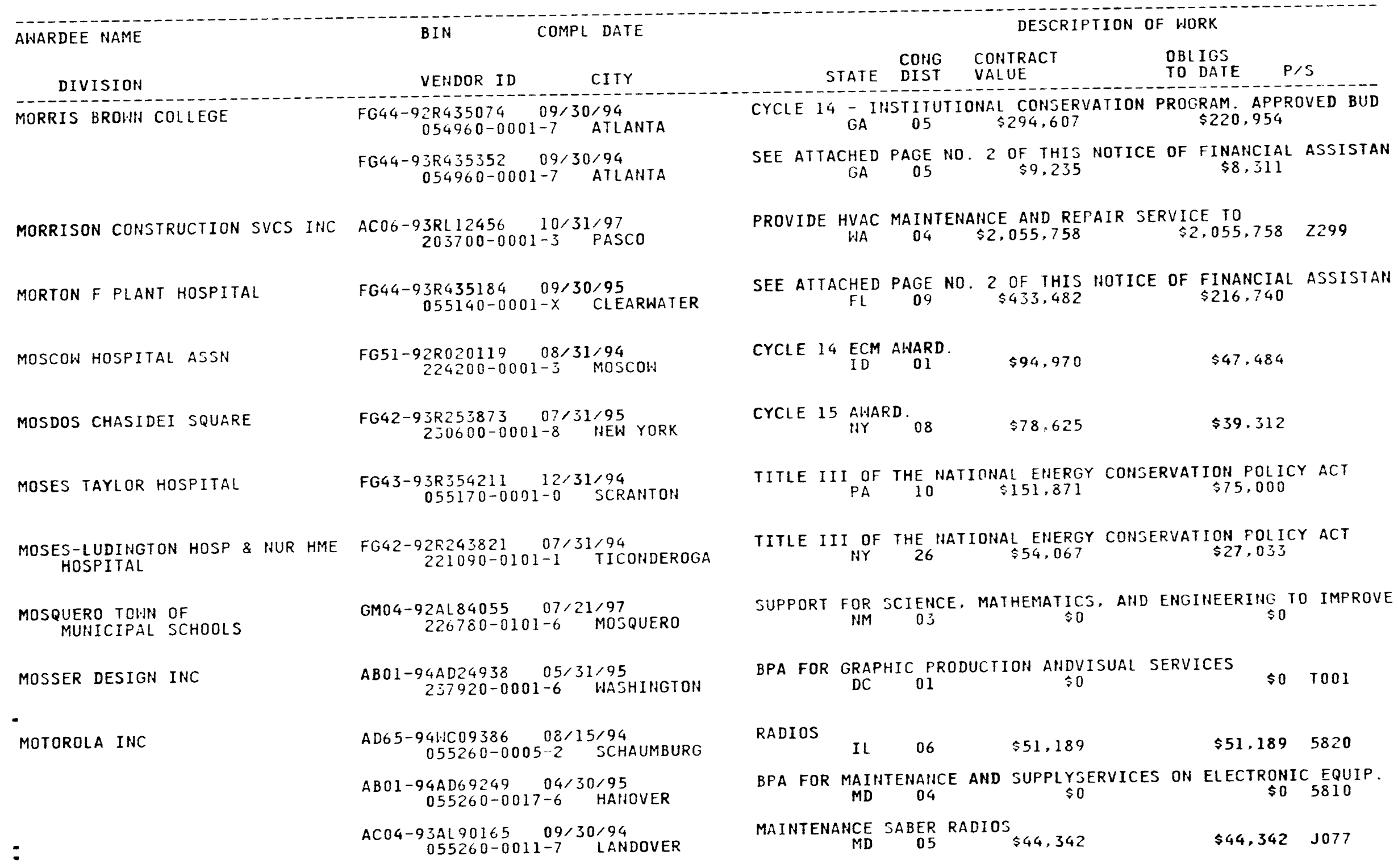


FROCUREMENT AND ASSISTANCE DATA NAMES BY NAME. CITY \& STATE - ACTIVE AWARDS

PAGE NO. 318

\begin{tabular}{|c|c|}
\hline AWARDEE NAME & COMPL DATE \\
\hline DIVISION & VENDOR ID \\
\hline $\begin{array}{l}\text { MOUNT HOLYOKE COLLEGE } \\
\text { PHYSICS DEPT }\end{array}$ & $\begin{array}{c}\text { FGO2-90ER4055j } 01 / 31 / 95 \\
055410-0201-X \text { SOUTH HADLEY }\end{array}$ \\
\hline MOUNT SINAI HOSPITAL & $\begin{array}{c}\text { FG43-92R344212 } 12 / 31 / 94 \\
055530-0003-3 \text { PHILADELPHIA }\end{array}$ \\
\hline \multirow[t]{3}{*}{ MOUNT SINAI SCHOOL OF MED } & $\begin{array}{c}\text { FG02-86ER13559 00/30/95 } \\
055560-0002-6 \text { NEW YORK }\end{array}$ \\
\hline & $\begin{array}{c}\text { FG02-88CH10351 } 03 / 31 / 95 \\
055560-0002-6 \text { NEW YORK }\end{array}$ \\
\hline & $\begin{array}{c}\text { FG02-88ER60675 } 04 / 22 / 95 \\
055560-0002-6 \text { NEW YORK }\end{array}$ \\
\hline MOUNT ST JOSEPH ACADEMY & $\begin{array}{c}\text { FG43-93R354212 } 12 / 31 / 94 \\
123590-0002-0 \text { FLOURTOHN }\end{array}$ \\
\hline \multirow[t]{3}{*}{ MOUNT ST JOSEPH HIGH SCHOOL } & $\begin{array}{c}\text { FG43-92R343208 } 08,31 / C 4 \\
222540-0001-2 \text { BALIIMORE }\end{array}$ \\
\hline & $\begin{array}{r}\text { FG43-93R353205 } 03 / 31 / 95 \\
222540-0001-2 \text { BALTIMORE }\end{array}$ \\
\hline & $\begin{array}{c}\text { FG43-93R553104 08/31/94 } \\
222540-0001-2 \text { BALTIMORE }\end{array}$ \\
\hline MOUNT WHEELER POWER & $\begin{array}{c}A C 08-78 D P 01567 \quad 09 / 30 / 94 \\
055640-0001-3 \quad E L Y\end{array}$ \\
\hline $\begin{array}{l}\text { MOUNTAIN BROOK CITY OF } \\
\text { - } \quad \text { EDUCATION BOARD OF }\end{array}$ & $\begin{array}{c}\text { FG } 44-93 R 455294 \\
230770-0101-2 \text { BIRMINGHAM }\end{array}$ \\
\hline MOUNTAIN SCHOOL PROGRAM & $\begin{array}{c}\text { FG41-92R196409 } 09 / 30 / 94 \\
211350-0001-8 \text { VERSHIRE }\end{array}$ \\
\hline MOUNTAIN STATES TACTICAL OFF & $\begin{array}{c}\text { GM07-94ID11149 } 12 / 31 / 99 \\
236900-0001-7 \text { GARDINER }\end{array}$ \\
\hline MOUNTAINAIR TOWN OF & GMO4-92AL84053 \\
\hline
\end{tabular}

DESCRIPTION OF WORK

CONG
STATE DINTRACT
VISTE

SUPPORT FOR SCIENCE, MATHEMATICS, AND ENGINEERING TO IMPROVE 


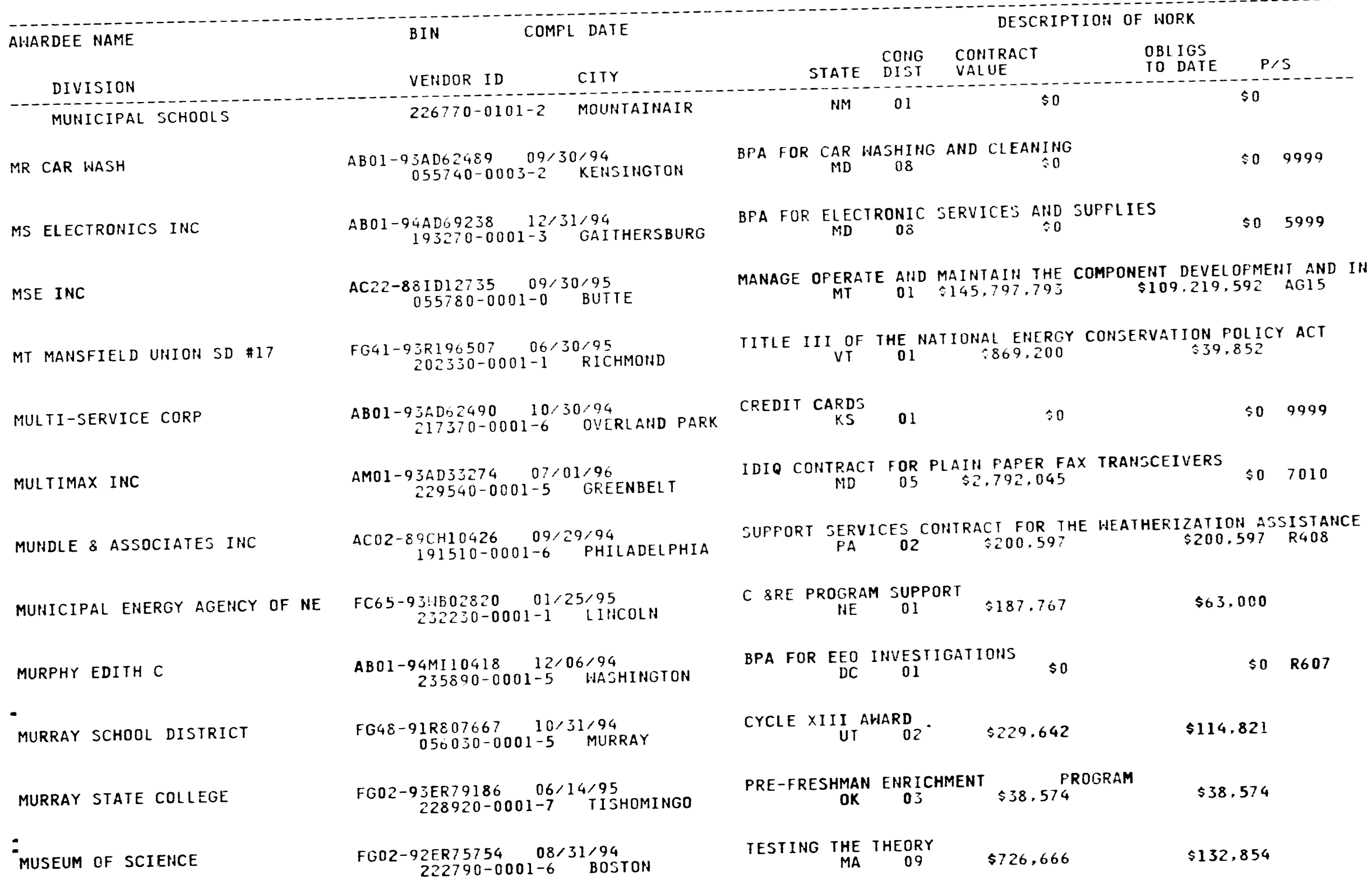




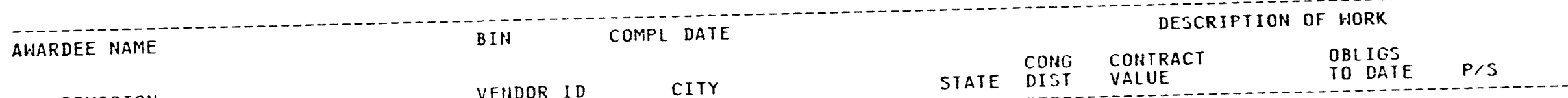

DIVISION VENDOR ID

STATE DIST TO DATE

(1)

FG02-94ER75958 12/14/94 222790-0001-6 BOSTON

MUSEUM OF SCIENCE \& INDUSTRY

FG02-94ER75952 $12 / 14 / 94$
$050080-0001-3$ CHICAGO

MYERS LINIAK \& BERENATO

MYERS ROSE \& LINIAK

MYRTLE WERTH MEDICAL CENTER

N T ENLOE HOSPITAL

HAMPA CITY OF

POLICE DEPT

NANOPTICS INC

NASA

AMES RESEARCH CENTER

: JPL-TE

ABO1-936C20324 02/31/95

FG45-9JR551820 07/31/95

FG49-92R914038 08/31/94 $222870-0001-1$ CHICO

GM07-92ID11097 09/30/99 $219950-0101-5$ HAMPA

A103-94ER61743 10/31/94

AI03-86SF16013 00/30/99

AI03-895F17905 09/15/94 $36 C 2032407 / 31 / 95$
$228970-0001-5$ BETHESDA

$A B 01-936 C 20313007 / 31 / 95$
$229050-0001-5$ BETHESDA SR 551820 07/31/95
$233420-0001-5$ MENOMONIE

FG05-93ER.81600 04/01/96
$138150-0002-x$ GAINESVILLE $\begin{array}{cccc}\text { HANDS-ON UNIVERSE EXHIBIT } & \text { KIOSK } \\ \text { MA } 09 & \$ 149.647\end{array}$

$\$ 149.647$

$\begin{array}{cccc}\text { PREPARE FILM USING COMPUTER VISUALIZATIONS ABOUT SOUND } \\ \text { IL } & 01 & \$ 5.000 & \$ 5.000\end{array}$

BPA FOR DOMESTIC PATENT APPLICATIONS

$\$ 0 \quad R 418$

NOVELTY SEARCHES

50

$\$ 0 \quad R 418$

ICP-NEH AWARD.

HI 03

$\$ 82.252$

$\$ 41,126$

TIILE III OF THE NATIONAL EHERGY CONSERVATION POLICY ACI CA $02 \quad \$ 408.830$

$\$ 204.415$

FIRING RANGE USE AGREEMENT WITH THE HAMPA POLICE DEPARTMENT ID 01

PHASE I SBIR AHARD - "HIGH RESOLUTION SCINTILLATOR BASED NEU $\begin{array}{cccc}\text { SBIR } & \text { AHARD - "HIGH RESOLUTION SCINTILLATOR } \\ \text { FL } & 06 & \$ 675,000 & \$ 1 / 5,000\end{array}$

HEH INIERAGEHCY AGREEMENT FOR A PROJECT ENTITLED: "MODELIHG NAVAL AI*CA $14 \quad \$ 668,000 \quad \$ 200,000$

EQUIPMENT S SERVICES FOR THE ANTIPROTON GRAVITY EXPERIMENT AI32-94AL96966 $03 / 31 / 95$
$056300-1312-2$ MOUNTAIN VIEW

AI01-92CE90056 08/26/97

$050300-0010-1$ PASADEHA

056300-2710-7 PAJADENA

AI05-920R2:030 04/30/95
AIC DIVISIOH "DIRECT COHVEFSIOH TECHHOLOGY"

AIC DIVISION "DIRECT COHVERSIOA TECHAOLOGY\$ \$105.000

ACT AS THE PROJECT OFFICE FOR THE SPIOO GES FROJECT.

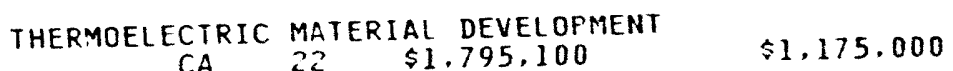

STUDY OF BIOCATALYTIC DESIGH BY CHEMICAL MODIFICATION

$$
\begin{array}{ccc}
\text { CA PROJECT OFFICE FOR THE SPIO0 } & \text { GES PROJECT } \\
\text { OI } \$ 73,398,000 & \$ 32.616 .671
\end{array}
$$




DIVISION
PROPULSION POHER \& ENERG

JET PROPULSION LABORATORY

ENERGY SYSTEMS DIV

ENERGY SYSTEMS DIV
COMPL DATE
DESCRIPTION OF HORK

CONG COHTRACT

STATE DIST VALUE

$C A \quad 22 \quad \$ 345,300$

OBLIGS

P/S

056300-0010-1 PASADENA

ATO4-93AL $94648 \quad 07 / 12 / 94$ $056300-2810-3$ PASADENA

AI05-940R22211 $04 / 30 / 95$ 056300-0410-7 PAJADENA

AI01-90CE50300 03/08/95 056300-0011-X WASHINGTON

AI01-91CE50306 12/31/96 056300-0111-6 WASHINGTON

AI01-04DP00376 09/01/94 056300-0011-X WASHINGTON

AI01-94DP00375 07/01/94 056300-0011-X WASHINGTON

AI02-94ER75989 10/31/97 056300-0011-X WASHINGTON

AI02-94ERI4424 02/28/99 $056300-0011-X$ HASHINGTON

AI02-93ER75887 10/31/97 $056300-0011-x$ WASHINGTON

AI02-93ER75888 10/31/97 $056300-0011-x$ HASHINGTON

AI04-80AL13137 01/01/99 056300-0111-6 HASHINGTON

AI05-79ET29372 12/31/95 056300-0011-X HASHINGTON

AIO1-94EH89485 06/01/96

AERONAUTICS \& SPACE TECHINOLOGY GODDARD SPACE FLIGHT CENTER

AI02-92FR61367 11/30/96 056300-0205-8 GREENBELT

AI 02-94ER61762 12/31/96 056300-0205-8 GREENBELT

: GODDARD SPACE FLIGHT CENTER GODDARD SPACE FLIGHT CENTER
$A I 32-88 A L 53197 \quad 09 / 30 / 94$ 056300-0205-8 GREENBELT
SPRAY COMBUTION MODEL ING

$$
\text { CA } 27 \quad \$ 100,000 \quad \$ 100,000
$$

WIDE AREA SURVEILLAIICE SYSTEM
CA
$\$ 32$

$\$ 35.700$ INTERAGENCY AGREEMENT TO SUPPORT ADVANCED TURBINE TECHNOLOGY
DC A

HEAVY DUTY TRANSPORT TECHNOLOGY PROGRAM $\$ 11.976 .195$

INTERAGENCY AGREEMENT TO PARTICIPAIE AS A MEMBER OF NATIONAL

INTERAGENCY AGREEMENT TO PARIICIPATE AS A MEMEFR OF NATIONAL

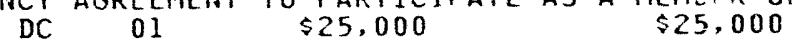

U.S. DOE FCCSET-SUMMEN $\$ 322,968$ TEACHING ENHAHCEMENT PROGRAM

CENTER FOR AEROSPACE RESEARCH AND EDUCATIOH FOR MINORITY

$$
\text { DC } 01012,445,000 \quad \$ 375,000
$$

DOE FUHDING TO SUPPORT NASA JPL SCIENCE SUMMER TEACHER DOE FUNDIHG TO SUPPORT HASA AERONAUTICS EDUCATION/SCIENCE
DC
01

SOLAR THERMAL DISTRIB SYS. PROG REF TASK ORDER

$$
\text { DC OI DISTB SYS. PROG REF TASK ORDER } \$ 0
$$

COMMUNICATIONS AND COHTROL FORFOWER DISTRIBUTION SYSTEMS

$$
\text { DC } 01 \text { \$9.068,000 } \$ 9,068,000
$$

DEVELOPMENT AND TESTING OF ADVANCED SAFETY AND HEALTH TECHNO $\begin{array}{lll}\text { DEVELOPMENT AND TESTIHG OF ADVANCED SAFETY AND HEALTH } & \$ 900,000 & \end{array}$

CIRRUS \& AEROSOL PROFILOMETER FOR RADIOMETRIC MEASUREMENTS

$M D \quad 05 \quad \$ 300,000 \quad \$ 185,000$

SCAHNING RAMAN LIDAR TO MEASURE HATE VAPOR \& AEROSOLS MD 05 \$ $\$ 24,000 \quad \$ 250,000$

COLLABORATORY IN SPACE PLASMA PHYSICS RESEARCH
MD
05
$\$ 4,900$
$\$ 4,900$ DC 01 \$11.000 $\$ 11.000$

$\begin{array}{llll}D C & 01 & \$ 322.968 & \$ 107.656\end{array}$ 


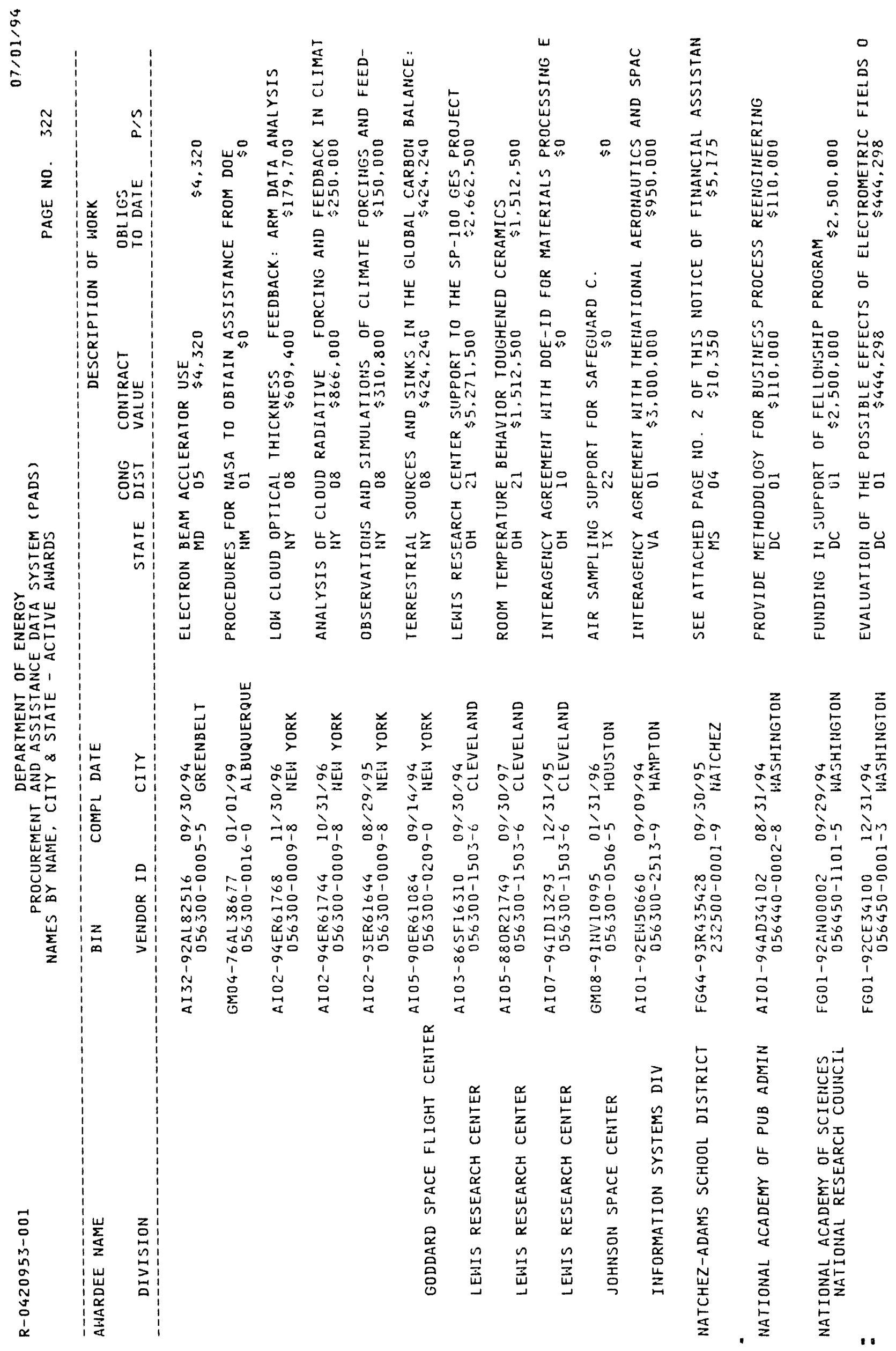


AI01-93CE50370 04/01/95 056450-0001-3 WASHINGTON

FG02-94ER14445 04/30/95 056450-0001-3 WASHINGTON FG02-93ER61547001/31/95 056450-0001-3 WASHINGTON FG02-921/E20609 09/30/94 $056450-0001-3$ HASHINGTON FG02-92ER14284 08/31/95 $056450-0001-3$ WASHINGTON

FG02-92ER61514 09/14/96 056450-0001-3 WASHINGTON FG02-93ER30209 11/30/94 $056450-0001-3$ HASHINGTON

FG02-94ER61782 01/14/95 $056450-0001-3$ WASHINGTON

FG02-94ER61793 02/28/95 $056450-0001-3$ HASHIHGTON FG02-94ER61757 11/30/94 $056450-0001-3$ HASHINGTON FG02-93ER25161 08/31/94 $056450-0001-3$ WASHINGTON FG02-93ER75903 06/30/98 $056450-0001-3$ WAJHINGTON FG02-93ER61702 12/14/94 $056450-0001-3$ WASHIHGTON

FG02-94ER61738 10/31/94 $056450-0001-3$ WASHINGTON

FG02-93ER40806 08/31/94 056450-0001-3 HASHINGTON
FG02-93ER40810 08/31/94
PROVIDE REPORT ON BIOBASED INDUSTRIAL PRODUCTS.

$$
\begin{array}{llll}
\text { REPORT ON } & \text { BIOBASED INDUSTRIAL PRODUCTS, } \\
\text { DC } & 01 & \$ 10,000 & \$ 10,000
\end{array}
$$

STUDY OF HIGH PERFORMANCE COMPUTING IN SEISMOLOGY

$$
\text { DC } 01 \quad \$ 19,400 \quad \$ 19,400
$$

SUPPORT OF THE WATER SCIENCE AND TECHNOLOGY BOARD

$$
\text { DC }{ }_{01} \$ 50,166 \quad \$ 50,166
$$

NATIONAL ACADEMY OF SCIENCE STUDY, "MANAGING \& DISPOSING

$$
\text { DC } 01 \quad \$ 390,000 \quad \$ 390,000
$$

DEPARTMENT OF ENERGY INTEGRATDMANUFACTURING FELLONSHIP

$$
\text { DC } 01 \text { \$1,544,000 } \$ 1,063,040
$$

SUPPORT OF THE U.S. NATIONAL COMMITTEE FOR THE COMMITTEE ON

$$
\text { DC THE OI. NATIONAL } \$ 106,550 \text { COMMITTEE FOR THE COMM }
$$

STRATEGIES FOR MIXIMIZING U.S. INTERESTS IN SCIENTIFICE AND

$$
\text { DC FOR MIXIMIZING U.S. INTERESTS IN SCIENTIFII } \$ 200,000
$$

ESTIMATING GENE FREQUENCIES IHDHA FOREHSIC TYPING

DC 01 \$10,000 $\$ 10,000$

POLAR RESEARCH BOARD CORE SUPPORT

$$
\text { DC } \$ 48,500 \quad \$ 48,500
$$

SUPPORT FOR ACTIVITIES OF THE OCEAN STUDIES BOARD DC ACTIVITIES OF THE OCEAN STUDIES $\$ 98,000$

TECHROLOGY AND TELECOMMUTING: ISSUES AND IMPACTS

$$
\begin{aligned}
& \text { DC AND TELECOMMUTING: ISSUES AND IMPACTS } \\
& 01 \quad \$ 150,000
\end{aligned}
$$

DC $01 \quad \$ 608,460 \quad \$ 608,460$

WORKSHOP ON BIOLOGICAL DIVER. IN MARINE SYSTEMS TO BE HELD

$$
\text { DC } 01 \text { \$25,000 } \$ 25,000
$$

SUPPORT FOR ACITVITIES OF THE NATIONAL RESEARCH COUHCIL

$$
\text { DC } 01 \quad \$ 40,000 \quad \$ 40,000
$$

A RESEARCH BRIEFIHG ON NEUTRINS ASTROPHYPICS 
AWARDEE NAME

BIN

COMPL DATE

VENDOR ID

CITY

CONG CONTRACT

DESCRIPTION OF WORK

DIVISION

FG02-93ER61703 08/31/95

056450-0001-3 NASHINGTON

FG02-93ER14371 08/31/96

056450-0001-3 WASHINGTON

FG05-92ER6138201/31/95

056450-0001-3 WASHINGTON

NATIONAL RESEARCH COUNCIL

FG05-89ER14032 06/14/95 056450-1101-5 WASHINGTON

FG05-89ER14061 08/31/94 056450-1101-5 HASHINGTON

NATIONAL RESEARCH COUNCIL

FG05-89ER25080 07/31/95 056450-0001-3 WASHINGTON

FG05-90ER60960 03/31/95 056450-0001-3 HASHINGTON

FG05-92EH89138 07/31/94 056450-0001-3 WASHINGTON

FG05-92ER4546903/31/95 056450-000I-3 HASHINGTON

FG08-92NV11227 07/27/97 056450-0001-3 WASHINGTON

ENERGY ENGINEERING BOARD

FG22-93PC93035 09/29/94 056450-0501-5 WASHINGTON

FG42-92R208008 09/30/94 056450-0004-8 WASHINGTON

FG02-94ER14451 05/31/97 056450-0001-3 WASHINGTON

FG21-89MC26237 08/13/95 056450-0003-X MORGANTOWN

NATIONAL ACTION CNCL MINOR ENG FG02-94ER75985 03/14/97 NATIONAL ACTION CNCL MINOR ENG FG02-94ER75985 03/14/97
$207950-0001-4$ NEW YORK NATIONAL ASSN OF MINORITY ENG
FG02-94ER75999 03/22/95
MOLECULAR MARINE BIOLOGY AND MARINE BIOTECHNOLOGY RESEARCH DC O1 \$100,000 $\$ 50,000$

DEPARTMENT OF ENERGY INTEGRATED MANUFACTURING

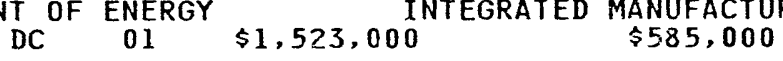
COMMITTEE ON BIOTECHIOLOGY
DC 01
$\$ 19,800$
$\$ 19,800$

BOARD ON CHEMICAL SCIENCES ANDTECHNOLOGY AND COMMITTEE ON N DC 01 \$1,115,000 $\$ 967,600$

ACTIVITIES IN GEOSCIENCES

DC 01 IN GEOSCIENCES $\$ 505,000 \quad \$ 505,000$

CORE SUPPORT FOR THE BOARD ON MATHEMATICAL SCIENCES ACTIVITIES OF THE NATIONAL ACADEMY OF SCIENCES IN RELATION T
DC $01 \$ 102,530,241$

INCEEMENTALLY FUND RADIOLOGICAL SAFETY IN THE MARSHALL ISLAN DC $01 \quad \$ 785,273$ RA $\$ 685,400$

NEW AWARD "AN ASSESSMENT OF NEUTRON SCATTERING SCIENCE"

STUDY ON FRACTURE CHARACTERIZATION AND FLUID FLOW.

DC 01 \$115,000 $\$ 115,000$

STRATEGIC ASSESSMENT OF THE U.S. DOE'S COAL PROGRAM
DC 01

DEPARTMENT OF ENERGY ORGANIZA TION ACT OF 1977 DC 01 \$ $\$ 150,000 \quad \begin{aligned} & 1977 \\ & \$ 150,000\end{aligned}$

COMMITTEE ON ATOMIC, MOLECULARAND OPTICAL SCIENCES DC $01, \quad \$ 129,000 \quad \$ 40,000$

$\begin{array}{ccc}\text { NRC/METC RESIDENT RESEARCH } \\ \text { WV } 02 & \$ 1,779,938 & \text { ASSOCIATESHIP PROGRAM } \\ \$ 557,257 & 02\end{array}$

PUBLIC SERVICES ADVERTISING CAMPAIGN $\begin{array}{lll}\text { NY } 14 & \$ 750,000 & \$ 242,500\end{array}$

NAMEPA 14TH ANNUAL CONFERENCE "PARTNERS IN THE PIPLINE: 


\begin{tabular}{|c|c|}
\hline AWARDEE NAME & COMPL DATE \\
\hline DIVISION & VENDOR ID \\
\hline & $237060-0001-6$ \\
\hline \multirow[t]{3}{*}{ NATIONAL ASSOC INC } & $\begin{array}{c}A B 01-94 P 01019803 / 31 / 95 \\
193760-0001-3 \text { WASHINGTON }\end{array}$ \\
\hline & $\begin{array}{c}\text { ABO1-94GC30728 } 03 / 30 / 95 \\
193760-0001-3 \text { HASHINGTON }\end{array}$ \\
\hline & $\begin{array}{c}\text { ABO1-94AD11099 } 03 / 31 / 95 \\
193760-0001-3 \text { WASHINGTO }\end{array}$ \\
\hline
\end{tabular}

NATIONAL ASSOC ST ENERGY DFF

NATIONAL BUREAU OF STANDARDS

MATERIALS SCIENCE CENTER

NATIONAL CANCER INSTITUTE

IIATIONAL COAL BOARD

NATIONAL COLOR INC

NATIONAL COMPUTER SYSTEMS
FG42-93R208011 $09 / 30 / 98$ 204230-0001-2 WASHINGTON

AI $21-86 M C 23120 \quad 09 / 30 / 94$ $004460-0001-9$ BOULDER

AI05-88ERI3823 11/30/95 $004460-0001-9$ BOUL DER

AI05-83ERI3108 07/31/95 004460-0002-7 WASHINGTON

AI05-850R21569 03/31/96 AI 05-86ER53237 10/14/94
$004460-0004-3 \quad$ GAITHERSBURG

AI05-92EH89188 09/30/94

GI22-76ET10372 03/31/95 6 ET10372 $03 / 31 / 95$
$056630-0003-8$ MIDDLE SEX

$A B 01-93 P P 00177 \quad 09 / 30 / 94$ $189270-0002-1$ HERINON

AD01-93AD64753 09/30/94 229170-0001-5 EAGAN 2EH89188 09/30/94
$222760-0001-5$ BETHESDA

DESCRIPTION OF WORK

CONG CONTRACT

IL 11 \$15,000

OBLIGS TO DATE P/S $\$ 15,000$

TEMPORARY SECRETARIES

SECRETARIES
DC 01

SECRETARIAL SERVICES

$D C$ OI $\$ 0$

TEMPORARY SECRETARIAL SERVICES
DC 01 $\begin{array}{rrr}\text { FY } 93 \text { AlHARD } & \text { FOR COLLECTING DATA ON STATE ALTERNATE FUELED FL } \\ \text { DC } & 01 & \$ 49,566\end{array}$

$\begin{array}{cccc}\text { GAS SEPARATION USING ION } & \text { EXCHANGE MEMBRANES FOR } \\ \text { CO } & 02 & \$ 1,384,358 & \$ 1,384,358\end{array}$

THERMOPHYSICAL FROPERTY MEASUREMENTS IN COMPLEX FLUID MIXTUR $\begin{array}{lll}\text { CO } 02 \$ 4,415,486 & \$ 3,772,486\end{array}$

PULSE RADIOLYTIC STUDIES OF INTER- AND INTRAMOLECULAR DC 01 \$2,245,270 $\$ 1,840,270$

CERAMIC CHARACTERIZATION AND STANDARDS FOR HEAT ENGINES $M D \$ 08 \quad \$ 5,499,000 \quad \$ 5,499,000$

TO DETERMINE ATOHIC DATA PERTINENT TO THE MAGNETIC FUSION EN MD $\quad 08 \quad \$ 5,111,500 \quad \$ 4,950,500$

NEW INTERAGENCY AGREEMENT US/SOVIET JOINT RESEARCH ON THE BI MD $07 \quad \$ 572,000$ $\$ 572,000$

PROVIDE COAL TECHNOLOGY SERVCS

$\$ 10,966,972$

BPA FOR VIDEO DUPLICATING SERVICES

$\$ 0 \quad 9999$

MAINTENANCE FOR GENIGRAPHIC COMPUTER EQUIPMENT

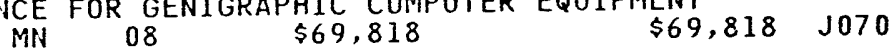




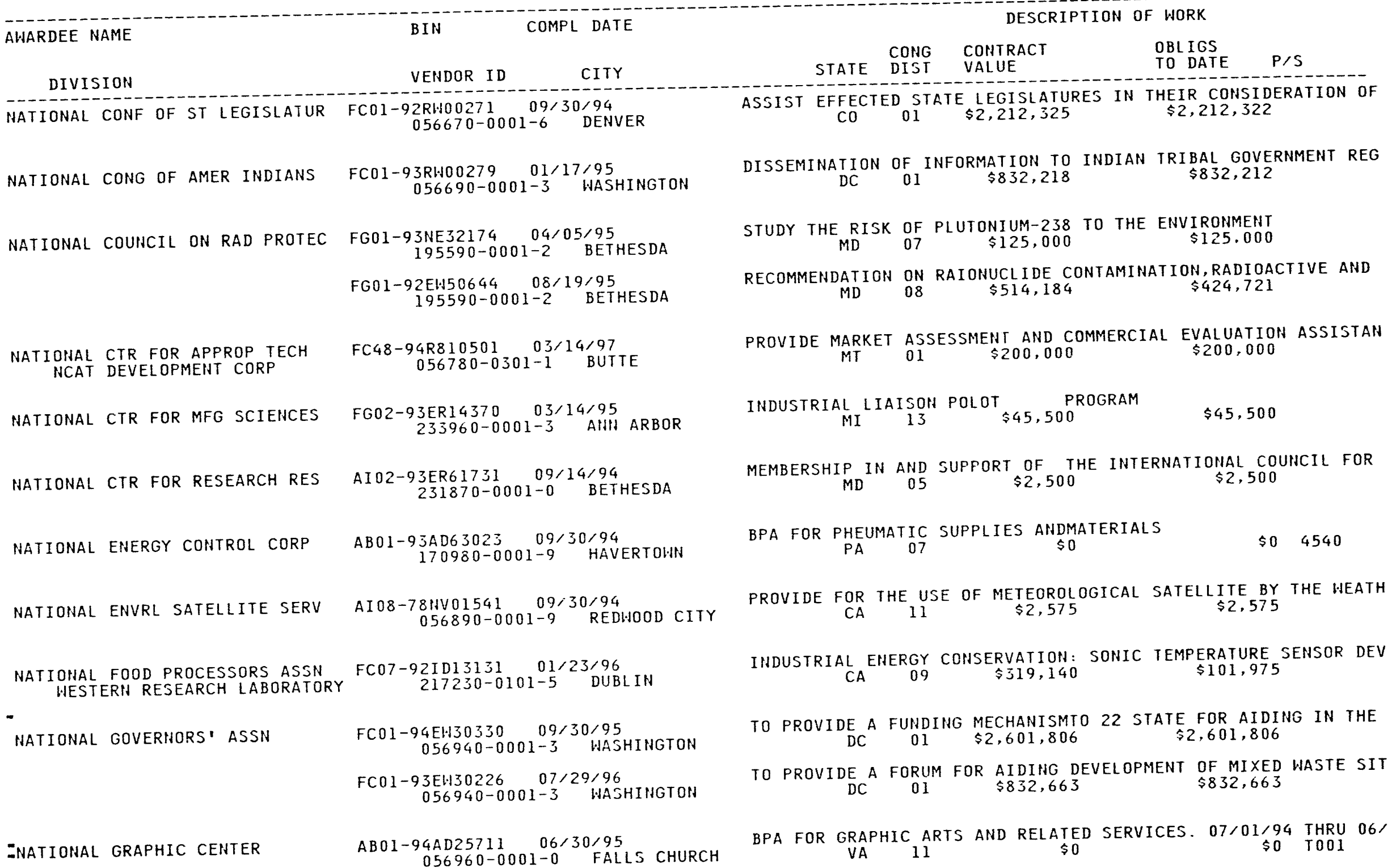


AWARDEE NAME COMPL DATE

DESCRIPTION OF WORK

BIN

CONG CONTRACT

OBLIGS
TO DATE

DIVISION VENDOR ID CITY STATE DIST VALUE $056960-0001-0$ FALLS CHURCH

NATIONAL INST FOR PROF DEV INC FG04-93AL94664 12/31/97 NATIONAL INST FOR PROF DEV INC FG04-93AL $94664012 / 31 / 97$
$205170-0001-6$ ALBUQUERQUE

PROJECT UPIIFT ISUAL SERVICES DESIGNAND FABRICATION 06/01/94-05/3 NM 01

NATIONAL INST OF STNDS \& TECH

CHEMISTRY

MATERIALS SCIENCE \& ENG LAB $180110-0001-2$ BOUL DER AI03-94ERI4420 12/31/96 $180110-0001-2$ BOUL DER

AI03-93ER54208 03/15/96 180110-0001-2 BOULDER

AI05-89ERI3992 01/31/95 $180110-0101-9$ BOUL DER

AI05-90ER54077 03/31/97 $180110-0801-3$ BOUL DER

AI05-930R22121 12/31/95 $180110-0001-2$ BOULDER

AI01-92CE23807 03/02/97 $180110-0002-0$ GAITHERSBURG

AI01-9ICE21042 03/05/96 $180110-0002-0$ GAITHERSBURG

AI01-91CE73060 04/17/96 $180110-0002-0$ GAITHERSBURG

AI01-91CEI5976 09/25/96 $180110-0002-0$ GAITHERSBURG

AI01-91CE23808 05/30/96 180110-0002-0 GAITHERSBURG

AI01-92EH89232 09/30/96 $180110-0002-0$ GAITHERSBURG

A101-92CE50354 09/30/97 $180110-0002-0$ GAITHERSBURG

AI01-94EH89436 09/30/95
$180110-0002-0$ GAITHERSBURG
NEW IA AGREEMENT "GELATION OF DENSE SILICA SUSPENS SUSPENSIONS: EFF

HIGH-TC SUPERCONDUCTOR - SEMICONDUCTOR INTEGRATION AND CONTA CO $02 \quad \$ 108,000 \quad \$ 108,000$

NEW INTERAGENCY AGREEMENT FOR PROJECT ENTITLED "ELECTROMECHA COSTEN AGREMENT FOR PROJECT ENTITLED "ELEC
$\$ 465,000$

INTEGRATED EXPERIMENTAL AND THEORETICAL STUDY OF THE THERMOP CO $02 \$ 1,662,010 \quad \$ 1,662,010$ CRYOGENIC STRUCTURAL MATERIALSFOR MFE MAGNET SYSTEMS

PRELIMINARY STUDY ON HEAT TRANSFER FOR MECHANISMS IN SUBCOOL

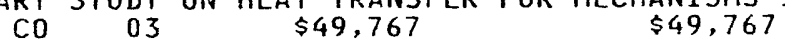

INTERAGENCY AGREEMENT W/NAT'L INSTITUTE DF STANDARDS \& TECHN MD 08 \$3,621,500 \$3,321,500

INDOOR AIR QUALITY INFILTIATION AND VEI " ILATION RESEARCH $M D \$ 88 \quad \$ 820,000 \quad \$ 820,000$ COMMUNICATIONS PROTOCAL FOR BUILDING CONTROLS

$$
\begin{array}{lll}
M D & 08 & \$ 321,000
\end{array}
$$

INVENTION EVALUATION BOTH FOR TECHNICAL MENT AND TECHNICAL I MD $08 \$ 10,000,000 \quad \$ 5,875,000$

CONDUCT RESEARCH OF CFC ALTERNATIVES SPECIHIALLY ZEOTROPIC R MD 03 \$1,786,000 $\$ 1,786,000$ PARTIAL SUPPORT OF U S/JAPAN PANEL ON WIND
MD
08 $\begin{gathered}\$ 60,000 \\ \$ 40,000\end{gathered}$

SUPPORT OF ADVANCE LUBRICATIDNAND SYSTEM RESEARCH

ESTABLISHED NEW IA FOR THE MQAPROGRAM UNDER NIST 
MD 08 \$GRENT $\$ 263,100$

$\$ 263,100$
$\$ 200,000$

ELECTRON PARAMAGHETIC RESOHANCE STUDY

COMBUSTION TECH. PROJECT FOCUSING ON THE INTERACTION OF FUEL $M D$ O6 $\$ 600,000 \quad \$ 100,000$

INTERAGENCY AGREEMENT AND SUPPORT OF RESEARCH PROJECTS FOR C INTERAGENCY AGREEMENT AND SUPPORT OF RESEARCH $\$ 350,000$ BPA FOR SUPPLIES \& REPAIR PARTS FOR TWO DOE-OWNED VEHICLES OPTICALLY DRIVENSURFACE REACTIONS: STATE-RESOLVED PROBES OPTICALLY DRIVENSURFACE REACTIONS: STATE-RESOLVED PR FAST MODULAR DATA AQUISITION SYSTEM $\$ \$ 200,000$ $\begin{array}{ccc}\text { DASTRUMENTATIOHAL FOR NUCLEAR APPLICATION } & \$ 98,000\end{array}$ ATOMIC COUNTING STANDARD AND ULTASENSITIVE RIMS $\begin{array}{ccc}\text { MD } 08 & \$ 312,000 \\ \end{array}$ NEUTRON INTERACTIONS WITH BIOLOGICAL TISSUE $\$ 80,000$ ION KINETICS \& THERMCHEMISTRY PERTIHENT TO MASS SPECTRO NEUTROH CROSS SECTION STANDARDS AND INSTRUMENTATION

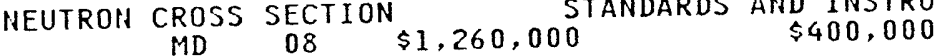
SPECTROSCOPIC INVESTIGATION OF THE VIBRATIONAL QUASI -

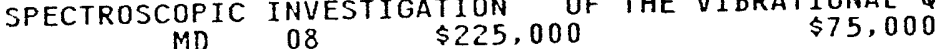
ABSOLUTE NEUTRON MEASUREMENTS

$\$ 505,000$

EUELITY OF RADON AND RADON ENERGY DEPOSITION \& RADIATION QUALITY OF RADON AND

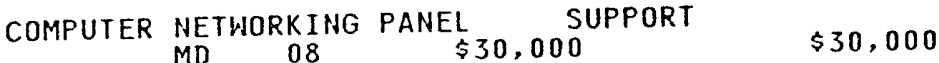




\begin{tabular}{|c|c|c|c|c|c|c|c|}
\hline AWARDEE NAME & BIN & $\angle$ DATE & & & DESC & F WORK & \\
\hline DIVISION & VENDOR ID & CITY & STATE & $\begin{array}{l}\text { CONG } \\
\text { DIST }\end{array}$ & $\begin{array}{l}\text { CONTRACT } \\
\text { VALUE }\end{array}$ & $\begin{array}{l}\text { OBLIGS } \\
\text { TO DATE }\end{array}$ & $P / S$ \\
\hline
\end{tabular}

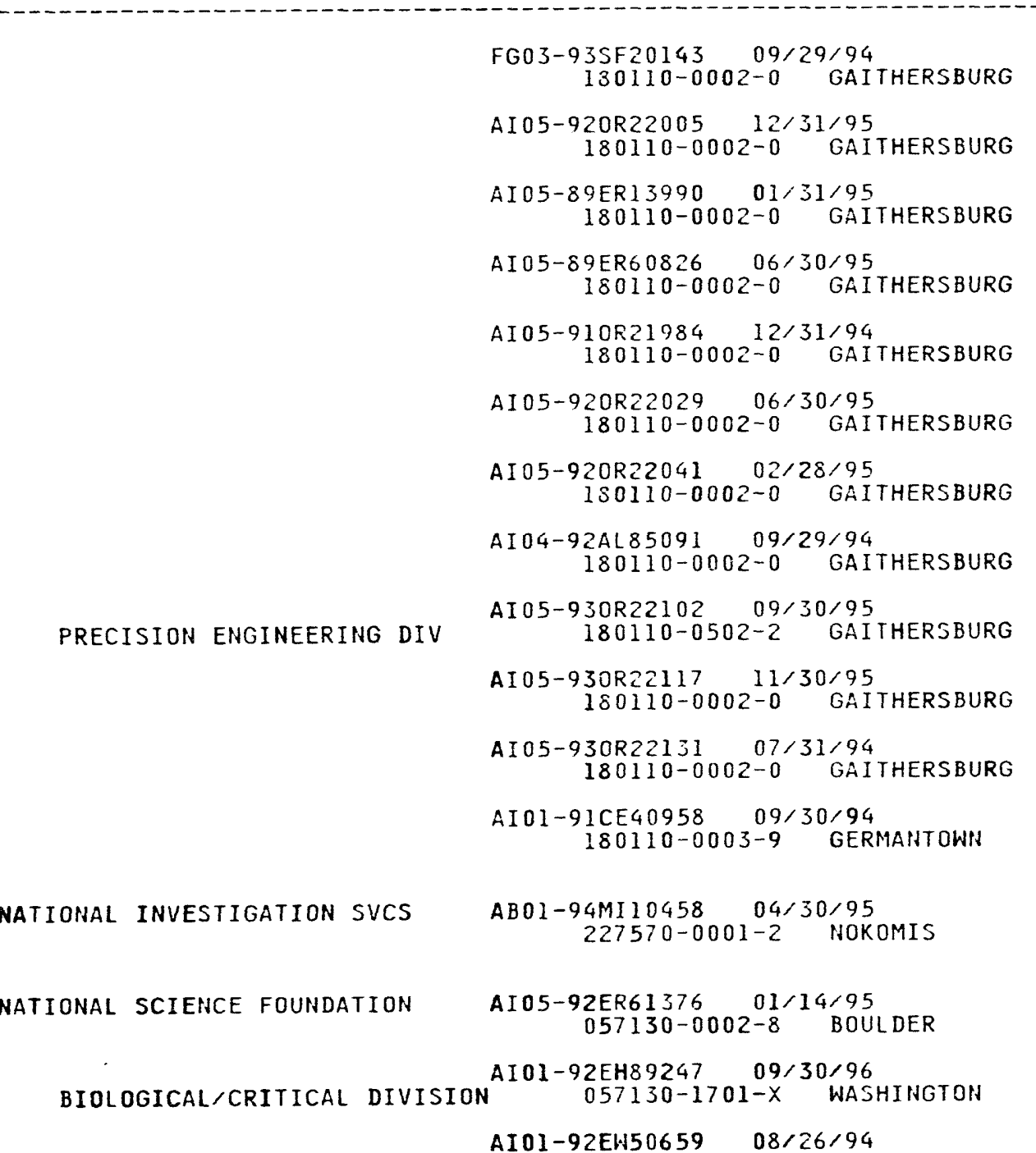

$\begin{array}{rrrr}\text { NLUF USER'S GRANT AWARD } & \text { - MEASUREMENT OF QUANTUM ELECTRODYNA } \\ \text { MD } & 08 & \$ 77,700 & \$ 77,700\end{array}$

NATIONAL VOLUNTARY LABORATORY ACCREDITATIOH PROGRAM

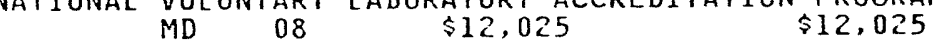

KINETICS DATA BASE FOR COMBUSTION MODELING
MD
08

IONIZING RADIATION-INDUCED DNADAMAGE AND ITS REPAIR IN HUMA $M D \quad 08 \quad \$ 458,618$ \$346,191

MOISTURE CONTROL GUIDELINES

THERMAL RESISTANCE MEASUREMENTS ON FOAM INSULATION AND POWDE

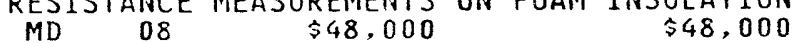

LOH-TEMPERATURE FABRICATIOH OFTRANSPARENT SILICON NITRIDE

$M D$ OS $\$ 332,000 \quad \$ 332,000$

TECHNOLOGY EVALUATION SERVICES
$M D O 8$
$\$ 175,000$
$\$ 175,000$

NVLAP ACCREDITATION OF $Y-12$ DIMENSIONAL STANDARDS LABORATORY $M D \quad 03 \quad \$ 850,000 \quad \$ 850,000$

DESIGN AND IMPLEMENTATION OF Y-12 CENTER FOR MANUFACTURING T MD $08 \quad \$ 80,000 \quad \$ 300,000$

PHASE DIAGRAMS FOR HIGH CRITICAL TEMPERATURE SUPERCONDUCTOR

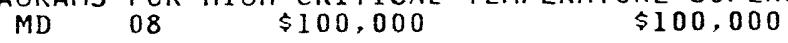

DETERMINE THE FATE OF METALS IN MUNICIPAL SOLID WASTE COMBUS MD 08 \$274,000 $\$ 274,000$

BPA FOR EEO INVESTIGATION 05/01/94-04/30/95
FL 13
$\$ 0$
\$0 R499

A HIERARCHICAL APPROACH TO IMPROVED CLOUD-RADIATION PARAMETE
CO
02 EXECUTIVE OF INTERAGENCY AGREEMENT AND FUNDING OF INTERAGENCY AGREEMENT AND FUNDING
$D C$
01 COLLABORATIVE RESEARCH FOR DEVELOPMENT AND DEMONSTRATION OF 


\section{DESCRIPTION OF WORK}

AWARDEE NAM

BIN
COMPL DATE

STATE DI

OHG

CONTRACT
VALUE $\$ 1,186,000$

OBLIGS TO DATE P/S $\$ 1,161,000$ $057130-0001-X$ HASHINGTOH AI 01-93CE50364 05/26/97 $057130-0001-X$ HASHINGTON AI02-92ER61516 09/29/95 $057130-0101-6$ HASHINGTON

ATMOSPHERIC SCIENCES DIV ATMOSPHERIC SCIENCES DIV OCEAN SCIENCES DIV

ATMOSPHERIC SCIENCES DIV ATMOSPHERIC SCIENCES DIV
AI02-93ER61589 09/26/95
$057130-0101-6$ WASHINGTON AI02-93ER61629 09/28/95 $057130-0301-9$ WASHINGTON AI02-93ER61631 08/31/96 $057130-0001-X$ WASHINGTON AI02-93ER61632 07/14/94 057130-0001-X WASHIHGTOH AI02-93ER61635 08/07/94 $057130-0101-6$ HASHINGTON

AI02-93ER61665 08/31/94 $057130-0101-6$ WASHINGTON

AI02-93ER61723 08/31/94 $057130-0001-X$ HASHINGTON

AI02-93ER61616 09/14/94 $057130-0001-X$ HASHINGTON

AI05-90ER61070 12/31/96 057130-0001-X WASHINGTON

AI05-91ER61210 09/14/94 $057130-0001-X$ HASHINGTON

AI05-91ER61149 09/15/94 $057130-0001-X$ WASHINGTON

AI05-01ER61224 08/31/94 $057130-0001-x$ HASHINGTON

AI05-92ER61389 02/28/95 $057130-0001-X$ WASHINGTOH

AI05-92ER61404 03/31/97 $057130-0001-X$ WASHIHGTON
DATION FOR RESEARCH TO DEVELOP DC 01 STIENE, $\$ 800$ $\$ 235,717$

PATIAL SUPPORT FOR THE CLIMATESYSTEMS MODELING PROGRAM DC OI TH50,000 \$300,000 RESEARCH ON GREEHOUSE-GAS- INDUCED CLIMATE CHANGE DC 01 \$300,000 $\$ 150,000$ SUPPORT FOR PLANNING THE GLOBAL OCEAH FLUX PROGRAM DC 01 \$30,000 $\$ 30,000$ ENHANCED RESEARCH PROGRAM ON THE LONG-RANGE CLIMATE DC 01 \$1,342,000 \$426,300 PARTIAL SUPPORT OF THE FY 93 COMMITTEE ON EARTH \& ENVIORNDC 01 \$207,000 $\$ 207,000$ 1993 GORDOH CONFERENCE ON ATMOSFHERIC CHEMISIRY AND A 1993 GORDOH CONFERENCE $\$ 15,250 \quad \$ 15.250$ PARTIAL SUPPORT FOR THE $\$ 52,600$ NATIOHAL ACADEMY OF SCIENCES HUMAN FRONTIER SCIENCE 000 PROGRAM $\$ 1,000,000$ SYMPOSIUM ON UNMAHNED AIRCRAFT IN GLOBAL CHANGE DC 01 \$34.883 $\$ 34.883$ DEVELOPMENT OF AH INTEGRATED DATA ASSIMILATION/SOUHDING SYST DEVELOPMENT OF AN TNTER 01 \$J,657,036 STUDY OF THE ATMOSPHERIC EFFECTS OF THE KUWAIT OIL FIRES DC 01 \$399,188 $\$ 334,001$ MEDICINE AT THE CROSSROADS $\begin{array}{lll}\text { AT THE CROSSROADS } & \\ \text { DC } 01 & \$ 600,000 & \$ 500,000\end{array}$ SCIENTIFIC DEVELOPMENT OF A MASSIVELY PARALLEL OCEAN CLIMATE DC $01 \quad \$ 297,490 \quad \$ 297,450$ BASIC ENERGY RESEARCH ON GLOBAL WARMING DEVELOPMENT AND TEST DC 01 \$887,550 $\$ 512,300$ SUPPORT FOR THE U.S. HOCE PROJECT OFFICE AT TEXAS A\&M UNIVER DC 01 \$125,000 $\$ 75,000$ 


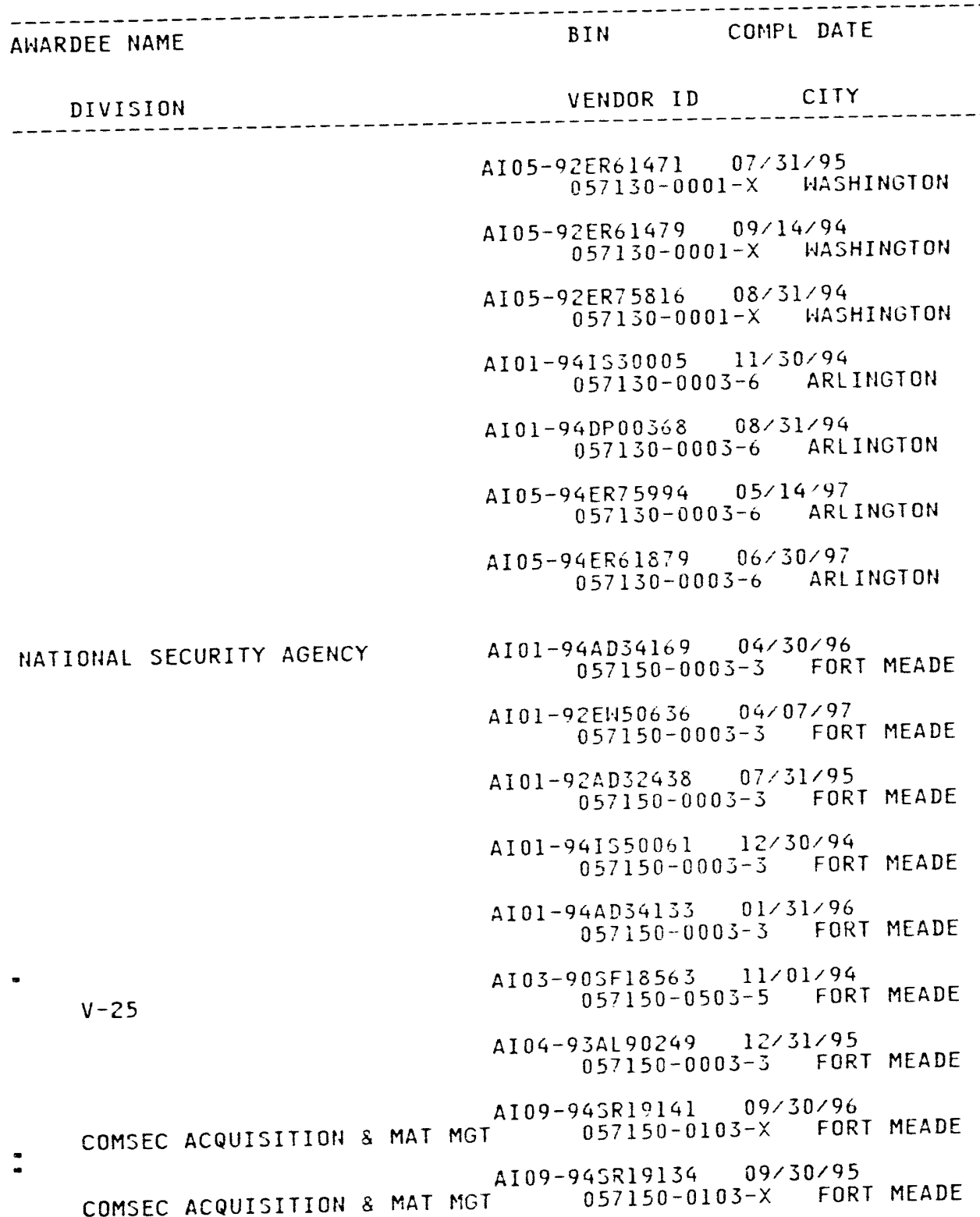

DESCRIPTION OF WORK

CONG CONTRACT
DIST VALUE

TO DATE P/S

IMPROVEMENT IN MOIST AHD RADIATIVE PROCESSES IN HIGHLY PARAL

IMPROVEMENT IN MOIST ATD RADIATIVE PROCESSES $\$ 504,976$ T33,142

CENTRAL EQUATORIAL PACIFIC EXPERIMENT (CEPEX)

$$
\begin{aligned}
& \text { QUATORIAL PACIFIC EXPERIMENT (CEPEX) } \\
& \text { DC } 01 \\
& \$ 1,200,000
\end{aligned}
$$

DOE FUNDING TO SUPPORT NSF SEIENTIST AHD ENGIHEER SURVEYS

DC $01 \quad \$ 450,000 \quad \$ 450,000$

AN INTERAGENCY AGREEMENT TO CONTINUE AN EXISTING STUDY ON SC $\begin{array}{rll}\text { AN INTERAGENCY AGREEMENT } & \text { T0 CONTINUE AN EXISTING } \\ \text { VA } & 08 & \$ 2,500\end{array}$

FUNDIHG

$$
\text { VA } 08 \quad \$ 13,000 \quad \$ 13,000
$$

MATHEMATICAL SCIENCES EDUCAIION BOARD (MJEB) CORE SUPPORT

ULTRAVIOLET RADIATION CLIMATOLOGY OF THE EARTH'S SURFACE AND VA $10 \quad \$ 287,303 \quad \$ 87,930$

KG-194 ENCRYPTION DEVICES

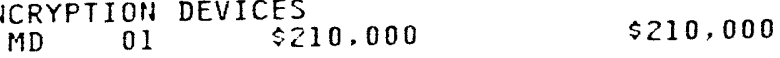

DATA FUSION HORKSTATION

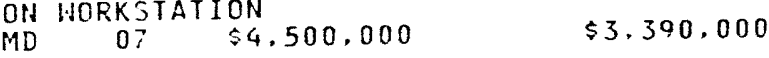

ENCRUPTION DEVICES $\$ 103,494 \quad \$ 103,494$

INTERAGENCY AGREEMENT FOR SECURE NETWORK SERVER SECURE MAIL MD 01 \$ $118.600 \quad \$ 118.600$

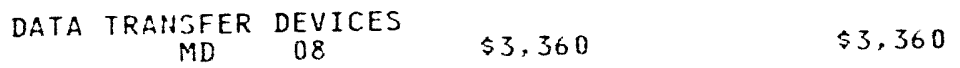

$\begin{array}{ccc}\text { TASK } 52 \text { IHCREMENTAL FUNDING } & & \\ & & \end{array}$

PURCHASE DATA TRANSFER DEVICE FR NSA

$\begin{array}{lll}\text { PURCHASE } & \text { CRYPTOGRAPHIC } & \text { EQUIPMENT } \\ \text { MD } 04 & \$ 11,200 & \$ 11.200\end{array}$

$\begin{array}{lll}\text { PURCHASE } & \text { CRYPTOGRAPHIC } & \text { EQUIPMENT } \\ \text { MD } 04 & \$ 11,200 & \$ 11,200\end{array}$ 


\section{DESCRIPTION OF WORK}

AWARDEE NAME

BIN

COMPL DATE

CONG CONTRACT

VENDOR ID

CITY

STATE

DIST

VALUE

OBLIGS
TO DATE P/S

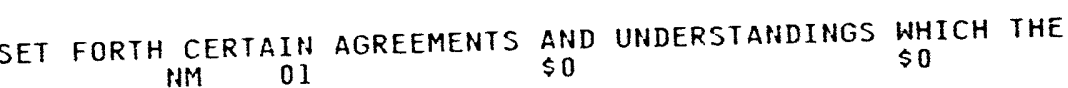

GM04-76AL02150 11/01/99
ALEUQUERQUE

ON-SITE AUTOMATICE DATA PROCESSING SUPPORT SERVICES

NATIONAL SYSTEMS \& RESEARCH CO AC75-89S1.24000 $07 / 31 / 94$

$$
\begin{array}{ccccc} 
& \\
\text { OUTOMATICE } & \text { DATA PROCESSING SUPPORT } & \text { SERVICES } & \\
\text { OK } 02 & \$ 3,094.693 & \$ 3,094.693 & \text { D301 }
\end{array}
$$$$
\text { 057190-0005-4 TULSA }
$$

NATIONAL TECHNICAL INFO SVCS

$A I 01-94 E 124143 \quad 12 / 31 / 94$
$213500-0001-9$ SPRINGFIELD

NATIONAL TRANSPORTATION SAF BD GM32-88AL53629 12/02/99 $179570-0001-0 \quad$ LOS ALAMOS

NATL ARCHIVES \& RECORDS ADMIN AI01-93FE62864 05/19/95 $228510-0001-2$ WASHINGTON

NATL CTR FOR HUMAN GENOME RES AI02-92ER61258 $11 / 14 / 95$
$237800-0001-6$
BETHESDA

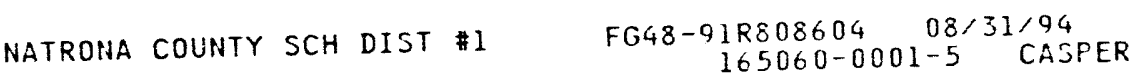

NATURAL SCIENCES ACADEMY OF

FG02-94ER30224 $03 / 31 / 95$
$000360-0002-7 \quad$ PHILADELPHIA

ACOS-94RL12930 $12 / 31 / 94$
$238690-0001-5$ RICHLAND

FG49-90SF18576 07/14/94
$115920-0002-9$ TSAILE

FG03-78C360001 $12 / 31 / 99$
$057380-0001-3$ FORT DEFIAHCE

FCO4-83AL16258 $09 / 30 / 94$

$057380-0002-1$ HIHDOW ROCK

: RENEWAL OF SUBSCRIPTIONS TO DIRECTORY OF COMPUTER SOFTWARE INVESTIGATION OF FUBLIC AIRCRAFT ACCIDENTS INVOLVIHG DOE

MICROFILMING OF CASE FILES

DC 01 O $\$ 42,277$

$\$ 42,277$

CHROMOSOMAL LOCALIZATIOH OF BRAIN CDHA'S $\$ 452,463$

CYCLE XIII AHARD

$\$ 88,078$

$\$ 54,338$

USE OF SEDIMENT DIATOM $\begin{gathered}\text { ASSELBLAGES TO IMFER MAJOR } \\ \$ 23.597\end{gathered}$

PA 02

$\$ 23.597$

STOKER GRADE COAL FOR 200 AREAAND 300 AREA

$\begin{array}{ccccc}\text { TOKER GRADE COAL FOR } 200 & \text { AREAAND } & 300 & \text { AREA } & \\ \text { H.HA } & 04 & \$ 2.995 .392 & \$ 2.995 .392 & 9999\end{array}$

GRANT FROGRAMS FOR SCHOOLS ANDHOSPITALS AND BUILDINGS OWHED

WEATHERIZATIOH PROGRAM IMSTAL LATION OF INSULLATION

COOPERATIVE AGREEMENT $\$ 962.212 \quad \$ 962,212$

WEATHERIZATION ASSISTANCE FOR LOW INCOME PERSONS

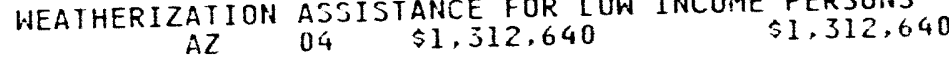
$A Z \quad 04 \quad \$ 62.833 \quad \$ 43.983$ NAVAJO HOUSING SERVICES DEPT FG49-895F18100 $057380-0102-8$ HIHDOH ROCK 


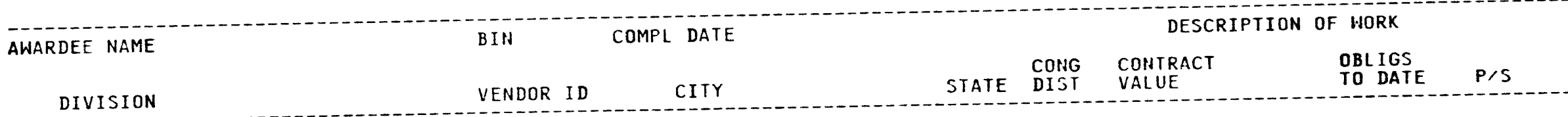

NAVAJO NATION HEALTH FDH SAGE HOSPITAL

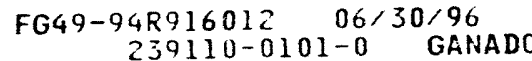

FC04-85AL26731 09/30/96 $134860-0001-2$ TUBA CITY

NAVAJO-HOPI TRIBE OF INDIANS

AC35-935C00017 06/01/97 225750-0001-7 WAXAHACHIE $\begin{array}{cccc}\text { CYCLE } 16 & \text { ICP } & \text { GRANT APPLICATION } \\ & \text { AZ } & 04 & \$ 51,529\end{array}$

NAVAJO-HOPI COOPERATIVE AGREEMENT $A Z \quad 03$ $\$ 593.40$ COLLIDER TEMPORARY CONSIRUCT

SUPERCONDUCTING SUPER $\$ 0$

MINICOMPUTER EQUIP IN ACCORD H/NAVAL AIR STATION KT W/PRC $A Z$
$A 2$ $\$ 178,512$ TIOT 7025

NAVY DEPARTMENT OF AD65-94WA12002 08/12/94 NAVAL COMPUTER \& TELECOM STA $057430-7659-6$ PHOENI

NAVAL AIR WARFARE CENTER $94 A L 9696703 / 31 / 95$
$057430-6406-7 \quad$ CHINA LAKE

AI01-93IN00622 09/30/94 057430-0015-8 MONTEREY

NAVAL POSTGRADUATE SCHOOL

AI05-91ER61223 08/31/94 $057430-1315-2$ MONTEREY

AI 01-92DP50092 04/13;95 $057430-6246-3$ POINT MUGU

WEAPONS

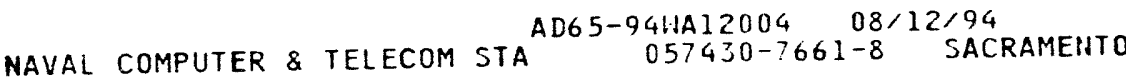
NAVAL OCEAN SYSTEMS CTR

AI01-91DP50084 05/31/96 $057430-1120-6$ SAN DIEGO

AI01-93DP20162 09/27/98 057430-0022-0 SEAL BEACH NAVAL COMPUTER \& TELECOM STA AD65-94WA12000 08/12/94 GEN NAVAL COMPUTER \& TELECOM STA AD65-94WA12044 09/22/94 057430-7655-3 GOLDEN AD65-94:14:2045,09/22/94 NAVAL COMPUTER \& TELECOM STA NAVAL COMPUTER \& TELECOM STA AD65-941.A12003 08/12/94
$057430-7660-X \quad$ LOVELAHD
LABOR. MATERIALS AND TRAVEL ASSOCIATED WITH INVESTIGATION OF CA 17 \$ $\$ 20,000 \quad \$ 20,000$

CONDUCT SYMPOSIA AND HORKSHOPS CA 07 \$250.000

$\$ 250,000$

SCIENTIFIC DEVELOPMENT OF A MASSIVELY PARALLEL OCEAN CLIMATE CA 16 \$ $\$ 396,500$ $\$ 396,500$

PROVIDIHG PROGRAM SUPPORT

MINICOMPUTER EQUIP IN ACCORD W NAVAL AIR STATION KT H/PRC $C A$ OJ $\$ 192,224$ $0192,224 \quad 7025$

RESEARCH IN THE AREA IN SENSORSYSTEMS CA 41 \$1,382,000

$\$ 635,000$

GIDEP WILL PROVIDE DATABASE SERVICES IN SUPPORT OF DEFENSE P $C A \quad 42 \quad \$ 1,030,000$ $\$ 234,000$

MINICOMPUTER EQUIP IN ACCORD W/NAVAL AIR STATION KT W/PRC $\begin{array}{cccc}\text { CO } & 06 & \$ 192,224 & \$ 192,224\end{array}$

ENHANCEMENTS TO MINICOMPUTER CO 06 \$16,005 $\$ 16,005 \quad 7025$ CO 06 S $\$ 9,501$ $\$ 9,501 \quad 7025$

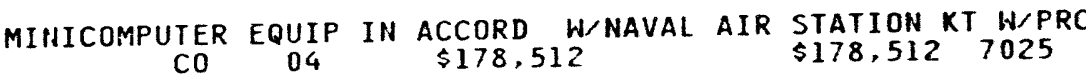

$$
\begin{array}{lll}
\text { PROGRAM SUPPORT } & \\
\text { CA } 05 & \$ 4.000,000 & \$ 3.696,000
\end{array}
$$
MINICOMPUTER EQUIP IN ACCORD W/NAVAL AIR STATION KT W/PRC 


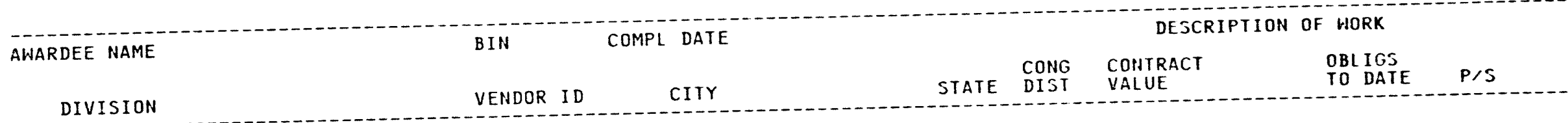

NAVAL COMPUTER \& TELECOM STA AD65-94WA12001 $08 / 12 / 94$

AI01-94CE50382 $03 / 13 / 99$
$057430-4125-3$ WASHINGTON

MARINE CORPS

NAVAL RESEARCH LAB

SPAWAR

NAVAL RESEARCH LAB

NAVAL RESEARCH LAB

NAVAL RESEARCH LAB

NAVAL RESEARCH LAB

NAVAL RESEARCH LAB

- naval REsearch lab

NAVAL MATERIAL COMMAND

NAVAL RESEARCH LAB

$=$
AI01-93EW53022 12/31/95

JEW53022 12,31/95 $057430-1425-6$ WASHINGTON

AI01-92EN40105 03/31/97

$2 E N 401050530-0025-5$
$057430-5 A S H I N G T O N$

AI01-93NE32170 09/30/97 $057430-0025-5$ HASHINGTON AI01-93CE50367 07/15/98 05743U-7825-4 HASHIHGION AI02-93ER54198 $10 / 31 / 95$
$057430-1425-6 \quad$ WASHINGTOH AI02-94ER54232 11/14/94 O57430-1425-6 WAJHINGTON

AI 02-93ER54214 05/14/95 057430-1425-6 HAJHINGTON A102-93ER40797 11/30/94 057430-0025-5 WAJHINGTON AI02-93ER40799 07/31/94 O57430-1425-6 HASHINGTON FG03-93SF20141 09/29/94 3SF 20141 09/29/94
$057430-1425-6$ HASHINGTON A103-94SF19892 09/30/95 057430-1425-6 HASHINGTON A107-79C550103 09/30/94 057430-1025-0 WASHINGTON AI07-92IDI3181 09/30/94 $057430-1425-6$ WASHIHGTON GM07-92ID11098 09/30/99 $057430-4147-4$ POCATELLO
MINICOMPUTER EQUIP IN ACCORD W/NAVAL AIR STATION KT H/PRC

CO 003 S $\$ 178,512$

INTERAGENCY TRAHSFER OF FUHDS FOR INCREMEHTAL COST OF ALTERH DC 01 \$1,563,263 $\$ 245,000$

INERAGEHCY AGREEMENT FOR HDA OF MIXED HASTE CONTAIHERS USIN DC 01 I $\$ 900,000$ $\$ 250.000$

DOE SHARE FUHDS FOR CERLA ACTION AND REMEDIAL INVESTIGATIOH DOE SHARE FUNDS FOR CER $\$ 750,000$ P PROVIDE AEROSPACE NUCLEAR SAFETY TECHNICAL SUPPORT FOR THE PROVIDE AEROI $\$ 1.750,000 \quad \$ 1.750,000$ U.S. NAVY (SPHARS) $\$ 1.000,000 \quad \$ 400,000$ THE INVESTIGATION OF ELECTRON -ION RADIOACIIVE \& DIELECT-YRO THE INVESTIGATION OF EL 0125.000 S 30,000 HEAVY IOH IGNITION $\begin{gathered}\text { ACCELERTOR THEORY AND } \\ \$ 12,000\end{gathered}$ RADIATIOH HARDENED FIBER OPTICS FOR FUSION REACTOR RADIATIOH HARDEHED FIBER $\$ 173,500 \quad \$ 173.500$ ADVANCED HIGH ENERGY LASER DRIVEN ACCELERAIOR SCHEMES AC 01 DVANCED $\$ 326,000 \quad \$ 326,000$ COMPUTER MODELIHG IN FOCUSED TRANSPORT OF INTENSE CHANGED COMPUTER MC 01 \$60,000 $\$ 60.000$ ILUF USER IS GRAHT AHARD - MORMAL - IHCIDICE MULTILAYER MIRROR NLUF USER'S GRANT AWARD - $\$ 139,978$ DC 01 \$139.978 HRL WILL CONDUCT A 225 MONTH PROGRAM TO CONTINUE TO DEVELOP NRL WILL CONDOI \$17.330,000 \$3,000,000 FEDERAL AGENCY E \& HV DEMONSTRATION PROJECT $\underset{D C}{01}{ }_{01}, 336,000 \quad \$ 1,516,000$ ATTEND MEETING OH URANIUM - ATOMIC VAPOR LASER ISOTOPE SEPAR $D C \quad 01 \quad \$ 36,963 \quad \$ 36,963$

FIRING RANGE USE AGREEMENT WITH UNITED STATES MARINE CORPS ID 02 \$O 
COMPL DATE

BIN VENDOR ID CITY
$92 R 110430--09 / 30 / 94--10$ SYSTEMS ACQUISITION REV CNCL PG1-92R110430--09/30/94 $057430-6548-9$ SALEM

AIO1-92EH50624 06/07/97

NAVAL SYSTEMS DEPARTMENT $057430-6343-5$ ANNAPOLIS COMPUTER \& INFORMATION SERVICE $057430-5433-9$ BETHESDA NAVAL RESEARCH LAB AI05-93ER61733 09/26/96 057430-1456-6 WAVELAND

NAVAL COMPUTER \& TELECOM STA AD65-94WA12005 $08 / 12 / 94$ BILINGS

GM04-78AL38703 09/30/99 057430-0035-2 AL BUQUERQUE GMO4-72AL 03131 09/30/99 057430-0035-2 ALBUQUERQUE GM04-83AL12413 09/30/99 $057430-0035-2$ AL BUQUERQUE GMO4-83AL23276 09/30/99 $057430-0035-2$ ALBUQUERQUE GM04-76AL38668 01/01/99 057430-0035-2 ALBUQUERQUE GM04-76AL38675 01/01/99 $057430-0035-2$ AL BUQUERQUE GM04-76AL38676 01/01/99 $057430-0035-2$ AL BUQUERQUE GM04-76AL $38680 \quad 01 / 01 / 99$ $057430-0035-2$ AL BUQUERQUE

GMO4-76AL38687 01/01/99 $057430-0035-2$ ALBUQUERQUE GM04-76AL38692 01/01/99 $057430-0035-2$ ALBUQUERQUE GMO4-82AL53606 12/24/99 $057430-0035-2$ AL BUQUERQUE
DESCRIPTION OF WORK

CONG CONTRACT

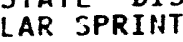

VALUE

OBL DATE P/S

06 $\$ 47,000$

DEVELOPMENT OF AIR CYCLONE SEPARATION TECHNOLOGY FOR REMOVAL MD 06 \$1.024,000 $\$ 194,000$

INTERAGEHCY AGREEMENT BETHEEN THE DEPT OF ENERGY AND US NAVY $M D \quad 08 \quad \$ 800,000$ $\$ 700.000$ NEW: "COUPLED ICE-OCEAN MODEL
MS FOR CLIMATE RESEARCH"
DI

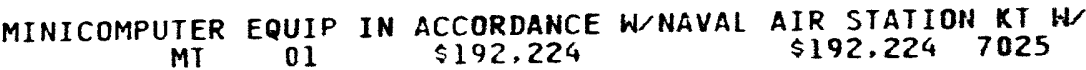
SET FORTH RESPONSIBILITIES FORFUNDING AND PRODUCTION OF THE NM 01

AEC SUPPORT TO THE NAVY IN THEIMPLEMENTATION OF THE NUCLEAR NM 01 $\$ 0$ ESTABLISHES THE DIVISION OF RESPONSIBILITY FOR DEVELOPMENT

DESIGN AND DEVELOPMENT OF AN $\underset{\$ 0}{\text { ARMING FUZIHG AND FIRING SYSTEM }}$ NM 01

AEC/NAVY HEW MATERIAL AND STOCKPILE LABORATORY TEST PROGRAM ESTABLISH PROCEDURES FOR DEFIHIHG \& CONTROLLING $\underset{\$ 0}{\text { THE }}$

QUALITY ASSURAICE SERVICE TESTPROGRAM \& NEW MATERIAL FIGHT

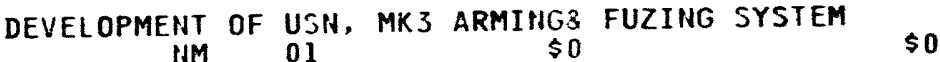

ESTABLISH JOINT HAVY/AEC NUCLEAR WEAPOHS STOCKPILE

PROVIDE GUIDELINES FOR UPGRADING MC2429 TO DEVELOP AN

DELINEATES RESPONSIBILITIES OFTHE USAF JCMP AND DOE IN THE HM 01$$
\$ 0
$$

SO

ESTABLISH BASIS FOR PRODUCTIONAND USE OF THE W88/MKS 


GM04-93AL8404301/05/98

MARINE CORPS RESERVE CENTER $3 A L 8404301 / 05 / 98$
$057430-7235-3 \quad$ AL BUQUERQUE GM04-93AL8404206/03/98 NAVAL SPECIAL WARFARE COMMAND $057430-7535-2$ ALBUQUERQUE SHIPS PARTS CONTROL CENTER AI11-94PII38008 09/30/94 $057430-3214-9$ MECHANICSBURG AI09-935R18277 03/09/95 NAVAL SHIP SYSTEMS ENGINEERING $057430-7318-x \quad$ PHILADELPHIA AI01-93PR10034 09/30/94 $057430-0002-6$ ARLINGTON AI01-93AD33615 09/30/94 057430-0002-6 ARLINGTON

NAVAL RESEARCH

AI05-94ER61836 04/30/96 $057430-2402-2$ ARLINGTON

FLEET SURVEILLANCE SUPPORT NC ALTERNATIVE ENERGY CORP

FG44-93R410607 09/30/94 $3 R 41060709 / 30 / 94$
$235300-0001-9$ DURHAM

NCR CORP

NEAL R GROSS \& CO INC

ACO1-93AD33605 09/30/94 213930-0001-0 ROCKVILLE

$A B 01-93 A D 24901 \quad 09 / 30 / 94$ $3 A D 24901$
$033920-0001-9$

-NEBRASKa INDIAN COMM COLLEge

FG47-92R703494 08/31/94 $197930-0001-5$ WIHHEBAGO

NEBRASKA PUBLIC POWER DISTRICT CR01-83HE44397 $03 / 15 / 30$ COLUMBUS CR01-83HEG4475 03/15/30 THIS AGREEMENT SETS FORTH THE GENERAL POLICY FOR THE CTA THIS AGREEMENT SETS FORTH THE $\underset{\$ 0}{\text { GENERAL POLICY FOR THE SCHEDUL }}$ RAILCAR REPAIRS AND PARTS.
PA 17
$\$ 30,200$
$\$ 30,200$

PROVIDE TECHHICAL DAAA AND EHGINEERING SERVICES TO SR PA OI PAA AND $\$ 5,842 \quad \$ 5,842$

CONTRACT FOR ADM. SERVICES $\underset{08}{\$ 43,000} \$ \$ 43,000$

PROVIDE DEPT OF ENERGY WITH AUTOMATED PERSONNEL ACTON (SF-52 PA $07 \quad \$ 70,000 \quad \$ 70,000$

NEH IA AWARD PARTICULATES IN THE WATER COLUMN IN OCEAN MARGI VA 10 \$ $\$ 106,398$ \$ $\$ 3,806$ MOU BETWEEN DOE $\&$ NAVY RELATIVE TO THE USE AND $\underset{\$ 0}{\substack{\$ 0 \\ \text { COHTROL }}}$ OF AM

INDUSTRIAL ELECTRIC MOTOR SYSTEMS RESOURCE CENTER

HARDWARE MAINTENANCE

MD 08 \$313,275

$\$ 313,275 \quad$ D301

BPA FOR COURT REPORTING, COPIES OF TRANSCRIPTS AND STENOGRAP DC $D 1$

$\$ \mathbf{0}$

$\$ 0 \quad$ R606

GRANT PROGRAMS FOR SCHOOLS ANDHOSPITALS AND FOR BUILDINGS 0 $\mathrm{NE} \quad 01$ \$13,001 $\$ 6,500$

DISPOSAL OF SPENT NUCLEAR FUELAND OR HIGH LEVEL RADIOACTIVE DISPOSAL OF SPENT NUCLEAR FUELAND/OR HIGH-LEVEL RADIOACTIVE $\mathrm{NE}$ S 


\begin{tabular}{|c|c|}
\hline AWARDEE NAME & COMPL DATE \\
\hline DIVISION & VENDOR ID \\
\hline $\begin{array}{l}\text { NEBRASKA STATE OF } \\
\text { ENERGY OFFICE }\end{array}$ & $\begin{array}{c}\text { FG47-92CE60440 06;30/95 } \\
057580-0301-8 \text { LINCOLN }\end{array}$ \\
\hline ENERGY OFFICE & $\begin{array}{c}\text { FG47-92CE60430 } 06 / 30 / 95 \\
057580-0301-8 \quad \text { LINCOLN }\end{array}$ \\
\hline ENERGY OFFICE & $\begin{array}{c}\text { FG47-92R701307 } 06 / 30 / 95 \\
057580-0301-8 \quad \text { LINCOLN }\end{array}$ \\
\hline ENERGY OFFICE & $\begin{array}{c}\text { FG47-92CE60410 06/30/95 } \\
057580-0301-8 \quad \text { LINCOLN }\end{array}$ \\
\hline $\begin{array}{l}\text { NEBRASKA UNIVERSITY OF } \\
\text { TECHNICAL AGRICULTURE C }\end{array}$ & $\begin{array}{c}\text { FG47-92R703489 } 08 / 31 / 94 \\
057590-1003-4 \quad \text { CURTIS }\end{array}$ \\
\hline PHYSICS DEPT & $\begin{array}{c}\text { FG02-86ER45262 } 06 / 18 / 95 \\
057590-0701-7 \quad \text { LINCOLN }\end{array}$ \\
\hline PHYSICS DEPT & $\begin{array}{c}\text { FG02-88ER60634 } 12 / 31 / 96 \\
057590-0701-7 \quad \text { LINCOLN }\end{array}$ \\
\hline \multirow[t]{9}{*}{ PHYSICS DEPT } & $\begin{array}{c}\text { FG02-88ER13955 07/31/94 } \\
057590-0701-7 \quad \text { LINCOLN }\end{array}$ \\
\hline & $\begin{array}{c}\text { FG02-90ER20002 } 05 / 31 / 96 \\
057590-0001-2 \quad \text { LIHCOLN }\end{array}$ \\
\hline & $\begin{array}{c}\text { FG02-91CH10475 09/08/95 } \\
057590-0001-2 \quad \text { LIHCOLN }\end{array}$ \\
\hline & $\begin{array}{c}\text { FG02-91ER20026 } 04 / 30 / 97 \\
057590-0001-2 \quad \text { LINCOLN }\end{array}$ \\
\hline & $\begin{array}{c}\text { FG02-91ER20053 } 03 / 24 / 95 \\
057590-0001-2 \quad \text { LIHCOLH }\end{array}$ \\
\hline & $\begin{array}{c}\text { FG02-91ER75675 09/29/94 } \\
057590-0001-2 \text { LIHCOLN }\end{array}$ \\
\hline & $\begin{array}{c}\text { FG02-9JER61701 } 09 / 01 / 96 \\
057590-0001-2 \quad \text { LIHCOLN }\end{array}$ \\
\hline & $\begin{array}{c}\text { FG05-92ER79133 } 07 / 31 / 94 \\
057590-0001-2 \text { LIHCOLN }\end{array}$ \\
\hline & $\begin{array}{c}\text { FG47-92R703508 } 08 / 31 / 94 \\
057590-0001-2 \quad \text { LINCOLN }\end{array}$ \\
\hline
\end{tabular}

DESCRIPTION OF HORK

STATE DIST VALUE

OBLIGS

TO DATE P/S

92 INSTIIUTIONAL CONSERUATIOH PROGRAM SEE ATTACHMENT A FOR

NE 01 \$168,000 $\$ 130,000$

92 ALLOCATION - HEATHERIZATIONASSISTANCE FOR LOH INCOME PER NE $01 \$ 10,942,884 \quad \$ 7,057,714$

AWARD FOR THE GOVENORS ETHAHOLCOALITION USING $\$ 30,000.00$ DO NE 01 \$ 169.535

$\$ 169,535$

92 STATE ENERGY CONSERVATION PROGRAM. SEE ATTACHMENT A FOR O NE 01 \$4,852,780 $\$ 396.660$

GRANT PROGRAMS FOR SCHOOLS ANDHOSPITALS AND FDR BUILDINGS 0

$$
\text { NE } 03 \quad \$ 19,316 \quad \$ 9,658
$$

MAGHETIC STUDIES OF IRON-RARE-EARTH-METALLOID ALLOYS

$$
\text { INE O1 } \$ 563,794 \quad \$ 563.792
$$

THEORY OF RELATIVE BIOLOGICAL EFFECTIVENESS

$$
\mathrm{NE} \quad 01 \quad \$ 506,950 \quad \$ 337,950
$$

DYNAMICS OF COLLISION PROCESS-ES

$\$ 392,100$ PHYSIOLOGY AND GENETICS OF METABOLIC FLUX RECULATION IN
NE 01

PROVIDE EQUIFMENT FOR THE PJEBRASKA CENTERS FOR SCIENCE \& IEC NE 01 \$5,700,000 $\$ 5,666,940$

INTERFERING RHAS ASSOCIATED WITH RHA PLANT VIRUSES $\begin{array}{ccc}\text { MECHANISTIC ENZMOLOGY OF CO DEHYDROGENASE FROM CLOSTRIDIUM } \\ \text { HE } 01 & \$ 440,758 & \$ 346.758\end{array}$

NEBRASKA EPSCOR PLANHIHG PROJECT WITH THE U.S. DOE.

THE DYNAMICS OF CARBOH EXCHANGIN VERTICALLY STRATIFIED CASNES OI $\$ 260,454 \quad \$ 79,579$

1992 INSTRUMENTATION - PURCHASE OF AN ELECTRON PARAMAGNETIC NE 01 \$326.550 $\$ 326.550$

GRANT FROGRAMS FOR SCHOOLS ANDHOSPITALS AND FOR BUILDINGS 0

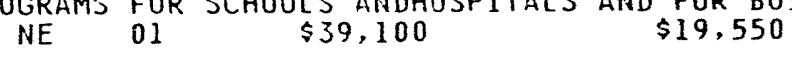




\begin{tabular}{|c|c|c|}
\hline AWARDEE NAME & COMPL DATE & DESCRIPTION OF WORK \\
\hline DIVISION & $\begin{array}{c}\text { VENDOR ID } \\
\text { FG47-9 CITY } \\
05755527-00-08 / 31 / 94-0001-2 \quad \text { LINCOLN }\end{array}$ & 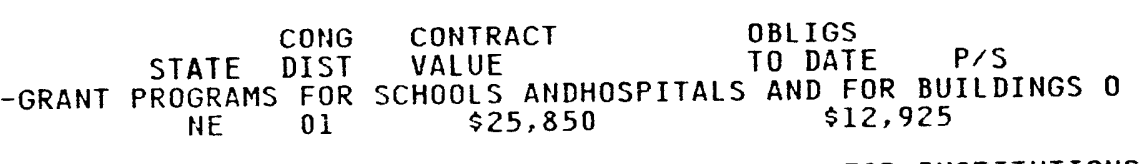 \\
\hline \multirow[t]{2}{*}{ CHEMICAL ENGINEERING DEPT } & $\begin{array}{l}\text { FC65-91WA08321 12/31/94 } \\
057590-1701-2 \text { LINCOLN }\end{array}$ & 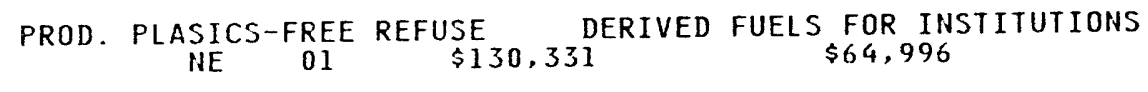 \\
\hline & $\begin{array}{l}\text { FG02-91ER61195 } 07 / 14 / 95 \\
057590-0002-0 \text { OMAHA }\end{array}$ & $\begin{array}{cccc}\text { MACRODIOSIMETRY \& MICRODOSIMETRY IN RADIOIMMUNOTHERAPY } \\
\mathrm{NE} & 02 & \$ 889,000 & \$ 721,000\end{array}$ \\
\hline \multirow[t]{3}{*}{ NEOS CORP } & $\begin{array}{c}\text { AC65-91WA07849 08/01/94 } \\
103100-0002-5 \text { LAFAYETTE }\end{array}$ & $\begin{array}{cccc}\text { CONSERVATION \& RENEWABLE } & \text { ENERGY DIRECT TECHNICAL ASSIST } \\
\text { CA } & 08 & \$ 3,585,483 & \$ 3,476,201 \quad \text { R } 421\end{array}$ \\
\hline & $\begin{array}{c}\text { AC65-901.A05637 11/13/94 } \\
103100-0004-1 \text { DENVER }\end{array}$ & 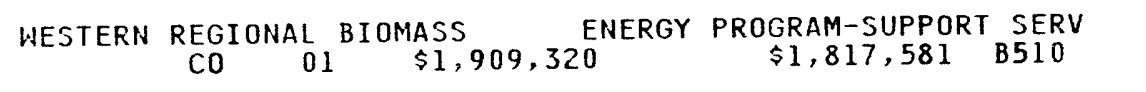 \\
\hline & $\begin{array}{c}\text { AC65-92WL } 03675 \quad 07 / 19 / 95 \\
103100-0003-3 \text { SALT LAKE CITY }\end{array}$ & $\begin{array}{c}\text { POWER MARKETING SUPPORT SVCS FOR SALT LAKE CITY AREA OFFICE } \\
\text { UT } 02\end{array}$ \\
\hline NESCAUM & $\begin{array}{l}\text { FG41-92R110449 } 09 / 30 / 95 \\
224580-0001-0 \quad \text { BOSTON }\end{array}$ & $\begin{array}{cccc}\text { IMPACT OF BATTERY POHERED VEHICLES ON IRP. } & \\
\text { MA } & 01 & \$ 50,000 & \end{array}$ \\
\hline NESS COUNTY HOSPITAL & $\begin{array}{c}\text { FG } 47-93 R 70271208 / 31 / 95 \\
232010-0001-9 \text { NESS CITY }\end{array}$ & $\begin{array}{cccc}\text { GRAIIT PROGRAMS } & \text { FOR } & \text { SCHOOLS ANDHOSPITALS AND FOR BUILDINGS } 0 \\
\mathrm{KJ} & 01 & \$ 51,961 & \$ 25,980\end{array}$ \\
\hline \multirow[t]{2}{*}{ NETWORK INC } & $\begin{aligned} \text { FG02-92ER75730 } 05 / 14 / 96 \\
218540-0001-2 \text { ANDOVER }\end{aligned}$ & $\begin{array}{rccc}\text { EVULATION \& } & \text { CAPACITY } & \text { BUILDING FOR DEPARTMENT OF ENERGY } & K-12 \\
\text { MA } & 05 & \$ 1,776,580 & \$ 1,055,241\end{array}$ \\
\hline & $\begin{array}{c}\text { FG02-93ER75920 07/31/94 } \\
218540-0001-2 \text { ANDOVER }\end{array}$ & 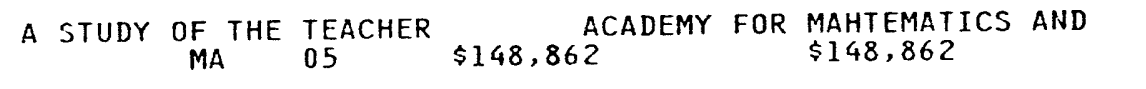 \\
\hline NETWORK SYSTEMS CORP & $\begin{array}{rl}\text { AD04-94AL } 90517 & 09 / 30 / 94 \\
160100-0001-7 \quad \text { ALBUQUERQUE }\end{array}$ & $\begin{array}{c}\text { GSA ORDER FOR SOFTWARE MAINTENANCE } \\
\text { NM } \\
01\end{array}$ \\
\hline "NEVADA OPERATIONS OFFICE & $\begin{array}{r}\text { GM04-90AL 58205 } 01 / 22 / 95 \\
181530-0001-2 \text { ALBUQUERQUE }\end{array}$ & 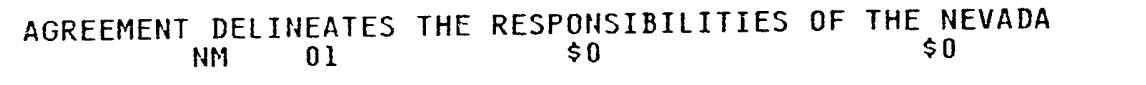 \\
\hline NEVADA POWER CO & $\begin{array}{l}\text { AC08-76NV00220 09/30/99 } \\
057760-0001-7 \text { LAS VEGAS }\end{array}$ & 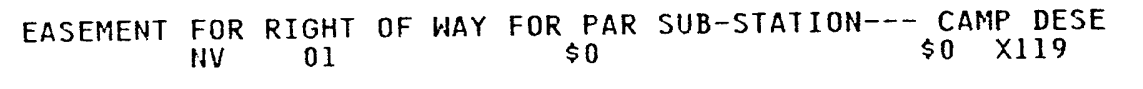 \\
\hline$=$ & $\begin{array}{c}\text { AC08-76NV00571 } 09 / 30 / 99 \\
057760-0001-7 \text { LAS VEGAS }\end{array}$ & 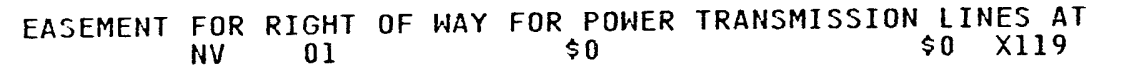 \\
\hline
\end{tabular}




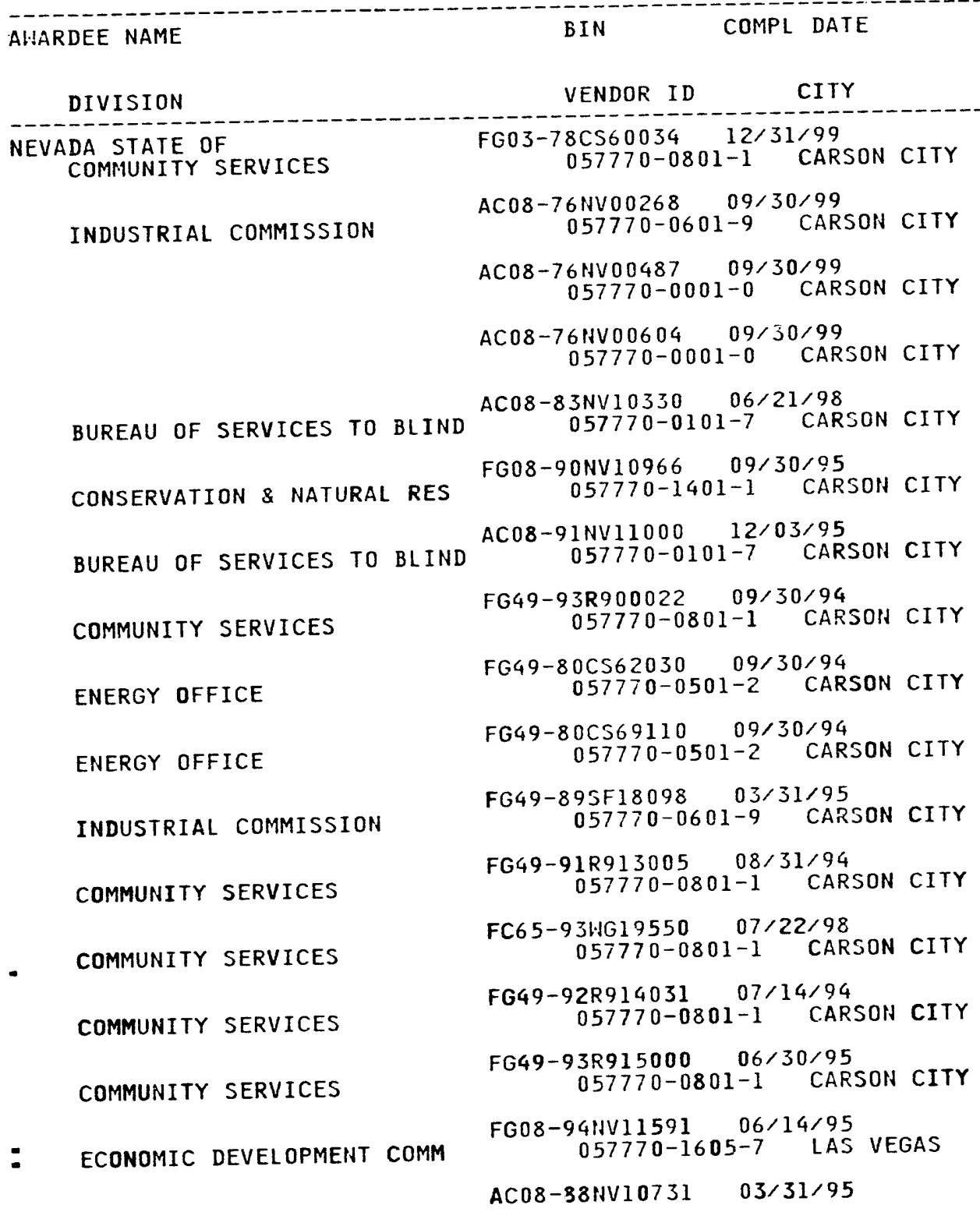

DESCRIPTION OF WORK

CONG CONTRACT OBLIGS HEATHERIZATION PROGRAM INSTAL LATION OF INSULATION

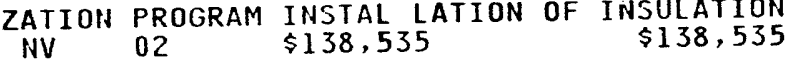
NORKMENS COMPENSATION COVERAGEFOR RADIATION HARM $\begin{gathered}\text { HO } \\ \text { NV } 01\end{gathered}$ PERMIT TO OPERATE CAFETERIA INTHE NVO HEADQUARTERS BUILDI MEMORANDUM OF UNDERSTANDING OFHAZARDOUS MATERIAL ACCIDENT NV 01 \$0 10025202

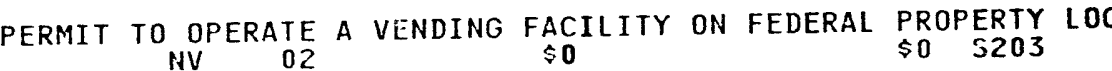
OVERSIGHT OF PUBLICH HEALTH AND ENVIRONMENTAL ISSUES REGARDI NV $02 \quad \$ 6,545,983 \quad \$ 3,790,483$

PERMIT FOR OPERATION OF THE C-I CAFETERIA IN NORTH LAS VEGAS NV 02 \$O \$O S203 HEAVY DUTY STATE/MUNICIPAL VEHICLE ALTERHATIVE FUEL DEMONSTR NEVADA STATE ENERGY CONSERVATION PROGRAM $\$ 1,781,366$ $\begin{array}{lll}\text { NEVADA ENERGY EXTENSION SERVICE } & \$ 1,312,577\end{array}$ WEATHERIZATION ASSISTANCE FOR LOW INCOME PERSONS WEATHERIZATION ASSISTANCE FOR $\$ 5,600,174$ OI $\$ 3,448,028$ ADMINISTRATIVE GRANT

NV $\underset{01}{\text { NIVENT }} \$ \$ 6,000 \quad \$ 30,000$

CONSERVATION \& RENEHABLE $\begin{array}{cc}\text { ENERGY FOR NEVADA } \\ \$ 641,666 & \$ 85,000\end{array}$ NV $02 \quad \$ 641,666 \quad \$ 85,000$ TITLE III OF THE NATIONAL ENERGY CONSERVATION POLICY ACT FY 193 ICP STATE ADMINISTRATIVE GRANT. (MATCH IS NO LONGER R NV STATE ADMINISTRATIVE GRANT. $\$$ M MATCH $\$ 50,000$ GRANT FOR COMMUHICATION SYSTEMTO LINK 12 PUBLIC/PRIVATE DEV NV $01 \quad \$ 200,000 \quad \$ 200,000$ PERMIT TO OPERATE A VENDING FACILITY CONSISTING OF A BOWLING 


AWARDEE NAME

AWARDEE NAME
VENDOR ID CITY

$057770-0104-1$ MERCURY

BUREAU OF SERVICES TO BLIND

NEVADA UNIVERSITY OF

$$
\begin{array}{r}
\text { FG02-91ER75667 09/29/94 } \\
057780-0002-2 \text { LAS VEGAS }
\end{array}
$$

MECHANICAL ENGINEERING DEPT

MECHANICAL ENGINEERING DEPT

FG06-94RL $12684 \quad 12 / 13 / 94$

FG06-94RL12752 11/26/94 $057780-1202-0$ LAS VEGAS

FC08-90NV10872 06/17/95 $057780-0002-2$ LAS VEGAS

FG08-92IVI11100 08/23/94 $057780-0002-2$ LAS VEGAS

FG08-93HV11375 10/08/94

ENVIRONMENTAL STUDIES PROGRAM FG08-93HV11375 057780-1602-6 LAS VEGAS

FG08-93IV11336 08/31/95 $057780-0002-2$ LAS VEGAS

GM08-92HV11414 04/13/97 $057780-0002-2$ LAS VEGAS

DESERT RESEARCH INSTITUTE

GM08-92IVII $416 \quad 07 / 26 / 97$

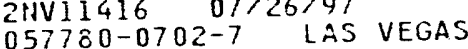

FG09-935RI8340 07/07/94 $057780-1202-0$ LAS VEGAS MECHANICAL ENGINEERING DEPT MECHANICAL ENGINEERING DEPT FG09-935P18347 08/02/94 $057780-1202-0$ LAS VEGAS

FG09-9 JSR18360 08/16/94 $057780-1202-0$ LAS VEGAS

RESEARCH ADMIN

FCO8-93NV $11399 \quad 08 / 31 / 98$ $057780-2002-3$ LAS VEGAS MECHANICAL ENGINEERING DEPT
FG21-94MC31231 03/25/95 VEGAS FG03-92ER45476 10/31/94
$057780-0001-4 \quad$ REHO

FG03-94ER61775 11/30/96

\section{DESCRIPTION OF WORK} TO DATE P/S $\$ 0$ \$0 M291

NV 0

NEVADA DOE/EPSCOR - PLANNING GRANT

$$
\text { NV } 01 \quad \$ 759,526
$$

$\$ 620,000$

USED ENERGY RELATED LABORATORYEQUIPMENT GRANT

$$
\text { HV } 01 \text { \$O }
$$

USED ENERGY RELATED LABORATORYEQUIPMENT GRANT

$$
\text { INV RELATED }
$$$$
\text { จ० }
$$

COOPERATIVE RESEARCH IN NUCLEAR WASIE DISPOSAL SITING.

$$
\text { IV } 01 \text { \$12,148,109 \$12,148,109 }
$$

SUPERCOMPUTING CALCULATIONAL SUPPORT.

$$
\text { NV } 01 \text { \$ } \$ 1,313,130
$$

$\$ 1,313,138$

STEP PROJECT FOR SOUTHERN NE VADA ENERGY/ENVIRONMENTAL RESI

$$
\text { IIV } 01 \text { SOUTHERN NE } \$ 30,195 \quad \$ 9,970
$$

EHVIROHMENTAL STUDIES COOPERATIVE EDUCATIOH CENTER.

$$
\text { IIV } 01 \quad \$ 219,642 \quad \$ 98.000
$$
MOU TO IMPLEMENT A COOPERATIVEEFFORT AT REACHING NATIONAL E

MOU TO IMPLEMENT A COOPERATIVEAGREEMENT AT REACHING NATIONA

$$
\text { NV MPLENT A COOPERATIVEAGREEMEHT AT REACHING }
$$

USED EHERGY RELATED LABORATORYEQUIPMENT

$$
\text { HV } 01
$$

$\$ 0$

USED ENERGY RELATED LABORATORYEQUIPMENT

$$
\text { HV OI }
$$

USED EHERGY RELATED LABORATORYEQUIPMENT

$$
\text { NV } 01
$$

$\$ 0$

COOPERATIVE AGREEMENT FOR RESEARCH AND EDUCATIONAL PROGRAM B $\begin{array}{ccc}\text { INE AGREEMENT } & \text { FOR RESEARCH AND EDUCATTONAL } \\ \text { IN } 01 & \$ 235,378 & \$ 235,378\end{array}$

USED ENERGY RELATED EQUIPMENT

HEH GRANT - PHOTOPHYSICAL PROCESSES OF TRIPLET STATES AND RA NV 02 \$276,250 $\$ 191,250$

HEH GRANT - "IMPROVEMENT OF THE MICRO PHYSICAL AND RADIATION 


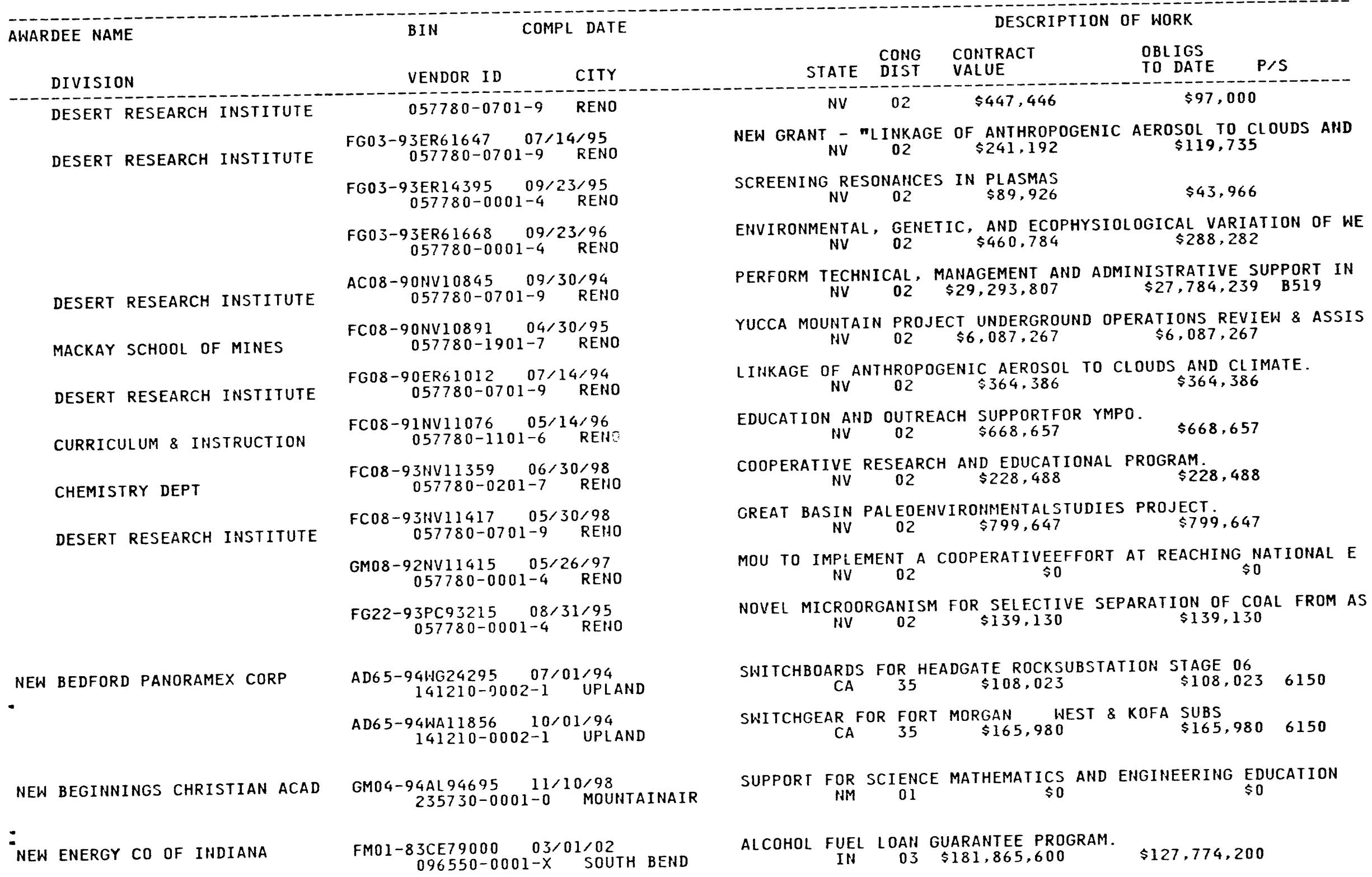




\section{AWARDEE NAME}

BIN COMPL DATE

DESCRIPTION OF WORK

DIVISION

VENDOR ID

CITY

CONG

TO DAT

VALUE

NEW ENGLAND GOVERNOR'S CONF

FG $41-91 R 110435 \quad 09 / 30 / 95$
$211330-0001-0$ BOSTON

$$
\begin{gathered}
\text { FCO1-91EI } 2278108 / 27 / 96 \\
058040-0001-2 \text { CONCORD }
\end{gathered}
$$$$
\begin{gathered}
\text { FG41-94R130434 } 03 / 31 / 99 \\
058040-1101-4 \quad \text { COHCORD }
\end{gathered}
$$$$
\begin{gathered}
\text { FG41-92R194418 08/31/94 } \\
058040-1101-4 \text { CONCORD }
\end{gathered}
$$$$
\begin{gathered}
\text { FG41-93R194550 08/31/95 } \\
058040-1101-4 \quad \text { COHCORD }
\end{gathered}
$$$$
\begin{gathered}
\text { FG41-93R194500 08/31/94 } \\
058040-1101-4 \text { CONCORD }
\end{gathered}
$$$$
\begin{gathered}
\text { FG41-93R130412 06/30/98 } \\
058040-0001-2 \text { CONCORD }
\end{gathered}
$$$$
\text { FG41-93R130442 06/30/98 }
$$$$
\text { 058040-0001-2 CONCORD }
$$

NEW HAMPSHIRE UNIVERSITY OF PHYSICS DEPT

$$
\begin{aligned}
& \text { FG02-88ER40410 12/31/96 } \\
& 058050-0401-1 \text { DURHAM } \\
& \text { FG02-90ER45433 08/31/94 } \\
& 058050-0001-6 \text { DURHAM } \\
& \text { FG02-90ER61083 09/14/94 } \\
& 058050-0001-6 \text { DURHAM } \\
& \text { FG02-93ER61685008/31/95 } \\
& 058050-0001-6 \text { DURHAM } \\
& \text { FG04-90CE40927 05/31/95 } \\
& 058050-0001-6 \text { DURHAM }
\end{aligned}
$$

NEW HANOVER COUNTY BD OF EDUCN FG44-90R434241 $12 / 30 / 94$ HINGTON

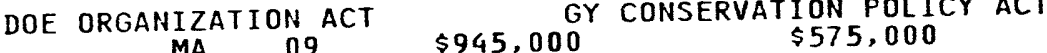

$$
\text { MA } 09 \quad \$ 945,000 \quad \$ 575,000
$$

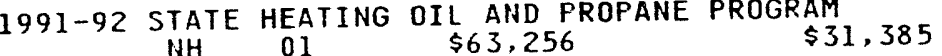

FY'94 AWARD.

$$
\text { NH } \quad 01 \quad \$ 2,128,612 \quad \$ 1,628,612
$$

AWARD NEW FUNDS FOR CYCLE 14 ECMS

$$
\begin{array}{lll}
\text { FUNDS FOR CYCLE } 14 \text { ECMS } & \$ 80,624 & \$ 241,604
\end{array}
$$

TITLE III OF THE NATIONAL ENERGY CONSERVATION POLICY ACT $\mathrm{NH} 01$ \$1,014,907 $\$ 196,693$

TITLE III OF THE NATIONAL ENERGY CONSERVATION POLICY ACT

FY'93 AWARD.
NH
01
$\$ 1,286,811$
$\$ 105,888$

FY 193 AWARD.

$01 \quad \$ 77,296$

$\$ 50,296$ NUCLER STRUCTURE INVESTIGA- TIONS ON SPHERICAL NUCLEI
NH

EFFECTS OF FRACTURE SURFACE $\underset{01}{\$ 304,056} \stackrel{\text { INTERFERENCE ON SHEAR CRACK }}{\$ 304,056}$

TERRESTRIAL SOURCE AND SINKS IN THE GLOBAL CARBON BALANCE

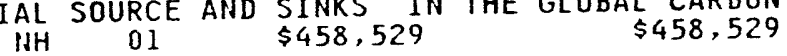

STRESS RESPONSES OF SUBSURFACE BACERIA: GENE

THERMAL SHING ABSORPTION $\$ 453,940$ SYSTEM $\quad \$ 293,647$

CYCLE 12 INSTITUTIONAL CONSERVATION PROGRAM. SPECIAL TERMS A CYCLE 12 INSTITOTIONAL $\$ 7668,562 \quad \$ 342,509$

SEE ATTACHED PAGE NO. 2 OF THIS NOTICE OF FINANCIAL ASSISTAN NC
07 


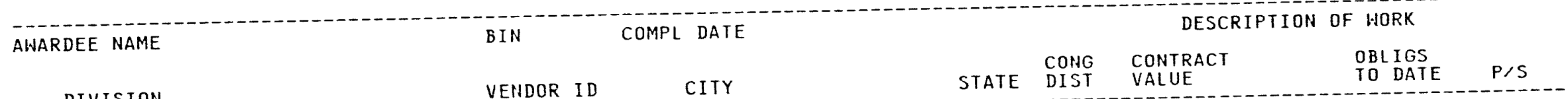

VENDOR ID

PRE-FRESHMAN ENRICHMENT $\underset{03}{\text { PROGRAM }}$

NEW HAVEN UNIVERSITY OF

NEW JERSEY INSTITUTE OF TECH MATHEMATICS DEPT

NEW JERSEY STATE OF PUBLIC UTILITIES BOARD OF

ENERGY DIV

ENERGY DIV

COMMUNITY AFFAIRS DEPT

ENERGY OFFICE OF

INEW MATERIAL CONCEPTS

NEW MEXICO COMM FOR THE BLIND

NEW MEXICO INST OF MIN \& TECH
FG02-93ER79189 06/15/95
$058090-0001-0$ WEST HAVEN

FG02-94ER25196 01/31/97

058110-0601-2 HEWARK

FG02-93ER79188 06/14/95

058110-0001-4 NEWARK

FCO1-91EI22782 08/07/96 058130-0201-4 NEWARK

FG42-80C56911200/30/95 058130-0001-1 NEWARK

FG42-80CS62032 06/30/95 -7 NEWARK FG42-80R200018 06/30/96

FG42-80R208002 $03 / 31 / 95$

FG42-92R208007 06/30/95 058130-1002-5 TRENTON

FG02-93ER816i5 $07 / 14 / 94$ 233680-0001-2 TEWKSBURY

GM32-89AL57298 12/22/99 180120-0001-6 LOS ALAMOS

ACO4-93AL94646 03/03/98 $180120-0002-4$ SAIITA FE

FG03-92ER61484 08/31/95 058200-0001-3 SOCORRO

FG03-92ER61488 08/31/95 058200-0001-3 SOCORRO
APPLIED MATH PROBEMS IN MICRO-HAVE PROCESSING OF CERAMIC

$\begin{array}{lll}\text { NJ } 10 & \$ 166,896 & \$ 52,686\end{array}$

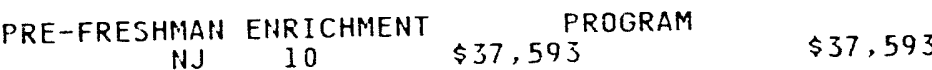

$$
\text { NJ } 10 \text { \$37,593 }
$$

COLLECTION AHD AHALYSIS OF DATA RELATED TO THE ENERGY INFORM

$$
\begin{array}{lll}
N \text { AND ANALYSIS OF DATA RELATED TO } & \$ 12,041
\end{array}
$$

ENERGY EXTENSIOH SERVICE PRO GRAM (NEW JERSEY

NJ 10 \$5, $077,667 \quad \$ 3,106,368$

STATE ENERGY CONSERVATION PROGRAM

$$
\text { NJ } 10 \text { ₹ } 16,056,874
$$$$
\$ 8,490,970
$$

INSTITUTIOHAL COHSERVATION PROGRAM - ADMINISTRATION - NEH

$$
\text { NJ } 10 \text { \$ } 10,473.090 \quad \$ 3,241,551
$$

NEATHERIZATION ASSISTANCE FOR LOW INCOME PERSONS - NEH JER

$$
\text { NJ } 04 \$ 67,790,949 \quad \$ 67,698,311
$$

HICE3 AHARD. $01 \quad \$ 1,006.940 \quad \$ 399,000$

DIRECT THERMAL TO OPTICAL ENERGY CONVERTER

$$
\begin{array}{ccc}
\text { ENERGY TO OPTICAL } & \text { ENINERTER } \\
\$ 74,918 & \$ 74,918
\end{array}
$$

OPERATE CERTAIN VENDING FACILITIES ON CERTAIN FEDERAL

$$
\text { NM } 03
$$

OPERATION OF A VENDING FACILIIY AT THE CENTRAL TRAINING NM OS

NEW GRAHT - "SMALL SCALE LABOFATORY STUDIES OF FLOW
NM
03

NEH GRANT - "A GEOLOGICAL AFPROACH TO CHARACTERIZING AQUIFER

$$
\begin{array}{rrr}
- & \text { NM GEOLOGICAL AFPROACH TO CHARACTERIZING } \\
03 & \$ 421,195 & \$ 132,500
\end{array}
$$




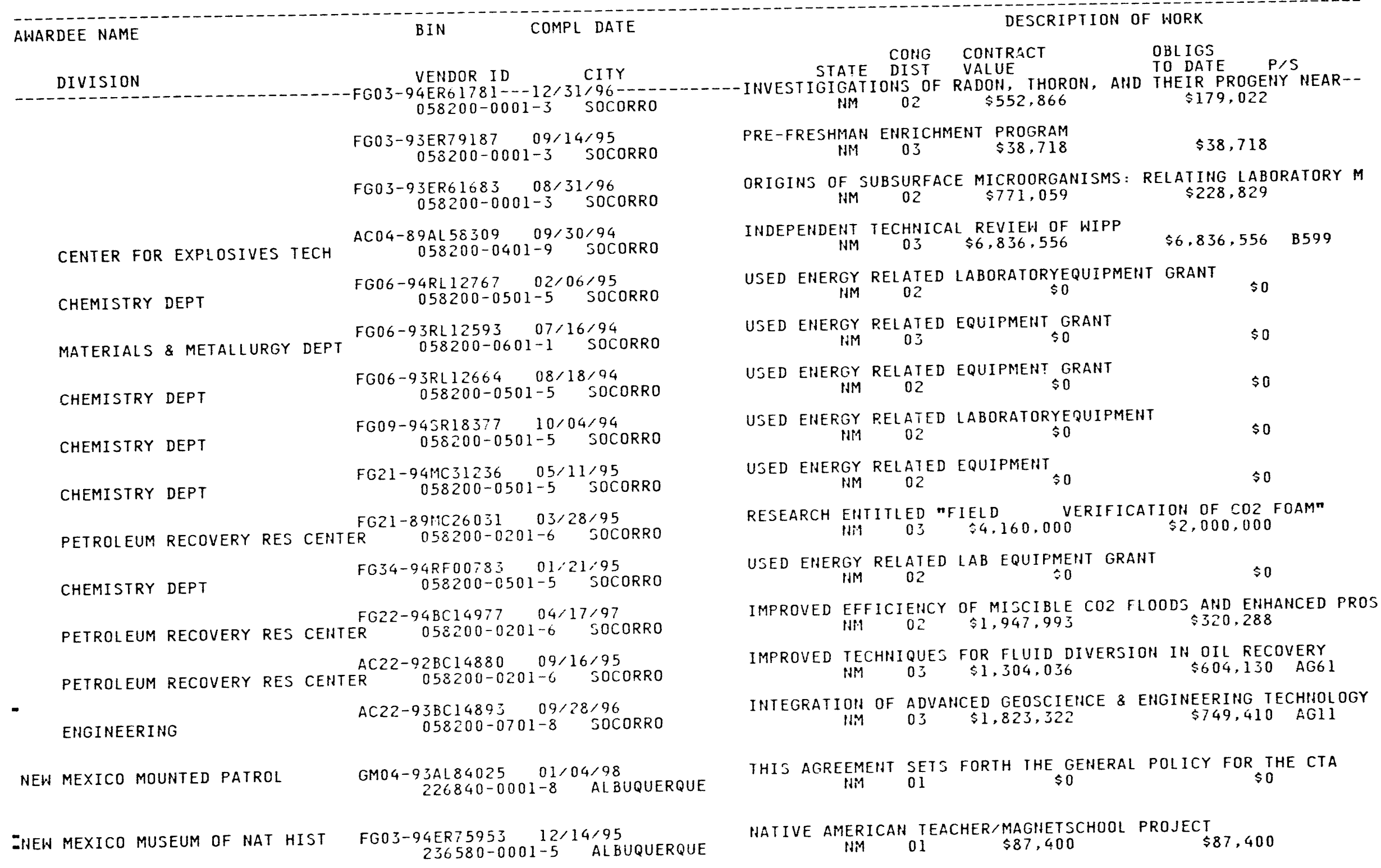




\section{AWARDEE NAME}

BIN

COMPL DATE

CITY

DESCRIPTION OF WORK

CONG CONTRACT

VALUE OBL IGS
TO DATE
NEW MEXICO OFFICE FURNITURE AB04-9JAL91276 10/31/94 $A B 04-93 A L 91277 \quad 10 / 31 / 94$ 095950-0001-9 ALBUQUERQUE

NEW MEXICO PUBLIC SERVICE CO

AC04-90AL57284 10/04/94 125190-0001-5 ALBUQUERQUE ACO4-89AL57511 10/01/94 $125190-0001-5$ AL BUQUERQUE ACO4-90AL58305 12/13/94 $125190-0001-5$ AL BUQUERQUE AC04-85AL27436 12/31/94 $125190-0003-1$ LOS ALAMOS

NEW MEXICO STATE OF REVENUE DEPT

PUBLIC SAFETY DEPT

PUBLIC SAFETY DEPT

ENVIRONMENTAL IMPROVEMENT DIV

GM04-83AL22606 12/28/99 3AL $22606210-1201-5$ ALBUQUERQUE GMO4-92AL58221 03/19/97 058210-1101-9 ALBUQUERQUE GM04-93AL84027 02/16/98 058210-1101-9 ALBUQUERQUE GM32-88AL53204 07/20/99 ENVIRONMENTAL IMPROVEMENT DIV

FC04-85AL20533 09/30/94 058210-1003-9 SAHTA FE FG04-91AL75237 09/30/94 $058210-1403-4$ SANTA FE

HEALTH \& ENVIRONMENT DEPT

HEALTH \& ENVIRONMENT DEPT FG04-91AL65779 09/30/95 $058210-1403-4$ SANTA FE ENERGY MINRLS \& NATRL RES DEPT $058210-1303-8 \quad$ SANTA FE FG46-93R649302 06/30/95 ENERGY MINRLS \& NATRL RES DEPT 058210-1303-8 SANTA FE
PROVIDE STEELCASE SYSIEMS FURNITURE AND PARTS
$\$ 325,000$ $\begin{array}{cccc}\text { ESTABLISH A BPA FOR DESIGN SERVICES FOR STEELCASE SYSTEM } \\ \text { NM } & 01 & \$ 210,000 & \$ 210,000\end{array}$ $\$ 471.386 \quad \$ 112$

$115 \mathrm{KV}$ SERVICE FACILITIES

$$
\text { NM } 01 \quad \$ 571.386 \quad \$ 112
$$

TRANSMISSION SERVICES KAFB

$\$ 0 \quad M 249$

ELECTRIC SERVICE TO KAFB

$$
\text { NM } 01 \text { \$371.697,000 }
$$

ELECTRICAL SERVICE HHEELING \& WHPA REPLACEMENT POHER $N M \$ 03 \$ 17,475,000$

THE PURPOSES OF THIS AGREEMENTARE TO FACILITATE THE

$$
\text { NMS } 01 \text { \$O }
$$

MUTUAL ASSISTANCE AND EMERGENCY MANAGEMENT

THIS AGREEMENT SETS FORTH THE GENERAL POLICY FOR THE CTA

$$
\text { IH } 01
$$$$
\$ 0
$$
SANTA FE/LOS ALAMOS ALTERNATE HIGHWAY ENVIRONMENTAL IMPACT
NM
03

UMTRA AMBROSIA LAKE, NEW MEXICO

$\$ 769,332$

LOS ALAMOS CANCER RATE STUDY

$\$ 562,809$

AGREEMENT IN FRINCIPLE FOR ENVIRONMENTAL OVERSIGHT. MONITOR $N M \quad 03 \quad \$ 6,783,205$ $\$ 6,783,205$

SI, 18,172 NEW FEDERAL FUNDS UNDER NEW HEATHERIZATION UM 03 \$10,237,606 $\$ 6,960,745$

AHARDS $\$ 30,000$ NEW DOE FUNDS, AUTHORIZES $\$ 30,000$ STATE MATCH NM 03 S $\$ 150,262$ $\$ 68,695$

WIPP - EMERGENCY RESPONSE ISSUES 


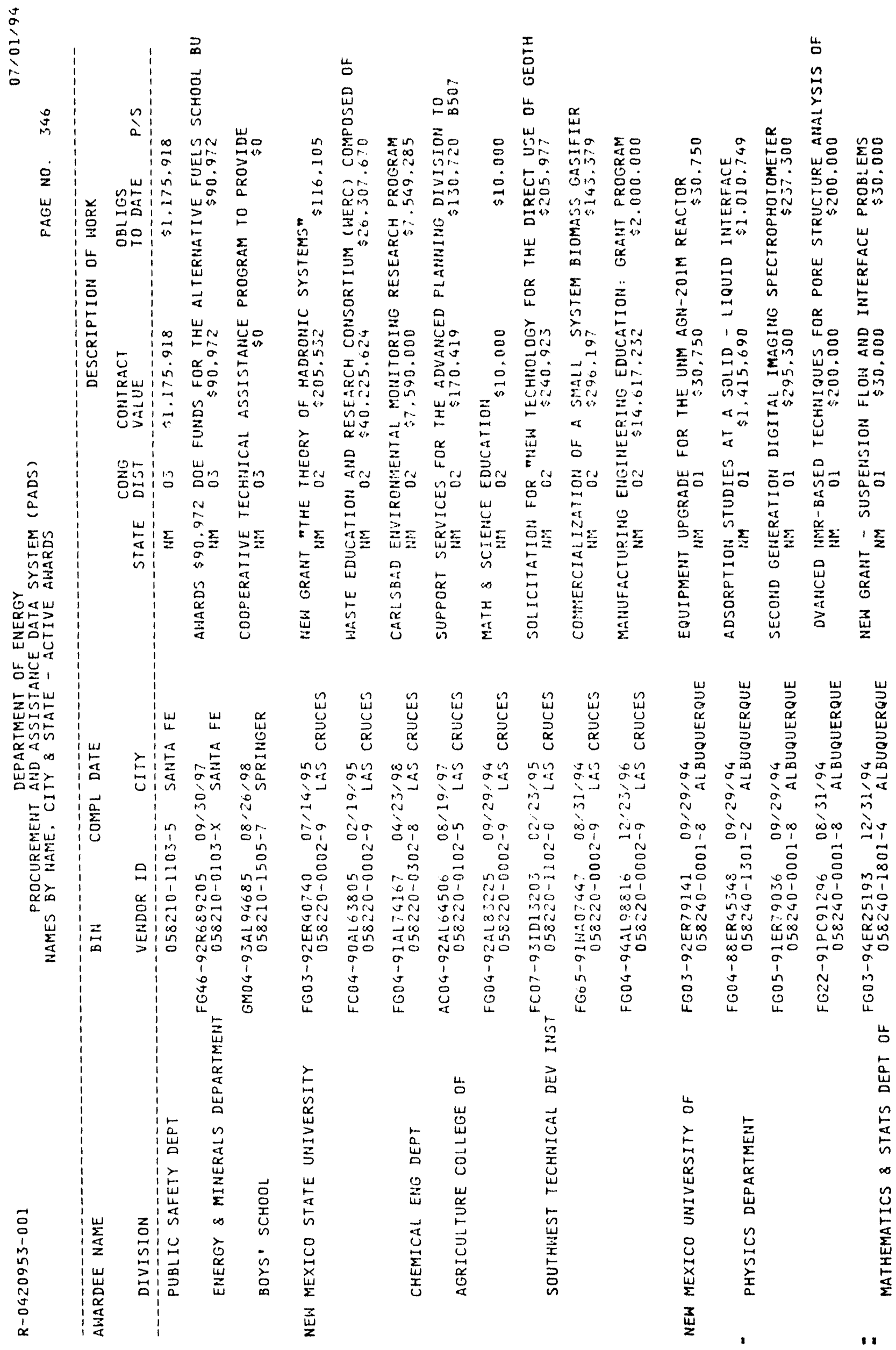


AWARDEE NAME

BIN

DESCRIPTION OF HORK

CONG CONTRACT OBLIGS

STATE DIST VALUE TO DAIE P/S VENDOR ID 4ER4U820---12/31/94_-.--058240-0001-8 AL BUQUERQUE FG03-93ER75881 12/31/94 058240-0001-8 AL BUQUERQUE

FG04-8SER40395 11/30/94

PHYSICS \& ASTRONOMY DEPARTMENT 058240-0801-9 ALBUQUERQUE FG03-92ER61520 05/24/95 $058240-0001-8$ AL BUQUERQUE FG03-92ER40732 02/28/95 $058240-0001-8$ AL EUQUERQUE FG03-92ER14296 02/14/95 058240-0001-8 AL BUQUERQUE FG03-92ER54182 09/14/95 058240-0001-8 AL BUQUERQUE FG03-93ER25163 05/31/95 058240-0001-8 AL BUQUERQUE

FG03-93ER75877 04/14/95 058240-0001-8 AL BUQUERQUE FG03-93ER14326 01/31/96

PHYSICS \& ASTRONOMY DEPARTMENT $058240-0801-9$ ALBUQUEROUE FG03-93ER45498 07/31/96 058240-0001-8 ALBUQUERQUE

FCO4-94AL $98934 \quad 05 / 05 / 96$ 058240-2601-7 ALBUQUERQUE

ASSISTIVE \& TRAINING TECH

PHYSICS \& ASTRONOMY DEPARTMEITT 058240-0801-9 ALBUQUERQUE FG04-89AL58502 09/27/98 058240-0001-8 ALBUQUERQUE GM04-93AL84023 09/16/98 058240-2501-0 AL BUQUERQUE

ARMY ROTC

MILITARY SCIENCE DEPARTMENT OF $\begin{array}{ll}\text { GM04-93AL84044 01/28/98 } \\ \text { OF }\end{array}$ HOSPITAL NM 0 $\$ 35,000$
CO-SPONSORTHIP OF

NEH GRANT - "MULTIGROUP DISCRETE ORDINATES SOLUTION OF BOLTZ NM 01 \$ $\$ 57,282$ ORDTHTES $\$ 57,282$

NUCLEAR PHYSICS AT INTERMEDIATE ENERGIES

$$
\text { IMM } 01 \text { \$1,623.000 \$1,268,000 }
$$

LUNG CANCER IN URANIUM MINERS: A TISJUE RESOURCE - PILOT IN

$$
\text { III } 01 \text { \$ } \$ 299.949 \text { \$299, } 949
$$

NEW MEXICO CENTE FOR PARTICLE PHYSICS: STUDIES OF FUNDAMENT

$$
\text { IIM } 01 \text { \$ } 010.000 \quad \$ 610,000
$$

NEW GRANT - "IMPACT DEPOSITS AT THE MAHJON IMPACT STRUCTURE"

$$
\text { HM } 01 \text { \$ \$86.081 } \$ 86.081
$$

RESEARCH TO DEVELOP AN IMFROVED NEUTRAL DIFFUSION MODEL FOR

$$
\text { HM } 01 \text { \$ } \$ 108,889 \text { \$ } \$ 50,182
$$

NEH GRANT - "REGULAR AND CHAOTIC DYNAMICS ARISING IN NONLIHE NM 01 \$ $\$ 3,000$ \$83,000

NEW GRANT - "EXPERIMENTAL AHD THEORETICAL

$$
\text { NM "EXPERIMENTAL } 0100.000 \text { \$100,000 }
$$

NEW GRANT - "H- SPECTROSCOPY";P.I. - HOWARD BRYANT

PARTICLE-INDUCED AMORPHIZATIONOF CRYSTALLINE SILICATES, COM HM 01 \$3

ASSISTIVE DEVICES. NEW MEXICO TECHNOLDGY DEPLOYMENT PILOT PR NM OI $\$ 4.756,396 \quad \$ 1.886,150$

STRANGE PARTICLES AND HEAVY IOH PHYSICS $\$ \$ 250,000$ NEH MEXICO MATHEMATICS, EHGINEERING AND SCIENCE ACHIEVEMENT
HM 01
$\$ 268,238$

THIS AGREEMENT SET FORTH THE $\underset{\$ 0}{\text { GM }}$. POLICY FOR THE SCHEDULED

THIS AGREEMENT SETS FORTH THE GENERAL POLICY FOR THE CTA

MUTUAL EMERGENCY RESPONSE 01 \$0 
ALIARDEE NAME

BIN

COMPL DATE

STATE DOHG CONIRACT

VENDOR ID

CITY VALUE

OBL IGS

DIVISION

GM32-94AL $96950 \quad 03 / 26 / 99$ $058240-2101-5$ AL BUQUERQUE

HOSPITAL

GM04-92AL84078 06/22/97 $058240-1903-7$ LOS LUNAS

VALENCIA BRANCH

NEW ORLEANS UNIVERSITY OF RESEARCH \& DEVELOPMENT PARK

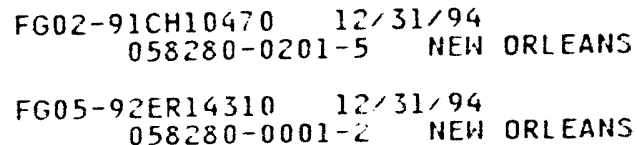

NEW PRAIRIE UNITED SCHOOL CORP

FG45-91R530075 12:31/94 196870-0001-5 NEW CARLISLE

NEW YORK ACADEM) OF SCIENCES

FG02-93EF61649 07/14/94 $058390-0001-9$ HEH YORK

NEW YORK STATE ELECTRIC \& GAS

FC22-93PC92642 06/30/98 058540-0002-4 LAHSIHG

NEW YORK STATE ENERGY OFFICE FG01-9CCE21001 12/31/94

NEW YORK STATE OF ENVIRONIAENTAL CONSERVATION ENERGY SERVICES DIVISION OF FG02-91CH10493 10/01/94 053550-1901-2 AL BANY FG42-94R260024 03/31/95 $058550-2001-0$ ALBANY FG42-94R260023 $03 / 31 / 95$ $058550-2001-0$ ALBAHY ENERGY SERVICES DIVISION OF ENERGY SERVICES DIVISION OF FG42-94R260022003/31/95 FG42-78R20001907/31/95 ENERGY OFFICE FG42-80C562034 03/31/95 - ENERGY OFFICE

PROVIDE MEDICAL CARE \& TREATMENT OF PATIENTS FROM DOE'S LANL NM 01 \$

SUPPORT FOR SCIENCE, MATHEMATICS, AND ENGINEERING 0

DESIGN \& CONSTRUCTIOH OF A CENTER FOR ENERGY RESOURCE MANAGE LA $02 \$ 20.665 .342 \quad \$ 9.942 .000$

ELECTROHIC AHD NUCLEAR FACTORJIN INTRAMOLECULAR CHAHGE AHD $L A$ OA 01 \$268.000

ICP-NEW AWARD. $03 \quad \$ 320,000$

$\$ 50,000$

NEW YORK ACADEMY OF SCIENCE CONFERENCE: "DHA DAMAGES:

$$
\text { NY } 08 \text { \$ } \$ 5,000 \quad \$ 5,000
$$

FORCED OXIDATION, LIMESTOHE, COHCURRENT/COUHTER-CURRENT FGD NY $25 \$ 261.802 .609 \quad \$ 45,000,000$

ANALYSIS OF THE EFFECTIVENESS OF ENERGY REIROFITS IN MULTIFA IIY $04 \quad \$ 214.757$ \$100.092

GRANT FOR STATE OVERSIGHT OF REMEDIAL ACTIVITIES UHDER CERCL HY $23 \quad \$ 2.000 .000 \quad \$ 448.028$

CYCLE 16 TECHHICAL ASSISTANCE AHARD IYY 23 AS $\$ 500,000 \quad \$ 500,000$

CYCLE 16 MARKETING AHARD

$$
\text { MARKETING AHARD } 23000000 \$ 400,000
$$

FY 94 PROGRAM ASSISTANCE AHARD NY $23 \quad \$ 912,577$

$\$ 912,577$

INSTITUTIOHAL COHSERVATIOH PROGRAM - ADMINISTRATION - NEH HY $23 \$ 50,745,327 \quad \$ 6,626,524$

STATE EHERGY COHSERVAIION PROGRAM (NEH YORK) HY $23 \$ 121,039.528 \quad \$ 15,303.915$ 


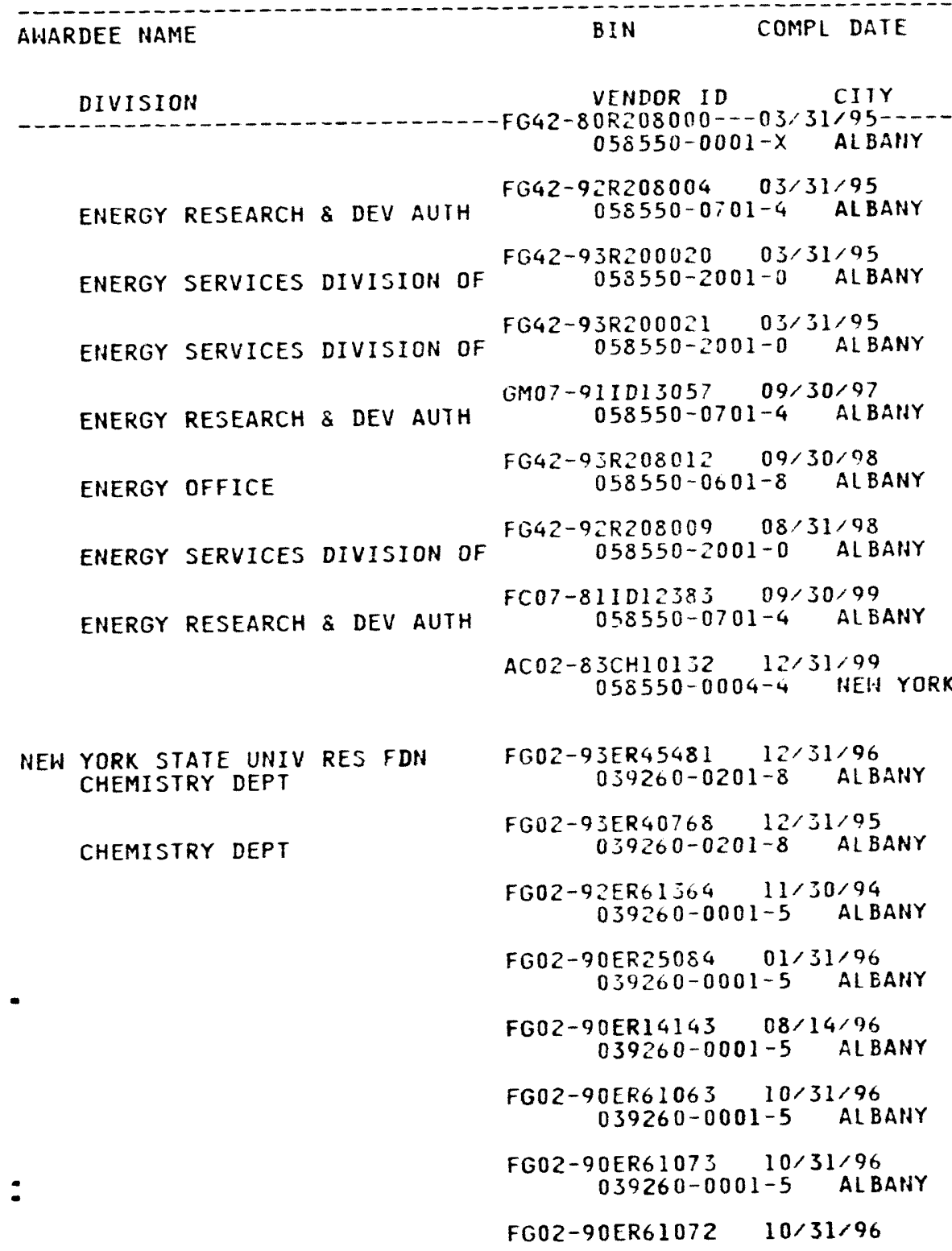

\section{DESCRIPTION OF HORK}

\section{CONG CONIRACT OBLIGJ}

IO DATE P/S

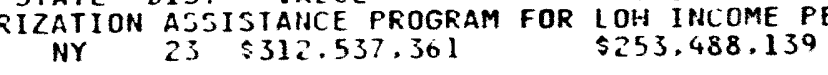
AHARD FUHDS FOR BUILDING TECHNOL OGY RSD. \$200.000 FY 93 AWARD FOR FROGRAM ASSISTANCE.

FY 93 AWARD 23 FORAM $\$ 41.073 \quad \$ 471.073$ FY 93 ICP MARKETIHG AHARD.

$\$ 359,327$ MEMORANDUM OF UNDERSIANDING FOR PREPARING A JOINT ENVIROIIMEN AFV HEAVY DUTY STATE/MUHICIPALVEHICLE PROJECT. FY 93 AHARD NY OI $\$ 544.524 \quad \$ 86.894$

ALTERHATE FUEL VEHICLE 3 THOOL BUSES AHARD. $\$ 64.936$ OPERATION OF HESIERN HEN YORK NUCLEAR SERVICES CEHTER SUPPLY OF ELECIRICAL SERVICES \$0 $\$ 112$

DETERMIHATIOH OF CONCEMIRATIONPROFILES AT IMTERFACES THE THEORY OF RELATIVISIIC HEAVY ION COLLISIONS

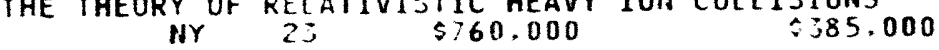
UJE OF CLQUD OBjERVAIIONJ AHD MESOSCALE METEROLOCY MODELS TO IIY $23 \quad \$ 284,458 \quad \$ 284.458$

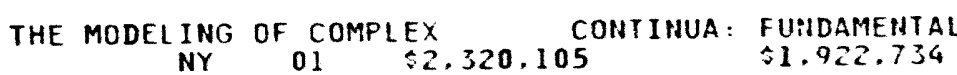
DETERMINAIION OF SALVATION KINETIES AIID SUPERCRITICAL SCIENCE TEAM PARTICIPATION IN THE ATMOSPHERIE RADIATION NY 01 \$3 38.162 \$211.941

TREATMENT OF CLOUD RADIATIVE EFFECTS IN GENERAL CIRCULATION NY 23 \$2.032.709 $\$ 1.317 .960$

DEVELOPMEHT ROTATING SHADOH- BAN SPECTRAL RADIOMETERS 


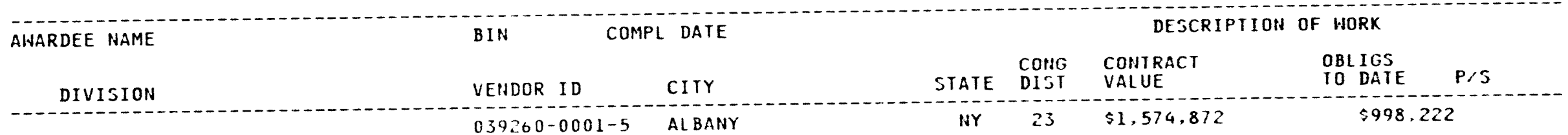

PHYSICS DEPT

PHYSICS DEPT

PHYSICS DEPT

CHEMISTRY DEPT

CHEMISTRY DEPT

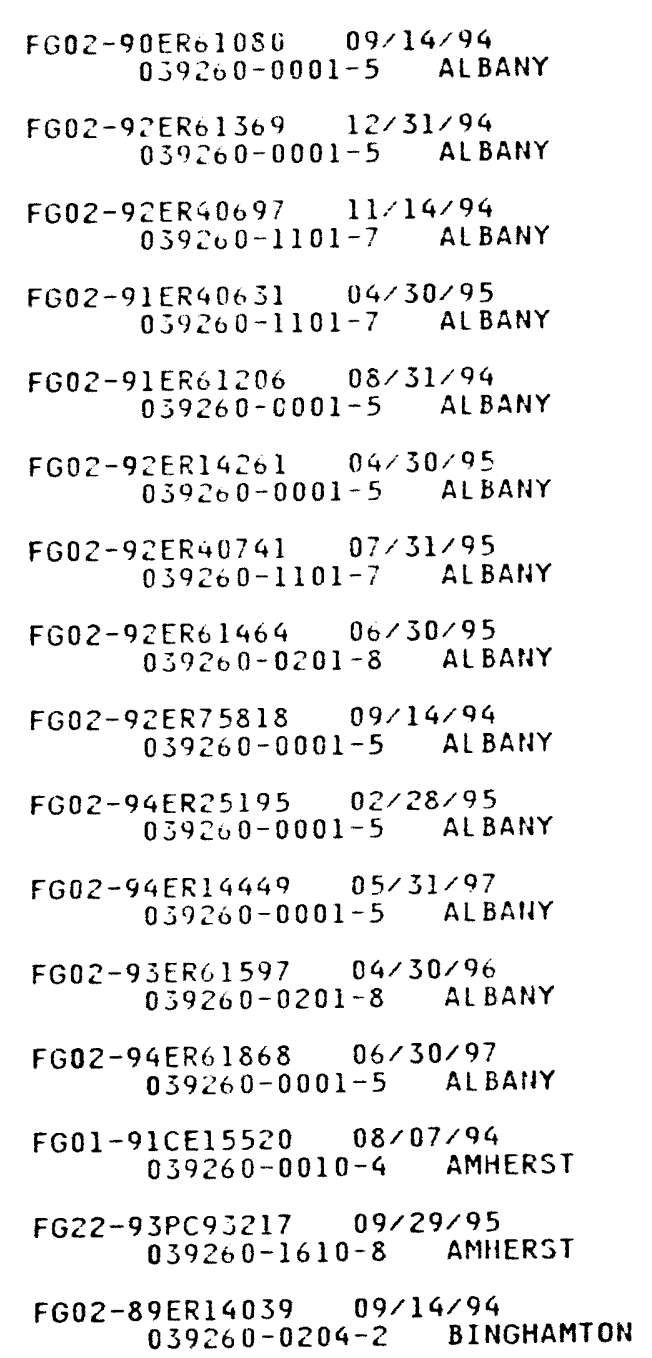

TERRESTRIAL SOURCES AND SIUKS IN THE GLOBAL CARBON BUDGET: NY $2 \mathrm{~J} \quad \$ 523.158 \quad \$ 520.002$

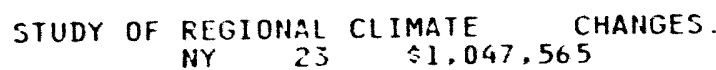

CALORIMETER BASED DETECTORS FOR HIGHT ENERGY HADRON NY $23 \quad \$ 2,474.744 \quad \$ 2,474.744$ STUDY OF HEAVY FLAVORED PARTICLES

HY 23 \$ 1.195 .800 \$ $\$ 1.195 .800$

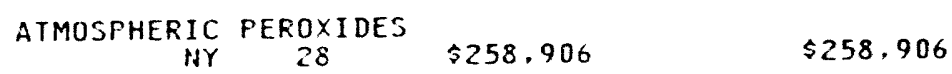
THREE DIMEHSIOHAL IMAGING OF DRICE CORE SAMPLES USING HY 23 S $\$ 63.760$ \$O 3.760 STUDIES IN QUANTUM FIELD 23 THY THEORY $\$ 108.000$ \$71.000 MULTIPLE TRACER MEASURENENT OF THE COUPLIHG BETHEEN HY $23 \quad \$ 420.879 \quad \$ 420,879$

REACTOR EDUCATION CENTER $\$ 43.413 \quad \$ 41.413$

H/A HY $23 \quad \$ 10,000 \quad \$ 10,000$

N/A NY $23 \quad \$ 377,896 \quad \$ 120.995$

CHEMISTRY INDRUCED BY THE SELECIIVE EXCITATION OF CORE IIY $23 \quad \$ 405,000 \quad \$ 266,100$

$N / A$
NY 23
$\$ 325,879$
$\$ 110.650$

CARBON FIBER REINFORCED TIN-SUPER CONDUCTOR COMPOSITES SUPERIOR CATALYSTS FOR SELECIIVE CATALYTIC REDUCTION OF NITR PHOTOCHEMISTRY OF CATALYSTS FOR SELECTIVE CATALYTIC REDUCTION
NY 31
$\$ 176.454$

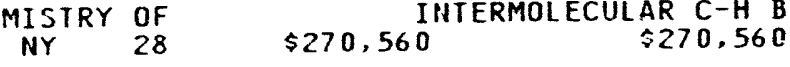




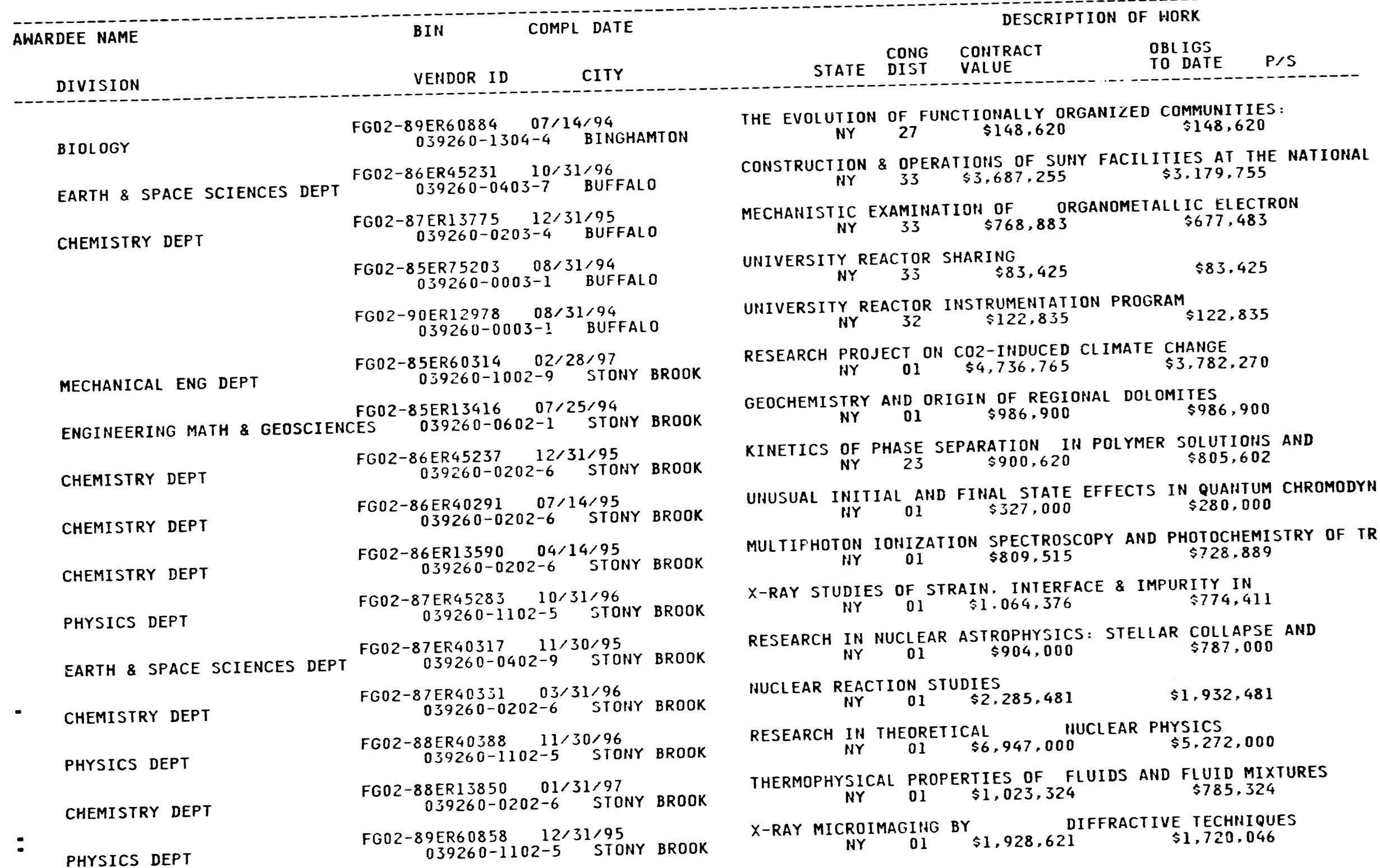


PROCUREMENT AEDARSISTANCE DATA SYSTEM (PADS) NAMES BY NAME, CITY \& STATE - ACTIVE AWARDS

PAGE NO. 352

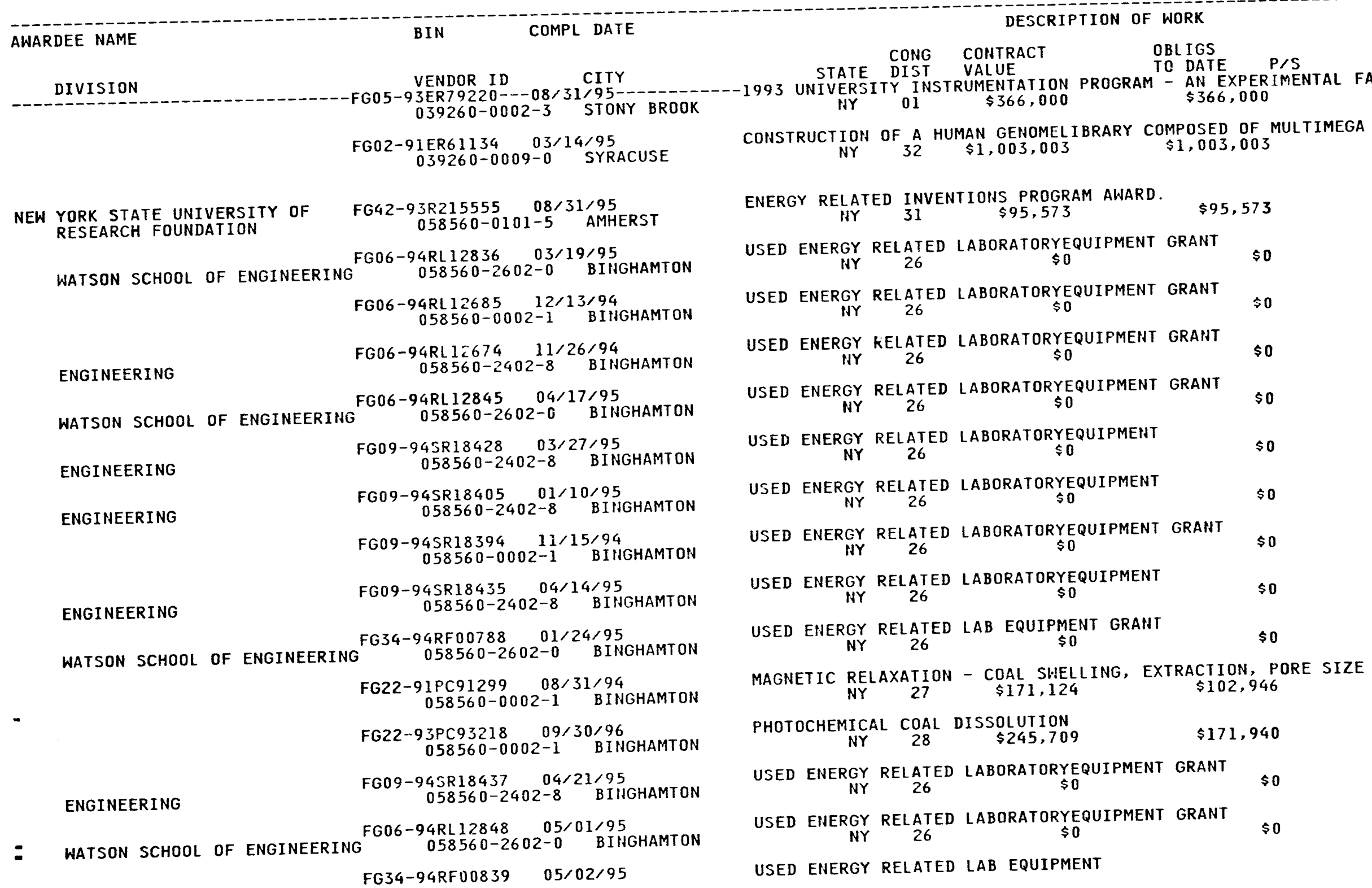




\begin{tabular}{|c|c|c|}
\hline \multicolumn{2}{|c|}{ AWARDEE NAME } & COMPL DATE \\
\hline & DIVISION & VEIIDOR ID \\
\hline & WATSON SCHOOL OF ENGINEERING & $058560-2602-0$ \\
\hline & ENG INEER ING & $\begin{array}{c}\text { FG34-94RF00896 } 05 / 11 / 95 \\
058560-2402-8 \quad \text { BINGHAMTON }\end{array}$ \\
\hline \multirow[t]{3}{*}{ NEW } & $\begin{array}{l}\text { YORK UNIV MEDICAL CENTER } \\
\text { ENVIROHMENTAL MEDICINE DEPT }\end{array}$ & $\begin{array}{c}\text { FG02-87ER60547 05/31/96 } \\
058580-0101-7 \text { HEW YORK }\end{array}$ \\
\hline & CELL BIOLOGY & $\begin{array}{c}\text { FG02-88ER60656 03/31/97 } \\
058530-0201-3 \text { NEW YORK }\end{array}$ \\
\hline & ENVIRONMENTAL MEDICINE DEPT & $\begin{array}{c}\text { FG02-87ER60592 } 05 / 14 / 96 \\
058580-0103-3 \text { TUXEDO }\end{array}$ \\
\hline \multirow[t]{7}{*}{ NEW } & $\begin{array}{l}\text { YORK UNIVERSITY } \\
\text { CHEMISTRY DEPT }\end{array}$ & $\begin{array}{c}\text { FG02-86ER60405 12/31/94 } \\
058590-0401-X \text { NEW YORK }\end{array}$ \\
\hline & COURANT INST OF MATH SCIENCE & $\begin{array}{l}\text { FG02-86ER53223 c 12/31/94 } \\
\text { ES } 058590-0501-6 \text { NEH YORK }\end{array}$ \\
\hline & APPLIED SCIENCE DEPT & $\begin{array}{c}\text { FG02-90ER61014 07/14/95 } \\
058590-0201-7 \text { HEH YORK }\end{array}$ \\
\hline & BIOLOGY DEPT & $\begin{array}{c}\text { FGO2-92ER2007 } 05 / 31 / 95 \\
058590-0301-3 \text { HEW YORK }\end{array}$ \\
\hline & • & $\begin{array}{c}\text { FG02-92ER25127 06/14/95 } \\
058590-0001-4 \text { NEH YORK }\end{array}$ \\
\hline & & $\begin{array}{c}\text { FG02-92ER54165 06/30/95 } \\
058590-0001-4 \text { NEW YORK }\end{array}$ \\
\hline & MATHEMATICAL SCIENCES & $\begin{array}{c}\text { FG02-92ER25139 } 02 / 14 / 95 \\
058590-0601-2 \text { NEW YORK }\end{array}$ \\
\hline & BIOLOGY DEPT & $\begin{array}{c}\text { FG02-90ER60931 02/29/96 } \\
058590-0301-3 \text { NEW YORK }\end{array}$ \\
\hline & COURANT INST OF MATH SCIENCE & $\begin{array}{l}\text { FG02-93ER25160 04/30/96 } \\
\text { ES } 058590-0501-6 \text { NEW YORK }\end{array}$ \\
\hline & COURANT INST OF MATH SCIENCE & $\begin{array}{l}\text { FG02-93ER54217 } 07 / 14 / 96 \\
\text { ES } 058590-0501-6 \quad \text { NEH YORK }\end{array}$ \\
\hline & MATHEMATICAL SCIENCES & $\begin{array}{c}\text { FG02-88ER25053 12/31/96 } \\
058590-0601-2 \text { NEW YORK }\end{array}$ \\
\hline
\end{tabular}

MEASUREMENT AND APPORTIONMENT OF RADON SOURCE TERMS FOR

CELLULAR MORPHUMETRY OF THE BRONCHI OF HUMAN AND DOG LUNGS NY $15 \quad \$ 941,829 \quad \$ 633,629$

PARTICLE DEPOSITION IN HUMAN AND CANINE TRACHEOBRONCHIAL

$$
\text { NY } 26 \quad \begin{array}{ll}
\$ 1,002,876 & \$ 838,976
\end{array}
$$

THE PHOTOPHYSICS AND CHEMISTRYOF PAH COMPOUNDS

HY 18 \$2,810,449 \$2,810,449

PLASMA PROPERTIES

CLOUD AND OCEAN EFFECTS ON GLOBAL GREENHOUSE WARMING NY 15 \$1, 094,650 $\$ 874,681$

ASPARAGINE SYNTHETASE GENE REGULATION \& PLANT NITROGEN NY 17 \$296,940 $\$ 296,940$

DEMAIN DECOMPOSITION \& PARALLEL COMPUTING FOR

BOUNDARY PLASMA MODELLING FOR ITER

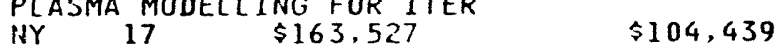

$\$ 104,439$ ADAPTIVE $\underset{N Y}{\text { METHODOL OGIES }} \underset{17}{\text { FOR }} \underset{\$ 444,000}{\text { COMPUTATIONAL FLUID DYNAMICS }}$

MOLECULAR UNDERSTANDING OF MUTAGENICITY USING POTENTIAL NY $15 \quad \$ 339,028 \quad \$ 272,052$

COMUTATIONAL METHODS FOR FUSION
NY 17
$\$ 225,000$
$\$ 147,750$

3D PLASMA FLUID SIMULATIONS IN DIVERTOR TOKAMAKS

ANALYTICAL AND COMPUTATIONAL MATHEMATICAL PHYSICS

MATHEMATICAL SCIENCES

$88 E R 25053$
$058590-0601-2$ 


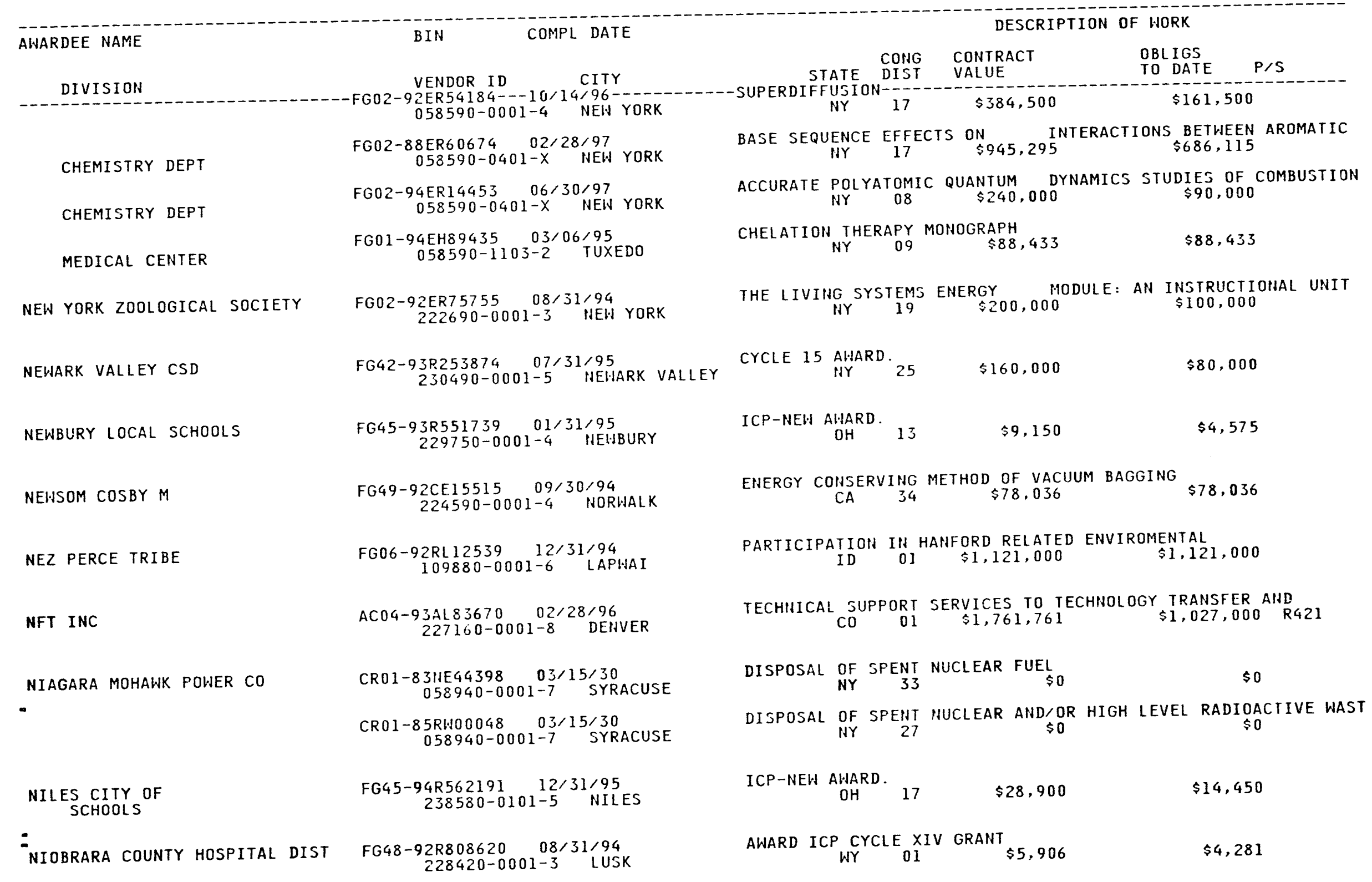




\begin{tabular}{|c|c|c|c|c|c|c|c|}
\hline AWARDEE NAME & BIN & $\angle$ DATE & & & DESCR & $=$ WORK & \\
\hline & VENDOR ID & CITY & STATE & $\begin{array}{l}\text { CONG } \\
\text { DIST }\end{array}$ & $\begin{array}{l}\text { CONTRACT } \\
\text { VALUE }\end{array}$ & $\begin{array}{l}\text { OBLIGS } \\
\text { TO DATE }\end{array}$ & $P / S$ \\
\hline
\end{tabular}

\section{NLO INC}

NONLINEAR SCI APPLCTNS INST OF

NORFOLK STATE UNIVERSITY CHEMISTRY DEPARTMENT

NORTH AMERICAN TRANSFORMER

NORTH ANDOVER SCHOOL DEPT

NORTH ATTLEBOROUGH TOHN OF PUBLIC SCHOOLS

NORTH BOLIVAR COUNTY SCHOOLS

NORTH BRADFORD TOWN OF EDUCATION BOARD OF

NORTH CAROLINA A \& T STATE U

- chemical engineering

CHEMICAL ENGINEERING

- north carolina abt state univ CHEMICAL ENGINEERING
AC05-760R01156 09/30/94 059080-0001-9 CINCINNATI

FG02-91ER54130 10/31/94
$216440-0001-6$ SHARON

FG05-89ER40525 06/30/95 $185200-0101-7$ NORFOLK

AC65-93HA11517 08/01/94 165990-0001-0 MILPITAS

FG41-93R193503 11/30/94 059330-0001-9 NORTH ANDOVER

$F G 41-93 R 193520 \quad 07 / 31 / 95$ 232380-0101-9 NORTH ATTLEBOROUGH

FG44-88R433669 12/31/94 177210-0001-7 SHELBY

FG41-93R191507 08/31/95 232340-0101-4 NORTHFORD

FG05-930R22119 09/23/94 $30 R 2211909 / 23 / 94$
$059420-0001-8$ GREENSBORO FG22-93MT93008 08/31/96 O59420-0501-X GREENSBORO FG22-93MT93009 08/31/94 $059420-0501-X$ GREENSBORO FG44-92R435008 08/31/94 $059420-0001-8$ GREENSBORO

FG22-91PC91300 08/31/94 $059420-0501-X$ GREENSBORO
FEED MATERIALS PRODUCTION CENTER (FMPC) MANAGEMENT AND OPERA $\mathrm{OH} 02 \$ 891,413,256 \quad \$ 878,274,618 \mathrm{M181}$

CHAOTIC DYNAMICS IN PLASMAS: METHOD OF SYMBOLIC KINETIC EQU MA 04 \$144,000 $\$ 144,000$

STUDY OF FEH-NUCLEON SYSTEMS HITH HADRONIC AHD ELECTROMAGNET VA $02 \quad \$ 379,000 \quad \$ 376,000$

POI.JER TRANSFORMERS FOR DEL TO VAR SUBS CA $10 \quad \begin{array}{lll}\$ 1,219,280 & \$ 1,219,280 & 6120\end{array}$

FY:93 ICP AHARD

$\$ 20,831$

ITL III OF THE NATIONAL ENERGY CONSERVATION POLICY ACT 03 \$129,822

CYCLE X - INSTITUTIONAL CONJERVATION PROGRAM. APPROVED BUDG MS 02 INSTITUTIONAL $\$ 59,822 \quad \$ 38.883$

INITIAL AHARD. $\underset{\text { CT }}{03} \$ \$ 659,960 \quad \$ 47,395$

THE EFFECT OF SAMPLE SIZE AND FINISH ON THE TENSILE CHARACTE NC 06 \$100,000 $\$ 100,000$

PREPARATION AND CHARACTERIZATION OF COMPOSITE MEMBRANE FOR HC 06 \$196,450 $\$ 196,450$

SIMULTANEOUS SO2/HO SEPARAIIONFROM FLUE GAS USING HFCLM NC $06 \quad$ \$10,000 $\$ 10,000$

CYCLE 14 - INSTITUTIONAL COHSERVATION PROGRAM. APPROVED BUD NC $06 \quad \$ 77,326 \quad \$ 38,663$

THERMODYNAMIC AND RHEOLOGICAL PROPERTIES OF SOLID-LIQUID SYS NC 06 R $\$ 160,000 \quad \$ 160,000$ 

NAMES BY NAME, CITY \& STATE - ACTIVE AWARDS

PAGE NO. 356

\begin{tabular}{|c|c|c|c|c|c|c|c|}
\hline AWARDEE NAME & BIN & L DATE & & & DESCR & F HORK & \\
\hline $15 S T$ & VENDOR ID & CITY & STATE & $\begin{array}{l}\text { COHG } \\
\text { DIST }\end{array}$ & $\begin{array}{l}\text { CONTRACT } \\
\text { VALUE }\end{array}$ & $\begin{array}{l}\text { OBLIGS } \\
\text { TO DATE }\end{array}$ & $P / S$ \\
\hline
\end{tabular}

CHEMICAL ENGINEERING

NORTH CAROLINA CENTRAL UNIV

FG22-92MT92020 08/31/95 $059420-0501-X$ GREENSBORO

FG44-93R435391 09/30/95 059440-0001-5 DURHAM

NORTH CAROLINA STATE OF ECONOMIC \& CMTY DEV DEPT OF

ENERGY DIV

ENERGY DIV

NORTH CAROLINA STATE UNIV MATERIALS ENGINEERINO

MATERIALS SCIENCES

PHYSICS DEPT

PHYSICS DEPT

PHYSICS DEPT

PHYSICS DEPT

- chemical eng dept
FCO1-91EI22716 08/07/96

FG44-93R410612 06/30/95 $059460-0302-X$ RALEIGH

FG44-80CS69115 06/30/95 $059460-0302-x$ RALEIGH

FG05-84ER45115 08/31/96 059470-0901-3 RALEIGH

FG05-86ER45259 06/30/95 059470-1301-0 RALEIGH

FG05-88ER40441 03/31/97 059470-0701-0 RALEIGH

FG05-88ER40461 05/31,97 059470-0701-0 RALEIGH

FG05-89ER45384 12/14/94 059470-0701-0 RALEIGH

FG05-90ER4053911/30/95 $059470-0701-0$ RALEIGH

FG05-90ER45436 11/30/96 059470-0101-2 RALEIGH

FG05-91ER14181 05/31/97 $059470-0001-6$ RALEIGH

FG05-92ER20085 07/14/95 059470-0001-6 RALEIGH

FG05-92ER75784 09/29/94 $059470-0001-6$ RALEIGH
IMPROVEMENT OF HYDROGEN SOLUBILITY AND ENTRAINMENT IN HYDROC NC $06 \quad \$ 198,114 \quad \$ 198,114$

SEE ATTACHED PAGE NO. 2 OF THIS NOTICE OF FINANCIAL ASSISTAN HC 02 \$80.044 $\$ 40,000$

STATE HEATING OIL AND PROPAHE PROGRAM
03
$\$ 17,115$
$\$ 10,695$

ALTERNATIVE FUEL SCHOOL BUS PROGRAM/SOLICITATION FOR HEAVY D NC 06 \$283,840 $\$ 96,812$

ENERGY EXTENSION SERVICE
$04 \quad \$ 6,341.392$
$\$ 2,428,525$

MICROSTRUCTURAL EFFECTS IN SOLID PARTICLE EROSION BAHD ELECTRONIC STRUCTURES ANDCRYSTAL PACKING FORCES OF ET BAHD ELECTRONIC $04 \quad \$ 936,002$ \$ $\$ 811,002$ NUCLEAR STRUCIURE RESEARCH AT THE TRIANGLE UNIVERSITIES NUCL HC 04 \$2,674,000 $\$ 1,887,000$ THEORETICAL NUCLEAR REACTION AND STRUCTURE STUDIES USING HYP IIC OS R $0493,000 \quad \$ 537,000$

RESEARCH AT AND OPERATION OF THE MATERIAL SCIENCE X-RAY ABSO HC $04 \$ 2,127,500 \quad \$ 2,127,500$

QUAHTUM CHROMODYNAMIC QUARK MODEL STUDY OF HADRON AND FEW HA NC $04 \quad \$ 301,000 \quad \$ 235,000$

THEORETICAL STUDIES OF SUPFCE REACTIONS ON METALS AND ELECTR HC $04 \quad \$ 508,000 \quad \$ 318,000$

THEORETICAL TREATMENT OF THE BULK AND SURFACE PROPERTIES OF NC $04 \quad \$ 626,167 \quad \$ 419,167$

NEW ALAPD TRANSCRIPTION FACTO RS REGULATING LIGNIN BIOSYNTHE NC $04 \quad \$ 299,734 \quad \$ 194,910$

NEW AWARD - A STUDY OF HYDROGEN EFFECTS ON FRACTURE BEHAVIOR NC ${ }_{04} \quad \$ 191,828 \quad \$ 191,828$ 


AIIARDEE NAME BIN COMPL DATE

\section{DESCRIPTION OF WORK}

CONG CONTRACT

FG05-93ER.20113 06/14/96

CHEMICAL ENG DEPT

CHEMICAL ENG DEPT

FG22-92PC92538 09/20/95

NORTH CAROLINA UNIVERSITY OF

CHEMISTRY DEPT

CHEMISTRY DEPT

CHEMISTRY DEPT

CHEMISTRY DEPT

PHYSICS DEPT

PHYSICS DEPT

BIOCHEMISTRY

$:$
VENDOR ID CITY
TERT5808--09/29/94 2ER75808-0 -09/29/94---
$059470-0001-6$ RALEIGH 059470-0001-6 RALEIGH

FG05-93ER79236 08/31/95 059470-0001-6 RALEIGH AC22-90PC90043 12/31/94 059470-0101-2 RALEIGH 059470-0I0I-2 RALEIGH

FG05-93ER75865 03/21/96 $3 E R 7586503 / 21 / 96$
$059490-0007-2$ ASHEVILLE

FG44-93R435400 09/30/95 059490-0007-2 ASHEVILLE

FG05-88ER13950 08/31/94 059490-0201-6 CHAPEL HILL

FG05-92ER61413 04/30/95 $059490-0001-3$ CHAPEL HILL

STATE DIST VALUE

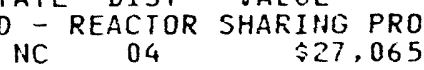

OBLIGS TO DATE P/S $\$ 27,065$ NEW AWARD BIOENERGETIC AND PHYSIOLOGICAL STUDIES OF HYPERTHE NC $04 \quad \$ 276,536 \quad \$ 182,456$

1993 UNIVERSITY INSTRUMENTATION PROGRAM - ADVAHCED GROHTH AN HC $04 \quad \$ 330.000 \quad \$ 330.000$

NOVEL APPROACH TO THE PRODUCTION OF HIGHER ALCOHOLS FROM SYN HC $04 \quad \$ 903,655 \quad \$ 903,655$ AG2I

BIOENERGEHIC STUDIES OF COAL SULFUR OXIDATION BY EXTREMELY T NC $04 \quad \$ 228,131 \quad \$ 199.941$

SUPPORT FOR THE 7TH, 8TH, AND 9TH NATIONAL CONFERENCE ON UND NC 11 \$75,000 $\$ 25,000$

SEE ATTACHED PAGE NO. 2 OF THIS NOTICE OF FINAICIAL ASSISTAN IIC II NO. 2 OF THIS NOTICE OF FINANCIAL $\$ 40.000$

PEPICO STUDIES OF ION DISSOCIATIONS: THE STRUCTURE AND HEAT NC 04 $\$ 535.852$

$\$ 535,852$ $\begin{array}{cccc}\text { CARBON CYCLING ON THE CONTINENTIAL MARGIN } & \& \text { EVIDENCE FROM SE } \\ \text { HC } & 04 & \$ 423,255 & \$ 423,255\end{array}$

FG05-85ER13430 11/14/95 059490-0201-6 CHAPEL HILL

FG05-86ER13633 11/14:95 $059490-0201-6$ CHAPEL HILL

F $505-87$ ERI3675 03/31/96 059490-0201-6 CHAPEL HILL FG05-85ER40219 04/30/97 $059490-0801-4$ CHAPEL HILL FG05-88ER40442 03/31/97 059490-0801-4 CHAPEL HILL FG05-91ER61135 05/31/97 059490-1601-7 CHAPEL HILL FG05-94ER40827 01/14/97 059490-0001-3 CHAPEL HILL

FG05-94ER14459 11/30/97 REDUCTIVE COUPLIHG OF CARBON MOHOXIDE TO C2 PRODUCTS
NC
$\begin{gathered}\$ 889 \\ \$ 784,082\end{gathered}$

ENERGY COHVERSION PROCESSES BASED ON MOLECULAR EXCITED STATE IIC $04 \$ 1,182,000 \quad \$ 1.027,000$

SOLID-STATE VOLTAMMETRY AND SENSORS IN GASES AND OTHER NON-I HC $04 \quad \$ 1,117,000 \quad \$ 989,500$

HIGH ENERGY THEORETICAL PHYSICS REJEARCH

$$
\text { NC } 04 \quad \$ 2,554,000 \quad \$ 2,032,000
$$

STUDIES OF NUCLEAR PROCESSES

$$
\text { IIC } 04 \quad \$ 3.179 .000 \quad \$ 2.304 .000
$$

DEVELOPMENT OF A HUMAN VIRAL-BASED GENOMIC LIBRARY OF 150-20 NC 03 \$1,001,392 $\$ 503,268$

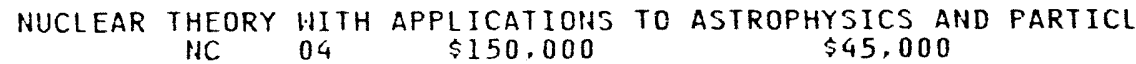

MECHANISTIC STUDIES OF TRAHSITIONAL METAL-CATALYZED ALTERNAT 


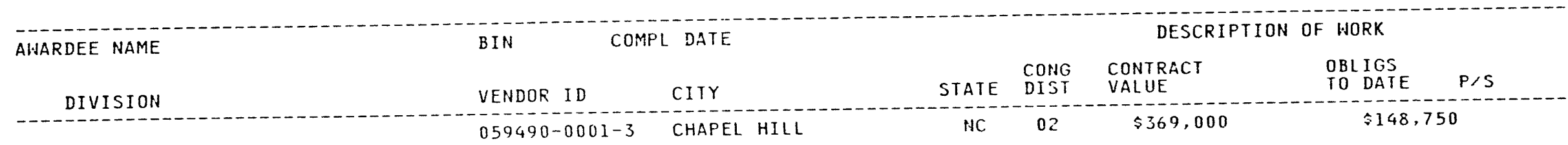

CHEMISTRY DEPT FG05-84ER13263 $08 / 31 / 96$
$059490-0202-4$ CHARLOTTE FG05-94ER75967 12/14/94 059490-0002-1 CHARLOTTE

MARINE SCIENCES

FG02-92ER61425 05/31/95 059490-1305-0 RALEIGH

NORTH CLARION COUNTY OF SCHOOL DISTRICT

NORTH COLLEGE HILL SCHOOLS

$$
\begin{gathered}
\text { FG43-94R364105 05/31/95 } \\
238680-0101-8 \text { TIONESTA }
\end{gathered}
$$

FG45-93R55174101/31/95 229770-0001-1 CINCINHATI

NORTH DAKOTA STATE OF HEALTH DEPT

FCO4-83AL20536 09/30/94 $\begin{array}{ll}3 A L 20536 & 09 / 30 / 94 \\ 059660-0201-0 \quad B I S M A R C K\end{array}$

INTERGOVERHMENTAL ASSISTANCE FG48-92R805710 0 07/31/95 $059660-0301-7 \quad$ BISMARCK

INTERGOVERNMENTAL ASSISTANCE FG48-90R805672 $09 / 30 / 94$

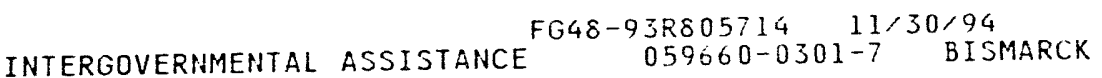

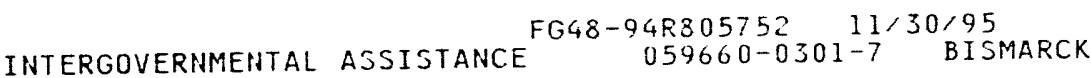

NORTH DAKOTA STATE UNIVERSITY

CHEMISTRY

$$
\begin{gathered}
\text { FG06-94ER75964 12/14/95 } \\
059670-0001-1 \text { FARGO } \\
\text { FG06-93ER14590 09/14/96 } \\
059670-0201-4 \text { FARGO } \\
\text { FG01-91CE15474008/07/94 } \\
059670-0002-X \quad \text { LAHGDON }
\end{gathered}
$$

FNORTH DAKOTA UNIVERSITY OF FG02-91CH10480 12/31/94 ENERGY \& ENVIROHIENTAL RES CTR 059680-0901-2 GRAHD FORKS
OLIGOMER AND MIXED-METAL COMPOUNDS, POTENTIAL MULTIELECTRON NC 03 \$1, 104,718 S $\$ 893,718$

PROGRAM TO ENRICH SCIENCE AND MATHEMATICS EXPERIENCE OF HIGH NC 09 SCIENC $\$ 38,058 \quad \$ 38,058$

A STUDY OF SEDIMEHT MOTIUHI \& BOTTOM BOUNDARY LAYER DYNAMICS HC $04 \quad \$ 6 ? 1,160 \quad \$ 671,160$

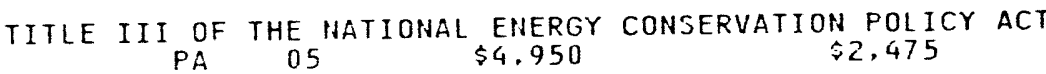

ICP-NEW AWARD.
$\mathrm{OH}$
$\$ 25,146$
$\$ 12,572$

01

URIANIUM MILL TAILIHGS REMEDIAL ACTION PROJECT

AWARD CYCLE XIV ICP COORDINATING AGENCY GRANI

GRANT PROGRAMS FOR SCHOOLS ANDHOSPITALS AND BUILDINGS OHNED $\begin{array}{lll}\text { IND } & 01 & \$ 1,739.770\end{array}$

AHARD CYCLE XV ICP COORDIHATING AGENCY GRANT

$$
\begin{array}{rrrr}
\text { CLE XV ICP } & \text { COORDIHATING AGENCY GRANT } \\
\text { HD } & 01 & \$ 440,169 & \$ 202.482
\end{array}
$$

AHARD CYCLE XVI GRAITS
$\$ 377,963$
\$ 188,105

IID

SCIENCE MAJORS AS TEACHING INTERNS

$\$ 22,779$ $\begin{array}{llll}\text { INFRARED LASER STUDIES OF IHE COMBUSTION } & \text { CHERISTRY OF } \\ \text { NDD } & 01 & \$ 240,402 & \$ 107.506\end{array}$ TEST AHD GATHER DATA ON SWEEP SPIKE COMBINATION TILLAGE TOOL
ND 01

DESIGN \& CONSTRUCTION OF AN ENERGY \& ENVIRONMENTAL RESEARCH HD $01 \quad \$ 6,994.617 \quad \$ 3,470,494$ 


\section{AWARDEE NAME}

BIN

COMPL DATE

VENDOR ID CITY

CONG COHTRACT

DESCRIPTION OF WORK

DIVISION

ENERGY \& ENVIRONMENTAL RES CTR

$059680-0901-2$ GRAND FORKS

ENERGY \& ENVIRONMENTAL RES CTR $059680-0901-2$ GRAND FORKS

FC21-86MC10637 09/30/94

ENERGY TECHNOLOGY CENTER

059680-0201-8 GRAND FORKS

ENERGY \& ENVIRONMENTAL RES CTR $059680-0901-2$ GRAND FORKS

ENERGY \& ENVIRONMENTAL RES CTR $059680-0901-2$ GRAND FORKS

NORTH DAKOTA UNIVERSITY SYST

FGC2-91ER75673 09/29/94

IER75673 $09 / 29 / 94$
$215300-0001-7$

NORTH DICKINSON COUNTY OF SCHOOL DISTRICT

NORTH GEORGIA COLLEGE

NORTH LITTLE ROCK SCHOOL DIST

NORTH MIAMI COMMUNITY SCHOOLS

NORTH OTTAHA COMMUNITY HOSP

NORTH PANOLA CONSOLIDATED SCH

NORTH PENN HOSPITAL

NORTH STAR RESEARCH CORP
FG45-92R530178 $12 / 31 / 94$
$217350-0101-5$ IRON MOUNTAIN

FG44-93R435353 09/30/94 $059740-0001-3$ DAHLONEGA

FG46-92R613532 $12 / 31 / 94$
$059820-0001-9$ NORTH LITTLE RO

FG45-93R551591 08/31/95

219470-0001-2 DEWVER

FG45-93R551641 08/31/95

232820-0001-4 GRAHD HAVEN

FG44-89R434016 09/30/94

$187240-0001-9$ SARDIS

FG43-93R354213 12/31/94

$230910-0001-X$ LANSDALE

FG05-91ERE1209 02/28/95 $175190-0001-X$ ALBUQUERQUE
STATE DIST VALUE
ENERGY AND ENVIRONMENTAL

ND $01 \$ 25,535,429$

OBLTGS

TO DATE P/S

(JSR) RESEARCH EMPHASIZING---
$\$ 3.055 .772$

ENERGY AND ENVIRONMENTAL RESEARCH EMPHASIZING LOW-RANK

$$
\text { ND } 01 \$ 10,908,814 \quad \$ 4,908,814
$$

THIS RESEARCH PROJECT SEEKS TOENHANCE AND DEVELOP WHERE

HD 01 \$4 $, 511,157 \quad \$ 35,814,574$

CATALYTIC FABRIC FILTRATION FOR SIMULTANEOUS NOX AND PARTICU ND 01 \$1,194,293 $\$ 1,194,293$ AG11

RESEARCH TITLED "IRACE ELEMENTEMISSIOHS"
ND
01
$\$ 948,956$
$\$ 948,956 \quad A G 92$

$\begin{array}{ccc}\text { PLANNING GRANT } & \text { FOR } & \text { DOE } \\ \text { NDPSCOR } & 01 & \$ 157,030\end{array}$

$\$ 116,500$

$\$ 47,200$

$\$ 19,112$

SEE ATTACHED FAGE NO. 2 OF THIS NOTICE OF FINANCIAL ASSISTAN GA 09 \$31,614 $\$ 15,800$

THIS IS A NEH CYCLE 14 ECM GRANT. INCLUDED IS THE NFAA, BUD ROCK

ICP-NEW AHARD

$\underset{\text { IN }}{\text { AN.IARD. }} 05 \quad \$ 100,096$ \34, 954

ICP-NEH AHARD.

MI 02

$\$ 202,000$

$\$ 27,506$

ICP-NEW AWAR

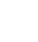

CYCLE 11 INSTITUTIONAL CONSERVATION PROGRAM. AFPROVED BUDGE
MS 01

TITLE III OF THE NATIONAL ENERGY CONSERVATION POLICY ACT
PA
$\$ 70,000$

ADVANCED PULSED POWER CIRCUIISFOR ION LINEAR INDUCTION ACCE

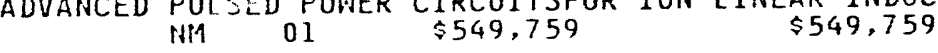


$R-0420953-001$

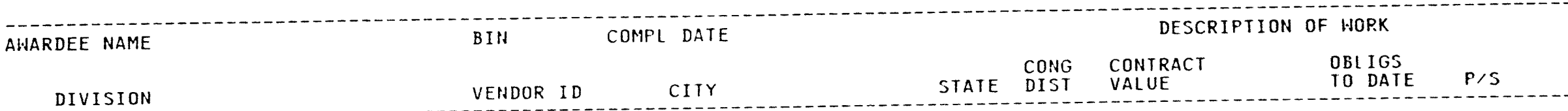

NORTH TEXAS UNIVERSITY OF

FG03-93ER45497 01/31/98
$059970-0001-X$ DENTON

NORTHEAST UTILITIES SERVICE CO

NORTHEASTERN UNIVERSITY PHYSICS DEPT

\section{BARNETT INSTITUTE}

MECHANICAL ENGINEERINO

-NORTHEASTERN haYNe SCHOOL CORP

NORTHERN ARIZONA UNIVERSITY

INORTHERN CUMBERLAND MEM HOSP

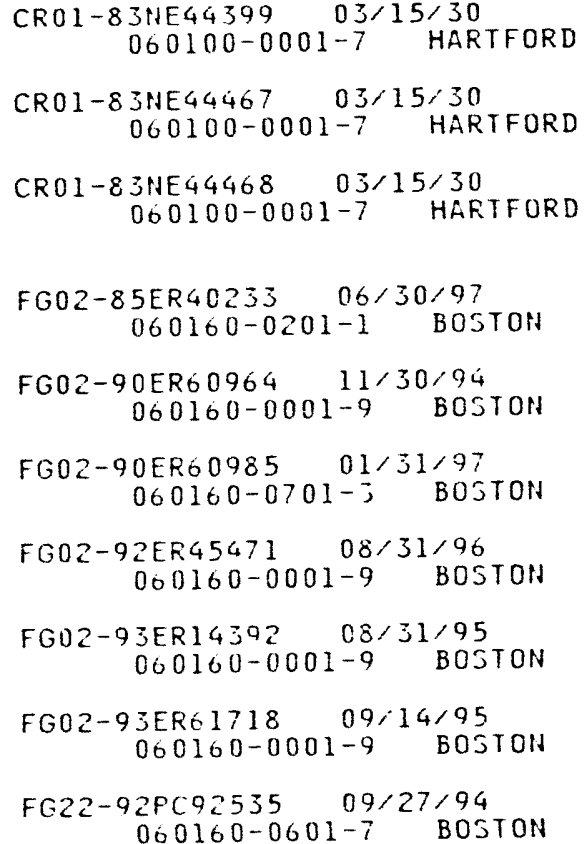

FG45-94R562011 06/30/95 238100-0001-9 FOUNTAIN CITY

FG03-94EP.6184905/31/97 060180-0001-6́ FLAGSTAFF

FG41-94R192611 06/30/96 BRIDGTON
IMFURITY-INDUCED CORROSION AT GRAIN BOUNDARIES. METAL-OXIDE TX $26 \quad \$ 474.646 \quad \$ 150,000$

DISPOSAL OF SPENT NUCLEAR FUELAND OR HIGH LEVEL RADIOACTIVE CT SPENT NUCLEAR FUELAND

DISPOSAL OF SPENT NUCLEAR FUEL

$\begin{array}{rrrr}\text { DISPOSAL OF } & \text { SPENT NUCLEAR FUEL } \\ \text { CT } & 01 & \$ 0\end{array}$

TOPICS IH GAUGE THEORIES AND UNIFICATION OF ELEMENTARY PARTI $M A$ S 09 \$ 1.573 .000 \$1, 423,000

NEW FLUORESCENCE METAODOLOGY FOR DETECTINY DHA ADDUCTS

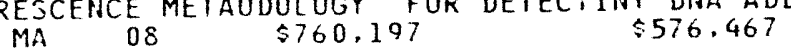

DEVELOFMEHT OF OPTIMIZED CAPILLARY GEL ELECTRO-PHORETIC MA $09 \$ 1.897,963$ \$1, 034,365

COMPUTEF MODELIHG OF SOLIDIFICATION MICROSTRUCTURE MA 08 \$ $\$ 270.526$ \$

SUPER-RESOLUTIOH IN SHALLOW EEOPHYSICS
MA 08 \$ 144,967

EFFICIENT IPTELLIGENT SYSTEMS FOR NAUIGATING THE BIOLOGICAL IHATELLIGENT SYSTEMS FOR RAUIGATING $\$ 250,000$ T171.250

CONTROL OF COAL COMBUSTION SOZAND NOX EMISSIONS BY IN BOILE MA 08 \$212,088

ICP - NEW AWARD ${ }_{\text {IN }} \quad \$ 2,835 \quad \$ 3,917$

GENETIC AMALYSIS OF INTERACTING TROPHIC LEVELS III A STRESSED AZ $06 \quad$ \$594.000 $\$ 194.000$

FY'94 ICP AHARD ${ }_{\text {ME }} \quad \$ 34.801 \quad \$ 17.400$ 


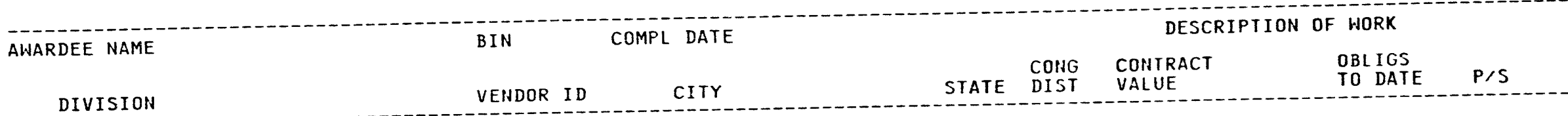

NORTHERN ILLINOIS GAS CO

AC02-76ET10758 06/30/99 060240-0001-4 AURORA

NORTHERN ILLINOIS UNIVERSITY

FG02-91ER40641 06/30/95 060250-0001-8 DE KALB

NORTHERN IOWA UNIVERSITY OF

METAL CASTING CENTER

NORTHERN MARIANA IS CMNHLTH OF

ENERGY OFFICE

ENERGY OFFICE

ENERGY OFFICE

ENERGY OFFICE

- energy office

ENERGY OFFICE

NORTHERN NEF

$$
:
$$

NORTHERN STATES POWER CO

FG02-91CHI0476 12/31/94

FC07-921D13164 07/08/94

FC07-94ID13279 02/15/96

AM06-94RL12943 06/07/95 105510-0001-5 CEDAR FALLS 105510-0001-5 CEDAR FALLS 105510-0101-1 CEDAR FALLS

$$
\begin{gathered}
\text { FG49-89SF } 1823508 / 31 / 94 \\
159860-0001-3 \text { SAIPAN } \\
\text { FG49-90SF } 18698008 / 31 / 94 \\
159860-0001-3 \text { SAIPAN } \\
\text { FG49-91R913021 } 08 / 31 / 94 \\
159860-0101-X \quad \text { SAIPAN } \\
\text { FG49-92R914021 } 08 / 31 / 94 \\
159860-0101-X \quad \text { SAIPAN } \\
\text { FG49-92R914020 08/31/94 } \\
159860-0101-X \quad \text { SAIPAN } \\
\text { FG49-92R900010 } 09 / 30 / 94 \\
159860-0101-X \quad \text { SAIPAN } \\
\text { FG49-93R915020 } 08 / 31 / 95 \\
159860-0101-X \quad \text { SAIPAN } \\
\text { FG49-93R915021 } 08 / 31 / 95 \\
159860-0101-X \quad \text { SAIPAN }
\end{gathered}
$$
COLORADO SPRINGS

FURNISH GAS SERVICE FOR ANL 1 UNDER RATE $4120 \underset{\$ 0}{\text { THERMS PER H }}$

STUDIES OF HIGH ENERGY $\begin{gathered}\text { PHENOMENA USING MUONS } \\ \$ 938,000\end{gathered}$

DESIGN \& CONSTRUCTION OF THE CENTER FOR ENERGY \& ENVIROHMEN

DESIGN \& CONSTRUCTION $\$ 3,976,800 \quad \$ 3.976,800$

METAL CASTING COMPLETITIVENESSRESEARCH PROGRAM

METAL CASTING $03 \quad \$ 1,848,349 \quad \$ 928,754$

"A NATIOHAL ASSISTANCE EXTENSION PROGRAM FOR METAL CASTING:

$$
\text { IA } 02 \quad \$ 3,800,000 \quad \$ 920,376
$$

GRAHT PROGRAMS FOR SCHOOLS AHDHOSPITALS AND BUILDIHGS OHNED

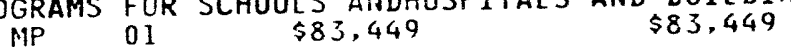

GRANT PROGRAMS FOR SCHOOLS AHDHOSPITALS AND BUILDINGS OHNED

$$
\text { MP } 01 \quad \$ 94,244 \quad \$ 94,244
$$

CYCLE XIII GRANT

CYCLE XIV ADMINISTRATIVE GRANT
MP OI

CYCLE XIV ECM GRANT AHARD

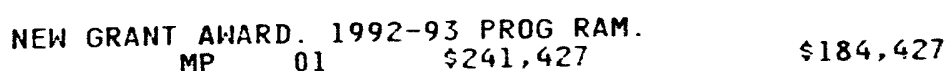

$\begin{array}{lll}\text { CYCLE XV ADMINSTRATIVE GRANT. } & \text { MP } 01 & \$ 35,204\end{array}$

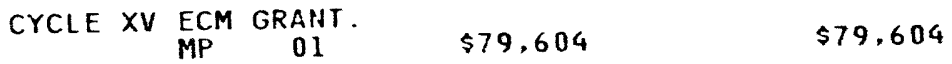

TO INSURE A SAFE WORK IHVIRONMENT THROUGH THE

DISPOSAL OF SPENT NUCLEAR FUELAND OR HIGH LEVEL RADIOACTIVE

DISPOSAL OF SPENT NUCLEAR FUELANID OR HIGH LEVEL RADIOACTIVE




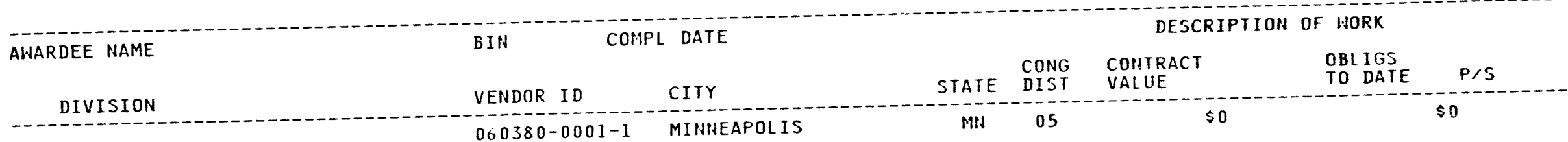

CROI-83NE44473 $03 / 15 / 30$ 060380-0001-1 MINNEAPOLIS

CRO1-83NE44474 03/15/30 060380-0001-1 MIINEAPOL IS

NORTHFIELD MOUNT HERMON SCHOOL

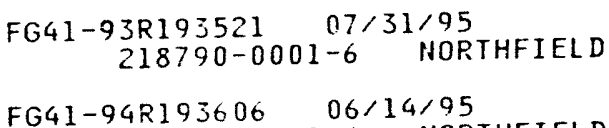

NORTHFIELD VILLAGE OF HIGH SCHOOL DISTRICT \#225

NORTHROP CORP

RESCH \& TECHNOLOGY CTR

NORTHWEST GEORGIA REG HOSP

NORTHHEST IOWA MEDICAL CENTER

NORTHWEST MISSOURI STATE UHIV APPLIED RESEARCH CENTER FOR

NORTHWEST NAZARENE COLLEGE

NORTHWESTERN COLLEGE

NORTHWESTERN UNIVERSITY 060690-0201-6 MARYVILLE

FG05-91ER79033 09/29/94
FG45-93R55152708/31/95 $060450-0102-8$ GLENVIEW

CRO1-83NF $44486 \quad 03 / 15 / 30$ 060490-0101-4 PALOS VERDES ESTATES

FG44-93R435354 09/30/95 175210-0001-3 ROME

FG47-92R704984 08/31/94 $212680-0001-9$ SHELDON

FGO1-92CE50350 09/29/94

FG51-93R02029300/30/94 $060700-0001-3$ NAMPA

FG47-92R704989 08/31/94 ORANGE CITY 060760-0001-5 EVANSTON

FG05-92ER79121 07/31/94 $060760-0001-5$ EVANSTON
DISPOSAL OF SPENT NUCLEAR FULE

$\$ 0$

DISPOSAL OF SPEHT HUCLEAR FUELAHD/OR HIGH LEVEL RADIOACTIVE MN 01 $\$ 0$ $\$ 0$

TIILE III OF THE NATIONAL ENERGY CONSERVATION POLICY ACT TILE III OF THE NATIOHAL ENERGY CONSERVATION POLICY ACT ITLE III OF THE NATIOHAL $\$ 10,800$ MA ICP-NEH AWARD

IL $09 \quad \$ 6.675$

$\$ 3.337$

NUCLEAR FUELAHD/OR HIGH LEVEL RADIOACTIVE

SEE ATTACHED PAGE NO. 2 OF THIS NOTICE OF FINANCIAL
$\$ 20,169$

RANT PROGRAMS FOR SCHOOLS AHCHOSPITALS AND FOR BUILDINGS 0

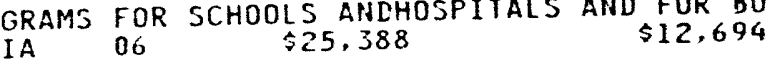
AVAILABLE ETHANOL/GASOLINE
MO COMMERCIALLY

CYCLE 15 TAS AWARD. $\$ 9,500 \quad \$ 4,750$

GRAUT PROGRAMS FOR SCHOOLS ANDHSOPTIALS AND FOR BUILDINGS O IA $06 \quad \$ 12,314$

MULTILAYER OPTICAL SPECIROSCOPIC SCANNER

INSTRUMENTATION - HIGH ENERGY PHYSICS RESEARCH INTO THE BASI IL 09 \$128,455 $\$ 128,455$ 


\begin{tabular}{|c|c|c|c|c|c|c|c|}
\hline AWARDEE NAME & BIN & DATE & & & DESC & F HOFK & \\
\hline DIVISION & VENDOR ID & CITY & STATE & $\begin{array}{l}\text { CONG } \\
\text { DIST }\end{array}$ & $\begin{array}{l}\text { COIITRACT } \\
\text { VALUE }\end{array}$ & $\begin{array}{l}\text { OBLIGS } \\
\text { IO DATE }\end{array}$ & $P / S$ \\
\hline
\end{tabular}

CHEMISTRY DEPT

CHEMISTRY DEPT

CHEMISTRY DEPT

ELECTRICAL ENGINEERING DEPT

MATERIALS SCI \& ENG DEPT

CHEMISTRY DEPT

PHYSICS \& ASTROHOMY

PHYSICS \& ASTROHOMY

MATERIALS SCI \& ENG DEPT

MATERIALS SCI \& ENG DEPT

PHYSICS \& ASTROHOMY

CIVIL ENG DEPT

$$
\begin{aligned}
& \begin{array}{c}
\text { FG02-85ER45220 12/31/94 } \\
060760-0301-4 \text { EVANSTON }
\end{array} \\
& \text { FG02-91ERI4228 11/30/94 } \\
& \text { 000760-0301-4 EVANSTON } \\
& \text { FG45-92R540080 12,31/94 } \\
& \text { 060760-0001-5 EVANSTON } \\
& \text { FG02-86ER13641 09/14/95 } \\
& \text { 060760-0001-5 EVANSTON } \\
& \begin{array}{l}
\text { FG02-87ER13654 01/06/95 } \\
060760-0301-4 \text { EVANSTON }
\end{array} \\
& \begin{array}{c}
\text { FG02-87ER2504707/31/95 } \\
060760-1301-X \text { EVAHSTON }
\end{array} \\
& \text { FG02-89ER45403 08/31/95 } \\
& \text { 060760-0501-7 EVANSTON } \\
& \text { FG02-92ER14303 09/14/95 } \\
& \text { 050760-0001-5 EVAHSTON } \\
& \text { FG45-93R551535 08/31/95 } \\
& \text { 060760-0001-5 EVAHSTON } \\
& \text { FG02-86ER13511 11/14/95 } \\
& \text { 060760-0301-4 EVANSTON } \\
& \text { FG02-87ER40344 11/30/95 } \\
& \text { 060760-0801-6 EVANSTON } \\
& \text { FG02-91ER40684 11/30/95 } \\
& \text { 060760-0801-6 EVANSTON } \\
& \text { FG02-92ER45475 12/31/95 } \\
& \text { 060760-0501-7 EVAHSTON } \\
& \text { FG02-84ER45097 05/31/96 } \\
& \text { 060760-0501-7 EVANSTON } \\
& \text { FG02-84ER4512501/31/96 } \\
& \text { 060760-0801-6 EVANSTON } \\
& \text { FG02-86ER13484 01/31/96 } \\
& \text { 060760-0401-0 EVANSTON }
\end{aligned}
$$
INIERFACIAL ELECTROCHEMICAL TRANSPORT IN BATIERIES EMPLOYIHG

VIBRATIONS DYHAMICS IN PHOTOINDUCED ELECIRON IRANSFER.

$\begin{array}{llll}\text { IL } 09 & \$ 327.655 & \$ 327.655\end{array}$

ICP - NEH AHARD.

$\$ 35,000$

$\$ 22.750$

THIHIING AND RUPTURE OF A LIQUID FILM ON A HORIZONTAL HEATED IL 09 S1,081.829 FILM ON A $\$ 922,953$

CHEMICAL INTERACIIOHS IN MULTIMETAL/ZEOLITE CATALYSTS IL 09 \$ $\$ 916,453 \quad \$ 916.453$

OPTIMIZATION SOFTHARE AND PARALLEL EIGENVALUE

$$
\text { IL } 09 \text { \$ } \$ 587.056 \quad \$ 510.136
$$

ATOMIC STRUCTURE AND CORUPOSITIONS OF IHIERNAL

$$
\text { IL } 09 \quad \$ 682,474 \quad \$ 591.618
$$

STABLILTY \& DYNAMICS OF SPATIOTEMPARAL STRUCTURE

IL $09 \quad \$ 215,236 \quad \$ 142.872$

ICP-NEH AWARD. $09 \quad \$ 488,700 \quad \$ 217,028$

SUPPORTED F-ELEMENT ORGAHOMETALLIC COMPLEXES: SURFACE

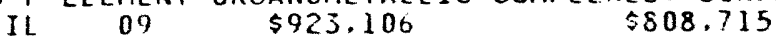

NUCLEAR PHYSICS STUDIES WITH MEDIUM ENERGY PROBES

SILICON TRACKER PROFOSAL FOR THE DO UPGRADE-OUTSTANDING JUHI

OS 09 \$4.596.000

ATOMIC RESOLUTIOH ANALYTICAL ELECTROH MICROSCOPY GF GRAIN

$$
\text { IL } 09 \text { \$272,011 } \$ 200,085
$$

POINT DEFECT CLUSTERS \& ELECTRICAL BEHAVIOR IN TRANSITON MET IL 09 \$2,313.699 $\$ 2,115,491$

STUDIES OF THE SHEAR RESPOHSE

$$
\text { IL } 09 \text { \$1,352,497 \$1,244,347 }
$$

"EFFECTS OF CRACK GEOMEIRY ANDHEAR-CRACK MATERIAL BEHAVIOR 


\section{DIVISION}

APPLIED MATH

CHEMICAL ENGINEERING DEPT

MATERIALS SCI \& ENG DEPT

CIVIL ENG DEPT

CIVIL ENGINEERING DEPT

MATERIALS SCI \& ENG DEPT

MATERIALS SCI \& ENG DEPT

CHEMISTRY DEPT

CHEMISTRY DEPT

ENGINEERING DEPT

PHYSICS \& ASTRONOMY

\section{NORWICH CITY OF}

PUBLIC SCHOOLS

NOTRE DAME CONSOLIDATED SCHOOL
VENDOR ID CITY $87 E R 25027--03 / 31 / 96-\cdots$
$060760-1201-3 \quad$ EVANSTON

FG02-87ER13725 05/31/96 060760-0201-8 EVANSTON FG02-87ER45314 06/30/96 060760-0501-7 EVANSTON FG02-90ER45434 01/27/96 060760-0001-5 EVANSTON FG02-91ER45460 01/15/96 060760-0401-0 EVAHSTOH FG02-93ER14344 04/14/96 060760-0401-0 EVANSTON FG02-86ER45229 11/30/96 060760-0501-7 EVANSTON FG02-85ER45209 04/30/97 060760-0501-7 EVANSTON FG02-86ERI3640 04/30/97 060760-0301-4 EVANSTOH FG02-87ER13808 05/14/97 060760-0301-4 EVANSTON FG02-88ERI3927 05/31/97 060760-1401-6 EVANSTON FG02-88ER45372 03/31/97 060760-0801-6 EVANSTON

FG41-93R193430 07/15/94 $060760-0003-1$ BOSTON

FG41-92R191406 08/31/94 $060820-0201-6$ NORHICH

FG41-94R195602 06/30/96 $184110-0002-8$ CENTRAL FALLS FG41-94R192600 04/30/95
$237540-0001-2$ SACO

\section{CONG CONTRACT}

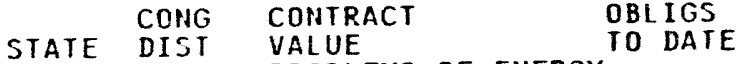
IN NONL INE

$\$ 989,795$ $P / S$ SOLID STAIE, SURFACE AND CATALYTIC STUDIES OF OXIDES PLASMA, PHOTON AHD BEAM SYNTHESIS OF DIAMOND FILMS AND IL $\quad 09 \quad \$ 773,145 \quad \$ 616,671$ DEPOSTION AHD FROPERTIES OF NOVEL NITIDE SUPERLATTICE IL 09 \$508.263 $\$ 422,11$ ? $\begin{array}{ccc}\text { THE EFFECT OF MOISTURE ON THE MICROSTRUCTURE OF CEMENT-BASED } \\ \text { IL } & 09 & \$ 588.593\end{array}$ SHEAR STRAIN LOCALIZATION AND FRACTURE EVOLUATIN IN ROCKS IL 09 \$238,577 \$147,565

"Use OF AHOMALOUS SMALL AHGLE X-RAY SCATTERING TO INUESTIGAT IL 09 SMAL $\$ 993,428$ S

DEFECT STRUCTURE OF SEMICONDUCTING AND INSULATING OXIDES IL 09 \$ $\$ 929,453$ OF ORGANOMETALLIC SURFACE CHEMISTRY
IL
09 จ 781.737 INTRAMOLECULAR ELECTRON TRANSFER RATES IL $09 \$ 1.215,854 \quad \$ 925.854$ ATTENUATION OF WAVES IN PARTIALLY SATURATED POROUS IL 09 \$ $\$ 473,957$ \$ 360,987

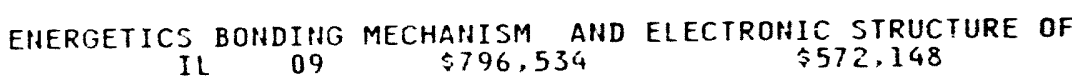

TITLE III OF THE NATIOHAL ENERGY CONSERVATION POLICY ACT

TITLE III OF THE NATIONAL ENERGY CONSERVATION POLICY ACT $C T$ TI $02 \quad \$ 196,995 \quad \$ 98,497$

FY'94 ICP AWARD.

RI $\quad 01 \quad \$ 10,550$

$\$ 9,495$

FY 1994 ICP AWARD.

$\$ 2,564$

$\$ 2,308$ 
AWARDEE NAME

BIN

COMPL DATE

VENDOR ID

CITY STATE

DIVISION

\section{DESCRIPTION OF WORK}

AAO2-76CHOO177 12/31/99 $060900-0001^{-9}$ SOUTH BEND

NOTRE DAME UNIVERSITY OF

RADIATION LAB

CHEMICAL ENGINEERING DEPT

PHYSICS DEPT

PHYSICS DEPT

PHYSICS DEPT

CHEMICAL ENGINEERING DEPT

PHYSICS DEPT

CIVIL ENGINEERING DEPT FG22-94PC94209 $08 / 31 / 97$
CHEMISTRY \& BIOCHEMISTRY DEPT $060900-0601-7$

CHEMICAL ENGINEERING DEPT

NOTUS SCHOOL DISTRICT 135

NOVA COLOR LABS

NOVAK ENGINEERING INC
ACO2-76ERO0038 12/31/95 $060900-0401-4$ SOUTH BEND

FG02-88ERI3913 10/31/94 060900-0501-0 SOUTH BEND

FG02-88ER45373 06/14/96 060900-0301-8 SOUTH BEND

FG02-91ER40640 07/31/94 060900-0301-8 SOUTH BEND

FG02-91ER40649 07/31/94 060900-0301-8 SOUTH BEND

FG02-92ER61403 03/31/95 060900-0001-9 SOUTH BEND

FG02-92ER14269 06/14/95 060900-0501-0 SOUTH BEND

FG02-92ER14283 10/31/95 $060900-0301-8$ SOUTH BEND

FG02-93ER14391 08/31/96 $060900-0101-5$ SOUTH BEND

FG22-92PC92529 09/14/95 060900-0501-0 SOUTH BEND FG51-92R020131 08/31/94
$188600-0001-7$

AB01-94AD25725 06/30/95 095960-0002-0 CARLISLE

AC08-92NV10980 04/06/96 $218050-0001-2$ JACKSON
EQUIPMENT ${ }_{\text {IN }}$ OAN TO DATE

P/S

MULTIPROGRAM RADIATION LABORATORY PRIMARILY INVOLVED IN

$$
\text { IN } 03 \text { \$ } \$ 93,946,371 \quad \$ 76,461,343 \text { AG51 }
$$

STUDY OF INTERFACIAL BEHAVIOR IN COCURRENT GAS-LIQUID FLOWS IN $D 3 \quad \$ 331,142 \quad \$ 331,142$

SIHGLE - ELECTRON CHARGING $\underset{\text { IN }}{\$ 4}$ EFFECTS

$\$ 366,775$

THEORETICAL STUDIES OF NUCLEARSTRUCTURE.

$$
\text { IN } 03 \quad \$ 154,000 \quad \$ 154,000
$$

RESEARCH IN HIGH ENERGY SSC PHYSICS

$$
\text { IN } 03 \text { S225,000 }
$$

$\$ 225,000$

HYDRODYNAIE CONTROLS ON PARTICLE TRANSPORT THROUGH IN 03 S $\$ 348,118 \quad$ PARTICLE TRANSPORT $\$ 345,058$

WAVE DYHAMICS ON TALLIHG AND ITS EFFECTS ON HEAT/MASS

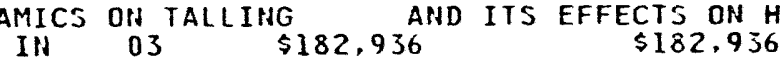

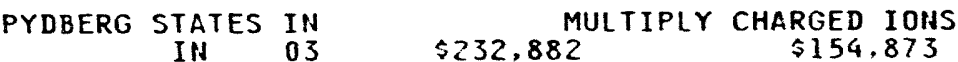

ENERGY PARTINING OF SESMIC WAVES IN FRACTURED

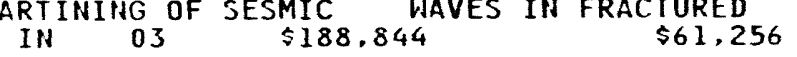

REGULATION OF COAL DEGRADATIOHBY FUNGI IN 03 \$312,122

$\$ 199,998$

TRANSIENT STUDIES OF LOW TEMPERATURE METALLO-OXIDE CATALYSTS IN 03 \$198,218 $\$ 192,218$

CYCLE 14 ECM AWARD. TOTAL AWA RD INCLUDES HARDSHIP AND EXXON ID $01 \quad \$ 92,044 \quad \$ 16,101$

BPA FOR PHOTO AND RELATED SERVICES
PA 19
$\$ 0 \quad T 015$

DESIGN AND INSPECTION SERVICES(A/E) FOR A POWER DISTRIUBUTI

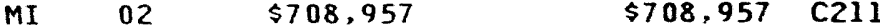




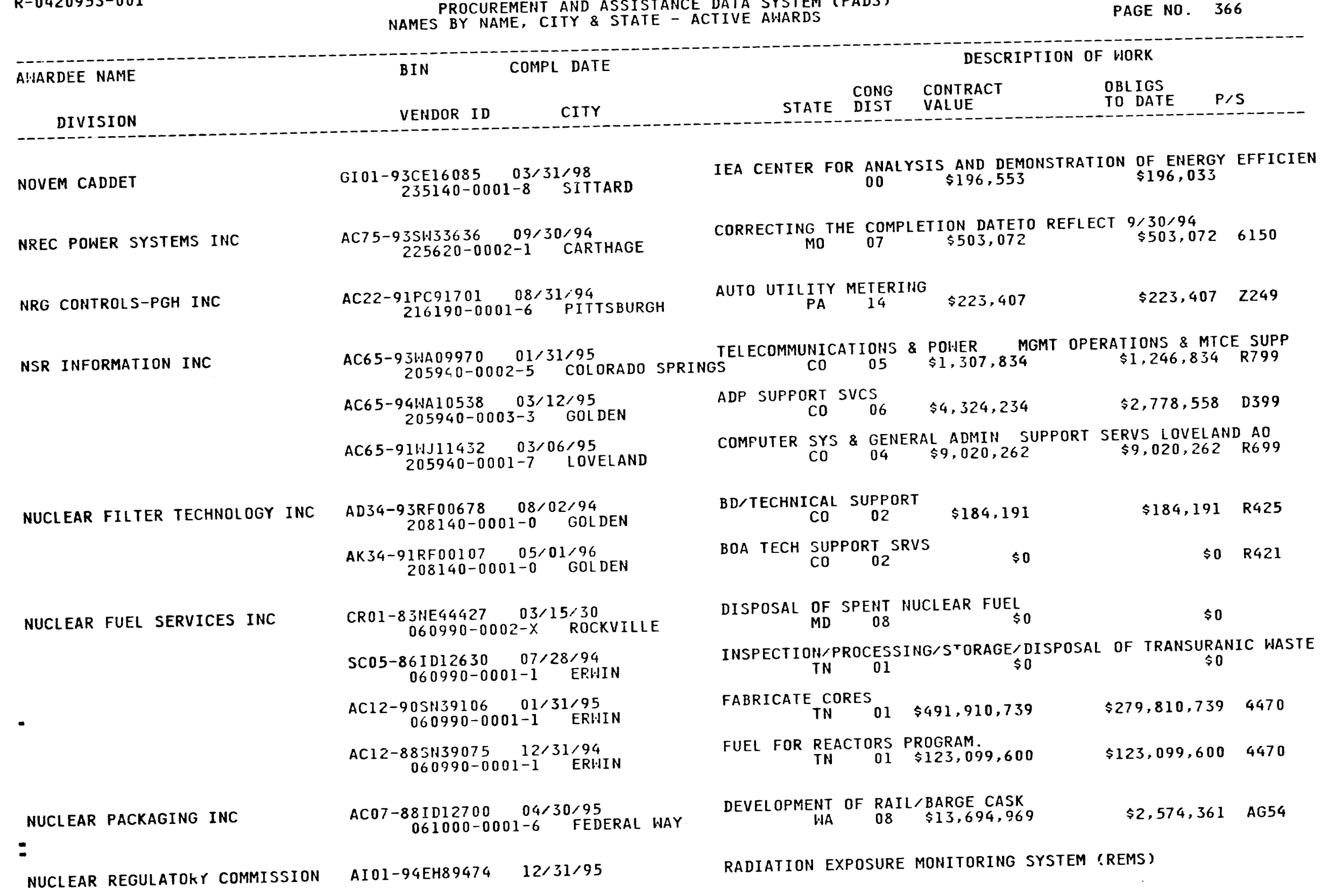




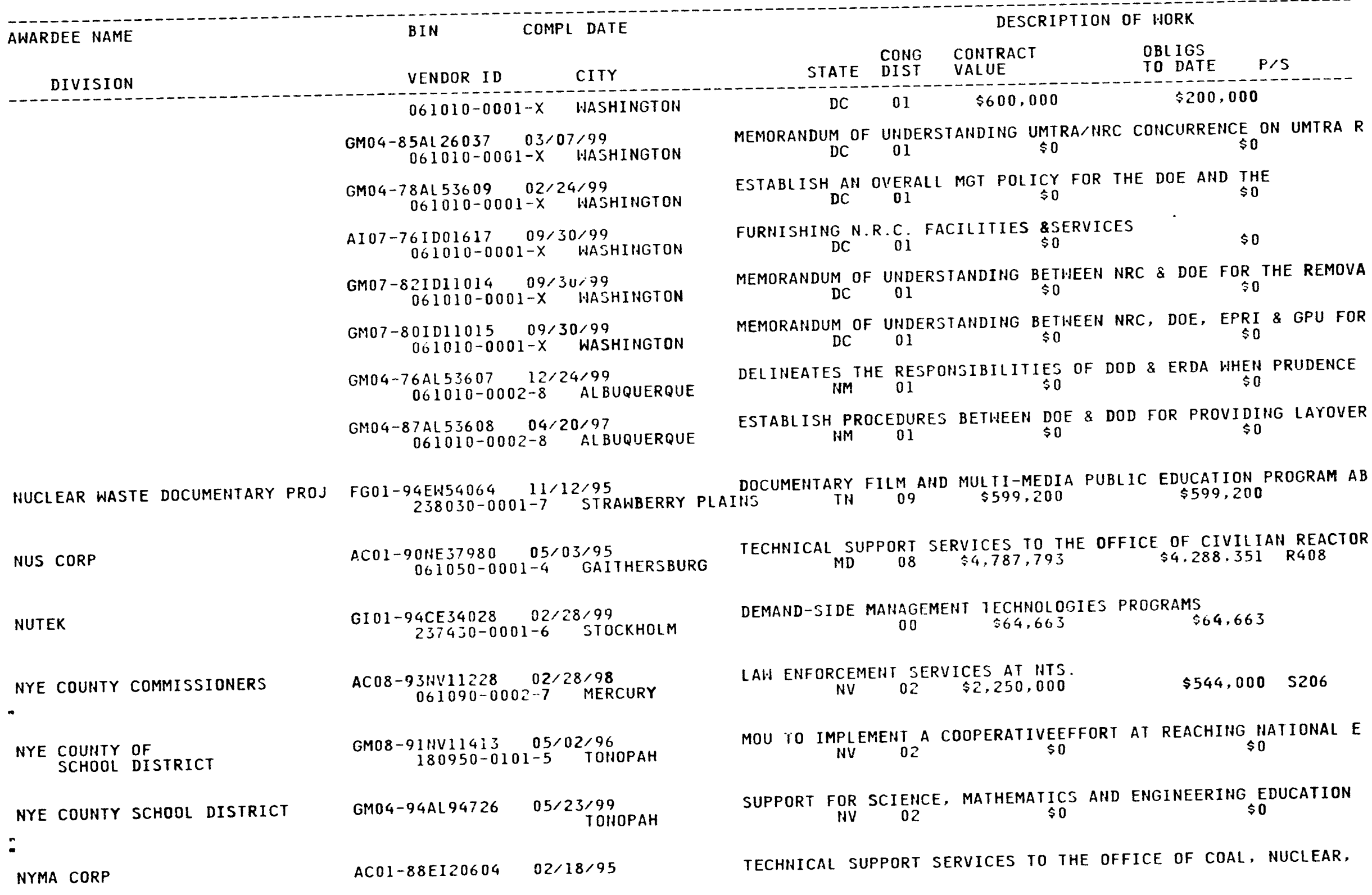




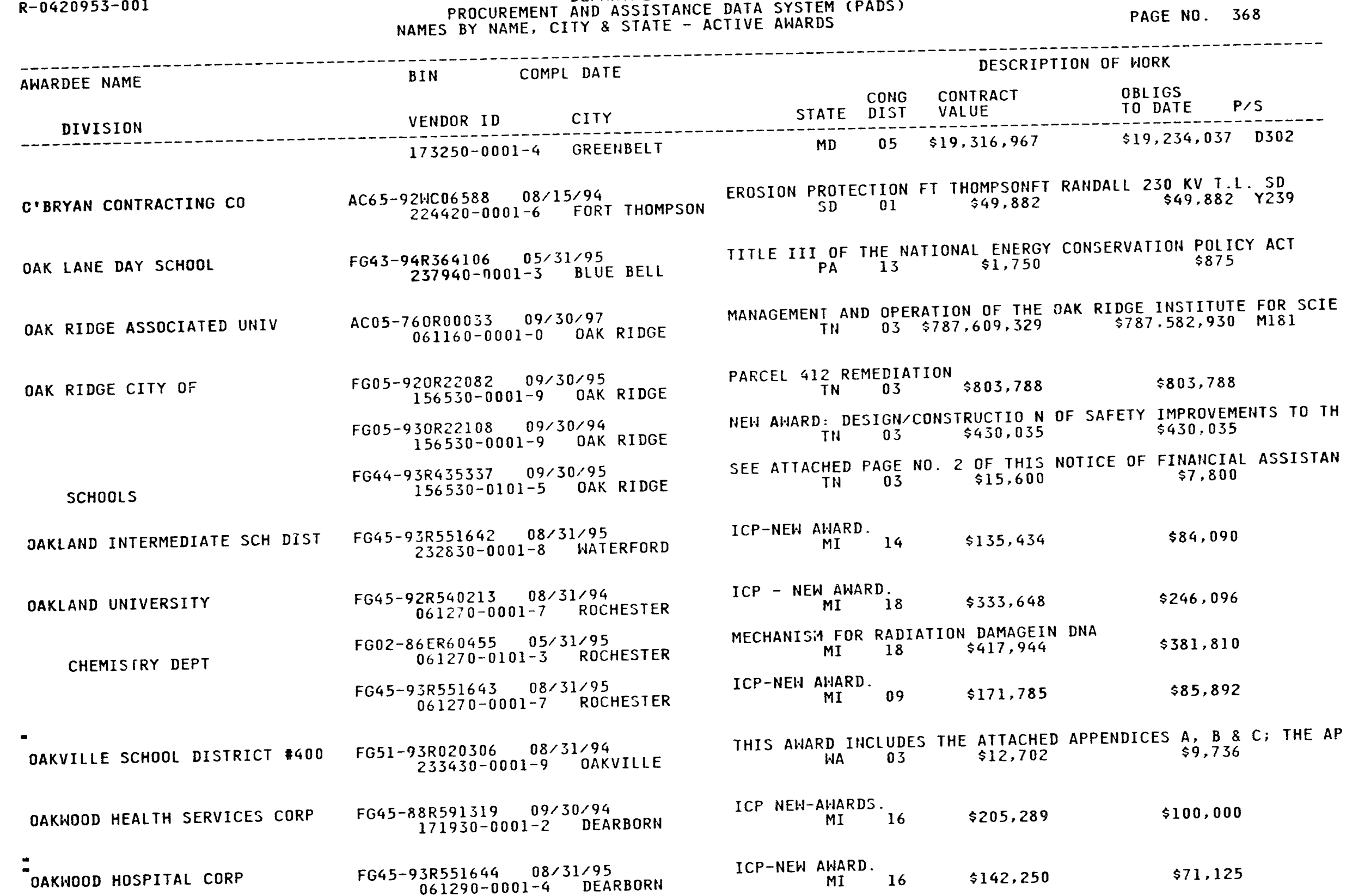




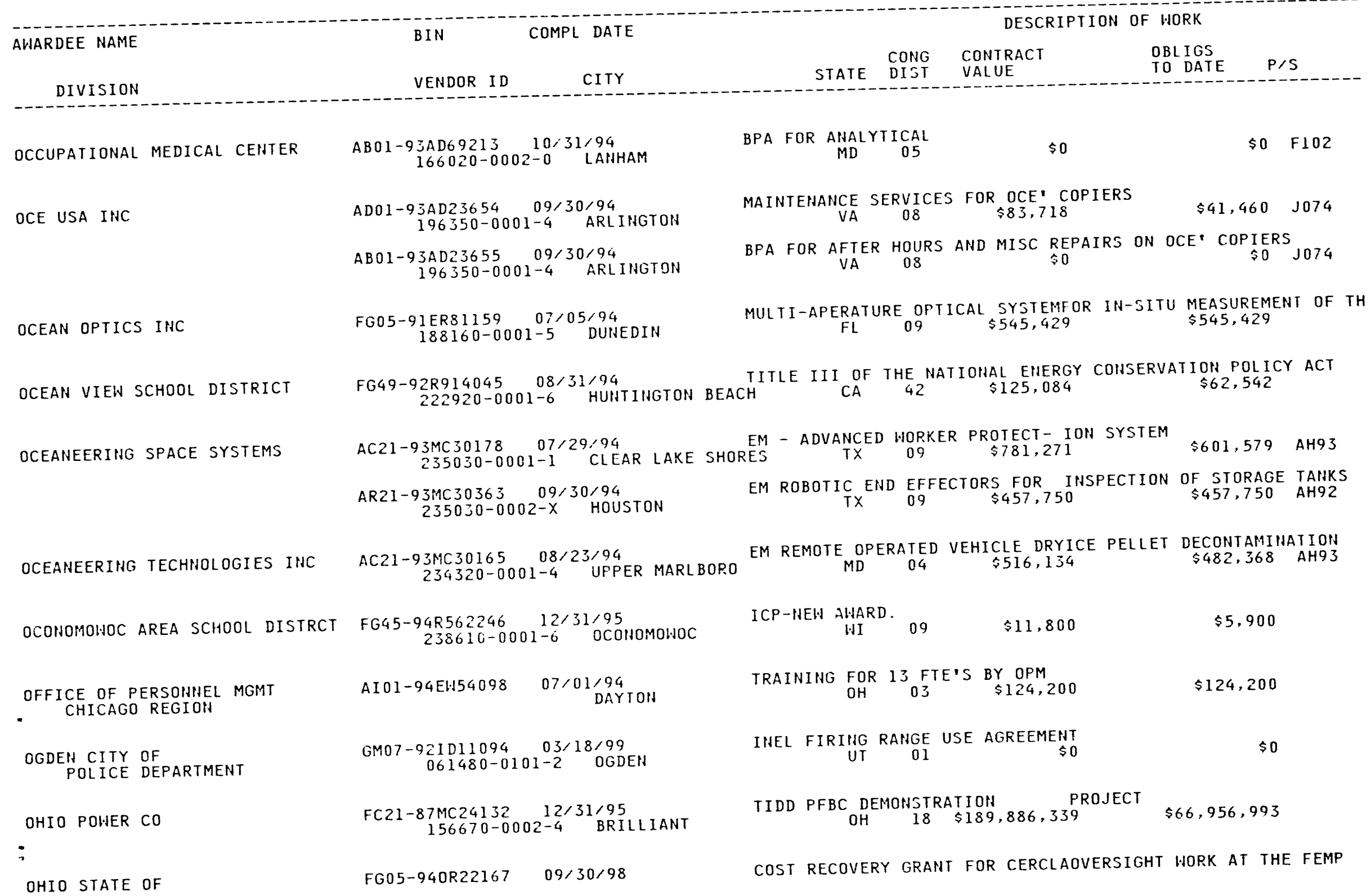




AWARDEE NAME

DIVISION

COMPL DATE

DESCRIPTION OF WORK

$061570-0003-1$ CINCINHATI

PUBLIC UTILITIES COMMISIION FCO1-91E122717 $08 / 07 / 96$
$061570-0901-2$ COLUMBUS FG04-93AL97373 $09 / 29 / 97$
ENVIRONMENTAL PROTECTION AGCY $061570-1501-2$ COLUMBUS ENERGY $R$ \& D DIV FG45-76C560015 09/30/94
$061570-0601-3$ COLUMBUS ENERGY R \& D DIV FG45-80CS69118 09/30/94
$061570-0601-3$ COLUMBUS ECONOMIC \& CMTY DEVELOP DEPT FG45-89CH10413 $03 / 31 / 95$ COLUMBUS FG45-92R530274 12/31/94 DEVELOPMENT DEPT $2 R 530274$
$061570-1201-3$ COLUMBUS YOUTH SERVICES DEPARTMENT OF $F G 45-94 R 562188 \quad 12 / 31 / 95$
$061570-1601-9$ COLUMBUS FG05-940R22206 01/31/99 061570-0002-3 MIAMISBURG

OHIO STATE UNIV RESEARCH FDH CHEMISTRY DEPT

PHYSICS DEPT

MICROBIOLOGY DEPT

. HELDING ENGINEERING

PHYSICS DEPT

PHYSICS DEPT

: PHYSICS DEPT
FG02-86ER13529 12/31/95 061580-0101-5 COLUMBUS FG02-86ER45271 10/31/96 061580-0601-7 COLUMBUS FG02-87ER13731 06/30/95 $061580-0501-0$ COLUMBUS FG02-87ER13749 06/30/96 061580-0701-3 COLUMBUS FG02-87ER45326 02/14/96 061580-0601-7 COLUMBUS FG02-88ER13916 09/30/94 061580-0601-7 COLUMBUS FG02-88ER45347 11/14/95 061580-0601-7 COLUMBUS

FG02-89ER14004 03/31/95

\begin{tabular}{|c|c|c|c|c|}
\hline STATE & $\begin{array}{l}\text { COHG } \\
\text { DIST }\end{array}$ & $\begin{array}{l}\text { CONTRACT } \\
\text { VALUE }\end{array}$ & $\begin{array}{l}\text { OBLIGS } \\
\text { TO DATE }\end{array}$ & $P / S$ \\
\hline $\mathrm{OH}$ & 04 & $\$ 8,200,000$ & $\$ 1,497$, & \\
\hline
\end{tabular}

COLLECTION \& ANALYSIS OF DATA RELATED TO THE ENERGY INFO ADM $O H=15 \quad \$ 30,814 \quad \$ 15,407$

DOE COST RECOVERY GRANT TO PROVIDE COSTS FOR OVERSIGHT $\begin{array}{lll}\mathrm{R} \quad 07 & \$ 1,903,451 & \$ 1,903,451\end{array}$

OHIO BASE STATE ENERGY COHSERVATION PLAN OH $10 \$ 24,459,644 \quad \$ 15,345,308$ STATE OF OHIO ENERGY EXTENSIONSERVICE $\mathrm{OH} 10 \mathrm{9} \quad \$ 7,103,636 \quad \$ 4,05 ? 466$ WEATHERIZATION ASSISTANCE FOR LOW INCOME PERSONS - STATE OF $\mathrm{OH} \quad 15 \$ 116,239,883 \quad \$ 64,914,464$

NATIONAL INDUSTRIAL COMPETITIVENESS THROUGH ENVIRONMENT, ENE $O H$ IS $1,900,000 \quad \$ 441,000$

ICP-NEW AWARD

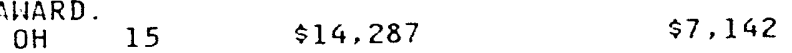

AGREEMENT IN PRIHCIPLE OVERSIGHT AND SUPPORT OF DOE'S ENVIRO $\mathrm{OH} \quad 03$ \$1 $1,000,000 \quad \$ 2,113,885$

MOLECULAR ORBITAL STUDIES OF THE BONDING IN HEAVY ELEMENT

$$
\mathrm{OH} 15 \text { \$ } \$ 581,893 \quad \$ 507,913
$$

MOLECULAR FERROMAGNETISM

$$
\text { OH } 15 \$ 1,760,515 \quad \$ 1,334,223
$$

STRUCTURE AHD REGULATIOH OF METHANOGEN GENES

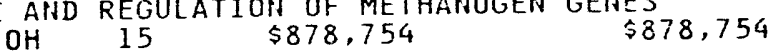

INVESTIGATIONS OF ULTRASONIC SURFACE WAVE INTERACTION WITH

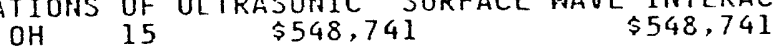

THEORETICAL STUDIES OF DYHAMICS AHD CORRELATIONS IN OH
STUDIES OF

PHYSICS OF CELLULAR AUTOMATA AND COHEREHCE AND CHAOS IN $\mathrm{OH} 15 \quad \$ 400,030$ \% $\$ 400,033$

STRONGLY INTERACTING FERMION SYSTEM

MOLECULAR CHARACTERISTICS OF THE LIGHIN FORMING PEROXIDASE 


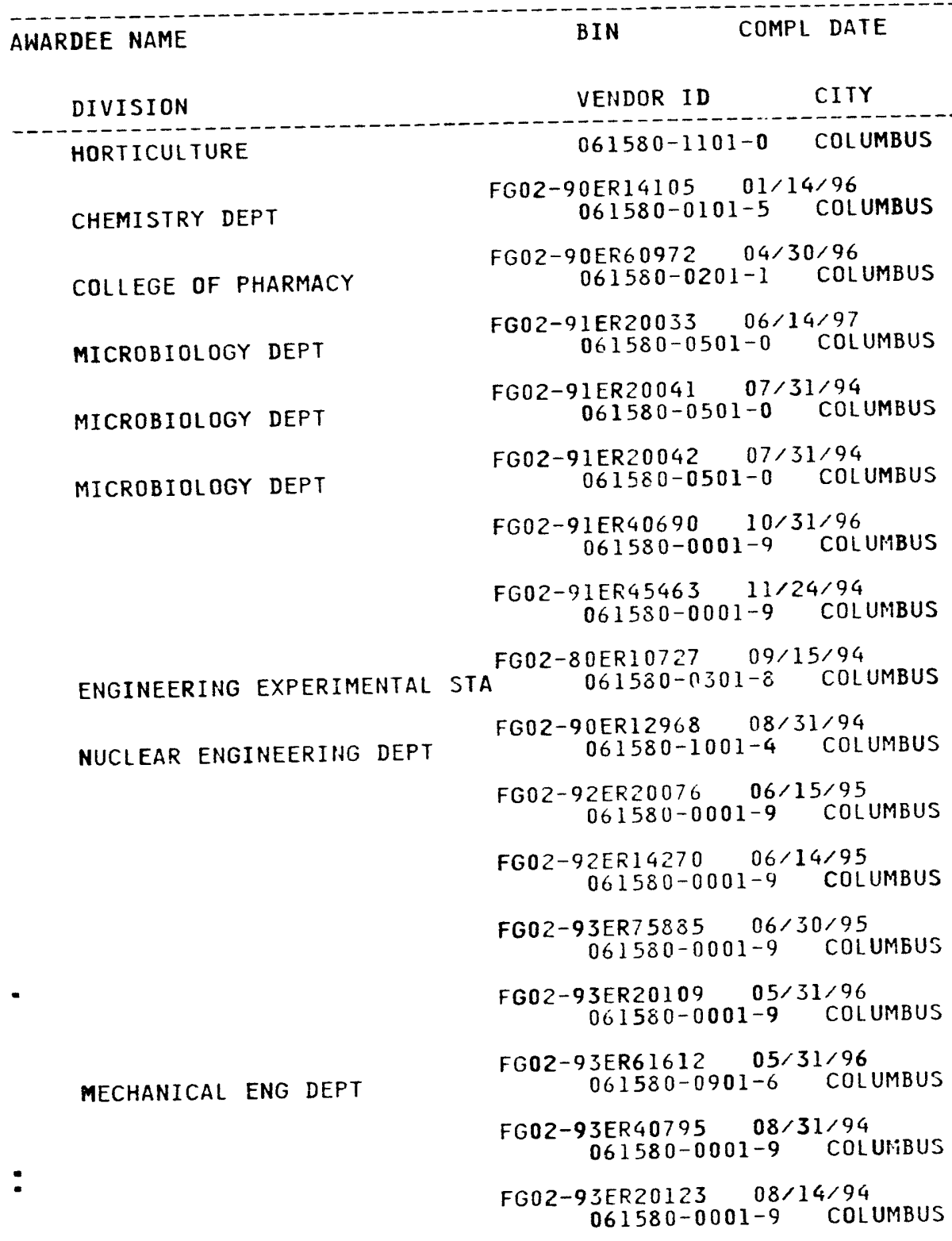

DESCRIPTION OF WORK

CONG CONTRACT

OH 15 \$581,121 $\$ 581,121$

PHOTO UNDUCED ELECTRON $\$ 548,000$ TRANSFER REACTIONS IN

SYNTHESIS \& EVALUATION OF BARON COMPOUNDS FOR NEUTRON

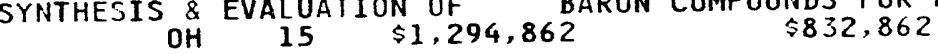

REGULATION OF ALTERNATIVE CO2 FIXATION PATHWAYS IN PRO-CARYO

OH 15 \$567,000 $\$ 467,000$

STRUCTURE \& REGULATION OF AN ARCHAEBACTERIAL PROMOTER: AN IN OH 15 \& $\$ 246,535 \quad \$ 246,535$

TRANSMETHYLATION REACTIONS DURING METHANOGENESIS FROM ACETAT OH 15 \$243,000 $\$ 243,000$

PROTOTYPE STUDY OF A NEH CENTRAL DRIFT CHAMBER FOR CLEO II \& $O H \quad 15$ \$11,892,000 $\$ 6,334,500$

NEW CARBOHYDRATE-BASED MATERIALS.

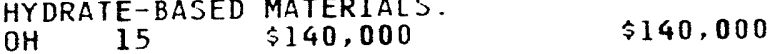

UNIVERSITY REACTOR SHARING
$\mathrm{OH}$
$\$ 377,000$
$\$ 377,000$

UNIVERSITY REACTOR INSTRUMENTATION PROGRAM

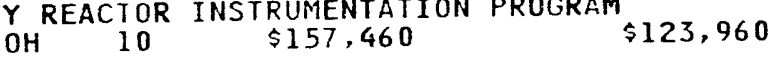

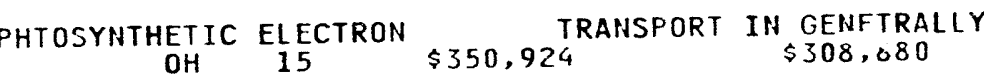
THE EVOLUTION OF A HELE-SHAW INTERFACE \& RELATED PROBLEMS
OH

PROPOSAL TO PLAN FOR AND SHIP HEU FUEL FROM THE OHIO STATE $\mathrm{OH} 15 \quad \$ 39,534 \quad \$ 39,534$

BIOSYNTHESIS OF HYDROCARBONS

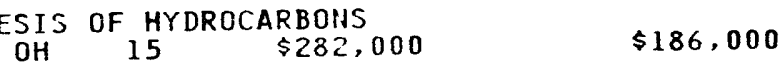

AN ACCELERATOR NEUTRON SOURCE FOR BNCT $\$ \$ 403,700$

SILICON DRIFT CHAMBER STUDIES FOR THE RHIC STAR EXPERIMENT SILICON DRIFT CHAMBER STO50,000 $15 \quad \$ 50,000$

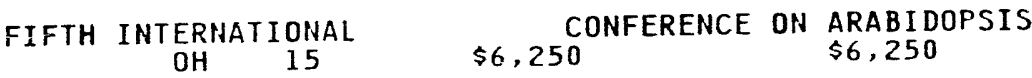




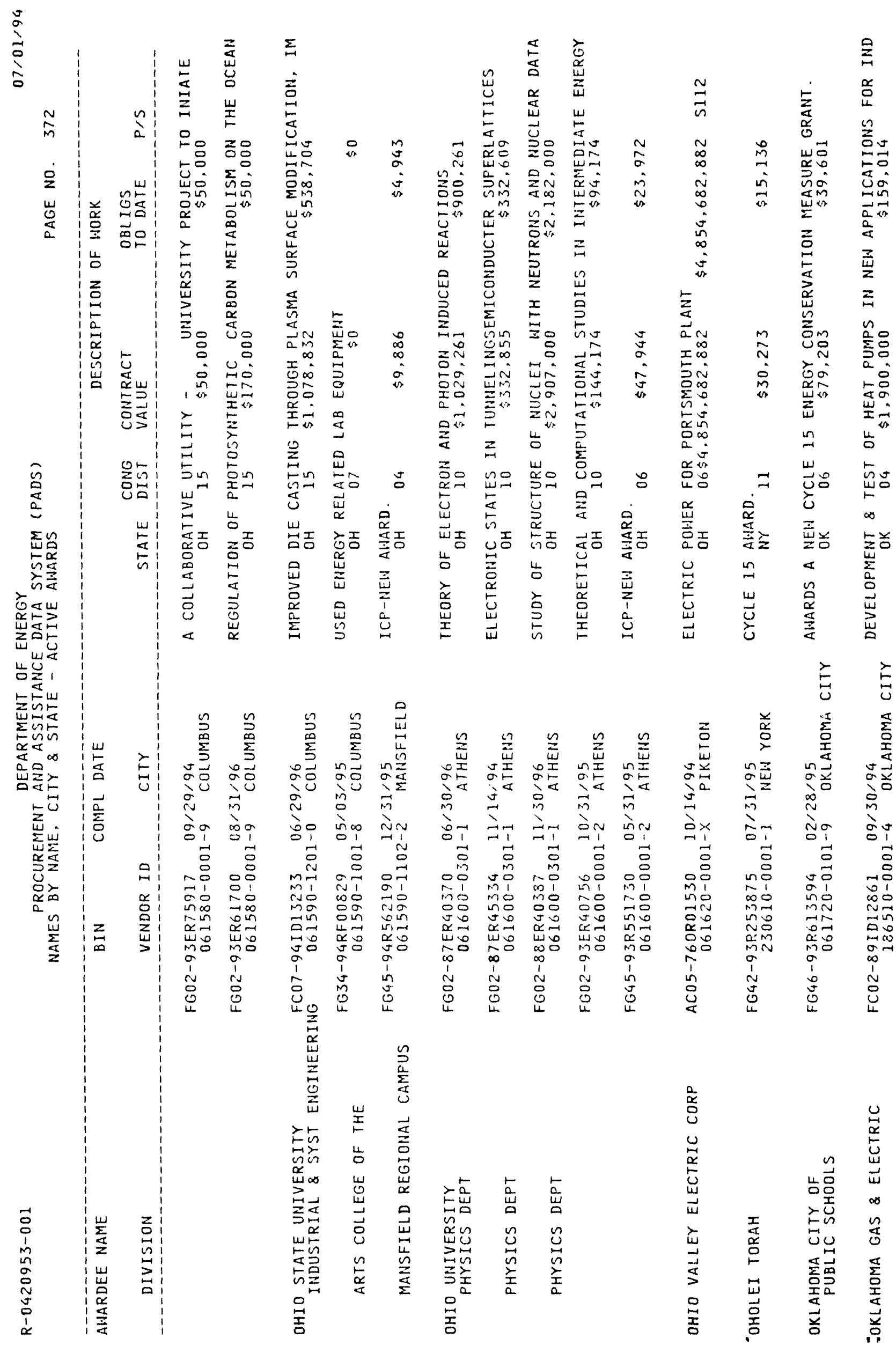




\section{AWARDEE NAME}

BIN

COMPL DATE

DESCRIPTION OF WORK

DIVISION

OKLAHOMA STATE OF COMMERCE DEPT

OKLAHOMA STATE UNIVERSITY

AGRICULTURE DIVISION DF

COLL OF ARTS \& SCIENCES

ENGINEERING RESEARCH OFFICE OF $\begin{array}{ccc}\text { FG46-94R689410 } 03 / 31 / 95 \\ 061770-0701-5\end{array}$ FG02-93ER20102 $03 / 31 / 96$
$061770-0001-0$ STILLWATER FG02-93ER61680 08/31/96 061770-0001-0 STILLWATER

FG02-92CH10534 04/30/97 $061770-0001-0$ STILLWATER

FG02-94ER40852 04/30/98 061770-0001-0 STILLHATER

OKLAHOMA UNIVERSITY OF -

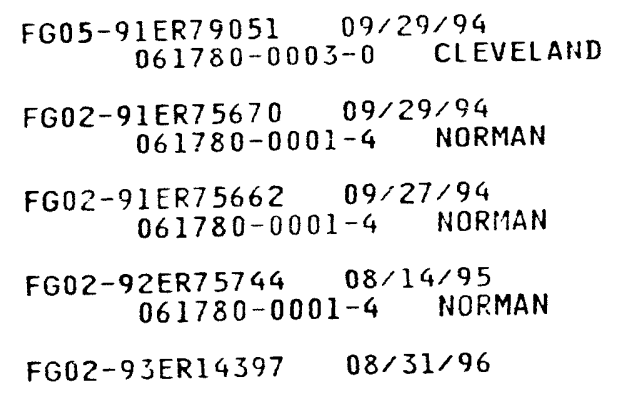

AWARDS \$1,599,672NEH FEDERAL FUNDS UNDER NEW WEATHERIZATION CONG CONTRAC
STATE DIST VALUE

$O B L I G S$ TO DATE P/S

MINORITY HONORS TRAINING \& INDUSTRIAL ASSISTANCE PROGRAM OK 02 \$828,900 $\$ 391,568$ ENERGY RELATED RESEARCH IN THEAREAS OF ENERGY CONSERVATION $O K=03 \quad \$ 4,322,175 \quad \$ 3,393,655$

COMBUSTION OF COTTON GIN TRASHTO PROVIDE PARTIAL DRYING OK $O 3 \quad \$ 134.997 \quad \$ 63.000$ THEORETICAL STUDIES IN WEAK E LECTROMAGNETIC AND SIRONG INTE THEORETICAL STUDIES IN $\$ 9613,036 \quad \$ 801,000$

RHEO-OPTICAL STUDIES OF MODEL "HARD SPHERE" SUSPENSIOHS RHEO-OPTICAL STUS 03 \$467,459 $\$ 425,306$ AWARDS $\$ 30,000$ NEH FEDERAL FUNDS FOR THE SUPPORT OF A TELECO $0 \mathrm{~K} \quad 03 \quad \$ 30,000 \quad \$ 30,000$

THE STRUCTURE OF PEETINS FROM COTTON SUSPENSIOH CULTURE CELL OK ${ }_{03} \$ 310,932 \quad \$ 208,646$

COMPARATIVE EVOLUTION OF THE REC A GENE OF SURFACE AND DEEP OK 03 \$118,223 $\$ 118,223$

PLANNING DESIGN \& CONSTRUCTIOHOF THE ADVANCED TECHNOLOGY OK 03 \$ $\$ 19,845,345 \quad \$ 6,812,345$ $\begin{array}{rcc}\text { THEGRETICAL STUDIES IN WEAK, ELECTROMAGNETIC AND STRONG } \\ \text { OK } \\ 03 & \$ 280,000 & \$ 70,000\end{array}$

FUHDS FOR PURCHASE OF GAS CHROMATOGRAPH-ISOTOPE RATIO MASS S OK $02 \quad \$ 300,000 \quad \$ 150,000$

PLAINING PROPOSAL FOR DOE/EPSCOR PROGRAM.

OK $04 \quad \$ 140,333$ P $\$ 120,000$

DOE/EPSCOR TRAINEESHIP PROPOSAL

$\$ 500,000$

EHERG, ENVIRONMENT \& POLICY CHOICES: SUMMER INSTITUTES FOR

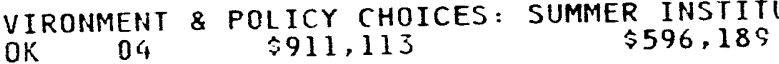

$N / A$ 


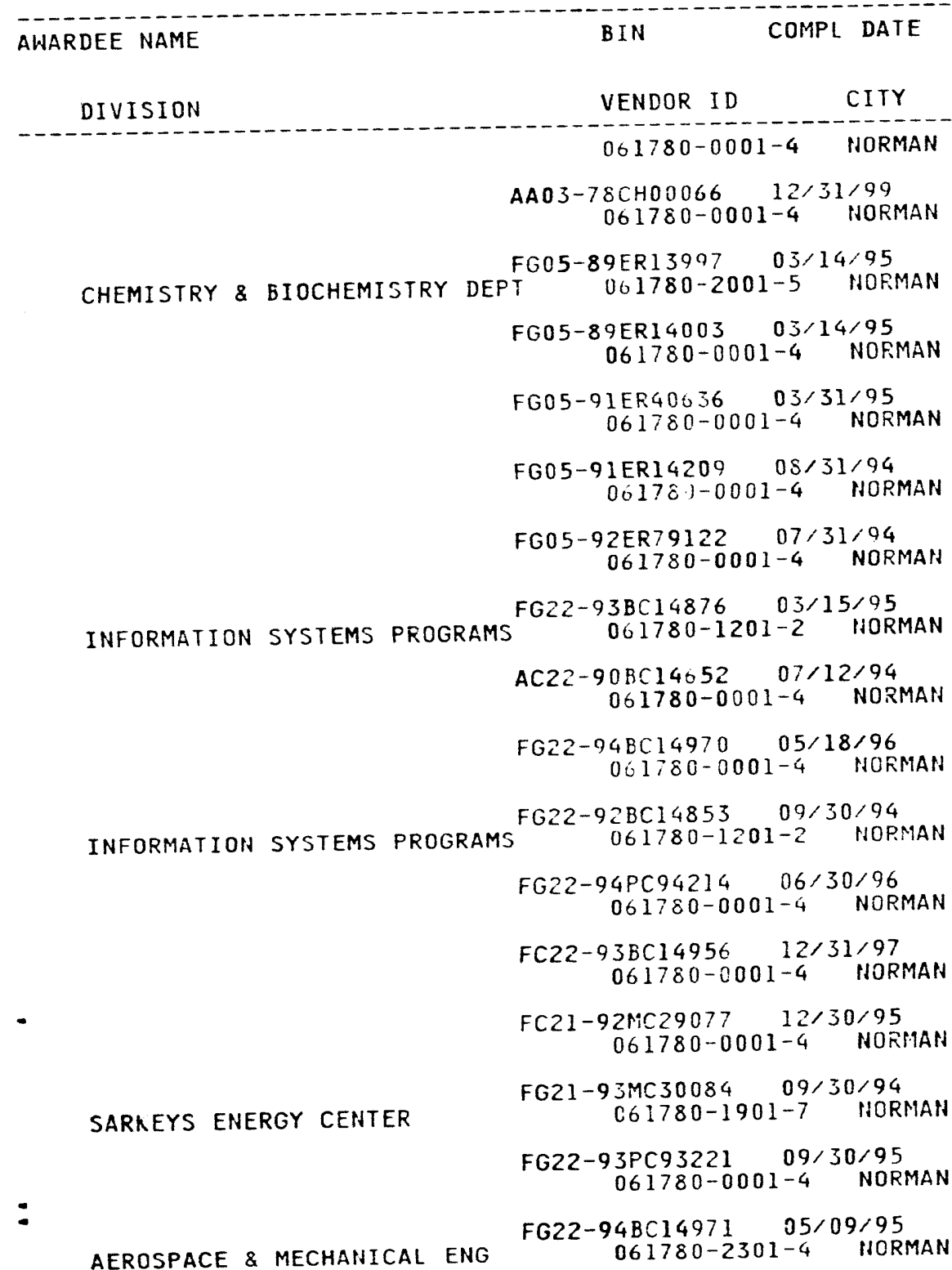

DESCRIPTION OF WORK

TO DATE P/S $\$ 247,259$

SRSA BASIC AGREEMENT OK 04

$\$ 247,259$

$\$ 0$

TRANSITION METAL-MEDIATED THERMAL AND PHOTOCHEMICA EFFECTS OF COMMUNITY STRUCTUREON THE KINETICS OF AHAEROBIC

HADROPRODUCTION OF CHARMED ANDBOTTOM MESONS
OK 04
จ $2.025,000$
$\$ 2,010,000$

HYDROCARBON MIGRATION EVENTS: DEVELOPMENT AHD APPLICL $O K=04 \quad \$ 221.316 \quad \$ 221.316$

1992 INSTRUMENTATIOH - HIGH ENERGY PHYSICS VLSI INSTRUMENTAT $\begin{array}{llll}0 K & 04 & \$ 128,670 & \$ 128,670\end{array}$

FLUVIAL-DOMINATED DELTAIL RESERVOIRS IN THE SOUTHERH MIDCONT OK 04 \$2 3532 ?

AN INVESTIGATION OF DISPERSIVITY AS A RESERVOIR ROCK CHARACT $\begin{array}{llll}0 K & 04 & \$ 439,9 u J & \$ 332,871 \text { AGll }\end{array}$ GYPSY FIELD PROJECT IN RESERVOIR CHARACTERIZATION

NATURAL RESERVOIRS INFORMATIONSYSTEM (NRIS) FOR THE STATE 0 DK $\$ 952.661$ \$700,000

ORGANOSULFUR COMPOUNDS IN COALS AS DETERMINED BY REACTION HI OK $04 \quad \$ 134,048 \quad \$ 134,048$

IDENTIFICATION AND EVALUATION OF FLUVIAL-DOMINATED DELTAIC R OK $04 \quad \$ 2,781,504 \quad \$ 1,390,752$

FRACTURING FLUID CHARACTER- IZATION FACILITY OK $04 \$ 13,785,048 \quad \$ 3.931,000$ FEASIBILITY ASSESSMENT OF A U.S. NATURAL GAS PRODUCTION $\begin{array}{lll}O K & 04 & \$ 720,020\end{array}$ COMBUSTION OF PULYERIZED COAL IN VORTEX STRUCTURES LIQUID NATURAL GAS AS A TRANSPORTATION FUEL IN THE HEAVY TRU

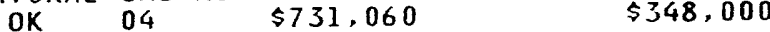




AHARDEE NAME
DIVISION
OKTAHA PUBLIC SCHOOLS

BIN COMPL DATE

VENDOR ID CITY STATE DIST DESCRIPTION OF HORK FG46-93R613597 02/28,95 $F G 46-93 R 61358908 / 31 / 94$
$232150-0001-6 \quad$ OKTAHA FG46-93R613595 02/28, 95 $232150-0001-6$ OKTAHA

OLA PUBLIC SCHOOLS

$F G 46-93 R 613603 \quad 02 / 28 / 95$
$232100-0001-8 \quad 0 L A$

OLD DOMINION UNIV RESEARCH FDN FG05-90ER54093 $10 / 31 / 94$ NOLK FG05-92ER79067 12/31/94 $167200-0001-2$ NORFOLK

OLD DOMINION UNIVERSITY

FG05-94ER40832 02/14/97 $061840-0001-2$ NORFOLK FG05-93ER4550510/31/96 061840-0001-2 NORFOLK FG05-94EF25216 06/14/97 061840-0001-2 NORFOLK

OLDHAM COUNTY BOARD OF EDUCN FG44-91R434520 08/31/94 061880-0001-7 LA GRANGE

AB01-92GC20275 07/22/94 197750-0001-7 ALEXANDRIA

OLIFF \& BERIDGE

OLIN CORP

WINCHESTER GROUP

OLIVET NAZARENE UNIVERSITY
AB01-93GC20314 07/31/95 $936 C 2031407 / 31 / 95$
$197750-0001-7$ ALEXANDRIA

AMO4-93AL $94355 \quad 12 / 22 / 94$ 061900-0201-3 EAST ALTON

FG45-93R551542 08/31/95
AWARDS A NEW CYCLE 15 ENERGY CONSERVATION MEASURE GRANT. OK $04 . \$ 127,625 \quad \$ 62,105$

$\begin{array}{rrrr}\text { AWARDS A } & \text { NEH CYCLE } & 15 & \text { TECHNICAL ASSISTANCE GRANT } \\ 0 \mathrm{~K} & 02 & \$ 6,426 & \$ 3.213\end{array}$ AWARDS A NEH CYCLE 15 ENERGY CONSERVATION MEASURE GRANT. $\begin{array}{lccc}N \text { NEW } & \text { CYCLE } & 15 & \text { ENERGY } \\ 0 K & 02 & \$ 42,389 & \$ 21,194\end{array}$ AMARDS A NEW CYCLE 15 ENERGY CONSERVATION MEASURE GRANT.

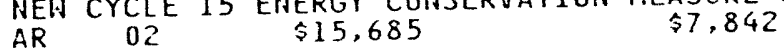

SCATTERING FROM ALFVEN HAVE FLUCTUATIOHS DRIVEN BY THERMONUC SCATTERING FROM ALFVEN WAVE FL 02 S68,800 PRE-FRESHMAN EHRICHMENT PROGRAM (PREP) - 1992

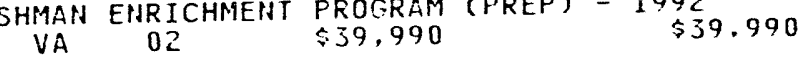

NEH AHARD TOPICS IN THEORETICAL NUCLEAR PHYSICS NA 02 \$163,000 $\$ 40,000$ DYHAMICS OF SURFACE MELTING FARALLEL SPANSE MATRIX COMPUTATIOHS
VA 02 $\begin{array}{cccc}\text { CYCLE } 13-\text { INSTITUTIOHAL CONSERVATION PROGRAM. AFPROVED BUD } \\ \mathrm{KY} & \$ 24,000 & \$ 12,000\end{array}$ BPA FOR NUVELTY SEARCHES ETC 08 \$0

NOVELTY SEARCHES 50

\$0 R418

OLIN-WINCHESTER AMMUNITION ST ANDARDIZATIOH EFFORT IL 21 \$O 


\begin{tabular}{|c|c|c|c|c|c|c|c|}
\hline AWARDEE NAME & BIN & L DATE & & \multicolumn{3}{|c|}{ DESCRIPTION OF WORK } & \multirow[b]{2}{*}{$P / S$} \\
\hline \multirow[t]{2}{*}{ DIVISION } & VENDOR ID & CITY & STATE & $\begin{array}{l}\text { CONG } \\
\text { DIST }\end{array}$ & $\begin{array}{l}\text { CONTRACT } \\
\text { VALUE }\end{array}$ & $\begin{array}{l}\text { OBLIGS } \\
\text { TO DATE }\end{array}$ & \\
\hline & $184630-0001-0$ & KANKAKEE & IL & 15 & $\$ 79,403$ & $\$ 51$. & 3 \\
\hline
\end{tabular}

OMAHA OPPORTUNITIES IND CTR

OMAHA PUBLIC POWER DISTRICT

OMATA \& ARAKI PATENT OFFICE

OMEGA CONTROLS

OMEGA-P INC

ONEIDA CITY OF

HOSPITALS

OHSL OW COUNTY OF

SCHOOLS

OPHIR CORP

- OPTICAL SOCIETY OF AMERICA

OPTIPHASE INC

:OPTISENSORS INC
$F G 47-92 R 703504 \quad 08 / 31 / 94$
$213750-0001-2 \quad$ OMAHA

CRO1-83NE44401 03/15/30 061940-0001-5 OMAHA

$A B O 1-926 C 20300 \quad 09 / 30 / 94$
$135050-0001-9 \quad$ TOKYO

$\begin{array}{cc}A B 01-93 G C 20338 & 09 / 30 / 95 \\ 135050-0001-9 & \text { TOKYO }\end{array}$

AM21-93MC30301 $06 / 22 / 95$
$229100-0001-\times \quad$ SOUTH CHARLESTO

FG05-92ER.81338 02/17/95 061990-0001-3 INEW HAVEN

$F G 42-92 R 24382201 / 31 / 95$
$220910-0101-5$ ONEIDA

FG44-93R435392 09/30/94 $231310-0101-1$ JACKSONVILLE

FG03-92ER81318 05/24/95 062120-0002-6 LITTLETON

FG02-94ERG $183204 / 30 / 95$ $137020-0001-1$ HASHINGTON

FG03-94ER81675 07/17/94 236120-0001-2 VAII NUYS

FG02-90ER81033 09/20/95 198620-0uU1-5 CAMBRIDGE

GRANT PROGRAMS FOR SCHOOLS ANDHOSPITALS AND FOR BUILDINGS 0

$$
\mathrm{NE} \quad 02 \quad \begin{array}{lll}
\$ 4.795 & \$ 2,397
\end{array}
$$
DISPOSAL OF SPENT NUCLEAR FUELAND FOR HIGH LEVEL $\underset{\$ 0}{\text { RADIOACTIVE }} \underset{\$ 0}{\text { IIE }}$

PROVIDE PATENT SERVICES

BPA FOR JAPANESE PATENTS
ริ) R499

\$0 R499

INDEFINITE QUANTITY CONTRACT FOR SCREHED BALL VALVES HARMONIC CONVERTER IO PRODUCE HIGH-POWER 10-30 GHZ MICROHAVE $C T \quad 03 \quad \$ 549,995 \quad \$ 408,868$

TITLE III OF THE NATIOHAL ENERGY CONSERVATIOH FOLICY ACT NYY $25 \quad \$ 239,385 \quad \$ 119,692$

SEE ATTACHED PAGE NO. 2 OF THIS NOTICE OF FINANCIAL ASSISTAN HC $0330.2 \$ 10,238 \quad \$ 6,048$

PHASE I AHARD FOR OPHIR CORF TITLE: "AH AIRBORNE IMAGING RA CO $06 \quad \$ 549,801 \quad \$ 338,600$

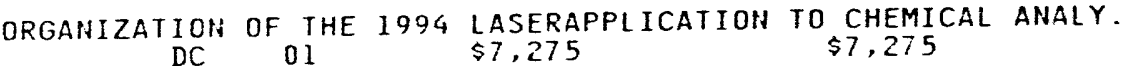

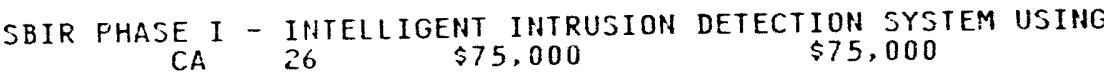

INTEFEROMETIRC FIBER OPTIC ACCELEROMETER MA $05 \quad \$ 547,894 \quad \$ 547,894$ 


AWARDEE NAME

DIVISION

BIN

COMPL DATE

VENDOR ID

FGO3-91ER81134 07/05/94 214540-0001-5 PALO ALTO

OPTIVISION INC

FG02-93ER81616 07/17/94 062180-0004-4 TOPSFIELD

OPTRA INC

ORACLE CORP

ORCAS POWER \& LIGHT CO

$$
\begin{gathered}
\text { ADO1-931G00372 09/30/94 } \\
116300-0001-9 \text { REDHOOD CITY }
\end{gathered}
$$$$
\text { ADO1-94EI24200 09/30/95 }
$$$$
116300-0001-9 \text { REDHOOD CITY }
$$

AD01-94EI24199 09/30/95 $116300-0001-9$ REDWODD CITY

AD65-941:A11655 10/31/94 116300-0005-1 GOLDEH

ADO1-93EI23461 09/30,94 $116300-0002-7$ BETHESDA

AD01-93AD34064 09/30/94 $116300-0002-7$ BETHESDA

AD01-94EH89455 05/05/95 $116300-0002-7$ BETHESDA

ORDELA INC

FC07-91ID13071 04/29/96 II D13071 $04 / 29 / 96$
I $80340-0001-9$ EASTSOUND

AC05-88ER80626 10/22/94 $175270-0001-5$ OAK RIDGE

$\begin{array}{llc}\text { OREGOH GRADUATE CENTER } & \text { FG03-935F1988 } & 08 / 09 / 94 \\ \text { ELECTRICAL ENGIHEERING DEPT OF } 062280-0501-4 & \text { BEAVERTON }\end{array}$ FG06-92ER20093 08/17/94 CHEMICAL BIOL \& ENV SCI DEPT $\begin{array}{cc}\text { FG06-92ER20093 } 08 / 17 / 94 \\ 062280-0401-8\end{array}$ BEAVERTON FG06-92ER20065 05/14/95
$062280-0101-9$ BEAVERTON

: environmental SCi dept FG06-89ER60845 06/30/96
DESCRIPTION OF WORK

CONG CONTRACT

OBLIGS

$P / S$

SBIR - PHASE I AHARD FOR - PHOTONIC SHITCHING FOR INTERCONNE CA 1 AIARD FOR $\$ 546,479 \quad \$ 546,479$

ABSOLUTE DIODE LASER $\begin{gathered}\text { HAVELENGTH STANDARD } \\ \$ 74,008\end{gathered}$

PREVENTIVE MAINTENAIICE FOR PROPRIETARY SOFTHARE FOR FY 1994 CA $12 \quad \$ 6.683 \quad \$ 6.683 \quad \mathrm{D} 399$ RENEHAL OF MAINIENANCE FOR ORACLE PROPRIETARY SOFTHARE (MAIN CA $12 \quad$ \$34,500 RENEWAL OF MAINTENANCE SOFTHARE $\$ 21.367 \quad 7030$ COHSULTATION SVCS $\$ 35.200$ $\$ 35.200 \quad 7045$ SOFTWARE MAINTEHANCE AND VENDOR SUPPORT $\$ 38.215 \quad 7030$ SOFTHARE MAINTENANCE AND VENDOR SUPPORT $\$ 74.568 \quad 7030$ SOFTWARE LICENSES $\$ 25,440$ $\$ 25,440 \quad 0399$

PARTICIPATIOH IN THE U.S. DEPARTMENT OF ENERGY'S ELECTRIC \& WA 02 \$I89,100 $\$ 74,978$

DEVELOPMENT OF A METHOD FOR G REATLY INCREASING THE COUNT-RA TH ${ }_{03} \begin{array}{ccc}\$ 544,355 & \$ 544,355 \text { AG91 }\end{array}$

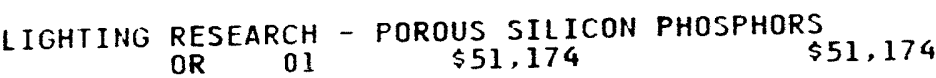

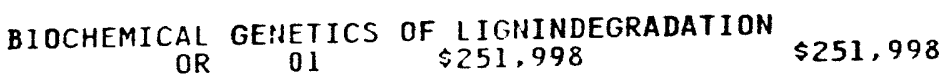
OXIDATIVE ENZYMES INVOL VED IN FUNGAL
OR OI ADSORPTON OF WASTE MIXTURES INAQUIFER MATERIALS 
- D - D - - - - -

AWARDEE NAME

BIN

COMPL DATE

VENDOR ID

CITY

ENVIRONMENTAL SCI DEPT

062280-0101-9 BEAVERTON

FG06-85ER60313 02/28/97

$062280-0001-2$ BEAVERTON

OREGON HEALTH SCIENCES UNIV

FG02-89CH10354 12/31/95

$179830-0001-4$ PORTLAND

FG02-92CH10505 01/31/96 $179830-0001-4$ PORTLAND

OREGON INSTITUTE OF TECHNOLOGY FG07-90ID13040 09/30/95 GEO HEAT CENTER

OREGOH SHORT LINE RR UNION PACIFIC RAILROAD

UNION PACIFIC RAILROAD

OREGON STATE OF

ENERGY DEPT

ENERGY DEPT

CONSERVATION RESOURCES DIV

ENERGY DEPT

HOUSING \& COMMUNITY SVCS

OREGON STATE UNIVERSITY

:

OCEANOGRAPHY DEPT
$01 D 1304009 / 30 / 95$
$062290-0101-2$

AC07-79ID01824 09/30/99 062310-0101-6 POCATELLO

AC07-76ID01293 12/31/99 $062310-0101-6$ FOCATELLO FCO4-84AL20534 09/30/94

AC06-89RL $1172208 / 31 / 94$

FG06-94RL12803 09/30/94 $062320-0202-4$ SALEM

FG51-93R020324 09/30/96

FG51-93R020323 03/30/96 $062320-0202-4$ SALEM

FG51-94R020020 03/31/95 062320-1202-X SALEM

FG05-91ER79037 09/29/94 062330-0001-7 CORVALLIS

FG06-93ER61523 10/31/95 $062330-0701-1$ CORVALLIS
DESCRIPTION OF WORK

CONG CONTRACT
STATE DIST VALUE

OR 01 \$536,062

OBLIGS

TO DATE $P / S$ $\$ 338,039$

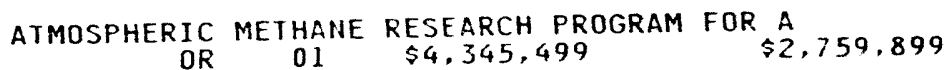

ALTERATIOHS TO BASIC SC BLDG

$O R \quad 04 \quad \$ 66,610,000$

$\$ 44.287 .500$

DESIGN \& CONSTRUCTION OF AMBULATORY RESEARCH \&

$O R \quad 01 \$ 33,000,000$

$\$ 20,000,000$

GEOTHERMAL DIRECT-HEAT UTILIZATION ASSISTAHCE

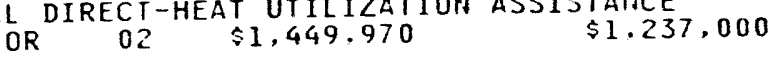

CONSTRUCT RAILROAD CROSSING ATSCOVILLE IDAHO

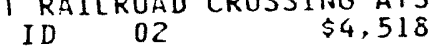

$\$ 4.518 \times 293$

COHSTRUCTION OF RR CROSSIHG OHUP MACKAY BRANCH LINE

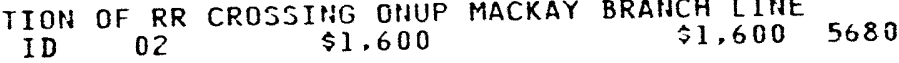

URANIUM MILL TAILINGS REMEDIALACTION PROJECT

OR 05 S $0547,136 \quad \$ 847,136$

RESEARCH AID DEVELOPMENT HANFORD HIGH LEVEL HASTE PROGRAM AR DEVELOPMENT HANFORD HIGH LEVEL $\$ 2.000,000 \mathrm{AZ11}$

INITIAL FUNDIHG FOR A GRANT FOR FIVE YEAR PLAN
$\begin{gathered}\text { OR } 05 \\ \$ 421,000\end{gathered}$

HICE3: HYDROCHLORIC ACID RECD VERY SYSTEM $\$ 97,000$

PRGRAM: NICES CULLET FEEDSTOCK PURIFICATIOH USING ADVAHCED NICES, CULLET FEEDSTOCK PURIFICATION $\$ 348,000$
$04 \$ 1,023,575$

IILE IV PART A, HEATHERIZATIOH ASSISTANCE FOR LOH INCOME $P$

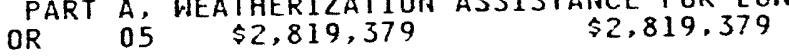

INDUCTIVELY COUPLED PLASMA-MASS SPECTROMETER

INVESTIGATIOII OF THREE PHASE TRANSPORT IN OHE

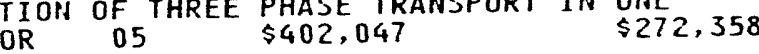




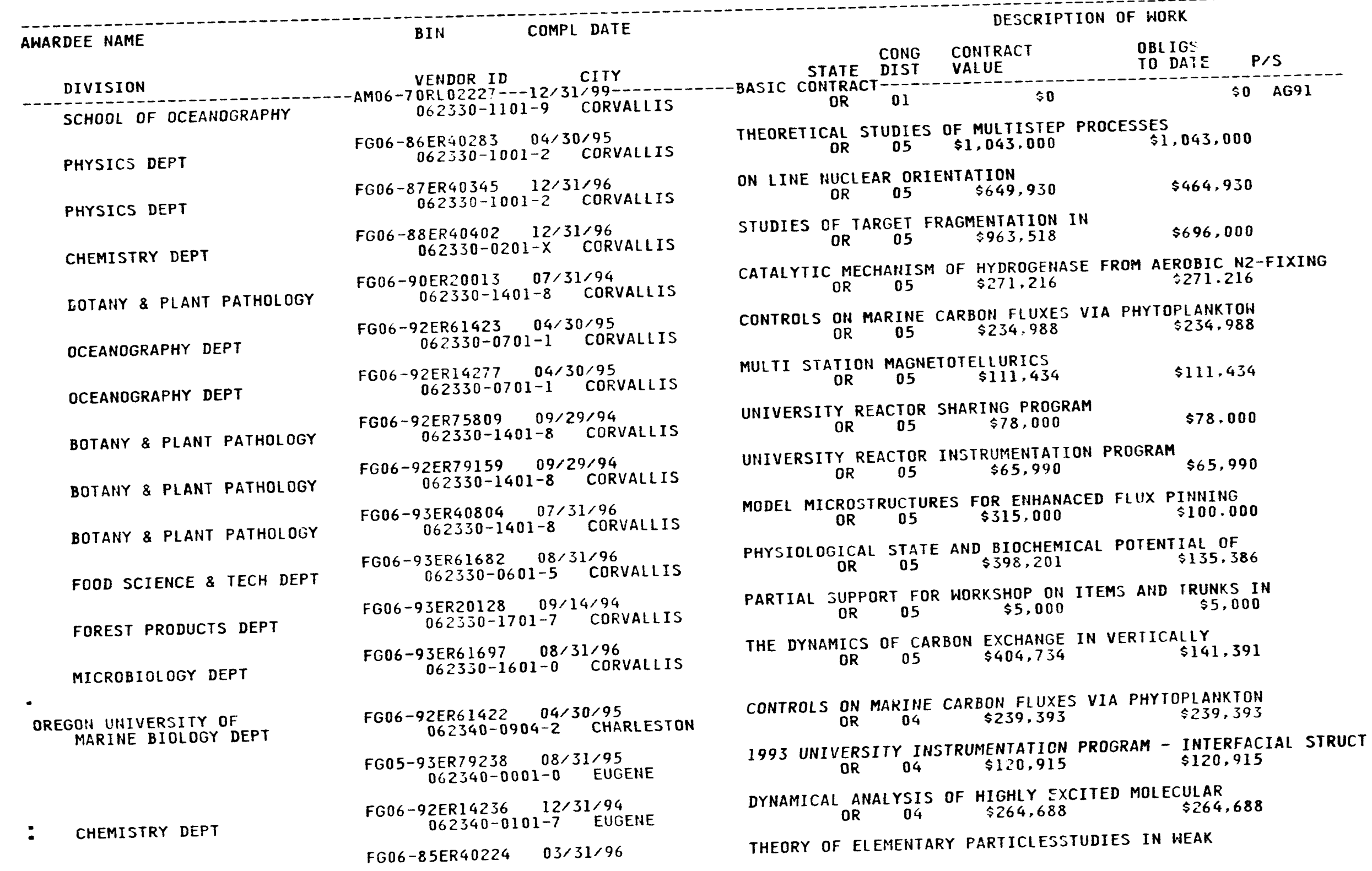



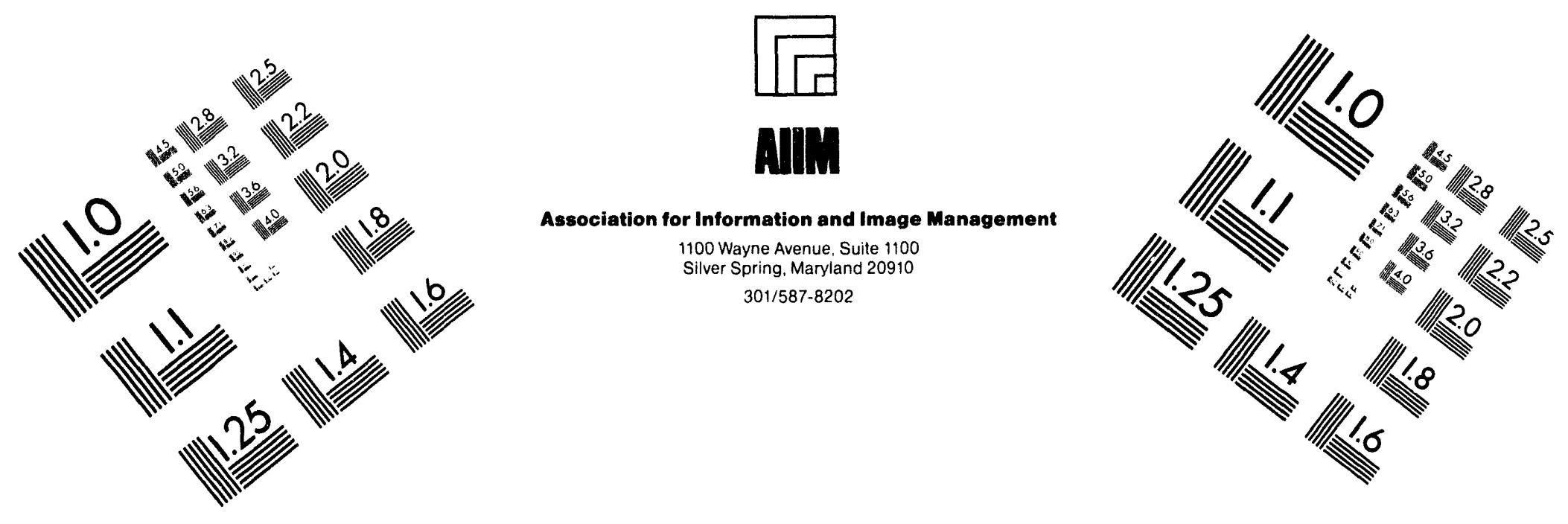

\section{Centimeter}

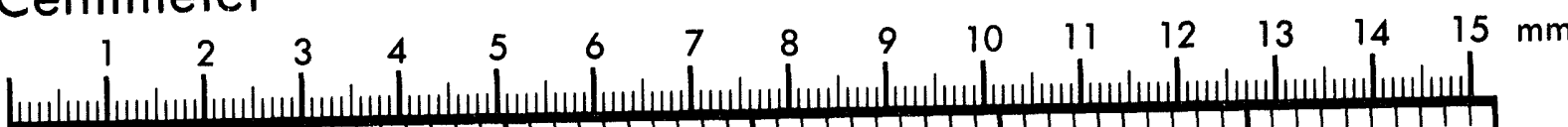

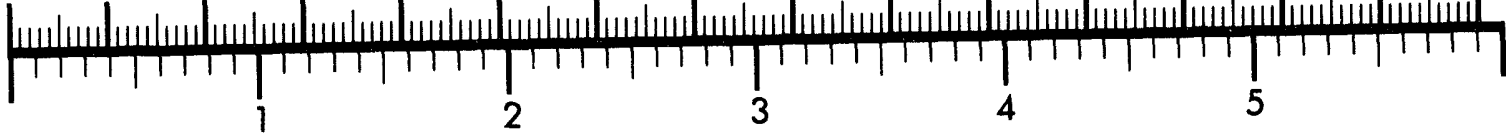
Inches
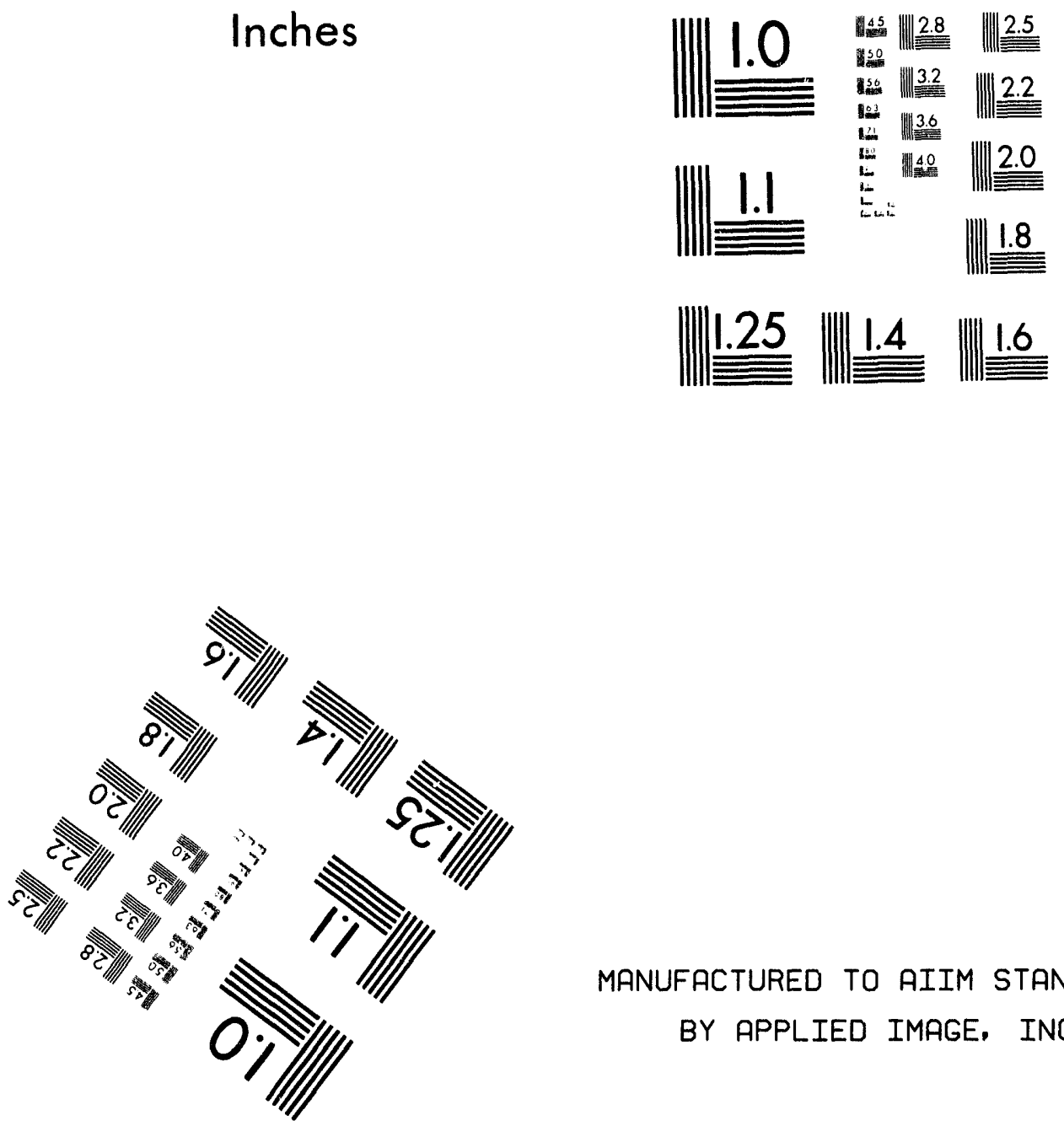

MANUFACTURED TO AIIM STANDARDS

BY APPLIED IMAGE, INC.

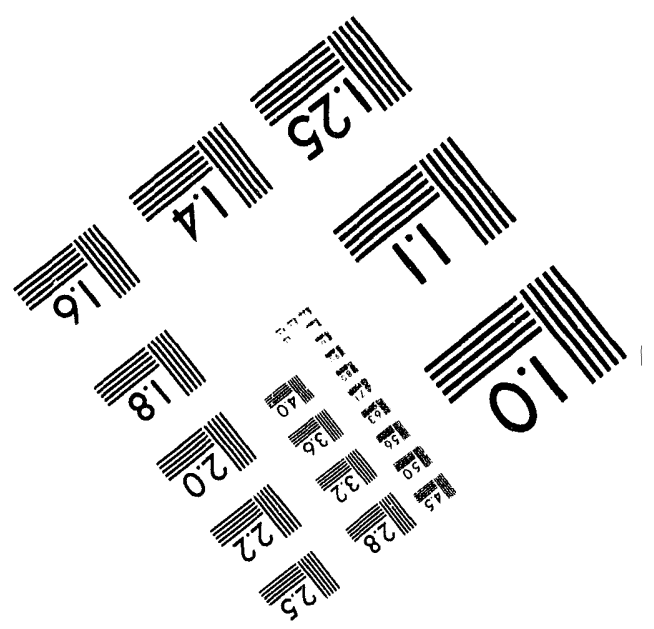



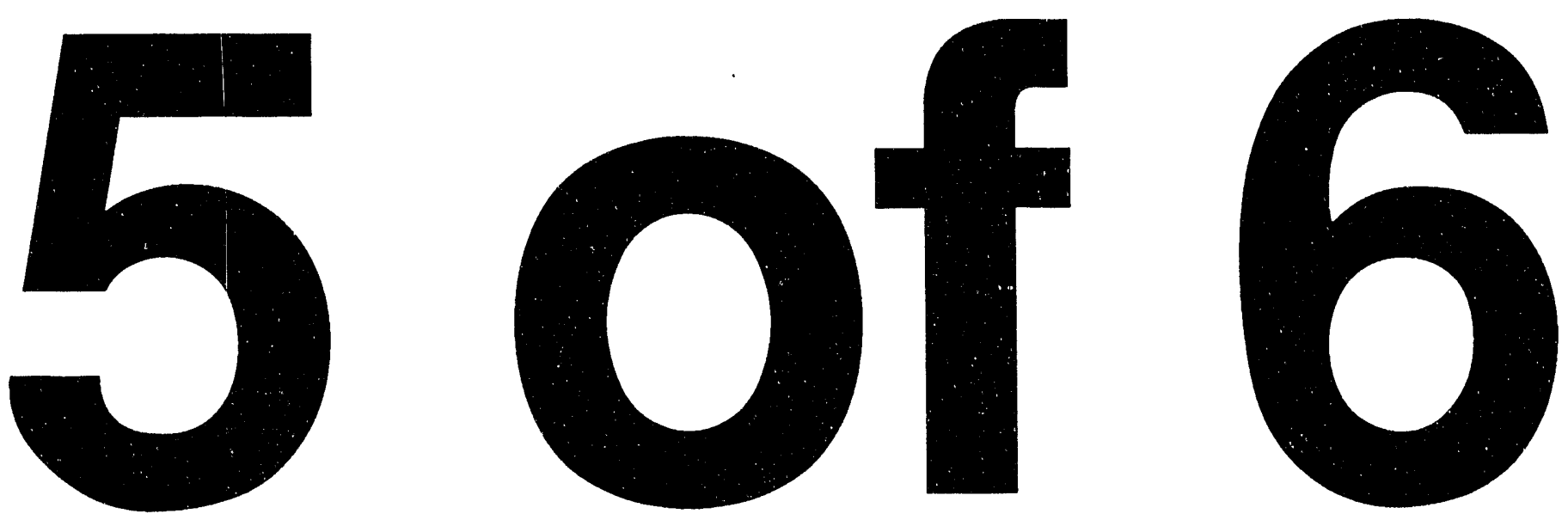


AWARDEE NAME BIN COMPL DATE

DESCRIPTION OF HORK

BIN

$062340-0401-6$

INST OF THEORETICAL SCIENCE

PHYSICS DEPT

FG06-86ER45275 11/30/95 062340-0201-3 EUGENE

CHEMISTRY DEPT

FG06-86ER45273 10/31/95
$062340-0101-7$ EUGENE

FG06-91ER40637 04/30/96

INST OF THEORETICAL SCIENCE

MOLECULAR BIOLOGY

BIOLOGY DEPT

INST OF THEORETICAL SCIENCE

ORFI SYSTEMS INC

ORG FOR ECONOMIC COOP \& DEV

FG06-91ER20054 08/31/95
$062340-0701-5$ EUGENE

FG06-92ER61417 04/30/95 06234:-0801-1 EUGEHE

FG06-94ER14406 11/14/96 4ER14406 11/14/96
$062340-0401-6$ EUGENE

AC43-92CH10510 03/26/95
$062350-0003-0$ MALVERN

GI01-92PE10020 $01 / 19 / 95$
$217430-0001-4 \quad$ PARIS

FG02-92ER35166 $06 / 14 / 95$
$217430-0001-4$ PARIS

$A C 05-930 R 2203501 / 19 / 95$
$149910-0002-5$ SAH DIEGO

FGO3-92ER81301 $02 / 17 / 95$
$221300-0001-0$ SAll DIEGO

AC01-90EI20713 09/25/95 062370-0001-1 SILVER SPRING

ORKAND CORP

ORTEK ENVIRONMENTAL LAB

ORZECHOWSKI KAREN L
AC06-92RL12342002/25/95
$217690-0001-1$ GREEN BAY

AB01-92GC20276 07/22/94
STATE CONG CONTRACT

SURFACE AND INTERFACE STRUCTURE

$O R \quad 04 \$ 1,325,184$

MONITORING INTERFACIAL DYNAMICS BY

OR $\quad 04 \quad \$ 942,574$

RELATIVISTIC HEAVY ION COLLISIONS

TO DATE P/S

$\$ 5,052,500$

$\$ 1,079,359$

$\$ 751,634$

$\$ 233,000$

ROLES OF NUCLEAR GENE PRODUCTSIN PLASTID DEVELOPMENT OR $04 \quad \$ 352,000 \quad \$ 352,000$ $\begin{array}{cccc}\text { PHYTOPLANKTON EXCRETION REVISITED HEALTHY } & \text { CELLS MAY } \\ \text { OR } & 04 & \$ 280,616 & \$ 280,616\end{array}$

PHOTOCHEMICAL WATER-SPLITTING USING ORGANOMETALLIC OXIDES AS

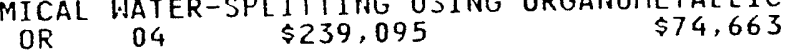

SUPPORT SERVICES CONTRACT FOR THE INSTITUTIONAL CONSERVATION PA 05 \$387,851 $\$ 105,588$ R421

INTERNATIONAL AGREEMENT FOR WORK PERFORMED BY OECD

FORUM ON MEGASCIENCE PROJECTS

$00 \quad \$ 297,000 \quad \$ 297,000$

MANAGEMENT AND ORGANIZATION DEVELOPMENT CONSULTING SUPPORT S $\begin{array}{llll}\text { CAND ORGANIZATION DEVELOPMENT CONSUUTING } & \text { SUPPORT } & \$ 127,465 & \text { R699 }\end{array}$

FAST PROCESSOR OF TOKAMAK ION TEMPERATURES

$$
\begin{array}{llll}
C A & 41 & \$ 555,954 & \$ 408,010
\end{array}
$$

TO PROVIDE OPERATONS AND SYSTEM SUPPORT SERVICES FOR THE ENE $M D \quad 06 \$ 26,130,964 \quad \$ 24,027,2077030$

ANALYTICAL LABORATORY SERVICES

HI 08 \$ $\$ 1,000,000$

จ $1,000,000 \quad A Z 11$

BPA FOR NOVELTY SEARCHES ETC 


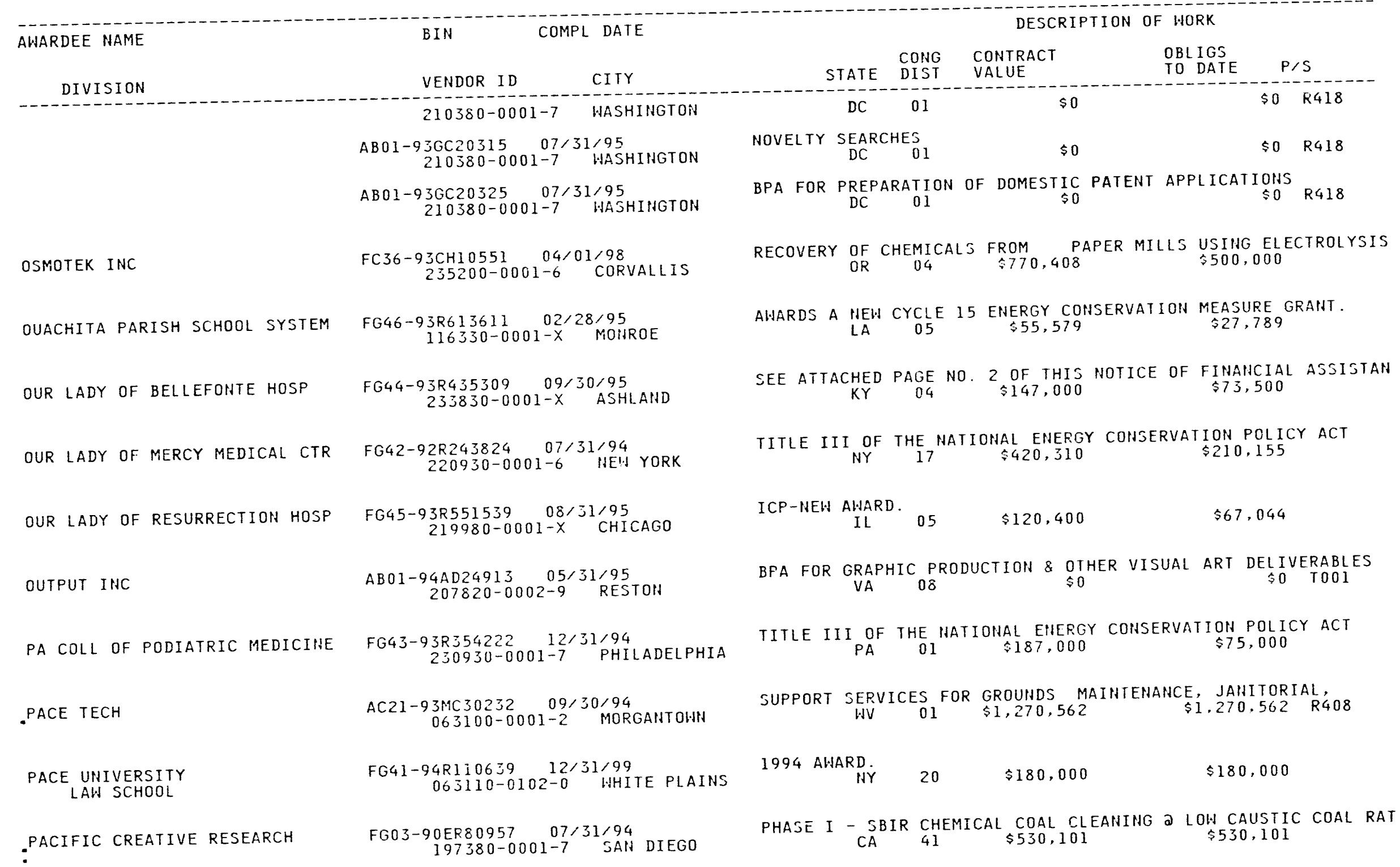




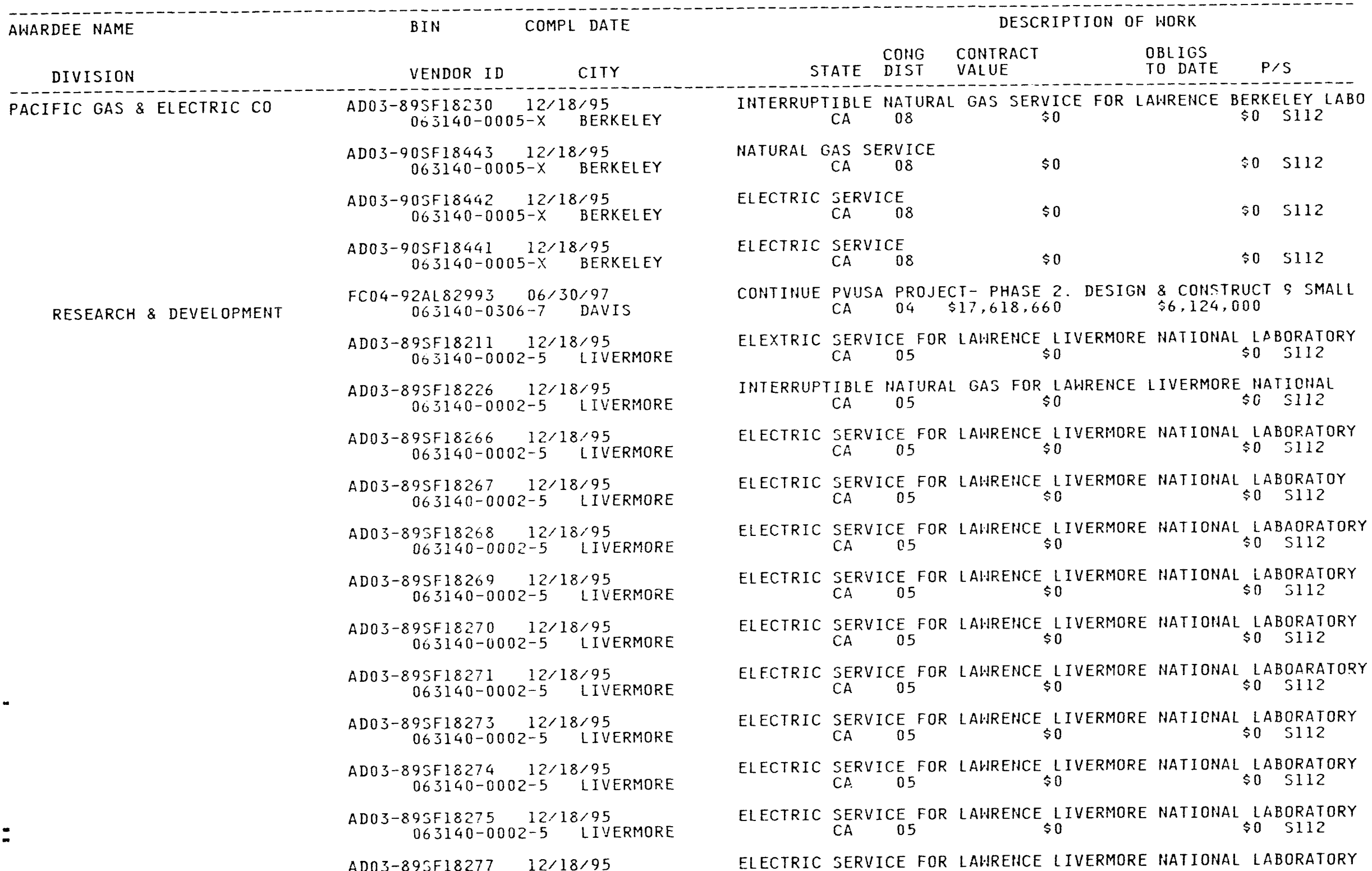




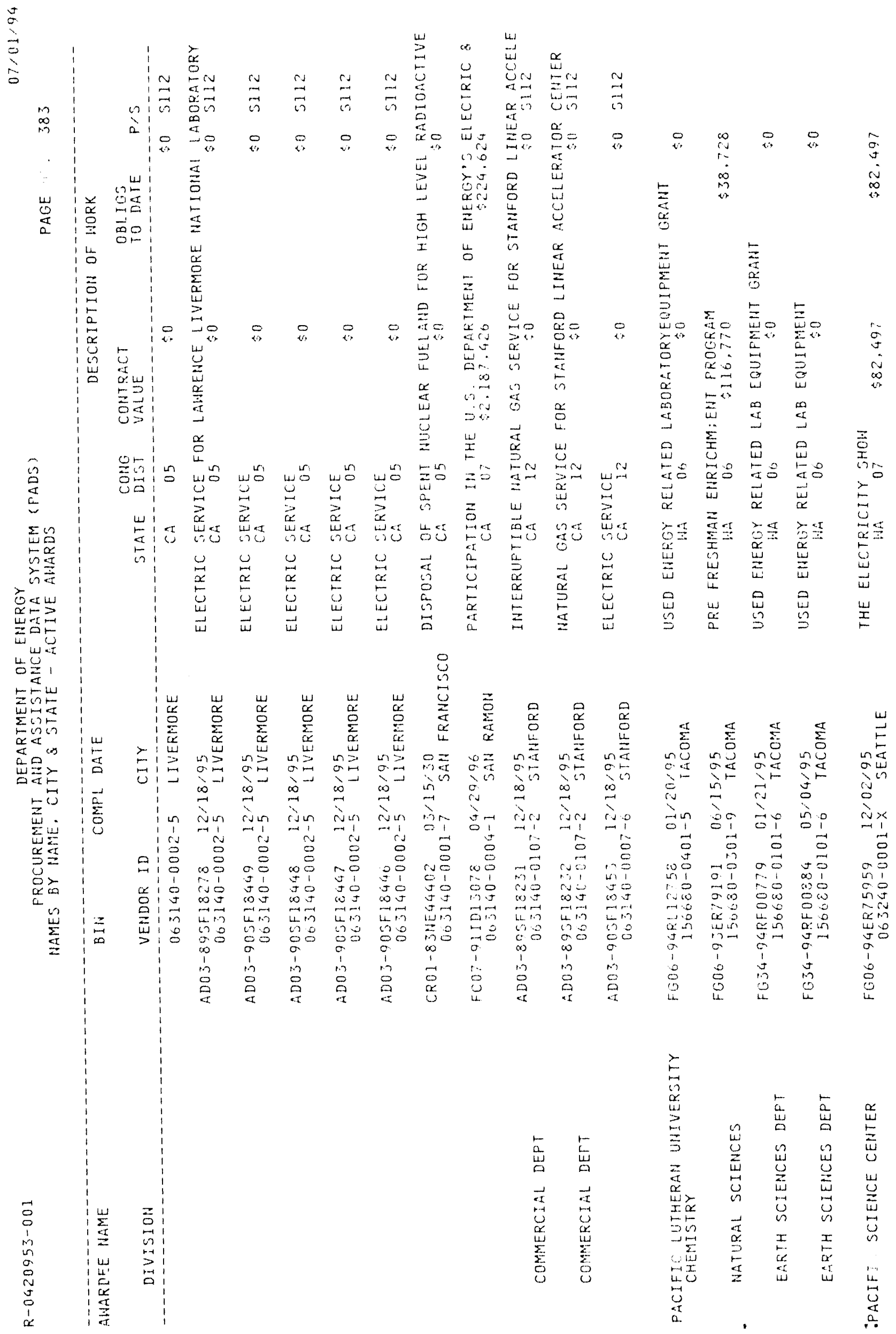




\begin{tabular}{|c|c|c|}
\hline A!IARDEE NAME & COMPL DATE & DESCRIPIION OF WORK \\
\hline DIVISION & VENDOR ID & $\begin{array}{l}\text { OBLIGS } \\
\text { TO DATE }\end{array}$ \\
\hline PACIFIC SIERRA RESEARCH CORP & $\begin{array}{c}\text { ACO1-90IE10747 } 09 / 27 / 94 \\
165160-0001-8 \text { ARLINGTON }\end{array}$ & $\begin{array}{ccccc}\text { TECH SUPPORT SEERV FOR THE OFFICE OF NUCLEAR NCN PROLIFE RAT } & \text { THE } \\
\text { VA } & 08 & \$ 431,437 & \$ 411,988 & \text { B549 }\end{array}$ \\
\hline PACIFIC UNIVERSITY OF THE & $\begin{array}{l}\text { FGO3-93ER79190 06/14/95 } \\
197410-0001-4 \text { STOCKTON }\end{array}$ & $\begin{array}{ccc}\text { PRE-FRESHMAH ENRICHMENT } & \text { PROGRAM } \\
\text { CA } & 13 & \$ 38,534\end{array}$ \\
\hline PACIFIC WESTERN TECHNOLOGIES & $\begin{array}{c}A C 65-921.1 A 09375 \quad 09 / 30 / 95 \\
224070-0001-3 \text { GOLDEN }\end{array}$ & $\begin{array}{cccc}\text { GENERAL ADMIN SUPPORT SVCS FORHAPA } & \text { HEADQUARTERS } \\
\text { CO } & 06 & \$ 4,177,394 & \$ 2.890,090\end{array}$ \\
\hline \multirow[t]{8}{*}{ PAI CORP } & $\begin{array}{c}\text { ACO3-90SF18504 } 01 / 01 / 95 \\
160150-0002-3 \text { MARTINEZ }\end{array}$ & $\begin{array}{ccc}\text { PROVIDE ESQA } & \text { TECHNICAL SUPPORT } \\
\text { CA } & 03 & \$ 1,940,104\end{array}$ \\
\hline & $\begin{array}{l}\text { AK34-9IRF00106 05/01/96 } \\
160150-0003-1 \quad \text { GOLDEN }\end{array}$ & $\begin{array}{c}\text { BOA FOR TECH. SUPPORT SVCS. } \\
\text { CO } \\
06\end{array}$ \\
\hline & $\begin{aligned} \text { ACO4-91AL72747 09/30/06 } \\
160150-0001-5 \text { ALEUQUERQUE }\end{aligned}$ & $\begin{array}{c}\text { PROVIDE TECHIICAL SUPPURT TO TSD IN ORDER TO MEET ES\&H } \\
\qquad \mathrm{NM} \text { OI } \\
\end{array}$ \\
\hline & $\begin{array}{l}\text { AC08-89NV10793 } 01 / 19 / 95 \\
160150-0004-X \text { LAS VEGAS }\end{array}$ & 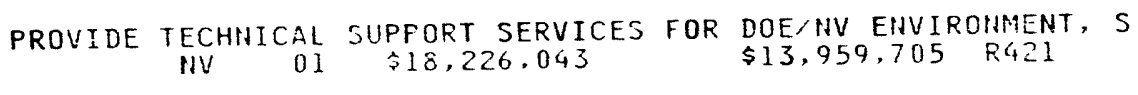 \\
\hline & $\begin{array}{l}\text { ACO8-9211V11095 } 01 / 16 / 95 \\
160150-0004-X \text { LAS VEGAS }\end{array}$ & 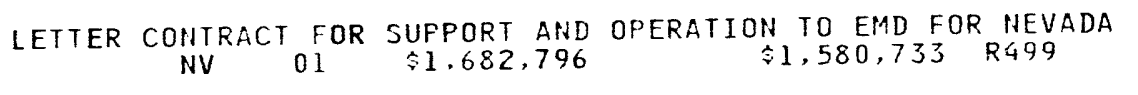 \\
\hline & $\begin{array}{c}\text { AC09-913R18221 } 08 / 13 / 94 \\
160150-0005-8 \text { AIKEN }\end{array}$ & $\begin{array}{rrrrr}\text { TECHHICAL SUPPORT SERVICES FORTHE ASSISTANT MAHAGER FOR } \\
\text { SC } 03 & \$ 3.132,065 & \$ 3.048 .000 & \text { R425 }\end{array}$ \\
\hline & $\begin{array}{r}A C 05-880 F .21794 \quad 08 / 07 / 96 \\
160150-0001-5 \text { OAK RIDGE }\end{array}$ & $\begin{array}{c}8 \text { (A) TO PROVIDE TECHNICAL ASSISTANCE AND STUDIES IH SUPPORT } \\
\text { TH } 030 \$ 24.698,711\end{array}$ \\
\hline & $\begin{array}{l}\text { AC06-92RL12083 } 09 / 30 / 94 \\
160150-0006-6 \quad \text { RICHLAND }\end{array}$ & $\begin{array}{ccccc}\text { SUPPORT SERVICES FOR HEALTH SAFETY ENVIRONMENT } & \text { HA } \\
\text { WA } 04 & \$ 5,237,170 & \$ 4,146,830 & \text { AZ11 }\end{array}$ \\
\hline \multirow[t]{2}{*}{-PAINE COLLEGE } & $\begin{array}{c}\text { FG09-943R18439 } 06 / 20 / 96 \\
063350-0001-6 \text { AUGUSTA }\end{array}$ & $\begin{array}{ccc}\text { PRE-FRESHMAN ENRICHMENT } & \text { PROGRAM } \\
\text { GA } & 10 & \$ 48,808\end{array}$ \\
\hline & $\begin{aligned} \text { FG09-943R18451 } 06 / 20 / 95 \\
063350-0001-6 \text { AUGUSTA }\end{aligned}$ & $\begin{array}{ccc}\text { FIFTH GRADERS } & \text { MOVING UP } & \text { PROJECT } \\
\text { GA } & 10 & \leqslant 135,579\end{array}$ \\
\hline AJ BUSINESS & $\begin{array}{l}A B 01-93 A D 00012010 / 31 / 94 \\
224900-0001-2 \text { SILVER SPRING }\end{array}$ & $\begin{array}{c}\text { BPA FOR TEMPORARY SECRETARIAL WORK } \\
\text { MD } 08 \text { \$0 }\end{array}$ \\
\hline
\end{tabular}




\begin{tabular}{|c|c|}
\hline AWARDEE NAME & COMPL DATE \\
\hline DIVISION & VENDOR ID \\
\hline PAL TECH INC & $\begin{array}{c}A C 01-93 E H 3923606 / 10 / 95 \\
228940-0001-4 \text { RESTON }\end{array}$ \\
\hline PAL-TECH INC & $\begin{array}{c}\text { AC02-9111P00257 } 08 / 22 / 94 \\
213910-0001-3 \quad \text { RESTON }\end{array}$ \\
\hline PALATINE TOWNSHIP HIGH SCHOOL & $\begin{array}{c}\text { FG45-92R540073 12/31/94 } \\
063390-0001-0 \text { PALATINE }\end{array}$ \\
\hline $\begin{array}{l}\text { PALAU REPUB!IC OF } \\
\text { PUBLIC WORKS BUREAU OF }\end{array}$ & $\begin{array}{c}\text { FG49-92R900008 } 09 / 30 / 94 \\
167360-0102-1 \quad \text { KOFOR }\end{array}$ \\
\hline PALL CORP & $\begin{array}{c}\text { FC34-94RF00865 05/02/95 } \\
237990-0001-1 \text { GLEN COVE }\end{array}$ \\
\hline PALMER COLLEGE & $\begin{array}{c}\text { FG } 47-93 R 707037 \\
232110-0001-1 \quad \text { DAVENPORT }\end{array}$ \\
\hline PALMER PUBLIC SCHOOLS & $\begin{array}{c}\text { FG41-91R193328 } 08 / 31 / 94 \\
203090-0001-7 \quad \text { PALMER }\end{array}$ \\
\hline PALO ALTO INST OF MOLE MED & $\begin{array}{c}\text { FG03-94ER20136 } 03 / 14 / 95 \\
237090-0001-7 \text { MOUHTAIN VIEW }\end{array}$ \\
\hline PAN INTERIATIONAL INC & $\begin{array}{c}\text { ABO1-94AD25712 } 05 / 3 i / 95 \\
147170-0001-3 \quad \text { ALEXANDRIA }\end{array}$ \\
\hline & $\begin{array}{c}A B 01-94 A D 2491405 / 31 / 95 \\
147170-0001-3 \quad \text { ALEXAHDRIA }\end{array}$ \\
\hline PANHANDLE CITY OF & $\begin{array}{c}\text { GM04-92AL33283 } 08 / 12 / 97 \\
228440-0001-0 \text { AMARILLO }\end{array}$ \\
\hline $\begin{array}{l}\text { PAPER SCIENCE \& TECH INST OF } \\
\text { CHEMICAL SCIENCE DIV }\end{array}$ & $\begin{array}{c}\text { FG02-90CE40936 } 09 / 29 / 94 \\
203800-0101-2 \text { ATLANTA }\end{array}$ \\
\hline NEERING \& PAPER SCI & $\begin{array}{c}\text { FG02-90CE40937 } 09 / 29 / 94 \\
203800-0201-9 \text { ATLANTA }\end{array}$ \\
\hline
\end{tabular}

SPECIALIZED ADMINISTRATIVE TRAINING SUPPORT SERVICES FOR NEH

COOPERATIVE AGREEMENT BETWEEN DOE, EG\&G, AND PARTICIPANT FOR NY 05 \$O

GRANT PROGRAMS FOR SCHOOLS ANDHOSPITALS AND FOR BUIDLINGS 0 $\begin{array}{llll}\text { IA } & 01 & \$ 37,043 & \$ 18,520\end{array}$

TITLE III OF THE NATIONAL ENERGY CONSERVATION POLICY ACT ANALYSIS OF PHOTOSYNTHESIS
MA THE
$\$ 30,424$
$\$ 19,416$

$$
\text { CA PHOTOSYNTHESIS } \$ 115,430
$$

$\$ 115,430$

BPA FOR GRAPHIC ARTS AHD RELATED SERVICES. 07/01/94 THRU 06/ $\$ 0$ TO01 BPA FOR GRAPHIC PRODUCTION ANDOTHER VISUAL ART DELIVERABLES MUTUAL ASSISTAHCE IN FIRE FIGHTING

TX 13 BLACK LIQUOR COMBUSTION- VALIDATED RECOVERY BOILER GA 05 \$2,085,000 \$1,905,000 DEVELOPMENT \& PILOT TESTING OF MODULAR DYNAMICS TMP MODEL ENGINEERING \& PAPER SCI UIV 203800-0201-9 ATLANTA 


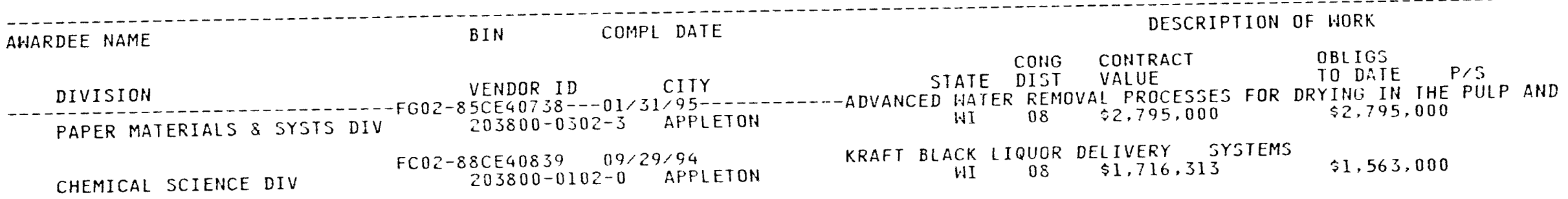

PAR ENTERPRISES INC

PARADISE VALLEY USD \#69

PARAMAGNETIC LOGGING INC

PARAMOUNT PETPOLEUM CORP

PARK COUNTY SCH DIST \#6

PARK RIDGE HOSPITAL

PARMET DR HERMAN OPTOMETRIST

PARSONS ENVIROHMENTAL SVCS INC

PARSONS MAIN INC

-PARSONS-BRINKERHOFF-QUADE

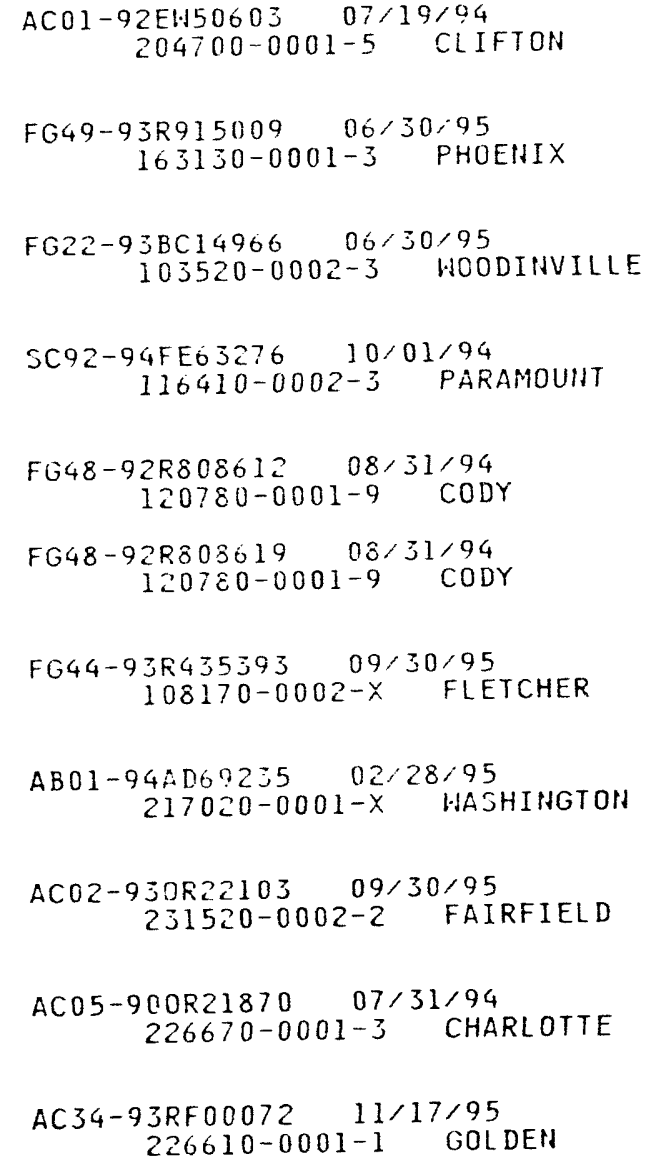

TO PROVIDE TECHHICAL, SURVEY, AHD ADMINISTRATIVE SUPPORT TO VA 08 \$767,198

CYCLE 15 ICP GRAHT AWARD

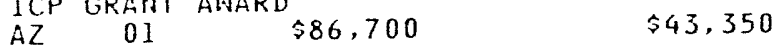

FABRICATIOH AHD DOHIHHOLE TESTING OF MOVING THROUGH CASING RE WA AND 1 \$ 1822,435 \$109,000 NPR-1 CRUDE OIL SALES CONTRACTEST REV
CA
09

AWARD ICP CYCLE XIV GRANT

$$
\text { AWARD ICF CYCLE XIV GRAHT }
$$

SEE ATTACHED PAGE NO. 2 OF THIS NOTICE OF FINANCIAL ASSISTAN
BFA FOR SAFETY GLASSES
$\$ 0$
$\$ 0 \quad 6540$

A-E SERVICES IN SUPPORT OF THEDOE ENVIRONMENTAL REMEDIATION $\mathrm{OH} 08 \quad \$ 3,529,677 \quad \$ 3,529.677 \quad$ C219

A-E SERVICES FOR PRODUCTION WASTE TREATMENT FACILITIES AT DO NC $09 \quad \$ 3,008,819 \quad \$ 3,008,819$ C211

RR/TECH SERVICES IN SUPPORT OFTHE SITE-HIDE ENVIRONMENTAL C0 $02 \quad \$ 8,162,557 \quad \$ 2,324,000 \quad R 425$ 


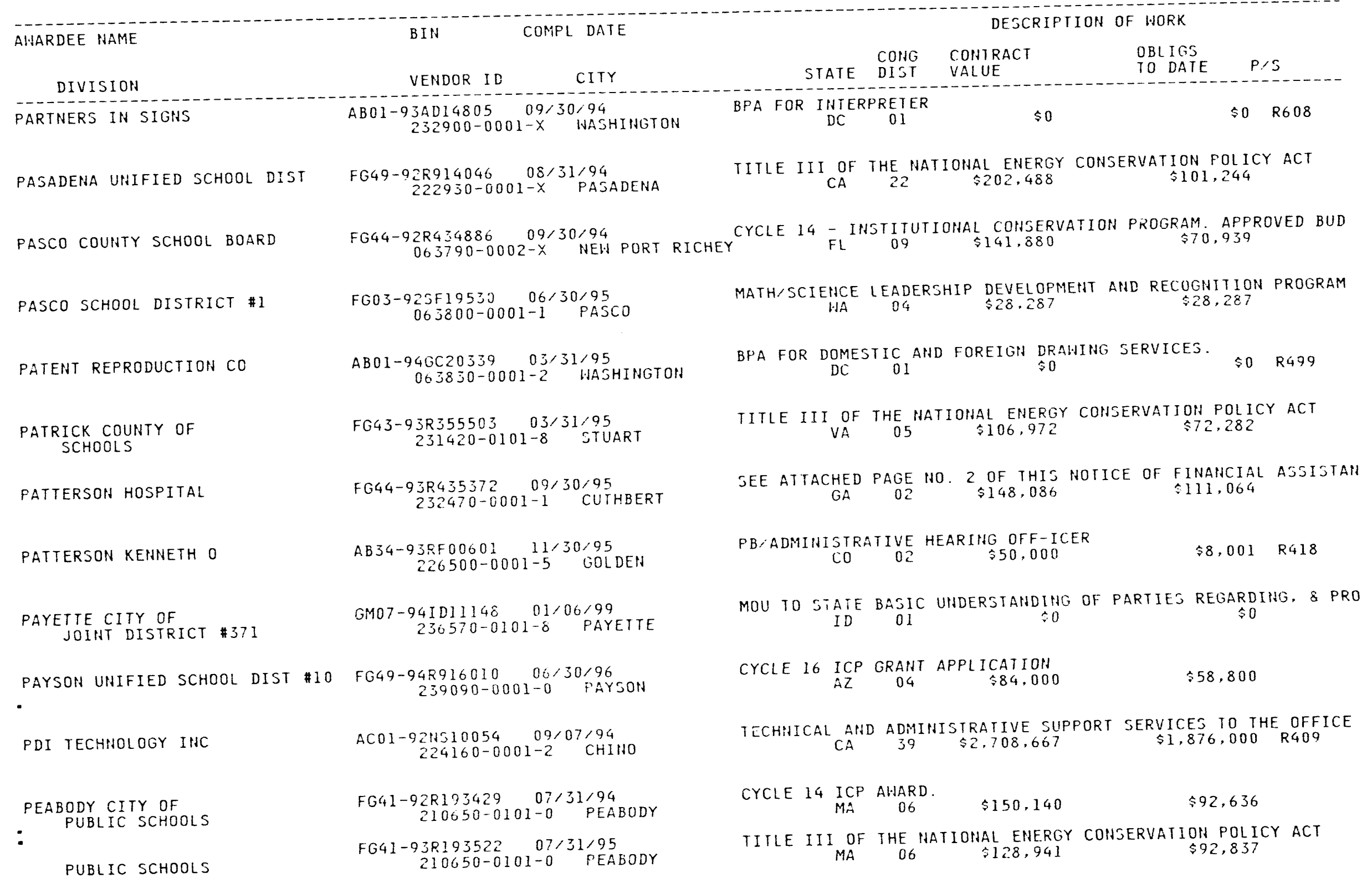




\section{AWARDEE NAME}

COMPL DATE

DESCRIPTION OF WORK

DIVISION

BIN

OBLIGS

VENDOR ID

CITY

CONG CONTRACT

TO DATE

$P / S$

PECOS INDEPENDENT SCHOOLS

PED INC

PEDDADA CONSULTANTS INC

PEI ASSOC INC

PEMBROKE STATE UNIVERSITY BUSINESS AFFAIRS OFFICE OF

BUSINESS AFFAIRS OFFICE OF

BUSIHESS AFFAIRS OFFICE OF

PEN KEM INC

PENASCO INDEPENDENT SCH DIST

PENDER COMMUNITY HOSPITAL DIST

PENDER COUNTY OF EDUCATION BOARD OF

$$
\begin{array}{r}
\text { GMO4-93AL84052 11/24,97 } \\
150020-0001-0 \quad \text { PECOS }
\end{array}
$$

AC65-94NC09123 08/31/94 $411 C 09123$
$237370-0002-6$

AC01-92FES2174 04/29/95 $185550-0002-5$ GAITHERSBURG AC11-93FE62708 03/31/97 $185550-0002-5$ GAITHERSBURG

ACO1-90MA00251 02/13/95 135140-0001-8 CINCINNATI

FG44-92R435009 08/31,94 2ก8080-0101-9 PEMBROKE

FG44-93R43540300/30/95 208080-0101-9 PEMBROKE FG44-93R4j5394 09/30/94 208080-0101-9 PEMBROKE

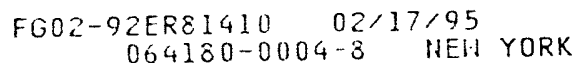

GM04-92AL84051 07/21/97 178330-0001-9 TAOS

$F G 47-92 R 703491 \quad 08 / 31 / 94$ 213720-0001-1 PENDER

FG44-92R435011 08/31/94
$220150-0101-8$ BURGAW

FG44-93R435395 09/30/95 220150-0101-8 BURGAN

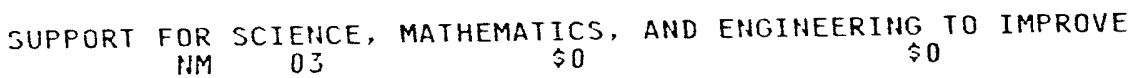

CONCRETE PAVEMENT - HURON SVC CENTER $\$ 38.144 \quad Y 224$

BUSINESS, ECONOMIC, TECHNICAL, ENGINEERING \& SCIENTIFIC SUPP $M D \quad 08$ \$3,141,090 \$1,672,058 R799

MAHAGEMENT \& ADMIN SUFPORT SERVICES TO THE OFFICE OF FOSSIL $M D=08$ \$2,995.452 $\$ 1,674,716 \quad$ R699

SUPPORT SERVICES FOR FARAMETRIC COST ANALYSIS AND COST ESTIM $O H \quad 01 \quad \$ 2,447,909 \quad \$ 1,595,000$ R425

CYCLE 14 - INSTITUTIOHAL COHVERSATION PROGRAM. APPROVED BUD NC 07 \$N3,238 $\$ 36,619$

SEE ATTACHED FAGE NO. 2 OF THIS NOTICE OF FINANCIAL ASSISTAN NiC 07 \$3, $\$ 18,559$

SEE ATTACHED FAGE HO. 4 OF THIS NOTICE OF FINANCIAL ASSISTAN HC 07 \$7.192 $\$ 3,596$

DEVELOPMENT OF A DIELECTRIC SPECTROSCOPY PROBE FOR CHARGE IIY 21 \$549,400 $\$ 408,443$ SUPPORT FOR SCIENCE. MATHEMATICS AND ENGIHEERING

GRANT PROGRAMS FOR SCHOOLS ANDHOSPITALS AND FOR BUILDINGS HE 01 \$9,500 $\$ 8,550$

CYCLE.14 - INSTITUTIONAL CONSERVATION PROGRAM. AFPROVED BUD NC $03 \quad \$ 69,476 \quad \$ 38,214$

SEE ATTACHED PAGE NO. 2 OF THIS HOTICE OF FINANCIAL ASSISTAN IIC 033 \$68,140 $\$ 40,000$ 
$\frac{5}{i}$

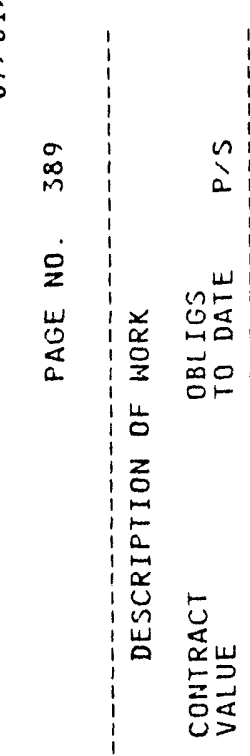

高

는

$i \frac{\pi}{4}$

¿ष山

运出

山洝

岂导

昆它

嵌政

$\sum_{i \infty}^{n}$

岩是

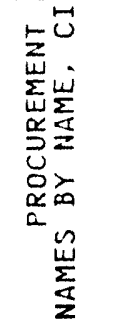

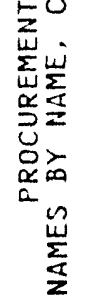

竞

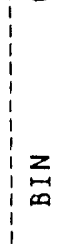

品:

站

5

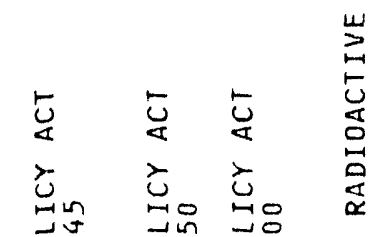

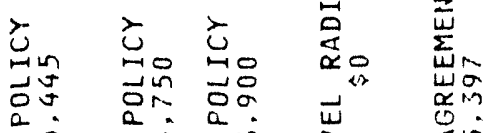

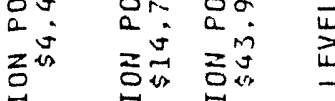

E

总点点

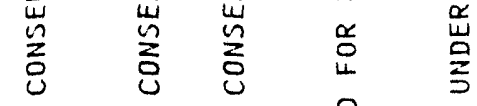

万人

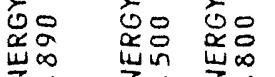

㟧品

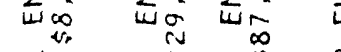

吉

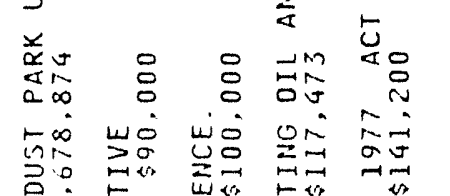

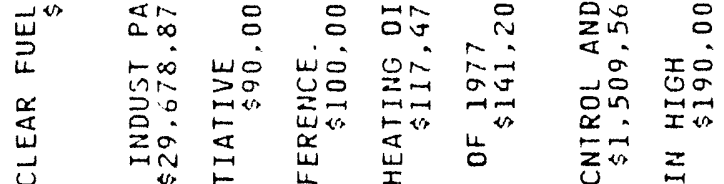

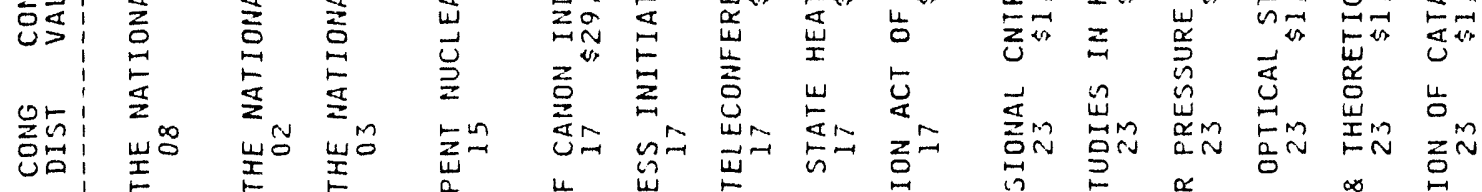

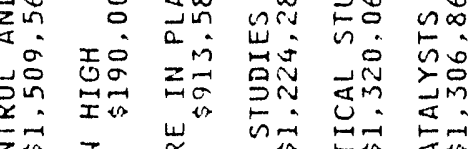

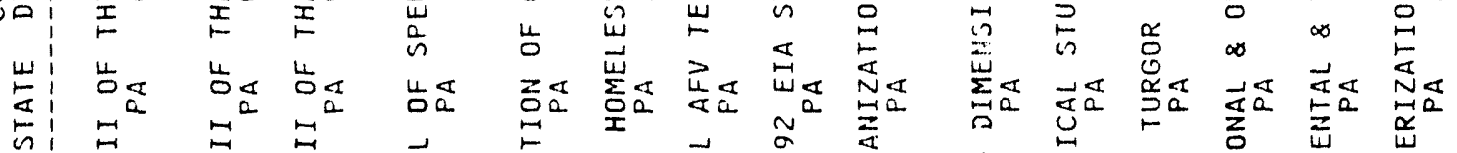

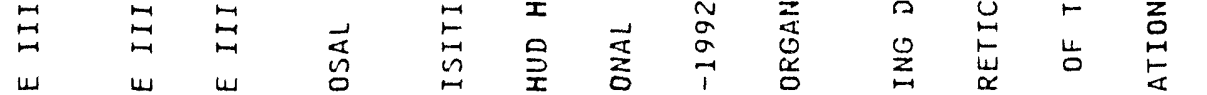

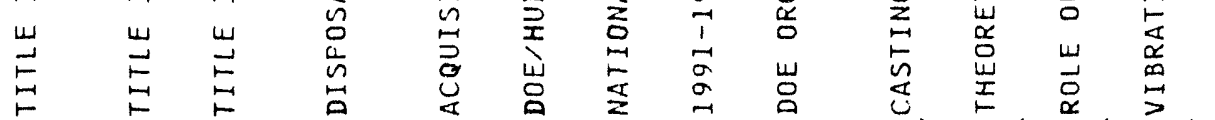
(n)

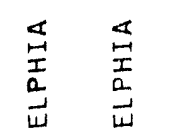 \\ 西}

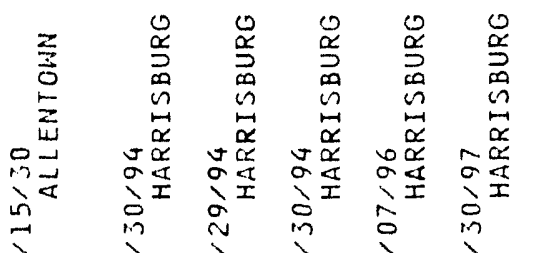

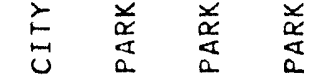

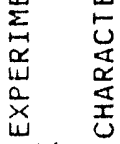

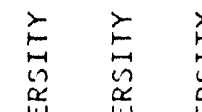

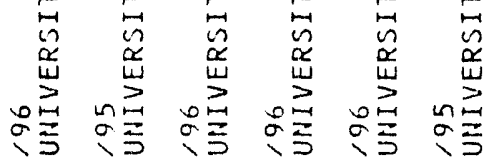

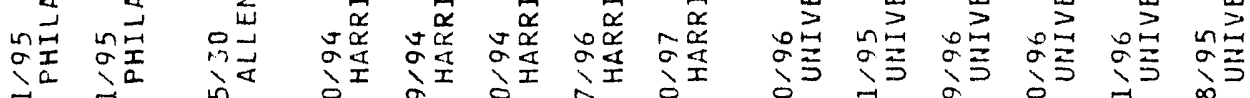
ले ले ले मे

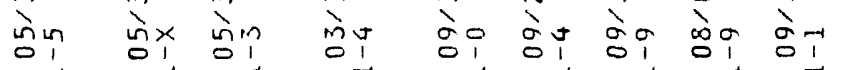

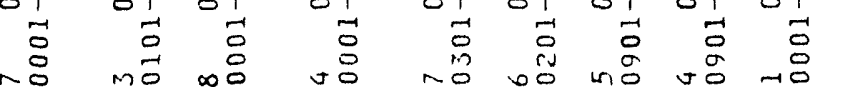

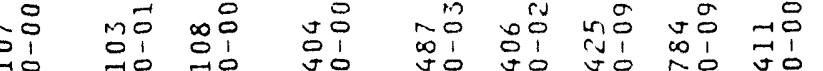

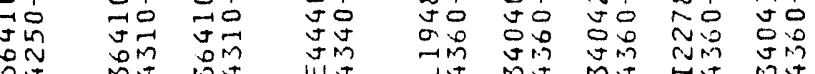
尔。 离

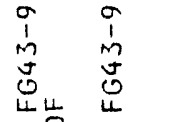
$\infty$
1
1
0
0
0 $\begin{array}{lll} & 0 & 0 \\ 1 & 1 & 0 \\ 0 & 0 & 0 \\ 0 & 0 & 0 \\ u & 0 & 0 \\ 4 & 4 & 4\end{array}$

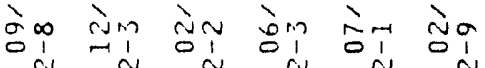

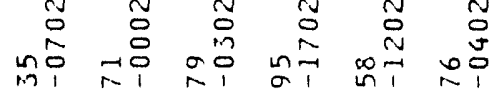

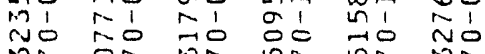
min

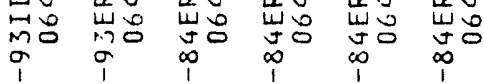

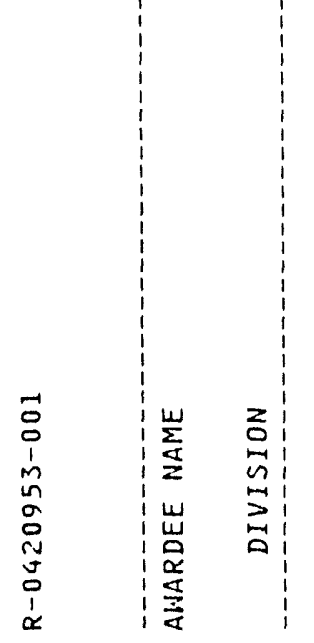

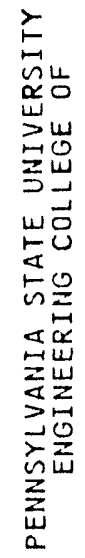

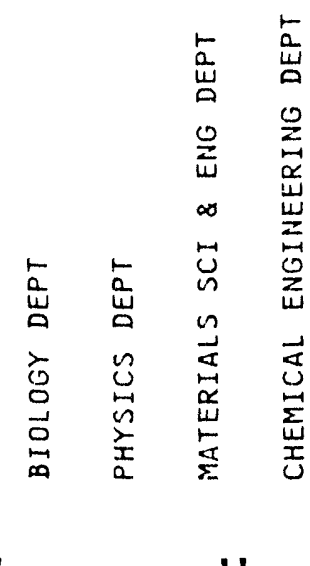


DESCRIPTION OF HORK

AWARDEE NAME

COMPL DATE

\section{TRACT}

STATE DIST

VALUE

VENDOR ID

CITY

TO DATE

$\mathrm{P} / \mathrm{S}$

DIVISION

FG02-84ER13295 $11 / 30 / 95$

TRANSITION METAL CATALYZED TRANSFORMATIONS OF UNSATURATED MO

CHEMISTRY DEPT

MATERIALS RESEARCH LAB

MATERIALS SCI \& ENG DEPT

PHYSICS DEPT

GEOSCIENCES

BIOLOGY DEPT

CHEMISTRY DEPT

CHEMISTRY DEPT

PHYSICS DEPT

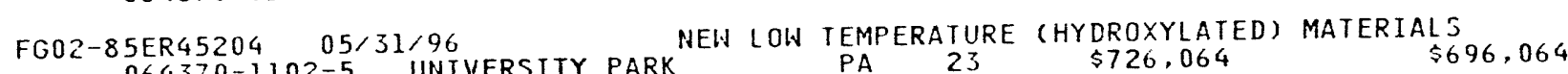
DETERMINATION OF THE DISTRIBUTION OF HYDROGEN IN COAL BY ELECTRON TRANSFER, IOHIZATION AND EXCITATION IN ATOMIC

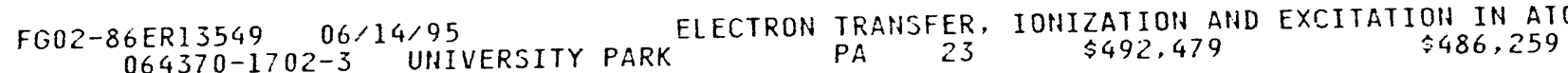

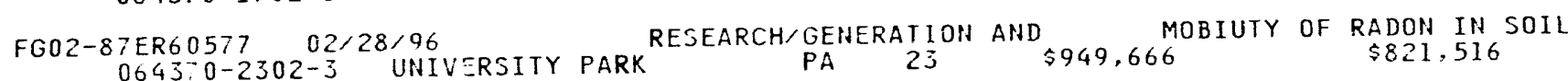
FG02-87ER13690 $03 / 14 / 95$
$064370-0302-2$ UNIVERSITY PARK ENZYMOLOGY AND MOLECULAR BIOLOGY OF LIGNIN DEGRADATION

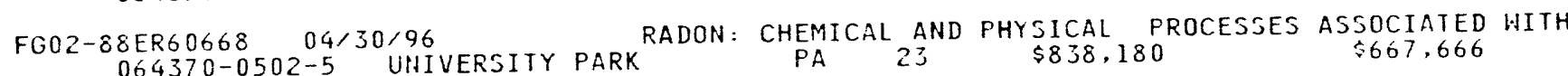

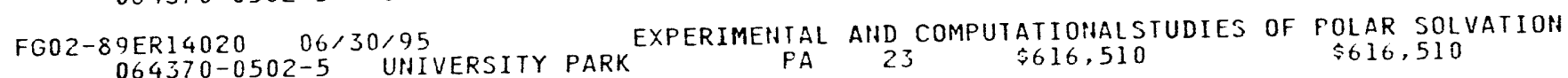

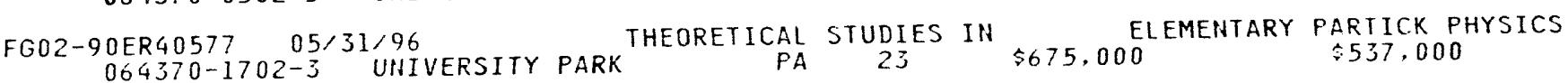

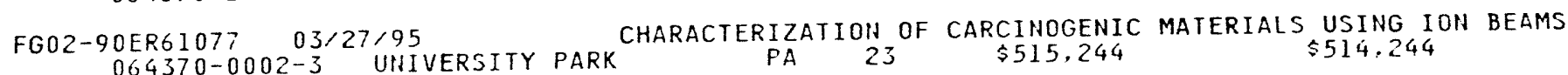

FG02-90ER61071 10/31/96 AN INTEGRATED CLOUD OBSERVATION \& MODELING INVESTIGSTION $064370-0002-3$ UHIVERSITY PARK AN INTEGRATED CLOUD OBJERVATION \& MODELIN $\$ 2,410,693$

FG02-91ER.61197 07/31/94 FIELD-SCALE VALIDATION \& DEVELOPMENT OF A THREE-DIMENSIONAL $064370-0002-3$ UNIVERSITY PARK PA 23 \$ 38,806 FG02-91ER20050 $08 / 31 / 94$
$066370-0002-3$ UNIVERSITY PARK ROLE OF CA++/CALMODULIN IN THEREGULATION OF MICROTUBULES IN

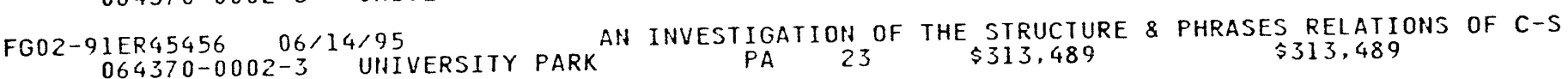

FG02-9IER45461 09/14/94 FUNDAMENTAL STUDIES OF PASSIVITY \& PASSIVITY BREAKDOHN.

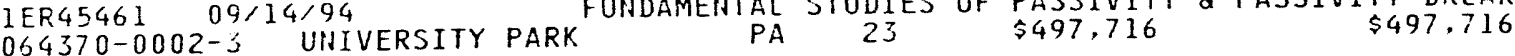

FG02-80ER10728 09/29/94 UNIVERSITY REACTOR SHARING

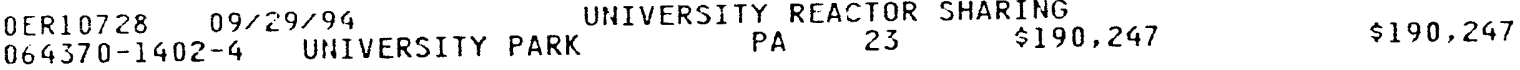

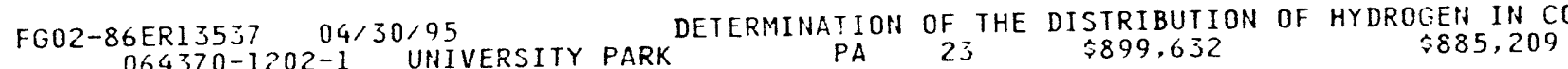


DIVISION VENDOR ID CITY CONG CONTRACT OBLIGS STATE DIST VALUE CONTRACT
VALUE TO DATE P/S $90 E R 12966--09 / 29 / 94-1402-4$
$064370-1402-4$ $90 E R 12966-09 / 29 / 94$
$064370-1402-4$ NUCLEAR ENGINEERING DEPT

\section{GEOSCIENCES} FG02-92ERI4251 $11 / 14 / 94$ UNIVERSITY PARK PA 23 $\$ 239,513$

$\$ 196,576$ FG02-92ER14258 04/30/95
$064370-0002-3$ UNIVERSITY PARK FG02-92ER14302 08/31/95 FG02-92ER75804 $09 / 29 / 94$
$064370-0002-3$ UNIVERSITY PARK APFLICATION FOR THE FG02-94ER20132 $01 / 06 / 95$
$064370-0002-3$ UNIVERSITY PARK

WORKSHOP ON VEGATATIVE

FG02-94ER61773 $11 / 30 / 96$
$064370-0002-3$ UNIVERSITY PARK FG02-94ER54231 $10 / 31 / 96$
$064370-0002-3$ UNIVERSITY PARK

FG02-94ER20137 $03 / 31 / 97$
$064370-0002-3$ UNIVERSITY PARK FG02-93ER61602 04/30/96 UNIVERSITY PARK 064370-0002-3 UNIVERSITY PARK FG02-93ER20117 06/30/95 UNIVERSITY PARK $\$ 263,457$ $\$ 26 \dot{3}, 457$ $\$ 343,000$ \begin{tabular}{ccc} 
METAL CLUSTER ALLOYS \& & OXIDES \\
\hline PA & 23 & $\$ 343,000$
\end{tabular} THE HVOF THERMAL DYNAMICS OF THE HVOF
$\$ 207,210$ $\$ 90,000$ MATCHING GRANTS PROGRAM $\begin{array}{lll}P A & 05 & 16,50\end{array}$ DEVEL OPMENT $\$ 90,000$ AN INTERGRATED CUMULUS $\$ 404,374$ ENSEMBLE/TURBULENCE RAPID RELATIVISTIC CALCULATIONOF ATOMIC DATA FOR PLASMA IGHT ENERGY TRANSDUCTION IN GREEN SULFURE BACTERIA YANMICS OF INITIAL IONIZATIONEVENTS IN BIOLOGICAL MOLECULTS FG02-93ER $14358006 / 30 / 96$
$064370-0002-3$ UIIIVERSITY PARK CARBON DEPOSITION AND PA 10 FG02-93ER $2518407 / 31 / 94$ UNIVERSITY PARK 064370-0002-3 UHIVERSITY PARK FG02-93ER $14374008 / 31 / 96$
$064370-0002-3$ UNIVERSITY PARK FG02-93ER $1438709 / 14 / 96$ UNIVESITY PARK SEVENTH INTERNATIONAL PA 05 $\$ 10.000$ TRANSFER REACTIONS INMICROPORUS SOLIDS
PA 05 $\underset{\$ 43 j, 770}{\$ 165,107}$ PA
DEVELOPMENT OF NOVEL $\$ 606,000$ FGC2-93ER61717 09/14/94 REGIONAL CLIMATES IN GCM:S 064370-0002-3 UNIVERSITY PARK $\quad$ PA $05 \quad \$ 57,824 \quad \$ 57,824$ FG04-91AL75029 $06 / 30 / 95$ UNIVERSITY PARK IN-CYLINDER STUDY OF THE IHTERDEPENDENCY OF SOOT AND NOX IN 


\begin{tabular}{|c|c|c|c|c|c|c|c|}
\hline AWARDEE NAME & BIN & L DATE & & & DESC & F HORK & \\
\hline DIVISION & VENDOR ID & CITY & STATE & $\begin{array}{l}\text { CONG } \\
\text { DIST }\end{array}$ & $\begin{array}{l}\text { CONTRACT } \\
\text { VALUE }\end{array}$ & $\begin{array}{l}\text { OBL IGS } \\
\text { TO DATE }\end{array}$ & $\mathrm{P} / \mathrm{S}$ \\
\hline
\end{tabular}
VENDOR ID STATE DIST VALUE TO DATE

FG05-91ER79047 09/29/94 SECOND GENERATION DIGITAL TMAGING SPECTROPHOTOMETER 1ER79047 $09 / 29 / 94$ VERTTY PARK SECOND GENERATION DIGITAL TMAGING SPECTROPHOTOMETER

FG05-92ER79113 07/31/94 INSTRUMENTATION - WATER, AEROSAL, VAPOR EXPERIMENT LIDAR AND $064370-0002-3$ UNIVERSITY PARK

FG05-93ER79218 08/31/95 1993 UNIVERSITY INSTRUMENTATION PROGRAM - A FEMTOSECOND LASE $064370-0002-3$ UNIVERSITY FARK 05 PA 05 \$176,080

FG05-93ER79226 08/31/95 1993 UNIVERSITY INSTRUMENTATION PROGRAM - ACQUISITION OF A C

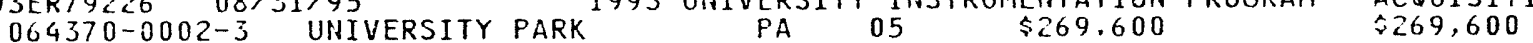

FG05-93ER7923908/31/95 1993 UNIVERSITY INSTRUMENTATION PROGRAM - DYNAMIC IMAGING AN $064370-0002-3$ UNIVERSITY PARK

FC22-89PC88697 03/01/95 SUPERCLEAN COAL-HATER SLURRY COMBUSTION TESTING IN AN OIL-FI

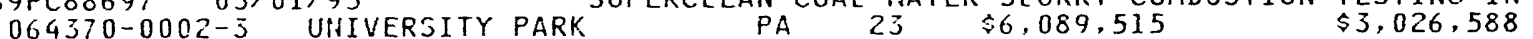

AC22-90PC90054 12/26́/94 NOVEL NAHODISPERSED COAL LIQUEFACTION CATALYSTS MOLECULAR DE $064370-0002-3$ UNIVERSITY PARK $P A \quad 23 \quad \$ 434,121 \quad$ PA

MINERAL ENGINEERING DEPT

FUEL SCIENCE DEPT

MATERIALS RESEARCH LAB

EARTH \& MINERAL SCIENCES

FUEL SCIENCE DEPT

- MINERAL ENGINEERING DEPT

FUEL SCIENCE DEPT

$=$
AC22-9211992008 09,29,94 DEVELOPMENT OF A COST-EFFECTIVE ENVIROHENTAL COMPLIANCE TEC

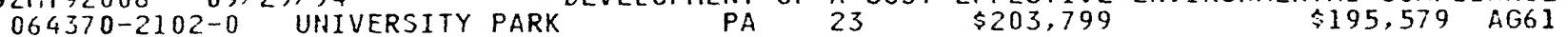

AC22-91PC91042 09/17/94 EFFECTS OF LOW TEMPERATURE CATALYTIC PRETREATMENTS ON COAL S 064570-0802-4 UNIVERSITY PARK.

FG22-91PC91302 09/18/94 HYDROTHERMAL REACTIONS OF FLYASH 064370-1102-5 UHIVERSITY PARK

FG22-9IPCOI303 09/10/94 SEMICONDUCTOR ELECROCHEMISIRY OF COAL PYRITE

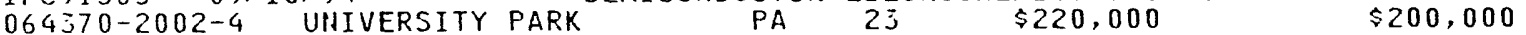

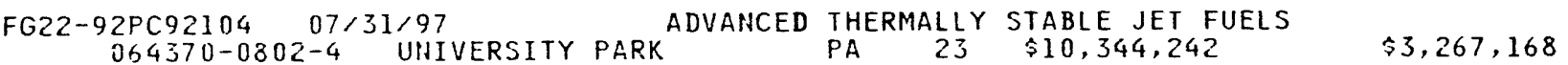

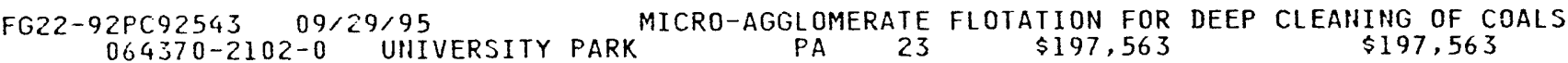
$\begin{array}{cccc}\text { FC22-92PC } 92162 & 09 / 27,95 \\ 064370-0002-3 & \text { UNIVERSITY PARK THE DEVELOPMENT OF COAL-BASED FUEL TECHHOLOGIES FOR DEPARTME } & \text { PA } 23 \text { \$18,500,000 } & \$ 9,770,000\end{array}$

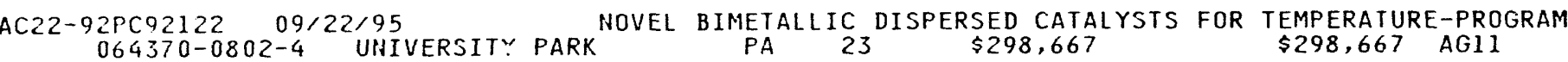

AC22-93PC93051 09/28/98 MAINTENAISCE OF THE COAL SAMPLEBANK \& DATABASE $064370-0602-1$ UNIVERSITY PARK 
AIIARDEE NAME
BIN

COMPL DATE
DESCRIPTION OF WORK

DIVISION

VENDOR ID CITY

$196--$

9 S'PC $93223-1-08$

UNI I

FG41-92R110542 06/30/95 064370-0002-3 UNIVERSITY PARK MISCELLANEOUS FEDERAL

CONG COHTRACT VALUE

\$OTO-0XIDI
$\$ 197,778$

$\$ 135,000$

D DATE $\$ 197,778$

ALTOONA CAMNUS

FG43-94R364109 05/31/95 064370-2802-5 UNIVERSITY PARK

TITLE III OF THE NATIONAL ENERGY CONSERVATION FOLICY ACT

FG43-94R364110 05/31/95

TITLE III OF THE NATIONAL ENERGY CONSERVATION POLICY ACT

FAYETTE CAMPUS 064370-2902-1 UNIVERSITY PARK

PENNSYLVANIA UNIVERSITY OF CHEMICAL ENG DEPT

FG02-85ER13350 03/31/97
$064380-0201-1$ PHILADELPHIA

MATERIALS SCIENCE \& ENG DEPT AC02-76ER03071 $12 / 31 / 94$

PHYSICS DEPT

FG02-84ER45118 01/31/97 064330-1201-7 PHILADELPHIA

MATLS SCI \& ENG DEPT

PHYSICS DEPT

MATLS SCI \& ENG DEPT

CHEMISTRY DEPT

CHEMISTRY DEPT

BIOLOGY

MATLS SCI \& ENG DEPT

CHEMISTRY DEPT

: CHEMISTRY DEPT
FG02-85ER45188 01/14/95
$064380-0701-3$ PHILADELPHIA 064380-0701-3 PHILADELPHIA

FG02-86ER40264 02/28/95
$064380-1201-7$ PHIL

FG02-86ER45254 05/31/95 $064380-0701-3$ PHILADELPHIA

FG02-86ER13584 03/31/95 064380-0401-4 PHILADELPHIA

FG02-86ER13615 12/14/95 064380-0401-4 PHILADELPHIA

FG02-87ER13680 02/14/95 064380-1801-5 PHILADELPHIA 064380-0701-3 PHILADELPHIA

FG02-87ER1 $3732 \quad 11 / 30 / 96$ 064380-0401-4 PHILADELPHIA

FG02-87ER13792008/14/96 064380-0401-4 PHILADELPHIA

FG02-88ER13825 01/14/97
FG02-87ER4529506/30/96
DESORPTION AND REACTION KINETICS STUDIES ON MODEL SUPPORTED $P A \$ 2 \$ 1,070,112$ \$861,882

$\begin{array}{ccc}\text { CONDUCT THEORETICAL AND EXPERIMENTAL RESEARCH PROGRAMS IN } \\ \text { FA } 01 \text { \$ } \$ 77,227,068 & \$ 77,073,068 \text { AG9 }\end{array}$

INTRINSIC SURFACE PHOHONS ON RECONSTRUCTED SEMICONDUCTOR SUR PA 01 \$755,602 $\$ 632,320$

FUNDAMENTALS OF HARDENIHG AND DECOHESION BEHAVIOR IN TIME-DE PA 01 \$1,729,168 $\$ 1,454,487$

"THEORETICAL RESEARCH IN NUCLEAR STRUCTURE AND NUCLEAR PA 01 \$1, $128,000 \quad \$ 1,121,000$

STAGING IN LAYER INTERCALATES

$$
\text { PA } 01 \text { LAYER INTERCALATES } \$ 1,252,231 \quad \$ 1,252,231
$$

DYHAMICS OF VIBRATIONALLY EXCITED POLYATOMIC MOLECULES AND T PA $01 \quad \$ 929,250 \quad \$ 921,930$

CATALYTIC HYDROGEHATION OF CARBON MONOXIDE

$$
\text { PA OI \$1,197,600 } \$ 956,460
$$

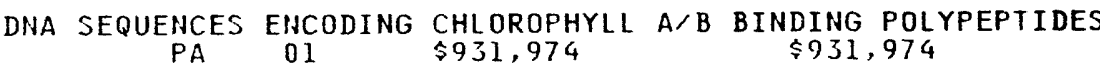

"ATOMISTIC STUDIES OF GRAIN BOUNDARIES IN ALLOYS AND

"SYNTHESIS AND PROPERTIES OF NEW PRECERAMIC MATERIALS" PA 01 \$1,005,400 \$764,695

HALF COLLISION STUDIES OF INELASTIC ENERGY TRANSFER

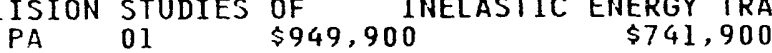

THERMODYNAMICS OF SYSTEMS HITHVERY MANY COMPONENTS PHASE 


\begin{tabular}{|c|c|c|}
\hline AWARDEE NAME & COMPL DATE & DESCRIPTION OF HORK \\
\hline DIVISION & VEHDOR ID & $\begin{array}{l}\text { OBLIGS } \\
\text { TO DATE }\end{array}$ \\
\hline CHEMICAL ENG DEPT & $064380-0201-1$ & $\$ 685,747$ \\
\hline PHYSICS DEPT & $\begin{array}{c}\text { FG02-88ER50642 } 02 / 14 / 97 \\
064380-1201-7 \text { PHILADELPHIA }\end{array}$ & $\begin{array}{ccccc}\text { POSITION RING SYSTEM USING } & \text { ANGER - TYPE DETECTORS } \\
\qquad \begin{array}{c}\text { PA } \\
01\end{array} & \$ 4,317,107 & \$ 2,679,785\end{array}$ \\
\hline \multirow[t]{4}{*}{ MATLS SCI \& ENG DEPT } & $\begin{array}{c}\text { FG02-90ER45428 } 09 / 14 / 96 \\
064380-0701-3 \quad \text { PHILADELPHIA }\end{array}$ & 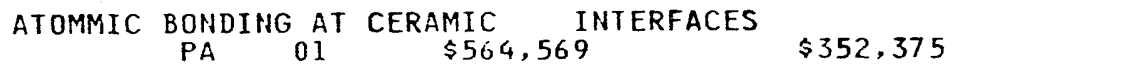 \\
\hline & $\begin{array}{c}\text { FG02-92ER61371 } 01 / 31 / 96 \\
064380-0001-9 \quad \text { PHILADELPHIA }\end{array}$ & 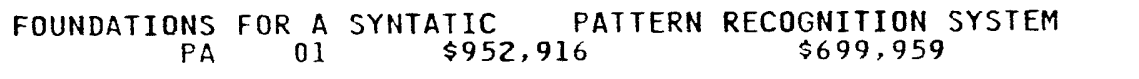 \\
\hline & $\begin{array}{c}\text { FG02-91ER14173 08/31/94 } \\
064380-0001-9 \text { PHILADELPHIA }\end{array}$ & $\begin{array}{ccc}\text { CATALYTIC SYNTHESIS OF SILICONCARBIDE PRECERAMIC POLYMERS : } & \text { PRT } \\
\text { PA } 01 & \$ 309,519 & \$ 309,519\end{array}$ \\
\hline & $\begin{array}{c}\text { FG02-91ER20052 08/31/94 } \\
064380-1801-5 \text { PHILADELPHIA }\end{array}$ & $\begin{array}{cccc}\text { MEMBRANE-ATTACHED } & \text { ELECTRON CARRIER IN PHOTOSYNTHESIS \& RESPI } \\
\text { PA } 01 & \$ 419,635 & \$ 419,635 & \end{array}$ \\
\hline \multirow[t]{2}{*}{ BIOLOGY } & $\begin{array}{c}\text { FG02-91ER20055 08/31/94 } \\
064380-1801-5 \text { PHILADELPHIA }\end{array}$ & $\begin{array}{cccc}\text { TRANSPORT FUNCTION \& REACTION MECHANISM OF VACUOLAR H+- TRAN } & \text { NACULA } \\
\text { PA } 01 & \$ 260,996 & \$ 260,996\end{array}$ \\
\hline & $\begin{array}{c}\text { FG02-91ER40680 } 08 / 31 / 94 \\
064380-0001-9 \text { PHILADELPHIA }\end{array}$ & $\begin{array}{r}\text { HIGH ENERGY PHYSICS RESEARCH } \\
\text { PA } 01 \text { \$1, } 486,300\end{array}$ \\
\hline MECHANICAL ENGINEERING DEPT & $\begin{array}{l}\text { FG02-92ER14271 } 06 / 14 / 95 \\
064380-0801-X \quad \text { PHILADELPHIA }\end{array}$ & $\begin{array}{ccc}\text { ACTIVE CONTROL OF CONVECTION } \\
\text { PA } & 01 & \hat{\$} 305,073\end{array}$ \\
\hline \multirow[t]{4}{*}{ BIOLOGY } & $\begin{array}{c}\text { FG02-93ER20104 } 04 / 30 / 96 \\
064380-1801-5 \quad \text { PHILADELPHIA }\end{array}$ & $\begin{array}{c}\text { MOLECULAR AND GENETIC ANALYSISOF CTRI; A NEGATIVE REGULATOR } \\
\text { FA } 01 \\
\quad \$ 288,090\end{array}$ \\
\hline & $\begin{array}{c}\text { FG02-93ER61657 01/31/97 } \\
064380-0001-9 \text { PHILADELPHIA }\end{array}$ & $\begin{array}{cccc}\text { PRECEPTOR SPECIFIC LIGAHDS } & \text { FOR SPEC IMAGING } \\
\text { PA } & 01 & \$ 737,116 & \$ 287,768\end{array}$ \\
\hline & $\begin{array}{c}\text { FG05-92ER79123 } 07 / 31 / 94 \\
064380-0001-9 \text { PHILADELPHIA }\end{array}$ & $\begin{array}{c}1992 \text { INSTRUMENTATIOH } \\
\text { PA } \\
\begin{array}{c}\text { PIGH SPEED MIXED AHALAY AND DIGITAL I } \\
\$ 227,000\end{array}\end{array}$ \\
\hline & $\begin{array}{c}\text { FG05-93ER79222 } 08 / 31 / 95 \\
064380-0001-9 \text { PHILADELPHTA }\end{array}$ & 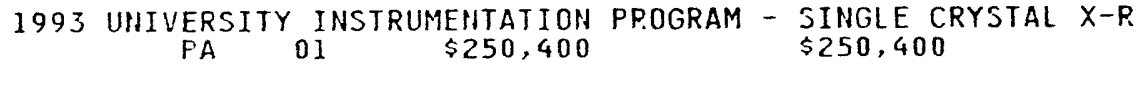 \\
\hline NOBSCOT BAY MEDICAL CENTER & $\begin{array}{rl}\text { FG4 } 4-93 R 192506 & 06 / 30 / 95 \\
064400-0001-2 & R O C K L A N D\end{array}$ & TITLE III DF THE NATIOHAL ENERGY CONSERVATION POLICY ACT \\
\hline EOPLESWORKS & $\begin{array}{r}A B 01-941911045904 / 30 / 95 \\
237490-0001-8 \text { LOS ANGELES }\end{array}$ & 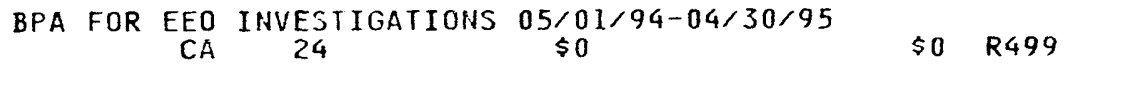 \\
\hline RCHETTI I & $\begin{array}{c}\text { AC08-78NV01606 08/31/94 } \\
064500-0001-5 \text { TOHOPAH }\end{array}$ & 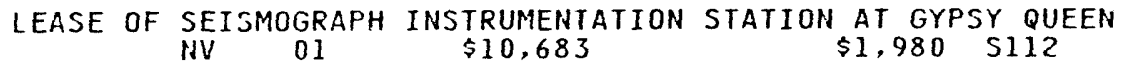 \\
\hline
\end{tabular}




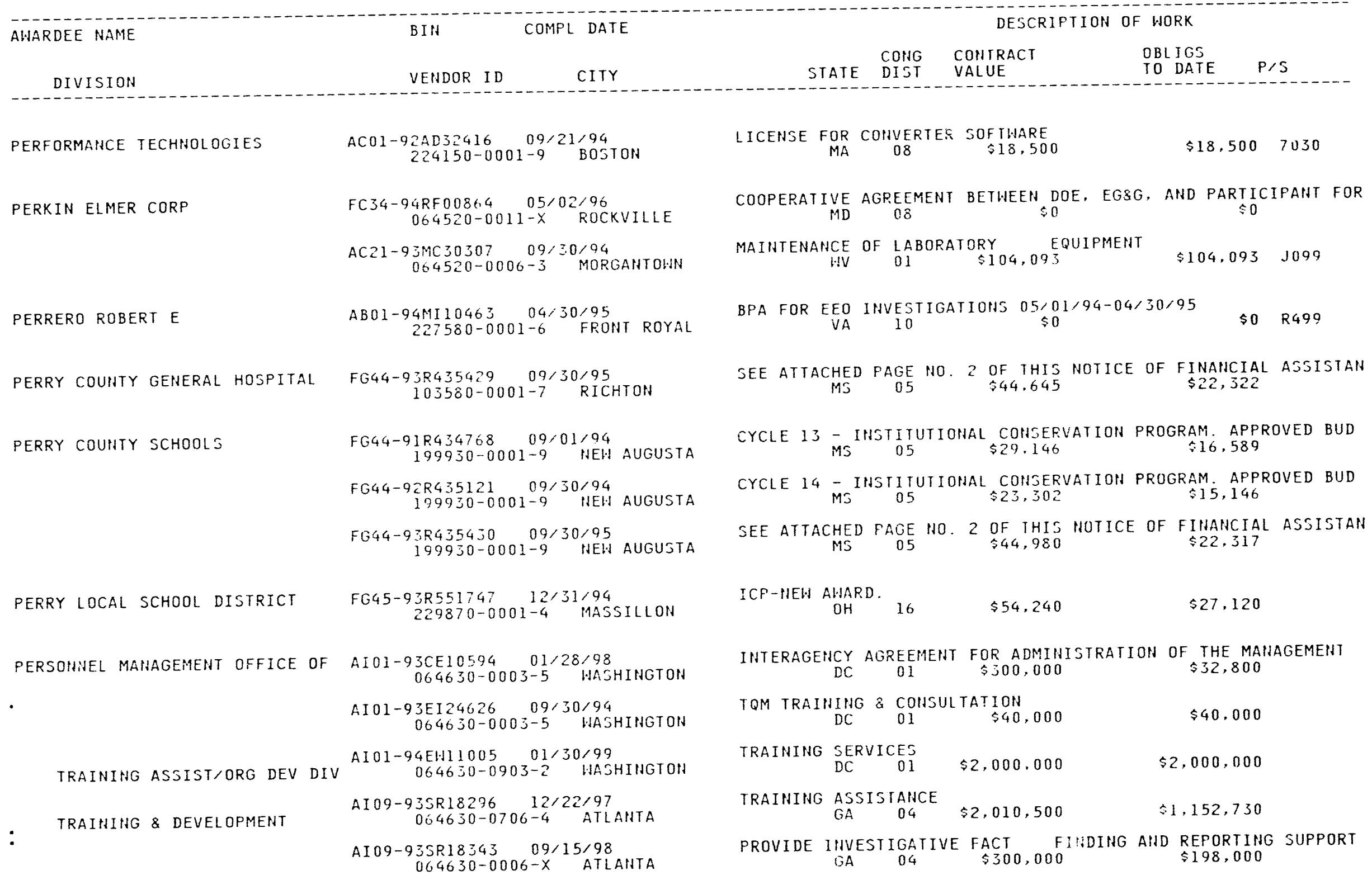


$R-0420953-001$

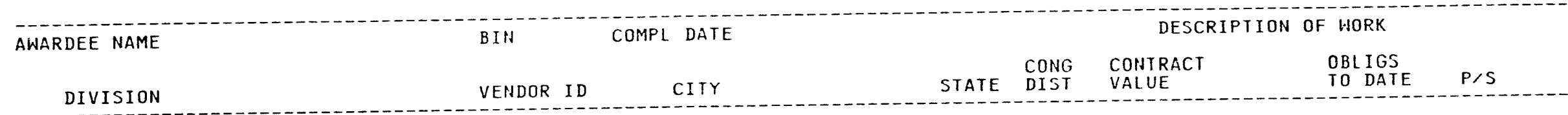

\section{PERU GOVERNMENT OF}

PERU STATE COLLEGE

PETAL SEPARATE SCHOOL DISTRICT

PETRO-DIAMOND INC

PETROLEUM CONSULTING SERVICES

PETROLEUM ENGIHEERS SOCIETY OF

\section{PHILADELPHIA CITY OF}

PHILADELPHIA ELECTRIC CO

$$
\begin{gathered}
\text { AI09-945R18447007/31/94 } \\
064630-0006-x \text { ATLANTA } \\
\begin{array}{c}
\text { AI01-941600393 } 09 / 02 / 94 \\
064630-0008-6 \quad \text { OAK RIDGE }
\end{array} \\
\begin{array}{c}
\text { AI65-9311410980 } 07 / 31 / 96 \\
064630-0007-8 \quad \text { DALLAS }
\end{array}
\end{gathered}
$$$$
\begin{array}{r}
\text { FC02-94P010129 11/16/95 } \\
235760-0001-1 \quad \text { LIMA }
\end{array}
$$$$
\begin{gathered}
F G 47-9 j R 703521008 / 31 / 94 \\
004650-0001-6 \quad \text { PERU }
\end{gathered}
$$

FG44-90R434388 01/31/95
$202340-0001-5$ PETAL

SC92-93FE63090 09/30/94 $158130-0002-X$ IRVINE

AC21-90MC26025 11/10/94
$195610-0001-6 \quad$ CANTON

FG22-94BC14873 07/30/94 230740-0001-5 RICHARDSON

FG43-92R340407 09/30/94 C:4930-0001-7 PHILADELPHIA

FG43-9 JR340422 09/30/95 064930-0001-7 PHILNDELPHIA

CR01-83NE44405 $03 / 15 / 30$
$064940-0001-0$ PHILADELPHIA

CRO1-83NE44470 03/15/30 064940-0001-0 PHILADELPHIA
PROVIDE POSITION CLASSIFICATION AND STAFFING SUPPORT TO SR

$$
\text { GA } 04 \quad \$ 100,000 \quad \$ 100,000
$$

INTERAGENCY AGREEMENT FOR SPACES/TRAINING SEMINARS

PROVIDE FACILITIES SVCS

PROVIDE FACILITIES SVCS $\$ 221,835$

$\$ 104,225$

CLIMATE CHANGE STUDY

$\$ 714,750$

$\$ 332,350$

GRANT PROGRAMS FOR SCHOOLS ANDHOSPITALS AND FOR BUILDINGS 0

$$
\text { HE } 01 \text { \$1.116 \$558 }
$$

CYCLE 12 INSTITUTIONAL COHSERVATION PROGRAM. AFPROVED FUNDED $\begin{array}{lll}\text { MS } 05 & \$ 36.453 & \end{array}$

NPR-1 LIQUID PRODUCTS SALE ESTIMATED CONIRACT AMOUNT: $\$ 102,8$ $C A \quad 40 \quad \$ 0 \quad \$ 0$

PRODUCTION VERIFICATION TESTS

$$
\text { OH } 16 \text { \$2,196,802 } \$ 2,196,802 \quad \text { AG22 }
$$

NINTH SYMPOSIUM ON IMPROVED OIL RECOVERY $\$ 15,000$

$$
T X \quad 03 \quad \$ 26,000 \quad \$ 15.000
$$

DEVELOPMENT OF METHODOLOGY FOR EXTERNALLY FINACED ENERGY $P A \$ 2 \quad \$ 70,000 \quad \$ 25,822$

PHILADELPHIA CLEAI CITIES

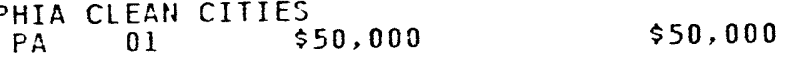

DISPOSAL OF SPENT NUCLEAR FUEL AND FOR HIGH LEVEL

TO AHARD CONTRACT FOR DISPOSALOF SPENT NUCLEAR FUEL AND $\angle O R$ 


\begin{tabular}{|c|c|c|c|c|c|c|c|}
\hline AWARDEE NAME & BIN & DATE & & & DESCF & F HORK & \\
\hline DIVISION & VENDOR ID & CITY & STATE & $\begin{array}{l}\text { COHG } \\
\text { DIST }\end{array}$ & $\begin{array}{l}\text { CONTRACT } \\
\text { VALUE }\end{array}$ & $\begin{array}{l}\text { OBLIGS } \\
\text { TO DATE }\end{array}$ & $\mathrm{P} / \mathrm{S}$ \\
\hline
\end{tabular}

PHILADELPHIA SCHOOL DISTRICT FG43-937340426 09930195 $064970-0001-1$ PHILADELPHA

PHILLIPS MOORE LEMPIO \& FIHLEY AC03-925F19335 09/29/94 $25 F 193350090200001-0$
$065 A N$ FRANCISCO CHOOL DISIRICT OF PHILADEIPHIA'S PARTNERSHIP IN ENERGY/ENVI

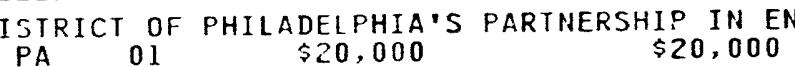

PROVIDE SUPPORT SERVICES TO OFFICE OF PATENTS IN THE OFFICE

PHILLIPS PETROLEUM CO AC96-87P060116 $12 / 01 / 96$
$065030-0003-0$ FREEPORT EXPLORATION PRODUCTION GROUP FC22-94BC14991 $01 / 02 / 01$ PHOTOCRAFT INC PHYSICAL OPTICS CORP
TERMINAL DISTRIBUTIOH SERVICES
TX $22 \quad \$ 10,106,811$

$\$ 10.106,811 \quad \mathrm{~V} 119$

DESIGN \& IMPLEMENTATION OF A CO2 FLOOD UTILIZING ADVANCED RE

BPA FOR GRAPHIC ARTS

\begin{tabular}{|c|c|c|c|c|c|}
\hline $\begin{array}{l}\text { GRAPHI } \\
\text { MAD }\end{array}$ & $\begin{array}{l}C \text { AR } \\
08\end{array}$ & 15 & $\$ 0$ & $\$ 0$ & T001 \\
\hline $\begin{array}{l}\text { PHOTO } \\
\text { MD }\end{array}$ & $\begin{array}{r}\text { AND } \\
08\end{array}$ & 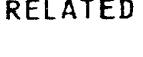 & $\begin{array}{c}\text { SERVICE } \\
\$ 0\end{array}$ & $\$ 0$ & T015 \\
\hline
\end{tabular}

BPA FOR PHOTO AND RELATED SERVICES
MD
08

BPA FOR GRAPHIC PRODUCTION AIJDOTHER VISUAL ART DELIVERABLES

SBIR - PHASE I AHARD FOR - HIGH DATA RATE PARALLEL-BIT OPTIC CA $42 \quad \$ 549,943$ DATA RATE $\$ 549,943$

LARGE CORE SILICA FIBER OPTIC SYSTEMS ARE UNDER DEVELOPMENT

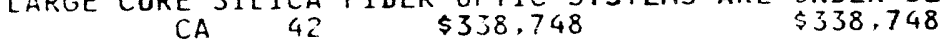
EXPERIMENTS HECESSITATES DATA PROCESSING TECHHIQUES CAPABLE CA $42 \quad \$ 549,982 \quad \$ 408,858$

HIGHLY VERSATILE TUHABLE MULTI-SPECTRAL IMAGING SENSOR BASED CA 42 \$74.980 $\$ 74,980$

MULTIMODE SENSOR NETWORK MEDIA $\$ 74,983$

HIGH POHER COAXIAL GYROIROHS MA $05 \quad \$ 549,991$

$\$ 549,991$

NITRO AHD EXPLOSIVES MONITOR FOR CONE PENETROMETER WASTE
MA 05 \$75,000

TIME RESOLVED NITRIC OXIDE $\underset{05}{\$ 75,000}$ MEASUREMENTS IN COMBUSITOH
FG02-91ER81190 09/30/94
$065140-0001-0$ AHDOVER FG02-94ER81678 07/17/94
$065140-0001-0$ ANDOVER

FG02-93ER81617 07/17/94 065140-0001-0 ANDOVER 


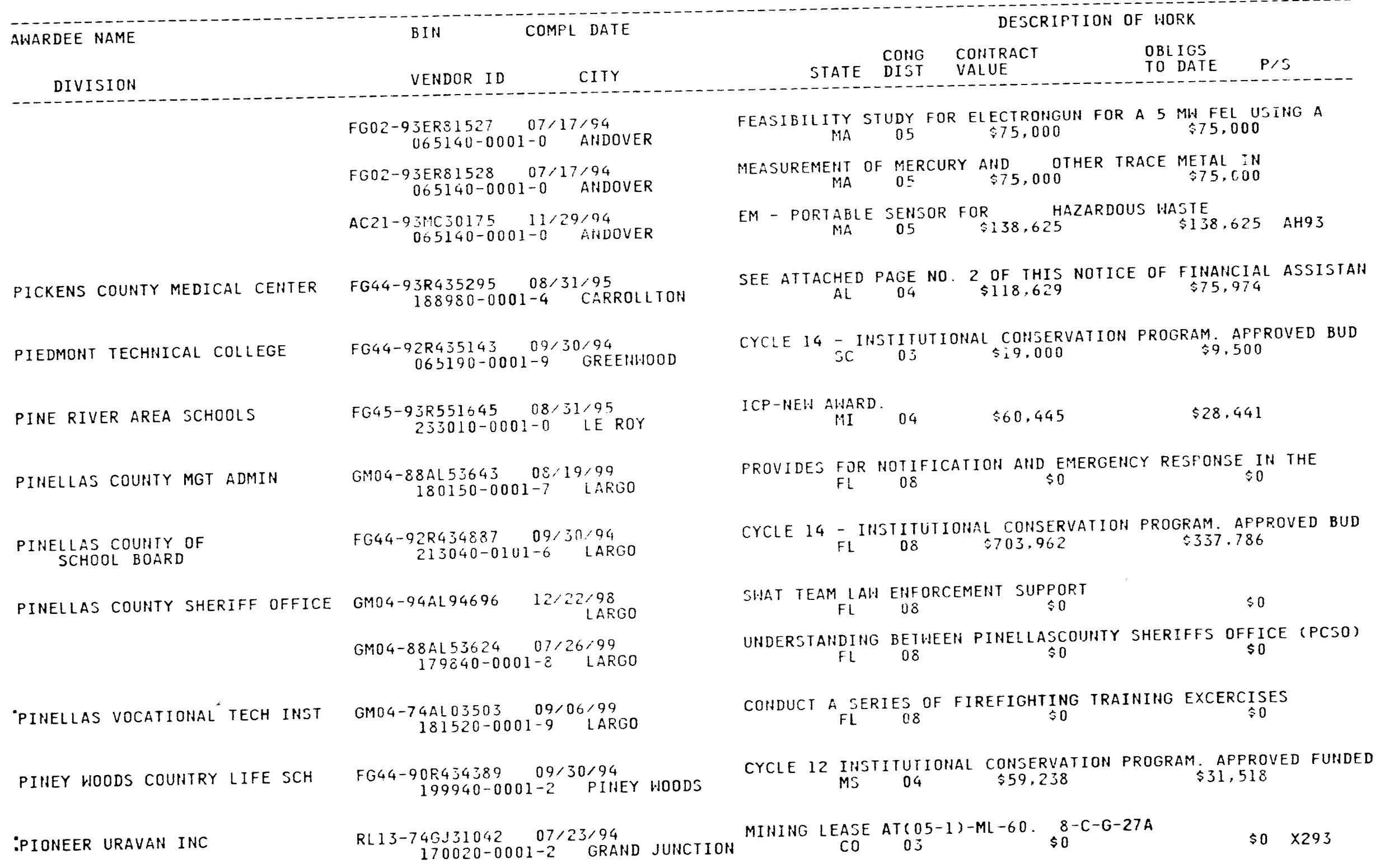




\begin{tabular}{|c|c|}
\hline AWARDEE NAME & COMPL DATE \\
\hline DIVISION & VENDOR ID \\
\hline PIPESTONE COUNTY MEDICAL CTR & $\begin{array}{c}\text { FG45-93R551709 } 0 ? / 31 / 95 \\
233200-0001-2 \text { PIP STONE }\end{array}$ \\
\hline PIQUA CITY OF & $\begin{array}{c}\text { ACO2-76EV01798 12/31/94 } \\
065420-0001-1 \quad \text { PIQUA }\end{array}$ \\
\hline PITTSBURG \& MIDHAY COAL & $\begin{array}{c}\text { ACO5-76ET10104 } 05 / 31 / 97 \\
065400-0002-4 \quad \text { KAHSAS CITY }\end{array}$ \\
\hline PITTSBURG STATE UNIVERSITY & $\begin{array}{c}\text { FG02-92CH10494 } 10 / 06 / 96 \\
103630-0001-1 \quad \text { PITTSBURG }\end{array}$ \\
\hline $\begin{array}{l}\text { PITTSBURGH UNIVERSITY OF } \\
\text { PHYSICS \& ASTRONOMY DEPT }\end{array}$ & $\begin{array}{c}\text { FG02-84ER451j1 } 10 / 31 / 96 \\
065490-1001-2 \text { PITTSBURGH }\end{array}$ \\
\hline PHYSICS DEPT & $\begin{array}{c}\text { FG02-87ER40363 } 04 / 30 / 97 \\
065490-1101-9 \quad \text { PITTSBURGH }\end{array}$ \\
\hline CHEMISTRY DEPT & $\begin{array}{c}\text { FG02-87ER13781 } 01 / 31 / 95 \\
065490-0301-6 \quad \text { PITTSBURGH }\end{array}$ \\
\hline CHEMISTRY DEPT & $\begin{array}{c}\text { FG02-88ER1 } 3864 \quad 03 ; 31,97 \\
005490-0301-6 \quad \text { PITTSBURGH }\end{array}$ \\
\hline MATERIALS \& ENGINEERING & $\begin{array}{c}\text { FG02-89ER45390 0 } 05 / 31>95 \\
065490-1301-1 \quad \text { PITTSBURGH }\end{array}$ \\
\hline CHEMISTRY DEPT & $\begin{array}{c}\text { FG02-89ERI4002 } 05 / 31 / 96 \\
065490-0301-t \quad \text { PITISBURGH }\end{array}$ \\
\hline MATERIALS \& ENGINEERING & $\begin{array}{c}\text { FGO2-90ER45438 } 09 / 24 / 96 \\
065490-1301-1 \text { PITTSBURGH }\end{array}$ \\
\hline PHYSICS DEPT & $\begin{array}{c}\text { FG02-91EF.40646 } \begin{array}{c}10 / 31 / 95 \\
065490-1101-9\end{array} \text { PITTSBURGH }\end{array}$ \\
\hline CHEMISTRY DEPT & $\begin{array}{c}\text { FC02-92CE40981 07/31/94 } \\
065490-0301-6 \text { PITTSBURGH }\end{array}$ \\
\hline & $\begin{array}{c}\text { FG02-92ERS1480 09/14/94 } \\
065490-0001-7 \text { PITTSBURGH }\end{array}$ \\
\hline & FG22-90PC90305 $08 / 15 / 94$ \\
\hline
\end{tabular}

CHEMICAL \& PETROLEUM ENG DEPT $062-90 P C 9030501080101-3$ FITTSBURGH

ICP-NEH AWARD.
MIN

$\begin{array}{ccc}\text { DEACTIVATION OF PNPF INCLUDINGENTOBMENT OF REACTOR VESSEL } \\ \$ 98.808 & \$ 98.808 \text { AZII }\end{array}$

FORT LEHIS PILOT PLANT

$$
\begin{aligned}
& \text { MO PILOT PLANT } \\
& 05 \text { \$193,047.186 }
\end{aligned}
$$

DESIGH AHD CONSTRUCTION OF THEAUTOMOTIVE. TECHNOLOGY BUILDING KS $055 \$ 8.096,000 \quad \$ 4,000,000$

THE PHYSICS OF PATIERH FORMATION AT LIQUID INTERFACES

THE PHYSPA 14 \$ $\$ 1,366,527$ AT $\$ 1,122,459$

"STUDIES OF RELATIVISTIC HEAVYION COLLISIONS AT THE AGS" FA 14 \$2, 114,000 \$1,643.000 $\begin{array}{cc}\text { STUDIES OF SUPPORTED } \\ \text { PA } 14 & \$ 14 \text { HYRODESULFURIZATION CATALYSTS }\end{array}$

VIBRATIOHAL SPECTROSCOPIC STUDIES OF SURFACE CHEMICAL

MICROSTRUCTURE AND MAGHETIC PROPERTIES IN HIGH-ENERGY $\begin{array}{llll}\text { PA } & 14 & \$ 659.096 & \$ 659,096\end{array}$

TIME RESULVED STUDIES OF CARRIER RECOMBINATION AT THE $\begin{array}{lll}\text { PA } 14 & \$ 595,000 & \$ 492,150\end{array}$

COMPUTER SIMULATIONS FOR THE ABSORTION OF FOLYMERS ONTO FA 14 \$ $\$ 303,607 \quad \$ 167,413$

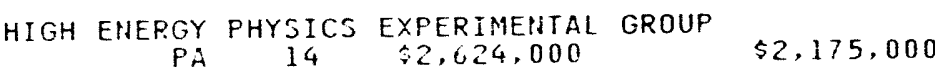
PRODUCTION OF BISPHENOL A POLYCABONATE USING CARBON
$\$ 200,000$ MDSRS STUDIES OF GAS-AEROSOL INTERACTION KINETIC.S PROBE MOLECULE STUDIES: ACIIVESPECIES IN ALCOHOL SYNTHESIS 


AWARDEE NAME
MEIVISION
MECHANICAL ENGINEERING

PLAINVIEW HOSPITAL

PLASMA PROCESSES

PLATTE RIVER POWER AUTHORITY

PLEASAHTOH UHIFIED SCHOOL DIST

PLEASAITVILLE CITY OF

EDUCATION BOARD OF

PLM TECHHOLOGIES INC

POCAHONTAS COUNTY BD OF EDUCN :

POCATELLO READY MIX INC
COMPL DATE

CONG CONTRACT

DESCRIPTION OF HORK

BIN COMPL DATE

VENDOR ID CITY

STATE DISI VALUE OBL IGS
TO DATE

$\mathrm{P} / \mathrm{S}$

A RHEOMETER FOR TIEASURING THE MATERIAL MODULI OF GRANULAR SO PA 14 \$ $\$ 196,846$ \$ 196,846

AHEORETICAL AND NUMERICAL STUDY OF THE FLON OF GRANULAR MA PA $14 \quad \$ 224,250 \quad \$ 224,250$ AGI

LH SEVERITY UPGRADING OF F-T HAXES WITH SOLID SUFERACIDS

$$
\text { PA } 14 \text { \$267,424 \$199,98 }
$$

EFFECT OF PRETREATING OF HOST OIL IN CO-PROCESSING

$$
\text { PA } 14 \quad \$ 377.643
$$

$A C 22-91 P C 91054 \quad 06 / 30 / 95$
$065490-0001-7 \quad$ PITTSBURGH

FG43-93F354220 12/31/94
$065490-0001-7$ PITTSBURGH

FG4J-93R354221 12/31/94
$065490-0001-7$ PITTSBURGH

FG47-92R703488 08,31/94
$065590-0001-X \quad$ PLAINVIEH

FG05-9JERE156j 04/01/96 35590-0001-3 HUNTSVILLE TITLE III OF THE NATIOHAL ENERGY CONSERVATION POLICY ACT

IITLE III OF THE NATIONAL ENERGY CONSERVATION FOLICY ACT $P A$ T4 $\$ 151,125$ \$75,000 GRAHT PROGRAMS FOR SCHOOLS AHDHOSFITALS AND FOR BUILDINGS 0 NE 03 O

HIGHIY EFFICIENT, HEAT-DISSIPATING, FUNCTIONAL!Y GRADIENT PL AL 05 HEAT-DISSIPATING, FUNCTIONALI, \$174,935

PARTICIPATIOH III THE DEPI OF ENERGY'S ELECTRIC AND HYBRID VE $\begin{array}{lll}\text { CO } & 04\end{array}$ $\$ 257,521$

FC07-91ID13070 02/24/90
$155820-0001-1$ FORT COLLINS

GM04-94AL94702 12/14/98
PLEASANTON

SUPPORT FOR SCIEHCE. MATHEMATICS \& ENG. EDUC.

$F G 42-94 R 26390306 / 30 / 95$
$238950-0101-5$ PLEASANTVILLE

CYCLE 16 AWARD.

$\$ 15,315$ AC65-91WL02822 $02 / 08 / 95$
$177360-0102-2$ SALT LAKE CITY TITLE I, II ATE SERVICES

ITLE I, II A/E SERVICES

$\$ \xi, 618,601 \quad C 211$

FG43-93R356202001/31/95

ITLE III DF THE NATIOHAL ENERGY CONSERVATION FOLICY ACT WV $03 \quad \$ 86,396$ $\$ 43.198$

AUTHORIZE LICENSE AGREEMENT FOR A CONCRETE BATCH PLANT AT TH 


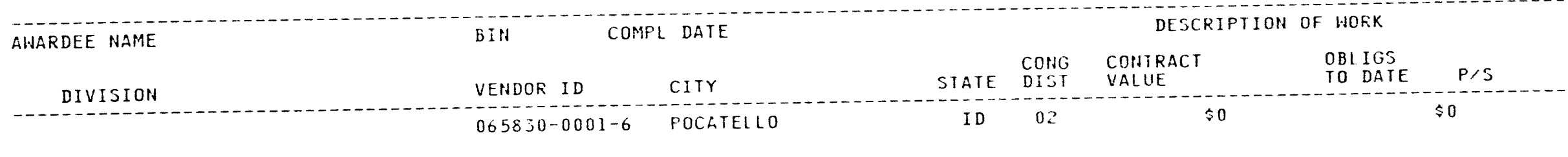
POCATELLO REGIONAL MEDICAL CTR GM07-91ID11081006/18/97 $209720-0001-1$ FOCATELLO

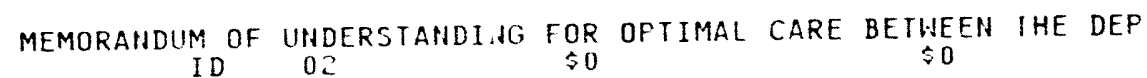

POILLON FLOREHCE

POINT BLANK BODY ARMOR INC

POINT PARK COLLEGE

POJOAQUE SCHOOLS

POLAND GOVERHMENT OF

POLE MAINTEIANCE CO

POLLOCK VANDE SAHDE \& PRIDDY

POLYTECHIIC INST OF NEH YORK PHYSICS DEPT

PHYSICS DEPT

POLYTECHNIC UNIVERSITY

AB01-94EH89437 01/31/95 SIVER SPRING TECHIICAL EDITING

$\$ 0$

\$0 9999

ADO4-93AL9114j $09 / 30 / 96$ I $57680-0002-X$ LAUREL

FG43-93R354214 12/31/94 $005870-0001-0$ PITTSBURGH

FG46-94F613647 06/30/95 226990-0101-5 POJOAQUE

GMO4-93AL84066 11/24/97 $226990-0101-5$ SAHTA FE

$F C 02-93 F 010095009 / 29 / 95$
$234370-0001-2$ WARSAH $A C 65-931121025 \quad 10 / 15 \% 94$
$065890-0010-7 \quad$ CASPER

$A B O 1-936 C 20327 \quad 07 / 31 / 95$ 198410-0001-6 HASHINGTON

FG02-84ER45127 01/31/97 $065960-0101-0$ BRDOKLYN FG02-87ER45301 05/31/96 BRDOKLYH HEIGHTS

SOFT BODY ARMOR

$\$ 143.450$

$\$ 143.450 \quad 1080$

TITLE III DF THE HATIONAL EHERGY CONSERVATION POLICY ACT PA IG S147.455 $\$ 73.377$

TITLE III OF THE WATIOHAL ENERGY COHJERVATION FOLICY ACT SUPPORT FOR SCIENCE, MATHEMATICS, AHD EINGIHEERING TO IMPROVE IIM 03

POLAHD CLIMATE CHAHGE COUHTRY JIUDY

$\$ 584.800$

IHSPECT \& TREATMENT OF HOOD POLES TLINES IN WYOMIHG WY 01 \$237.521 $\$ 237.521 \quad 2239$

BPA FOR PREPARAIION OF DOMESTIC PATENT APPLICATIONS
DC 01
00 MIXED VALENT BEHAVIOR IN THE ACTIHIDES \& THE RELATIONSHIP TO SCANIIIIG TUNHELING MICROSPECTROSCOPY OF SOLIDS AND SURFACES TUNHELING MICROSPECTROSCOPY OF SOLIDS AND
IHY 14 \$1, 34,291

FG02-92ER75786 09/29/94
$221850-0001-2$ HEW YORK FG02-93ER45499 02:15/97

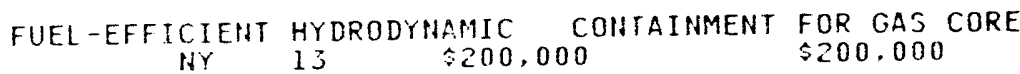

PROCESSING, DEFORMATION AND MICROSTRUCTURE OF SINGLE 


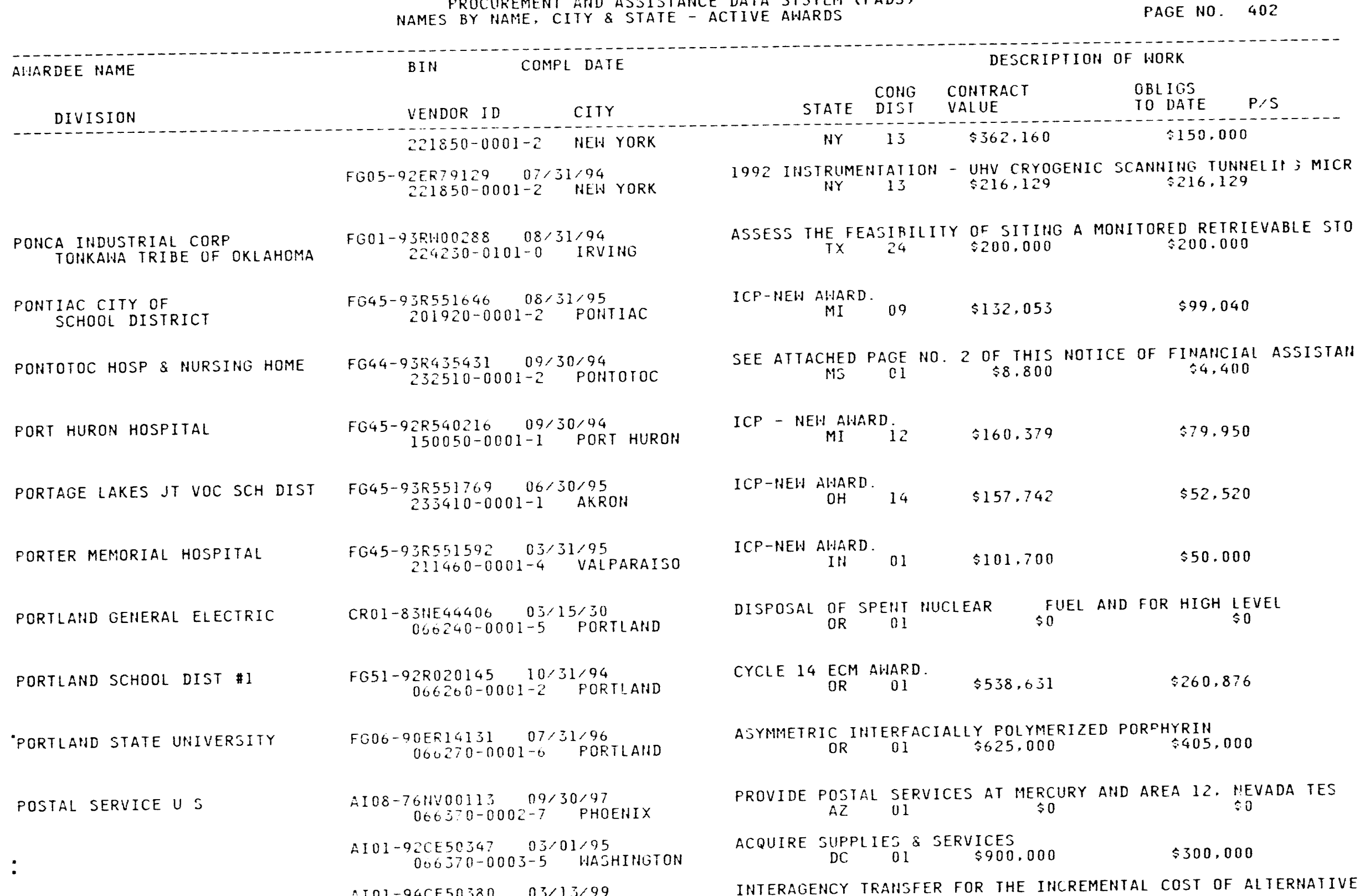


AWARDEE NAME

COMPL DATE

DESCRIPTION OF HORK

DIVISION

BIN

STATE CONG CONTRACT

VENDOR ID CITY

DC 01 \$1,701,126

OBL IGS

$066370-0003-5$ WASHIHGTON

HY $26 \quad \$ 494,225$

$\$ 228,776$

CONSOLIDATED SCHOOL DISTRICT FG42-92R243825 $207 / 31 / 94$ POTSDAM

POTTS KERR \& CO

$A B 01-926 C 20297 \quad 09 / 30 / 94$
$066440-0003-7 \quad$ MERSEYSIDE

PROVIDE PATENT SERVICES IN EUROPE

$\$ 0 \quad R 418$

$A B O 1-946 C 20341 \quad 03 / 30 / 96$

BFA FOR PATENT APPLICATIONS \& SERVICES

$066440-0003-7$ MERSEYSIDE UNITED KING* 00

$\$ 0$

$\$ 0 \quad R 498$

POWER AUTHORITY STATE OF NY

CRO1-83NE44407 03/15/30

$\begin{array}{ccccc}\text { DISPOSAL OF SPEHT HUCLEAR } & \text { FUEL AND FOR HIGH LEVEL } \\ & \text { NY } & & \$ 0 & \end{array}$

$066480-0001-5$ HEL YORK

BUILDING 84, PHASES II AND III

$\$ 3,101,293 \quad \Upsilon 199$

POWER CONTRACTING INC

AC22-91PC91601 08/31/94
$205370-0001-1$ PITTSBURGH

AC22-92PC92930 10/31/94

$205370-0001-1$ PITTSBURGH

BUILDING 922 WEST END RENOVATIONS

$\$ 3,877,307 \quad Y 199$

POHER ENGINEERING CORP

AC65-92WA10063 $10 / 26 / 96$

$103710-0002-5$ MEDFORD

POWER TRANSFORMER FOR DEER CREEK SWITCHYARD

POWER REACTOR \& NUCLEAR FUEL

GM04-81AL17110 09/30/99 $066530-0002-8$ ALBUQUERQUE

PR NEWSWIRE

AB01-94PP00189 12/31/94 $195990-0003-X$ HASHINGTON

AC05-910R21936 09/30/94 199990-0001-0 MCLEAI

PRAGMATICS INC

FG45-91R530069 IJ/31/94 $196880-0001-9$ LAGRANGE FG $45-94 R 562249 \quad 12 / 31 / 95$
$238630-0001-3$ RACINE

PRAIRIE SCHOOL

OR

$\$ 79,104 \quad 6120$

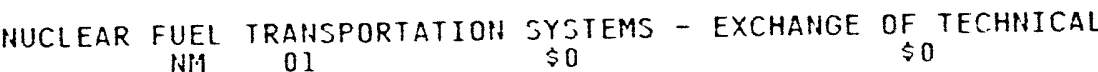

BPA FOR VERBATIUM TRANSMISSIOHOF HEHS RELEASES
DC

$\$ 0 \quad D 317$

SUPPORT SERVICE CONTRACT TO PROVIDE PROFESSINAL SUPPORT SERV $\checkmark A \quad 10 \quad \$ 1,538,071 \quad \$ 1,337,000 \quad R 421$

ICP-NEW AWARD

IN $04 \quad \$ 177,360$

$\$ 66,789$

ICP-NEW AWARD.

WI 01

₹ 10,000

$\$ 5,000$ 


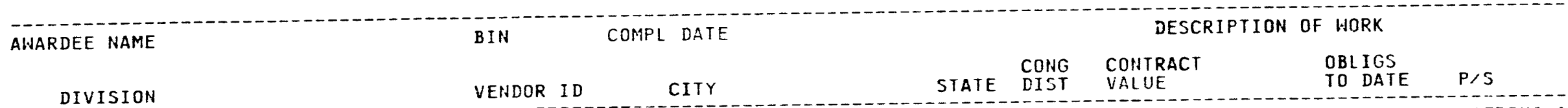

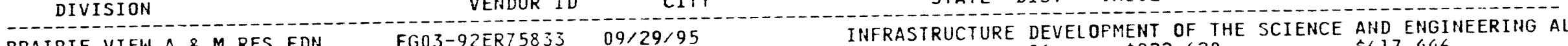

PRAIRIE VIEW A \& M RES FDN FG03-92ER75833 $09 / 29 / 95$ ER40728 $03 / 14 / 95$ COLLE STAT) FG05-92ER40728 03/14/95
$177390-0001-9$ COLLEGE STATION FG03-92ER54189 08/14/95 $177390-0002-7$ PRAIRIE VIEW

PRAIRIE VIEW A \& M UNIV

FG05-90ER54107 08/31/94 066640-0001-6 PRAIRIE VIEW FG04-87AL43436 09/29/96 066640-0001-6 PRAIRIE VIEW

PRC ENVIROHMENTAL MGMT INC

AC09-925R18258 05/13/95 219210-0001-5 AIKEN

PRC INC

ADO1-94CE41111 09/30/94
$236890-0001-7$ RESTON

PREFERRED TEMPORARY SERVICES

ABO1-94P010196 03/31/95 $193720-0001-9$ HAJHINGTON

ABO1-94DP00365002/09/95 193720-0001-9 WASHIHGTON

ABO1-94AD11100 03/31/95 $193720-0001-9$ HASHINGTON

ABO1-94AD38000 10/31/94 193720-0001-9 HASHIHGTOH

-PRENTICE hall finANCIAL LEGAL

ADC1-93AD34025 09/30/94 $186120-0001-7$ HASHINGTOH

PRENTICE HALL INC

ADO1-94AD35029 09/30/95 $066720-0002-X$ HASHIHGTON

AC02-76IR03438 12/30/99 066720-0001-1 EHGLEHOOD CLIFFS
INFRASTRUCTURE DEVELOPMENT 06 \$922.620 $\$ 617,446$

SUPPORT OF HYDROPRODUCTIOH OF BOTTOM USING THE 800 DEV/C PRI $\begin{array}{lll}\text { IX } 06 & \$ 914,000 & \$ 679,000\end{array}$

LOCAL HEAT TRANSFER AND CHF FOR SUBCOOLED FLON BOILING $T X \quad 06 \quad \$ 470,000 \quad \$ 470,000$

THE DEVELOPMENT OF A CHEMICAL KIHETIC MEASUREMENT APPARATUS $T X$ OO $\$ 165,000 \quad \$ 165,000$

GRANT FOR HISTORICALLY BLACK COLLEGES AHD UHIVERSITIES $T X \quad 14 \quad \$ 49,229 \quad \$ 49,229$

TECHNICAL SUPPORT SERVICES FORASST MGR FOR ENVIRONMEHTAL SC 03 \$1 $3,383,180$

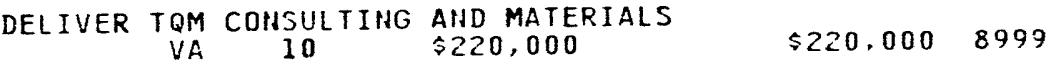

TEMPORARY SECRETARIES

SECRETARIES
DC 01

\$0 R699

BPA FOR TEMP. SECRETARIES

\$0 R699

TEMPORARY SECRETARIAL SERVICES

\$0 R499

BPA FOR TEMPORARY SECRETARIES

$\$ 0 \quad R 699$

ACCESS TO PRENTICE ON LINE DATA BASE
$D C \quad 01$ \$1,000
$\$ 1.000 \quad 7030$

TIMESHARING SERVICES

$01 \quad \$ 2,000$

\$0 D305

PUB COMMERCIAILY AND MARKET A TECH BOOK FURNISHED BY COMMISS

TECH BOOK FURNISHED BY COMMIS

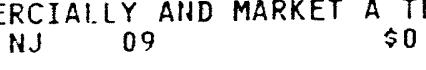




\begin{tabular}{|c|c|c|}
\hline AWARDEE NAME & COMPL DATE & DESCRIPTION OF WORK \\
\hline DIVISION & VENDOR ID & $\begin{array}{l}\text { COHIRACT } \\
\text { VALUE }\end{array}$ \\
\hline PRESBYTERIAN HEALTHCARE SVC & $\begin{array}{r}\text { GM04-93AL84016 04/28/98 } \\
228150-0001-6 \text { AL BUQUERQUE }\end{array}$ & PROVIDE DOE WITH EMERGENCY MEDIAL SERVICES FOR $\underset{\$ 0}{\text { INJURIES AND }}$ \\
\hline PRESS ASSOC & $\begin{array}{c}\text { ADO1-93AD34003 09/30/94 } \\
066780-0001-3 \text { NEH YORK }\end{array}$ & $\begin{array}{clr}\text { ACCESS TO NEWS } & \text { WIRE SERVICES } \\
\text { NY } & 10 & \$ 22,592\end{array}$ \\
\hline PRESTSii MLAONIA! HOSPITAL & $\begin{array}{c}\text { FG43-92R346200 01/31/95 } \\
222010-0001-8 \text { KINGWOOD }\end{array}$ & TITLE III OF THE NATIONAL ENERGY CONSERVATION POLICY ACT \\
\hline PRESTON SCHOOL DISTRICT $\# 201$ & $\begin{array}{c}\text { FG51-93R020287 } 09 / 30 / 94 \\
066800-0001-7 \quad \text { PRESTON }\end{array}$ & $\begin{array}{c}\text { CYCLE } 15 \text { ECM AWARD. } \\
\text { ID } 02\end{array}$ \\
\hline PRIME TEMPS INC & $\begin{array}{c}\text { ABO1-93AD00017 } 10 / 31 / 94 \\
234490-0001-2 \text { WASHINGTON }\end{array}$ & $\begin{array}{c}\text { BPA FOR TEMPORARY SECRETARIAL WORK } \\
\text { DC } 01\end{array}$ \\
\hline \multirow[t]{2}{*}{ PRIMEENERGY CORP } & $\begin{array}{l}\text { AC21-91MC27260 } 12 / 30 / 94 \\
215640-0001-X \quad \text { STAMFORD }\end{array}$ & 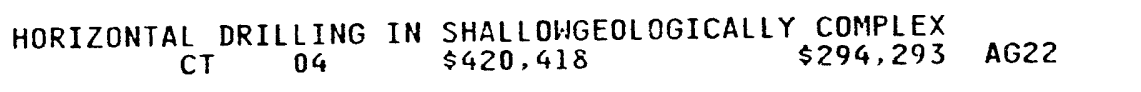 \\
\hline & $\begin{array}{c}\text { AC21-91MC28239 } 12 / 30 / 94 \\
215640-0001-X \text { STAMFORD }\end{array}$ & 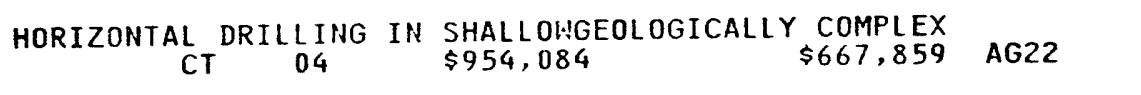 \\
\hline PRINCE GEORGES CTY PUBLIC SCH & $\begin{array}{l}\text { FG02-93ER75907 } 06 / 30 / 95 \\
229410-0001-1 \quad \text { UPPER MARLBORO }\end{array}$ & 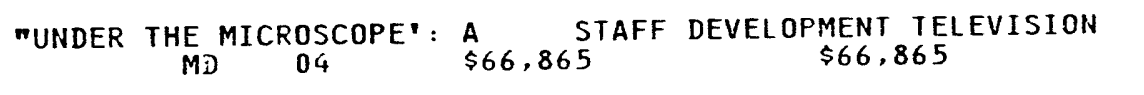 \\
\hline \multirow[t]{3}{*}{ PRINCETON ECONOMIC RES INC } & $\begin{array}{c}\text { ACO1-93CE20000 12/14/94 } \\
166360-0003-1 \quad \text { ROCKVILLE }\end{array}$ & 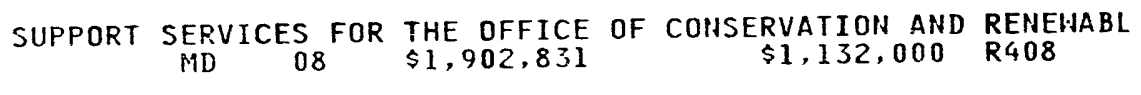 \\
\hline & $\begin{array}{l}\text { ACO1-93CE16070 09/14/95 } \\
166360-0003-1 \text { ROCKVILLE }\end{array}$ & 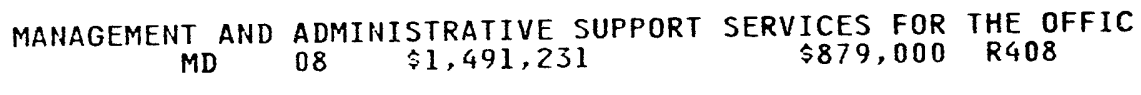 \\
\hline & $\begin{array}{c}\text { ACO1-93CE35060 09/14/96 } \\
166360-0003-1 \text { ROCKVILLE }\end{array}$ & $\begin{array}{cccc}\text { TECHNICAL \& } & \text { MANAGEMENT SUPPORTSERVICES FOR OFFICE OF UTILIT } & \text { OF } \\
\text { MD } & 08 & \$ 7,703,795 & \$ 4,110,685\end{array}$ \\
\hline PRINCETON PROFESSIONAL PARK & $\begin{array}{l}\text { FG02-93ER12129 } 11 / 18 / 95 \\
066900-0001-X \quad \text { PRINCETON }\end{array}$ & 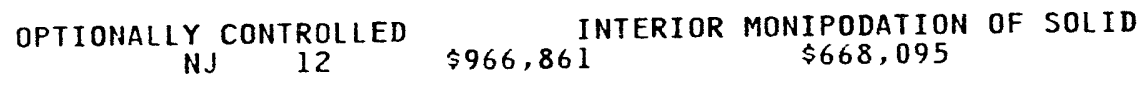 \\
\hline RINCETON SCIENTIFIC INST INC & $\begin{array}{c}\text { FG05-92ER81401 } 02 / 17 / 95 \\
221410-0001-7 \text { MOHMOUTH JUHCT }\end{array}$ & 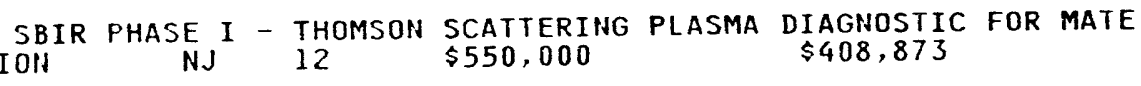 \\
\hline
\end{tabular}




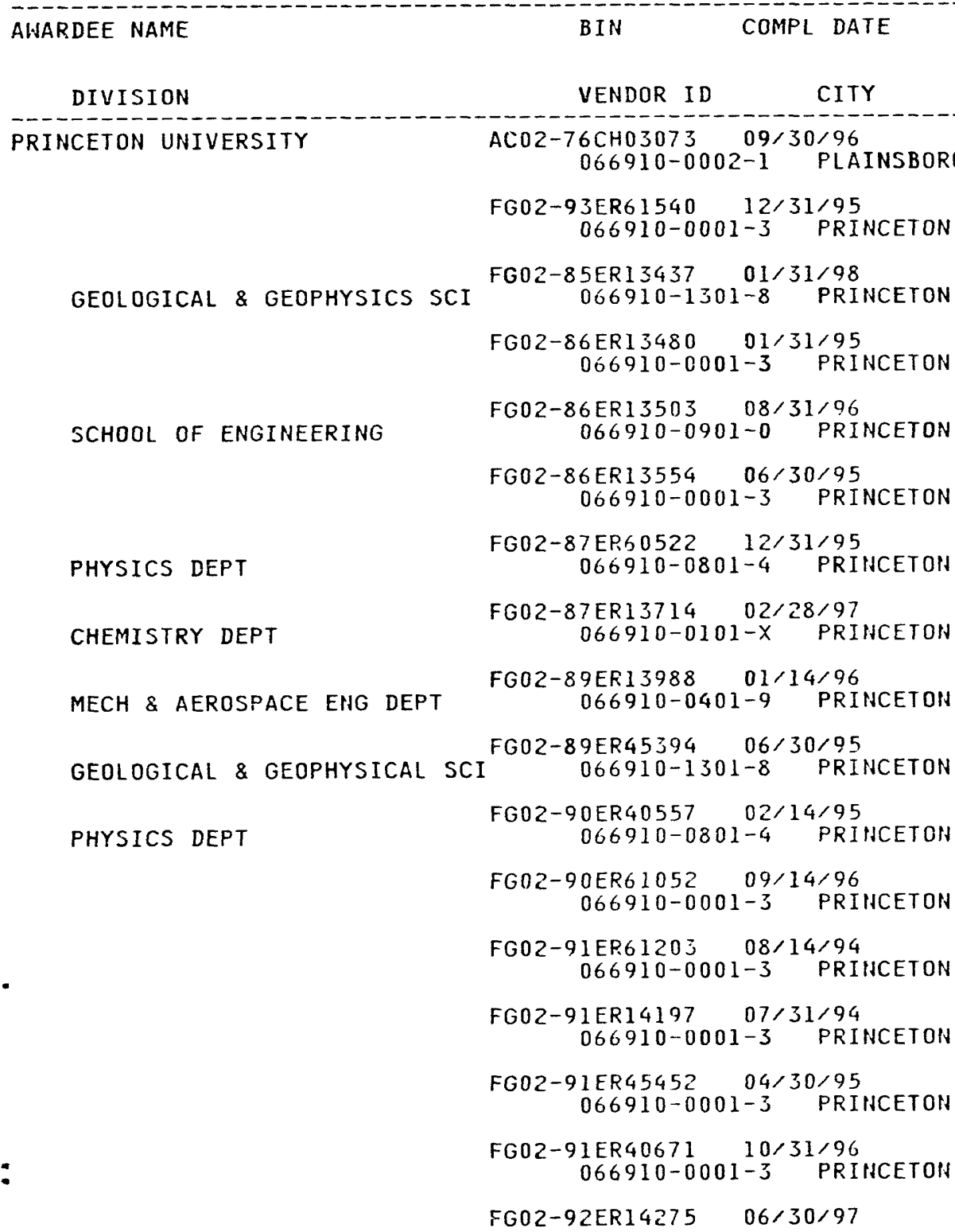

STATE DIST VONTRAC

DESCRIPTION OF HORK

NERGY RESEARCH

$\begin{gathered}\text { FUSION ENERGY RESEARCH } \\ \mathrm{NJ}\end{gathered} 12 \$ 2,460,142,364 \quad \$ 2,153,312,326 \quad$ AG51

OBLIGS

$\mathrm{P} / \mathrm{S}$

GLOBAL SURVEY OF CARBON DIOXIDE IN THE OCEAN

MELTS AND GLASSES: THERMOCHEM ICAL STUDIES

NJ 12 \$1,606,186 \$1,156,186

DYNAMICAL STUDIES OF MOLECULARSYSTEMS
$\$ 1,135,259$
$\$ 1,040,746$

COMPREHENSIVE MECHANISMS FOR COMBUSTION CHEMISTRY

$$
\text { NJ } 12 \$ 1,002,318 \quad \$ 784,318
$$

AROMATIC RADICAL OXIDATION KINETICS
NJ 12
$\$ 747,017$
$\$ 747,017$

DEVELOPMENT AND APPLICATIONS OF PHOTOSENSITIVE DEVISE

$$
\text { NJ } 12 \quad \$ 2,712,028 \quad \$ 2,182,028
$$

MOLECULAR DYNAMICS STUDIES

$$
\begin{array}{lll}
\text { NJ } 12 & \$ 615,829 & \$ 473,829
\end{array}
$$

STRUCTURE AND STABILIZATION OFPREMIXED AND DIFUSION FLAMES

$$
\text { NJ } 12 \quad \$ 673,838 \quad \$ 565,838
$$

THERMO CHEMISTRY OF PHASES RELATED TO OXIDE SUPER

$$
N J 12 \quad \$ 614,095
$$

THE PHYSICS OF LASER POLARIZEDMUONIC ATOMS

$$
\text { NJ } 12 \quad \$ 501,762 \quad \$ 496,762
$$

SIMULATION OF THE GLOBAL CARBON CYCLE AND ANTHROPOGENIC

REFLECTIOH IMAGING X-RAY LASERMICROSCOPE \& ITS BIOLOGICAL

THE EFFECTS DF NATURAL \& RADIATION INDUCED DEFECTS ON NOBLE IJ $12 \quad \$ 102,543 \quad \$ 102,543$

VISCOELASTICITY OF POLYMEN MELTU

A SILICON-DRIFT-CHAMBER, A PARALLEL COMPUTER FORM, \& READOUT
NJ

TRANSPORT PROPERTIC OF DISORDERED POROUS MEDIA FROM 


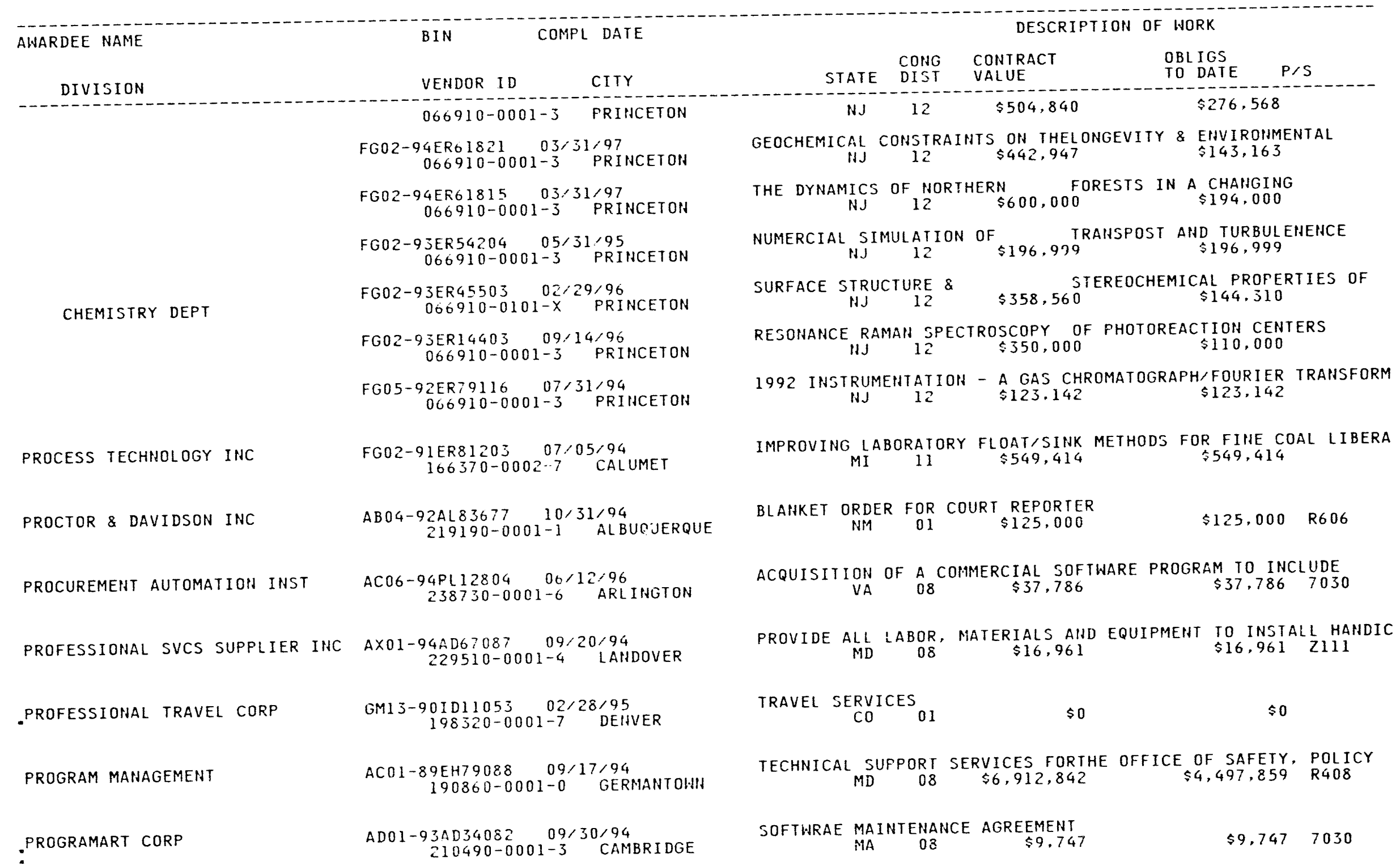




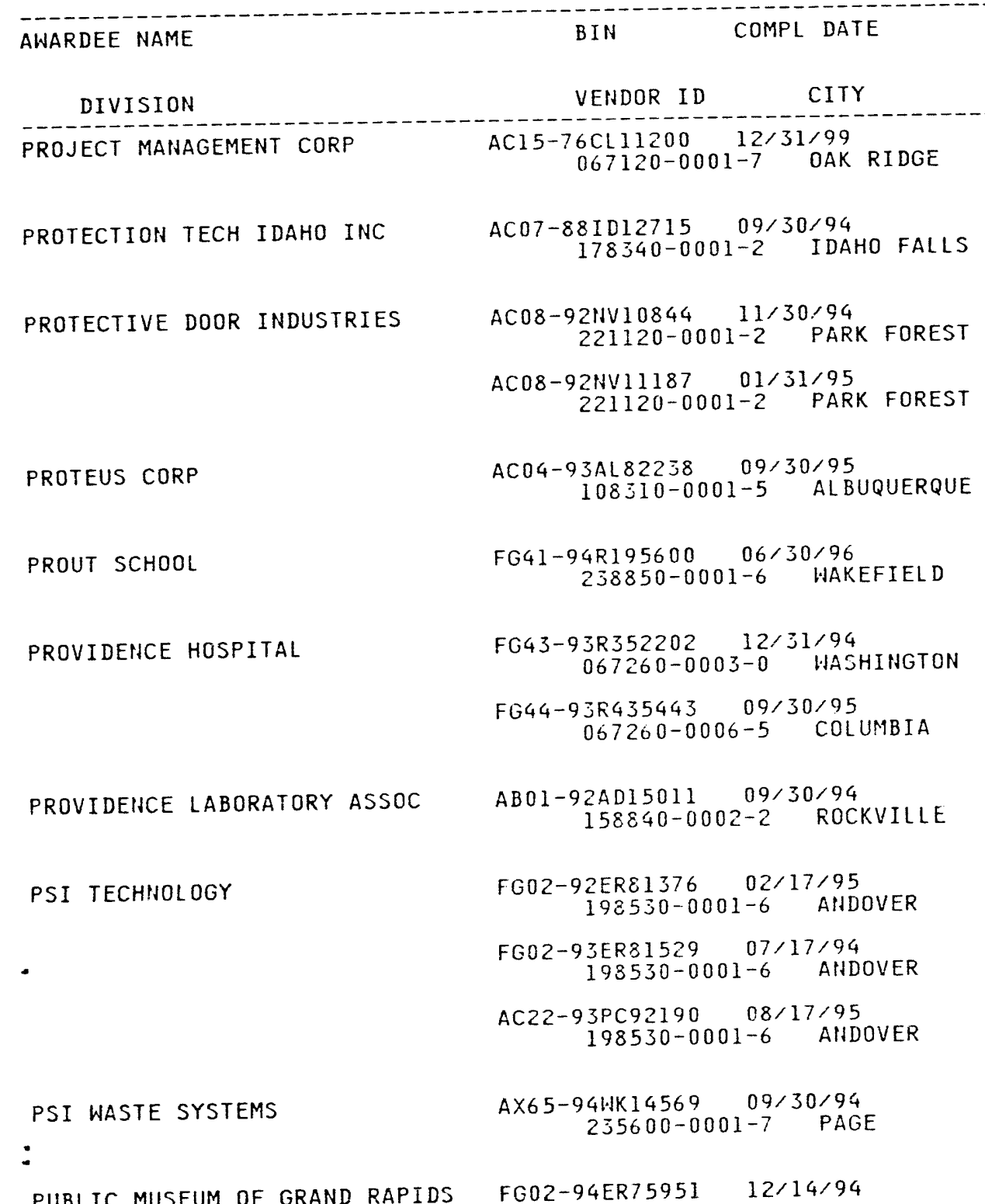

DESCRIPTION OF WORK

$\begin{array}{ll} & \text { CONG COLIIGS } \\ \text { STATE DIST VALUE } & \text { TO DATE }\end{array}$

PRINCIPAL PROJECT AGREEMENT DESIGN, CONSTRUCTION AND $\$ 030$

M\&O CONTRACT FOR SECURITY PROTECTIVE SERVICES, FIRST YEAR FU

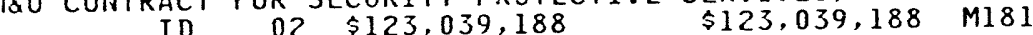

DESIGN AND FABRIATE SPECIAL DOORS FOR THE DEVICE ASSEMBLY FA $04 \quad \$ 8,787,712 \quad \$ 8,787,7129999$

IHSTALLATION OF SPECIAL DOORS IN THE DEVICE ASSEMBLY FACILIT

SECOM SUPPORT $\$ 2,121,859 \quad \$ 1,232,450 \quad$ R699

FY'94 ICP AHARD.

TITE III OF THE NATIOHAL EHERGY CONSERVATION POLICY ACT

DC 01 \$36,590 $\$ 18,345$

SEE ATTAHCED PAGE NO. 2 OF THIS NOTICE OF FINANCIAL ASSISTAN SC 02 \$232,571 \$116,285

BPA FOR LABORATORY HORK

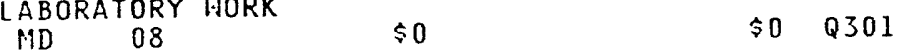

ADVAISED ANALYTICAL METHODS FOR SELECTION OF COALS CONTROL OF MERCURY \& OTHER VOLATILE TRACE METALS IN FOSSI $\begin{array}{lll}\text { MA } 05 & \$ 75,000 & \$ 75,000\end{array}$ FUHDAMENTAL STUDY OF ASH FORMATIOH AND DEPOSITION: EFFECT 0 $\begin{array}{lllll}\text { MA } & 05 & \$ 898,558 & \$ 653,714 \text { AGII }\end{array}$

GARBAGE P-UP FOR GLEN CANYON $\begin{array}{llll}-U P & \text { FOR } & & \end{array}$

THE FURHITURE CITY: A

HISTORY OF SCIENCE AHD EHERGY 


\begin{tabular}{|c|c|}
\hline AWARDEE NAME & COMPL DATE \\
\hline DIVISION & VENDOR ID \\
\hline & GRAIID $R$ \\
\hline \multirow[t]{4}{*}{ PUBLIC SERVICE CO OF COLORADO } & $\begin{array}{c}\text { FC22-91PC90550 11/30/94 } \\
067390-0001-8 \text { DENVER }\end{array}$ \\
\hline & $\begin{array}{c}A D 13-901 D 12995 \quad 10 / 31 / 98 \\
067390-0001-8 \text { DEIIVER }\end{array}$ \\
\hline & $\begin{array}{r}\text { FR22-91PC90585 } 01 / 09 / 17 \\
067390-0001-8 \text { DENVER }\end{array}$ \\
\hline & $\begin{array}{c}\text { CRO1-83HE44408 03/15/30 } \\
067390-0001-8 \text { DENVER }\end{array}$ \\
\hline
\end{tabular}

PUBLIC SVC CO OF NEW HAMPSHIRE CRO1-86RHO0111 03/15/30 667410-0002-X SEABROOK

PUBLIC SVC ELECTRIC \& GAS CO

PUEBLO OF SAN IIDELFONSO

PUEBLO DF SANTA CLARA

PUERTO RICO COMMONWEALTH OF COMPUUNITY AFFAIRS DEPT OF

- OFFICE OF ENERGY

ENERGY OFFICE DF

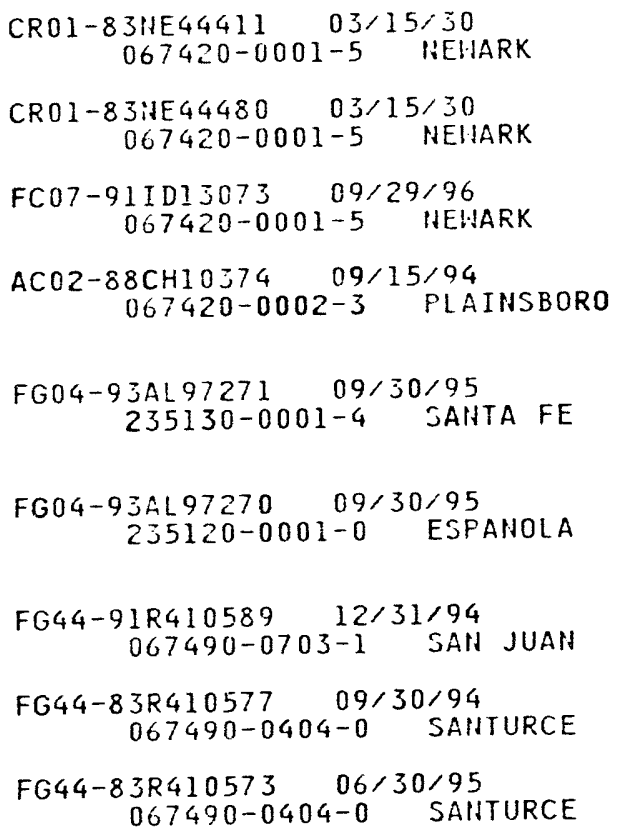

\section{DESCRIPTION OF WORK}

COHG COHTRACT

MI $05 \quad \$ 97,000$

OBLIGS
TO DATE P/S $\$ 97,000$

$$
\text { CO } 01 \$ 27,411,462 \text { SRY } \$ 13,705,731
$$

ELECTRICAL UTILITIES FOR GRANDJUNCTIOH
CO 01
\$0 $\$ 112$

REPAYMENT AGREEMENT INTEGRATEDDRY NOX/SO2 EMISSION CONTROL $\begin{array}{ccrl}\text { DISPOSAL OF SPENT } & \text { HUCLEAR FUELAND FOR HIGH LEVEL RADOACTIVE } \\ \text { CO } & 05 & \$ 0 & \$ 0\end{array}$ DISPOSAL DF SPENT NUCLEAR AND $\begin{gathered}\text { \$OR } \\ 00\end{gathered}$

DISPOSAL OF SPENT NUCLEAR FUEL

TO AHARD CONIRACT FOR DISPOSALOF SPENT NUCLEAR FUEL AND $\$ O R$ PARTICIPATIOH IH THE US DEPARIMENT OF EHERGY'S ELECIRIC \&

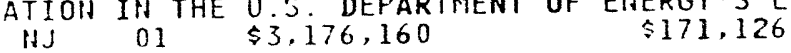

PRUVIDE ELECTRIC SERVICE TO THE PRINCETON PLASMA FHYSICS NJ 04 $\$ 0 \quad 5112$

BASIC AHARD-LOS ALAMOS PUEBLO PROJECT CHARTER FUEBLOS

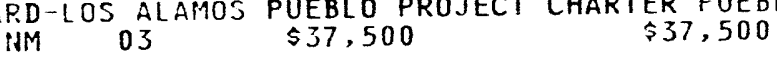

BASIC AWARD-LOS ALAMOS PUEBLO PROJECT

$$
\text { IM } 03 \text { OLAMOS PUEBLO PROJECT } \$ 12,500 \quad \$ 12,500
$$

APPROVED BUDGET PLAN AHD SPECIAL TERMS AND CONDITIONS ATTACH PR $01 \quad \$ 168,000 \quad \$ 100,000$ $\begin{array}{ccc}\text { GRANT PROGRAM FOR SCHOOLS, HOSPITALS, BUILDINGS OWNED BY UNI } \\ \text { PR } \\ 01 & \$ 11,162.826 & \$ 762,923\end{array}$

ENERGY EXTENSION SERVICE
PR 01 \$1.696,961
$\$ 1.035 .006$ 


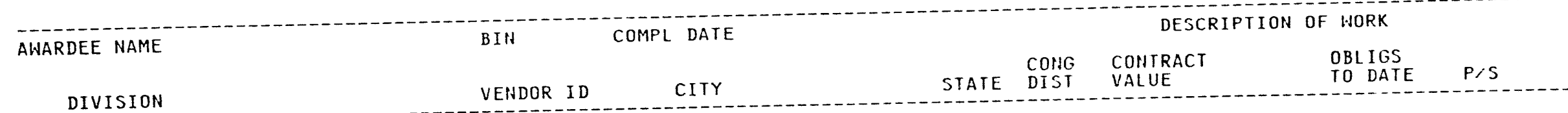

PUERTO RICO UNIVERSITY OF

FG44-89R434024 12/31/94 $067500-0004-5$ BAYAMON

FG05-94ER40842 03/31/97 067500-0001-0 MAYAGUEZ

FG05-9JER40798 07/14/94 $067500-0001-0$ MAYAGUEZ

FG44-8\&R433652 02/28/95 $067500-0003-7$ RIO PIEDRAS

FG44-93R435414 09/30/95 $067500-0003-7$ RIO PIEDRAS

FG02-91ER75674 09/29/94 $067500-0002-9$ SAN JUAN

FG06-94RL12770 $02 / 06 / 95$
SAIN JUAN

FG44-92R435131 09/30/94 $067500-0002-9$ SAIN JUAH

MEDICAL SCIENCES CAMPUS

FG44-93R435413 09/30/95
$067500-0402-4$ SAN JUAN

FG09-945R18454 06/15/95 $067500-0002-9$ SAH JUAN

PULSAR DATA SYSTEMS INC

ACO1-93AD33277 04/27/95 $232580-0001-8$ LAHHAM

PUMA CONSTRUCTION CO

ADO4-94AL97258 07/13/94
$231410-0001-8$ AL BUQUERQUE
CYCLE 11 INSTITUTIONAL CONSERVATION PROGRAM. APPROVED BUDGE $P R \quad 01$ \$88,000 $\$ 44,000$

CHARM DECAYS AIID HIGH ENERGY PHOTOPRODUCTIOH

CHARM DECAYS AIN HIGH ENERGY PHOTOPRODUCTION $\$ 53,000$

RING IMAGING CEREHKOV STUDIES

$\$ 6,000$

CYCLE $x$ - INSTITUTIONAL CONJERVATION PROGRAM. APPROVED BUDG

CYCLE $X$ PR 01
INSTIST

SEE ATTACHED PAGE NO. 2 OF THIS NOTICE OF FINANCIAL ASSISTAN

$$
\text { PR OI } \$ 278,873 \text { \$IJ9,436 }
$$

PLANNING PROPOSAL TO DEVELOP AN EPSCOR IN ENERGY-RELATED

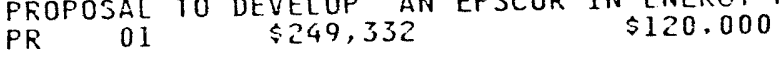

USED ENERGY RELATED LABORATORYEQUIPMENT GRANT

$$
\text { PR OI } \$ 0
$$

CYCLE 14 - INSTITUTIONAL COHSERVATION PROGRAM. APPROVED BUD CYCLE 14 PR 01 INSTITUTIONAL CONSERVATION PROGRAM, $\$ 162,450 \quad 225$

SEE ATTACHED PAGE NO. 2 OF THIS NOTICE OF FINAHCIAL ASSISTAC

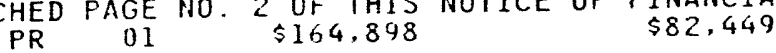

USED ENERGY RELATED LABORATORYEQUIPMENT

$$
\begin{array}{ccc}
\text { PR } & 01 & \$ 0
\end{array}
$$

ADP HARDWARE SOFTWARE AHD SOFTHARE SUPPORT

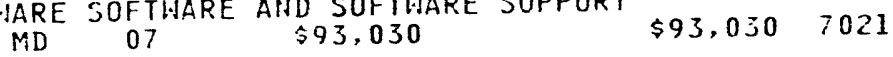

REPAIR TSD BUHKER

$\$ 137,099$

$\$ 137,099 \quad Y 299$

READY ROOM ADDITION

$$
\text { HM } 01 \text { ODDITION } \$ 377,638
$$

$\$ 377.638 \quad Y 119$

REBURISH/RENOVATE ALO COMPLEX $\leqslant 0$

$\$ 0 \quad Y 119$

AM04-93:196969004/14/95
$231410-0001-8$ ALBUQUERQUE

ADO4-94AL97269 $11 / 07 / 95$
$231410-0001-8$ AL BUQUERQUE
DISTANCE LEARHIHG CENTER

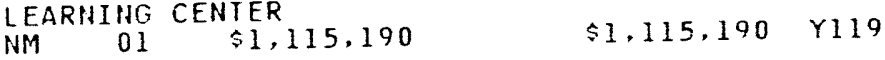


PAGE NO. 411

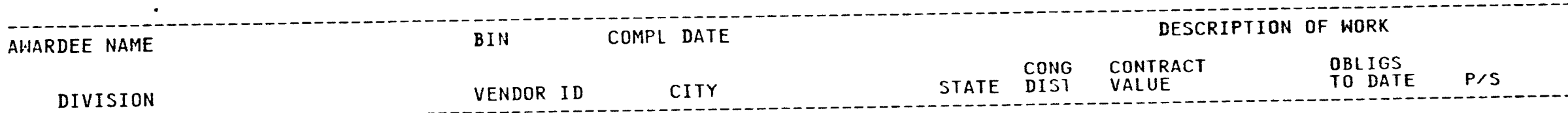

PURCELL PUBLIC SCHOOLS

FG46-91R61350108/01/94 211630-0001-9 PURCELL

PURDUE RESEARCH FOUNDATION

AGRICULTURAL ENGINEERING

MATERIALS ENGINEERING

MATERIALS ENGINEERING

PHYSICS

AGRONOMY \& CHEMISTRY

CHEMISTRY DEPT

MECHANICAL ENGINEERING DEPT

CHEMISTRY DEPT

CHEMISTRY DEPT

PHYSICS

BOTANY \& PLANT PATHOLOGY DEPT

BIOLOGICAL SCIENCES DEPT

CHEMISTRY DEPT

MATERIALS ENGINEERING $\begin{array}{cc}\text { FG09-93SR } 18352 & 08 / 02 / 94 \\ 067560-0003-9 & \text { FORT WAYNE }\end{array}$

FG02-85CE40772 $02 / 28 / 97$
$067560-0801-3$ WEST LAFAYETTE

FG02-84ER $4513312 / 31 / 94$
$067560-0201-5$ WEST LAFAYETTE

FG02-85ER45183 01/31/97
$067560-0201-5$ NEST LAFAYETTE

FG02-85ER45199 01/15/95
$067560-0301-1$ WEST LAFAYETTE

FG02-85ER60310 06/30/97
$067560-0901-X$ HEST LAFAYETTE

FG02-87ER $4034605 / 31 / 96$
$067560-0501-4$ WEST LAFAYETTE

FG02-87ER13759 $07 / 31 / 96$
$067560-1001-8$ HEST LAFAYETTE

FG02-87ER13766 01/31/95 $067560-0501-4$ WEST LAFAYETTE

FG02-88ER40408 12/31/96
$067560-0501-4$ WEST LAFAYETTE

FG02-88ER40412 12/31/96 WEST LAFAYETTE

FG02-88ER13903 04/30/96 06 HEJT LAFAYETTE

FG02-89ER14028 05/31/95
$067560-1601-6$ WEST LAFAYETTE

FG02-90ER14121 10/31/96 $067560-0501-4$ WEST LAFAYETTE $\begin{array}{rl}\text { FG02-90ER45427 } 07 / 23 / 94 & 074 \\ 067560-0201-5 & \text { WEST LAFAYETTE FORMATION OF THE MIDHEST SUPERCONDUCTIVITY CONSORT }\end{array}$

CYCLE 13 ECM DOE GRANT.

OK $\underset{04}{ }{ }^{2} \$ 163,879$

USED ENERGY RELATED LABORATORYEQUIPMENT

$$
\text { IN RELATED LABORATORYEQUTPMENT } 04 \text { \$0 }
$$

CONVERSION OF FOOD PROCESSING WASTE TO ETHANOL
IN 02 \$N $\$ 2,603,780$

STUDY OF MULTICOMPOHENT DIFFUSION \& TRANSPORT PHENOMENA

IN 07 \$ $04,04,978$ \& $\$ 949,877$

MATERIALS RESEARCH UTILIZING NSLS

$\$ 3,651,089$

INELASTIC SCATIERING IN CONDENSED MATTER WITH HIGH INTENSITY SCATTERING IN CONDENSED MATTER WITH HIGH
IN 07
$\$ 751,877$

SUPERCOMPUTER SIMULATION OF CLAY-ORGAHIC-WATER INTERACTIONS IN 07 \$2,113,945 \$1,589,009

STUDIES OF YRAST AHD CONTIHUUMSTATES IN $A=140-160$ NUCLEI $\begin{array}{lll}\text { OF YRAST AHD CONTINUUNSTATES IN } & A=140-160 \\ \text { IN } 07 & \$ 1,542,000 & \$ 1,344,000\end{array}$ EFFECT OF FORCED AND NATURAL CONVECTION ON SOLIDIFICATION

REACTIONS OF METAL IONS AND THEIR CLUSTERS IN THE GAS IN 02 \$N $\$ 813,530$ \$793,230 DEEXCITATION PROCESSES IN IN 07 \$ $1,736,000$

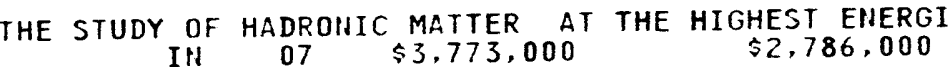
BIOSYNTHSIS AND ASSEMBLEY OF CELL WALL POLYSACCHARIDES IN IN $07 \quad \$ 746,200 \quad \$ 650,200$ REGULATION OF PHOTOSYNTHETIC MEMBRANE COMPONENTS IN IN 07 \$ $\$ 513,920 \quad \$ 513,920$ FUNDAMENTAL STUDIES OF REAC- TIVE INTERMEDIATES IN FORMATION OF THE MIDHEST SUPERCONDUCTIVITY CONSORTIUM 


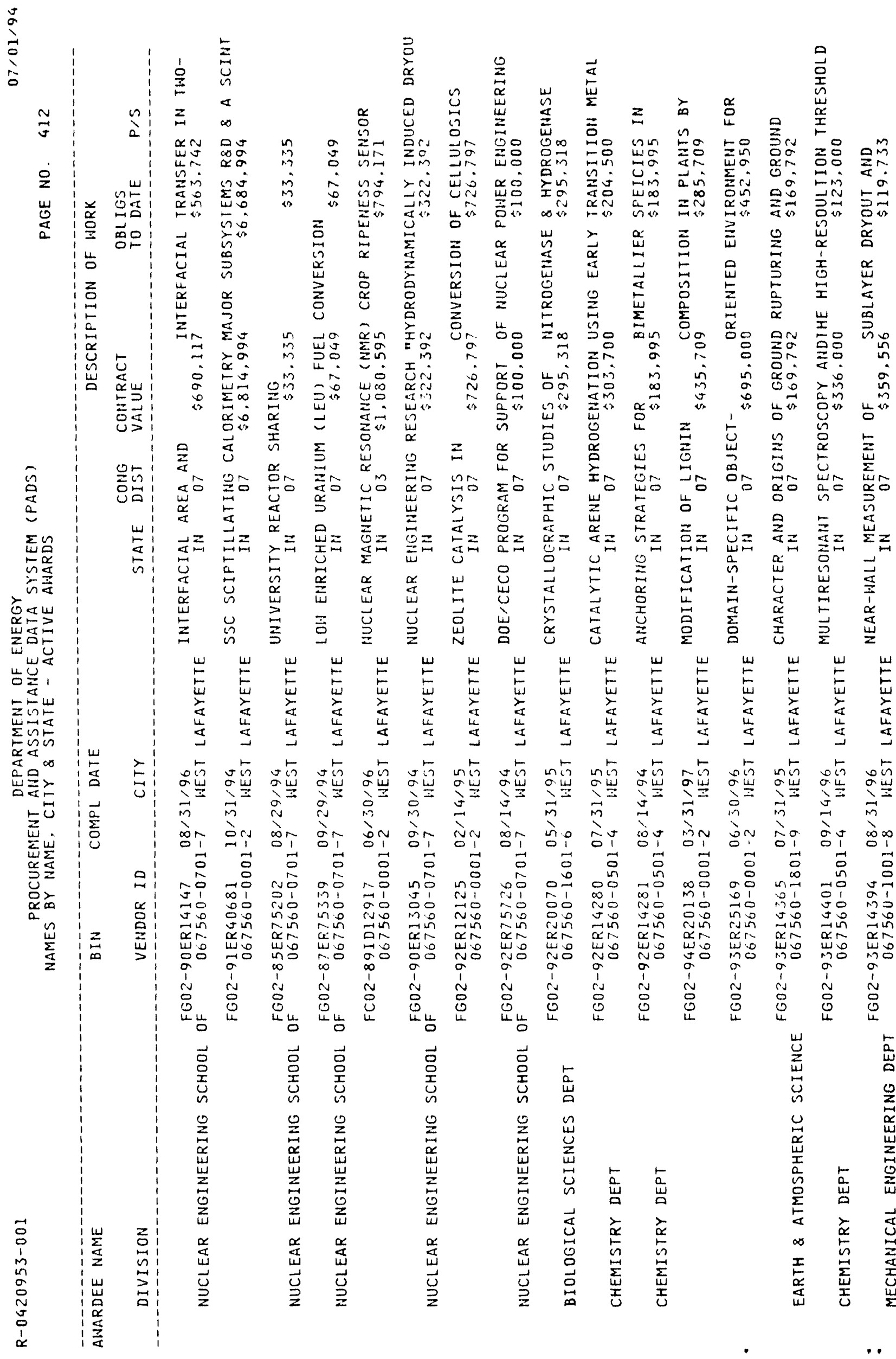


$\frac{5}{\vdots}$

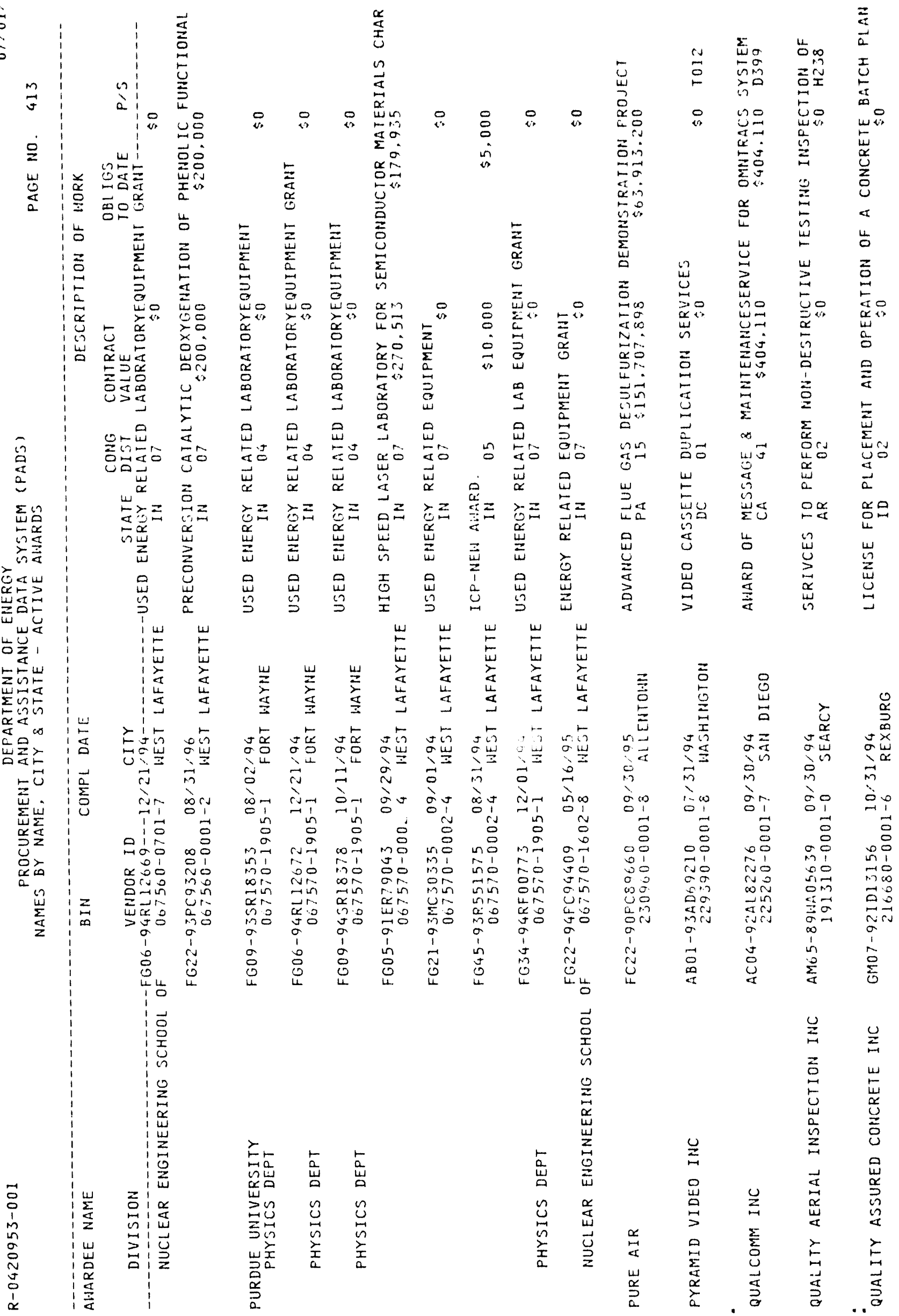


AWARDEE NAME

BI:1 COMPL DATE

DESCRIFTION OF WORK

DIVISION

VEHDOF in

GTATE DIS CONIRAC VALUE

OBLIGS
TO DATE P/S

QUANTUM GROUP INC

QUANTUM MAGNETICS INC

QUARTET OVONICS

QUE PUBLISHING

QUEENSBOROUGH COMMUNITY COLL

QUEMADO INDEPENDENT SCH DIST

QUEST INTEGRATED INC

QUESTA TOWH OF MUHICIPAL SCHOOLS

\section{QUINCY CITY OF} SCHOOL DEPT

QUITMAN COUNTY SCHOOL DISTRICT FG44-92R435122 09/30/94

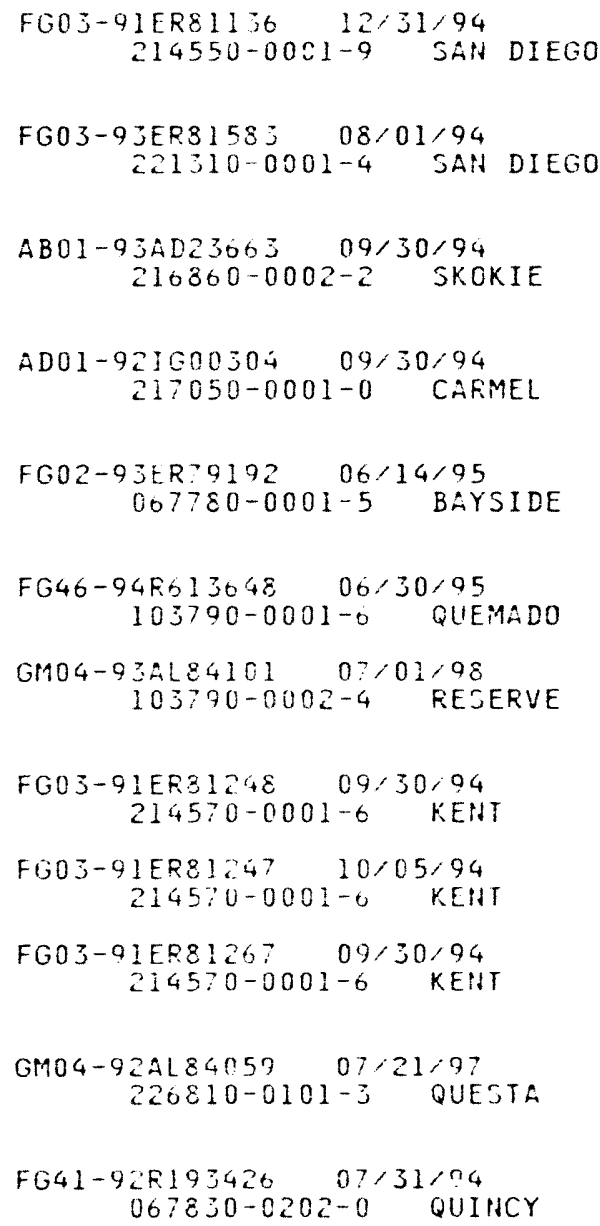

TEIR - PHASE I AHARD FOR - THERMOFHOTOVOLIAIC COGEIERAIORS F

$$
\text { CA } 41 \text { \$549.732 }
$$

NEW SBIR GRANT - "IHIENSITY AND POSITION MONITOR FOR PARTICL

$$
\begin{aligned}
& \text { GRANT - "IHTENSITY AND POSITION MONITOR FOR } \\
& \text { CA } 41 \text { \$673.081 }
\end{aligned}
$$

BPA FOR MISCELLANEOUS REFAIRS AHD SERVICE ON COFIERS

$$
\text { IL } 12 \text { IISELS REFAIRS AHD SERVICE OH COF } \$ 0003610
$$

USING ENABLE/OA \& EHABLE 2 AN D 2.15

$$
\text { IN } 00 \text { \& }
$$

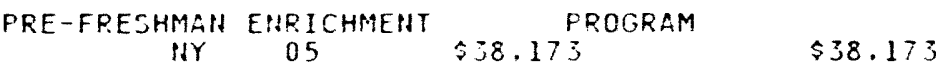

TITLE III OF THE WATIOHAL ENERGY CONSERVATIOH POLICY ACT HM 02 TMTIONAL $\$ 9.720 \quad \$ 4.800$

SUPPORT FOR SCIEHCE MATHEMATICS AHD EHGIHEERIHG EDUCATIOH HM OOJ

SBIR - PHASE I AHARD FOR - MOTORLESS DIRECIIOHAL DRILL FOR O WA 08 \$ $\$ 546.997 \quad \$ 546.997$

SBIR - PHASE I AHARD FOR - IHTEGRATED DRILLIHI/COMPLEIION SY HA 08 \$ $\$ 527.672$ \$527,672

SBIR - PHAJE I AHARD FOR - DEEF HOLE DRILLIHG IN REFRACTORY

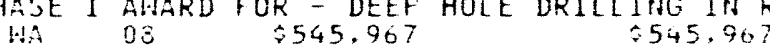

SUPPORI FOR SCIENCE, MATHEMATICS. AHD ENGIHEERING TO IMPROVE IIM 03300

CYCLE 14 ICP AHARD.

$\$ 94.860$

CYCLE 14 - IUSTITUTIONAL CONSERVATION FROGRAM. APPROVED BUD MS $02 \quad \$ 28.875 \quad \$ 20.212$ 
$\sum_{0}^{0}$

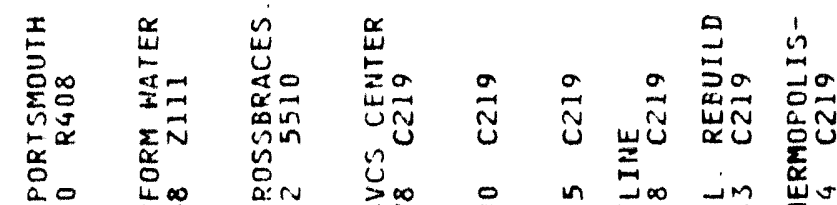

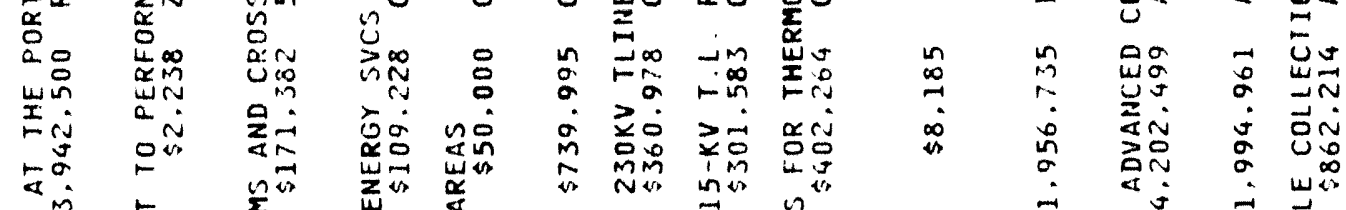

온

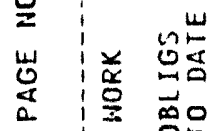

के

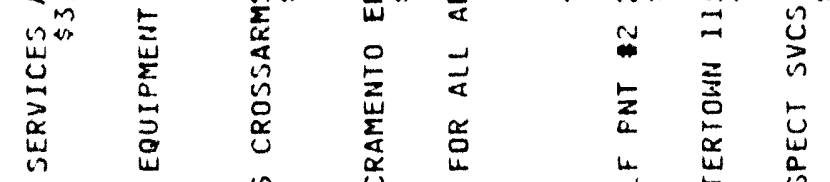

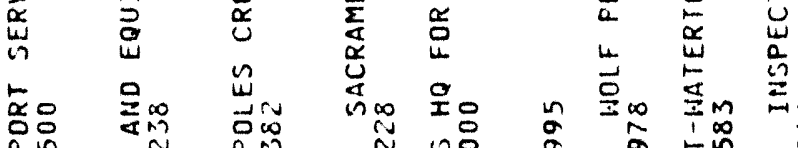

苛

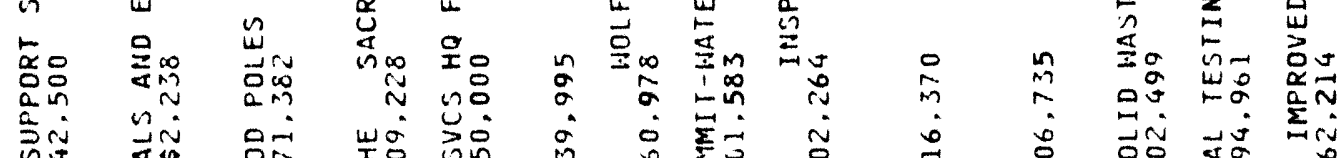

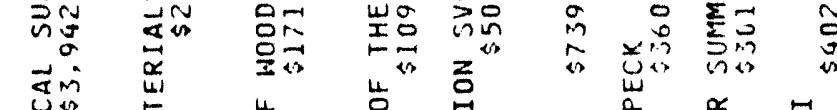

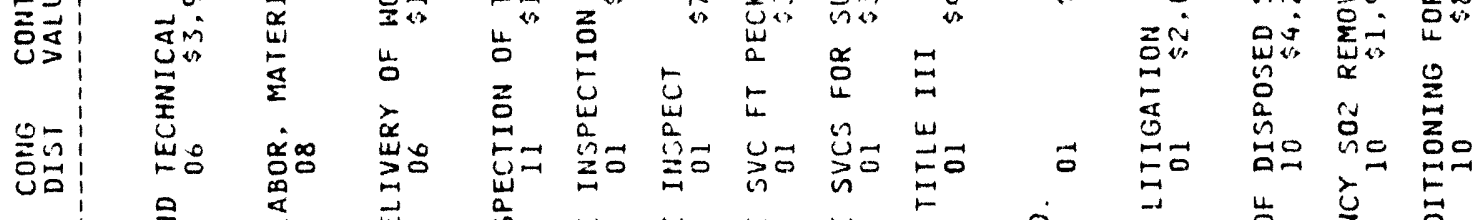

$\hat{n}$

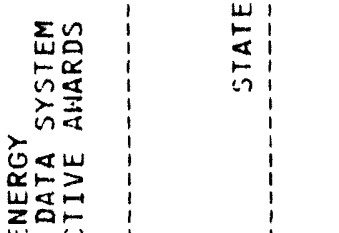

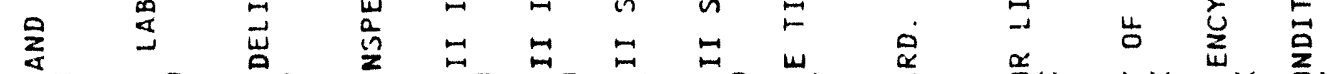

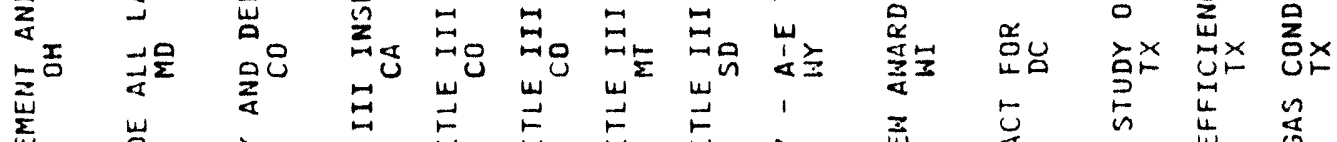

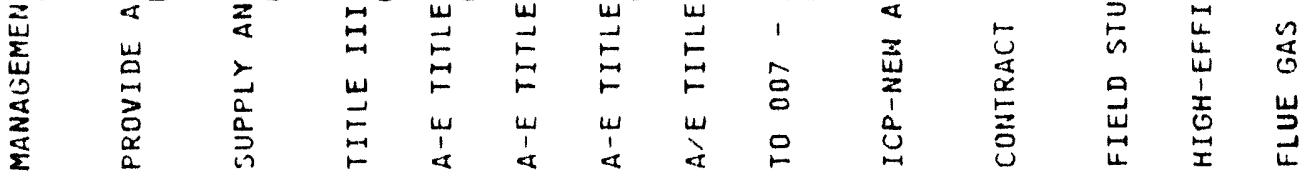

敌

约

岁里

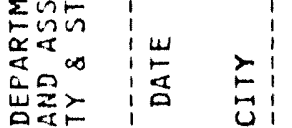

近

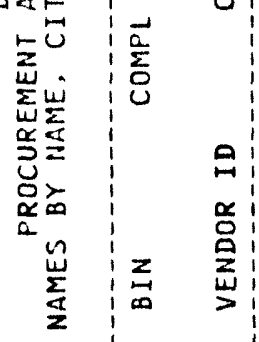

至点

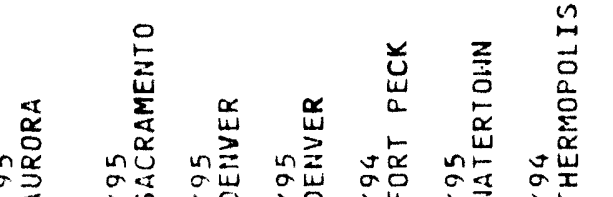

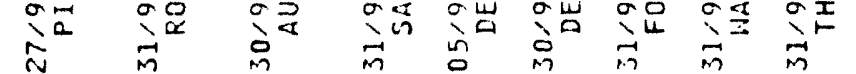

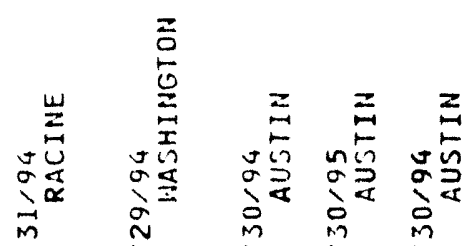

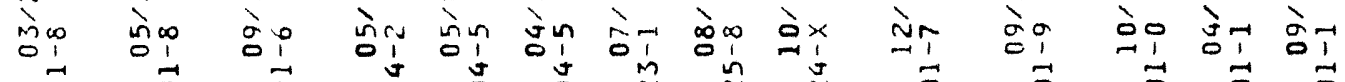

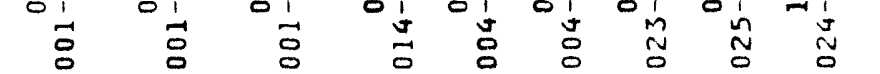

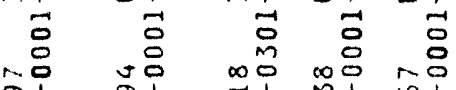

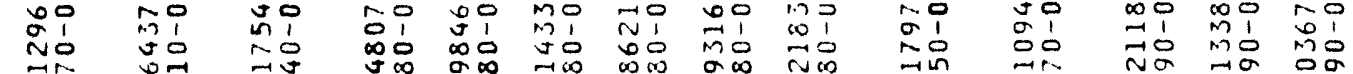
罂

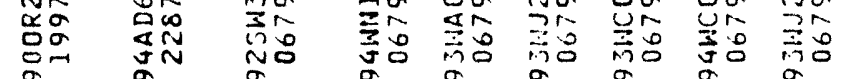

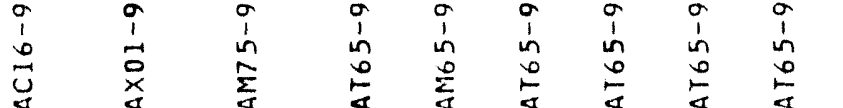

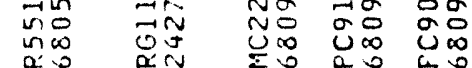

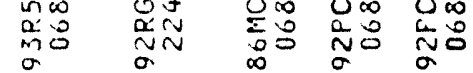

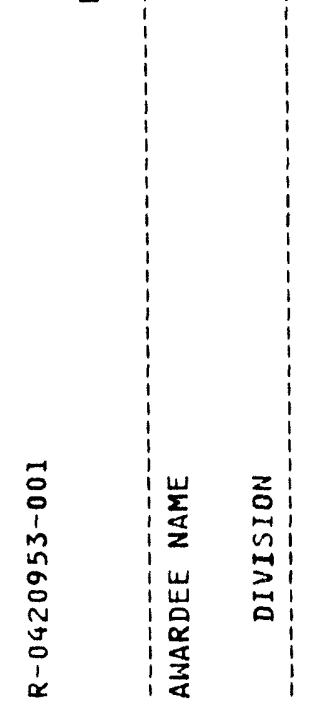

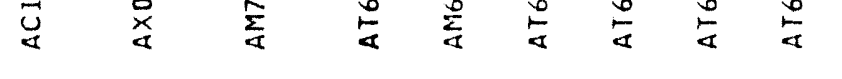

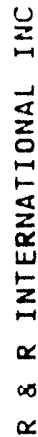

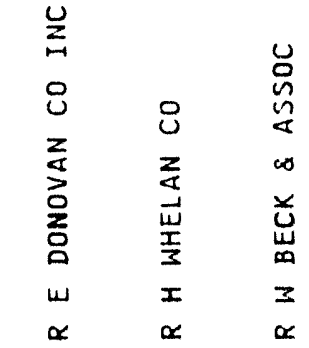

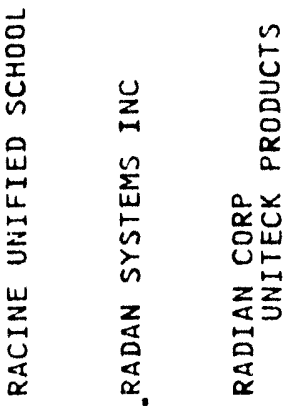




\begin{tabular}{|c|c|c|c|c|c|c|c|}
\hline AWARDEE NAME & BIN & L DATE & & & DESCF & $=$ HORK & \\
\hline DIVISION & VENDOR ID & CITY & STATE & $\begin{array}{l}\text { CONG } \\
\text { DIST }\end{array}$ & $\begin{array}{l}\text { COHTRACT } \\
\text { VALUE }\end{array}$ & $\begin{array}{l}\text { OBLIGS } \\
\text { TO DATE }\end{array}$ & $\mathrm{P} / \mathrm{S}$ \\
\hline
\end{tabular}

RADIATION MONITORING DEVICES

RADIO TV REPORTS INC

\section{RAI-RAPID INC}

RALPH G MOORE \& ASSOC

RALPH M PARSONS CO

RAND CORP

- Rand technologies corp

RAHDALL COUNTY

:RANDOLPH COUNTY HOSPITAL ASSH
$A C 22-93 P C 93253 \quad 12 / 15 / 94$ $068090-0001-1$ AUSTIS

FG02-94ER81680 07/17/94 $063100-0001-1$ HATERTOHN

FG02-94ER81679 07/17/94 068100-0001-1 WATERTOWH

FG02-93ER81530 07/17/94 068100-0001-1 WATERTOWH

$A C 01-89 C P 12015 \quad 07 / 13 / 94$ DO8 140-0002-4 HASHINGTOH

ACOB-94NV11101 04/03/95
$237290-0001-2$ LAS VEGAS

ACO2-90CH10433 08/31/95 $202620-0001-6$ CHICAGO

AC45-92R540000 09/30/94 202620-0001-6 CHICAGO

AC07-93ID13209 10/12/97 $068240-0003-5$ IDAHO FALLS

FG03-93ER61734 09/29/94 JER61734 09/29/94
$068340-0001-1$ JANTA MONICA

FG05-94ER81681 07/17/94 4ER81681 $07 / 17 / 94$

GM04-88AL32385 01/13/98 $206070-0001-5$ AMARILLO

FG44-91R434754 12/30/94 $1 R 434754$
$211140-0001-9$ HEDOWEE A STUDY OF TOXIC EMISSIONS FROM A COAL-FIRED POHER FLANT UTI HANDHELD CDTE SPECTROMETER FORNUCLEAR SAFEGUARDS INSPECTIOHS
MA
04

MINIATURE CHARGE SENSIIIVE PREAMFLIFIER USING PALSED AN INTEGRAIED VIDEO \& GAMMA RAY IMAGING SYSIEM FOR

TV AHD RADIO REPORTS ON ENERGY

$D C \quad 01 \quad \$ 125,000 \quad \$ 125,000 \quad R 603$

ADMINISTRATIVE SUFFORT SERVICES. (SUPERSEDES COHIRACT NO: D NV $01 \quad \$ \$ 10,463 \quad \$ 200,000 R 699$

ADP SUPPORT SERVICES FOR THE CHICAGO OPERATION OFFICE SUPFORT SERVICES FOR ON-SITE PROGRAMMATIC COAPLIANCE
IL 07

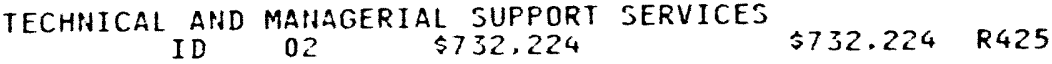
NEW GRANT ENTITLED "TRADEOFFS AMONG GREEHOUSE GAS EMISSIONS:
CA
29

NEH AWARD DEVELOFMENT OF RF TAGGING METHOD

VA 11 \$ $\$ 4.898$ M $\$ 74,898$ $\underset{\text { COOPERTIOH OF EMERGEHCY MATTERS OF MUTUAL CONCERN AHD TO }}{\$ 0} \underset{\$ 0}{13}$ CYCLE 13 - INSTIIUTIONAL CONSERVATION PROGRAM. APPROVED BUD 


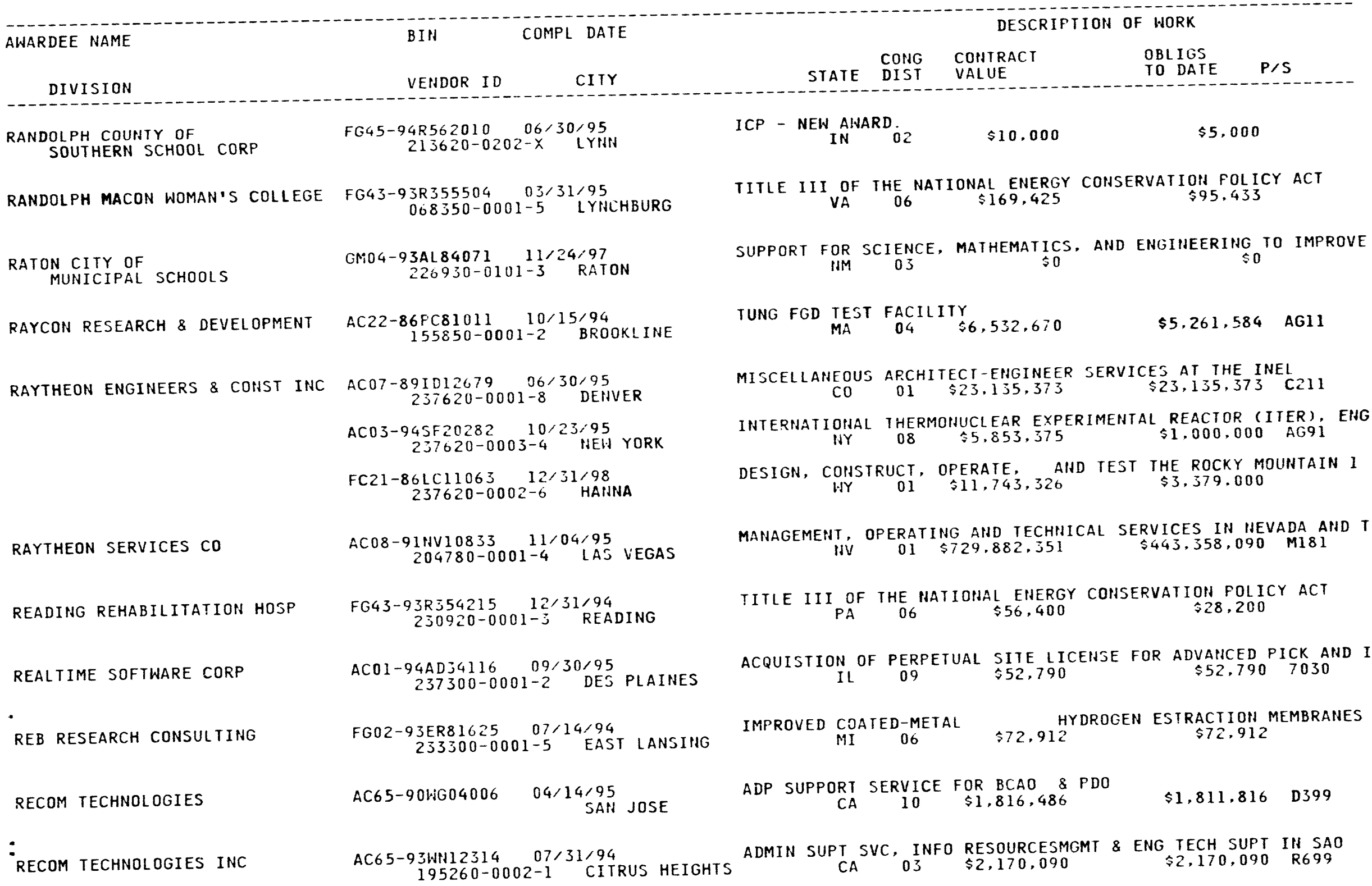


AWARDEE MAME

BIN

COMPL DATE

DESCRIPTION OF WORK

DIVISION

VENDOR ID CITY

STATE CONG

CONTRACT

OBLIGS

DIVISION

DATE P/S

CONTRACT AHARD PAO SUPPORT SVC

RED BANK BOROUGH BD OF EDUCN

AC65-91WG16339 $08.31 / 94$
$195260-0001-3$ SAN JOSE

FG42-93R253858 06/30/95

$116900-0001-5$ RED BANK

CYCLE 15 AHARD.

$\$ 4,243,820$

R699

RED ROCKS COMMUNITY COLLEGE

FC34-94RF00861 05/02/98
$237820-0001-3$ LAKEHOOD

AD01-940C12208 07/31/94

238510-0001-3 MIDLAND

FG41-93R110585 07/30/98

$231700-0001-2$ GARDINER

FG48-92R808607 08/31/94 $224290-0001-6$ SHERIDAH

FC36́-93CH10580 04/15/96 $234980-0001-2$ HESTBOROUGH

REMOTEC INC

RENSSELAER POLYTECHNIC INST MECHANICAL ENG DEPT

MATERIALS ENG DEPT

CHEMISTRY DEPT

NUCLEAR ENG DEPT

:

FG05-92ER81437 $02 / 17 / 95$
$221740-0001-6$ OAK RIDGE
FG02-86ER13566 $07 / 31 / 94$
$009260-1101-x$ TROY
FG02-89ER52161 $08 / 31 / 94$
$069260-0901-5$ TROY
FG02-91ER12117 $07 / 31 / 94$
$069260-0301-7$ TROY
FG02-91ID13084 $09 / 29 / 94$
$009260-1201-6$ TROY
FG02-90ER13032 $09 / 29 / 94$
$069260-0001-8$ TROY
FG02-93ER75923 $09 / 29 / 94$

COOPERATIVE AGREEMENT BETWEEN
CO
00

$$
\text { CO } 00
$$

SUBSCRIPTIOHS

GA 11

$\$ 568$

$\$ 568$

9999

NEW FY 1993 AHARD.

$\$ 175,000$

$\$ 175.000$

AHARD ICP CYCLE XIV GRAHT

$$
\text { WYY } 01 \text { \$12.768 } \$ 6,384
$$

DESIGN. CONSTRUCT AHD TEST 100 HP HTS MOTOR

$$
M A \quad 03 \text { \$3,102,079 } \$ 2,124,924
$$

ENVIRONMENTAL HARDENING OF MOBILE MANIPULATOR ROBOTIC SYSTEM

$$
\text { IN } 03 \quad \$ 799,899 \quad \$ 408,790
$$

INELASTIC DEFORMATION AHD DAMAGE AT HIGH TEMPERATURE

$$
\text { NY } 23 \quad \$ 1.017 .941 \text { \$1.017.941 }
$$

AQUEOUS CORROSION STUDIES IN SUPPORT OF ITER ACTIVITY

$$
\text { IIY } 23 \text { \$4 } \$ 46.000 \text { \$4 }
$$

CATIOHICALLY POLYMERIZABLE MONOMERS DERIVED FROM RENEWABL UHIVERSITY REACTOR INSTRUMEHTATION GRANT

NUCLEAR ENGINEERING RESEARCH "PROPOSAL FOR THE DEVELOPMENT 0 SHARIHG OF THE REISSELAER POLYTECHNIC IHSTITUTE 


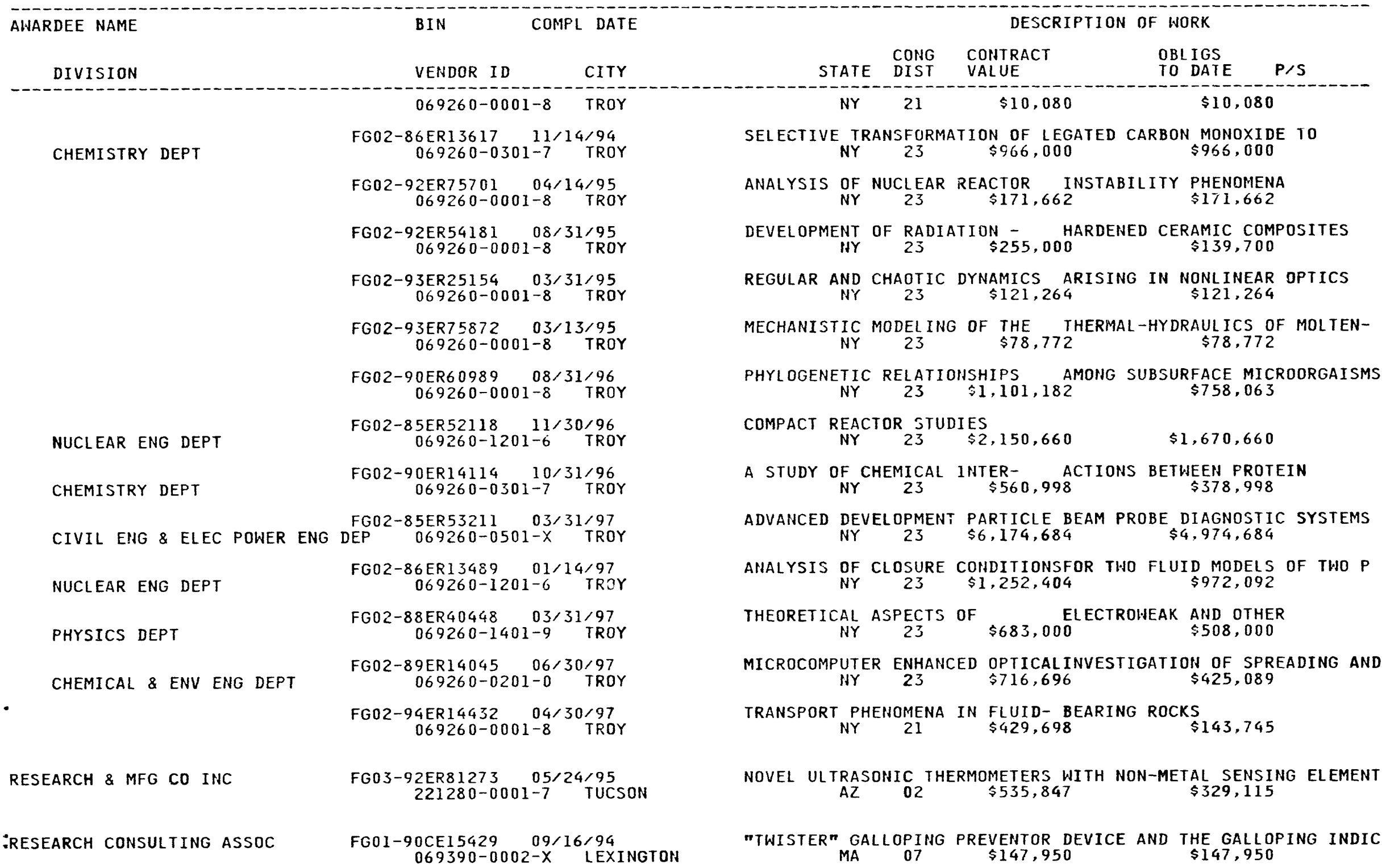




\begin{tabular}{|c|c|c|}
\hline AHARDEE NAME & COMPL DATE & DESCRIPTION OF WORK \\
\hline DIVISION & VENDOR ID & $\begin{array}{l}\text { CONTRACT } \\
\text { VALUE }\end{array}$ \\
\hline RESEARCH INTERNATIONAL INC & $\begin{array}{c}\text { FG03-94ER81682 } 07 / 17 / 94 \\
236460-0001-5 \text { WOODINVILLE }\end{array}$ & $\begin{array}{cccc}\text { FIBER OPTIC } & \text { SENSORS FOR HEAVY METAL CATIONS } & \text { HE } \\
\text { WA } & \$ 65,156 & \$ 65,156\end{array}$ \\
\hline RESEARCH MGMT CONSULTANTS INC & $\begin{array}{c}\text { AC01-92CE40956 09/29/94 } \\
210410-0002-2 \text { CAMARILLO }\end{array}$ & 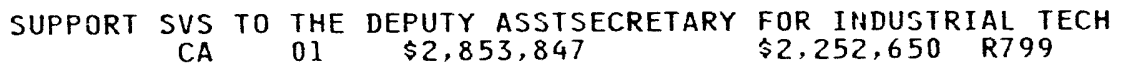 \\
\hline & $\begin{array}{c}A C 01-92 A D 25568001 / 05 / 95 \\
210410-0001-4 \text { MCLEAN }\end{array}$ & 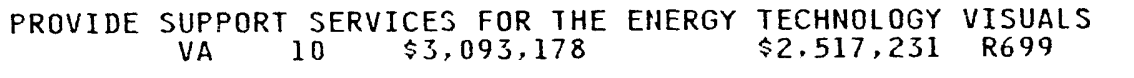 \\
\hline & $\begin{array}{c}\text { AC01-92NS10055 09/16/95 } \\
210410-0001-4 \text { MCLEAN }\end{array}$ & 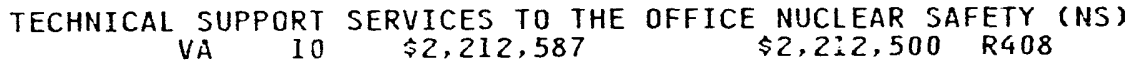 \\
\hline RESEARCH PLANNING INC & $\begin{array}{c}\text { ACOI-931N510037 05/13/95 } \\
233930-0001-2 \text { ARL INGTON }\end{array}$ & 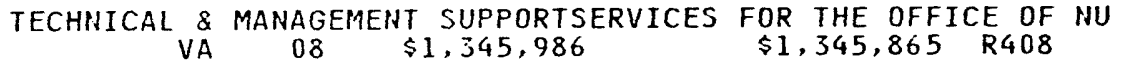 \\
\hline \multirow[t]{5}{*}{ RESEARCH TRIANGLE INSTITUTE } & $\begin{array}{c}\text { FCO1-93EF10029 } 09 / 30 / 94 \\
069450-0002-8 \text { DURHAM }\end{array}$ & 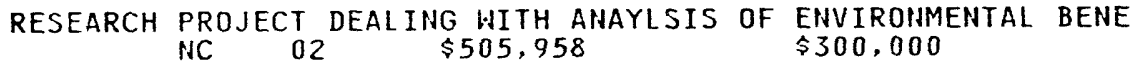 \\
\hline & $\begin{array}{rl}A C 21-93 N C 30010 & 12 / 30 / 94 \\
069450-0002-8 \quad \text { DURHAM }\end{array}$ & $\begin{array}{ccccc}\text { RESEARCH ENTITLED } & \text { "BENCH-SCALEDEMONSTRATION OF HOT-GAS } \\
\text { NC } & 02 & \$ 826,190 & \$ 826,190 & \text { AG15 }\end{array}$ \\
\hline & $\begin{array}{c}A C 21-8814 C 25006 \quad 11 / 21 / 94 \\
069450-0002-8 \text { DURHAM }\end{array}$ & 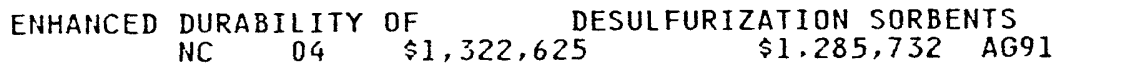 \\
\hline & $\begin{array}{c}\text { AC21-94MC } 31258 \quad 09 / 20 / 94 \\
069450-0002-8 \text { DURHAM }\end{array}$ & $\begin{array}{ccccc}\text { RESEARCH ENTITLED "ADVANCED } & \text { SULFUR CONTROL CONCEPTS FOR } \\
& \text { NC } & 02 & \$ 62,836 & \$ 62,836\end{array}$ \\
\hline & $\begin{array}{l}\text { AC21-92MC29011 02/15/95 } \\
069450-0001-X \text { DURHAM }\end{array}$ & 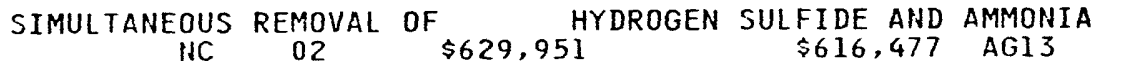 \\
\hline RESERVOIR ENGINEERING RES INST & $\begin{aligned} \text { FG22-93BC14875 } 09 / 29 / 96 \\
216480-0001-0 \text { PALO ALTO }\end{aligned}$ & $\begin{array}{l}\text { RESEARCH CONSORTIUM ON FRACTURAL PETROLEUM RESERVOIRS } \\
\qquad \begin{array}{lll}\text { CA } 12 & \$ 1,289,834 & \$ 50,000\end{array}\end{array}$ \\
\hline RESOURCE DYNAMICS CORP & $\begin{array}{c}A C 01-92 F E 62489 \quad 09 / 29 / 97 \\
130980-0002-3 \text { VIEHNA }\end{array}$ & $\begin{array}{crcc}\text { TECHNICAL SUPPORT SERVICES CONIRACT IN SUPPORT OF FE-4 } & \text { SE FE } \\
\text { VA } 10 & \$ 1,462,931 & \$ 829,100 & \text { R425 }\end{array}$ \\
\hline RESOURCE TECHNOLOGY CORP & $\begin{array}{c}\text { AC22-9211T92009 08/05/95 } \\
222390-0001-5 \text { LARAMIE }\end{array}$ & 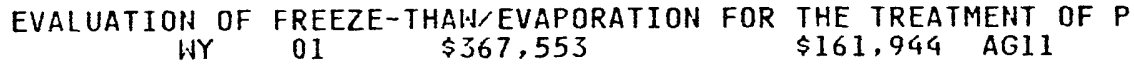 \\
\hline RESOURCES FOR THE FUTURE & $\begin{array}{c}\text { FG02-93ER61710 09/14/95 } \\
069550-0001-2 \text { WASHINGTON }\end{array}$ & 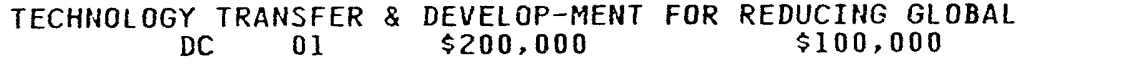 \\
\hline
\end{tabular}




\section{AWARDEE NAME}

BIN

COMPL DATE

DESCRIPTION OF WORK

DIVISION

VENDOR ID

CITY

STATE COHG COHTRACT

DIST
VALUE

OBLIGS

TO DATE P/S

RESPONSE ANALYSIS CORP

$$
\begin{gathered}
\text { ACO1-92EI21948 } 12 / 01 / 96 \\
069580-0001-3 \quad \text { PRINCETON }
\end{gathered}
$$

REUTERS AMERICA INC

$$
\begin{gathered}
\text { ADO1-93AD34035 09/30/94 } \\
225680-0001-5 \text { WASHINGTON }
\end{gathered}
$$$$
\text { AD01-93AD34045 09/30/94 }
$$$$
225680-0001-5 \text { WASHINGTON }
$$

REXBURG CITY OF

POLICE DEPARTMENT

$$
\begin{array}{r}
\text { GM07-94ID11142 } 12 / 30 / 99 \\
235930-0101-2 \text { REXBURG }
\end{array}
$$

AC08-94IJV11432 12/31/95 $069650-0001-5$ LAS VEGAS

REYNOLDS ELEC \& ENG CO INC

REYNOLDS METALS CO
MANUFACTURING TECHNOLOGY LAB FC07-90ID13038
$069690-0104-0$

RHODE ISLAND AEC

FG02-86ER75270 12/30/94
$069790-0101-9$ NARRAGANSETT

HUCLEAR SCIENCE CENTER

NUCLEAR SCIENCE CENTER

RHODE ISLAND COLLEGE

$$
\begin{gathered}
\text { FG02-93ER75922 } 09 / 29 / 94 \\
069790-0101-9 \text { NARRAGANSETT }
\end{gathered}
$$

$$
\begin{array}{r}
\text { FG41-91R195307 11/30/94 } \\
069810-0001-6 \text { PROVIDENCE }
\end{array}
$$$$
\text { FG41-92R195402 12/31/95 }
$$$$
\text { 069810-0001-6 PROVIDEHCE }
$$

FG41-93R195502 06/30/95 069810-0001-6 PROVIDENCE

FG41-94R195601 06/30/96 069810-0001-6 PROVIDENCE

RHODE ISLAND NUCLEAR SCI CTR

FG09-935R18346 08/01/94 231000-0001-3 HARRAGANSETT
COLIECTION AND ANALYSIS OF STATISTICAL DATA ON ENERGY END US NJ $12 \$ 32,194,720$

$\$ 10,625,993$ Dj99

ACCESS TO ENERGY $2000+$ REUTERLINK DATABASE

DC $01 \quad \$ 55,570 \quad \$ 55,570$

ACCESS TO REUTERS NEWS HIRE

$$
\begin{array}{ll}
\text { RE RETERS NEWS HIRE } & \$ 10
\end{array}
$$

FIRING RANGE USE AGREEMENT WITH THE REXBURG POLICE DEPT ID 02

MANAGEMENT, OPERATION AHD MAINTENANCE SERVICES IN SUPPORT OF NV́ $01 \$ 675,000,000 \quad \$ 259,442,000 \quad M 181$

EVALUATION OF TIB2-G CATHODE COMPONENTS

$$
\begin{array}{lll}
N \text { OF TIB2-G CATHODE COMPONENTS } & \$ 3,187,737
\end{array}
$$

LOW ENRICHED URANILM (LEU) FUEL CONVERJION $\begin{array}{lll}\text { RI } 02 & \$ 103,058 & 103,058\end{array}$ SUPPORT OF UTLIZTION OF THE RHODE ISLAND NUCLEAR-SCIENCE $\begin{array}{lll}\text { RI } & 02 & \$ 15,000\end{array}$ TITLE II OF THE NATIONAL ENERGY CONSERVATION POLICY ACT TITLE III OF THE NATIONAL $\$ 97,279$ CYCLE $14 \underset{R I}{\operatorname{RICP}} \underset{02}{\operatorname{AHARD} .} \$ 153,213 \quad \$ 75,000$ TITLE III OF THE NATIONAL ENERGY CONSERVATION POLICY ACT FY'94 ICP AHARD. $\$ 136,542 \quad \$ 68,271$ 


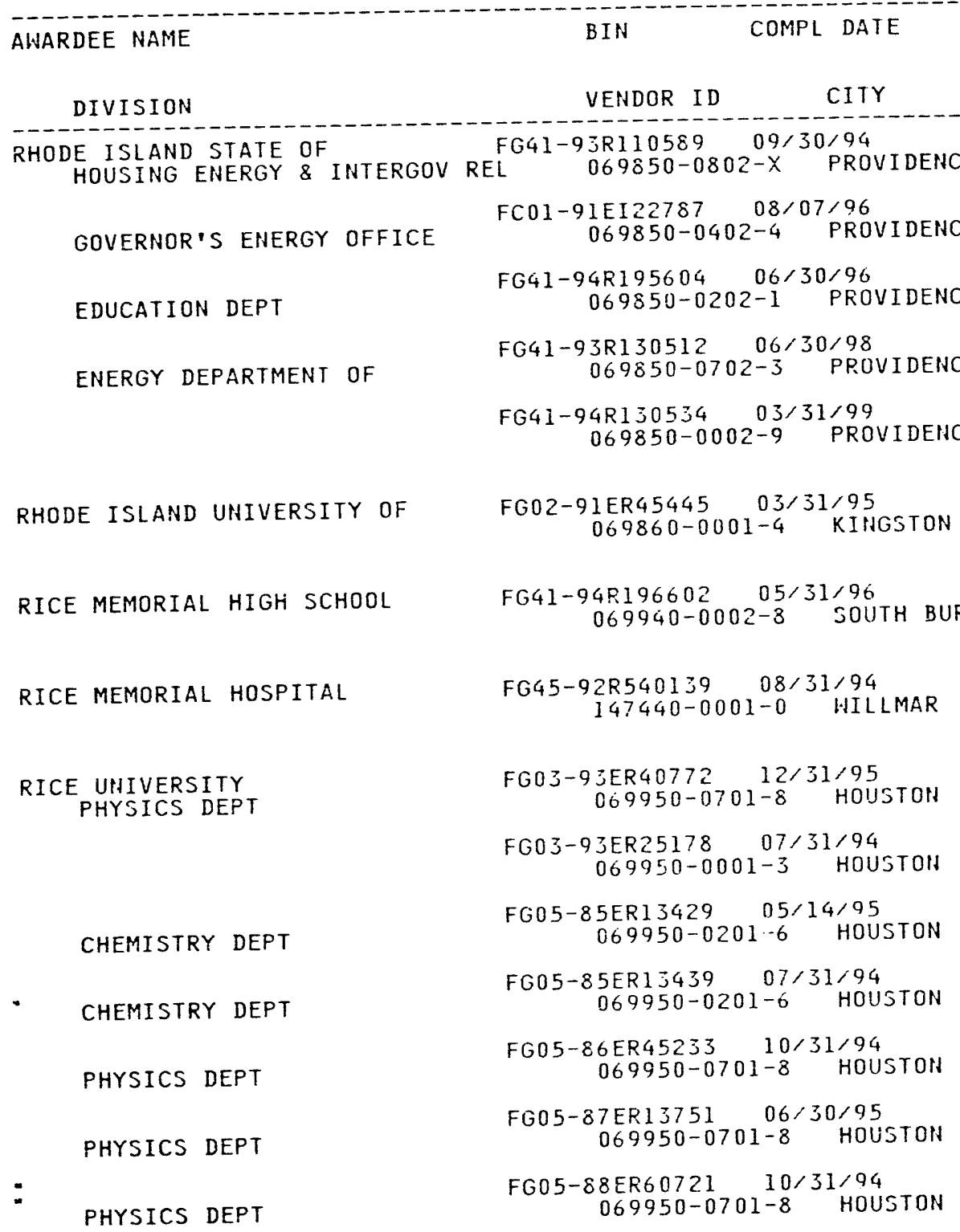

DESCRIPTION OF WORK

$\begin{array}{llll} & \text { CONG } & \text { CONTRACT } & \text { OBLIGS } \\ \text { STATE } & \text { DIST } & \text { VALUE } & \text { TO DATE }\end{array}$

IST

$\begin{array}{rrrr}\text { ALTERNATIVE FUELS DEMO } & \text { PROGRAM. } & \\ \text { RI } & 01 & \$ 348,636 & \$ 90,416\end{array}$
COLLECTIOH \& ANALYSIS OF DATA RELATED TO THE ENERGY INFO ADM

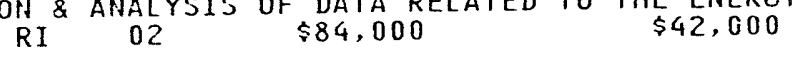

FY.94 ICP AWARD. RI $_{02} \$ 163,800 \quad \$ 75,000$

AWARD NEW FY' 93 FUNDS.

$$
\text { RI } 01 \text { FUNDS } \$ 1,390,988 \quad \$ 278,139
$$

WEATHERIZATION ASSISTANCE - FY1994 GRANT.

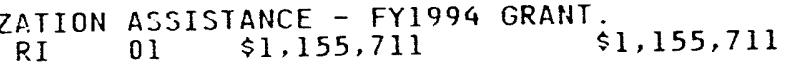
$\begin{array}{cccc}\text { SURFACE PHYSICS WITH COLD AND THERMAL NEUTRON REFLECTOMETRY. } & \text { NHT } \\ \text { RI } & 02 & \$ 235,785 & \$ 235,185\end{array}$

TITLE III OF THE NATIONAL ENERGY COHSERVATION POLICY ACT

TITLE III OF THE NATIONAL ENT 01 \$33,910

$$
\text { ICP - NEW AWARD }{ }_{\text {MNN }} \quad \$ 118,900 \quad \$ 59,450
$$

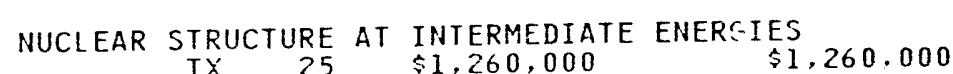
OPTIMIZATION INFRASTRUCTURE FOR INVERSE PROBLEMS IN AQUIFER

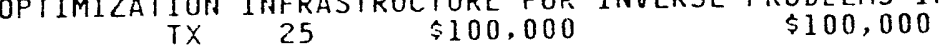
SUPERSOHIC BARE METAL CLUSTER BEAMS INFRARED ABSORPIION SPECTROSCOPY AND CHEMICAL KINETICS OF FR IIJFRARED $\mathrm{TX} 18 \quad \$ 701,076 \quad \$ 698,576$ STUDIES OF ULTRATHIN MAGNETIC FILMS AND PARTICLE-SURFACE INT TX 25 \$1,976,555 $\$ 1,918,395$

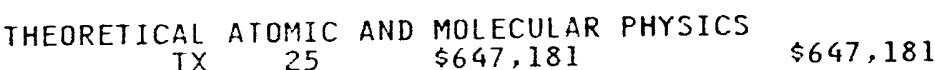
QUASI-ELECTRONIC NEUTRON SCATTERING STUDIES OF PROIEIN DYNAM QUASI-ELECTRONIC NX $25 \quad \$ 219,920 \quad \$ 165,688$ 
AWARDEE NAME

BIN COMPL DATE

VENDOR ID CITY $069950-0001-3$ HOUSTON

FG05-91ERI4213 07/14/94 $069950-0001-3$ HOUSTON

MATHEMATICAL SCIENCES DEPT FG05-91ER14215 08/31/94
$069950-0501-5$ HOUSTON FG05-92ER40717 02/28/97 T W BONNER NUCLEAR LABORATORY 2ER $4071702 / 28 / 97$
$069950-0901-0$ HOUSTON BIOCHEMISTRY DEPARTMENT

FG05-92ER20092 07/31/95
$069950-1001-9$ HOUSTON FG05-92ER25144 08/31/95 $069950-0001-3$ HOUSTON FG05-92ERI4295 08/31/95 $069950-0001-3$ HOUSTON

CHEMICAL ENGINEERING DEPT FG22-91PC91307 09/01/94 $069950-0101-X$ HOUSTON FG46-93R613639 02/28/95 $069950-0001-3$ HOUSTON FG46-93F613630 08/31/94 069950-0001-3 HOUSTON

RICHARD HEATH \& ASSOC INC AC49-93R900020 09/30/95 $168710-0002-X$ FRESNO

RICHLAND CITY OF

AM06-90RL11854 10/31/99
$070050-0001-3 \quad$ RICHLAND

AD06-94RL12806 12/31/94 $070050-0001-3$ RICHLAND

AD06-93RL12705 12/3!/95 $070050-0001-3$ RICHLAND

AD06-94RL12979 01/31/97 $070050-0001-3 / 31 / 97$ RICHLAHD

FG44-93R435397 09/30/95 $231320-0001-9$ ROCKINGHAM
RICHMOND MEMORIAL HOSPITAL

\section{DESCRIPTION OF WORK}

CONG CONTRACT

OBLIGS
TO DATE FOR LARGE SCALE EIG

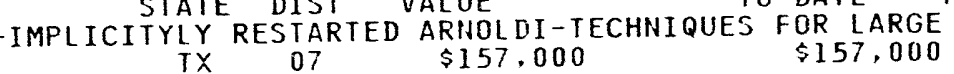
RADIATIVE PROPERTIES OF STRONGLY MAGNETIZED PLASMAS STOCHASTIC QUADRATIZATION FOR PROBABILISTIC ANALYSIS OF LARG $\begin{array}{lll}\text { IC QUADRATIZATION FOR PROBABILISTIC ANALIYSIS } \\ \text { TX } 07 & \$ 256,154 & \$ 256,154\end{array}$

HIGH ENERGY PHYSICS $\$ 4,146,300 \quad \$ 1,686,300$

GENETIC AND BIOCHEMICAL ANALYSIS OF SOLVENT FORMATION IN CLO TX $07 \quad \$ 273,000 \quad \$ 180,000$

INTEL EQUIPMENT FOR WILLIAM MARCH RICE UNIVERSITY, CONSORTIU TX $07 \quad \$ 600,000 \quad \$ 600,000$

TRANSITION METAL CATALYSIS IN THE GENERATION AND PETROLEUM A $T X \$ 07$ \$327,409 $\$ 226,185$

COAL COMBUSTION: EFFECT OF PROCESS CONDITIONS ON CHAIH REACT TX 25 \$199,983 $\$ 199,983$

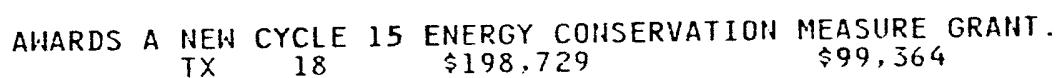

$\begin{aligned} \text { AWARDS A NEW CYCLE } 15 \text { TECHNICAL ASSISTANCE GRANT. } & \$ 20.574 \\ & \$ 10,286\end{aligned}$

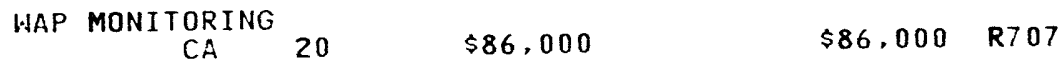

$\begin{array}{llll}\text { UTILITY SERVICES } & \$ 0 & \$ 0 & \$ 119\end{array}$

TREATMENT OF SANITARY SEWAGE FROM THE 300 AREA IN

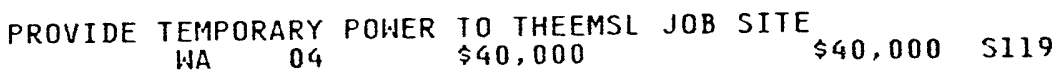

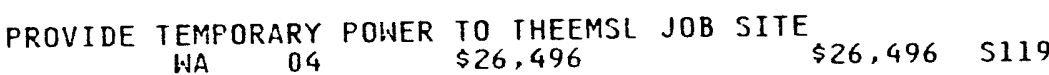

SEE ATTACHED PAGE NO. 2 OF THIS NOTICE OF FINANCIAL ASSISTAN NC 08 \$ $\$ 180,148 \quad \$ 40,000$ 


\begin{tabular}{|c|c|c|c|c|c|c|c|}
\hline AWARDEE NAME & BIN & DATE & & & DESCF & F WORK & \\
\hline & VENDOR ID & CITY & STATE & $\begin{array}{l}\text { CONG } \\
\text { DIST }\end{array}$ & $\begin{array}{l}\text { COHTRACT } \\
\text { VALUE }\end{array}$ & $\begin{array}{l}\text { OBLIGS } \\
\text { TO DATE }\end{array}$ & $\mathrm{P} / \mathrm{S}$ \\
\hline
\end{tabular}

RICHMOND UNIVERSITY OF PHYSICS DEPT

RICOH CORP

RIEGER COMMUNICATIONS INC

RILEY STOKER CORP RILEY RESEARCH CENTER

RINGS LEIGHTON LTD

RIO GRANDE MEDICAL TECH INC

RIO RANCHO POLICE DEPARTMENT

RITZ INSTRUMENT TRANSFORMERS

RIVER FOREST TOWN OF PUBLIC SCHOOL DISTRICT \#90

- RIVERSIDE health SERVICES INC

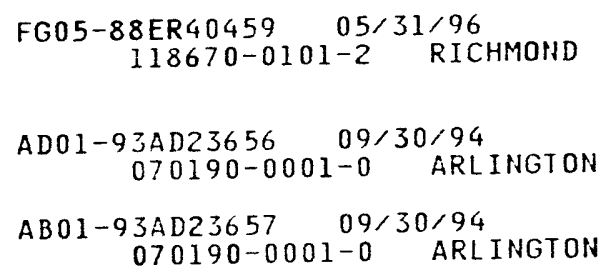

FG47-93R702705 08/31/95 232000-0001-5 WICHITA
COMPLETE AND INCOMPLETE FUSIONIN $40 C A+12 C$ AND $285 I+24 M G$ VA $03 \quad \$ 633,000 \quad \$ 535,000$

MAINTENANCE SERVICES FOR RICOHCOPIERS

$\$ 1,668 \quad j 074$

BPA FOR CONTINUED MISCELLANEOUS MAINTENANCE AND $\begin{array}{r}\$ 0 R \\ \text { PA PARTS ON }\end{array}$ VA 08

BPA FOR PHOTO AND RELATED SERVICES

MD 08

$\$ 0$

$\$ 0 \quad 1015$

ENGINEERING DEVELOPMENT OF ADVANCED COAL-FIRED LOW-EMISSION MA $01 \$ 56,400,262$

$\$ 3,800,851$ AGII

BPA FOR GRAPHIC PRODUCTION ANDVISUAL SERVICES
DC
01
\$ 0

\$0 T001

A SYSTEM FOR NONIMVASIVE ARTERIAL BLOOD GAS MEASUREMFNT NM $01 \quad \$ 2,436,952 \quad \$ 1,006,952$

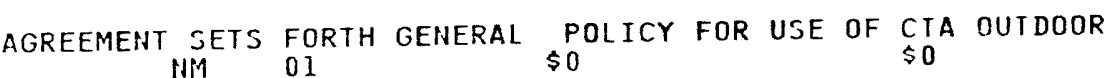

COUPLING CAPACITOR VOLTAGE TRANSFORMERS FOR MEAD STAGE 06

$$
\text { GA } 01 \quad \$ 55,110
$$

1106120

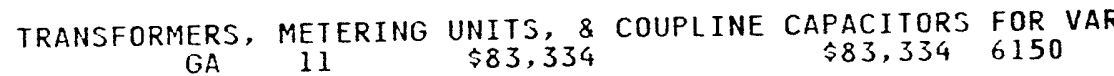

$\begin{array}{cccc}\text { CURRENT TRANSFRMR FOR MEAD SUBST } & (005 \text { ONLY) } \\ & \$ 89,400 & \$ 89,400 & 6120\end{array}$

ICP-NEW AWARD
IL
$\$ 56,800$
$\$ 31,339$

GRANT PROGRAMS FOR SCHOOLS ANDHOSPITALS AND FOR BUILDINGS O KS $04 \quad \$ 186,390 \quad \$ 93,195$ 


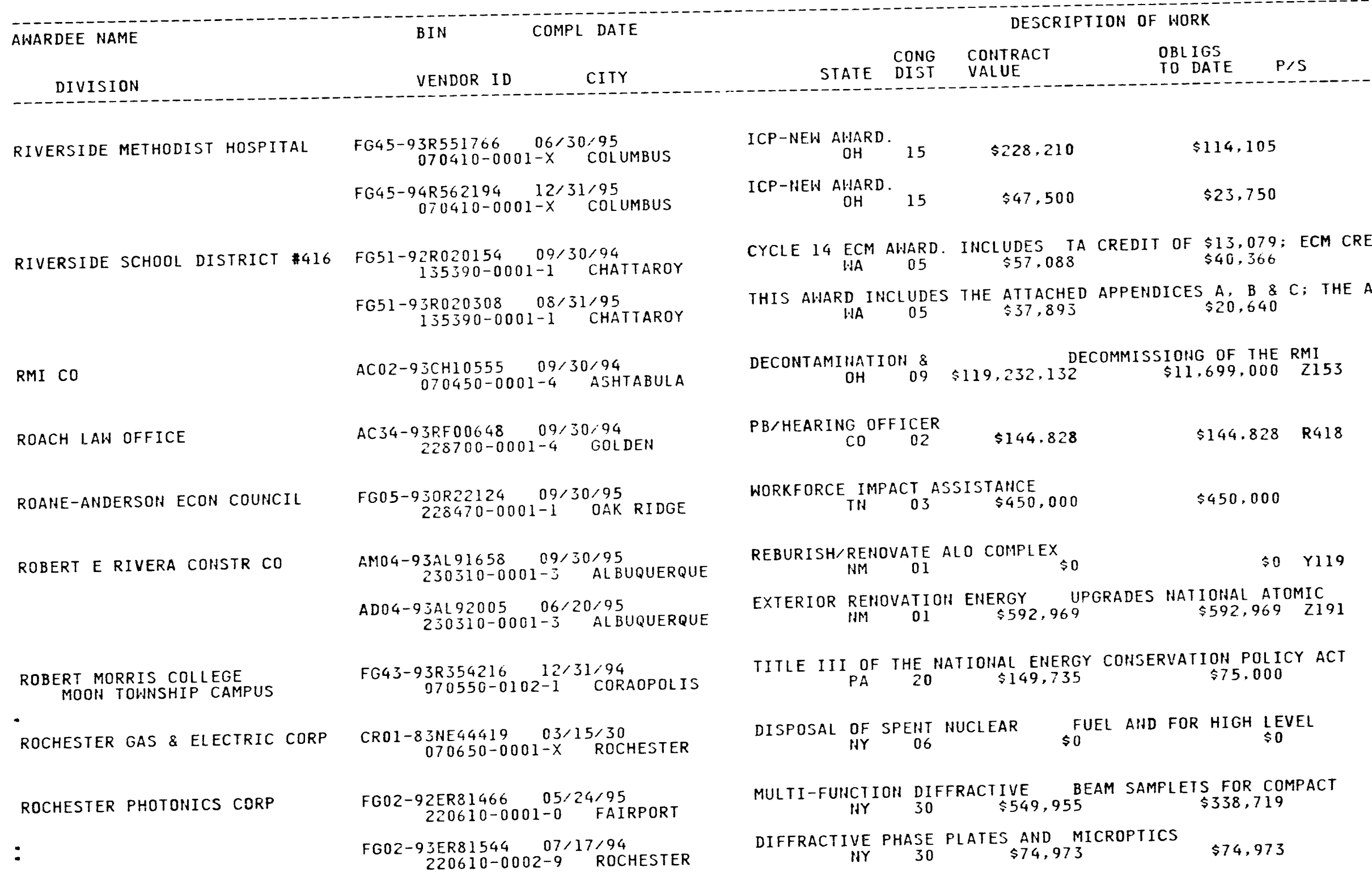




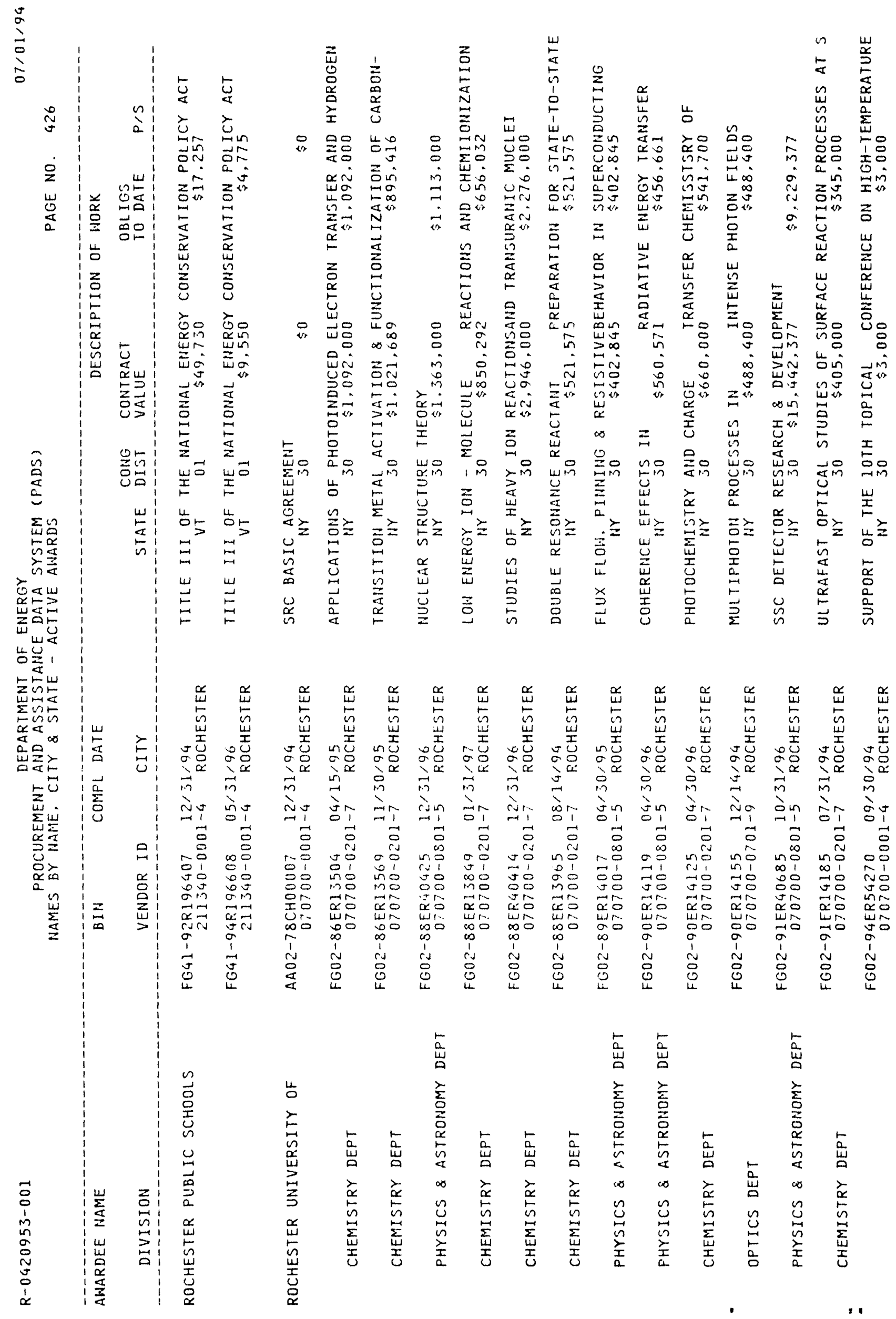




\begin{tabular}{|c|c|c|}
\hline AWARDEE NAME & COMPL DATE & DESCRIPTION OF WORK \\
\hline DIVISION & VENDOR ID & $\begin{array}{l}\text { COHTRACT } \\
\text { VALUE }\end{array}$ \\
\hline & $\begin{array}{c}\text { FG02-94ER79242 } 06 / 14 / 96 \\
070700-0001-4 \text { ROCHESTER }\end{array}$ & $\begin{array}{cccc}\text { PRE-COLLEE } & \text { EXPERIENCE IN } & \text { PHYSICS } & \text { (PREP) AT THE } \\
\text { NY } & 30 & \$ 37,581 & \$ 37,581\end{array}$ \\
\hline CHEMISTRY DEPT & $\begin{array}{c}\text { FG02-93ER61596 } 07 / 31 / 94 \\
070700-0201-7 \text { ROCHESTER }\end{array}$ & 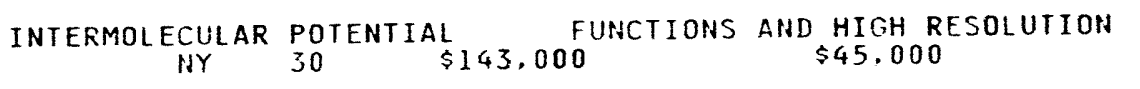 \\
\hline LAB FOR LASER ENERGETICS & $\begin{array}{c}\text { FCO3-925F19460 } 09 / 30 / 97 \\
070700-1801-0 \text { ROCHESTER }\end{array}$ & 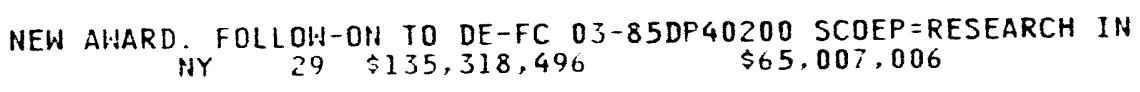 \\
\hline & $\begin{array}{l}\text { FG05-91ER79048 } 09 / 29 / 94 \\
070700-0001-4 \quad \text { ROCHESTER }\end{array}$ & 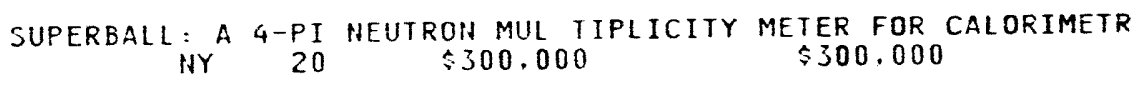 \\
\hline ROCK COUNTY HEALTH CARE CENTER & $\begin{aligned} & \text { FG45-94R562251 } 12 / 31 / 95 \\
& 238640-0001-7 \quad \text { JANESVILLE }\end{aligned}$ & ICP-NEH AHARD. \\
\hline ROCKDALE HOSPITAL & $\begin{array}{rl}F G 44-93 R 435373 & 09 / 30 / 95 \\
232480-0001-5 \text { COHYERS }\end{array}$ & 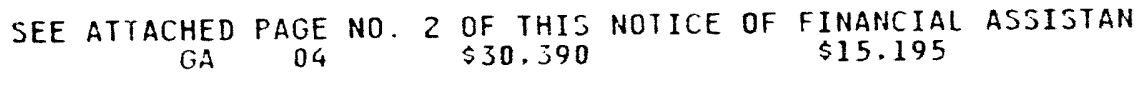 \\
\hline $\begin{array}{l}\text { ROCKEFELLER UNIVERSITY } \\
\text { FHYSICS DEPT }\end{array}$ & $\begin{array}{c}\text { FG02-88ER13847 02/28/95 } \\
070770-0301-9 \text { NEW YORK }\end{array}$ & $\begin{array}{cccc}\text { SOME BASIC RESEARCH PROBLEMS RELATED TO ENERGY } \\
\text { NY } & 15 & \$ 494,328 & \$ 494,328\end{array}$ \\
\hline & $\begin{array}{c}\text { FG02-91ER40651 01/31/95 } \\
0 \% 0770-0001-X \text { HEW YORK }\end{array}$ & $\begin{array}{cccc}\text { SUBSYSTEM PROPOSAL FOR PRE-SHOHER \& } & \text { SHOWER-MAXIMUM DETECTORS } \\
\text { NY } & 15 & \$ 3,499.500 & \$ 3.499,500\end{array}$ \\
\hline & $\begin{array}{c}\text { FG02-94ER20142 04/30/97 } \\
070770-0001-X \text { NEW YORK }\end{array}$ & $\begin{array}{c}\text { CHARACTERIZATION OF THE } \\
\text { IYY } 15\end{array}$ \\
\hline ROCKFORD COLLEGE & $\begin{aligned} \text { FG02-93ER79194 } 06 / 14 / 95 \\
199010-0001-3 \quad \text { R.OCKFORD }\end{aligned}$ & \begin{tabular}{cccc} 
PRE-FRESHMAN ENRICHMENT & \multicolumn{2}{c}{ PROGRAM (PREF) } \\
IL & 16 & $\$ 38,736$ & $\$ 38,736$
\end{tabular} \\
\hline & $\begin{array}{c}\text { FG45-90R520247 } 11 / 30 / 94 \\
199610-0001-3 \text { ROCKFORD }\end{array}$ & ICP - NEH AHARD \\
\hline $\begin{array}{l}\text { ROCKINGHAM COUNTY OF } \\
\text { BOARD OF EDUCATION }\end{array}$ & $\begin{array}{c}\text { FG44-92R435014 08/31/94 } \\
220840-0101-3 \text { WEHTWORTH }\end{array}$ & $\begin{array}{c}\text { CYCLE } 14 \text { - INSTITUTIOHAL COHSERVATION PROGRAM. APPROVED BUD } \\
\mathrm{NC} 050143.843\end{array}$ \\
\hline ROCKWELL INTERHATIOHAL CORP & $\begin{array}{c}\text { ACO3-881NE32129 04/06/99 } \\
070870-0002-0 \text { CAHOGA PARK }\end{array}$ & $\begin{array}{c}\text { DYNAMIC ISOTAPE POHER SUBSYSTEM DEMONSTRATION } \\
\text { CA } 01 \$ 226,710,216\end{array}$ \\
\hline ENERGY SYSTEMS GROUP & $\begin{array}{c}\text { ACO3-765F00700 09/30/94 } \\
070870-0902-8 \quad \text { CANOGA PARK }\end{array}$ & 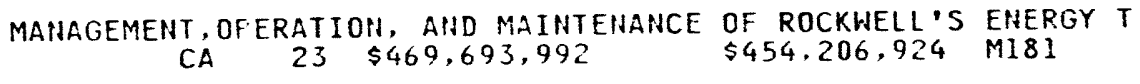 \\
\hline
\end{tabular}




AWARDEE NAME

DESCRIPTION OF HORK

BINTOR ID

COHG COHTRACT

OBI IGS

DIVISION
FOSSIL ENERGY CONVERSION SYS

ACO3-86́SF16021005/31/97

ROCKETDYNE DIV 070870-1502-8 CAHOGA PARK

FOSSIL ENERGY CONVERSION SYS

FG03-89ER5216j 03/24/95

070870-1102-2 CANOGA PARK

ROCKETDYNE DIV

AC03-925F19138 01/24/95
$070870-1502-8$

ROCKETDYNE DIV

$81 D 12749 \quad 09 / 30 / 94$ $070870-1502-8$ CAHOGA PARK

FG03-89ER45400 08/14/9G 070870-1610-5 THOUSAHD DAKS

SCIENCE CENTER

ROCKY MOUNTAIN BANKCARD SYSTEM ADO1-94AD63071 11/29/94
$185870-0001-2$ DENVER

ADO3-945F20257 11/29/94

$S F / F A D$

AD07-941D10596 04/11/95
$185870-0001-2$ DENVER

AD22-94FC94407 11/01;94 $185870-0001-2$ DENVER

ROCKY MOUNTAIN COLLEGE

GM04-94AL94727 06/06/99 BILLINGS

ROCKY RESEARCH

$A C 02-90 C E 40923 \quad 08 / 31 / 94$ $179010-0001-5$ BOULDER CITY

ROFF TOUN OF

PUBLIC SCHOOLS

FG46-93R613596 02/28/95
$221870-0101-6$ ROFF

$A B 01-94 A D 24924 \quad 05 / 31 / 95$ $219030-0001-7$ ROCKVILLE

ROGAY INC

FG05-9JER54218 02/31/94

FUEL DECLADDING FROJECT

$$
\begin{array}{llll}
A D & & & \\
C A & \$ 30,558,513 & \$ 28,694,301 & A G 51
\end{array}
$$

HELIUM GENERATION IN FUSION REACTOR MATERIALS

$$
\begin{array}{ccc}
\text { ENERATION IN FUSION REACTOR MATERIALS } \\
\text { CA } 23 & \$ 49,964 & \$ 49,964
\end{array}
$$

AHARD AND FUHDING OF LETTER COHTRACT

$$
\begin{array}{ccc}
\text { FUHDIIIG OF LETTER COHTRACT } & \$ 4,618,686 & \text { AC24 }
\end{array}
$$

SPACE NUCLEAR FOWER SUPPLY

$\begin{array}{llll}C A & 2 j & \$ 889.650 & \$ 889.650 \quad \text { AC21 }\end{array}$

MECHANISMS OF MECHANICAL FATIQUE IN CERAMICS
CA 21

CREDIT CARD SERVICES
CO OI
$\$ 0$
\$0 $\quad \mathbf{R} 799$

VISA BANK CARDS

$₹ 105,000$

$\$ 105,000 \quad R 799$

CREDIT CARD SERVICES PROVIDED UNDER FED SUFPLY SCHED COHT.

CREDIT CARD DELIVERY ORDER \& FUHDING FROM MAR 4 - MAR 17, 19

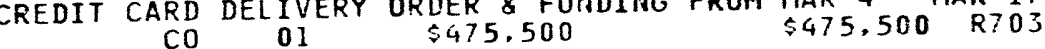

SUPPORT FOR SCIENCE, MATHEMATICS AND EHGINEERING EDUCATION

MT OI

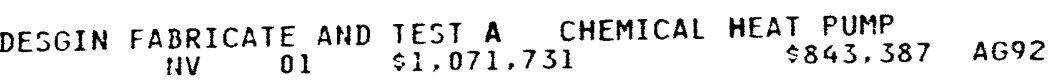

AHARDS A HEH CYCLE 15 ENERGY CONSERVATION MEASURE GRANT

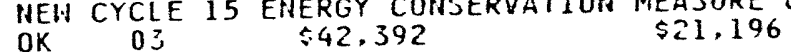

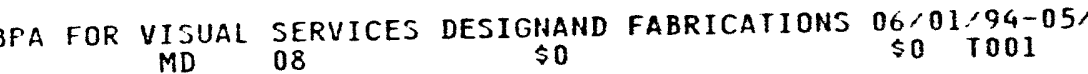

NEW RESEARCH GRANT : THEORY OF ELECTION - ION COLLISIONS
FL
05 ROLLINS COLLEGE 070990-0001-2 HIHTER PARK 


AHARDEE NAME BIN COMPL DATE

DESCRIPTION OF WORK

AWARDE NAME

VENDOR ID

CITY

STATE DING

COHTRAC VALUE

OBL IGS
TO DATE $P / S$

\section{DIVISION}

ROMAN FRANCISCO A

ROSEBUD SYNCOAL PARTNERSHIP

ROSELLE PARK BOARD OF EDUCN

ROSS AVIATION

ROSS COMPUTATIONAL RESOURCES

ROSS JEROME

ROW SCIENCES INC

ROY F WESTON INC

ROYBAL \& ASSOC
AB01-93ADI4807 $09 / 30 / 94$
$227840-0001-\times \quad$ CAPITOL HEIGHTS

FC22-90PC89604 03/12/96
$203590-0001-0$ COLSTRIP

FG42-942263914 06/30/96 071160-0001-1 ROJELLE PARK

AC04-89AL52318 02/28/96 $89 A L 52318$
$071230-0001-3 \quad 28$

FG02-93ERE1562 $07 / 17 / 94$
$233260-0001-4$

AB01-94AD18770 08/31/94

ACO1-9JEHS9302 06/01/96 JEH $29230200001-X \quad$ ROCKVILLE
2200010

$A C 01-92 R+100227 \quad 08 / 23 / 95$ $0 ? 1320-0003-9$ WASHINGTOH AC01-92R1:00259 07/22/94 D7 1320-0003-9 HASHINGTON ACO2-9ZER31192 09/06/95 $071320-0002-0$ HEST CHESTER AC22-93PC93255 12/14/94 $071320-0002-0$ HEST CHESTER

AC75-9054/26156 09/30/94 $192880-0002-x$ TULSA AC75-9051126364 01/31/95

CYCLE 16 ANARD.

BPA FOR ARBITRATOR
DESIGH AND OPERATE A FACILITY TO DEMONSIRATE HECO'S ADVANCED MT $02 \quad \$ 69.000 .000 \quad \$ \$ 4.500,000$

$\$ 84.937$

$\$ 42.468$

MANA SEMENT AND OPERATIOH OF ALAIR TRAHSPORTATIOH

$$
\text { IMM } 01 \text { \$? } 1.020 .781 \quad \$ 49.868 .128 \quad M 129
$$

EARTHLAB: A TELEVISUALIZATIOH ENUIRONMIET FOR LEARHINO HI 02 \$75,000 $\$ 75.000$

SO

\$0 R499

HEALTH COMMUNICATION

$M D \quad 08 \quad \$ 2,119,118$

$\$ 1,108,000 \quad R 426$

TECHNICAL SUPPORT TO THE OFFICE OF CIVILIAH RADIOACIIVE HAST DC 01 \$3 30.204 .696 $\$ 24.607 .126$ R425 TECHNICAL SUPPCRT TO THE OFFICE OF CIVILIAN RADIOACTIVE WAST DC 01 T $\$ 8,728.683 \quad \$ 8.273 .683$ R425 SUPPORT JERVICES FOR TECHNICALAHD ADMINISIRAIIVE ASSISTANCE PA 16 \$3,971,821 $\$ 2,000,000 \quad R 499$ ASSESSMENT OF TOXIC EMISSIOHS FROM TWO POHER PLANTS. ONE UTI $P A \quad 05 \quad \$ 2,956,611 \quad \$ 1,944,737$ AG11

STAFF AND OPERATE THE SHPA ENGINEERING DRAHIHG AHD RECORDS S

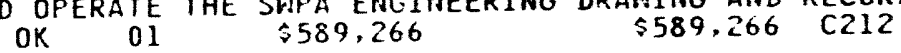

ON-SITE GENERAL SUPPORT SERVICES OK $01 \quad \$ 2.977 .958 \quad \$ 2.101 .808 \quad R 699$ 


AHARDEE NAME

BIN

COMPL DATE

DESCRIPTION OF HORK

DIVISION

VENDOR ID

CITY

CONG COHTRACT

OBLIGS

IO DATE $P / S$

RUSH PRESBYT ST LUKE'S MED CTR FG45-92R540083 12/31/94
$071430-0001-9$
CHICAGO

ICP - NEH AHARD.

IL OS $\$ 325,700$

$\$ 148,846$

RUSSELL COUNTY BD OF EDUCN

FG44-88R433609 09/30/94 $071470-0001-3$ PHENIX CITY

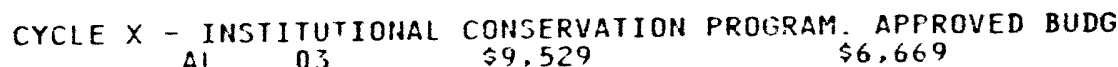
FG44-92R435030 08/15/94
$148290-0001-5$ ALEXAHDER CITY

$$
\text { AL } 03 \quad \$ 9.529
$$

CYCLE 14 - INSTITUTIOHAL COHSERVATION PROGRAM. APPROVED BUD

FC02-93P010118 07/29/96

$234350-0001-1$ MOSCOH

CLIMATE CHANGE COUNTRY STUDY.

RUSSIAN FEDERATION

RUST FEDERAL SERVICES

RUST GEOTECH INC

RUTGERS THE STATE UNIVERSITY

CERAMICS DEPT ACO7-941D13306 11/08/94
$237810-0001-X$ GRAHD JUHCTION

FEASIBILITY STUDY FOR TREATMENT OF ALPHA CONTAMIMATED MIXED ACO4-861012584 03/30/95 SUCCESSOR COHIRACT FOR OPERATION
$236440-0001-8$ GRAND JUNCTION

FG02-92ER61453 07/31/94
$071510-0001-4$ HEH BRUHSHICK

FG05-87EF13794 08/31/94 $0 ? 1510-0001-4$ NEH BRUHSHICK

FG05-88ER45368 $03 / 31 / 95$ $071510-0201-7$ NEW BRUHSHICK

FCO1-92CE41022 09/17/94 071510-0002-2 PISCATALAY

FG02-9JER14331 02/14/96 JER14331 $02 / 14 / 96$
$071510-0002-2$ PISCATAWAY

FG02-93ER14353 05/31/96 OT1510-0002-2 PISCATAHAY

FG02-93ER14356 06/14/96 071510-0002-2 PISCATANAY

FG02-93ER25179 $07 / 31 / 96$
$071510-0002-2$ PIJCATAHAY

AN EVALUATIN OF THE APPROPRATELAND-SURFACE RESOLUTIN FOR
NJ
06

CELLULASE - A KEY EHZYME FOR FERMENTATION FEEDSTOCKS NJ 06 O648.257 $\$ 422,709$

MULTICOMPONENT GLASS SURFACES: STRUCTURE AHD ADSORPTION NJ $06 \quad \$ 679.165 \quad \$ 679,165$

FIELD COORDINATION OF ENERGY ANALYSIS AHD DIAGHOSTIC CENTER $\begin{array}{lll}\text { HJ } 06 & \$ 4,249,590 & \$ 4.108 .407\end{array}$ MORPHOLOGICAL IHSTABILITY IN MODEL THIN FILM CATALYSTS: HJ OU $\$ 321,000 \quad \$ 210,000$ CARBON-HYDROGEN BOHD FUNCIIONALIZATION CATALYZED HJ 06 \$J \$277,800 \$90.000 INTRAMOLECULAR ELECTRON TRANSFER ACROSS SYNTHETIC HJ $06 \quad \$ 270,000 \quad \$ 90,000$ VISUALIZATION AND QUANTIFICATION OF EVOLVING

$071510-0002-2$ PISCATALAY NISUALIZAT $06 \quad \$ 480,000 \quad \$ 310,000$ 


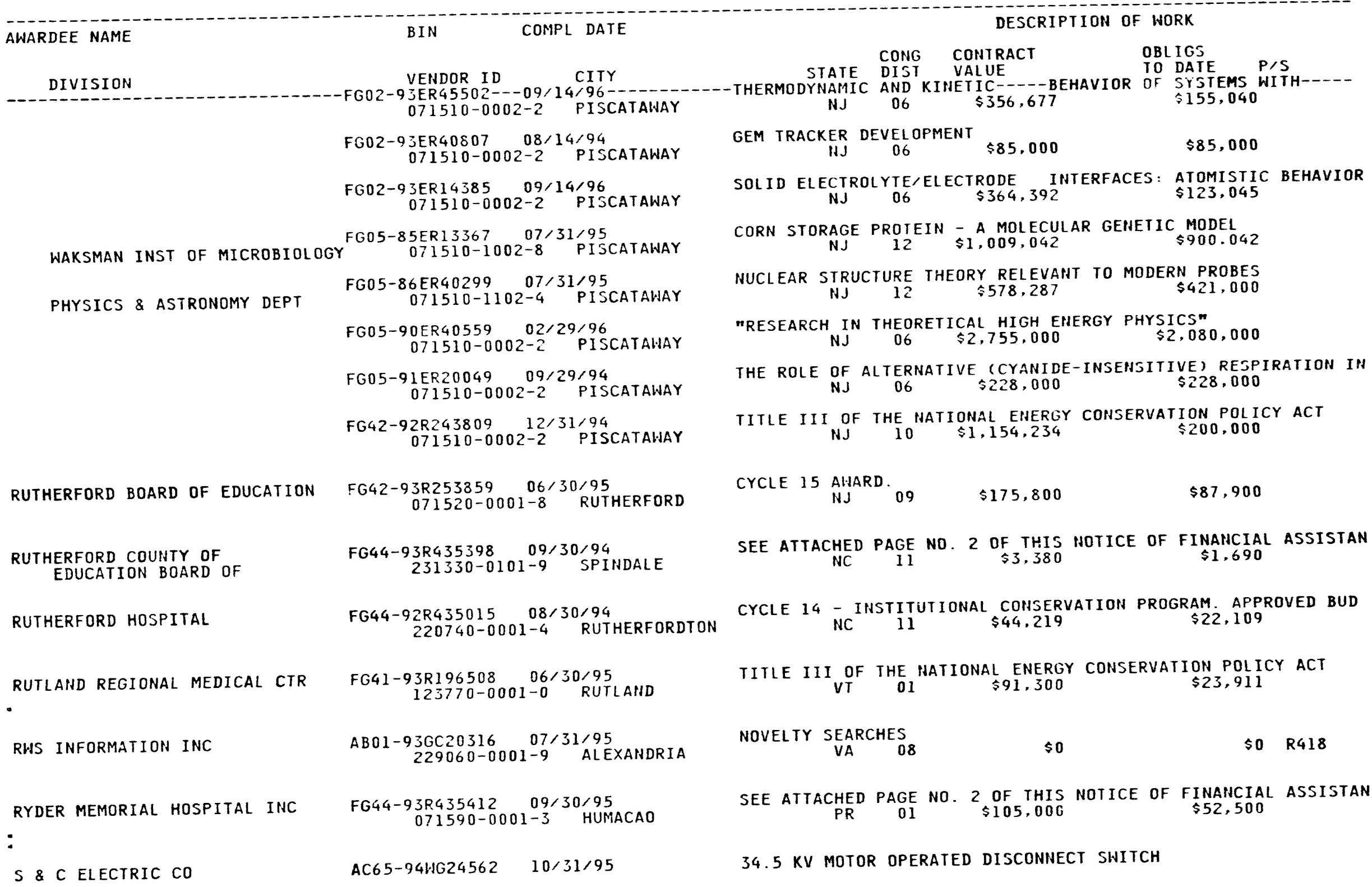




\section{AWARDEE NAME}

BIN

COMPL DATE

$071610-0001-7$ CHICAGO

\section{S \& H PHOTO}

$S$ E LACKEY MEMORIAL HOSPITAL

S POWELL CONSTRUCTION CO INC

SACRAMENTO MUNICIPAL UTIL DIST

SACRED HEAFT SCHOOL

SACRED HEART UNIVERSITY OF

SAG CORP

SAGE CORP

SAGINAW VALLEY STATE UNIV

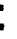

SAINT AGNES CHURCH
$071610-0001-7 \quad$ CHICAGO

AC65-93WA11253 12/01/95
$071610-0001-7$ CHICAGO

AB01-94AD25727 06/30/95 205300-0001-6 GAITHERSBURG

FG44-91R43476903/01/95 $131110-0001-X \quad$ FOREST

AC21-9411C31259 07/15/94 237240-0001-4 MORGANTOHN

$\begin{array}{ccc}\text { FC65-93WA } 0897 & 03 / 31 / 95 \\ 071770-0003-8 & \text { FOLSOM }\end{array}$

CRO1-83NE44415 03/15/30 $071770-0002-X$ SACRAMENTO

FG47-92R703499 08/31/94 071880-0019-0 FALLS CITY

FG44-93R435338 09/30/94 $071880-0020-4$ LAWIRENCEBURG

FG02-93ER7919506/14/95 071900-0001-1 SAlITUROE

AB01-93AD24902 09/30/94 178380-0001-7 HASHINGTON

FG05-93ER8154504/01/96 233570-0001-6 PISCATAWAY

$\begin{array}{cc}\text { FG45-92R540225 } & 08 / 31 / 94 \\ 223330-0001-5 & \text { SAGINAH }\end{array}$

FG42-94R263904 06/30/95

\section{DESCRIPTION OF HORK}

STATE DIST CONTRACT

OBLIGS

TO DATE P/S

IL 13

ITEMS 005 - 010 INTERPTR SHITCHITH MOTOR OFERATOR VAR SUBST

IL 03 S $\$ 396,042$

$\$ 396,042 \quad 5925$

BPA FOR PHOTO AND RELATED SERVICES

MD 08

$\$ 0$

\$0 T015

CYCLE 13 - INSIITUTIONAL CONSERVATION PROGRAM. APPROVED BUD $M J \quad 03 \quad \$ 15,710 \quad \$ 7,855$

DEMOLISH AIN REMOVE GASIFIER AHD STRETFORD PADS

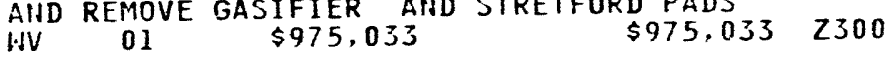

ANAEROBIC DIGESTER/BIOGAS PROJ J
CA $04 \quad \$ 807,500$

$\$ 65,000$

DISPOSAL FO SPENT NUCLEAR FUELAHD FOR HIGH LEVEL RADIOACTIVE $C A \quad 04 \quad \$ 0$

GRANT PROGRAMS FOR SCHOOLS ANDHOSPITALS AND FOR BUILDIHGS NE 01 \$14,988 \$7,494

SEE ATTACHED PAGE NO. 2 OF THIS NOTICE OF FINANCIAL ASSISTAN TN 04 \$3,849 $\$ 1,924$

PRE-FRESHMAN EHRICHMENT PROGRAM

$$
\text { DO } \$ 38.740
$$

$\$ 38.740$ BPA FOR STEHOGRAPHIC TYPIST AND COPIES OF TRANSCRIPTS

NEW AWARD - ADVANCED WINDOHS GLAZING TECH NJ $06 \quad \$ 674,571 \quad \$ 174,571$

ICP - NEW AWARD ${ }_{\text {MI }}$ \$8 $\$ 111,500$

$\$ 26,401$

CYCLE 16 AWARD 


\begin{tabular}{|c|c|c|c|c|c|c|}
\hline AWARDEE NAME & BIN $\quad C$ & COMPL DATE & & & DESCRIF & F WORK \\
\hline DIVISION & VENDOR ID & CITY & STATE & $\begin{array}{l}\text { CONG } \\
\text { DIST }\end{array}$ & $\begin{array}{l}\text { COHTRACT } \\
\text { VALUE }\end{array}$ & $\begin{array}{l}\text { OBLIGS } \\
\text { TO DATE }\end{array}$ \\
\hline & $238960-0001-$ & ATLANTIC HIGHL & ANDS & 06 & $\$ 4,860$ & $\$ 2,430$ \\
\hline SAINT ANTHONY HOSPITAL & $\begin{array}{r}F G 45-93 R 5515380 \\
199470-0001^{-}\end{array}$ & $\begin{array}{l}08 / 31 / 95 \\
-x \quad \text { CHICAGO }\end{array}$ & $\begin{array}{l}\text { ICP-NEW AWARD. } \\
\text { IL }\end{array}$ & 07 & $\$ 132,800$ & $\$ 67,044$ \\
\hline SAINT BARNABUS SCHOOL & $\begin{array}{r}F G 45-93 R 5515790 \\
229610-0001-\end{array}$ & $\begin{array}{l}07 / 31 / 94 \\
-7 \text { INDIANAPOLIS }\end{array}$ & $\begin{array}{l}\text { ICP-NEW AWARD. } \\
\text { IN }\end{array}$ & 10 & $\$ 2,600$ & $\$ 1,300$ \\
\hline $\begin{array}{l}\text { SAINT BERHARD CATHOLIC CHURTH } \\
\text { SCHOOLS }\end{array}$ & $\begin{array}{r}F G 45-92 R 5401411 \\
184130-0101-\end{array}$ & $\begin{array}{l}12 / 31 / 94 \\
-3 \\
\text { ST. PAUL }\end{array}$ & ICP - NEW AWAR & $\mathrm{RD}_{4}$ & $\$ 30,203$ & $\$ 22,651$ \\
\hline SAINT ELIZABETH MEDICAL CENTER & $\begin{array}{r}\text { FG45-9 } 3 R 5517680 \\
233400-0001-\end{array}$ & $\begin{array}{l}03 / 31 / 95 \\
-8 \text { DAYTON }\end{array}$ & $\begin{array}{c}\text { ICP-NEW AHARD. } \\
\text { OH }\end{array}$ & 03 & $\$ 682,283$ & $\$ 258,500$ \\
\hline SAINT ELIZABETH SETON GR SCH & $\begin{array}{r}\text { FG45-93R551711 } 1 \\
233370-0001-\end{array}$ & $\begin{array}{l}12 / 31 / 95 \\
-0 \text { MIPNEAPOLIS }\end{array}$ & $\begin{array}{c}\text { ICP-HEW AHARD. } \\
\text { MNN }\end{array}$ & 05 & $\$ 27,120$ & $\$ 23,052$ \\
\hline SAINT FRANCIS HEALTH CARE CTR & $\begin{array}{r}F G 45-94 R 562195 \\
238420-0001-\end{array}$ & $\begin{array}{l}12 / 31 / 96 \\
-4 \text { GREEN SPRINGS }\end{array}$ & $\begin{array}{c}\text { ICP-NEH AWARD. } \\
\text { OH }\end{array}$ & 05 & $\$ 10,875$ & $\$ 5.437$ \\
\hline SAINT FRANCIS HIGH SCHOOL & FG49-94R916031 & $\begin{array}{l}06 / 30 / 96 \\
\text { HONOLULU }\end{array}$ & CYCLE XVI & $\begin{array}{l}\text { GRAHT } \\
01\end{array}$ & $\begin{array}{l}\text { AHARD } \\
\qquad \$ 35,184\end{array}$ & $\$ 17,592$ \\
\hline SAINT FRANCIS OF ASSISI PARISH & $\begin{array}{r}F G 45-93 R 551647 \\
219700-0001-\end{array}$ & $\begin{array}{l}08 / 31 / 95 \\
-5 \text { AlNH ARBOR }\end{array}$ & $\begin{array}{c}\text { ICP-NEW AWARD. } \\
\text { MI }\end{array}$ & 13 & $\$ 16,363$ & $\$ 12.271$ \\
\hline SAINT FRANCIS XAVIER GRADE SCH & $\begin{array}{r}F G 45-93 R 551712 \\
233380-0001-6\end{array}$ & $\begin{array}{l}12 / 31 / 95 \\
-4 \quad \text { BUFFALO }\end{array}$ & $\begin{array}{c}\text { ICP-NEW AWARD. } \\
\text { MH }\end{array}$ & 02 & $\$ 12,953$ & $\$ 6,476$ \\
\hline SAINT GREGORY'S PARISH & $\begin{array}{r}F G 45-94 R 562245 \\
238600-0001\end{array}$ & $\begin{array}{l}12 / 31 / 95 \\
-2 \quad \mathrm{KIEL}\end{array}$ & $\begin{array}{c}\text { ICP-HEW AWARD. } \\
\text { WI }\end{array}$ & 06 & $\$ 4,000$ & $\$ 2,000$ \\
\hline SAINT JOHN VIANNEY SCHOOL & FG49-94R916032 & $\begin{array}{r}06 / 30 / 96 \\
\text { KAILUA }\end{array}$ & CYCLE XVI $\underset{H I}{E C M}$ & $\begin{array}{l}\text { GRANT } \\
02\end{array}$ & $\begin{array}{l}\text { AWARD } \\
\qquad 17,674\end{array}$ & $\$ 8,837$ \\
\hline SAINT JOHN'S CATHOLIC SCHOOL & $\begin{array}{r}F G 45-93 R 551810 \\
230000-0001-\end{array}$ & $\begin{array}{l}01 / 31 / 95 \\
-1 \quad \text { EDGAR }\end{array}$ & $\begin{array}{c}\text { ICP-NEW AHARD. } \\
\text { WI }\end{array}$ & 07 & $\$ 1,800$ & $\$ 1,350$ \\
\hline
\end{tabular}




\section{AWARDEE NAME}

BIN

COMPL DATE

DESCRIPTION OF WORK

DIVISION

VENDOR ID

CITY

STATE DIST

CONTRACT VALUE

OBLIGS O DATE

P/S

SAINT LOUIS SCHOOL

SAINT LUKE GRADE SCHOOL

SAINT LUKE'S HOSPITAL INC

SAINT MARY COLLEGE OF

SAINT MARY SCHOOL

SAINT MARY'S CENTRAL SCHOOL

SAINT MARY'S CHURCH

SAINT MARY'S PARISH

SAINT MICHAEL'S HOSPITAL

SAINT NICHOLAS' HOSPITAL

. SAINT PATRICK SCHODL $\begin{array}{lccc} & \text { ICP-NEW AWARD. } \\ 32551737 & 01 / 31 / 95 & \text { AHE } \\ 229730-0001-7 & \text { CLEVELAND HEIGHTS } & \text { OH }\end{array}$

$\$ 2,350$

$\$ 1,175$

FG45-93R551714 12/31/95 20 ST. PAUL

ICP-NEW AWARD

MN 04

$\$ 11,510$

$\$ 9,208$

FG45-93R551798 12/31/95

ICP-NEW AWARD

WI $01 \quad \$ 34,000$

$\$ 17,000$ $\begin{array}{cc}F G 47-93 R 703518 & 08 / 31 / 95 \\ 223360-0001-6 & \text { OMAHA }\end{array}$

FG47-93R703525 08/31/94 223360-0001-6 OMAHA FG $45-93 R 551743001 / 31 / 95$
$229790-0001-9$
LORAIN

FG45-92R540143 12/31/94 $22300-0001-2$ OHATONHA

GRANT PROGRAMS FOR SCHOOLS ANDHOSPITALS AND FOR BUILDINGS $\mathrm{NE} \quad 02 \quad \$ 114,832 \quad \$ 57,416$

GRANT PROGRAMS FOR SCHOOLS ANDHOSPITALS AND FOR BUILDINGS 0 $\mathrm{NE} \quad 02 \quad \$ 3,650 \quad \$ 1,825$

ICP-NEN AHARD
$\mathrm{OH} 13$
$\$ 2,700$
$\$ 1,350$

ICP - NEW AWARD

$\$ 37,237$

$\$ 26,065$

FG41-93RI91508 08/31/95

$232350-0001-1$ MILFORD

INITIAL AHARD.

$\$ 34,800$

$\$ 17,400$

FG45-93R551799 12/31/94 235640-0001-1 FOND DU LAC

ICP-NEW AWARD

HII 06

$\$ 4,000$

$\$ 2,000$

FG45-93R551801 12/31/94 $229960-0001-3$ STEVENS POINT

ICP-NEH AHARD.

WI $07 \quad \$ 29,500$

$\$ 14,750$

FG45-93R551800 12/31/94 $229950-0001-X$ SHEBOYGAN

ICP-NEW AWARD

WI 09

$\$ 17,500$

$\$ 8,750$

FG45-92R530045 10/31/94 $199600-0001-X$ ROCKFORD
ICP - NEH AHARD ${ }_{\text {IL }}$

$\$ 10,860$

$\$ 6,516$ 


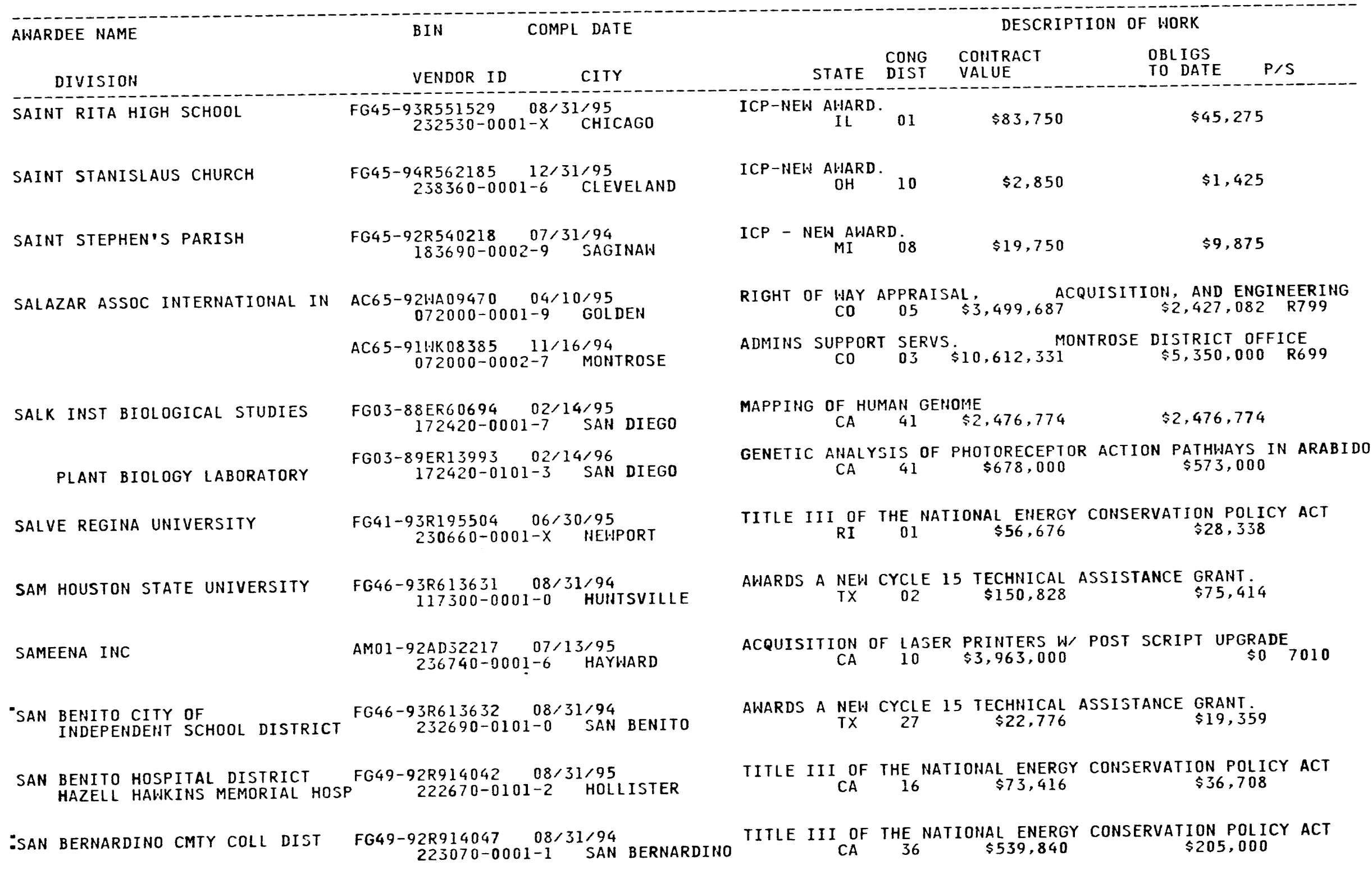




\begin{tabular}{|c|c|c|}
\hline \multicolumn{2}{|c|}{ AWARDEE NAME } & COMPL DATE \\
\hline & DIVISION & VENDOR ID \\
\hline SAN & CARLOS HOSPITAL & $\begin{array}{r}\text { FG44-92R435130 } 09 / 30 / 94 \\
226290-0001-X \quad M O C A\end{array}$ \\
\hline SAN & $\begin{array}{l}\text { DIEGO MISSION } \\
\text { SCHOOLS }\end{array}$ & $\begin{array}{r}\text { GM04-93AL84075 } 11 / 24 / 97 \\
117320-0102-2 \text { GALLUP }\end{array}$ \\
\hline SAN & DIEGO SPACE \& SCIENCE FDN & $\begin{array}{c}\text { FG03-92ER75756 08/31/94 } \\
222810-0001-X \text { SAN DIEGO }\end{array}$ \\
\hline \multirow[t]{3}{*}{ SAN } & DIEGO STATE UNIVERSITY & $\begin{array}{c}\text { FG03-86ER60479 } 08 / 31 / 94 \\
0 ? 2350-0001-5 \text { SAN DIEGO }\end{array}$ \\
\hline & & $\begin{array}{c}\text { FG03-93ER61669 08/31/96 } \\
072350-0001-5 \text { SAH } \Gamma: E G 0\end{array}$ \\
\hline & & $\begin{array}{c}\text { FG03-93ER61715 } 08 / 31 / 96 \\
072350-0001-5 \text { SAN DIEGO }\end{array}$ \\
\hline SAN & $\begin{array}{l}\text { FRANCISCO CITY OF } \\
\text { UNIFIED SCHOOL DISTRICT }\end{array}$ & $\begin{array}{c}\text { GMO4-94AL } 94699 \quad 11 / 29 / 98 \\
236210-0101-8 \text { SAN FRANCISCO }\end{array}$ \\
\hline SAN & FRANCISCO STATE UNIVERSITY & $\begin{array}{c}\text { FG03-92ER40711 } 01 / 31 / 96 \\
072370-0001-2 \text { SAH FRANCISCO }\end{array}$ \\
\hline SAN & JACINTO COLLEGE & $\begin{array}{rl}\text { FG } 46-93 R 613640 & 02 / 28 / 95 \\
072390-0001-X \quad \text { PASADENA }\end{array}$ \\
\hline SAN & JON SCHOOLS & $\begin{array}{c}\text { FG04-93AL } 9683907 / 08 / 96 \\
230860-0001-5 \text { SAN JON }\end{array}$ \\
\hline 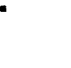 & & $\begin{array}{c}\text { GM04-93AL84106 07/01/98 } \\
230860-0001-5 \text { SAN JON }\end{array}$ \\
\hline SAN & JOSE STATE UNIVERSITY & $\begin{array}{c}\text { FG03-91ER } 40630 \quad 10 / 31 / 94 \\
072440-0001-4 \text { SAN JOSE }\end{array}$ \\
\hline$N$ & MATEO COMMUNITY COLL DIST & $\begin{array}{c}\text { FG49-92R } 91404808 / 31 / 94 \\
222940-0001-3 \text { SAN MATEO }\end{array}$ \\
\hline
\end{tabular}

$\begin{array}{cccc}\text { CYCLE } 14 & - \text { INSTITUTIONAL CONSERVATION PROGRAM. APPRO } \\ \text { PR } 01 & \$ 437,427 & \$ 218,713\end{array}$

SUPPORT FOR SCIENCE, MATHEMATICS, AND ENGINEERING TO IMPROVE NM 03

DESCRIPTION OF WORK

NEW GRANT: "STARFOWER", AN OM NIMAX FILM ON THERMOHUCLEAR FU

$$
\text { CA } 41 \quad \$ 204,400 \quad \$ 102,200
$$

RESPONSE OF TUIIDRA ECOSYSTEMS TO ELEVATED ATMOSPHERIC CO2

$$
\text { CA } 41 \text { \$2,419,000 } \$ 2,044,000
$$

RESFONSES OF SOIL MICROORGANISM AND MICROBIAL-MEDIATED PROCE CA SOIL MICROORGANISM AND MICROBIAL-MEDIATE
49

RESPONSE OF MEDITERRANEAN-TYPEECOSYSTEMS TO ELEVATED ATMOSP CA $49 \quad \$ 600,000 \quad \$ 400,000$

SUPPORT FOR SCIENCE, MATHEMATICS \& ENG. EDUCATION

SUPPORTS A PROGRAM OF RESEARCHIN HIGH ENERGY PHYSICS AT SAN $\begin{array}{lccc}A & P R O G R A M & \text { OF } & \text { RESEARCHIN } \\ C A & 06 & \$ 483,016 & \$ 211,008\end{array}$

AWARDS A NEW CYCLE 15 ENERGY CONSERVATION MEASURE GRANT.

EDUCATIOH OUTREACH

$$
\text { NM OUTREACH } 02 \quad \$ 75,000 \quad \$ 25,000
$$
$\begin{array}{cccc}\text { SUPPORT FOR } & \text { SCIENCE MATHEMATICS AND ENGINEERING } \\ \mathrm{NM} & 02 & \$ 0 & \text { EDUCATION } \\ & 02 & \end{array}$

DEVELOPMENT OF AN ELECTRONIC NUCLEAR DATABASE ENVIRONMENT FO CA $\quad 10 \quad \$ 175,000 \quad \$ 175,000$

TITLE III OF THE NATIONAL ENERGY CONSERVATION POLICY ACT

ITLE III OF THE NATIONAL $\$ 276,144 \quad \$ 138,072$ 
AWARDEE NAME

BIN

COMPL DATE

VENDOR ID COMPL DATE

DESCRIPTION OF WORK

DIVISION

VENDOR ID

SAND SPRINGS PUBLIC SCHOOLS

FG46-91R613502 08/31/94

$211640-0001-2$ SAND SPRINGS

FG46-92R613542 07/31/94

211640-0001-2 SAHD SPRINGS

FG46-93R613598 02/28/95

211640-0001-2 SAND SPRINGS

SANDIA CORP

GM04-89AL58201 09/29/94
$206460-0001-2$ ALBUQUERQUE

ACO4-94AL85000 09/30/98

206460-0001-2 ALBUQUERQUE

SANDIA NATIONAL LABORATORIES

GM04-87AL58197 04/02/97 206450-0001-9 ALBUQUERQUE

GMO4-93AL84097 10/28/98 206450-0001-9 AL BUQUERQUE

SANFORD TOWN OF

SCHOOL DEPARTMENT

SANTA BARBARA COTTAGE HOSPITAL

SANTA BARBARA COUNTY APCD

SANTA CRUZ VALLEY UNION HSD

SANTA FE INSTITUTE

$$
\begin{gathered}
\text { FG41-93R192519 06/30/95 } \\
230420-0101-6 \text { SANFORD }
\end{gathered}
$$

FG49-91R913031 02/28/95 190050-0001-5 SAINTA BARBARA

FG02-93CE41026 06/22/97
$229110-0001-3$ GOLETA FG49-93R915010 06/30/95
$229460-0001-X \quad$ ELOY

FG03-94ER61835 04/19/95 161790-0001-8 SANTA FE FG03-94ER14425 12/31/94 161790-0001-8 SANTA FE

FG03-93ER61639 08/14/96 161790-0001-8 SANTA FE
CYCLE 13 DOE ECM GRANT

OK 01 TRANT $\$ 561,230$

TOLIGS

$\mathrm{P} / \mathrm{S}$

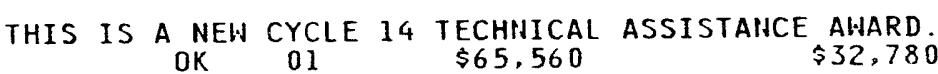

AWARDS A NEW CYCLE 15 ENERGY CONSERVATION MEASURE GRANT. OK 01 \$37,812 $\$ 18,906$

DRUG-FREE WORKPLACE
NM 01

$\$ 0$

MANAGE AND OPERATE THE GOVERNMENT-OHNED SAHDIA NATIONAL LABO HM $01 \$ 3,293,536,157 \quad \$ 2,004,036,157$ M181

SETS FORTH $\underset{N M}{H}$ THE UNDERSTANDING $\underset{\$ 1}{\text { AND }}$ RESPONSIBILITIES OF TSD OSB COMPUTER SUPPORT

TITLE III OF THE NATIONAL ENERGY CONSERVATION POLICY ACT ME 01 N $\$ 205,546 \quad \$ 37,411$

CYCLE XIII GRANT

$\$ 258,384$

$\$ 132.392$

\begin{tabular}{rlll} 
THE GRANTEE WILL RESUME TO & IMPLEMENT & \multirow{2}{*}{ DEMONSTRATE THE } \\
CA & 23 & $\$ 3,625,493$ & $\$ 1,277,000$
\end{tabular}

CYCLE 15 ICP GRAHT AHARD

$$
\begin{array}{lccc}
\text { ICP } & \text { GRAIIT } & \text { AWARD } \\
\text { AZ } & 04 & \$ 131,920 & \$ 88,300
\end{array}
$$

CONFERENCE ON SEQUENCE DESIGN
NM
03

$\$ 19,933$

COMPLEX SYSTEMS IN THE EARTH SCIENCES: PREDICTABILITY, MITI

ADAPTION TO THE EDGE OF CHAOS IN CELLS \& ECOSYSTEMS 


\begin{tabular}{|c|c|c|c|c|c|c|c|}
\hline AWARDEE NAME & BIN & DATE & & & DESCF & $=110 \mathrm{RK}$ & \\
\hline DIVISION & VENDOR ID & CITY & STATE & $\begin{array}{l}\text { CONG } \\
\text { DIST }\end{array}$ & $\begin{array}{l}\text { CONTRACT } \\
\text { VALUE }\end{array}$ & $\begin{array}{l}\text { OBLIGS } \\
\text { TO DATE }\end{array}$ & $P / S$ \\
\hline
\end{tabular}

SANTA FE NATIONAL FOREST

AI32-77DP03792 09/30/94 $072720-0001-5$ LOS ALAMOS

SANTA ROSA CONSOLIDATED SCHS

GM04-92AL58:31 07/21/97 $215830-0001-1$ SANTA ROSA

SANWA BANK OF CALIFORNIA

AC03-93SF $19689 \quad 09 / 30 / 98$ 232410-0001-X SAIN FRAHCISCO

SARAH BUSH LINCOLN HEALTH CTR

FG45-93R551:25 $08 / 31 / 94$
$232520-0001-6$

SAS INSTITUTE INC

$$
\begin{gathered}
\text { ABO1-93E123379 } 12 / 31 / 94 \\
103650-0001-8 \mathrm{CARY} \\
\begin{array}{r}
\text { AB01-94E124146 12/31/94 } \\
108650-0001-8 \mathrm{CARY}
\end{array} \\
\begin{array}{r}
\text { ACO1-93E123473 } 09 / 30 / 94 \\
108650-0001-8 \mathrm{CARY}
\end{array} \\
\begin{array}{r}
\text { ACO1-93AD34057 09/30/94 } \\
108650-0001-8 \mathrm{CARY}
\end{array}
\end{gathered}
$$

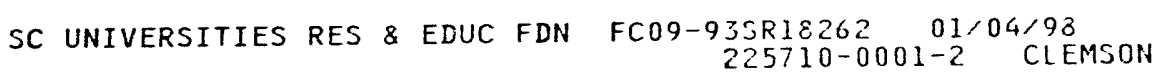

$$
\begin{gathered}
\text { AC21-92MC29115 09/30/95 } \\
225710-0001-2 \text { CLEMSON }
\end{gathered}
$$

-SCARBOROUGH SCHOOL DEPT

FG41-94R192607 06/30/96 SCARBOROUGH

SCHLESINGER ARKWRIGHT GARVEY

ABO1-93GC20317 $07 / 31 / 95$ 229070-0001-2 ALEXANDRIA

:SCHMELTZER APTAKER \& SHEPARD
SERVICES OF A PROFESSIO-HAL FORESTER AT LOS ALAMOS

$$
\mathrm{HM} 03 \quad \$ 296,620 \quad \$ 296,620
$$

SUPPORT FOR SCIENCE, MATHEMATICS, AHD ENGINEERING TO IMPROVE NM 01

$\$ 0$

CHECKS - PAID METHOD OF LETTEROF CREDIT FIHANCIHG FOR SLAC PAID METHOD OF LETTEROF CREDIT FINANCTHG $\$ 0$ R703

ICP-HEW AWARD.

$$
\text { IL } 15 \quad \$ 117,562
$$

$\$ 58,781$

REHEH BLAHKET PURCHASE AGREEMENT FOR TECHNICAL MANUALS AN SA IIC
BPA FOR USER GUIDES AHD TECHNICAL PUBLICATIONS $\$ 07630$ FY 94 PROPRIETARY SOFTHARE LICENSES RENEWAL FOR DOE FORRESTA RENENED LICENSE AND MAINTENANCE AGREEMENT FOR PROPRIETARY SA
NC 04
$\$ 49,995$

EDUCATIONAL OUTREACH TECHNOLOGY TRANSFER, TRAINING \& RESEARC SC 03 \$18,963,692 $\$ 6,538,176$

EM-AN INTELLIGENT INSPECTION AND SURVEY ROBOT

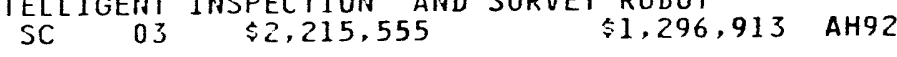

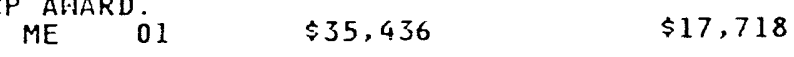

NOVELTY SEARCHES

$\$ 0$

$\$ 0 \quad$ R418

LEGAL SUPPORT SERVICES IN LABOR RELATIOHS LAW

$$
\begin{gathered}
\text { AC01-94GC30725 } 07 / 17 / 94 \\
236830-0001-5 \text { WASHINGTON }
\end{gathered}
$$

$\begin{array}{lllll}\text { DC } & 01 & \$ 612,625 & \$ 520,000 & R 418\end{array}$ 


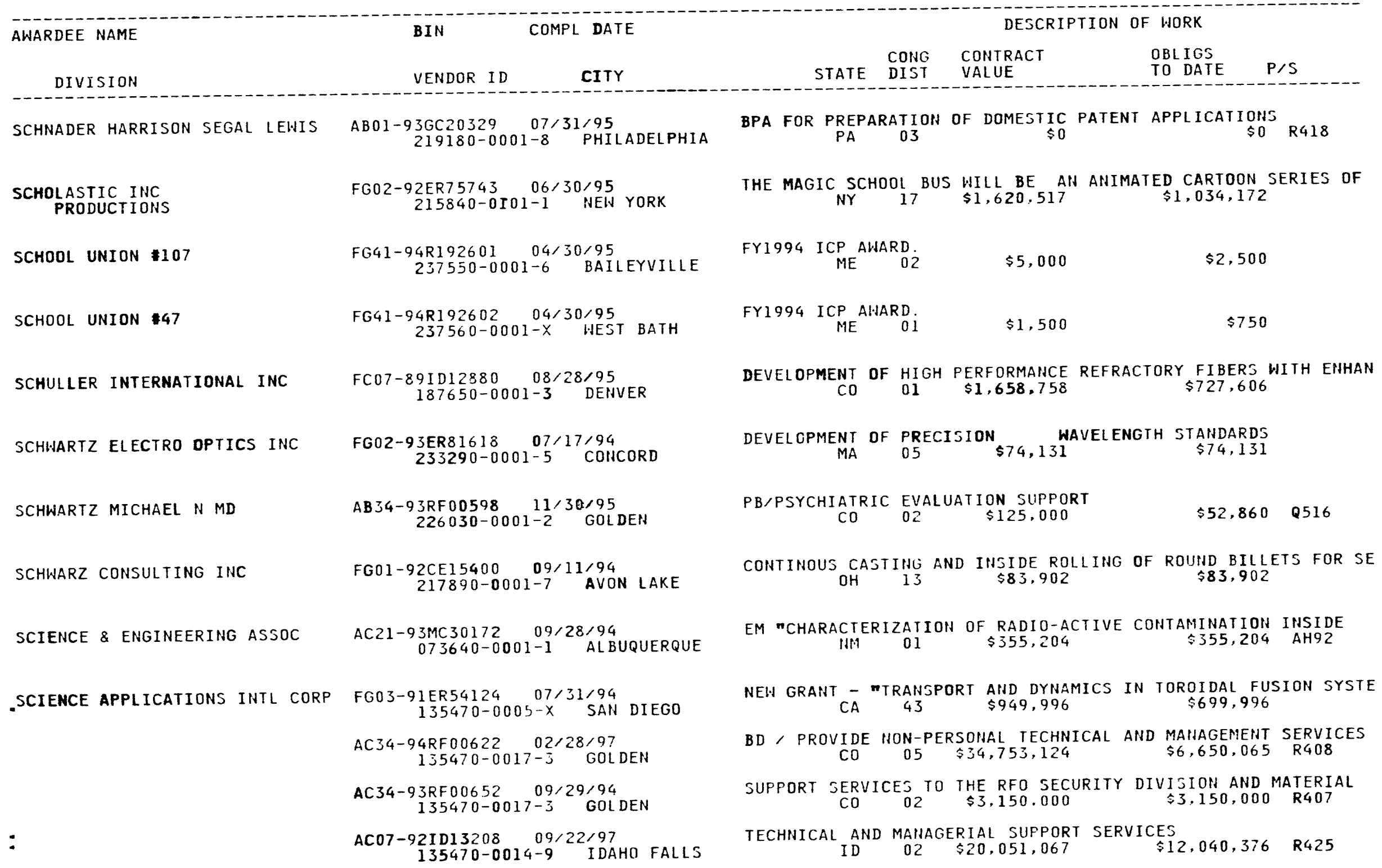




\begin{tabular}{|c|c|c|}
\hline \multirow{13}{*}{ DIVISION } & COMPL DATE & $\begin{array}{l}\text { DESCRIPTION OF WORK } \\
\text { OBLIGS }\end{array}$ \\
\hline & $\begin{array}{c}\text { VENDOR ID } \\
\text {-ACO1-92DP70056--12/12/94Y } \\
135470-0013-0 \text { GERMANTOHN }\end{array}$ & $\begin{array}{clll}\text { CONG } & \text { CONTRACT } & \text { OBLIGS } \\
\text { STATE DIST } & \text { VALUE } & \text { DO DATE S } \\
\text { - -TECHNICAL AHALYTICAL AND ADMINISTRATIVE SUPPORT SERVICES TO }\end{array}$ \\
\hline & 135470-0013-0 GERMANTOINN & 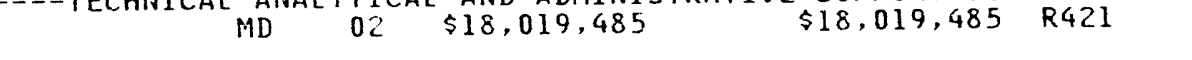 \\
\hline & $\begin{array}{c}\text { ACO1-94DF70067 } 04 / 12 / 97 \\
135470-0013-0 \text { GERMANTOWN }\end{array}$ & $\begin{array}{c}\text { TECHNICAL, AHALYTICAL AND ADMINISTRATIVE SUPPORT SERVICES FOR } \\
\text { MD } 06 \quad \$ 17,893,905\end{array}$ \\
\hline & $\begin{array}{l}\text { AC04-91AL58678 09/30/94 } \\
135470-0012-2 \text { ALBUQUERQUE }\end{array}$ & 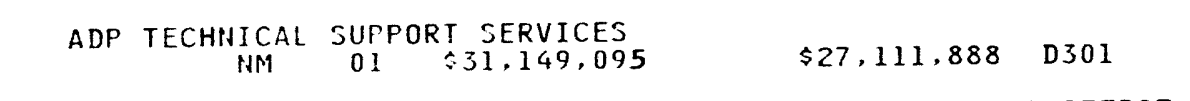 \\
\hline & $\begin{array}{c}\text { ACO1-93R!100250 04/27/96 } \\
135470-0011-4 \text { LAS VEGAS }\end{array}$ & 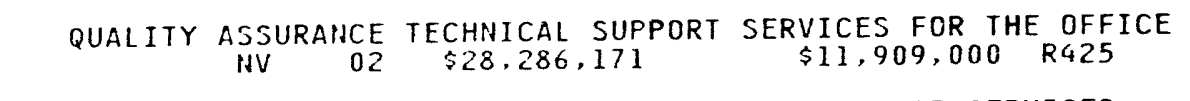 \\
\hline & $\begin{array}{c}A C 09-91 S R 18168 \quad 05 / 31 / 96 \\
135470-0007-6 \text { AIKEN }\end{array}$ & $\begin{array}{c}\text { SAFEGUARDS AND SECURITY } \\
\text { SC TECHNICAL SUPPORT SERVICES } \\
03 \$ 22,842,520\end{array}$ \\
\hline & $\begin{array}{r}\text { AC05-91MA40061 } 09 / 30 / 94 \\
135470-0008-4 \text { OAK RIDGE }\end{array}$ & 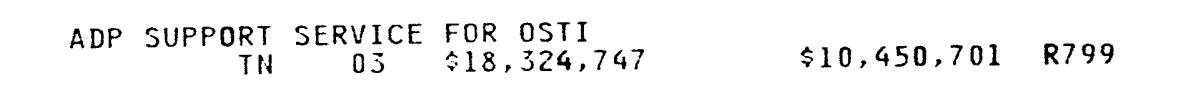 \\
\hline & $\begin{array}{rl}A C 05-910 R 21950 & 04 / 30 / 98 \\
135470-0008-4 \quad O A K & \text { RIDGE }\end{array}$ & $\begin{array}{l}\text { CERCLA/ MEPA RCRA CONTRACT TO FLAN \& MANAGE THE EXECUTION OF } \\
\text { TH } 03 \$ 39,230,857 \\
\end{array}$ \\
\hline & $\begin{array}{c}\text { FG02-92ER61368 } 02 / 28 / 95 \\
135470-0001-7 \quad \text { MCLEAiN }\end{array}$ & 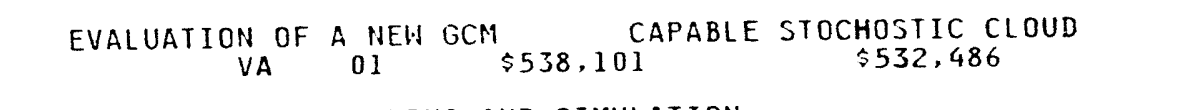 \\
\hline & $\begin{array}{c}\text { FG05-93ER54201 } 02 / 28 / 96 \\
135470-0001-7 \quad \text { MCLEAN }\end{array}$ & $\begin{array}{c}\text { ICRF ANTENHA MODELING AND SIMULATION } \\
\text { VA } 10 \\
\end{array}$ \\
\hline & $\begin{aligned} \text { ACO5-91ER40625 } 04 / 14 / 95 \\
135470-0001-7 \text { MCLEAN }\end{aligned}$ & 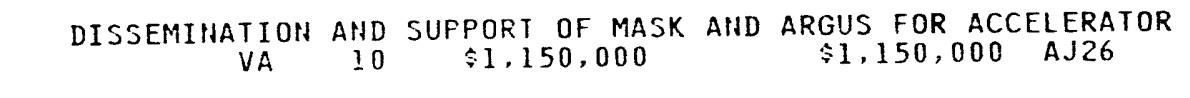 \\
\hline & $\begin{array}{rl}A C O 1-93 S A 10043 & 03 / 03 / 96 \\
& 135470-0015-7 \quad \text { RESTOH }\end{array}$ & 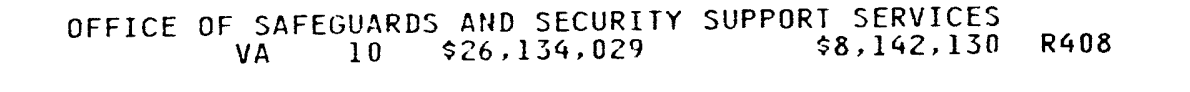 \\
\hline SCIENCE MUSEUM OF MINNESOTA & $\begin{array}{r}\text { FG02-92ER75758 } 08 / 31 / 94 \\
223520-0001-7 \text { ST. PAUL }\end{array}$ & 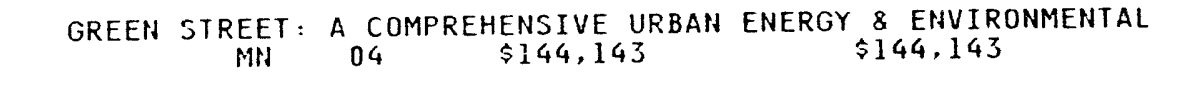 \\
\hline SCIENCE POLICY ASSOC INC & $\begin{array}{c}\text { FGO2-94ER61788 } 09 / 30 / 94 \\
236850-0001-2 \text { WASHINGTON }\end{array}$ & $\begin{array}{cccc}\text { BIODIVERSITY UNCERTAINTIES \& RESEARCH NEEDS } \\
\qquad C \quad 01 & \$ 25,000 & \$ 25,000\end{array}$ \\
\hline SCIENCE RESEARCH LAB INC & $\begin{array}{c}\text { FG02-91ER } 12120 \quad 09 / 14 / 94 \\
160370-0001-8 \text { SOMERVILLE }\end{array}$ & 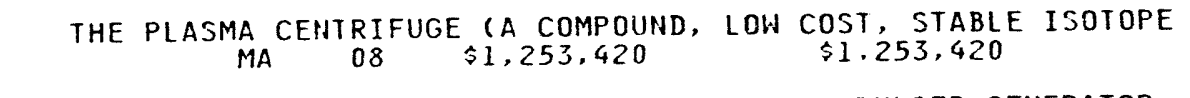 \\
\hline - & $\begin{array}{l}\text { FG02-91ER81194 } 03 / 31 / 95 \\
160370-0001-8 \text { SOMERVILLE }\end{array}$ & $\begin{array}{ccc}\text { DEV. OF AIR-WATER COOLED, ALL-SOLID-STATE PULSED GENERATOR } \\
\text { MA } 08 & \$ 549,940 & \$ 549,940\end{array}$ \\
\hline & $\begin{array}{c}\text { FG02-91ER81195 } 07 / 05 / 94 \\
160370-0001-8 \quad \text { SOMERVILLE }\end{array}$ & $\begin{array}{c}\text { A NEW HIGH-FIELD CRYOGENIC TARGET FOR } \\
\text { MA } 08\end{array}$ \\
\hline
\end{tabular}




\begin{tabular}{|c|c|c|c|c|c|c|c|}
\hline AWARDEE NAME & BIN & DATE & & & DESCF & F WORK & \\
\hline & VENDOR ID & CITY & STATE & $\begin{array}{l}\text { CONG } \\
\text { DIST }\end{array}$ & $\begin{array}{l}\text { CONTRACT } \\
\text { VALUE }\end{array}$ & $\begin{array}{l}\text { OBLIGS } \\
\text { TO DATE }\end{array}$ & $P / S$ \\
\hline
\end{tabular}

SCIENTECH INC

SCIENTIFIC ECOLOGY GROUP INC

SCITECH SCITEX DIGITAL PRINTING

$$
\begin{gathered}
\text { FG02-92ER81383 } 05 / 24 / 95 \\
160370-0001-8 \text { SOMERVILLE } \\
\text { FG02-93ER81531007/17/94 } \\
160370-0001-8 \text { SOHERVILLE } \\
\text { FG02-93ER81532007/17/94 } \\
160370-0001-8 \text { SOMERVILLE }
\end{gathered}
$$$$
\begin{aligned}
& \text { ACO1-92DP70055 11/12/94 } \\
& 120960-0001-7 \text { IDAHO FALLS }
\end{aligned}
$$$$
\text { AC07-92ID13143 12/29/96 }
$$$$
120960-0001-7 \text { IDAHO FALLS }
$$$$
\begin{gathered}
\text { AC07-901D12916 03/31/95 } \\
120960-0001-7 \text { IDAHO FALLS }
\end{gathered}
$$$$
\begin{aligned}
& \text { ACO1-91EH89095 11/16/94 } \\
& 120960-0004-1 \text { ROCKVILLE }
\end{aligned}
$$$$
\begin{gathered}
\text { ACO1-92DP00248 05/10/97 } \\
120960-0004-1 \quad \text { ROCKVILLE }
\end{gathered}
$$$$
\begin{gathered}
\text { ACO1-92N510052 05/11/95 } \\
120960-0004-1 \text { ROCKVILLE }
\end{gathered}
$$$$
\begin{array}{cc}
\text { AC02-9014P00029 11/08/94 } \\
120960-0004-1 \quad \text { ROCKVILLE }
\end{array}
$$$$
\text { ACO4-93AL } 82548 \quad 09 / 24 / 94
$$$$
120960-0003-3 \text { AL BUQUERQUE }
$$

$\begin{array}{cr}A C 07-94 I D 13305 & 11 / 08 / 94 \\ 155910-0001-0 & \text { OAK RIDGE }\end{array}$

FG02-92ER75757 $09 / 20 / 94$
$213550-0001-7$ AURORA

FGO2-94ER75950 12/14/95
$213550-0001-7$ AURORA

ADO1-94AD24934 $07 / 18 / 94$
$238050-0001-4 \quad$ DAYTON
A HIGH AVERAGE POHER HIGH PEAK POHER SUBHULLMETER LASER

$$
\text { MA } 08 \text { \$549.928 } \$ 338.690
$$

PASSIVE OPTICAL MONITOR FOR TOKAMAK PLASMA IHHOMOGENITIES

$$
\text { MA } 08 \text { \$74,945 } \$ 74,945
$$

HIGH REPETITIOH-RATE, HIGH POWER, ALL-SOLID-STATE PULSED

$$
\text { MA } 08 \text { S } \$ 74,940 \text { \$74,940 }
$$

EXECUTE LETTER CONTRACT NITH FUNDING

$$
\begin{array}{llll}
\text { ETTER CONTRACT WITH FUHDING } & \$ 4,942,971 & \text { R421 }
\end{array}
$$

SUPPORT SERVICES TO INCLUDE MANAGEMENT, TRAINING, QUALITY AS $\begin{array}{llll} & \end{array}$

TECHNICAL AND MALAGEMENT ASSISTANCE IN $R \&$ $D$

$\begin{array}{cccc}\text { ID } & 02 & \$ 4,018,535 & \$ 3.059,299 \\ \text { ADMINISTRATIVE AND TECHNICAL SUPPORT SERVICE FOR THE OFFICE }\end{array}$ ADMINISTRATIVE AND TECHNICAL SUPPORT SERVICE FOR THE OFF ADMINISTRATIVE AND TECHHICAL SUPPORT TO $\underset{\$ 1 \text { THE OFFICE OF DEFENS }}{\$ 1,827,522}$ R421 TECHNICAL ANA. ADMIN. SUPPORT SERVICE FOR OFFICE OF NUCLEAR TECHNICAL ANA. ADMIN. SUPPORT SERVICE FOR $\$ 2,948,000$ R408 TECHNICAL SUPPORT SERVICES IN THE AREA OF EVALUATION OF SAFE TECHNICAL SUPPORT SERVICES IN THE AREA OF EVALUATI $\$ 12,997,773$ R421 TECHNICAL AND MANAGEMENT SUPPORT FOR DOE/AL AND LAAO NM $01 \quad \$ 7,979,080 \quad \$ 5,478,134 R 408$

FEASIBILITY STUDY FOR ALPHA COHTAMINATED MIXED LOW LEVEL WAS TN 03 STUDY FOR ALPHA CONTAM

E=MC2 A HAHDS-ON EXPLORATION OF EISTEIN'S

$\begin{aligned} & \text { IL } 04 \$ 226,395 \\ & \text { MUSEUM IN A SCHOOL EDUCATIOHALFROGRAM }\end{aligned}$

MUSEUM IN A SCHOOL EDUCATIONALFROGRAM $\$ 14,057$ S74,057

INKJET IMAGING SYSTEM $\$ 64,831 \quad \$ 64,831 \quad 7010$ 


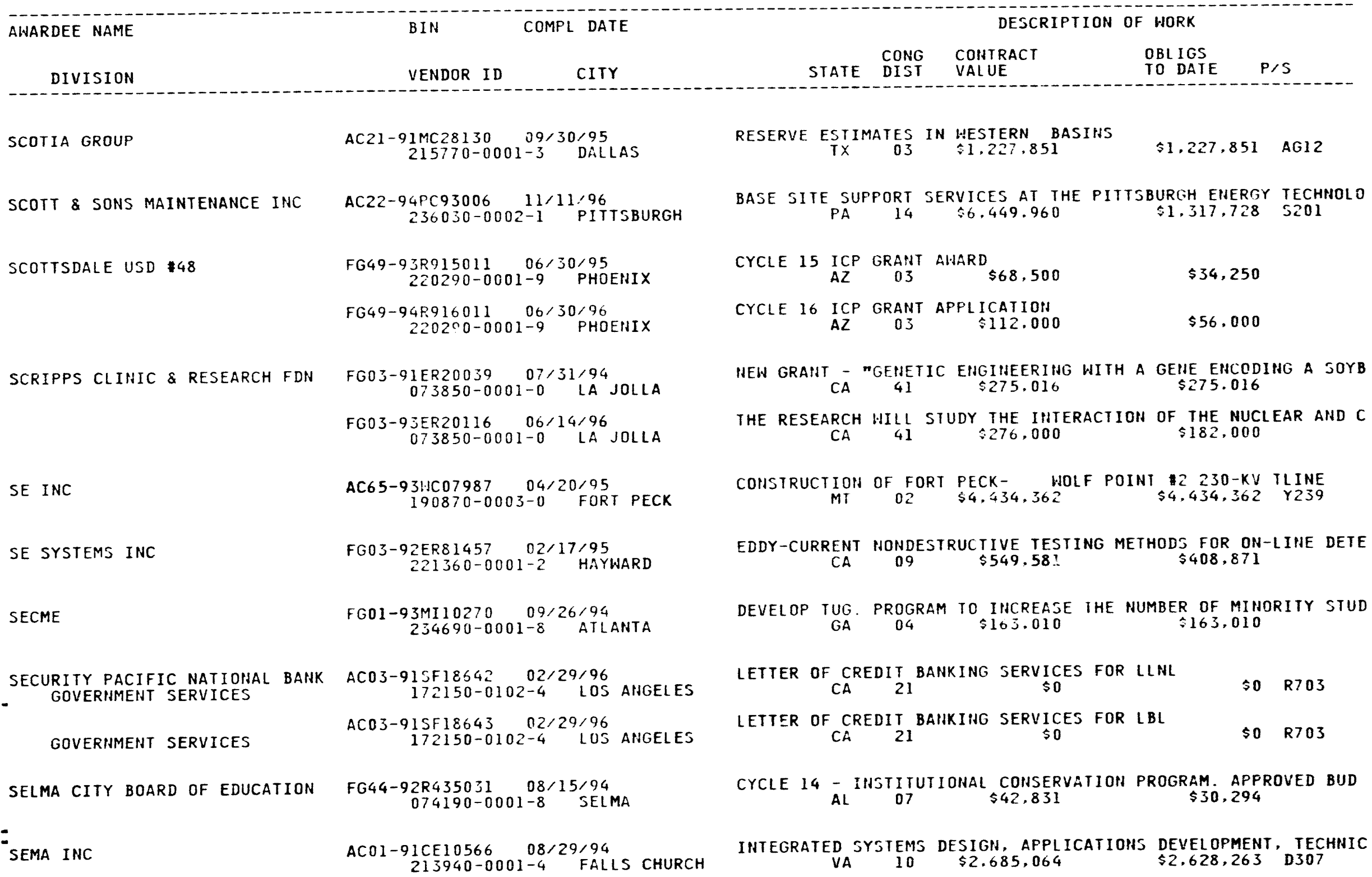




\begin{tabular}{|c|c|}
\hline AWARDEE NAME & COMPL DATE \\
\hline DIVISION & VENDOR ID \\
\hline SEMINOLE VOLUNTEER FIRE DEPT & $\begin{array}{r}\text { GM04-79AL53644 06/30/99 } \\
180190-0001-1 \quad \text { LARGO }\end{array}$ \\
\hline \multirow[t]{2}{*}{ SENTINAL CLEANERS } & $\begin{array}{c}A B 01-93 A D 69204 \quad 07 / 05 / 94 \\
225570-0001-9 \text { ALEXANDRIA }\end{array}$ \\
\hline & $\begin{array}{c}A B O 1-94 A D 69253 \\
225570-0001-9\end{array}$ \\
\hline
\end{tabular}

SENTRAL COMMUNITY SCHOOL DIST

SEQUATCHIE COUNTY OF
EDUCATION BOARD OF

SERRANO PORTABLE T'ILET SVC

SETON HALL UNIVERSITY

SEVENTH DAY ADVENTIST SCHOOL

SEVERN GRAPHICS INC

SEWICKLEY VALLEY HOSPITAL

SFA INC

SGI FUELS INC
FG47-93R707039 0 08/31/94
$212750-0001-0$ FENTON

FG44-93R435339 09/30/95 231230-0101-6 DUNLAP

A $665-941.1 K 1456209 / 30 / 94$ $41.1 K 1456209 / 30 / 94$
$235570-0001-\times \quad$ BLOOMFIELD

FG02-9JER79196 07/14/95

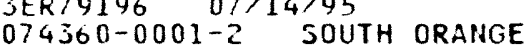
FG44-93R435418 $09 / 30 / 95$
$074410-0002-5 \quad$ CHARLOTIE AMALIE

AB01-94AD25723 07/01/94
$117530-0001-7$ GLEN BURHIE

$A B 01-94 A D 25713 \quad 06 / 30 / 95$ 117530-0001-7 GLEN BURNIE

FG43-93R354217 12/31/94 $074450-0001-1$ SEHICKLEY

FG05-90ER81034 11/19/94

AC21-91MC27240 09/04/94 $199320-0001-9$ LAHDOVER

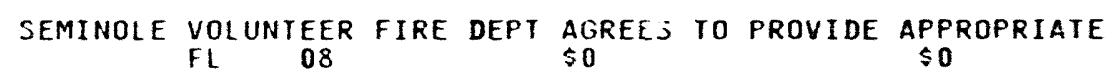

BPA FOR CLEANING SERVICES

$$
\text { VA OS \$O \$O } \$ 209
$$

BPA FOR CLEAIING SERVICES

$\$ 0$

$\$ 0 \$ 209$

GRANT PROGRAMS FOR SCHOOLS ANDHOSPITALS AND FOR BUILDIHGS 0

$$
\text { IA } 05 \quad \$ 3.500 \quad \$ 1.750
$$

SEE ATTACHED PAGE NO. 2 OF THIS NOTICE OF FINANCIAL ASSISIAN
TH
03

PORTABLE TOILET SVC - WATERFIOSUBST

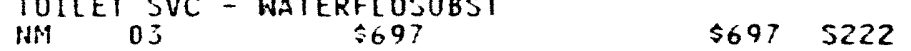

PRE-FRESHMAN ENRICHMENT
$\mathrm{NJ}$
10

SEE ATTACHED PAGE NO. 2 OF THIS NOTICE OF FINANCIAL ASSISTAN VIS PAGE NO. 2 OF THIS NOTICE OF FINAHCIAL
VI
$\$ 52.845$

BPA FOR PHOTO AND RELATED SERVICES BPA FOR GRAFHIC ARTS AND RELAIED SERVICES. 07/01/94 THRU 06，
MD 01

TITLE III OF THE NATIONAL ENERGY CONSERVATION POLICY ACT $P A \quad 20 \quad \$ 222,773 \quad \$ 75,000$

PHYSICAL APPLICATIONS OF CHANNELING RADIATION AS A NOVEL. IN MD $05 \quad \$ 534.910 \quad \$ 534.910$

PRODUCTION OF MILD

GASIFICATION COPRODUCTS 
$R-0420953-001$

\begin{tabular}{|c|c|}
\hline AWARDEE NAME & BIN COMPL DATE \\
\hline DIVISION & VENDOR ID \\
\hline & $215050-0001-7$ \\
\hline \multirow[t]{3}{*}{ SHARP ELECTRONICS CORP } & $\begin{array}{r}\text { ADO1-93AD23661 } 09 / 30 / 94 \\
074540-0003-7 \quad \text { ALEXANDRIA }\end{array}$ \\
\hline & $\begin{array}{c}\text { ABO1-93AD23662 } 09 / 30 / 94 \\
074540-0003-7 \text { ALEXANDRIA }\end{array}$ \\
\hline & $\begin{array}{c}\text { ADOI-94AD23693 } 07 / 31 / 94 \\
074540-0003-7 \quad \text { ALEXANDRIA }\end{array}$ \\
\hline
\end{tabular}

SHELDON CITY OF FG47-935707040 $08,31 / 94$
CONSOLIDATED SCHOOL DISTRICT $232080-0101-0 \quad$ SHELDON

SHLESINGER ARKIHRIGHT GARVEY

$A B O 1-020 C 20277$
$170450-0001-4$

$A B \cup 1-93 C C 20330 \quad 07 / 31 / 95$ $170450-0001-4$ ARLIHGTON

SHOSHONE-BANNOCK TRIBES

$$
\begin{gathered}
\text { FG07-93ID132225 } 02 / 28 / 98 \\
075020-0001-1 \text { FCRT HALL } \\
\text { FC07-91IDI3066 09/30/86 }
\end{gathered}
$$$$
\text { 075020-0UU1-1 FORT HALL }
$$

SHOSHOHE-MONTGOMERY MINE

BULLFROG ENTERPRISES

SHOW LOW CONSTRUCTION IHC

$$
\begin{gathered}
\text { ACO3-83HV10326 } 03 / 16: 95 \\
075040-0101-5 \text { BEATTY }
\end{gathered}
$$

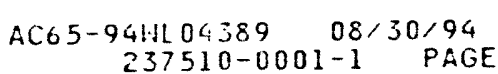

SIDNEY CENTRAL SCHOOL DISTRICT

\section{SIEMENS}

SIEMENS ENERGY \& AUTOMAT INC
FG42-93P253878 07/31/95
$230520-0001-2$ SIDHEY

AC65-904.1103204 07/01/94 $190410-0002-x$ ERLANGEN

AC65-92HA10115 01/01/95 161840-0008-X. EHGLEHOOD
DESCRIPIION OF HORK

OBLIGS

STATE CONG COHTRACT

VALUE

$\$ 430.548$

$\$ 430.548$ AGI2

MAIUTEHAHCE AGREEMEIIT FOR REPAIR DF THREE SHARP 9800 COPIERS

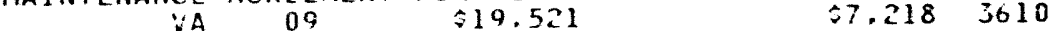

BPA FOR REPAIRS AHD MAIHTENAHCE OH COPIERS

$$
\text { VAPAIRS AHD MAIMTENAHCE OH COPIERS } 08 \text { SO \$0 } 3610
$$

PURCHASE OF COPIER

$\$ 12.102$

$\$ 12.102 \quad 3610$

GRANT PROGRAMS FOR SCHOOLS ANDHOSPIIALS AND FOR BUILDINGS 0

$$
\text { IA } 050 \text { \$2.150 } \$ 1.075
$$

BPA FOR HOVELTY SEARCHES ETC. 10

S0 R418

BFA FOR FREFARATION OF DOMESTIC PATENT AFPLICATIOHS

$$
\begin{aligned}
& \text { FREFARATIOH OF DOMESTIC PATENT AFPLICATIOHS } \\
& \text { VA } 10
\end{aligned}
$$

GRANT FUNDING TO IMPLEMENT PROVISIOHS OF

ID 02 \$1.500.00

"HORKING AGREEMENT"

WIPP INDIAH GOVERHMENT FROGRAM

$\$ 160.058$

COMPENSATIOH FOR PRODUCTIOH LOSS AND CREH SALARIES HHEN HO

COMPENSATION FOR PRODUCTIOH LOJS AND CREH SALARIES NHEN
HV 01

GLEN CANYON CONSOLIDATION

CYCLE 15 AHARD. $23 \quad \$ 60.697 \quad \$ 30.348$

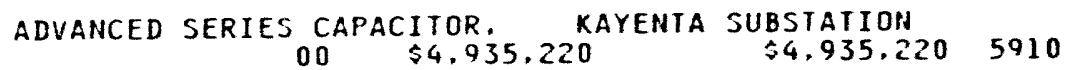

POWER CIRCUIT BREAKERS

CO $02 \quad \$ 4,038,460 \quad \$ 4,038.460 \quad 5925$


AWARDEE NAME

BIN

COMPL DATE

DESCRIPTION OF HORK

DIVISION

VENDOR ID

STATE COHG

CONIRACT

OBL IGS

DIVISION

AC65-90WA08249 12/23/94

$101840-0001-2$ RICHLAND

AC65-93HA11129 05/01/95

161840-0001-2 RICHLAND

POWER CIRCUIT BREAKERS

MS 03 \$1,706.556

$\$ 1,706,556 \quad 5925$

POHER CIRCUIT BREAKERS FOR VAR LOCATIONS

$\begin{array}{lllll}\text { MS } & \text { BREAKERS FOR } & \text { VAR LOCATIONS } & & \\ & \$ 1,898,068 & \$ 1,898,068 & 5925\end{array}$

SIERRA ENERGY CO

FC22-93BC14950 12/31/94
$226160-0001-6$ CDD

ENHANCED OIL RECOVERY UTILIZING HIGH-ANGLE WELLS IN THE FRON

TITLE III OF THE NATIOHAL ENJERGY CONSERVATION POLICY ACT

SIERRA KINGS DISTRICT HOSPITAL FG49-92R914049 $08 / 31 / 94$
$222950-0001-7$
REEDLEY

SIERRA NEVADA COLLEGE

FG03-93ER79197 06/14/95
$213220-0001-8$ IHCLIHE VILLAGE

SIERRA NEVADA MEMORIAL HOSP

FG49-91F913033 02/28/95

214910-0001-5 GRASS VALLEY

PRE-FRESHMAN EHRICHMEHT PROSRAM

$\$ 39,631$

CYCLE XIII GRAIT

$\$ 269,922$

$\$ 142,311$

SIERRA PACIFIC POWER CO

FC21-92MC29309 $07 / 31 / 00$
$075150-0002-3 \quad$ SPARKS

FR21-92AC29392 07/31/20

$075150-0002-3$ SPARKS DEMONSIRATIOH OF AN INTEGRATEDCOAL GASIFICATION COMBINED
NV $02 \$ 269.793 .100$

REPAYMENT AGREEMENT
NV 02

$\$ 0$

ABO1-93AD14806 09/30/94 $140200-0002-6$ SILVER SPRING

BPA FOR INTERPRETER MD 08

$\$ 0$

SIGN LANGUAGE ASSOC

$A B 01-937 D 14804 \quad 09 / 30 / 94$ 232890-0001-X SPRINGFIELD

BPA FOR INTERPRETER VA 08

sิo

\$0 R608

SIGNING HANDS FG04-93AL96836 07/08/96
$230840-0001-8$ SILVER CITY

SILVER CONSOLIDATED SCHOOLS

GM04-93AL84103 07/01/98 230840-0001-S SILVER CITY

-SIMPLEX TIME RECORDER CO

AD01-93ADS9206 09/30/94 075330-0003-X LANHAM
EDUCATION OUTREACH

IIM 02

$\$ 100.000$

SUPPORT FOR SCIEHCE MATHMATICSAND ENGINNERING EDUCATION IIM 02

MAINTENANCE AGREEMENT RENEHAL

MD $07 \quad \$ 950$

$\$ 950 \quad J 099$ 


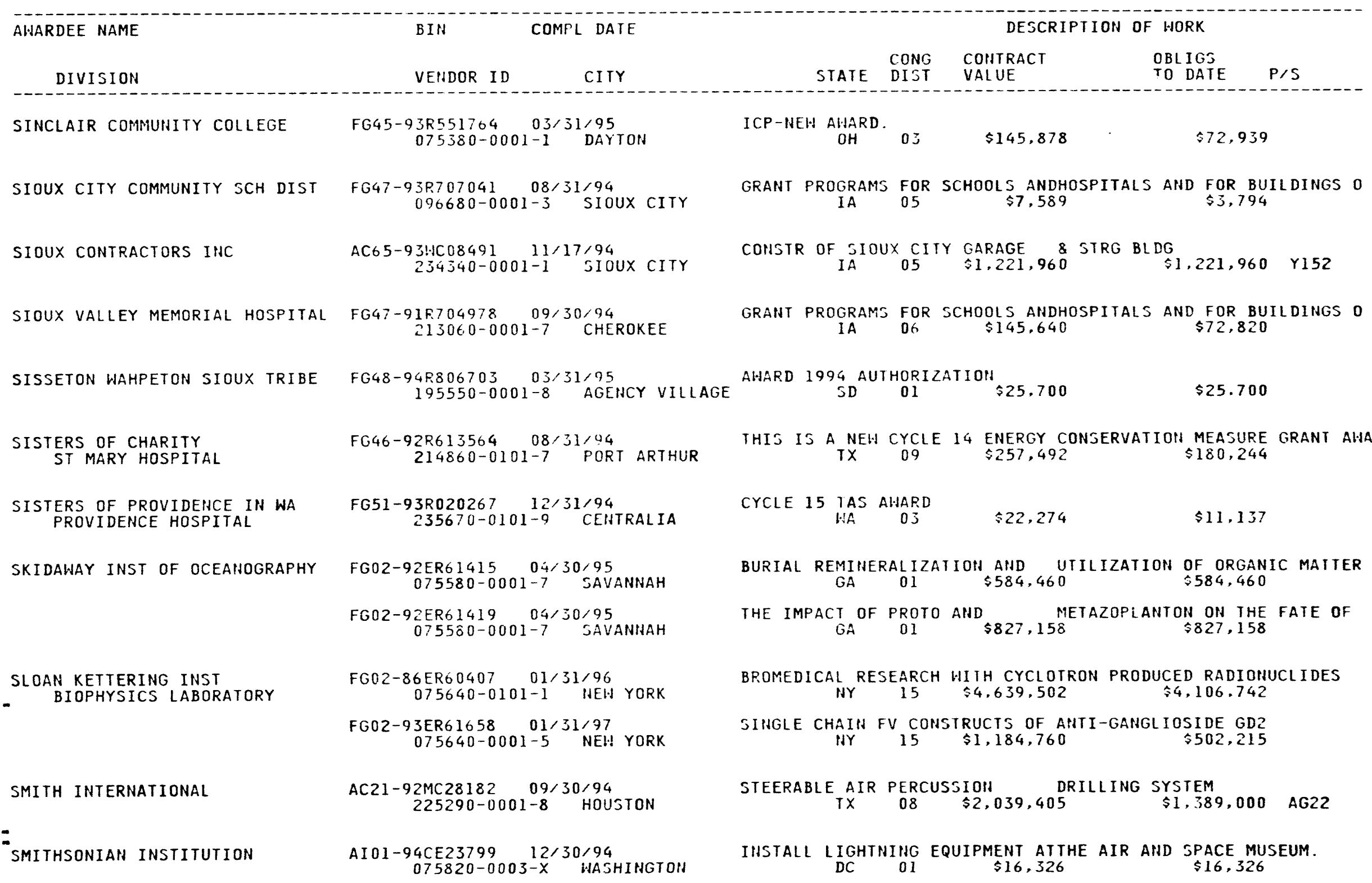




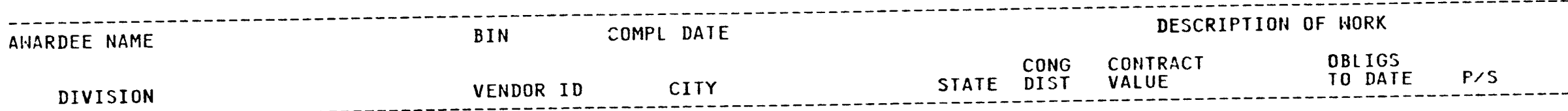

FG02-94ER61791 02/28/95 $02003-X$ WASHINGTON
$075820-0003-x$

FG05-85ER60374 $09 / 19 / 94$
ENVIRONMENTAL RESEARCH CENTER $075820-1003-5$ WASHINGTON

ASTROPHYSICAL OBSERVATORY

FG02-91ER40635 04/30/95 $075820-0101-X$ CAMBRIDGE

SOCIETY FOR ADV OF CHICANOS

SINSHEIMER LABORATORIES

SOCIETY FOR IND \& APPLIED MATH

$$
\begin{aligned}
& \text { FG03-92ER75832 09/14/94 } \\
& 223990-0101-X \text { SANTA CRUZ }
\end{aligned}
$$

$$
\begin{gathered}
\text { FG02-94ER25197 03/31/95 } \\
212650-0001-8 \text { FHILAL }
\end{gathered}
$$$$
\text { FG02-94ER25211 05/31/95 }
$$$$
\text { 212650-0001-8 PHILADELPHIA }
$$$$
\text { FG02-94ER25210 05/31/95 }
$$$$
\text { 212650-0001-8 PHILADELPHIA }
$$$$
\text { FG02-93ER25185 08/15/94 }
$$$$
\text { 212650-0001-8 FHILADELPHIA }
$$

SOCIOTECHNICAL RESEARCH APPL

$$
\begin{gathered}
\text { ACO1-92PE10017 11/14/94 } \\
217560-0001-8 \text { ARLINGTON }
\end{gathered}
$$

GMO4-9JAL84069 11;24/97
$226920-0101-X \quad$ SOCORRO

SOC.ORRO CITY OF

MUNICIPAL SCHOOLS

SODA SPRINGS SCHOOL DIST \#150

SOFTWARE AG INC

SOLANCO SCHOOL DISTRICT

$$
\begin{gathered}
\text { ADO1-94EI24192 } 09 / 30 / 95 \\
076110-0001-2 \text { RESTON } \\
\text { ADO1-93E123455 09/30/94 } \\
076110-0001-2 \text { RESTON }
\end{gathered}
$$

FG43-94R364111 05/31/95
MECHANICAL INTERACTIONG $\$ 15,000$

EXHIBITS ON BIOTECHNOL OGY FOR

A FIELD STUDY OF THE EFFECTS OF ELEVATED CO ON THE ECOSYSTEM DC 01 \$2,121.754 $\$ 1,881,971$

THE APPLICATION OF THO DIMENSIONAL IMAGING TO VERY HIGH ENER

$$
\text { MA } 08 \quad \$ 1,070,647 \quad \$ 996,000
$$

NEH GRANT - U.S. HISPANICS ANDAMERICA 2000: A PLAN FOR MAT

CA 16 \$76,028 $\$ 63,028$

SIAM CONFERENCE ON APPLIED LINEAR ALGEBRA

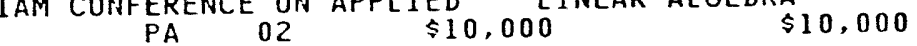

SIAM SYMPOSIUM ON CONTROL PROBLEMS IN INDUSTRY TO BE

PA 02 CONTROL $\$ 9,700$ \$9.700

THE 3RD SIAM FOUM TO BE HELD JULY $23,1994 \$ \$ 8,342,34$

$\begin{array}{ccc}\text { SIAM CONFERENCE ON } & \text { GEOMETRIC DESIGH } \\ \text { PA } 02 & \$ 10,000 & \$ 10,000\end{array}$

OFFICE OF POLICY PLANNING \& AHALYSIS (PE) SUPPORT SERVICES

SUPPORT FOR SCIENCE, MATHEMATICS, AND ENGINEERING TO IMPROVE NMI 03 \$O $\$ 0$

STATE THE BASIC UNDERSTANDING OF THE PARTIES REGARDING, \& PR GMO7-94ID11151 $03 / 17 / 99$
$076070-0001-1 \quad$ SODA SPRINGS 076170-0001-4 QUARRYVILLE

\section{RENEWAL OF MANINTENACE OF COMPUTER SOFTWARE}

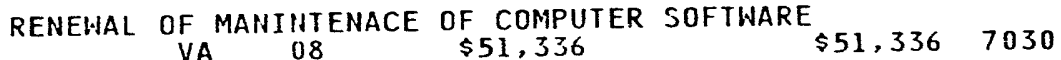

SOFTWARE UPDATES

$\$ 51,336$

$\$ 51,336 \quad 7030$

TITLE III OF THE NATIONAL ENERGY CONSERVATION POLICY ACT 


\begin{tabular}{|c|c|c|}
\hline AWARDEE NAME & COMPL DATE & DESCRIPTION OF HORK \\
\hline DIVISION & VENDOR ID & $\begin{array}{l}\text { CONTRACT } \\
\text { VALUE }\end{array}$ \\
\hline SOLAR DESIGN ASSOC INC & $\begin{array}{c}\text { FC36-93CH10572 } 09 / 30 / 97 \\
229200-0001-2 \text { HARVARD }\end{array}$ & $\begin{array}{l}\text { MODULE DEVEL OPMENT } \\
\$ 375,977\end{array}$ \\
\hline SOLAR ELECTRIC LIGHT FUND & $\begin{array}{c}\text { FG41-92R110431 } 03 / 31 / 95 \\
225510-0001-7 \text { WASHINGTON }\end{array}$ & $\begin{array}{l}\text { ASSISTANCE ACTION } \\
\$ 47,500\end{array}$ \\
\hline \multirow[t]{2}{*}{ SOLAR ENERGY INDUSTRIES ASSN } & $\begin{array}{c}\text { FG41-93R110588 } 09 / 30 / 94 \\
076270-0001-7 \text { WASHINGTON }\end{array}$ & $\begin{array}{r}\text { NEH AHARD - SOLAR BIOMASS } \\
\text { DC } \\
1\end{array}$ \\
\hline & $\begin{array}{c}\text { FG41-93R110544 } 06 / 30 / 95 \\
076270-0001-7 \text { WASHINGTON }\end{array}$ & $\begin{array}{c}\text { SOLTECH CONFERENCE AHD EARTH DAY. } \\
\text { DC } 01\end{array}$ \\
\hline \multirow[t]{5}{*}{$\begin{array}{l}\text { SOLAR TURBINES INC } \\
\text { PROD DEVELOPMENT CENTER }\end{array}$} & $\begin{array}{r}A C .21-88 M C .2501009 / 30 / 94 \\
076430-0201-0 \text { SAN DIEGO }\end{array}$ & 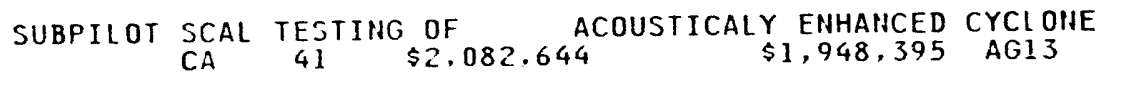 \\
\hline & $\begin{array}{c}A C 02-92 C E 4096004 / 30 / 95 \\
076430-0001-8 \text { SAN DIEGO }\end{array}$ & $\begin{array}{rccc}\text { DEVELOPMENT OF CERAMIC } & \text { STATIONARY GAS TURBINE } \\
\text { CA } & 44 \$ 21,151,616 & \$ 9.192,700 \text { AG81 }\end{array}$ \\
\hline & $\begin{array}{r}A C 21-93 M C 30246 \quad 05 / 03,95 \\
076430-0001-8 \text { SAN DIEGO }\end{array}$ & $\begin{array}{l}\text { ADVANCED TURBINE SYSTEMS FRO- GRAM - - CONCEPTUAL DESIGN AND } \\
\begin{array}{llll}\text { CA } 49 & \$ 2.380 .470 & \$ 1,785.352 & \text { AG23 }\end{array}\end{array}$ \\
\hline & $\begin{array}{rl}A C 02-87 C E 40812 & 09 / 30 / 96 \\
076430-0001-8 & \text { SAN DIEGO }\end{array}$ & $\begin{array}{l}\text { HIGH PERFORMANCE STEAM PROOF OF CONCEPT PHASES COGENERATION } \\
\text { CA } 44 \$ 13,537,928\end{array}$ \\
\hline & $\begin{array}{r}\text { FCO2-88ID12799 05/29/96 } \\
076430-0001-8 \text { SAN DIEGO }\end{array}$ & $\begin{array}{rrrr}\text { DEVEL OFMENT OF HIGH PRESSURE HEAT } & \text { EXCHANGE SYSTEMS } \\
\text { CA } & 41 & \$ 11,645,084 & \$ 2,669,193\end{array}$ \\
\hline SOLID WASTE SERVICES & $\begin{array}{c}\text { A } 665-94 \text { HCO8857 } 09 / 30 / 94 \\
236360-0001-2 \text { HINTOH }\end{array}$ & GARBAGE PICKUP \\
\hline $\begin{array}{l}\text { SOLON CITY OF } \\
\text { SCHOOL DISTRICT }\end{array}$ & $\begin{aligned} \text { FG45-94R562186 } 12 / 31 / 95 \\
238370-0101-6 \quad \text { JOLOH }\end{aligned}$ & $\begin{array}{c}\text { ICP-NEW AHARD. } \\
\text { OH }\end{array}$ \\
\hline SOLVENT INC & $\begin{array}{c}\text { SC92-94FE63277 } 10 / 01 / 94 \\
237030-0001-5 \quad \text { BAKEPSFIELD }\end{array}$ & 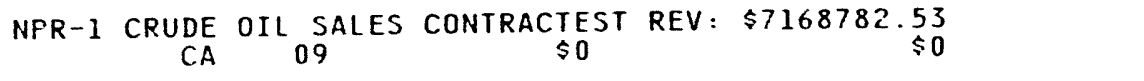 \\
\hline SOMERSET HOSP CTR FOR HEALTH & $\begin{array}{l}\text { FG43-94R364112 } 05 / 31 / 95 \\
237950-0001-7 \text { SOHERSET }\end{array}$ & 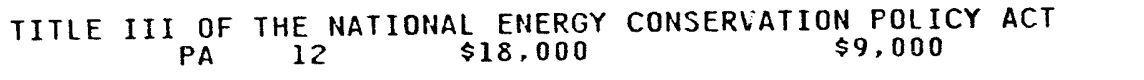 \\
\hline
\end{tabular}




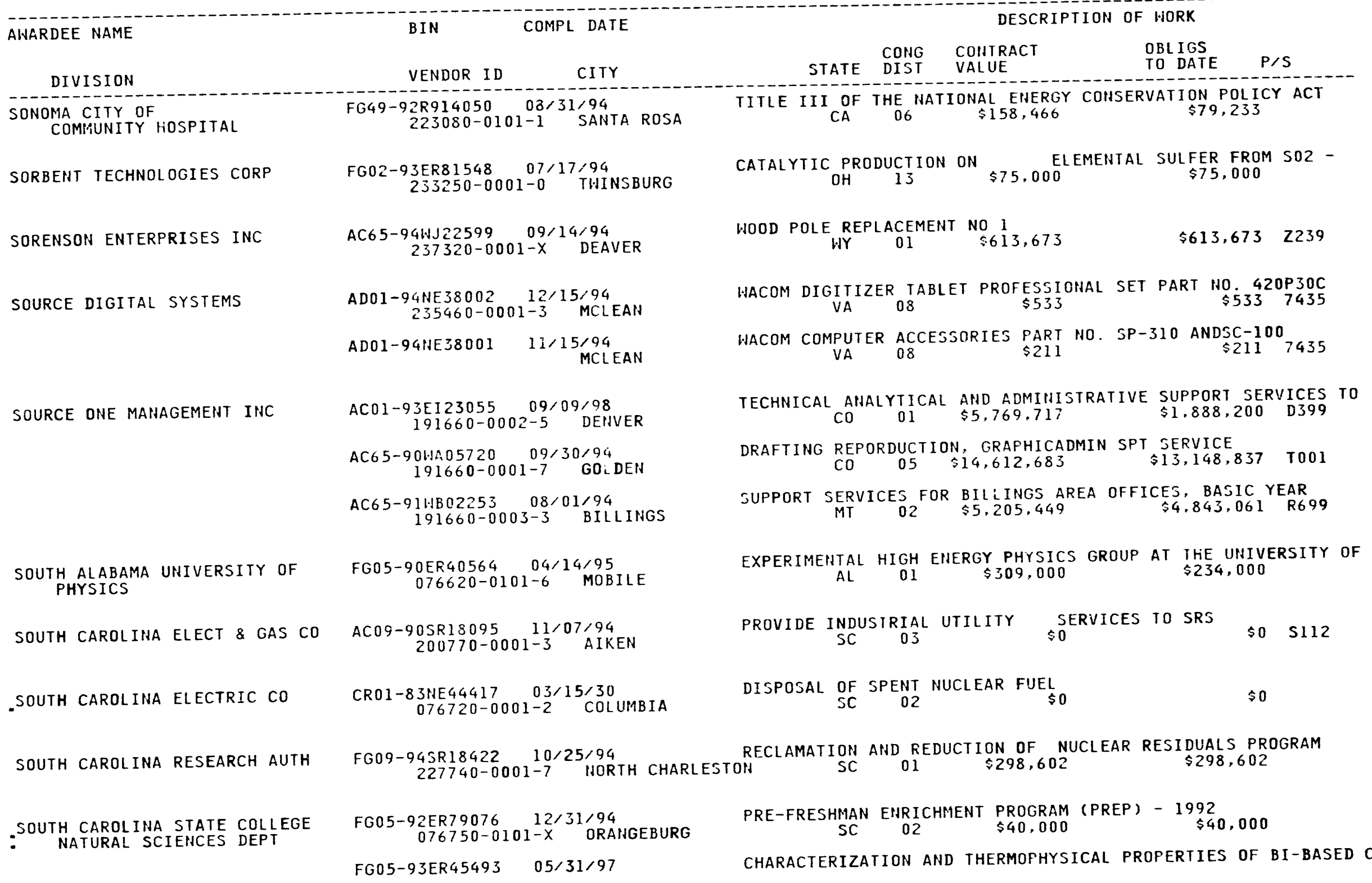


à
$\vdots$
$i$
0

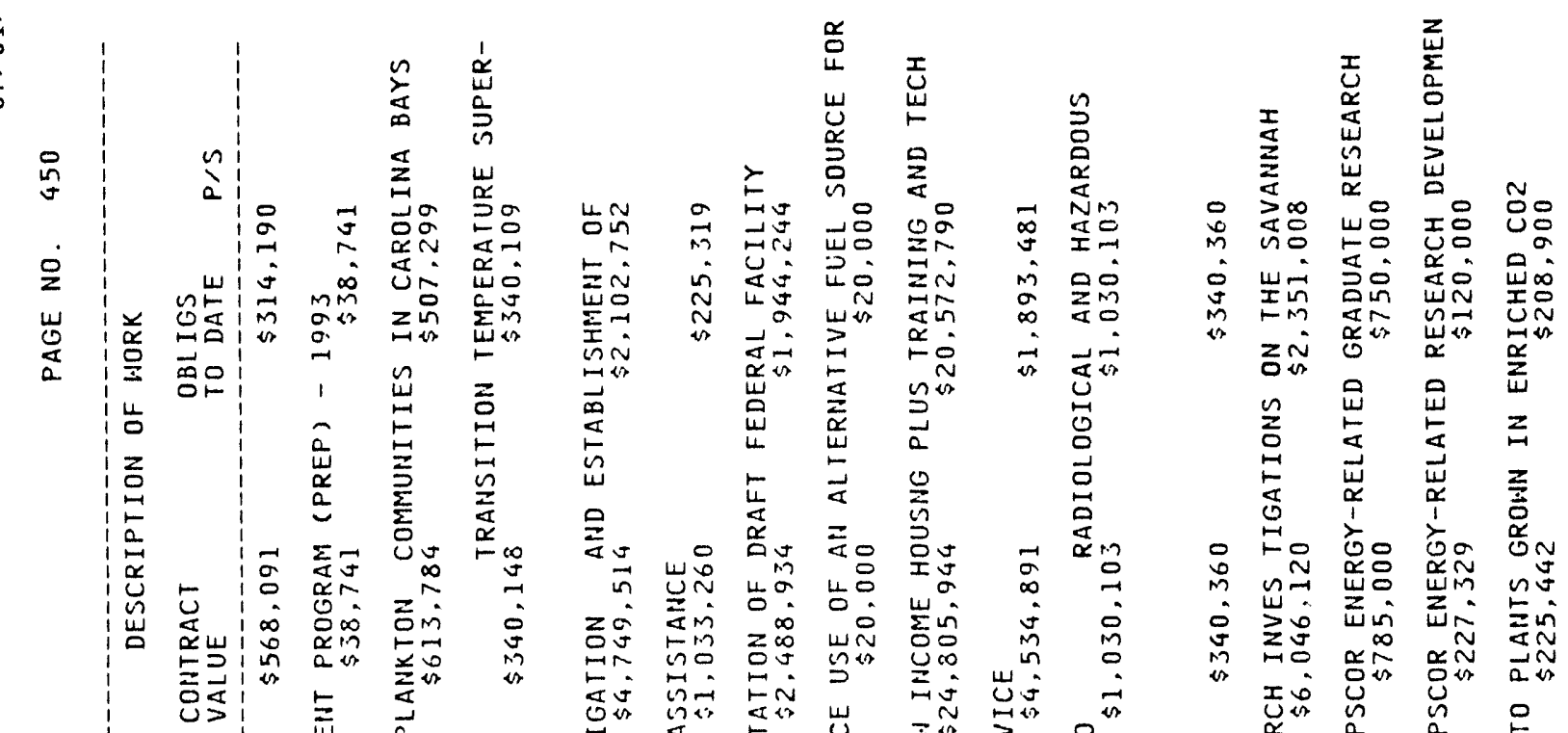

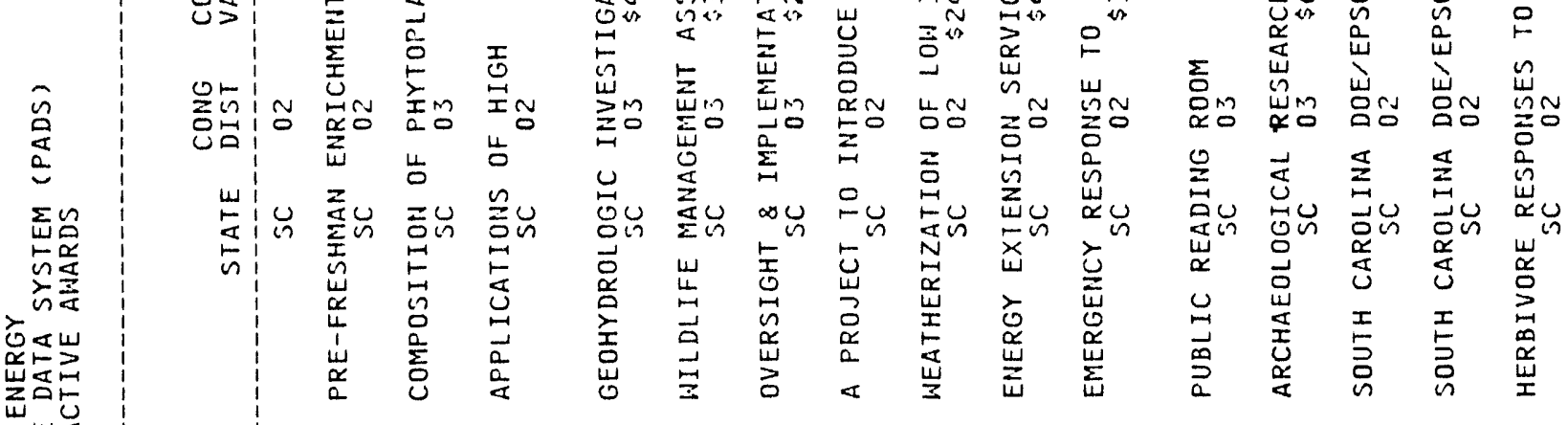
岂

送 0

点芯

$\sum_{\substack{\infty \\ \alpha}}$

$\sum_{a}^{\infty}$

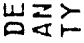

密

tr:

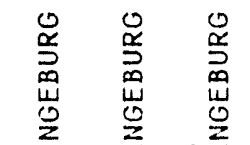

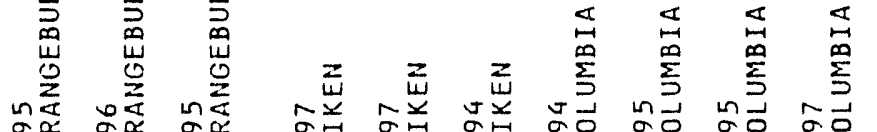

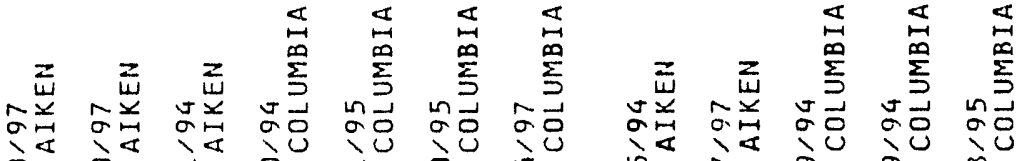

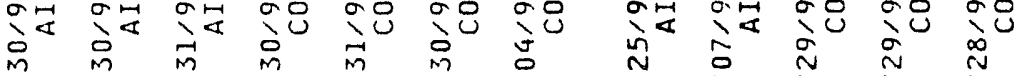
min

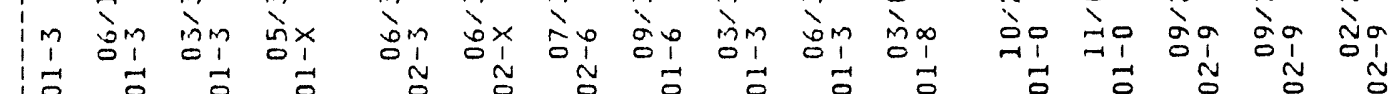

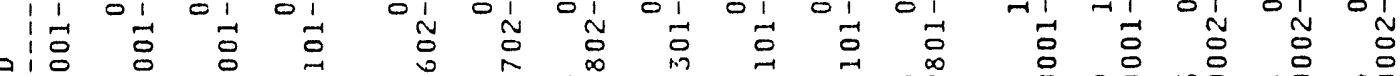

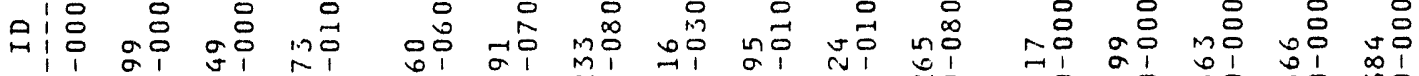

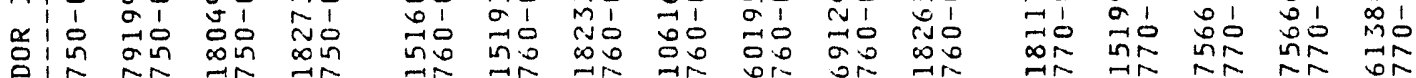

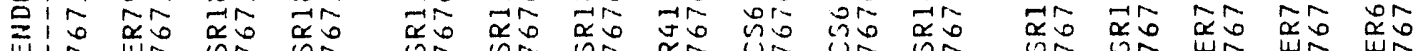

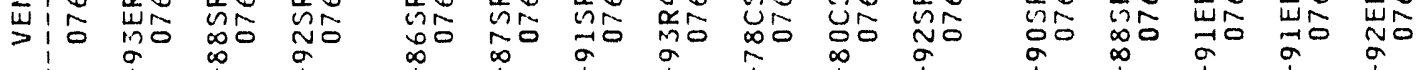
1
1
0
0
0
4

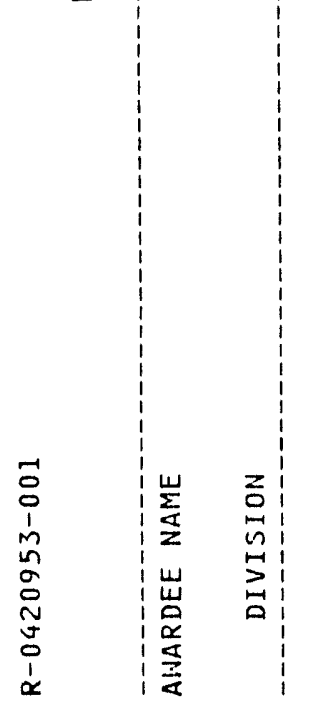
in 


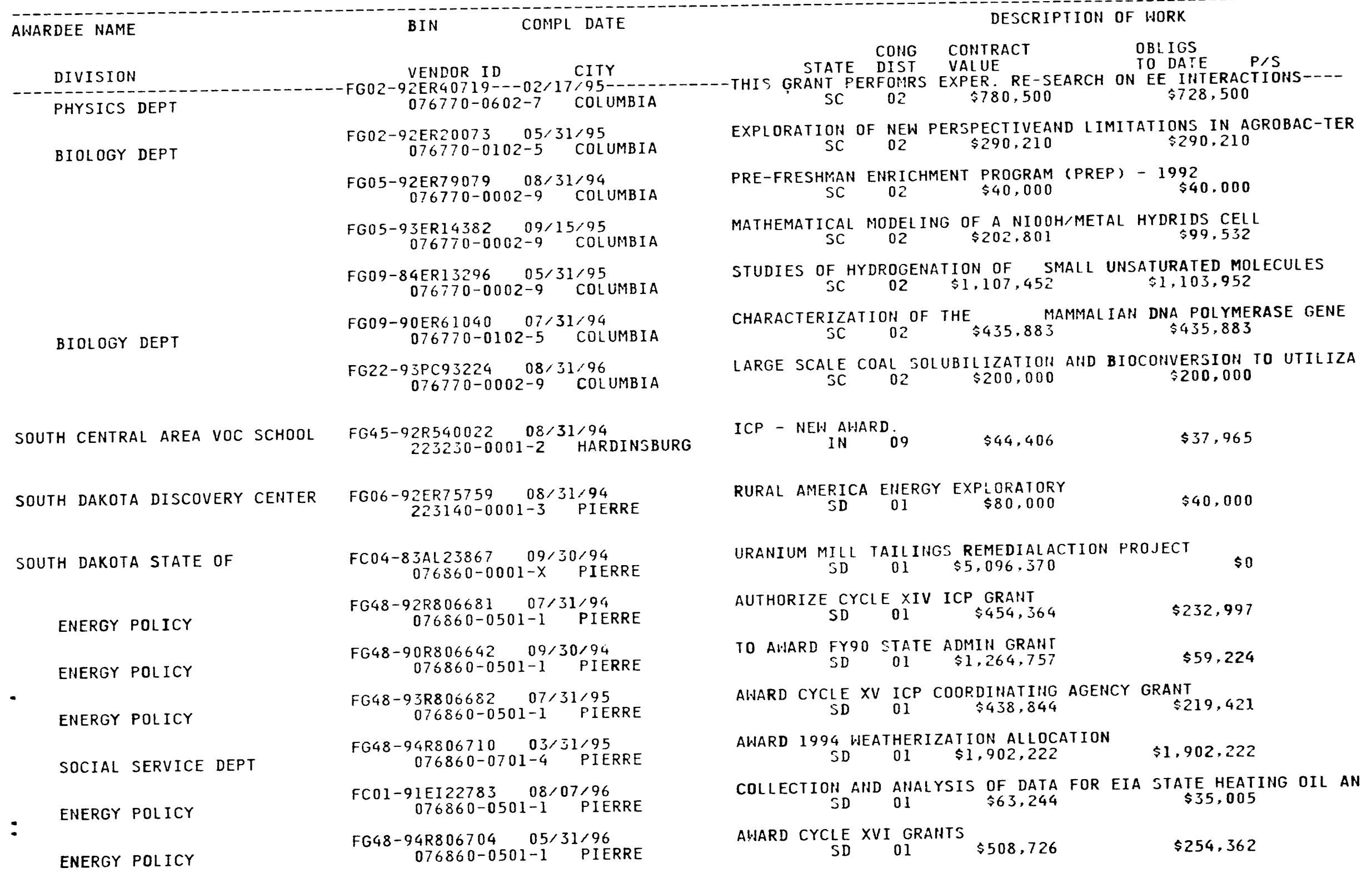




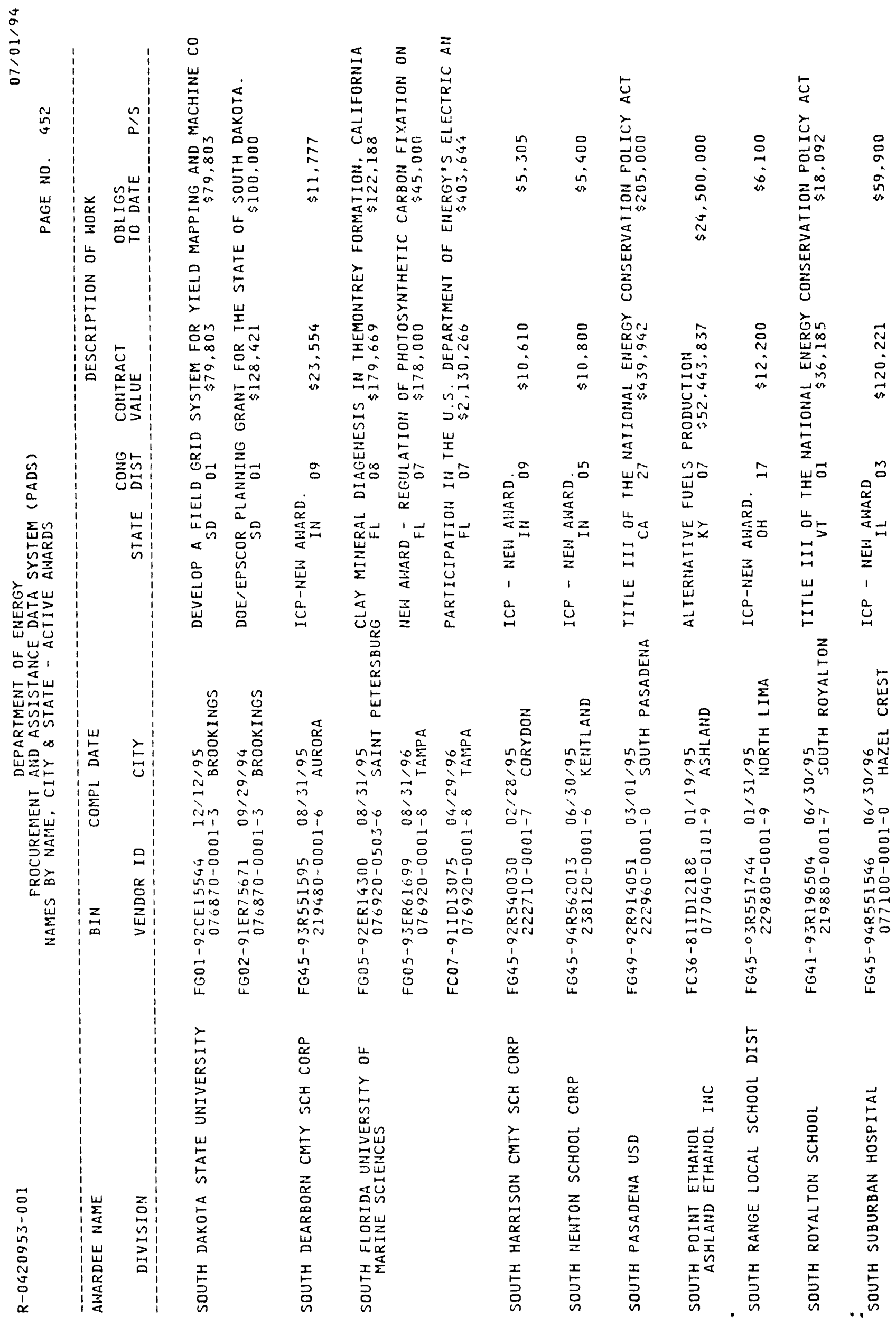




\begin{tabular}{|c|c|c|c|c|c|c|c|}
\hline AWARDEE NAME & BIN & DATE & & & DESCR & F HORK & \\
\hline DIVISI & VENDOR ID & CITY & STATE & $\begin{array}{l}\text { COHG } \\
\text { DIST }\end{array}$ & $\begin{array}{l}\text { CONTRACT } \\
\text { VALUE }\end{array}$ & $\begin{array}{l}\text { OBLIGS } \\
\text { TO DATE }\end{array}$ & $P / S$ \\
\hline
\end{tabular}

SOUTH UMPQUA SCHOOL DIST \#19

FG51-91R020033 12/31/94
$200060-0001-0$ MYRTLE CREEK

SOUTHAMPTON HOSPITAL ASSOC

SOUTHEAST COMMUNITY COLLEGE MILFORD CAMPUS

SOUTHEAST LOCAL SCHOOLS

SOUTHEASTERN UNIV RESCH ASSN

SOUTHERN CALIFORNIA EDISON CO

SOUTHERN CALIFORNIA RES CORP

SOUTHERN CALIFORNIA UNIV OF

PHYSICS DEPT

SCHOOL OF ENGINEERING

: SCHOOL OF ENGINEERING

$$
\begin{gathered}
\text { FG42-91R233771 } 07 / 31 / 94 \\
077490-0001-1 \quad \text { SOUTHAMPTON }
\end{gathered}
$$

FG47-93R703529 08/31/94

$231760-0101-0$ MILFORD

FG45-93R551746 01/31/95
$229860-0001-0$ RAVENHA AC05-84ER $4015000 / 30 / 94$
$098620-0001-5 \quad$ NEWPORT NEWS

CRO1-83NE44418 $03 / 15 / 30$
$077230-0001-4 \quad$ ROSEMEAD

FC36-93CH10550 01/31/99
$077230-0001-4$ ROSEMEAD

FC07-911D13077 08/23/96
$077230-0001-4$ ROSEMEAD

FG49-93CE15600 03/31/95
$204090-0001-9$ SAH RAFAEL

AMO3-76SF00113 12/31/99 077240-0001-8 LOS ANGELES

FG03-84ER40168 02/28/95 $077240-1301-2$ LOS ANGELES

FG03-87ER13807 09/14/95 $077240-1101-x$ LOS ANGELES FG03-88ER13959 03/31/95
$077240-1101-X$ LOS ANGELES

FG03-89ER45407 12/14/95
CYCLE 13 ECM AWARD. INCLUDES $\$ 3,776$ HARDSHIP FUNDS. TRI-CIT

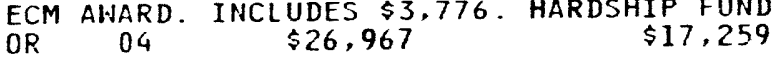

TITLE III OF THE NATIONAL ENERGY CONSERVATION POLICY ACT

$$
\begin{array}{rrr}
\text { OF THE NATIONAL ENERGY CONSERVATION POLICY } \\
\text { NY } 01
\end{array}
$$

GRANT PROGRAMS FOR SCHOOLS ANDHOSPITALS AND FOR BUILDINGS NE 01 \$28,579 $\$ 11,001$

ICP-NEW AWARD

$$
\mathrm{OH} \text { 14 } \$ 15,250 \quad \$ 7,625
$$

R\&D AND ADVANCED ENGINEERING STUDIES - CEBAF

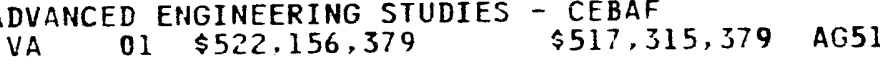

DISPOSAL OF SPENT NUCLEAR FUELAND FOR HIGH LEVEL RADIOACTIVE UPGRADE THE SOLAR ONE FACILITYFROM A WATER/STEAM CENTRAL CA $13 \quad \$ 48,500,000 \quad \$ 7,610,000$ PARTICIPATION IN THE U.S. DEPARTMENT OF ENERGY'S ELECTRIC AN CA 17 \$3,000,000 $\$ 681,123$

DEVELOP NEW METHOD FOR CUTTINGSTEAM LOSSED DURIHG CYCLING S

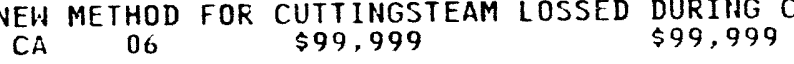

THIS IS A BASIC CONTRACTWORK IS DEFINED BY SEPA RATE PROJECT $C A \quad 25$ \$O $\$ 0$ AG91

PREOHS, SUPERSYMMETRY NON-PERTERBATIHE FIELD THEORY $28 \$ 1,604,000$ \$ $\$ 1,579,000$

"CSDP: SEISMOLOGY OF CONTINE NTAL THERMAL REGIMES" CA 25 \$1,316,992 $\$ 1,105,135$

REACTIONS OF CARBOH ATOMS USING PULSED MOLECULAR BEAMS CA $28 \quad \$ 571,666$ P

SYNTHESIS OF NOVEL ASSOCIAIINGWATER-SOLUBLE COPOLYMERS 
AWARDEE NAME

BIN

COMPL DATE

VENDOR ID

CITY

$077240-1101-x$ LOS ANGELES

SCHOOL OF ENGINEERING

CHEMICAL ENGINEERING DEPT

SOUTHERN CO SERVICES INC
FG03-91ER1422j 08/31/94 077240-0001-8 LOS ANGELES
FG03-91ER61236 08/31/95 077240-0001-8 LOS ANGELES
FG03-92ER45472 05/31/96 077240-0001-8 LOS AHGELES
FG03-92ER14266 10/31/94 077240-0001-8 LOS ANGELES
FG03-92ER40745 08/14/95 077240-0001-8 LOS ANGELES
FG03-93ERI4346 04/30/96 077240-0001-8 LOS ANGELES
FG03-93ER14360 07/14/96 077240-0001-8 LOS ANGELES
FG03-93ER61663 07/31/94 077240-0001-8 LOS ANGELES
FG05-93ER79223 08/31/95 077240-0001-8 LOS ANGELES
FG22-92FC92527 08/31/95 077240-0201-0 LOS ANGELES
FC22-90PC87650 01/01/95 $077260-0001-5$ BIRMINGHAM
FC22-90PC89651 12/31/94 $077260-0001-5$ BIRMINGHAM
FC22-90PC89652 10/31/95 077260-0001-5 BIRMINGHAM
FC21-90iAC25140 03/13/96 $077260-0001-5$ BIPMINGHAM
AC22-90PC90033 01/27/95 077260-0002-3 WILSONVILLE

DESCRIPTION OF HORK

$\begin{array}{ll}\text { CONG CONTRACT } & \text { COATE DIST VALUE } \\ \text { STATE }\end{array}$

$\$ 576,881$

TO DATE P/S $\$ 488,518$

NEW GRANT - PARTICLE PRESSURESIN FLUIDIZED BEDS - P.I. CHAR CA $28 \quad \$ 337,586 \quad \$ 337,586$

DIRECT DETECIION OF SOMATIC MUTATIONS IN HUMAN MITOCHONDRIAL CA $28 \quad \$ 260.535 \quad \$ 260,535$

DEVELOP AN UHDERSTANDING OF THE FLOW AND FRACTURE BEHAVIOR O CA 28 \$424,646 $\$ 300,880$

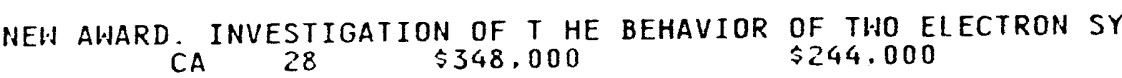

EXPERIMENTAL, THEORETICAL AND COMPUTATIONAL STUDIES OF PLASM CA 28 \$259,999 $\$ 169,999$

POROUS MEDIA FL OHJ $\$ 3259,998 \quad \$ 251,098$

AMPLITUTDE MODULATION OF ATOMIC WAVE FUNCTIONS

$$
\text { CA } 28 \quad \$ 276,000 \quad \$ 90,000
$$

PLANNING FOR HUGO INTERHATIOHAL YEARBOOK

$$
\begin{array}{rrrr}
\text { FOR HUGO } & \text { INTERHATIOHAL } \\
\text { CA } & 32 & \$ 14,976 & \$ 14,976
\end{array}
$$

1993 UNIVERSITY INSTRUMEHTATION PROGRAM - LARGE AREA MICROCH CA 23 \$136,184 \$136,184

HINDERED DIFFUSIOH OF COAL LIQUIDS

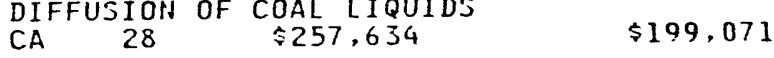

DEMONSTRAATION OF INNOVATIVE APPLICATIONS OF TECHNOLOGY FOR

$$
A L=06 \quad \$ 43,074,996 \quad \$ 21,085,211
$$

DEMONSTRATION OF ADVANCED WALL-FIRED COMBUSTION TECHHIQUES

$$
\text { AL } 06 \text { \$13.234,909 } \$ 5.977 .526
$$

DEMONSTRATIOH OF SELECTIVE CATALYTIC REDUCTION CSCRI TECHNOL

$$
\text { AL } 06 \$ 22,901,041 \quad \$ 9,406,672
$$

HOT GAS CLEANUP TEST FACILITY FOR GASIFICATION AND

$$
\text { AL } 06 \$ 113,159,613 \quad \$ 64,939,049
$$

OPERATION OF THE WILSONVILLE ADVANCED COAL LIQUEFACTION RESE $A L=07 \$ 18,678,749 \quad \$ 13,795,240$ AG1 


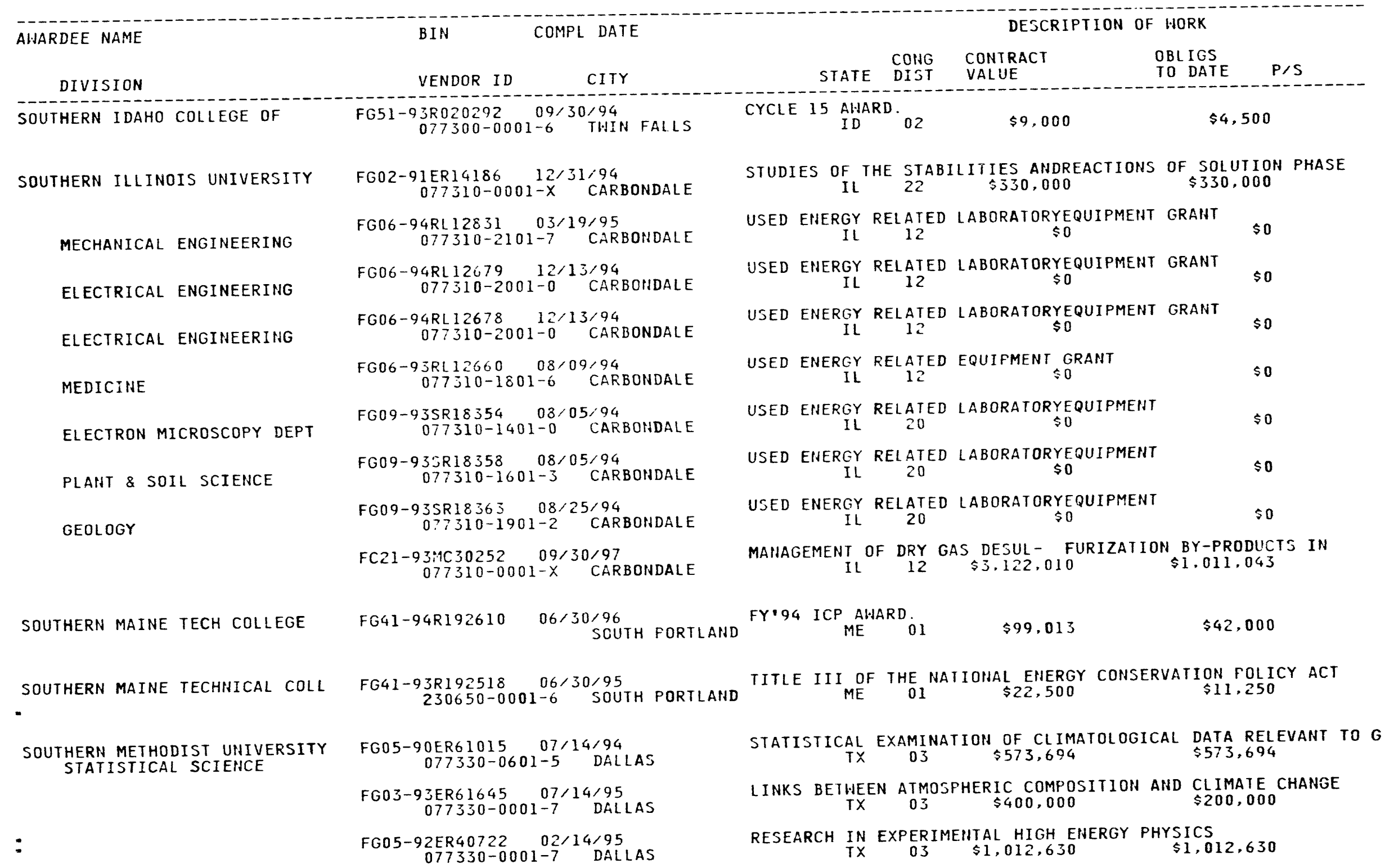




\begin{tabular}{|c|c|c|c|c|c|c|c|}
\hline AIIARDEE NAME & BIN & L DATE & & & DESC & F HORK & \\
\hline DIVISION & VENDOR ID & CITY & STATE & $\begin{array}{l}\text { COHG } \\
\text { DIST }\end{array}$ & $\begin{array}{l}\text { COHTRACT } \\
\text { VALUE }\end{array}$ & $\begin{array}{l}\text { OBLIGS } \\
\text { TO DAIE }\end{array}$ & $\mathrm{P} / \mathrm{s}$ \\
\hline
\end{tabular}

SOUTHERN MISSISSIPPI UHIV OF

POLYMER SCIENCE DEPT

SOUTHERN NEVADA CMTY COLL OF

SOUTHERN NEVADA COURIER SVC

SOUTHERN OHIO MEDICAL CENTER

-SOUTHERN RESEARCH INSTITUTE

$\Xi$

SOUTHERN STATES EHERGY BOARD
FG02-91ER75660 09/29/94 077340-0001-0 HATTIESBURC FG44-92R4351111 10/31/94 $077340-0001-0$ HATTIESBURG FG02-92ER61443 05/31/95 $077340-0001-0$ HATTIESBURG AC22-92BC14882 09/21/95 $077340-0201-3$ HATTIESBURG FG44-93R435434 09/30/95 $077340-0001-0$ HATTIESBURG FG08-93HV11385 $09 / 22,95$
$228820-0002-2$ LAS VEGAS

FG08-91NV11007 12/31;95 $228020-0001-4$ IORTH LAS VEGAS

GM08-9JNV11361 02/21/98 20130 HAS VEGAS

AC08-93HV11162 02/14,95 $077350-0001-4$ LAJ VEGAS AC08-931V11358 02:28/95 $077 \div 50-0001-4$ LAS VEGAS

FG45-93R551745 01/31/95 $3 R 55174501 / 31 / 95$
$229810-0001-2 \quad$ PORTSMOUTH

$A C 21-89 M C 26239 \quad 08 / 22 / 94$ $077 \div 90-0001-9$ BIRMINGHAM AC22-91PC90365 09/04/94 $077390-0001-9$ BIRMINGHAM AC22-93FC93254 01/10/95 $077390-0001-9$ BIRMINGHAM

FCO1-92RH00247 01/31/97
MISSISSIPPI STATEHIDE DOE/EPSCOR ENERGY-RELATED GRAUDATE TRA MS $05 \$ 1.109 .472$ $\$ 750,0 J 0$

CYCLE 14 - INSTITUTIONAL COHSERVATION PROGPAM. APPROVED BUD MS 05 \$79,000 $\$ 33,430$

DEVELOPMEHI OF A MULTI- SEHSOR IN SITU FIBER OPTIC MS $05 \quad \$ 380.441 \quad \$ 380.441$

RESPONSIVE COPOLYMERS FOR ENHAHCED PETROLEUM RECOVERY

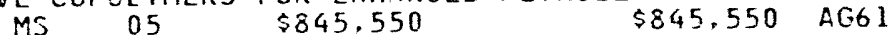

SEE ATTACHED PAGE NO. 2 OF THIS NOTICE OF FINANCIAL ASSISTAN MS $05 \quad \$ 53.375 \quad \$ 26.687$

ENVIRONMENTAL RESTORATIO:A TECHNOL OGY PROGRAM.

$$
\text { IV } 01 \text { \$129.648 } \$ 64.824
$$
GEOGRAPHICAL IIHFORHATIOH SYSTEPS TRAIHING PROGRAM. MOU TO IMPLEMEHT A COOPERATIVEEFFORT AT REACHING WATIOHAL E SHUTTLE SERVICE HITHIH THE GREATER LAS VEGAS AREA UHDER SBA PICK-UP \& 81 TIVEFY SERVICES HITHIN THE GREATER LAS VEGAS ARE

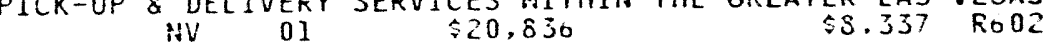

ICP-HEH AH:AR.D. $06 \quad \$ 25,000 \quad \$ 12,500$

RESEARCH AHD DEVELOPMEIIT FOR PARTICLE COHTROL TECHHOLOGIES $A L \quad 06 \quad \$ 2,499,877 \quad$ \$2,499,877 AG92

FUHDAMENTAL MECHANISMS IN FLUEGAS CONDITIONINO AL $00 \quad \$ 730.090 \quad \$ 730,090$ AG11 CHARACTERIZING TOXIC EMISSIONSFRAM A COAL-FIRED POHER PLANT $\begin{array}{llll}A L & 07 & \$ 3,386,050 & \$ 2,525,210\end{array}$

NUCLEAR MATERIAL TRANSPORTATION PROJECT 


\begin{tabular}{|c|c|c|}
\hline AWARDEE NAME & COMPL DATE & DESCRIPTION OF HORK \\
\hline \multirow[t]{4}{*}{ DIVISION } & VENDOR ID & $\begin{array}{l}\text { CONTRACT } \\
\text { VALUE }\end{array}$ \\
\hline & $077410-0002-0$ & $\$ 480,325$ \\
\hline & $\begin{array}{c}\text { FGO1-92FE62466 } 03 / 16 / 97 \\
077410-0002-0 \text { NORCROSS }\end{array}$ & $\begin{array}{ccc}\text { TO SUPPORT A REGIONAL EFFORT TO PROMOTE THE INCREASED USE OF } \\
\text { GA } 10 & \$ 237,826 & \$ 237,826\end{array}$ \\
\hline & $\begin{array}{c}\text { FCO4-93AL82966 } 05 / 23 / 98 \\
077410-0002-0 \text { NORCROSS }\end{array}$ & 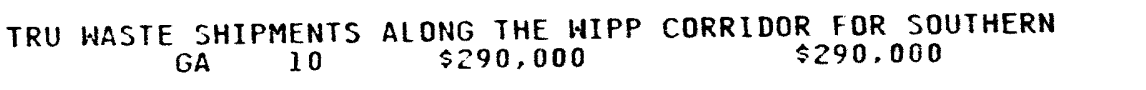 \\
\hline \multirow[t]{2}{*}{ SOUTHERN UNIVERSITY } & $\begin{array}{c}\text { FG03-905F18721 } 09 / 29 / 95 \\
077440-0001-3 \text { BATON ROUGE }\end{array}$ & $\begin{array}{l}\text { MINORITY UNDERGRADUATE TRAINIHG N ENERGY-RELATED CAREERS } \\
\qquad \begin{array}{lll}\text { LA } 08 & \$ 388,082 & \$ 363,532\end{array}\end{array}$ \\
\hline & $\begin{array}{l}\text { FG05-94ER45509 } 01 / 14 / 97 \\
077440-0001-3 \text { BATON ROUGE }\end{array}$ & 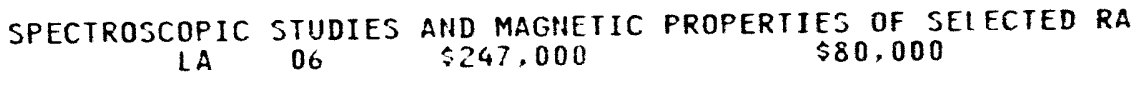 \\
\hline SOUTHERN WELLS COMMUNITY SCHS & $\begin{array}{c}\text { FG45-93R551596 } 03 / 31 / 95 \\
232790-0001-7 \text { POHETO }\end{array}$ & ICP-NEW AHARD. 04 \\
\hline SOUTHFACE ENERGY INJTITUTE INC & $\begin{array}{l}\text { FG } 44-93 R 410610 \text { 09/30/94 } \\
234850-0001-9 \text { ATLANTA }\end{array}$ & $\begin{array}{ccc}\text { ENERGY EFFICIENT AFFORDABLE HOUSING. ATLANTA'S PRG OLYMPIC } \\
\text { GA } 04 & \$ 25,000 & \$ 25,000\end{array}$ \\
\hline \multirow[t]{2}{*}{ SOUTHWEST PUBLIC SERVICE } & $\begin{array}{c}\text { ACO4-91AL } 56554 \quad 11 / 29 / 95 \\
077610-0001-8 \text { AMARILLO }\end{array}$ & $\begin{array}{l}\text { ELECTRIC SERVICE TO THE HIPP SITE } \\
\text { TX } 13 \$ 16.275,000\end{array}$ \\
\hline & $\begin{array}{c}\text { ACO4-93AL93259 04/05/05 } \\
077610-0001-8 \text { AMARILLO }\end{array}$ & $\underset{T X}{\text { PANTEX ELECTRIC SERVICE }}{ }_{13} \$ 36.433 .000$ \\
\hline SOUTHWEST RESEARCH INSTITUTE & $\begin{array}{c}\text { FG36-93CH10582 } 09 / 30 / 94 \\
077640-0001-9 \text { SAN AHTOHIO }\end{array}$ & $\begin{array}{c}\text { GAS FUELED RAILHAY RESEARCH } \\
\qquad X \quad 20 \text { PROGRAM }\end{array}$ \\
\hline INSTRUMENTATION \& SPACE RES & $\begin{array}{l}\text { FG05-91ER14180 10,31/94 } \\
077640-1401-X \text { SAH ANTOHIO }\end{array}$ & 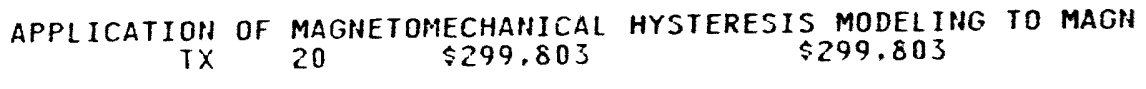 \\
\hline \multirow[t]{2}{*}{ MATERIALS SCIENCES DEPT } & $\begin{array}{c}\text { FG05-84ER45063 } 04 / 14 / 95 \\
077640-0901-6 \text { SAN ANTONIO }\end{array}$ & $\begin{array}{l}\text { CHARACTERIZATION OF PORE EVDLUTION IN CERAMICS DURING CREEP } \\
\qquad \begin{array}{lll}\text { TX } 20 & \$ 1,471,875 & \$ 1,471,875\end{array}\end{array}$ \\
\hline & $\begin{array}{c}\text { FGO4-93AL } 97070 \text { 09/29/96 AHTONIO } \\
077640-0001-9 \text { SAH AHTO }\end{array}$ & $\begin{array}{cccc}\text { PARTICULATE FORMATION IN } & \text { DIESEL ENGINE COMBUSTION } \\
\text { TX } & 20 & \$ 100,000 & \$ 100,000\end{array}$ \\
\hline SOUTHWEST RESOURCE DEVELOPMENT & $\begin{array}{c}\text { AC04-92AL42123 } 06 / 11 / 97 \\
219810-0001-1 \text { AMARILLO }\end{array}$ & $\begin{array}{l}\text { OFFICE AND ADMINISTRATION SUPPORT TO TU AMARILLO AREA } \\
\qquad \mathrm{TX} 13 \mathrm{~s}, 775,634\end{array}$ \\
\hline SOUTHWEST SCIENCES INC & FG03-91ER81211 & SBIR - PHASE I AHARD FOR - MEAJUREMEHT OF COMBUSTION RADICAL \\
\hline
\end{tabular}


BIN
AWARDEE NAME

DESCRIPTION OF HORK

DIVISION

VENDOR ID COMPL DATE

CITY

160420-0001-2 SANTA FE

FG03-92ER81404 05/24/95 160420-0001-2 SANTA FE

SOUTHWESTERN INDIAN POLY INST

FG03-94ER75947 11/14/96 236430-0001-4 AL BUQUERQUE

FG06-93RL12592 07/16/94 077730-0201-0 LAFAYETTE

THWESTERN LOUISIANA UNIV OF
CHEMICAL ENGINEERING DEPT

SOUTHWESTERN STATE HOSPITAL

FG44-93R435356 09/30/95 077760-0001-9 BAINBRIDGE

SPACE EXPLORATION ASSOC

SPACE POWER INC

SPARKIE MAINTENANCE INC

SPARKS PERSONNEL SERVICES INC

SPARKS REGIONAL MEDICAL CENTER

SPARKS STATE TECHNICAL COLLEGE

SPARKTECH

$$
\begin{gathered}
A B 01-94 A D 1110103 / 31 / 95 \\
206390-0002-9 \text { BETHESDA }
\end{gathered}
$$

AB01-94PP00187 11/30/94 206390-0002-9 BETHESDA

FG02-93ER81549 07/17/94 220990-0001-8 CEDARVILLE

AC03-92SF19441 01/15/96 077810-0002-1 SAIH JOSE

ACO4-91AL71676 09/28/94 215760-0001-X ALBUQUERQUE

$2 R 61353308 / 31 / 94$ $077860-0001-1$ FORT SMITH

FG02-92CH10500 09/30/94 218060-0001-6 EUFAULA

FG49-93CE15595 03/31/95 $234270-0001-X$ DUARTE
CONG CONTRACT

INM $03 \quad \$ 549,042$

$$
\text { HM OI } \$ 1,089,740^{\circ} \quad \$ 351,442
$$

USED ENERGY RELATED EQUIPMENT GRAIT

$$
G A \begin{array}{ll}
\$ 147.226 & \$ 73.613
\end{array}
$$

IN-SITU ELECTRICAL TESTING OF MULTICELL THERMIONIC FUEL

AWARD AND FUNDING OF LETTER CONTRACT

CENTER FOR ADVANCE ELECTRONIC TECHNOLOGY

TO DATE P/S $\$ 537,516$

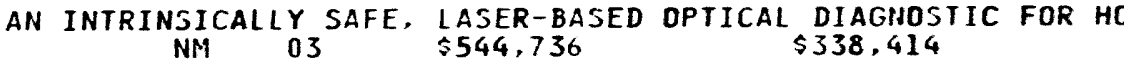

IMPROVING SCIENCE/MATHEMATICS EDUCATION IN WATIVE SCHOOLS

\$o

SEE ATTACHED PAGE NO. 2 OF THIS NOTICE OF FINAHCIAL ASSISTAN

$$
\text { CA } 10 \text { \$11.111.119 } \$ 3,923,999 \text { AC24 }
$$

JANITORIAL SERVICES FOR AL COMPLEX

$\$ 1.742 .686 \quad \$ 201$

TEMPORARY SECRETARIAL SERVICES
MD 08

BPA FOR SECRETARIAL SUPPORT SERVICES

\$0 R499

$\$ 0 \quad R 609$

THIS IS A NEW CYCLE 14 ECM GRANT AWARD. INCLUDED IS THE HFA

$$
\text { AL } 02 \text { ADVANCE ELECTRONIC TECHNOLOGY } \$ 1,600,000
$$

ACOUSTIC HUMIDITY SENSOR
28
$\$ 99,540$
$\$ 99,540$ 


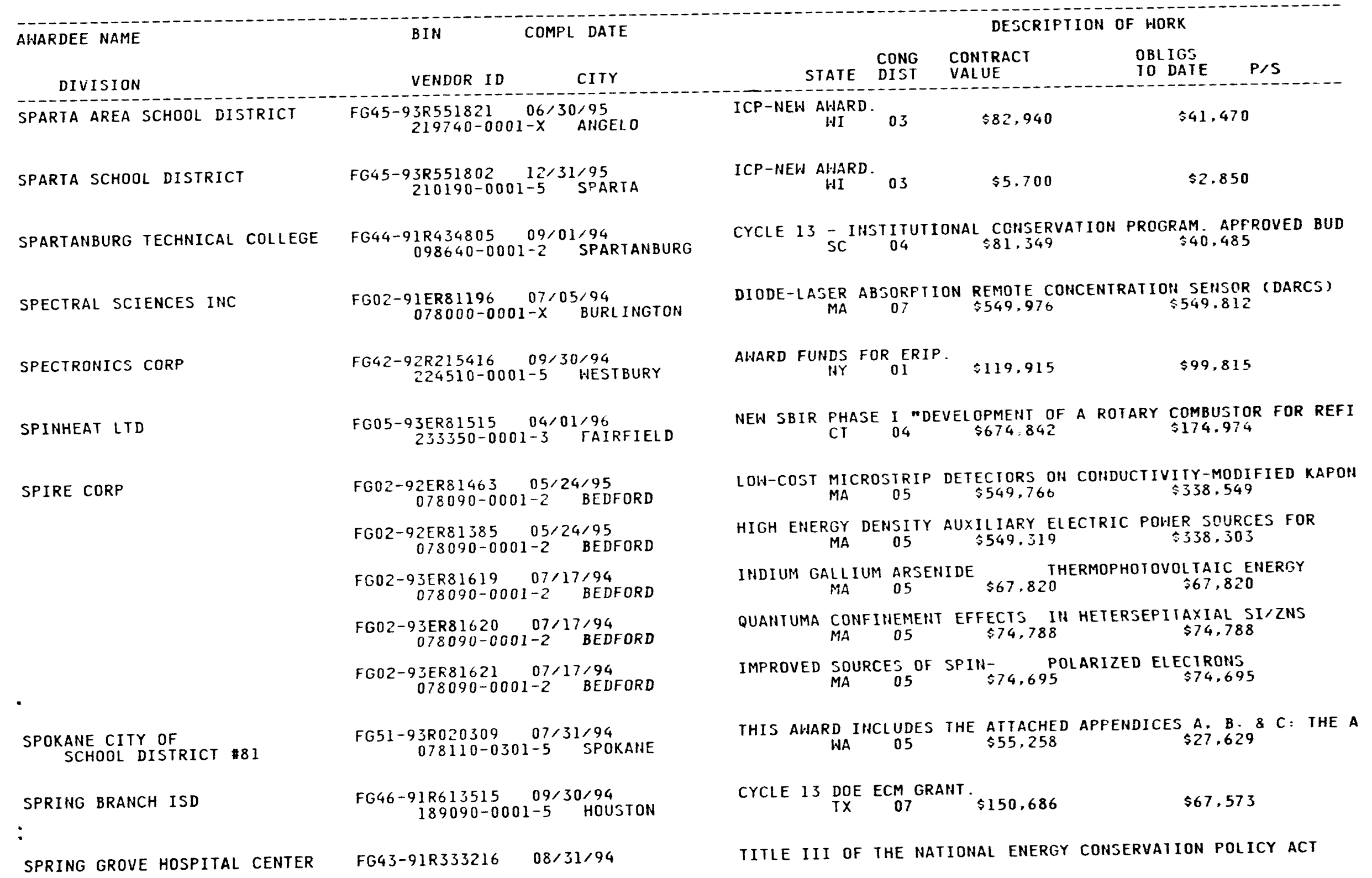


$j$
$\vdots$
$i$
$\vdots$

울

感

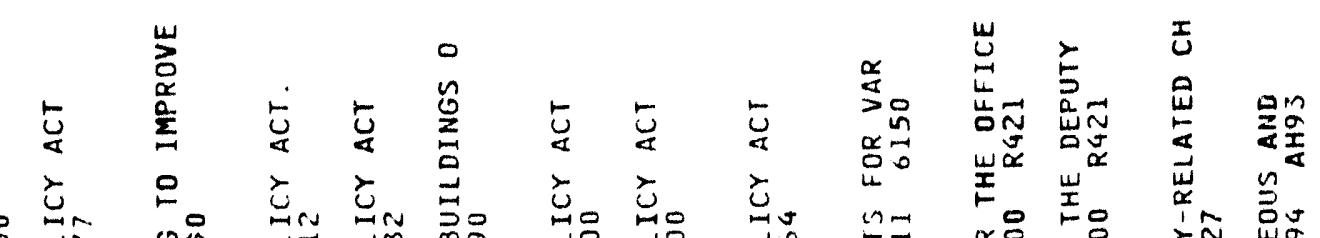

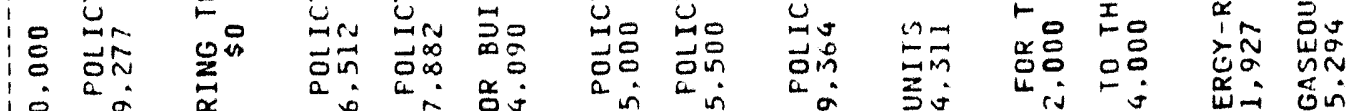

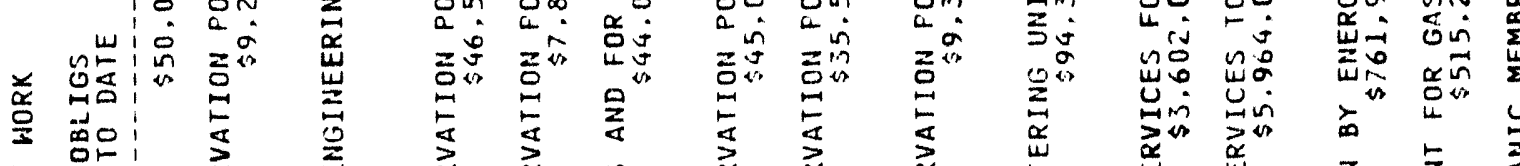

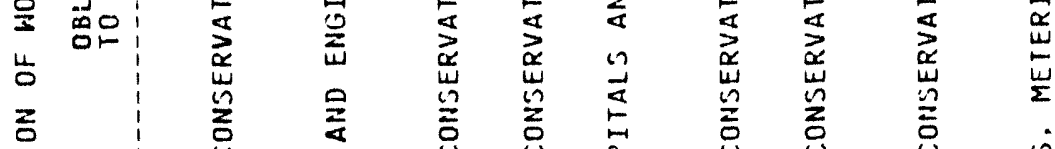

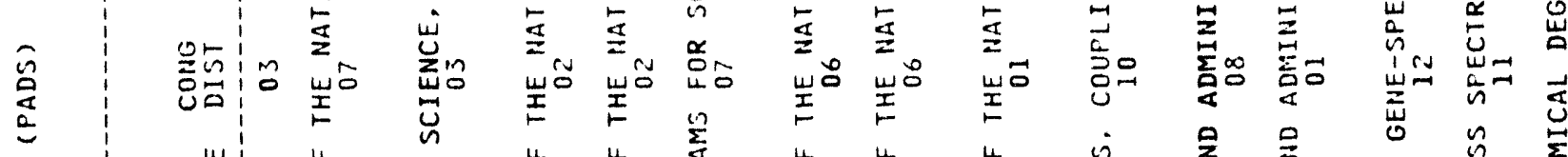

至要

过岁

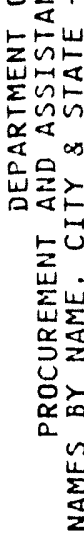

宏:是

$$
\frac{a}{n} i^{\Sigma}
$$

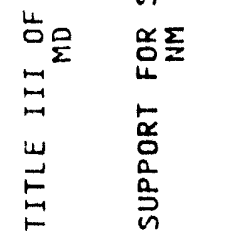

紋

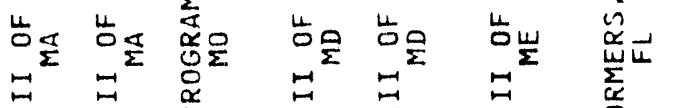
$\exists \Xi \stackrel{\alpha}{a}$

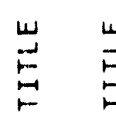

$\stackrel{\Perp}{\Perp}$

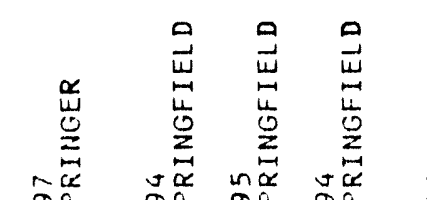

崩岂

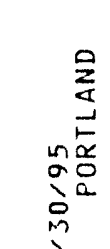

总

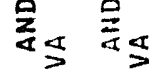

on

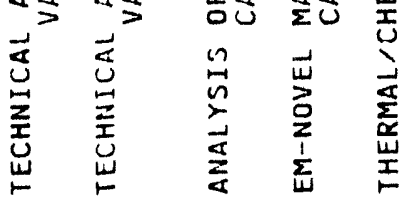

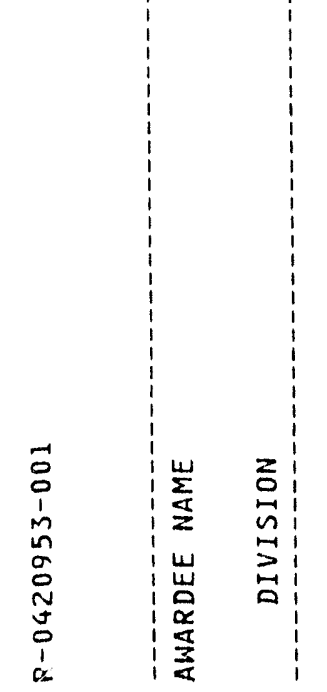

Nin

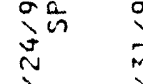

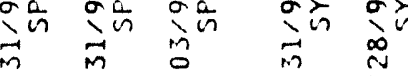

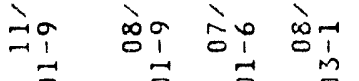

के సें क्ष

के के

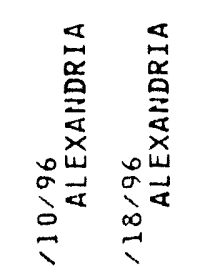

总

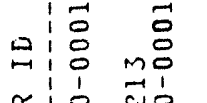

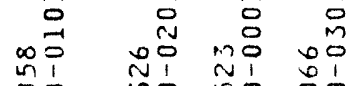

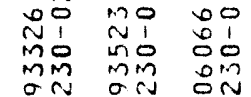

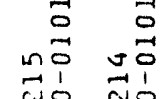

夏

r.:

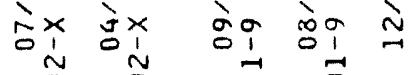

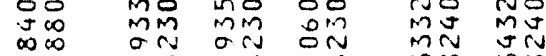

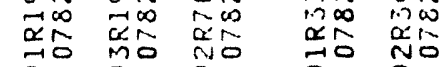

$\sin$

吉

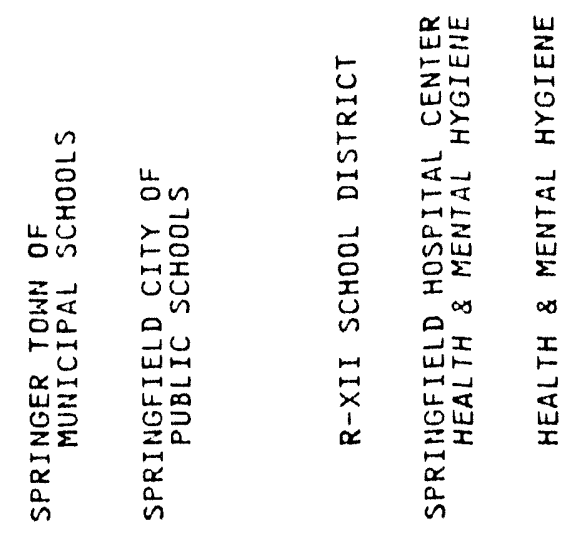

(5)

总

s.

NU

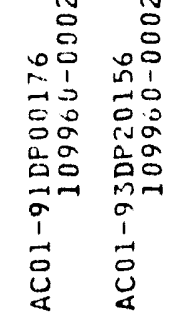

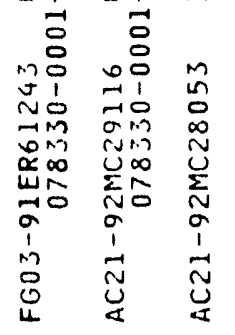

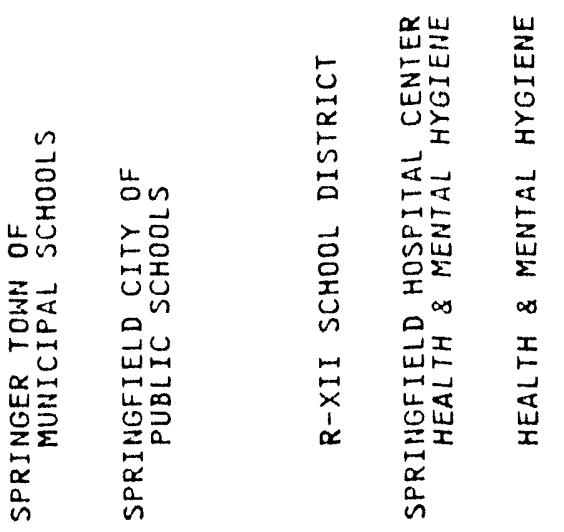

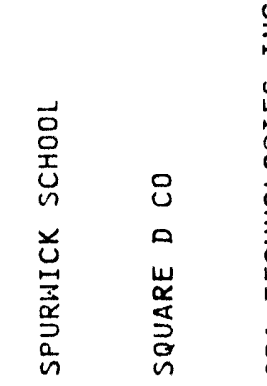

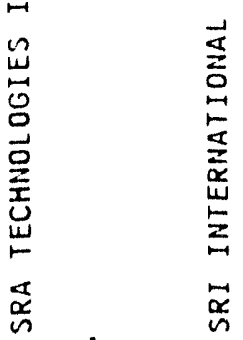




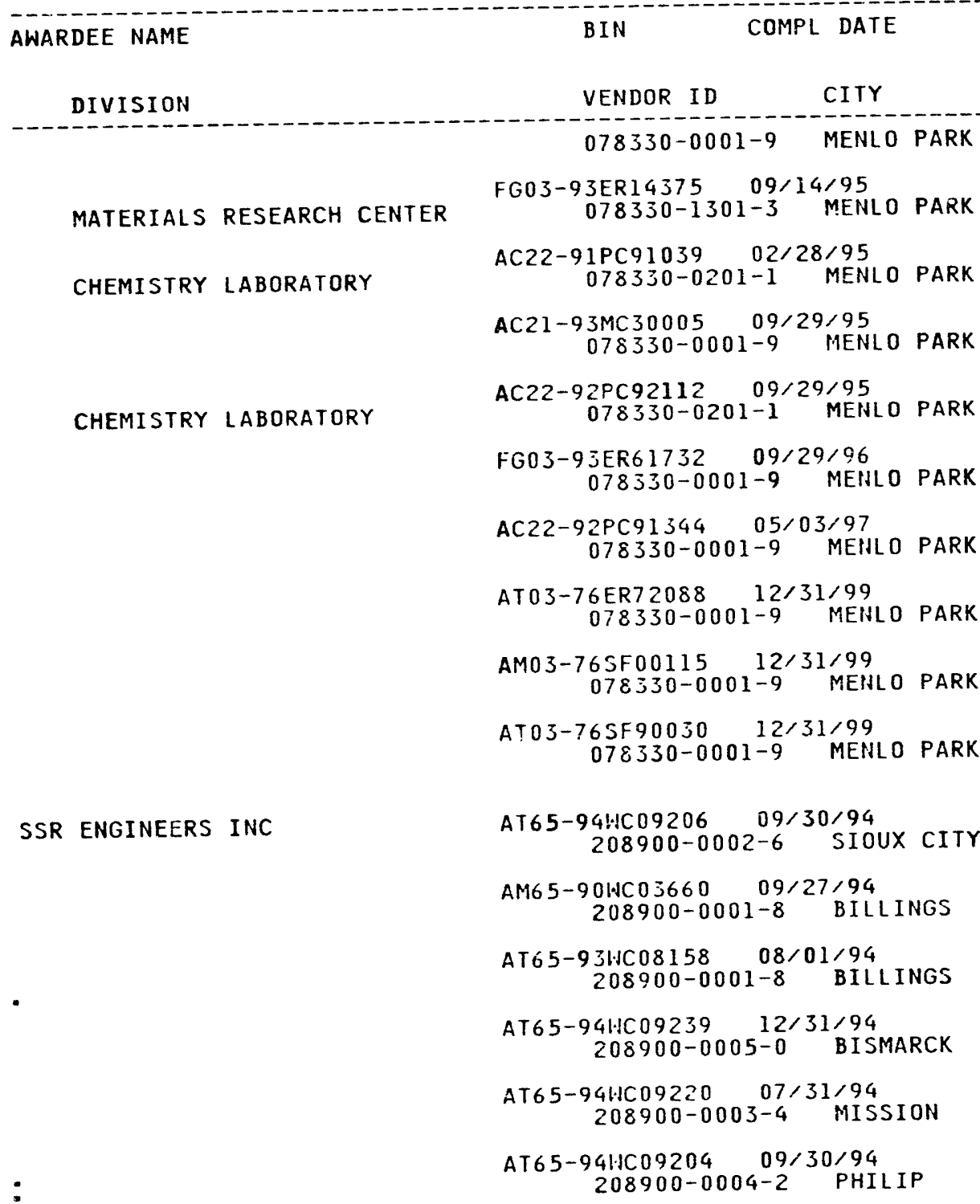

STATE DIST VONTRACT

CA 11

$\$ 520,798$ AG92

DEVELOPMENT OF A LITHIUM BATTERY WITH A NONFLAMMABLE ELECTRO

CA 12 A $\$ 470,150$ W $\$ 230,754$

DISPERED CATALYSTS FOR COAL LIQUEFACTION

$$
\text { CA } 12 \quad \$ 575,106
$$

$\$ 575,106 \quad A G 11$

DEVELOPMENT OF DISPOSABLE SOR-BENTS FOR CHLORIDE REMOVAL $\begin{array}{ccc}\text { CA OF DISPOSABLE SOR-BENTS FOR CHLORIDE REMOVAL } \\ 14 & \$ 605,704 & \$ 375,000 \text { AGI2 }\end{array}$

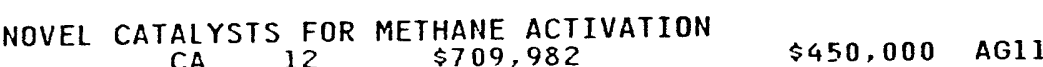

SENSITIVITY AND UNCERTAINTY ANALYSIS DF ATMOSPHERIC OZONE PH CA 12 \$600,001 $\$ 200,001$

ADVANCED SEPARATION TECHNOLOGYFOR FLUE GAS CLEANUP

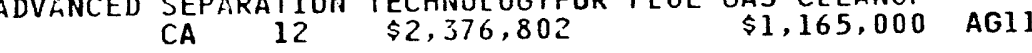
EQUATION OF STATE OF ROCKS

$$
\begin{array}{llll}
\text { OF } & \text { STATE OF ROCKS } \\
\text { CA } & 12 & \$ 138,023 & \$ 138,023 \text { AG91 }
\end{array}
$$

THIS IS A BASIC CONTRACTHITH SEPARATE PROJECTS

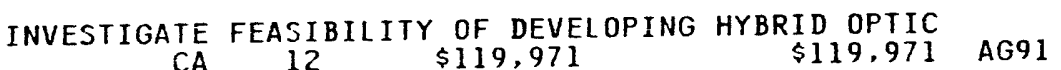

TITLE III SVCS FOR SIOUX CITY SUB 2 GARAGE \& MTNCE BLDG \& M TITLE I, II,III A/E SERVS $\underset{M T}{02} \begin{gathered}\text { FOR BILLINGS AREA } \\ \$ 0\end{gathered}$ TITLE III SVCS FOR STATIC VAR \& MISC PROJECTS

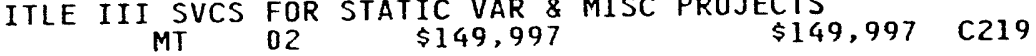

TITLE III SVCS FOR COMMUNICATION TOWERS CONSTRUCTION PROJECT ND $01 \quad \$ 62,964 \quad \$ 62,964$ C219

TITLE III INSPECTION SVCS FOR MICROWAVE COMMUNICATIONS TOWER

$\begin{array}{ccccc}\text { TITLE III SVCS FOR PHILIP SVC CTR MTNCE BLDG } \& \text { MISC WORK } & \text { MV W } \\ \text { SD } & 01 & \$ 69,036 & \$ 69,036 & \text { C219 }\end{array}$ 


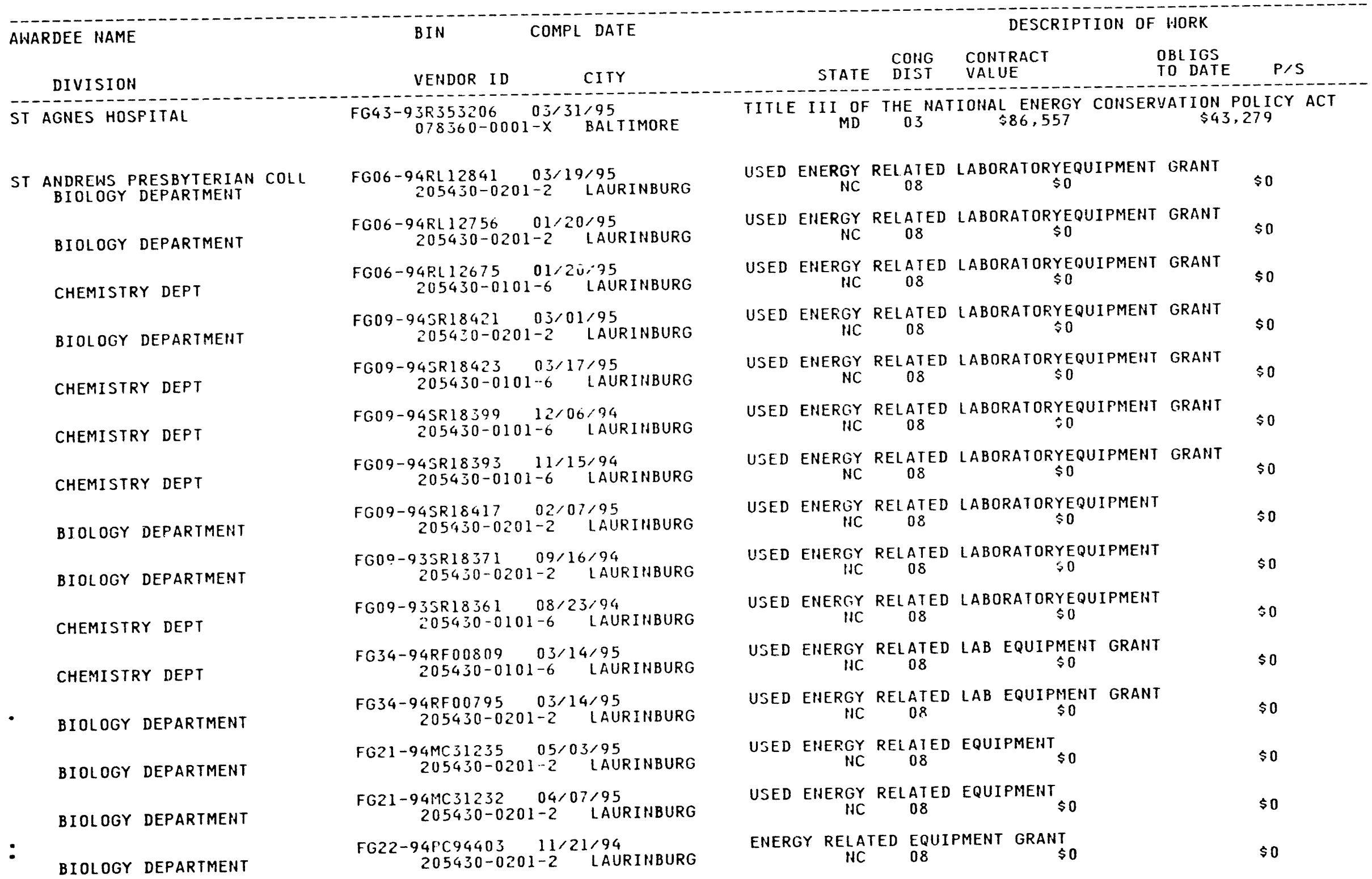




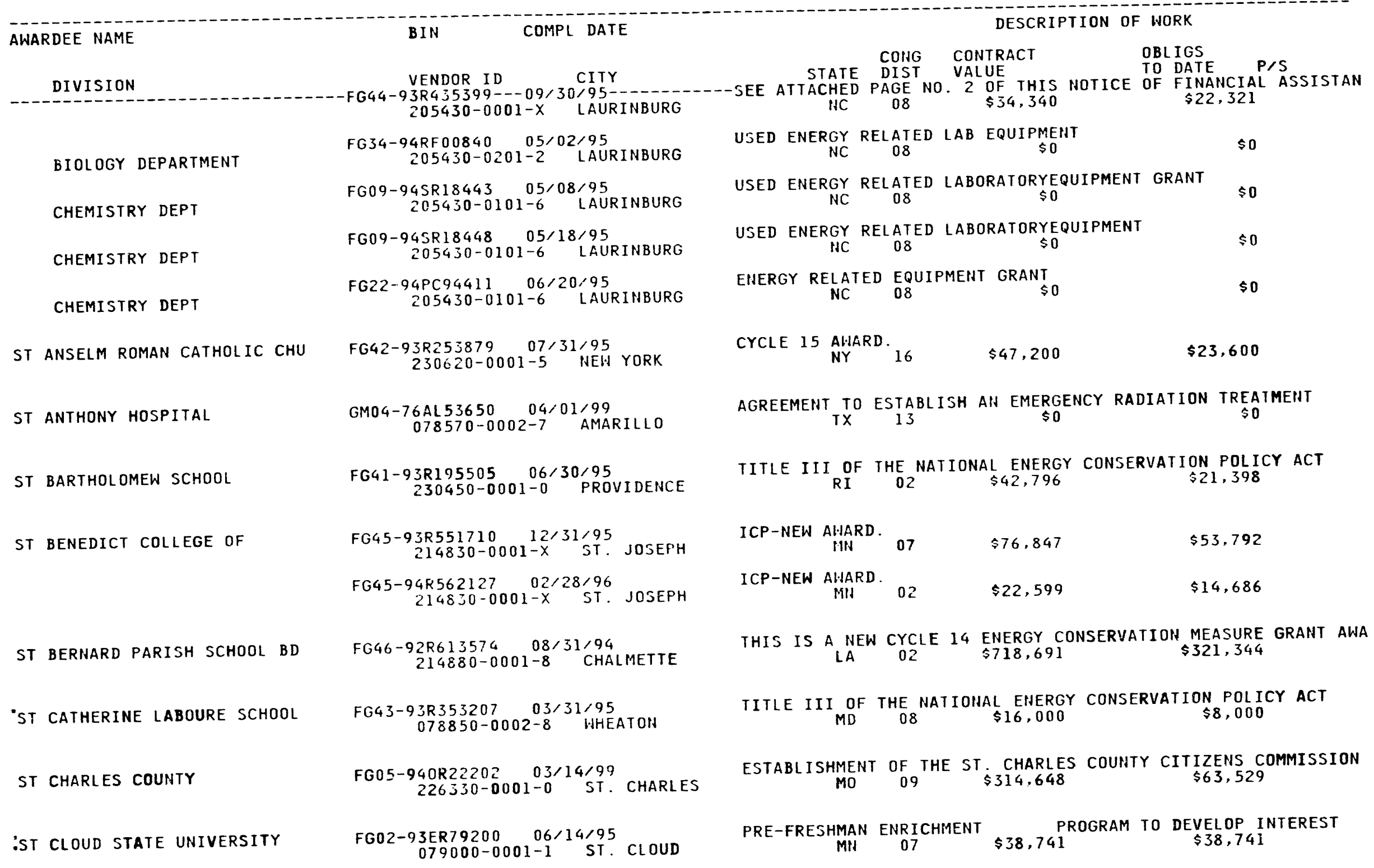




\section{AWARDEE NAME}

BIN COPPL DATE

DESCRIPTION OF HORK

DIVISION

VENDOR ID

CONG CONTRACT
STATE DIST VALUE

OBLIGS O DATE

$P / S$

ST CROIX VALLEY CONS CTHC SCH FG45-92R540142 12/31/94

222290-0001-2 STILLWATER

ICP - NEH AWARD

$\$ 24,309$

$\$ 18,237$

ST CYRIL \& METHODIUS CHURCH

FG45-94R562187 12/31/95
$238380-0001-3$ LAKEWOOD

$3 R 19251306 / 30 / 95$

2R192513 06/30/95
$218140-0001-1$

ST DOMINIC'S REGIONAL HIGH SCH

ST EDMOND HIGH SCHOOL

FG47-92R704995 $08 / 31 / 94$
$104420-0001-4$ FORT DODGE

ST FRANCIS DE SALES SCHOOL

FG43-93R352201 02/28/95
$079210-0001-0$ WASHINGTON

ST FRANCIS HOSP \& HEALTH CTR

FG45-93R551537 08/31/95 232850-0001-5 BLUE ISLAND

ST FRANCIS HOSP \& MEDIC

ST JAMES GRADE SCHOOL

ST JAMES SCHOOL

ST JOHN THE BAPTIST SCHOOL

ST JOHN THE EVANGELIST CHURCH

ST JOHN-WESTSHORE HOSPITAL
FG47-92R702694 08/31/94
$079220-0001-4$ TOPEKA

FG42-94R263915 06/30/96

VENTNOR CITY

FG41-94R192614 06/30/96

B I DDEFORD

FG42-92R243827 07/31/94 079670-0005-6 SYRACUSE

FG42-93R253880 07/31/95 230530-0001-6 MAHOPAC

FG45-94R562193 12/31/95 239060-0001-X WESTLAKE
ICP-NEW AHARD.

$\mathrm{OH} \quad 10 \quad \$ 1,700$

$\$ 850$

TITLE III OF THE NATIONAL ENJERGY CONSERVATION POLICY ACT $M E \quad 02 \quad \$ 4,155 \quad \$ 3,740$

GRANT PROGRAMS FOR SCHOOLS ANDHOSPITALS AND FOR BUILDINGS IA $05 \quad \$ 75,682 \quad \$ 61,425$

TITLE III OF THE NATIONAL ENERGY CONSERVAIION FOLICY ACT DC $01 \quad \$ 7,200 \quad \$ 6,120$

ICP-NEW AWARD

IL $02 \quad \$ 151,781 \quad \$ 67,044$

GRANT PROGRAMS FOR SCHOOLS ANDHOSPITALS AND FOR BUILDINGS 0 KS $02 \quad \$ 384,053 \quad \$ 100,000$

$\begin{array}{lll}\text { CYCLE } 16 \underset{\mathrm{NJ}}{\text { ANARD. }} 02 & \$ 17,255 & \$ 10,733\end{array}$

FY'94 ICP AHARD DI $M$ OI $^{\text {A }} \quad \$ 10,810 \quad \$ 9,729$

TITLE III OF THE NATIONAL ENERGY CONSERVATION POLICY ACT HY $27 \quad \$ 83,176 \quad \$ 41,588$

CYCLE 15 AHARD

NY $19 \quad \$ 57,735 \quad \$ 28,867$

$\underset{O H}{\text { ICP-NEW AWARD }} 10 \quad \$ 27,800$

$\$ 13,900$ 


\begin{tabular}{|c|c|c|}
\hline \multicolumn{2}{|c|}{ AHARDEE NAME } & BIN COMPL DATE \\
\hline & DIVISION & VENDOR ID \\
\hline \multirow[t]{3}{*}{ ST } & JOSEPH HOSPITAL & $\begin{array}{c}\text { FG } 44-91 R 43472208 / 01 / 95 \\
079910-0005-2 \text { MEMPHIS }\end{array}$ \\
\hline & & $\begin{array}{c}\text { FG44-92R435064 08/15/94 } \\
079910-0005-2 \text { MEMPHIS }\end{array}$ \\
\hline & & $\begin{array}{c}\text { FG44-93R435340 09/30/95 } \\
079910-0005-2 \text { MEMPHIS }\end{array}$ \\
\hline ST & $\begin{array}{l}\text { JOSEPH HOSPITAL \& HEALTH } \\
\text { SISTERS DF ST FRANCIS }\end{array}$ & $\begin{array}{c}\text { FG51-93R020311 } 11 / 30 / 94 \\
233440-0101-9 \text { TACOMA }\end{array}$ \\
\hline \multirow[t]{2}{*}{ ST } & JOSEPH HOSPITAL HEALTH CTR & $\begin{array}{c}\text { FG45-92R540028 } 12 / 31 / 94 \\
222990-0001-1 \text { KOKOMO }\end{array}$ \\
\hline & & $\begin{array}{c}\text { FG45-93R551593 } 0 j / 31 / 95 \\
222990-0001-1 \text { KOKOMO }\end{array}$ \\
\hline \multirow[t]{2}{*}{ ST } & JOSEPH'S HOSPITAL & $\begin{array}{c}\text { FG } 44-91 R 434648 \quad 09 / 01 / 94 \\
080060-0009-3 \quad \text { TAMPA }\end{array}$ \\
\hline & & $\begin{array}{r}\text { FG44-92R435016 } 08 / 30 / 94 \\
080060-0016-6 \quad \text { ASHEVILLE }\end{array}$ \\
\hline ST & JOSEPH'S MEDICAL CENTER & $\begin{array}{r}\text { FG } 45-93 R 55171308 / 31 / 95 \\
080080-0002-3 \text { BRAINERD }\end{array}$ \\
\hline ST & JOSEPH'S ROMAN CATH CHURCH & $\begin{array}{c}\text { FG } 42-94 R 26391607 / 01 / 96 \\
239040-0001-2 \text { WEST MILFORD }\end{array}$ \\
\hline ST & LANDRY PARISH SCHOOL BOARD & $\begin{array}{r}\text { FG46-91R613523 09/30/94 } \\
181790-0001-X \text { OPELOUSAS }\end{array}$ \\
\hline ST & LEO CHURCH CORP & $\begin{array}{c}\text { FG41-94R195603 } 06 / 30 / 96 \\
238860-0001-X \quad \text { PAWTUCKET }\end{array}$ \\
\hline ST & LOUIS COMMUNITY COLLEGE & $\begin{array}{c}\text { FG47-92R706064 } 08 / 03 / 94 \\
080210-0001-5 \quad \text { ST. LOUIS }\end{array}$ \\
\hline ST & LUKE'S HOSPITAL & GM04-79AL 38645 \\
\hline
\end{tabular}

DESCRIPTION OF WORK

STATE DIST VONTRACT

OBLIGS
TO DATE P/S CYCIE 13 - INSTITUTIONAL CONSERVATION PROGRAM. APPROVED BUD $\begin{array}{lll} & \\ \text { TN } 07 & \$ 229,109 & \$ 118,452\end{array}$ CYCIE 14 - INSTITUTIONAL CONSERVATION PAROGRAM. APPROVED BU CYCLE 14 TN INSTITUTIONAL 07 \$242,018 $\$ 162,804$ SEE ATTACHED PAGE NO. 2 OF THIS NOTICE OF FINANCIAL ASSISTAN

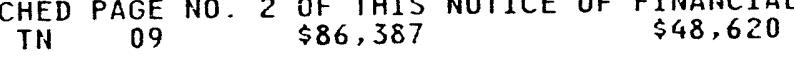

CYCLE 15 ECM GRANT.

$\$ 428,902$

$\$ 257,870$

ICP - NEW AWARD.

$\$ 98,600$

$\$ 49$, siv

ICP-NEH AWARD
IN
05
$\$ 35,050$
$\$ 17,525$

CYCLE 13 - INSTITUTIONAL CONSERVATION PROGRAM. APPROVED BUD $\begin{array}{llll}\mathrm{FL} & 07 & \$ 650.994 & \$ 267,756\end{array}$

CYCLE 14 - INSTITUTIONAL COHSERVATION PROGRAM. APPROVED BUD NC $11 \quad \$ 154,890 \quad \$ 40,000$

ICP-NEW AWARD.
MIN
$\$ 123.530$
$\$ 61,765$

CYCLE 16 AHARD.

$\$ 29,257$

CYCLE 13 DEPARTMENT OF ENERGY GRANT.

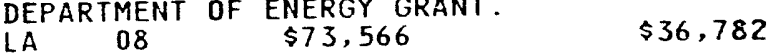

FY:94 ICP AWARD.

$\$ 33,512$

$\$ 29,323$

GRANT PROGRAMS FOR SCHOOLS ANDHOSPITALS AND FOR BUILDINGS 0 MO $01 \quad \$ 111,264 \quad \$ 55,632$

TO PROVIDE TREATMENT FOR PERSONNEL INVOLVED IN A SERIOUS 
$\frac{5}{2}$

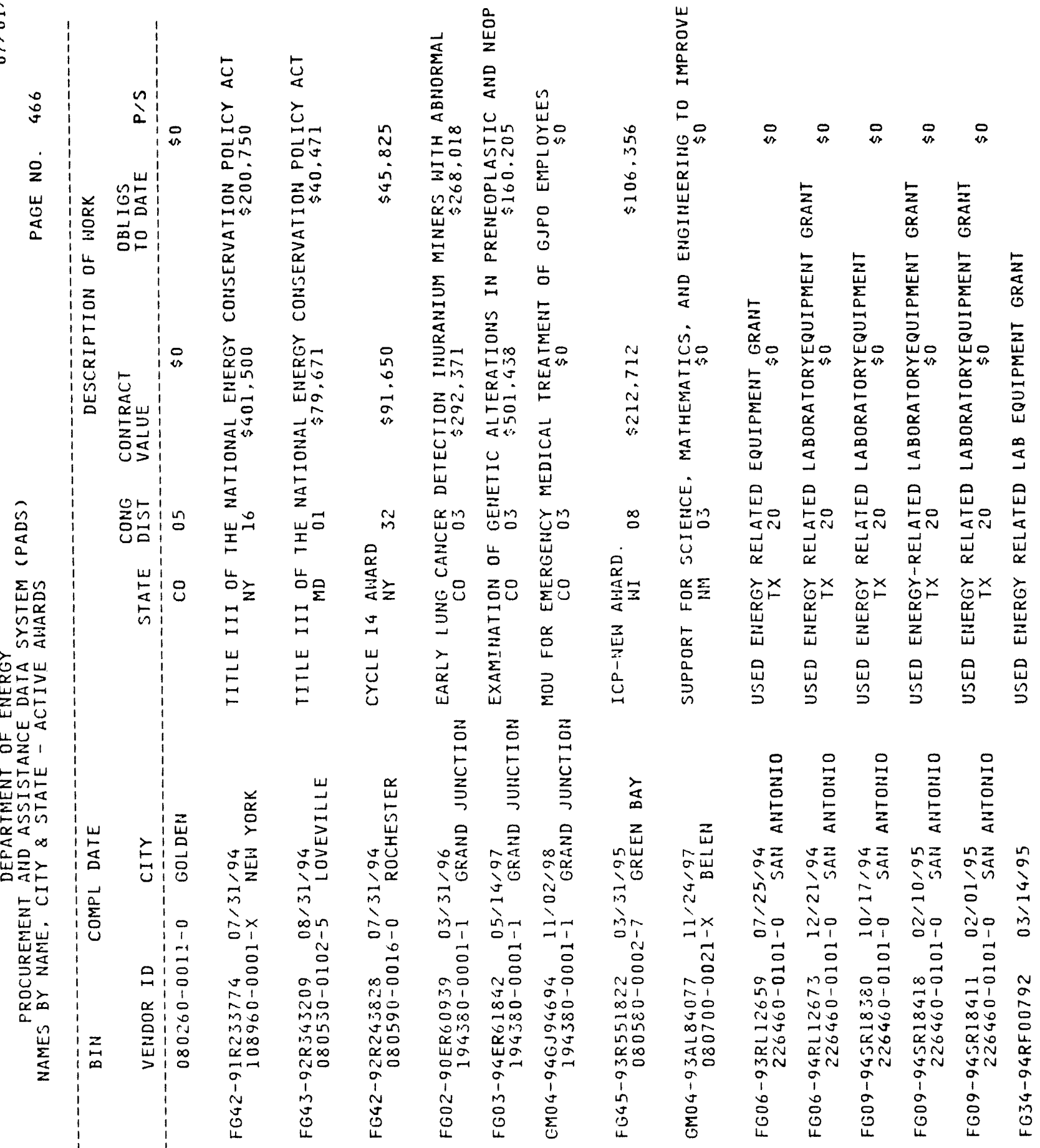
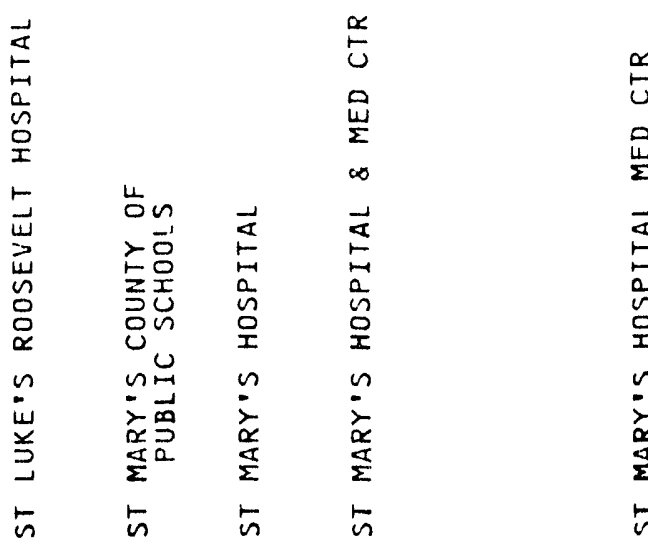


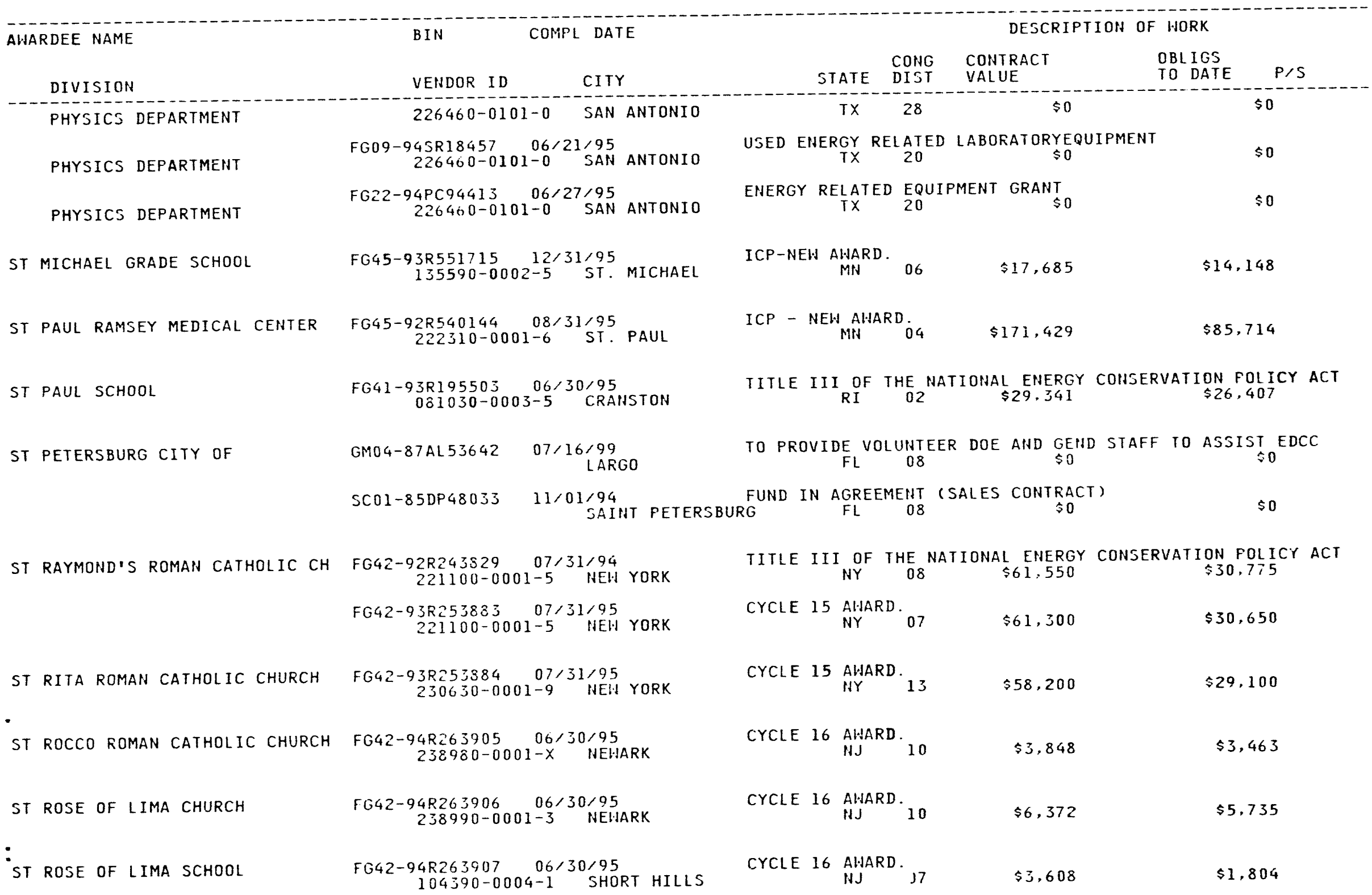




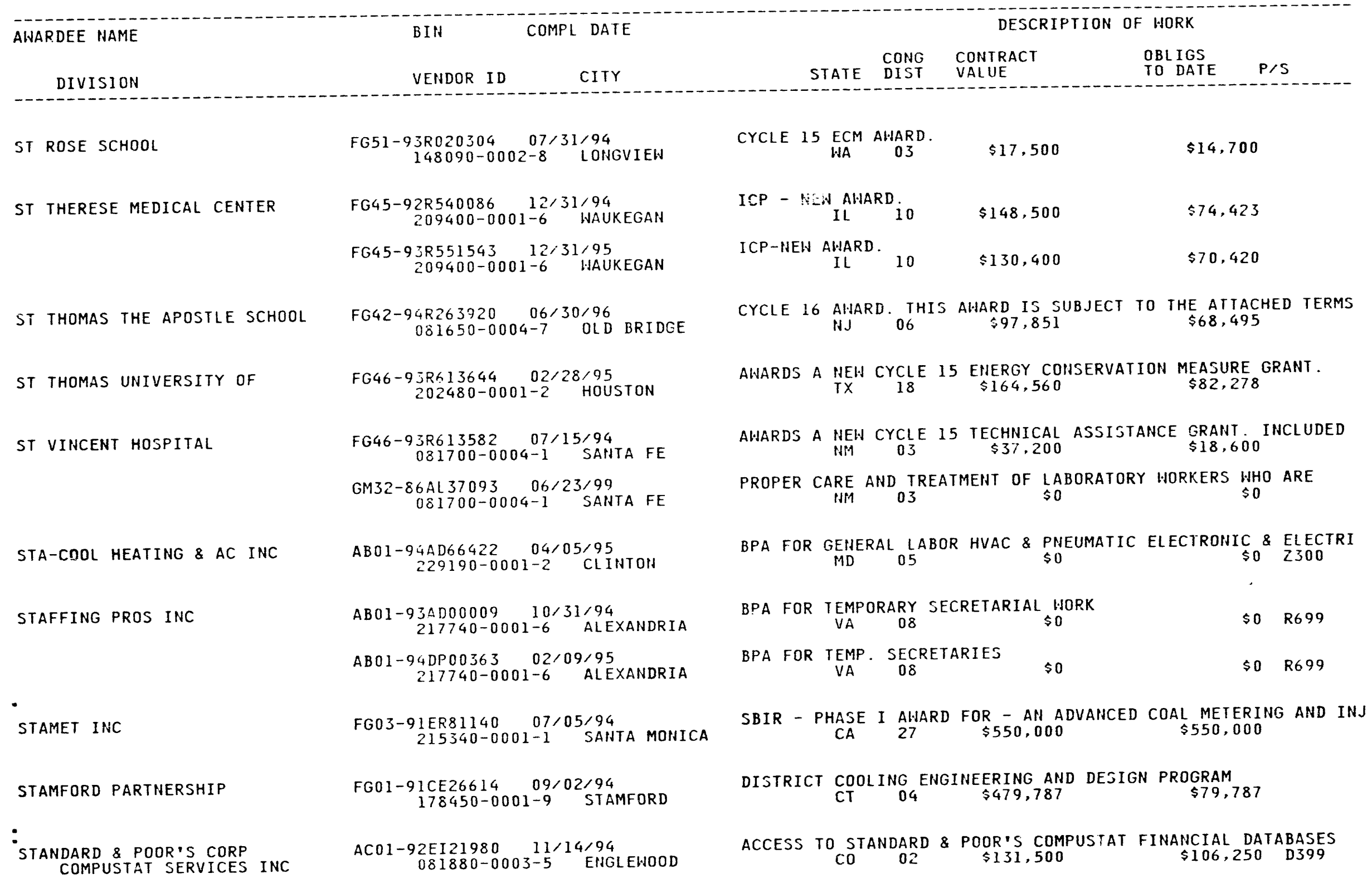




\begin{tabular}{|c|c|}
\hline AWARDEE NAME & COMPL DATE \\
\hline DIVISION & VENDOR ID \\
\hline \multirow[t]{12}{*}{$\begin{array}{l}\text { STANFORD UHIVERSITY } \\
\text { SPONSORED PROJECTS OFFICE }\end{array}$} & $\begin{array}{l}01 / 31 / 95 \\
\text { PASADENA }\end{array}$ \\
\hline & $\begin{array}{c}\text { FG01-91EI22504 } 08 / 15 / 94 \\
081920-0001-X \text { STANFORD }\end{array}$ \\
\hline & $\begin{array}{c}\text { ACO3-76SF00515 09/30/94 } \\
\text { OS1920-0001-X STAHFORD }\end{array}$ \\
\hline & $\begin{array}{r}\text { FG03-90ER14154 09/14/94 } \\
081920-1101-1 \text { STANFORD }\end{array}$ \\
\hline & $\begin{array}{r}\text { FGO3-90ER14152 } 07 / 14 / 94 \\
081920-0001-X \text { STAHFORD }\end{array}$ \\
\hline & $\begin{array}{c}\text { FG03-91EF14196 07/31/94 } \\
081920-0001-X \quad \text { STANFORD }\end{array}$ \\
\hline & $\begin{array}{c}\text { FG03-91ER20048 } 07 / 31 / 94 \\
081920-0001-X \quad \text { STAHFORD }\end{array}$ \\
\hline & $\begin{array}{r}\text { FG03-93ER61684 09/19/94 } \\
081920-0001-X \text { STANFORD }\end{array}$ \\
\hline & $\begin{array}{r}\text { FG03-93ER61724 09/14/94 } \\
081920-0001-X \text { STAHFORD }\end{array}$ \\
\hline & $\begin{array}{r}\text { FG05-91ER79053 } 09 / 29 / 94 \\
081920-0001-X \quad \text { STANFORD }\end{array}$ \\
\hline & $\begin{array}{c}\text { FG05-92ER79119 0 07/31/94 } \\
081920-0001-X \quad \text { STANFORD }\end{array}$ \\
\hline & $\begin{array}{r}\text { FG22-91PC91284 09/19/94 } \\
031920-0001-x \quad \text { STANFORD }\end{array}$ \\
\hline \multirow[t]{3}{*}{ GEOPHYSICS DEPT } & $\begin{array}{cc}A C 21-91 M C 28087 & 09 / 30 / 94 \\
081920-1201-8 & \text { STAHFORD }\end{array}$ \\
\hline & $\begin{array}{c}\text { FG03-86ER13468 } 12 / 31 / 94 \\
031920-0001-x \text { STANFORD }\end{array}$ \\
\hline & $\begin{array}{r}\text { FG03-87ER13757 } 11 / 01 / 94 \\
081920-1701-x \quad \text { STANFORD }\end{array}$ \\
\hline : MECHANICAL ENG DEPT & $\begin{array}{c}\text { FG03-86ER13479 } 04 / 30 / 95 \\
081920-0001-X \text { STANFORD }\end{array}$ \\
\hline
\end{tabular}

DESCRIPTION OF HORK

CONG CONTRACT OBLIGS

TO DATE PIS

A QUEST FOR A NEN SUPERCONDUCTING STATE

THE OBJECTIVE OF THIS GRANT ISTO HELP SUPPORT THE CONTINUAT CA 12 \$1,366,938 $\$ 816,004$

OPERATION AND MAINTENANCE

$$
\begin{array}{lll}
\text { AND MAINTENANCE } & \\
\text { CA } & 12 \$ 2,684,341,974 & \$ 2,383.750,906 \quad M 181
\end{array}
$$

NEH GRANT ${ }_{C A} 11 \quad \$ 358,854 \quad \$ 358,854$

NEW AWARD OF A FROJECT ENTITLED "SEISMICITY INDUCED BY HYDRO CA 11 \$407,180 $\$ 237,165$

NEH GRANT - "STABILITY \& STRESS ANALYSES OF SURFACE MORPHOLO HEH GRANT - ENZYMOLOGY AND MOLECULAR BIOLOGY OF CELL WALL BI ROLE OF STARVATION GENES IN THE SURVIVAL OF DEEP SUBSURFACE
CA 12 IGPB-GCTE SYMPOSIUM ON TERRESTRIAL ECOSYSTEM RESPOHSE TO ELE IGPB-GCTE SYMPOSIUM ON TE $\$ 25,000 \quad \$ 25,000$ ASJAYIHG OF MOLECULAR SIGHALS AND RESPONJES IN THE RHIZOBIUM OF MOLECULAR SIGNALS AND RESPONSES TN THE
CA 12

1992 INSTRUMENTATION - A PRESSURIZED THERMOGRAVIMETERIC AMAL $\begin{array}{lll}\text { RUMENTATION - A PRESSURIZED THERMOGRAVTMETER } \\ \text { CA } 12 & \$ 122,335 & \$ 122,335\end{array}$

NITRATION OF POLYHUCLEAR AROMATIC HYDROCARBONS IN COAL COMBU CA $12 \quad \$ 180,006 \quad \$ 180,006$ INTEGRATED SEISMIC STUDY OF NATURALLY FRACTURED TIGHT GAS RPTION AND HETEROGE "MOLECULAR BEAM STUDIES OF ACTIVATED ADSORPTION AN
$\$ 1,221,516$

"FLUTD DYHAMICS OF DOUBLE DIFFUSIVE SYSTEMS"

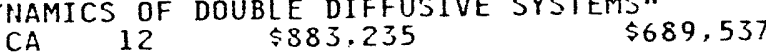
REDUCTION OF DISSIPATIOH IN CONBUSTION AND ENGINES 


\begin{tabular}{|c|c|c|c|c|c|c|c|}
\hline AWARDEE NAME & BIN & L DATE & & & DESCR & F HORK & \\
\hline DIVISION & VENDOR ID & CITY & STATE & $\begin{array}{l}\text { CONG } \\
\text { DIST }\end{array}$ & $\begin{array}{l}\text { CONTRACT } \\
\text { VALUE }\end{array}$ & $\begin{array}{l}\text { OBLIGS } \\
\text { TO DATE }\end{array}$ & $P / S$ \\
\hline
\end{tabular}

MECHANICAL ENG DEPT

PETROLEUM RES INST

- mechanical eng dept

MECHANICAL ENG DEPT
FG03-87ER1 3662 01/31/95 OS1920-0001-X STANFORD FG03-87ER60527 03/31/95 081920-0001-X STANFORD FG03-89ER45387 03/31/95 081920-1701-X STANFORD FGOJ-90ERI4157 09/14/95 $081920-0001-X$ STAHFORD

FG03-92EF25116 04/14/95 081920-0001-X STALFORD

FG03-92ER25117 04/14/95 081920-0001-X STÂHFORD

FGO3-92ER12126 04/30/95 081920-0001-X STAIFORD

FG03-92ER20091 07/14/95 081920-0001-X STAHFORD

FG03-93ER61729 09/14/95 081920-0001-X STAHFORD

FG05-93ER79230 08/31/95 081920-0001-X STAHFORD

FG05-93ER79232 08/31/95 OE1920-0001-X STAHFORD

FG07-901012934 06/30/95 OE19ट0-2101-7 STAHFORD

FG22-92BC14852 09/29/95 $081920-1701-X$ STAHFORD

FG22-92PC92528 $08 / 17 / 95$ OS1920-1701-X STANFORD

FG49-90SF $18680 \quad 02 / 28 / 95$ $081920-0001-X$ STANFORD

FG09-94SR18459 06/21/95 081920-1701-X STANFORD
NEW SRG "ENERGY CHANGES IN TRANSFORMING SOLIDS" (FORMALLY ER $\mathrm{CA} 12$ \$1, 382,030 \$1, 188,440

K-EDGE SUBTRACTION ANGIOGRAPHYHITH SYNCHROIRON X-RAYS $C A \begin{array}{lll}2 & \$ 2,093,816 & \$ 1,961,316\end{array}$

MECHANICAL PROPERTIES OF MATERIAL WITH NANOMETER SCALE MICRO CA 12 \$ $\$ 09,255$ \$909,255

ESTABLISHMENT OF A NEH GRANT ENTITLED "OPTICAL TECHNIQUES FO CA 12 N $\$ 822,227 \quad \$ 672,227$

NEW AWARD $\quad 12 \quad \$ 222,750 \quad \$ 222,750$

NEW AWARD

THE GOAL OF THIS RESEARCH IS TO DEVELOP A HOVEL AHD SIMPLE T CA 12 \$1,081,447 $\$ 1,081,447$

HEW GRANT - "THE EFFECT OF OLIGOSACCHARIDES ON GLYCOFROTEIH CÁ "THE EFFECT OF OLIGOSACCHARIDES $\$ 12$ \$285,000,000

TECHHICAL IHNOVATION AHD DIFFUSION IN LARGE-SCALE ENERGY MOD $\mathrm{CA} \quad 12 \quad \$ 200,385 \%$

1993 UHIVERSITY INSTRUMENTATIOH PROGRM - IHFRA-RED OPTICAL P CA $12 \quad \$ 179,926$ \$ 179,926

1993 UHIVERSITY INSTRUMENTATION PROGRM - AUTOMATED TRIAXIAL CA 12 \$240,000 $\$ 240.000$

GEOTHERMAL RESEARCH \& DEVELOPMENT - STANFORD RESERVOIR AND I CA $12 \$ 1,700,000 \quad \$ 1,564,452$

SCALE-UP OF MISCIBLE FLOOD PROCESSES FOR HETEROGENEOUS RESER CA 12 MIST $12,049,960 \quad \$ 699,964$

CHAR PARTICLE FRAGMENTATION AND ITS EFFECT ON UNBURNED CARBO CA $12 \quad \$ 200,000$ T2 $\$ 20,000$

GRANT PROGRAMS FOR SCHOOLS ANDHOSPITALS AND BUILDINGS OHNED CA 12 S 1226,767

$\$ 113,382$

USED ENERGY RELATED LABORATORYEQUIPMENT

CA 14 


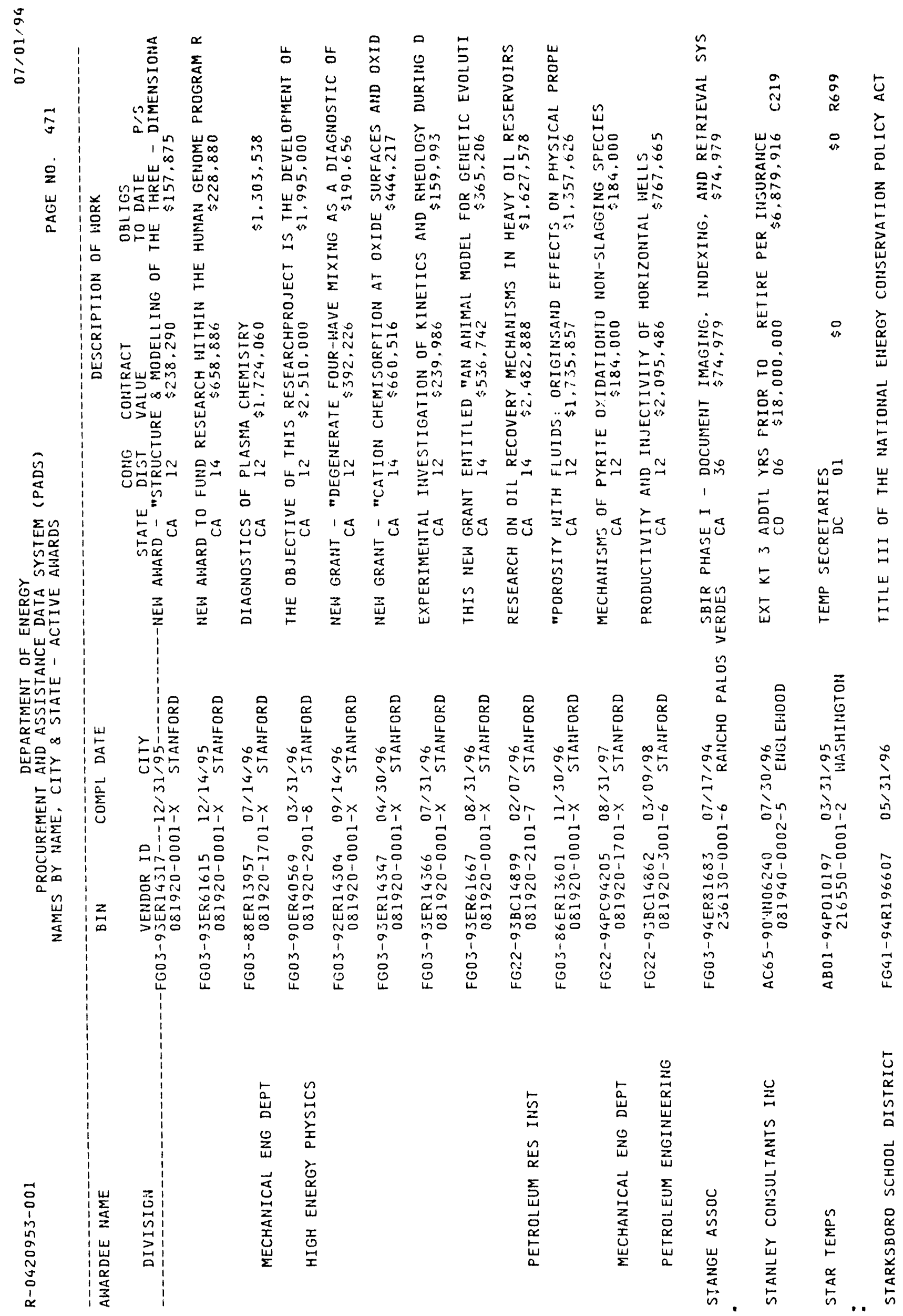




AWARDEE NAME
DIVISION
VENDOR ID
STARLAB
ABO1-93AD69209 $07 / 31 / 94$
220520-0001-1 ROCKVILLE

\section{STARPOINT CENTRAL SCHOOL DIST FG42-93R253885 $07 / 31 / 95$
$082000-0001-X$
LOCKPORT}

STATE DEPARTMENT OF AI01-91AD00266 02/28/96 FOREIGN BLDGS OPERATION OFF OF 082060-0702-4 WASHIHGTON AI01-9JIEI1210 09/30/97 082060-0002-X HASHINGTON

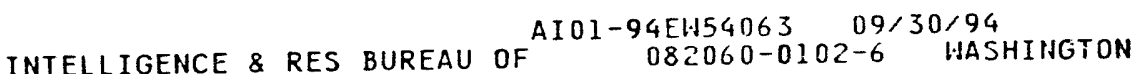
AI01-94IS30003 09/30/94$$
\text { AI01-93NE20693 } 09 / 30 / 95
$$
$082060-0002-X$ HASHINGTON

MAN \& BIOSPHERE COMMITTEE ON AI02-94ER61756 12/31/94 $082060-1102-1$ HASHINGTON A101-94I530018 05/31/95 $082060-0002-X$ WASHINGTON

STATE FAIR COMMUNITY COLLEGE FG47-92R706062 $08 / 03 / 94$
$104900-0001-0 \quad$ SEDALIA

STATE GOVERNMENTS COUNCIL OF MI DWESTERN OFFICE

STATE OF HAWAII HILO HOSPITAL STATE OF NEBRASKA
NEBRASKA ENERGY OFFICE
FC01-94RW00286 $12 / 31 / 94$
$236370-0101-2 \quad$ LOMBARD

FG49-94R916030 06/30/96

FG47-94R701317 06/30/95 LINCOLN
DESCRIPTION OF HORK

CONG CONTRACT

$\frac{\text { STATE DIST VALUE }}{\text { VT }} 01$ 1

OBLIGS

TO DATE P/S $\$ 12,754$
VIDEO CASSETTE DUPLICATION SERVICES

MD 08

$\$ 0$

$\$ 94,810$

$\$ 47,405$

CYCLE 15 AHARD.

$$
\text { NYY } 29
$$

INTERAGENCY AGREEMENT WITH THEDEPARTMENT OF STATE

$$
\text { DC } 01 \text { \$180,000 } \$ 180.000
$$

ADMINSTRATIVE SUPPORT TO THE DOE'S OFFICES IN: PARIS, FRANCE

$$
\text { DC } 01 \text { \$2,330,000 } \$ 540,000
$$

COORDINATION OF SALES OF MAPS AND RELATED DOCUMENIS

$$
\text { DC } 01 \quad \$ 5,000 \quad \$ 5,000
$$

INTERAGENCY AGREEMENT TO PROVIDE DOE THE CAPABILITY TO ACCES DC 01 \$78,500 S78, 500

INTERPRETATION \& TRANSLATION SUPPORT FOR THE U.S. LISBON NUC DC 01 \$500,000 $\$ 500,000$

JOINT SUPPORT FOR THE U.S. MANAND THE BIOSPHERE PROGRAM

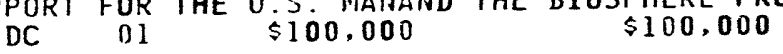

INTERAGENCY W/DEPT OF STATE

$$
\begin{array}{ccc}
\text { DCY } & W \text { DEPT OF STATE } & 01
\end{array}
$$

GRANT PROGRAMS FOR SCHOOLS ANDHOSPITALS AND FOR BUILDINGS 0

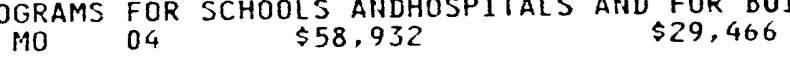

MIDHESTERN HIGH LEVEL RADIOACTIVE HASTE TRANSPORTATION PROJE

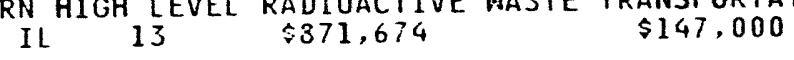

CYCLE XVI ECM GRANT AHARD.
NE 0
01
$\$ 87,278$
$\$ 87,278$ 


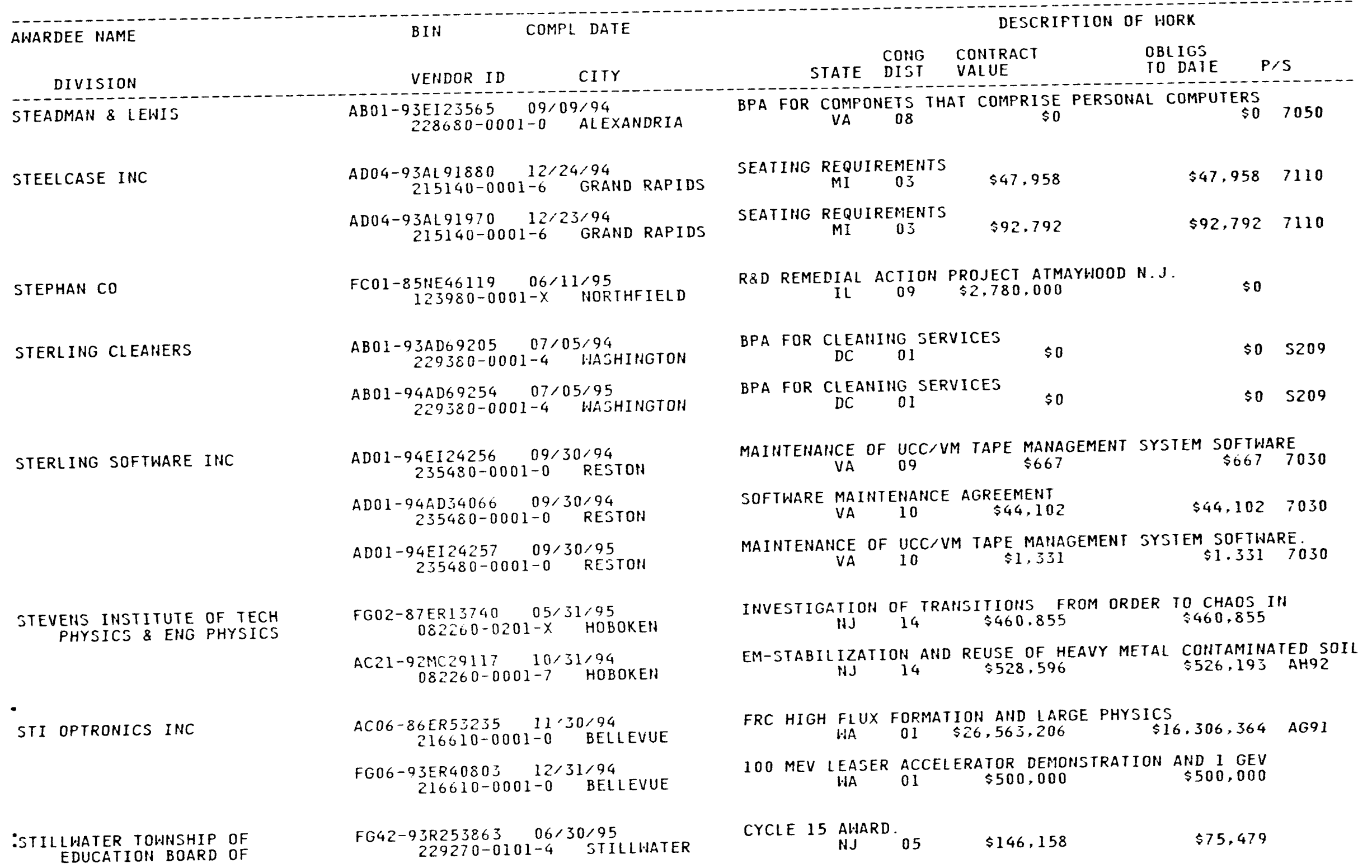


$\frac{0}{0}$

逭

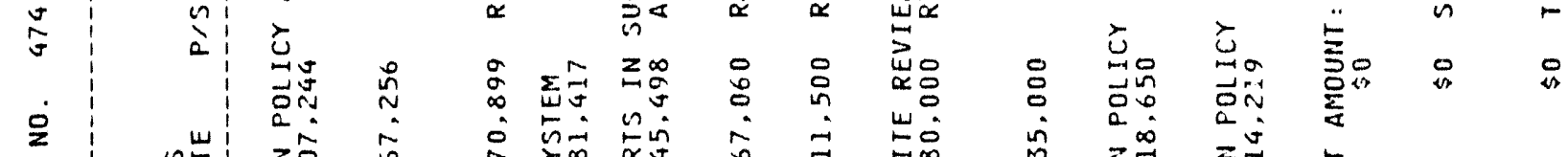

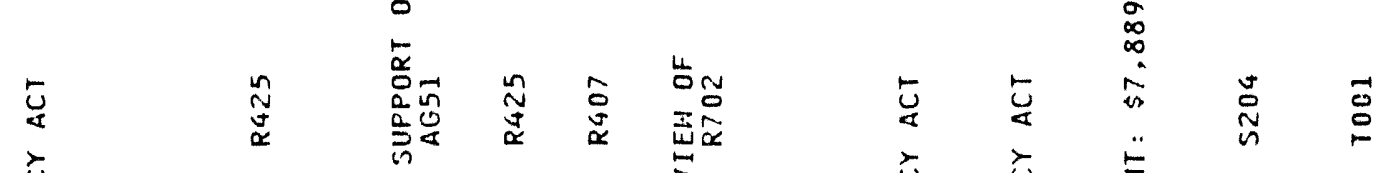

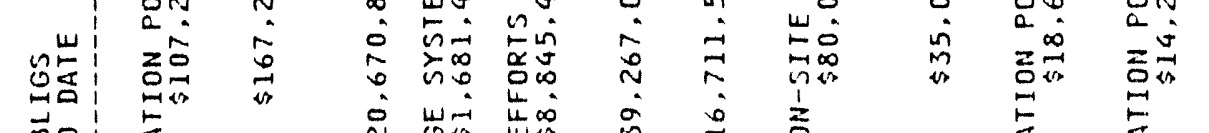

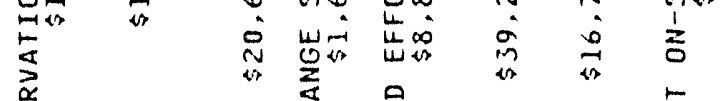

ầ

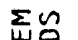

跎

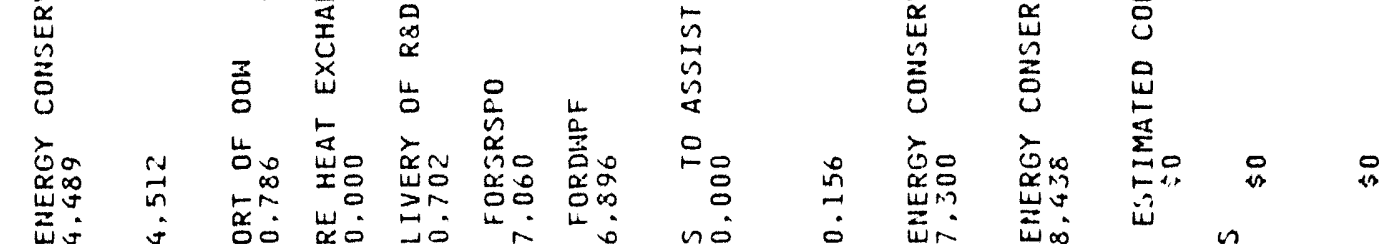

它䟘

总

崖

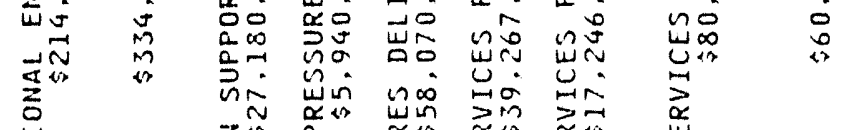

.

$\stackrel{5}{2}$

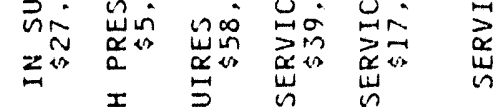

要

똥

蛋

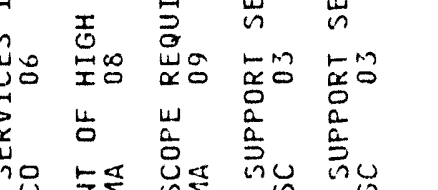

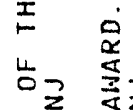

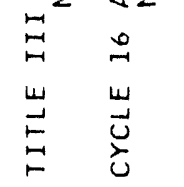

逽

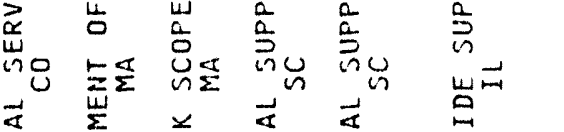

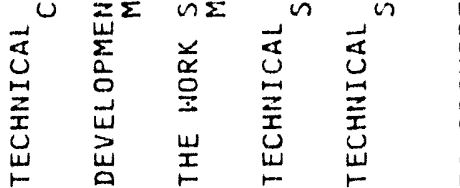

䓵

密战

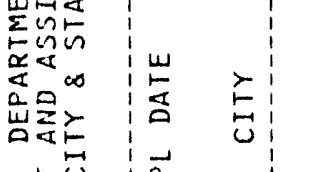

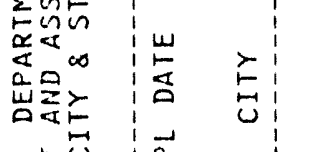

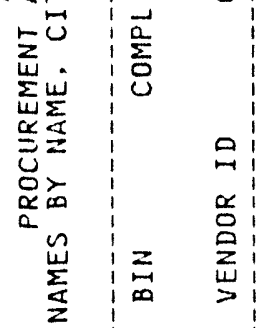

$\stackrel{\frac{8}{2}}{\frac{0}{2}}$

भin

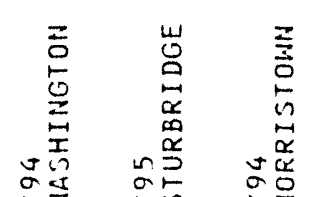

焉

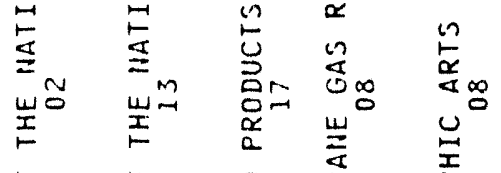

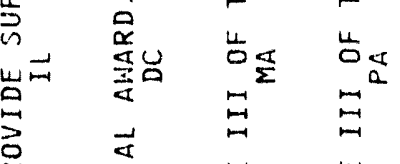

至

品点

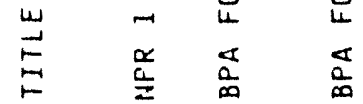

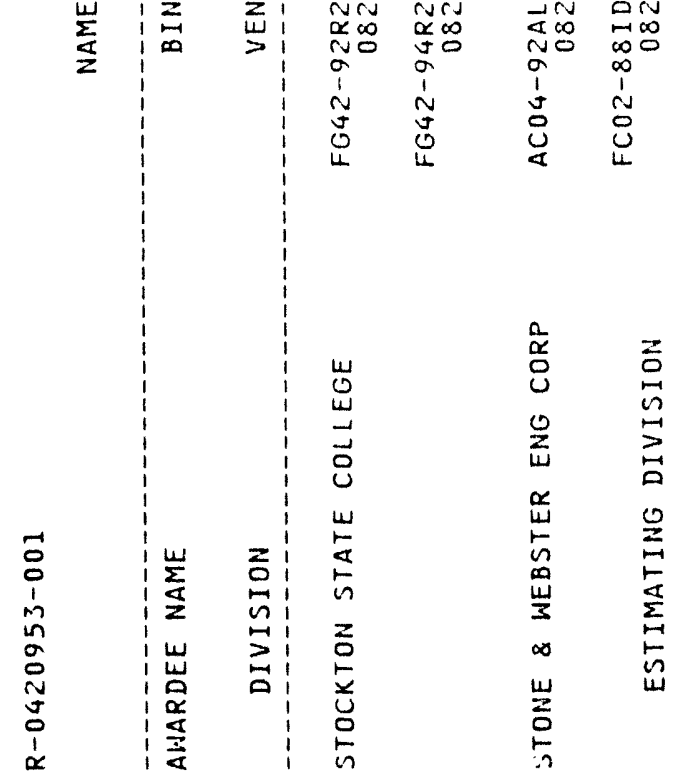

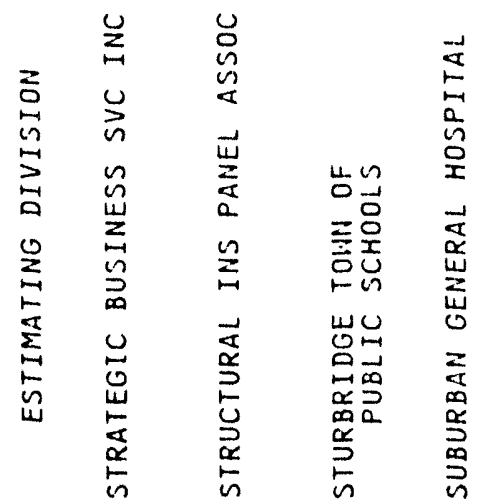

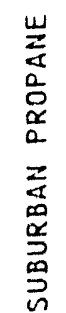

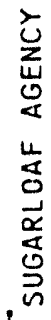



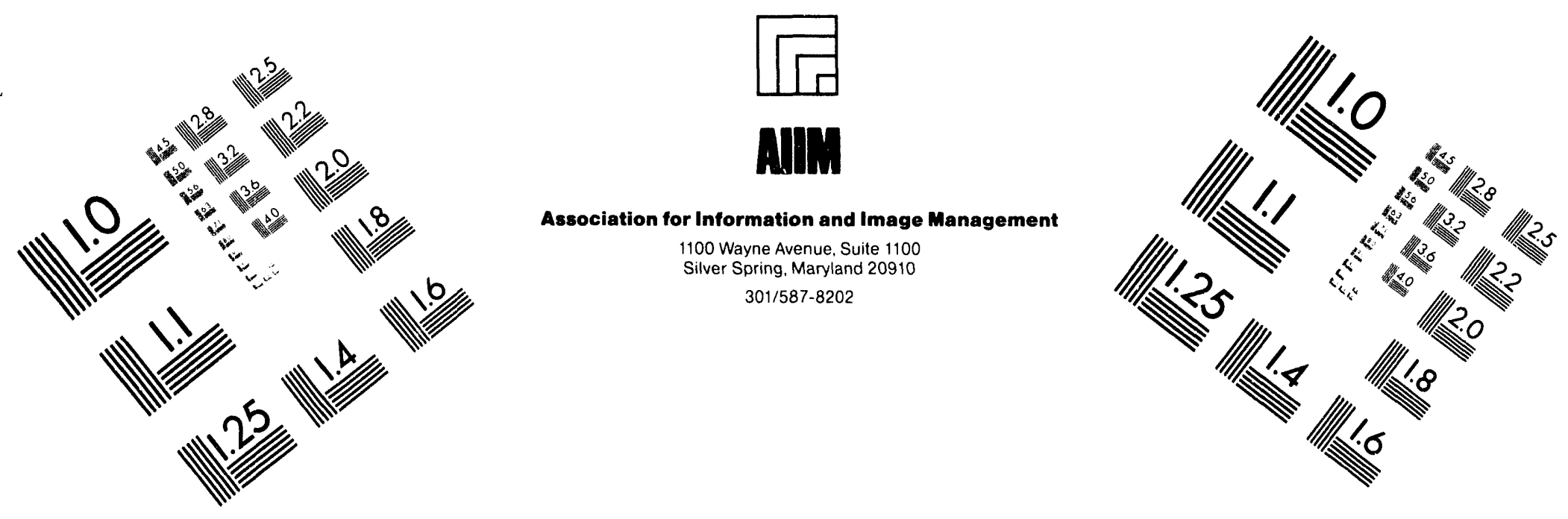

\section{Centimeter}

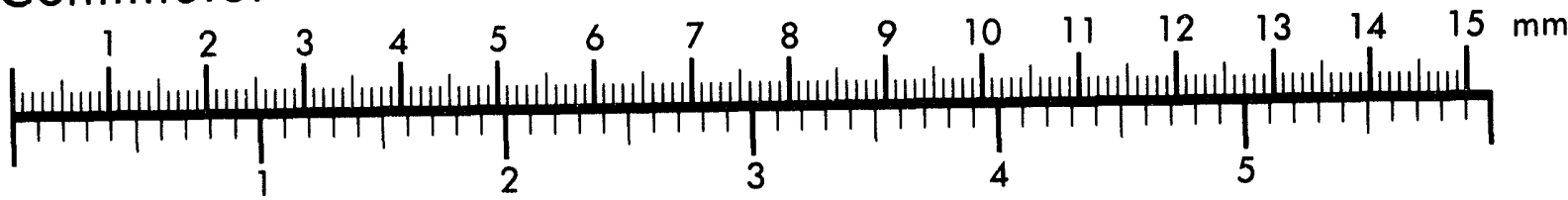
Inches
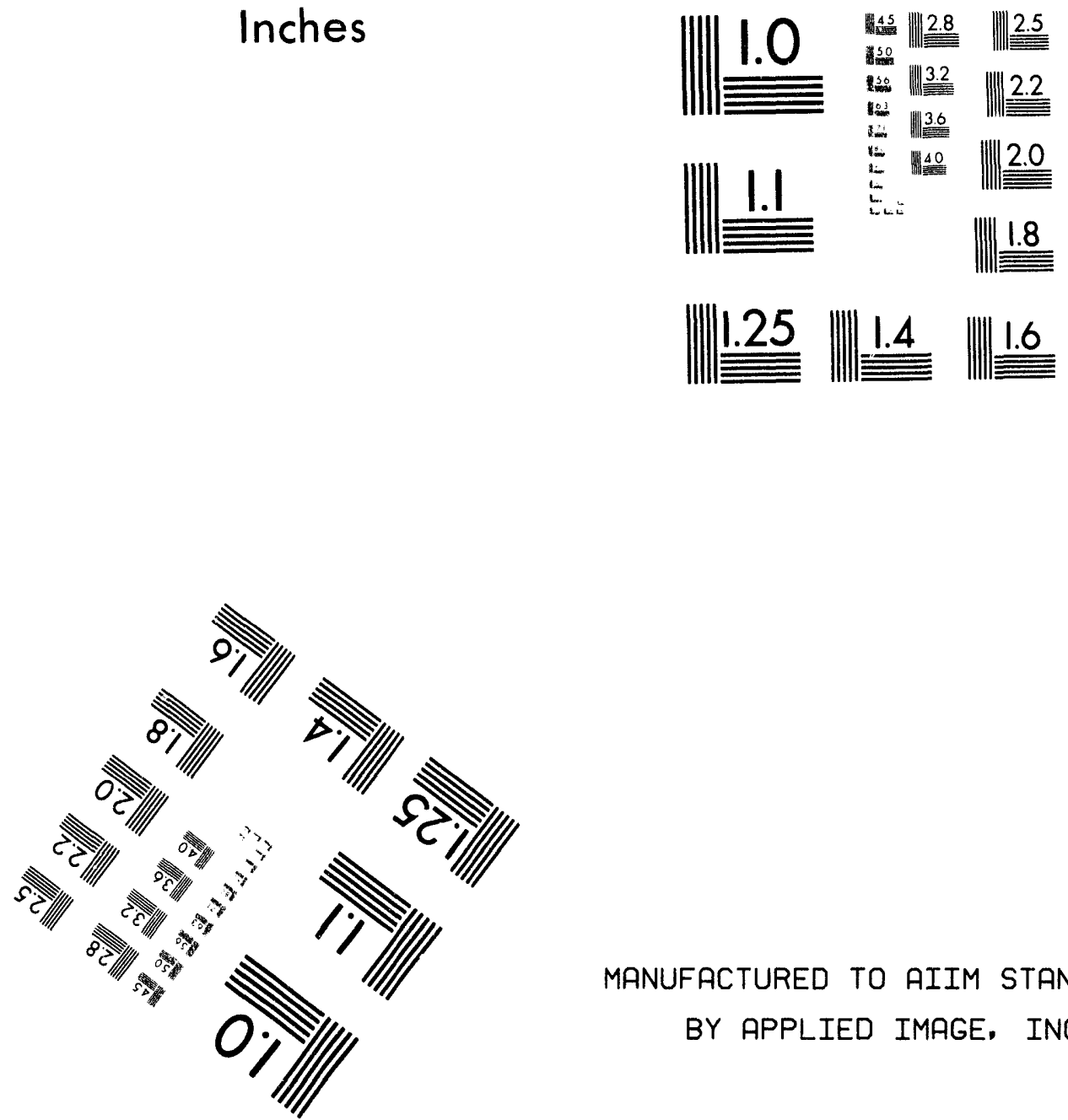

MANUFACTURED TO AIIM STANDARDS

BY APPLIED IMAGE, INC.

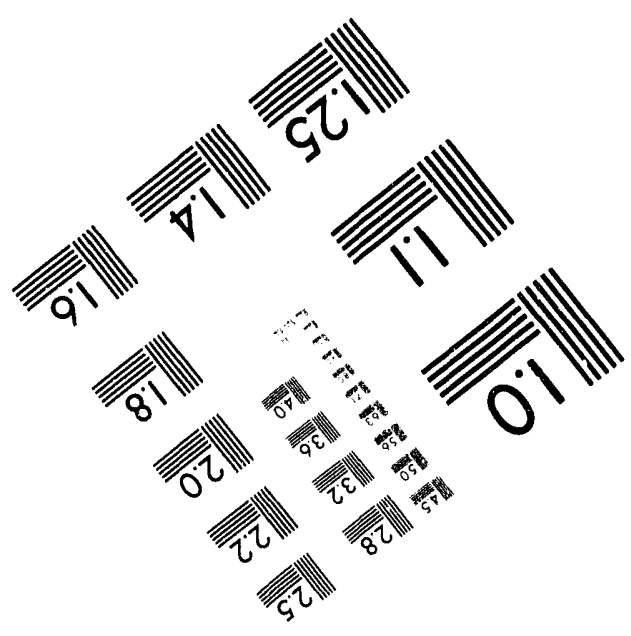



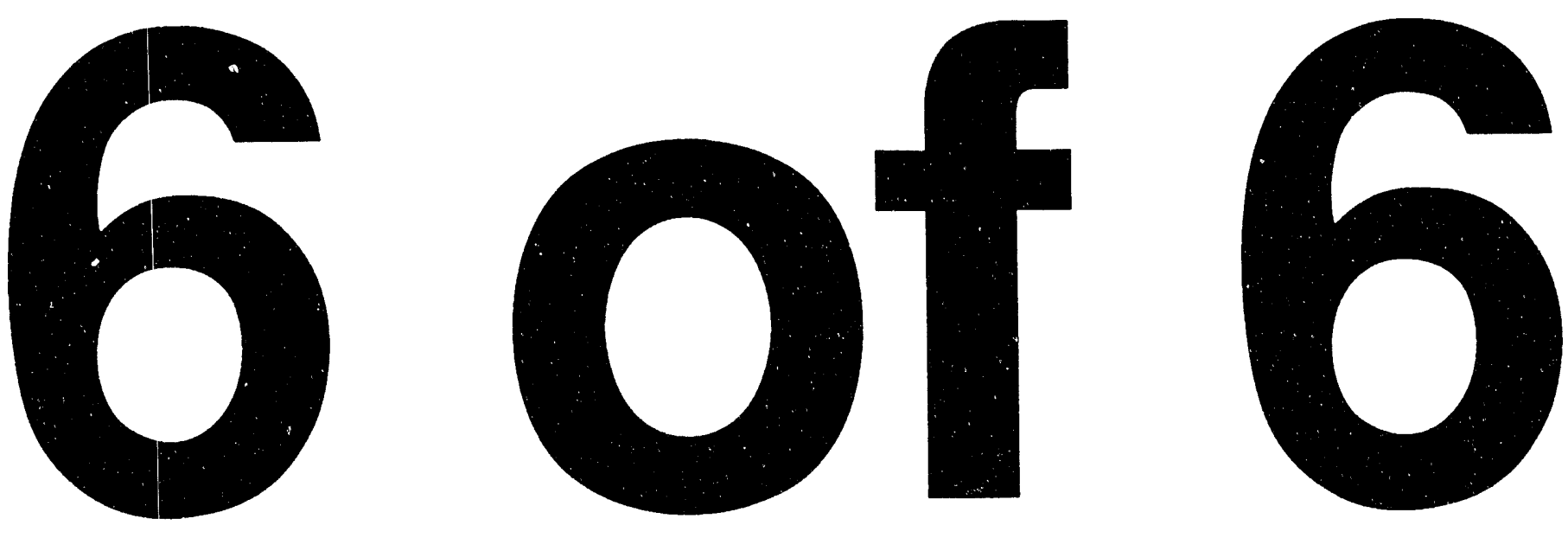


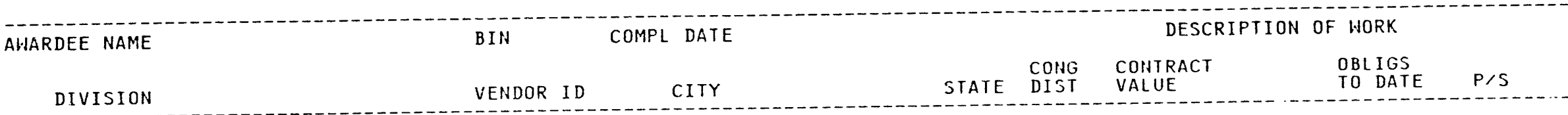

SUMMITEC CORP SUINGARD DIV

SUN PIPE LINE CO

PUBLIC SCHOOL DISTRICT

SUN REFINING \& MARKETING CO APPLIED RESEARCH \& DEV

SUNLAND REFINING CORP

SUPERCON INC
SUN INFORMATIOM SERVICES

SUN MARINE TERMINALS INC

\section{SUN PRAIRIE CITY OF}

ACO5-910R21959 07/21/96
$213450-0001-4$ OAK RIDGE

ACOI-92EI22059 0]/31/95 $083010-0101^{-1}$ PHILADELPHIA

AC $96-78 P 002837 \quad 09 / 30 / 94$ $083020-0001-9$ NEDERLAND

AC96-89F014136 12/05/98 $179870-0001-9$ INEDERLAHD AC96-89P015280 07/31/94 179870-0001-9 NEDERLAND

FG45-92R540267 09/30/95 $083060-0101-X$ SUI PRAIRIE

FC21-90MC26029 01/30/97 225720-0101-2 MARCUS HOOK

SC92-94FE63278 10/01/94 $121470-0002-7$ BAKERSFIELD

FG02-91ER81199 09/30/94 083260-0002-7 JHREHSBURY

FG02-92ER81387 02/17/95 $083260-0002-7 \quad$ SHREWSBURY

FG02-93ER8153j 04/01/96 083260-0002-7 SHREHSBURY

FG02-93ER8 $1622 \quad 07 / 17 / 94$ 083260-0002-7 SHREHSBURY

SSUPERIOR TOWN OF
UNIFIED SCHOOL DISTRICT \#15 $\begin{array}{cc}\text { FG49-93R915012 } 06 / 30 / 95 \\ 229470-0101-X\end{array}$ SUPERIOR
TO INITIATE CONTRACT WITH SUMMITEC CORPORATION ( 8 (A) TO PROV TN $03 \quad \$ 2,784,260 \quad 32,437,807$ R699

COMPUTER DISASTER RECOVERY SERVICL'S FOR THE EIA'S COMFUTER F PA $01 \quad \$ 182,640 \quad \$ 182,587$ DJ01

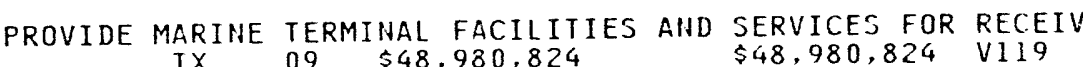

BIG HLL TIE-IN TERMINALLING SERVICES $\$ 25,626,379$ VIX 09 \$35,627,421

$\begin{array}{lll}\text { HEST HACKBERRY FILL SERVICES } & \\ \text { OYX } & 09 & \$ 2,342,201\end{array}$

$\$ 2,342,201 \quad$ V119

ICP - NEW AWARD.

$\$ 95,265$

$\$ 47,631$

CATALYTIC CONVERSION OF LIGHT ALKANES
PA 05 \$14,377.665

$\$ 3,153,222$

NPR-1 CRUDE OIL SALES CONIRACTEST REV $\$ 9050697.39$
CA
09

MULTIFILAMENTARY HBISN SUPERCOHDUCTING WIRE USING APC, COMPO HA $03 \quad \$ 549,895 \quad \$ 549,895$

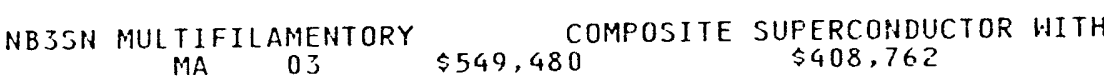

NBJSH SUPERCONDUCTING WIRE
MA 03 $\underset{\$ 674,968}{\text { HITH BULT-IN NB SURFACE }}$

$N / A \quad M A \quad 03 \quad \$ 74,984 \quad \$ 74,984$

CYCLE 15 ICP GRANT AWARD
$\$ 3,000$
$\$ 1,800$ 


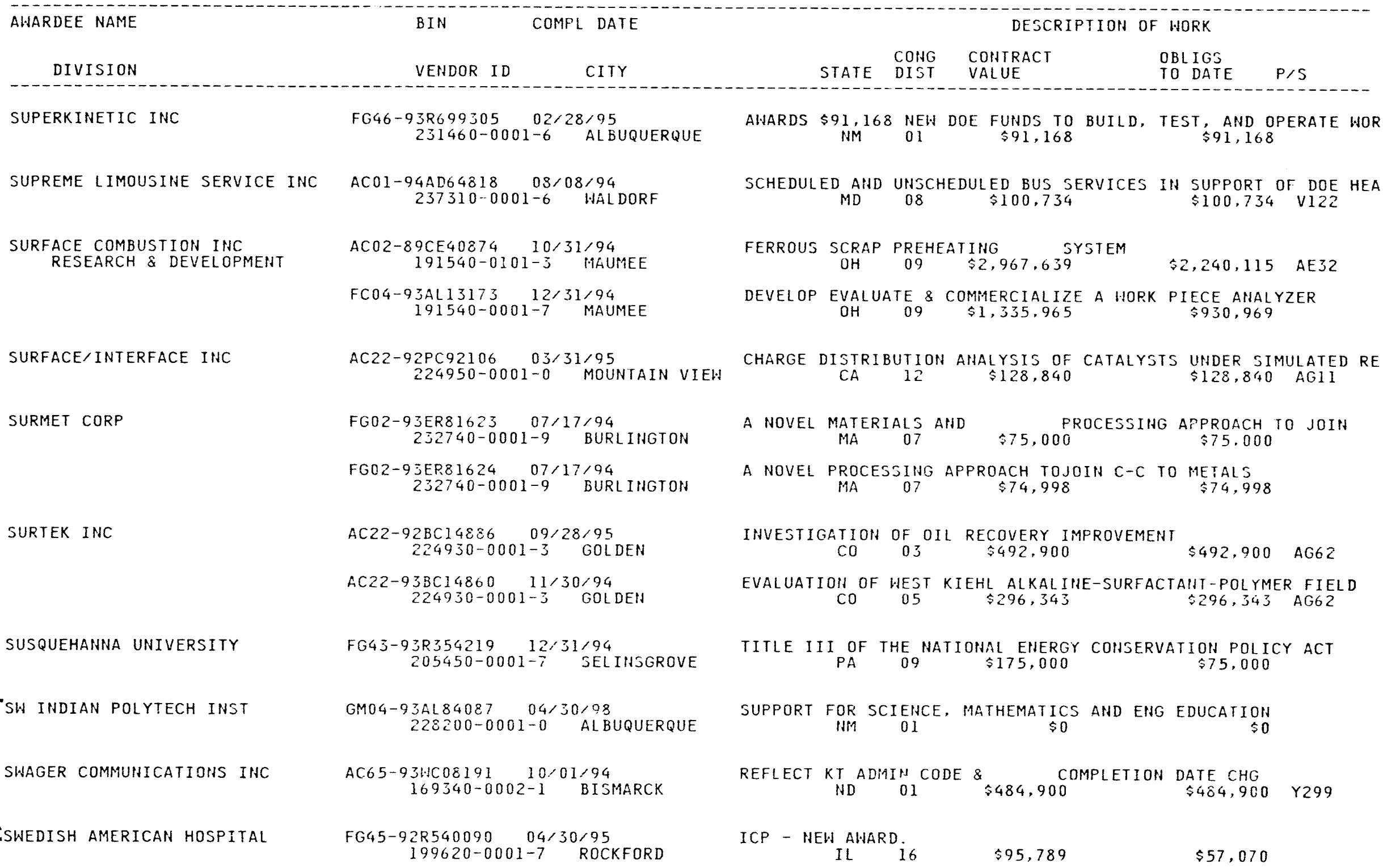




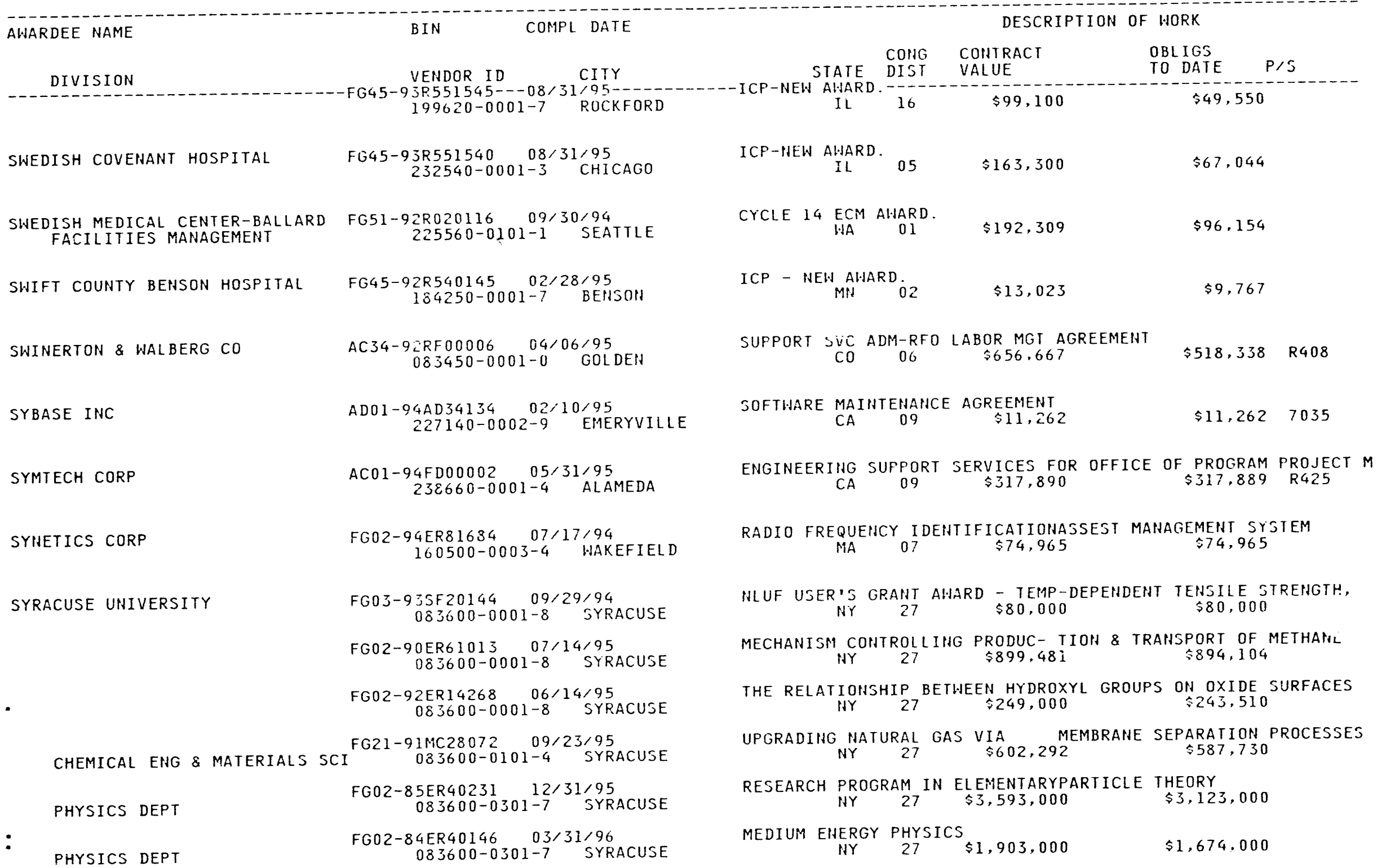


5
$\vdots$
0
0

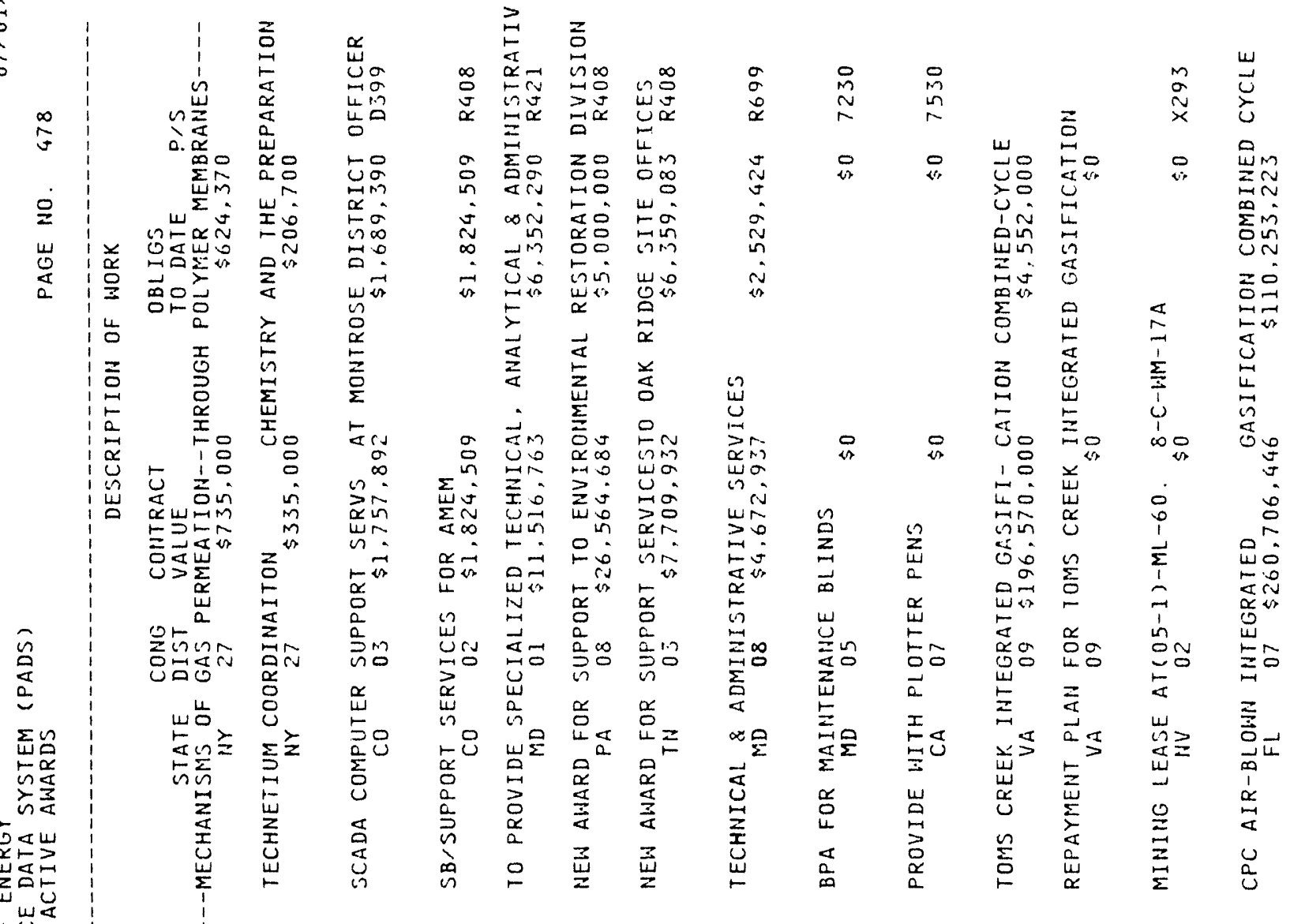

능닌

的
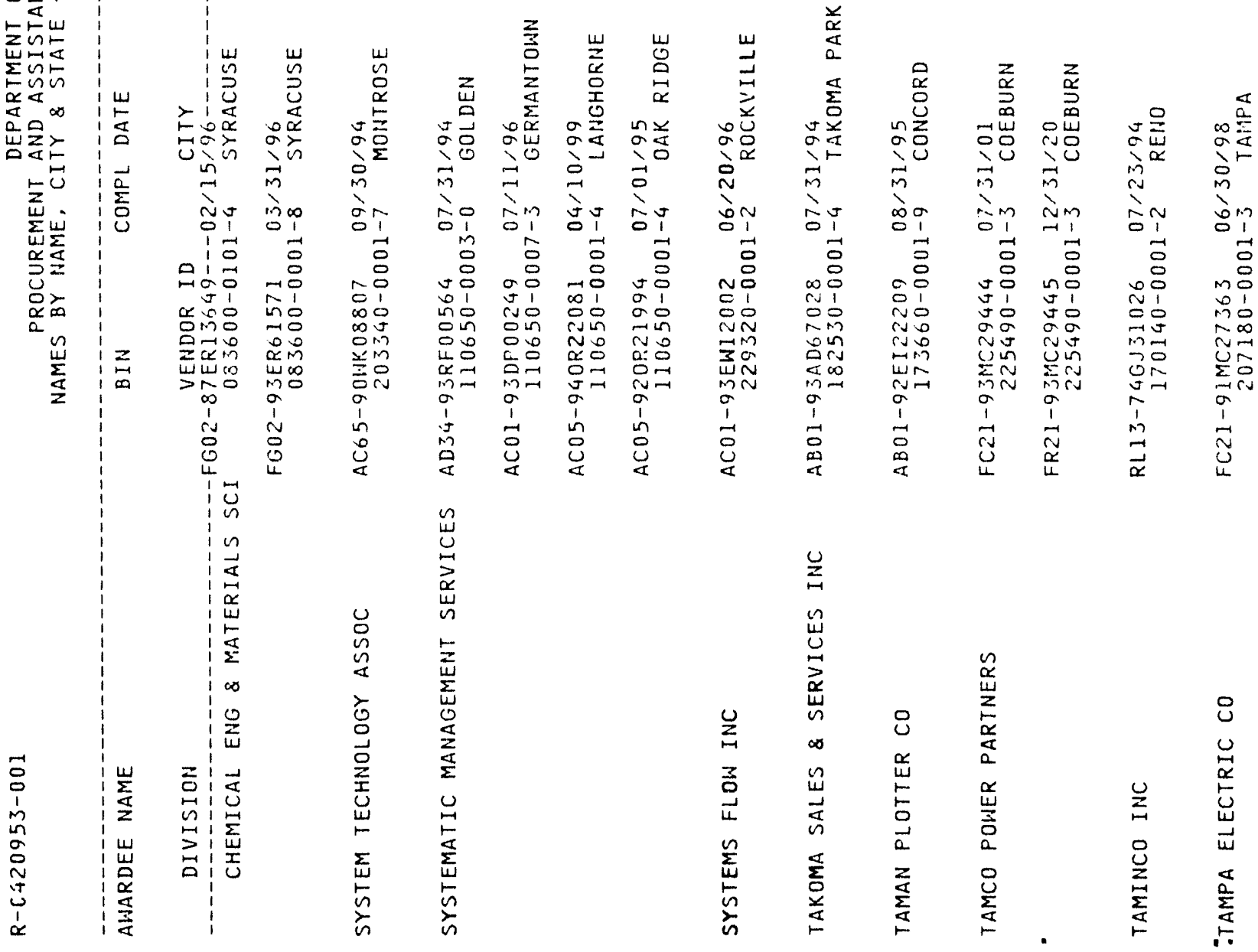
TAMPA GENERAL HOSPITAL

FG44-93R435209 09/30/95 $037890-0001-9$ TAMPA

TANGRAM ENTERPRISE SDLUTIONS

ABOI-94EI24147 12/31/94 $4 E I 24147$
$235600-0001-9$

TAYLOR JOHN P

TC ENTERPRISES INC

TCS INC

TDA RESEARCH INC AC03-92SF19605 09/29/94
$191030-0001-X$ SAH JOSE

AD04-9JAL $91539 \quad 07 / 15 / 94$ 231140-0001-0 ALBUQUERQUE AMO4-93AL91690 04/16/95 231140-0001-0 ALBUQUERQUE

FC22-94PC94117 09/13/96 237080-0001-3 KRAKOW

FG03-90ER80998 07/31/94 198710-0001-4 WHEAT RIDGE

FG03-92ER.31320 05/25/95 198710-0001-4 HHEAT RIDGE

FG03-92ER81322 05/24,95
$198710-0001-4$ 198710-0001-4 WHEAT RIDGE

TEACHERS ACADEMY OF MATH \& SCI FCO2-90ER75603 $11 / 30 / 94$
$200920-0001-0 \quad$ CHICAGO

TEAM INC

ACO7-94IDI3272 $12 / 17 / 95$
$190140-0003-0 \quad$ IDAHO FALLS

AC06-93RL12652 08/31/96 $190140-0002-2$ RICHLAND

TECHMATICS CORP ACOI-94DP20164 $12 / 12 / 96$
$084290-0003-8$ FAIRFAX
SEE ATTACHED PAGE NO. 2 OF THIS NOTICE OF FINANCIAL ASSISTAN $\mathrm{FL} \quad 11$ \$1, 249,938 $\$ \$ 450,000$

RENEW BLANKET PURCHASE AGREEMENT FOR TECHNICAL MANUALS OF AR

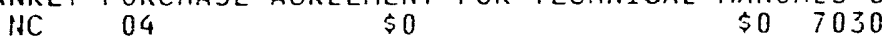

$\begin{array}{ccc}\text { PROVIDE SUPPORT SERVICES TO OFFICE OF PATEHTS IN THE OFFICE } \\ \text { CA } 10 & \$ 160,000 & \$ 82,000 \\ \text { R499 }\end{array}$

TCE TASK 30 HAM FIRE PROTECTION AND LIFE SAFTEY UFGRADES
HM 01 Y
$\$ 497,881$

REBURISH/RENOVATE ALO COMPLEX
NM 01
$\$ 0$
$\$ 0 \quad Y 119$

MICRONIZED COAL-FIRED RETROFITSYSTEMS FOR SOX REDUCTION $00 \$ 2,155,811$ \$1 36,746

$\begin{array}{cccc}\text { PHASE I SBIR AWARD FOR HIGH TEMPERATURE HYDROGEN SULFIDE REM } \\ \text { CO } & 06 & \$ 549,221 & \$ 549,221\end{array}$ SBIR PHASE I - "PREPARATION OFLOW DENSITY MICROCELLULAR MAT CO $03 \quad \$ 549.720 \quad \$ 338.476$

SBIR - PHASE I: "CONTINUOUS P RODUCTION OF FULLERENES FROM H

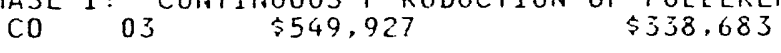

SUPPORT FDR MATH AND SCIEHCE TEACHERS

$$
\begin{aligned}
& \text { FDR MATH AND SCIEHCE TEACHERS } \\
& \text { IL } 01 \text { \$1 } \$ 1,267,339
\end{aligned}
$$

PROVIDE SOFTWARE AND ASSISTANCE IN DOE-ID'S 360 DEGREE PERFO
ID 02

SYSTEM AND SERVICES OF A CUSTOMIZED COMPUTER PROGRAM THAT HA $04 \quad$ \$152,000

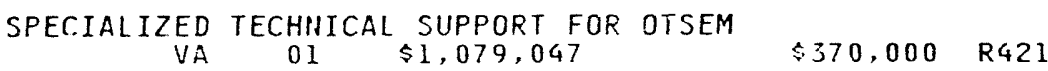


AWARDEE NAME

BIN

COMPL DATE

DIVISION

VENDOR ID

CITY

COISG CONTRACT

DESCRIPTION OF HORK

TECHNOCONCEPTS INC

TECHNOLOGY \& MANAGEMENT SVCS

TECHNOLOGY MANAGEMENT INC

TECOGEN INC

TEEC NAS POS SCHOOL

TEIKYO MARYCREST UNIVERSITY

TEKTRONIX INC

TELCOM MARKETING INC

TELEDYNE ENERGY SYSTEMS

TELEROBOTICS INTERNATIONAL INC TELSPAN INTERNATIONAL INC
FG03-92ER81459 02/17/95
$220820-0001-X$ HEWBURY PARK

$A C 01-92 F E 62041 \quad 04 / 30 / 95$

150630-0003-7 GERMANTOWN

ACO9-91SR18201 09/27/94

FG02-91ER81226 07/05/94 214440-0001-2 CLEVELAND

FC22-94PC94110 $03 / 31 / 95$
$169030-0003-8 \quad$ KRAKOW

AC22-93PC93156 $03 / 30 / 95$ 169030-0002-X WALTHAM GM04-93AL84074 I1/24/97
$227030-0001-4 \quad$ GALLUP

FG47-9 $3270704208 / 31 / 94$
$: 32120-0001-5$ DAVENPORT

AD34-94RF00808 09/30/94 084490-0015-7 GOLDEN

AC65-92WA09475 08/30/94 124050-0002-4 FORT SMITH

AC01-92HE32156 09/29/97 034560-0001-9 TIMONIUM

FG05-91ER81238 07/05/94 I52180-0001-9 KHOXVILLE

$A B 01-94 A D 69237 \quad 11 / 21 / 94$

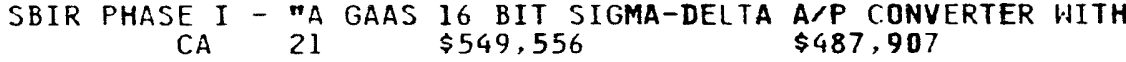

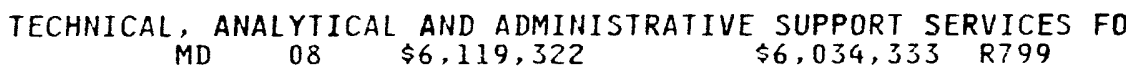

TECHNICAL SUPPORT SERVICES FURBUDGET BRANCH

$$
\text { SC } 03 \quad \$ 2,525,000 \quad \$ 2,525,000 \quad \text { R421 }
$$

SINGLE MANIFOLD, RADIAL FLOW, SOLID OXIDE FUEL CELL.

COMMERCIAL \& INDUSTRIAL BOILERMODIFICATION PROGRAM FOR IMPR INTEGRATION OF THICKENER UNDERFLOH INTO THERMAL DRYER CIRCUI MA $08 \quad \$ 799,718$ \$535,511 AGII

SUPPORT FOR SCIENCE, MATHEMATICS, AND ENGINEERING TO IMPROVE GRANT PROGRAMS FOR SCHOOLS ANDHOSPITALS AND FOR BUILDINGS 0 IA 01 \$10,336 $\$ 5,168$

CV $~$ PRINTER MAIHTENANCE
CO 02
$\$ 1,380$
$\$ 1.380 \quad 0301$

SUPPLY BATTERIES AND RACKS FORVARIOUS SUBSTATIONS

$$
A R \quad 03 \quad \$ 421,076 \quad \$ 421,076 \quad 6140
$$

RESEARCH DEVELOPMENT AND REFURBISHMENT OF RADIOISOTAPE THERM $M D \quad 02 \$ 13,065,626 \quad \$ 5,829,697$ AG55 POSITION CONTROL SYSTEM OF AN INTEGRAL FAST REMOTE HANDOING
TN 02

BPA FOR DUPLICATION OF VIDEO RASSETTES 


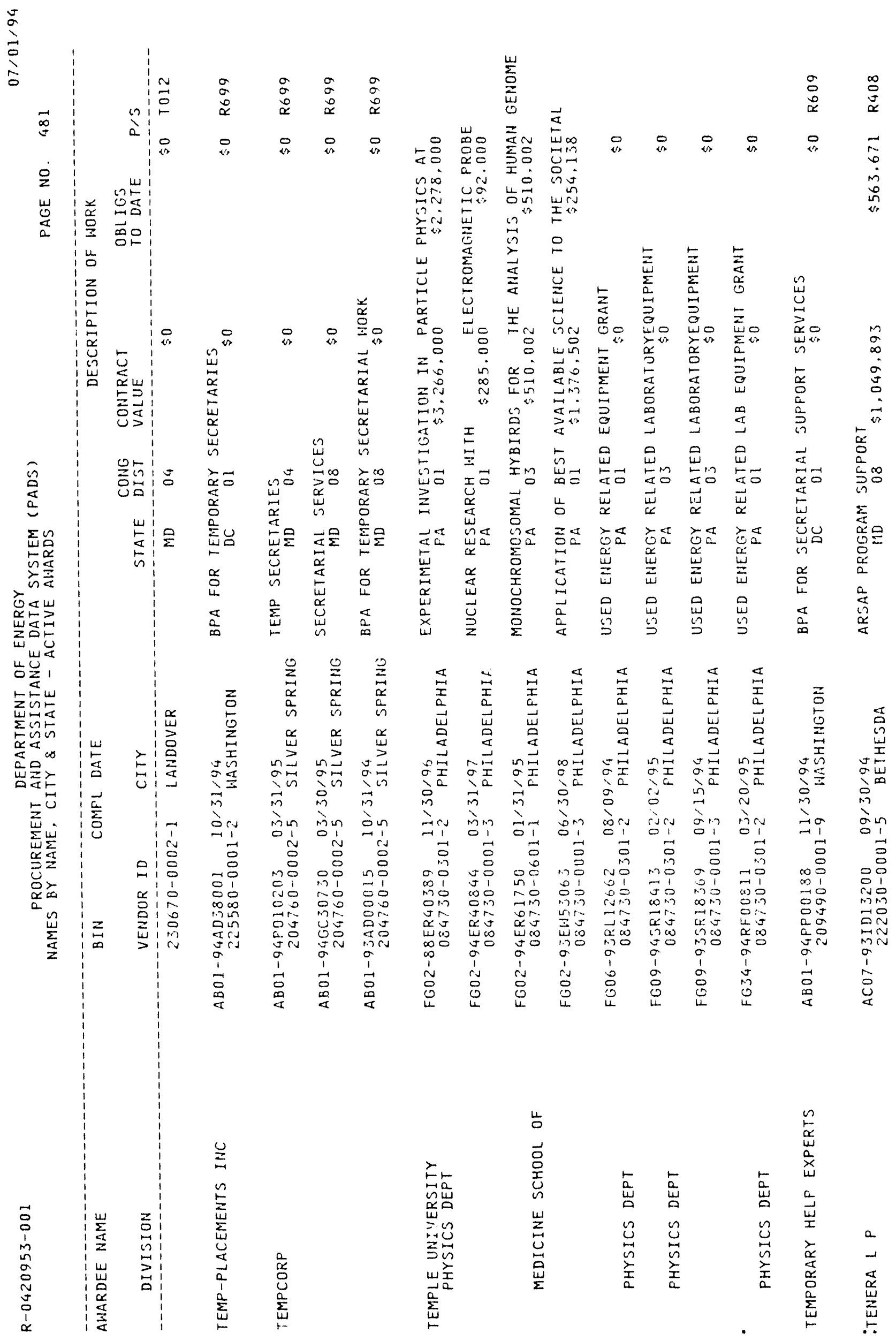




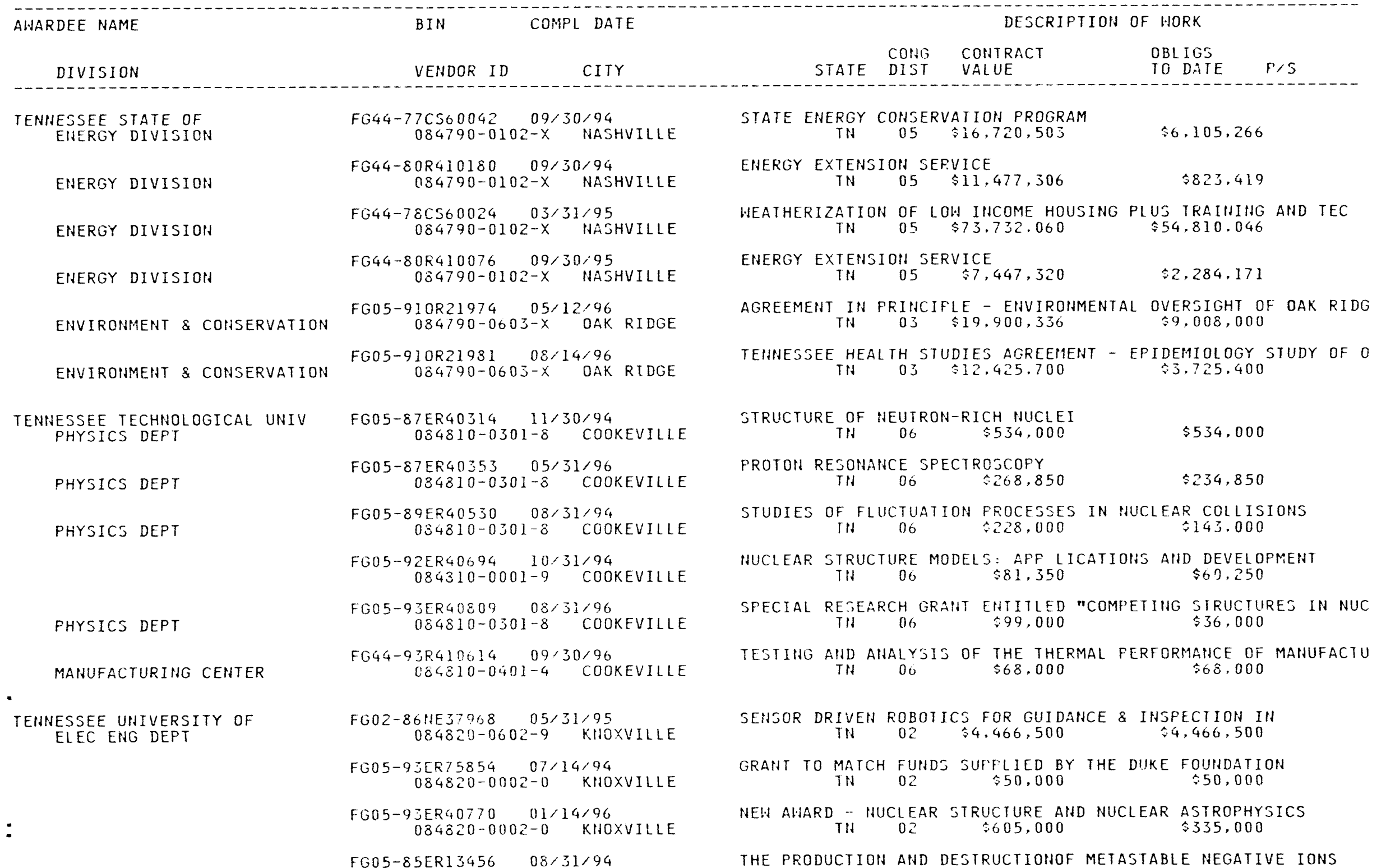




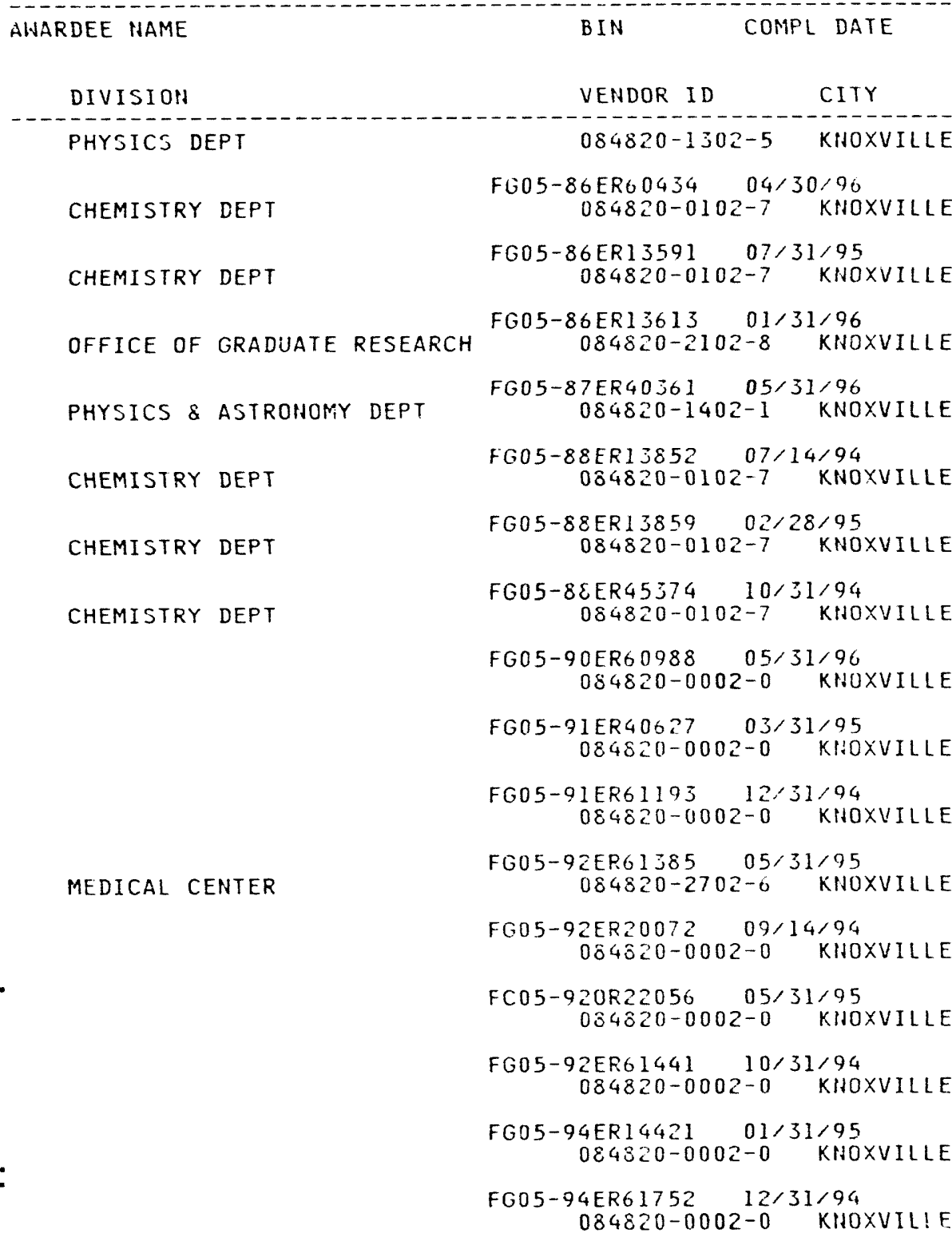

DESCRIPTION OF WORK

CONG COIITRACT OBLIGS

$\$ 587,500$

SYNTHESIS OF RADIOFHARMACEUTICALS CONTAINING SHORT-LIVED RAD

$$
\text { TN } 02 \$ 1,556,983 \quad \$ 1.348,883
$$

DUAL MECHANISM BIFUNCTIONAL POLYMERS: DESIGN, SYNTHESIS, AN

$$
\text { TN } 02 \quad \$ 811,733 \quad \$ 643,732
$$

DEVELOPMENT OF ELECTROKINETIC CAPILLARY SEPARATION AND SPECT

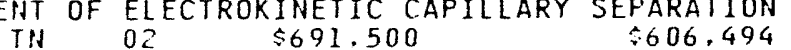

NUCLEAR SPECTROSCOPY STIIDIES

$$
\text { TH } 02 \quad \$ 3.446 .000 \quad \$ 2.545 .000
$$

STUDIES OF RADIATION-PRODUCED RADICALS AND RADICAL IONS

$$
\begin{aligned}
& \text { OF RADIATION-PRODUCED RADICALS AND RADICAL } \\
& \text { TN } 02 \quad \$ 693,390
\end{aligned}
$$

STUDY OF THE SURFACE PROPERTIES OF CERAMIC MATERIALS BY CHRO TN 02 \$ \$ $\$ 17,908$ \$

INVESTIGATIONS OF THE EFFECTS IN ISOTOPIC SUBSTITUTION AND $P$ TN 02 \$1, 100,552 \$ 342,948

BIOMASS, COMMUNITY STRUCIURE SNUTRITIONAL STATUS ATTRIBUTES

$$
\text { TN } 02 \quad \$ 546.000 \quad \$ 362,000
$$

ELEMENTARY PARTICLE INTERACTIONS

$$
\text { TN } 002 \quad \$ 3,161,400 \quad \$ 3.161 .400
$$

ON-LINE MONITORING OF AEROBIC BIOREMEDIATION WITH BIOLUMINES TiN 02 OF AEROBIC BIOREMEDIATION $\$ 470.000 \quad 370.000$

A COHSORTIUM TO DEVELOP THE MEDICAL USES OF NRM IMAGIHG, HMR IN 02 \$285,000 $\$ 190,000$

NEW AWARD - "PLAHT RECOGHITIONOF BRADRYHIZOEIUM JAFONICUM N

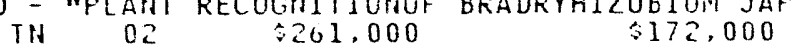

JOINT EDUCATIOH PROGRAM IN ENVIFOHMENTAL KESTORATION AND WAS in $02 \$ 2,805,774 \quad \$ 1,565,000$

NEW AHARD INTRAVEHOUS COROHARYAHGIOGRAPHY UTILIZING K-EMISS TN $02 \quad \$ 60.000 \quad \$ 60.000$

THEORETICAL AND EXPERIMENTAL STUDY OF MIXED SOLVENT ELECTROL HEW AWARD - SEVENTH INTERHATIOHAL SYMPOSIUM

HEW ANARD TN 02 \$15.000 
AWARDEE NAME NAMES BY NAME, CITY \& STATE - ACTIVE AWARDS

BIN COMPL DATE

DESCRIPTION OF HORK

DIVISION

VENDOR ID

CITY

STATE DINT CONTRACT

OBL IGS

FG05-93ER61610 $04 / 30 / 96$
$034820-0002-0$ KHOXVILLE

FG05-9jER25173 08/14/94 084820-2902-9 KNOXVILLE

MECH \& AEROSPACE ENG DEPT

FG22-91PC91306 08/31/94 084820-0102-7 KNOXVILLE

CHEMISTRY DEPT

FG05-94ER6 $1870 \quad 06 / 30 / 98$ 084820-0002-0 KNOXVILLE

SPACE INSTITUTE

FG22-93PC93225 09/27/96 084820-1605-9 TULLAHOMA

TENNESSEE VALLEY AUTHORITY

AI01-89CE31019 08/01/94 084830-0003-2 MUSCLE SHOALS

NATL FERTILIZER \& ENV RES CTR 0 165-92WA09429 $094830-1103-4 \quad$ MUSCLE SHOALS

AI65-93!:A10998 09,30,94 084830-0003-2 MUSCLE SHOALS

AI01-87CE79010 08/14/95 084830-0003-2 MUSCLE SHOALS

AT21-92MC29438 $05 / 06 / 95$ 084830-0003-2 MUSCLE SHOALS

NATIONAL FERTILIZER DEV CTR AI21-91MC28271 $09 / 20 / 96$ MUSCLE SHOALS

A $101-93$ CE1 $6078 \quad 02 / 28 / 98$ 084830-0003-2 MUSCLE SHOALS

FC22-92PC92647 07/01/96 $084830-0010 \cdots 5$ HEST PADUCAH

CR01-83NE44420 03/15/30 084830-0001-6 CHATTANOOGA

AC02-81FE10014 03/31/95 084830-0001-6 CHATTANOOGA
NEW AWARD MOLECULAR MEDICINE SYNTHESIS AND IN-VIVO DETECTION
TH 02
$\$ 320,365$ THE USE OF SYMBOLIC COMPUTATIONS IN RADIATIVE, ENERGY, AND N

SORPTION AND CHEMICAL TRANSFORMATION OF PAHS ON COAL FIY ASH TN $02 \quad \$ 200,000 \quad \$ 200,000$

FIELD RELEASE OF GENETICALLY ENGINEERED BIOLUMINESCENT REPOR TN 02 \$1, $946,343 \quad \$ 203,700$

HIGH PERFORMANCE MATERIAL IN COAL CONV/UTILIZATION

$$
\text { TN } 04 \quad \$ 518,841 \quad \$ 399,985
$$

RESEARCH AND DEV OF SOUTHEASTERN REGIONAL BIOMASS ENERGY $A L=05 \$ 4,421,000 \quad \$ 4,421,000$

FACILITIES ENVIFONMENTAL EVALUATIONS

AL $05 \$ 2,863,900 \quad \$ 2,863,900$

FACILITIES ENVIRN EVALUATION

$$
\text { AL } 05 \quad \$ 220,700 \quad \$ 220,700
$$
$\begin{array}{rrrrr}\text { TECHNICAL MONITORING } & \text { OF THE ALCOHOL FUELS OFFICE - ALCOHOL F } \\ \text { AL } 05 & \$ 6,300,000 & \$ 2,800,000\end{array}$

ENVIRONMENTAL TECHNICAL $\$ 70,000$ SUPPORT SERVICES; TASK ORDER

ENVIRONMENTAL TECHNICAL SUPPORT SERVICES

$$
A L \text { S } 05 \text { \$O } \$ 0
$$

REGIOHAL BIOMASS ENERGY SOUTHEASTERN REGIOH

MICRONIZED COAL REBURNING DEMONSTRATION FOR NOX CONTROL ON A $\begin{array}{lll}\text { KY COAL REBURNING DEMONSTRATION FOR NOX CONTI } \\ 01 & \$ 7,330,041 & \$ 3,514,755\end{array}$

DISPOSAL OF SPENT NUCLEAR

$\$ 0$

\$o

ELECTRIC POWER FAR DOE/MHD COAL-FIRED FLWO FACILITY

DESPOSAL OF RESIDUAL RADIO ACTIVE MATERIAL

$\$ 150,000$ 
AWARDEE NAME

BIN

COMPL DATE

VENDOR ID

CITY STATE DIST VALUE

DESCRIPTION OF WORK

ACח5-940R22208 03/04/04 084830-0001-6́ CHATTANOOGA

A122-94PC93228 $08 / 13 / 95$ 084830-0001-6 CHATTANOOGA

WATER RESOURCES DIVISION

AI05-920R22012 09/30/94 084830-0102-0 KNOXVILLE

AI 05-940R22332 10/31/94 084830-0002-4 KNOXVILLE

AI05-930R22093 09/50/96 084830-0002-4 KHOXVILLE

AI $05-910 R 21980 \quad 09 / 30 / 96$ 0848?0-0002-4 KNOXVILLE

WATER RESOURCES DIVISION

AI05-910R22007 09/30/97 $084830-0102-0 \quad$ KNOXVILLE

AI 05-930R221j3 $09 / 30 / 97$ 084830-0002-4 KNOXVILLE

AI 05-840R21467 06/30/95 $034830-0904-8$ OAK RIDGE

POWEP BRANCH

TENHOL INC

TEHSA SERVICES IHC

TERA RESEARCH INC

TERRITORY OF GUAM GUAM EHERGY OFFICE

GUAM ENERGY OFFICE

GUAM ENERGY OFFICE

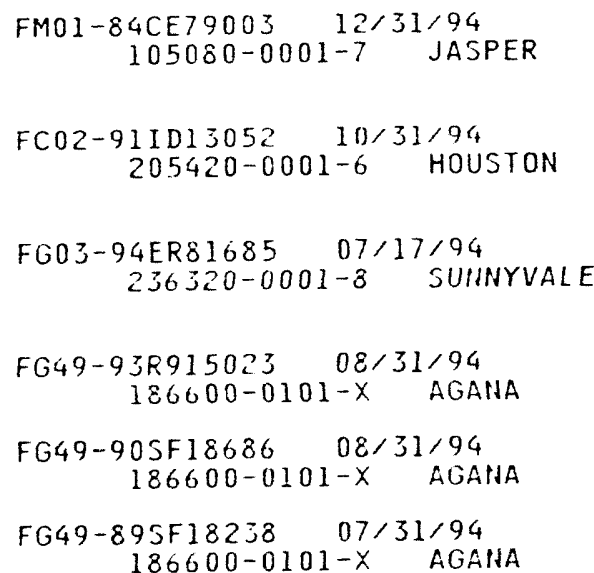
TN 03

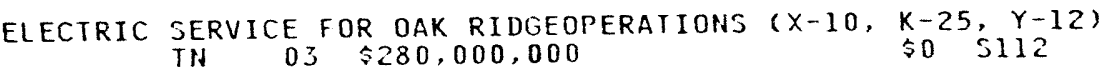

DN-SITE TECHNICAL ASSISTANCE AT COAL-FIRED POWER PLANTS IN I TN 03 \$695,200 $\$ 695.200$

DYHAMIC RESPONSES OF MATURE FOREST TREES TO CHEMICAL AND PHY TN 02 \$48, $200 \quad \$ 48,200$

$\begin{array}{ccc}\text { TECHHICAL ASSISTANCE FOR EDUCATIOHAL TECHHOLOGY } \\ \text { TH } 02 & \$ 12,340 & \$ 12,340\end{array}$

ROOT BIOMASS AND GROHTH ANALYSES ON THE WALKER BRANCH WATERS TN 02 \$ 127,000 \$127,000

ASSESSMENT OF EARTHQUAKE GROUND MOTIONS $\$ 130,000$

TECHNICAL ASSISTANCE FOR THE CLINCH RIVER RCRA FACILITY INVE TH $02 \quad \$ 2,514,100 \quad \$ 2,514,100$

DAK RIDGE RESERVATIOH DIGITAL SPATIAL DATA BASE

$$
\text { Til } 02 \text { \$3, } 580,000 \quad \checkmark 3,580,000
$$

INSTALLATION \& MAINTENANCE FORTVA BY DOE OF EQUIPMENT TO

ILCOHOL FUELS LOAN GURANTEE

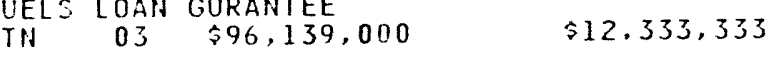

INITIATE PHASE II OF AGREEMENT. CHANGE PARIICIPAHT FROM LITH $\begin{array}{llrr}\text { PHASE } & \text { II } & \text { OF AGREEMENT. CHANGE PARIICIPAHT } \\ \text { TX } & 07 & \$ 964,000 & \$ 117,494\end{array}$

LOW-COST, REMOTELY OPERATED, SAFEGUARDS SURVEILLAHCE SATCOM CA 14 \$74,706 $\$ 74,706$

CYCLE XV TA GRAITT.

$\$ 9.109$

\$ 9.109

GRANT PROGRAMS FOR SCHOOLS ANDHOSPITALS AND BUILDIHGS OWNED GU $01 \quad \$ 636.460 \quad \$ 30.000$

GRANZ PROGRAMS FOR JCHOOLS ANDHOSPITALS AND BUILDINGS OHNED GU 01 \$75,059 $\$ 75,059$ 


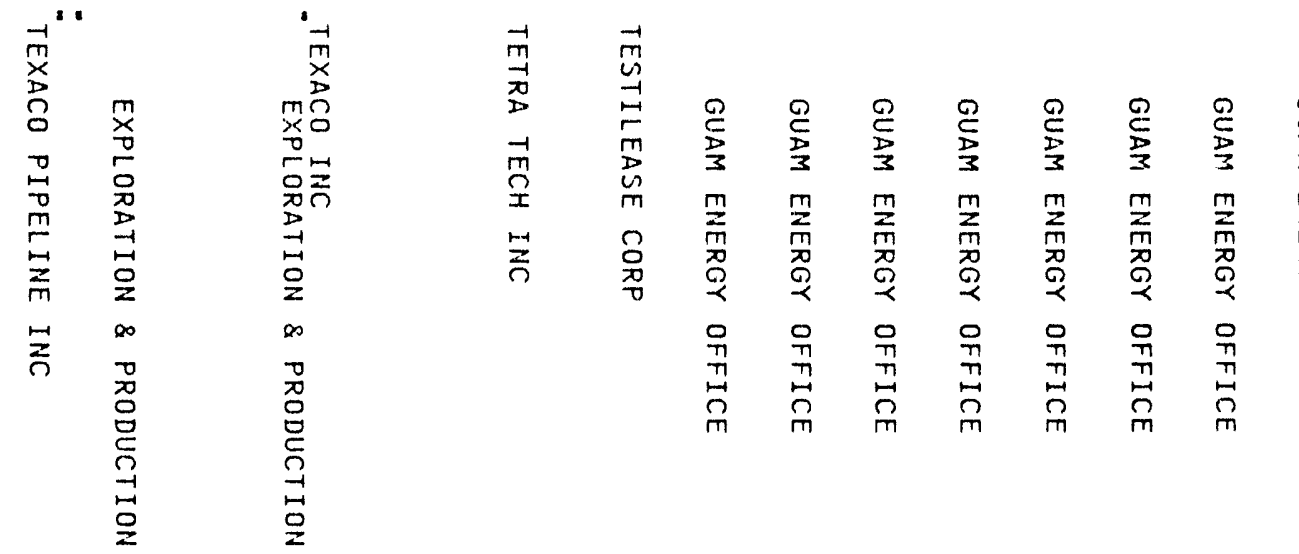

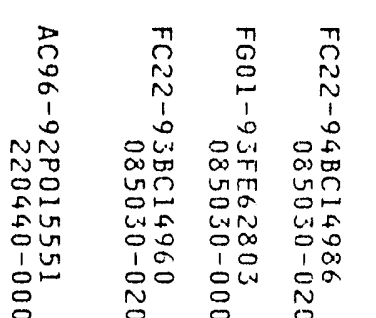

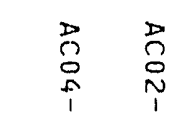

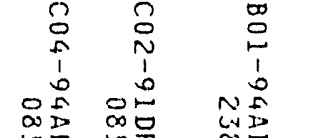

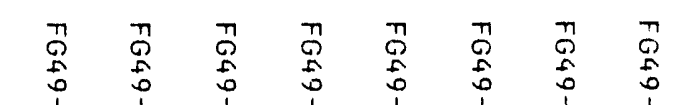

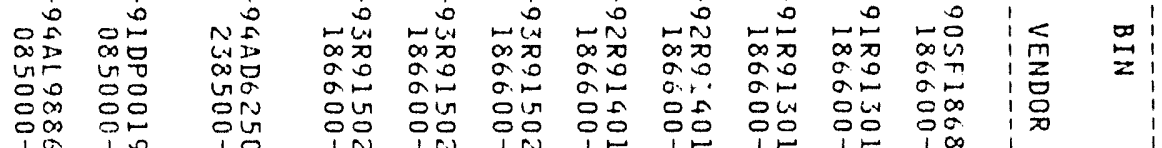

向

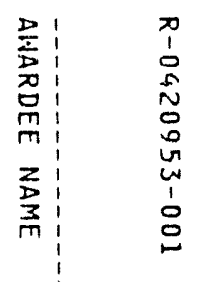

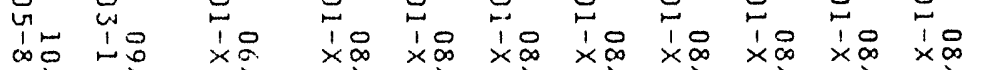

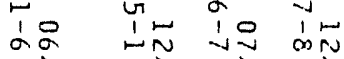

ì w

iे

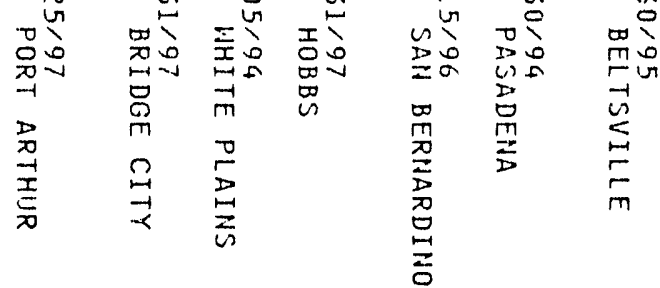

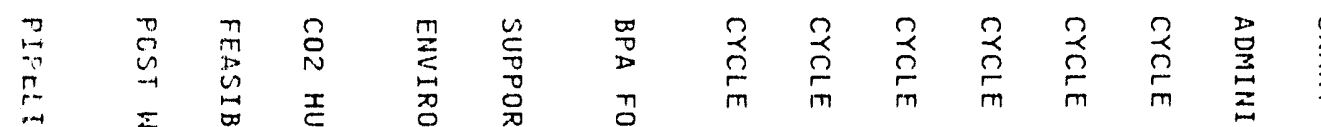

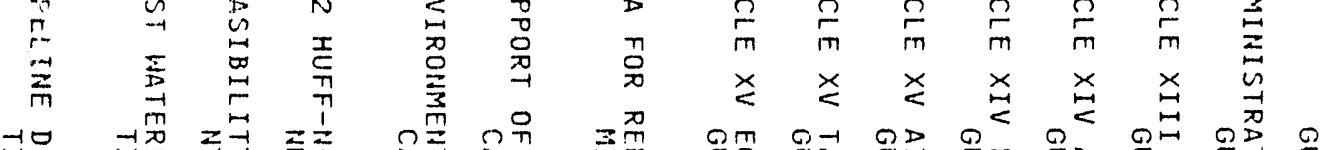

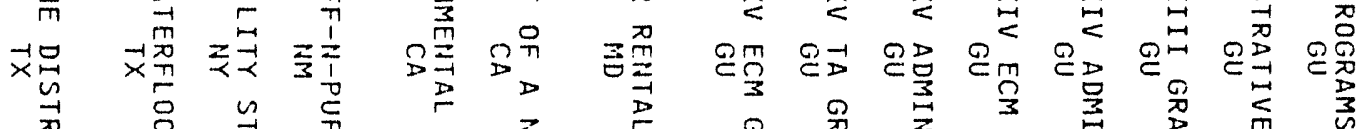

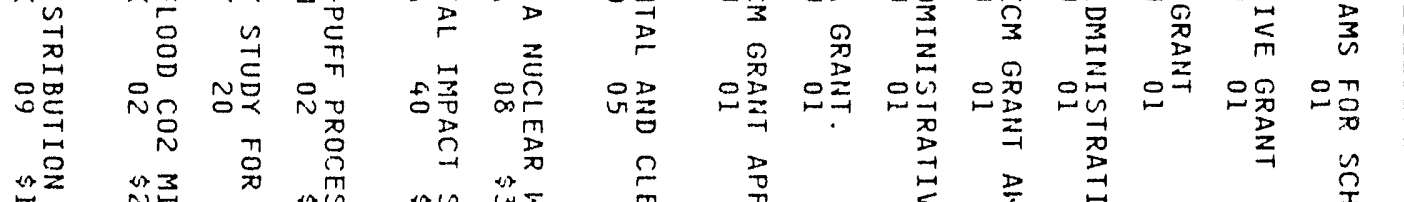

紊

몾즁

空员

草学

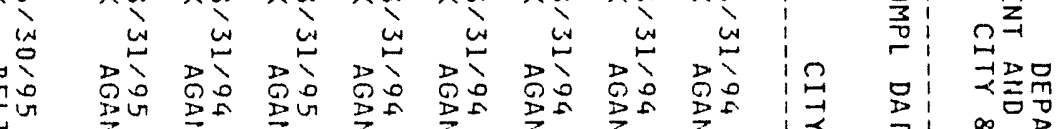

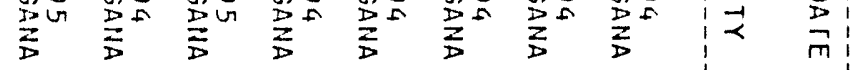

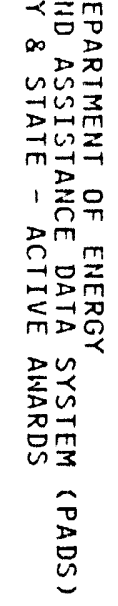

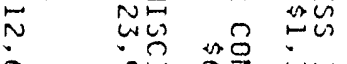

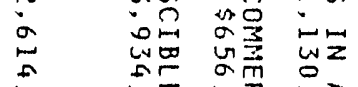

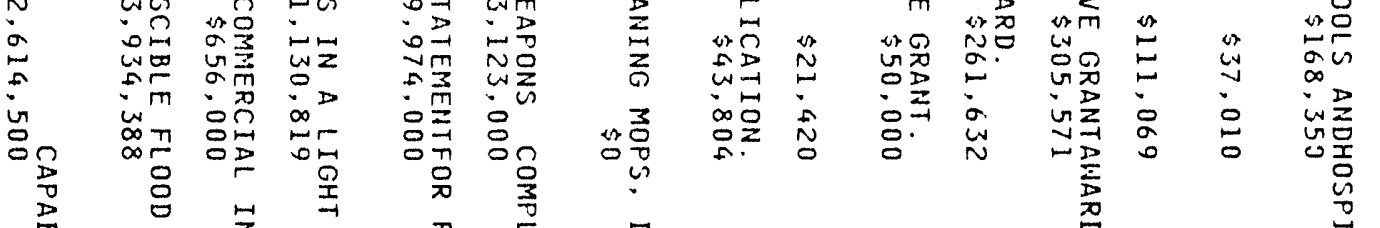

$\stackrel{m}{=}$

$\frac{2}{m}$

品:

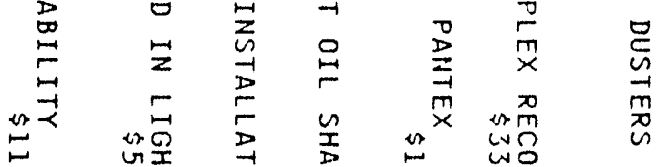

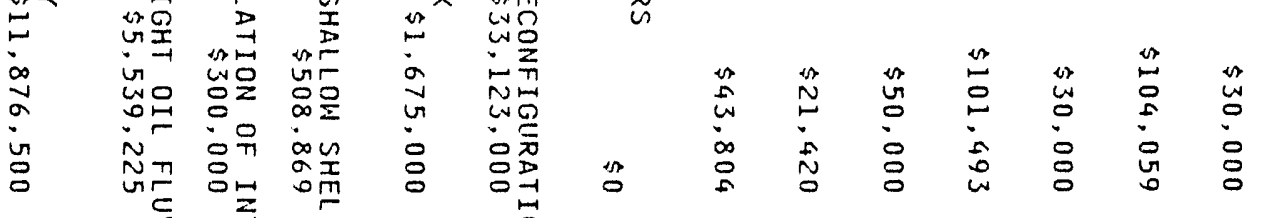

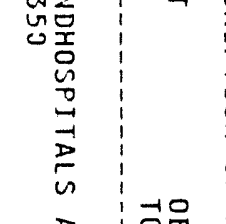

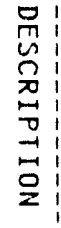

趇

可

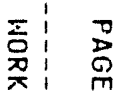

$<\quad \frac{\pi}{\pi} \pi$

$\underset{5}{5}$

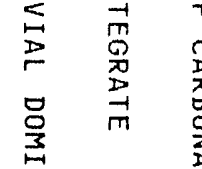

율

离

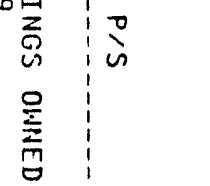

z

$\hat{\infty}$ 


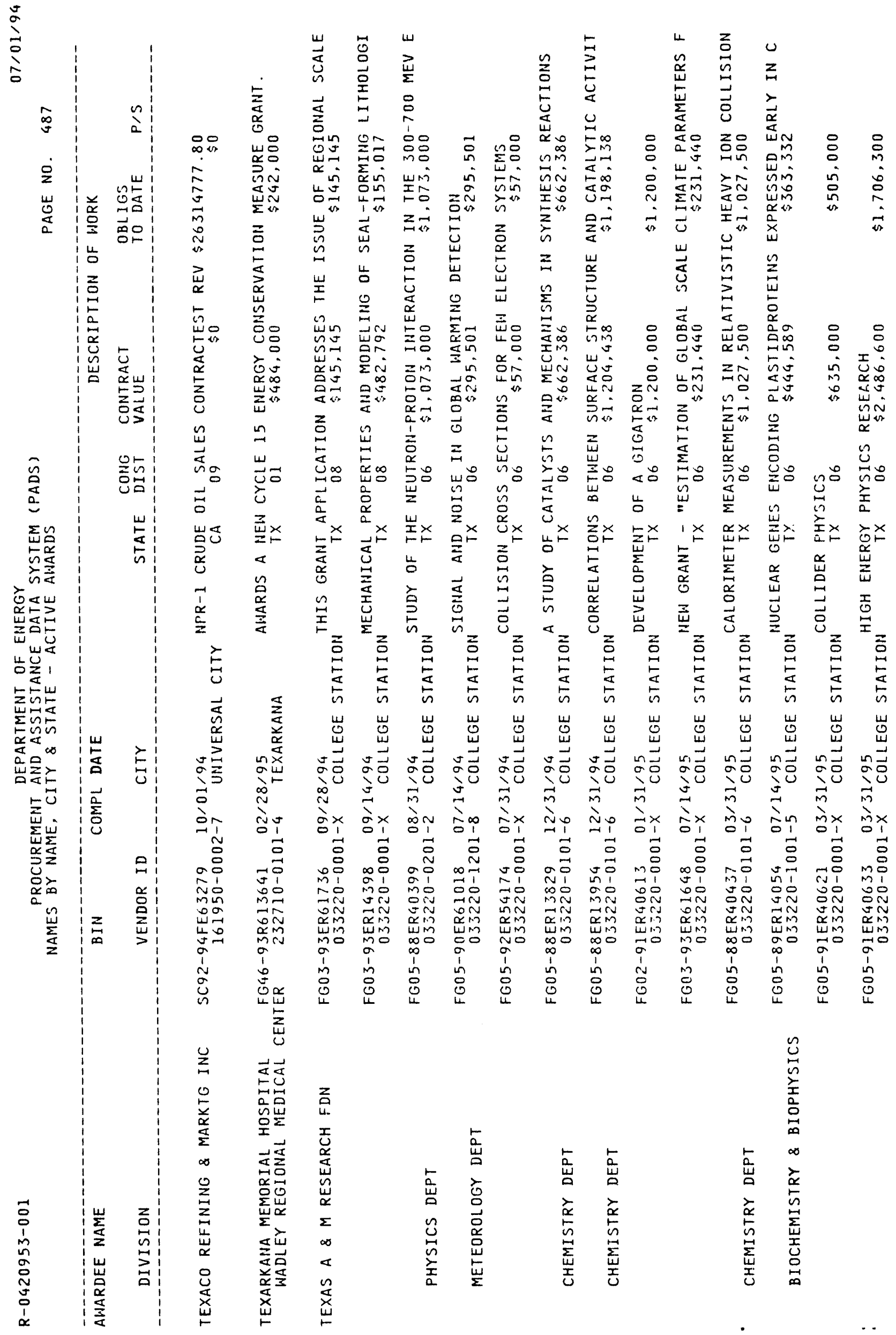




AWARDEE NAME

PHYSICS DEPT

CHEMISTRY DEPT

BIN COMPL DATE

DESCRIPTION OF HORK

CONG CONTRACT OBLIGS VENDOR ID STATE DIST VALUE TO DATE P/S

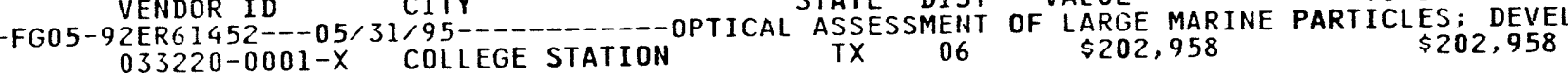

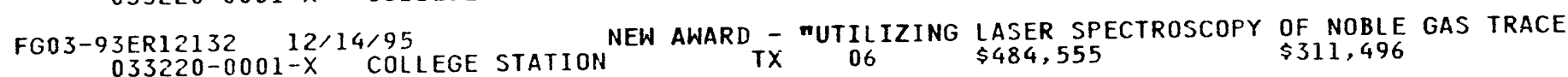
FG05-85ER13434 $11 / 30 / 95$ COLLEG STATION DEVELOPMENT OF LASER-ION PHOTODISSOCIATION METHODS FG03-93ER14354 06/30/96
$033220-0001-X$
COLLEGE STATION NEW GRANT - "SOLID STATE NMR STUDIES OF ZEOLITE ACIDITY" P.I FG03-93ER61636 06/30/96
$033220-0001-X \quad$ COLLEGE STATION NEW GRANT: MICROBIAL METHANE CYCLE IN SUBSURFACE SEDIMENTS
$\$ 272,351$

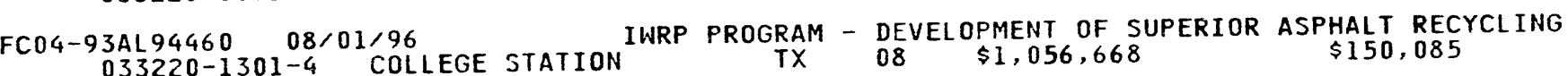
FG03-93ER40765 11/30/96 NEW AWARD - "STANDARD MODEL TESTS IN MU DECAY WITH MEGA DETE COLLEGE STATION TX S6 T220-0201-2 \$642,000

12/31/96 NEW GRANT - "A STUDY OF CATALYSTS AND MECHAN SMS IN SYNTHESI 033220-0101-6 COLLEGE STATION TX 08 T322,000

FG03-94ER61846 03/31/97 NEW GRANT - CONSIDERATIONS OF BETA AND ELECTRON TRANSPORT IN 033220-0001-X COLLEGE STATION

FG05-92ER61421 04/30/95
$033220-1403-7$ GALVESTON

THE PRODUCTION OF COLLOIDS IN THE BENTHIC BOUNDARY LAYER \& $P$

MARINE SCIENCES DEPT OF

FG03-93ER40773 12/31/95
$085070-0401-6$ COLLEGE STATION FG05-86ER40256 12/31/94 COLLEGE STATION NEW AHARD - "CYCLOTRON BASED NUCLEAR SCIENCE" PI - JOSEPH B

TEXAS A \& M UNIVERSITY CYCLOTRON INSTITUTE

PHYSICS DEPT FG05-92ER25143 08/31/95
$085070-1101-2$ COLLEGE STATION FG46-94R689409 03/31/95
$085070-1001-6$ COLLEGE STATION

AWARDS $\$ 62,000$ NEW DEPARTMENT OF ENERGY FUNDS TO INITIATE A TX 08 \$62,000

ENGINEERING EXPERIMENT STA FG46-93R613642 02/28/95
$085070-0001-0$ COLLEGE STATION FG46-93R613633 $08 / 31 / 94$ COLLEGE STATION AWARDS A NEN CYCLE 15 ENERGY CONSERVATION MEASURE GRANT. TECHNICAL ASSITTANCE GRANT 


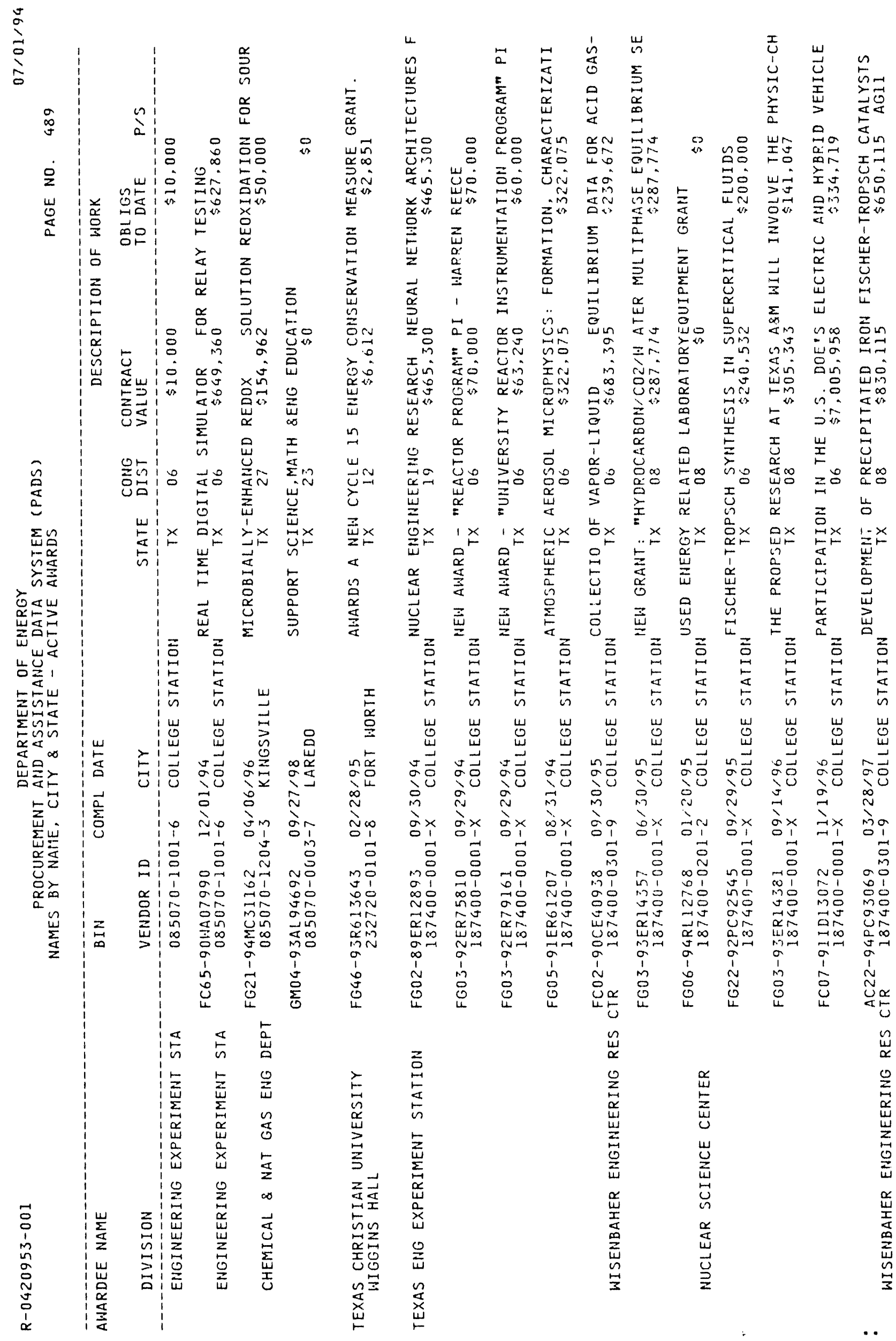


$\frac{j}{2}$

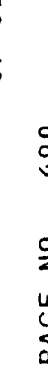

is

a

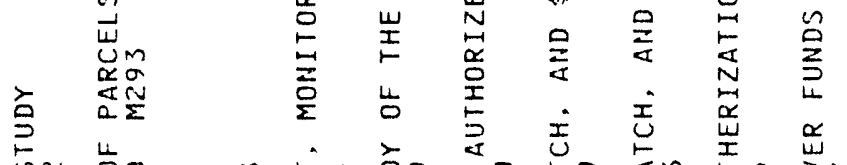

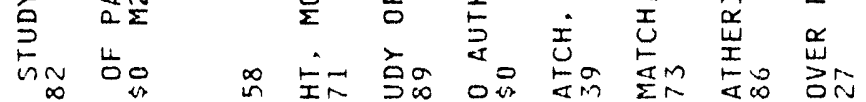

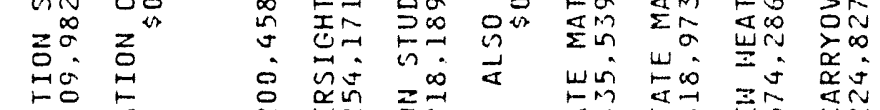

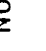

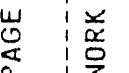

n荘!

武方吾

台

z

紫

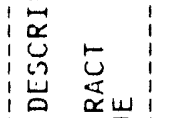

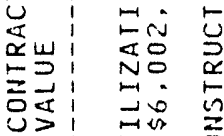

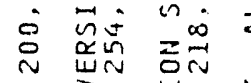

के वे 药

岩

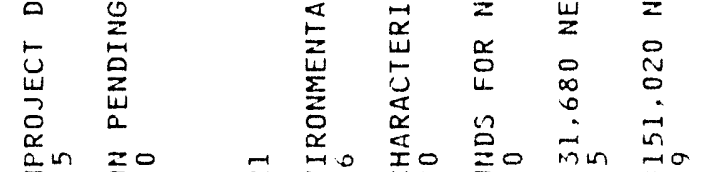

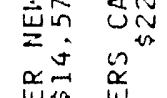

$\frac{2}{2}$

$\underset{\alpha}{a}$

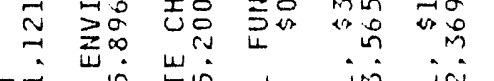

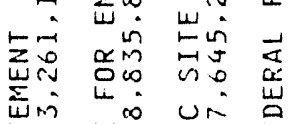

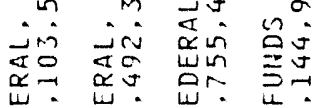

品 Ov

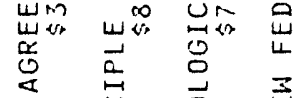

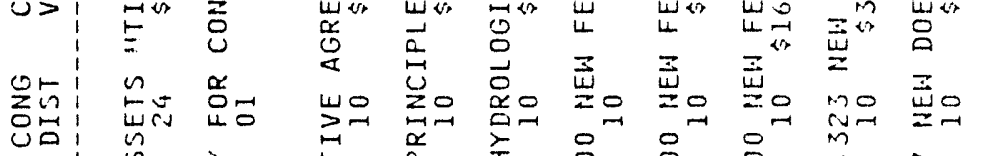

高

䑰

紋

לัญ

㲖起

山地

㟔异

䰹的

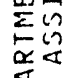

崖是文

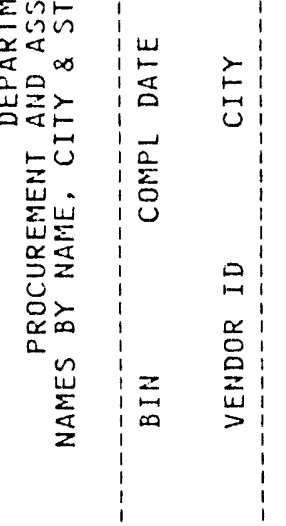

点

岁

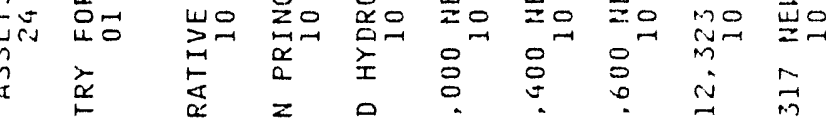

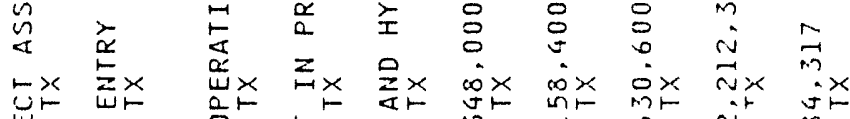

$\vec{w}$
0
0
$a$
0
in
n

${ }_{0}$

虫

강

䓌

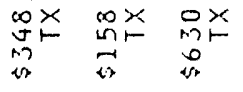

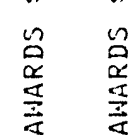

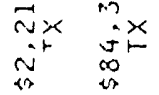

志

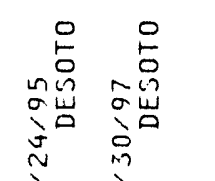

imm Iेm $_{0}$

藏

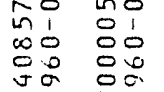

㖼离

$\begin{array}{ll}i & i \\ i & 1 \\ 0 & n \\ 0 & 0 \\ 0 & 0\end{array}$

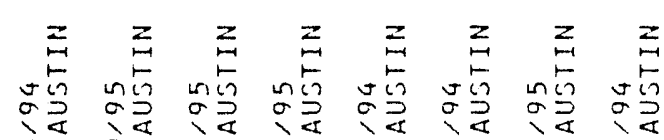

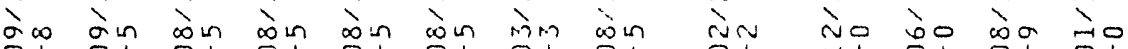

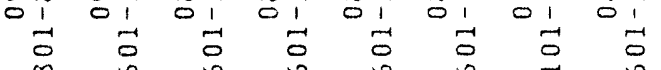

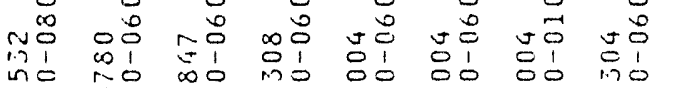

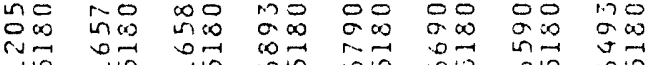

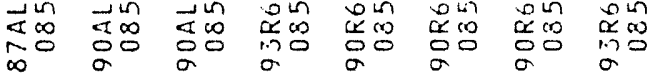

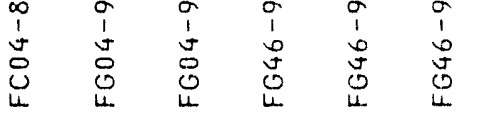

1
0
0
0
$L$

占

政

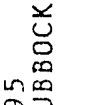

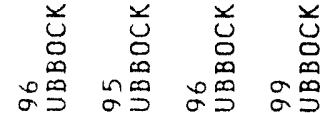

m.

$\sum_{0}$

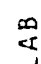

$\overrightarrow{0}$
a
z
0

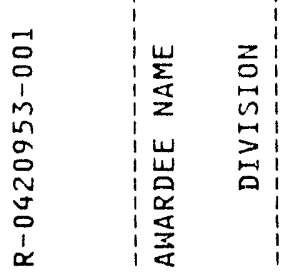

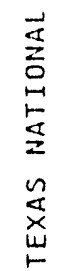

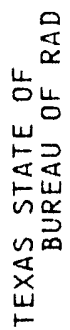

岕

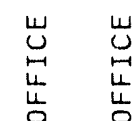

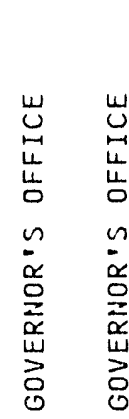

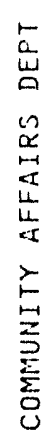

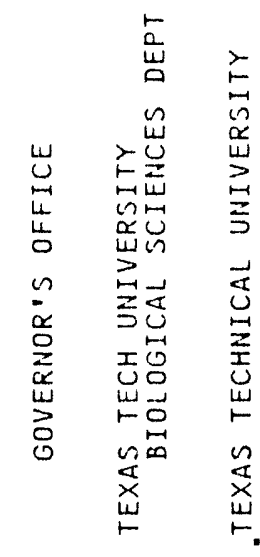

㟧

产

文 
\begin{tabular}{l}
\multirow{2}{0}{} \\
$\stackrel{2}{0}$ \\
0 \\
0
\end{tabular}

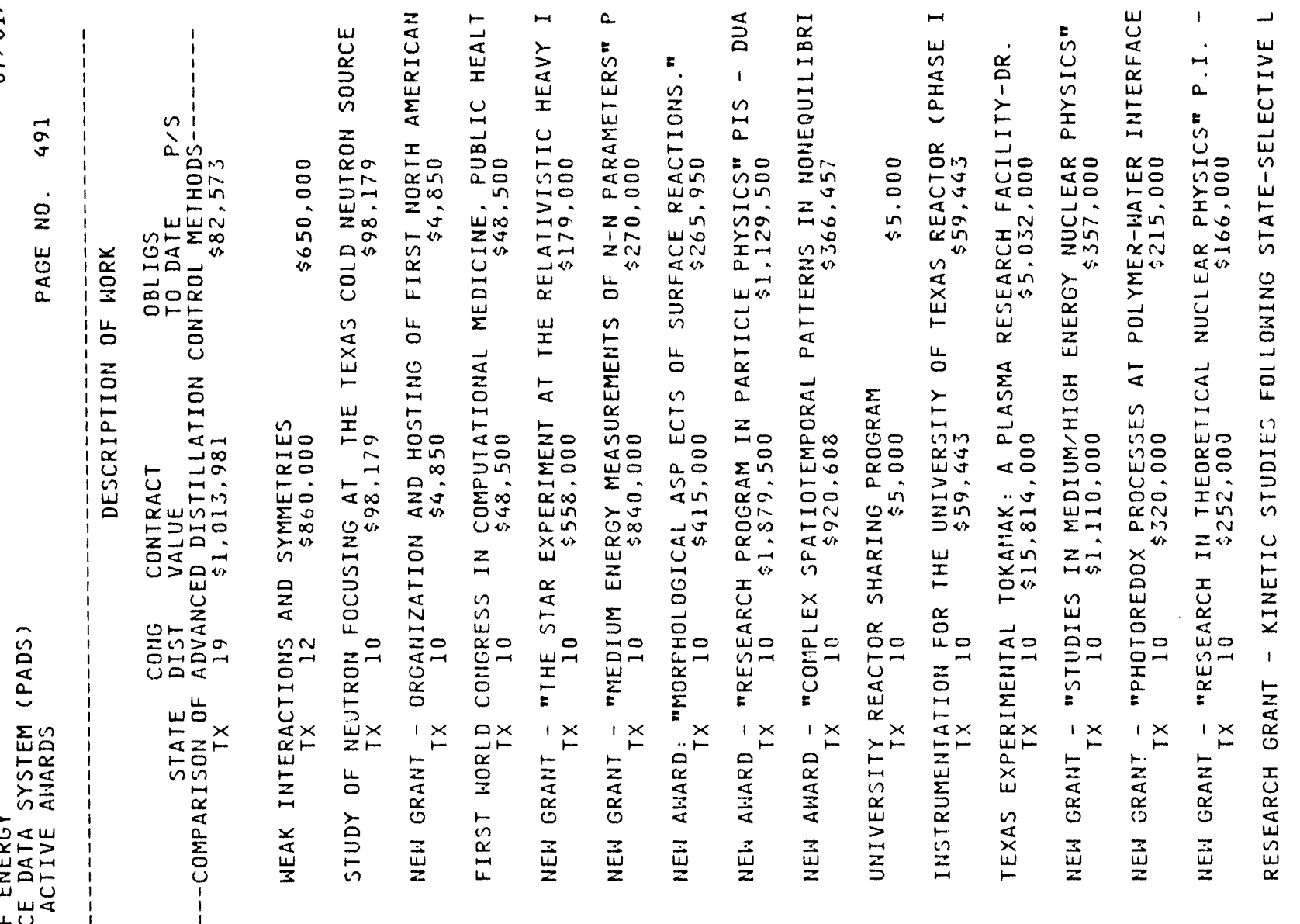

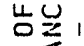

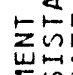

$\sum n$

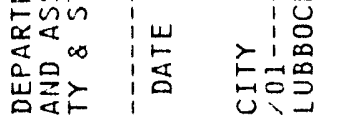

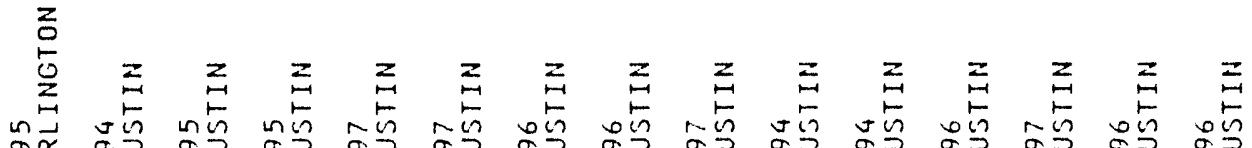
妾 金

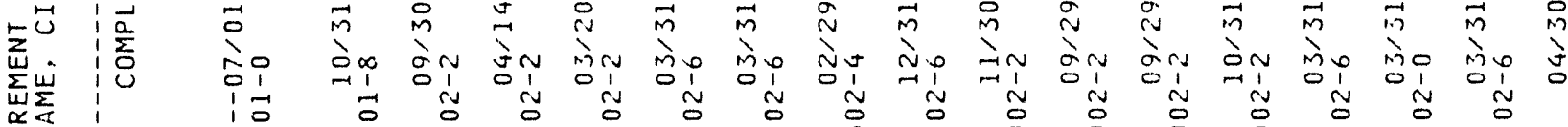

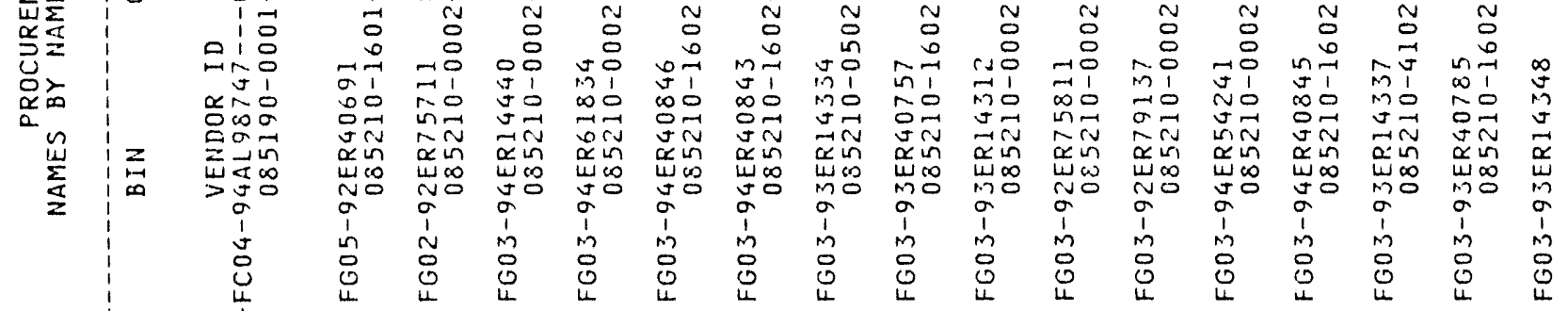

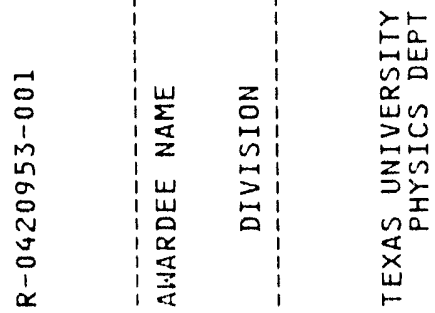

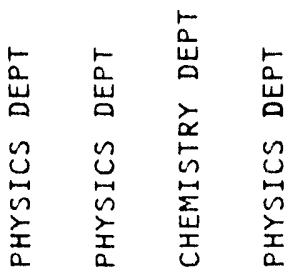

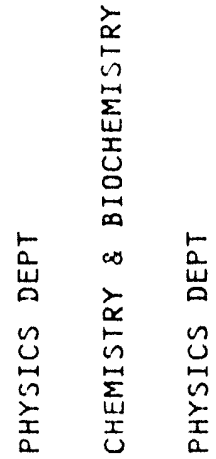




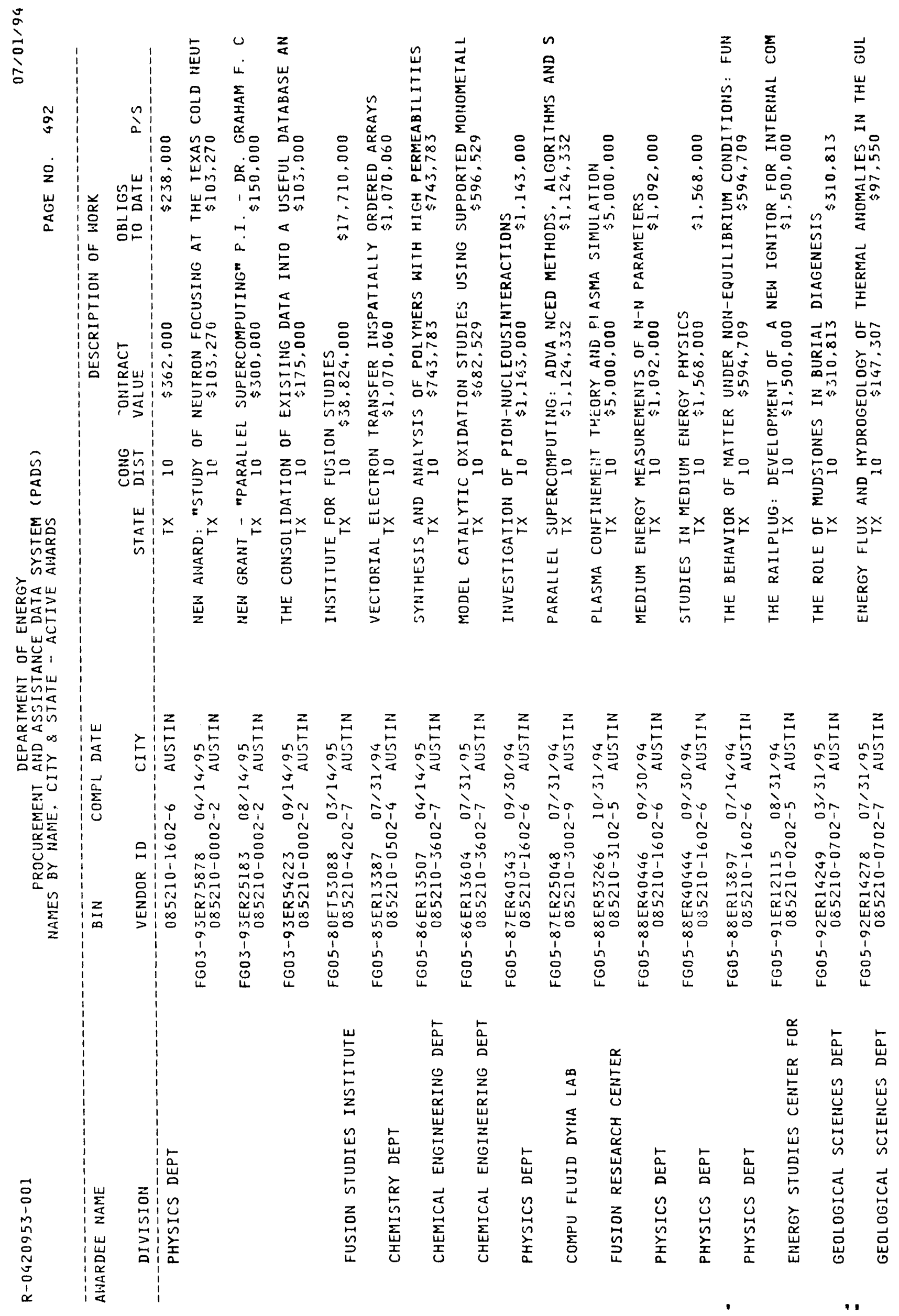




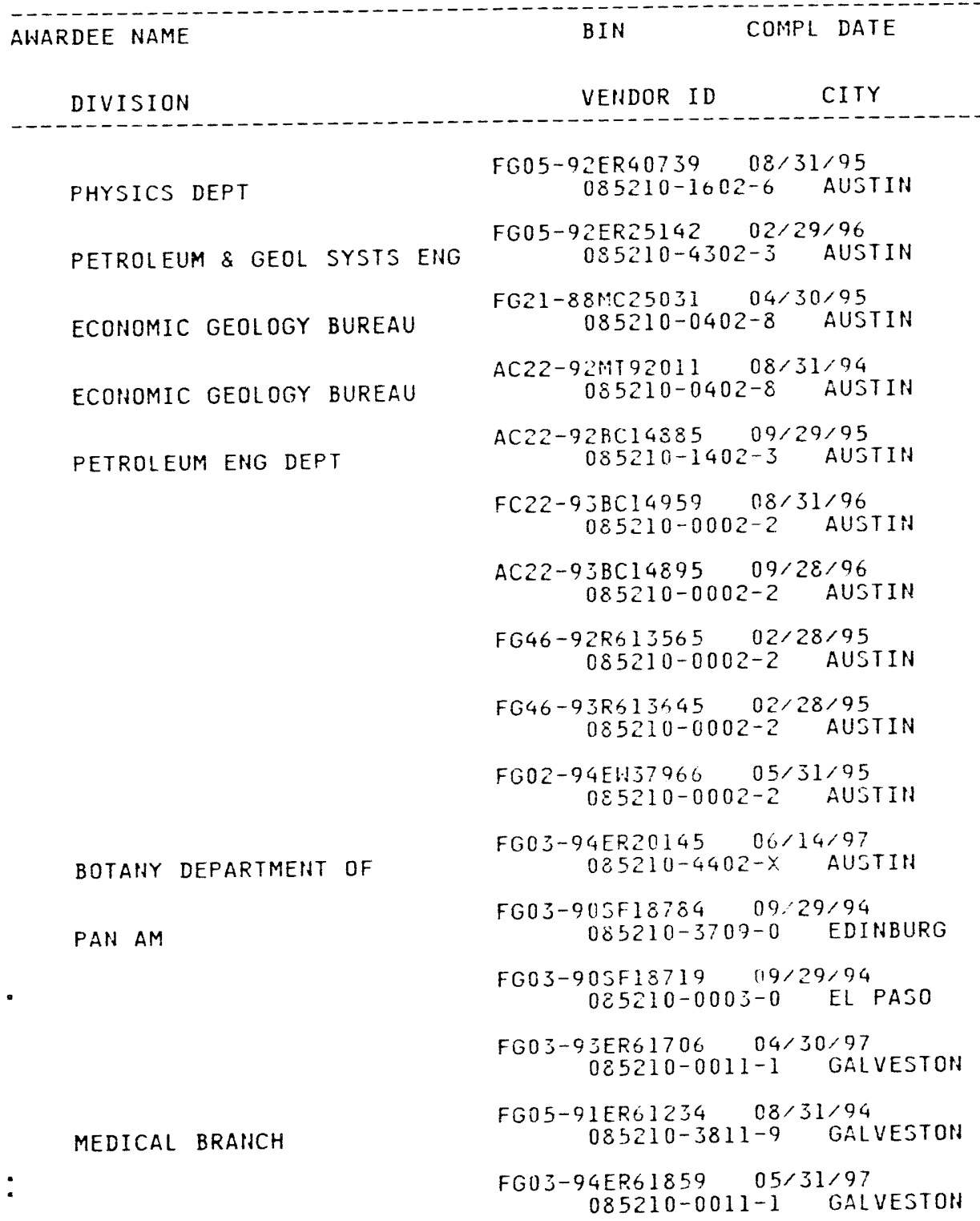

DESCRIPTION OF WORK

CONG CONTRACT OBLIGS

CALUE

DATE

$P / S$

LASER WAKEFIELD EXCITATION ANDMEASUREMENT ON A FEMTOSECOHD

$$
T X \quad 10 \quad \$ 450.000 \quad \$ 300,000
$$

PARALLEL ALGORITHMS FOR MODELING FLOW IN PERMEABLE MEDIA

$$
\text { TX } 10 \quad \$ 960,000 \quad \$ 650,000
$$

SECONDARY HATURAL GAS RECOVERYTARGETED TECHHOLOGY

HYDROGEOCHEMICAL AND PRODUCTIOH CONTROLS ON NORM IN OIL AND HYDROGEOCHEMICAL AND PRODUCTIOH CONTROLS $\$ 193,314$ N193,314 AGII DEVELOPMENT OF COST-EFFECTIVE SURFACTANT FLOODING TECHHOLOGY REVITALIZING A MATURE OIL PLAY: SIRATEGIES FOR FINDING AHD

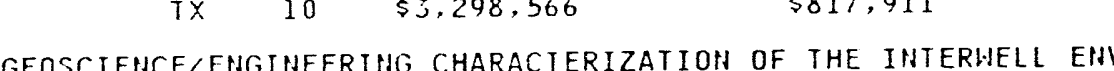
$\begin{array}{lll}T X & 10 & \$ 720,119\end{array}$ THIS IS A NEW CYCLE 14 ENERGY CONSERVATION GRAHT AHARD.
TX 10

AWARDS A NEW CYCLE 15 ENERGY CONSERVATION MEASURE GRANT

$$
\text { TX } 10 \quad \$ 155,355 \quad \$ 77,677
$$

DEVELOPMENT AND DEMOHSTRATION OF A TELECPERATED MODULAR

$$
\text { TX } 10 \quad \$ 850.000 \quad \$ 850,000
$$

HEH GRAHT - "MOLECILAR, GENETIC. AHD BIOCHEMICAL AHALYSIS OF TX $10 \quad \$ 255,840$ AND BIOCHEMICAL $\$ 166,840$

MINORITY UNDERGRADUATE TRAINING IN ENERGY-RELATED CAREERS

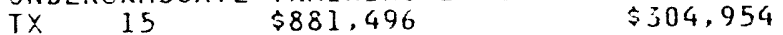
$\begin{array}{ccc}\text { MINORITY UNDERGRADUATE TRAINING IN ENERGY-RELATED CAREERS } \\ \text { TX } 16 & \$ 536.707 & \$ 363.593\end{array}$ THE HUMAN HOMOLOGS ARE BEING BIOCHEMICALLY ANALYZED AND CHEC
TX 09
$\$ 350,000$

CELLULAR GHD MOLECULAR MECHANISMS IN RADIATION CARCINOGENES TX $09 \quad \$ 654,328 \quad \$ 654,328$ NEN GRANT - "LATENT EXPRESSIONOF GENETIC DAMAGE IN HUMAN LU
TX
09 


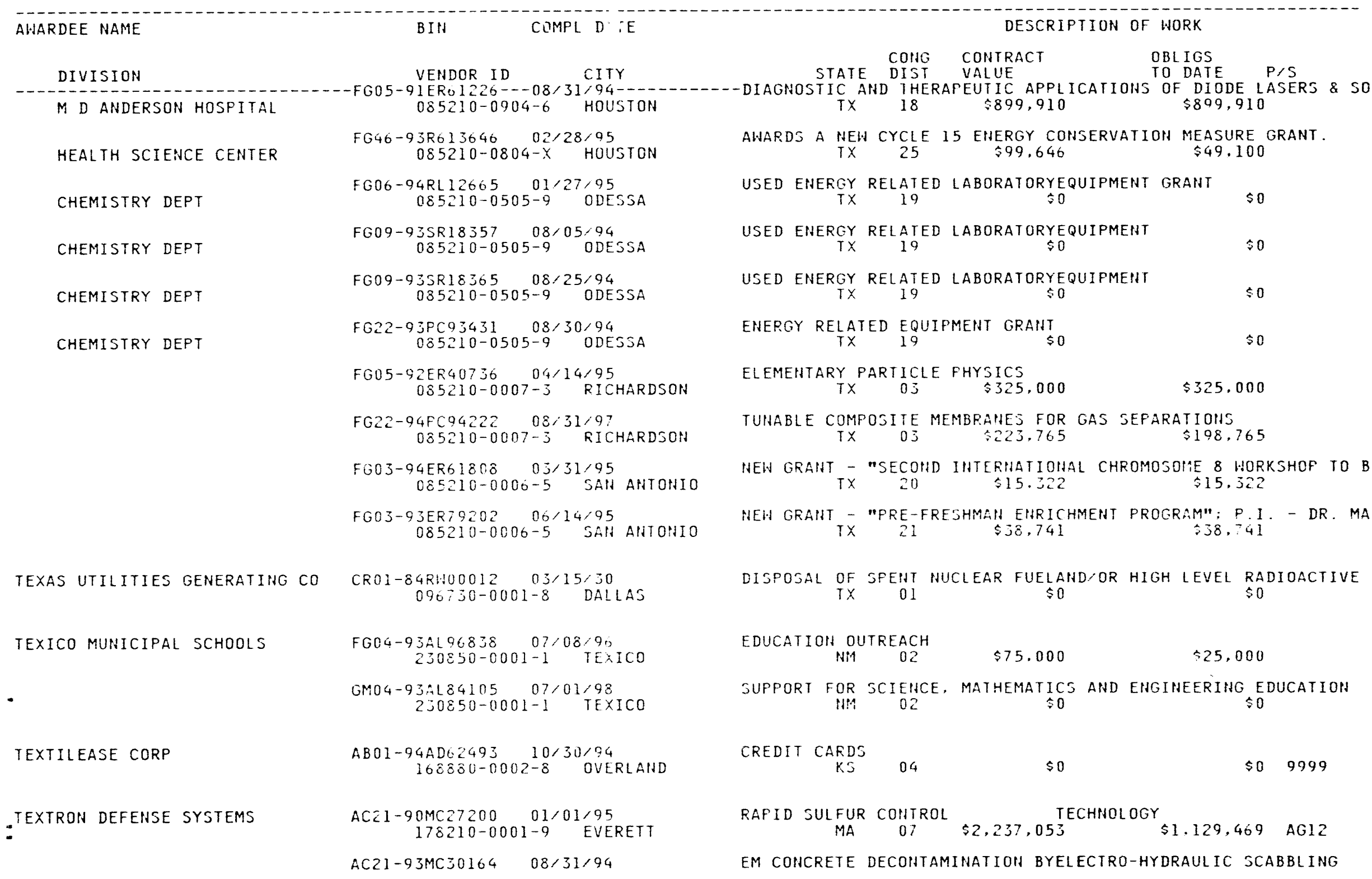




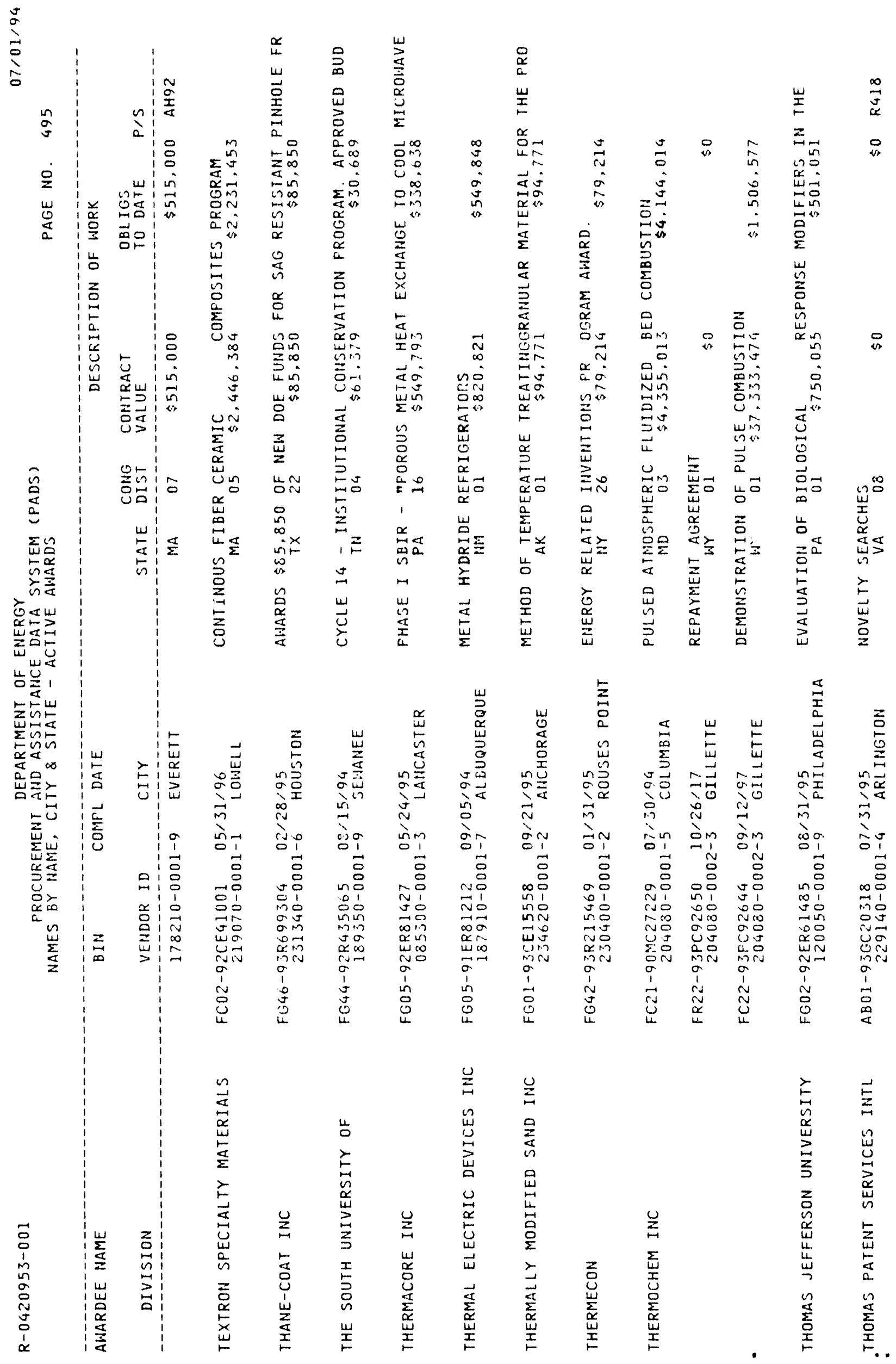


0
0
0
0
0

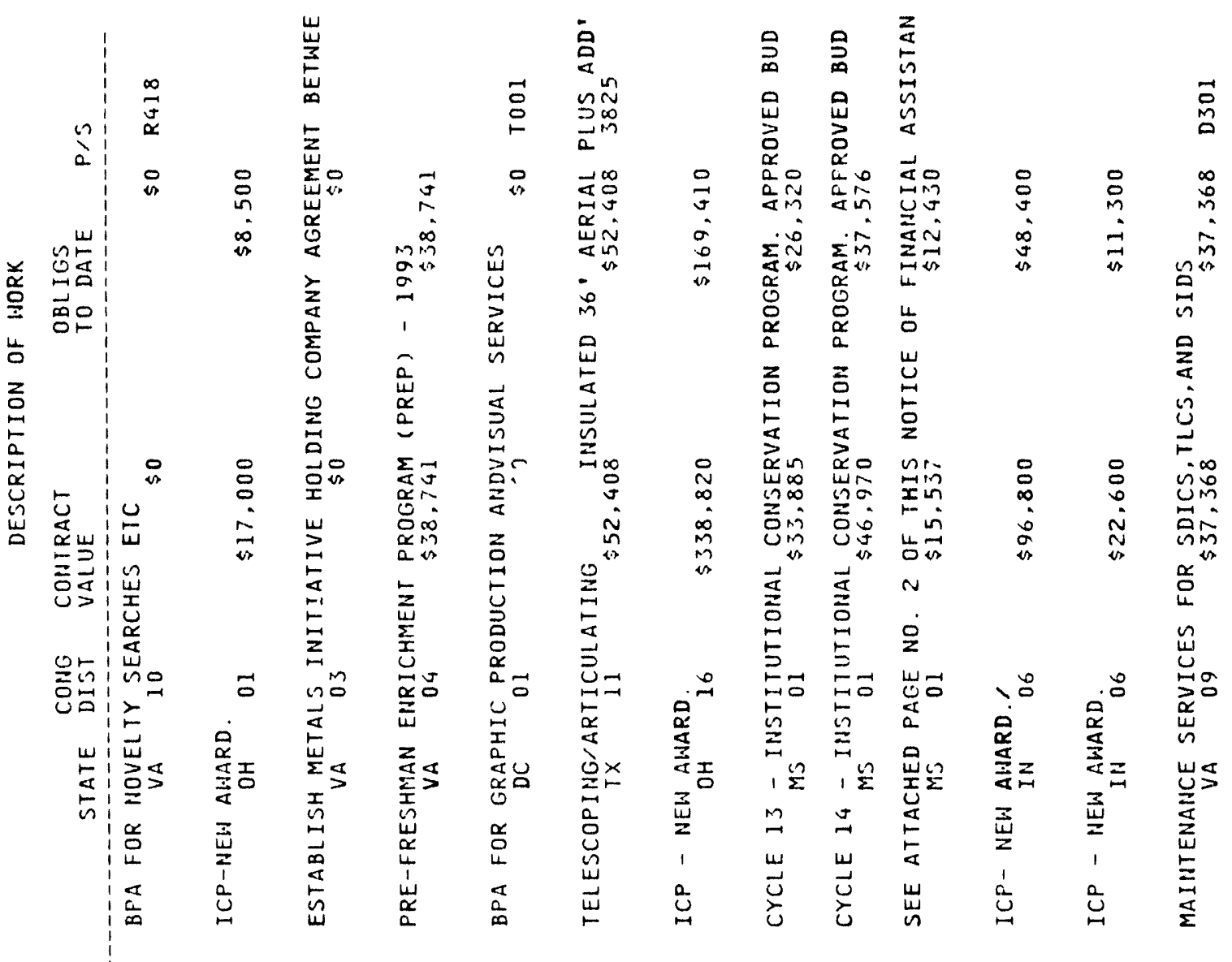

ర大षw

和㲾

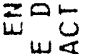

范岸

产的

崖出

sin

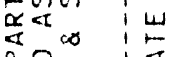

z

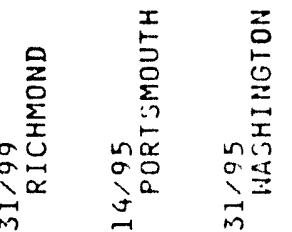

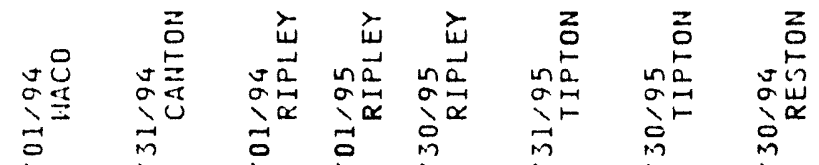

昰式

崖宗

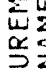

这.

㐌

ב:

点

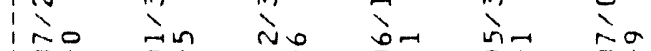

10

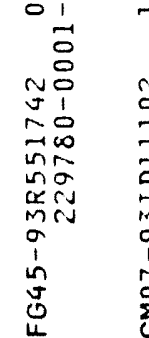

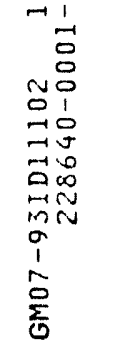

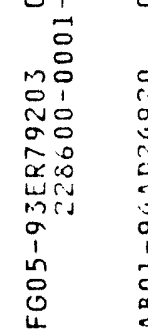

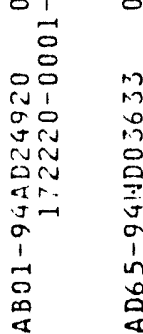

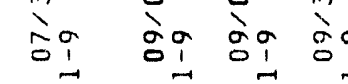

मै

音

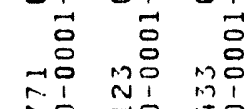

जis ñ nis

$\alpha$ iso

잉요

1.70 ơ

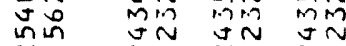

am

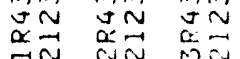

$\begin{array}{ccc}0 & 0 \\ 0 & 0 & 0 \\ 0 & 0 & 0 \\ 0 & 0 & 0 \\ 0 & 0 & 0\end{array}$

in

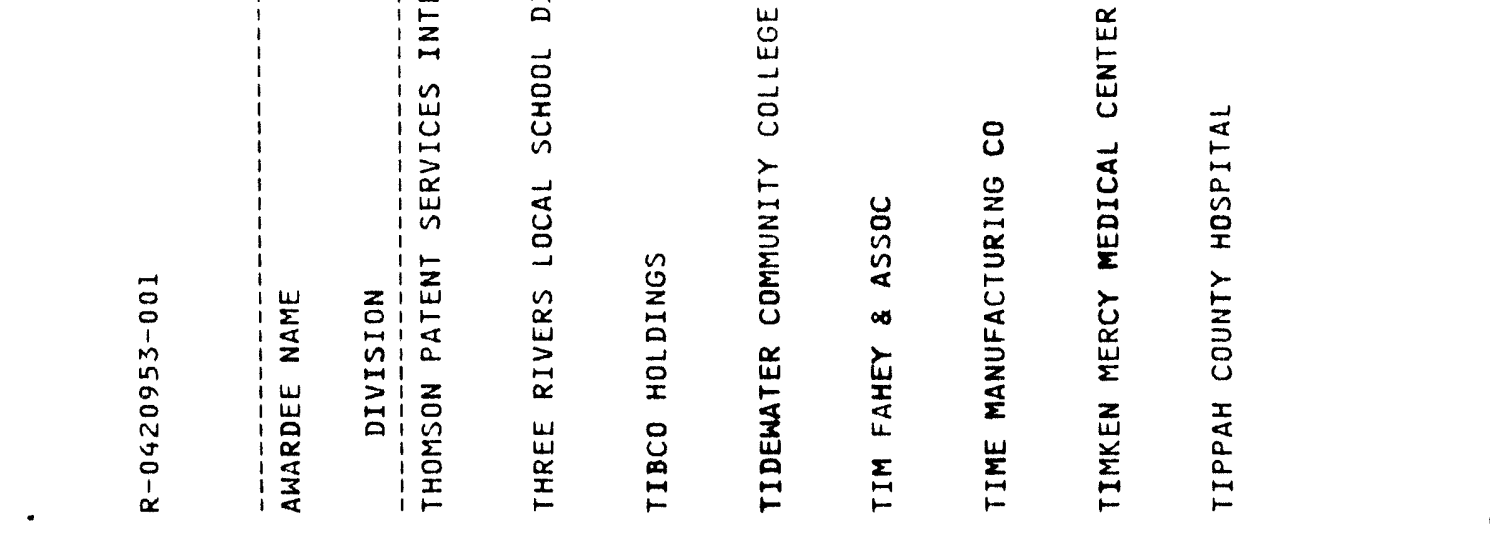

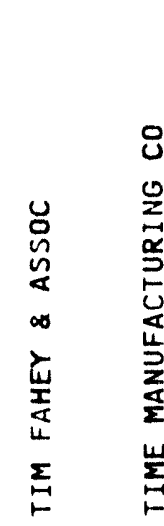

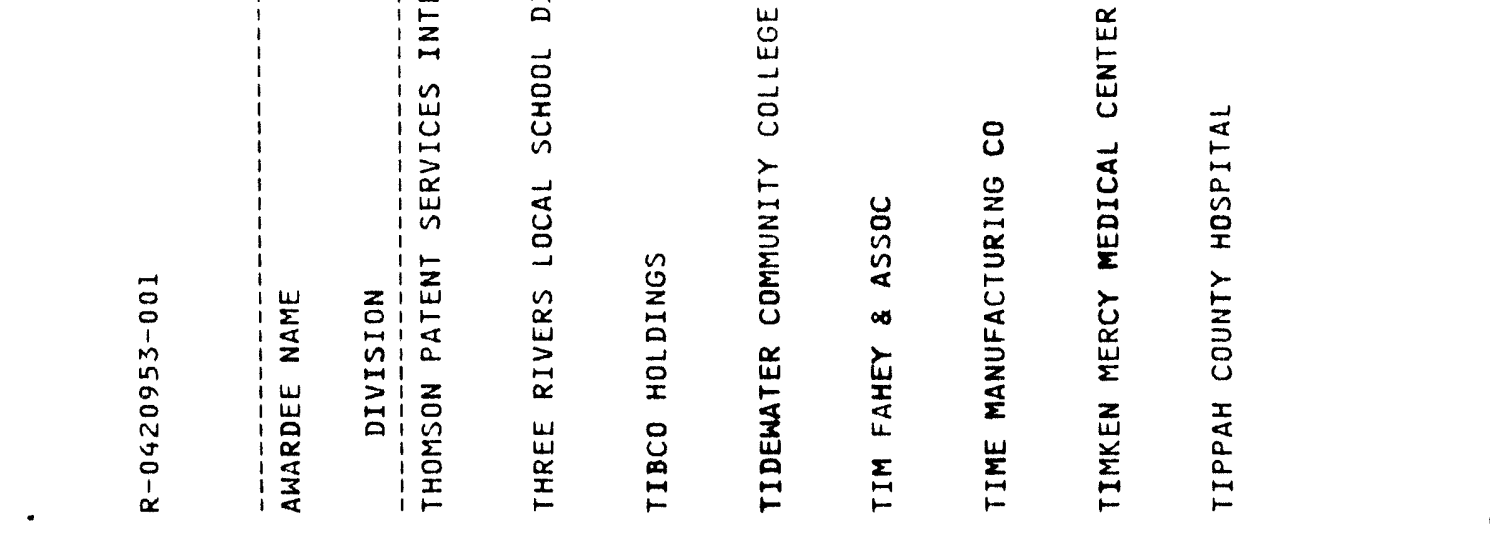

옹

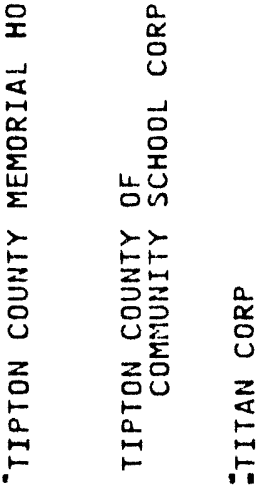




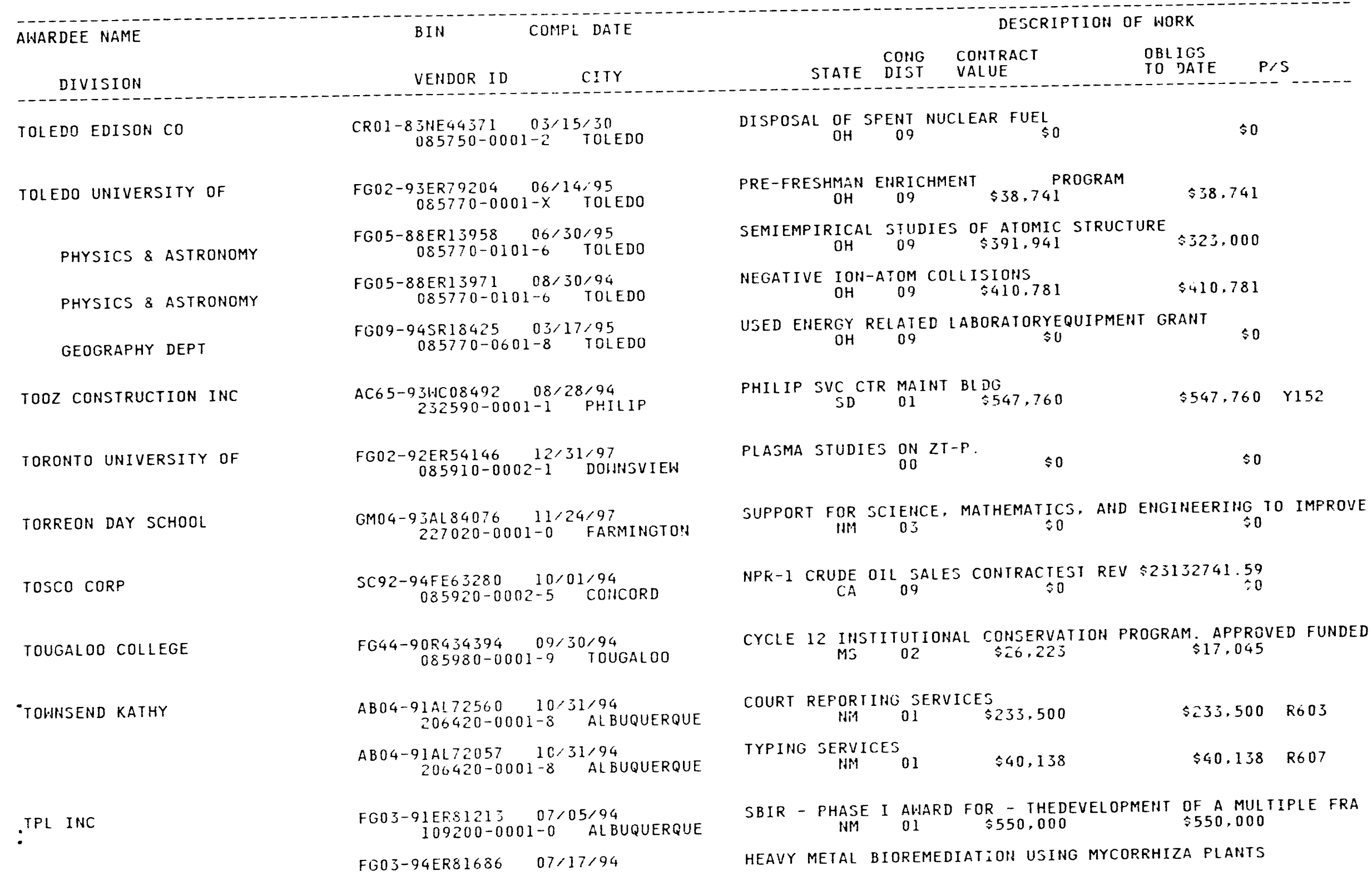




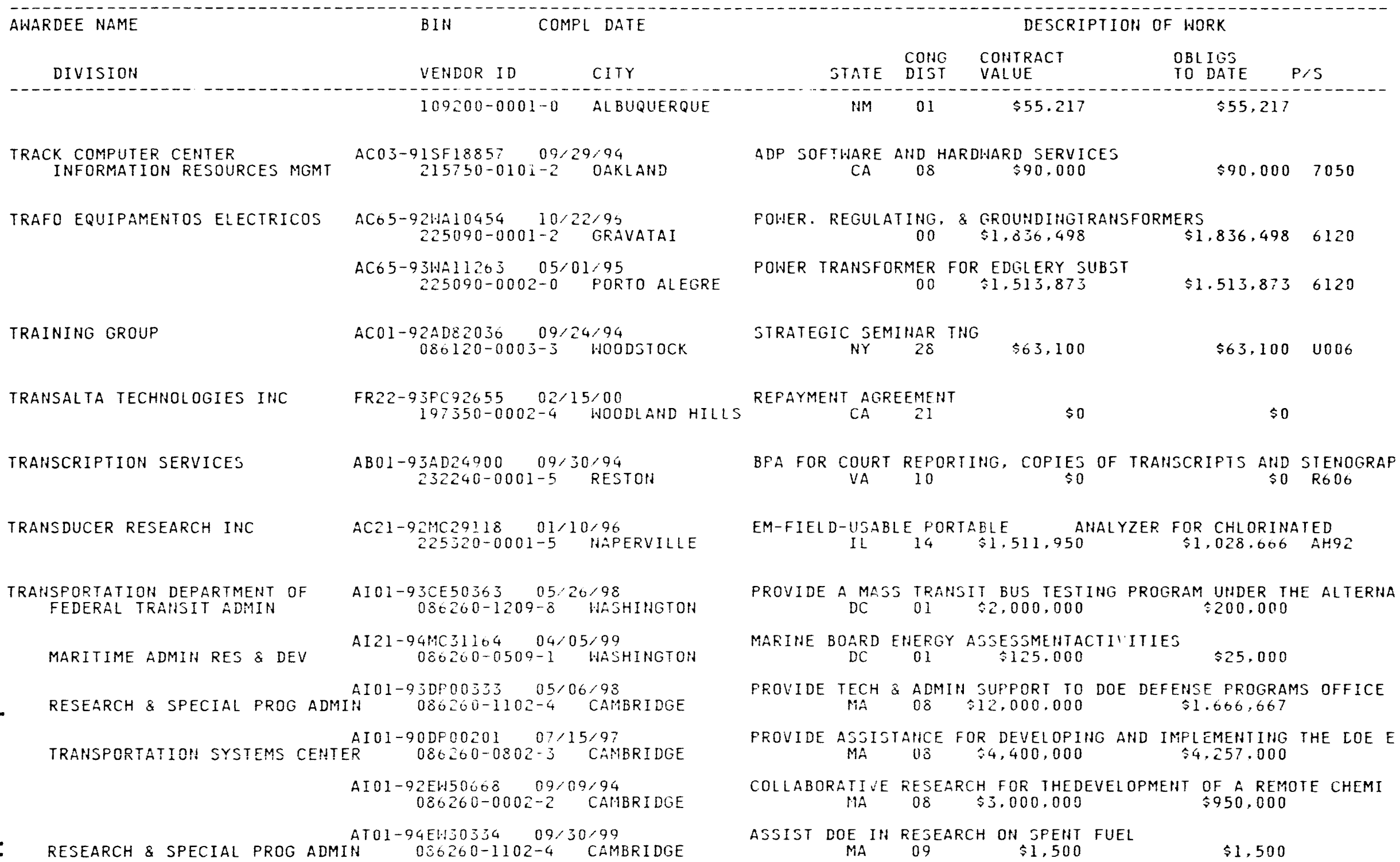




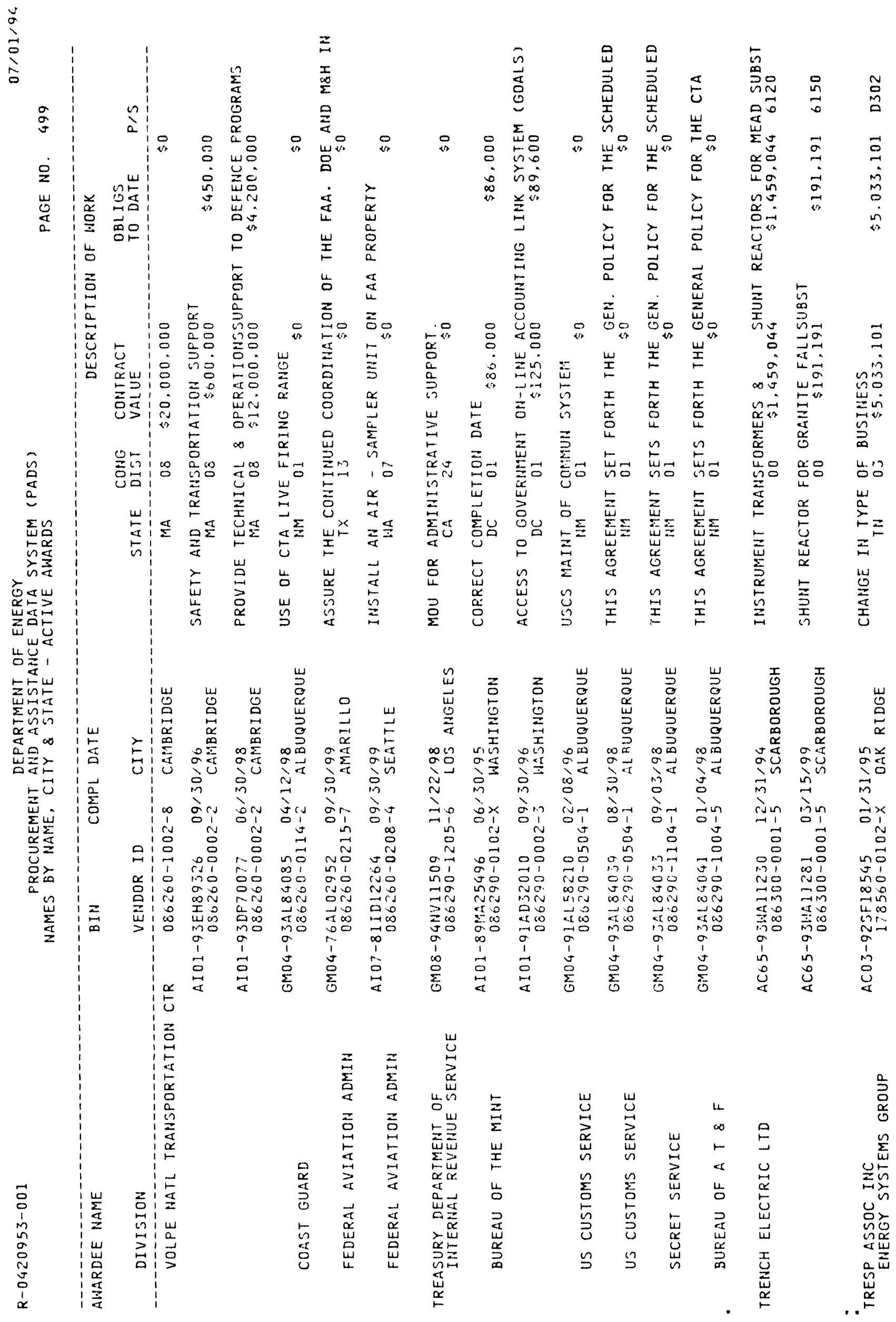




\begin{abstract}
AWARDEE NAME
\end{abstract}

BIN
COMPL DATE DESCRIPTION OF HORK

DIVISION

CITY
STATE COHG CONTRACT VALUE

AC01-94DP70073 12/10/96 178560-0001-5 ALEXANDRIA FG05-940R2221203/31/95 178560-0001-5 ALEXANDRIA AC05-880R21850 09/30/94 $178560-0001-5$ AL EXANDRIA AT21-94MC $31247 \quad 09 / 30 / 94$ 178560-0003-1 MORGANT OWN AT21-94MC 31165 11/30/94 178560-0003-1 MORGANTOWN AT21-93MC30378 $09 / 30 / 94$ 178560-0003-1 MORGANTOHN AT21-93MC30367 09/30/94 178560-0003-1 MORGANTOIN AM21-93MC $3031409 / 20 / 94$ $178560-0003-1$ MORGANTOHN AT21-94MC31335 07/29/94 $178560-0003-1$ MORGANTOHN

TREVECCA NAZARENE COLLEGE

$$
\begin{gathered}
\text { FG06-94RL12835 03/19/95 } \\
209600-0001-1 \text { NASHVILLE }
\end{gathered}
$$

FG09-93SR $18366 \quad 08 / 26 / 94$ 209600-0101-8 NASHVILLE

SCIENCE \& MATHEMATICS

SCIENCE \& MATHEMATICS TRI-ARK INDUSTRIES INC TRI-COR INDUSTRIES INC TRI-O INC

$$
\begin{aligned}
& 1 P C 90740 \quad 10 / 31 / 94 \\
& 215870-0001-6 \quad \text { PITTSBURGH }
\end{aligned}
$$

AC65-93WJ21469 10/09/94 195880-0002-5 THERMOPOL IS

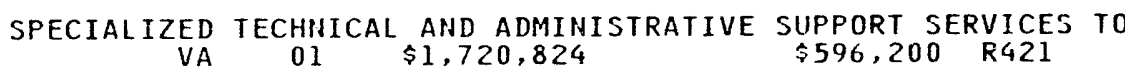
$\begin{array}{llll}\text { MINORITY OWNED BUSINESS TECHNOLOGY TRANSFER PILOT } \\ \text { VA } & 08 & \$ 300,000 & \$ 300,000\end{array}$ FUND ADP/TELECOMMUNICATION SUPPORT SERVICE CONTRACT (TRESP A $\begin{array}{llll}\text { VA } 08 & \text { \$22,161,290 } & \$ 18,632,869 & \text { D399 }\end{array}$

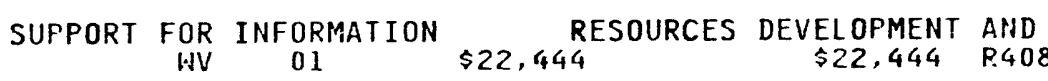

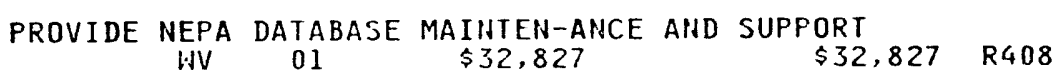
PROVIDE FOR THE MAI:: ENANCE OF DATA TO TRACK ASSESSMENTS FOR THE MAI: : ENANCE OF DATA TO TRACK ASSESSMENTS
WV 01 SUPPORT SERVICE OF INFORMATIONTECHNOLOGY RESOURCES AT METC
WV 01 SUPPORT SERVICES FOR INFORMA- TION TECHNOLOGY RESOURCES $\begin{array}{llllll}\text { PROVIDE FDDI } & \text { BACKBONE CABLE INSTALLATION } & & \\ \text { WV } & 01 & \$ 26,938 & \$ 26,938 & \text { R408 }\end{array}$

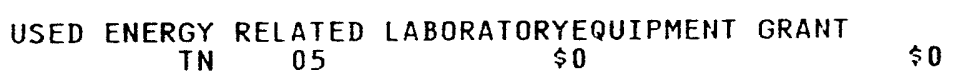
USED ENERGY RELATED LABORATORYEQUIPMENT
TH
06 USED ENERGY RELATED LAB EQUIPMENT GRANT

$\begin{array}{ccccc}\text { CLEANING AND UTILITY SERVICES FOR DOE FORRESTAL BUILDING } \\ \text { DC } & 01 & \$ 1,328,688 & \$ 1,328,688 & \text { S201 }\end{array}$ INFORMATION PROCESSING AHD AUTOMATED DATA PROCESSING SUPPORT PA 03 \$ $16,519,601 \quad \$ 7,731,649$ R421

THERMOPOLIS-CARTER MTN $230 \mathrm{KV}$ TLINE WY 01 \$4,659,836 $\$ 4,659,836 \quad$ Y239 


\begin{tabular}{|c|c|c|c|c|c|c|c|}
\hline AWARDEE NAME & BIN & L DATE & & & DESCF & $=$ WORK & \\
\hline DIVISION & VENDOR ID & CITY & STATE & $\begin{array}{l}\text { CONG } \\
\text { DIST }\end{array}$ & $\begin{array}{l}\text { CONTRACT } \\
\text { VALUE }\end{array}$ & $\begin{array}{l}\text { OBL IGS } \\
\text { TO DATE }\end{array}$ & $P / S$ \\
\hline
\end{tabular}

TRI-STATE ENGINEERING \& MGMT

TRI-TECHNEC INC

TRIANGLE COALITION FOR SCIENCE

TRIMBLE LOCAL SCHOOL DISTRICT

TRINITY BAY CONSERVATION DIST

TROPIX INC

TROY STATE UNIVERSITY

TROY SYSTEMS INC
AT21-94MC $31306 \quad 08 / 01 / 94$ 098730-0005-4 MORGANTOHN

AT21-94MC $31295 \quad 07 / 29 / 94$ $098730-0005-4$ MORGANTOHN AT21-94MC $3132108 / 10 / 94$ MARTINSVILLE

AT21-94MC31320 07/29/94 MARTINSVILLE

AM21-94MC $30329 \quad 11 / 07 / 94$ $098730-0002-X$ HEW MARTINSVILLE AC65-91WN08658 08/03/94
$216270-0001-1 \quad$ OAKDALE

FG02-94ER75946 09/14/94
$222090-0001-7$ COLLEGE PARK

FG02-93ER75896 08/14/94 222090-0001-7 COLLEGE PARK

FG45-93R551733 01/31/95 $229720-0001-3$ GLOUSTER

AC96-93P017036 02/04/98 $3 P 017036002 / 04 / 98$
$228180-0001-7$ ANAHUAC

FG05-92ER81389 05/24/95 $2 E R 81389$
$188280-0001-5 \quad$ BEDFORD

FG44-91R434753 12/31/94 086740-0001-0 TROY

AC01-92EI22264 09/01/94 $224050-0001-6$ ALEXANDRIA
INSTALLATION OF PHOTO LAB $\underset{\text { WV }}{01} \stackrel{\text { EXHAUST HOOD IN BUILDING }}{\$ 18,529}$

BACKUP POHER SYSTEM FOR THE B-2 COMPUTER ROOM

WV $01 \quad \$ 125,546 \quad \$ 125,546 \quad Z 111$

DESIGN OF AUTOMATIC WET SPRINKLER/ALARM SYSTEM

$\begin{array}{llll}\text { WV } 01 & \$ 23,725 & \$ 23,725 \mathrm{C} 1\end{array}$

DESIGN OF MOTOR FOOL GARAGE FACILITY

HV $01 \quad \$ 29,959 \quad \$ 29,959 \quad$ C21

ARCHITECT/ENGINEER SERVICES

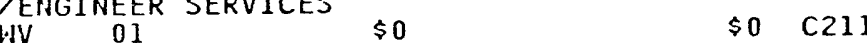

CONSTRUCTION OF HEW MELONES SHITCHING STATION STAGE 02
$\$ 771,589$

COHGRESSIONAL FELLOWS PROGRAM

$M D \quad 05 \quad \$ 50,440 \quad \$ 50,440$

ELURIBUS UNUM: ROLE OF COLLABORATION IN PERFORM IN MD $\quad 04 \quad \$ 17,282 \quad \$ 17,282$

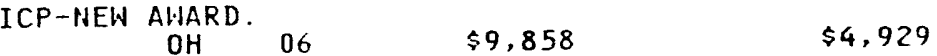

POTABLE WATER FOR BIG HILL
TX 09 \$1,106,240
$\$ 1,106,240 \quad \$ 114$

CHEMILUMINSECENT MULTIPRIMED DNA SEQUENCING

$M A \$ 3 \quad \$ 550,000 \quad \$ 378,716$

CYCLE 13 - INSTITUTIONAL COHSERVATION PROGRAM. APPROVED BUD

COMPUTER SECURITY SUPPORT SERVICES

VA $10 \quad \$ 606,628 \quad \$ 606,628 \quad$ D310 


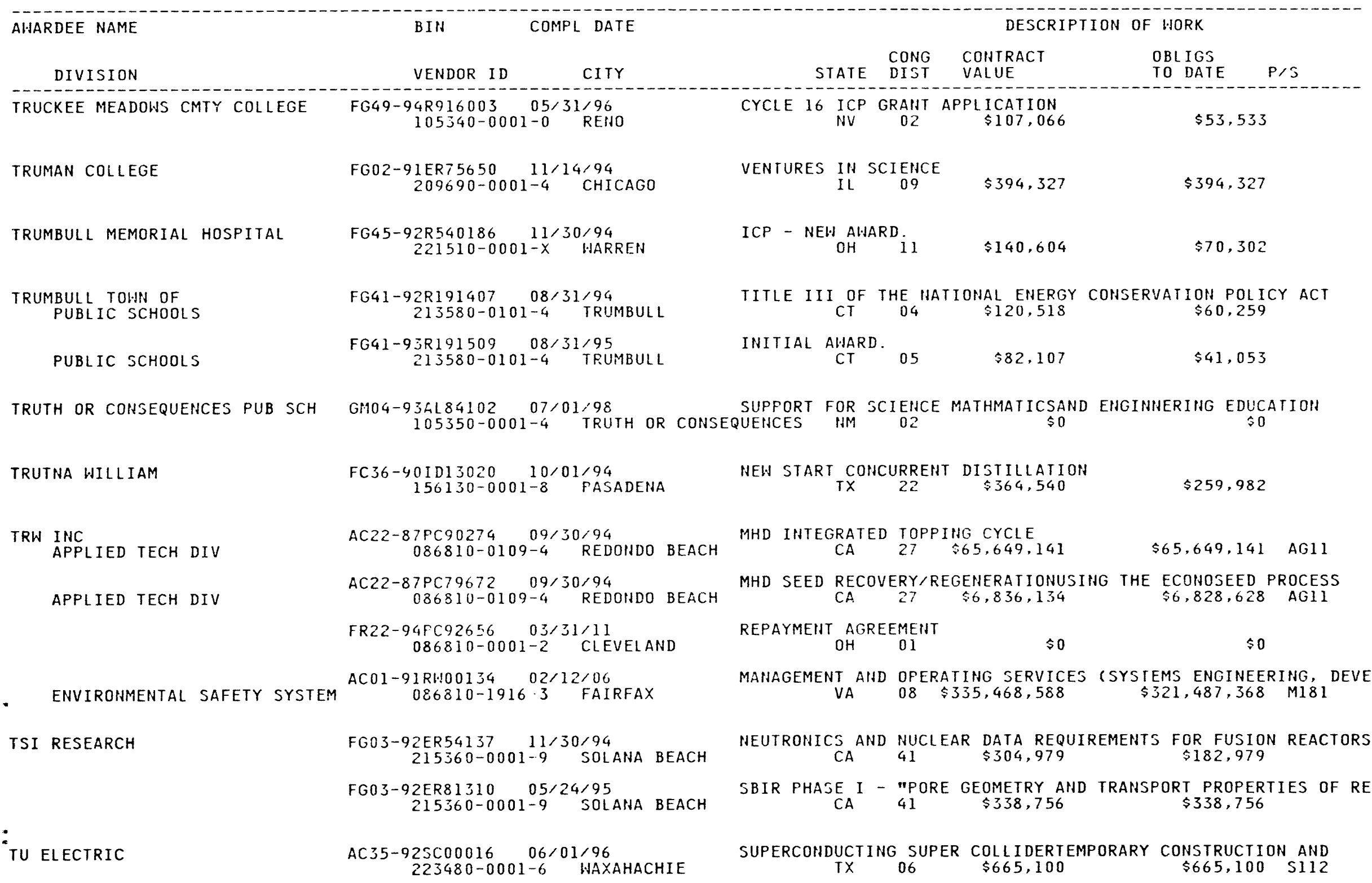




\begin{tabular}{|c|c|c|c|c|c|c|c|}
\hline AWARDEE NAME & BIN & L DATE & & & DESCF & F HORK & \\
\hline DIVISION & VENDOR ID & CITY & STATE & $\begin{array}{l}\text { CONG } \\
\text { DIST }\end{array}$ & $\begin{array}{l}\text { CONTRACT } \\
\text { VALUE }\end{array}$ & $\begin{array}{l}\text { OBLIGS } \\
\text { TO DATE }\end{array}$ & $P / S$ \\
\hline
\end{tabular}

\section{TUBA CITY USD \#15}

TUCKER \& ASSOC INC

TUCSON UNIFIED SCHOOL DISTRICT

TUCUMCARI PUBLIC SCHOOLS

TUFTS UNIVERSITY

MECHANICAL ENGINEERING DEPT

PHYSICS DEPT

VETERINARY MEDICINE SCHOOL OF

TULANE UNIVERSITY

CHEMICAL ENGINEERING

PHYSICS DEPT OF

CHEMISTRY DEPT

CHEMISTRY DEPT

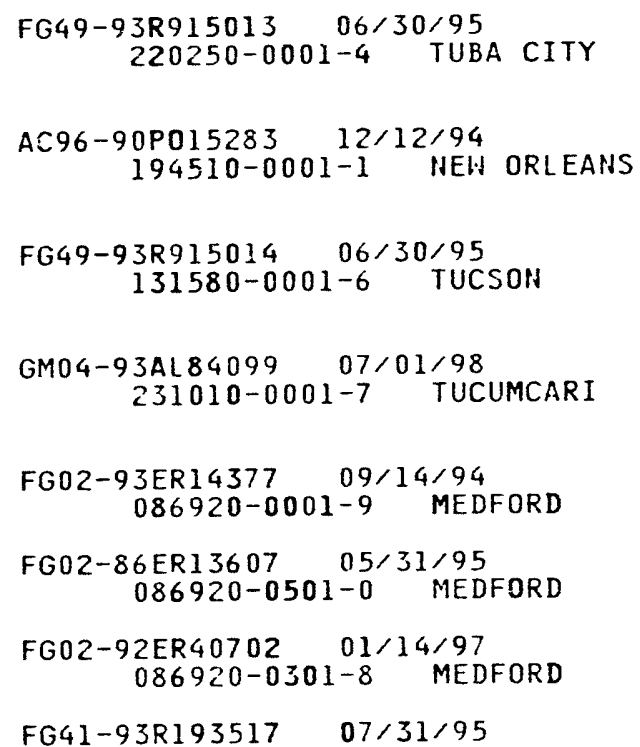
086920-0602-5 NORTH GRAFTON

FG01-93EW53023 12/30/97 O8693023 12/30/97 NEW ORLEANS

FC01-94EW54102 05/02/95 086930-0001-2 NEW ORLEANS

FG05-91ER14165 11/30/95 086930-0301-1 NEW ORLEANS

FG05-92ER14286 12/31/95 086930-0401-8 NEW ORLEANS

FG05-92ER14309 12/14/95 086930-0101-9 NEW ORLEANS

FG09-945R18398 03/08/95 086930-0101-9 NEH ORLEANS
CYCLE 15 ICP GRANT AWARD

$\begin{array}{lll}A Z & 04 & \$ 29,300\end{array}$

TECHNICAL AHD MANAGEMENT SUPPORT SERVICES LA $02 \$ 29,021,519 \quad \$ 24,799,834 \quad R 799$

CYCLE 15 ICP GRANT AHARD

AZ $\quad 02 \quad \$ 24,391$

SUPPORT FOR SCIENCE MATHEMATICS AND ENGINEERING EDUCATION IM 02 \$O

COLLABORATIVE RESEARCH ON ADVANCED MICROBATTERIES MA 07 \$ $\$ 135,535 \quad \$ 135,535$

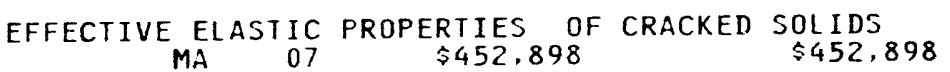
HIGH ENERGY PHYSICS RESEARCH
MA 07 \$3, 875,000
$\$ 2,165,000$

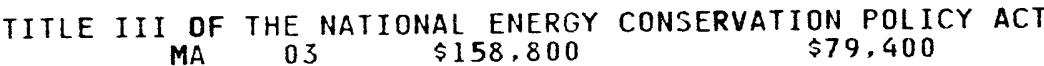

DETERMINE EFFECTS OF HAZARDOUSMATERIALS IN AQUATIC ENVIRONME LA $01 \$ 29,864,992$

$\$ 9,864,992$

CONSORTIUM FOR ENVIROMENTAL RISK ASSESSMENT

$$
\text { LA } 05 \text { \$6,000,000 } \$ 6,000,000
$$

THE FORMATION OF SUPPORTED BIMETALLIC CLUSTERS: THE EFFECT

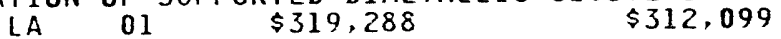

FEH ELECTRON TRANSITIONS IN ATOMIC COLLISIONS

$$
\text { LA } 010186,000 \quad \$ 120,140
$$

NEW AWARD PHOTOINDUCED ENERGY TRANSFER IN TRANSITION METAL C LA $01 \quad \$ 355,000 \quad \$ 257,150$

USED ENERGY RELATED LABORATORYEQUIPMENT GRANT 


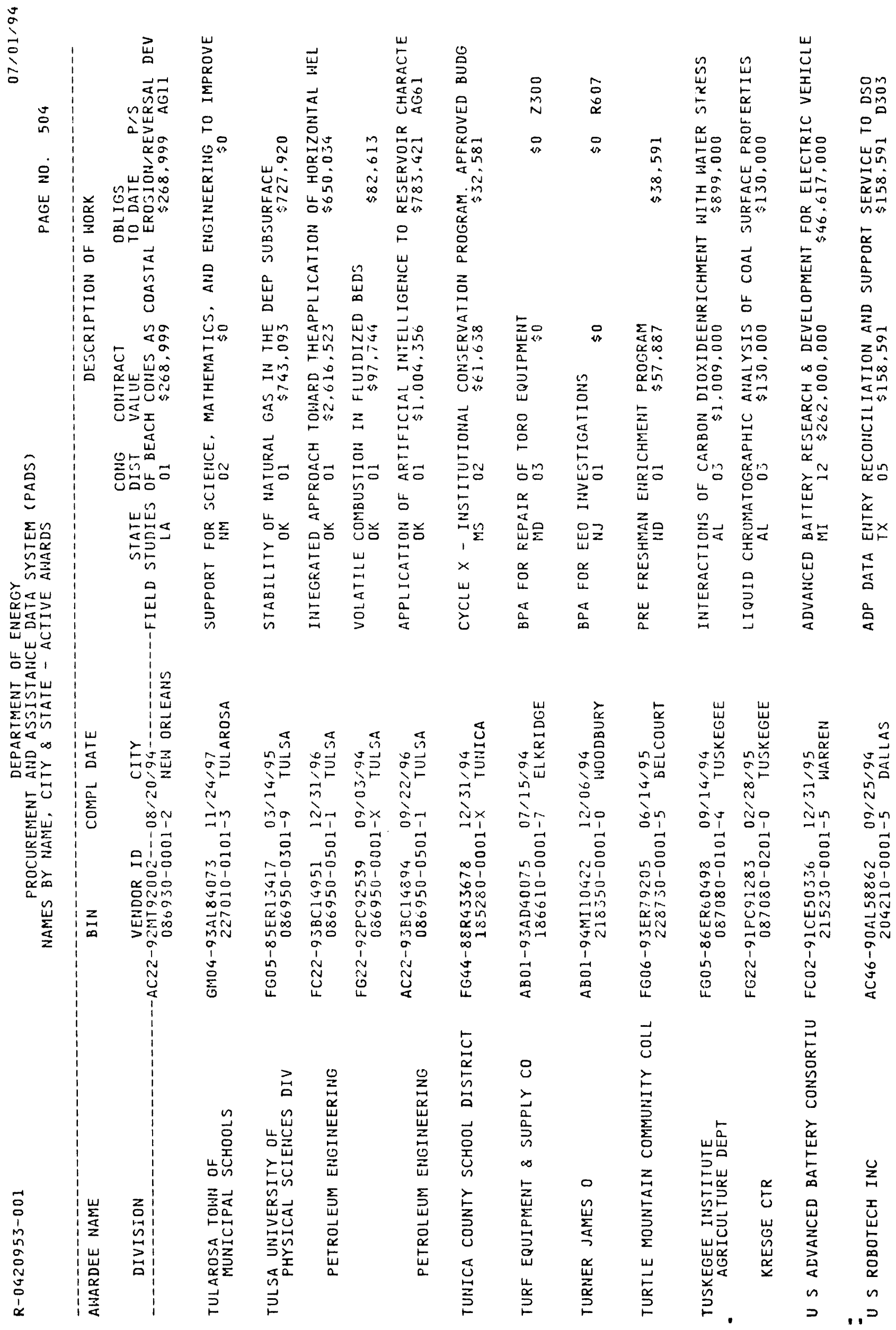




\section{AWARDEE NAME}

BIN

COMPL DATE

VENDOR ID

CITY

CONG
STATE DIST

DESCR
ITRACT
UE

UE\&C-CATALYTIC INC

ULTRAFLO CORP

ULTRAMET

UMETCO INC

UNDERGROUND INJECTION COUNCIL

UNGERMANN-BASS INC

UNICOI COUNTY BOARD OF EDUCN

UNIFIED CATHOLIC PARISH SCHS

UNION CARBIDE CORP

CHEMICALS \& PLASTICS

\section{AC06-90RL 11706 \\ $09 / 30 / 94$ \\ RICHLAND}

FGO1-93CE15028 09/21/95
$234600-0001-5 \quad$ SANDUSKY

FG03-93ER81584 07/01/94
$152190-0001-2$ PACOIMA

RL13-74GJ31022 07/23/94
$170170-0001-3 \quad$ GRAND JUNCTION

RL13-74GJ31025 07/23/94 $170170-0001-3$ GRAND JUNCTION

FG22-94MTO4003 179630-0001-9 OKLAHOMA CITY

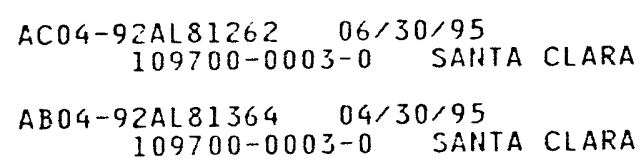
087710-0005-4 SOUTH CHARLESTOH
CONSIRUCTION/CONSTRUCTION MANAGEMENT SERVICES FOR THE

$\$ 36,891,630 \quad$ Y159

ULTRAFLO CONSERVATION WATER SYSTEM

$$
\mathrm{OH} 19 \quad \$ 99,985
$$

$\$ 99,985$

COATED MICROGRAIN CARBIDES FORWEAR RESISTANCE

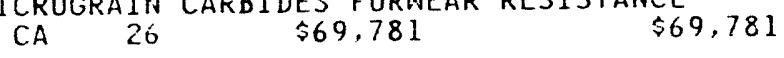

MINING LEASE AT $(05-1)-M L-60.8$-C-SR-15A

CO 03 SO

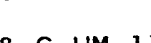

$\begin{array}{cc}\text { MINING LEASE AT }(05-1)-M L-60 & 8-\mathrm{C}-W M-17 \\ \text { CO } & 03\end{array}$

$\$ 0 \times 293$

$\$ 0 \times 293$

ASSISTANCE OF STATE UNDERGROUND INJECTION CONTROL PROGRAMS \&

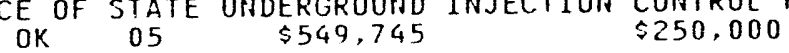

AWARD BASIC LAN SOFTWARE MAINTENANCE AND SUFPORT.

$\begin{array}{llll} & \end{array}$

BLANKET ORDER FOR UB SPARES/PARTS

$\$ 98,000 \quad 7050$

CYCLE 12 - INSTITUTIOHAL CONSERVATION PROGRAM. APPROVED BUD ICP-NEW AWARD.

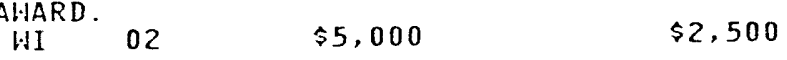

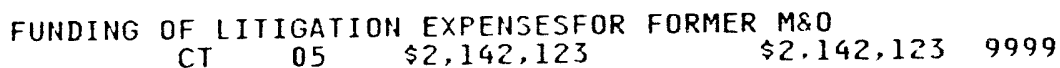

DISPOSAL OF SPENT NUCLEAR FUELAND/OR HIGH LEVEL RADIOACTIVE HETEROGENEOUS CATALYTIC PROCESS FOR ALCOHOL FUELS FROM SYNGA
HIV $03 \quad \$ 1,225,381$ 


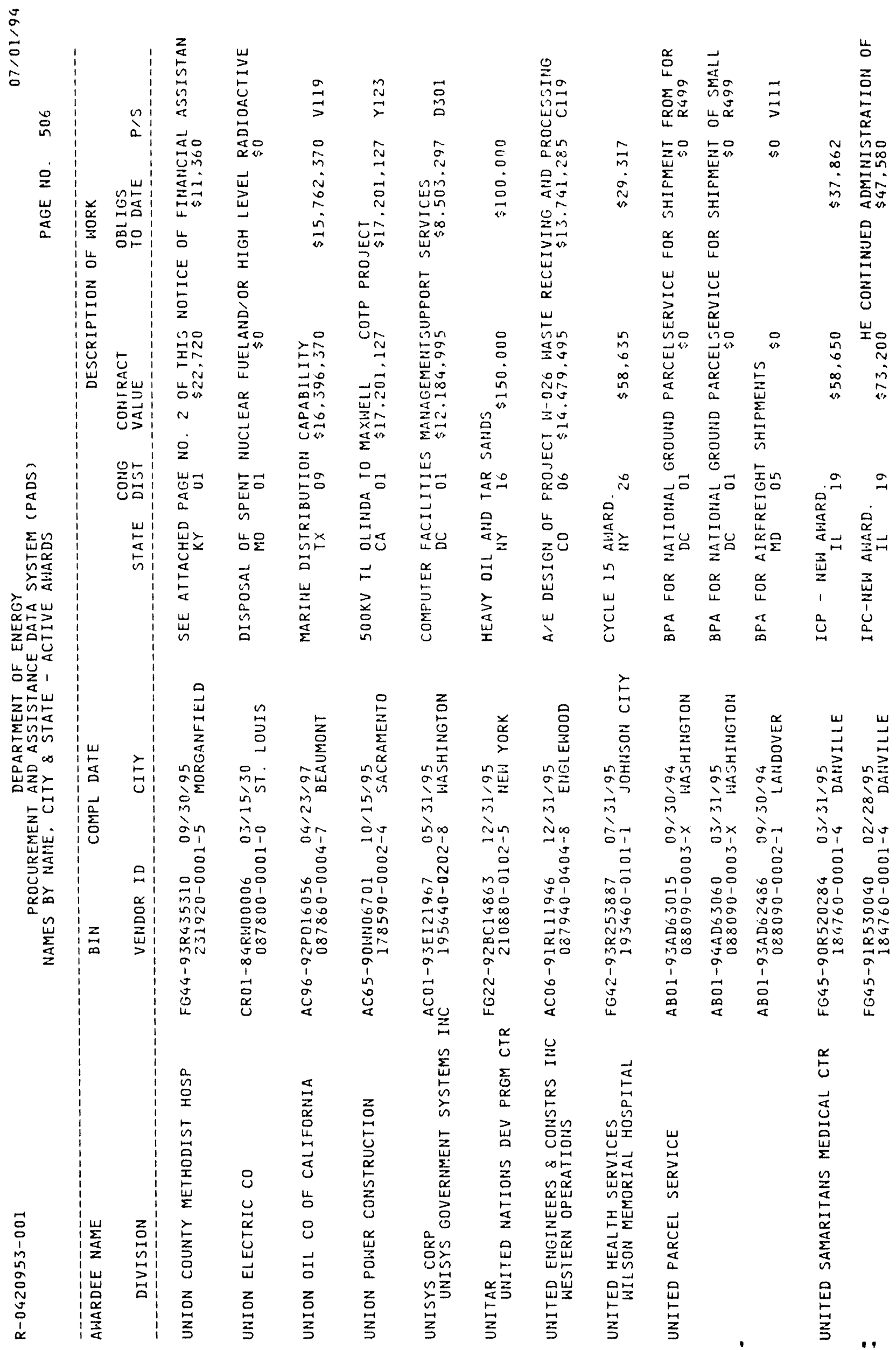




\begin{tabular}{|c|c|c|c|c|c|c|c|}
\hline WARDEE NAME & BIN & L DATE & & & $\mathrm{DESCP}$ & F HORK & \\
\hline DIVISION & VENDOR ID & CITY & STATE & $\begin{array}{l}\text { CONG } \\
\text { DIST }\end{array}$ & $\begin{array}{l}\text { CONTRACT } \\
\text { VALUE }\end{array}$ & $\begin{array}{l}\text { OBLIGS } \\
\text { TO DATE }\end{array}$ & $P / S$ \\
\hline
\end{tabular}

UNITED STATES ENERGY ASSOC

UNITED TALMUDICAL ACADEMY

UNITED TECHNOLOGIES CORP RESEARCH CENTER

RESEARCH CENTER

\section{UNITY COLLEGE}

UNIV \& CMTY COLL SYST OF NV DESERT RESEARCH INSTITUTE

UNIVERSAL BUILDING MAINTENANCE

UNIVERSAL FUEL DEV ASSOC

UNIVERSITIES RESEARCH ASSN

UHIVERSITY CITY SCIENCE CENTER

UNIVERSITY MICROFILMS INTL

UNIVERSITY OF PITTSBURGH BRADFORD CAMPUS FACIL DIV
FG02-93F010094 09/19/94
$234630-0001-6$ HASHINGTON

FG42-92R243531 $07 / 31 / 94$
$109260-0002-0$ MOHROE

AC22-92PC92176 07/20/94 088120-1101-2 EAST HARTFORD

AC22-92FC91155 07/26/94 $088120-1101-2$ EAST HARTFORD

FG41-94R192609 06/30/96 UNITY

FG05-92ER79110 07/31/94 221630-0101-6 REHO

AC08-89NV10799 09/30/94 $166860-0001-9$ LAS VEGAS
LS

AC21-8914C26255 10/31/94 914C26255 10/31/94
$175920-0001-6$ GRAND FORKS

$\mathrm{ACO2}-76 \mathrm{CHO} 3000 \quad 12 / 31 / 96$ $088210-0001-x$ BATAVIA

AC35-89ER40486 12/31/97 $088210-0002-\varepsilon$ WAXAHACHIE

FCO1-92CE40961 09/30/94 $088230-0001-7$ PHILADELPHIA

ACO1-93AD21136 09/30/94 $141390-0001-5$ ANII ARBOR

FG43-93R354203 12/31/94 230980-0101-1 PITTSBURGH
COHFERENCE ENTITLED "ENERGY FOR TOMORROWS WORLD"
$\$ 75,000$

TITLE III OF THE NATIOHAL ENERGY CONSERVATION POLICY ACT

$$
\text { NY } 22 \text { \$1 } 36,307 \quad \$ 68,153
$$

THERMODYNAMIC PROPERTIES OF PULVERIZED COAL DURING RAPID HEA CT $01 \quad \$ 294.480$ $\$ 294,480$ ASII

ENGINEERING DEVELOPMENT OF COAL-FIRED HIGH PERFORMANCE POWER $C T$ OT $\$ 5,870,524 \quad \$ 5,870,524$ AG13

FY'94 ICP A.IARD.

$$
M E \quad 01 \quad \$ 47,235 \quad \$ 41,311
$$

1992 INSTRUMENTATION - CLOUD COHDEHSATION NUCLEUS SPECTROME NV $02 \quad \$ 172.346 \quad \$ 172.346$

JANITORIAL SERVICES UNDER SBA $8(A)$ SUBCONIRACT NO. SB9-89-2NV $01 \quad \$ 3,354,171 \quad \$ 3.312,761$ \$201

GEOTECHNICAL/GEOCHEMICAL CHARACTERIZATION OF ADVAHCED HD 01 \$374.100 $\$ 374.100$ AG92

OPERATION OF FERMI NATIOHAL ACCELERATOR LAB

$$
\text { IL } 15 \$ 3.604,585,099 \quad \$ 3,602,035,595 \mathrm{Ml} 81
$$

MAHAGE AND OPERATE THE SJC LABORATORY

FIELD COORDINATION OF ENERGY ANALYSIS AND DIAGNOSTIC CENTER

$$
\text { PA } 01 \text { \$4,002,810 } \$ 4,002,810
$$

PROQUEST DATABASE SYSTEM

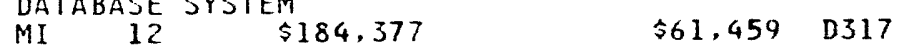

TITLE III OF THE NATIOHAL ENERGY CONSERVATION POLICY ACT

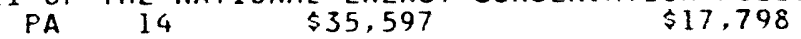




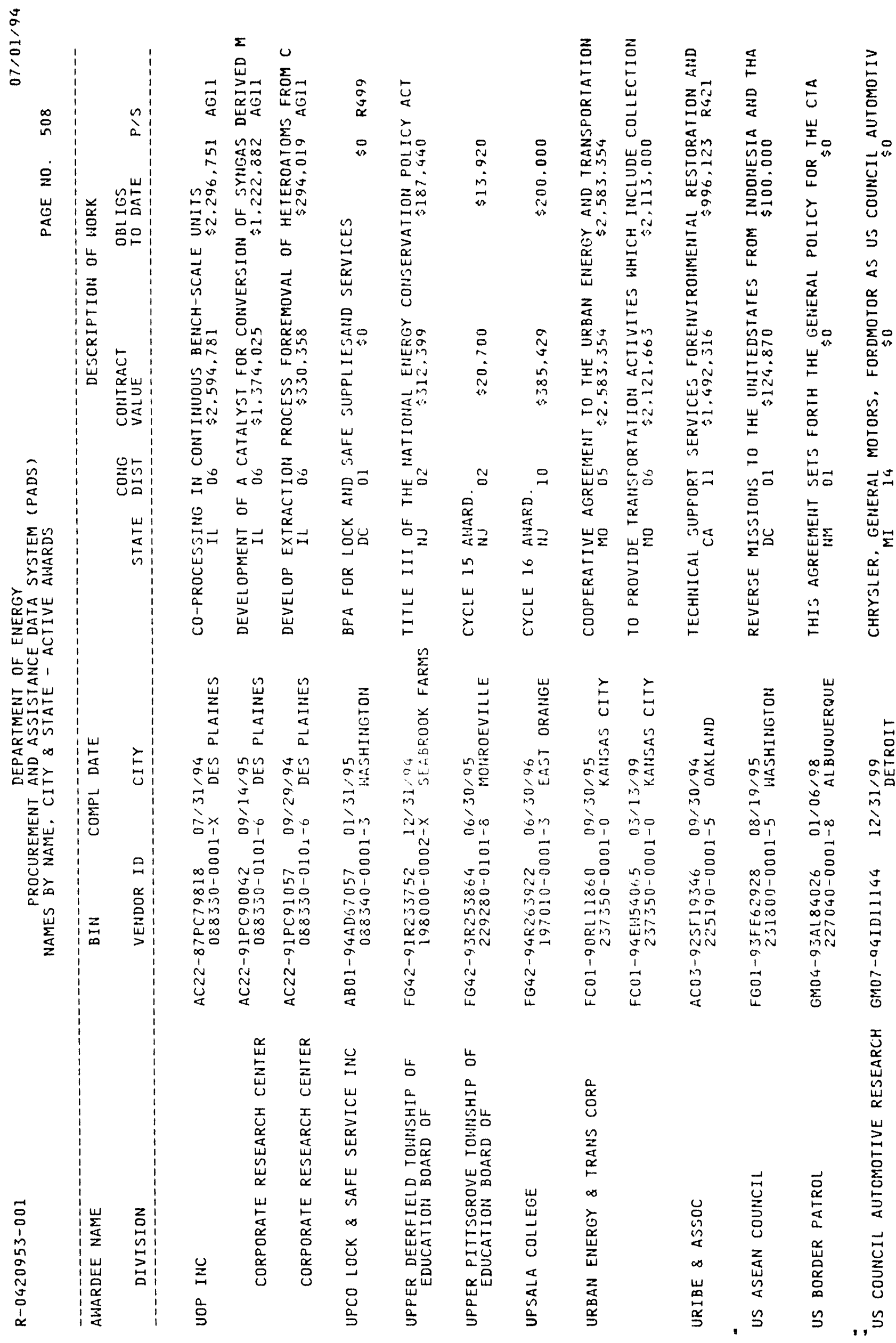




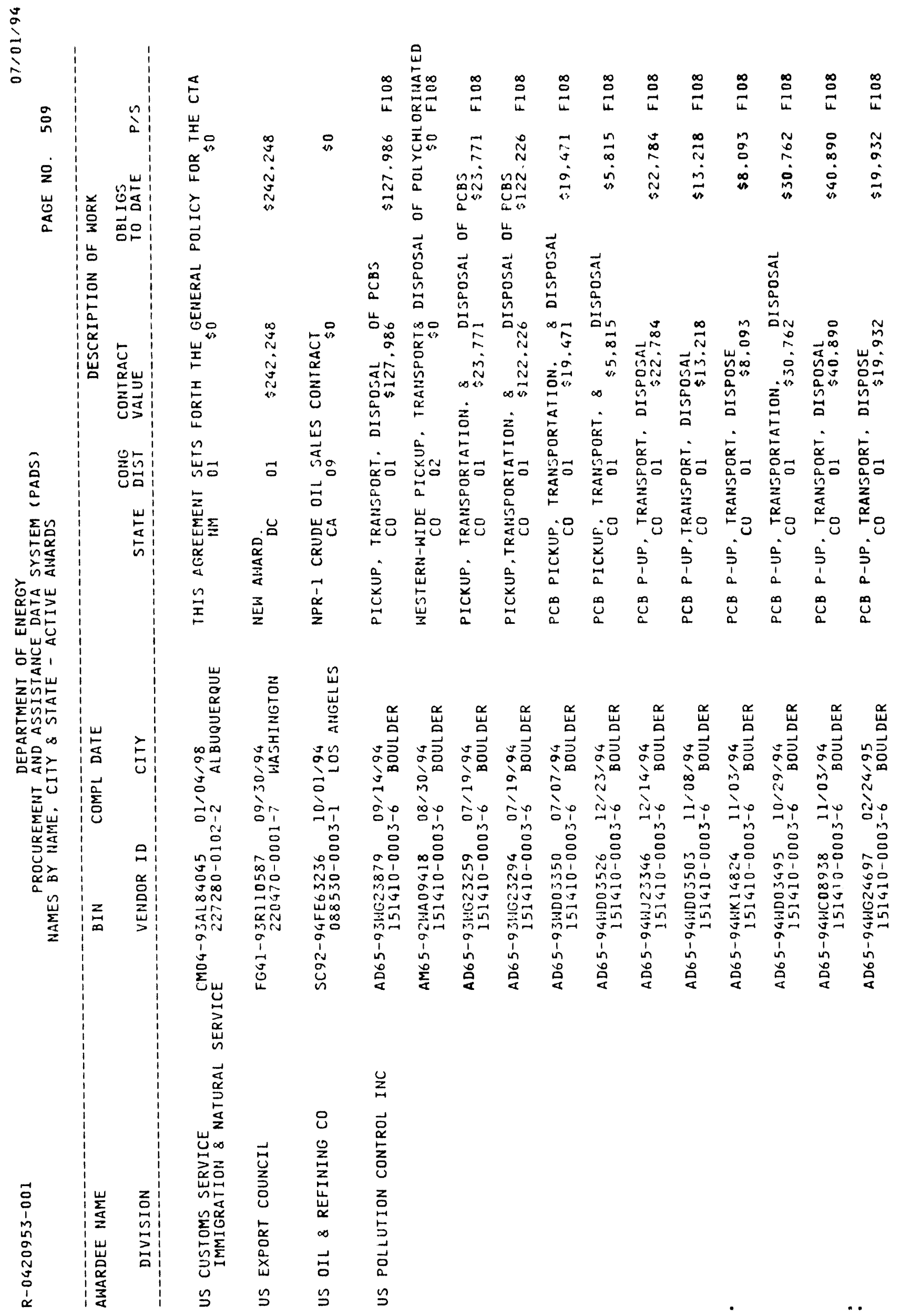




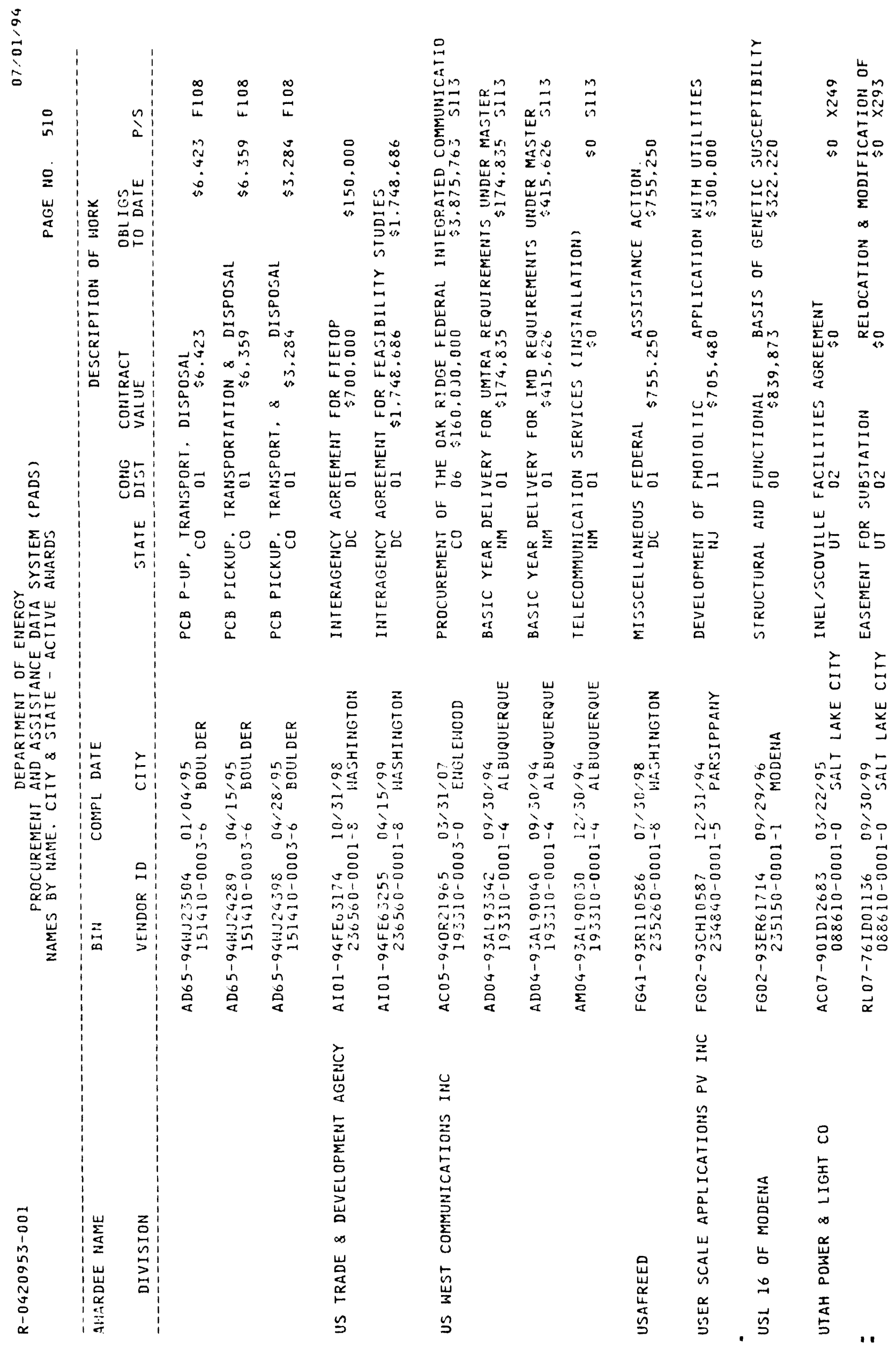




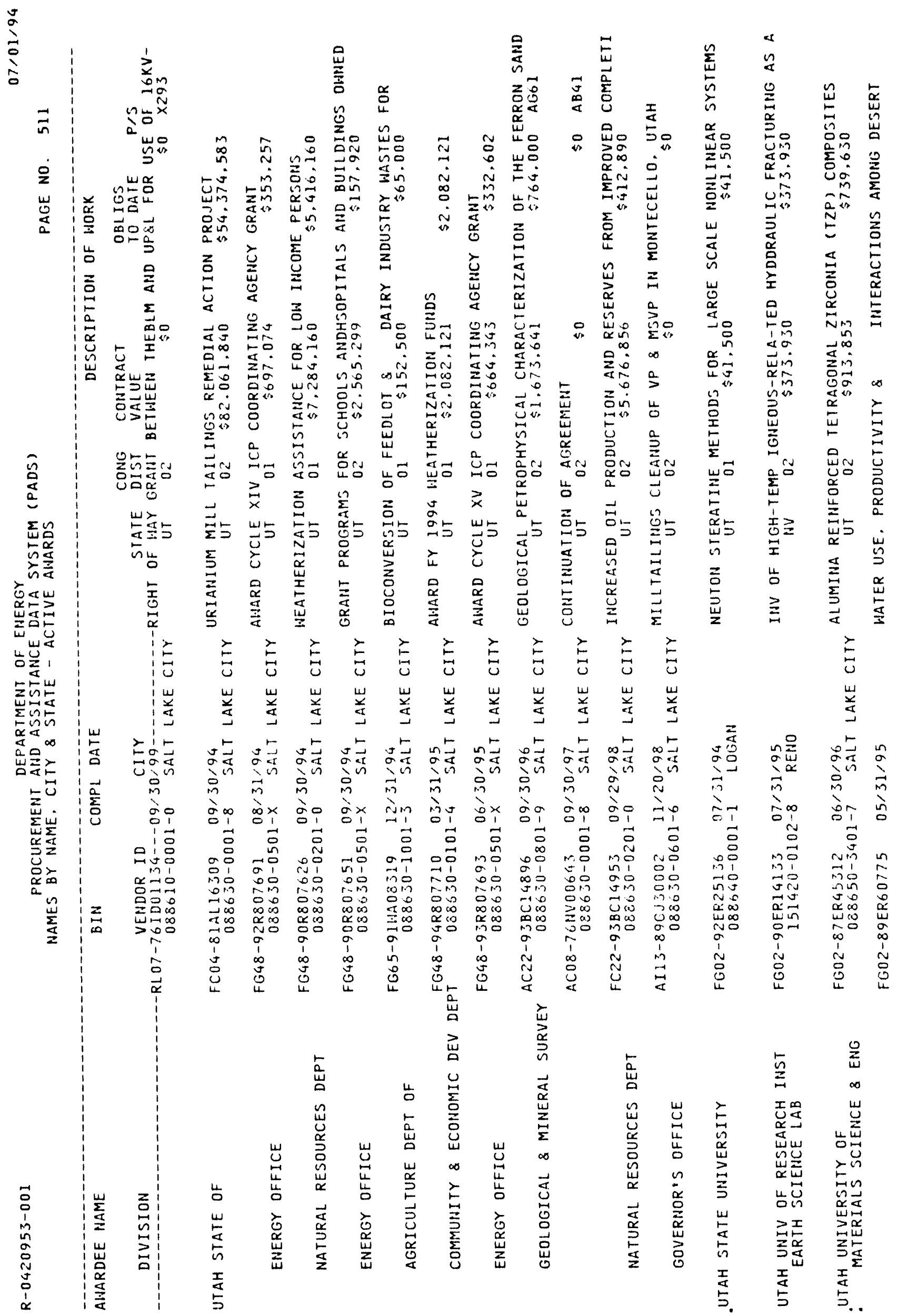




DIVISIOH
BIOLOGY DEPARTMENT
BIOLOGY DEPARTMENT

CHEMISTRY DEPARTMENT

CHEMISTRY DEPARTMENT

CHEMISTRY DEPARTMENT

CHEMISTRY DEPARTMENT

PHYSICS DEPARTMENT

BIN
COMPL DATE

DESCRIPTION OF HORK

VENDOR ID 088650-2501-8 SALT LAKE CITY STATE DOHG COHTRACT

UT $02 \quad \$ 1,121,682$

OBL IGJ TO DATE P/S \$958, 359 FG02-91ER14193 07/31/94 THERMOCHEMISTRY OF TRANSITION METAL CLUSTERS. 083650-0001-5 SALT LAKE CITY UT 02 S FG02-92ER20080 07/09/95 THE PLANT MITOCHOHDRIAL MAT-R GENE/NAD I GONE COMPLEX
$088650-2501-8$ SALT LAKE CITY UT 02 S $\begin{aligned} \text { FGO3-9JERI4333 } 02 / 29 / 96 & \text { NEW GRANT - "TIME RESOLVED AHALYTICAL METHODS FDR LIQUID/SOL } \\ 088650-0401-0 & \text { SALT LAKE CITY }\end{aligned}$ $088650-0001-5$ SALT LAKE CITY FABRICATION, PHASE TRAHSFORMATION STUDIES, AHD CHARACTERIZAT UT 02 \$253.874 $\$ 174,864$

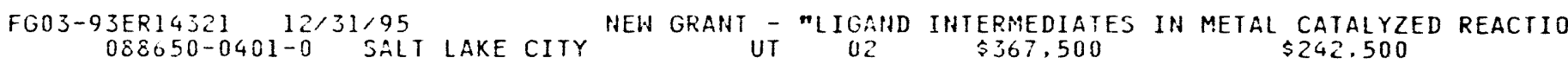
FG03-9 JER14315 12/14/95 NEH GRANT - "THE HYDROPHOBIC CHARACTER OF HOHSULFIDE MINERAL 088650-0001-5 SALT LAKE CITY UT $02 \quad \$ 336,658 \quad 5000$ FG03-93ERI4313 11/14/95 NEW GRANT - "HIGH RESOLUIION IMAGING OF ELECIRICAL COHDUCTIV 088650-0001-5 SALT LAKE CITY UT 02 \$181.897

FG0J-92ER75812 09/29/94 NEH GRAHT - "UHIVERSITY REACTOR SHARING PROGRAM" P.I. - DR.

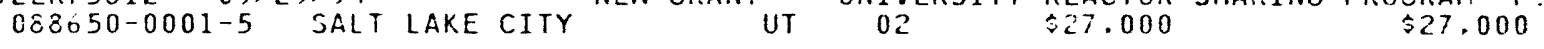

FG03-92ER79135 $09 / 29 / 94$
$083650-0001-5$
SALT LAKE CITY NEH GRANT - "UHIVERSITY OF UTAH HUCLEAR ENGINEERIHG LABORATO FG03-94ER61817 0 01/31/97 LAKE CITY NEH GRANT - ADVAHCD SEQUENCINGIECHNOLOGY P. I. - FROF. RAYMO
$038650-0001-5$ JALT LAKE FG03-94ER61769 11/30/96
$082650-0001-5$ SALT LAKE CITY HEH GRANT - "DEVELOPMENT AND TESTING OF A LIFE CYCLE MODEL A

FG03-94ER61747 10/31/96 CIRRUS CLOUD MEASUREMENT PROGRAM FOR IMPROVING SATELLIETE AN $088650-0001-5$ SALT LAKE CITY UT $02 \quad \$ 684,001 \quad \$ 339,500$

FG03-93ER45504 02/28/95 L THE SYNTHESIS OF MOLECULE/POLYMER-BASED MAGNETIC MATERIALS
$088650-0401-0$ JALT LAKE CITY OT

FG03-93ER45485 03/31/96 NEW GRANT - EXPLORIHG THE PHEHOMEHON OF ATOMIC-SCALE ORDERIH 088650-0001-5 3ALT LAKE CITY UT $02 \quad \$ 264.361$ S

FG03-93ER14340 03/31/96 NEW GRANT - "LASER FLASH PHOTOLYSIS, EPR AND RAMAN STUDIES O 088650-0401-0 SALT LAKE CITY UT $02 \quad \$ 260,000 \quad \$ 180,000$

FG03-93ER45490 03/31/96 NEN GRANT: "TRANSIENT AHD CH OPTICAL SIUDIES OF COHDUCTING 088650-2701-0 SALT LAKE CITY UT 02 \$316,000 


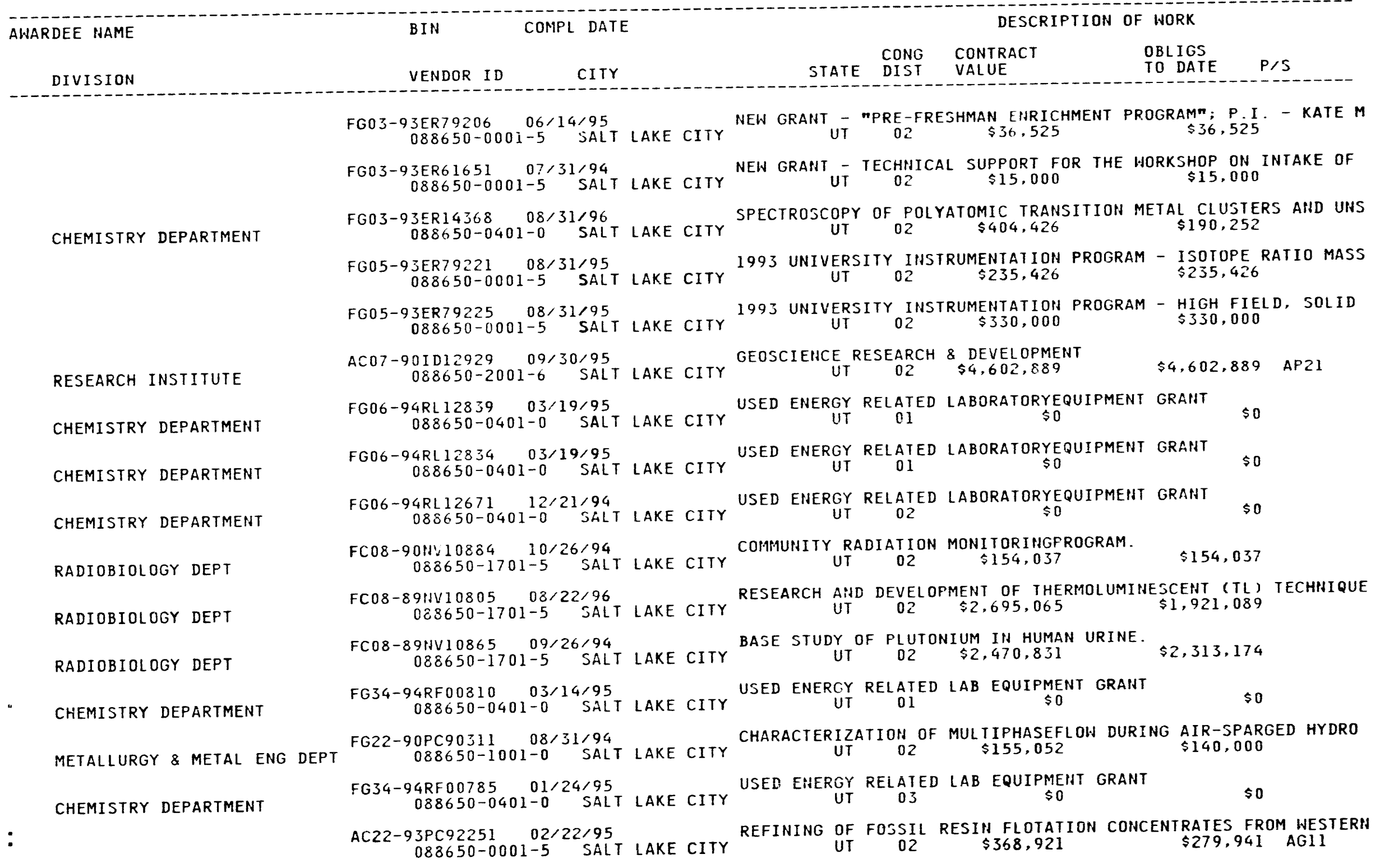


AWARDEE NAME

BIN

COMPL DATE

DESCRIPTION OF HORK

DIVISION COMPL DATE $\begin{array}{ll} & \text { CONG CONTRACT } \\ \text { STATE DIST VALUE }\end{array}$

OBLIGS

METALLURGY \& METAL ENG DEPT

VENDOR ID CITY

TO DATE

$\$ 194,535$
$\$ 194,535$ 088650-1001-0 SALT LAKE CITY

$\begin{array}{ccc}\text { UT } & 02 & \$ 194,535\end{array}$ AC22-92FC92121 09/27/95
$088650-0001-5$ SALT LAKE CITY HIGH COHVERSION OF COAL TO TRANSPORTATION FUELS FOR THE FUTU 088650-0001-5 SALT LAKE CITY UT 02 \$360,000

FUELS ENGINEERING DEPT FC21-93MC $30256 \quad 09 / 29 / 94$

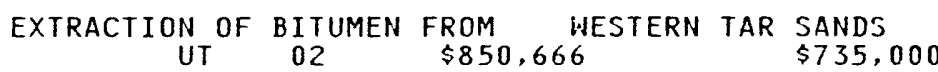

\section{CHEMISTRY DEPARTMENT} FG03-94ER14452 05/31/97 $088650-0401-0$ SALT LAKE CITY FG09-94SR18449 06/01/95 NEN GRANT - "CARBON-13 NMR OF SOLID STATE HYDROCARBONS AND R USED ENERGY RELATED LABORATORYEQUIPMENT
UT
02 $\$ 110,580$ $4581844906850-0401-0$
08861

CHEMISTRY DEPARTMENT FG34-94RF00927 06/06/95 088650-0441-0 SALT LAKE CITY

CHEMISTRY DEPARTMENT FG34-94RF00928 06/06/95
$088650-0401-0$ SALT LAKE CITY USED ENERGY RELATED LAB EQUIPMENT GRANT
UT
01

\section{\$0} USED ENERGY RELATED LAB EQUIPMENT GRANT
UT
01 FG34-94RF00937 066/09/95 LAKE CITY USED ENERGY RELATED LAB EQUIPMENT
$088650-0001-5$ SALT LAT

CHEMISTRY DEPARTMENT

UTD INC

AC21-92MC29119 08/15/94
$221570-0001-1$ NEWIHGTON

EM-INNOVATIVE DIRECTIONAL AHD POSITION SPECIFIC SAMPLING
VA
08 $\underset{\$ 603,561}{\$ 603,561}$ AH93

SC91-93FE63023 09/30/94 088740-0002-2 IDAHO FALLS $\underset{\text { NPR-3 LIQUID PRODUCTS SALES CONTRACT }}{\$ 0}$ 今 0 FG05-93ER79207 06/14/95 PRE-FRESHMAN ENRICHMENT PROGRAM (PREP) - 1993 $\begin{array}{ccccc}105550-0001-X \quad \text { VALDOSTA } & G A & 02 & \$ 38,221 & \$ 38,221\end{array}$

VALDOSTA STATE COLLEGE

GM04-93AL84110 08/23/98 232210-0101-0 ALBUQUERQUE

THIS AGREEMENT SETS FORTH THE GENERAL POLICY FOR THE

VALENCIA COUNTY OF

VALLEY ELECTRIC ASSOC

RL 08-94NV11444 06/30/98 $088860-0001-4$ PAHRUMP IIM 01 $\$ 0$

LEASE OF BUILDING ON SAHTOOTH MOUNTAIN. (SUPERSEDES CONTRAC $F G 49-93 R 915016 \quad 06 / 30 / 95$
$229480-0001-7 \quad$ MESA

CYCLE 15 ICP GRANT AWARD AZ $01 \quad \$ 108,086$

$\$ 54,043$
VALLEY LUTHERAN HOSPITAL -

VALLEY VIEW PUB SCH DIST \#365U FG45-91R530043 08/31/94
ICP-NEW AWARD. 


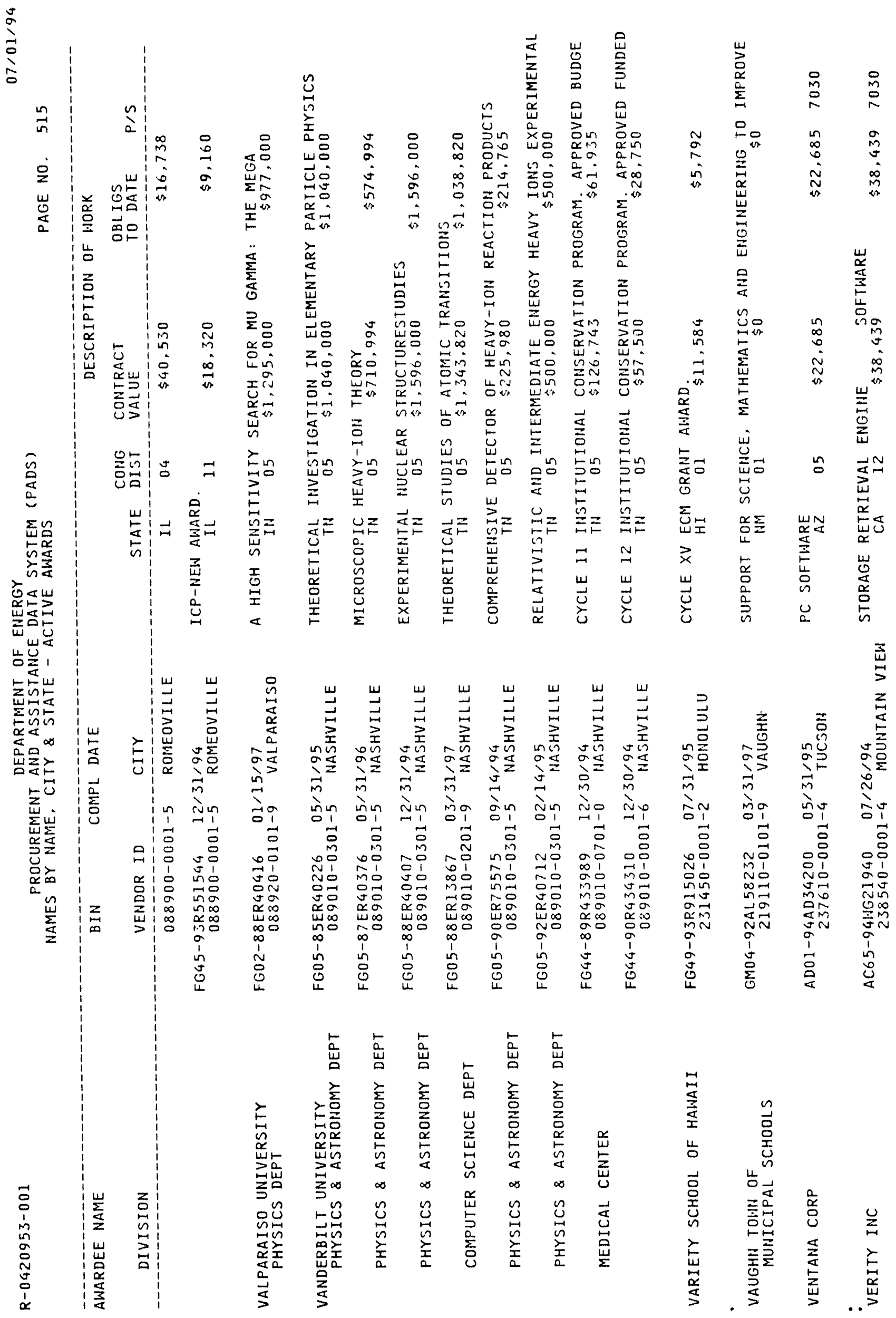




\begin{tabular}{|c|c|c|c|c|c|c|c|}
\hline AWARDEE NAME & BIN & L DATE & & & DESC & F HORK & \\
\hline DIVISION & VENDOR ID & CITY & STATE & $\begin{array}{l}\text { CONG } \\
\text { DIST }\end{array}$ & $\begin{array}{l}\text { CONTRACT } \\
\text { VALUE }\end{array}$ & $\begin{array}{l}\text { OBLIGS } \\
\text { TO DATE }\end{array}$ & $P / S$ \\
\hline
\end{tabular}

VERMILION LOCAL SCHOOLS

VERMONT STATE OF

PUBLIC SERVICE DEPT

PUBLIC SERVICE DEPT

PUBLIC SERVICE DEPT

ECONOMIC OPPORTUHITY OFFICE

VERMONT UNIVERSITY OF MEDICINE COLLEGE OF

VERMONT YAHKEE NUCLEAR POWER

VETERANS ADMIN

VETERANS ADMIN MEDICAL CENTER

VETERANS AFFAIRS DEPT OF

$$
\begin{aligned}
& \begin{array}{c}
\text { FG45-93R551738 01/31/95 } \\
229740-0001-0 \text { VERMILION }
\end{array} \\
& \begin{array}{c}
\text { FC01-91EI22718 } 08 / 07 / 96 \\
039210-0701-6 \quad \text { MONTPELIER }
\end{array} \\
& \text { FG41-92R110446 03/31/95 } \\
& \text { 089210-0701-6 MONTPELIER } \\
& \begin{array}{c}
\text { FG41-93R130612 06/30/98 } \\
089210-0001-1 \text { MONTPELIER }
\end{array} \\
& \begin{array}{c}
\text { FG41-93R130642 } 06 / 30 / 98 \\
089210-0701-6 \quad \text { MONT }
\end{array} \\
& \begin{array}{c}
\text { FG41-94R130634 03/31/99 } \\
089210-0802-0 \text { WATERBURY }
\end{array}
\end{aligned}
$$

CRO1-83NE44431 $03 / 15 / 30$
$089250-0001-6 \quad$ FRAMINGHAM

$$
\begin{gathered}
\text { AI01-90CE35035 } 06 / 18 / 95 \\
089340-0002-3 \quad \text { LOIA LINDA } \\
\text { AI } 02-76 \text { EV } 02327 \quad 12 / 31 / 99
\end{gathered}
$$
$089340-0001-5$ BUFFALO

FG41-92R191408 08/31/94
$096160-0004-7$ MERIDEN

GM04-88AL53611 03/22/99 096160-0002-0 ALBUQUERQUE

AI 01-93EH89275 $01 / 14 / 98$
$215210-0002-6 \quad$ BOJTON
ICP-NEW AWARD
OH

$\mathrm{OH}^{0} 05$

$\$ 23,650$

$\$ 11,825$

1991-1992 ENERGY INFO ADMIN. STATE HEATING $\begin{aligned} & \text { OIL AND PROPANE } P \\ & \$ 23,543\end{aligned}$

$\begin{array}{ccc}\text { ALTERHATIVE FUELS' SCHOOL BUS PROGRAM. } \\ \text { VT } \\ 01 & \$ 238,197\end{array}$

$\$ 33,197$

FY'93 AWARD
VT

238,197

$\$ 80,834$

FY'93 AWARD

$01 \quad \$ 798,530$

$\$ 46,900$

FY' 94 AHARD

$\$ 52,730$

$1,376,109$

THE DEVEL OPMENT OF IN VITRO MUTAGENICITY TESTING SYSTEMS
VT DI

AN IMMUNOCHEMICAL APPROACH TO THE STUDY OF DNA DAMAGE AND VT 01 \$1,593,502 $\$ 1,264,289$

DISPOSAL OF SPENT NUCLEAR FUEL

TISSUE INTERACTION WITH NON-IONIZING FIELDS

$$
\text { CA } 35 \text { \$2,400,000 } \$ 2,400,000
$$

RADIOISOTOPE POWERED CARDIAC PACEMAKER PROGRAM

$\$ \mathbf{0}$

TITLE III OF THE NATIONAL ENERGY CONSERVATION POLICY ACT

FORM A MANAGEMENT AND PROCEDURAL FRAMEHORK FOR PROVIDING
NM 01

CLINICAL SURVEILLANCE

MA 09 \$1,000,000 $\$ 850,000$




\begin{tabular}{|c|c|}
\hline AWARDEE NAME & COMPL DATE \\
\hline DIVISION & VENDOR ID \\
\hline AMARILLO MEDICAL CENTER & $\begin{array}{cc}\text { AI04-91AL42124 } & 06 / 30 / 96 \\
215210-0101-4 & \text { AMARILLO }\end{array}$ \\
\hline VIDALIA CITY SCHOOLS & $\begin{array}{c}\text { FG44-92R435076 } 09 / 30 / 94 \\
089420-0001-0 \quad \text { VIDALIA }\end{array}$ \\
\hline VILLA MARIE SCHOOL & $\begin{array}{c}\text { FG47-93R703513 } 08 / 31 / 95 \\
231720-0001-X \text { WAVERLY }\end{array}$ \\
\hline VINDICATOR CORP & $\begin{array}{r}\text { AC96-91P015535 01/28/95 } \\
210800-0001-1 \text { AUSTIN }\end{array}$ \\
\hline
\end{tabular}

DESCRIPTION OF WORK

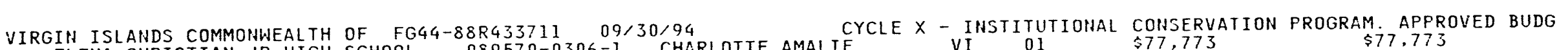
ELENA CHRISTIAN JR HIGH SCHOOL 089570-0306-1 CHARLOTTE AMALIE FG44-88R433712 09/30/94 CYCLE X - INSTITUTIOHAL CONSERVATIRIi PROGRAM. APPROVED BUDG IVANNA EUDORA KEAN HIGH SCHOOL $039570-0601-X$ CHARLOTTE AMALIE FG44-89R434179 09/30/94 CYCLE 11 INSTITUTIONAL CONSERVATION FPOGRAM. APPROVED BUDGE 089570-0501-3 CHARLOTTE AMALIE VI 01 CHARLOTTE AMALIE HIGH SCHOOL

ENERGY DFFICE

ENERGY OFFICE

VIRGIN ISLANDS MONTESSORI SCH

VIRGIN ISLANDS UNIVERSITY OF

ST THOMAS CAMPUS

VIRGINIA COMMOHHEALTH UHIV PHYSICS DEPT

VIRGINIA ELECTRIC \& POWER CO

\section{FG44-88R410583 09/30/94} 089570-0102-6 FREDERIKSTED

FG44-88R410584 09/30/94 089570-0102-6 FREDERIKSTED $\begin{array}{ccc}\text { ESTABLISH AND MAINTAIN AN EMERGENCY RADIATION TREATMENT } \\ \$ 1,200,000 & \$ 1,200,000\end{array}$ $\begin{array}{cccc}\text { CYCLE } 14 & \text { - INSTITUTIUNAL COHSERVATION PROGRAM. APPROVED BUD } \\ \text { GA } & 01 & \$ 87,005 & \$ 43,502\end{array}$

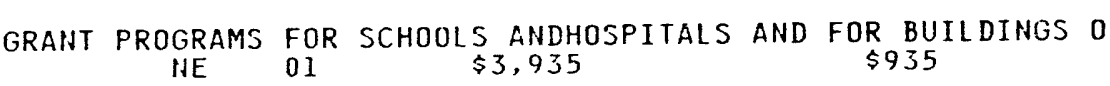

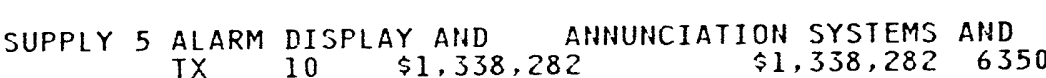




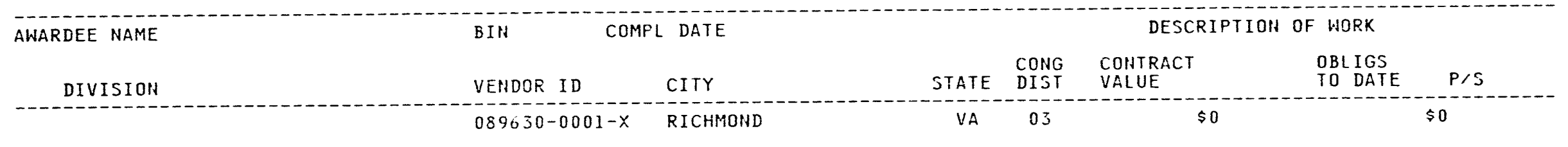

VIRGINIA POLYTECH INST/ST UNIV ACO1-93DP20166 $09 / 22 / 94$ 089660-0001-0 BLACKSBURG FG05-92ER40709 01/31/95 089660-0001-0 BLACKSBURG

PHYSICS DEPT

FG05-84ER40143 $08 / 31 / 94$ 089660-1201-9 BLACKSBURG

AIAEROBIC MICROBIOLOGY

ANAEROBIC MICROBIOLOGY

PHYSICS DEPT

GEOLOGICAL SCIENCES

COMPUTER SCIENCE DEPT

PHYSICS DEPT

- Mining \& Minerals ENG DEPT
FG05-85ER13368 05/31/97 089660-1401-1 BLACKSBURG

FG05-87ER13730 05/31/95 039660-1401-1 BLACKSBURG

FG05-88ER40454 04/30/07 D89660-1201-9 BLACKSBURG

FG05-88ER13951 07/31.94 089660-1001-6 BLACKSBURG

FG05-88ER25068 08/31/96 089660-1101-2 BLACKSBURG FG05-89ER14065 10/31/94 089660-0001-0 BLACKSBURG FG05-90ER14129 01/31/97 089660-0001-0 BLACKJBURG

F005-91ER40639 12/31/94 089660-1201-9 BLACKJBURG

FG05-93ER25175 07/31/95 089660-0001-0 BLACKSBURG

FG05-93ER79233 08/31/95 089660-0001-0 BLACKSBURG

FG05-93ER25189 09/14/96 089660-0001-0 BLACKSBURG AC22-91PC91164 07/31/94 $089660-0901-8$ BLACKSBURG

FG22-91PC91309 08/31/94
PROVIDE SPECIALIZED SERVICES TO DEVELOP \& EVALUATE COMPUTER VA 09 \$3,174,485 \$1,263,531 P.421 INVESTIGATIOHS OF DYNAMICS OF GAUGE THEORETICAL PARTICAL PHY VA 09 \$1,627,000

THEORETICAL STUDIES IN FEN-BODY INTERMEDIATE PHYSICS

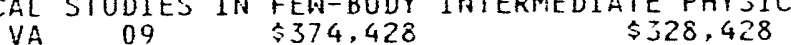

ENZYMOLOGY OF ACETONE-BUTAHOL-ISOPROPAHOL FORMATIOH VA $09 \quad \$ 954,278 \quad \$ 854,725$

ENZYMOLOGICAL STUDIES OF ONE-CARBOH REACTIOHS IN THE PAIHWAY VA 09 \$6 098,028 \$668,028

PARTIAL WAVE AHALYSIS OF HADRON SCATTERING BELOW 2 GEV VAE ANALYSIS OF HADRON SCATTERING BELOW $\$ 1,077,000$

ZIFCOHS AHD FLUIDS: AN EXPERI MENTAL IHVESTIGATION WITH APPL VA 09 AN \$394,307 \$394,307

PARALLEL MATHEMATICAL SOFTHARE

$\$ 535,835$

PVTY PROPERTIES OF FLUID SYSTEMS: NACI-CACI2-H2O

VA

INFLUENCE OF SURFACE DEFECTS AHD LOCAL STRUCTURE OH EXYGENAT VAF SURFACE DEFECTS AFD LOCAL STRUCTURE $\$ 295,020$

DEVELOPMENT OF A COMPENSATING SCINTILLATER PLATE CALORIMETER VA SF A COMPENSATING SCIMTILLATER PLATE CALO $\$ 125,000$

AHALYSES, ALGORITHMS, AND COMPUTATIONS FOR MODELS OF HIGH TE VALORITHMS, AND COMPUTATIONS FOR MODELS $\$ 90,000$

I993 UHIVERSITY IHSTRUMENTATION PROGRAM - ULTRAHIGH VACUUM S VASTY INSTRUMENTATION PROGRAM - ULTRAHIGH

RESEARCH EFFORT ENTITLED TOOLSAND ALGORITHMS FOR PARRALLEL

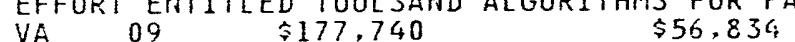

DEVELOPMENT OF THE SELECTIVE HYDROPHOBIC COAGULATIOH PROCESS VA 0 \$ 249,991 S

ALKALI CORROSION OF CERAMICS AT HIGH TEMPERATURES IN COAL GA 
5
$\vdots$
0
0

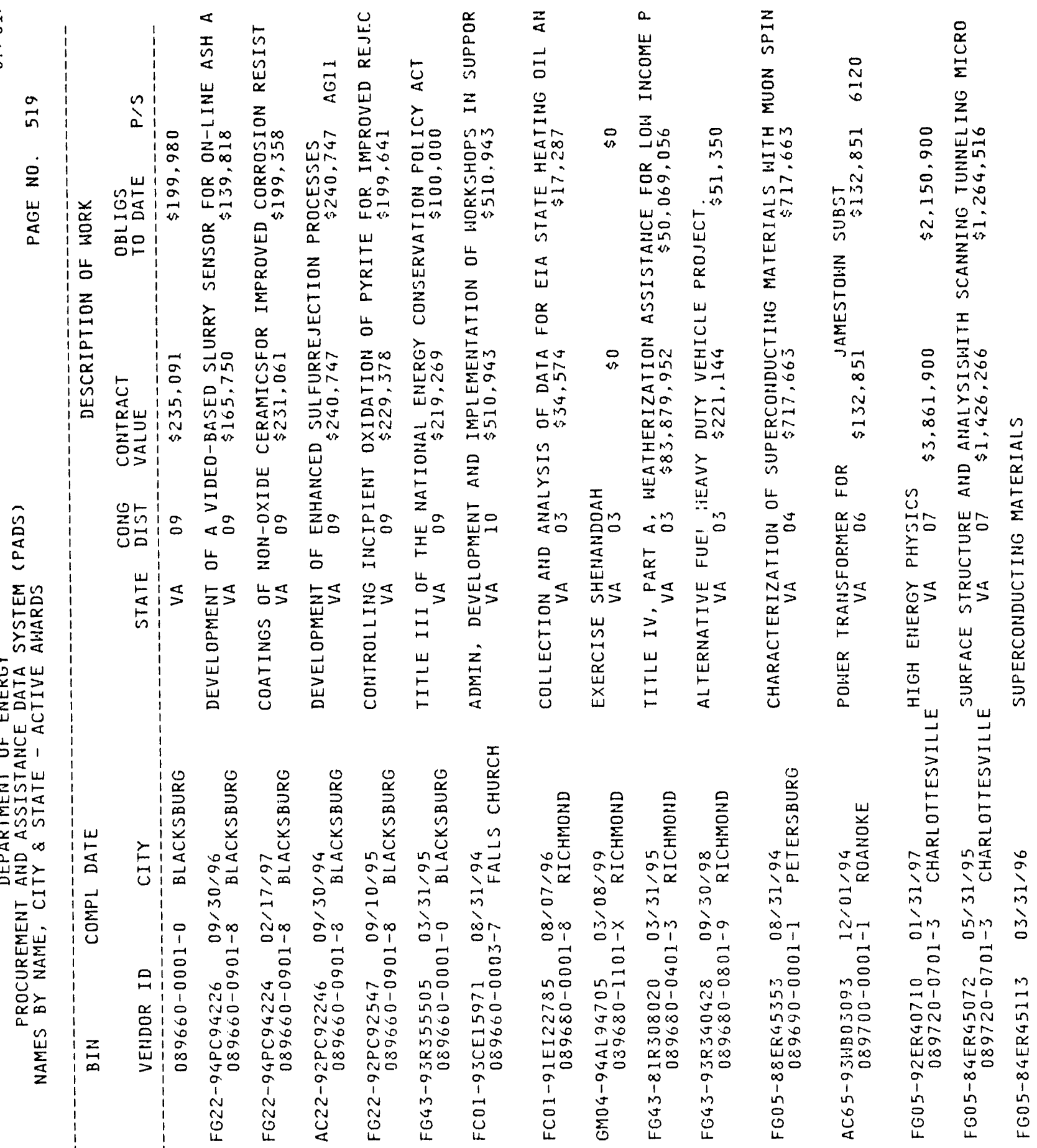
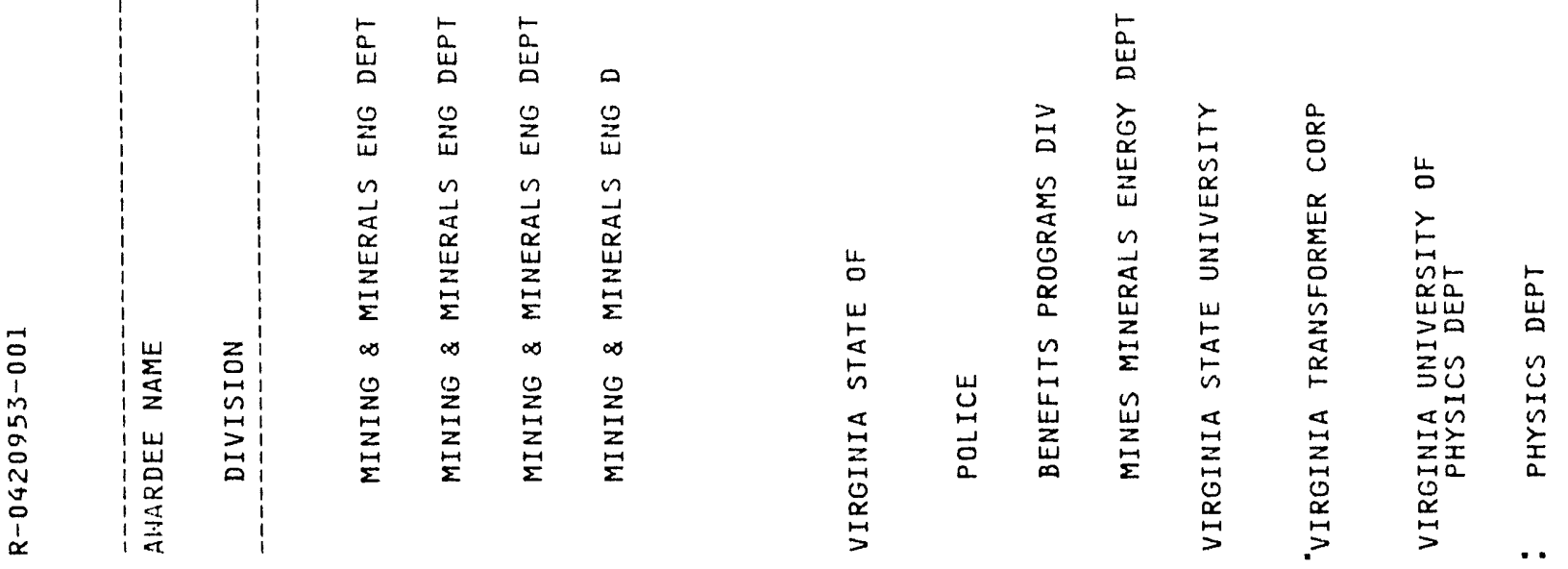


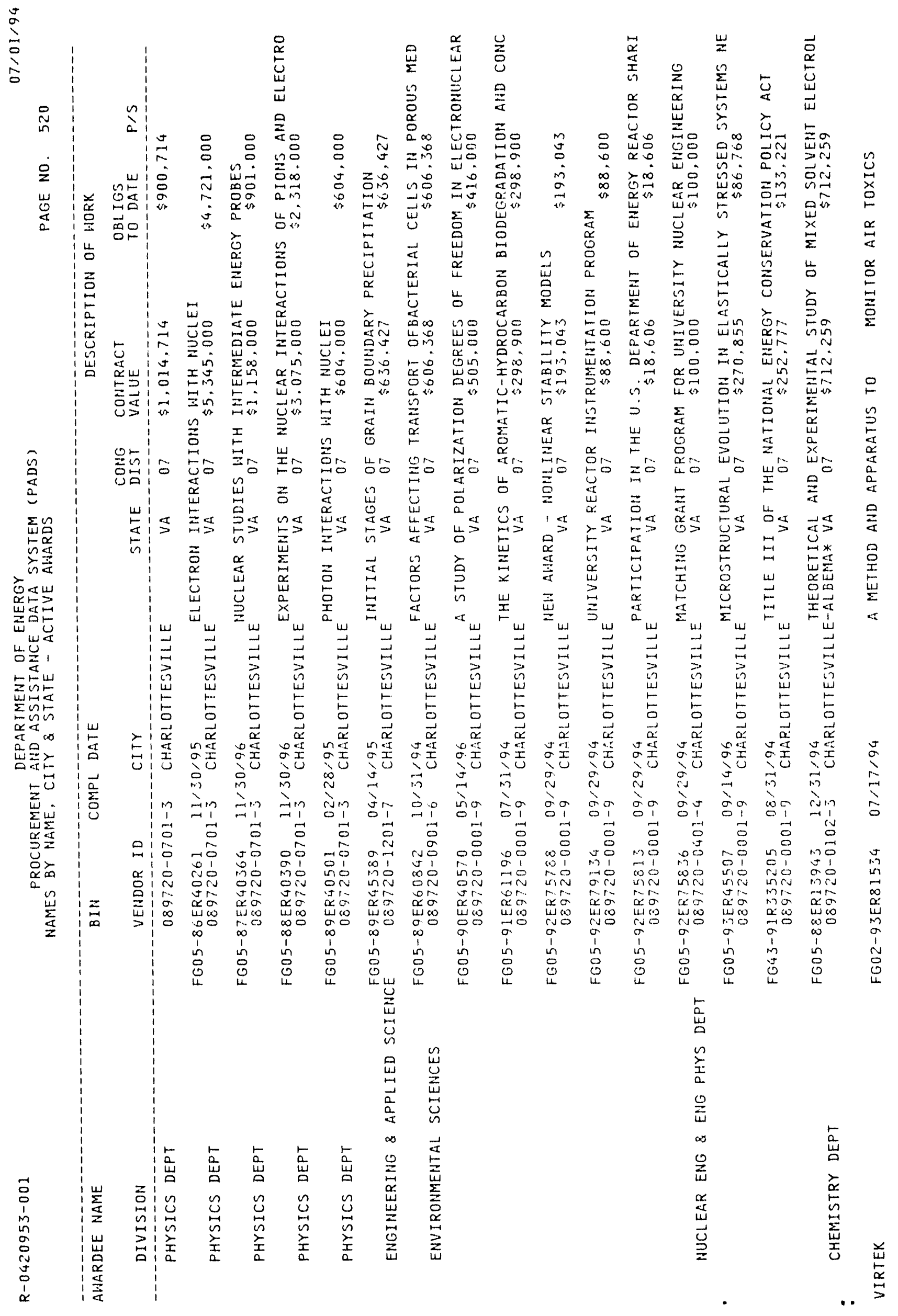




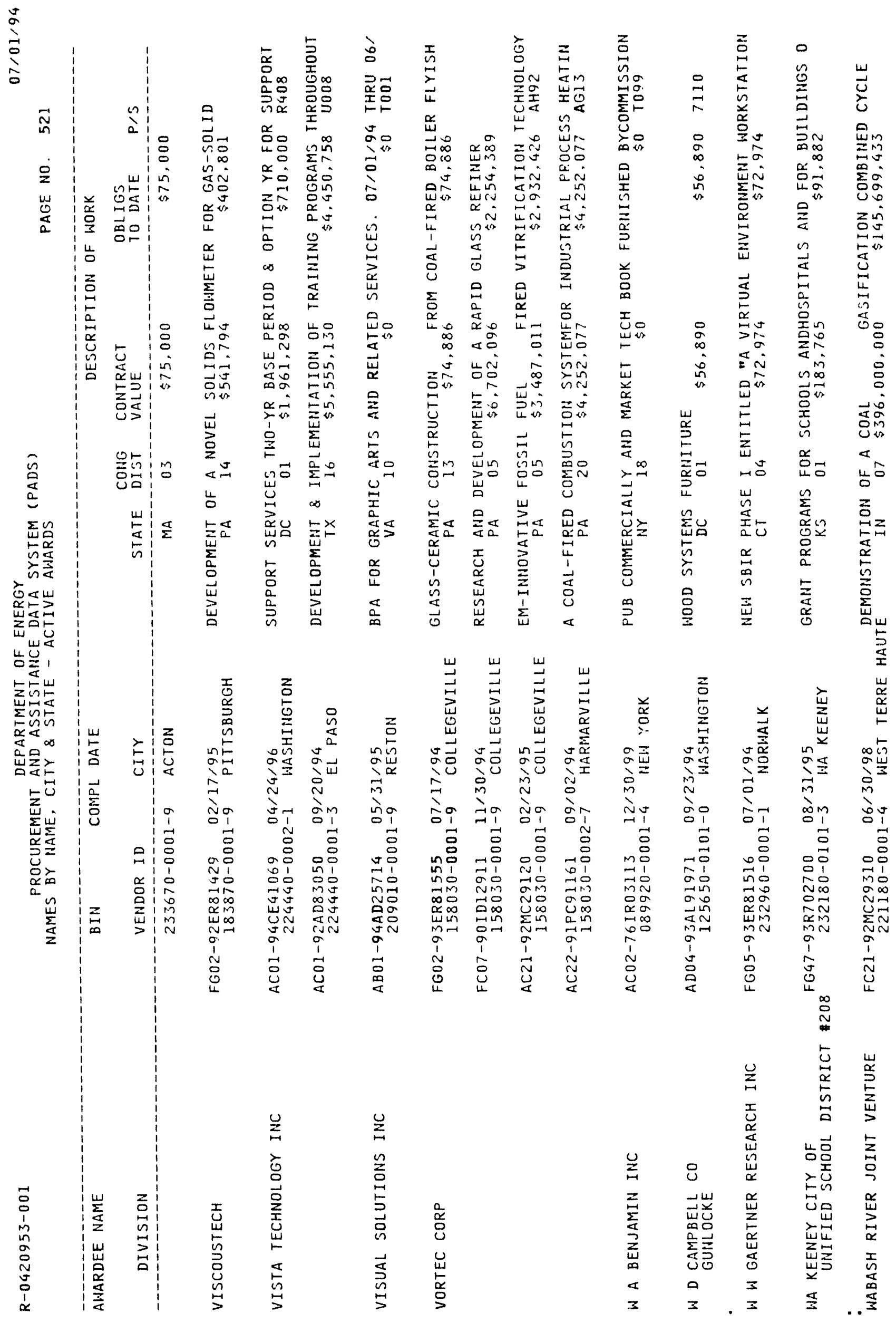


DIVISION

WABASH VALLEY MFG INC

MACKENHUT SERVICES INC

\section{WAGNER \& GEYER}

"WAGON MOUHD TOWH OF MUNICIPAL SCHOOLS

WAHIAWA GENERAL HOSPITAL

:WAKE FOREST UNIVERIITY

COMPL DATE

BIN COMPL DATE

STATE CONG COHTRACT

VENDOR ID

CITY

FR21-92MC29075 $06 / 30 / 18$
$221180-0001-4$

OBLIGS VALUE

TO DATE P P P

ADO1-93AD64786 10/31/94 PURCHASE OF PICNIE TABLES

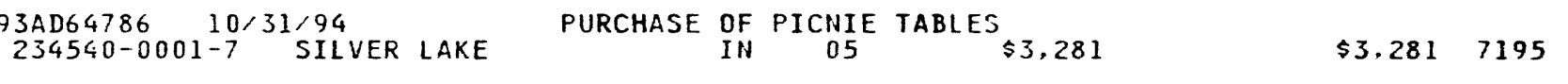

AC34-93RF00283 03/31/96
$090020-0005-7$ GOLDEN

AC04-94AL94463 04/30/99

090020-0003-0 AL BUQUERQUE

ACO4-90AL57353 06/30/95

090020-0002-2 AL BUQUERQUE

ACO8-9311V11011 09/30/97 090020-0004-9 LAS VEGAS

ACOS-93SR18292 09/30/98 090020-0001-4 AIKEN

AB01-93GC20334 09/30/95

$36 C 2033409 / 30 / 95$
$190240-0001-7$ MUHCHEN

$A B 01-93 G C 20336 \quad 09 / 30 / 95$
$190240-0001-7$ MUNCHEN

ABO1-926C20296 09/30/94

$190240-0001-7$ MUHICH

$A B 01-94 G C 20340 \quad 04 / 30 / 96$ $190240-0001-7$ MUNICH

PROTECTIVE FORCE FOR THE ROCKYFLATS PLANT

CO $02 \$ 63,936,742 \quad \$ 63,217,222 \$ 206$

TECHNICAL SERVICES IN SUPPORT OF PERSONHEL SECURITY DIVISION

OPERATION OF U.S. DOE CENTRAL TRAINING ACADEMY

NM OI $\$ 39,075,873 \quad \$ 28.633,910$ U009

SECURITY PROTECTIVE FORCE SERVICES.

NV $01 \$ 140,000,000 \quad \$ 45,677,999 \quad 2206$

PARAMILITARY SECURITY FORCES

SC SOS \$320,000,000 $\$ 74,655,000 \quad \$ 206$

BPA FOR GERMAN PATENTS

OO

$\$ \mathbf{D}$

\$0 $\mathbf{R} 499$

BPA FOR EUROPEAN PATENTS

DO

$\$ 0$

$\$ 0 \quad R 499$

PROVIDE PATENT SERVICES IN GERMANY

$\$ 0$

$\$ 0 \quad \mathbf{R} 418$

EUROPEAN PATENT SERVICES

$\$ 0$

$\$ 0 \quad R 499$

GM04-92AL84056 07/21/97 226790-0101-h WAGON MOUHD

SUPPORT FOR SCIENCE, MATHEMATICS, AND ENGINEERING TO IMPROVE IN 03 \$0 $\$ 0$

FG49-91R913025 08/31/94 $1 R 91302508 / 31 / 94$
$109330-0001-4$ WAHIAWA

CYCLE XIII GRAHT

$\$ 126,416$

$\$ 63,208$

FG44-93R435401 09/30/94 $090140-0001-4$ HIHSTON-SALEM

SEE ATTACHED PAGE NO. 4 OF THIS NOTICE OF FINANCIAL ASSISTAN

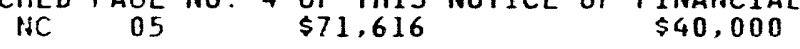




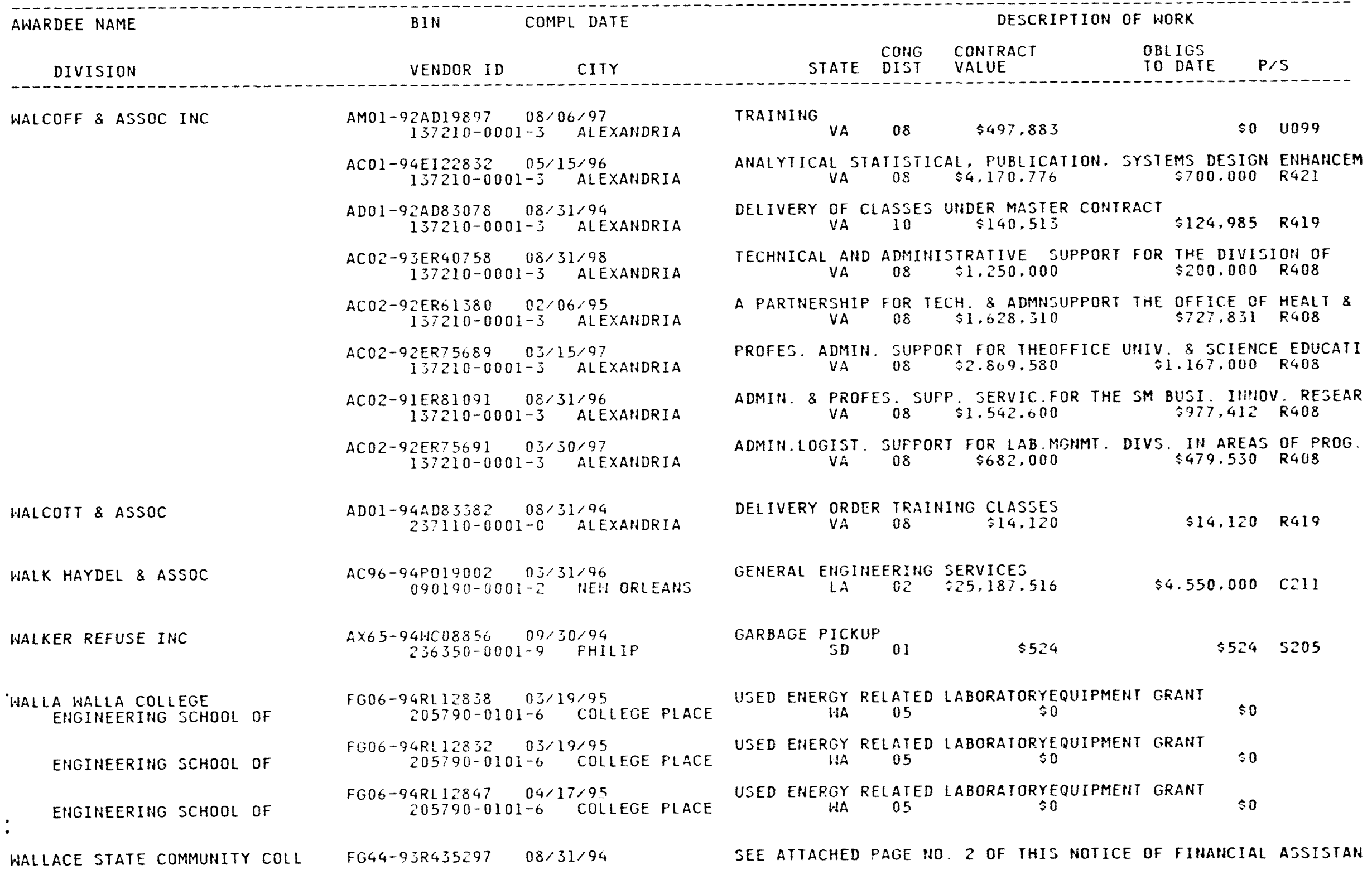




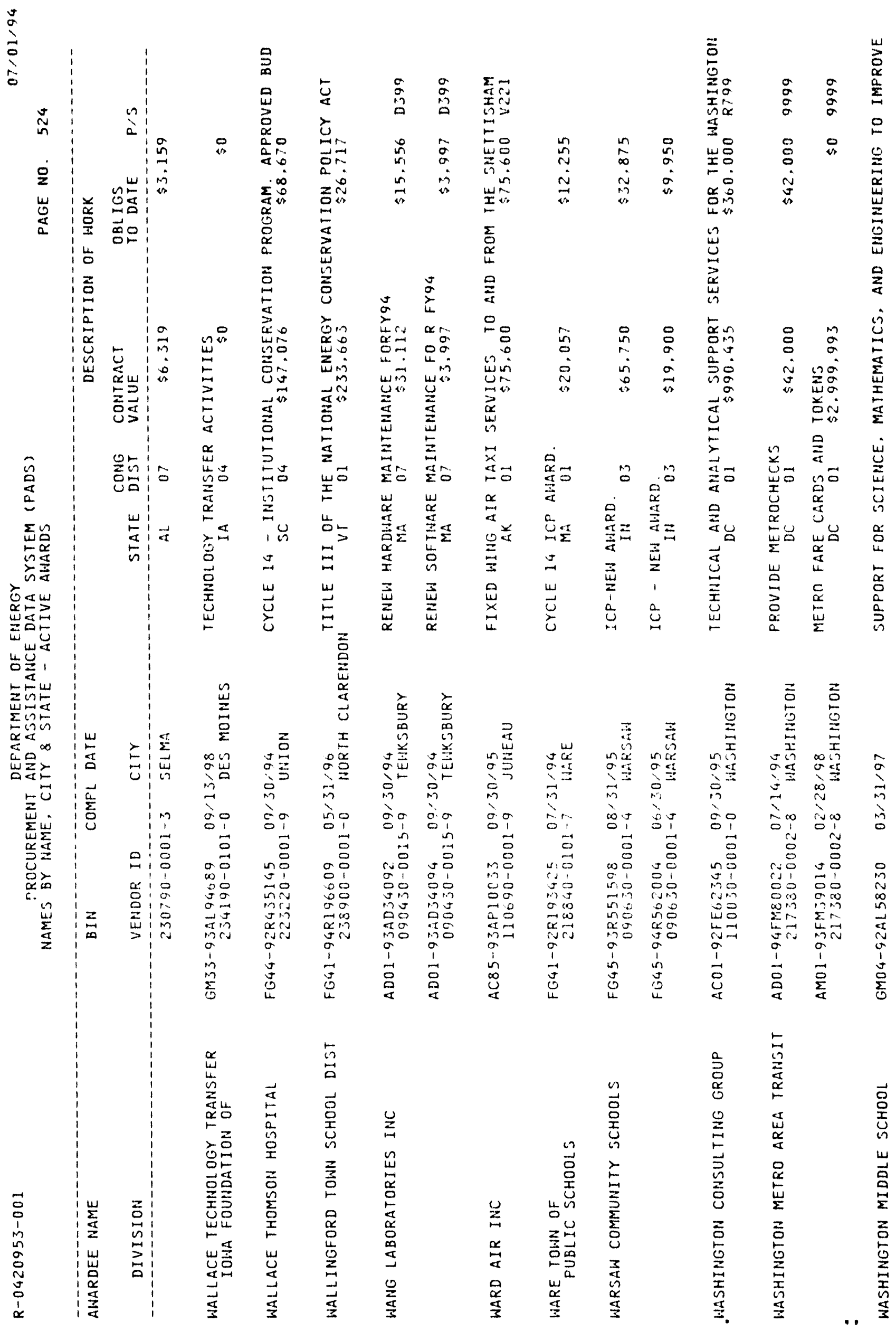


$\frac{\vdots}{0}$

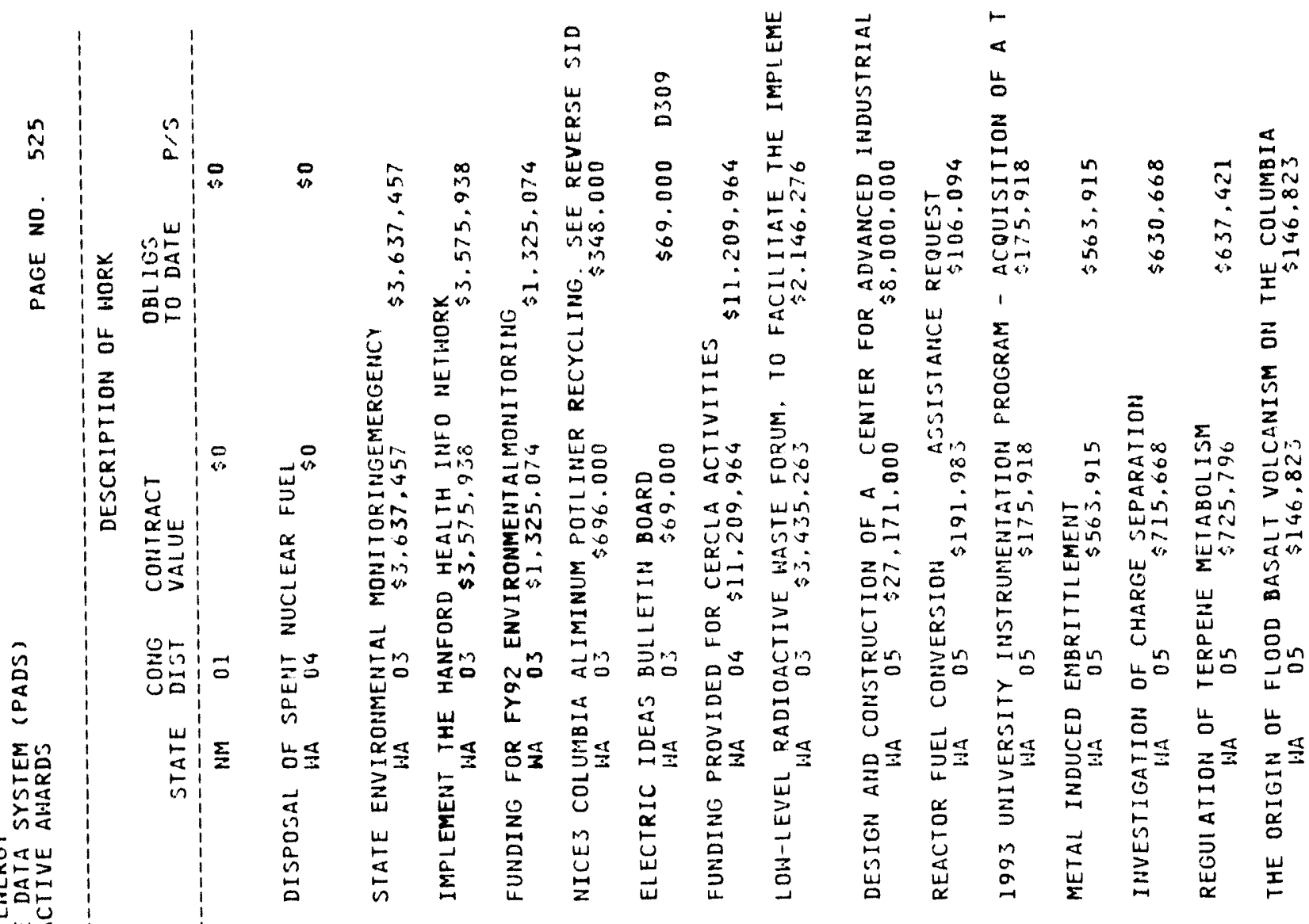

㟔预। $\stackrel{w}{2}$ 究点 व 崖藏

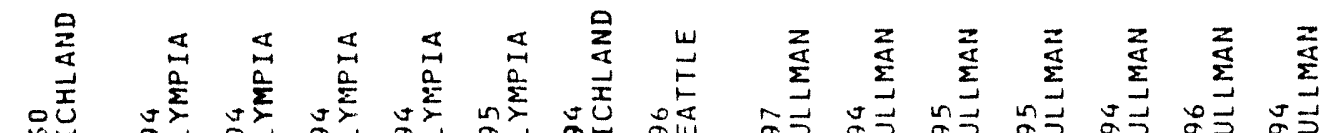

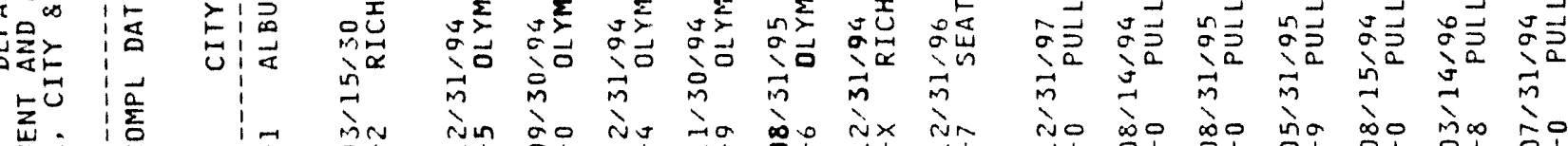

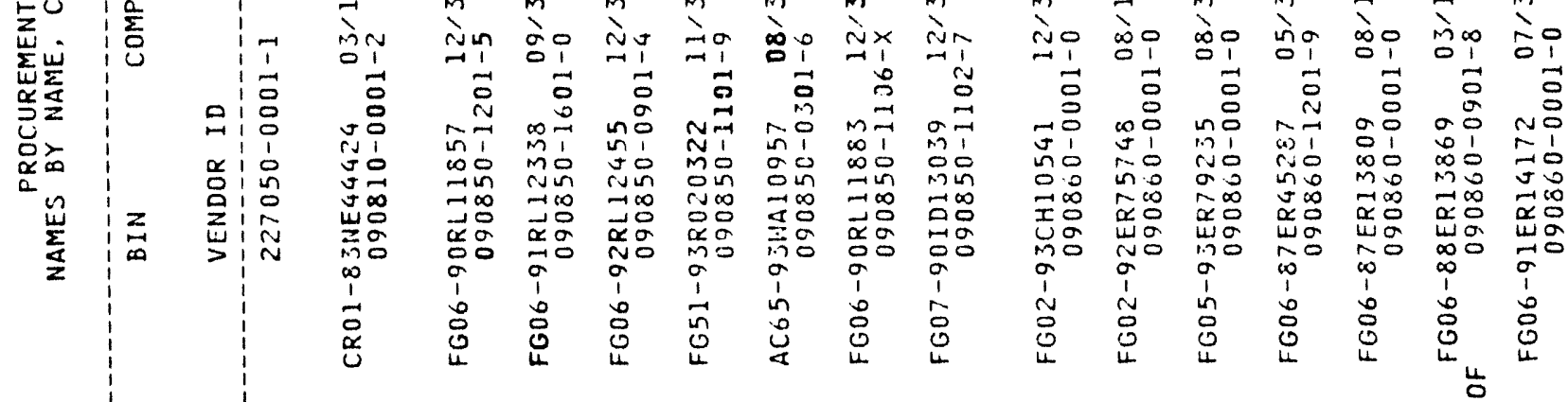

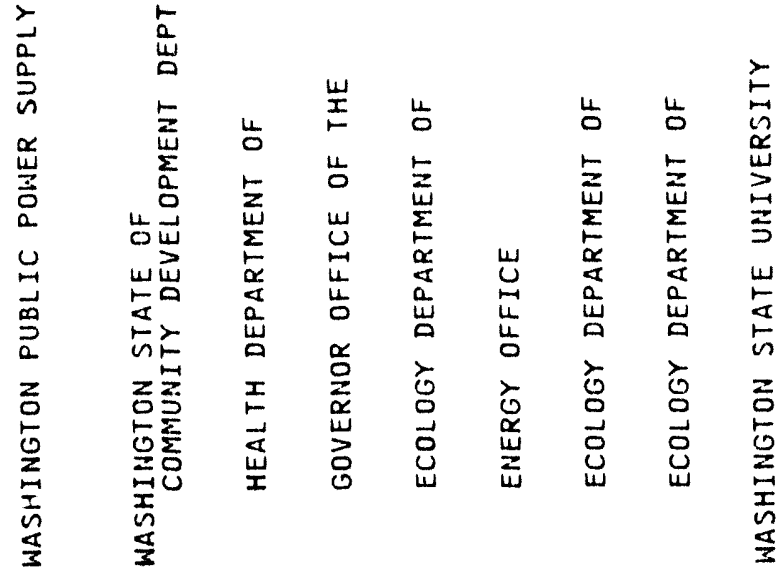

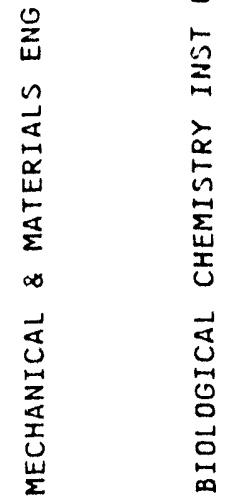




\begin{tabular}{|c|c|c|c|c|c|c|c|}
\hline AWARDEE NAME & BIIN & L DATE & & & DESCF & F HORK & \\
\hline DIVISION & VENDOR ID & CITY & STATE & $\begin{array}{l}\text { CONG } \\
\text { DIST }\end{array}$ & $\begin{array}{l}\text { COHIRACT } \\
\text { VALUE }\end{array}$ & $\begin{array}{l}\text { OBLIGS } \\
\text { TO DATE }\end{array}$ & $p / s$ \\
\hline
\end{tabular}

PHYSICS DEPT

PHYSICS DEPT

PHYSICS DEPT

MECHANICAL \& MATERIALS ENG MECHANICAL \& MATERIALS ENG

NORCUS

NORCUS

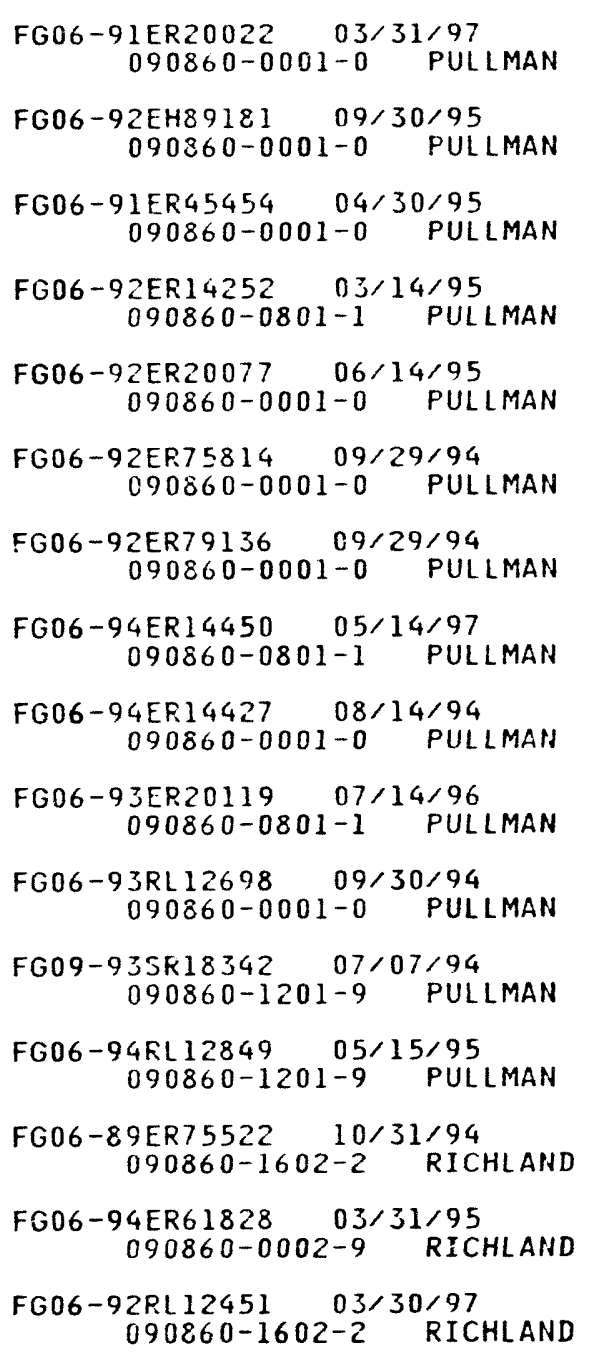

TOWARDS AN UNDERSTANDING OF STRUCTURAL VARIABILITY IN LIGNIN H.A $05 \$ 1,170,140$ $\$ 831,303$

PROPOSAL TO MANAGE AND OPERATETRANSURANIUM HA 05 \$4.762.179 $\$ 3.403 .954$ A STUDY OF TRANSIENT PARTICLE COARSENING
WA 05 \$188,769 $\$ 188,769$ UV LASER SURFACE INTERACTIONS RELEVANT TO $\$ 279,619$ MEMBRANE FUNCTION IN LIPID MUTANTS OF ARABIDOPSIS WA 05 T305,680 REACTOR SHARING PROGRAM HA 05 \$ $\$ 44,000 \quad \$ 44,000$ WSU RADIATION CENTER REACTOR AND RESEARCH EQUIPMENT UPGRADE HA 05 \$ $\$ 4,155$ R4,155

COUPLED PARTICLE DISPERIIOH BYTHREE DIMENSIONAL

$$
\text { HA } 05 \text { \$232,307 } \$ 73,683
$$

MEMBRANE BASED PHOTOCHEMICAL SYSTEMS AS MODELS

$$
\text { HA } 05 \text { \$ } \$ 50.000 \text { M } \$ 50.000
$$

CARBOH METABOI ISM IN SYMBIOTICHITROGEN FIXATION

$$
\text { WA } 05 \text { \$246,000 } \$ 162,000
$$

EXTABLISH A MESA 9TH GRADE TRANSITION PROGRAM TO DEVELOP WA MESA 05 TIH GRADE TRANSITION PROGRAM $\$ 40,000$

USED ENERGY RELATED LABORATORYEQUIPMENT

$$
\text { RA RELATED LABORATORYEQUIPMENT } 05 \text { \$0 \$0 }
$$

USED ENERGY RELATED LABORATORYEQUIPMENT GRANT

$$
\text { WA } 05
$$

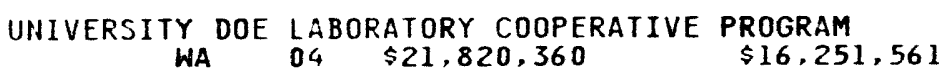
TECHNICAL SUPPORT FOR THE INTERHATIONAL SEMINAR ON WA 04 \$12,000 $\$ 12,000$

DOE UNIVERSITY LABORATORY COOPERATIVE PROGRAM

$$
\text { HA } 04 \$ 17,891,136 \quad \$ 17,891,136
$$




\begin{tabular}{|c|c|c|c|c|c|c|c|}
\hline AWARDEE NAME & BIN & $\angle$ DATE & & & DESCR & $=$ HORK & \\
\hline DIVIS & VENDOR ID & CITY & STATE & $\begin{array}{l}\text { CONG } \\
\text { DIJT }\end{array}$ & $\begin{array}{l}\text { CONTRACT } \\
\text { VALUE }\end{array}$ & $\begin{array}{l}\text { OBLIGS } \\
\text { TO DATE }\end{array}$ & $P / S$ \\
\hline
\end{tabular}

WASHINGTON UNIVERSITY RADIOL OGY DEPT

FG02-93ER61522 01/31/95 090880-0901-5 ST. LOUIS

MALLINCKRODT INST RADIOLOGY

BIOLOGY DEPT

PHYSICS DEPT

\section{CHEMISTRY DEPT}

MEDICINE SCHOOL OF

HEMATOLOGY \& ONCOLOGY DEPT

\section{CHEMISTEY DEPT}

SYSTEMS SCIENCE \& MATH DEPT

PHYSIOS DEPT

- BIOLOGY DEPT

MEDICINE SCHOOL OF

WASHINGTON UNIVERSITY OF CHEMISTRY DEPT FG02-84ER60218 $03 / 31 / 96$
$090880-0501-X \quad$ ST. LOUIS

FG02-84ER13255 05/31/97 090880-0101-4 ST. LOUIS

FG02-84ER45130 12/31/96 090880-0801-9 ST. LOUIS

FG02-87ER40316 12/31/95 $090880-0401-3$ ST. LQUIS

FG02-87ER60512 12/31/95 090880-1101-X ST. LOUIS

FG02-87ERI3704 04/14/96 $090880-1201-6$ ST. LOUIS

FG02-88ER40406 12/31/96 $090880-0401-3$ ST. LOUIS

FG02-90ER14140 07/14/96 090880-1001-3 ST. LOUIS

FG02-91ER40628 03/31/96 090880-0801-9 ST. LOUIS

FG02-92ER14297 09/14/95 $090380-0001-8$ ST. LOUIS

FG02-93ER61619 05/14/96 $090880-0001-8$ ST. LOUIS

FG02-93ER20105 08/14/94 090880-0101-4 ST. LOUIS

FGO2-93ER6158C 04/14/95 090880-1101-X ST. LOUIS

FG02-93ER61659 01/31/97 090880-0001-8 ST. LOUIS

FG06-91ER14224 09/14/94 090890-0502-1 SEATTLE

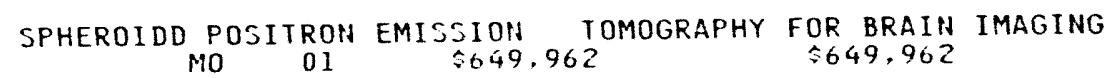

LABELING OF RECEPTOR LIGANDS WITH RADIOHALOGENS

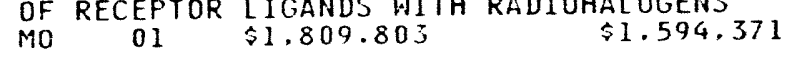

HYDROXYPROLINE - RICH GLYCOPROTEINS OF THE PLANT \& CELL HALL

$$
\text { MO } 01 \text { \$1.007.368 } \$ 932,236
$$

NON-EMPIRICAL INIERATOMIC FOTENIIALS FOR TRANSITION METALS MiO 01 \$ 856.214 S713,924

STUDIES JF COMPLEX FRAGMENT EMISSION IH HEAVY IOH REACTIONS MO 01 \$ $\$ 82,000 \quad \$ 840.000$

PREPARATIOH OF GALLIUM-68 RADIOPHARMACEUTICALS FOR POSITRON MO $01 \$ 1.623 .145$ \$ $\$ 1.341 .448$

PROCESSIHG \& TARGETING OF THE THIOL PROTEASE. ALEURAIN

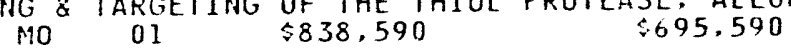

INVESTIGATIONS OF NUCLEAR STRUCTURE AND NUCLER REACTIONS MO 01 \$2, 500,000 $\$ 1.935 .000$

DYNAMICAL SYSTENS WITH IITIER - NAL STRUCTURE A HEW APPROACH

$\begin{array}{lll}\text { MO UI } & \$ 455,375 & \$ 294,375\end{array}$

STUDIES IN QUANTUM FIELD
MO OHEORY

THERMODYIAMICS PROPERTIES OF AQUEOUS SOLUTIONS AT SUPER-
MO
01

FIRST INTERNATIONAL WORKSHOP ON HUMAN CHROMOSOME? TO BE MO $01 \quad \$ 14,581 \quad \$ 14,581$

PARTICLA SUPPORT FOR A $\begin{gathered}\text { SYMPOSIUM ENTIILED "THE PAST } \\ \text { MO } 01\end{gathered}$

$\times$ CHROMOSOME WORKSHOP TO BE HELD MAY 9-12, 1993

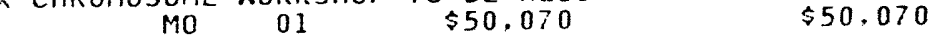

DETECTION \& ASSESSMENT USING POSITIOH EMISSION TOMOGRAPTIY MO $0 \mathrm{~J} \$ 1,350,211 \quad \$ 587.829$

ATOMIC PROBES OF SURFACE STRUCTURE

WA 07 \$309,000

$\$ 309,000$ 


\begin{tabular}{|c|c|c|c|c|c|c|c|}
\hline AHARDEE NAME & BIN & L DATE & & & $\mathrm{DESCF}$ & FORK & \\
\hline DIVISION & VENDOR ID & CITY & STATE & $\begin{array}{l}\text { CONG } \\
\text { DIST }\end{array}$ & $\begin{array}{l}\text { COHTRACT } \\
\text { VALUE }\end{array}$ & $\begin{array}{l}\text { OBL IGS } \\
\text { TO DATE }\end{array}$ & $P / S$ \\
\hline
\end{tabular}

CHEMISTRY DEPT

BIOLOGICAL STRUCTURE DEPT

PHYSICS DEPT

CHEMISTRY DEPT

APPLIED MATHEMATICS DEPT

MEDICINE SCHOOL OF

GEOLOGICAL SCIENCES DEPT

PHYSICS DEPT

OCEANOGRAPHY SCHOOL OF

PHYSICS DEPT

MEDICINE SCHOOL OF

CHEMISTRY DEPT

CHEMISTRY DEPT

MECHANICAL ENGINEERING DEPT

MEDICINE SCHOOL OF
FG06-93ER14372008/31/94 090890-0502-1 SEATTLE FG06-86ER60409 12/31/94 090890-0202-2 SEATTLE FG06-90ER45425 12/14/94 090890-1702-X SEATTLE

FG06-92ER14254 10/31/94 090890-0502-1 SEATTLE

FG06-92ER75775 12/31/94 090890-0002-X SEATTLE

FG06-86ER25019 07/31/95 090890-2702-5 SEATTLE

FG06-93ER61553 01/31/95 090890-2602-9 SEATTLE

FG06-92ER14231 02/28/95 090890-1102-1 SEATTLE

FG06-90ER $40561 \quad 02 / 28 / 95$
$090890-1702-X \quad$ SEATTLE

FG06-90ER61037 04/30/95 090890-1902-2 SEATTLE

FG06-91ER40655 07/14/95 090890-1702-X SEATTLE

FG06-92ER61487 02/28/95 090890-2602-9 SEATTLE

FG06-94ER76005 04/15/95 090890-0502-3 SEATTLE

FG06-93ER79208 06/14/95 090890-0502-1 SEATTLE

FG22-92PC92548 01/24/95 D90890-1402-0 SEATTLE

FG06-92ER61459 10/31/95 $090890-2602-9$ SEATTLE

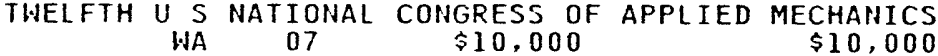
IMMUNIDEFICIENCY SYNDROMES: DISORDERS OF

$$
\text { MA } 07 \text { \$2,086,060 } \$ 1,800,308
$$

LOCAL ENVIROHMENT INDUCED BY IMPURITIES

$$
\begin{array}{lll}
\text { WA } 07 & \$ 352,600 & \$ 352,600
\end{array}
$$

HOMOLYTIC ACTIVATION OF HYDROCARBONS AND

$$
\text { WA } 07 \text { \$153,000 } \$ 153,000
$$

ENCOURAGING IHNOVATIOIN FOR WASHINGTON PRESERVICE

$$
\text { HA } 07 \text { \$ } \$ 77,566 \quad \$ 77,566
$$

NONL INEAR RESONANCE

$$
\text { WA } 07 \quad \$ 524,652 \quad \$ 524,643
$$

CHROMOSOME MAPPIHG BY FLUORESCENT IN SITU

$$
\text { WA } 07 \text { \$1, } 147,727 \quad \$ 1,147,727
$$

TWO AND THREE DIMENSIONAL MAGNETOTELLURIC

$$
\text { HA } 07 \text { \$205,435 } \$ 205,435
$$

NATIONAL INSTITUTE FOR NUCLEARTHEORY

$$
\text { \$A } 07 \quad \$ 5,855,460 \quad \$ 5,855,460
$$

MIXED-LAYER MODEL

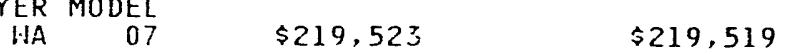

SSC MUON SUBSYSTEM R\&D PROGRAM

WA $07 \quad \$ 1.090,800 \quad \$ 1,090,800$

VERTICALLY INTEGRATED ANALYSISOF HUMAN DNA

$$
\text { WA } 07 \quad \$ 1,702,280 \text { HUMAN DNA } \$ 1,702,280
$$

1994 HEPAN ANNUAL CONFERENCE

$$
\begin{array}{lrr}
\text { WA } & 07 & \$ N N \text { COHFERENCE }
\end{array}
$$

PRE FRESHMAH EMRICHMENT PROGRAM

SUPRESSION OF FINE ASH FORMATION IN PULVERIZED COAL FLAMES

$$
\text { WA } 0 ? \quad \$ 181,281 \text { S } \$ 181,281
$$

IMPROVED RADIOIMMUNOTHERAPY OFHEMATOLOGIC
WA 07
$\$ 577,000$
$\$ 407,410$ 
DIVISION VENDOR ID CITY $\begin{array}{ll} & \text { CONG CONTRACT } \\ \text { STATE IGS }\end{array}$ DIVISION
MOLECULAR BIOTECHNOLOGY DEPT

CHEMISTRY DEPT

PHYSICS DEPT

PHYSICS DEPT

AERONAUTICS \& ASTRONAUTICS

CHEMISTRY DEPT

CHEMISTRY DEPT

CHEMISTRY DEPT

CHEMISTRY DEPT

CHEMISTRY DEPT

CHEMISTRY DEPT

NUCLEAR PHYSICS LABORATORY

\section{PHYSICS DEPT}

CHEMISTRY DEPT

AEROSPACE \& ENERGETICS RES

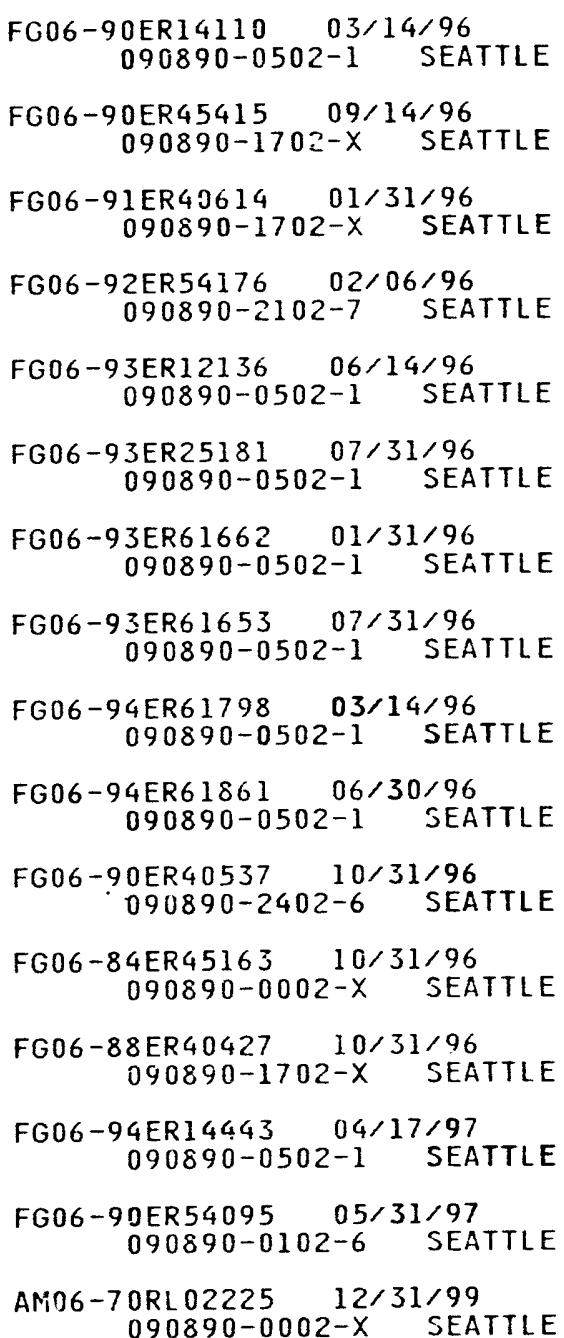

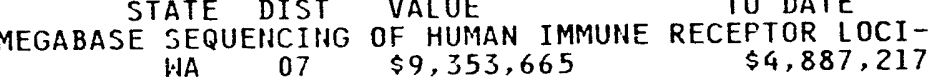

MODEL CU/ZNO CATALYSTS FOR METHANOL

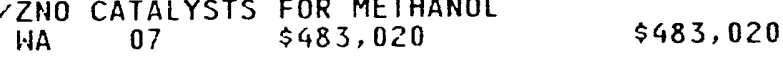

THEORETICAL STANDARDS OF XAFS

WA $07 \quad \$ 253,509$

$\$ 184,897$

EXPERIMENTAL AND THEORETICAL NUCLEAR PHYSICS

HA 07 \$5,518,117 $\$ 4,417,117$

TOKAMAK REFUELING BY ACCELERATED PLASMOIDS

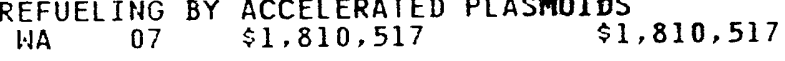

THE SUPERDONIC MIXING SHOCK WAVE REACTOR AN INNOVATIVE WA $07 \quad \underset{\$ 98,576}{\$ 677,346}$

IMMERSED INTERFACE METHODS

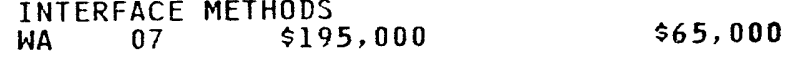

FLOW KARYOTYPING AND FLOW INSTRUMENTATION DEVELOPMENT

$$
\text { HA } 07 \quad \$ 1,068,181 \quad \$ 675,238
$$

PET IMAGING OF PROTEIN SYNTHESIS RIBOSOMES AND MRNA WA $07 \quad \$ 423,360 \quad \$ 138,354$

INVOLVEMENT OF HIGH SCHOOL STUDENTS IN THE

ANALYSES OF SHADOWBAND RADIOMETRIC DATA WITH EMPHASIS WA $07 \quad \$ 57,828 \quad \begin{aligned} & \$ 57,828 \\ & 07\end{aligned}$

EXPERIMENTAL NUCLEAR PHYSICS

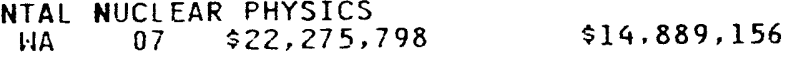

$X$-RAY SPECTROSCOPY OF SOLIDS UHDER PRESSURE

$$
\begin{array}{lll}
\text { WA } 07 & \$ 1,358,423 & \$ 1,114,987
\end{array}
$$

NUCLEAR THEDRY $07 \quad \$ 5,415,000 \quad \$ 4,115,000$

FEMTOSECOND SPECTROSCOPY OF ENERGY TRANSFER

FORMATION AND SUSTAIMMENT OF AVERY LOW ASPECT RATIO WA $07 \quad \$ 2,824,737 \quad \$ 1,841,543$

BASIC CONTRACT

WA $01 \quad \$ 0$




\section{AWARDEE NAME}

BIN

COMPL DATE

DESCRIPTION OF WORK

VENDOR ID

CONG CONTRACT

OBL IGS

$P / S$

WASHOE COUNTY SCHOOL DISTRICT

WASHOE MEDICAL CENTER

WASTE MGMT OF FOUR CORNERS

WASTE MGMT OF THE ROCKIES

\section{HASTE POLICY INSTITUTE}

\section{WATANABE \& HOTTA}

WATERWORKS DIST 9 OF WARD 4

WATKINS SECURITY AGENCY INC

WATSONVILLE COMMUNITY HOSPITAL

"WAYNE COUNTY OF
SCHOOL DISTRICT

EDUCATION BOARD OF

WAYNE STATE UNIVERSITY PHYSICS DEPT
FG08-93NVI1373 09/30/94 090900-0001-1 REND FG49-93R915004 05/31/95
$090910-0001-5$ REIIO

AX65-94HK14563 09/30/94 235580-0001-3 RIO RAHCHO AX65-94WK $1456409 / 30 / 94 \quad 04$ GARBAGE SVC $-\underset{03}{\text { HAYDE }}$

STEP PROJECT-TRAINING IN EXPERIMENTAL DESIGN FOR ELEMENTARY

CYCLE 15 ICP GRANT AWARD

$$
\text { NV } 02 \quad \$ 42,013 \quad \$ 22,006
$$

GARBAGE SVC $-\underset{N M}{\text { SHIPROCK SUBST }} \underset{03}{\$ 1,080}$

$\$ 1,080 \$ 205$ 235630-0001-8 STEAMBOAT SPRINGS

AC01-93EW50512 10/28/97 216620-0001-4 BLACKSBURG

TECHNICAL SUPPORT SERVICES FORENVIRONMENTAL RESTORATION \& W VA 09 \$40,176,157 $\$ 40,176,157$ R425 ABO1-93GC20335 $09 / 30 / 95$

AC96-88P015090 02/07/98 177710-0001-0 LAKE CHARLES

AC21-90MC27331 05/31/95 96440-0001-3 MORGANTOWN

BPA FOR GERMAN PATENTS
OO
\$0
\$0 $\quad \mathbf{R 4 9 9}$

HATER SERVICE FOR THE TEXOMA/ LAKE CHARLES METERING STATION

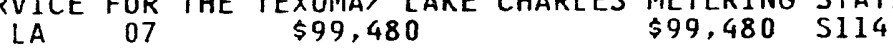

METC PHYSICAL SECURITY SERVICE

$\$ 1,992,450 \quad 5206$

FG49-92R914052 08/31/94 222680-0001-X WATSONVILLE TITLE III OF THE NATIONAL ENERGY CONSERVATION POLICY ACT

FG44-93R43543509/30/95 $210270-0303-6$ HAYNESBORO FG44-93R435066 01/31/95 210270-0202-1 HAYNESBORO

FG02-85ER40209 03/31/95 $091280-0401-9$ DETROIT

FG02-92ER40713 05/14/96 SEE ATTACHED PAGE NO. $2 \underset{\text { MS }}{2} \underset{\$ 5}{\text { OF THIS NOTICE OF FINANCIAL ASSISTAN }} \underset{\$ 64,938}{ }$

CYCLE 14 - INSTITUTIONAL CONSERVATION PROGRAM. APPROVED BUD
$\$ 48,770$
$\$ 9,990$

QUANTUM THEORY OF FIELDS

$\begin{array}{lll}M I & 13 & \$ 921,000\end{array} \$ 921,000$

RELATIVISTIC HEAVY ION RESEARCH 
PAGE NO. 531

\begin{tabular}{|c|c|c|}
\hline AWARDEE NAME & COMPL DATE & DESCRIPTION OF HORK \\
\hline DIVISION & VENDOR ID & $\begin{aligned} \text { CONG } \\
\text { STATE DIST }\end{aligned}$ \\
\hline \multirow{3}{*}{ PHYSICS DEPT } & $091280-0401-9$ & $\$ 1,533,000$ \\
\hline & $\begin{array}{l}\text { FG02-92ER20089 } 07 / 14 / 95 \\
091280-0001-3 \text { DETROIT }\end{array}$ & $\begin{array}{c}\text { MUTAGENESIS OF AN ENERGY } \\
M I\end{array} \underbrace{}_{13} \underset{251,103}{\text { TRANSDUCING }} \begin{array}{l}\text { MEMBRANE PROTEIN } \\
\$ 166,461\end{array}$ \\
\hline & $\begin{array}{l}\text { FG02-94ER61807 } 03 / 31 / 95 \\
091280-0001-3 \text { DETROIT }\end{array}$ & $\begin{array}{ccccc}\text { FIFTH INTERNATIONAL HUMAN } & \text { CHROMOSOME } & \text { WORKSHOP TO BE HELD } \\
\text { MI } & 15 & \$ 16,067 & \$ 16,067 & \end{array}$ \\
\hline PHYSICS DEPT & $\begin{array}{c}\text { FG02-94ER40831 } 01 / 31 / 96 \\
091280-0401-9 \text { DETROIT }\end{array}$ & $\begin{array}{cccc}\text { SPACE-TIME DESCRIPTION OF } & \text { UTRAVELATIVISTIC HEAVY ION } \\
\text { MI } & 15 & \$ 95,000 & \$ 45,000\end{array}$ \\
\hline WAYNE TOWNSHIP OF & $\begin{array}{c}\text { FG05-940R22210 } 08 / 31 / 94 \\
238770-0001-0 \text { WAYNE }\end{array}$ & $\begin{array}{c}\text { PAYMENT IN LIEU OF TAXES } 1991 \text { AND } 1992 \\
\mathrm{NJ} 12\end{array}$ \\
\hline \multirow[t]{2}{*}{ WAYNFLETE SCHOOL } & $\begin{array}{l}\text { FG41-93R192507 06/30/95 } \\
125660-0001-8 \text { PORTLAND }\end{array}$ & TITLE III OF THE NATIONAL ENERGY CONSERVATION POLICY ACT \\
\hline & $\begin{array}{l}06 / 30 / 96 \\
\text { PORTLAND }\end{array}$ & FY'94 ICP AHARD \\
\hline WEFA GROUP & $\begin{array}{rl}A C 01-93 E I 23551 & 09 / 30 / 94 \\
216160-0001-5 \quad B A L A-C Y N H Y D\end{array}$ & $\begin{array}{cccc}\text { COMPUTER TIMESHARING AND ECONOMETRIC FORCASTING SERVICES } \\
\text { PA } 01 & \$ 99,180 & \$ 99,180 & \text { DJ09 }\end{array}$ \\
\hline WEIZMANH INSTITUTE OF SCIENCE & $\begin{array}{l}\text { FG02-94ER61831 } 05 / 31 / 97 \\
238550-0001-8 \text { REHOVOT }\end{array}$ & 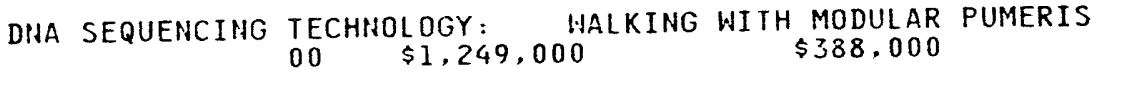 \\
\hline WELCH JAMES D & $\begin{array}{rl}F G 47-93 R 701314_{4} & 12 / 31 / 94 \\
230230-0001-8 \text { OMAHA }\end{array}$ & $\begin{array}{ccc}1993 \text { ENERGY } & \text { RELATED INVENTIONSPROGRAM. } \\
\text { NE } & 02 & \$ 85,000\end{array}$ \\
\hline WELDCOMPUTER CORP & $\begin{array}{c}\text { FG01-94CE15588 } 04 / 15 / 96 \\
237400-0001-5 \text { GREEN ISLAND }\end{array}$ & 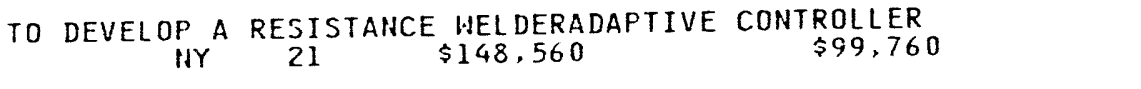 \\
\hline $\begin{array}{l}\text { HELDON CITY OF } \\
\text { SCHOOLS }\end{array}$ & $\begin{array}{c}\text { FG44-93R435402 } 09 / 30 / 94 \\
091610-0101-5 \text { HELDON }\end{array}$ & 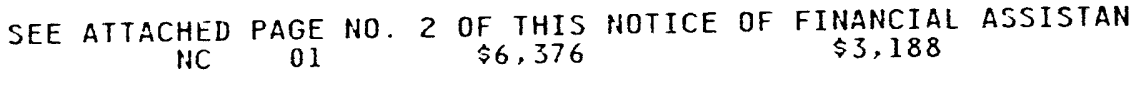 \\
\hline WELLS FARGO GUARD SERVICES & $\begin{array}{c}\text { AC65-93HG18630 02/28/95 } \\
091640-0003-6 \quad \text { BOULDER CITY }\end{array}$ & 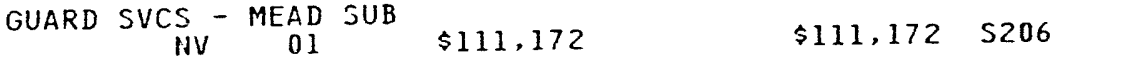 \\
\hline $\begin{array}{l}\text { WENATCHEE CITY OF } \\
\text { SCHOOL DISTRICT } \$ 246\end{array}$ & $\begin{array}{c}\text { FG51-93R020305 08/31/94 } \\
224020-0201-8 \text { WEHATCHEE }\end{array}$ & $\begin{array}{c}\text { CYCLE } 15 \text { ECM AWARD. } \\
\text { WA } 04\end{array}$ \\
\hline
\end{tabular}




\begin{tabular}{|c|c|c|c|c|c|c|c|}
\hline AWARDEE NAME & BIN & $\perp$ DATE & & & DESC & F WORK & \\
\hline DIVISICN & VENDOR ID & CITY & STATE & $\begin{array}{l}\text { CONG } \\
\text { DIST }\end{array}$ & $\begin{array}{l}\text { COHTRACT } \\
\text { VALUE }\end{array}$ & $\begin{array}{l}\text { OBL IGS } \\
\text { TO DATE }\end{array}$ & $\mathrm{P} / \mathrm{S}$ \\
\hline
\end{tabular}

WESLEYAN UNIVERSITY

WEST FELICIANA PARISH HOSPITAL

WEST FLORIDA UNIVERSITY OF

WEST GEORGIA COLLEGE

CHEMISTRY DEPT

CHEMISTRY

CHEMISTRY DEPT

CHEMISTRY DEPT

CHEMISTRY

CHEMISTRY

CHEMISTRY DEPT

. WEST HILLS CONSOL SCH DIST

\section{FG02-93ER61598 04/30/96 026930-0001-7 MIDDLETOWN}

STUDIES OF DNA DUPLEXES $\$ 370,000$ CONTAINING DAMAGED THYMINES

$3 R 613612 \quad 02 / 28 / 95$ AHARDS A NEN CYCLE 15 ENERGY CONSERVATION MEASURE GRANT.

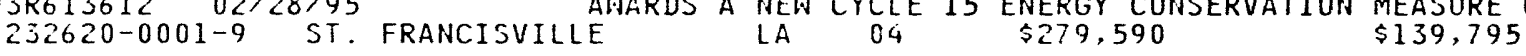

FG05-93ER79209 $06 / 14 / 95$ $091880-0001-x$ PEHSACOLA

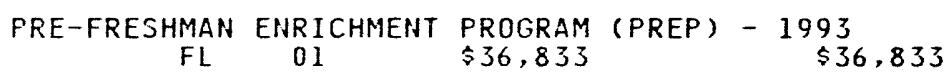
FG05-9 $\begin{gathered}\text { JER } 40808 \\ 136440-0001-8 / 31 / 96\end{gathered}$
CARROLLTON FG06-94RL12846 04/17/95
$136440-0101-4$ CARROLLTON FG06-93RL12667 09/05/94
$136440-0101-4 \quad$ CARROLLTON FG06-94RL12771 $02 / 06 / 95$
$136440-0101-4$ CARROLLTON FG06-94RL12764 02/06/95
$136440-0101-4$ CARROLLTON FG09-94SR18426 03/20/95
$136440-0001-8 \quad$ CARROLLTOH FG09-945R18383 10/20/94 136440-0001-8 CARROLLTOH FG09-93JRR18355 08/05/94 136440-0101-4 CARROLLTON FG09-935R18364 $08 / 25 / 94$ 136440-0101-4 CARROLLTOH FG34-94RF00889 05/04/95 $136440-0101-4$ CARROLLTON

FG49-91R913036 $02 / 28 / 95$ 214920-0001-9 COALINGA
LOW ENERGY POLARIZED PROTON CAPTURE IN LIGHT NUCLEI
GA
07

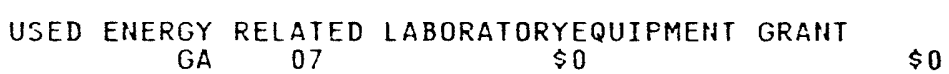

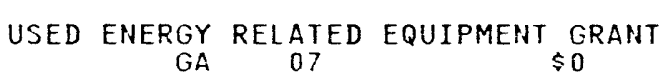
USED ENERGY RELATED LABORATORYEQUIPMENT GRANT USED ENERGY RELATED LABORATORYEQUIPMENT GRANT USED ENERGY RELAIED LABORATORYEQUIPMENT GRANT
G0 USED ENERGY RELATED LABORATORYEQUIPMENT USED ENERGY RELATED LABORATORYEQUIPMENT USED ENERGY RELATED LABORATORYEQUIPMENT USED ENERGY
GA
RELATED LAB EQUIPMENT
07

$$
\text { CA } 15 \quad \$ 137,321
$$




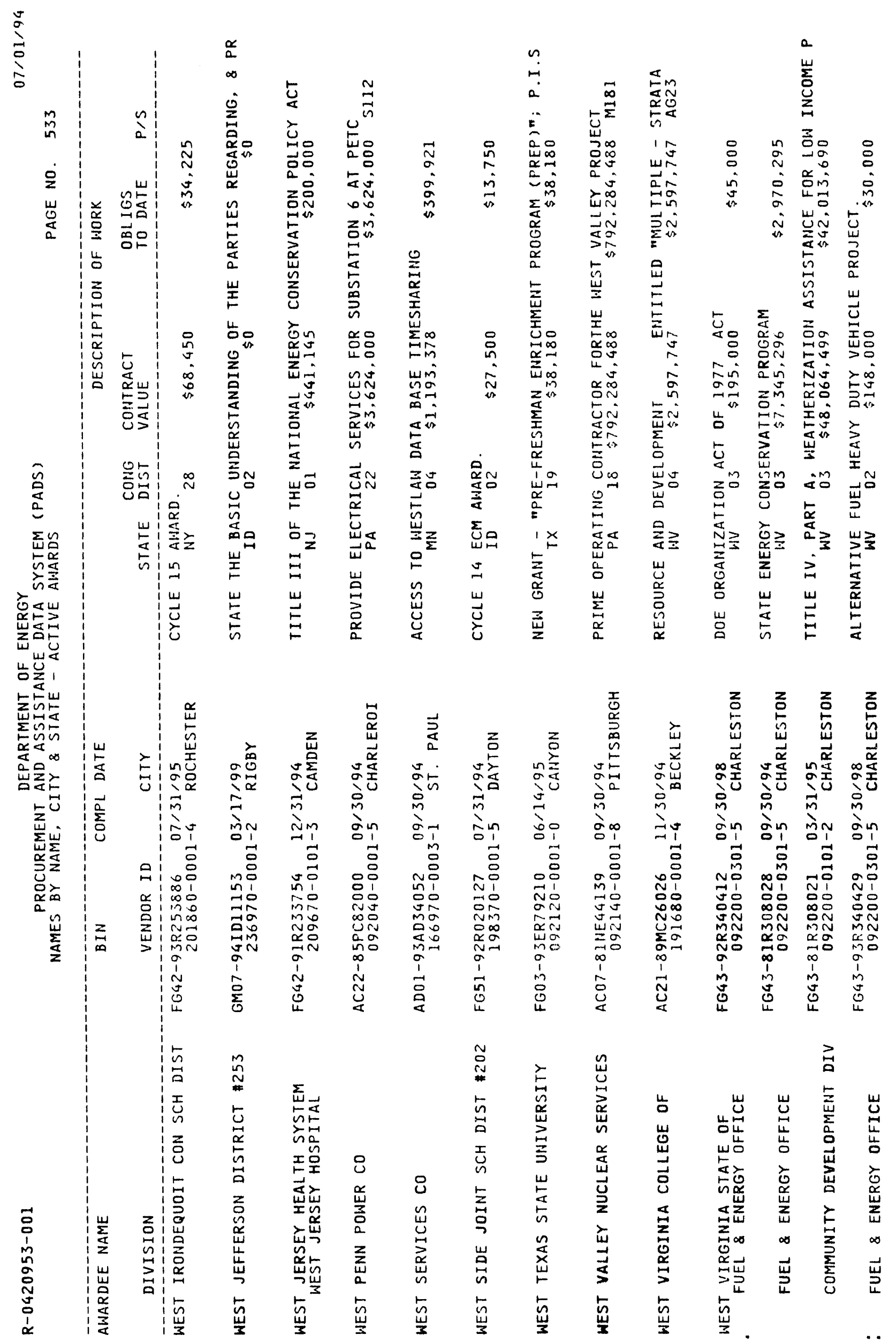


$\frac{⿱ 亠}{\sigma}$

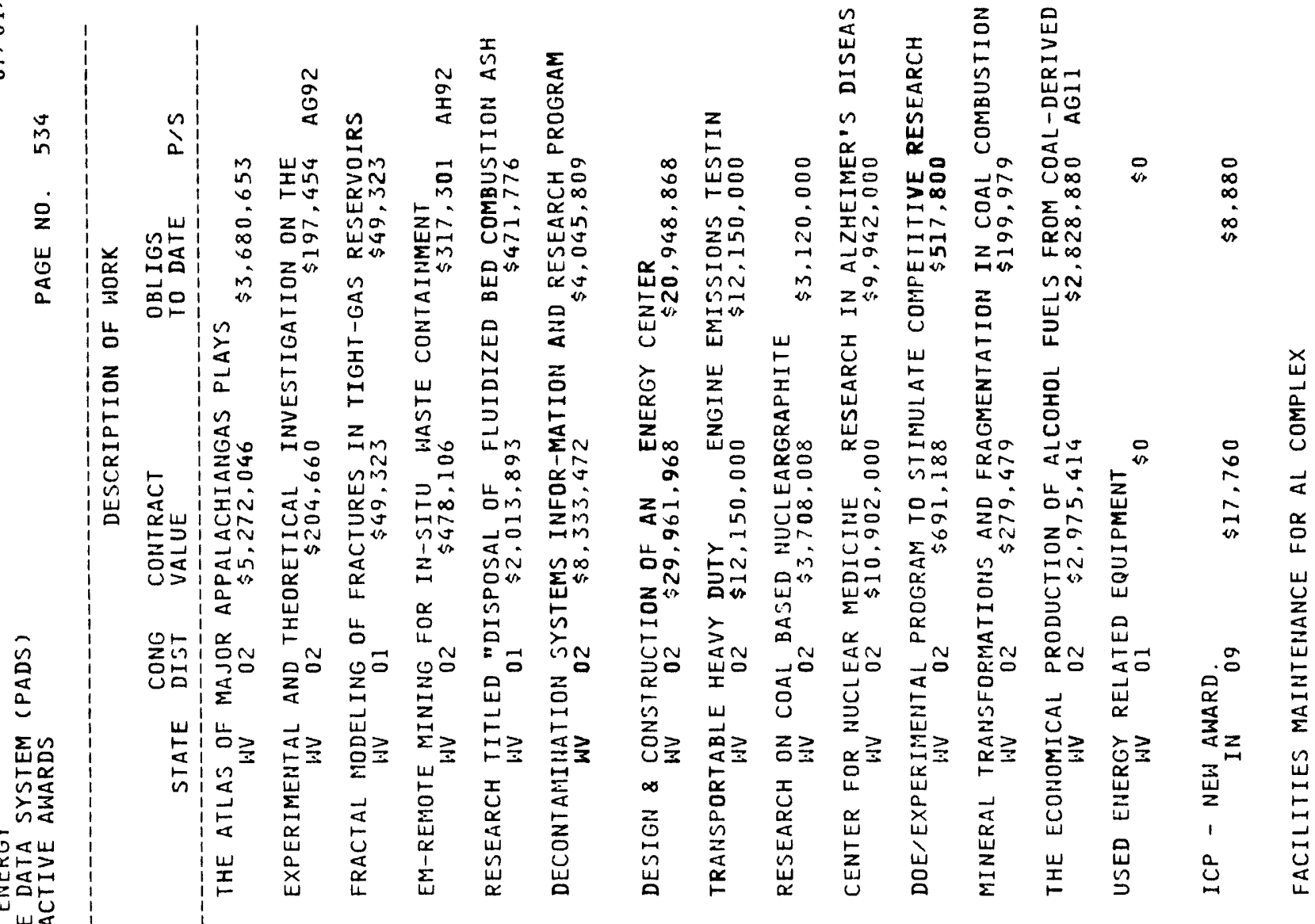

응

辣帒

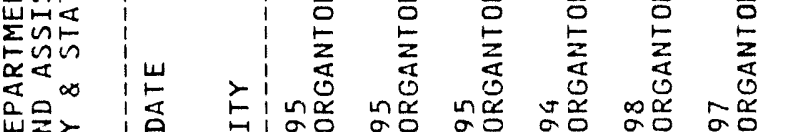

岩至己

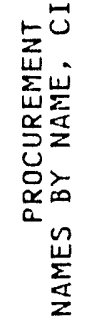

t)

遈

$\sum_{0}^{a}$

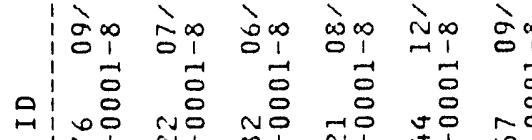

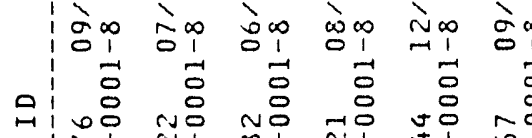

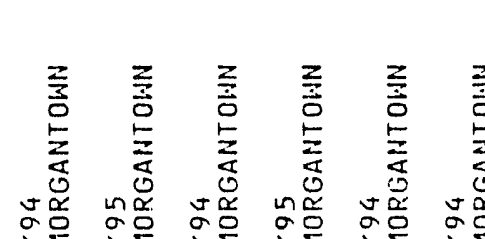

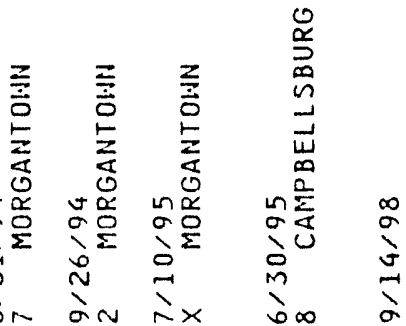

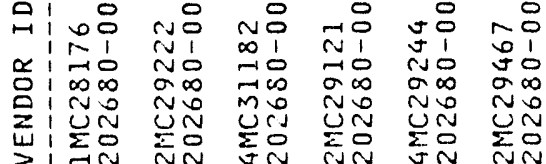

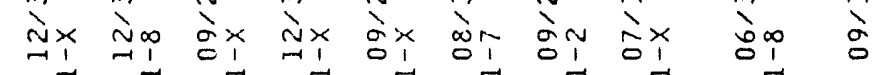

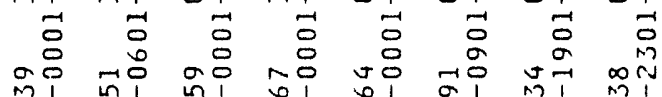

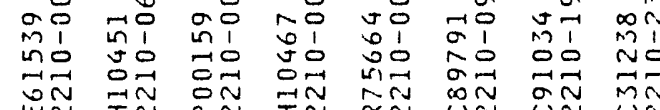

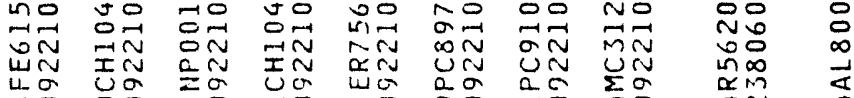

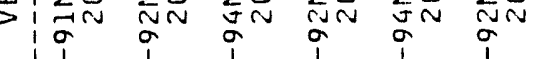

我

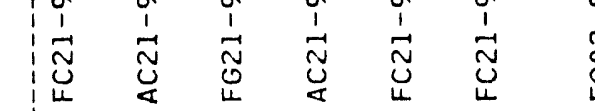

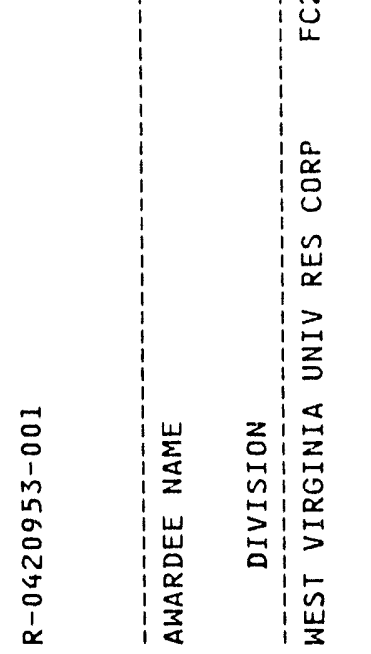

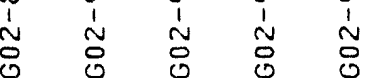

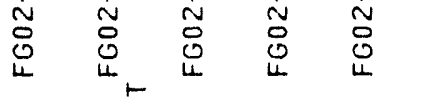

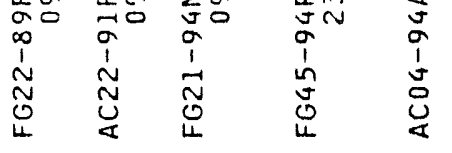
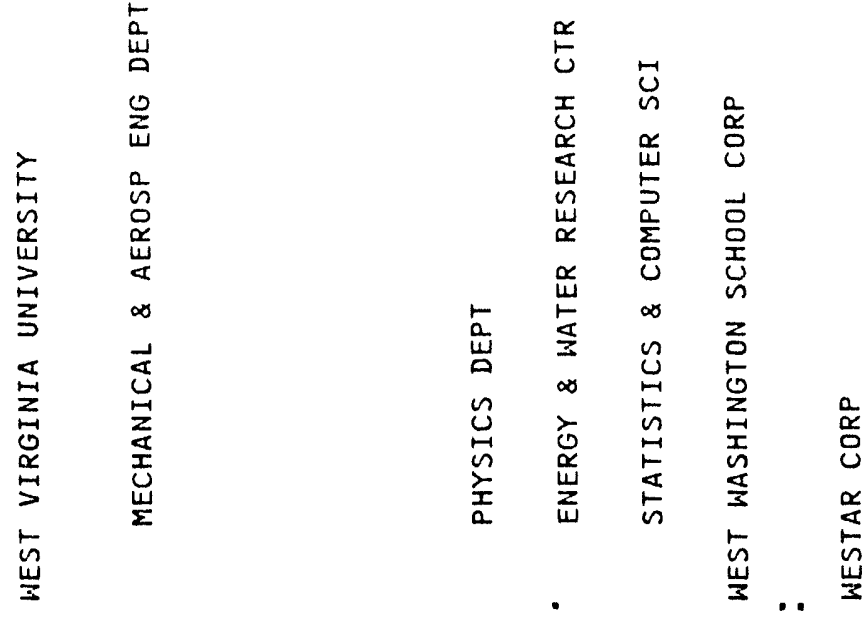
5
0
0
0
0

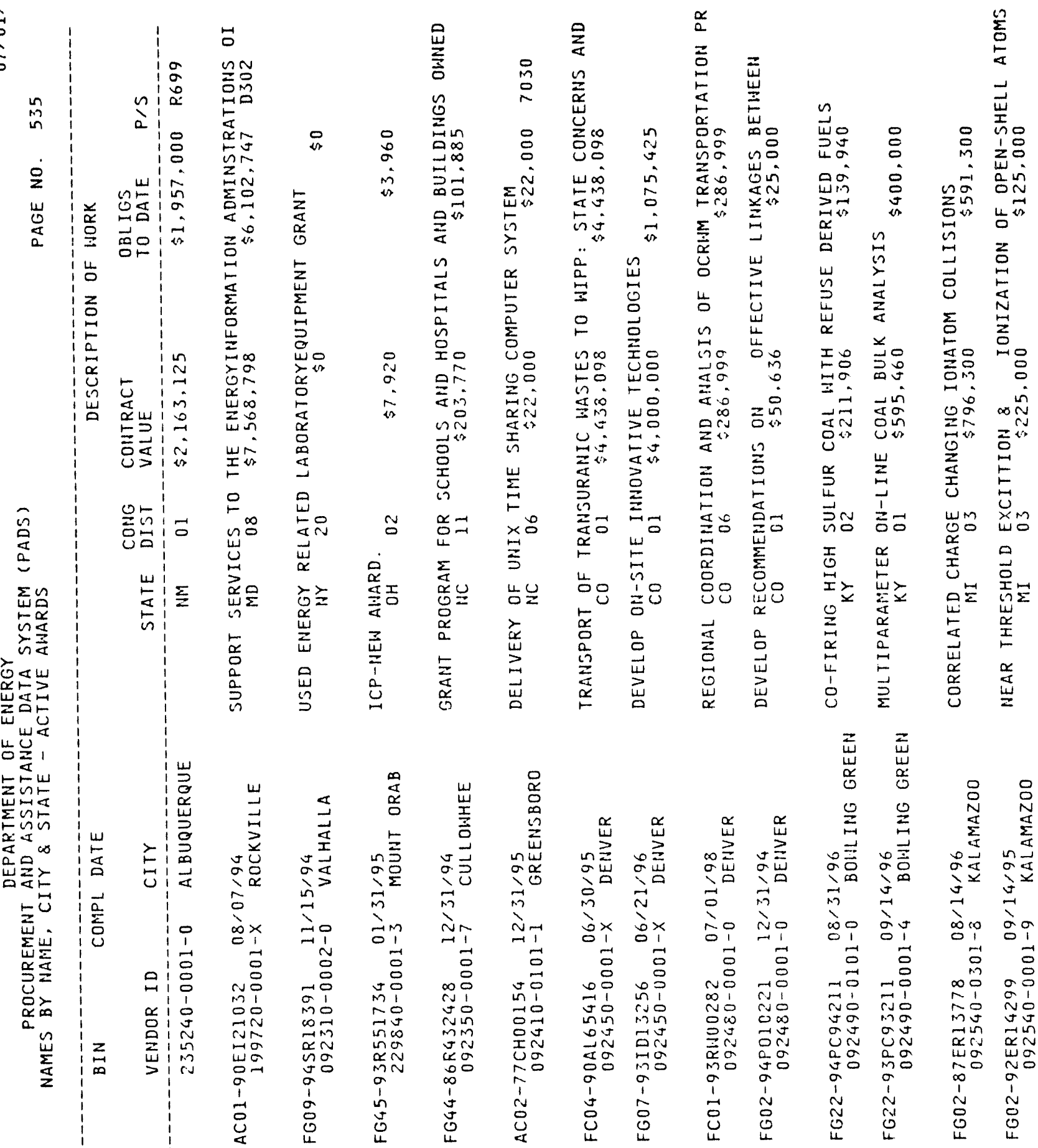

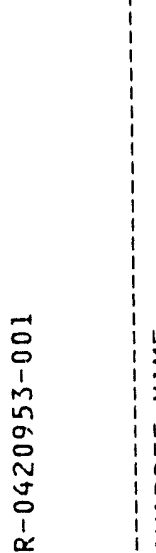

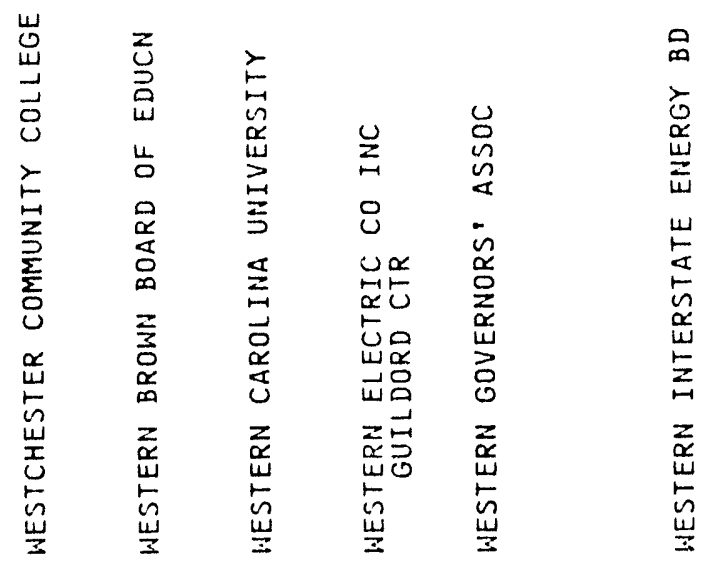

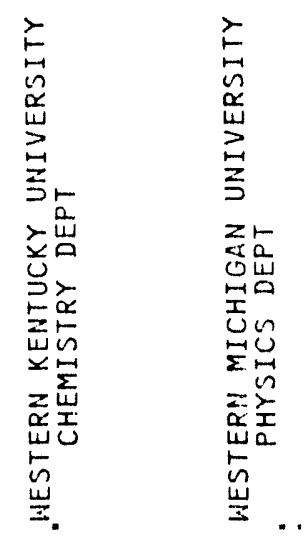


5
$\vdots$
0
0
0
0

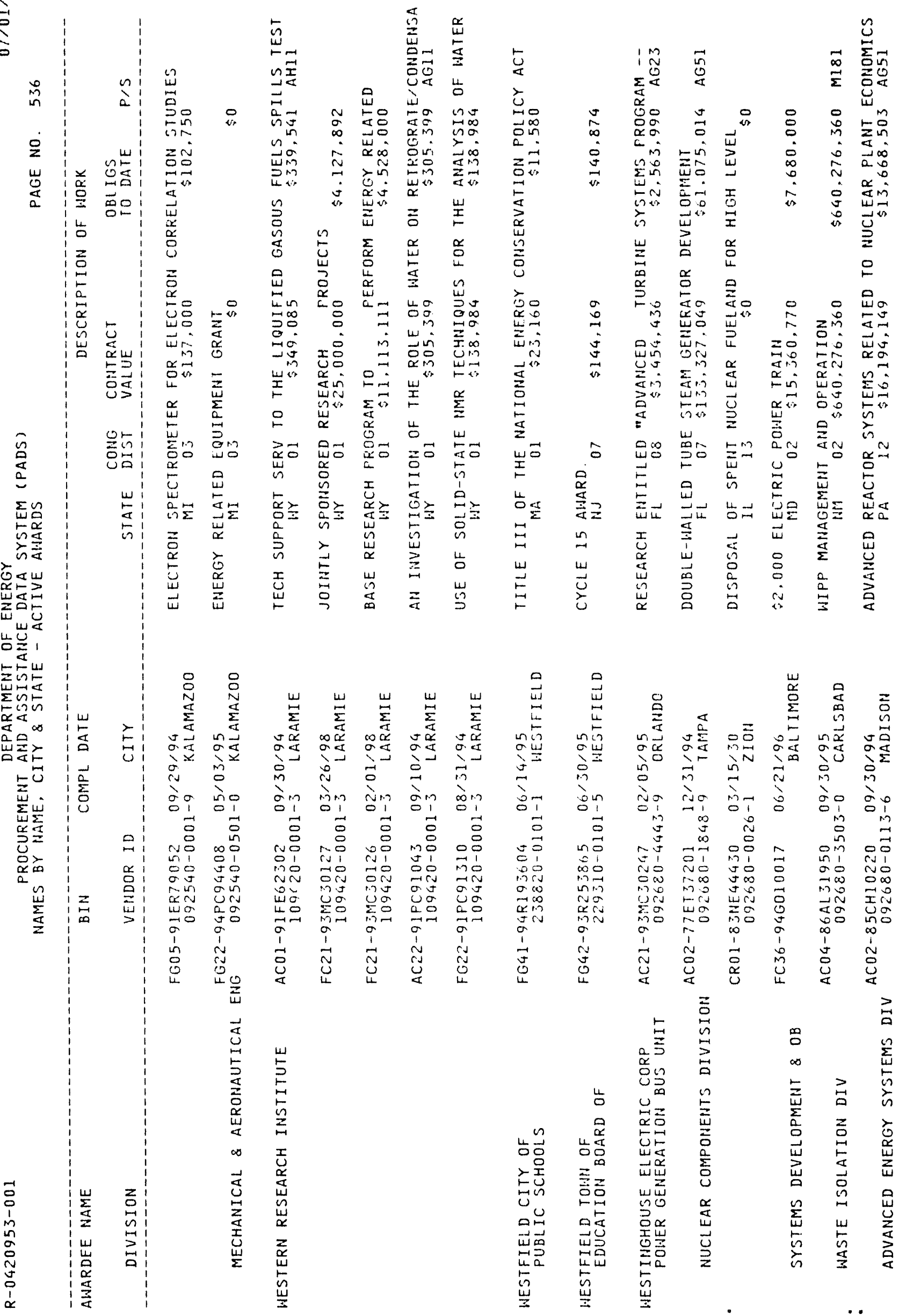


AWARDEE NAME

BIN

COMPL DATE

CITY

AC07-88ID12752 09/30/94 $092680-0013-X \quad$ MADISON

AC01-92NE32166 06/29/95 092680-0019-9 PITTSBURGH

AC03-93SFI9683 09/30/94 $092680-0019-9$ PITTSBURGH

NUCLEAR \& ADVANCED TECH DIV

ACO3-90SF $18495 \quad 12 / 31 / 97$ $092680-4319-x$ PITTSBURGH NUCLEAR WASTE DEPT

AC07-88ID12699 05/12/95 $092680-3619-3$ PITTSBURGH

SCIENCE \& TECHNOLOGY CENTER SCIENCE \& TECHNOLOGY CENTER SCIENCE \& TECHNOLOGY CENTER SCIENCE \& TECHNOLOGY CENTER SCIENCE \& TECHNOLOGY CENTER SCIENCE \& TECHNOLOGY CENTER SCIENCE \& TECHNOLOGY CENTER SCIENCE \& TECHNOLOGY CENTER AC21-86MC23252 $07 / 31 / 94$
$092680-3719-X \quad$ PITTSBURGH AC21-87MC24257 09/30/94 $092680-3719-X$ PITTSBURGH AC21-88MC25034 02/28/95 $092680-3719-X$ PITTSBURGH AC21-91MC27259 12/15/94 $092680-3719-X$ PITTSBURGH AC21-89MC26355 07/17/95 $092680-3719-x$ PITTSBURGH FC21-91MC28055 11/30/95 $092680-5719-x$ PITTSBURGH AC21-91MC28020 08/31/94 $092680-3719-X$ PITTSBURGH AC21-93MC $30177 \quad 08 / 30 / 94$ 092680-3719-X PITTSBURGH AC11-93PNउĒ195 09/30/98 BETTIS ATOMIC POWER LABORATORY 092680-0624-3 WEST MIFFLIN WESTINGHOUSE HANFORD CO $A C 06-87 R L 10930 \quad 03 / 31 / 96$ $092690-0001-X$ RICHLAND WESTINGHOUSE IDAHO NUCLEAR CO AC07-84ID12435 09/30/94

\section{DESCRIPTION OF WORK}

OBLIGS O DATE P/S

SPACE NUCLEAR FOHER SUPPLY

$P A \quad 12 \$ 1,168,311$

$\$ 1,168,311 \quad A C 21$

TECHHICAL SUPPORT SERVICES FOROFFICE OF SPECIAL APPLICATION PA $14 \$ 3,510,175$ $\$ 2,346,000 \quad R 408$

INITIATE HORK FOR PU COHSUMPTION $\$ 3,611.343 \quad A G 53$

DESIGN AND NRC CERTIFICATION OF ALHR PLANT OF HORK CASK SYSTEMS DEVELOPMENT, INITIATIVE I, DEVELOPMENT OF FROMPA 14 \$19,459.864 $\$ 4,443,399$ AG54 MECHANICAL AHALYSIS OF CROSS FLOW FILTERS PA 14 \$1.237.419 $\$ 1.237 .419$ AGI3 INTEGRATED LOW EMISSIONS $\begin{array}{ccc}\text { CLEANUP (ILEC) SYSTEM FOR } \\ \text { I4 }\end{array}$ THERMAL/CHEMICAL DEGRADATIOH OF CERAMIC FILTER MATERIAL PA 14 \$1, $467,106 \quad \$ 1.449,546$ AGI3 MOVING GRANULAR-BED FILTER DEVELOPMENT PROGRAM $\$$ \$1, 073,612 AG14 EVALUATION OF FUEL IMPURITY EFFECTS ON SOLID OXIDE FUEL DEVELOPMENT OF HIGH TEMFERATURE PA OF HIGH $14 \$ 143,860,892 \quad \$ 54.883 .709$ SMART STRUCTURES FOR APPLICATION IN CERAMIC BARRIER PA 14 \$417,993 $\$ 417,993$ AG14 EM - TREATABILITY STUDY $\$ 565,834$ USING PROMPT GAMMA NEUTRON MAHAGE AND OPERATE BETTIS ATOMIC PONER LABORATORY PA $18 \$ 1,661,914,414 \quad \$ 261,914,414 \quad M 181$

OPERATIONS AND ENGINEERING SERVICES FOR HANFORD $\begin{array}{llll} & \\ \text { HA } & 04 \$ 7,130,926,583 & \$ 7,130.926 .583 & M 181\end{array}$

MANAGEMENT OPERATION AND MAINTEHANCE OF ICPF 


\begin{tabular}{|c|c|}
\hline AHARDEE NAME & COMPL DATE \\
\hline DIVISION & VENDOR ID \\
\hline & $092700-0001-x$ \\
\hline WESTINGHOUSE SAVANNAH RIVER CO & $\begin{array}{c}\text { ACO9-89SR } 180 j 509 / 30 / 96 \\
228160-0001-x \quad \text { AIKEN }\end{array}$ \\
\hline $\begin{array}{l}\text { WESTMORELAND COUNTY OF } \\
\text { PUBLIC SCHOOLS }\end{array}$ & $\begin{array}{c}\text { FG43-93R355506 } 03 / 31 / 95 \\
231390-0101-0 \text { MONTROSS }\end{array}$ \\
\hline WESTPORT PUBLIC SCHOOLS & $\begin{array}{c}\text { FG41-93R191510 08/31/95 } \\
119070-0001-1 \text { HESTPORT }\end{array}$ \\
\hline & $\begin{array}{c}\text { FG41-94R191605 05/30/96 } \\
119070-0001-1 \text { WESTPORT }\end{array}$ \\
\hline
\end{tabular}

WESTHOOD COMMUNITY SCHOOL DIST
FG45-92R540222 09/30/94 $105860-0002-x$ INKSTER

WHALEN-SHAW DR MICHAEL

FCO1-94CE15502 04/27/9t
$232650-0001-9$ CIRCLE 237650-0001-9 CIRCLEVILLE

HHEATOH COLLEGE

FG41-94R193602 06/14/95
$176010-0001-\times$ HORTON

FG43-93R356204 01/31/95 $093020-0101-6$ WHEELING WHEELING COLLEGE
SIIHT COMPLEX WHITEHALL TOWN OF
CONSOLIDATED SCHOOL DISTRICT
$220950-0101-x$

'HHITLEY COUNTY OF EDUCATION BOARD OF

FG44-93R435311 09/30/95 $231930-0101-5$ HILLIAMJBURG

WHITTIER UHIOH HIGH SCH DIST BUSINESS SERVICES

:WHITWORTH COLLEGE
FG49-895F18335 07/31/94
$136510-0101-6$ WHITTIER

FG51-93R020310 08/31/94 $093240-0001-2$ SPOKAHE
DESCRIPTION OF WORK

OBLIGS STATE CONG CONTRACT

ID 0291.370 .988 .517

$\$ 1.370,829.935 \quad M 181$

OPERATIOHS OF SRS

$S C \quad 0310.490 .000 .000 \quad \$ 9.779 .508 .185 \quad M 159$

TIILE III OF THE NATIONAL ENERGY CONSERVAIION FOLICY ACT VA $01 \quad \$ 162.465 \quad \$ 100.000$

INITIAL AHARD.

$\$ 141,924$

$\$ 47.395$

TITLE III OF THE HATIOHAL EIERGY CONSERVATION POLICY ACT $C T \quad 04 \quad \$ 421.544 \quad \$ 210.772$

ICP - HEW AHARD

$\$ 135,795$

$\$ 67.897$

DEVELOP REPLACEMENT OF THERMALLY PRODUCED CALCINED CLAY WITH $\mathrm{OH} 04 \quad \$ 99.858 \quad \$ 99.858$

TITLE III OF THE NATIOHAL ENERGY CONSERVATION FOLICY ACT MA $04 \quad \$ 13.000 \quad \$ 0.500$

TITLE III OF THE NATIOHAL ENERGY CONSERVATION POLICY ACT HV $01 \quad \$ 80,000 \quad \$ 56,569$

TITLE III OF THE NATIOHAL ENERGY COHSERVATION POLICY ACT NY 22 \$84,000 $\$ 42,000$

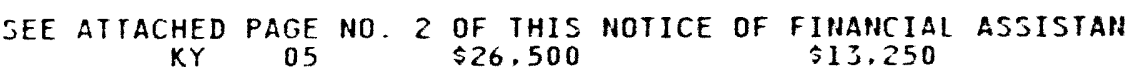

GRANT PROGRAMS FOR SCHOOLS ANDHOSPITALS AND BUILDINGS OHHED CA $34 \quad \$ 111,000 \quad \$ 55,500$

CYCLE 15 ECM AHARD

HA 05 \$36,051

$\$ 17.859$ 


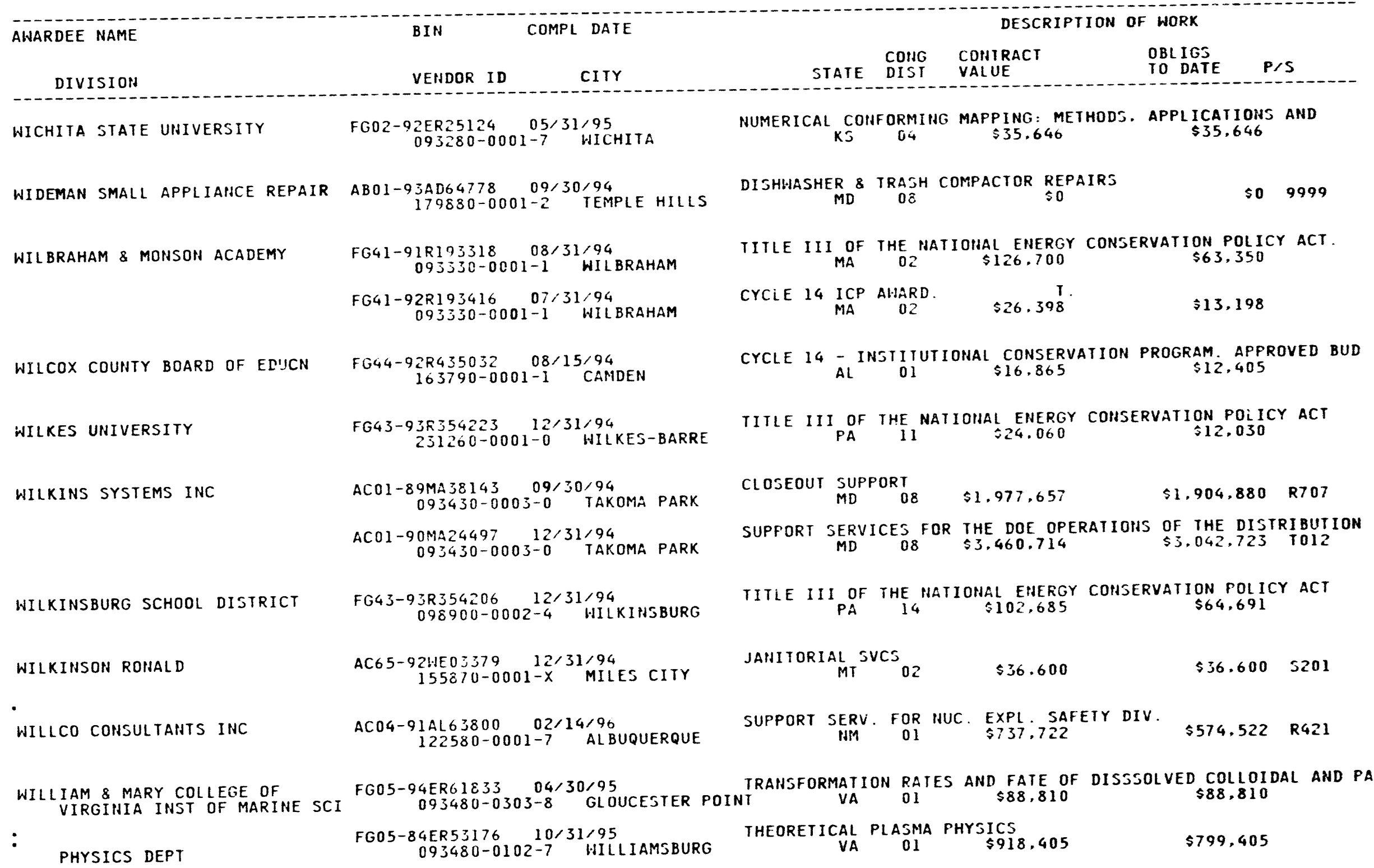


$\stackrel{\$}{i}$

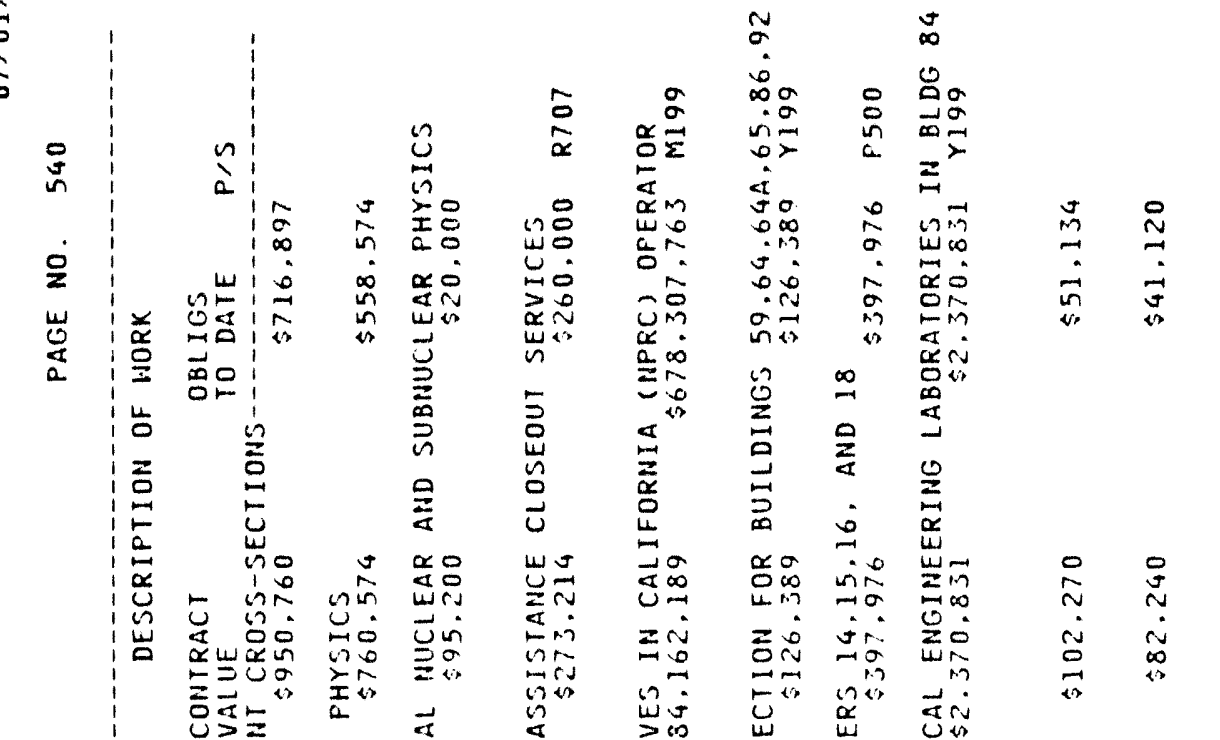

峞

造寻

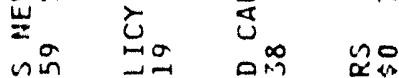

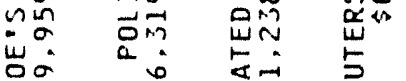

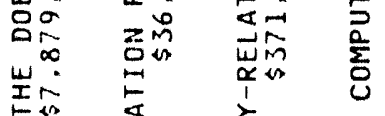

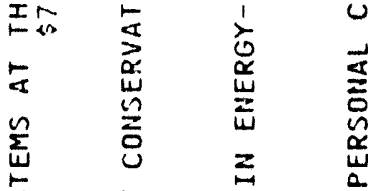

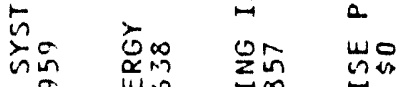

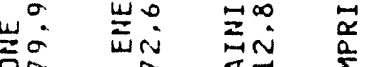

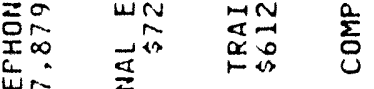

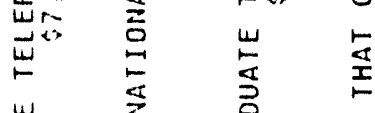

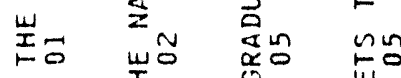

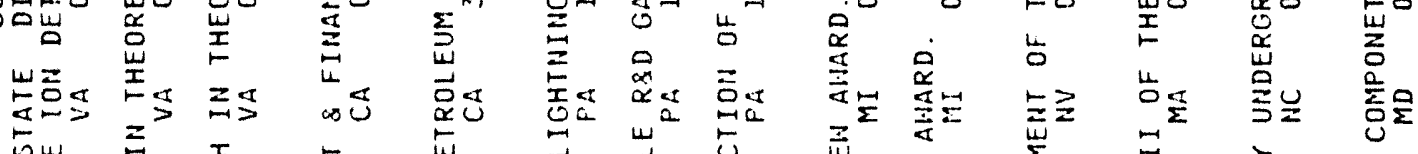

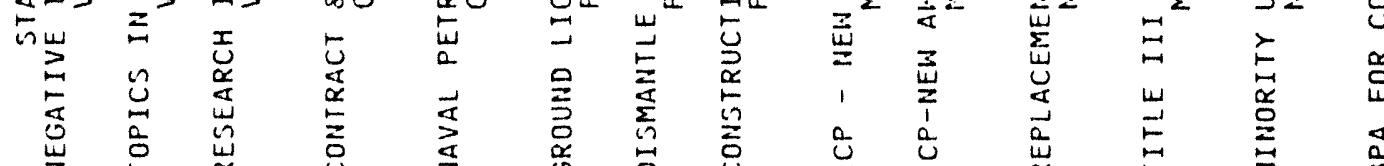

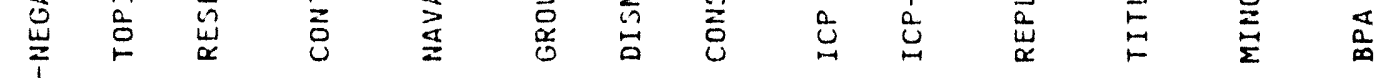

in

岁

气药

㟧心

煞

更我崖

岩是元

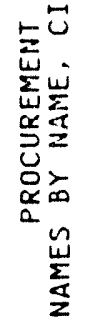

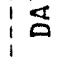

品

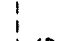

is

商

足

$\sum_{2}^{12} \sum_{5}^{10}$

$r=$

决

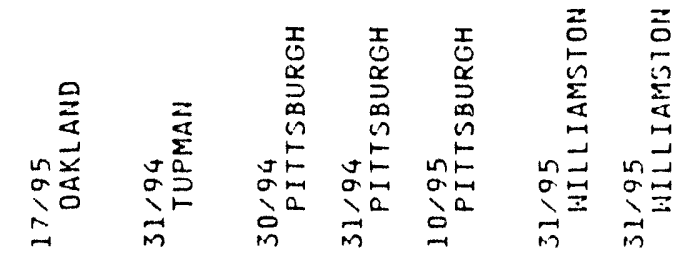

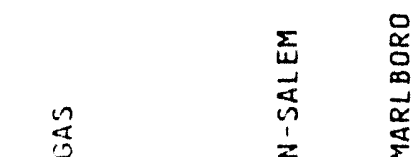

我

ने ले ले ने

cors cors

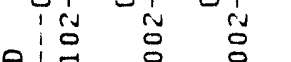

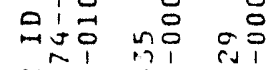

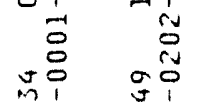

$\begin{array}{lll}7 & -1 & 0 \\ 0 & 0 & 0\end{array}$

$\begin{array}{lll}0 & 0 \\ 0 & 0 & 0 \\ 0 & 1 & 0 \\ 0 & 0 & 0 \\ 10 & 0 & 0 \\ 0 & 0 & 0 \\ 0 & 0 & 0\end{array}$

궁 No⿱8

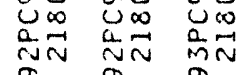

mo 0

mo 00

品: 000

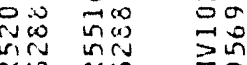

a

$\begin{array}{lll}1 & 1 \\ 0 & 0 \\ 0 & 0 \\ 0 & 0 \\ u & 0\end{array}$

$\geqq$ स्रे

$\infty$
1
$\infty$
0
0
0

更

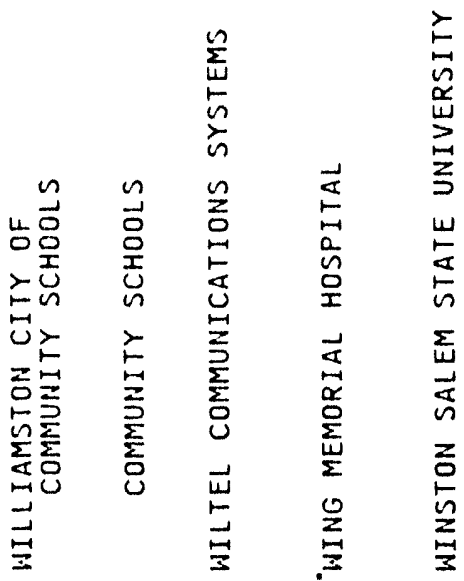

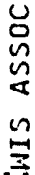




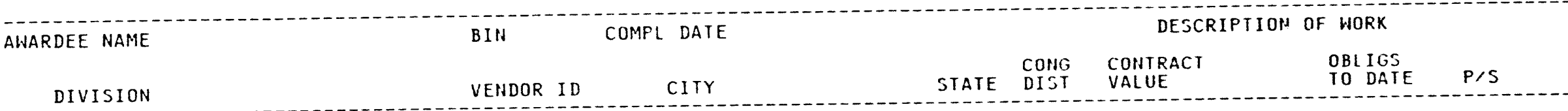
WISC CTR FOR DEMAND-SIDE RES FC47-93R7013150 09/30/95 $233630-0001-4$ MADISON

WISCONSIN ELECTRIC POWER

CRO1-83NE44425 03/15/30 094070-0001-X MILHAUKEE

WISCONSIN PUBLIC SERVICE CORP CROI-83NE44429 03/15/30 094100-0001-7 KEHAUHEE

FG45-94R562248 12/31/95 219790-0101-4 HISCONSIN RAPIDS

WISCOHSIN RAPIDS CITY OF PUBLIC SCHOOLS

WISCOHSIN STATE OF PUBLIC SERVICE COMMISSION

FCO1-91EI22725 08/06/96 $094110-0901-8$ MADISON

HEALTH \& SOCIAL SVCS DEPT OF

ADMINISTRATION DEPT

ADMINISTRATION DEPT

WISCOHSIN UNIVERSITY OF ENGINEERING COLLEGE OF

GEOLOGY \& GEOPHYSICS DEPT

ELECTRICAL \& COMPUTER ENG DEPT CO2-93ER54196 11/30/95 $094130-0801-9$ MADISON FG02-92ER61361 11/30/94 094130-0001-8 MADISOH

FG02-92ER61j65 12/14/94 094130-0001-8 MADISON

FG02-88ER $40438 \quad 02 / 28 / 95$
MIDHEST REGIONAL COLLABORAIIVEDEVELOPMENT FROJECT ON ENERGY WI 02 \$ 191,419 $\$ 154,000$

$\begin{array}{ccccc}\text { DISPOSAL OF SPENI NUCLEAR } & \text { FUEL AND FOR HIGH LEVEL } \\ \text { HI } & 09 & \text { SO } & \text { \$O }\end{array}$

$\begin{array}{cccc}\text { DISPOSAL OF SPENT NUCLEAR } & \text { FUEL AHD FOR HIGH } \\ \text { WI } & 08 & \$ 0 & \\ & & \$ 0\end{array}$

ICP-NEW AWARD.

WIAR.D. $07 \quad \$ 15,000 \quad \$ 7,500$

1991-92 STATE HEATING OIL AHD PROPANE PROGRAM

HI Ol 01 \$37.401 $\$ 18.684$

FY 1989 FUNDING FOR THE STATE OF HISCONSIN'S LOH-INCOME HEAT HI 02 \$ $75,031.553$ \$ 44.549 .136

AWARD OF FY 1989 FUNDIHG FOR ADMINISTRATION OF THE INSTITUTI HI 02 \$4,069,351 \$412,305

IHIS ACTION INITIATES THE AHARD OF $\$ 25,000$ OF DISCRETIONARY HI 02 \$75.000 $\$ 75.000$

TFTR EXPERIMENTAL DATA $\begin{gathered}\text { ANALYSIS COLLABORAIION } \\ \text { IJJ O4 }\end{gathered}$

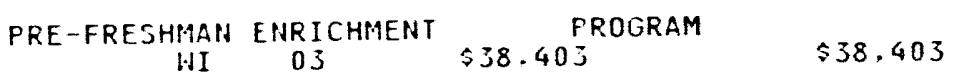

HRTEM INVESTIGATION OF $\$ 177,605$ INTERACTONS BETHEEN MINERALS

TFTR ION CYCLOTRON RANGE OF FREQUENCIES (ICRF) EXPERIMENT HI 02 \$207,000 \$131.710

PARAMEIERIZATION OF (GCM) 02 GENERAL CIRCULATION MODEL

ATMOSPHERIC RADIATION MEASUREMENT (ARM: FOURIER HI 02 \$ $\$ 582.458$ M $\$ 532.458$

DEVELOPMENT OF A HYDROGEN AND DEUTERIUM POLARIZED GAS TARGET 


AWARDEE NAME BIN COMPL DATE

DIVISION
PHYSICS DEPT
ACNDOR ID $02-76$ ER008 $8130-1901-0 \quad 12 / 31 / 94$

PHYSICS DEPT

CHEMICAL ENGINEERING DEPT

CHEMICAL ENGINEERING DEPT

RES ADMIN - FINANCIAL

METALLURGICAL \& MNRL ENG DEPT

MEDICAL PHYSICS DIV

CHEMISTRY DEPT

GENETICS DEPT

CHEMICAL ENGINEERING DEPT

METALLURGICAL \& MNRL ENG DEPT

HUMAN ONCOLOGY DEPT
AC02-76ER00881 12/31/94
$094130-1901-0$ MADISON

FG02-84ER13183 06/30/96 $094130-0301-7$ MADISON

FG02-84ER13291 08/31/94
$094130-0301-7$ MADISON FG02-85ER53198 10/31/96
$094130-0001-8$ MADISON FG02-85ER13406 10/31/94
$094130-0001-8$ MADISON FG02-85ER52122 08/31/94 094130-0001-8 MADISON FG02-85ER53212 10/31/94 $094130-0001-8$ MADISON

FG02-85CE40735 08/14/94 $094130-0001-8$ MADISON

FG02-86ER53218 11/15/94
$094130-2001-9$ MADISOH FG02-86ER52131 01/31/96 86 ER52131 01/31/96
$094130-1601-1$ MADISON FG02-86ER60417 01/31/95
$094130-1501-5$ MADISON FG02-86ERI3500 03/31/95 $094130-0401-3$ MADISON FG02-86ER13539 05/31/97 094130-1101-X MADISON

FG02-86ER13551 08/31/95
$094130-0301-7$ MADISON FG02-86ER45274 08/31/95 $094130-1601-1$ MADISON

FG02-87ER60507 12/31/95 $094130-1301-2$ MADISON

\section{DESCRIPTION OF HORK}

CONG CONTRACT OBLIGS

STATE DIST VALUE TO DATE P/S

WI $02 \quad \$ 878,000$

$\$ 878,000$

HIGH ENERGY EXPERIMENTAL AND THEORETICAL PHYSICS RESEARCH

$$
\text { WI } 02 \text { \$95,548,796 } \$ 93,948,796 \text { AG91 }
$$

ACID SITES FORMED BY DOPING CATIONS INTO OXIDE SURFACES. TH HI 02 \$I, 145,210 \$1,028,310

INTERPHASE TRANSPORT \& MULTISTAGE SEPARATIONS

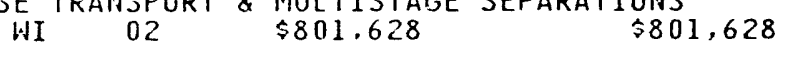

REVERSED FIELD PINCH STUDIES

$$
\text { WI } 02 \text { \$18,567,000 \$14,652,000 }
$$

ORGANOMETALLIC CHEMISTRY OF BIMETALLIC COMPOUNDS

$$
\text { WI } 02 \text { \$1,096,170 \$ } 1,096,170
$$

HIGH POHER MICROHAVE TRAHSMISSION SYSIEMS FOR ELECTRON

$$
\text { HI } 02 \text { \$1.805,180 } \$ 1,805,180
$$

THEORETICAL REVERSED FIELD PINCH STUDIES
HI
02

$\$ 548,000$

DEVELOPMENT OF A GRAVEL BED COMBUSTOR

$$
\text { WI } 02 \text { \$1,500,000 } \$ 1,271,500
$$

FUSION PLASMA THEORY

$$
\text { WI } 02 \quad \$ 3,095,000 \quad \$ 3,094,000
$$

MICROSTRUCTURAL UNDERSTANDING AND CRITICAL CURREHT $\begin{array}{lll}\text { HI } 02 & \$ 1,890,000 & \$ 1,636,000\end{array}$

FAST NEUTRON DOSIMETRY

$$
\text { WI } 02 \quad \$ 888,308
$$

UNIMOLECULAR REACTION DYHAMICSAND VIBRATIONAL OVERTONE

$$
\text { WI } 02 \quad \$ 988,470 \quad \$ 988,470
$$

ORGANIZATION OF THE R CHROMOSOME REGION IN MAIZE

$$
\text { HI } 02 \quad \underset{\$ 57,093}{\$ 587,093}
$$

THE DEVELOPMENT OF PROCESS DESIGN AND CONTROL STRATEGIES FOR

$$
\begin{array}{lll}
\text { HI } 02 & \$ 991,507 & \$ 864,507
\end{array}
$$

THERMODYNAMICS AND KINETICS OFPHASE FORMATION OF THIN-FILM

$$
\text { WI } 02 \quad \$ 919,732 \quad \$ 823,967
$$

RADIOGENIC NEOPLASIA IN THYROID \& MAMMARY CLONOGENS

$$
\text { WI } 02 \$ 2,614,483 \quad \$ 2,239,481
$$




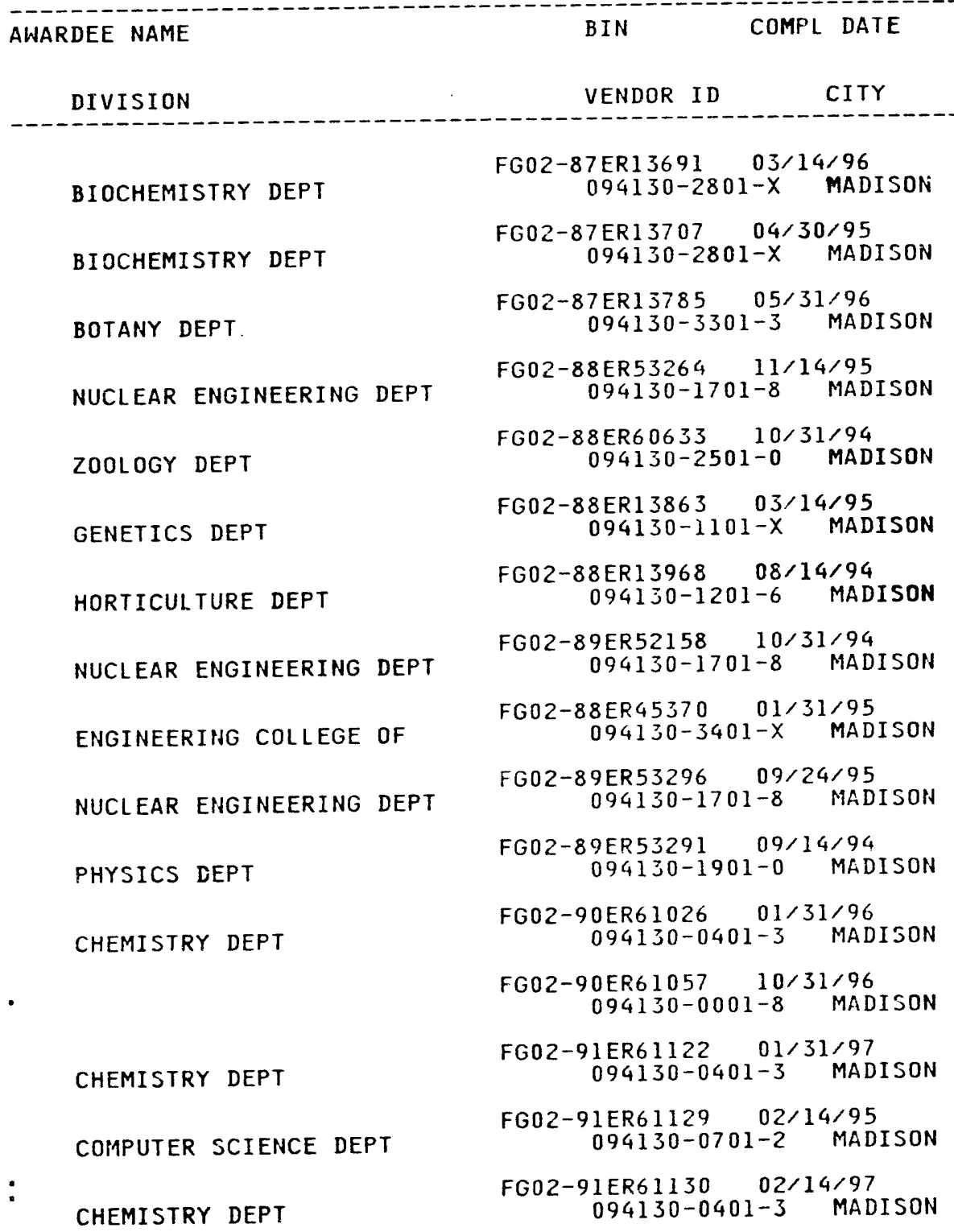

DESCRIPTION OF WORK

CONG CONTRACT

VALUE

CARBON MONOXIDE METABOLISM BY PHOTOSYNTHETIC BACTERIA

$$
\text { WI } 02 \quad \$ 717,000 \quad \$ 623,000
$$

ENZYMOLOGY OF BIOLOGICAL NITROGEN FIXATION

$$
\text { HI } 02 \quad \$ 383,595 \quad \$ 383,595
$$

GAS EXCHANGE CHARACTERISTICS OF INTACT LEAVES AS INDICATORS WI $02 \quad \$ 673,180$ $\$ 584,180$

$\begin{array}{cccc}\text { EXPERIMENTAL INVESTIGATION } & \text { OF ICRF EFFECTS } \\ \text { HI } & 02 & \$ 16,451,565 & \$ 13,506,000\end{array}$ MASS \& ENERGY BUDGETS OF ANIMALS BEHAVIORAL AND WI $02 \quad \$ 1,199,907 \quad \$ 1,199,907$

STARCH SYNTHESIS IN THE MAIZE ENDOSPERM AS AFFECTD BY WI 02 \$457,611 \$457,611

MECHAIISM OF SELECTIVE CONJUGATION OF UBIQUITIN TO WI $02 \quad \$ 499,950 \quad \$ 499,950$

FUSION REACTOR DESIGN STUDIES
NI $02 \$ 1,060,000$
$\$ 1,060,000$

MORPHOLOGICAL AILALYSIS OF
$\$ 657,096$
$\$ 657,096$

APPLICATION OF DPTICAL FLUCTU-ATION DIAGNOSTICS TO TRANSPORT WI $02 \$ 2,525,905$

$\$ 2,045,905$ THEORETICAL STUDIES OF
HI
02 $\stackrel{\substack{\text { TURBULENCE AND ANOMALOUS } \\ \$ 500,000}}{\$}$

A HIGH-SPEED AUTOMATED DNA SEQUENCER WI $02 \$ 1,881,744 \quad \$ 1,611,014$ HIGH SPECTRAL RESOLUTION RADIAHCE MEASUREMENTS HI $02 \quad \$ 2,379,251 \quad \$ 1,701,989$

AUTOMATION OF THE FRONT END OFDNA SEQUENCING WI 02 \$1,802,637 $\$ 1,102,432$

APPLYING MACHINE LEARNING TECHNIQUES TO DNA SEQUENCE ANALYSI HI $02 \quad \$ 682,804 \quad \$ 682,804$

HIGH SPEED DNA SEQUENCE AHALYSIS BY MATRIX-ASSISTED LASER WI $02 \quad \$ 1,390,579$ \$ 0266,579 


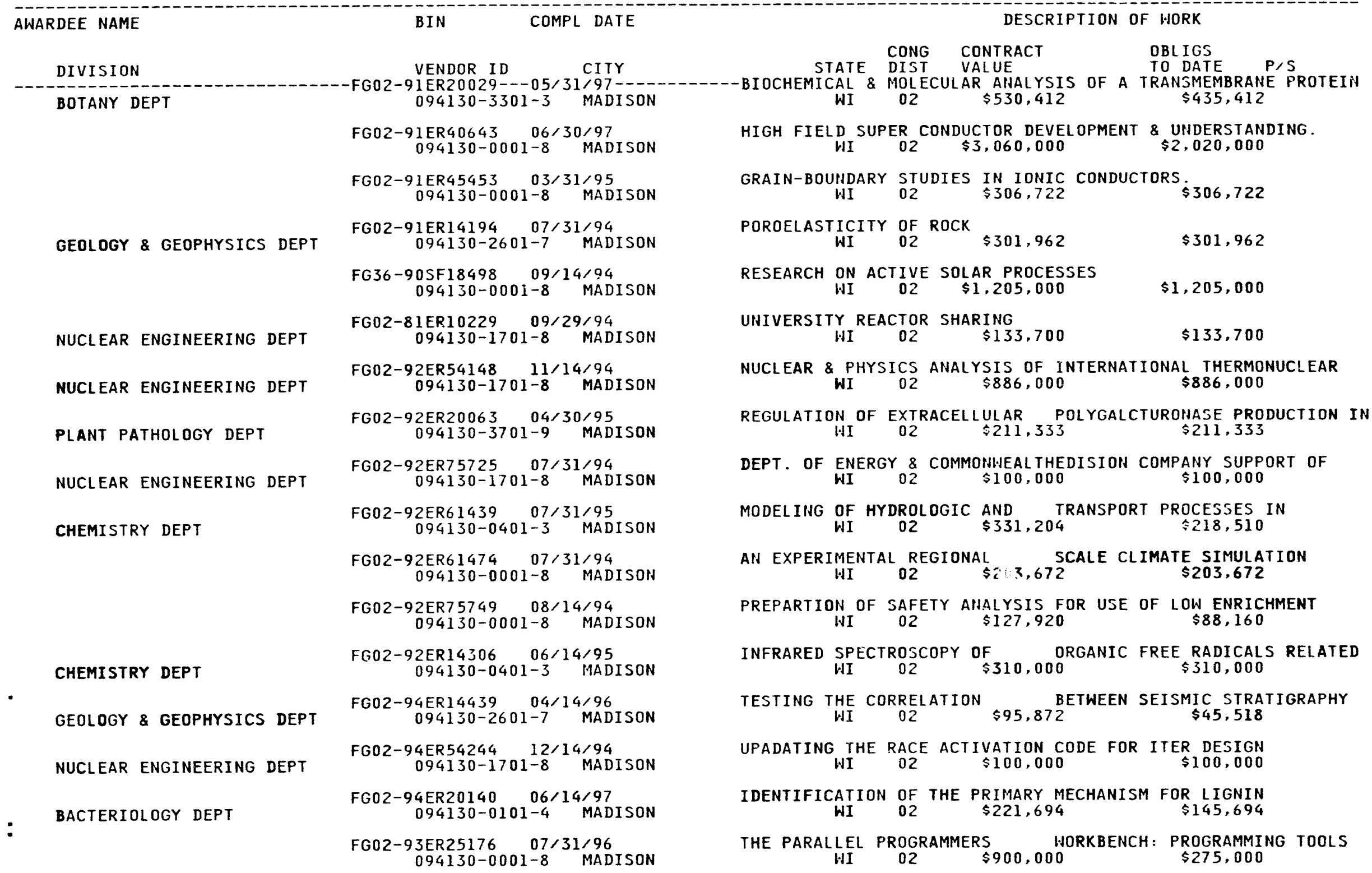




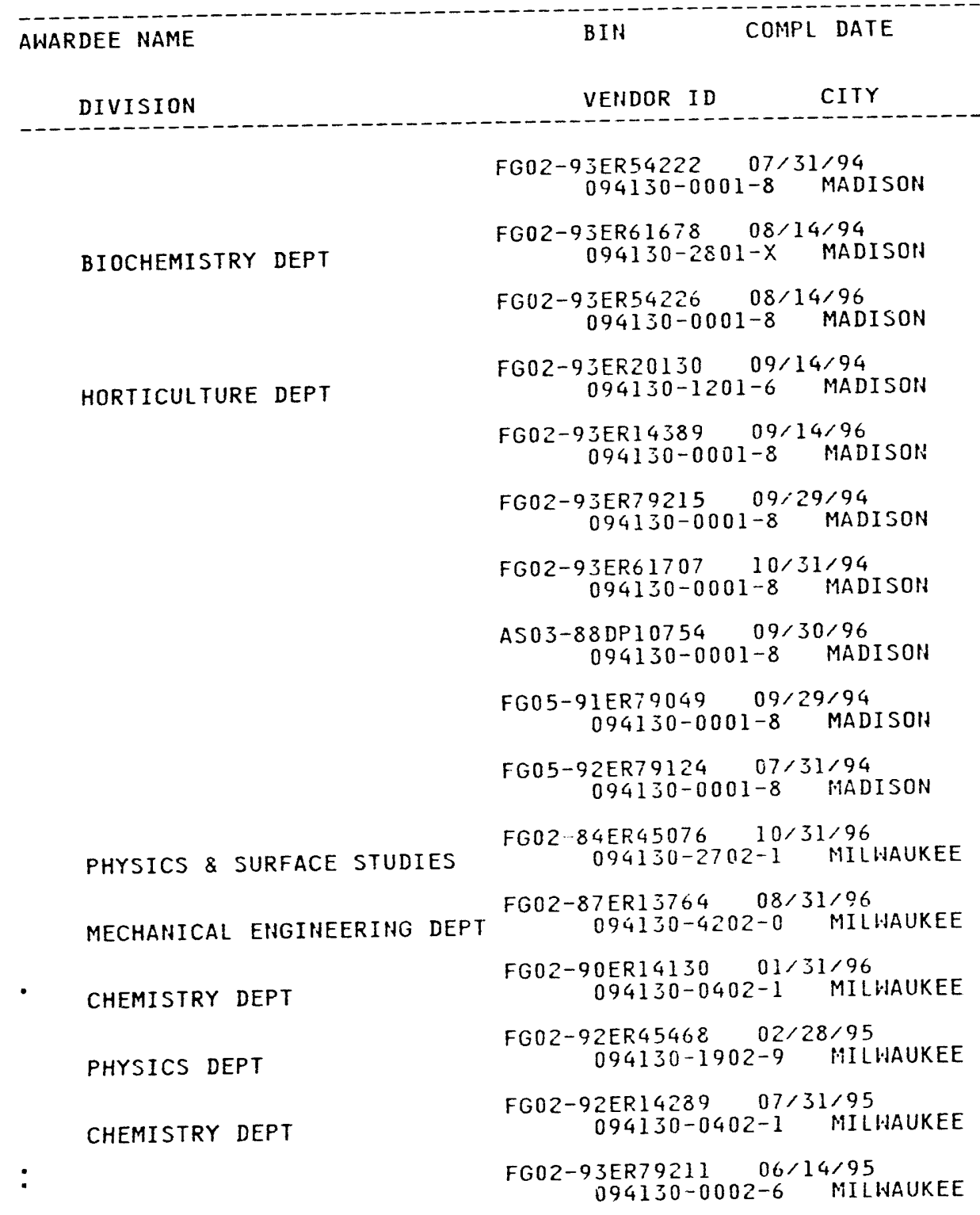

DESCRIPTION OF HORK

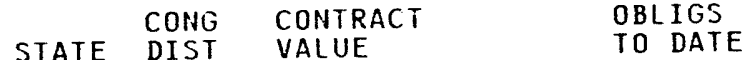

HSX; A HELICALLY SYMMETRIC TOROIDAL EXPERIMENT

SECOND E. COLI GENOME MEETING -1993 TO BE HELD

RF CURRENT DRIVE AHTENNA

$$
\text { WI DRIVE AHTENNA } \$ 84,000 \quad \$ 26,000
$$

PARTIAL SUPPORT FOR THE LIGHT IN CONTROLLED ENVIRONMENTS

$$
\text { WI } 02 \quad \$ 5,000 \quad \$ 5,000
$$

MICROANALYSIS OF STABLE ISOTOPE RATIOS IN GEOTHERMAL SYSTEMS $\begin{array}{lll}\text { YIS OF STABLE ISOTOPE RATIOS IN } & 02 & \$ 144,115\end{array}$

NUCLEAR REACTOR SUSTRUME $\$ 4,000$ UPGRADE $\$ 44,000$ ROLE OF X-RAY INDUCED $\$ 252,166$ TRANSCRIPTS IN ADAPTIVE RESEARCH ENTITLED. "DEVELOPMENT OF THE SYMMETRIC-ILLUMINATIO $\begin{array}{lll}\text { HI } & 02 & \$ 1,597,209\end{array}$ " $\$ 1,173,200$ AZ11

CLASS (CROSS-CHAIN ATMOSFHERICSOUHDING SYSTEM) FOR SUPPORTI $\begin{array}{lll}\text { WI } 02 & \$ 157,223 & \$ 125,778\end{array}$

1992 INSTRUMENTATION - DETECTOR DEVELOPMENT AND TEST FACILIT HI 02 \$251.193 $\$ 251.193$

SURFACE EXCITATIOHS AND THEIR INTERACTION WITH LOW ENERGY EL HI 05 \$1, 108,078 \$934,078

INTERFACIAL AREA AND INTERFACIAL TRANSFER IN TWOWI ${ }_{05} \quad \$ 969,445$ AND

ALUMINUM CORRDINATION AND AC- TIVE CATALYTIC SITES IN HI $05 \quad \$ 514,496 \quad \$ 423,496$

UNDULATOR SFECTRO-MICROSCOPY FACILITY AT THE ADVANCED LIGHT WI $05 \quad \$ 300,000 \quad \$ 300,000$

AN INVESTIGATIOH OF MOLYBDENUMAND MOLYBDENUM OXIDE CATALYZED IGATIOH OF MOLYBDENUMATD MOLYBDENUN
05 $\stackrel{\$ 310,557}{\$ 211,573}$

PRE-FRESHMAH ENRICHMENT $\$ 38,580 \quad$ PROGRAM $\$ 38,580$ 


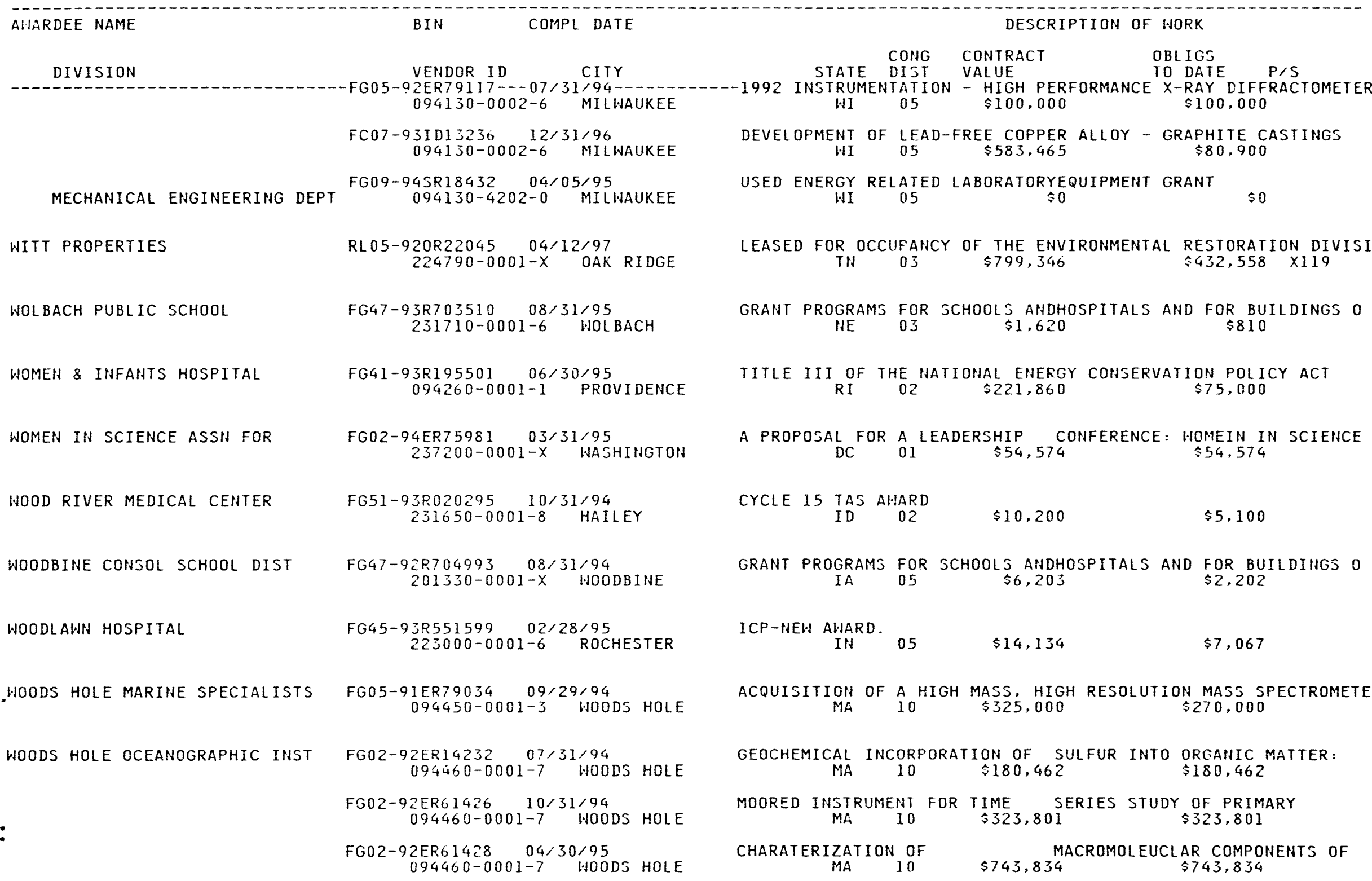




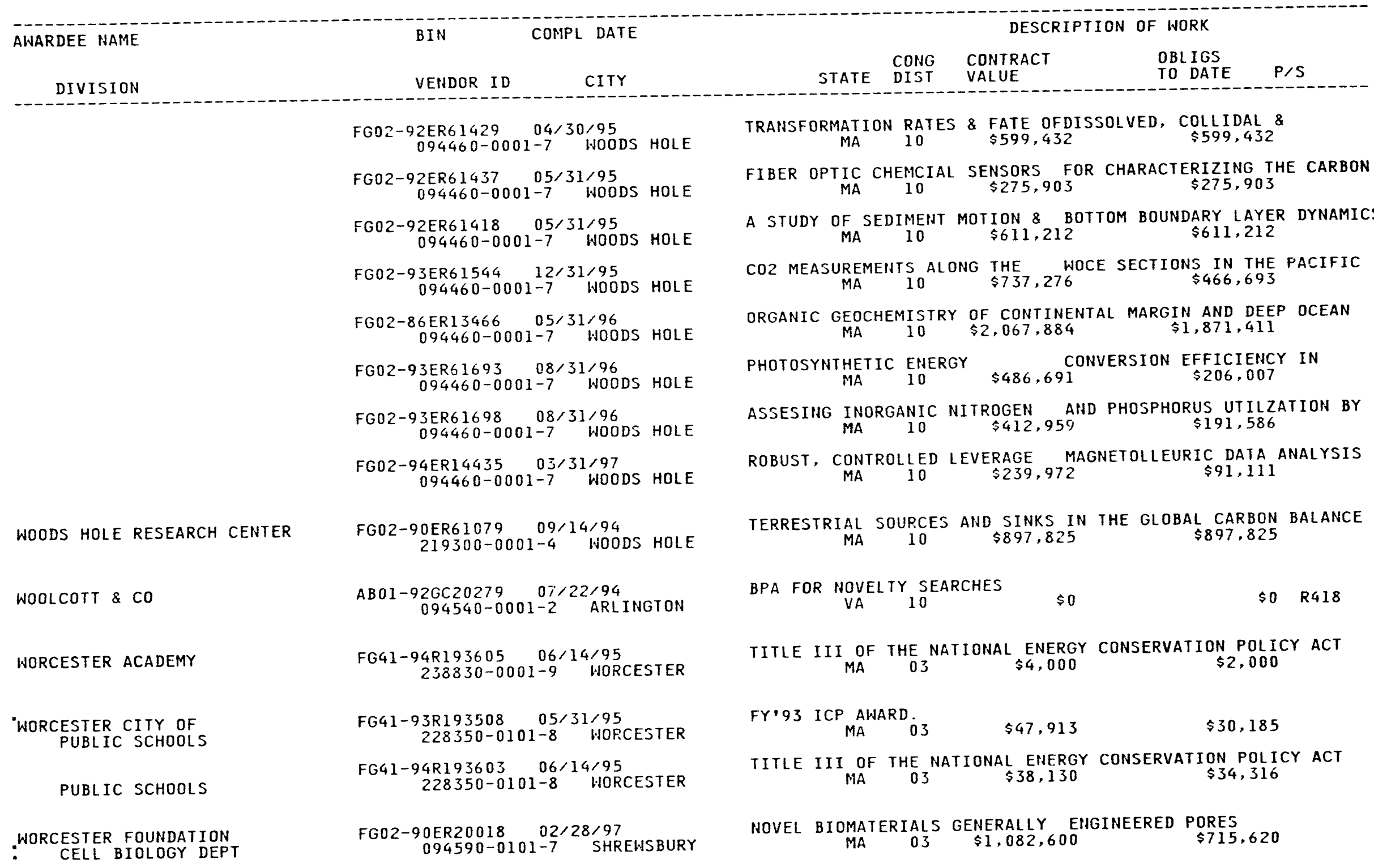




\begin{tabular}{|c|c|c|c|c|c|}
\hline AWARDEE NAME & COMPL DATE & \multicolumn{4}{|c|}{ DESCRIPTION OF WORK } \\
\hline DIVISION & VENDOR ID & $\begin{array}{l}\text { COHG } \\
\text { DIST }\end{array}$ & $\begin{array}{l}\text { CONTRACT } \\
\text { VALUE }\end{array}$ & $\begin{array}{l}\text { OBLIGS } \\
\text { TO DATE }\end{array}$ & $P / S$ \\
\hline \multirow[t]{5}{*}{$\begin{array}{l}\text { WORCESTER POLYTECHIIIC INST } \\
\text { NUCLEAR REACTOR FACILITY }\end{array}$} & $\begin{array}{c}\text { FG02-87ER75351 } 09 / 15 / 94 \\
094610-0301-3 \text { HORCESTER }\end{array}$ & UNIVERSITY REACTOR S & $\begin{array}{l}\text { SHARING } \\
\qquad \$ 64,000\end{array}$ & \multicolumn{2}{|l|}{$\$ 64.000$} \\
\hline & $\begin{array}{c}\text { FG02-93ER79214 } 09 / 14 / 94 \\
094610-0001-4 \quad \text { WORCESTER }\end{array}$ & $\begin{array}{r}\text { UNIVERSITY REACTOR } \\
\text { MA } 03\end{array}$ & \multicolumn{3}{|c|}{$\begin{array}{l}\text { INSTRUMENTATION PROGRAM } \\
00 \\
\$ 28,000\end{array}$} \\
\hline & $\begin{array}{c}\text { AC22-91PC90185 } 12 / 09 / 94 \\
094610-0001-4 \quad \text { HORCESTER }\end{array}$ & $\begin{array}{ll}\text { KINETIC THEORY AND } \\
\text { MA } & 0 J\end{array}$ & $\begin{array}{l}\text { BOUNDARY CONDITIONS } \\
\quad \$ 314,400\end{array}$ & $\begin{array}{l}\text { FOR FLOWS OF } \\
\$ 224,840\end{array}$ & $\begin{aligned} & \text { HIGHLY I } \\
& \text { AGII }\end{aligned}$ \\
\hline & $\begin{array}{c}\text { AC22-92PC92113 } 09 / 29 / 95 \\
094610-0001-4 \text { WORCESTER }\end{array}$ & METHAHE COUPLING BY & $\begin{array}{l}\text { CATALYTIC MEMBRANE } \\
\$ 747,840\end{array}$ & $\begin{array}{l}\text { REACTOR } \\
\quad \$ 460,000\end{array}$ & $A G 11$ \\
\hline & $\begin{array}{r}\text { FCO7-94ID13234 06/01/99 } \\
094610-0001-4 \text { HORCESTER }\end{array}$ & $\begin{aligned} & \text { CLEAN METAL CASTIHG } \\
& \text { MA } 03\end{aligned}$ & $\$ 2,843,485$ & $\$ 287,542$ & \\
\hline WORKMED & $\begin{array}{l}09 / 30 / 94 \\
\text { TULSA }\end{array}$ & MEDICAL SERVICES FOR & $\begin{array}{l}\text { R EMPL OYEE HEALTH P } \\
\quad \leqslant 162,480\end{array}$ & $\begin{array}{l}\text { ROGRAM } \\
\qquad 162,480\end{array}$ & $Q 201$ \\
\hline WORLD COMPUTER SYSTEMS IHC & $\begin{array}{r}A C 09-895 R 18051 \quad 12 / 31 / 94 \\
185610-0001-5 \text { AIKEN }\end{array}$ & $\begin{array}{c}\text { PERFORM GENERAL ADMI } \\
\text { SC } 03\end{array}$ & $\begin{array}{l}\text { INISTRATIVESUPPORT } \\
\$ 14,173,793\end{array}$ & $\begin{array}{l}\text { SERVICES FOR } \\
\$ 12,250,000\end{array}$ & $\begin{array}{l}\text { SRS } \\
\text { D399 }\end{array}$ \\
\hline \multirow[t]{2}{*}{ WRIGHT STATE UNIVERSITY } & $\begin{array}{c}\text { FG02-93ER79213 06/15/95 } \\
094700-0001-3 \text { DAYTON }\end{array}$ & \multicolumn{2}{|c|}{ 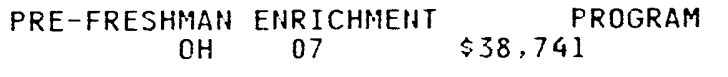 } & $\$ 38,741$ & \\
\hline & $\begin{aligned} \text { FG45-91R530103 } 10 / 31 / 94 \\
094700-0001-3 \text { DAYTON }\end{aligned}$ & $\begin{array}{c}\text { ICP-NEW AWARD. } \\
\text { OH }\end{array}$ & $\$ 97,580$ ING $\$ 12$ & \multicolumn{2}{|c|}{$\begin{array}{c}210.00 \text { IN STRIPPER WEL } \\
\$ 43.790\end{array}$} \\
\hline WYATT DATA SERVICES & $\begin{array}{c}A C 01-91 P R 38140 \quad 10 / 31 / 94 \\
212120-0001-3 \text { FORT LEE }\end{array}$ & $\begin{array}{c}\text { COMPENSATIOH SURVEY } \\
\text { HJ } 09\end{array}$ & $\$ 695,399$ & $\$ 695,399$ & R408 \\
\hline WYNFORD BOARD OF EDUCATION & $\begin{array}{rl}\text { FG } 45-94 R 562184 & 12 / 31 / 95 \\
238350-0001-2 \text { BUCYRUS }\end{array}$ & $\begin{array}{l}\text { ICP-NEW AWARD. } \\
\text { OH }\end{array}$ & $\$ 20,220$ & $\$ 10,110$ & \\
\hline WYOMING BOYS SCHOOL & $\begin{array}{c}\text { FG48-92R808610 08/31/94 } \\
150600-0001-X \text { HORLAND }\end{array}$ & $\begin{array}{l}\text { AHARD ICP CYCLE XIV } \\
\text { WY }\end{array}$ & $\begin{array}{l}\text { GRANT } \\
\quad \$ 26,380\end{array}$ & $\$ 13,190$ & \\
\hline WYOMING REPORTING SERVICE & $\begin{array}{c}A B 34-93 R F 00607 \quad 11 / 30 / 95 \\
094830-0002-5 \text { GOLDEN }\end{array}$ & $\begin{array}{ll}\text { PB/COURT } & \text { REPORTERS } \\
\text { CO } 02\end{array}$ & $\$ 0$ & $\$ 0$ & R606 \\
\hline WYOMING STATE HOSPITAL & $\begin{array}{c}\text { FG48-92R808621 } 08 / 31 / 94 \\
228430-0001-7 \text { EVANSTON }\end{array}$ & $\begin{array}{c}\text { AWARD ICP CYCLE XIV } \\
\text { WY }\end{array}$ & $\begin{array}{l}\text { GRANT } \\
\$ 30,760\end{array}$ & $\$ 15,380$ & \\
\hline
\end{tabular}




\begin{tabular}{|c|c|c|c|c|c|c|c|}
\hline AWARDEE NAME & BIN & L DATE & & & DESCR & HORK & \\
\hline DIVISION & VENDDR ID & CITY & STATE & $\begin{array}{l}\text { CONG } \\
\text { DIST }\end{array}$ & $\begin{array}{l}\text { CONTRACT } \\
\text { VALUE }\end{array}$ & $\begin{array}{l}\text { OBLIIGS } \\
\text { TO DATE }\end{array}$ & $P / S$ \\
\hline
\end{tabular}

WYOMING STATE OF

ENVIRONMENTAL QUALITY DEPT

WYOMING UNIVERSITY OF PHYSICS \& ASTRONOMY DEPT

CHEMISTRY DEPT

PHYSICS \& ASTRONOMY DEPT

PHYSICS \& ASTRONOMY DEPT

PHYSICS \& ASTRONOMY DEPT

: PHYSICS \& ASTRONOMY DEPT

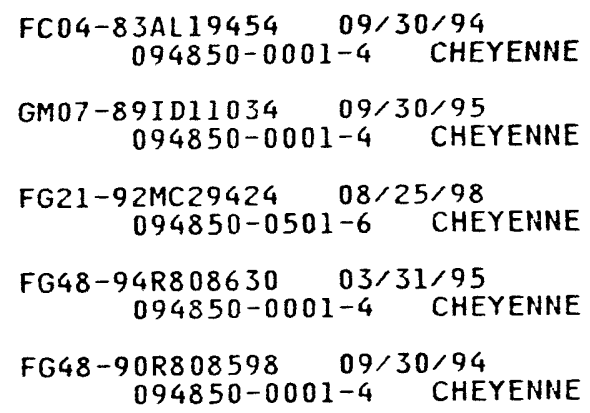

FG22-94PC94401 10/27/94 094860-1005-6 CHEYENNE

FG02-86ERI3547 06/14/95 094860-0201-0 LARAMIE

FG02-91ER75665 09/29/94 094800-0001-8 LARAMIE

FG02-91ER75680 09/29/94 $094860-0001-8$ LARAMIE

FG06-94RL12833 03/19/95 094860-1001-3 LARAMIE

FG06-94ER40835 01/31/97 094860-0001-8 LARAMIE

FG06-94RL12681 12/13/94 094860-1001-3 LARAMIE

FG06-94RL12680 12/13/94 094860-1001-3 LARAMIE

FG09-94SR18390 11/15/94 094860-0001-8 LARAMIE

FG34-94RF00786 03/13/95 094860-1001-3 LARAMIE

FG22-94PC94405 01/09/95
URIANIUM MILL TAILINGS REMEDIAL ACTION PROJECT PLANNING FOR AND PROVISION AND/OR COORDINATION OF FEDERAL RA

EVALUATION AND OVERSIGHT OF CLEAN-UP OF US DOE WASTE SITES WYY 01 \$306.950 $\$ 155,000$

AWARD FY 1994 WEATHERIZATION FUNDS

$$
\text { WY } 01 \quad \$ 2,231,674 \quad \$ 1,231,674
$$

AWARD FY9O PHASE II ADMIN. FUNDS

$\$ 69,341$

WY 01 \$ $\$ 40,348$

$\$ 0$

ENERGY RELATED EQUIPMENT GRANT

$$
\text { HY } 01 \text { \$O }
$$

$\begin{array}{rll}\text { SOLID-SURFACE LUMINESCENCE ANALYS } & \\ 01 & \$ 839,390\end{array}$

$\$ 750,000$

DOE/EPSCOR TRAINEESHIP PROGRAMFOR WYOMING.

$$
\text { WY } 01 \quad \$ 750,000 \quad \$ 750,000
$$

ENHANCEMENT \& COORDINATION OF ENERGY RESEARCH: A PLAN FOR

$$
\text { WY } 01 \text { \$ } \$ 268,740 \quad \$ 120,000
$$

USED ENERGY RELATED LABORATORYEQUIPMENT GRANT

WY 01 \$O

EXOTIC ATOMS

$01 \quad \$ 90,000$

$\$ 30,000$

USED ENERGY RELATED LABORATORYEQUIPMENT GRANT

WY 01 \$O

USED ENEPGY RELATED LABORATORYEQUIPMENT GRANT

$$
\text { WY RELATED LABORATORYEQUIPMENT GRANT \$0 }
$$

USED ENERGY RELATED LABORATORYEQUIPMENT GRANT

WY 01 \$O

USED ENERGY RELATED LAB EQUIPMENT GRANT

$$
\text { WY } 01 \text { \$O }
$$

ENERGY RELATED EQUIPMENT GRANT 


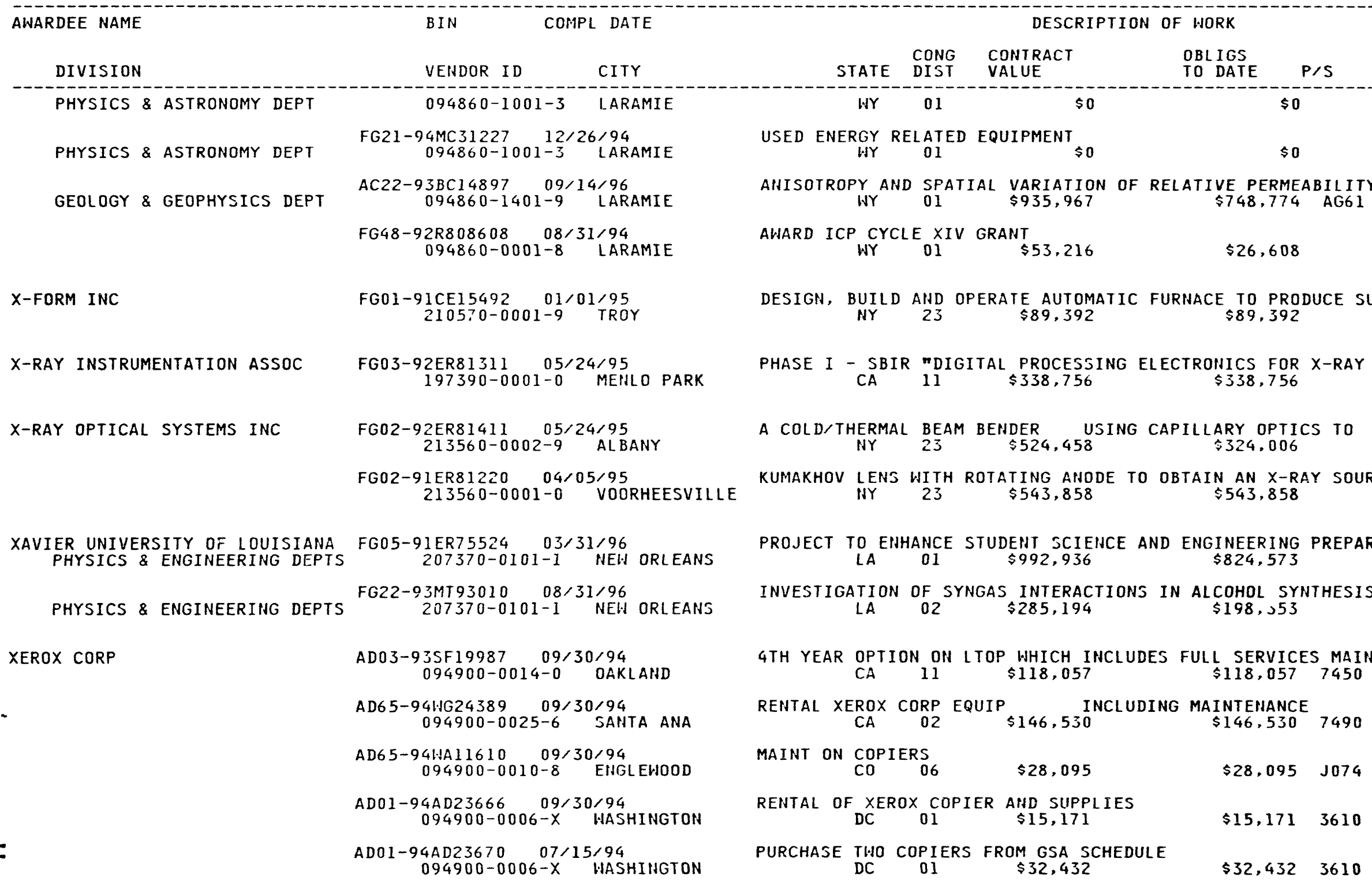




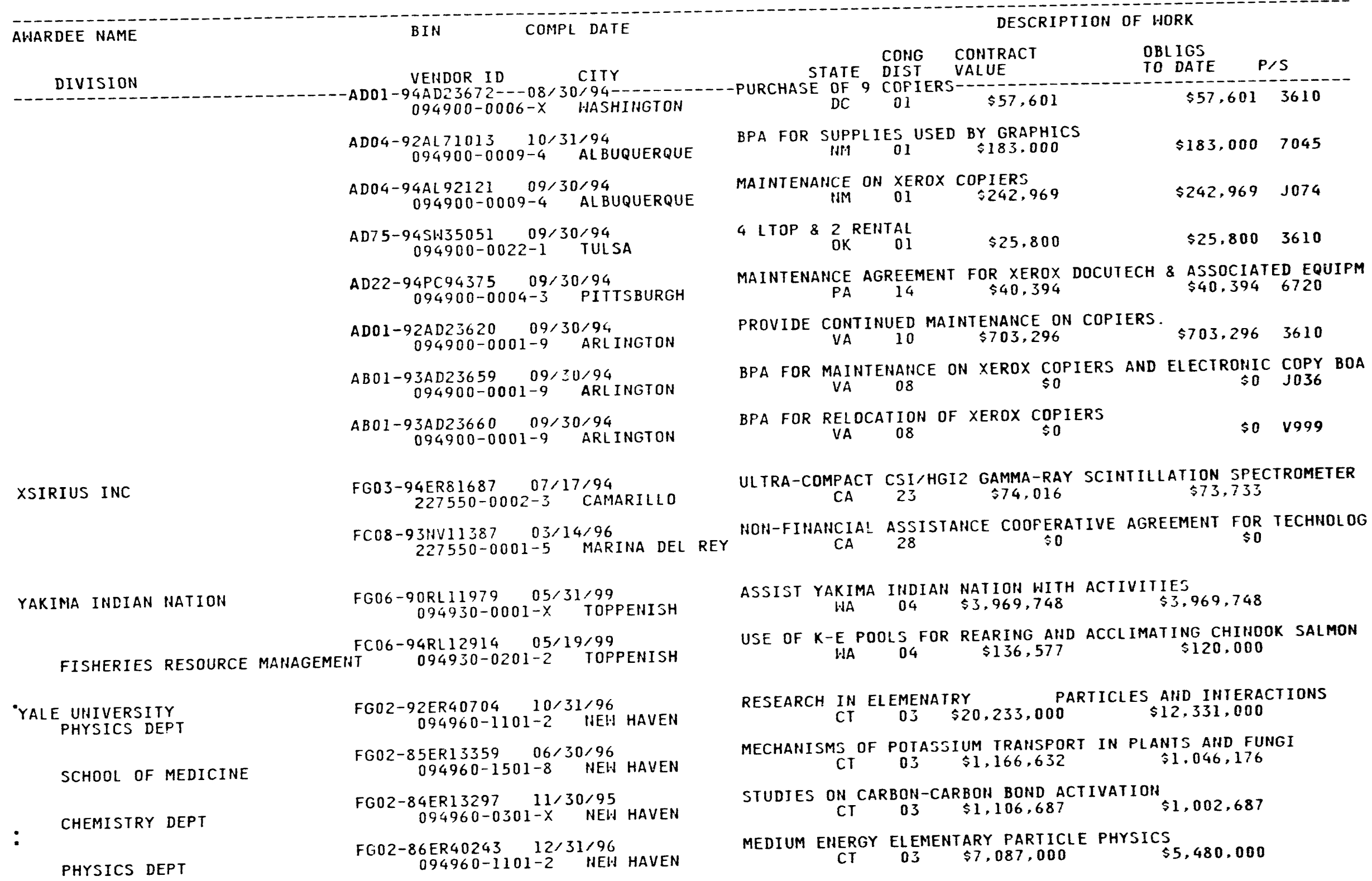




\begin{tabular}{|c|c|c|c|c|c|c|c|}
\hline \multirow{2}{*}{ AWARDEE NAME } & \multicolumn{2}{|c|}{ BIN COMPL DATE } & \multicolumn{5}{|c|}{ DESCRIPTION OF HORK } \\
\hline & VENDOR ID & CITY & STATE & $\begin{array}{l}\text { CONG } \\
\text { DISI }\end{array}$ & $\begin{array}{l}\text { CONTRACT } \\
\text { VALUE }\end{array}$ & $\begin{array}{l}\text { OBL IGS } \\
\text { TO DATE }\end{array}$ & PIS \\
\hline
\end{tabular}

BIOLOGY DEPT

APPLIED PHYSICS DEPT

GEOLOGY \& GEOPHYSICS DEPT

SCHOOL OF MEDICINE

CHEMICAL ENGINEERING DEPT

BIOLOGY DEPT

MECHANICAL ENGINEERING DEPT

PHYSICS DEPT

PHYSICS DEPT

BIOLOGY DEPT 094960-1401-1 NEH HAVEN

FG02-86ER53240 09/14,94 094960-1701-0 NEW HAVEN

FG02-87ER60576 03/14/96 $094960-0601-9$ NEW HAVEN FG02-87ER13734 05/31/96 $094960-1501-8$ HEH HAVEN FG02-88ER13836 01/14/97 094960-0201-3 HEW HAVEN FG02-88ER13947 07/14/94 094960-1401-1 NEW HAVEN FG02-88ER13966 05/31/95 $094960-0801-1$ NEW HAVEN

FG02-90ER40562 03/31/95 $094060-1101-2$ NEW HAVEN FG02-90ER14153 08/31/95 094960-0001-0 NEH HAVEN

FG02-91ER40608 12/31/94 $094760-0001-0$ HEW HAVEN

FG02-91ER40609 12/31/96 094960-0001-0 HEH HAVEN

FG02-91ER40650 08/31/94 094960-1101-2 NEH HAVEN

FG02-91ER20038 07/14/95 094960-1401-1 HEW HAVEN

FG02-92ER25121 04/30/95 $094960-0001-0$ NEH HAVEN

FG02-92ER14279 07/31/95 $094960-0001-0$ NEH HAVEN
FG02-94ER40824 01/14/97 094960-0001-0 NEW HAVEN
MOLECULAR CLONING AHD STRUCTURAL CHARACTERIZATION OF THE $C T$ CI 03 \$795.340 $\$ 696,520$

THEORETICAL AND HUMERICAL STUDIES IN MAGNETIC MIRROR FUSION CT 03 S $\$ 390,000 \quad \$ 390,000$

THE DETERMINATION OF 222RN FLUX FROM SOILS BASED ON $210 \mathrm{OB}$

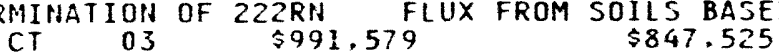

THE FIRST STEP OF CHLOROPHYLL SYNTHESIS: RHA IHVOLVEMENT

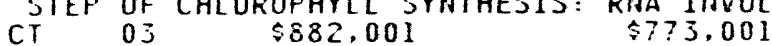

A SPECTROSCOPIC AND CATALYTIC INUESTIGATIOH OF ACTIVE PHASE CI 0 S $\$ 990.841$ \$748.841

ORGAHIZATION AND CONTROL OF GENES ENCODING CATABOLIC CT 03 \$487.866 \$487,860

COMPUTATIONAL AND EXPERIMENTALSTUDY OF LAMIMAR PREMIXED AHD

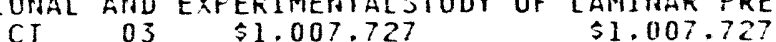

SEARCH FOR STRANGE QUARK MATTER AND AHTIMATIER PRODUCED CT $03 \quad \$ 625,000 \quad \$ 625,000$

REACTIVE FLUID FLOH MODELS AHDAPPLICATIOHS TO DIAGENESIS. CT 03 \$1, 063.424 \$6 39,051

THEORETICAL NUCLEAR PHYSICS

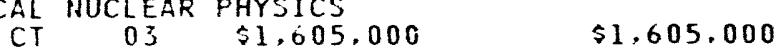

EXPERMEHTAL STUDIES

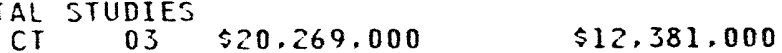

SSC PROPOSAL FOR STUDY OF PRE- SHOWER \& SHOWER MAXIMUM DETECT CT $03 \quad \$ 213.000 \quad \$ 213.000$

SPATIAL REGULATION OF CH GEHESIN C3 C4 AHD C3/C4 CI 003 OF CH GENESIN C3 C4 AND $\$ 392,000$

RESEARCH IN GEOMEIRY, SYMMETRY \& PHYSICS $\$ 125,000 \quad \$ 125,000$

CATALYIIC OXIDATION OF HYDROCARBONS BY BINUCLEAR $C T$ T 03 \$333.186 $\$ 219,159$

MEDIUM ENERGY ELEMENTARY PARTICLE MUONIUM CT 03 \$777,000 $\$ 311,000$ 
PROCUREMENT DEPARTMENT OF ENERGY NAMES BY NAME. CITY \& STATE - ACTIVE AWARDS

PAGE NO. 553

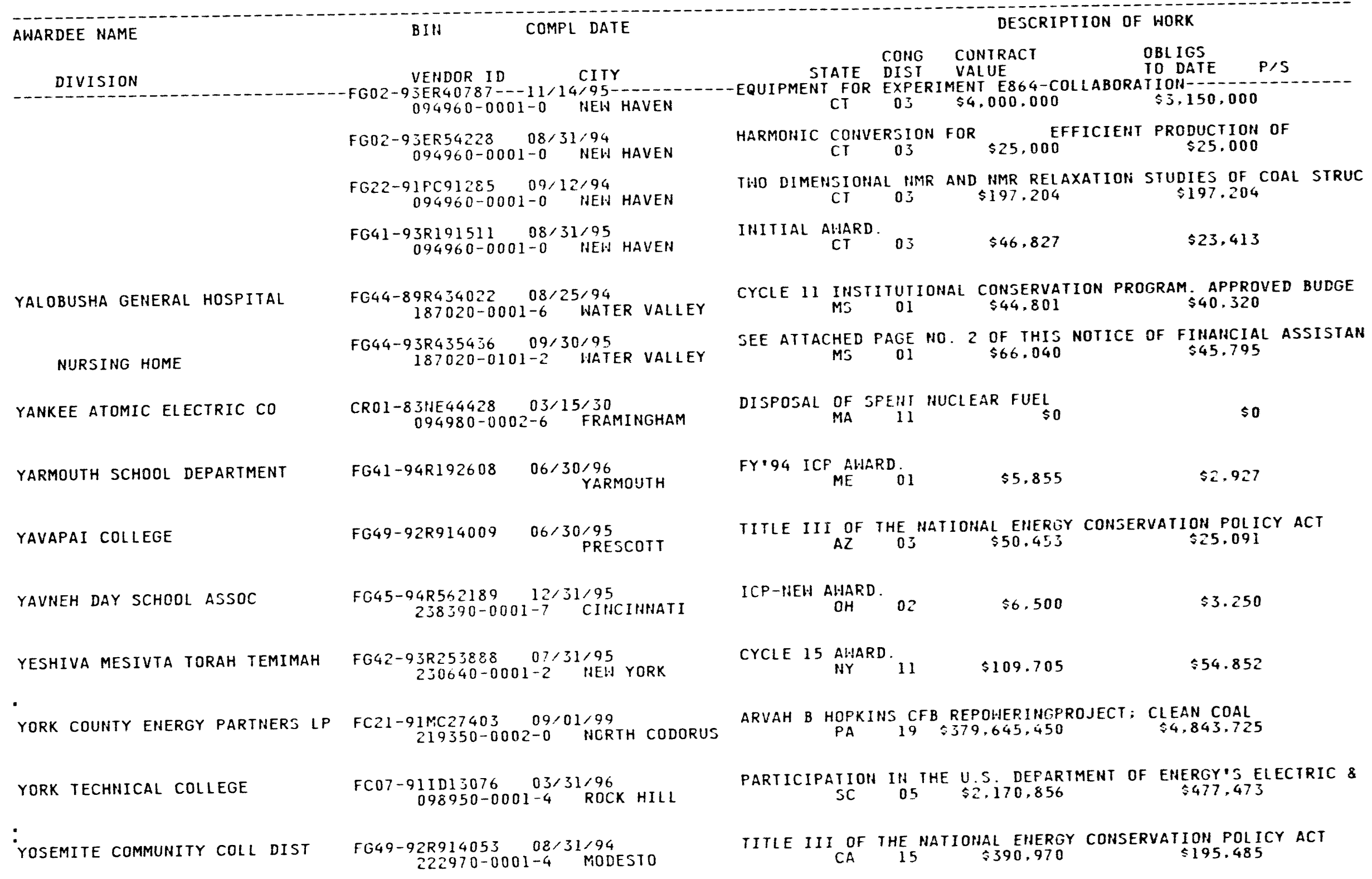




\begin{tabular}{|c|c|c|c|c|c|c|c|}
\hline AHARDEE NAME & BIN & $L$ DATE & & & DEJC & F HORK & \\
\hline DIVISION & VENDOR ID & CITY & STATE & $\begin{array}{l}\text { CCNG } \\
\text { DIST }\end{array}$ & $\begin{array}{l}\text { CONTRACT } \\
\text { VALUE }\end{array}$ & $\begin{array}{l}\text { OBLIGS } \\
\text { TO DATE }\end{array}$ & $P / S$ \\
\hline
\end{tabular}

YOUNG'S MANUFACTURING INC

YOUTH ENERGY CORP FOR

YUMA WORC CENTER

ZIMBABWE GOVERNMENT OF

ZIMMER GUNSUL FRASCA

ZION LUTHERAN CHURCH

ZUHI PUBLIC SCHOOL DISTRICT

351 SURGICAL HOSPITAL

$$
\begin{gathered}
\text { AM65-93HA10710 07/14/94 } \\
217150-0001-3 \text { MOHTROSE }
\end{gathered}
$$

FG49-92R900016 09/30/94 $224550-0001-X$ NEW YORK

$A C 65-93 W G 18628002 / 28 / 95$
$227930-0001-9$ YUMA

FC02-93P010098 09/29/95 $234400-0001-x$ HARARE

AC06-91RL11944 12/30/96 $1 R L 1194412 / 30 / 96$
$205930-0001-3 \quad$ PORTLAHD

FG45-94R562007 06/30/95 238090-0001-9 DECATUR

GM04-92AL58237 04/17/97 $095520-0002-5$ GALLLP

GM04-93AL $84030 \quad 01 / 04 / 98$ $227000-0001-5$ ALBUQUERQUE
PURCHAJE GALV LATTICE STEEL TLINE TOHERS

$50 \quad 5445$

TECHNICAL ADVICE TO COMMUNITY BASED ORG IN ENERGY CONSERVATI

$$
\text { NY } 18 \quad \$ 35,000 \quad \$ 35,000
$$

LAWN/GROUNDS MAINT FOR GILA SUBST

$$
A Z{ }_{02} \quad \$ 51.660 \quad \$ 51.660 \quad 5208
$$

Climate CHANGE COUNTY STUDY

00 \$352.442

$\$ 329,442$

ACQUIRE OFFSITE A/E SERVICES FOR EMSL PHASE I

$$
\begin{array}{lllll}
\text { OFFSITE A'E SERVICES FOR EMSL PHASE I } & \\
\text { OR } 01 & \$ 7.861,791 & \$ 7.861,791 & 0118
\end{array}
$$

ICP - NEH AHARD.
If 04
$\$ 2,200$
$\$ 1,100$

SUPPORT FOR SCIENCE, MATHEMATICS. AND ENGINEERING TO IMPROVE INM 03 , 30 . AND ENGINEERING $\$ 0$

THIS AGREEMENT SETS FORTH THE GENERAL POLICY FOR THE CTA 

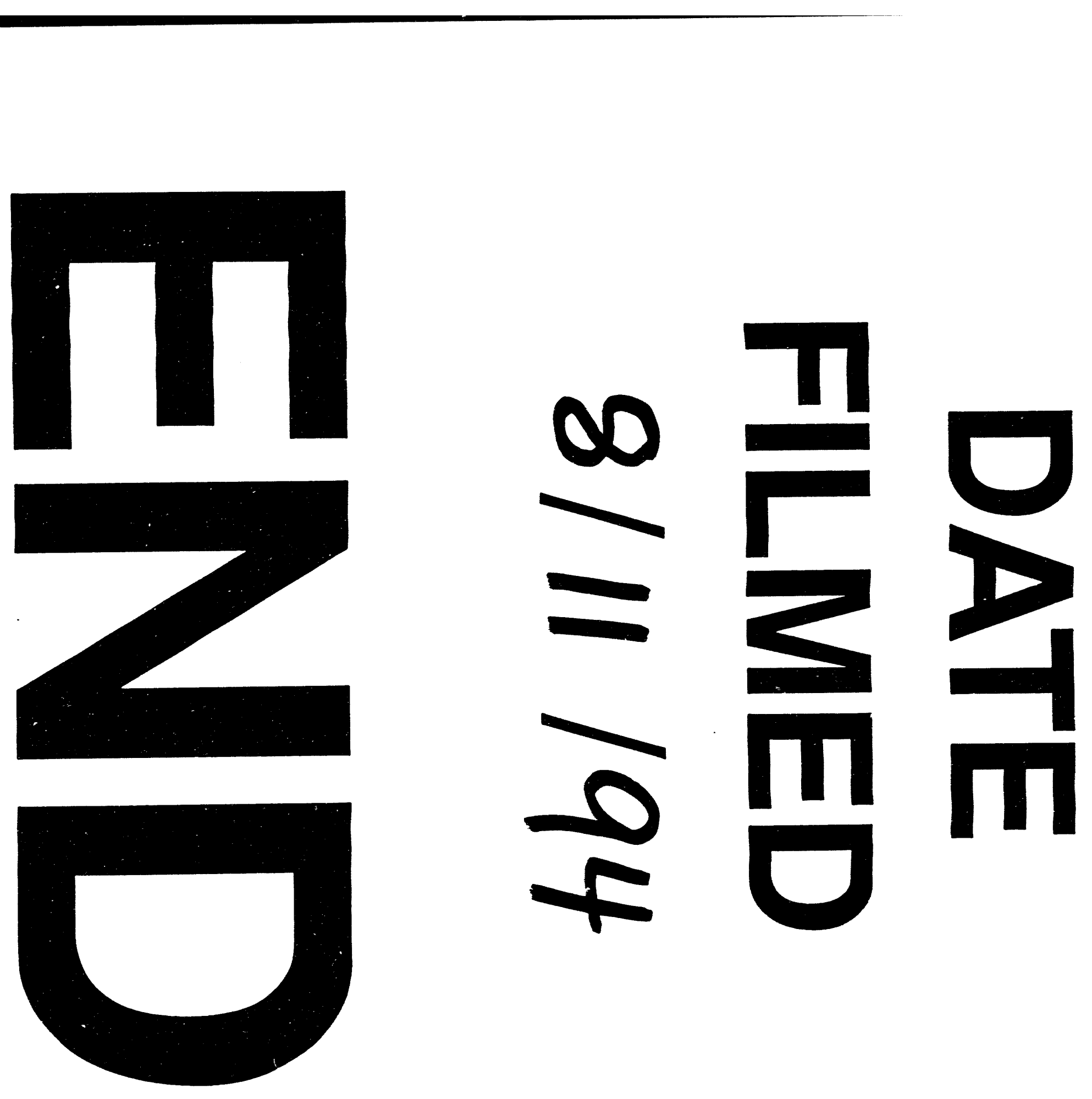